\title{
Evaluation of Test Specimen Geometry of Asphalt Mixes Tested with the Asphalt Mixture Performance Tester
}

\author{
Abha Dwivedy \\ West Virginia University, abdwivedy@mix.wvu.edu
}

Follow this and additional works at: https://researchrepository.wvu.edu/etd

Part of the Civil Engineering Commons, and the Transportation Engineering Commons

\section{Recommended Citation}

Dwivedy, Abha, "Evaluation of Test Specimen Geometry of Asphalt Mixes Tested with the Asphalt Mixture Performance Tester" (2020). Graduate Theses, Dissertations, and Problem Reports. 7685.

https://researchrepository.wvu.edu/etd/7685

This Dissertation is protected by copyright and/or related rights. It has been brought to you by the The Research Repository @ WVU with permission from the rights-holder(s). You are free to use this Dissertation in any way that is permitted by the copyright and related rights legislation that applies to your use. For other uses you must obtain permission from the rights-holder(s) directly, unless additional rights are indicated by a Creative Commons license in the record and/ or on the work itself. This Dissertation has been accepted for inclusion in WVU Graduate Theses, Dissertations, and Problem Reports collection by an authorized administrator of The Research Repository @ WVU.

For more information, please contact researchrepository@mail.wvu.edu. 
Evaluation of Test Specimen Geometry of Asphalt Mixes Tested with the Asphalt Mixture Performance Tester

Abha Dwivedy

Follow this and additional works at: https://researchrepository.wvu.edu/etd

Part of the Civil Engineering Commons, and the Transportation Engineering Commons 


\title{
Evaluation of Test Specimen Geometry of Asphalt Mixes Tested with the Asphalt Mixture Performance Tester
}

\author{
Abha Dwivedy \\ Dissertation submitted \\ to the Benjamin M. Statler College of Engineering and Mineral Resources \\ at West Virginia University \\ in partial fulfillment of the requirements for the degree of \\ Doctor of Philosophy in
}

Civil and Environmental Engineering

John Zaniewski, Ph.D., Chair

John Quaranta, Ph.D.

Fei Dai, Ph.D.

Yoojung Yoon, Ph.D.

Kashy Aminian, Ph.D

Department of Civil and Environmental Engineering

Morgantown, West Virginia

2020

Keywords: AMPT, Sample Dimensions, Air Void Uniformity, Inscribed Circles, FlexPAVE

Copyright 2020 Abha Dwivedy 


\section{Abstract \\ Evaluation of Test Specimen Geometry of Asphalt Mixes Tested with the Asphalt Mixture Performance Tester}

Abha Dwivedy

Asphalt concrete, AC, is a heterogeneous material that is modeled as a homogeneous material. The disparity between material structure and theory presents issue with performance testing with test sample geometries, resulting in variability. To accommodate this issue, the concept of Representative Volume Element, RVE, was developed. RVE is a term that expresses a concept that can be implemented through testing sample dimensions such as aspect ratios, diameter to aggregate size ratio, and gauge length. The laboratory test exhibit variabilities and therefore specimen size should be large enough to enable results representative of AC mix. As RVE of asphalt mix depends on aggregate size, shape, and orientation, it should be unique for each mix due to aggregate effect within the particular AC mix. The purpose of this research is to develop appropriate sample geometry for dynamic modulus, fatigue, and rutting test for accurate characterization of asphalt mix and ease of testing of three different NMAS mixes.

Four sample geometries were fabricated for three mix types. All specimens are fabricated at $7 \% \pm 0.5 \%$ air voids and tested for dynamic modulus, fatigue, and rutting in Asphalt Mixture Performance Tester, AMPT. The effect of sample geometry was statistically analyzed using laboratory test results and performance predictions using FlexPAVE. For ranking analysis all Sample Types, were compared to the full size AASHTO standard dimension used for up to $37.5 \mathrm{~mm}$ NMAS mixes for dynamic modulus and rutting test and for up to $25 \mathrm{~mm}$ NMAS mixes for fatigue test. Samples were also tested for air void uniformity using AASHTO R 83-17 for the three NMAS mixes. The lateral air void distribution from six different inscribed circles are also statistically analyzed. The MANOVA showed there is a statistical difference for different temperatures and frequencies in dynamic modulus test. This confirms that specific sample types be selected based on the mix NMAS for dynamic modulus test. More variability in air void distribution is observed axially than radially. The ST6, one 75X110 mm from one SGC pill, for $12.5 \mathrm{~mm}$ and $25 \mathrm{~mm}$ and ST4, three 50X110 $\mathrm{mm}$ from one SGC pill, for $19 \mathrm{~mm}$ NMAS mixes have uniform air void distribution from statistical analysis and performed comparable to ST1, one 100X150 mm from one SGC pill, from ranking analysis. These results provide detailed insight on correct sample dimension testing for performance prediction of $12.5 \mathrm{~mm}, 19$ $\mathrm{mm}$, and $25 \mathrm{~mm}$ NMAS asphalt mixes. 


\section{ACKNOWLEDGEMENTS}

I would like to take this opportunity to thank Dr. John Zaniewski for presenting me with an opportunity to pursue my $\mathrm{PhD}$. under his guidance. His constant support and advising has helped me achieve my aspirations. Dr. Zaniewski's faith in me and the hard work I put into this research has helped me accomplish my goals. In these few years of knowing and working for Dr. Zaniewski, I have learnt immensely, and I am so thankful for my growth professionally. I would also like to thank Dr. Quaranta, Dr. Dai, Dr. Yoon, and Dr. Aminian for being in my committee and providing their support and guidance.

I would like to extend my gratitude to WVDOT for providing me with the equipment and resources in the WVU lab. I would also like to also thank all the participating asphalt mix plants.

My education and hard work would not be possible without the endless love and support of my parents and my husband. Lastly, I would like to thank my fellow graduate students for all their insights and assistance in making this accomplishment. 


\section{Table of Contents}

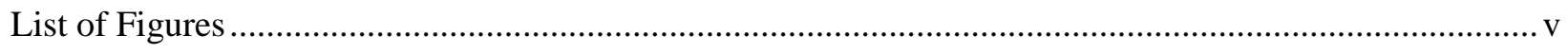

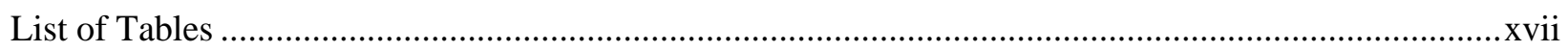

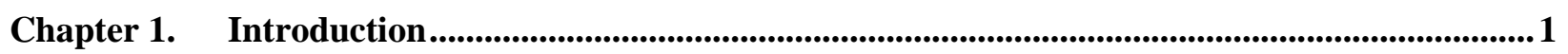

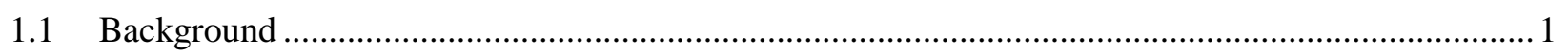

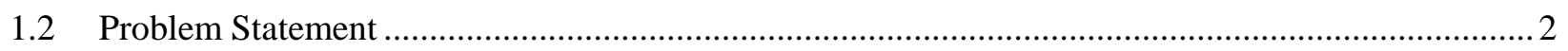

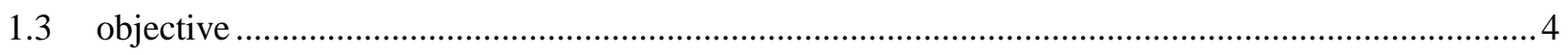

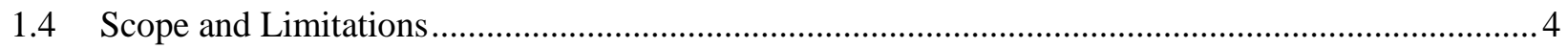

Chapter 2. Literature Review ...................................................................................................................5

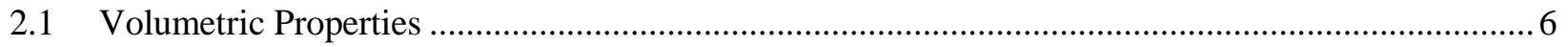

2.2 Asphalt mixture performance tester test methods .................................................................. 7

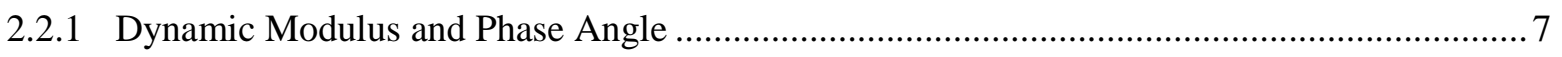

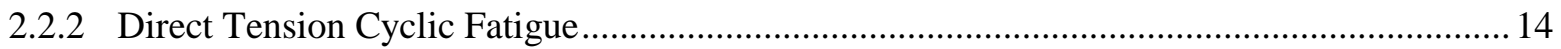

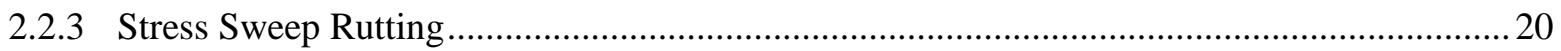

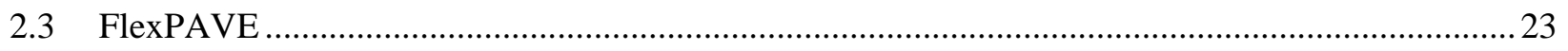

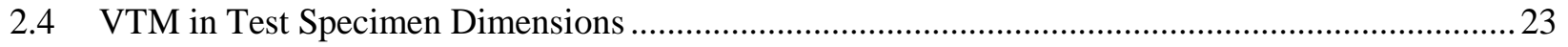

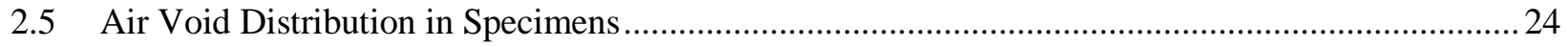

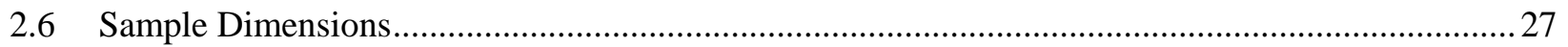

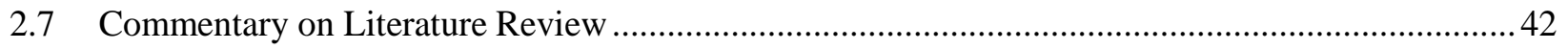

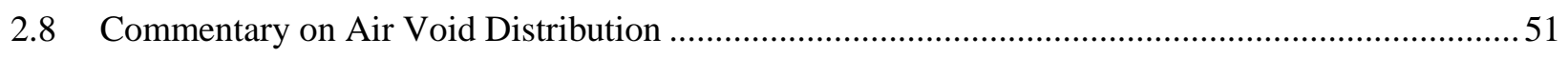

Chapter 3. Methodology …....................................................................................................................................5 53

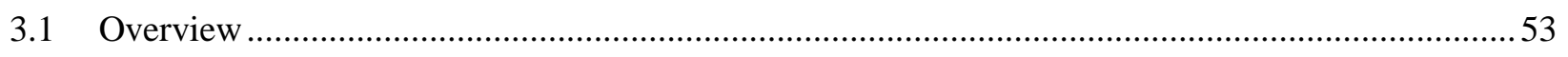

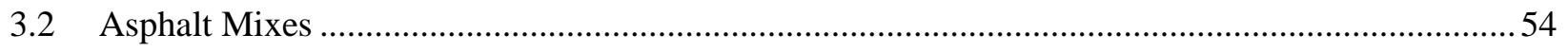

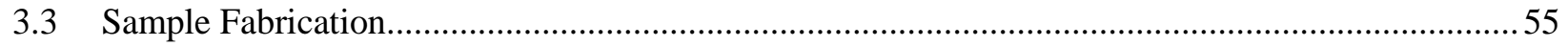

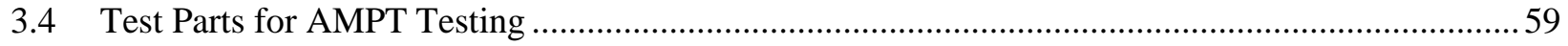

3.5 Fabrication of 50X110 mm and 75X110 mm Custom Parts .....................................................61

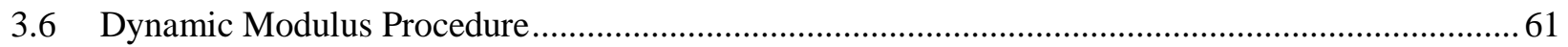

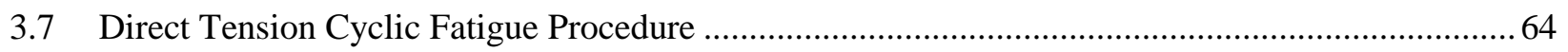

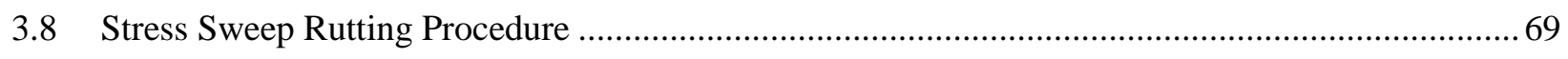

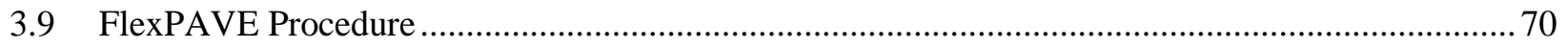




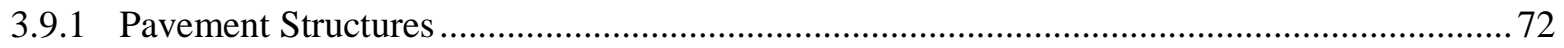

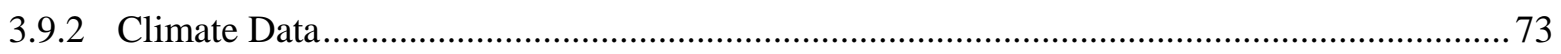

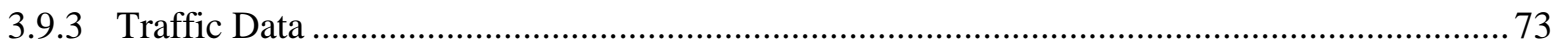

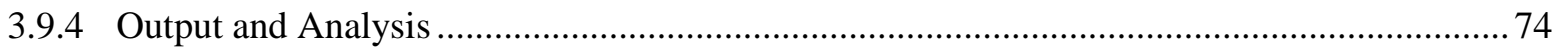

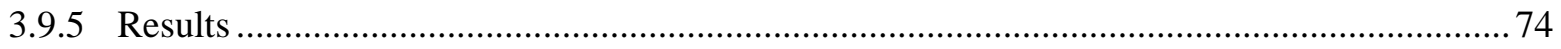

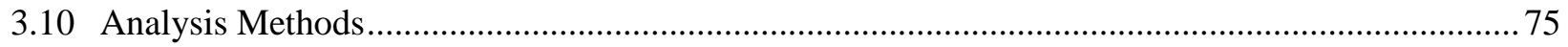

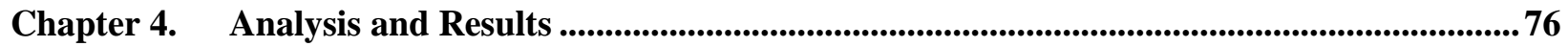

4.1 Ranking Analysis from Master Curve and FlexMAT Software ..................................................... 76

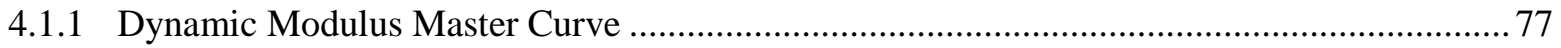

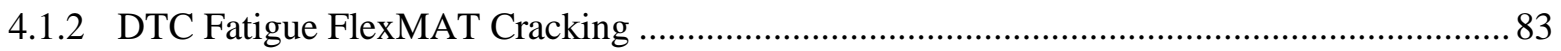

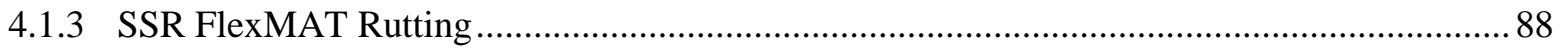

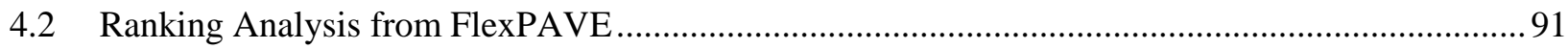

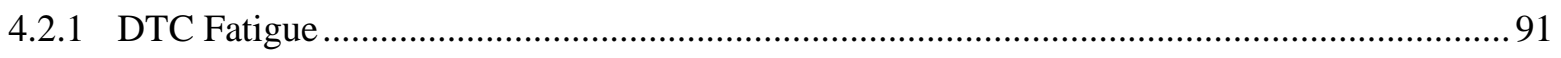

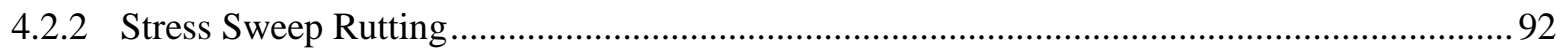

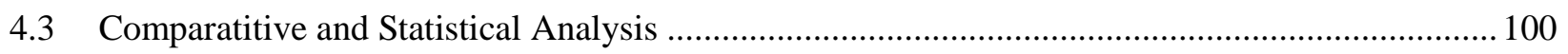

4.4 Within Sample Air Void Variability Analysis ........................................................................ 141

4.5 Within Inscribed Circles Air Void Variability Analysis ........................................................... 148

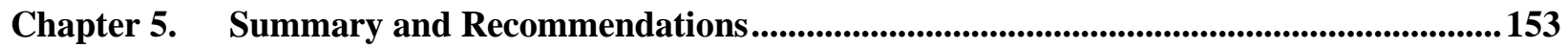

References................................................................................................................................................................................... 156

Test Methods Used........................................................................................................................................................ 160

Appendix A - General AMPT Test Setup …...................................................................................................... 162

Appendix B -Design Mix Sheet...................................................................................................................... 163

Appendix C -Mix Verification....................................................................................................................... 166

Appendix D Custom Parts Fabricated for Research ............................................................................... 168

Appendix E Test Procedure Steps .................................................................................................................. 182

Appendix F FlexPAVE Models Inputs.................................................................................................... 185

\section{List of Figures}

Figure 1: Asphalt Concrete Master Curve (Lee, 2013). 10

Figure 2: Relationship between Sum (1-C) and $\mathrm{N}_{\mathrm{f}}$ (Wang and Kim., 2019) 17 
Figure 3: Permanent Deformation of Asphalt Concrete versus Number of Load Cycles (Chilukwa and Lungu, 2019)

Figure 4: Bulk VTM as Cumulative Air Void Distribution (Bowers et al., 2015)

Figure 5: Compaction of HMA specimen resulting in "Cone Shape" (Thyagarajan et al., 2010)

Figure 6: $38 \mathrm{~mm}$ diameter specimens cored from $150 \mathrm{~mm}$ diameter specimen (Li and Gibson, 2012)

Figure 7: Sample Fabrication Process: a) $150 \mathrm{~mm}$ Field Core, b) Sublayer Thickness in the Core, c)

Horizontal Coring to Obtain Prismatic and Cylindrical small specimens, d) Prismatic and Small

Performance Test Specimens(Park and Kim, 2013)

Figure 8: $38 \mathrm{~mm}$ and $50 \mathrm{~mm}$ diameter specimens cored from SGC pill (Bowers et al., 2015) 36

Figure 9: Fabrication of Test Specimen (Lee el al., 2017b) 40

Figure 10: New Prismatic Specimen Proposed for DTC Fatigue Test (Karr, 2018) 41

Figure 11: Tapered Prismatic Test Specimen on DTC Fatigue Platen (Karr, 2018) 41

Figure 12: Inscribed Circle Diameter of $100 \mathrm{~mm}$ from $150 \mathrm{~mm}$ Diameter of SGC pill (AASHTO PP-19)

Figure 13: Flow Diagram of Unique Sample Number Preparation $\quad 57$

Figure 14: Gauge Point Fixing Jig 60

Figure 15: End Platen Gluing Jig a) 100X130 mm specimens b) 38X110 mm, 50X110 mm, and 75X110 mm specimens $\quad 60$

Figure 16: Dynamic Modulus Test Setup a) 100X150 mm Specimen b) 38X110 mm 62

Figure 17: Flow Diagram of Dynamic Modulus AMPT Data Uploaded into FlexMAT Cracking 63

Figure 18: DTC Fatigue Test Setup a) 100X130 mm Specimen b) 75X110 mm 65

Figure 19: Flow Diagram of DTC Fatigue AMPT Data Uploaded into FlexMAT Cracking 67

Figure 20: SSR Test Setup for 100X150 mm Specimen $\quad 69$

Figure 21: Flow Diagram of SSR AMPT Data Uploaded into FlexMAT Rutting 70

Figure 22: Flow Diagram of FlexPAVE

Figure 23: Output and Analysis Option in Performance Analysis of FlexPAVE 74

Figure 24: Master curves Design Binder Content, NAMS 12.5 mm, Target 7.0 \% VTM 78

Figure 25: Master curves Design Binder Content, NAMS 19 mm, Target 7.0\% VTM 78

Figure 26: Master curves Design Binder Content, NAMS 25 mm, Target 7.0 \% VTM 79

Figure 27: Comparison of Dynamic modulus Values for Rutting Considerations at $54.4^{\circ} \mathrm{C}$ and $5 \mathrm{~Hz} \quad 81$

Figure 28: Comparison of Dynamic modulus Values Fatigue Considerations at $20^{\circ} \mathrm{C}$ and $73.2 \mathrm{~Hz} \quad 82$

Figure 29: Fatigue Potential from $S_{\text {app }}$ Ranking at $21^{\circ} \mathrm{C}$ for Different Sample Types 84

Figure 30: Damage Characterization Curve Comparison of Samples from Different Sample Types to

Samples Obtained from Sample Type 1. 85 
Figure 31: RSI Ranking for 20 Years of Traffic for Different Sample Types 89

Figure 32: Average Permanent Microstrain, 12.5 mm NMAS 89

Figure 33: Average Permanent Microstrain, 19 mm NMAS 90

Figure 34: Average Permanent Microstrain, 25 mm NMAS 90

Figure 35: Comparison of Total Rut Depth at the end of 20 Years between different Models and

$\begin{array}{ll}\text { Structures } & 93\end{array}$

Figure 36: Total Rut Depth Comparison for different Models to Model 1 for each of the Three Structures

Figure 37: Comparison of Dynamic Modulus for STs fabricated with 12.5 mm NMAS 104

Figure 38: Comparison of Phase Angle for STs fabricated with 12.5 mm NMAS 106

Figure 39: Comparison of Dynamic Modulus for STs fabricated with 19 mm NMAS 108

Figure 40: Comparison of Phase Angle for STs fabricated with 19 mm NMAS 110

Figure 41: Comparison of Dynamic Modulus for STs fabricated with 25 mm NMAS 112

Figure 42: Comparison of Phase Angle for STs fabricated with 25 mm NMAS 114

Figure 43: Comparison of Dynamic modulus and phase angle for ST 1 fabricated from different NMAS

Figure 44: Comparison of Dynamic modulus and phase angle for ST 2 fabricated from different NMAS

Figure 45: Comparison of Dynamic modulus and phase angle for ST 3 fabricated from different NMAS

Figure 46: Comparison of Dynamic modulus and phase angle for ST 4 fabricated from different NMAS

Figure 47: Comparison of Dynamic modulus and phase angle for ST 5 fabricated from different NMAS

Figure 48: Comparison of Dynamic modulus and phase angle for ST 6 fabricated from different NMAS

Figure 49: General Schematic of AMPT Test Setup 162

Figure 50: Dynamic Modulus and SSR Test Bottom Platen Cross Section for 50X110 mm Specimen 170

Figure 51: Dynamic Modulus and SSR Test Bottom Platen Top View for 50X110 mm Specimen 170

Figure 52: Dynamic Modulus and SSR Test Stem Cross Section for 50X110 mm Specimen $\quad 170$

Figure 53: Dynamic Modulus and SSR Test Top Platen Cross Section for 50X110 mm Specimen 171

Figure 54: Dynamic Modulus and SSR Test Top Platen Top View for 50X110 mm Specimen 171

Figure 55: Dynamic Modulus and SSR Test Setup for 50X110 mm Specimen 171

Figure 56: Dynamic Modulus Test Bottom Platen Cross Section for 75X110 mm Specimen $\quad 171$ 
Figure 57: Dynamic Modulus Test Bottom Platen Top View for 75X110 mm Specimen

Figure 58: Dynamic Modulus Test Stem Cross Section for 75X110 mm Specimen

Figure 59: Dynamic Modulus Test Top Platen Cross Section for 75X110 mm Specimen

Figure 60: Dynamic Modulus Test Top Platen Top View for 75X110 mm Specimen

Figure 61: Dynamic Modulus Test Setup for 75X110 mm Specimen

Figure 62: Dynamic Modulus Test Setup Comparison of Four Different Specimen Geometries

Figure 63: SSR Test Setup Comparison for of Different Specimen Geometries

Figure 64: Gauge point gluing Jig spacer cross section for 50X110 mm Specimen

Figure 65: Gauge point gluing Jig spacer cross section for 75X110 mm Specimen

Figure 66: Top Fatigue End Platen Gluing Adapter Cross Section for 75X110 mm Specimen

Figure 67: Gauge point gluing Jig Comparison of Four Different Specimen Geometries

Figure 68: Top Fatigue End Platen Gluing Adapter Top View for 75X110 mm Specimen

Figure 69: Bottom Fatigue End Platen Gluing Adapter Cross Section for 75X110 mm Specimen

Figure 70: Bottom Fatigue End Platen Gluing Adapter Top View for 75X110 mm Specimen

Figure 71: DTC Fatigue Top and Bottom Platens Cross-Section for 50X110 mm Specimen

Figure 72: DTC Fatigue Top and Bottom Platens Top View for 50X110 mm Specimen

Figure 73: DTC Fatigue Top and Bottom Platens Cross-Section for 75X110 mm Specimen

Figure 74: DTC Fatigue Top and Bottom Platens Top View for 75X110 mm Specimen

Figure 75: DTC Fatigue Top and Bottom Spacer Cross Section for 50X110 mm and 75X110 mm

Figure 76: DTC Fatigue Top and Bottom Spacer Top View for 50X110 mm and 75X110 mm Specimen

Figure 77: DTC Fatigue End Platens Gluing Jig and Test Set Up for 100X130 mm Specimen 
Figure 89: General Information for Run 1.2 190

Figure 90: Design Structure of AC Layer for Run 1.2 190

Figure 91: Design Structure of Base 1 Layer for Run 1.2 191

Figure 92: Design Structure of Base 2 Layer for Run 1.2 191

Figure 93: Design Structure of Subgrade Layer for Run 1.2 192

Figure 94: Climate Data for Run 1.2 192

Figure 95: Traffic Data for Run 1.2 193

Figure 96: Output and Analysis Options for Run 1.2

Figure 97: General Information for Run 1.3

Figure 98: Design Structure of AC Layer for Run 1.3 195

Figure 99: Design Structure of Base 1 Layer for Run 1.3 195

Figure 100: Design Structure of Base 2 Layer for Run 1.3 196

Figure 101: Design Structure of Subgrade Layer for Run 1.3 196

Figure 102: Climate Data for Run 1.3

Figure 103: Traffic Data for Run $1.3 \quad 198$

Figure 104: Output and Analysis Options for Run 1.3

Figure 105: General Information for Run $1.4 \quad 199$

Figure 106: Design Structure of AC Layer for Run 1.4

Figure 107: Design Structure of Base 1 Layer for Run 1.4 200

Figure 108: Design Structure of Base 2 Layer for Run 1.4 201

Figure 109: Design Structure of Subgrade Layer for Run $1.4 \quad 201$

Figure 110: Climate Data for Run 1.4 202

Figure 111: Traffic Data for Run $1.4 \quad 203$

Figure 112: Output and Analysis Options for Run 1.4

Figure 113: General Information for Run 1.5 204

Figure 114: Design Structure of AC Layer for Run 1.5 205

Figure 115: Design Structure of Base 1 Layer for Run 1.5 205

Figure 116: Design Structure of Base 2 Layer for Run 1.5 206

Figure 117: Design Structure of Subgrade Layer for Run 1.5

Figure 118: Climate Data for Run 1.5 207

Figure 119: Traffic Data for Run 1.5 208

Figure 120: Output and Analysis Options for Run 1.5

Figure 121: General Information for Run 1.6 209

Figure 122: Design Structure of AC Layer for Run 1.6 
Figure 123: Design Structure of Base 1 Layer for Run 1.6

Figure 124: Design Structure of Base 2 Layer for Run 1.6

Figure 125: Design Structure of Subgrade Layer for Run 1.6

Figure 126: Climate Data for Run 1.6

Figure 127: Traffic Data for Run 1.6

Figure 128: Output and Analysis Options for Run 1.6

Figure 129: General Information for Run 1.7

Figure 130: Design Structure of AC Layer for Run 1.7

Figure 131: Design Structure of Base 1 Layer for Run 1.7

Figure 132: Design Structure of Base 2 Layer for Run 1.7

Figure 133: Design Structure of Subgrade Layer for Run 1.7

Figure 134: Climate Data for Run 1.7

Figure 135: Traffic Data for Run 1.7

Figure 136: Output and Analysis Options for Run 1.7

Figure 137: General Information for Run 1.8

Figure 138: Design Structure of AC Layer for Run 1.8

Figure 139: Design Structure of Base 1 Layer for Run 1.8

Figure 140: Design Structure of Base 2 Layer for Run 1.8

Figure 141: Design Structure of Subgrade Layer for Run 1.8

Figure 142: Climate Data for Run 1.8

Figure 143: Traffic Data for Run 1.8

Figure 144: Output and Analysis Options for Run 1.8

Figure 145: General Information for Run 1.9

Figure 146: Design Structure of AC Layer for Run 1.9

Figure 147: Design Structure of Base 1 Layer for Run 1.9

Figure 148: Design Structure of Base 2 Layer for Run 1.9

Figure 149: Design Structure of Subgrade Layer for Run 1.9

Figure 150: Climate Data for Run 1.9

Figure 151: Traffic Data for Run 1.9

Figure 152: Output and Analysis Options for Run 1.9

Figure 153: General Information for Run 1.10

Figure 154: Design Structure of AC Layer for Run 1.10

Figure 155: Design Structure of Base 1 Layer for Run 1.10 
Figure 157: Design Structure of Subgrade Layer for Run $1.10 \quad 231$

Figure 158: Climate Data for Run 1.10 232

Figure 159: Traffic Data for Run 1.10

Figure 160: Output and Analysis Options for Run 1.10

Figure 161: General Information for Run 1.11 234

Figure 162: Design Structure of AC Layer for Run 1.11

Figure 163: Design Structure of Base 1 Layer for Run 1.11 235

Figure 164: Design Structure of Base 2 Layer for Run 1.11

Figure 165: Design Structure of Subgrade Layer for Run 1.11 236

Figure 166: Climate Data for Run 1.11 237

Figure 167: Traffic Data for Run 1.11 238

Figure 168: Output and Analysis Options for Run 1.11

Figure 169: General Information for Run 1.12 239

Figure 170: Design Structure of AC Layer for Run 1.12

Figure 171: Design Structure of Base 1 Layer for Run 1.12

Figure 172: Design Structure of Base 2 Layer for Run 1.12

Figure 173: Design Structure of Subgrade Layer for Run 1.12

Figure 174: Climate Data for Run 1.12 242

Figure 175: Traffic Data for Run 1.12 243

Figure 176: Output and Analysis Options for Run 1.12

Figure 177: General Information for Run 2.1 245

Figure 178: Design Structure of AC Layer for Run 2.1 245

Figure 179: Design Structure of Base 1 Layer for Run 2.1

Figure 180: Design Structure of Base 2 Layer for Run 2.1 246

Figure 181: Design Structure of Subgrade Layer for Run 2.1 247

Figure 182: Climate Data for Run 2.1 247

Figure 183: Traffic Data for Run $2.1 \quad 248$

Figure 184: Output and Analysis Options for Run 2.1

Figure 185: General Information for Run 2.2

Figure 186: Design Structure of AC Layer for Run 2.2

Figure 187: Design Structure of Base 1 Layer for Run 2.2

Figure 188: Design Structure of Base 2 Layer for Run $2.2 \quad 251$

Figure 189: Design Structure of Subgrade Layer for Run $2.2 \quad 251$

Figure 190: Climate Data for Run 2.2 
Figure 191: Traffic Data for Run 2.2

Figure 192: Output and Analysis Options for Run 2.2 254

Figure 193: General Information for Run 2.3 254

Figure 194: Design Structure of AC Layer for Run 2.3

Figure 195: Design Structure of Base 1 Layer for Run 2.3

Figure 196: Design Structure of Base 2 Layer for Run $2.3 \quad 256$

Figure 197: Design Structure of Subgrade Layer for Run 2.3

Figure 198: Climate Data for Run 2.3 257

Figure 199: Traffic Data for Run $2.3 \quad 258$

Figure 200: Output and Analysis Options for Run 2.3 259

Figure 201: General Information for Run 2.4

Figure 202: Design Structure of AC Layer for Run 2.4

Figure 203: Design Structure of Base 1 Layer for Run 2.4 260

Figure 204: Design Structure of Base 2 Layer for Run 2.4 261

Figure 205: Design Structure of Subgrade Layer for Run 2.4

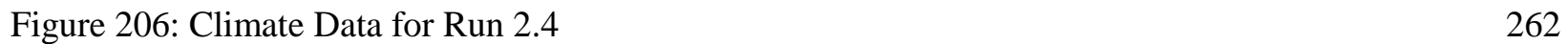

Figure 207: Traffic Data for Run 2.4

Figure 208: Output and Analysis Options for Run 2.4

Figure 209: General Information for Run 2.5 264

Figure 210: Design Structure of AC Layer for Run 2.5 265

Figure 211: Design Structure of Base 1 Layer for Run 2.5 265

Figure 212: Design Structure of Base 2 Layer for Run 2.5 266

Figure 213: Design Structure of Subgrade Layer for Run 2.5

Figure 214: Climate Data for Run 2.5 267

Figure 215: Traffic Data for Run 2.5 268

Figure 216: Output and Analysis Options for Run 2.5 269

Figure 217: General Information for Run 2.6 269

Figure 218: Design Structure of AC Layer for Run $2.6 \quad 270$

Figure 219: Design Structure of Base 1 Layer for Run $2.6 \quad 270$

Figure 220: Design Structure of Base 2 Layer for Run 2.6 271

Figure 221: Design Structure of Subgrade Layer for Run $2.6 \quad 271$

Figure 222: Climate Data for Run 2.6 272

Figure 223: Traffic Data for Run 2.6 273

Figure 224: Output and Analysis Options for Run 2.6 
Figure 225: General Information for Run 2.7 274

Figure 226: Design Structure of AC Layer for Run 2.7 275

Figure 227: Design Structure of Base 1 Layer for Run 2.7

Figure 228: Design Structure of Base 2 Layer for Run 2.7 276

Figure 229: Design Structure of Subgrade Layer for Run 2.7

Figure 230: Climate Data for Run 2.7 277

Figure 231: Traffic Data for Run 2.7 278

Figure 232: Output and Analysis Options for Run $2.7 \quad 279$

Figure 233: General Information for Run 2.8 279

Figure 234: Design Structure of AC Layer for Run $2.8 \quad 280$

Figure 235: Design Structure of Base 1 Layer for Run 2.8 280

Figure 236: Design Structure of Base 2 Layer for Run $2.8 \quad 281$

Figure 237: Design Structure of Subgrade Layer for Run $2.8 \quad 281$

Figure 238: Climate Data for Run 2.8

Figure 239: Traffic Data for Run 2.8

Figure 240: Output and Analysis Options for Run 2.8

Figure 241: General Information for Run 2.9 284

Figure 242: Design Structure of AC Layer for Run 2.9

Figure 243: Design Structure of Base 1 Layer for Run 2.9

Figure 244: Design Structure of Base 2 Layer for Run 2.9

Figure 245: Design Structure of Subgrade Layer for Run 2.9

Figure 246: Climate Data for Run 2.9

Figure 247: Traffic Data for Run $2.9 \quad 288$

Figure 248: Output and Analysis Options for Run 2.9 289

Figure 249: General Information for Run 2.10 289

Figure 250: Design Structure of AC Layer for Run 2.10 290

Figure 251: Design Structure of Base 1 Layer for Run 2.10 290

Figure 252: Design Structure of Base 2 Layer for Run 2.10 291

Figure 253: Design Structure of Subgrade Layer for Run 2.10 291

Figure 254: Climate Data for Run 2.10 292

Figure 255: Traffic Data for Run 2.10 293

Figure 256: Output and Analysis Options for Run 2.10

Figure 257: General Information for Run 2.11 294

Figure 258: Design Structure of AC Layer for Run 2.11 
Figure 259: Design Structure of Base 1 Layer for Run 2.11

Figure 260: Design Structure of Base 2 Layer for Run 2.11

Figure 261: Design Structure of Subgrade Layer for Run 2.11

Figure 262: Climate Data for Run 2.11

Figure 263: Traffic Data for Run 2.11

Figure 264: Output and Analysis Options for Run 2.11

Figure 265: General Information for Run 2.12

Figure 266: Design Structure of AC Layer for Run 2.12

Figure 267: Design Structure of Base 1 Layer for Run 2.12

Figure 268: Design Structure of Base 2 Layer for Run 2.12

Figure 269: Design Structure of Subgrade Layer for Run 2.12

Figure 270: Climate Data for Run 2.12

Figure 271: Traffic Data for Run 2.12

Figure 272: Output and Analysis Options for Run 2.12

Figure 273: General Information for Run 3.1

Figure 274: Design Structure of AC Layer for Run 3.1

Figure 275: Design Structure of Base 1 Layer for Run 3.1

Figure 276: Design Structure of Base 2 Layer for Run 3.1

Figure 277: Design Structure of Subgrade Layer for Run 3.1

Figure 278: Climate Data for Run 3.1

Figure 279: Traffic Data for Run 3.1

Figure 280: Output and Analysis Options for Run 3.1

Figure 281: General Information for Run 3.2

Figure 282: Design Structure of AC Layer for Run 3.2

Figure 283: Design Structure of Base 1 Layer for Run 3.2

Figure 284: Design Structure of Base 2 Layer for Run 3.2

Figure 285: Design Structure of Subgrade Layer for Run 3.2

Figure 286: Climate Data for Run 3.2

Figure 287: Traffic Data for Run 3.2

Figure 288: Output and Analysis Options for Run 3.2

Figure 289: General Information for Run 3.3

Figure 290: Design Structure of AC Layer for Run 3.3

Figure 291: Design Structure of Base 1 Layer for Run 3.3 
Figure 293: Design Structure of Subgrade Layer for Run 3.3

Figure 294: Climate Data for Run 3.3

Figure 295: Traffic Data for Run 3.3 318

Figure 296: Output and Analysis Options for Run 3.3 319

Figure 297: General Information for Run 3.4

Figure 298: Design Structure of AC Layer for Run 3.4

Figure 299: Design Structure of Base 1 Layer for Run 3.4

Figure 300: Design Structure of Base 2 Layer for Run 3.4

Figure 301: Design Structure of Subgrade Layer for Run 3.4

Figure 302: Climate Data for Run 3.4

Figure 303: Traffic Data for Run 3.4

Figure 304: Output and Analysis Options for Run 3.4

Figure 305: General Information for Run 3.5 324

Figure 306: Design Structure of AC Layer for Run 3.5 325

Figure 307: Design Structure of Base 1 Layer for Run 3.5 325

Figure 308: Design Structure of Base 2 Layer for Run 3.5 326

Figure 309: Design Structure of Subgrade Layer for Run 3.5

Figure 310: Climate Data for Run 3.5 327

Figure 311: Traffic Data for Run 3.5 328

Figure 312: Output and Analysis Options for Run 3.5

Figure 313: General Information for Run 3.6 329

Figure 314: Design Structure of AC Layer for Run 3.6

Figure 315: Design Structure of Base 1 Layer for Run 3.6 330

Figure 316: Design Structure of Base 2 Layer for Run 3.6 331

Figure 317: Design Structure of Subgrade Layer for Run 3.6

Figure 318: Climate Data for Run 3.6 332

Figure 319: Traffic Data for Run 3.6 333

Figure 320: Output and Analysis Options for Run 3.6 334

Figure 321: General Information for Run 3.7 334

Figure 322: Design Structure of AC Layer for Run 3.7

Figure 323: Design Structure of Base 1 Layer for Run 3.7

Figure 324: Design Structure of Base 2 Layer for Run 3.7

Figure 325: Design Structure of Subgrade Layer for Run 3.7

Figure 326: Climate Data for Run 3.7 
Figure 327: Traffic Data for Run 3.7

Figure 328: Output and Analysis Options for Run 3.7

Figure 329: General Information for Run 3.8 339

Figure 330: Design Structure of AC Layer for Run $3.8 \quad 340$

Figure 331: Design Structure of Base 1 Layer for Run $3.8 \quad 340$

Figure 332: Design Structure of Base 2 Layer for Run 3.8

Figure 333: Design Structure of Subgrade Layer for Run $3.8 \quad 341$

Figure 334: Climate Data for Run 3.8

Figure 335: Traffic Data for Run 3.8

Figure 336: Output and Analysis Options for Run 3.8

Figure 337: General Information for Run 3.9

Figure 338: Design Structure of AC Layer for Run 3.9

Figure 339: Design Structure of Base 1 Layer for Run 3.9

Figure 340: Design Structure of Base 2 Layer for Run 3.9 346

Figure 341: Design Structure of Subgrade Layer for Run 3.9

Figure 342: Climate Data for Run 3.9

Figure 343: Traffic Data for Run 3.9

Figure 344: Output and Analysis Options for Run 3.9

Figure 345: General Information for Run 3.10 349

Figure 346: Design Structure of AC Layer for Run 3.10 350

Figure 347: Design Structure of Base 1 Layer for Run 3.10

Figure 348: Design Structure of Base 2 Layer for Run 3.10 351

Figure 349: Design Structure of Subgrade Layer for Run 3.10 351

Figure 350: Climate Data for Run 3.10 352

Figure 351: Traffic Data for Run 3.10 353

Figure 352: Output and Analysis Options for Run 3.10 354

Figure 353: General Information for Run 3.11 354

Figure 354: Design Structure of AC Layer for Run 3.11

Figure 355: Design Structure of Base 1 Layer for Run 3.11

Figure 356: Design Structure of Base 2 Layer for Run 3.11

Figure 357: Design Structure of Subgrade Layer for Run 3.11

Figure 358: Climate Data for Run 3.11

Figure 359: Traffic Data for Run 3.11 358

Figure 360: Output and Analysis Options for Run 3.11 
Figure 361: General Information for Run 3.12 359

Figure 362: Design Structure of AC Layer for Run 3.12 360

Figure 363: Design Structure of Base 1 Layer for Run 3.12 360

Figure 364: Design Structure of Base 2 Layer for Run 3.12 361

Figure 365: Design Structure of Subgrade Layer for Run $3.12 \quad 361$

Figure 366: Climate Data for Run 3.12

Figure 367: Traffic Data for Run 3.12 363

Figure 368: Output and Analysis Options for Run 3.12 364

\section{List of Tables}

Table 1: Mix Types Used by WVDOH (MP 401.02.22) 1

Table 2: Sample Dimensions and Maximum NMAS for asphalt mixes used in AMPT Testing per AASHTO Standards

Table 3: Recommended Test Temperatures and Frequencies for 100X150 mm Dynamic Modulus Specimen (AASHTO T 378-17)

Table 4: Recommended Test Temperatures and Frequencies for 38X110 mm Dynamic Modulus Specimen (AASHTO TP 132-19)

Table 5: Data Quality Statistics for 100X150 mm and 38X110 mm Dynamic Modulus Specimen (AASHTO T 378-17 and AASHTO TP 132-19)

Table 6: Example Dynamic Modulus Test Summary (AASHTO T 378-17 and AASHTO TP 132-19) 9

Table 7: SSR Low Test Temperature for PG Grades 20

Table 8: Failure Cycles for 100X150 mm and 38X110 mm in Cyclic Fatigue Test (Li and Gibson, 2012)

Table 9: Summary of Diameter to MAS Ratio on Dynamic Modulus Test from Different Studies in

Literature

Table 10: Summary of Aspect Ratio on Dynamic Modulus Test from Different Studies in the Literature 46

Table 11: Summary of Diameter to MAS Ratio on DTC Fatigue Test from Different Studies in Literature

Table 12: Summary of Aspect Ratio on DTC Fatigue Test from Different Studies in the Literature 50 Table 13: Failure Cycles for 100X150 mm and 38X110 mm in Cyclic Fatigue Test (Li and Gibson, 2012)

Table 14: Summary of Sample Types

Table 15: Aspect Ratio and Diameter to MAS Ratio for AASHTO and Researched Geometries 54

Table 16: Summary of Plant Produced Asphalt Mix Obtained for This Research 54 
Table 17: Summary of Asphalt Mix Verification

Table 18: Multiple Test Specimens Fabricated from Inscribed Diameter within SGC Pill 56

Table 19: Experimental Design Variables and Unique Sample Numbers 58

Table 20: Test Specimens and Extra Specimens obtained from SGC Pill for AMPT testing 59

Table 21: Test Parameters for Dynamic Modulus Test 63

Table 22: Approximate Time to Perform Dynamic Modulus Test in AMPT 64

Table 23: Approximate Time to Perform Cyclic Fatigue Test in AMPT 68

Table 24: Approximate Time to Perform Stress Sweep Rutting in AMPT 70

Table 25: Pavement Thicknesses for Three Pavement Structures used in FlexPAVE Analysis 72

Table 26: Models for Each of the Three Pavement Structures used in FlexPAVE Analysis 73

Table 27: Master curve Fitting Parameters

Table 28: Dynamic Modulus for Rutting Considerations at $54.4^{\circ} \mathrm{C}$ and $5 \mathrm{~Hz}$

Table 29: Dynamic Modulus for Fatigue Considerations at $20^{\circ} \mathrm{C}$ and $5 \mathrm{~Hz}$

Table 30: $\mathrm{S}_{\text {app }}$ Values at $21^{\circ} \mathrm{C}$ for Different Sample Types for Each NMAS asphalt Mix 83

Table 31: RSI Value for 20 Years of Traffic for Different Sample Types for Each NMAS asphalt Mix 88

Table 32: Percentage Damage at the end of 20 Years from FlexPAVE 92

Table 33: Total Rut Depth at the end of 20 Years from FlexPAVE 93

Table 34: Dynamic Modulus and Phase Angle for 12.5 mm NMAS Unique Replicate Samples 101

Table 35: Dynamic Modulus and Phase Angle for 19 mm NMAS Unique Replicate Samples 102

Table 36: Dynamic Modulus and Phase Angle for 25 mm NMAS Unique Replicate Samples 103

Table 37: t-test of Dynamic Modulus for STs fabricated from $12.5 \mathrm{~mm}$ NMAS at $4{ }^{\circ} \mathrm{C} \quad 119$

Table 38: t-test of Phase Angle for STs fabricated from 12.5 mm NMAS at $4^{\circ} \mathrm{C} \quad 120$

Table 39: t-test of Dynamic Modulus for STs fabricated from $12.5 \mathrm{~mm}$ NMAS at $20^{\circ} \mathrm{C} \quad 120$

Table 40: t-test of Phase Angle for STs fabricated from $12.5 \mathrm{~mm}$ NMAS at $20^{\circ} \mathrm{C} \quad 121$

Table 41: t-test of Dynamic Modulus for STs fabricated from $12.5 \mathrm{~mm}$ NMAS at $40^{\circ} \mathrm{C} \quad 121$

Table 42: t-test of Phase Angle for STs fabricated from $12.5 \mathrm{~mm} \mathrm{NMAS}$ at $40^{\circ} \mathrm{C}$

Table 43: t-test of Dynamic Modulus for STs fabricated from $19 \mathrm{~mm}$ NMAS at $4^{\circ} \mathrm{C}$

Table 44: t-test of Phase Angle for STs fabricated from $19 \mathrm{~mm} \mathrm{NMAS}$ at $4^{\circ} \mathrm{C}$

Table 45: t-test of Dynamic Modulus for STs fabricated from $19 \mathrm{~mm}$ NMAS at $20^{\circ} \mathrm{C}$

Table 46: t-test of Phase Angle for STs fabricated from $19 \mathrm{~mm} \mathrm{NMAS}$ at $20^{\circ} \mathrm{C}$

Table 47: t-test of Dynamic Modulus for STs fabricated from 19 mm NMAS at $35^{\circ} \mathrm{C} \quad 124$

Table 48: t-test of Phase Angle for STs fabricated from $19 \mathrm{~mm} \mathrm{NMAS}$ at $35^{\circ} \mathrm{C}$

Table 49: t-test of Dynamic Modulus for STs fabricated from $25 \mathrm{~mm}$ NMAS at $4^{\circ} \mathrm{C} \quad 125$

Table 50: t-test of Phase Angle for STs fabricated from $25 \mathrm{~mm} \mathrm{NMAS}$ at $4^{\circ} \mathrm{C}$ 
Table 51: t-test of Dynamic Modulus for STs fabricated from $25 \mathrm{~mm}$ NMAS at $20^{\circ} \mathrm{C}$

Table 52: t-test of Phase Angle for STs fabricated from $25 \mathrm{~mm}$ NMAS at $20^{\circ} \mathrm{C}$

Table 53: t-test of Dynamic Modulus for STs fabricated from $25 \mathrm{~mm}$ NMAS at $35^{\circ} \mathrm{C}$

Table 54: t-test of Phase Angle for STs fabricated from $25 \mathrm{~mm}$ NMAS at $35^{\circ} \mathrm{C}$

Table 55: ANOVA of STs for Dynamic Modulus and Phase Angle from $12.5 \mathrm{~mm}$ NMAS

Table 56: ANOVA of STs for Dynamic Modulus and Phase Angle from 19 mm NMAS

Table 57: ANOVA of STs for Dynamic Modulus and Phase Angle from 25 mm NMAS

Table 58: MANOVA of NMAS and STs for Dynamic Modulus and Phase Angle at $4^{\circ} \mathrm{C}$ and $0.1 \mathrm{~Hz}$

Table 59: MANOVA of NMAS and STs for Dynamic Modulus and Phase Angle at $4^{\circ} \mathrm{C}$ and $1 \mathrm{~Hz}$

Table 60: MANOVA of NMAS and STs for Dynamic Modulus and Phase Angle at $4^{\circ} \mathrm{C}$ and $10 \mathrm{~Hz} \quad 135$

Table 61: MANOVA of NMAS and STs for Dynamic Modulus and Phase Angle at $20^{\circ} \mathrm{C}$ and $0.1 \mathrm{~Hz} 136$

Table 62: MANOVA of NMAS and STs for Dynamic Modulus and Phase Angle at $20^{\circ} \mathrm{C}$ and $1 \mathrm{~Hz} \quad 137$

Table 63: MANOVA of NMAS and STs for Dynamic Modulus and Phase Angle at $20^{\circ} \mathrm{C}$ and $10 \mathrm{~Hz} \quad 138$

Table 64: MANOVA of NMAS and STs for Dynamic Modulus and Phase Angle at $35^{\circ} \mathrm{C}$ and $0.1 \mathrm{~Hz} 139$

Table 65: MANOVA of NMAS and STs for Dynamic Modulus and Phase Angle at $35^{\circ} \mathrm{C}$ and $1 \mathrm{~Hz} \quad 140$

Table 66: MANOVA of NMAS and STs for Dynamic Modulus and Phase Angle at $35^{\circ} \mathrm{C}$ and $10 \mathrm{~Hz} \quad 141$

Table 67: Comparison of Sample Third Air Void to Entire Sample Air Void for 12.5 mm NMAS mix 142

Table 68: Comparison of Sample Third Air Void to Entire Sample Air Void for 19 mm NMAS mix 143

Table 69: Comparison of Sample Third Air Void to Entire Sample Air Void for 25 mm NMAS mix 144

Table 70: Statistical Analysis of Air Void Variability for Different STs in 12.5 mm NMAS Mix 145

Table 71: Statistical Analysis of Air Void Variability for Different STs in 19 mm NMAS Mix 146

Table 72: Statistical Analysis of Air Void Variability for Different STs in 25 mm NMAS Mix 147

Table 73: Data Set Comparisons for Different Inscribed Circles used in Sample Types

Table 74: Statistical Analysis of Air Void Variability within different inscribed Circles of Sample Types in 12.5 mm NMAS Mix

Table 75: Statistical Analysis of Air Void Variability within different inscribed Circles of Sample Types in 19 mm NMAS Mix

Table 76: Statistical Analysis of Air Void Variability within different inscribed Circles of Sample Types in 25 mm NMAS Mix

Table 77: Recommendations of this Research for AASHTO Tests performed in AMPT

Table 78: Description of Vertical Space in AMPT Testing Chamber for Dynamic Modulus, DTC Fatigue, and SSR Test

Table 79: Summary of Custom Parts for Dynamic Modulus and SSR Test for This Research 


\section{List of Abbreviations}

AASHTO = American Association of State Highway and Transportation Officials

$\mathrm{AC}=$ Asphalt Concrete

ASTM $=$ American Society for Testing and Materials

AMPT $=$ Asphalt Mixer Performance Tester

$\mathrm{CT}=$ Computed Tomography

DTCF $=$ Direct Tension Cyclic Fatigue Test

$\mathrm{DM}=$ Dynamic Modulus

DTT $=$ Direct Tension Tensile test

$\mathrm{ESAL}=$ Equivalent Single Axle Load

FHWA $=$ Federal Highway Administration

$G_{m b}=$ Bulk specific gravity of compacted mix

$G_{m m}=$ Theoretical maximum specific gravity of loose mixture

$G_{s b}=$ Specific gravity of bulk aggregate

HMA $=$ Hot Mix Asphalt

ITS = Indirect Tensile Strength

LVDT $=$ Linear Variable Displacement Transducers

MAS = Maximum Aggregate Size

NMAS $=$ Nominal Maximum Aggregate size

NCHRP $=$ National Cooperative Highway Research Program

$\mathrm{NCSU}=$ North Carolina State University

$P_{S}=$ Percentage of stone/aggregate in mix

RAP $=$ Reclaimed Asphalt Pavement

$\mathrm{RVE}=$ Representative Volume Element

SSR $=$ Stress sweep Rutting Test

$\mathrm{SSD}=$ Saturated Surface Dry

SGC $=$ Superpave Gyratory Compactor

SHARP $=$ Strategic Highway Research Program

SPT $=$ Simple Performance Tester

S-VECD $=$ Simplified Viscoelastic Continuum Damage

$\mathrm{USACE}=$ U.S. Army Corps of Engineers

$\mathrm{VTM}=$ Voids in Total Mix

VMA $=$ Voids in Mineral Aggregate

VFA $=$ Voids Filled with Asphalt

WVDOH $=$ West Virginia Department of Highway 


\section{CHAPTER 1. INTRODUCTION}

\subsection{BACKGROUND}

The U.S. highway system encompasses over 4 million miles of public roads (FHWA, 2019). The West Virginia Department of Highways (WVDOH) is responsible for about 36,000 miles of highway network and despite the small size of the state, it is the $6^{\text {th }}$ biggest network of state-maintained highways in the nation (WVDOH, 2016). Roads are a crucial part of transportation system and with limited resources the Department of Transportation must continue to deliver services and facilitate country's demand.

The WVDOH uses two asphalt mix design methods, Marshall and Superpave. The Superpave mixes are designated by Nominal Maximum Aggregate size (NMAS). The NMAS is one sieve size larger than the first sieve to retain at least 10\% of the aggregate blend and Maximum Aggregate size (MAS) is one sieve size larger than NMAS (AASHTO R 35-17). The NMAS of asphalt mix ranges from $4.75 \mathrm{~mm}$ to $37.5 \mathrm{~mm}$. The WVDOH designates Marshall Mixes as either wearing or base mix followed by a number that uniquely identifies the NMAS of the mix. The mix types used by the WVDOH are summarized in Table 1 (WVDOT, 2011).

Table 1: Mix Types Used by WVDOH (MP 401.02.22)

\begin{tabular}{|c|c|c|c|}
\hline Marshall & Superpave & NMAS (mm) & MAS (mm) \\
\hline Wearing III $^{\mathbf{1}}$ & 4.75 & 4.75 & 9.5 \\
\hline Wearing I & 9.5 & 9.5 & 12.5 \\
\hline- & 12.5 & 12.5 & 19 \\
\hline Base II Wearing IV $^{2}$ & 19 & 19 & 25 \\
\hline- & 25 & 25 & 37.5 \\
\hline Base I & 37.5 & 37.5 & 50 \\
\hline
\end{tabular}

${ }^{1}$ The WVDOH also has pavement preservation specialty mixes for both Marshall and Superpave with a NMAS of $4.75 \mathrm{~mm}$

2 The Wearing IV mix requires the gradation pass above the primary sieve control point as defined for a Superpave $19 \mathrm{~mm}$ mix 


\subsection{PROBLEM STATEMENT}

Performance testing of asphalt mix is growing with an expectation of accurately measuring engineering properties of pavement materials. Performance testing is important because it ensures the quality of Hot Mix Asphalt (HMA). The measured engineering properties of material can then be used for structural design of pavement. It also provides potential method for evaluating performance expectation in mix design process.

The Asphalt Mixture Performance Tester (AMPT) is a servo-hydraulic test device. FHWA in partnership with state highway agencies uses AMPT for asphalt mix performance testing. AMPT test methods were developed by Witczak under National Cooperative Highway Research Program (NCHRP Report 513, 2003) to test dynamic modulus and permanent deformation of asphalt concrete. Witczak (2000) analyzed multiple sample dimensions and concluded that $100 \mathrm{X} 150 \mathrm{~mm}$ was a good geometry to test dynamic modulus and permanent deformation of asphalt mix. In 2009, AASHTO published 100X150 mm geometry for dynamic modulus and flow number testing under AASHTO TP 79. As per FHWA (2013), fatigue testing in AMPT was developed by Christensen and Bonaquist (2009) and Hou et al. (2010). In 2014, AASHTO TP107-14 required 100X130 mm sample for Direct Tension Cyclic (DTC) fatigue testing.

In 2019, AASHTO published three testing procedures based on the work of Kim at NC State University (NCHRP IDEA Project 181, 2017):

- $\quad$ AASHTO TP 132-19- 38X110 mm samples for dynamic modulus tests

- $\quad$ AASHTO TP 133-19-38X110mm samples for DTC fatigue tests

- AASHTO TP 134-19- 100X150 mm samples for Stress Sweep Rutting (SSR) tests In addition to the research that produced test methods, several researches have investigated the issue of sample dimensions relative to the aggregate size in the mix (Kutay et al., 2009, Li and Gibson, 2012, Bower et al., 2015, Lee et al., 2017a, and Lee et al., 2017b). Each research recommended different sample geometries. The lack of uniformity in sample size recommendations demonstrates that sample size is an unresolved issue for AMPT testing to evaluate the performance characteristics of asphalt mix.

An issue associated with the NMAS of asphalt mix versus sample dimensions is the distribution of air voids within the sample. Air voids distribution within the sample can impact the evaluation of performance of asphalt mix. Kassem et al. (2011) found air void variability within a sample affected the variability of fatigue test results. Additionally, Lee et al. (2017b) investigated the possibility of end failure in DTC fatigue specimens as a result of air void gradient in the test specimen. To test the air void uniformity within the specimen, AASHTO R 83-17 recommends slicing 100X150 mm test samples into 3 
disks, top, middle, and bottom, and using a Student's t test to check for statistically significant difference in the vertical air void distribution. The AASHTO PP 99-19 for 38X110 mm does not require checking air void uniformity. Therefore, there is a need for research to investigate if the air void distribution within 38X110 mm (4 cored from a single Superpave Gyratory Compacted (SGC) pill and 1 cored from the center of a single SGC pill), 50X110 mm (4 cored from a single SGC pill and 1 cored from the center of a single SGC pill), $75 \mathrm{X} 110 \mathrm{~mm}$, and $100 \mathrm{X} 150 \mathrm{~mm}$ is statistically same or not.

AASHTO R 83-17 requires coring 100X150 mm and 100X130 mm specimens from the center of 150X180 mm SGC pills and AASHTO PP 99-19 requires coring four 38X110 mm specimens within a $100 \mathrm{~mm}$ diameter of $150 \mathrm{X} 180 \mathrm{~mm}$ SGC pill. No research was reported on obtaining the small size specimens outside the $100 \mathrm{~mm}$ diameter of SGC pill. By fabricating three 50X110 mm samples within $120 \mathrm{~mm}$ diameter of SGC pill and four 38X110 mm samples within $104 \mathrm{~mm}$ diameter of SGC pill, total air void distribution within the sample can be compared to 100X150 mm and 75X110 mm specimen, which is obtained from the center of SGC pill. This would help find if total air void in specimen geometry obtained from $120 \mathrm{~mm}, 104 \mathrm{~mm}, 100 \mathrm{~mm}$ or the center is statistically same or not.

For ease of sample fabrication and testing, small samples are preferred. However, for a composite material that are a blend of aggregates and binder, the minimum sample dimension is constrained by the size of the aggregates. ASTM D- $3497^{3}$ recommends two sample dimension requirements:

- The minimum ratio of specimen height to diameter should be 2:1

- The minimum ratio of specimen dimeter to MAS should be $4: 1$

In the development of AASHTO TP 79, Witczak (2000) followed the recommendation for the minimum ratio of specimen dimeter to MAS but not for minimum ratio of specimen height to diameter (aspect ratio). Contrarily, Park and Kim (2013), and Lee et al., (2017b) followed the recommendation for the minimum aspect ratio but not for minimum ratio of specimen dimeter to MAS for the development AASHTO TP 132-19 and TP 133-19.

Some researches referred to these minimum specimen dimensions and minimum gauge length to measure sample deformation as Representative Volume Element, RVE. Several researches (Witczak et al., 2000, Chehab et al., 2000, and Weissman et al., 1999) have investigate RVE from laboratory performance test and concluded different results for specimen dimension: aspect ratio and diameter to MAS ratio. This indicates sample dimension is an unresolved issue for AMPT testing and more research is needed to examine sample geometry by analyzing dynamic modulus, fatigue, and rutting of different

${ }^{3}$ Although ASTM D-was withdrawn, it is a referenced cited by some researchers. 3 
asphalt mixes. This research can help define RVE requirements and sample dimensions for reliable performance testing with the AMPT.

\subsection{OBJECTIVE}

The objective of this research is to determine appropriate sample geometry for dynamic modulus, fatigue, and rutting test for accurate characterization of asphalt mix and ease of testing.

\subsection{SCOPE AND LIMITATIONS}

The scope of this research is divided into three parts:

1. Effect of new specimen geometries $(50 \mathrm{X} 110 \mathrm{~mm}$ and $75 \mathrm{X} 110 \mathrm{~mm})$ yielding different aspect and diameter to MAS ratio for dynamic modulus, DTC fatigue, and SSR test.

2. Effect of vertical coring three $50 \mathrm{X} 110 \mathrm{~mm}$ specimens from $120 \mathrm{~mm}$ and four $38 \mathrm{X} 110 \mathrm{~mm}$ specimens from $104 \mathrm{~mm}$ diameter within the SGC pill. Additionally, effect of vertical coring one 75X110 $\mathrm{mm}$ specimen from the center of SGC pill.

3. Investigate air void variability within sample.

Most researches have evaluated 100X150 mm, 100X130 mm and 38X110 mm specimens therefore, the purpose of this research is to analyze another small scale specimen of 50X110 mm as this dimension would provide better Representative Volume Element (RVE) for higher NMAS mixes such as 19 and $25 \mathrm{~mm}$, yet yield three specimens per a single SGC pill. Additionally, 75X110 mm specimens are researched as this may provide better air void uniformity compared to 100X150 mm and 100X130 mm specimens. This research also fabricates three 50X110 mm specimens from a single SGC pill and compare the permanent strain results to $100 \mathrm{X} 150 \mathrm{~mm}$ specimens.

The air void uniformity is tested and compared between different geometries obtained from a single SGC pill to investigate the reduction in air void from coring within 120- and 104-mm diameter of SGC pill.

The air void uniformity within each specimen is tested by sawing the specimens in three equal sections.

Due to extensive geometry testing, only three different plant produced asphalt mixes with different NMAS is tested. The three plant produced asphalt mixes are: $12.5 \mathrm{~mm}$, Marshall Wearing 4 (19 $\mathrm{mm}$ ), and $25 \mathrm{~mm}$. Additionally, only the equipment available in WVU asphalt lab are used for this research with a single operator. 


\section{CHAPTER 2. LITERATURE REVIEW}

This chapter discusses AMPT testing and data analysis. For AMPT test, SGC is used to compact asphalt mix into cylindrical test pills of $150 \mathrm{~mm}$ in diameter and $180 \mathrm{~mm}$ in height, called SGC pill ${ }^{4}$. The pills are then trimmed to specific dimensions required for the different tests. Table 2 summarizes AASHTO sample dimensions for the dynamic modulus, Direct Tension Cyclic (DTC) fatigue, and Stress Sweep Rutting (SSR) AMPT tests.

Table 2: Sample Dimensions and Maximum NMAS for asphalt mixes used in AMPT Testing per AASHTO Standards

\begin{tabular}{|c|c|c|c|c|}
\hline Test & AASHTO Method & $\begin{array}{c}\text { Max. NMAS, } \\
\mathrm{mm}\end{array}$ & Diameter, mm & $\begin{array}{c}\text { Height, } \\
\mathrm{mm}\end{array}$ \\
\hline \multirow{2}{*}{ Dynamic Modulus } & T 378-17* & 37.5 & 100 & 150 \\
\cline { 2 - 5 } & TP $132-19 * *$ & 19 & 38 & 110 \\
\hline \multirow{2}{*}{ DTC Fatigue } & TP $107-18$ & 25 & 100 & 130 \\
\cline { 2 - 5 } & TP $133-19 * *$ & 19 & 38 & 110 \\
\hline SSR & TP 134-19 & 37.5 & 100 & 150 \\
\hline
\end{tabular}

*This standard replaced TP 79 that was commonly cited by researchers.

**Four samples are obtained from a single SGC pill.

Meaningful use of the AMPT necessitates analysis to estimate material parameters for pavement performance. For the analysis of the dynamic modulus tests, the Excel template developed by Bonaquist (2011) was used to compute master curve parameters. The analysis of the dynamic modulus can also be performed with DTC fatigue and SSR tests in FlexMAT Cracking and Rutting templates developed by Kim (2018) at North Carolina State University (NCSU). The output of these templates is used for the material inputs to the NCSU FlexPAVE program for mechanistic analysis and pavement deterioration predictions (FlexPAVE 1.1 Alpha., 2018).

This chapter also discusses volumetric properties of asphalt mix in dynamic modulus, fatigue, and rutting. The literature on air void distribution within the SGC pill is discussed in addition to specimen geometries and different asphalt mix NMAS used by different researches to test the outcome of performance test.

\footnotetext{
${ }^{4}$ In some cases, the samples are obtained using 6-inch diameter core of the pavements. Test results from field core are not discussed here in.
} 
The AASHTO standards provide protocols for AMPT testing for dynamic modulus, DTC fatigue, and SSR test. However, some researchers used testing methods that were not performed with current AASHTO protocol. These include:

- Push-Pull fatigue test- uniaxial tension compression test performed in controlled stress and strain loading mode. The stress control is set at $\pm 610 \mathrm{kPa}$ and strain control is \pm 300 microstrain. The strain level is controlled using LVDT actuator with an initial specimen strain of 300 microstrain (Kutay et al., 2009).

- Uniaxial compressive test- stiffness test conducted per ASTM D 3497 by applying haversine loading between 0 to 35 psi. This test is conducted either unconfined or with a confining pressure 30 psi. The test temperatures and frequencies are:

\begin{tabular}{|c|c|}
\hline Temperature, ${ }^{\circ} \mathrm{C}$ & Frequency, $\mathrm{Hz}$ \\
\hline \multirow{2}{*}{5} & 1 \\
\cline { 2 - 2 } & 4 \\
\cline { 2 - 2 } & 16 \\
\hline \multirow{2}{*}{25} & 1 \\
\cline { 2 - 2 } & 4 \\
\hline \multirow{3}{*}{40} & 16 \\
\hline \multirow{2}{*}{4} & 1 \\
\cline { 2 - 2 } & 4 \\
\hline
\end{tabular}

- Crosshead rate monotonic test until failure- conducted in uniaxial tension with crosshead rate of 0.0004 strains $/ \mathrm{sec}$ and temperature between $-30^{\circ} \mathrm{C}$ to $40^{\circ} \mathrm{C}$ (Chehab et al., 2000).

\subsection{VOLUMETRIC PROPERTIES}

The volumetric properties used in this research are:

- Bulk Specific Gravity of asphalt mix $\left(\mathrm{G}_{\mathrm{mb}}\right)$ is determined per AAASHTO T 166.

- Maximum Specific Gravity of AC mix $\left(G_{m m}\right)$ is determined per AASHTO T 209.

- Bulk Specific Gravity of Aggregate $\left(\mathrm{G}_{\mathrm{sb}}\right)$ is determined per AASHTO T 84-13 (2017)).

- Voids in Total Mix (VTM) is determined per AASHTO T 269-14.

- Voids in Mineral Aggregate (VMA) is determined per AASHTO R 35-17

- Voids Filled with Asphalt (VFA) is determined per AASHTO R 35-17 


\subsection{ASPHALT MIXTURE PERFORMANCE TESTER TEST METHODS}

Asphalt Mixture Performance Testing (AMPT) machine can perform multiple tests such as dynamic modulus, cyclic fatigue, Stress Sweep Rutting (SSR), and flow number. The SSR test was developed as an alternative to the flow number test (Kim and Kim, 2017).

\subsubsection{Dynamic Modulus and Phase Angle}

Dynamic Modulus Test Protocol

AMPT subjects specimens to controlled sinusoidal (haversine) compressive ${ }^{5}$ stress and the stress values are calculated by setting peak to peak microstrain between 75 and 125 for unconfined 100X150 mm specimen (per AASHTO T 378) and 50 to 75 microstrain for unconfined 38X110 mm specimen (per AASHTO TP 132). At least two replicate specimens are required for dynamic modulus testing (per AASHTO T 378 and AASHTO TP 132). The test specimen air void uniformity is performed on 100X150 mm dynamic modulus specimen (per AASHTO R-83 17) for which three replicate specimens are required.

The test temperature and frequencies in AMPT relate to traffic conditions and road temperature for example low frequency refers to long duration of load. The recommended test temperature and loading frequency for 100X150 mm specimen per AASHTO R 84-17 and for 38X110 mm specimen per AASHTO TP 132 are shown in Tables 3 and 4.

The data quality statistics requirements as per AASHTO T 378-17 and AASHTO TP 132-19 are shown in Table 5. If the data quality does not fall within the range, sample should be discarded, and test should be repeated with a new sample. The possible causes and solution to obtain data quality statistics are provided in the test standard (AASHTO T 378 and AASHTO TP 132).

\footnotetext{
${ }^{5}$ The AASHTO T-378-17 specifications uses sinusoidal to describe the load pulses. The AMPT actually uses a haversine load pulse for compressive tests. 
Table 3: Recommended Test Temperatures and Frequencies for 100X150 mm Dynamic Modulus Specimen (AASHTO T 378-17)

\begin{tabular}{|c|c|c|c|c|c|}
\hline \multicolumn{2}{|c|}{ PG 58-XX and Softer } & \multicolumn{2}{c|}{ PG 64-XX and PG 70-XX } & \multicolumn{2}{c|}{ PG 76-XX and Stiffer } \\
\hline $\begin{array}{c}\text { Temperature, } \\
{ }^{\circ} \mathrm{C}\end{array}$ & $\begin{array}{c}\text { Loading } \\
\text { Frequencies, } \\
\mathrm{Hz}\end{array}$ & $\begin{array}{c}\text { Temperature, } \\
{ }^{\circ} \mathrm{C}\end{array}$ & $\begin{array}{c}\text { Loading } \\
\text { Frequencies, } \\
\mathrm{Hz}\end{array}$ & $\begin{array}{c}\text { Temperature, } \\
{ }^{\circ} \mathrm{C}\end{array}$ & $\begin{array}{c}\text { Loading } \\
\text { Frequencies, } \\
\mathrm{Hz}\end{array}$ \\
\hline 4 & $10,1,0.1$ & 4 & $10,1,0.1$ & 4 & $10,1,0.1$ \\
\hline 20 & $10,1,0.1$ & 20 & $10,1,0.1$ & 20 & $10,1,0.1$ \\
\hline 35 & $\begin{array}{c}10,1,0.1, \\
\text { and } 0.01\end{array}$ & 40 & $\begin{array}{c}10,1,0.1, \\
\text { and } 0.01\end{array}$ & 45 & $\begin{array}{c}10,1,0.1, \\
\text { and } 0.01\end{array}$ \\
\hline
\end{tabular}

Table 4: Recommended Test Temperatures and Frequencies for 38X110 mm Dynamic Modulus Specimen (AASHTO TP 132-19)

\begin{tabular}{|c|c|c|c|}
\hline \multicolumn{2}{|c|}{ PG 58-XX and Softer } & \multicolumn{2}{c|}{ PG 64-XX and Stiffer } \\
\hline $\begin{array}{c}\text { Temperature, } \\
{ }^{\circ} \mathrm{C}\end{array}$ & $\begin{array}{c}\text { Loading Frequencies, } \\
\mathrm{Hz}\end{array}$ & $\begin{array}{c}\text { Temperature, } \\
{ }^{\circ} \mathrm{C}\end{array}$ & $\begin{array}{c}\text { Loading Frequencies, } \\
\mathrm{Hz}\end{array}$ \\
\hline 4 & $10,1,0.1$ & 4 & $10,1,0.1$ \\
\hline 20 & $10,1,0.1$ & 20 & $10,1,0.1$ \\
\hline 35 & $10,1,0.1$ & 40 & $10,1,0.1$ \\
\hline
\end{tabular}

Table 5: Data Quality Statistics for $100 X 150 \mathrm{~mm}$ and 38X110 mm Dynamic Modulus Specimen (AASHTO T 378-17 and AASHTO TP 132-19)

\begin{tabular}{|c|c|}
\hline Data Statistics & Limit \\
\hline Load Standard Error & $>10 \%$ \\
\hline Deformation Standard Error & $>10 \%$ \\
\hline Deformation Uniformity & $>30 \%$ \\
\hline Phase Uniformity & $>3$ degrees \\
\hline
\end{tabular}

\section{Dynamic Modulus and Phase Angle Analysis}

The stresses and strains measured during the AMPT dynamic modulus are used to compute dynamic modulus and phase angle (Witczak et al., 2000). The dynamic modulus is computed as:

$$
\left|E^{*}\right|=\frac{\sigma_{0}}{\varepsilon_{0}}
$$

Where,

$\left|E^{*}\right|=$ Dynamic modulus, $\mathrm{N} / \mathrm{m}^{2}$ or $\mathrm{psi}$; 
$\sigma_{0}=$ Peak stress amplitude, $\mathrm{N} / \mathrm{m}^{2}$ or $\mathrm{psi}$

$\varepsilon_{0}=$ Peak amplitude of axial strain

The peak stress amplitude, $\sigma_{0}$, is the peak load applied over sample cross section area. The peak axial strain amplitude, $\varepsilon_{0}$, is the maximum change in length divided by initial gauge length of $70 \mathrm{~mm}$ (Faridmehr et al., 2014).

Phase angle $(\delta)$ represents viscous and elastic property of asphalt mix and ranges from $0^{\circ}$ to $90^{\circ}$. The phase angle of $0^{\circ}$ represents an elastic material and $90^{\circ}$ represents a viscous material (Witczak et al., 2000). The phase angle is computed as:

$$
\delta=\frac{t_{i}}{t_{p}} \times 360
$$

Where,

$\delta=$ Phase angle, degrees;

$t_{i}=$ Average time lag between stress and strain cycles, seconds;

$t_{p}=$ Average time for stress cycles, seconds

Following the test at each temperature, AMPT creates a summary file with the dynamic modulus and phase angle, and other results about the test as shown in Table 6.

Table 6: Example Dynamic Modulus Test Summary (AASHTO T 378-17 and AASHTO TP 132-19)

\begin{tabular}{|l|r|r|r|}
\hline Test results summary & & & \\
\hline & $10 \mathrm{~Hz}$ & $1 \mathrm{~Hz}$ & $0.1 \mathrm{~Hz}$ \\
\hline Dynamic modulus (MPa) & 13043 & 9879 & 6825 \\
\hline Phase angle (Degrees) & 10.13 & 13.44 & 18.33 \\
\hline Average temperature ( ${ }^{\circ} \mathrm{C}$ ) & 4.1 & 4.1 & 4.1 \\
\hline Average confining pressure (kPa) & 0.6 & 0.7 & 0.6 \\
\hline Average micro-strain & 97 & 97 & 100 \\
\hline Load drift (\%) & 0 & 0 & 0 \\
\hline Load standard error (\%) & 0.7 & 0.4 & 0.5 \\
\hline Average deformation drift (\%) & -42.5 & -70.4 & -134.6 \\
\hline Average deformation standard error (\%) & 2.9 & 2.6 & 2.8 \\
\hline Deformation uniformity (\%) & 28.5 & 26.2 & 24.3 \\
\hline Phase uniformity (Degrees) & 0.5 & 0.8 & 0.9 \\
\hline
\end{tabular}

The dynamic modulus test results from each temperature-frequency pair and from replicate samples are then used to determine a master curve which defines the modulus versus frequency 
relationship for the asphalt concrete relative to a reference temperature. The concept for transforming the dynamic modulus test results to a master curve is shown in Figure 1.

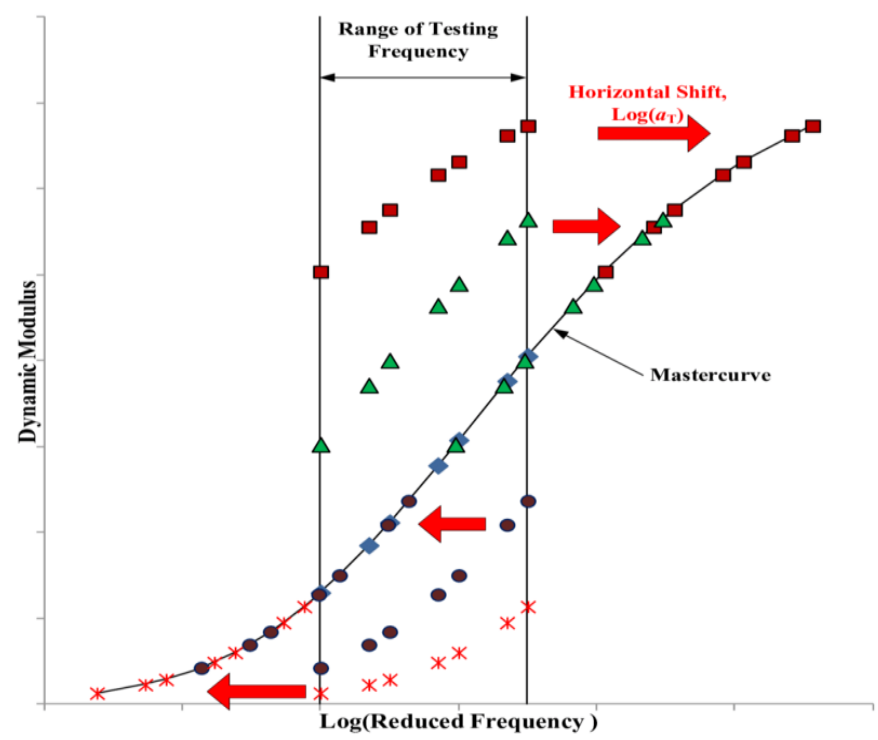

Note: the reference temperature is for the master curve. The test results points are plotted versus actual frequency

\section{Figure 1: Asphalt Concrete Master Curve (Lee, 2013).}

Figure 1 shows data points obtained from AMPT above and below the fitted line, and points on the fitted line were for tests performed at the reference temperature of the master curve. The master curve is constructed as per time temperature superposition principle which relates frequency to temperature for viscoelastic materials. Master curve is developed by shifting dynamic modulus data for various temperatures with respect to reduced frequency to merge into a smooth curve. The amount of horizontal shift for a given temperature and shift frequency is referred as reduced frequency. The shift factor is the amount of shift that occurs at each temperature to the reference temperature, to form master curve. The dynamic modulus of asphalt mix is determined from master curve at a particular temperature and frequency (Lee, 2013).

The master curve equation from AASHTO R 84 is:

$$
\log \left|\mathrm{E}^{*}\right|=\delta+\frac{\left(\left|\mathrm{E}^{*}\right|_{\max }-\delta\right)}{1+\mathrm{e}^{\beta+\gamma\left\{\log \left[\mathrm{f}_{\mathrm{r}}\right]\right\}}}
$$

Where,

$\left|E^{*}\right|=$ Dynamic modulus, psi; 
$\delta, \beta$, and $\gamma=$ Fitting parameters;

$\left|E^{*}\right|_{\max }=$ Limiting maximum asphalt mixture dynamic modulus, psi;

$f_{r}=$ Reduced frequency, $\mathrm{Hz}$

Bonaquist (2011) developed the MasterSolver Excel template for computing the master curve fitting parameters from the AMPT dynamic modulus test. An examination of the equation used by Bonaquist $^{6}$ is:

$$
\log \left|E^{*}\right|=\log (\text { Min })+\frac{\log (\operatorname{Max})-\log (\text { Min })}{1+e^{\beta+\gamma\left\{\log \left[\mathrm{f}_{\mathrm{r}}\right]\right\}}}
$$

Where,

$\left|E^{*}\right|=$ Dynamic modulus, psi;

Min $=$ Limiting minimum modulus $(\delta$ in Equation 3$), \mathrm{ksi}$;

Max = Limiting maximum modulus $\left(\left|\mathrm{E}^{*}\right|_{\max }\right.$ in Equation 3), ksi;

$\beta$, and $\gamma=$ Fitting parameters;

$f_{r}=$ Reduced frequency, $\mathrm{Hz}$

$$
\log \left(\mathrm{f}_{\mathrm{r}}\right)=\log (\mathrm{f})+\log [\alpha(\mathrm{T})]
$$

Where,

$f_{r}=$ Reduced frequency, $\mathrm{Hz}$;

$f=$ Frequency, $\mathrm{Hz}$;

$\alpha(T)=$ Temperature shift factor function

$$
\log [\alpha(\mathrm{T})]=\frac{\Delta \mathrm{E}_{\mathrm{a}}}{19.14714}\left(\frac{1}{\mathrm{~T}}-\frac{1}{\mathrm{~T}_{\mathrm{r}}}\right)
$$

Where,

$\alpha(T)=$ Temperature shift factor function

$\Delta \mathrm{E}_{\mathrm{a}}=$ Activation energy (fitting parameter), $(\mathrm{J} / \mathrm{mol})$;

$\mathrm{T}=$ Test temperature, $\left({ }^{\circ} \mathrm{K}\right)$;

$T_{r}=$ Reference temperature, $\left({ }^{\circ} \mathrm{K}\right)$

${ }^{6}$ Dr. Bonaquist verified equation 3 is correct in an email to Dr. Zaniewski, February 17, 2020. 
Combining equations 5 and 6, reduced frequency is computed using Arrhenius equation:

$$
\begin{gathered}
\log \left(\mathrm{f}_{\mathrm{r}}\right)=\log (\mathrm{f})+\frac{\Delta \mathrm{E}_{\mathrm{a}}}{19.14714}\left(\frac{1}{\mathrm{~T}}-\frac{1}{\mathrm{~T}_{\mathrm{r}}}\right) \\
\left|\mathrm{E}^{*}\right|_{\max }=\mathrm{P}_{\mathrm{c}}\left[\begin{array}{c}
4,200,000\left(1-\frac{\mathrm{VMA}}{100}\right)+435,000\left(\frac{\mathrm{VFA} \times \mathrm{VMA}}{10,000}\right) \\
\left.+\frac{1-\mathrm{P}_{\mathrm{c}}}{\frac{\left(1-\frac{\mathrm{VMA}}{100}\right)}{4,200,000}+\frac{\mathrm{VMA}}{435,000(\mathrm{VFA})}}\right]
\end{array}\right.
\end{gathered}
$$

Where,

$$
P_{c}=\frac{\left(20+\frac{435,000(V F A)}{V M A}\right)^{0.58}}{650+\left(\frac{435,000(V F A)}{V M A}\right)^{0.58}}
$$

Where,

$\left|E^{*}\right|_{\max }=$ Limiting maximum asphalt mixture dynamic modulus, psi;

$V M A=$ Voids in mineral aggregate, $\%$;

$V F A=$ Voids filled with asphalt, $\%$

Note that equations 9 and 10 use the volumetric parameters VMA and VFA. These are determined using standard mix design tests (AASHTO R 35-17).

The AMPT dynamic modulus data are analyzed by FlexMAT Cracking Excel template, which generates Prony series and linear viscoelastic properties of asphalt mix (Kim, 2018). 
The Prony series was developed to represent long term complex viscoelastic behavior of asphalt mix (Zhang et al.,2018). The Wiechert model is used to obtain Prony series expression of the relaxation modulus. The Wiechert model consist of m Maxwell Models and a parallel spring, to describe the mechanical behavior of asphalt mix. The Prony series is determined as:

$$
\mathrm{E}^{*}=E_{e}+\sum_{i=1}^{m} \frac{i \omega \rho_{i} E_{i}}{i \omega \rho_{i}+1}
$$

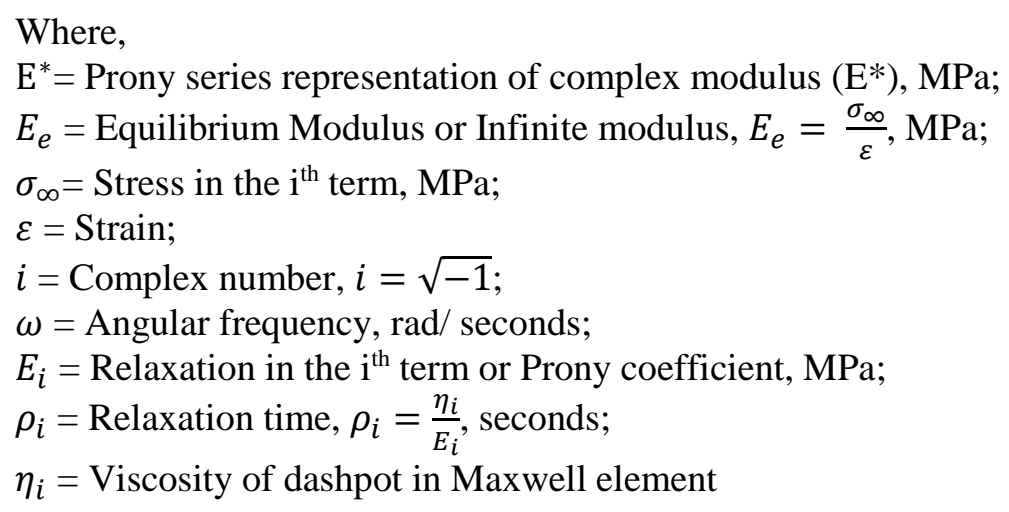

Viscoelastic properties in FlexMAT Cracking Excel template include relaxation modulus, Poisson's ratio, and shift factors. Relaxation modulus is the ratio of stress at any time divided by instantaneous strain. When instant strain is fixed and remains constant, the applied stress is relaxed over time. The reduction in stress is called stress relaxation (Sun et al., 2018). The relaxation modulus is computed as:

$$
E(t)=E_{e}+\sum_{i=1}^{m} E_{i} \exp \left(-t / \rho_{i}\right)
$$

Where,

$\mathrm{E}(\mathrm{t})=$ Relaxation modulus, $\mathrm{MPa}$;

$E_{e}=$ Equilibrium modulus or Infinite modulus, $E_{e}=\frac{\sigma_{\infty}}{\varepsilon}, \mathrm{MPa}$;

$\sigma_{\infty}=$ Stress in the $\mathrm{i}^{\text {th }}$ term, MPa;

$\varepsilon=$ Strain;

$E_{i}=$ Relaxation modulus in the $\mathrm{i}^{\text {th }}$ term or Prony coefficient, $\mathrm{MPa}$;

$t=$ Total time, seconds;

$\rho_{i}=$ Relaxation time, $\rho_{i}=\frac{\eta_{i}}{E_{i}}$, seconds;

$\eta_{i}=$ Viscosity of dashpot in a Maxwell element, Pa.sec 
The derivation of Prony is series is well described by Sun et al. (2018), and Zhang et al (2018).

FlexMAT Cracking is also used to analyze DTC fatigue data from the AMPT. FlexMAT Cracking generates output files for dynamic modulus and DTC fatigue separately for input into FlexPAVE, a 3-D finite element program for analyzing pavement structures (FlexPAVE 1.1 Alpha., 2018).

\subsubsection{Direct Tension Cyclic Fatigue}

\section{Direct Tension Cyclic Fatigue Test Protocol}

The cyclic fatigue test in AMPT is performed per AASHTO TP 107-18 and ASHTO TP 133-19. The cyclic fatigue test is a pull-pull (tension-tension) actuator displacement test. It begins by determining a linear viscoelastic fingerprint. This test is performed at a frequency of $10 \mathrm{~Hz}$ and target microstrain of 50 to 75 . The fingerprint test determines mix's sensitivity to fatigue damage and records time history, applied load, and axial deformation (AASHTO TP 107-18 and ASHTO TP 133-19). It is used to calculate Dynamic Modulus Ratio (DMR) to ensure linear viscoelastic behavior for Simplified-Viscoelastic Continuum Damage (S-VECD) analysis (Sabouri and Kim, 2014). The S-VECD model characterizes cumulative fatigue damage under external loading in asphalt mix from different loading histories and model parameters (Wang, 2019). The AASHTO TP 107-18 for 100X130 mm does not specify the DMR range however, AASHTO TP 133 for $38 \mathrm{X} 10 \mathrm{~mm}$ requires three replicate samples to be in the range of 0.9 to 1.1 .

The master curve from MasterSolver is used to calculate the initial dynamic modulus value at a particular temperature and frequency for DTC fatigue test. DMR is computed as:

$$
D M R=\frac{\left|E^{*}\right|_{\text {fingerprint }}}{\left|E^{*}\right|_{L V E}}
$$

Where,

$\left|E^{*}\right|_{\text {fingerprint }}=$ Fingerprint dynamic modulus;

$\left|E^{*}\right|_{L V E}=$ Computed from master curve at test temperature and $10 \mathrm{~Hz}$ frequency

DTC fatigue test is performed at $10 \mathrm{~Hz}$ and temperature for binder grade selected for 98 percent reliability level using LTPP Bind Version 3.0. The test temperature is calculated as: 


$$
\mathrm{T}^{\circ} \mathrm{C}=\frac{\text { High Temperature } P G \text { Grade Binder }- \text { Low Temperature PG Grade Binder }}{2}-3
$$

Three replicate specimens are tested. The seating load and the minimum number of cycle requirements before failure are:

\begin{tabular}{|c|c|c|c|}
\hline AASHTO & Dimension, mm & Seating Load & $\begin{array}{c}\text { Minimum Number of Load } \\
\text { Cycles }\end{array}$ \\
\hline TP 107-18 & $100 \mathrm{X} 130$ & $0.09 \mathrm{kN}(20 \mathrm{lbs})$. & 500 \\
\hline TP 133-19 & $38 \mathrm{X} 110$ & $0.01 \mathrm{kN}(2.2 \mathrm{lbs})$. & $2,000-80,000$ \\
\hline
\end{tabular}

If the minimum cycles are not achieved, the data and specimen must be discarded, and test must be repeated on new specimen. In DTC fatigue test, the macrocrack must develop within the gauge points, known as middle failure. A macrocrack outside the gauge length is an end failure. Per AASHTO TP 10718 and AASHTO TP- 133- 19, the data must be discarded, and test must be repeated on new specimen.

\section{Direct Tension Cyclic Fatigue Test Protocol}

The FlexMAT Cracking computes the S-VECD fatigue properties as input for FlexPAVE software. The model describes material property as fatigue damage grows. The S-VECD embedded in FlexPAVE computes fatigue damage. The detail of the process is well documented (Hou, 2009, Underwood et al. 2009, Zhang, 2012, Underwood et al., 2012, Witczak et al., 2013, and Sabouri, 2014).

The AMPT test (AASHTO TP 107-18 and ASHTO TP 133-19) generates entire test run and initial five cycles run files. These files contain detailed S-VECD data on axial stress, axial micro strain, peak to peak strain for each LVDT, and dynamic modulus and phase angle at each cycle. FlexMAT computes S-VECD fatigue properties: $C_{11}, C_{12}, \alpha, \mathrm{G}^{\mathrm{R}}$ and $\mathrm{D}^{\mathrm{R}}$ to be imported into FlexPAVE software.

According to Wang and Kim (2019), the material loses its stored pseudo strain energy in cyclic fatigue as the damage accumulates. The difference between undamaged state of material and current stored pseudo strain energy is referred to as total released pseudo strain energy denoted as $W^{R}$. This dissipated pseudo strain energy is related to accumulated amount of damage (S) over a given time under fatigue loading as:

$$
\frac{\partial S}{\partial t}=\left(-\frac{\partial W^{R}}{\partial S}\right)^{\alpha}
$$


Where,

$\mathrm{S}=$ Accumulated amount of damage;

$\mathrm{t}=$ Time, second;

$W^{R}=$ Dissipated pseudo strain energy;

$\alpha=\mathrm{S}-$ VECD Model Parameter

The accumulated damage is related to pseudo stiffness as:

$$
C=1-C_{11}\left(S^{C_{12}}\right)
$$

Where,

$\mathrm{S}=$ Accumulated amount of damage;

$\mathrm{C}=$ Pseudo stiffness;

$C_{11}$ and $C_{12}=$ S-VECD Model Parameters

FlexPAVE analyses use either $\mathrm{G}^{\mathrm{R}}$ or $\mathrm{D}^{\mathrm{R}}$ based failure criterion to predict fatigue damage. Sabouri and $\operatorname{Kim}(2014)$ developed $\mathrm{G}^{\mathrm{R}}$ failure criteria to have power relationship with number of cycles to failure, $N_{f}$. The research defined $\mathrm{G}^{\mathrm{R}}$ as rate of change of average released pseudo strain energy (per cycle) throughout the entire loading history until failure.

$$
\mathrm{G}^{\mathrm{R}}=\gamma\left(N_{f}\right)^{\delta}
$$

Where,

$\mathrm{G}^{\mathrm{R}}=$ Rate of change of average released pseudo strain energy (per cycle);

$\gamma$ and $\delta=$ Coefficients of material properties;

$N_{f}=$ Number of cycles to failure

Sabouri and Kim (2014) stated that dissipation of pseudo strain energy results in reduction of pseudo stiffness. The reduction in pseudo stiffness is considered to be caused by damage. There is a log$\log$ relation between $\mathrm{G}^{\mathrm{R}}$ and number of cycles to failure is shown as: 


$$
G^{R}=\frac{\int_{0}^{N_{f}} \frac{1}{2}(1-C)\left(\varepsilon_{0, \text { ta }}^{R}\right)^{2} d N}{\left(N_{f}\right)^{2}}
$$

\footnotetext{
Where,

$\mathrm{G}^{\mathrm{R}}=$ Rate of change of average released pseudo strain energy (per cycle);

$N_{f}=$ Number of cycles to failure;

$\mathrm{C}=$ Pseudo stiffness;

$\varepsilon_{0, \text { ta }}^{\mathrm{R}}=$ Pseudo strain amplitude for the duration of the stress in tension;

$\mathrm{N}=$ Number of loading cycles
}

According to Wang et al. (2018) the exponential relationship for $\mathrm{G}^{\mathrm{R}}$ makes this failure criteria highly sensitive to test variability. This shortcoming of $G^{R}$ failure criteria let to the development of the $D^{R}$ failure criteria (Wang et al., 2018). The linear relationship between sum (1-C) to failure versus $N_{f}$ is denoted as $\mathrm{D}^{\mathrm{R}}$ as shown in Figure 2. The sum (1-C) represents summation of reduction in pseudo stiffness.

Wang et al. (2018) defines $\mathrm{D}^{\mathrm{R}}$ failure criteria as average integrity loss per cycle or reduction in pseudo stiffness up to failure, i.e. the slope of the line in Figure 2. It is independent of mode of loading, stress/strain amplitude, and temperature.

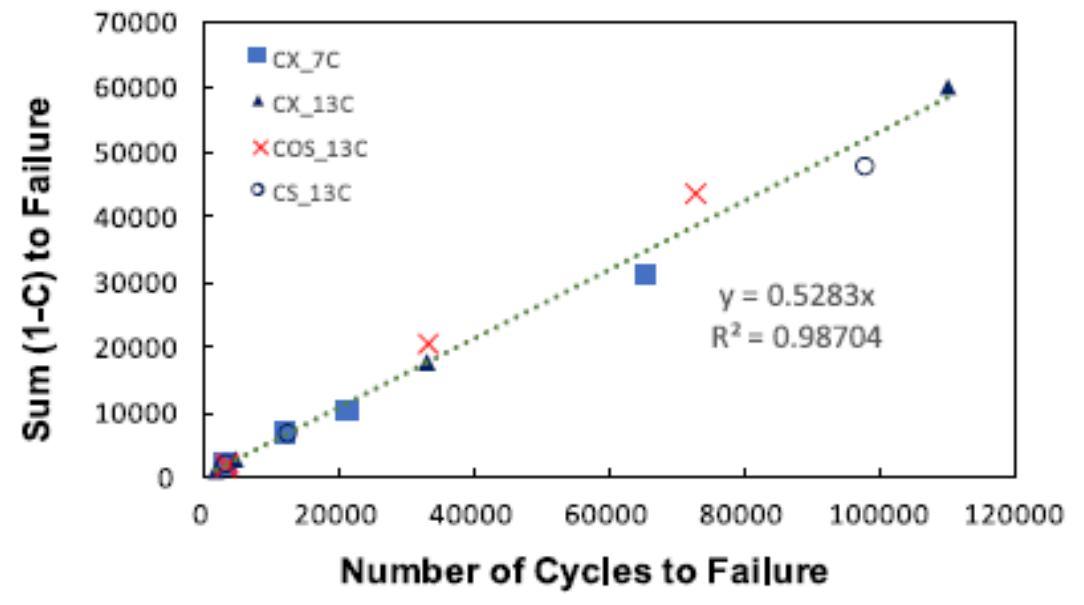

Figure 2: Relationship between Sum (1-C) and $N_{f}$ (Wang and Kim., 2019)

Wang et al. (2018) defines $\mathrm{D}^{\mathrm{R}}$ failure criteria as: 


$$
\mathrm{D}^{\mathrm{R}}=\frac{\int_{0}^{\mathrm{N}_{\mathrm{f}}}(1-\mathrm{C}) \mathrm{dN}}{N_{f}}=\frac{\operatorname{sum}(1-C)}{N_{f}}
$$

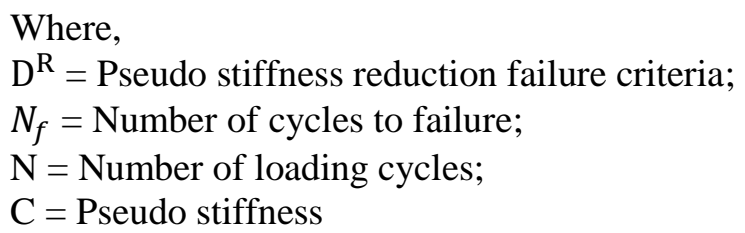

Since application of $\mathrm{D}^{\mathrm{R}}$ is on an arithmetic scale, the application may be less sensitive to variability and reduce possible error. The $\mathrm{D}^{\mathrm{R}}$ offers more reliability over $\mathrm{G}^{\mathrm{R}}$, if same number of tests are used for characterization (Wang et al., 2018) and (Wang and Kim, 2019).

The model parameters from $\mathrm{G}^{\mathrm{R}}$ or $\mathrm{D}^{\mathrm{R}}$ based failure criterion are used to predict fatigue damage. To account for cracking potential in asphalt mixtures, Wang (2019) developed S-VECD model-based index parameter called $S_{\text {app. }}$. Wang (2019) found two main factors that affect cracking potential of asphalt mixtures: modulus and toughness. Modulus represents stiffness of asphalt mix and toughness is the ability to absorb energy without fracturing. The research highlights these two factors because when comparing asphalt mixes under same load amplitude, asphalt mix with poor cracking resistance can either have high modulus with low toughness or low modulus with high toughness. Therefore, $S_{\text {app }}$ was developed to address the effect of these parameters on fatigue cracking.

Wang (2019) found the modulus effect can be determined from the damage characterization curve by plotting pseudo stiffness, $\mathrm{C}$ with respect to damage, $\mathrm{S}$. The mix with higher modulus value appears higher on the plot compared to mix with low modulus value. Mixes with same modulus value but different toughness can be estimated with higher $D_{R}$ value where $D_{R}$ is equal to $1-C_{\text {avg }}$.

$$
C_{\text {avg }}=\frac{\int_{0}^{N_{f}} C d N}{N_{f}}
$$

Where,

$C_{\text {avg }}=$ Average pseudo stiffness;

$N_{f}=$ Number of cycles to failure;

$\mathrm{C}=$ Pseudo stiffness; 


\section{$\mathrm{N}=$ Number of loading cycles}

With increase in load cycles, pseudo stiffness, $\mathrm{C}$ decreases and as a result average pseudo stiffness, $\mathrm{C}_{\mathrm{avg}}$ is also low throughout the entire fatigue life of mix. The corresponding $\mathrm{S}$ value when $\mathrm{C}_{\mathrm{avg}}$ is located on the damage characterization curve is defined as representative fatigue damage, $S_{\text {rep }}$.

$$
S_{\text {rep }}=\left(\frac{a_{T}^{\frac{C_{12}}{\alpha+1}}}{C_{11}} 1-C_{a v g}\right)^{\frac{1}{C_{12}}}
$$

Where,

$\mathrm{S}_{\text {rep }}=$ Representative fatigue damage;

$\alpha_{\mathrm{T}}=$ Time-Temperature superposition shift factor (Climatic PG Grade $-3^{\circ} \mathrm{C}$ );

$\alpha, \mathrm{C}_{11}, \mathrm{C}_{12}=\mathrm{S}-\mathrm{VECD}$ model parameters;

$\mathrm{C}_{\mathrm{avg}}=$ Average pseudo stiffness

To evaluate fatigue-based failure, $S_{\text {rep }}$ parameter is related to number of cycles to failure, $N_{f}$.

$$
\mathrm{N}_{\mathrm{f}}=\left(\frac{1}{\mathrm{~m}}+1\right)^{\mathrm{m}} \times \mathrm{N}_{\mathrm{S}_{\mathrm{rep}}}
$$

$$
\mathrm{p}=1-\alpha \mathrm{C}_{12}+\alpha
$$

Where,

$\mathrm{N}_{\mathrm{f}}=$ Number of cycles to failure;

$\mathrm{N}_{\mathrm{S}_{\text {rep }}}=$ Number of cycles for $\mathrm{S}$ to be equal to $\mathrm{S}_{\text {rep }}$;

$\mathrm{m}=$ Material constant equal to $\mathrm{p} / \mathrm{C}_{12}$;

$\alpha, \mathrm{C}_{12}=\mathrm{S}-\mathrm{VECD}$ model parameters

Wang (2019) proposed an empirical adjustment as $S_{\text {rep }}$ failed to accurately represent damage behavior and modulus. The empirical adjusted parameter is called the apparent damage capacity, $\mathrm{S}_{\text {app. }}$. For 
the adjustment, mixture dynamic modulus value at $10 \mathrm{~Hz}$ and reference temperature was introduced. A power term of $\alpha / 4$ was applied on dynamic modulus. Additionally, $1000^{\frac{\alpha}{2}-1}$ was applied to keep $S_{\text {app }}$ value in the range of 0 to 50 . The final formula to calculate $S_{\text {app }}$ of an asphalt mixture is:

$$
S_{a p p}=1000^{\frac{\alpha}{2}-1} \frac{a_{T^{\frac{1}{\alpha+1}}}\left(\frac{D^{R}}{C_{11}}\right)^{\frac{1}{C_{12}}}}{\left|E^{*}\right|^{\frac{\alpha}{4}}}
$$

Where,

$S_{a p p}=$ Apparent damage capacity (In the range of 0-50);

$a_{T}=$ Time-temperature shift factor for target temperature (Local climatic PG Grade minus $3^{\circ} \mathrm{C}$ );

$\alpha, C_{11}, C_{12}=\mathrm{S}$-VECD model parameters

$D^{R}=$ Average reduction in pseudo stiffness up to failure;

$\left|E^{*}\right|=$ Material dynamic modulus at $10 \mathrm{~Hz}$ and reference temperature

\subsubsection{Stress Sweep Rutting}

|Stress Sweep Rutting Test Protocol

The SSR test (AASHTO TP 134-19) was developed for AMPT to predict permanent strain as a function of load time, temperature, and deviator stress for 100X150 mm specimens. The test requires four replicates at target air void content of $7 \% \pm 0.5 \%$. Two specimens are tested at low temperature and two specimens are tested at high temperature. The test is conducted at constant confining pressure of $69 \mathrm{kPa}$ (10 psi) with the deviator stresses for each temperature. The low-test temperature is determined from the PG grade:

Table 7: SSR Low Test Temperature for PG Grades

\begin{tabular}{cccccccc}
\hline & \multicolumn{7}{c}{ Test Temperature, ${ }^{\circ} \mathrm{C}$} \\
\cline { 2 - 7 } PG Low & \multicolumn{7}{c}{ PG High Temperature, ${ }^{\circ} \mathrm{C}$} \\
\cline { 2 - 7 } & 46 & 52 & 58 & 64 & 70 & 76 & 82 \\
\hline-10 & 23 & 26 & 29 & 32 & 32 & 32 & 32 \\
-16 & 20 & 23 & 26 & 29 & 32 & 32 & 32 \\
-22 & 17 & 20 & 23 & 26 & 29 & 32 & 32 \\
-28 & 17 & 17 & 20 & 23 & 26 & 29 & 32 \\
-34 & 17 & 17 & 17 & 20 & 23 & 26 & 29 \\
-40 & 17 & 17 & 17 & 17 & 20 & 23 & 26 \\
-46 & 17 & 17 & 17 & 17 & 17 & 20 & 23 \\
\hline
\end{tabular}

The high-test temperature, determined from the depth of the top of the pavement layer and degree-days obtained from LTPPBind Version 3.1, is calculated as: 


$$
T_{h}=0.87 \times\left(58+7 \times \frac{D D}{1000}-15 \times \log (H+45)\right)
$$

Where,

$T_{h}=$ High test temperature, ${ }^{\circ} \mathrm{C}$;

$\mathrm{DD}=$ Degree Days $>10^{\circ} \mathrm{C}$ obtained from LTPPBind;

$\mathrm{H}=0$ for surface layer and design depth to top of layer for base layers, $\mathrm{mm}$

Three levels of deviator stress are applied for 200 cycles each in the order as:

\begin{tabular}{|c|c|c|c|}
\hline Temperature, ${ }^{\circ} \mathrm{C}$ & Deviator Stress & Load Time, sec & Rest Time, sec \\
\hline High, $T_{h}$ & $\begin{array}{c}689 \mathrm{kPa}(100 \mathrm{psi}), \\
483 \mathrm{kPa}(70 \mathrm{psi}), \text { and } \\
896 \mathrm{kPa}(130 \mathrm{psi})\end{array}$ & 0.4 & 3.6 \\
\hline Low, $T_{l}$ & $\begin{array}{c}483 \mathrm{kPa}(70 \mathrm{psi}), \\
689 \mathrm{kPa}(100 \mathrm{psi}), \text { and } \\
896 \mathrm{kPa}(130 \mathrm{psi})\end{array}$ & 0.4 & 1.6 \\
\hline
\end{tabular}

|Stress Sweep Rutting Analysis

The FlexMAT Rutting computes SSR model coefficients for input into FlexPAVE. AMPT test (AASHTO TP 134-19) generates entire test run for each test specimen. These files contain detailed SSR data on deviator stress, permanent microstrains, deviator load, contact load, and permanent deformation. FlexMAT rutting computes SSR model coefficients: $p_{1}, p_{2}, d_{1}, d_{2}, \beta, \varepsilon_{o}, N_{1}$, and $T_{r e f}$ for use in FlexPAVE (FlexPAVE 1.1 Alpha., 2018).

Kim and Kim (2017) states that shift model was developed to calculate viscoplastic strain from deviatoric stress, load time, and temperature. The shift model comprises of viscoplastic strain master curve and shift functions as presented in equation 26 through equation 28 (Kim and Kim, 2017).

$$
\varepsilon_{v p}=\frac{\varepsilon_{o} N_{r e d}}{\left(N_{1}+N_{r e d}\right)^{\beta}}
$$

Where,

$\varepsilon_{v p}=$ Viscoplastic strain or permanent strain;

$1-\beta=$ Slope of permanent strain versus number of loading cycles in log-log space;

$\varepsilon_{o}=$ Intercept of permanent strain versus number of loading cycles in log-log space; 
$N_{1}=$ Number of cycles at which secondary region begins as show in Figure 3;

$N_{\text {red }}=$ Reduced number of cycles at reference loading conditions;

$$
N_{\text {red }}=N^{\text {Physical }} \times 10^{a_{10}}
$$

Where,

$N_{\text {red }}=$ Reduced number of cycles at reference loading conditions;

$N^{\text {Physical }}=$ Actual number of load cycles

$$
a_{10}=p_{1} \log _{10}\left(\xi_{p}\right)+p_{2}+\left(d_{1} T_{r e f}+d_{2}\right)\left(\log _{10}\left(\frac{\sigma_{v}}{p_{o}}\right)-0.877\right)
$$

Where,

$p_{1}$ and $p_{2}=$ Coefficients of reduced load time shift factors;

$d_{1}$ and $d_{2}=$ Coefficients of vertical stress shift factors;

$\xi_{p}=$ Reduced load Time, seconds;

$T_{\text {ref }}=$ Reference temperature, ${ }^{\circ} \mathrm{C}$;

$\sigma_{v}=$ Vertical stress due to vertical loading, $\mathrm{kPa}$;

$p_{o}=$ Atmospheric pressure, psi

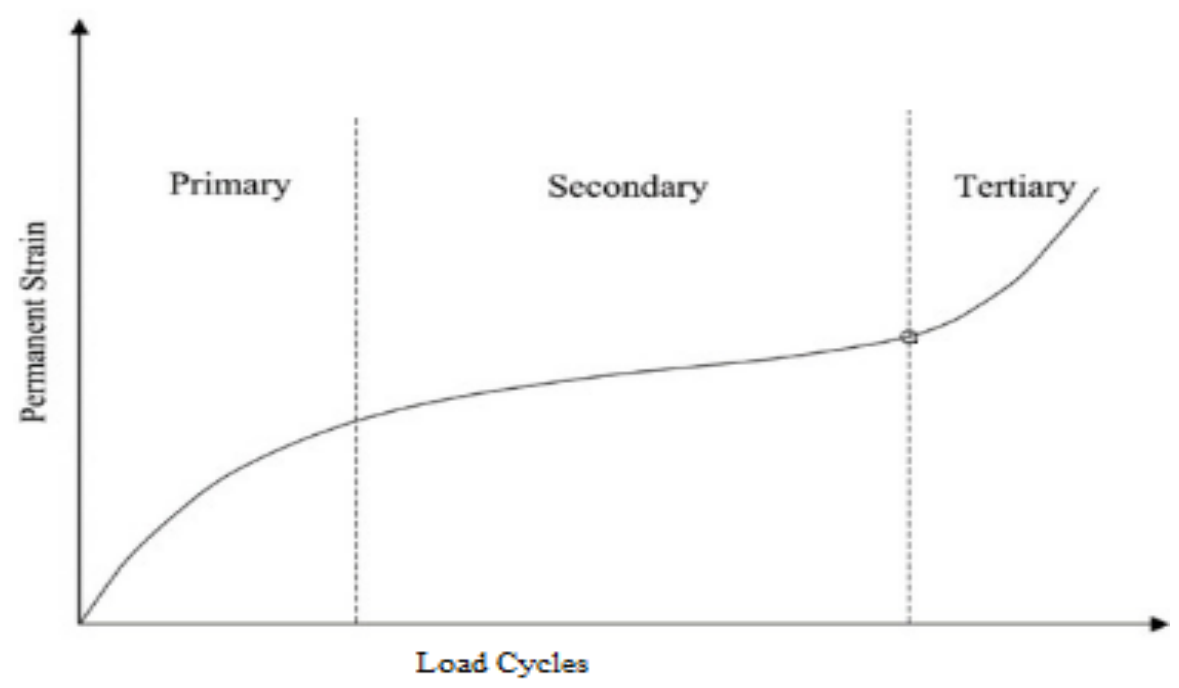

Figure 3: Permanent Deformation of Asphalt Concrete versus Number of Load Cycles (Chilukwa and Lungu, 2019) 


\subsection{FLEXPAVE}

The NCSU researchers developed the FlexPAVE 3-D finite element program to predict pavement distress. The program has two analysis options:

1. Pavement Response Analysis computes stresses, strains and displacements.

2. Pavement Performance Analysis estimates fatigue cracking, damage evolution and rut depth.

FlexPAVE uses data imported from FlexMAT Cracking and FlexMAT Rutting along with user defined parameters for the pavement structure and traffic to analyze the pavement structure. The output and analysis option lets the user specify evaluation point coordinates. The pavement response output shows time history and spatial distribution. Time history graphs show selected response history at evaluation points. Spatial distribution shows contours of selected response at any time within pavement cross section. The pavement performance output shows time history and spatial distribution as part of response results. Fatigue failure criteria are based on pseudo stiffness value of the asphalt. The damage factor distribution over pavement life depicts the growth of damage within the pavement. Damage evolution plots percentage versus pavement life. Finally, rut depth of each layer and total rut depth can be plotted with respect to pavement life (FlexPAVE 1.1 Alpha, 2018).

While FlexPAVE performance analysis is a use tool for evaluating the effect of different mixes on pavement distresses, it does not provide an effective tool for ranking the rutting potential of different mixes. To this end, Ghanbari et. al. (2020) developed a Rutting Strain Index, RSI, for rating the relative performance of mixes. The essence of the RSI is the predication of the rutting level at 20 years of traffic for a standard pavement structure using FlexPAVE. This recently developed method should be evaluated for ranking mixtures.

\subsection{VTM IN TEST SPECIMEN DIMENSIONS}

Kutay et al. (2009) conducted DTC Fatigue test on 38.1X100 mm and 71.4X150 mm specimen with target VTM of 7\%. The average VTM ranged from 6.9 to $8 \%$. VTM of $7 \%$ was chosen because it was a good representation of field compaction (Kutay et al., 2009).

Bower et al. (2015) conducted dynamic modulus test on 38X135, 50X135, 38X110, 50X110 and 100X150 mm specimen. The intent was to fabricate the specimens with target air voids of $7 \%$ to $8 \%$. However, VTM for some specimens was as high as $12 \%$ and as low as $2 \%$. Figure 4 shows bulk air void content as cumulative percent. The VTM of $50 \%$ of specimens ranged between $6.5 \%$ and $7 \%$ (Bowers et al., 2015). 


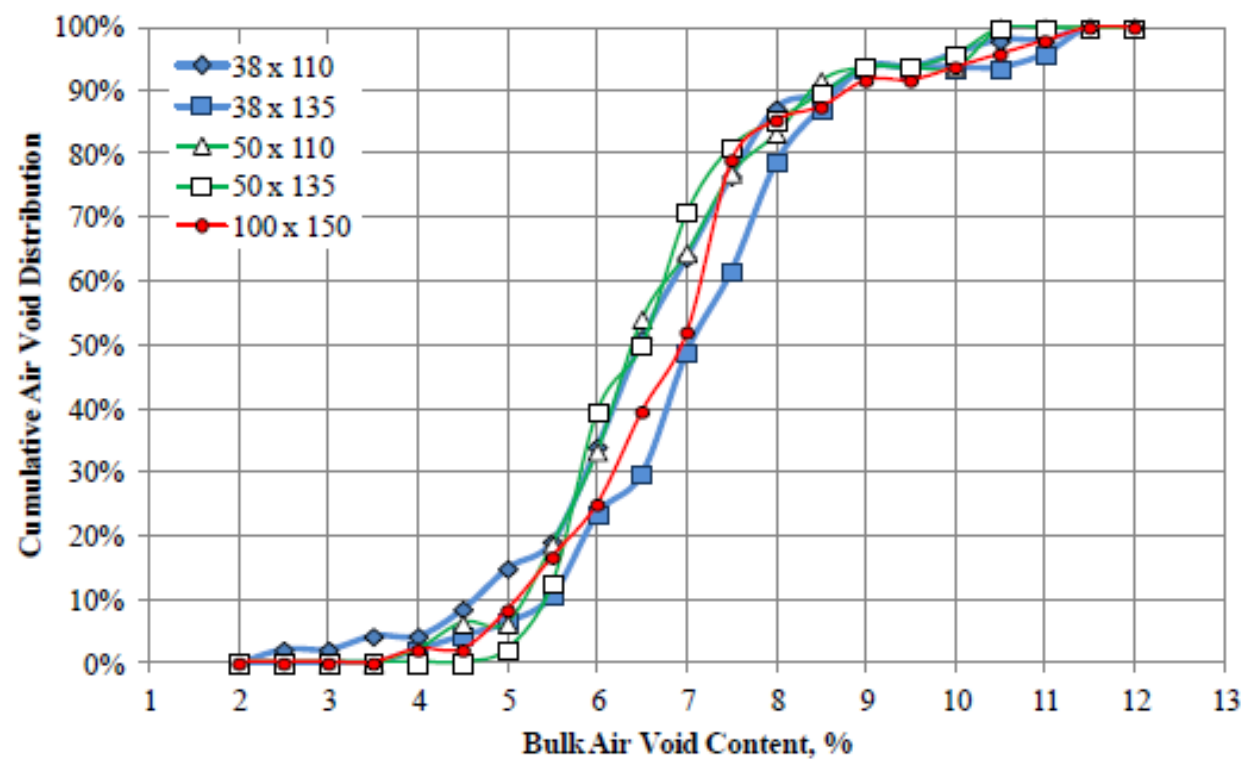

Figure 4: Bulk VTM as Cumulative Air Void Distribution(Bowers et al., 2015)

Blankenship and Anderson (2010) conducted laboratory test to evaluate pavement performance from various air void levels between $4 \%$ and $11.5 \%$. The effect of VTM on fatigue performance was evaluated in accordance with AASHTO T 321-07. The results showed that as VTM increased from 7\% to $11.5 \%$, fatigue performance at 350 microstrain reduced by $42 \%$. The research concluded that with $1 \%$ increase in VTM, reduction in fatigue life is $9.2 \%$. The effect of VTM on rutting performance was also evaluated using flow number test in accordance with AASHTO TP 79. The results showed that with increase in VTM from $7 \%$ to $8.5 \%$, rutting resistance of mixture decreased by $34 \%$. The research corresponded reduction in rutting resistance to $22.7 \%$ for $1 \%$ increase in VTM (Blankenship and Anderson, 2010).

\subsection{AIR VOID DISTRIBUTION IN SPECIMENS}

Thyagarajan et al. (2010) tested vertical and lateral air void distribution for $12.5 \mathrm{~mm}$ NMAS asphalt mix in SGC pill from Pine Compactor. The study tested:

\begin{tabular}{|c|c|c|}
\hline Test & Diameter, $\mathrm{mm}$ & Height, $\mathrm{mm}$ \\
\hline \multirow{3}{*}{ Vertical Heterogeneity } & \multirow{2}{*}{150} & 150 \\
\cline { 3 - 3 } & & 100 \\
\cline { 3 - 3 } & & 50 \\
\hline
\end{tabular}




\begin{tabular}{|c|c|}
\hline Test & Radius from the center of specimen, $\mathrm{mm}$ \\
\hline \multirow{3}{*}{ Lateral Heterogeneity } & 25 \\
\cline { 2 - 2 } & 35 \\
\cline { 2 - 2 } & 43 \\
\cline { 2 - 2 } & 50 \\
\hline
\end{tabular}

The results showed that higher air void content and more uniformity was seen in middle sawed sections of specimens. This was due to "cone effect" as shown in Figure 5. As specimen height increases, cones move away from each other leaving middle section more heterogeneous.

The air void non-uniformity is highest along the circumference where mix is in contact with the mold. Air void heterogeneity exponentially increases for cored specimens moving towards the SGC pill circumference. Lateral heterogeneity index was higher than vertical heterogeneity index as large surface area is in contact with the SGC wall compared to top and bottom plates. Although it was anticipated that cutting the SGC pill to desired height reduced vertical air void heterogeneity and coring the SGC pill to obtain desired diameter specimen affects lateral air void heterogeneity, coring and cutting the SGC pill significantly reduced vertical air void heterogeneity. Out of different compactors tested, pine compactor had highest lateral but lowest vertical air void heterogeneity. The effect of compaction plate on air void heterogeneity was observed even after trimming $10 \mathrm{~mm}$ from top and bottom of SGC pill (Thyagarajan et al., 2010).

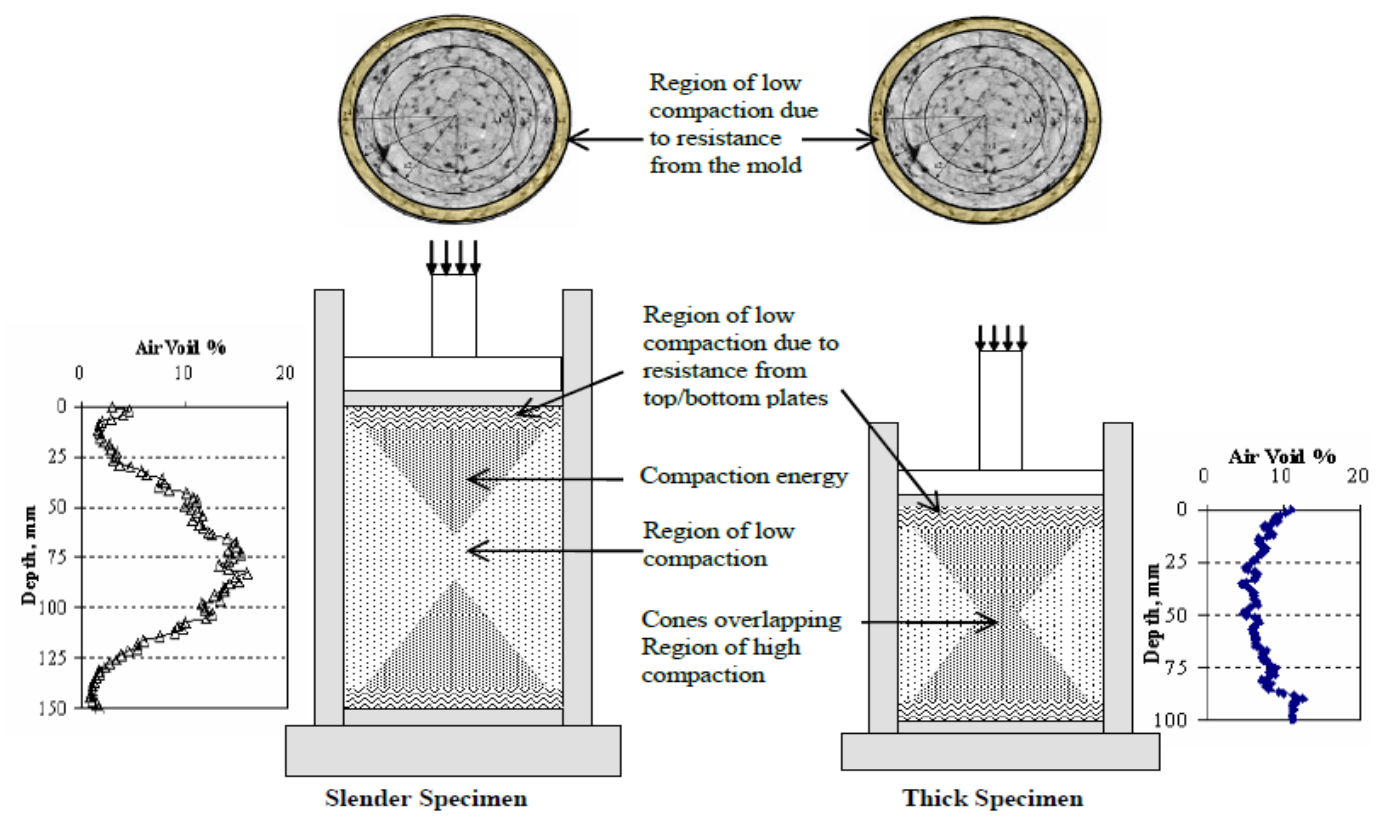

Figure 5: Compaction of HMA specimen resulting in "Cone Shape" (Thyagarajan et al., 2010) 
Chehab et al., (2000) performed a detailed air void distribution for the samples obtained from SGC pill as:

\begin{tabular}{|c|c|}
\hline SGC pill & Test Sample \\
\hline $150 X 175 \mathrm{~mm}$ & $75 \times 150 \mathrm{~mm}$ \\
\hline $150 \times 140 \mathrm{~mm}$ & $75 \times 115 \mathrm{~mm}$ \\
\hline $150 \times 115 \mathrm{~mm}$ & $75 \times 76 \mathrm{~mm}$ \\
\hline $150 \times 175 \mathrm{~mm}$ & $100 \times 150 \mathrm{~mm}$ \\
\hline
\end{tabular}

The results of air void variation from SGC pill and test samples is shown as:

\begin{tabular}{|c|c|}
\hline SGC pill & SGC Pill VTM, $\%$ \\
\hline $150 X 175 \mathrm{~mm}$ & 5.8 \\
\hline $150 X 140 \mathrm{~mm}$ & 7.0 \\
\hline $150 \times 115 \mathrm{~mm}$ & 6.9 \\
\hline $150 X 175 \mathrm{~mm}$ & 5.0 \\
\hline
\end{tabular}

\begin{tabular}{|c|c|}
\hline Test Sample & Top to bottom Sectional VTM, \% \\
\hline \multirow{4}{*}{ 75X150 mm } & 4.3 \\
\cline { 2 - 2 } & 2.8 \\
\cline { 2 - 2 } & 3.6 \\
\cline { 2 - 2 } & 3.5 \\
\cline { 2 - 2 } & 2.9 \\
\hline \multirow{4}{*}{$75 \times 115 \mathrm{~mm}$} & 4.2 \\
\cline { 2 - 2 } & 4.5 \\
\cline { 2 - 2 } & 3.4 \\
\cline { 2 - 2 } & 3.7 \\
\hline \multirow{3}{*}{$75 \times 76 \mathrm{~mm}$} & 3.1 \\
\cline { 2 - 2 } & 4.5 \\
\hline \multirow{4}{*}{ 100X150 mm } & 4.3 \\
\cline { 2 - 2 } & 4.2 \\
\cline { 2 - 2 } & 4.1 \\
\cline { 2 - 2 } & 3.4 \\
\cline { 2 - 2 } & 3.4 \\
\cline { 2 - 2 } & 3.2 \\
\cline { 2 - 2 } & 2.1 \\
\hline
\end{tabular}

It was found that air void was highest on the top, bottom, and around the wall of SGC pill. The specimens were cut in 6 equal sections. 
- Top and bottom sections of 75X150 mm specimens had higher air voids compared to other sections.

- $100 X 150 \mathrm{~mm}$ specimen sections had less air voids than the $75 \times 150 \mathrm{~mm}$ specimens.

- The lower sections of 100X150 mm specimens has lower air voids compared to the top sections.

- $75 X 115 \mathrm{~mm}$ and 75X76 mm had the best air void uniformity.

Aguilar (2015) thesis also tested air void uniformity in 100X150 mm specimens (per

AASHTO PP 60) for $9.5 \mathrm{~mm}$ and $12.5 \mathrm{~mm}$ NMAS asphalt mix. All specimens were fabricated at $4 \% \pm$ $0.5 \%$ from the center of $150 \times 180 \mathrm{~mm}$ SGC pill. It was concluded that samples from SGC did not have uniform air void distribution. On average, VTM in middle section was $1.15 \%$ greater than VTM in top and bottom sections for Corelok data (Aguilar, 2015).

Dalton (2016) thesis analyzes air void uniformity in 100X150 mm specimens (per AASHTO PP 60) for $9.5 \mathrm{~mm}$ and $19 \mathrm{~mm}$ NMAS asphalt mix. All specimens were fabricated at $7 \% \pm 0.5 \%$ from the center of 150X180 mm SGC pill. It was concluded that the VTM of sections were higher than VTM of intact samples by $0.4 \%$. More frequently, the top section had highest VTM followed by middle and bottom section. The t-test compared VTM of top-middle, middle-bottom, and top-bottom sections. Topmiddle and middle-bottom with p-value between 0.06 and 0.6 failed to reject the null hypothesis of equal means for these comparisons. However, $\mathrm{p}$-value of 0.03 in top-bottom comparison resulted in sufficient evidence to reject null hypothesis of equal means for this comparison (Dalton, 2016).

\subsection{SAMPLE DIMENSIONS}

Asphalt is inherently heterogeneous; however, analysis theories assume homogeneous properties. This disparity between the material structure and the theory assumptions presents issues with test sample size and variability. To accommodate this issue, the concept of a representative volume element, RVE, was developed. This concept has been addressed by several researchers (Weissman et al., 1999, Mitchell et al., 2010, and Lee et al., 2017a). The importance of RVE is stated as: Weissman et al. (1999) stated that consistent results are likely to be obtained when specimen size is larger than RVE. Many laboratory test exhibit variabilities and therefore specimen size should be large enough to enable results representative of AC mix. As RVE of asphalt mix depends on aggregate size, shape, and orientation, it should be unique for each mix due to aggregate effect within particular AC mix (Weissman et al., 1999).

Mitchell et al. (2010) and Lee et al. (2017a) stated RVE as the proper dimension of specimen size and gauge length. The research refers ASTM D 3497 to state two primary requirements for proper specimen dimensions:

$>$ The minimum height to diameter ratio should be $2: 1$. 
The minimum diameter to MAS ratio should be 4:1

In essence, RVE is a term that expresses a concept that can be implemented through test sample dimensions such as aspect ratios, diameter to MAS ratio, and gauge length. These dimensional parameters should be altered for type of test, rate of loading and test temperature (Weissman et al., 1999 and Mitchell et al., 2010).

The following literature review concentrates on tests that can be replicated with the AMPT. Research using other testing modes, such as Indirect Tensile (IDT) test and Repeated Simple Shear Test at Constant Height (RSST-CH) are not in the scope of this review.

Witczak et al. (2000) tested asphalt mixes of $12.5 \mathrm{~mm}, 19 \mathrm{~mm}$, and $37.5 \mathrm{~mm}$ NMAS with PG 64-22 binder. Uniaxial compression tests were conducted with 70, 100, and $150 \mathrm{~mm}$ diameters and aspect ratios of 1, 1.5, 2, and 3. Samples were tested for complex modulus and repeated load permanent deformation. The complex modulus test was performed using controlled stress with nominal stress of 145 psi for high stiffness and 5 psi for low stiffness mixes to produce an axial strain in the range of 75 to 200 microstrain. According to Fremont et al. (2015), high stiffness asphalt mix ranges between 14,000 MPa and $23,000 \mathrm{MPa}$ at $15^{\circ} \mathrm{C}$ and $10 \mathrm{~Hz}$. Anything below 14,000 MPa is considered low stiffness asphalt mix. The repeated load permanent deformation test was conducted at $40^{\circ} \mathrm{C}$ with stress level of $20 \mathrm{psi}$ and 6000 pulse load cycles. Duration of load cycles was 0.1 seconds with rest time of 0.9 seconds. Strain was measured with two LVDTs mounted 180 degree apart in the middle of the specimen with gauge length equal to specimen diameter.

All specimens were fabricated from 150X160 mm SGC pills. For test specimens greater than 150 $\mathrm{mm}$ in height, specimens were stacked and glued with epoxy under 5 psi load to achieve the required aspect ratio. Two replicates were tested for each aspect ratio. The target air voids were:

\begin{tabular}{|c|c|}
\hline NMAS, mm & VTM, \% \\
\cline { 1 - 2 } 412.5 & \multirow{2}{*}{$4 \% \pm 0.5 \%$} \\
\cline { 1 - 2 } 37.5 & $4 \% \pm 1 \%$ \\
\hline
\end{tabular}

The results showed an increase in dynamic modulus value and increase in standard deviation of data for high stiffness test for specimens with aspect ratio of 1. It was suggested that this was due to the effect of friction, alignment and roughness from the specimen ends. The LVDT's for specimens with aspect ratio of 1 was mounted few millimeters below the loading platen and specimen interface. As a result, greater variability was anticipated from specimen roughness and stress or strain concentrations caused by misalignment as measurements were made closer to specimen ends. However, analysis of 
variance in low and high stiffness conditions concluded no significant difference in dynamic modulus due to specimen diameter or specimen aspect ratio. Although no statistically significant difference in dynamic modulus was identified due to aspect ratio, it was concluded that specimens should be tested with an aspect ratio of at least 1.5 .

For low and high stiffness conditions, phase angle decreased with increase in diameter suggesting as the diameter increases, specimen response is more elastic. The analysis of variance concluded no significant effect of aspect ratio at any temperature, but significant effect of diameter was found at $4^{\circ} \mathrm{C}$ and $40^{\circ} \mathrm{C}$. No explanation for the diameter effect on phase angle was found however, it was hypothesized that it may be caused due to radial changes in the structure of SGC pill.

The onset of tertiary flow is defined as flow number as it indicates resistance of mix to rutting. The flow number of samples with an aspect ratio of 1 was significantly higher than aspect ratios of $1.5,2$, and 3. Also, flow number for $70 \mathrm{~mm}$ diameter samples was less than $100 \mathrm{~mm}$, and $150 \mathrm{~mm}$ diameter samples. This suggested a $100 \mathrm{~mm}$ diameter with aspect ratio of 1.5 or greater is good for testing flow number. The coefficient of variance was large for $100 \mathrm{~mm}$ diameter specimen with an aspect ratio of 1.5. The coefficient of variance was minimized with an aspect ratio of 2 . The overall variance increased greatly for $19 \mathrm{~mm}$ and $37.5 \mathrm{~mm}$ NMAS asphalt mix compared to $12.5 \mathrm{~mm}$ NMAS asphalt mix.

Although 70X140 mm specimen was shown to provide low standard error in dynamic modulus test and low variability, 100X150 mm was recommended for permanent deformation test. Therefore, 100X150 mm specimen was recommended for both complex modulus and permanent deformation test (Witczak et al., 2000).

Chehab et al. (2000) analyzed four different geometries with $12.5 \mathrm{~mm}$ NMAS asphalt mix and 5.2\% PG 70-22 binder for dynamic modulus and constant crosshead-rate monotonic test until failure. Three replicates were used for each geometry with target air void content of $4 \% \pm 0.5 \%$. The test specimens were cored from SGC pill as:

\begin{tabular}{|c|c|}
\hline SGC Pill, mm & Specimen Dimension, mm \\
\hline $150 X 140$ & $75 \times 115$ \\
\hline $150 X 175$ & $75 \times 150$ \\
\cline { 2 - 2 } & $100 \times 150$ \\
\hline $\begin{array}{c}100 X 150 \text { plus two 100X25 } \\
\text { slices }\end{array}$ & $100 \times 200^{*}$ \\
\hline
\end{tabular}

*Two 100X25 mm sections were glued on top and bottom of 100X150 mm specimen. 
The specimens were preconditioned by applying 50 haversine loading at $10 \mathrm{~Hz}$ and $120 \mathrm{kPa}$ and tested for dynamic modulus and constant crosshead-rate monotonic test until failure. Each specimen was first tested for dynamic modulus followed by 2 hours of rest time to perform constant crosshead-rate monotonic test until failure. The dynamic modulus test was performed with 100 cycles for each loading frequency of $1 \mathrm{~Hz}, 2 \mathrm{~Hz}, 5 \mathrm{~Hz}, 10 \mathrm{~Hz}$, and $20 \mathrm{~Hz}$ at $40^{\circ} \mathrm{C}$. The stress level for dynamic modulus corresponding to strain of 40 microstrain was chosen to keep response within linear viscoelastic range. A 5 minutes rest time was used between each test frequency. For constant crosshead-rate monotonic test until failure, the loading rate was $0.0004 \mathrm{strains} / \mathrm{sec}$. The specimens were rested for two hours for sufficient material relaxation after dynamic modulus test and before crosshead-rate monotonic test. Four LVDTs were mounted at 90 degrees from each other. The gauge length was $57.5 \mathrm{~mm}$ for the $75 \mathrm{X} 115 \mathrm{~mm}$ specimen and $50 \mathrm{~mm}$ for other geometries.

The results showed that dynamic modulus for $100 \mathrm{X} 200 \mathrm{~mm}$ specimens was considerably higher than that of other geometries. The research attributed this to the effect of glue deforming between two sections, thereby relieving AC in the middle of specimen from applied stress. Other observations from dynamic modulus test were:

- At $1 \mathrm{~Hz}$, dynamic modulus increased with increase in diameter and aspect ratio.

- At $20 \mathrm{~Hz}$, dynamic modulus decreased with increase in aspect ratio and increased with increase in diameter.

- At 2 and $5 \mathrm{~Hz}$, phase angle of 100X150mm specimen was higher than that of 75X150 mm specimen.

- At $10 \mathrm{~Hz}, 100 \mathrm{X} 150 \mathrm{~mm}$ specimen had higher dynamic modulus than 75X150 mm specimen. Following observations were made for constant crosshead-rate monotonic test:

- The 75X115 mm and 100X200 mm specimens exhibited lowest strength from stress versus strain curves.

- The 75X150 and 100X150 mm specimens exhibited similar stress versus strain curves.

The study recommended 75X150 mm and 100X150 mm geometry and concluded that it may not be universally applied to other AC mixes and SGC pills (Chehab et al., 2000).

Kutay et al. (2009) conducted dynamic modulus test and cyclic push-pull fatigue test on AMPT for $12.5 \mathrm{~mm}$ NMAS asphalt mix with following binder types: 


\begin{tabular}{|c|}
\hline Binder Types \\
\hline PG 72-23 \\
\hline PG 79-28 \\
\hline PG 74-31 \\
\hline PG 74- 28 \\
\hline PG 70-22 \\
\hline PG 73-23 \\
\hline
\end{tabular}

The target air void of lab compacted specimens was $7 \%$ with average VTM ranging from 6.9 to $8 \%$. The $38.1 \mathrm{X} 150 \mathrm{~mm}$ specimens were cored horizontally from field slabs for push-pull fatigue test.

Dynamic modulus test was performed on only $100 \mathrm{X} 150 \mathrm{~mm}$ specimens at following temperatures and loading frequencies:

\begin{tabular}{|c|c|}
\hline Temperatures, ${ }^{\circ} \mathrm{C}$ & Frequency, $\mathrm{Hz}$ \\
\hline 4 & 20 \\
\hline 19 & 10 \\
\hline 31 & 5 \\
\hline 46 & 0.5 \\
\hline 58 & 0.1 \\
\hline
\end{tabular}

Cyclic push pull tests were performed on 71.4X150 mm and 38.1X100 mm samples cored from the center of SGC pill. Three replicates for each geometry were tested. In cyclic push-pull test, 71.4X150 $\mathrm{mm}$ specimen was tested in stress controlled of $\pm 610 \mathrm{kPa}$ and strain controlled of $\pm 300 \mu \epsilon$. Additionally, $38.1 \mathrm{X} 150 \mathrm{~mm}$ specimen was tested in strain controlled of $\pm 300 \mu \epsilon$.

The dynamic modulus results from 100X150 mm specimens were in good agreement for all 12.5 mm NMAS mixes with different binder types. Dynamic modulus from the master curve at $19^{\circ} \mathrm{C}$ and 10 $\mathrm{Hz}$ ranked $12.5 \mathrm{~mm}$ asphalt mix with different binder from low to high modulus as:

\begin{tabular}{|c|}
\hline Binder Types \\
\hline PG 74-31 \\
\hline PG 74-28 \\
\hline PG 79-28 \\
\hline PG 72-23 \\
\hline PG 73-23 \\
\hline PG 70-22 \\
\hline
\end{tabular}

The damage characterization curve for $71.4 \mathrm{X} 150 \mathrm{~mm}$ and $38.1 \mathrm{X} 100 \mathrm{~mm}$ samples fabricated from different binder types except PG 74-28, collapsed on a single curve. This indicated that $38.1 \mathrm{X} 100 \mathrm{~mm}$ 
samples can provide same information as $71.4 \mathrm{X} 150 \mathrm{~mm}$ samples. The research concluded that $38.1 \mathrm{X} 100$ mm samples can be used to characterize asphalt pavements (Kutay et al., 2009).

Li and Gibson (2012) conducted dynamic modulus on seven plant and lab produced asphalt mixes of $4.75 \mathrm{~mm}, 9.5 \mathrm{~mm}, 12.5 \mathrm{~mm}$, and $19 \mathrm{~mm}$ NMAS asphalt mix. Dynamic modulus was tested on three replicates for $100 \times 150 \mathrm{~mm}, 38 X 140 \mathrm{~mm}$, and $38 X 110 \mathrm{~mm}$ with $70 \mathrm{~mm}$ gauge length at following test temperatures and frequencies.

\begin{tabular}{|c|c|}
\hline Temperature, ${ }^{\circ} \mathrm{C}$ & Frequency, $\mathrm{Hz}$ \\
\hline 4.4 & 25 \\
\hline 21.1 & 10 \\
\hline 37.8 & 5 \\
\hline 54.4 & 1 \\
\hline & 0.5 \\
\cline { 2 - 2 } & 0.1 \\
\hline
\end{tabular}

The pull-pull cyclic fatigue test was performed on four plant and lab produced asphalt mixes of $4.75 \mathrm{~mm}, 9.5 \mathrm{~mm}$, and $19 \mathrm{~mm}$ NMAS asphalt mix. The cyclic fatigue test was performed on 100X150 $\mathrm{mm}$ and $38 \mathrm{X} 110 \mathrm{~mm}$ specimens with a $70 \mathrm{~mm}$ gauge length at $21^{\circ} \mathrm{C}$ and $10 \mathrm{~Hz}$. For target failure cycle of 1,000 to 10,000 , the range of microstrain was set between 50 and 70 . The cyclic fatigue data was used to predict fatigue life at $200 \mu \epsilon$ and $21^{\circ} \mathrm{C}$. The binder ranged from PG 58-34 to PG 82-22. Target air voids were $3.5 \%, 6.5 \%, 7 \%$, and $9 \%$ for dynamic modulus and fatigue tests. SGC pills were compacted at $150 \mathrm{X} 185 \mathrm{~mm}$ to fabricate $100 \mathrm{X} 150 \mathrm{~mm}, 38 \mathrm{X} 110 \mathrm{~mm}$, and 38X140 mm samples. The 100X150 mm specimen was obtained from the center of SGC pills and for $38 \mathrm{~mm}$ diameter specimens, six samples were cored from each pill as shown in Figure 6.

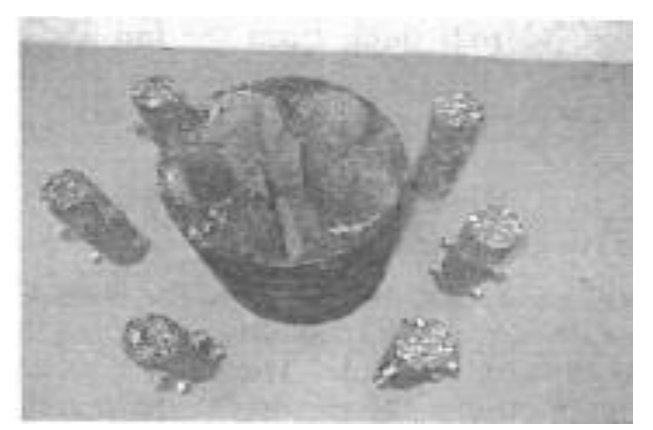

Figure 6: $38 \mathrm{~mm}$ diameter specimens cored from $150 \mathrm{~mm}$ diameter specimen (Li and Gibson, 2012)

The dynamic modulus results showed: 
- $38 X 110 \mathrm{~mm}$ and 38X140 mm specimens had lower dynamic modulus value at high temperature and low frequency. 38X110 mm and 38X140 mm specimens had higher phase angle compared to 100X150 mm specimen.

- The $9.5 \mathrm{~mm}$ NMAS mix had statistically different dynamic modulus value for all frequencies at $4.4{ }^{\circ} \mathrm{C}$ and $21.1^{\circ} \mathrm{C}$. For other mixes, statistically different dynamic modulus value was found at $37.8^{\circ} \mathrm{C}$ and $54.4^{\circ} \mathrm{C}$. The statistical difference in dynamic modulus value was thought to be due to aspect ratio of specimen geometries.

The 100X150 mm and 38X110 mm specimens predicted same fatigue life ranking for $4.75 \mathrm{~mm}$, $9.5 \mathrm{~mm}$, and one of the two $19 \mathrm{~mm}$ NMAS asphalt mix. The other $19 \mathrm{~mm}$ NMAS asphalt mix with PG 58-34 binder showed high fatigue resistance for 100X150 mm specimens compared to 38X110 mm sample. This was attributed to testing at $21^{\circ} \mathrm{C}$ for all four mixes instead of testing at temperature based on PG grade of binder. Li and Gibson concluded that although the fatigue life for 100X150 mm sample was higher than that of $38 \mathrm{X} 110 \mathrm{~mm}$ samples, the values were comparable as shown in Table 8 .

Table 8: Failure Cycles for 100X150 mm and 38X110 mm in Cyclic Fatigue Test (Li and Gibson, 2012)

\begin{tabular}{|c|c|c|c|c|c|c|c|c|}
\hline & \multicolumn{2}{|c|}{$4.75 \mathrm{~mm}$ NMAS } & \multicolumn{2}{|c|}{$9.5 \mathrm{~mm}$ NMAS } & \multicolumn{2}{|c|}{$19 \mathrm{~mm}$ NMAS } & \multicolumn{2}{|c|}{$19 \mathrm{~mm}$ NMAS } \\
\hline & $\begin{array}{c}100 X 150 \\
\mathrm{~mm}\end{array}$ & $\begin{array}{c}38 X 110 \\
\mathrm{~mm}\end{array}$ & $\begin{array}{c}100 X 150 \\
\mathrm{~mm}\end{array}$ & $\begin{array}{c}38 X 110 \\
\mathrm{~mm}\end{array}$ & $\begin{array}{c}100 \mathrm{X} 150 \\
\mathrm{~mm}\end{array}$ & $\begin{array}{c}38 X 110 \\
\mathrm{~mm}\end{array}$ & $\begin{array}{c}100 X 150 \\
\mathrm{~mm}\end{array}$ & $\begin{array}{c}38 X 110 \\
\mathrm{~mm}\end{array}$ \\
\hline $\begin{array}{l}\text { Failure } \\
\text { Cycles } \\
\text { (x1M) }\end{array}$ & 24,000 & 22,800 & 5,730 & 9,170 & 3,210 & 6,250 & 4,620 & 512 \\
\hline
\end{tabular}

Park and Kim (2013) tested uniaxial dynamic modulus test and controlled crosshead cyclic direct tension test on 9.5 mm NMAS asphalt mix with PG 70-22 SCS modified binder. The research investigated two prismatic specimens of $25 \mathrm{~mm}$ thick, $50 \mathrm{~mm}$ wide, and $100 \mathrm{~mm}$ long, two 38X100 mm specimens, and one 75X150 mm specimen obtained from $150 \mathrm{~mm}$ diameter field core as shown in Figure 7. 


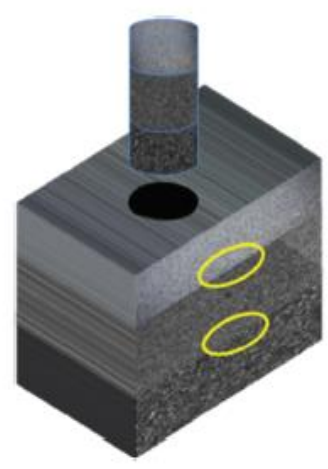

(a)

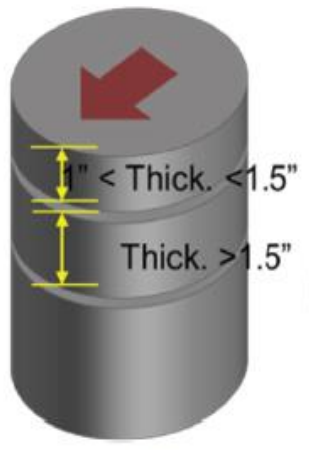

(b)

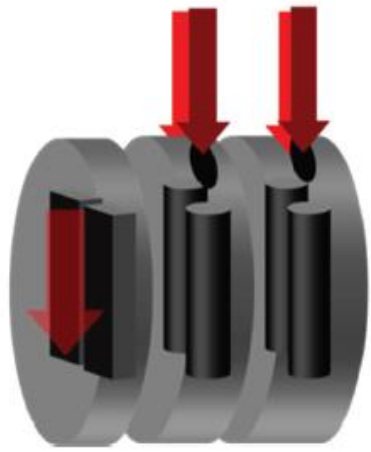

(c)

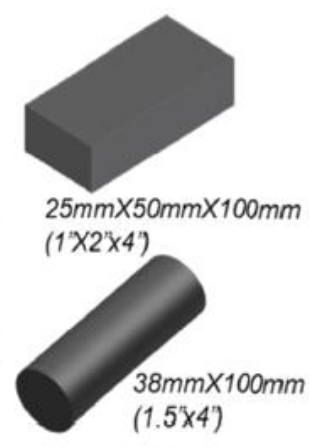

(d)

Figure 7: Sample Fabrication Process: a) $150 \mathrm{~mm}$ Field Core, b) Sublayer Thickness in the Core, c) Horizontal Coring to Obtain Prismatic and Cylindrical small specimens, d) Prismatic and Small Performance Test Specimens(Park and Kim, 2013)

Controlled crosshead cyclic direct tension tests were performed at $19^{\circ} \mathrm{C}$ and $10 \mathrm{~Hz}$ and dynamic modulus was conducted at:

\begin{tabular}{|c|c|}
\hline Temperature, ${ }^{\circ} \mathrm{C}$ & Frequency, $\mathrm{Hz}$ \\
\hline \multirow{6}{*}{$5^{\circ} \mathrm{C}$} & $25 \mathrm{~Hz}$ \\
\hline & $10 \mathrm{~Hz}$ \\
\hline & $5 \mathrm{~Hz}$ \\
\hline & $1 \mathrm{~Hz}$ \\
\hline & $0.5 \mathrm{~Hz}$ \\
\hline & $0.1 \mathrm{~Hz}$ \\
\hline \multirow{6}{*}{$20^{\circ} \mathrm{C}$} & $25 \mathrm{~Hz}$ \\
\hline & $10 \mathrm{~Hz}$ \\
\hline & $5 \mathrm{~Hz}$ \\
\hline & $1 \mathrm{~Hz}$ \\
\hline & $0.5 \mathrm{~Hz}$ \\
\hline & $0.1 \mathrm{~Hz}$ \\
\hline \multirow{6}{*}{$40^{\circ} \mathrm{C}$} & $25 \mathrm{~Hz}$ \\
\hline & $10 \mathrm{~Hz}$ \\
\hline & $5 \mathrm{~Hz}$ \\
\hline & $1 \mathrm{~Hz}$ \\
\hline & $0.5 \mathrm{~Hz}$ \\
\hline & $0.1 \mathrm{~Hz}$ \\
\hline
\end{tabular}

The dynamic modulus results showed that dynamic modulus values were very close for different geometries and tested statistically the same. The damage characterization curve for $75 \times 150 \mathrm{~mm}$ specimens was below 38X100 mm specimens however, they were statistically same. For the ease of testing, 38X100 mm specimens were recommended (Park and Kim, 2013). 
Bower et al. (2015) conducted dynamic modulus test (per AASHTO TP 79) on 38X135 mm, 50X135 mm, 38X110 mm, 50X110 mm and 100X150 mm specimens with LVDT gauge length of 70 mm. Plant produced asphalt mixes of $9.5 \mathrm{~mm}, 12.5 \mathrm{~mm}, 19 \mathrm{~mm}$, and $25 \mathrm{~mm}$ NMAS were used with binder grades of PG 64-22, PG 70-22, and PG 76-22. Three replicate specimens were tested. The cored samples were prepared with a target air voids of $7 \%$ to $8 \%$. However, only $50 \%$ of the sample VTM ranged between $6.5 \%$ and $7 \%$. The 38X135 mm, 50X135 mm, 38X110 mm, and 50X110 mm specimens were horizontally cored from SGC pill as shown in Figure 8. Generally, three small specimens were cored from SGC pill however, if the pill was less than $200 \mathrm{~mm}$ in height, only two specimens were obtained. The dynamic modulus was tested at following temperatures and frequencies with peak to peak microstrain between 75 and 125 . 


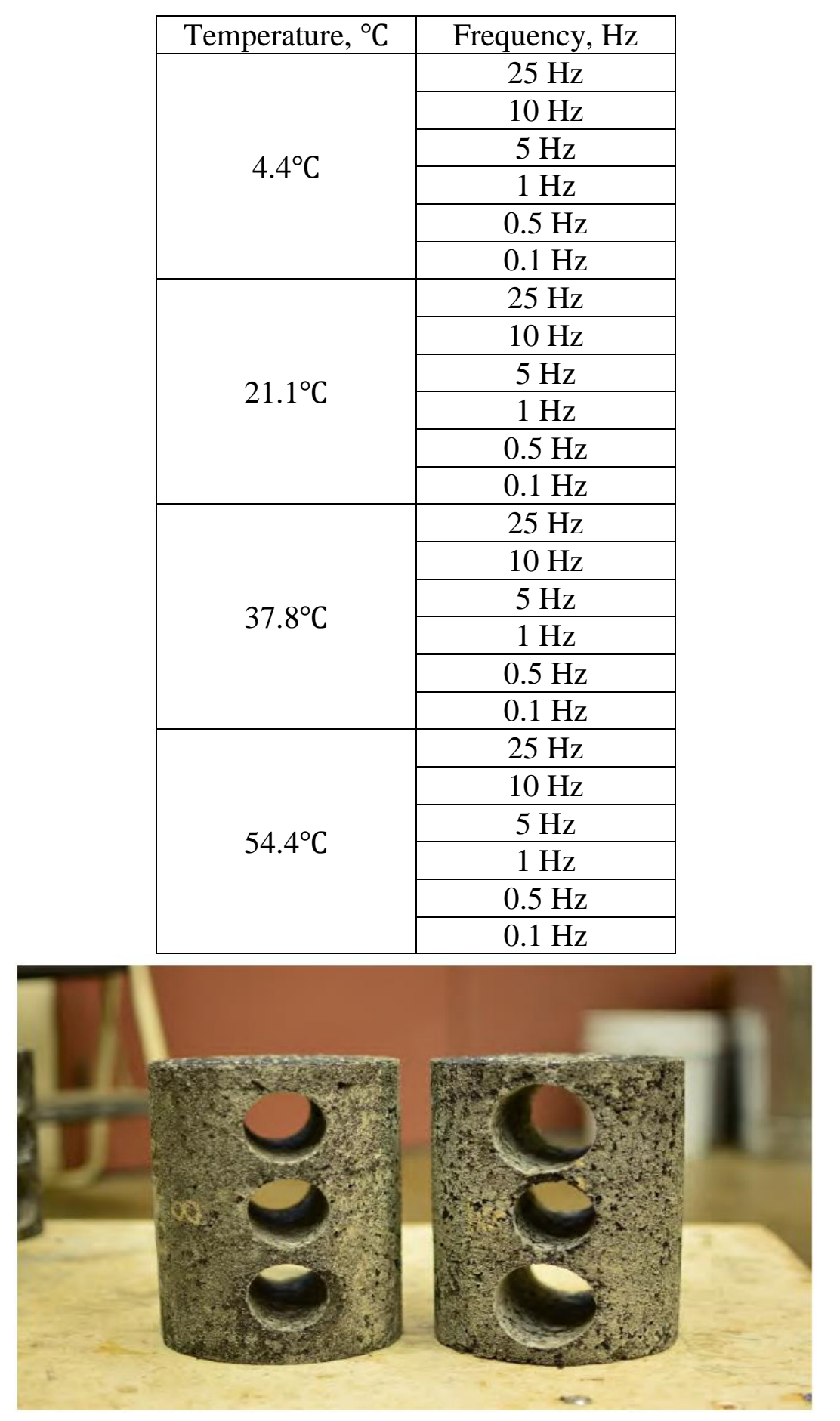

Figure 8: $38 \mathrm{~mm}$ and $50 \mathrm{~mm}$ diameter specimens cored from SGC pill (Bowers et al., 2015)

The results showed that although sample to sample variability was highest for small scale specimens (38X135 mm, 50X135 mm, 38X110 mm, and 50X110 mm) at $54.4^{\circ} \mathrm{C}$, they were statistically same to 100X150 mm sample for all mixes. Further investigation into aggregate anisotropy, effects of air voids, and NMAS was recommended. The analysis recommended $38 \mathrm{~mm}$ diameter specimens to be used 
for $9.5 \mathrm{~mm}$ and $12.5 \mathrm{~mm}$ NMAS mix and $50 \mathrm{~mm}$ diameter specimens to be used for $19 \mathrm{~mm}$ and $25 \mathrm{~mm}$ NMAS diameter mix (Bowers et al., 2015).

Lee et al. (2017a) conducted dynamic modulus and DTC fatigue test on 9.5 mm NMAS asphalt mixture with 5.2\% virgin PG 70-22 binder and 19\% Reclaimed Asphalt Pavement (RAP). The 1.2\% by weight of PG 64-22 binder was obtained from the RAP. The same research was also published under NCHRP Idea Project 181 by Castorena et al. (2017)

The dynamic modulus test was conducted (per AASHTO TP 79) on three replicates of only 100X150 mm specimens with $70 \mathrm{~mm}$ gauge length, microstrain levels between 50 and 75 and temperatures and frequencies as:

\begin{tabular}{|c|c|}
\hline Temperature, ${ }^{\circ} \mathrm{C}$ & Frequency, $\mathrm{Hz}$ \\
\hline \multirow{6}{*}{$5^{\circ} \mathrm{C}$} & $25 \mathrm{~Hz}$ \\
\hline & $10 \mathrm{~Hz}$ \\
\hline & $5 \mathrm{~Hz}$ \\
\hline & $1 \mathrm{~Hz}$ \\
\hline & $0.5 \mathrm{~Hz}$ \\
\hline & $0.1 \mathrm{~Hz}$ \\
\hline \multirow{6}{*}{$20^{\circ} \mathrm{C}$} & $25 \mathrm{~Hz}$ \\
\hline & $10 \mathrm{~Hz}$ \\
\hline & $5 \mathrm{~Hz}$ \\
\hline & $1 \mathrm{~Hz}$ \\
\hline & $0.5 \mathrm{~Hz}$ \\
\hline & $0.1 \mathrm{~Hz}$ \\
\hline \multirow{6}{*}{$40^{\circ} \mathrm{C}$} & $25 \mathrm{~Hz}$ \\
\hline & $10 \mathrm{~Hz}$ \\
\hline & $5 \mathrm{~Hz}$ \\
\hline & $1 \mathrm{~Hz}$ \\
\hline & $0.5 \mathrm{~Hz}$ \\
\hline & $0.1 \mathrm{~Hz}$ \\
\hline \multirow{6}{*}{$54.4^{\circ} \mathrm{C}$} & $25 \mathrm{~Hz}$ \\
\hline & $10 \mathrm{~Hz}$ \\
\hline & $5 \mathrm{~Hz}$ \\
\hline & $1 \mathrm{~Hz}$ \\
\hline & $0.5 \mathrm{~Hz}$ \\
\hline & $0.1 \mathrm{~Hz}$ \\
\hline
\end{tabular}

The DTC fatigue was conducted on 75X130 mm, 75X150 mm, 100X130 mm, and 100X150 mm samples. Four replicates were tested (per AASHTO TP 107) at $18^{\circ} \mathrm{C}$ and $10 \mathrm{~Hz}$ to determine damage characterization curves. 
The results showed that semi-log scale better demonstrated dynamic modulus values at low temperature and high frequency conditions. The log-log scale better demonstrated dynamic modulus values at high temperature and low frequency conditions.

The results showed that $75 \times 150 \mathrm{~mm}$ and $100 \mathrm{X} 150 \mathrm{~mm}$ specimens had end failure. Despite the end failure, damage characterization curve for 75X150 mm and 100X150 mm samples collapsed with 75X130 mm and 100X130 mm samples. Additionally, 100X150 mm and 100X130 mm samples' damage characterization curves were statistically same as those of 75X150 mm and 75X130 mm samples.

The end failure can cause significant errors and high variability for failure criteria as data is not recorded effectively by LVDTs. Although, damage characterization curves was not affected by end failure, the on-specimen deformation error was large, indicating the importance of new geometry that increases the propensity for failure within the gauge length. Although, 75X130 and 100X130 samples met RVE requirement (per ASTM D-3497) of minimum diameter to MAS ratio of 4 to 1 and an aspect ratio of 2 to $1,100 \mathrm{X} 130 \mathrm{~mm}$ specimen was recommended as it will satisfy RVE requirements for higher NMAS asphalt mixes (Lee et al., 2017a).

Lee et al. (2017b) tested dynamic modulus on $9.5 \mathrm{~mm}, 12.5 \mathrm{~mm}, 19 \mathrm{~mm}$, and $25 \mathrm{~mm}$ NMAS asphalt mixes with PG 64-22 and PG 76-22 binder types. The DTC fatigue test was performed on 9.5 mm, $19.5 \mathrm{~mm}$, and $25 \mathrm{~mm}$ NMAS mixes with PG 64-22 binder. The target air void content for all specimens was $4 \% \pm 0.5 \%$, however, only $60 \%$ of small test specimens had acceptable air voids. The dynamic modulus test (per AASHTO TP 79) was performed on 38X110 mm and 100X150 mm specimen with target on specimen microstrain of 63 with allowable microstrain range between 50 and 75 . Three replicate specimens were tested with $70 \mathrm{~mm}$ gauge length at temperatures and frequencies: 


\begin{tabular}{|c|c|}
\hline Temperature, ${ }^{\circ} \mathrm{C}$ & Frequency, $\mathrm{Hz}$ \\
\hline \multirow[t]{6}{*}{$5^{\circ} \mathrm{C}$} & $25 \mathrm{~Hz}$ \\
\hline & $10 \mathrm{~Hz}$ \\
\hline & $5 \mathrm{~Hz}$ \\
\hline & $1 \mathrm{~Hz}$ \\
\hline & $0.5 \mathrm{~Hz}$ \\
\hline & $0.1 \mathrm{~Hz}$ \\
\hline \multirow[t]{6}{*}{$20^{\circ} \mathrm{C}$} & $25 \mathrm{~Hz}$ \\
\hline & $10 \mathrm{~Hz}$ \\
\hline & $5 \mathrm{~Hz}$ \\
\hline & $1 \mathrm{~Hz}$ \\
\hline & $0.5 \mathrm{~Hz}$ \\
\hline & $0.1 \mathrm{~Hz}$ \\
\hline \multirow[t]{6}{*}{$40^{\circ} \mathrm{C}$} & $25 \mathrm{~Hz}$ \\
\hline & $10 \mathrm{~Hz}$ \\
\hline & $5 \mathrm{~Hz}$ \\
\hline & $1 \mathrm{~Hz}$ \\
\hline & $0.5 \mathrm{~Hz}$ \\
\hline & $0.1 \mathrm{~Hz}$ \\
\hline \multirow[t]{6}{*}{$54.4^{\circ} \mathrm{C}$} & $25 \mathrm{~Hz}$ \\
\hline & $10 \mathrm{~Hz}$ \\
\hline & $5 \mathrm{~Hz}$ \\
\hline & $1 \mathrm{~Hz}$ \\
\hline & $0.5 \mathrm{~Hz}$ \\
\hline & $0.1 \mathrm{~Hz}$ \\
\hline
\end{tabular}

DTC fatigue test was performed (per AASHTO TP 107) with 100X130 mm, $38 X 110 \mathrm{~mm}$, and prismatic specimens of $25 \mathrm{~mm}$ in thickness, $50 \mathrm{~mm}$ in width, and $110 \mathrm{~mm}$ in height at $18^{\circ} \mathrm{C}$ and $10 \mathrm{~Hz}$. The fabrication of specimens is shown in Figure 9.

The dynamic modulus and phase angle master curves for 38X110 mm and 100X150 mm specimens were statistically same for different NMAS asphalt mixes at low and intermediate temperatures. However, at high temperature, $38 X 110 \mathrm{~mm}$ specimen displayed higher dynamic modulus and lower phase angle values compared to $100 \mathrm{X} 150 \mathrm{~mm}$ specimens.

Damage characterization curves were in good agreement for all test geometries however two test specimen results demonstrating outlier behavior were omitted in demonstrating damage characterization curves for $9.5 \mathrm{~mm}$ NMAS mix. It was concluded that additional $38 \mathrm{X} 110 \mathrm{~mm}$ specimens will be required to obtain representative damage curve and failure criterion. 


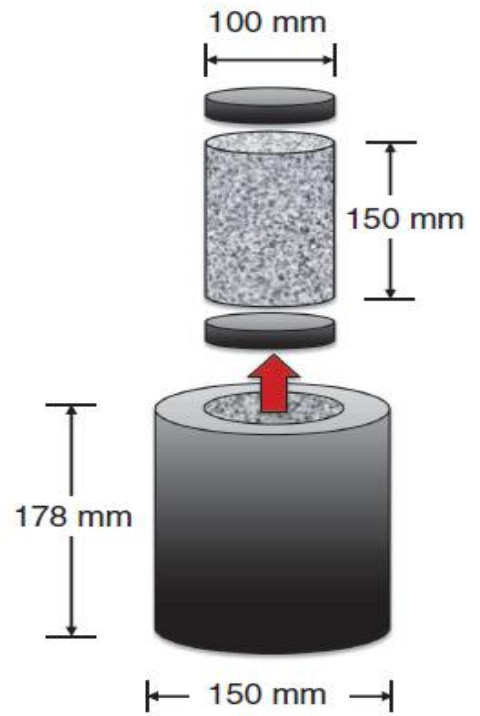

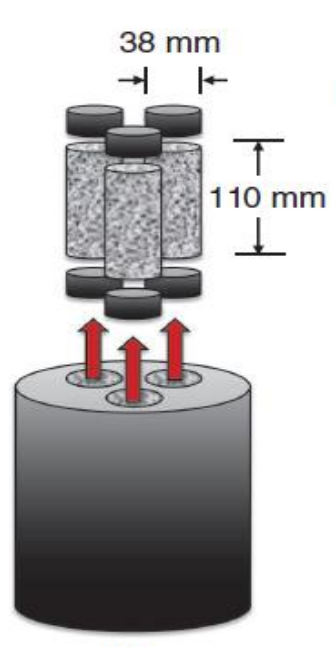

(b)

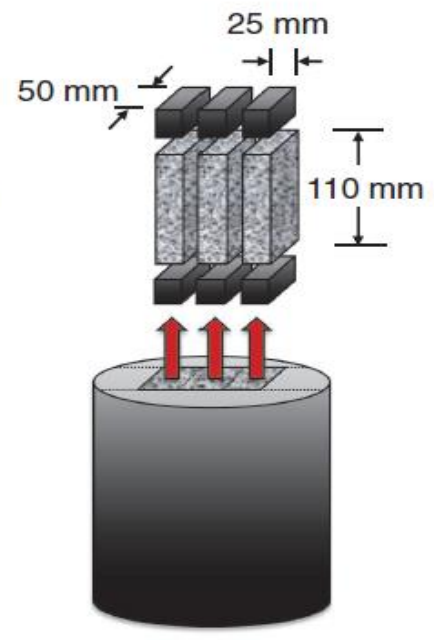

(c)

(a)

Figure 9: Fabrication of Test Specimen (Lee el al., 2017b)

The damage characterization curve for 19 mm NMAS asphalt mix showed good agreement between 100X130 mm and 38X110 mm specimens however, two out of four 38X110 mm test specimens resulted in end failure. The research attributed end failure to damage localization near larger aggregate present close to the edge of specimen. A high sample to sample variability was observed in $25 \mathrm{~mm}$ NMAS mix for 38X110 mm and 100X130 mm specimens in DTC fatigue test. Six out of eight 100X130 $\mathrm{mm}$ specimen of $25 \mathrm{~mm}$ NMAS resulted in end failure. The 38X110 mm specimen was recommended due to ease of fabrication (Lee et al., 2017b).

Karr (2018) proposed new test geometry with the goal of reducing end failure in DTC fatigue test specimens from field cores. The research used four types of asphalt mixes:

\begin{tabular}{|c|c|c|c|}
\hline Asphalt Mix Name & NMAS, mm & Binder Type & \% Binder \\
\hline STIV & 12.5 & \multirow{2}{*}{ PG 64-16 } & 6.2 \\
\cline { 4 - 4 } STIV-R & 12.5 & & 6.0 \\
\hline FAA3Q & 12.5 & PG 70-22 SBS-modified & 5.8 \\
\hline TLO & 9.5 & PG 72-22 SBS-modified & 7.5 \\
\hline
\end{tabular}

The STIV-R mix contained 20\% RAP. The prismatic specimen was sliced horizontally from 150 $\mathrm{mm}$ diameter cylindrical pill and dimensions of $110 \mathrm{~mm}$ long by $100 \mathrm{~mm}$ wide with thickness between 25 to $38 \mathrm{~mm}$, depending on the thickness of the pavement layer, as shown in Figure 10. 

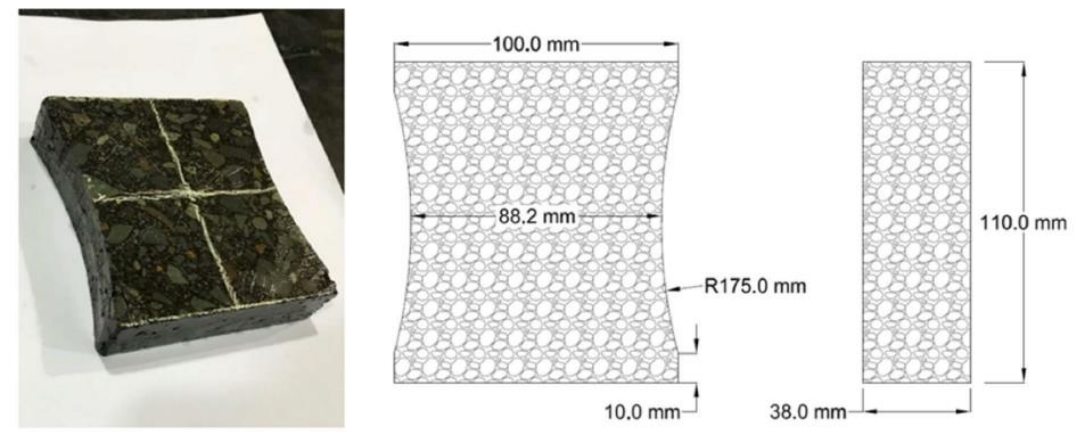

Figure 10: New Prismatic Specimen Proposed for DTC Fatigue Test (Karr, 2018)

The specimens were fabricated by cutting a horizontal slice from the SGC pill and discarding two end slices. The horizontal slices were cut into a rectangular shape. Finally, the radius of $175 \mathrm{~mm}$ was achieved from a jig with arched back that cut the specimen about the blade. Up to three specimens were fabricated from a SGC pill and took up to 1 to 2 hours to cut the desired specimen geometries from one pill. A typical glued test specimen on the fatigue platen is shown in Figure 11.

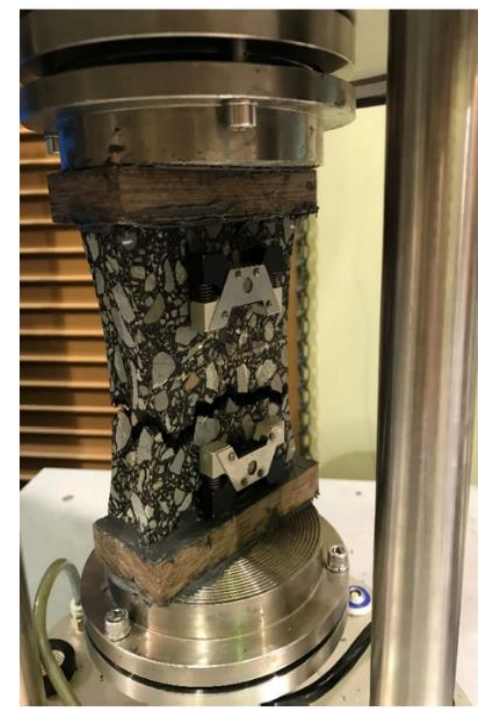

\section{Figure 11: Tapered Prismatic Test Specimen on DTC Fatigue Platen (Karr, 2018)}

The results showed that tapered prismatic specimen reduced the end failure drastically. The TLO and FAA3Q mixes with modified binder had higher number of cycles to failure compared to STIV and STIV-R. Karr (2018) also tested the effect of air voids on fatigue test and concluded that increase in VTM reduces fatigue life. One of the drawbacks was alignment of the specimen onto the platen and de-bonding issues. The specimen had to be fabricated properly because any eccentricity resulted in separation of specimen from platen and wastage of that specimen (Karr, 2018). 


\subsection{COMMENTARY ON LITERATURE REVIEW}

The summary of aspect ratio and diameter to MAS ratio from literature is shown in Table 9, Table 10, Table 11, and Table 12.

Witczak et al. (2000) performed very detailed dynamic modulus testing with different NMAS asphalt mix and aspect ratios. However, did not perform a similar extent of research for DTC fatigue test in AMPT. Additionally, the smallest specimen size in this research was $70 X 70 \mathrm{~mm}$. More research is required to prove if $38 \mathrm{X} 110 \mathrm{~mm}$ specimens will perform similar results.

Chehab et al. (2000) analyzed only $12.5 \mathrm{~mm}$ NMAS asphalt mix with smallest specimen size of $75 \mathrm{X} 115 \mathrm{~mm}$. More research is required for higher NMAS mix with smaller specimen size such as $38 \times 110 \mathrm{~mm}$.

Kutay (2009) tested 71.4X150 mm and 38.1X100 mm specimens for 12.5 mm NMAS mix. No statistical analysis was performed to conclude that $38.1 \mathrm{X} 100 \mathrm{~mm}$ samples can provide same information as $71.4 \mathrm{X} 150 \mathrm{~mm}$ samples because damage characterization curves from different binder types except PG 74 - 28 collapsed on a single curve. Additionally, it is important to verify if small sample geometry will provide significant results for larger NMAS mix such as $19 \mathrm{~mm}$ and $25 \mathrm{~mm}$ NMAS. More research is needed to compare results of small specimen geometry such as $71.4 \mathrm{X} 150 \mathrm{~mm}$ and $38.1 \mathrm{X} 100 \mathrm{~mm}$ to standard full-size specimens of 100X150 mm and 100X1130 mm.

Li and Gibson (2012) cored six 38X110 mm specimens very close to the outside edge of SGC pill. This procedure may have resulted in non-uniform air void distribution within the test sample. No further test was done to assess test specimen uniformity by cutting the specimen into equal sections. More research is needed to verify how far away from the center of SGC pill can small test specimens be cored and sawed to result in less air void non-uniformity. Additionally, although Li and Gibson (2012) conducted dynamic modulus and DTC fatigue testing for small size and large specimens on larger NMAS of up to $19 \mathrm{~mm}$, the results were not accurately described. For the DTC fatigue testing, it was concluded that $38 \mathrm{X} 110 \mathrm{~mm}$ and $100 \mathrm{X} 150 \mathrm{~mm}$ specimens predicted same fatigue life ranking for $4.75 \mathrm{~mm}, 9.5 \mathrm{~mm}$, and one of the two $19 \mathrm{~mm}$ NMAS asphalt mix. The other $19 \mathrm{~mm}$ NMAS AC mix showed high fatigue resistance for 100X150 mm specimens compared to 38X110 mm sample. This was not supported by the data as shown in Table 13. Additionally, the research concluded that fatigue life was comparable between 38X110 mm and 100X150 mm specimens however, no statistical analysis was performed.

Park and Kim (2013) tested 38X100 mm and 75X150 mm specimens for only $9.5 \mathrm{~mm}$ NMAS asphalt mix. Although the results were meaningful, more research is needed to prove if $38 \mathrm{X} 110 \mathrm{~mm}$ 
specimens can be used for higher NMAS mix. Additionally, this research did not compare the results to standard full-size specimens of 100X150 mm and 100X1130 mm.

Bower (2015) compacted SGC pill to $150 \mathrm{X} 200 \mathrm{~mm}$ to be cored and sawed to desired test specimens. The height of the SGC mold is $250 \mathrm{~mm}$, with the top plate inside the mold. In our experience it was very difficult to fit higher NMAS of $19 \mathrm{~mm}$ and $25 \mathrm{~mm}$ inside the mold and achieve $200 \mathrm{~mm}$ height with $7 \pm 0.5 \%$ VTM. However, this can be done if mix is put into the mold in lifts and each lift is rodded into the lift below. Smaller NMAS mix such as $4.75 \mathrm{~mm}, 9.5 \mathrm{~mm}$, and $12.5 \mathrm{~mm}$ might be able to fit in to the mold with little or no rodding action to achieve 7+_0.5\% VTM. Although, Bower (2015) results are promising for dynamic modulus test, more research is needed to evaluate how 50X110 mm small specimens will perform in DTC fatigue test. Also, since Bower (2015) cored the specimens horizontally, it is important to see how $50 \mathrm{~mm}$ specimens vertically cored from SGC pill will affect dynamic modulus and fatigue results

Lee et al., (2017a) performed dynamic modulus test on only 100X150 $\mathrm{mm}$ specimen. The smallest test specimen for DTC fatigue testing used in this research was $75 \mathrm{X} 130 \mathrm{~mm}$. Additionally, this research was done on $9.5 \mathrm{~mm}$ NMAS asphalt mix. More research is needed to prove if 38X110 mm will provide similar results for higher NMAS mix.

Lee et al., (2017b) compared 38X110 mm specimens with 100X150 mm specimens for smaller and larger NMAS asphalt mix. In DTC Fatigue testing, 38X110 mm specimens did not perform well in 19 mm and 25 mm NMAS asphalt mix. The research manipulated the data by omitting two outliers to demonstrate damage characterization curves for $9.5 \mathrm{~mm}$ NMAS mix. No statistical analysis was performed in dynamic modulus or DTC fatigue test to determine agreement between 100X130 mm and 38X110 mm specimens. More research is needed to determine a good small size specimen for higher NMAS.

Karr (2018) performed DTC fatigue test on tapered prismatic new geometry however did not compare results to standard full-size geometry of $100 \mathrm{X} 150 \mathrm{~mm}$ and $100 \mathrm{X} 130 \mathrm{~mm}$. It is unknown how the results would compare. Although the results were promising for $9.5 \mathrm{~mm}$ and $12.5 \mathrm{~mm}$ NMAS asphalt mix, this research could be extended to larger NMAS asphalt mixes such as $19 \mathrm{~mm}$ and $25 \mathrm{~mm}$. 
Table 9: Summary of Diameter to MAS Ratio on Dynamic Modulus Test from Different Studies in Literature

\begin{tabular}{|c|c|c|c|c|c|}
\hline & $\begin{array}{c}\text { Diameter } \\
(\mathrm{mm})\end{array}$ & $\begin{array}{c}\text { NMAS } \\
(\mathrm{mm})\end{array}$ & $\begin{array}{l}\text { MAS } \\
(\mathrm{mm})\end{array}$ & $\begin{array}{l}\text { Diameter: } \\
\text { MAS }\end{array}$ & Summary \\
\hline \multirow{4}{*}{$\begin{array}{c}\text { Current } \\
\text { specification }\end{array}$} & 38 & 4.75 & 9.5 & $4.0: 1$ & \multirow{4}{*}{ N/A } \\
\hline & 38 & 19 & 25 & $1.5: 1$ & \\
\hline & 100 & 4.75 & 9.5 & $10.5: 1$ & \\
\hline & 100 & 25 & 37.5 & $2.7: 1$ & \\
\hline \multirow{9}{*}{$\begin{array}{l}\text { Witczak et } \\
\text { al. }(2000)\end{array}$} & 70 & 12.5 & 19 & $3.7: 1$ & \multirow{9}{*}{$\begin{array}{c}\text { For low and high stiffness } \\
\text { conditions, phase angle decreased } \\
\text { with increase in diameter } \\
\text { suggesting that as the diameter } \\
\text { increases, specimen response } \\
\text { becomes more elastic. Flow } \\
\text { number for } 70 \text { mm diameter } \\
\text { samples was less than } 100 \mathrm{~mm} \\
\text { and } 150 \mathrm{~mm} \text { diameter samples. } \\
\text { The overall variance in flow } \\
\text { number increased greatly for } 19 \\
\text { mm and } 37.5 \text { mm NMAS asphalt } \\
\text { mix compared to } 12.5 \text { mm } \\
\text { NMAS asphalt mix. }\end{array}$} \\
\hline & 70 & 19 & 25 & $2.8: 1$ & \\
\hline & 70 & 37.5 & 50 & $1.4: 1$ & \\
\hline & 100 & 12.5 & 19 & $5.3: 1$ & \\
\hline & 100 & 19 & 25 & $4.0: 1$ & \\
\hline & 100 & 37.5 & 50 & $2.0: 1$ & \\
\hline & 150 & 12.5 & 19 & $7.9: 1$ & \\
\hline & 150 & 19 & 25 & 6.0:1 & \\
\hline & 150 & 37.5 & 50 & 3.0:1 & \\
\hline \multirow[b]{2}{*}{$\begin{array}{l}\text { Chehab et } \\
\text { al. (2000) }\end{array}$} & 75 & 12.5 & 19 & $3.9: 1$ & \multirow{2}{*}{$\begin{array}{c}\text { At } 1 \mathrm{~Hz} \text {, dynamic modulus } \\
\text { increased with increase in } \\
\text { diameter. At } 20 \mathrm{~Hz} \text {, dynamic } \\
\text { modulus increased with increase } \\
\text { in diameter. }\end{array}$} \\
\hline & 100 & 12.5 & 19 & 5.3:1 & \\
\hline $\begin{array}{l}\text { Kutay et al. } \\
\text { (2009) }\end{array}$ & 100 & 12.5 & 19 & 8.0:1 & $\begin{array}{l}\text { The dynamic modulus results } \\
\text { from } 100 X 150 \text { mm specimens } \\
\text { were in good agreement for all } \\
12.5 \text { mm NMAS mix with } \\
\text { different binder types. }\end{array}$ \\
\hline \multirow{8}{*}{$\begin{array}{l}\text { Li and } \\
\text { Gibson } \\
(2012)\end{array}$} & 38 & 4.75 & 9.5 & $4.0: 1$ & \multirow{8}{*}{$\begin{array}{l}\text { The } 9.5 \mathrm{~mm} \text { NMAS mix had } \\
\text { statistically different dynamic } \\
\text { modulus value for all frequencies } \\
\text { at } 4.4^{\circ} \mathrm{C} \text { and } 21.1^{\circ} \mathrm{C} \text {. The } \\
\text { statistical difference in } 9.5 \mathrm{~mm} \\
\text { dynamic modulus value was } \\
\text { thought to be due to aspect ratio } \\
\text { of specimen geometries. For other } \\
\text { mixes, the statistical difference } \\
\text { was found at } 37.8^{\circ} \mathrm{C} \text { and } 54.4^{\circ} \mathrm{C} \text {. }\end{array}$} \\
\hline & 38 & 9.5 & 12.5 & $3.0: 1$ & \\
\hline & 38 & 12.5 & 19 & $2.0: 1$ & \\
\hline & 38 & 19 & 25 & $1.5: 1$ & \\
\hline & 100 & 4.75 & 9.5 & 10.5:1 & \\
\hline & 100 & 9.5 & 12.5 & $8.0: 1$ & \\
\hline & 100 & 12.5 & 19 & $5.3: 1$ & \\
\hline & 100 & 19 & 25 & 4.0:1 & \\
\hline
\end{tabular}




\begin{tabular}{|c|c|c|c|c|c|}
\hline \multirow{2}{*}{$\begin{array}{l}\text { Park and } \\
\text { Kim (2013) }\end{array}$} & 38 & 9.5 & 12.5 & $3.0: 1$ & \multirow{2}{*}{ N/A } \\
\hline & 75 & 9.5 & 12.5 & $6: 01$ & \\
\hline \multirow{12}{*}{$\begin{array}{l}\text { Bower et al. } \\
(2014)\end{array}$} & 38 & 9.5 & 12.5 & $3.0: 1$ & \multirow{12}{*}{$\begin{array}{l}\text { The analysis recommended } 38 \mathrm{~mm} \\
\text { dimeter specimens to be used for } 9.5 \\
\text { and } 12.5 \mathrm{~mm} \text { NMAS mix and } 50 \mathrm{~mm} \\
\text { diameter specimens to be used for } 19 \\
\text { and } 25 \mathrm{~mm} \text { NMAS diameter mix }\end{array}$} \\
\hline & 38 & 12.5 & 19 & $2.0: 1$ & \\
\hline & 38 & 19 & 25 & $1.5: 1$ & \\
\hline & 38 & 25 & 37.5 & $1.0: 1$ & \\
\hline & 50 & 9.5 & 12.5 & $4.0: 1$ & \\
\hline & 50 & 12.5 & 19 & $2.6: 1$ & \\
\hline & 50 & 19 & 12.5 & $2.0: 1$ & \\
\hline & 50 & 25 & 37.5 & $1.3: 1$ & \\
\hline & 100 & 9.5 & 12.5 & $8.0: 1$ & \\
\hline & 100 & 12.5 & 19 & $5.3: 1$ & \\
\hline & 100 & 19 & 25 & $4.0: 1$ & \\
\hline & 100 & 25 & 37.5 & $2.7: 1$ & \\
\hline \multirow{2}{*}{$\begin{array}{l}\text { Lee, J.S. et } \\
\text { al. (2017a) }\end{array}$} & 75 & 9.5 & 12.5 & $6.0: 1$ & \multirow{2}{*}{ N/A } \\
\hline & 100 & 9.5 & 12.5 & $8.0: 1$ & \\
\hline \multirow{8}{*}{$\begin{array}{l}\text { Lee, K.P. et } \\
\text { al. (2017b) }\end{array}$} & 38 & 9.5 & 12.5 & $3.0: 1$ & \multirow{8}{*}{$\begin{array}{l}\text { The dynamic modulus and phase } \\
\text { angle master curves were statistically } \\
\text { same for different NMAS asphalt } \\
\text { mixes at low and intermediate } \\
\text { temperatures. }\end{array}$} \\
\hline & 38 & 12.5 & 19 & 2.0:1 & \\
\hline & 38 & 19 & 25 & $1.5: 1$ & \\
\hline & 38 & 25 & 37.5 & 1.0:1 & \\
\hline & 100 & 9.5 & 12.5 & $8.0: 1$ & \\
\hline & 100 & 12.5 & 19 & $5.3: 1$ & \\
\hline & 100 & 19 & 25 & 4.0:1 & \\
\hline & 100 & 25 & 37.5 & $2.7: 1$ & \\
\hline
\end{tabular}


Table 10: Summary of Aspect Ratio on Dynamic Modulus Test from Different Studies in the Literature

\begin{tabular}{|c|c|c|c|c|}
\hline & $\begin{array}{l}\text { Diameter } \\
(\mathrm{mm})\end{array}$ & $\begin{array}{l}\text { Height } \\
(\mathrm{mm})\end{array}$ & Aspect Ratio & Summary \\
\hline \multirow{3}{*}{$\begin{array}{c}\text { Current } \\
\text { specification }\end{array}$} & 38 & 110 & $0.4: 1$ & \multirow{3}{*}{$\mathrm{N} / \mathrm{A}$} \\
\hline & 100 & 130 & $1.3: 1$ & \\
\hline & 100 & 150 & $1.5: 1$ & \\
\hline \multirow{20}{*}{$\begin{array}{l}\text { Witczak et } \\
\text { al. (2000) }\end{array}$} & 70 & 70 & $1.0: 1$ & \multirow{20}{*}{$\begin{array}{l}\text { The results showed an increase in } \\
\text { dynamic modulus value and increase in } \\
\text { standard deviation of data for high } \\
\text { stiffness test for specimens with aspect } \\
\text { ratio of } 1 \text {. Although no statistically } \\
\text { significant difference in dynamic } \\
\text { modulus was identified due to aspect } \\
\text { ratio, it was concluded that specimens } \\
\text { should be tested with an aspect ratio of } \\
\text { at least } 1.5 \text {. The flow number of samples } \\
\text { with an aspect ratio of } 1 \text { was } \\
\text { significantly higher than aspect ratios of } \\
1.5,2 \text {, and } 3 \text {. Flow number for } 70 \text { mm } \\
\text { diameter samples was less than } 100 \text { mm } \\
\text { and } 150 \text { mm diameter samples. This } \\
\text { suggested a } 100 \text { mm diameter with } \\
\text { aspect ratio of } 1.5 \text { or greater is good for } \\
\text { testing flow number. The coefficient of } \\
\text { variance was large for } 100 \text { mm diameter } \\
\text { specimen with aspect ratio of } 1.5 \text {. The } \\
\text { coefficient of variance was minimized } \\
\text { with aspect ratio of } 2 . \text { Although } 70 X 140 \\
\text { mm specimen was shown to provide low } \\
\text { standard error in dynamic modulus test } \\
\text { and low variability, } 100 X 150 \text { mm was } \\
\text { recommended for both complex } \\
\text { modulus and permanent deformation } \\
\text { test. }\end{array}$} \\
\hline & 70 & 105 & $1.5: 1$ & \\
\hline & 70 & 140 & $2.0: 1$ & \\
\hline & 70 & 210 & $3.0: 1$ & \\
\hline & 100 & 100 & $1.0: 1$ & \\
\hline & 100 & 150 & $1.5: 1$ & \\
\hline & 100 & 200 & $2.0: 1$ & \\
\hline & 100 & 300 & $3.0: 1$ & \\
\hline & 150 & 150 & 1.0:1 & \\
\hline & 150 & 225 & $1.5: 1$ & \\
\hline & 150 & 300 & $2.0: 1$ & \\
\hline & 150 & 450 & $3.0: 1$ & \\
\hline & 100 & 100 & $1.0: 1$ & \\
\hline & 100 & 69 & $1.5: 1$ & \\
\hline & 100 & 35 & $3.0: 1$ & \\
\hline & 100 & 17 & $6.0: 1$ & \\
\hline & 150 & 150 & $1.0: 1$ & \\
\hline & 150 & 100 & $1.5: 1$ & \\
\hline & 150 & 50 & $3.0: 1$ & \\
\hline & 150 & 25 & $6.0: 1$ & \\
\hline
\end{tabular}




\begin{tabular}{|c|c|c|c|c|}
\hline \multirow[b]{4}{*}{$\begin{array}{l}\text { Chehab et } \\
\text { al. }(2000)\end{array}$} & 75 & 115 & $1.5: 1$ & \multirow{4}{*}{$\begin{array}{l}\text { Dynamic modulus for } 100 \mathrm{X} 200 \mathrm{~mm} \\
\text { specimens was considerably higher than } \\
\text { that of other geometries. At } 1 \mathrm{~Hz}, \\
\text { dynamic modulus increased with } \\
\text { increase in aspect ratio. At } 20 \mathrm{~Hz}, \\
\text { dynamic modulus decreased with } \\
\text { increase in aspect ratio. At } 2 \text { and } 5 \mathrm{~Hz} \text {, } \\
\text { phase angle of } 100 \mathrm{X} 150 \mathrm{~mm} \text { was higher } \\
\text { than that of } 75 \mathrm{X} 150 \mathrm{~mm} \text { specimen. At } \\
10 \mathrm{~Hz}, 100 \mathrm{X} 150 \mathrm{~mm} \text { specimen had } \\
\text { higher dynamic modulus than } 75 \mathrm{X} 150 \\
\mathrm{~mm} \text {. The study recommended } 75 \mathrm{X} 150 \\
\mathrm{~mm} \text { and } 100 \mathrm{X} 150 \text { mm geometry and } \\
\text { concluded that it may not be universally } \\
\text { applied to other AC mixes and SGC } \\
\text { pills. }\end{array}$} \\
\hline & 100 & 150 & $1.5: 1$ & \\
\hline & 75 & 150 & 2.0:1 & \\
\hline & 100 & 200 & 2.0:1 & \\
\hline $\begin{array}{l}\text { Kutay et al. } \\
(2009)\end{array}$ & 100 & 150 & $1.5: 1$ & N/A \\
\hline \multirow{3}{*}{$\begin{array}{l}\mathrm{Li} \text { and } \\
\text { Gibson } \\
\text { (2012) }\end{array}$} & 38 & 110 & $2.9: 1$ & \multirow{3}{*}{$\begin{array}{l}38 X 110 \mathrm{~mm} \text { and } 38 X 140 \mathrm{~mm} \\
\text { specimens had lower dynamic modulus } \\
\text { value at high temperature. } 38 X 110 \mathrm{~mm} \\
\text { and } 38 X 140 \mathrm{~mm} \text { specimens had higher } \\
\text { phase angle compared to } 100 X 150 \mathrm{~mm} \\
\text { specimen. }\end{array}$} \\
\hline & 38 & 140 & $3.7: 1$ & \\
\hline & 100 & 150 & $1.5: 1$ & \\
\hline \multirow{2}{*}{$\begin{array}{l}\text { Park and } \\
\text { Kim (2013) }\end{array}$} & 38 & 100 & 2.6:1 & \multirow{2}{*}{$\begin{array}{l}\text { The dynamic modulus results showed } \\
\text { that dynamic modulus values were very } \\
\text { close for different geometries and tested } \\
\text { statistically the same. }\end{array}$} \\
\hline & 75 & 150 & $2: 01$ & \\
\hline \multirow{5}{*}{$\begin{array}{l}\text { Bower et al. } \\
\text { (2014) }\end{array}$} & 38 & 110 & $2.9: 1$ & \multirow{5}{*}{$\begin{array}{l}\text { Sample to sample variability was } \\
\text { highest for small scale specimens } \\
\text { (38X135, 50X135, 38X110, and } \\
50 \mathrm{X} 110 \mathrm{~mm}) \text { at } 54.4^{\circ} \mathrm{C} \text {. However, they } \\
\text { were statistically same to } 100 \mathrm{X} 150 \mathrm{~mm} \\
\text { sample for all mixes. }\end{array}$} \\
\hline & 38 & 135 & 3.6:1 & \\
\hline & 50 & 110 & $2.2: 1$ & \\
\hline & 50 & 135 & $2.7: 1$ & \\
\hline & 100 & 150 & $1.5: 1$ & \\
\hline
\end{tabular}




\begin{tabular}{|c|c|c|c|c|}
\hline $\begin{array}{l}\text { Lee, J.S. et } \\
\text { al. (2017a) }\end{array}$ & 100 & 150 & $1.5: 1$ & $\begin{array}{l}\text { The semi-log scale better demonstrates } \\
\text { dynamic modulus values at low } \\
\text { temperature and high frequency } \\
\text { conditions. The log-log scale better } \\
\text { demonstrates dynamic modulus values } \\
\text { at high temperature and low frequency } \\
\text { conditions. }\end{array}$ \\
\hline & 38 & 110 & $2.9: 1$ & \multirow[b]{2}{*}{$\begin{array}{l}\text { The dynamic modulus and phase angle } \\
\text { master curves for } 38 \mathrm{X} 110 \mathrm{~mm} \text { and } \\
100 \mathrm{X} 150 \mathrm{~mm} \text { specimens were } \\
\text { statistically same at low and } \\
\text { intermediate temperatures. However, at } \\
\text { high temperature, } 38 \mathrm{X} 110 \mathrm{~mm} \\
\text { specimen displayed higher dynamic } \\
\text { modulus and lower phase angle values } \\
\text { compared to 100X150 mm specimens. }\end{array}$} \\
\hline $\begin{array}{l}\text { Lee, K.P. et } \\
\text { al. (2017b) }\end{array}$ & 100 & 150 & $1.5: 1$ & \\
\hline
\end{tabular}


Table 11: Summary of Diameter to MAS Ratio on DTC Fatigue Test from Different Studies in Literature

\begin{tabular}{|c|c|c|c|c|c|}
\hline & $\begin{array}{c}\text { Diameter } \\
(\mathrm{mm})\end{array}$ & $\begin{array}{c}\text { NMAS } \\
(\mathrm{mm})\end{array}$ & $\begin{array}{l}\text { MAS } \\
(\mathrm{mm})\end{array}$ & $\begin{array}{l}\text { Diameter: } \\
\text { MAS }\end{array}$ & Summary \\
\hline \multirow{4}{*}{$\begin{array}{c}\text { Current } \\
\text { specification }\end{array}$} & 38 & 4.75 & 9.5 & $4.0: 1$ & \multirow[b]{4}{*}{ N/A } \\
\hline & 38 & 19 & 25 & $1.5: 1$ & \\
\hline & 100 & 4.75 & 9.5 & 10.5:1 & \\
\hline & 100 & 25 & 37.5 & $2.7: 1$ & \\
\hline \multirow{2}{*}{$\begin{array}{l}\text { Chehab et } \\
\text { al. }(2000)\end{array}$} & 75 & 12.5 & 19 & $3.9: 1$ & \multirow{2}{*}{ N/A } \\
\hline & 100 & 12.5 & 19 & $5.3: 1$ & \\
\hline \multirow{2}{*}{$\begin{array}{c}\text { Kutay et al. } \\
(2009)\end{array}$} & 38.1 & 12.5 & 19 & $3.0: 1$ & \multirow[b]{2}{*}{ N/A } \\
\hline & 71.4 & 12.5 & 19 & $5.7: 1$ & \\
\hline \multirow{6}{*}{$\begin{array}{l}\text { Li and } \\
\text { Gibson } \\
(2012)\end{array}$} & 38 & 4.75 & 9.5 & 4.0:1 & \multirow{6}{*}{$\begin{array}{l}\text { The } 100 \mathrm{X} 150 \mathrm{~mm} \text { and } 38 \mathrm{X} 110 \\
\text { mm predicted same fatigue life } \\
\text { ranking for } 4.75 \mathrm{~mm}, 9.5 \mathrm{~mm} \text {, } \\
\text { and one of the two } 19 \mathrm{~mm} \\
\text { NMAS asphalt mix. The other } \\
19 \mathrm{~mm} \text { NMAS AC mix with } \\
\text { PG } 58-34 \text { binder showed high } \\
\text { fatigue resistance for } 100 \mathrm{X} 150 \\
\text { mm specimens compared to } \\
38 \mathrm{X} 110 \mathrm{~mm} \text { sample. }\end{array}$} \\
\hline & 38 & 9.5 & 12.5 & $3.0: 1$ & \\
\hline & 38 & 19 & 25 & $1.5: 1$ & \\
\hline & 100 & 4.75 & 9.5 & $10.5: 1$ & \\
\hline & 100 & 9.5 & 12.5 & $8.0: 1$ & \\
\hline & 100 & 19 & 25 & $4.0: 1$ & \\
\hline \multirow{2}{*}{$\begin{array}{c}\text { Park and } \\
\text { Kim (2013) }\end{array}$} & 38 & 9.5 & 12.5 & $3.0: 1$ & \multirow{2}{*}{ N/A } \\
\hline & 75 & 9.5 & 12.5 & 6:01 & \\
\hline \multirow{2}{*}{$\begin{array}{l}\text { Lee, J.S. et } \\
\text { al. (2017a) }\end{array}$} & 75 & 9.5 & 12.5 & $6.0: 1$ & \multirow[b]{2}{*}{ N/A } \\
\hline & 100 & 9.5 & 12.5 & $8.0: 1$ & \\
\hline \multirow{6}{*}{$\begin{array}{l}\text { Lee, K.P. et } \\
\text { al. }(2017 b)\end{array}$} & 38 & 9.5 & 12.5 & $3.0: 1$ & \multirow{6}{*}{$\begin{array}{l}\text { A high sample to sample } \\
\text { variability was observed in } 25 \\
\text { mm NMAS mix. Six out of } \\
\text { eight } 100 X 130 \text { mm specimen } \\
\text { of } 25 \text { mm NMAS resulted in } \\
\text { end failure. The damage } \\
\text { characterization curve for } 19 \\
\text { mm NMAS asphalt mix } \\
\text { showed good agreement } \\
\text { between 100X130 mm and } \\
38 X 110 \text { mm specimens } \\
\text { however, two out of four } \\
\text { 38X110 mm test specimens } \\
\text { resulted in end failure. }\end{array}$} \\
\hline & 38 & 19 & 25 & $1.5: 1$ & \\
\hline & 38 & 25 & 37.5 & $1.0: 1$ & \\
\hline & 100 & 9.5 & 12.5 & $8.0: 1$ & \\
\hline & 100 & 19 & 25 & $4.0: 1$ & \\
\hline & 100 & 25 & 37.5 & $2.7: 1$ & \\
\hline
\end{tabular}


Table 12: Summary of Aspect Ratio on DTC Fatigue Test from Different Studies in the Literature

\begin{tabular}{|c|c|c|c|c|}
\hline & $\begin{array}{c}\text { Diameter } \\
(\mathrm{mm})\end{array}$ & $\begin{array}{c}\begin{array}{c}\text { Height } \\
(\mathrm{mm})\end{array} \\
\end{array}$ & Aspect Ratio & Summary \\
\hline \multirow{3}{*}{$\begin{array}{c}\text { Current } \\
\text { specification }\end{array}$} & 38 & 110 & $0.4: 1$ & \multirow{3}{*}{ N/A } \\
\hline & 100 & 130 & 1.3:1 & \\
\hline & 100 & 150 & $1.5: 1$ & \\
\hline \multirow{4}{*}{$\begin{array}{l}\text { Chehab et } \\
\text { al. (2000) }\end{array}$} & 75 & 115 & $1.5: 1$ & \multirow{4}{*}{$\begin{array}{l}\text { The } 75 \times 115 \mathrm{~mm} \text { and } 100 \mathrm{X} 200 \mathrm{~mm} \\
\text { specimen exhibited lowest strength from } \\
\text { stress versus strain curves. The } 75 \mathrm{X} 150 \\
\text { and } 100 \mathrm{X} 150 \mathrm{~mm} \text { specimens exhibited } \\
\text { similar stress versus strain curves. The } \\
\text { study recommended } 75 \mathrm{X} 150 \mathrm{~mm} \text { and } \\
100 \mathrm{X} 150 \mathrm{~mm} \text { geometry and concluded } \\
\text { that it may not be universally applied to } \\
\text { other AC mixes and SGC pills }\end{array}$} \\
\hline & 100 & 150 & $1.5: 1$ & \\
\hline & 75 & 150 & $2.0: 1$ & \\
\hline & 100 & 200 & $2.0: 1$ & \\
\hline \multirow[t]{2}{*}{$\begin{array}{c}\text { Kutay et al. } \\
\text { (2009) }\end{array}$} & 38.1 & 100 & 2.6:1 & \multirow{2}{*}{$\begin{array}{l}\text { The damage characterization curve for } \\
71.4 \mathrm{X} 150 \mathrm{~mm} \text { and } 38.1 \mathrm{X} 100 \mathrm{~mm} \\
\text { samples fabricated from different binder } \\
\text { types except PG } 74-28 \text {, collapsed on a } \\
\text { single curve. This indicated that } \\
38.1 \mathrm{X} 100 \mathrm{~mm} \text { samples can provide } \\
\text { same information as } 71.4 \mathrm{X} 150 \mathrm{~mm} \\
\text { samples. The research concluded that } \\
\text { 38.1X100 mm samples can be used to } \\
\text { characterize asphalt pavements }\end{array}$} \\
\hline & 71.4 & 150 & $2.1: 1$ & \\
\hline \multirow{2}{*}{$\begin{array}{l}\text { Li and } \\
\text { Gibson } \\
(2012)\end{array}$} & 38 & 110 & $2.9: 1$ & \multirow{2}{*}{$\begin{array}{l}\text { Although the fatigue life for } 100 \mathrm{X} 150 \\
\mathrm{~mm} \text { sample was higher than } 38 \mathrm{X} 110 \\
\mathrm{~mm} \text {, the values were reasonable and } \\
\text { comparable }\end{array}$} \\
\hline & 100 & 150 & $1.5: 1$ & \\
\hline \multirow[b]{2}{*}{$\begin{array}{c}\text { Park and } \\
\text { Kim (2013) }\end{array}$} & 38 & 100 & $2.6: 1$ & \multirow{2}{*}{$\begin{array}{l}\text { The damage characterization curve for } \\
75 \times 150 \mathrm{~mm} \text { specimens was below } \\
38 \times 100 \mathrm{~mm} \text { specimens however, they } \\
\text { were statistically same. For the ease of } \\
\text { testing, } 38 \mathrm{X} 100 \mathrm{~mm} \text { specimens were } \\
\text { recommended }\end{array}$} \\
\hline & 75 & 150 & 2:01 & \\
\hline
\end{tabular}




\begin{tabular}{|c|c|c|c|c|}
\hline \multirow{5}{*}{$\begin{array}{l}\text { Lee, J.S. et } \\
\text { al. }(2017 \mathrm{a})\end{array}$} & 75 & 150 & $2.0: 1$ & \multirow{5}{*}{$\begin{array}{l}\text { The results showed that } 75 X 150 \mathrm{~mm} \\
\text { and } 100 \mathrm{X} 150 \mathrm{~mm} \text { specimens had end } \\
\text { failure. Despite the end failure, damage } \\
\text { characterization curve for } 75 \mathrm{X} 150 \mathrm{~mm} \\
\text { and } 100 \mathrm{X} 150 \mathrm{~mm} \text { samples } \\
\text { collapsed with } 75 \mathrm{X} 130 \mathrm{~mm} \text { and } \\
100 \mathrm{X} 130 \mathrm{~mm} \text { samples. Additionally, } \\
100 \mathrm{X} 150 \mathrm{~mm} \text { and } 100 \mathrm{X} 130 \mathrm{~mm} \\
\text { sample's damage characterization } \\
\text { curves were statistically same to those } \\
\text { of } 75 \mathrm{X} 150 \mathrm{~mm} \text { and } 75 \mathrm{X} 130 \mathrm{~mm} \text {. } \\
\text { Although, } 75 \mathrm{X} 130 \text { and } 100 \mathrm{X} 130 \mathrm{met} \\
\text { RVE requirement of minimum diameter } \\
\text { to MAS ratio of } 4 \text { to } 1 \text { and aspect ratio } \\
\text { of } 2 \text { to } 1,100 \mathrm{X} 130 \mathrm{~mm} \text { specimen was } \\
\text { recommended as it will satisfy RVE } \\
\text { requirements for higher NMAS asphalt } \\
\text { mixes }\end{array}$} \\
\hline & 100 & 150 & $1.5: 1$ & \\
\hline & 75 & 130 & $1.7: 1$ & \\
\hline & 100 & 130 & $1.3: 1$ & \\
\hline & 100 & 150 & $1.5: 1$ & \\
\hline \multirow{2}{*}{$\begin{array}{l}\text { Lee, K.P. et } \\
\text { al. }(2017 b)\end{array}$} & 38 & 110 & $2.9: 1$ & \multirow{2}{*}{$\begin{array}{l}\text { The } 38 X 110 \mathrm{~mm} \text { specimen was } \\
\text { recommended due to ease in fabrication. }\end{array}$} \\
\hline & 100 & 130 & $1.3: 1$ & \\
\hline
\end{tabular}

Table 13: Failure Cycles for $100 X 150 \mathrm{~mm}$ and $38 X 110 \mathrm{~mm}$ in Cyclic Fatigue Test (Li and Gibson, 2012)

\begin{tabular}{|c|c|c|c|c|c|c|c|c|}
\hline & \multicolumn{2}{|c|}{$4.75 \mathrm{~mm}$ NMAS } & \multicolumn{2}{|c|}{$9.5 \mathrm{~mm}$ NMAS } & \multicolumn{2}{|c|}{$19 \mathrm{~mm}$ NMAS } & \multicolumn{2}{|c|}{$19 \mathrm{~mm}$ NMAS } \\
\hline & $\begin{array}{c}100 \mathrm{X} 15 \\
0 \mathrm{~mm}\end{array}$ & $\begin{array}{c}38 \mathrm{X} 11 \\
0 \mathrm{~mm}\end{array}$ & $\begin{array}{c}100 \times 15 \\
0 \mathrm{~mm}\end{array}$ & $\begin{array}{c}38 \mathrm{X} 11 \\
0 \mathrm{~mm}\end{array}$ & $\begin{array}{c}100 X 15 \\
0 \mathrm{~mm}\end{array}$ & $\begin{array}{c}38 X 11 \\
0 \mathrm{~mm} \\
\end{array}$ & $\begin{array}{c}100 \mathrm{X} 15 \\
0 \mathrm{~mm} \\
\end{array}$ & $\begin{array}{c}38 \mathrm{X} 11 \\
0 \mathrm{~mm} \\
\end{array}$ \\
\hline $\begin{array}{l}\text { Failure } \\
\text { Cycles } \\
(\mathrm{x} 1 \mathrm{M})\end{array}$ & 24,000 & 22,800 & 5,730 & 9,170 & 3,210 & 6,250 & 4,620 & 512 \\
\hline $\begin{array}{c}\mathrm{N}_{\mathrm{f}} \\
\text { large/N } \\
\text { f small }\end{array}$ & \multicolumn{2}{|c|}{1.05} & \multicolumn{2}{|c|}{0.625} & \multicolumn{2}{|c|}{0.532} & \multicolumn{2}{|c|}{9.02} \\
\hline
\end{tabular}

\subsection{COMMENTARY ON AIR VOID DISTRIBUTION}

The AMPT testing on different size test specimens must be conducted with uniform target VTM of $7 \% \pm 0.5 \%$ unlike Bower et al. (2015) where VTM of test specimens ranged from $2 \%$ to $12 \%$. As concluded by Blankenship and Anderson (2010), this can cause variation in fatigue and rutting performance between replicates. With difference of as small as 1\% VTM, disparity was seen in performance results 
Target VTM of 7\% $\pm 0.5 \%$ is selected as Kutay et al. (2009) stated 7\% VTM a good representation of field compaction to test lab compacted specimens for fatigue and rutting performance.

Researches (Thyagarajan et al., 2010 and Chehab et al., 2000) have investigated air void distribution in SGC pill and cored samples to conclude that air void gradient is the highest around mold wall, top, and bottom of SGC pill. Although Li and Gibson (2012) cored six 38X110 mm specimens from single SGC pill, no details were provided on how far away from center the small specimens were obtained. Although Thyagarajan et al. (2010) analyzed lateral air void distribution, no cores were obtained from the analyzed radius: $25 \mathrm{~mm}, 35 \mathrm{~mm}, 43 \mathrm{~mm}$, and $50 \mathrm{~mm}$. The research concluded that since coring and cutting the specimens result in erroneous interpretation of air void on core and cut selections, nondestructive method such as X-Ray Computed Tomography is efficient to study microstructure of asphalt mix. Romero and Masad (2001) used X-Ray Computed Tomography to quantify internal structure of asphalt mix by creating a 3-D analysis of aggregate structure and air void distribution (Romero and Masad, 2001).

Research (Thyagarajan et al., 2010) concluded that air void heterogeneity exponentially increases for cored specimens moving towards the circumference of SGC pill and AASHTO PP 99- 19 requires cored small specimens within $100 \mathrm{~mm}$ diameter. However, no research has been conducted to compare air void distribution in specimens obtained from within $100 \mathrm{~mm}$ diameter to those obtained outside $100 \mathrm{~mm}$ diameter. Researchers (Thyagarajan et al., 2010, and Chehab et al., 2000) have analyzed air void distribution within samples from single NMAS asphalt mix. Although (Dalton, 2016 and Aguilar, 2015) researches have analyzed multiple NMAS asphalt mix, only one 100X150 mm specimen was cored from the center of 150X180 mm SGC pill. It is important to look at different NMAS asphalt mixes for different specimen dimensions and conclude if the lateral and vertical air void heterogeneity behavior compares. 


\section{CHAPTER 3. METHODOLOGY}

\subsection{OVERVIEW}

This research focuses on one new small specimen geometry of 50X110 mm to be compared with $38 \mathrm{X} 110 \mathrm{~mm}$ and one new full specimen geometry of $75 \mathrm{X} 110 \mathrm{~mm}$ to be compared with $100 \mathrm{X} 150$ or 100X130 mm specimen. All performance tests were performed with the AMPT as described in Appendix A.

With the development of FlexPAVE, this research analyzes rutting using SSR test on a new geometry of 50X110 mm to be compared with $100 \mathrm{X} 150 \mathrm{~mm}$ specimen. The summary of sample geometries with different inscribed circle is referred as Sample Type (ST). The inscribed circle defines the surface diameter from which all cored specimens are taken The ST used in this research are shown in Table 14. All samples are fabricated from 150X180 mm SGC pills.

Table 14: Summary of Sample Types

\begin{tabular}{|c|c|c|c|}
\hline $\begin{array}{c}\text { Sample } \\
\text { Types }\end{array}$ & $\begin{array}{c}\text { Dynamic } \\
\text { Modulus Test, } \\
\text { mm }\end{array}$ & $\begin{array}{c}\text { DTC Fatigue } \\
\text { Test, } \\
\text { mm }\end{array}$ & $\begin{array}{l}\text { Stress Sweep } \\
\text { Rutting Test, } \\
\text { mm }\end{array}$ \\
\hline 1 & $\begin{array}{c}\text { 100X150- Three } \\
\text { Samples from } \\
\text { Three Pills }\end{array}$ & $\begin{array}{l}\text { 100X130- Three } \\
\text { Samples from } \\
\text { Three Pills }\end{array}$ & $\begin{array}{l}100 X 150-\text { Four } \\
\text { Samples from } \\
\text { Four Pills }\end{array}$ \\
\hline 2 & $\begin{array}{l}\text { 38X110- Four } \\
\text { Samples from } \\
\text { One Pill }\end{array}$ & $\begin{array}{l}\text { 38X110- Four } \\
\text { Samples from } \\
\text { One Pill }\end{array}$ & N/A \\
\hline 3 & $\begin{array}{c}\text { 38X110- Three } \\
\text { Samples from } \\
\text { Three Pills }\end{array}$ & $\begin{array}{c}38 X 110-\text { Three } \\
\text { Samples from } \\
\text { Three Pills }\end{array}$ & N/A \\
\hline 4 & $\begin{array}{c}\text { 50X110- Three } \\
\text { Samples from } \\
\text { One Pill }\end{array}$ & $\begin{array}{c}\text { 50X110- Three } \\
\text { Samples from } \\
\text { One Pill }\end{array}$ & $\begin{array}{c}\text { 50X110- Four } \\
\text { Samples from } \\
\text { Two Pills } \\
\end{array}$ \\
\hline 5 & $\begin{array}{c}\text { 50X110- Three } \\
\text { Samples from } \\
\text { Three Pills }\end{array}$ & $\begin{array}{c}\text { 50X110- Three } \\
\text { Samples from } \\
\text { Three Pills }\end{array}$ & N/A \\
\hline 6 & $\begin{array}{c}\text { 75X110- Three } \\
\text { Samples from } \\
\text { Three Pills }\end{array}$ & $\begin{array}{c}\text { 75X110- Three } \\
\text { Samples from } \\
\text { Three Pills }\end{array}$ & N/A \\
\hline
\end{tabular}

The diameter to MAS ratio and aspect ratio for the geometries as per AASHTO standards and proposed geometries are shown in Table 15. 
Table 15: Aspect Ratio and Diameter to MAS Ratio for AASHTO and Researched Geometries

\begin{tabular}{|c|c|c|c|c|c|c|}
\hline \multirow{5}{*}{ Data Set } & \multirow{5}{*}{$\begin{array}{l}\text { Diameter, } \\
\mathrm{mm}\end{array}$} & \multirow{5}{*}{$\begin{array}{c}\text { Height, } \\
\text { mm }\end{array}$} & \multirow{5}{*}{$\begin{array}{c}\text { Aspect } \\
\text { Ratio }\end{array}$} & \multicolumn{3}{|c|}{ D: MAS Ratio } \\
\hline & & & & \multicolumn{3}{|c|}{ NMAS, mm } \\
\hline & & & & 12.5 & 19 & 25 \\
\hline & & & & \multicolumn{3}{|c|}{ MAS, mm } \\
\hline & & & & 19 & 25 & 37.5 \\
\hline AASHTO T 378-17 & 100 & 150 & $1.5: 1$ & \multirow{2}{*}{$5.3: 1$} & \multirow{2}{*}{$4.0: 1$} & \multirow{2}{*}{$2.7: 1$} \\
\hline AASHTO TP 107-18 & 100 & 130 & $1.3: 1$ & & & \\
\hline $\begin{array}{c}\text { AASHTO TP 132-19 } \\
\text { and AASHTO TP } \\
133-19\end{array}$ & 38 & 110 & 2.9:1 & $2.0: 1$ & $1.5: 1$ & $1.0: 1$ \\
\hline \multirow{2}{*}{ Current research } & 50 & 110 & 2.2 & 2.6 & 2.0 & 1.3 \\
\hline & 75 & 110 & 1.5 & 4.0 & 3.0 & 2.0 \\
\hline
\end{tabular}

\subsection{ASPHALT MIXES}

Three asphalt mixes, Marshall and Superpave, are used to test dynamic modulus, fatigue, and SSR for different geometries. These mixes are plant produced and are obtained from two paving companies. The mix design sheets are in Appendix B and are summarized in Table 16.

Table 16: Summary of Plant Produced Asphalt Mix Obtained for This Research

\begin{tabular}{|c|c|l|c|c|c|c|c|}
\hline Company & $\begin{array}{c}\text { Plant } \\
\text { Location }\end{array}$ & Mix Type & $\begin{array}{c}\text { NMAS, } \\
\text { mm }\end{array}$ & $\begin{array}{c}\text { HMA } \\
\text { Type }\end{array}$ & $\begin{array}{c}\text { Binder } \\
\text { Content, } \\
\%\end{array}$ & $\begin{array}{c}\text { Binder } \\
\text { Type }\end{array}$ & $\begin{array}{c}\text { Design } \\
\text { Traffic }\end{array}$ \\
\hline $\begin{array}{c}\text { West } \\
\text { Virginia } \\
\text { Paving, } \\
\text { Inc. }\end{array}$ & $\begin{array}{c}\text { Poca, } \\
\text { WV }\end{array}$ & Superpave & 12.5 & $\begin{array}{c}12.5 \mathrm{~mm} \\
\text { Skid- } \\
\text { RAP }\end{array}$ & 5.5 & $\begin{array}{c}\text { PG } \\
64 \mathrm{H}-22\end{array}$ & $\begin{array}{c}3 \text { to }<30 \\
\text { Million }\end{array}$ \\
\hline $\begin{array}{c}\text { J. F. Allen } \\
\text { Company }\end{array}$ & $\begin{array}{c}\text { Elkins, } \\
\text { WV }\end{array}$ & Marshall & 19 & $\begin{array}{c}\text { Wearing } \\
-4 \text { Skid }\end{array}$ & 4.9 & $\begin{array}{c}\text { PG 64- } \\
22\end{array}$ & Heavy \\
\hline $\begin{array}{c}\text { J. F. Allen } \\
\text { Company }\end{array}$ & $\begin{array}{c}\text { Lorentz, } \\
\text { WV }\end{array}$ & Superpave & 25 & $\begin{array}{c}25 \mathrm{~mm}- \\
\text { RAP }\end{array}$ & 4.4 & $\begin{array}{c}\text { PG } \\
64 S-22\end{array}$ & $\begin{array}{c}3 \text { to < } 30 \\
\text { Million }\end{array}$ \\
\hline
\end{tabular}

The $12.5 \mathrm{~mm}$ and $25 \mathrm{~mm}$ NMAS mixes contain Reclaimed Asphalt Pavement (RAP). The percentage of aggregate and binder of RAP in the mix are: 


\begin{tabular}{|c|c|c|c|}
\hline Company & Mix NMAS, mm & RAP aggregate, $\%$ & Binder in RAP, \% \\
\hline West Virginia Paving, Inc. & 12.5 & 15 & 4.7 \\
\hline J. F. Allen Company & 25 & 15 & 3.4 \\
\hline
\end{tabular}

The mix obtained from the plant was transported and stored in West Virginia University (WVU) Asphalt Technology Laboratory. Upon obtaining the mix, an inventory was prepared based on box number and weight of each box. This is done such that the box can be randomly selected from the inventory. An approximate amount of mix mass obtained was:

\begin{tabular}{|c|c|}
\hline Mix NMAS, mm & Mix Mass, Kg \\
\hline 12.5 & 1,059 \\
\hline W-4 (19) & 448 \\
\hline 25 & 494 \\
\hline
\end{tabular}

Each mix was verified as per AASHTO T 312 for Superpave and AASHTO R 68 for Marshall Mixes. The steps for verification are summarized in Appendix C. For verification of Superpave and Marshall asphalt mix, $G_{s b}, P_{b}$, and $G_{m m}$ are obtained from T-400. The verification data, Table 17, demonstrate the mixes are within the $4 \pm 0.5$ percent tolerance.

Table 17: Summary of Asphalt Mix Verification

\begin{tabular}{|c|c|c|c|c|}
\hline NMAS, mm & $G_{s b}$ & $P_{b}, \%$ & $G_{m m}, \mathrm{Kg} / \mathrm{m}^{3}$ & $\mathrm{VTM}, \%$ \\
\hline \multirow{2}{*}{12.5} & & & & 3.7 \\
\cline { 5 - 5 } & 2.605 & 5.5 & 2.450 & 4.2 \\
\cline { 5 - 5 } 19 & & & & 4.1 \\
\hline \multirow{2}{*}{25} & 2.639 & \multirow{2}{*}{4.9} & 2.501 & 4.1 \\
\cline { 5 - 5 } & & & & 4.2 \\
\hline & 2.686 & \multirow{2}{*}{4.4} & 2.545 & 4.1 \\
\cline { 5 - 5 } & & & & 3.8 \\
\hline
\end{tabular}

\subsection{SAMPLE FABRICATION}

All specimens are fabricated at target VTM of 7\% $\pm 0.5 \%$. The $150 \times 180 \mathrm{~mm}$ SGC pills are compacted to achieve a VTM of $8 \% \pm 1.0 \%$ so that upon coring and sawing, the target VTM of $7 \% \pm$ $0.5 \%$ can be achieved. To fabricate $100 \times 130 \mathrm{~mm}, 100 \times 150 \mathrm{~mm}$ and $75 \times 110 \mathrm{~mm}$ specimens, specimens are cored from the center of a SGC pill. Additionally, for one set of 50X110 mm and one set of 38X110mm specimens, specimens are cored form the center of a SGC pill. For other sets, a coring template is prepared such that three $50 \mathrm{X} 110 \mathrm{~mm}$ and four $38 \mathrm{X} 110 \mathrm{~mm}$ specimens are cored from a single SGC pill. After coring, the samples are sawed to desired height. 
For multiple specimens fabricated from SGC pill, Table 18 shows inscribed circle diameter within SGC pill from which samples are cored and spacing between consecutive cored samples. The inscribed circle diameter is shown in Figure 12.

Table 18: Multiple Test Specimens Fabricated from Inscribed Diameter within SGC Pill

\begin{tabular}{|c|c|c|}
\hline $\begin{array}{c}\text { Sample } \\
\text { Type }\end{array}$ & $\begin{array}{c}\text { Inscribed circle } \\
\text { Diameter, } \mathrm{mm}\end{array}$ & $\begin{array}{c}\text { Spacing between } \\
\text { samples, mm }\end{array}$ \\
\hline 1 & 100 & N/A \\
\hline 2 & 104 & 8.5 \\
\hline 3 & 38 & N/A \\
\hline 4 & 120 & 11.0 \\
\hline 5 & 50 & N/A \\
\hline 6 & 75 & N/A \\
\hline
\end{tabular}

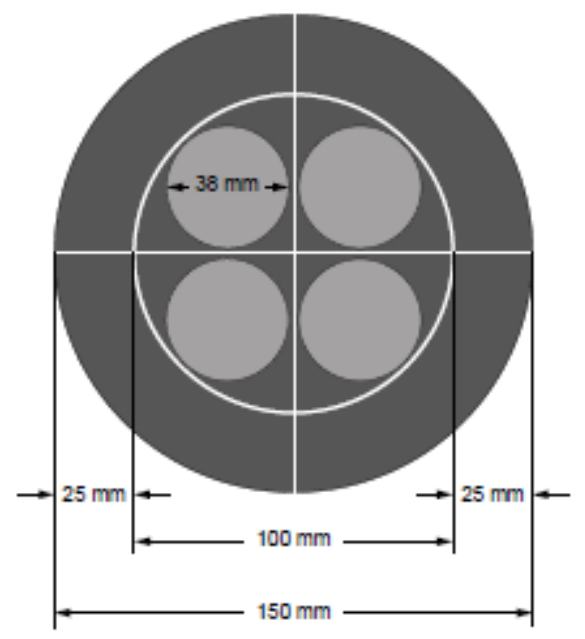

Figure 12: Inscribed Circle Diameter of $100 \mathrm{~mm}$ from $150 \mathrm{~mm}$ Diameter of SGC pill (AASHTO PP19)

Once specimens are cored and sawed, bulk specific gravity is measured by drying the sample in the Coredry and then performing Saturated Surface Dry (SSD) test (per AASHTO T 166). The samples are then dried overnight and placed in a zip lock bag and stored in a dark cabinet for up to 14 days. The samples are allotted a unique number based on the process in Figure 13. 


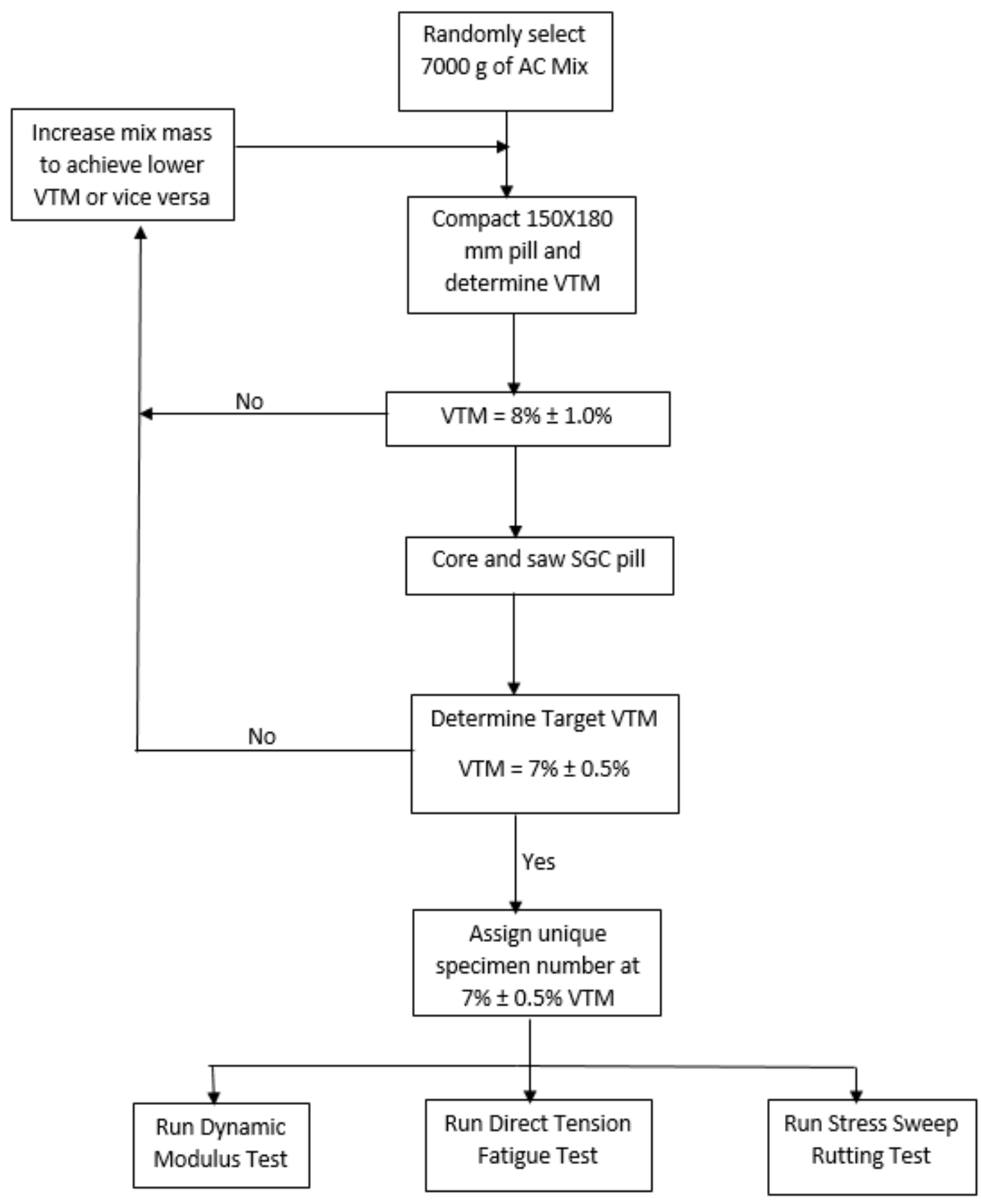

Figure 13: Flow Diagram of Unique Sample Number Preparation 
The unique numbers assigned to test specimens are shown in Table 19

Table 19: Experimental Design Variables and Unique Sample Numbers

\begin{tabular}{|c|c|c|c|c|c|c|c|c|c|c|c|c|c|c|c|c|c|c|}
\hline \multirow{4}{*}{$\begin{array}{l}\mathbb{8} \\
\mathbb{8} \\
\mathbb{8}\end{array}$} & \multicolumn{6}{|c|}{$\mathrm{NMAS}, \mathrm{mm}$} & \multicolumn{4}{|c|}{12.5} & \multicolumn{4}{|c|}{19} & \multicolumn{4}{|c|}{25} \\
\hline & \multicolumn{6}{|c|}{ MAS, mm } & \multicolumn{4}{|c|}{19} & \multicolumn{4}{|c|}{25} & \multicolumn{4}{|c|}{37.5} \\
\hline & \multirow{2}{*}{ Diameter } & \multirow{2}{*}{ Height } & \multirow{2}{*}{$H / D$} & \multicolumn{3}{|c|}{ D/MAS } & \multirow{2}{*}{$D M$} & \multirow{2}{*}{ DTC } & \multirow{2}{*}{\multicolumn{2}{|c|}{$S S R$}} & \multirow{2}{*}{$D M$} & \multirow{2}{*}{ DTC } & \multirow{2}{*}{\multicolumn{2}{|c|}{$S S R$}} & \multirow{2}{*}{ DM } & \multirow{2}{*}{ DTC } & \multirow{2}{*}{\multicolumn{2}{|c|}{ SSR }} \\
\hline & & & & MAS, $19 \mathrm{~mm}$ & MAS, $25 \mathrm{~mm}$ & MAS, $37.5 \mathrm{~mm}$ & & & & & & & & & & & & \\
\hline \multirow{7}{*}{$\begin{array}{l}\text { Tे } \\
5 \\
0 \\
0 \\
\frac{5}{5} \\
\text { है } \\
\text { दे }\end{array}$} & \multirow{4}{*}{100} & \multirow{4}{*}{150} & \multirow{4}{*}{1.5} & \multirow{7}{*}{5.26} & & & 1 & & & & 8 & & & & 15 & & & 18 \\
\hline & & & & & & & 2 & $n / a$ & & & 9 & $n / a$ & & & 16 & $n / a$ & & 19 \\
\hline & & & & & & & & & & & & & & & & & & 20 \\
\hline & & & & & 4.00 & 2.67 & 3 & & & & 10 & & & & 17 & & & 21 \\
\hline & & & & & & & & 22 & & & & 25 & & & & 28 & & \\
\hline & 100 & 130 & 1.3 & & & & $n / a$ & 23 & & & $n / a$ & 26 & & & $n / a$ & 29 & & $1 / a$ \\
\hline & & & & & & & & 24 & & & & 27 & & & & 30 & & \\
\hline & & & & & & & $31 \mathrm{a}$. & $32 \mathrm{a}$. & & & $33 \mathrm{a}$. & 34 a. & & & 35 a. & $36 \mathrm{a}$. & & \\
\hline$\frac{E}{\bar{y}}$ & 38 & 110 & & & & & $31 \mathrm{~b}$. & $32 \mathrm{~b}$. & & & $33 \mathrm{~b}$. & $34 \mathrm{~b}$. & & & $35 \mathrm{~b}$. & $36 \mathrm{~b}$. & & $1 / a$ \\
\hline & & & 289 & 200 & 152 & 101 & $31 \mathrm{c}$. & $32 c$. & & & $33 c$. & $34 c$. & & & $35 c$. & $36 c$. & & \\
\hline & & & 2.89 & 2.00 & 1.52 & 1.01 & 37 & 40 & & & 43 & 46 & & & 49 & 52 & & \\
\hline & 38 & 110 & & & & & 38 & 41 & & & 44 & 47 & & & 50 & 53 & & $1 / a$ \\
\hline & & & & & & & 39 & 42 & & & 45 & 48 & & & 51 & 54 & & \\
\hline & & & & & & & 55 a. & $56 \mathrm{a}$. & 57 a. & $58 \mathrm{a}$ & 59 a. & $60 \mathrm{a}$. & 61 a. & $62 \mathrm{a}$ & $63 \mathrm{a}$. & $64 \mathrm{a}$. & 65 a. & $66 \mathrm{a}$. \\
\hline & 50 & 110 & & & & & 55 b. & $56 \mathrm{~b}$. & $57 \mathrm{~b}$ & $58 \mathrm{~b}$ & $59 \mathrm{~b}$. & $60 \mathrm{~b}$. & $61 \mathrm{~b}$ & $62 \mathrm{~b}$ & $63 \mathrm{~b}$. & $64 \mathrm{~b}$. & $65 \mathrm{~b}$ & $66 \mathrm{~b}$. \\
\hline$\frac{\pi}{0}$ & & & 2.20 & 2.63 & 200 & 133 & $55 \mathrm{c}$. & $56 c$. & & & $59 c$. & $60 \mathrm{c}$. & 010. & 620 & $63 c$. & $64 c$. & 650. & 660. \\
\hline द & & & & & 2.00 & 1.05 & 67 & 70 & & & 73 & 76 & & & 79 & 82 & & \\
\hline & 50 & 110 & & & & & 68 & 71 & & & 74 & 77 & & & 80 & 83 & & $/ a$ \\
\hline & & & & & & & 69 & 72 & & & 75 & 78 & & & 81 & 84 & & \\
\hline & & & & & & & 85 & 88 & & & 91 & 94 & & & 97 & 100 & & \\
\hline & 75 & 110 & 1.47 & 3.95 & 3.00 & 2.00 & 86 & 89 & & & 92 & 95 & & & 98 & 101 & & $1 / a$ \\
\hline & & & & & & & 87 & 90 & & & 93 & 96 & & & 99 & 102 & & \\
\hline
\end{tabular}


A total of 102 SGC pills are compacted for this research to fabricate 132 specimens. Table 20 shows minimum replicates required for each test dimension, total number of SGC pill compacted to produce replicates, number of specimens fabricated from SGC pill by coring and sawing, and number of extra specimens fabricated.

Table 20: Test Specimens and Extra Specimens obtained from SGC Pill for AMPT testing

\begin{tabular}{|c|c|c|c|c|c|}
\hline Test & Dimension, mm & Replicates & $\begin{array}{c}\text { Total SGC Pill } \\
\text { compacted }\end{array}$ & $\begin{array}{l}\text { Specimens from } \\
\text { SGC Pill }\end{array}$ & Extra \\
\hline \multirow{6}{*}{$\begin{array}{c}\text { DM } \\
\text { and } \\
\text { DTC } \\
\text { Fatigue }\end{array}$} & $100 X 150$ & \multirow{6}{*}{3} & 3 & 3 & None \\
\hline & $38 X 110$ & & 1 & 4 & 1 \\
\hline & $50 \times 110$ & & 1 & 3 & None \\
\hline & $75 \times 110$ & & 3 & 3 & None \\
\hline & 50X110 (center) & & 3 & 3 & None \\
\hline & $38 X 110$ (center) & & 3 & 3 & None \\
\hline \multirow{2}{*}{ SSR } & $50 \times 110$ & \multirow[t]{2}{*}{4} & 2 & 6 & 2 \\
\hline & $100 \times 150$ & & 4 & 4 & None \\
\hline
\end{tabular}

\subsection{TEST PARTS FOR AMPT TESTING}

After the specimens are fabricated and the VTM verified, gauge points are attached using gauge point fixing jig as shown in Figure 14. The glue jig was designed for $100 \mathrm{~mm}$ samples. For the samples with a smaller diameter, arm extensions were fabricated.

Gauge points are glued to the specimen 120 degree apart. The gauge length is set at $70 \mathrm{~mm} \pm$ $1 \mathrm{~mm}$, where axial deformation is measured using LVDT. The gauge points are glued using "clearweld professional grade quick setting epoxy". The dynamic modulus and SSR test specimen are ready to be tested after application of gauge points. However, for DTC fatigue test the end platens are glued on using the gluing jig, Figure 15. 


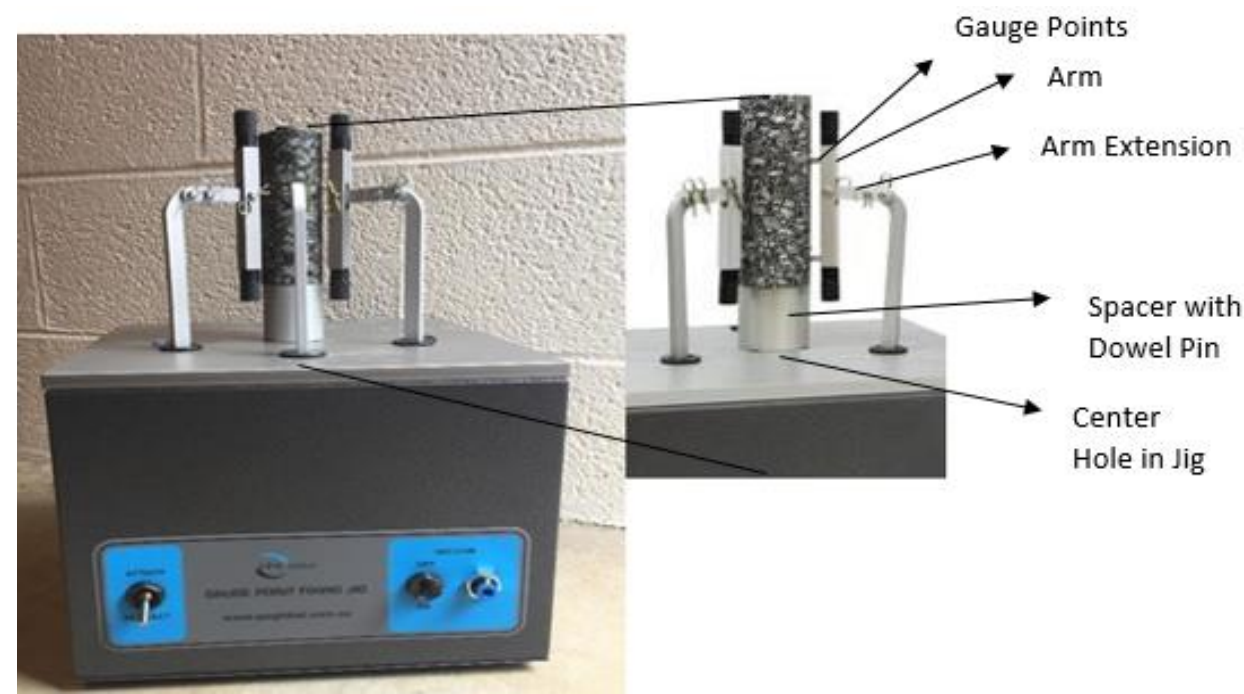

Figure 14: Gauge Point Fixing Jig

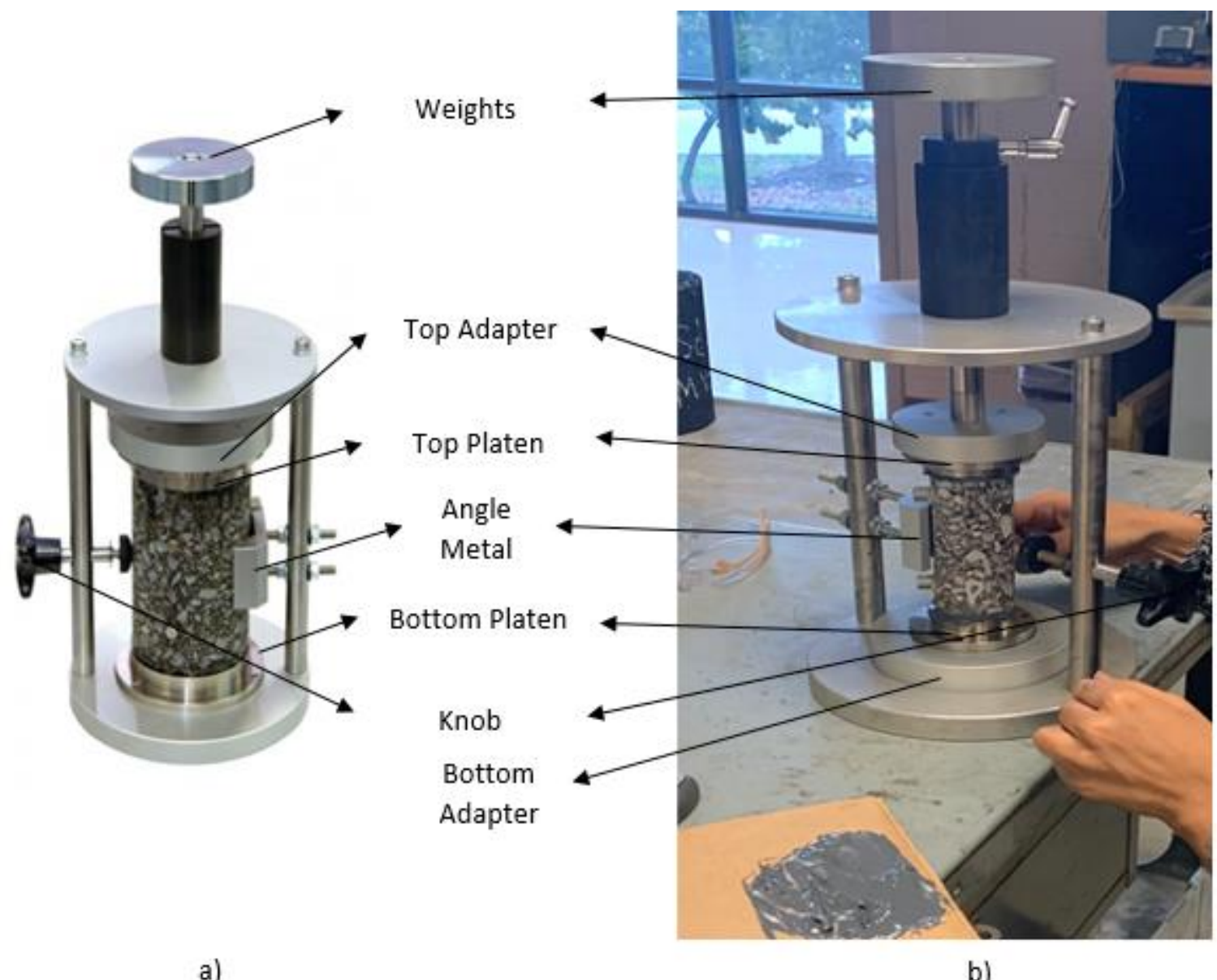

a)

b)

Figure 15: End Platen Gluing Jig a) 100X130 mm specimens b) $38 X 110 \mathrm{~mm}, 50 X 110 \mathrm{~mm}$, and 75X110 mm specimens

The jig ensures that end platen and specimen are centered, and specimen is standing perpendicular to two parallel end platens. The Top and bottom adapter holds end platen such that they are parallel to each 
other. Top adapter also provides extra weight for specimen and platens while the epoxy cures. The bottom adapter provides additional height for $110 \mathrm{~mm}$ height specimens (38X110 mm, 50X110 mm, and 75X110 $\mathrm{mm}$ ). This can be seen in Figure 14 where 100X130 mm specimen does not have bottom adapter because the specimen does not need an additional height for top adapter with weight to hit top end platen. However, for 38X110 mm, 50X110 mm, and 75X110 mm specimens, bottom adapter provides extra height.

The angle metal and plastic knob centers the specimen to the end platen. The weights used for each specimen dimension is shown as:

\begin{tabular}{|c|c|c|}
\hline Specimen, mm & Weight, $\mathrm{kN}$ & Weight, $\mathrm{lb}$ \\
\hline $100 \times 130$ & 0.045 & \multirow{2}{*}{10} \\
\hline $75 \times 110$ & & \\
\cline { 1 - 1 } $50 \times 110$ & 0.02 & 4.5 \\
\hline 38X110 & & 4.5 \\
\hline
\end{tabular}

The amount of epoxy used for different specimens is:

\begin{tabular}{|c|c|c|c|c|c|}
\hline $\begin{array}{c}\text { Dimension, } \\
\mathrm{mm}\end{array}$ & Epoxy & $\begin{array}{c}\text { Resin } \\
\text { Mass, g }\end{array}$ & $\begin{array}{l}\text { Hardner } \\
\text { Mass, g }\end{array}$ & $\begin{array}{l}\text { Hardening } \\
\text { Time }\end{array}$ & $\begin{array}{l}\text { Cure Time Before } \\
\text { Testing, Hrs. }\end{array}$ \\
\hline $100 \times 130$ & Devcon 10120 & 53.75 & 6.25 & 4 Hours & N/A \\
\hline $75 \times 110$ & \multirow{3}{*}{ Devcon 10240} & 40 & 4.65 & \multirow{3}{*}{5 Min. } & 1 \\
\hline $38 \times 110$ & & 4 & 2.16 & & 1 \\
\hline $50 \times 110$ & & 12 & 6.5 & & 1 \\
\hline
\end{tabular}

\subsection{FABRICATION OF 50X110 MM AND 75X110 MM CUSTOM PARTS}

The dynamic modulus and SSR test platens work interchangeably, with the only exception of drainage tube in SSR. Samples are simply placed on the platens and not glued to the platens in dynamic modulus and SSR test. A ball is used in both dynamic modulus and SSR test. The 50X110 mm is the only new specimen that is tested for both dynamic modulus and SSR. The new test parts designed for this research are described Appendix D.

The AMPT test specimen parts for 50X110 mm and 75X110 mm specimens are modeled such that they follow the same AMPT actuator movement as that of 100X150 mm or 100X130 mm and 38X110 mm specimens in dynamic modulus, fatigue, and SSR test.

\subsection{DYNAMIC MODULUS PROCEDURE}

The dynamic modulus test is conducted per AASHTO 378-17 for 100X150 mm and AASHTO 132-19 for 38X110 mm specimens. These test specifications require a minimum of two replicate 
specimens. However, since for this research air void distribution within dynamic modulus samples is evaluated per AASHTO R 83-17, three replicate specimens are tested for dynamic modulus test. The test setup for dynamic modulus test is shown in Figure 16.

The high test temperature is determined from adjusted binder type calculated per Section 2.2.1 (per AASHTO 378-17 and AASHTO 132-19) in Tables 3 and 4. The adjusted binder is calculated from LTPPBind at layer depth, mm and 98\% reliability for Morgantown, WV.

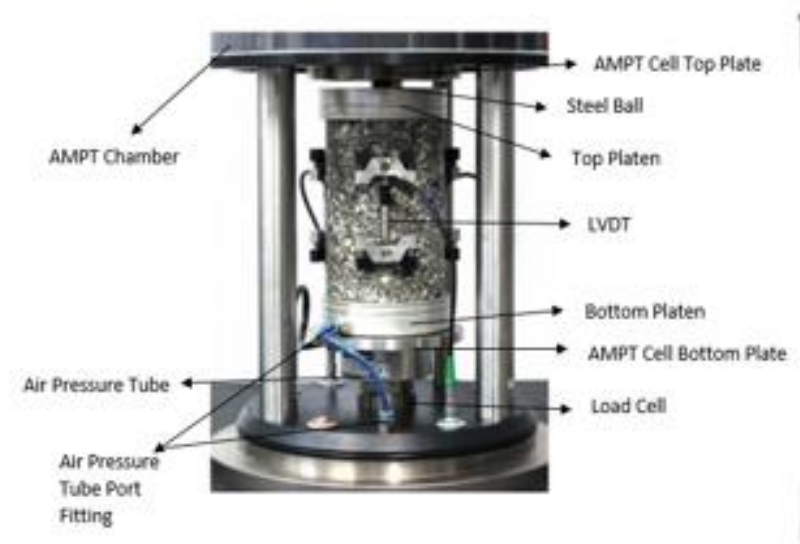

a)

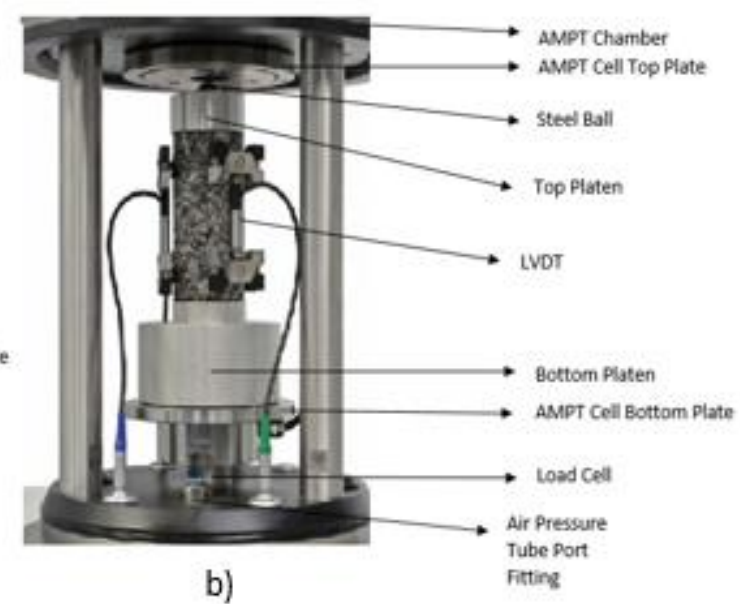

b)

Figure 16: Dynamic Modulus Test Setup a) 100X150 mm Specimen b) $38 X 110 \mathrm{~mm}$

The layer depth used in LTPPBind, the adjusted binder types and high-test temperatures are:

\begin{tabular}{|c|c|c|c|c|c|}
\hline NMAS, mm & $\begin{array}{c}\text { Layer Thickness, } \\
\text { inch }\end{array}$ & $\begin{array}{c}\text { Layer Depth, } \\
\text { inch }\end{array}$ & $\begin{array}{c}\text { T-400 } \\
\text { Binder Type }\end{array}$ & $\begin{array}{c}\text { Adjusted } \\
\text { Binder Type }\end{array}$ & $\begin{array}{c}\text { High test } \\
\text { Temp. C }\end{array}$ \\
\hline 12 & 2 & 2 & $64 \mathrm{H}-22$ & $70-22$ & 40 \\
\hline 19 & 3 & 5 & $64-22$ & $58-22$ & 35 \\
\hline 25 & 6 & 11 & $64 \mathrm{~S}-22$ & $58-16$ & 35 \\
\hline
\end{tabular}

The test parameters used for the dynamic modulus test are summarized in Table 21. The test parameters: microstrain range and test frequencies for 50X110 mm and 75X110 mm specimens are same as for 38X110 mm (AASHTO 132-19). 
Table 21: Test Parameters for Dynamic Modulus Test

\begin{tabular}{|c|c|c|c|c|}
\hline $\begin{array}{l}\text { Dimension, } \\
\mathrm{mm}\end{array}$ & $\begin{array}{l}\text { Mix NMAS, } \\
\mathrm{mm}\end{array}$ & Microstrain & $\begin{array}{c}\text { Temperature, } \\
{ }^{\circ} \mathrm{C}\end{array}$ & $\begin{array}{c}\text { Frequencies, } \\
\mathrm{Hz}\end{array}$ \\
\hline \multirow{3}{*}{ 100X150 } & 12.5 & \multirow{3}{*}{$75-125$} & 4,20 , and 40 & \multirow{3}{*}{$\begin{array}{l}0.01,0.1,1 \\
\quad \text { and } 10\end{array}$} \\
\hline & 19 & & & \\
\hline & 25 & & & \\
\hline \multirow{3}{*}{$38 X 110$} & 12.5 & \multirow{9}{*}{$50-75$} & 4,20 , and 40 & \multirow{9}{*}{$0.1,1$, and 10} \\
\hline & 19 & & 420 and 35 & \\
\hline & 25 & & 4,20, and 55 & \\
\hline \multirow{3}{*}{$50 X 110$} & 12.5 & & 4,20 , and 40 & \\
\hline & 19 & & \multirow{2}{*}{4,20, and 35} & \\
\hline & 25 & & & \\
\hline \multirow{3}{*}{$75 X 110$} & 12.5 & & 4,20 , and 40 & \\
\hline & 19 & & \multirow{2}{*}{4,20, and 35} & \\
\hline & 25 & & & \\
\hline
\end{tabular}

The steps for dynamic modulus testing and air void uniformity are described in Appendix E.

The dynamic modulus and phase angle data from sum files (AASHTO T 378-17 and AASHTO TP 132-19) generated by the AMPT are uploaded into the FlexMAT Cracking Excel template with the number of test specimen replicates and average of VMA and VFA of replicates. The output of FlexMAT Cracking are linear viscoelastic properties and Prony series data. These are then imported to the FlexPAVE software. The flow diagram of this process is shown in Figure 17.

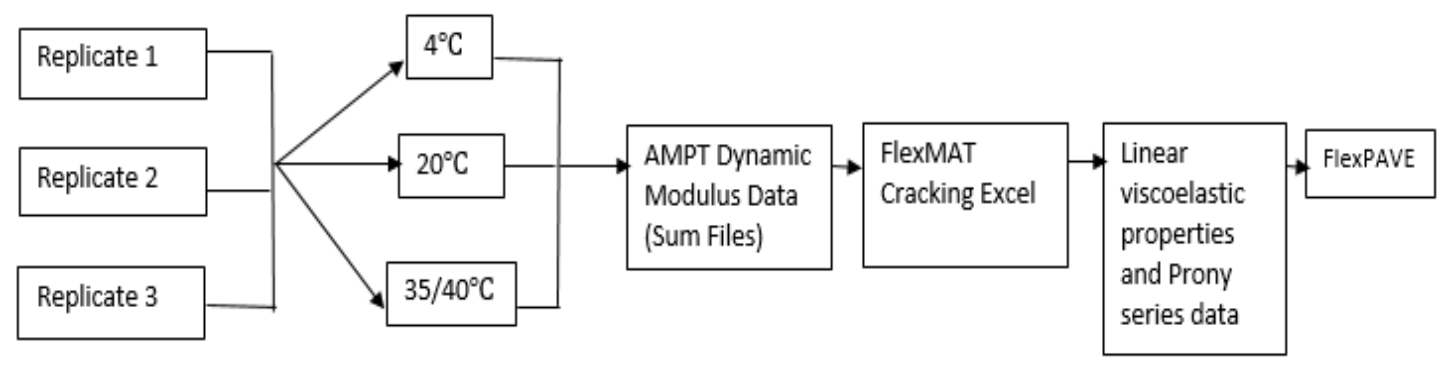

Figure 17: Flow Diagram of Dynamic Modulus AMPT Data Uploaded into FlexMAT Cracking 
The Table 22 shows approximate time to perform dynamic modulus test for different geometries.

Table 22: Approximate Time to Perform Dynamic Modulus Test in AMPT

\begin{tabular}{|c|c|c|c|c|c|c|c|c|}
\hline \multirow{2}{*}{$\begin{array}{l}\text { Dimension, } \\
\mathrm{mm}\end{array}$} & \multirow{2}{*}{$\begin{array}{c}\text { Quarter } \\
\text { and } \\
\text { Compact, } \\
\text { hrs. }\end{array}$} & \multirow{2}{*}{$\begin{array}{c}\text { SSD Before } \\
\text { Fabrication, } \\
\text { hr. }\end{array}$} & \multirow{2}{*}{$\begin{array}{l}\text { Coring and } \\
\text { Sawing, } \\
\text { hrs. }\end{array}$} & \multirow{2}{*}{$\begin{array}{c}\text { SSD After } \\
\text { Fabrication, } \\
\text { hr. }\end{array}$} & \multicolumn{3}{|c|}{$\begin{array}{c}\text { Dynamic } \\
\text { Modulus Test, } \\
\text { hrs. }\end{array}$} & \multirow{2}{*}{$\begin{array}{c}\text { Total } \\
\text { Time, } \\
\text { hrs. }\end{array}$} \\
\hline & & & & & $4 \mathrm{C}$ & $20 \mathrm{C}$ & $\begin{array}{l}40 / \\
35 \mathrm{C}\end{array}$ & \\
\hline $100 \times 150$ & 4.5 & 1 & 1.5 & 1 & 5.5 & 2.5 & 5 & 21 \\
\hline $38 X 110$ & 3.5 & 1 & 1 & 1 & 4 & 2 & 2 & 14.5 \\
\hline $\begin{array}{l}38 X 110 \\
\text { (Center) }\end{array}$ & 4.5 & 1 & 1.5 & 1 & 4 & 2 & 2 & 16 \\
\hline $50 \times 110$ & 3.5 & 1 & 1 & 1 & 4.5 & 2 & 2.5 & 15.5 \\
\hline $\begin{array}{l}\text { 50X110 } \\
\text { (Center) }\end{array}$ & 4.5 & 1 & 1.5 & 1 & 4.5 & 2 & 2.5 & 17 \\
\hline $75 \times 110$ & 4.5 & 1 & 1.5 & 1 & 5 & 2.5 & 3 & 18.5 \\
\hline
\end{tabular}

\subsection{DIRECT TENSION CYCLIC FATIGUE PROCEDURE}

The DTC Fatigue test is conducted per AASHTO TP 107 for 100X130 mm and AASHTO TP 133 for $38 X 110 \mathrm{~mm}$ specimens. A minimum of three replicates are required for DTC fatigue testing. The test setup is shown in Figure 18.

Sample fabrication and testing for different specimen geometries is done per AASHTO specification as:

\begin{tabular}{|c|c|c|}
\hline Dimension, mm & Fabrication Standard & Test Standard \\
\hline $100 \times 130$ & AASHTO R 83-17 & AASHTO TP 107 \\
\cline { 1 - 1 } $38 \mathrm{X} 110$ & AASHTO PP 99-19 & AASHTO TP 133 \\
\cline { 1 - 1 } $50 \mathrm{X} 110$ & & \\
\hline
\end{tabular}

After specimen fabrication and gluing gauge points, the samples are glued to the platens which have been heated to $40^{\circ} \mathrm{C}$. Heating the platen helps specimens from debonding. Prior to testing, glued sample and platen are temperature conditioned at target test temperature. 


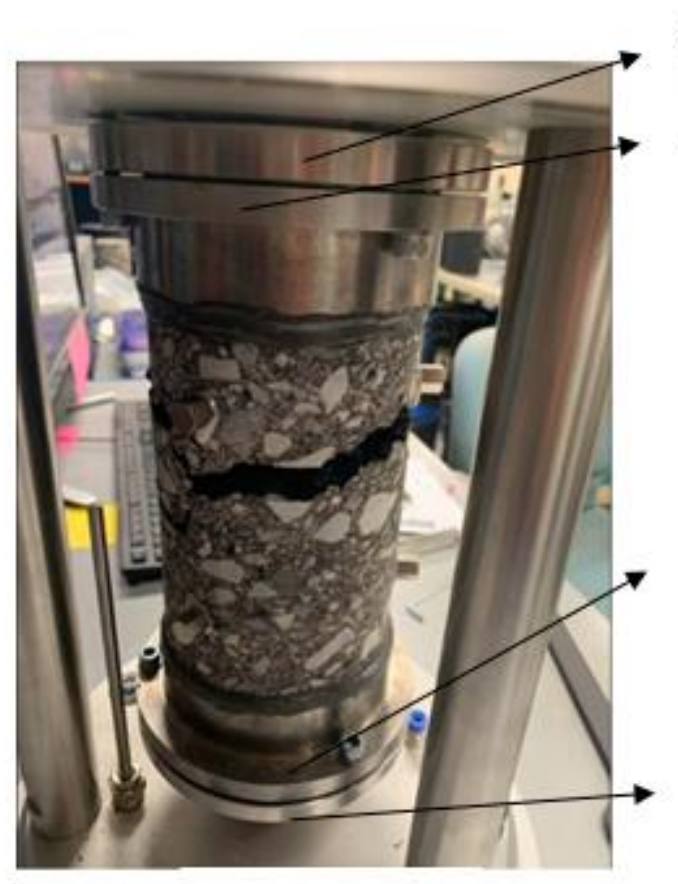

a)

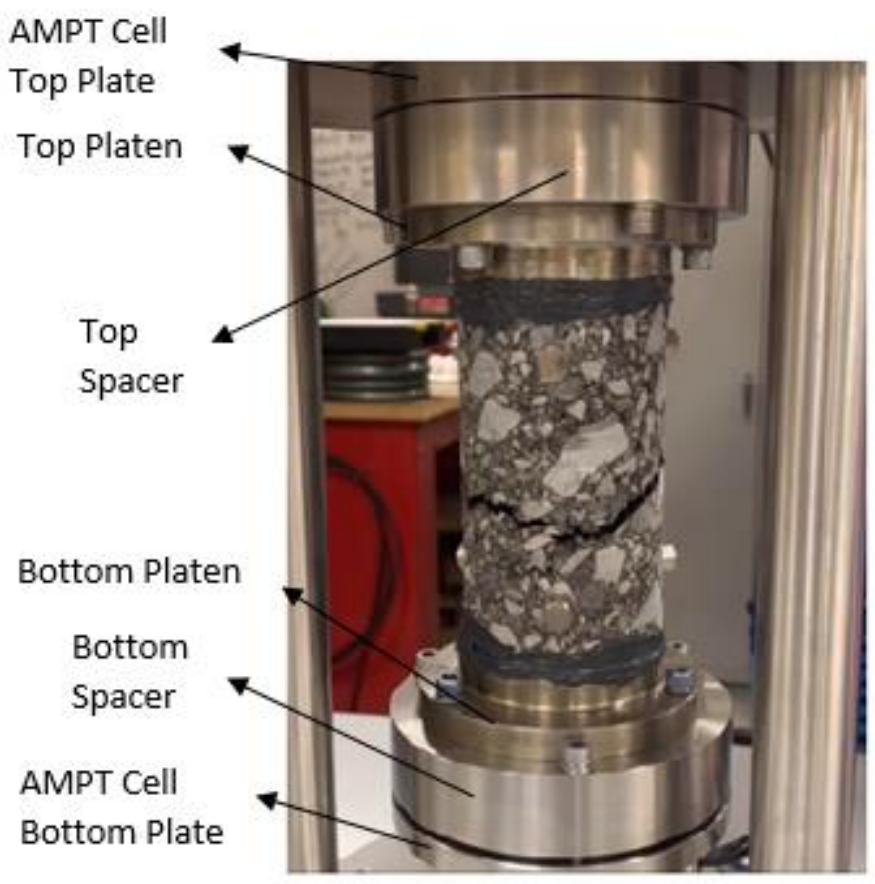

b)

Figure 18: DTC Fatigue Test Setup a) $100 X 130 \mathrm{~mm}$ Specimen b) $75 \times 110 \mathrm{~mm}$

The test temperature is determined from adjusted binder type per Section 2.2.2, Equation 14 . Adjusted binder is calculated from LTPPBind at layer depth, mm and 98\% reliability for Morgantown, WV. The layer depth used in LTPPBind, adjusted binder type and test temperature are:

\begin{tabular}{|c|c|c|c|c|c|}
\hline $\begin{array}{c}\text { NMAS, } \\
\text { mm }\end{array}$ & $\begin{array}{c}\text { Layer } \\
\text { Thickness, inch }\end{array}$ & $\begin{array}{c}\text { Layer } \\
\text { Depth, inch }\end{array}$ & $\begin{array}{c}\text { T-400 Binder } \\
\text { Type }\end{array}$ & $\begin{array}{c}\text { Adjusted } \\
\text { Binder Type }\end{array}$ & $\begin{array}{c}\text { Test } \\
\text { Temperature, }{ }^{\circ} \mathrm{C}\end{array}$ \\
\hline 12 & 2 & 2 & $64 \mathrm{H}-22$ & $70-22$ & 21 \\
\hline 19 & 3 & 5 & $64-22$ & $58-22$ & 15 \\
\hline 25 & 6 & 11 & $64 \mathrm{~S}-22$ & $58-16$ & 18 \\
\hline
\end{tabular}

DTC fatigue test starts with dynamic modulus fingerprint test. The fingerprint test is performed at $10 \mathrm{~Hz}$ and a range of 50 to 75 microstrain for all dimension test specimens. The initial modulus value required for fingerprint test is computed using the master curve from the dynamic modulus test and at DTC fatigue test temperature. The unique sample numbers for dynamic modulus test that were used in MasterSolver at DTC fatigue test temperature to obtain initial estimated modulus for DTC fatigue test specimens are shown as: 


\begin{tabular}{|c|c|c|c|c|}
\hline $\begin{array}{c}\text { Dynamic } \\
\text { Modulus } \\
\text { Specimens }\end{array}$ & $\begin{array}{l}\text { Initial Estimated } \\
\text { Modulus, } \mathrm{MPa}\end{array}$ & $\begin{array}{l}\text { DTC Fatigue } \\
\text { specimen }\end{array}$ & $\begin{array}{c}\text { Initial } \\
\text { Fingerprint, } \\
\mathrm{MPa}\end{array}$ & $\begin{array}{c}\text { Final } \\
\text { Fingerprint, } \\
\mathrm{MPa}\end{array}$ \\
\hline \multirow{3}{*}{1,2 , and 3} & \multirow{3}{*}{6995.5} & 22 & 7126 & 6642 \\
\hline & & 23 & 7539 & 7527 \\
\hline & & 24 & 8562 & 7771 \\
\hline \multirow{3}{*}{8,9, and 10} & \multirow{3}{*}{8845.7} & 25 & 7636 & 7015 \\
\hline & & 26 & 8990 & 9057 \\
\hline & & 27 & 9696 & 9516 \\
\hline \multirow{3}{*}{15,16, and 17} & \multirow{3}{*}{10165.6} & 28 & 6285 & 6210 \\
\hline & & 29 & 9329 & 8866 \\
\hline & & 30 & 9355 & 8448 \\
\hline \multirow{3}{*}{$31 \mathrm{a}, \mathrm{b}$, and c } & \multirow{3}{*}{7004.2} & $32 \mathrm{a}$ & 5518 & 5499 \\
\hline & & $32 \mathrm{~b}$ & 7274 & 7198 \\
\hline & & $32 \mathrm{c}$ & 7315 & 7251 \\
\hline \multirow{3}{*}{$33 \mathrm{a}, \mathrm{b}$, and $\mathrm{c}$} & \multirow{3}{*}{9575.5} & $34 \mathrm{a}$ & 7132 & 7117 \\
\hline & & $34 \mathrm{~b}$ & 8480 & 8437 \\
\hline & & $34 \mathrm{c}$ & 7464 & 7335 \\
\hline \multirow{3}{*}{$35 \mathrm{a}, \mathrm{b}$, and $\mathrm{c}$} & \multirow{3}{*}{9230.9} & $36 \mathrm{a}$ & 6591 & 6587 \\
\hline & & $36 \mathrm{~b}$ & 9640 & 9554 \\
\hline & & $36 \mathrm{c}$ & 7285 & 7251 \\
\hline \multirow{3}{*}{37,38 , and 39} & \multirow{3}{*}{7306.6} & 40 & 5296 & 5265 \\
\hline & & 41 & 7285 & 7223 \\
\hline & & 42 & 8085 & 8004 \\
\hline \multirow{3}{*}{43,44, and 45} & \multirow{3}{*}{7724.0} & 46 & 8274 & 8186 \\
\hline & & 47 & 8481 & 8409 \\
\hline & & 48 & 6570 & 6551 \\
\hline \multirow{3}{*}{49,50, and 51} & \multirow{3}{*}{9138.1} & 52 & 9367 & 9314 \\
\hline & & 53 & 8864 & 8819 \\
\hline & & 54 & 8580 & 8528 \\
\hline \multirow{3}{*}{$55 \mathrm{a}, \mathrm{b}$, and c } & \multirow{3}{*}{7934.9} & $56 \mathrm{a}$ & 6026 & 5988 \\
\hline & & $56 \mathrm{~b}$ & 6491 & 6433 \\
\hline & & $56 \mathrm{c}$ & 6640 & 6582 \\
\hline \multirow{3}{*}{$59 \mathrm{a}, \mathrm{b}$, and $\mathrm{c}$} & \multirow{3}{*}{9144.6} & $60 \mathrm{a}$ & 7957 & 7904 \\
\hline & & $60 \mathrm{~b}$ & 8869 & 8789 \\
\hline & & $60 \mathrm{c}$ & 8002 & 7944 \\
\hline \multirow{3}{*}{$63 \mathrm{a}, \mathrm{b}$, and $\mathrm{c}$} & \multirow{3}{*}{9235.2} & $64 a$ & 7100 & 7041 \\
\hline & & $64 \mathrm{~b}$ & 7092 & 7075 \\
\hline & & $64 \mathrm{c}$ & 7229 & 7213 \\
\hline \multirow{3}{*}{67,68, and 69} & & 70 & 6300 & 6229 \\
\hline & 6753.2 & 71 & 8365 & 8253 \\
\hline & & 72 & 8593 & 8478 \\
\hline & & 76 & 6549 & 6528 \\
\hline 73,74 , and 75 & 7954.0 & 77 & 6776 & 6731 \\
\hline & & 78 & 8893 & 8820 \\
\hline
\end{tabular}




\begin{tabular}{|c|c|c|c|c|}
\hline $\begin{array}{l}\text { Dynamic } \\
\text { Modulus } \\
\text { Specimens }\end{array}$ & $\begin{array}{l}\text { Initial Estimated } \\
\text { Modulus, MPa }\end{array}$ & $\begin{array}{l}\text { DTC Fatigue } \\
\text { specimen }\end{array}$ & $\begin{array}{c}\text { Initial } \\
\text { Fingerprint, } \\
\mathrm{MPa}\end{array}$ & $\begin{array}{c}\text { Final } \\
\text { Fingerprint, } \\
\mathrm{MPa}\end{array}$ \\
\hline \multirow{3}{*}{79,80, and 81} & \multirow{3}{*}{9898.2} & 82 & 8147 & 8148 \\
\hline & & 83 & 9424 & 9357 \\
\hline & & 84 & 8654 & 8621 \\
\hline \multirow{3}{*}{85,86, and 87} & \multirow{3}{*}{7648.6} & 88 & 7592 & 7498 \\
\hline & & 89 & 8361 & 8109 \\
\hline & & 90 & 7388 & 7297 \\
\hline \multirow{3}{*}{91,92 , and 93} & \multirow{3}{*}{8360.8} & 94 & 9591 & 9477 \\
\hline & & 95 & 9412 & 9251 \\
\hline & & 96 & 8986 & 8878 \\
\hline \multirow{3}{*}{97,98 , and 99} & \multirow{3}{*}{9252.3} & 100 & 7180 & 7136 \\
\hline & & 101 & 7796 & 7722 \\
\hline & & 102 & 8165 & 8034 \\
\hline
\end{tabular}

The test parameters used for the DTC fatigue test are:

\begin{tabular}{|c|c|c|c|}
\hline AASHTO & Dimension, mm & Seating Load & $\begin{array}{c}\text { Minimum Number of Load } \\
\text { Cycles }\end{array}$ \\
\hline TP 107-18 & $100 X 130$ & $0.09 \mathrm{kN}(20 \mathrm{lbs})$. & 500 \\
\hline \multirow{2}{*}{ TP 133-19 } & $38 \mathrm{X} 110$ & \multirow{2}{*}{$0.01 \mathrm{kN}(2.2 \mathrm{lbs})}$. & $2,000-80,000$ \\
\cline { 2 - 2 } & $50 \mathrm{X} 110$ & \\
\cline { 2 - 2 } & $75 \times 110$ & & \\
\hline
\end{tabular}

The file with all cycle data and file with initial cycle data (AASHTO T 378-17 and AASHTO TP 132-19) generated by the AMPT are uploaded into the FlexMAT Cracking Excel template with the number of test specimen replicates. The output of FlexMAT Cracking is S-VECD fatigue properties. These are further imported into FlexPAVE software. The flow diagram of this process is shown in Figure 19.

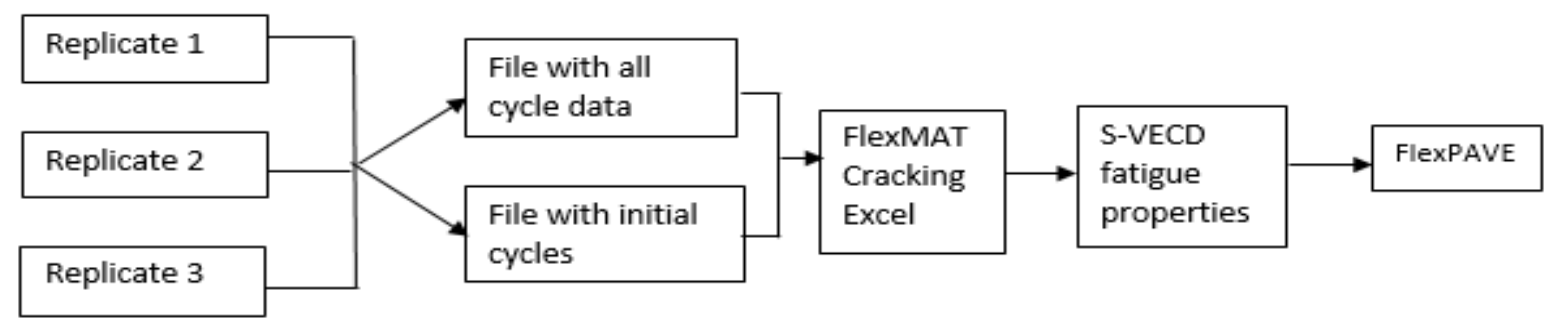

Figure 19: Flow Diagram of DTC Fatigue AMPT Data Uploaded into FlexMAT Cracking

The end failure data is discarded, and the data is analyzed only for samples with middle failure.

The number of samples that resulted in end failure is shown as: 


\begin{tabular}{|c|c|c|}
\hline Mix, mm & Sample Type & $\begin{array}{l}\text { Number of End } \\
\text { Failures }\end{array}$ \\
\hline 12.5 & \multirow{3}{*}{1} & none \\
\hline 19 & & 1 \\
\hline 25 & & 2 \\
\hline 12.5 & \multirow{3}{*}{2} & 1 \\
\hline 19 & & none \\
\hline 25 & & 2 \\
\hline 12.5 & \multirow{3}{*}{3} & none \\
\hline 19 & & none \\
\hline 25 & & none \\
\hline 12.5 & \multirow{3}{*}{4} & none \\
\hline 19 & & none \\
\hline 25 & & none \\
\hline 12.5 & \multirow{3}{*}{5} & none \\
\hline 19 & & none \\
\hline 25 & & none \\
\hline 12.5 & \multirow{3}{*}{6} & none \\
\hline 19 & & none \\
\hline 25 & & none \\
\hline
\end{tabular}

The Table 23 shows approximate time to perform dynamic modulus test for different geometries.

Table 23: Approximate Time to Perform Cyclic Fatigue Test in AMPT

\begin{tabular}{|c|c|c|c|c|c|c|c|}
\hline \multirow[b]{2}{*}{$\begin{array}{l}\text { Dimension, } \\
\mathrm{mm}\end{array}$} & \multirow{2}{*}{$\begin{array}{c}\text { Quarter } \\
\text { and } \\
\text { Compact, } \\
\text { hrs. }\end{array}$} & \multirow[b]{2}{*}{$\begin{array}{c}\text { SSD Before } \\
\text { Fabrication, } \\
\text { hr. }\end{array}$} & \multirow{2}{*}{$\begin{array}{c}\text { Coring } \\
\text { and } \\
\text { Sawing, } \\
\text { hrs. }\end{array}$} & \multirow[b]{2}{*}{$\begin{array}{c}\text { SSD After } \\
\text { Fabrication, } \\
\text { hr. }\end{array}$} & \multicolumn{2}{|c|}{$\begin{array}{c}\text { DTC Fatigue } \\
\text { Test, hrs. }\end{array}$} & \multirow[b]{2}{*}{$\begin{array}{c}\text { Total } \\
\text { Time, } \\
\text { hrs. }\end{array}$} \\
\hline & & & & & $\begin{array}{c}\text { Gluing } \\
\text { and } \\
\text { Resting, } \\
\text { hrs. }\end{array}$ & $\begin{array}{l}\text { Test, } \\
\text { hrs. }\end{array}$ & \\
\hline $100 \times 150$ & 4.5 & 1 & 1.5 & 1 & 14.5 & 4.5 & 27 \\
\hline $38 \times 110$ & 3.5 & 1 & 1 & 1 & 4.5 & 4.5 & 15.5 \\
\hline $\begin{array}{l}38 X 110 \\
\text { (Center) }\end{array}$ & 4.5 & 1 & 1.5 & 1 & 4.5 & 4.5 & 17 \\
\hline $50 \times 110$ & 3.5 & 1 & 1 & 1 & 4.5 & 4.5 & 15.5 \\
\hline $\begin{array}{l}50 X 110 \\
\text { (Center) }\end{array}$ & 4.5 & 1 & 1.5 & 1 & 4.5 & 4.5 & 17 \\
\hline $75 \times 110$ & 4.5 & 1 & 1.5 & 1 & 4.5 & 4.5 & 17 \\
\hline
\end{tabular}

The steps for DTC Fatigue testing using AMPT are described in Appendix E. 


\subsection{STRESS SWEEP RUTTING PROCEDURE}

The SSR test is conducted per AASHTO TP 134-19 for 100X150 mm. A minimum of two replicates are tested for each of low and high temperatures. The test setup is shown in Figure 20. Sample fabrication and temperature conditioning are done as per AASHTO R 83-17 and TP 134-19 respectively.

The test temperature is determined from adjusted binder type per Section 2.2.3, Table 7 and Equation 25. Adjusted binder adjusted binder is calculated from LTPPBind at layer depth, mm and 98\% reliability for Morgantown, WV. Although $19 \mathrm{~mm}$ and $25 \mathrm{~mm}$ have same T-400 binder type, the adjusted binder is not same because layer depth for $19 \mathrm{~mm}$ NMAS is different from layer depth for $25 \mathrm{~mm}$ NMAS. The test setup is shown in Figure 20.

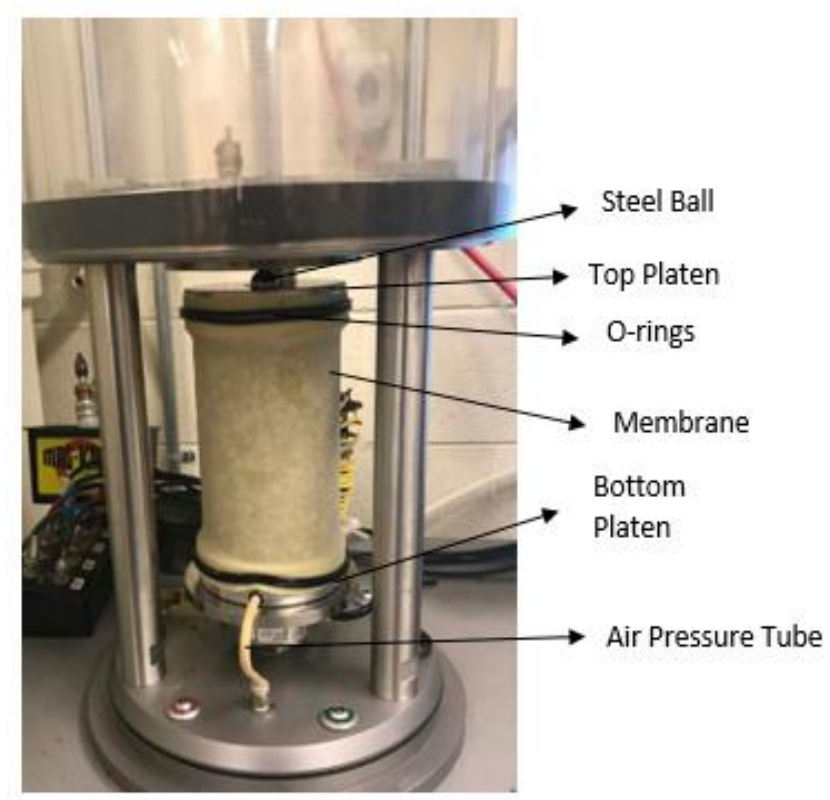

Figure 20: SSR Test Setup for 100X150 mm Specimen

The layer depth used in LTPPBind, adjusted binder type and test temperatures are:

\begin{tabular}{|c|c|c|c|c|c|c|}
\hline $\begin{array}{c}\text { NMAS, } \\
\text { mm }\end{array}$ & $\begin{array}{c}\text { Layer } \\
\text { Thickness, } \\
\text { inch }\end{array}$ & $\begin{array}{c}\text { Layer } \\
\text { Depth, } \\
\text { inch }\end{array}$ & $\begin{array}{c}\text { T-400 } \\
\text { Binder } \\
\text { Type }\end{array}$ & $\begin{array}{c}\text { Adjusted } \\
\text { Binder } \\
\text { Type }\end{array}$ & $\begin{array}{c}\text { Low } \\
\text { Temperature, } \\
{ }^{\circ} \mathrm{C}\end{array}$ & $\begin{array}{c}\text { High } \\
\text { Temperature, } \\
{ }^{\circ} \mathrm{C}\end{array}$ \\
\hline 12 & 2 & 2 & $64 \mathrm{H}-22$ & $70-22$ & 29 & 46 \\
\hline 19 & 3 & 5 & $64-22$ & $58-22$ & 23 & 38 \\
\hline 25 & 6 & 11 & $64 \mathrm{~S}-22$ & $58-16$ & 26 & 35 \\
\hline
\end{tabular}

The SSR test for 50X110 is conducted as per AASHTP TP 134-19. The deviatoric stress, confining pressure, load time and rest time for 50X110 mm specimens remain same as that for 100X150 $\mathrm{mm}$ specimens. The test parameters are shown in section 2.2.3. 
The steps for SSR testing using AMPT are described in Appendix E.

Once SSR test is performed in AMPT, the entire test run data is output to an Excel file. The files for replicates are saved in different folders based on low and high temperature for each mix and each test geometry. These folders are then uploaded into FlexMAT Rutting. The SSR model coefficients generated from FlexMAT Rutting is used in FlexPAVE software. The flow diagram of this process is shown in Figure 21.

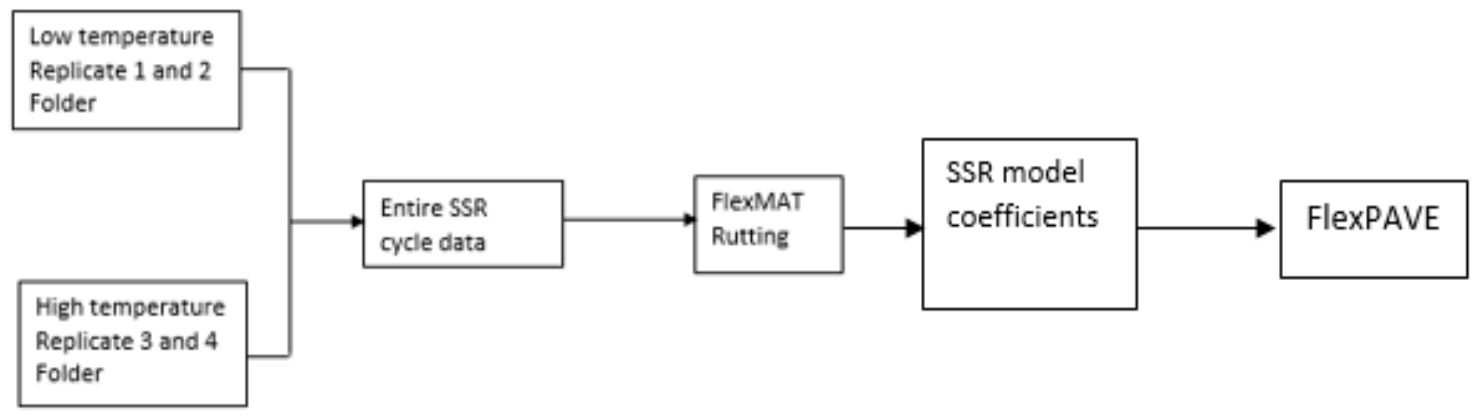

Figure 21: Flow Diagram of SSR AMPT Data Uploaded into FlexMAT Rutting

The Table 24 shows approximate time to perform SSR test for different geometries.

Table 24: Approximate Time to Perform Stress Sweep Rutting in AMPT

\begin{tabular}{|c|c|c|c|c|c|c|c|}
\hline \multirow[b]{2}{*}{$\begin{array}{l}\text { Dimension, } \\
\mathrm{mm}\end{array}$} & \multirow{2}{*}{$\begin{array}{c}\text { Quarter and } \\
\text { Compaction, } \\
\text { hrs. }\end{array}$} & \multirow{2}{*}{$\begin{array}{c}\text { SSD Before } \\
\text { Fabrication, } \\
\text { hrs. }\end{array}$} & \multirow{2}{*}{$\begin{array}{c}\text { Coring } \\
\text { and } \\
\text { Sawing, } \\
\text { hrs. }\end{array}$} & \multirow{2}{*}{$\begin{array}{l}\text { SSD After } \\
\text { Fabrication, } \\
\text { hrs. }\end{array}$} & \multicolumn{2}{|c|}{ SSR Test, hrs. } & \multirow{2}{*}{$\begin{array}{c}\text { Total } \\
\text { Time, } \\
\text { hrs. }\end{array}$} \\
\hline & & & & & $\begin{array}{l}\text { Low } \\
\text { Temp. }\end{array}$ & $\begin{array}{l}\text { High } \\
\text { Temp. }\end{array}$ & \\
\hline $100 \times 150$ & 4.5 & 1 & 1.5 & 1 & 4 & 4 & 16 \\
\hline $50 \times 110$ & 3.5 & 1 & 1 & 1 & 2.5 & 2.5 & 11.5 \\
\hline
\end{tabular}

\subsection{FLEXPAVE PROCEDURE}

The flow diagram of FlexPAVE process is shown in Figure 22. FlexPAVE is used to conduct response analysis or performance analysis for all specimen dimension sets. 


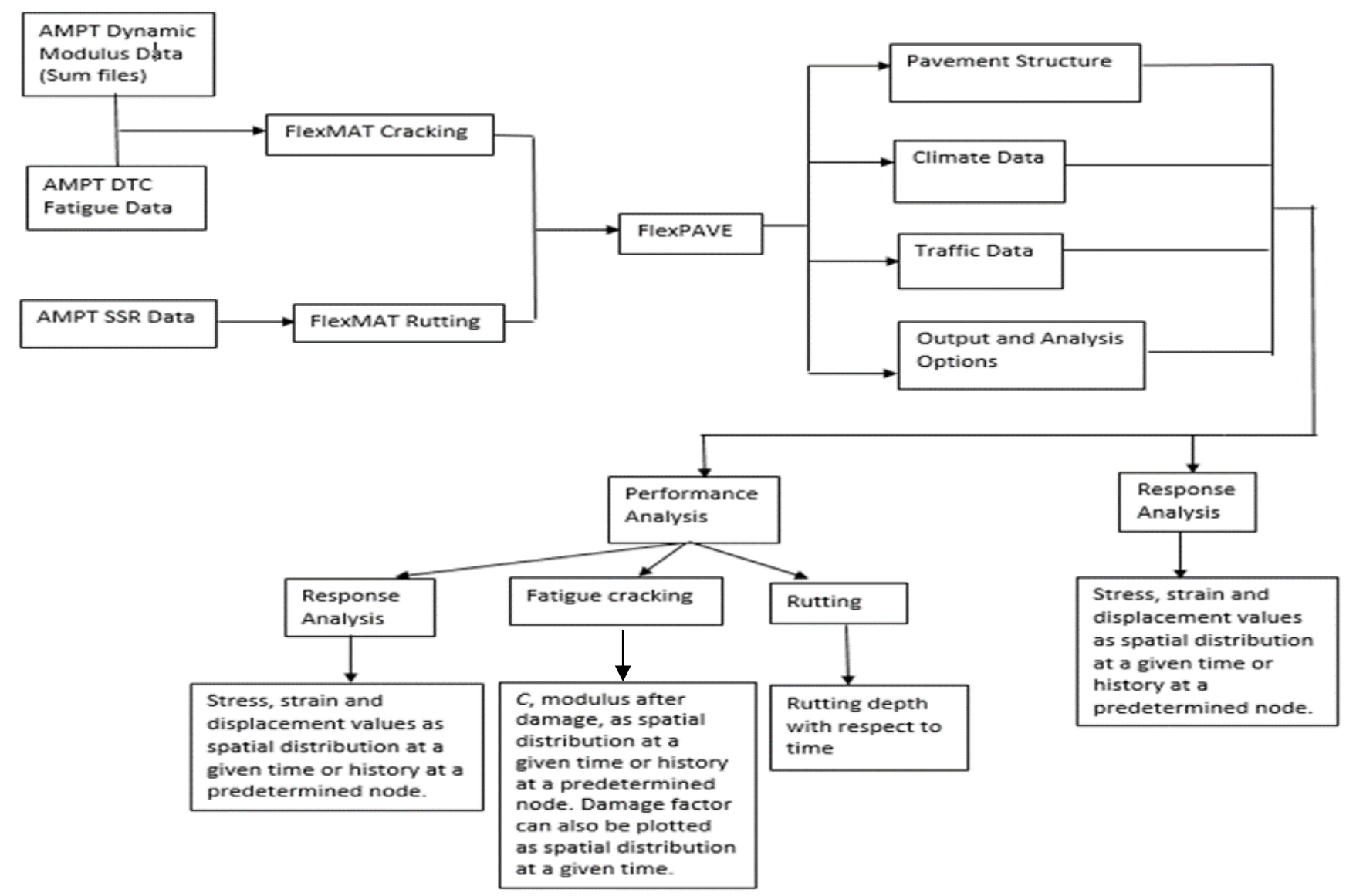

Figure 22: Flow Diagram of FlexPAVE 
The program lets the user conduct response analysis in which only stress, strain, and displacement values within the pavement are computed. The performance analysis also computes responses and additionally estimates fatigue cracking and rutting. In this research, performance analysis is conducted for six specimen dimension sets fabricated from three asphalt mixes.

\subsubsection{Pavement Structures}

Three pavement structures are used for the analysis. The three Structural Numbers of the pavement represent light, medium, and heavy-duty structures. The layer thicknesses and approximate AASHTO 93 structural number, using a structural coefficient of 0.45 is shown Table 25. For each pavement structure, twelve models are analyzed as shown in Table 26.

Table 25: Pavement Thicknesses for Three Pavement Structures used in FlexPAVE Analysis

\begin{tabular}{|c|c|c|c|c|c|c|c|}
\hline \multirow[b]{2}{*}{ Layer } & \multirow{2}{*}{$\begin{array}{c}\text { Mix } \\
\text { Types } \\
\mathrm{mm} \\
\end{array}$} & \multicolumn{2}{|c|}{$\begin{array}{l}\text { FlexPave } \\
\text { Example- } \\
\text { Structure 1 } \\
\end{array}$} & \multicolumn{2}{|c|}{$\begin{array}{l}\text { Structure assumed } \\
\text { for testing- } \\
\text { Structure } 2 \\
\end{array}$} & \multicolumn{2}{|c|}{$\begin{array}{l}\text { Heavy duty } \\
\text { pavement- } \\
\text { Structure } 3\end{array}$} \\
\hline & & $\mathrm{cm}$ & inch & $\mathrm{cm}$ & inch & $\mathrm{cm}$ & inch \\
\hline Wearing & 12.5 & 5 & 1.9 & 5 & 2 & 6.4 & 2.5 \\
\hline Base 1 & 19 & 5 & 1.9 & 7.6 & 3 & 8.9 & 3.5 \\
\hline Base 2 & 25 & 30 & 11.8 & 15.3 & 6 & 40.6 & 16 \\
\hline Subgrade & N/A & 380 & 150 & 380 & 150 & 380 & 150 \\
\hline $\begin{array}{c}\text { Total } \\
\text { thickness }\end{array}$ & N/A & 40 & 15.7 & 27.9 & 11.0 & 55.9 & 22.0 \\
\hline $\begin{array}{c}\text { Approximate } \\
\text { Structural } \\
\text { Number }\end{array}$ & N/A & & & & & & \\
\hline
\end{tabular}


Table 26: Models for Each of the Three Pavement Structures used in FlexPAVE Analysis

\begin{tabular}{|c|c|c|c|}
\hline Model & $\begin{array}{c}\text { Dynamic } \\
\text { Modulus } \\
\text { Specimen, mm }\end{array}$ & $\begin{array}{c}\text { DTC Fatigue } \\
\text { Specimen, } \\
\text { mm }\end{array}$ & $\begin{array}{c}\text { SSR } \\
\text { Specimen, } \\
\text { mm }\end{array}$ \\
\hline 1 & \multicolumn{3}{|c|}{ ST1 } \\
\hline 2 & \multicolumn{2}{|c|}{ ST2 } & ST1 \\
\hline 3 & \multicolumn{2}{|c|}{ ST3 } & ST1 \\
\hline 4 & \multicolumn{3}{|c|}{ ST4 } \\
\hline 5 & \multicolumn{2}{|c|}{ ST5 } & ST4 \\
\hline 6 & \multicolumn{2}{|c|}{ ST6 } & ST1 \\
\hline 7 & \multicolumn{2}{|c|}{ ST1 } & ST4 \\
\hline 8 & \multicolumn{2}{|c|}{ ST2 } & ST4 \\
\hline 9 & \multicolumn{2}{|c|}{ ST3 } & ST4 \\
\hline 10 & \multicolumn{2}{|c|}{ ST4 } & ST1 \\
\hline 11 & \multicolumn{2}{|c|}{ ST5 } & ST1 \\
\hline 12 & \multicolumn{2}{|c|}{ ST6 } & ST4 \\
\hline
\end{tabular}

Once pavement structure is designated, Prony series, fatigue data, and rutting data are imported for each layer. Pavement structure with material properties are designed and imported for all 12 models shown in Table 26. Each of the three pavement structures are designed for 12 models making total of 36 runs. The subgrade is linearly elastic with modulus value of $95 \mathrm{MPa}(13.78 \mathrm{ksi})$ and Poisson ratio of 0.4.

\subsubsection{Climate Data}

The climate data is selected for Morgantown, WV from EICM (Enhanced Integrated Climatic Model) database (NCHRP Report 602, 2008).

\subsubsection{Traffic Data}

A speed of $60 \mathrm{mph}$ was assumed for the analysis.

FlexPAVE allows single axle analysis or single wheel analysis. Single axle represents entire axle and single wheel represents half an axle. An axle load of $18,000 \mathrm{lb}(80 \mathrm{kN})$ with dual tires, a tire pressure of $110 \mathrm{psi}(827.37 \mathrm{kPa})$, and a spacing of 12 inches $(30.47 \mathrm{~cm})$ with a rectangular contact area was assumed. A single wheel analysis is used with 9,000 lbs on each side of the axle and 4,500 lbs. on each tire.

The daily Equivalent Single Axle Load (ESAL) used for this research is 2700 with linear growth rate of $0.4 \%$. 


\subsubsection{Output and Analysis}

The $\mathrm{Y}$ axis is the longitudinal axis in the direction of traffic. The output and analysis option in FlexPAVE are performed at several ZX planes where Y position is fixed. The longitudinal axis is converted into time by design velocity. Thus, instead of having several spatial segments in longitudinal axis, there are several time segments for different ZX planes. Add and replace mesh means that the user can change the display points of the ZX mesh of results, where user can add or replace different meshes depending on desired evaluation points. By using the auto generating evaluation point tool ( $\ldots$ ), evaluation points are generated at both wheels in terms of asphalt pavement depth as shown in Figure 23.

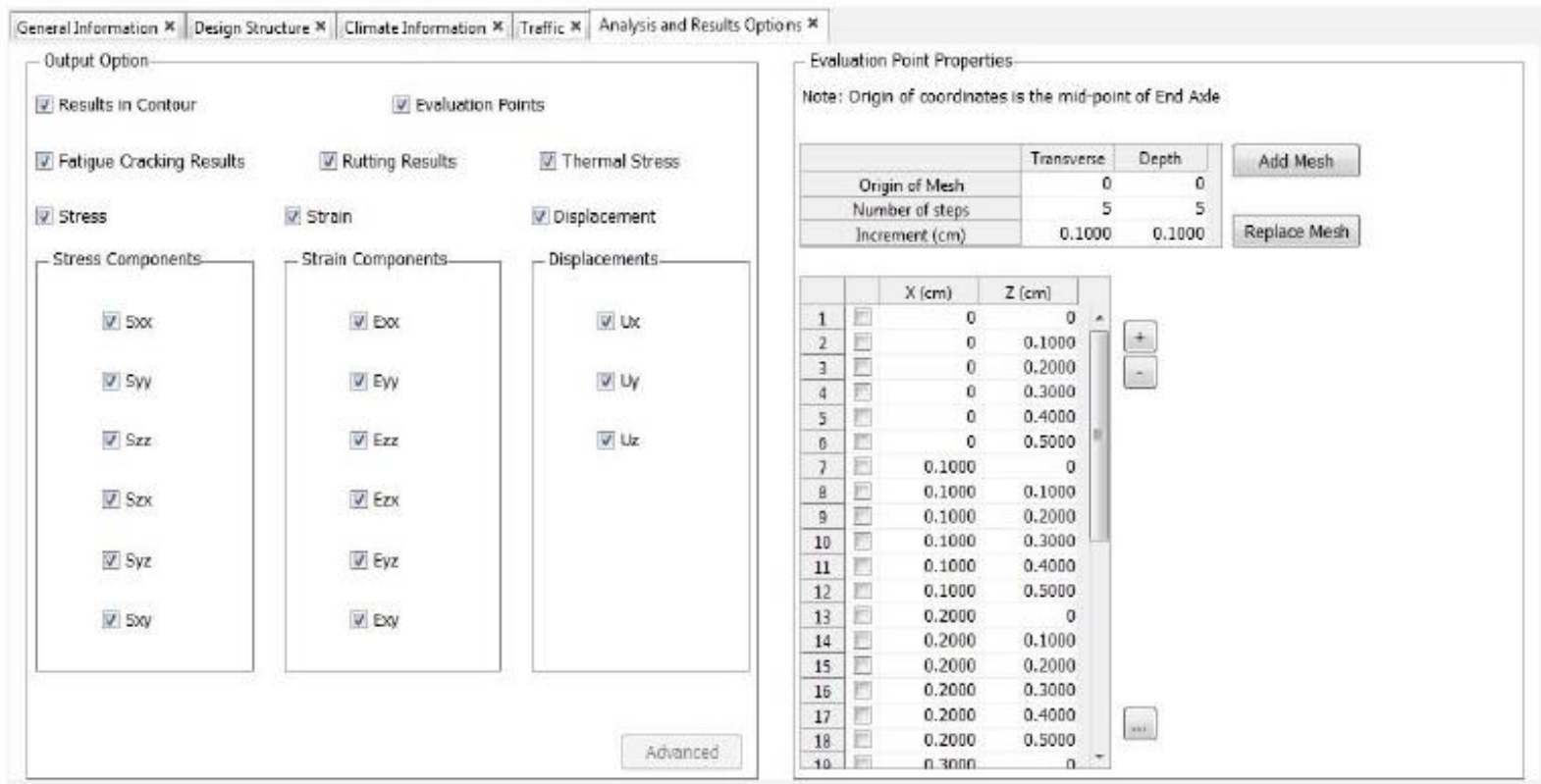

Figure 23: Output and Analysis Option in Performance Analysis of FlexPAVE

\subsubsection{Results}

Response results (stress, strain, and displacement values), fatigue cracking (damage factor and pseudo stiffness), and rut depths can be investigated in FlexPAVE. For analyzing response results, user can choose a certain time and plot stress, strain, and displacement values. Contour and history plots can also be plotted for stress, strain, and displacement at predetermined nodes. For fatigue cracking, contour and history plots can also be plotted for modulus after damage (C). Additionally, percentage damage evolution of whole pavement can be plotted as a function of time. For rutting, rut depth is plotted as a function of time. 


\subsection{ANALYSIS METHODS}

The objective of this research is to determine appropriate sample geometry for dynamic modulus, fatigue, and rutting test for accurate characterization of asphalt mix and ease of testing. To accomplish this objective, effect of sample geometry is analyzed using statistical analysis of the test results and performance predictions using FlexPAVE. The data analysis is divided into 5 analysis:

1) The Analysis 1 consists of ranking six samples types for three NMAS asphalt mixes used for three different tests performed in AMPT:

a) Dynamic modulus values from master curve are ranked for six sample types from three NMAS asphalt mixes. This also includes evaluating rutting potential from dynamic modulus master curve at a temperature of $54.4{ }^{\circ} \mathrm{C}$ and frequency of $5 \mathrm{~Hz}$. The fatigue potential is evaluated from dynamic modulus master curve at a temperature of $20{ }^{\circ} \mathrm{C}$ and frequency of $73.2 \mathrm{~Hz}$.

b) Fatigue $-S_{\text {app }}$ parameter from FlexMAT cracking Excel is used to rank six sample types from three NMAS asphalt mixes.

c) Rutting -Rutting Strain Index (RSI) from FlexMAT rutting Excel is used to rank six sample types from three NMAS asphalt mixes.

2) The Analysis 2 consists of ranking 12 models for three pavement structures in FlexPAVE. Since the purpose of the performance is to have the ability to estimate pavement performance, it is important to examine how different sample dimensions affect pavement performance. This analysis determines if the sample dimensions affect pavement performance predictions.

3) The Analysis 3 performs comparative and statistical analysis of dynamic modulus and phase angle values of each replicate for six STs fabricated form three different NMAS mixes.

4) The Analysis 4 evaluates air void uniformity for samples tested for dynamic modulus test in AMPT using AASHTO R 83-17 for six STs each for three different NMAS mixes.

5) The Analysis 5 statistically compares air voids of STs for six different inscribed circles each for three different NMAS mixes. 


\section{CHAPTER 4. ANALYSIS AND RESULTS}

\subsection{RANKING ANALYSIS FROM MASTER CURVE AND FLEXMAT SOFTWARE}

The Analysis 1 incorporates ranking of six Sample Types (STs) for three different NMAS asphalt mixes used for three different AMPT tests. The summary of sample geometries with unique inscribed circles are shown as:

\begin{tabular}{|c|c|c|c|}
\hline Sample Types & Dynamic Modulus Test, mm & DTC Fatigue Test, mm & $\begin{array}{c}\text { Stress Sweep Rutting } \\
\text { Test, mm }\end{array}$ \\
\hline 1 & $\begin{array}{l}\text { 100X150- Inscribed Circle of } \\
100 \text { mm- AASHTO T } 378\end{array}$ & $\begin{array}{c}\text { 100X130- Inscribed Circle of } \\
100 \text { mm- AASHTO TP 107-18 }\end{array}$ & $\begin{array}{l}\text { 100X150- Inscribed } \\
\text { Circle of } 100 \text { mm- } \\
\text { AASHTO TP 134-19 }\end{array}$ \\
\hline 2 & \multicolumn{2}{|c|}{$\begin{array}{c}\text { 38X110- Inscribed Circle of } 104 \text { mm- AASHTO TP 132-19 } \\
\text { and AASHTO 133-19 }\end{array}$} & Not Tested \\
\hline 3 & \multicolumn{2}{|c|}{$38 X 110$ - Inscribed Circle of $38 \mathrm{~mm}$} & Not Tested \\
\hline 4 & \multicolumn{2}{|c|}{ 50X110- Inscribed Circle of $120 \mathrm{~mm}$} & $\begin{array}{l}\text { 50X110- Inscribed Circle } \\
\text { of } 120 \mathrm{~mm}\end{array}$ \\
\hline 5 & \multicolumn{2}{|c|}{ 50X110- Inscribed Circle of $50 \mathrm{~mm}$} & Not Tested \\
\hline 6 & \multicolumn{2}{|c|}{ 75X110- Inscribed Circle of $75 \mathrm{~mm}$} & Not Tested \\
\hline
\end{tabular}

The ranking involves dynamic modulus values form master curve, $S_{\text {app }}$ parameter from FlexMAT cracking for fatigue, and Rutting Strain Index (RSI) from FlexMAT rutting for rutting. For ranking analysis all STs are compared to ST1 as it is full size AASHTO standard dimension used for up to 37.5 mm NMAS mixes for dynamic modulus and rutting test and for up to $25 \mathrm{~mm}$ NMAS mixes for fatigue test. Data from replicates were combined, so it is not possible to perform statistical analysis since there is no variance term. Therefore, ranking is performed in this analysis. In dynamic modulus test, ranking is performed from master curves. Dynamic modulus values from master curve are ranked for six STs each for three NMAS asphalt mixes.

In DTC fatigue test, ranking is performed from FlexMAT Cracking Excel. The AMPT data for sample replicates were imported into FlexMAT cracking Excel to produce S-VECD properties. As there 
is no variance term within S-VECD properties, $S_{\text {app }}$ was chosen to rank the six sample types for each if the three NMAS asphalt mixes.

In SSR test, ranking was performed from FlexMAT Rutting Excel. The AMPT data for sample replicates were imported into FlexMAT Rutting Excel to produce shift factor model and RSI for layer type and location. As there is no variance term within shift factors, RSI was chosen to rank the six sample types for each of the three NMAS asphalt mixes.

\subsubsection{Dynamic Modulus Master Curve}

The fitting parameters for six STs for each NMAS mix using MasterSolver are in Table 27. Figures 24 to 26 show master curves for six STs for each NMAS mix. Master curve for all STs are compared to that of ST 1. In general, for $12.5 \mathrm{~mm}$ and $25 \mathrm{~mm}$ NMAS asphalt mix, dynamic modulus values for ST6 were close to the ST1 samples. This is in line with expectations as aspect ratio of sample type 1 and 6 is 1.5. For 19 mm NMAS mix, ST 4 and ST6 are closest to ST1. Again, this is in line with expected as aspect ratio of ST 4 is 2.2 which is next closest to 1.5 aspect ratio of ST 1 and ST 6.

Table 27: Master curve Fitting Parameters

\begin{tabular}{|c|c|c|c|c|c|c|c|}
\hline \multirow{2}{*}{$\begin{array}{l}\Xi \\
\sum \\
\sum \\
\sum \\
\sum\end{array}$} & \multirow{2}{*}{ 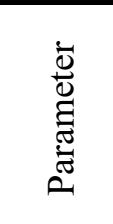 } & \multicolumn{6}{|c|}{ Sample Type } \\
\hline & & 1 & 2 & 3 & 4 & 5 & 6 \\
\hline \multirow{5}{*}{$\stackrel{n}{\simeq}$} & $\beta$ & -1.53217 & -1.40253 & -1.61880 & -1.35363 & -1.34512 & -1.41801 \\
\hline & $\gamma$ & -0.39159 & -0.41499 & -0.39802 & -0.46195 & -0.41845 & -0.41542 \\
\hline & $\Delta \mathrm{EA}$ & 252480 & 216740 & 230558 & 182030 & 231442 & 216602 \\
\hline & Emax & 3156.8 & 3176.1 & 3171.1 & 3142.0 & 3139.3 & 3148.8 \\
\hline & Emin & 0.7 & 2.4 & 0.9 & 5.4 & 3.0 & 2.3 \\
\hline \multirow{5}{*}{$\stackrel{2}{=}$} & $\beta$ & -1.43638 & -1.07081 & -1.18714 & -1.52446 & -0.79246 & -1.79099 \\
\hline & $\gamma$ & -0.34949 & -0.47837 & -0.43304 & -0.39357 & -0.51742 & -0.35928 \\
\hline & $\Delta \mathrm{EA}$ & 308118 & 219342 & 199053 & 226800 & 186464 & 225883 \\
\hline & Emax & 3242.9 & 3249.0 & 3204.2 & 3201.4 & 3211.9 & 3207.0 \\
\hline & Emin & 0.3 & 5.5 & 1.1 & 0.5 & 9.2 & 0.03 \\
\hline \multirow{5}{*}{ 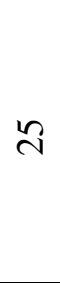 } & $\beta$ & -1.67224 & -1.58035 & -1.33911 & -1.48435 & -1.35888 & -1.80115 \\
\hline & $\gamma$ & -0.40097 & -0.44358 & -0.50749 & -0.47628 & -0.53105 & -0.42424 \\
\hline & $\Delta \mathrm{EA}$ & 338923 & 239440 & 213144 & 217844 & 210030 & 218329 \\
\hline & Emax & 3255.4 & 3211.3 & 3220.0 & 3254.4 & 3267.0 & 3236.3 \\
\hline & Emin & 0.5 & 0.7 & 2.1 & 1.0 & 2.8 & 0.1 \\
\hline
\end{tabular}




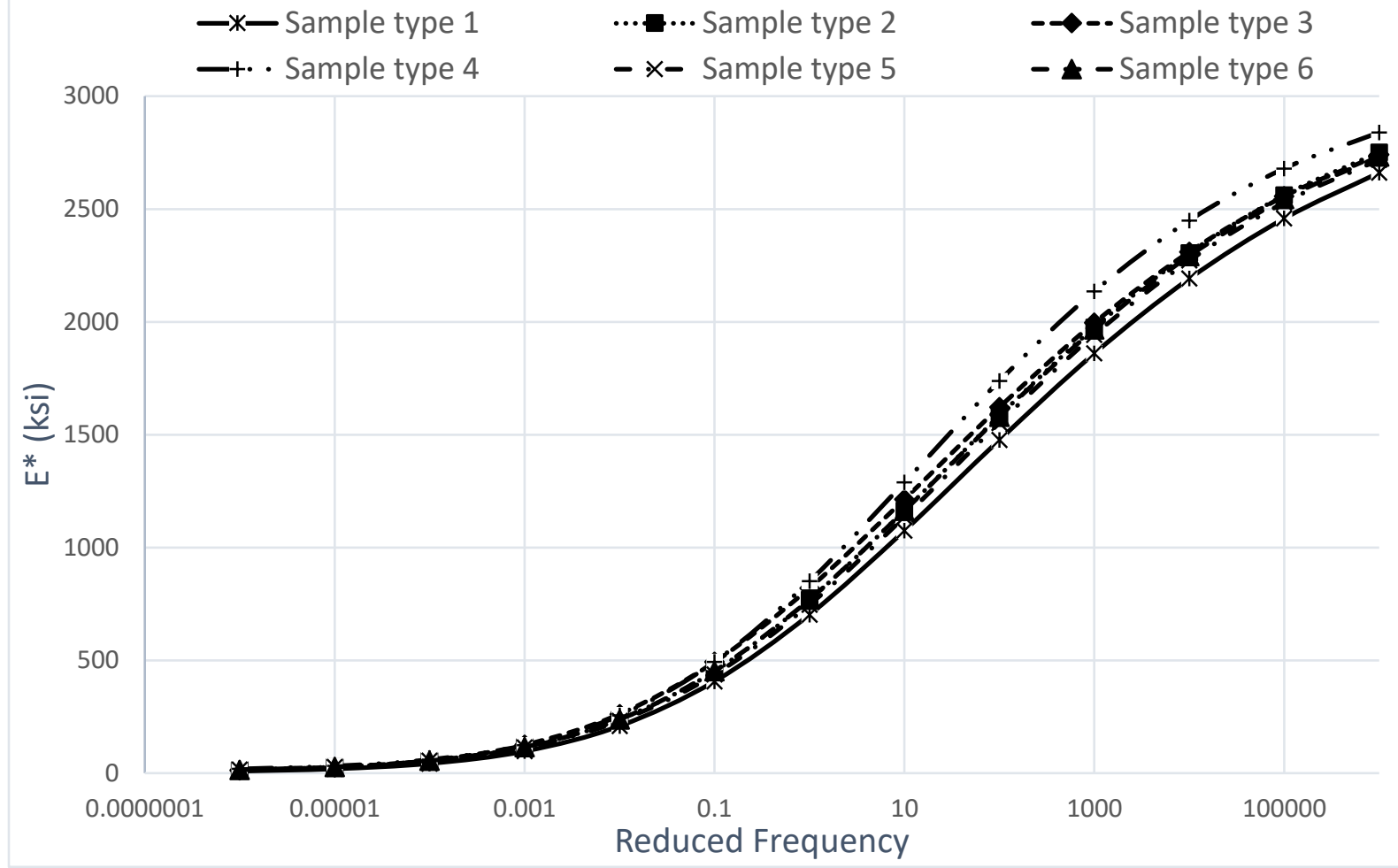

Figure 24: Master curves Design Binder Content, NAMS 12.5 mm, Target 7.0 \% VTM

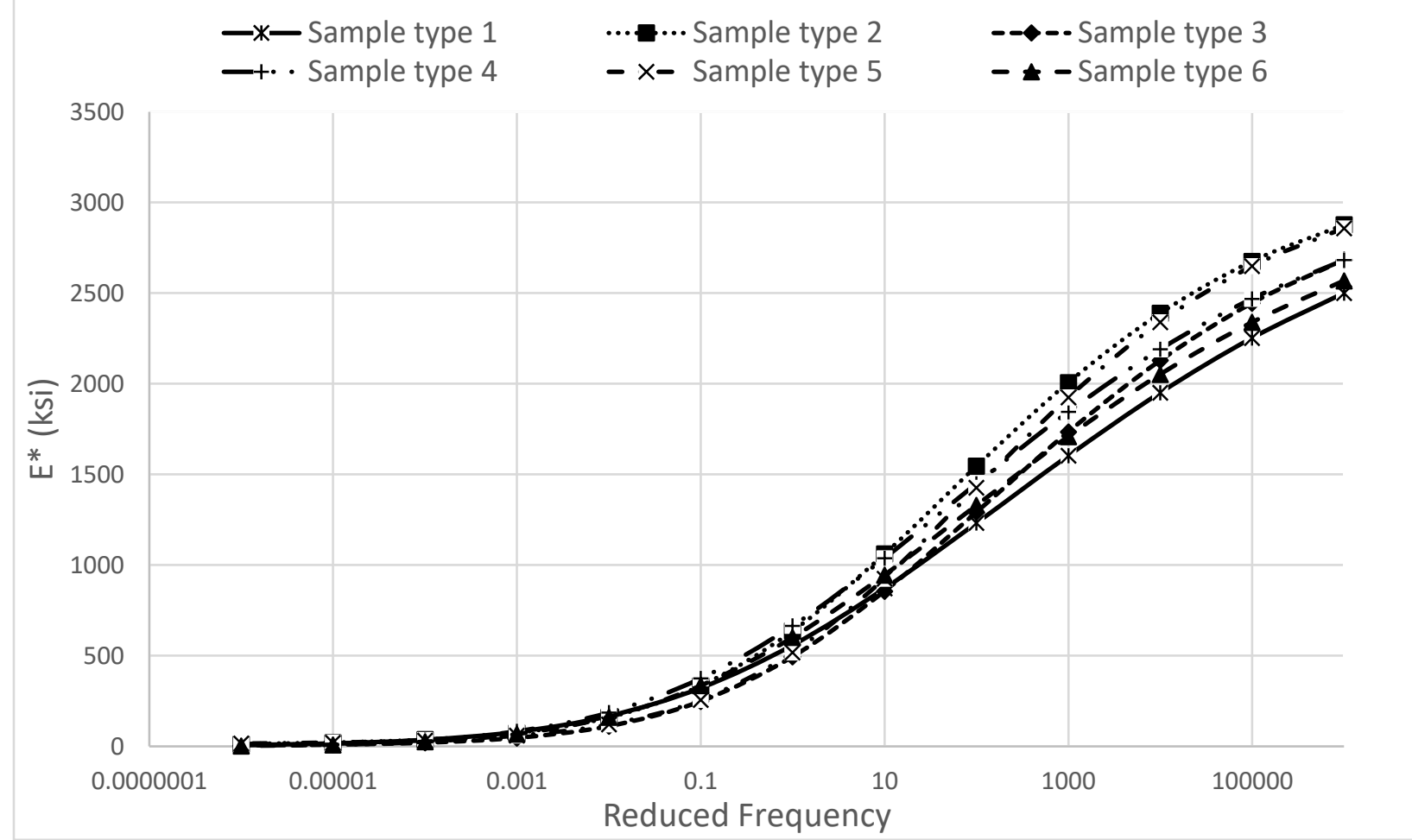

Figure 25: Master curves Design Binder Content, NAMS 19 mm, Target 7.0 \% VTM 


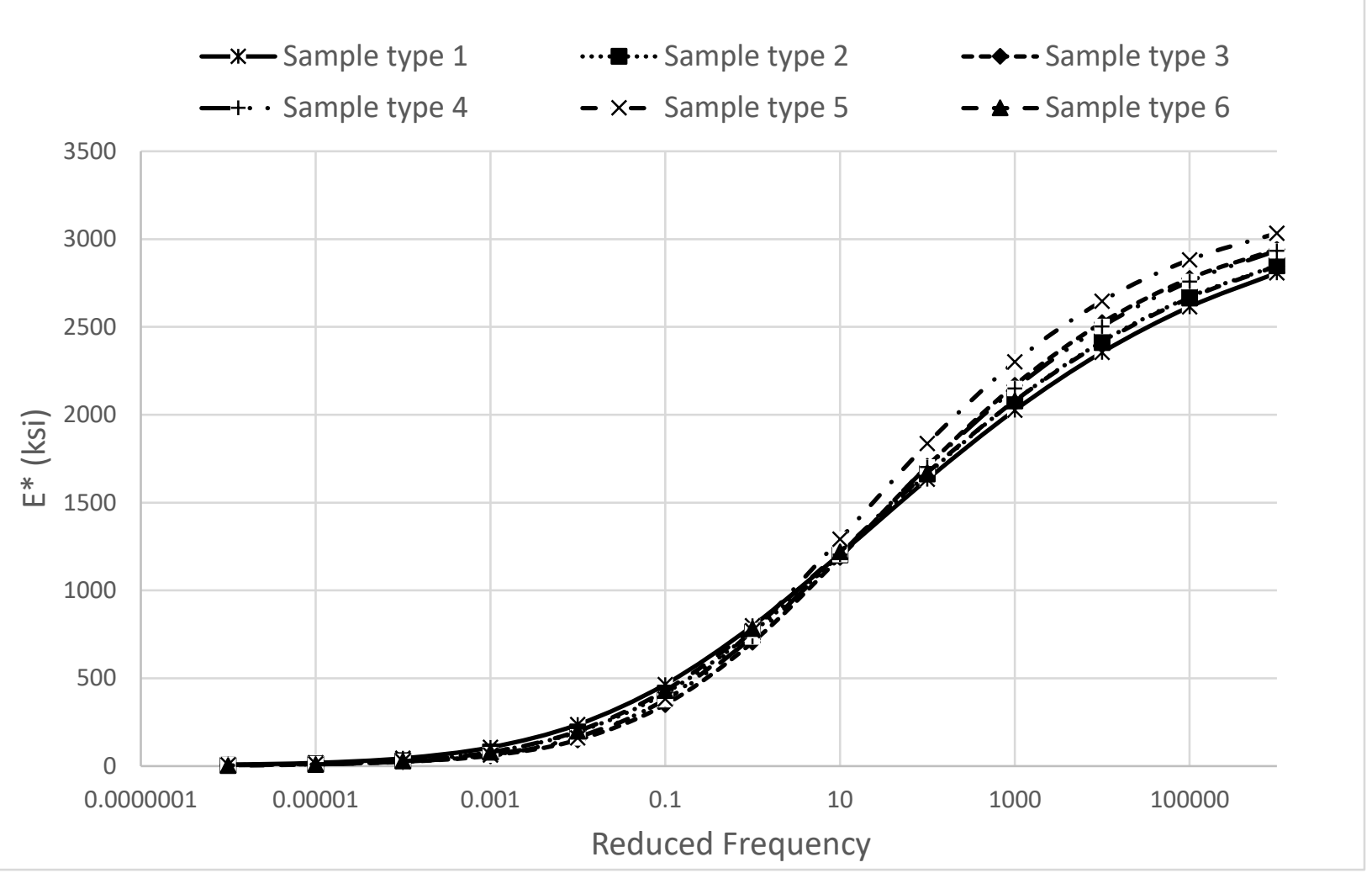

Figure 26: Master curves Design Binder Content, NAMS 25 mm, Target 7.0 \% VTM

\section{|Rutting Consideration from Master Curve}

Pellinen and Witczak. (2002) recommended evaluating rutting potential from dynamic modulus master curve at a temperature of $54.4{ }^{\circ} \mathrm{C}$ and frequency of $5 \mathrm{~Hz}$. The $54.4{ }^{\circ} \mathrm{C}$ represents high pavement temperature susceptible to rutting. The $5 \mathrm{~Hz}$ is approximately equivalent to a speed of $3.8 \mathrm{mph}$ for a 6inch layer per the Ullitz method. For this combination of temperature - frequency, dynamic modulus values for six STs were computed from the master curves with the fitting parameters shown in Table 27 to produce the results in Table 28. Bennert (2009) recommended the threshold dynamic modulus of 94 ksi to limit rutting potential of asphalt mixtures for less than 10 million ESAL's at $54.4{ }^{\circ} \mathrm{C}$ and $5 \mathrm{~Hz}$.

The modulus values comparisons are shown in Figure 27, (a) $9.5 \mathrm{~mm}$ mixes (b) $12.5 \mathrm{~mm}$ and (c) $25 \mathrm{~mm}$ mixes. The results show a highly variable effect of sample sizes on dynamic modulus values. In $12.5 \mathrm{~mm}$ mix, only ST4 crossed the minimum dynamic modulus threshold to limiting rutting. In $19 \mathrm{~mm}$ and $25 \mathrm{~mm}$ mixes, none of the STs crossed the threshold. The estimated dynamic modulus closest to ST 1 for $12.5 \mathrm{~mm}$ mix is ST 5, for $19 \mathrm{~mm}$ is ST 6, and for $25 \mathrm{~mm}$ mix is ST 2. Overall, dynamic modulus obtained from ST 3 was consistently close to that of ST 1 for all three NMAS mixes. While there is not a definite answer as to why the specimens exhibit this behavior, several factors may apply. These include: 
- The ST 3 cored specimens likely had rather uniform air voids throughout the specimen.

- The air voids for each ST were at $7 \% \pm 0.5 \%$. The $\pm 0.5 \%$ difference may have caused significant difference in dynamic modulus values for STs in $19 \mathrm{~mm}$ and $25 \mathrm{~mm}$ asphalt mixes.

Table 28: Dynamic Modulus for Rutting Considerations at $54.4^{\circ} \mathrm{C}$ and $5 \mathrm{~Hz}$

\begin{tabular}{|c|c|c|}
\hline NMAS & Sample Type & Estimated Dynamic Modulus (ksi) \\
\hline \multirow{4}{*}{12.5} & 1 & 41.5 \\
\cline { 2 - 3 } & 2 & 88.4 \\
\cline { 2 - 3 } & 3 & 76.2 \\
\cline { 2 - 3 } & 4 & 152.5 \\
\cline { 2 - 3 } & 5 & 71.4 \\
\hline \multirow{4}{*}{19} & 6 & 88.2 \\
\cline { 2 - 3 } & 1 & 15.5 \\
\cline { 2 - 3 } & 2 & 55.6 \\
\cline { 2 - 3 } & 3 & 45.9 \\
\cline { 2 - 3 } & 4 & 52.1 \\
\hline \multirow{4}{*}{25} & 5 & 68.2 \\
\cline { 2 - 3 } & 1 & 40.8 \\
\cline { 2 - 3 } & 2 & 10.60 \\
\cline { 2 - 3 } & 3 & 38.60 \\
\cline { 2 - 3 } & 4 & 45.40 \\
\cline { 2 - 3 } & 5 & 45.30 \\
\hline
\end{tabular}




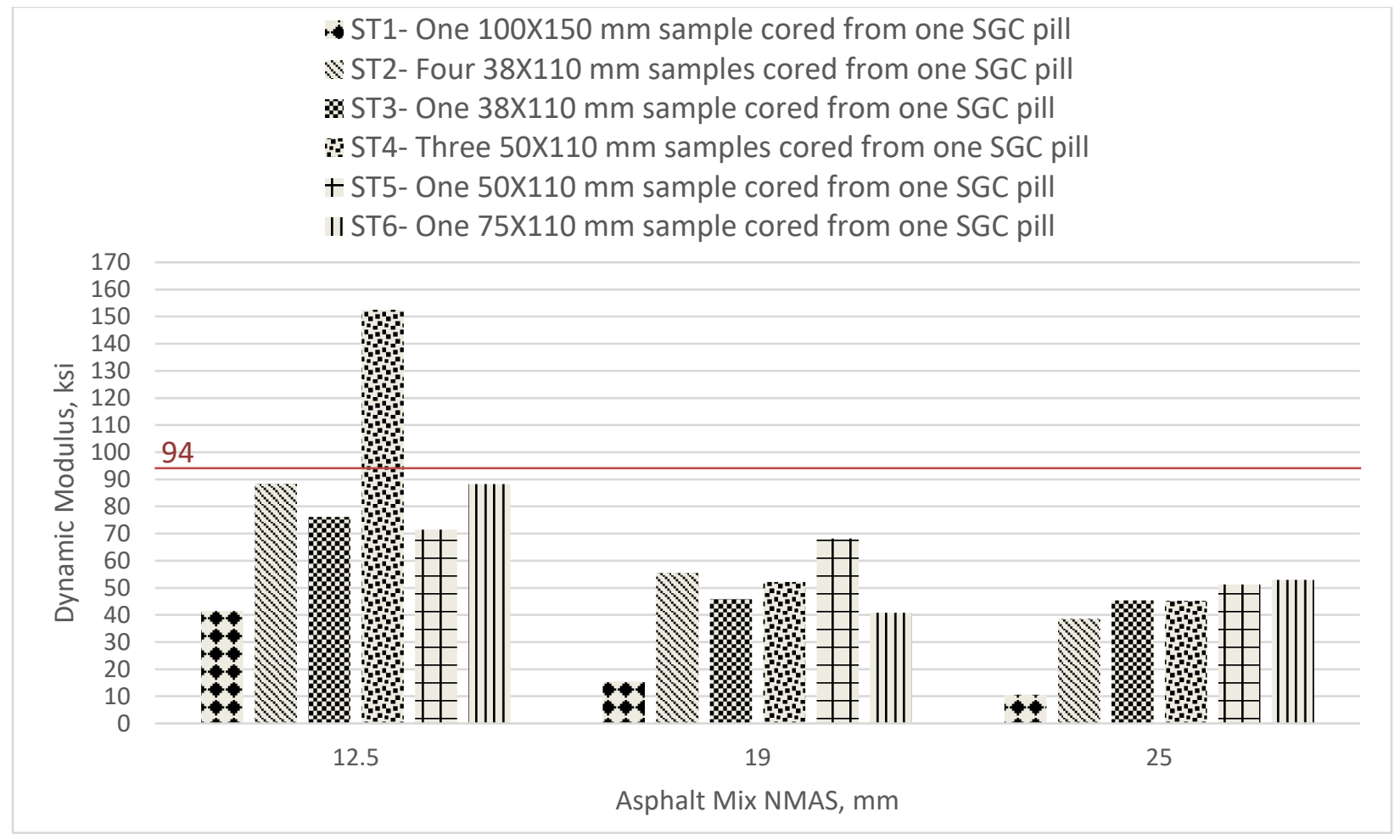

Figure 27: Comparison of Dynamic modulus Values for Rutting Considerations at $54.4^{\circ} \mathrm{C}$ and $5 \mathrm{~Hz}$

\section{Fatigue Consideration from Master Curve}

The fatigue potential was evaluated from dynamic modulus master curve at a temperature of $20^{\circ} \mathrm{C}$ and frequency of $5 \mathrm{~Hz}$. The $5 \mathrm{~Hz}$ was used to compare dynamic modulus from this research to the threshold dynamic modulus value at $20^{\circ} \mathrm{C}$ and $5 \mathrm{~Hz}$ obtained from Bennert (2009). Bennert (2009) performed detailed research on development of limiting fatigue cracking potential at $20^{\circ} \mathrm{C}$ and different loading frequencies. In this research, the combination of temperature - frequency, dynamic modulus values for six STs were computed from the master curves with the fitting parameters shown in Table 27 to produce the results in Table 29. Bennert (2009) recommended the threshold dynamic modulus of $670 \mathrm{ksi}$ to limit fatigue cracking potential of asphalt mixtures for general cracking resistance at $20^{\circ} \mathrm{C}$ and $5 \mathrm{~Hz}$.

The modulus values comparisons are shown in Figure 28 and all STs crossed the fatigue cracking potential threshold. Estimated dynamic modulus closest to ST 1 for $12.5 \mathrm{~mm}$ mix is ST 5, for $19 \mathrm{~mm}$ is ST 3, and for $25 \mathrm{~mm}$ mix is ST 2. These results follow very closely to that of rutting considerations for master curve with the exception of sample type 6 instead of sample type 3 for 19 mm NMAS. When compared sample 3 and 6 in 19 mm NMAS mix, the dynamic modulus values for both sample types were very close with a difference of $3.7 \%$ and comparable to sample type 1 . Overall, dynamic modulus obtained from sample type 6 was consistently close to that of sample type 1 for all three NMAS mixes. 
Table 29: Dynamic Modulus for Fatigue Considerations at $20^{\circ} \mathrm{C}$ and $5 \mathrm{~Hz}$

\begin{tabular}{|c|c|c|}
\hline \multirow{4}{*}{ NMAS } & $\begin{array}{c}\text { Sample } \\
\text { Type }\end{array}$ & $\begin{array}{c}\text { Estimated Dynamic } \\
\text { Modulus (ksi) }\end{array}$ \\
\hline \multirow{4}{*}{12.5} & 1 & 956.9 \\
\cline { 2 - 3 } & 2 & 1039.7 \\
\cline { 2 - 3 } & 3 & 1157.0 \\
\cline { 2 - 3 } & 4 & 1135.6 \\
\cline { 2 - 3 } & 5 & 1013.0 \\
\hline \multirow{5}{*}{19} & 6 & 1039.6 \\
\cline { 2 - 3 } & 1 & 770.3 \\
\cline { 2 - 3 } & 2 & 923.5 \\
\cline { 2 - 3 } & 3 & 737.5 \\
\cline { 2 - 3 } & 4 & 919.8 \\
\hline \multirow{5}{*}{25} & 5 & 789.6 \\
\cline { 2 - 3 } & 1 & 835.8 \\
\cline { 2 - 3 } & 2 & 1079 \\
\cline { 2 - 3 } & 3 & 1064 \\
\cline { 2 - 3 } & 4 & 1034 \\
\cline { 2 - 3 } & 5 & 1053 \\
\cline { 2 - 3 } & 6 & 1128 \\
\hline
\end{tabular}

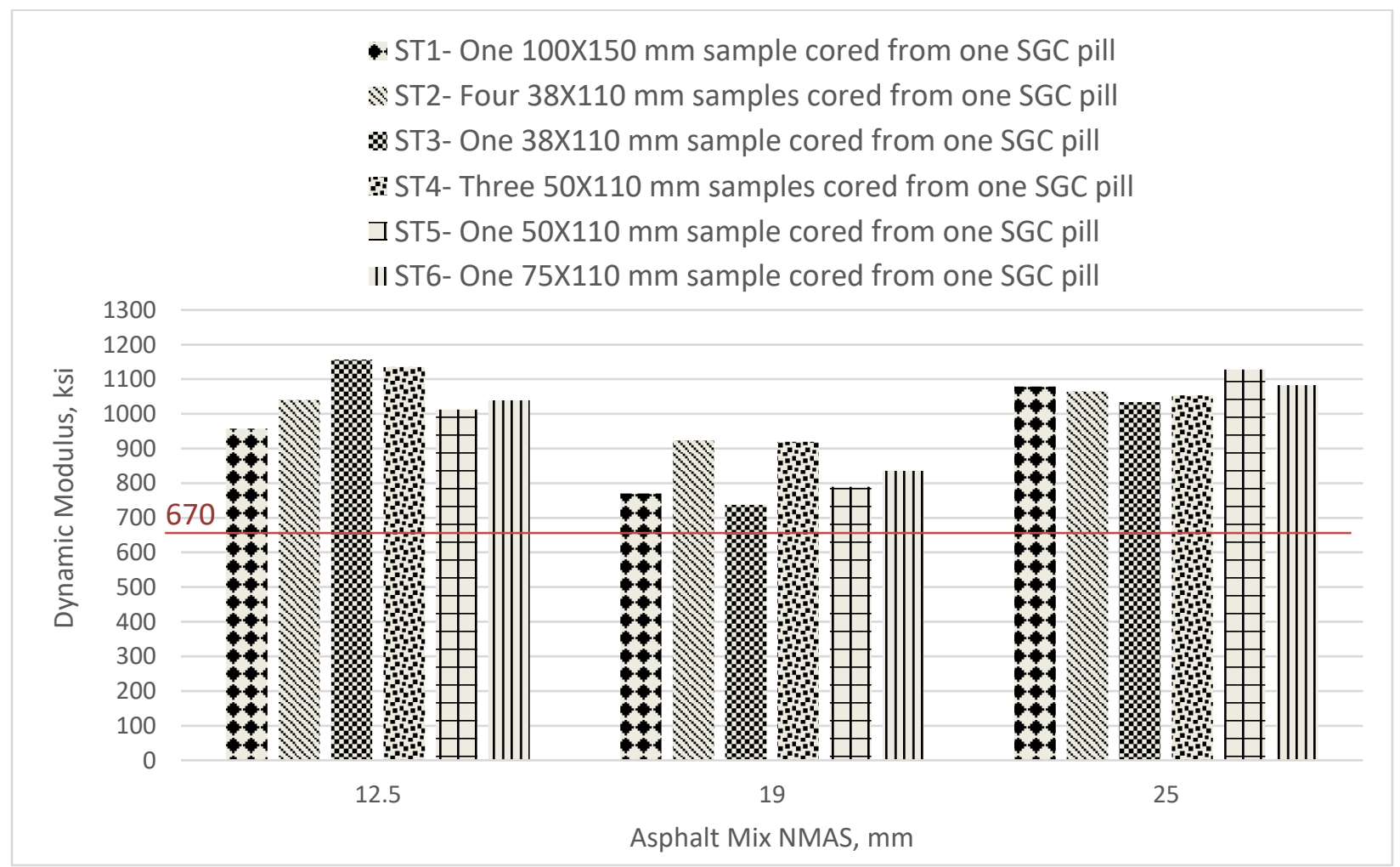

Figure 28: Comparison of Dynamic modulus Values Fatigue Considerations at $20^{\circ} \mathrm{C}$ and $73.2 \mathrm{~Hz}$ 


\subsubsection{DTC Fatigue FlexMAT Cracking}

The fatigue potential was evaluated from the $S_{\text {app }}$ parameter obtained from FlexMAT Cracking Excel at $21^{\circ} \mathrm{C}$. Table 30 shows $\mathrm{S}_{\text {app }}$ value for six different $\mathrm{STs}$ each for three NMAS mixes. The values comparisons are shown in Figure 29, (a) $9.5 \mathrm{~mm}$ mixes (b) $12.5 \mathrm{~mm}$ and (c) $25 \mathrm{~mm}$ mixes.

The $S_{\text {app }}$ was developed by Wang (2019) to address the effect of modulus and toughness parameter on fatigue cracking. Wang (2019) recommended threshold value of $S_{\text {app }}$ of greater than 8 for less than 10 million ESALs. To compare apparent damage capacity of asphalt mix, the $S_{\text {app }}$ values from different STs in FlexMAT Cracking for different NMAS mixes are compared to ST1. The $S_{\text {app }}$ value closest to ST 1 for $12.5 \mathrm{~mm}$ mix is ST 3, for $19 \mathrm{~mm}$ is ST 4, and for $25 \mathrm{~mm}$ mix is ST 4. When compared sample 3 and 4 in $12.5 \mathrm{~mm}$ NMAS mix, the $S_{\text {app }}$ values for both STs were very close with a difference of $8.2 \%$ and comparable to ST 1. Overall, $S_{\text {app }}$ value obtained from ST 4 was consistently close to that of ST 1 for all three NMAS mixes. This is in line with the expectation because literature review shows that specimens with uniform air void distribution reduces test variability in terms of resistance to fatigue cracking. Additionally, results from $S_{\text {app }}$ ranking do not match with the fatigue results considered from dynamic modulus master curves. Therefore, latter may not be a true representation of fatigue resistance of sample types.

Table 30: $\mathrm{S}_{\text {app }}$ Values at $21^{\circ} \mathrm{C}$ for Different Sample Types for Each NMAS asphalt Mix

\begin{tabular}{|c|c|c|}
\hline NMAS & Sample Type & $\mathrm{S}_{\text {app }}$ at $21^{\circ} \mathrm{C}$ \\
\hline \multirow{4}{*}{12.5} & 1 & 18.80 \\
\cline { 2 - 3 } & 2 & 13.50 \\
\cline { 2 - 3 } & 3 & 17.97 \\
\cline { 2 - 3 } & 4 & 16.61 \\
\cline { 2 - 3 } & 5 & 14.00 \\
\hline \multirow{4}{*}{19} & 6 & 14.91 \\
\cline { 2 - 3 } & 1 & 16.36 \\
\cline { 2 - 3 } & 2 & 20.72 \\
\cline { 2 - 3 } & 3 & 18.67 \\
\cline { 2 - 3 } & 4 & 17.84 \\
\hline \multirow{4}{*}{25} & 5 & 14.48 \\
\cline { 2 - 3 } & 6 & 17.91 \\
\cline { 2 - 3 } & 2 & 11.55 \\
\cline { 2 - 3 } & 3 & 14.76 \\
\cline { 2 - 3 } & 4 & 18.07 \\
\cline { 2 - 3 } & 5 & 12.36 \\
\hline
\end{tabular}




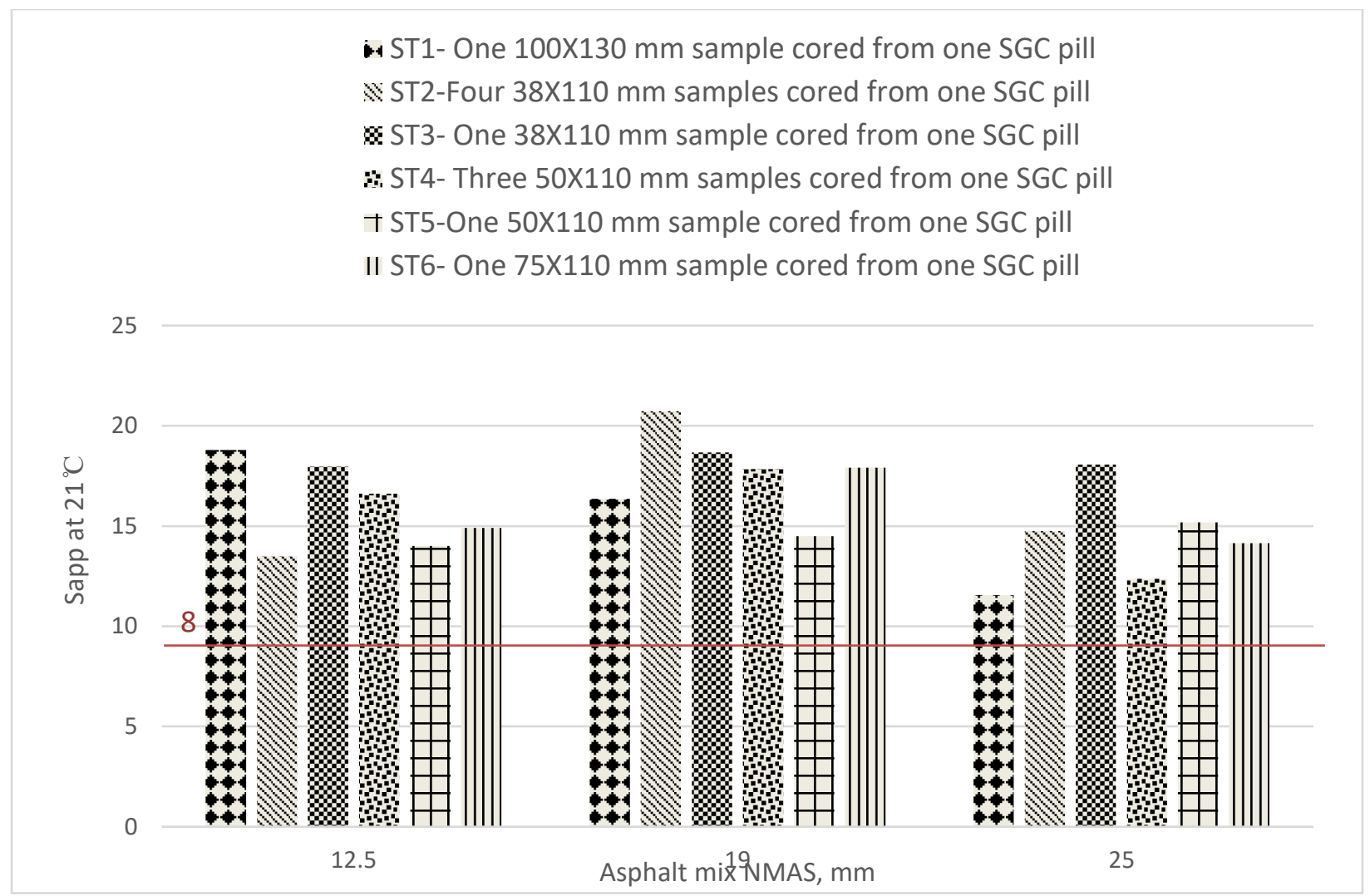

Figure 29: Fatigue Potential from $S_{\text {app }}$ Ranking at $21^{\circ} \mathrm{C}$ for Different Sample Types

The damage characterization curves (C versus $\mathrm{S}$ ) are visually evaluated for different samples fabricated from each STs and compared to that of samples obtained from ST 1. The C and S values are obtained from FlexMAT Cracking Excel. The graph comparisons for different STs to ST1 for three different NMAS mixes are shown in Figure 30. As this is a ranking analysis, visually, least sample to sample variability and closest trend to ST 1 is seen in ST5 for $12.5 \mathrm{~mm}$ NMAS mix. This is because in the first column consisting of five graphs, all three grey lines, which are replicates for ST 5, fall very closely with all three black lines, which are replicates of ST1. Similarly, for 19 mm NMAS mix, ST6 show least sample to sample variability and closest trend to ST 1. For $25 \mathrm{~mm}$ NMAS mix, ST2 and ST6 show least sample to sample variability and closest trend to ST 1 .

The damage characterization curve is used to predict damage response for any given loading history using test results. Therefore, when STs, where grey lines for its replicates, fall closest to black line for ST1 replicates, it shows that the two STs will predict similar damage response for a given loading. 

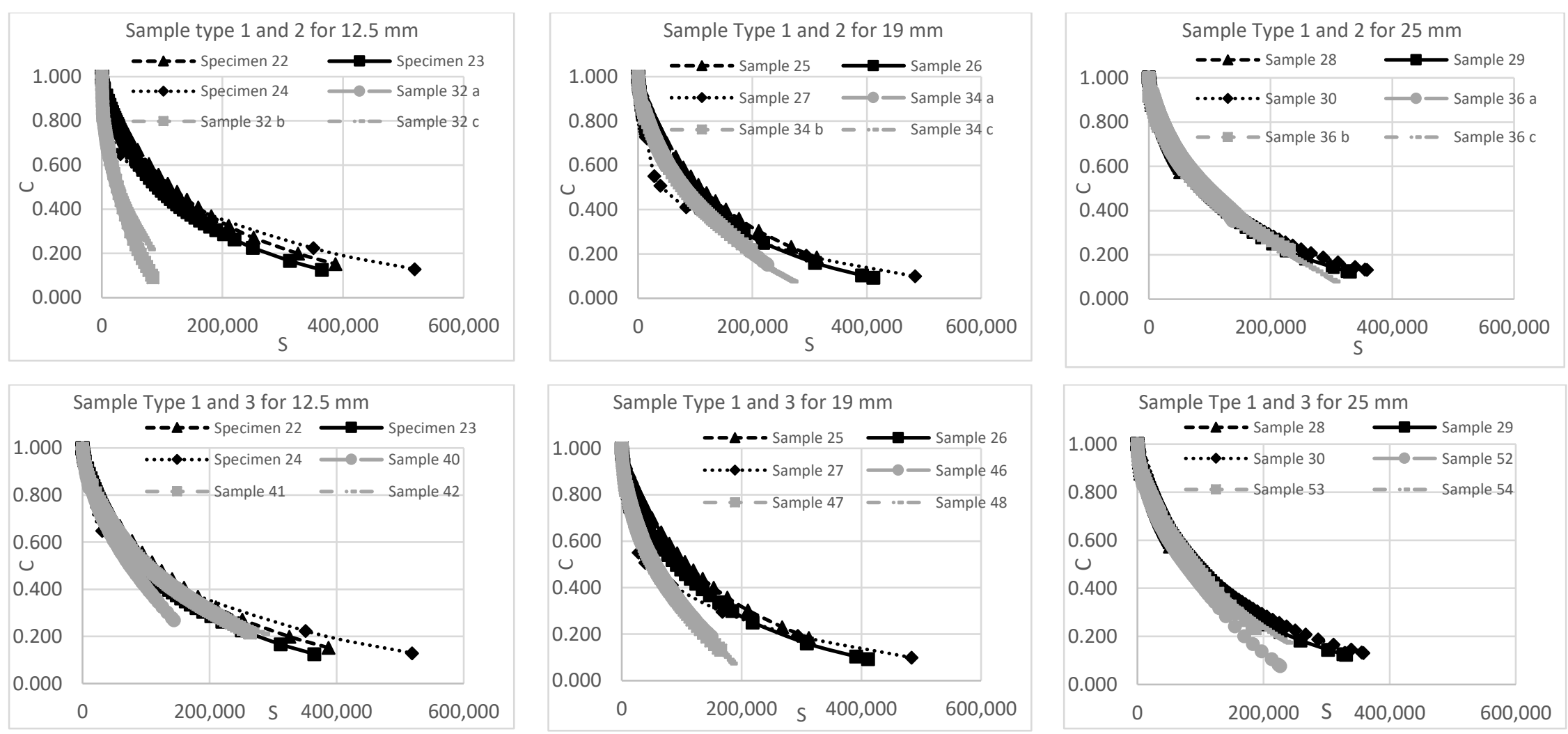

Figure 30: Damage Characterization Curve Comparison of Samples from Different Sample Types to Samples Obtained from Sample Type 1. 


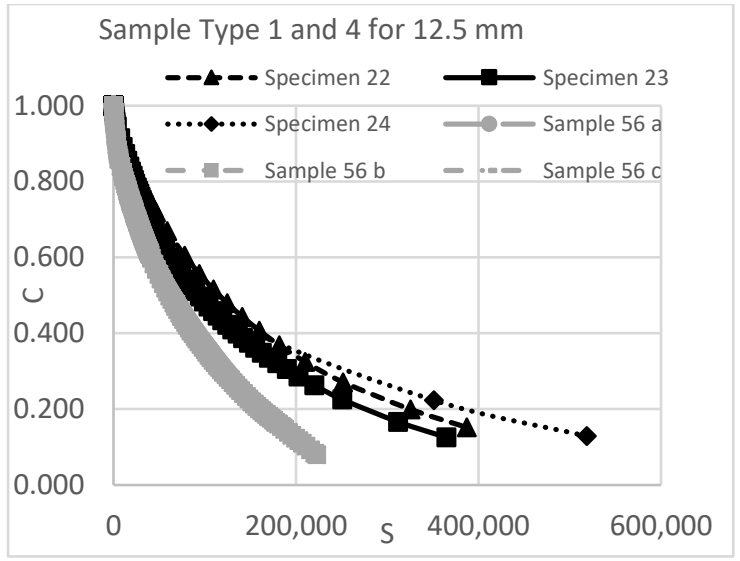

Sample Type 1 and 5 for $12.5 \mathrm{~mm}$

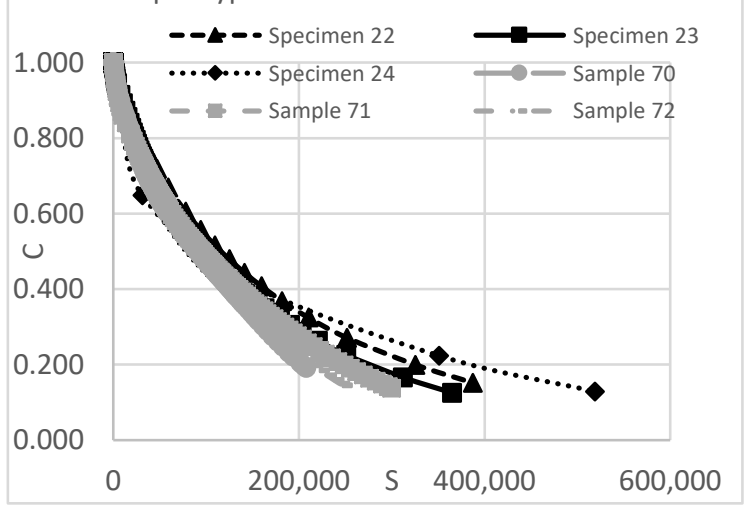

Figure 30 Cont.: Damage Characterization Curve Comparison of Samples from Different Sample Types to Samples Obtained from Sample

Type 1.
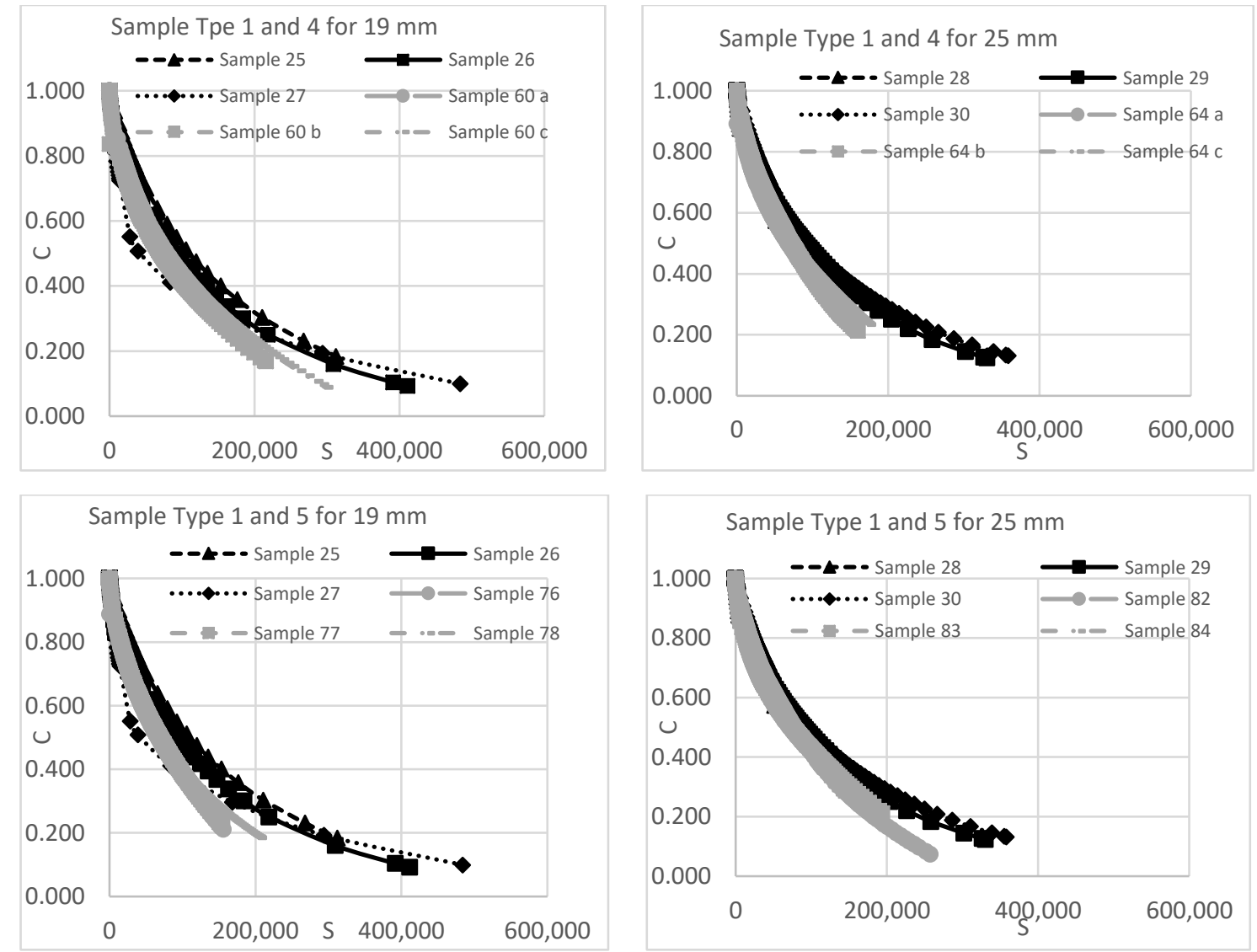

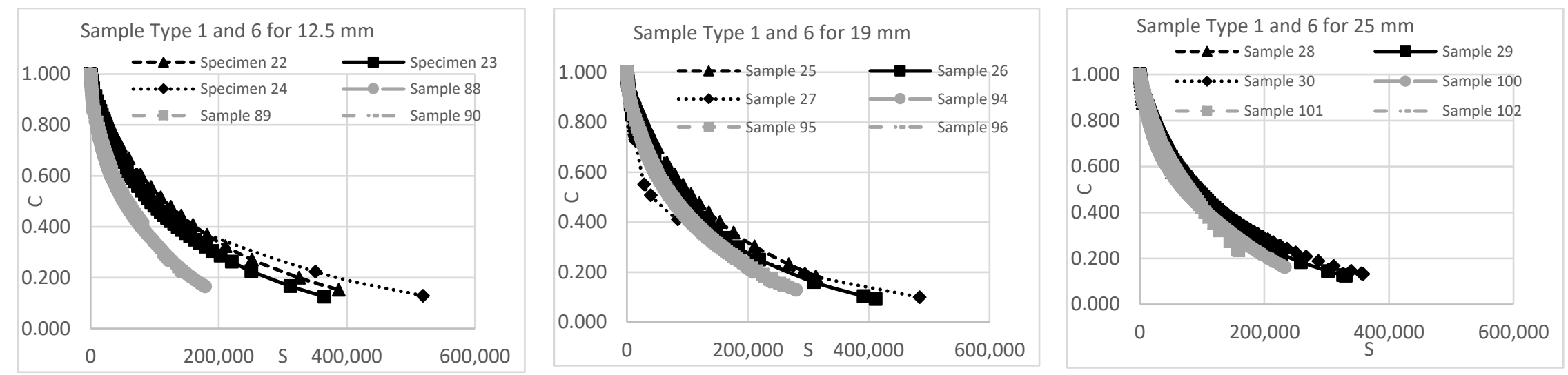

Figure 30 Cont.: Damage Characterization Curve Comparison of Samples from Different Sample Types to Samples Obtained from Sample Type 1. 


\subsubsection{SSR FlexMAT Rutting}

The rutting potential is evaluated from Rutting Strain Index (RSI) obtained from FlexMAT Rutting Excel at $21^{\circ} \mathrm{C}$. The RSI is developed by Ghanbari et. al. (2020) to predict the rutting level at 20 years of traffic for a standard pavement structure using FlexPAVE. Ghanbari et. al. (2020) recommended RSI threshold of less than 12 for less than 10 million ESALs. Table 31 shows RSI value for two STs for three NMAS mixes. The values comparisons are shown in Figure 31, (a) $9.5 \mathrm{~mm}$ mixes (b) $12.5 \mathrm{~mm}$ and (c) $25 \mathrm{~mm}$ mixes. Figures 32 to 34 show average permanent microstrain for two STs for each NMAS mix.

All STs fall well below the threshold of $12 \%$. The RSI for ST 4 varied widely from ST 1 in 12.5 $\mathrm{mm}$ and $19 \mathrm{~mm}$ NMAS mixes. This is in line with expectation as from the literature review (Blankenship and Anderson, 2010), rutting resistance is less sensitive to air void distribution and more influenced by air void percent of test sample. As ST 4 is expected to have more uniform air void distribution compared to ST 1, results may vary widely due to difference in air void content of specimen in $12.5 \mathrm{~mm}$ and $19 \mathrm{~mm}$ NMAS mix. ST 4 had higher air void content than ST 1 in 12.5 mm NMAS mix and ST1 had higher air void content than ST4 in 19 mm NMAS mix. Therefore, higher air void percent caused higher RSI indicating higher rutting level at 20 years of traffic.

The closest RSI comparison between ST 1 and ST 4 is in $25 \mathrm{~mm}$ NMAS mix, which is counter intuitive. ST 1 and 4 had similar air void content and although ST 4 may have air void uniformity, the RSI ranking results were affected by air void content.

Table 31: RSI Value for 20 Years of Traffic for Different Sample Types for Each NMAS asphalt Mix

\begin{tabular}{|c|c|c|}
\hline NMAS & Sample Type & RSI for 20 Years of Traffic, \% \\
\hline \multirow{2}{*}{12.5} & 1 & 0.4 \\
\cline { 2 - 3 } & 4 & 1.4 \\
\hline \multirow{2}{*}{19} & 1 & 1.8 \\
\cline { 2 - 3 } & 4 & 0.9 \\
\hline \multirow{2}{*}{25} & 1 & 0.5 \\
\cline { 2 - 3 } & 4 & 0.6 \\
\hline
\end{tabular}




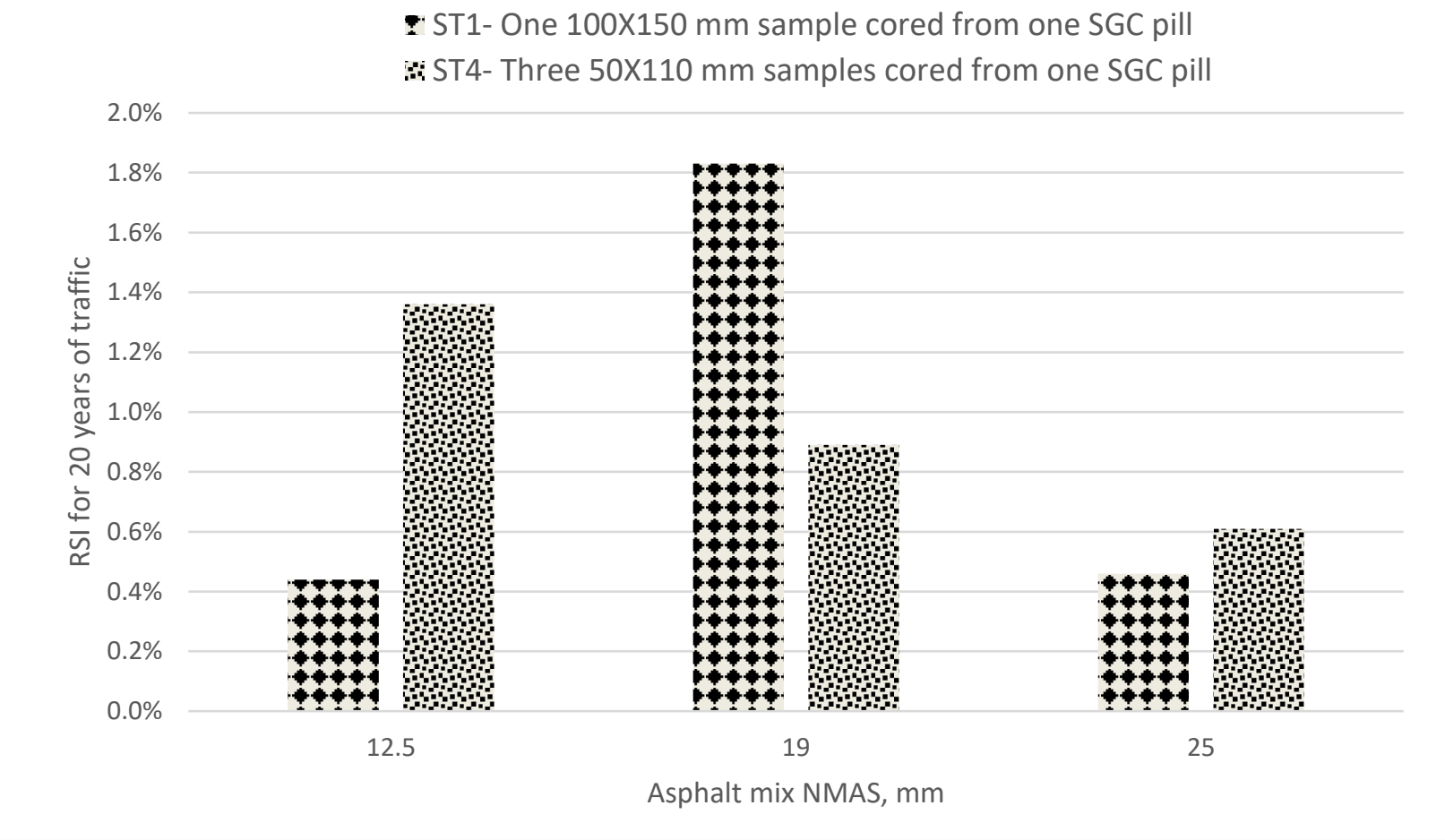

Figure 31: RSI Ranking for 20 Years of Traffic for Different Sample Types

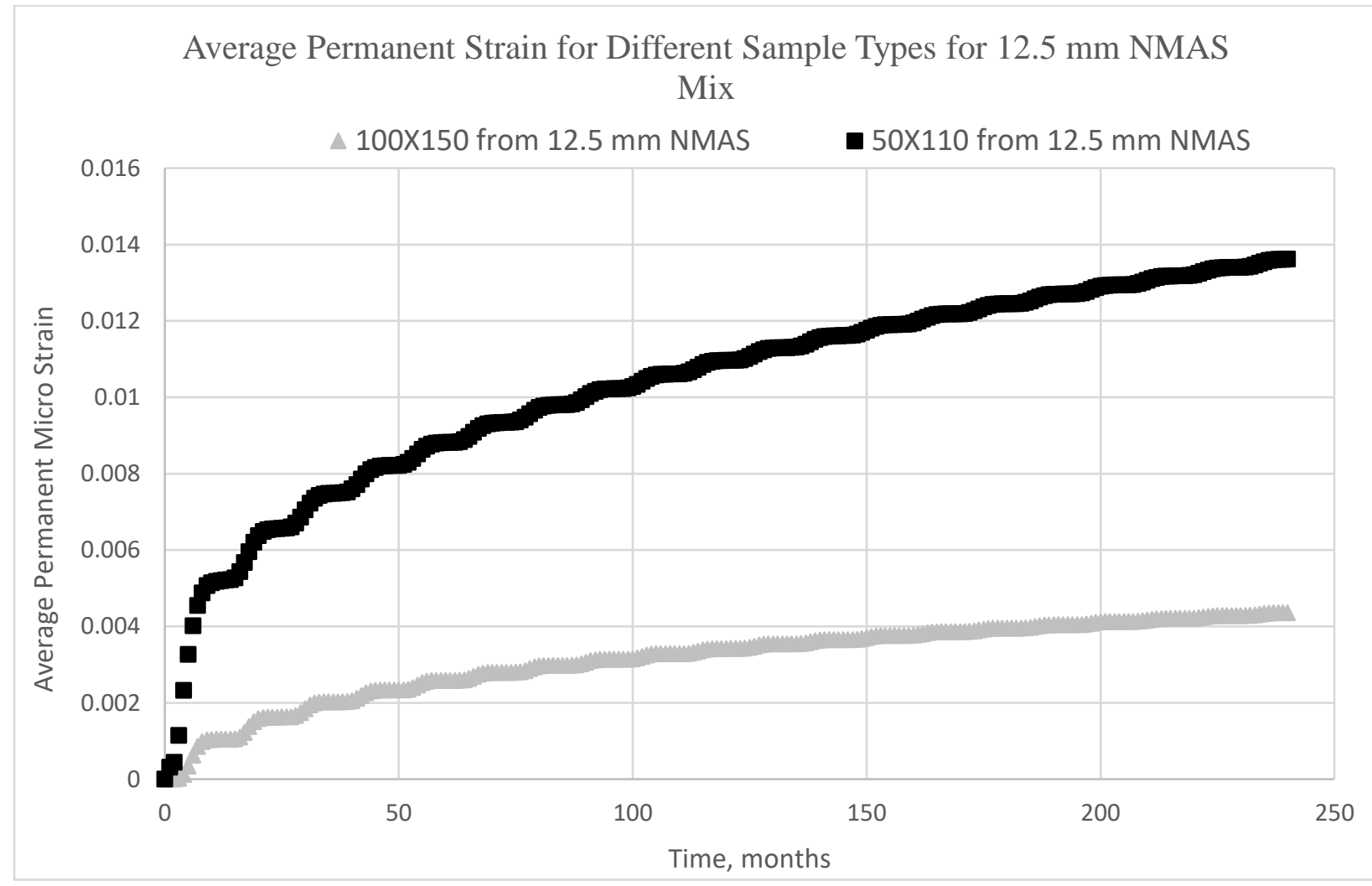

Figure 32: Average Permanent Microstrain, 12.5 mm NMAS 


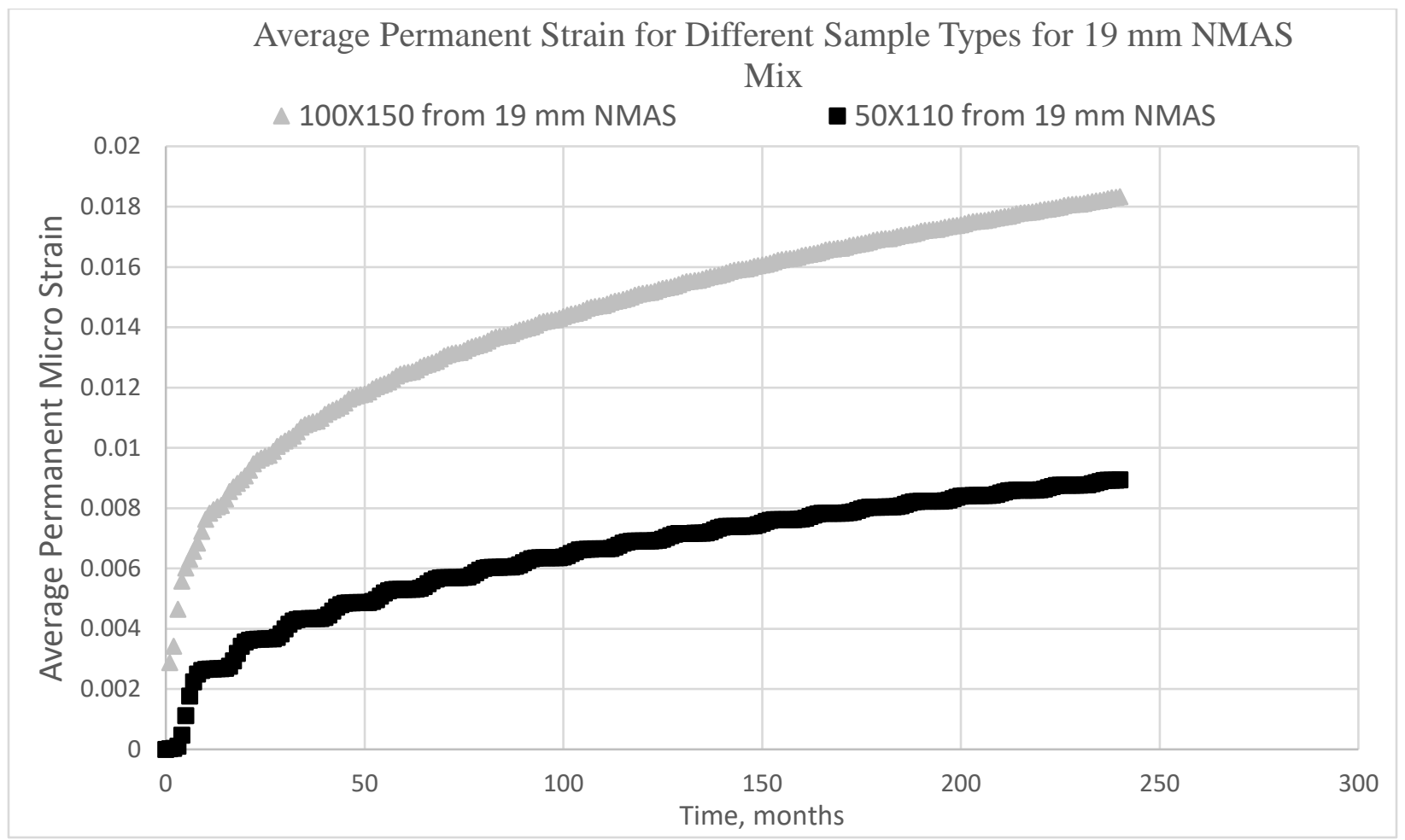

Figure 33: Average Permanent Microstrain, 19 mm NMAS

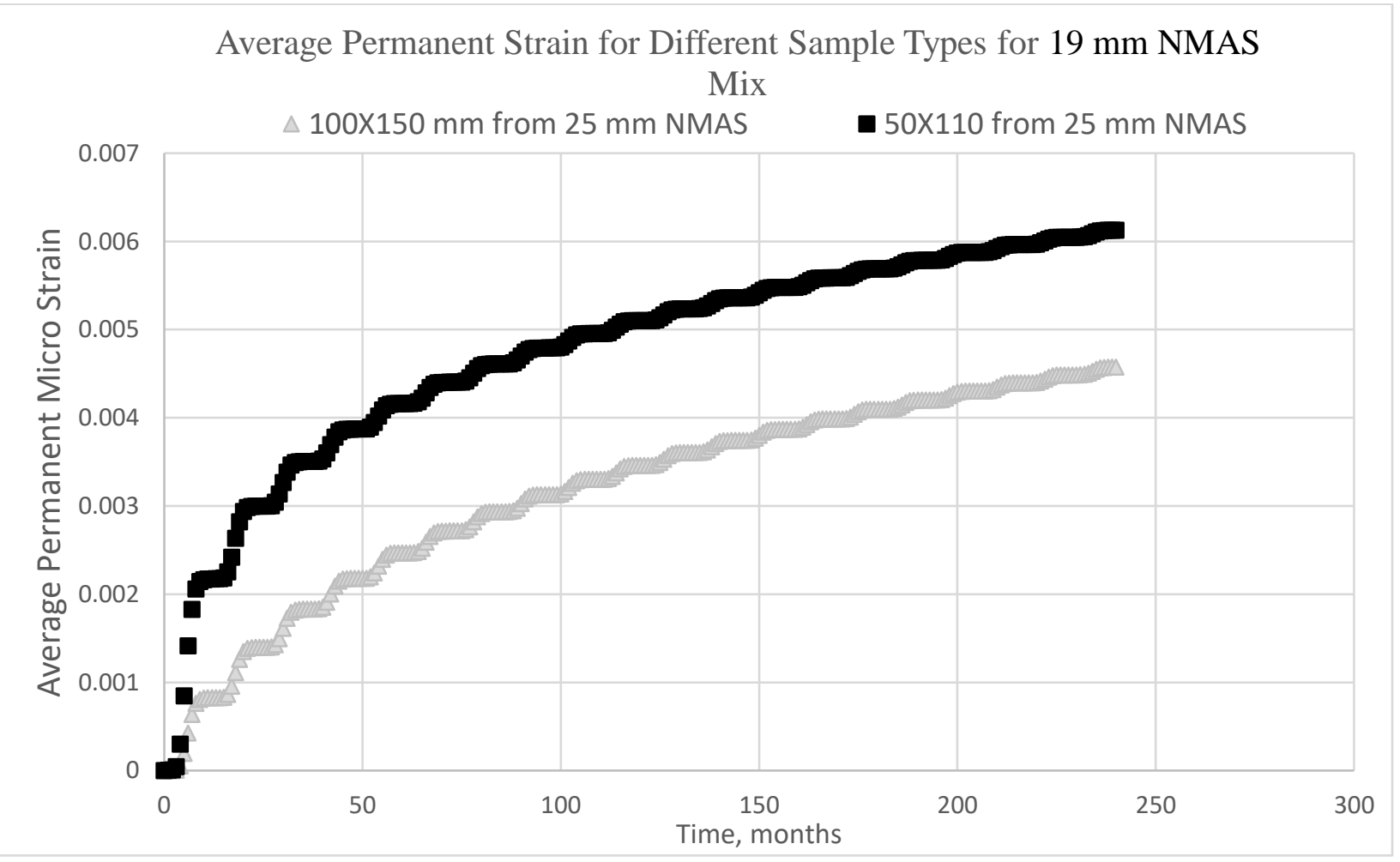

Figure 34: Average Permanent Microstrain, 25 mm NMAS 


\subsection{RANKING ANALYSIS FROM FLEXPAVE}

The Analysis 2 consists of ranking 12 models for three pavement structures in FlexPAVE. Since data is combined from replicates, it is not possible to perform statistical analysis as there is no variance term. Therefore, ranking is performed in analysis 2. The terminology of Run X.Y is used in this analysis where, $\mathrm{X}$ stands for one of the three different structures and $\mathrm{Y}$ stands for one of the twelve different models. This is also detailed in Table 25 and Table 26. The performance analysis is performed in FlexPAVE and for analysis of outputs, percentage damage for fatigue cracking as a function of time and rut depth development are compared.

\subsubsection{DTC Fatigue}

One of the outputs for ranking analysis from FlexPAVE is percentage damage for fatigue cracking as a function of time. As there is wide difference in the values obtained from each model ranging from $2.26 \mathrm{E}-05$ to 1.9 , it is not possible to depict them in bar chart.

As STs used for dynamic modulus and DTC fatigue in model 1 to 6 are same as that in model 7 to 12 , values for percentage damage at the end of the 20 years remains same. The results show that the highest percentage damage at the end of 20 years was shown in ST2, four 38X110 mm samples fabricated from one SGC pill. The same geometry, 38X110 mm, but different inscribed circle in ST3, one 38X110 $\mathrm{mm}$ samples fabricated from one SGC pill, showed significantly lower percentage damage. The percentage damage at the end of the 20 years value for model 5 values were consistently close to model 1 for all three pavement structures. 
Table 32: Percentage Damage at the end of 20 Years from FlexPAVE

\begin{tabular}{|c|c|c|c|}
\hline $\begin{array}{c}\text { \% Damage at the end of 20 } \\
\text { years }\end{array}$ & $\begin{array}{c}\text { Structure } \\
1\end{array}$ & $\begin{array}{c}\text { Structure } \\
2\end{array}$ & $\begin{array}{c}\text { Structure } \\
3\end{array}$ \\
\hline Model 1 & 0.00011 & 0.00078 & 0.00002 \\
\hline Model 2 & 0.80850 & 1.89730 & 0.41560 \\
\hline Model 3 & 0.00080 & 0.00340 & 0.00025 \\
\hline Model 4 & 0.00640 & 0.02490 & 0.01880 \\
\hline Model 5 & 0.00047 & 0.00240 & 0.00014 \\
\hline Model 6 & 0.04740 & 0.15840 & 0.01720 \\
\hline Model 7 & 0.00011 & 0.00078 & 0.00002 \\
\hline Model 8 & 0.80850 & 1.89730 & 0.41560 \\
\hline Model 9 & 0.00080 & 0.00340 & 0.00025 \\
\hline Model 10 & 0.00640 & 0.02490 & 0.00220 \\
\hline Model 11 & 0.00047 & 0.00240 & 0.00014 \\
\hline Model 12 & 0.04740 & 0.15840 & 0.01720 \\
\hline & & & \\
\hline
\end{tabular}

\subsubsection{Stress Sweep Rutting}

One of the other outputs for ranking analysis as shown in Table 33 and Figure 35 obtained from FlexPAVE is total rut depth at the end of 20 years. Additionally, rut depth development over 20 years trend in Figure 35 for different models is compared to model 1 for all three different structures.

The total rut depth at the end of 20 years for model 2 and 10 values were same as that of model 1 for all three structures. Although model 4 values were consistent across all three structures, the rut depth is different model 1. From the trend observed in Figure 36, models that use 50X110 mm for SSR visually cause difference in trend from that of model 1 for all three structures. Additionally, structure 1, medium duty pavement, shows less rutting than structure 3, heavy duty pavement. The general trend is that as the thickness of pavement increases, total rut depth decreases. As the results of flexPAVE total rut depth in structure 1 compared to structure 3 does not follow this trend, the developers of the software are contacted to follow up on the counter intuitive results. The model 5 results for different structures are consistent with the trend of decrease in rut depth with increase in pavement thickness.

${ }^{7}$ Kim's geometry consisting of 38X110 mm for dynamic modulus and DTC fatigue testing and 100X150 $\mathrm{mm}$ for SSR testing. 
Table 33: Total Rut Depth at the end of 20 Years from FlexPAVE

\begin{tabular}{|c|c|c|c|}
\hline $\begin{array}{c}\text { Total Rut Depth at the end of 20 } \\
\text { years }\end{array}$ & $\begin{array}{c}\text { Structure } \\
1\end{array}$ & $\begin{array}{c}\text { Structure } \\
2\end{array}$ & Structure \\
\hline Model 1 & 0.59 & 0.67 & 0.64 \\
\hline Model 2 & 0.59 & 0.67 & 0.64 \\
\hline Model 3 & 0.59 & 0.68 & 0.65 \\
\hline Model 4 & 0.65 & 0.67 & 0.65 \\
\hline Model 5 & 0.62 & 0.66 & 0.66 \\
\hline Model 6 & 0.59 & 0.67 & 0.65 \\
\hline Model 7 & 0.61 & 0.65 & 0.65 \\
\hline Model 8 & 0.61 & 0.65 & 0.66 \\
\hline Model 9 & 0.62 & 0.66 & 0.66 \\
\hline Model 10 & 0.59 & 0.67 & 0.64 \\
\hline Model 11 & 0.59 & 0.68 & 0.65 \\
\hline Model 12 & 0.61 & 0.66 & 0.66 \\
\hline
\end{tabular}

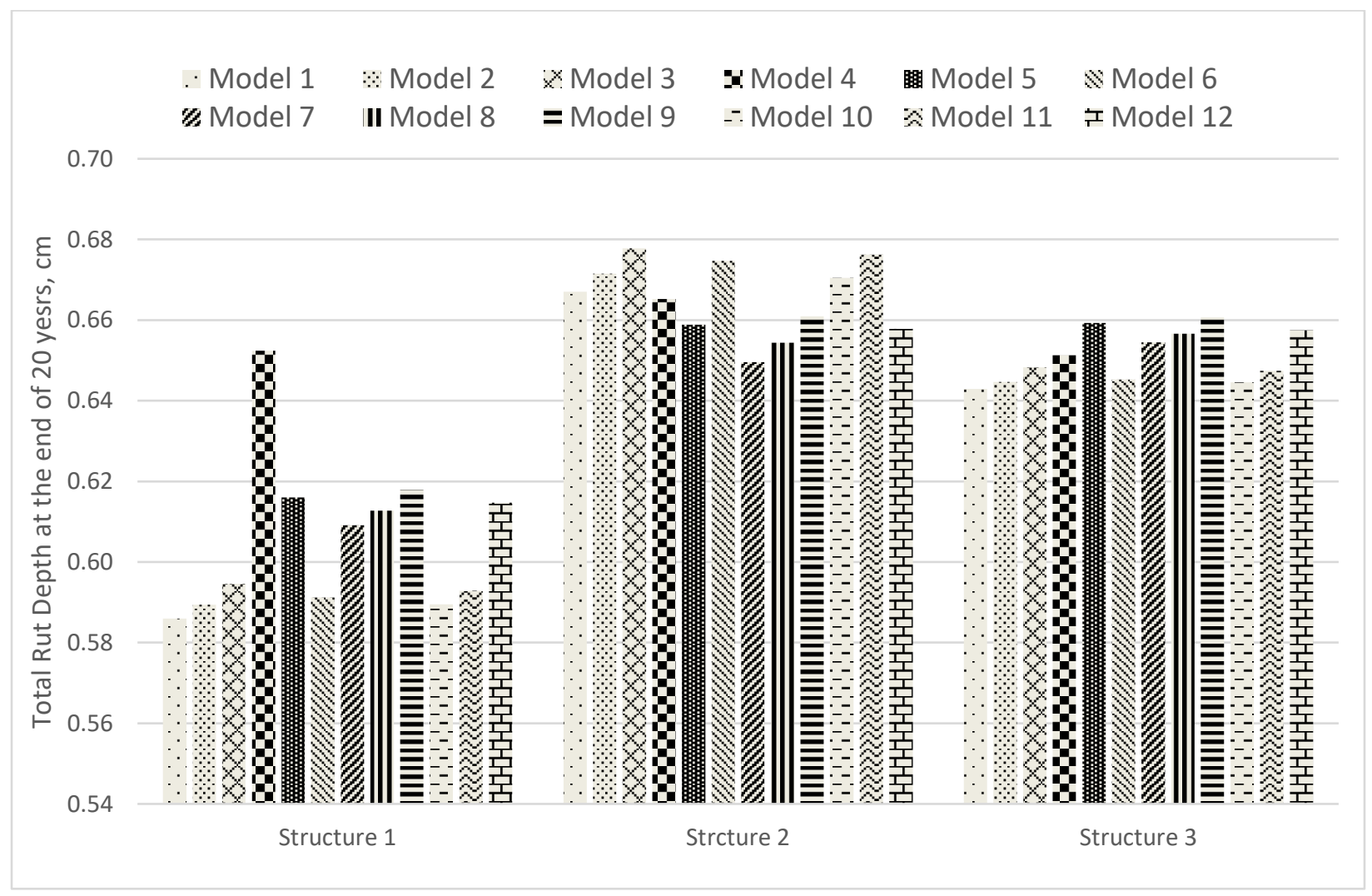

Figure 35: Comparison of Total Rut Depth at the end of 20 Years between different Models and Structures 

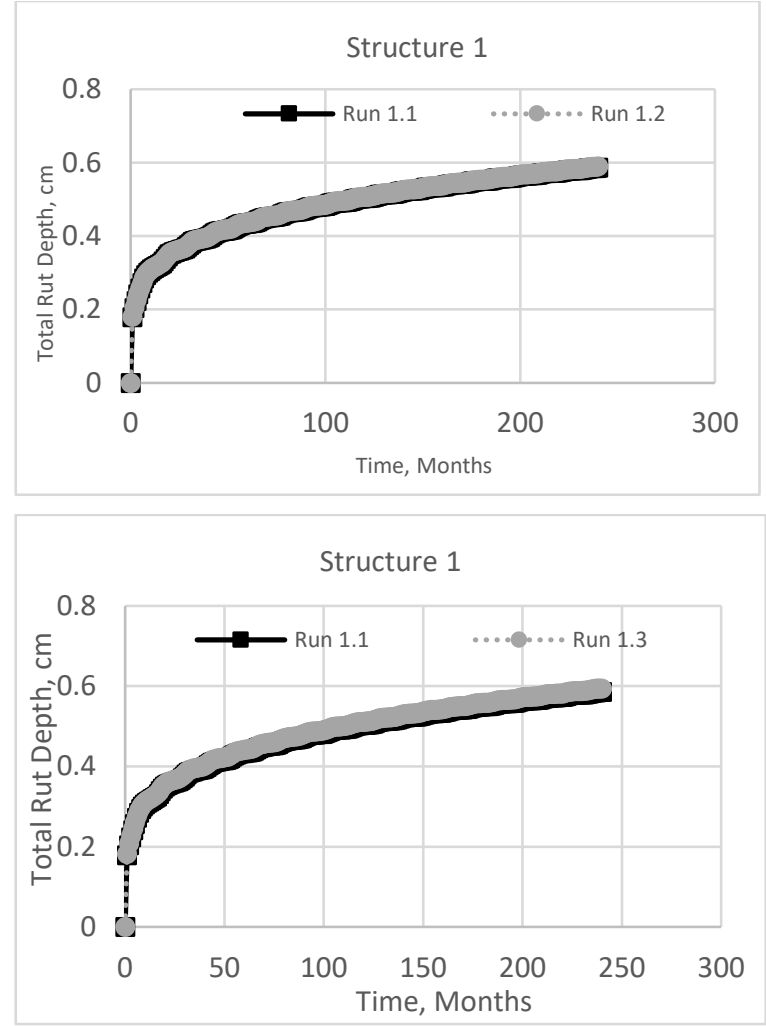
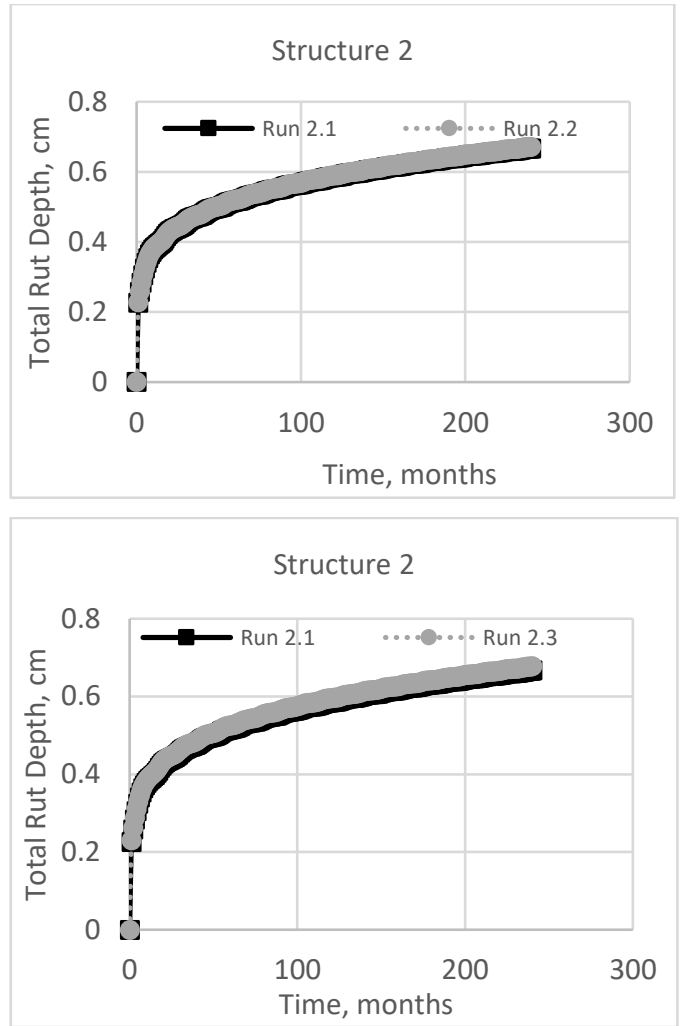
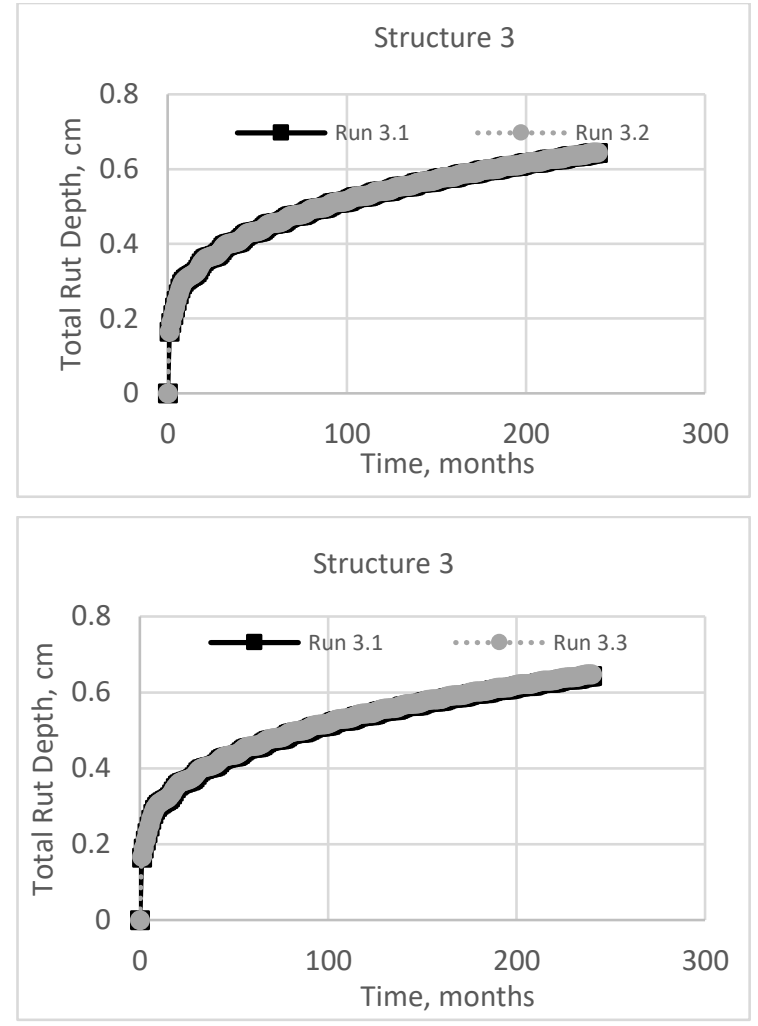

Figure 36: Total Rut Depth Comparison for different Models to Model 1 for each of the Three Structures 

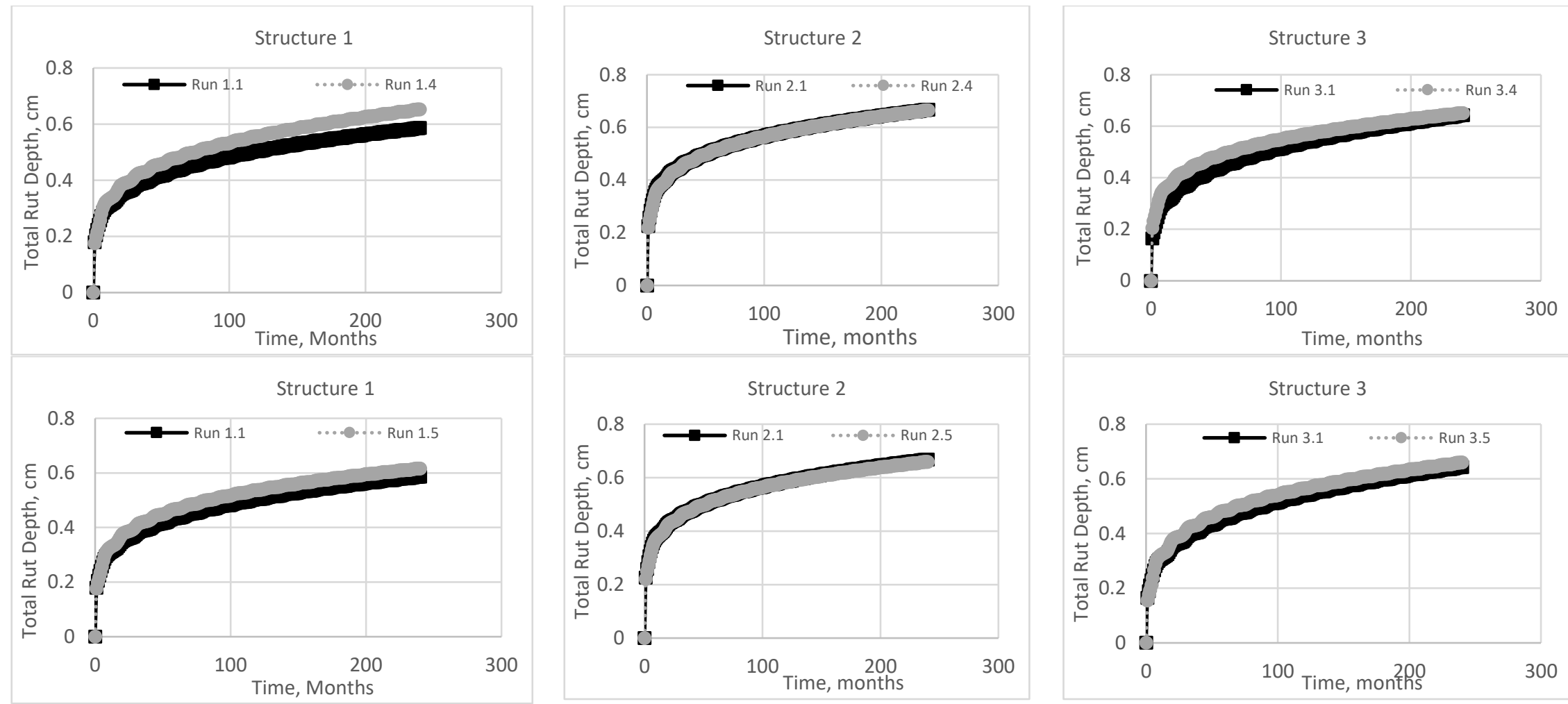

Figure 36 Cont.: Total Rut Depth Comparison for different Models to Model 1 for each of the Three Structures 

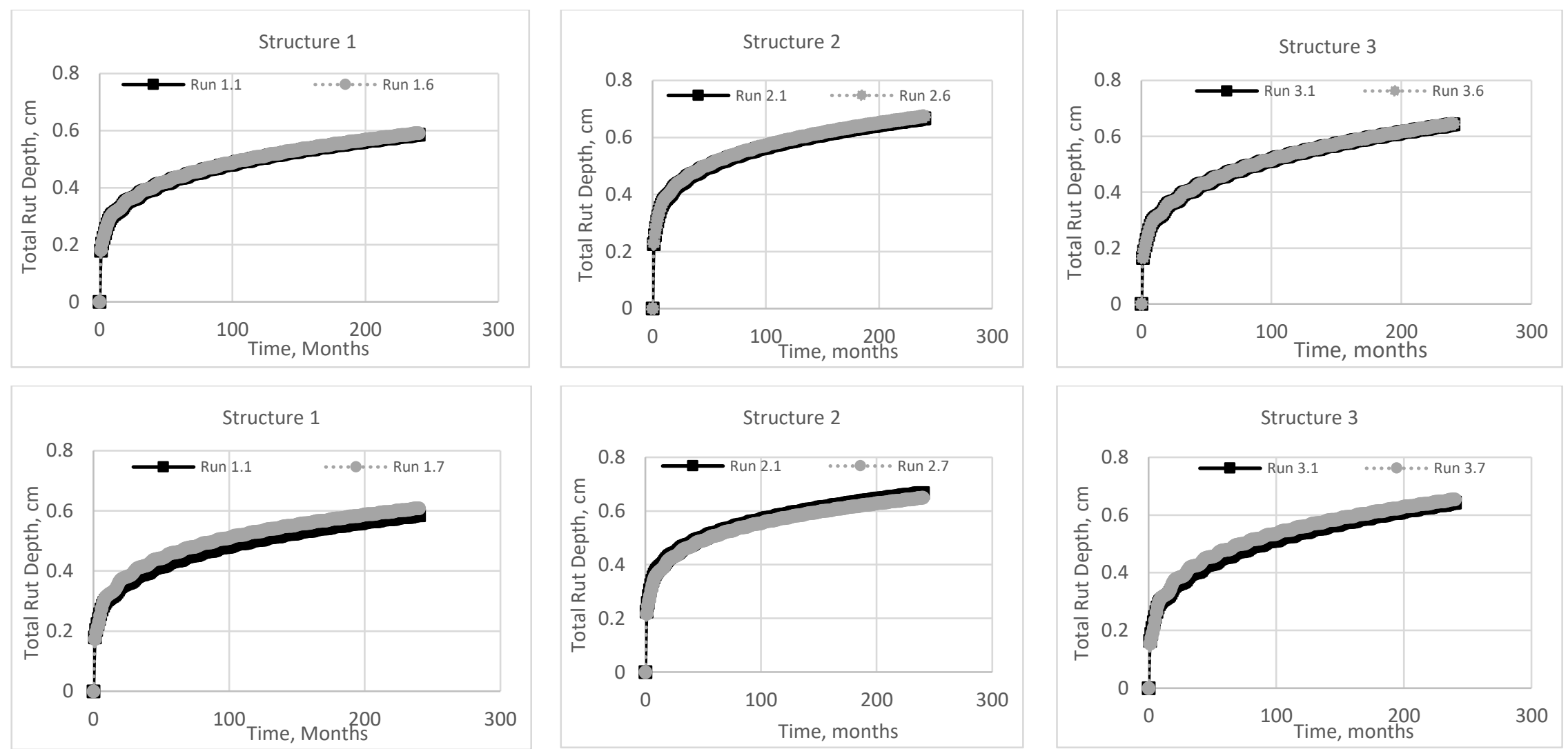

Figure 36 Cont.: Total Rut Depth Comparison for different Models to Model 1 for each of the Three Structures 

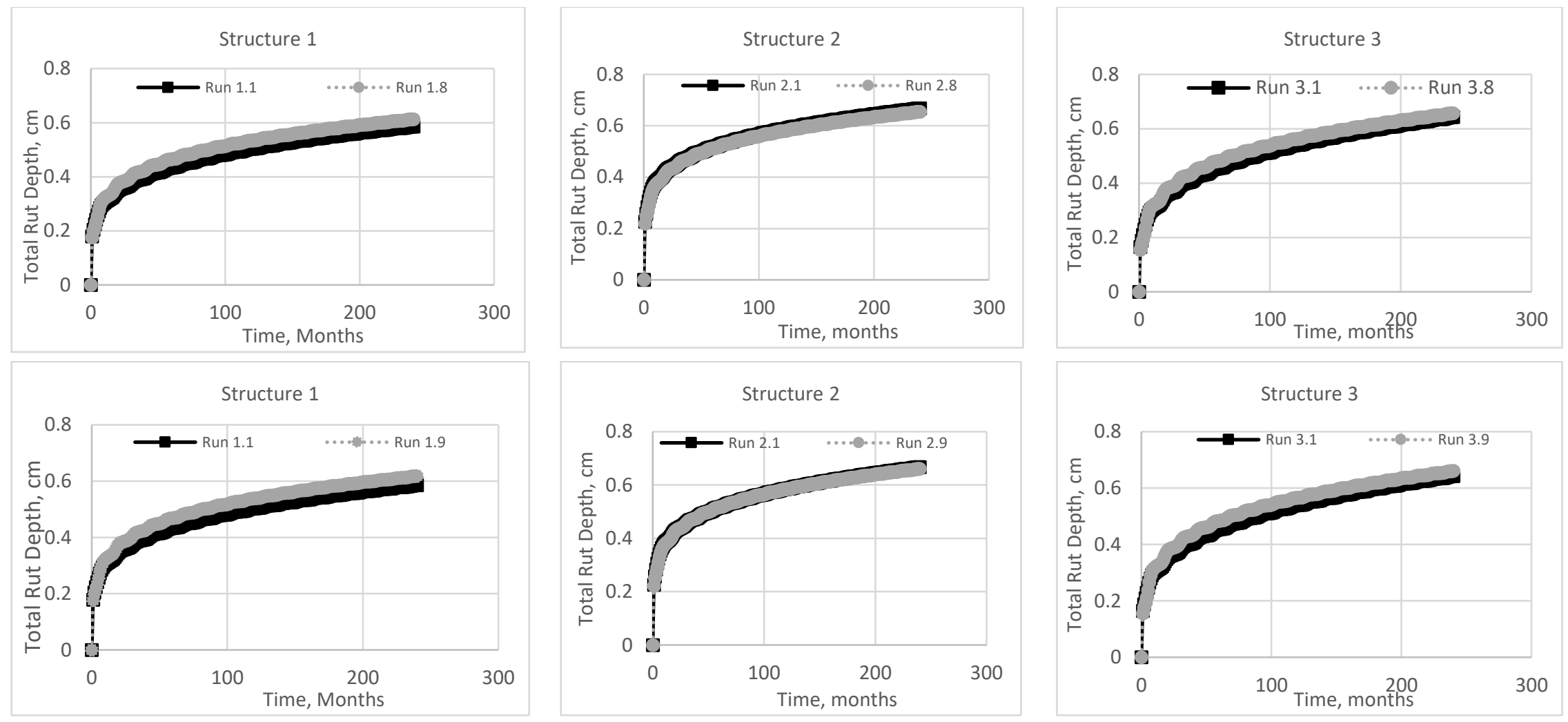

Figure 36 Cont.: Total Rut Depth Comparison for different Models to Model 1 for each of the Three Structures 

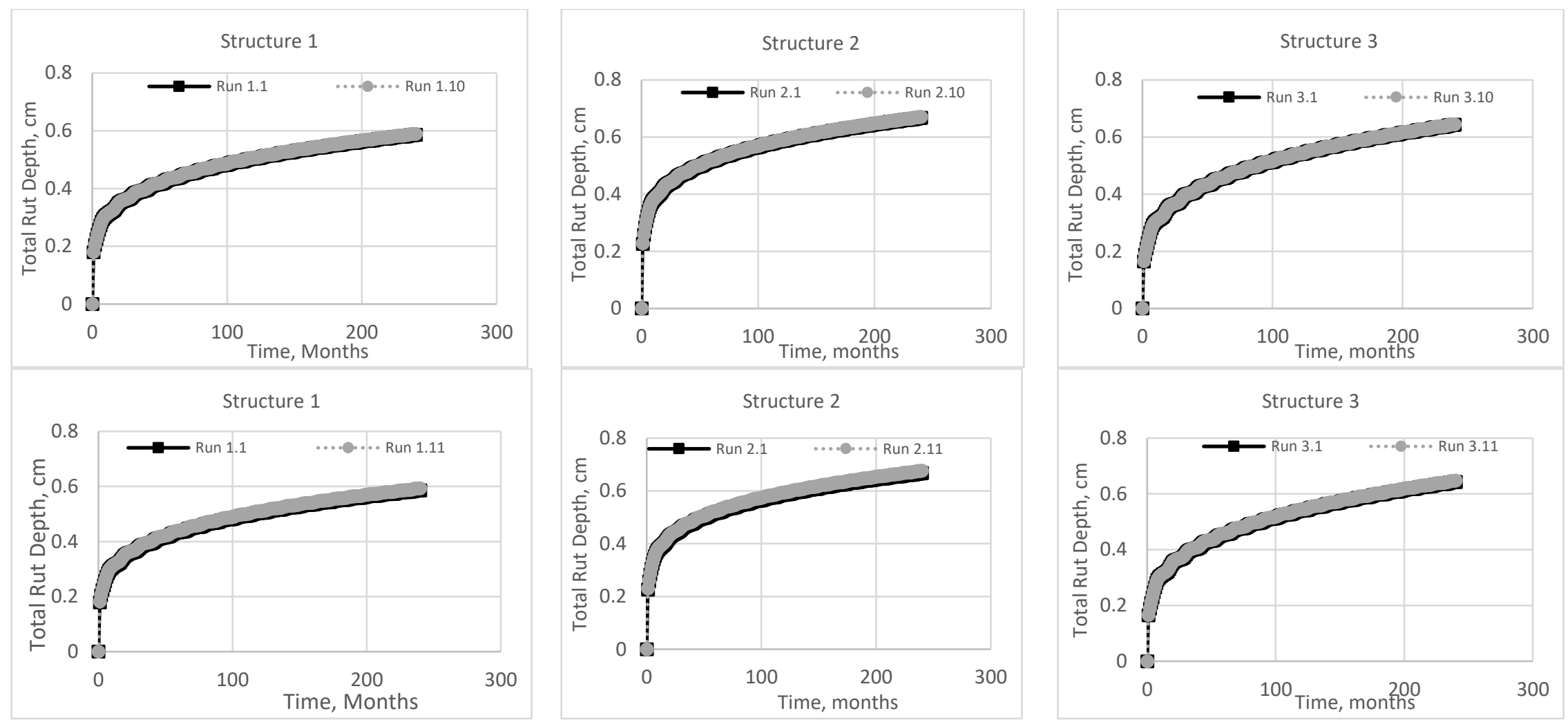

Figure 36 Cont.: Total Rut Depth Comparison for different Models to Model 1 for each of the Three Structures 

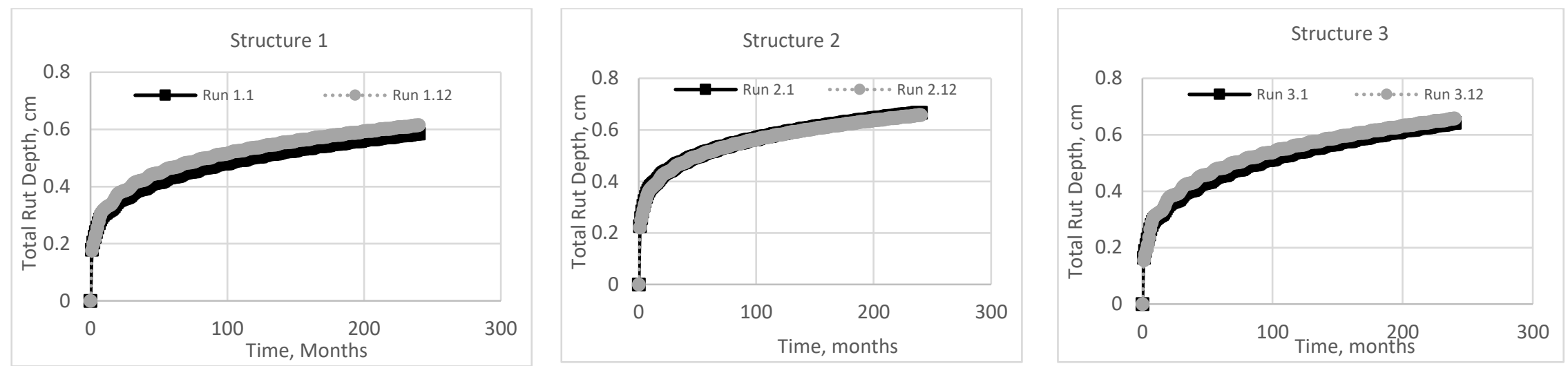

Figure 36 Cont.: Total Rut Depth Comparison for different Models to Model 1 for each of the Three Structures 


\subsection{COMPARATITIVE AND STATISTICAL ANALYSIS}

The Analysis 3 consists of performing comparative and statistical analysis of dynamic modulus and phase angle values obtained from master curve for six different STs fabricated form three different NMAS. In AMPT dynamic modulus test, three replicates are used for each sample type. Therefore, variance term exists for this analysis and statistical analysis can be performed to compare dynamic modulus and phase angle values obtained from AMPT.

As each replicate for sample types is assigned a unique number, Tables 34 to 36 show dynamic modulus and phase angle values for six STs for each of the three NMAS mix. Figures 37 to 48 illustrate the effects of temperature on dynamic modulus and phase angle for all STs fabricated from each NMAS. Although it is important to see how six different STs perform for each NMAs mix, it is also important to see how each ST yield different DM and PA values across different NMAS mix. The former analysis is done because per current AASHTO standard, full size sample (sample type 1) is used for up to $37.5 \mathrm{~mm}$ NMAS mix.

In addition to comparative analysis t-test, ANOVA, and MANOVA are also performed in this analysis. Table 37 to 54 show t-tests performed to compare dynamic modulus and phase angle values of STs to that of ST1 for each NMAS at different temperature and frequency pairs. Table 55 to 57 show that ANOVA is performed to compare dynamic modulus and phase angle values for all STs fabricated for each NMAS at different temperature and frequency pairs. Table 58 to 66 show that MANOVA is

performed in SPSS Statistical Software to compare dynamic modulus and phase angle from STs to that of ST1 fabricated from three different NMAS at different temperature and frequency pairs. 
Table 34: Dynamic Modulus and Phase Angle for 12.5 mm NMAS Unique Replicate Samples

\begin{tabular}{|c|c|c|c|c|c|c|c|c|c|c|c|c|c|c|c|c|c|c|c|}
\hline \multirow{3}{*}{\multicolumn{2}{|c|}{\begin{tabular}{|c|} 
NMAS \\
Sample Type \\
Unique Sample Number
\end{tabular}}} & \multicolumn{18}{|c|}{12.5} \\
\hline & & \multicolumn{3}{|c|}{1} & \multicolumn{3}{|c|}{2} & \multicolumn{3}{|c|}{3} & \multicolumn{3}{|c|}{4} & \multicolumn{3}{|c|}{5} & \multicolumn{3}{|c|}{6} \\
\hline & & 1 & 2 & 3 & $31 \mathrm{a}$. & $31 \mathrm{~b}$. & $31 \mathrm{c}$. & 37 & 38 & 39 & 55 a. & $55 \mathrm{~b}$. & $55 \mathrm{c}$. & 67 & 68 & 69 & 85 & 86 & 87 \\
\hline 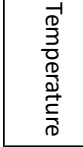 & $\begin{array}{l}\frac{T}{1} \\
\frac{1}{0} \\
\frac{1}{1} \\
\stackrel{D}{2} \\
2\end{array}$ & \multicolumn{18}{|c|}{ Dynamic Modulus (ksi) } \\
\hline \multirow{3}{*}{4} & 0.1 & 1228.3 & 1109.7 & 1281.8 & 1349.4 & 1345.5 & 1426.3 & 1430.8 & 1385.7 & 1288.7 & 1372.8 & 1361.8 & 1366.983 & 1250.5 & 1388.9 & 1411.2 & 1349.4 & 1345.5 & 1426.3 \\
\hline & 1 & 1643.4 & 1494.8 & 1688.1 & 1720.6 & 1700.6 & 1800.6 & 1820.7 & 1754.8 & 1674.8 & 1759.0 & 1737.6 & \begin{tabular}{|l|}
1741.181 \\
\end{tabular} & 1627.8 & 1769.0 & 1796.9 & 1720.6 & 1700.6 & 1800.6 \\
\hline & 10 & 2051.1 & 1876.8 & 2080.6 & 2108.6 & 2061.0 & 2179.2 & 2207.2 & 2125.1 & 2074.0 & 2136.4 & 2109.6 & 2104.936 & 2028.1 & 2148.4 & 2186.4 & 2108.6 & 2061.0 & 2179.2 \\
\hline \multirow{3}{*}{20} & 0.1 & 439.5 & 389.4 & 466.4 & 454.5 & 469.9 & 417.0 & 558.0 & 501.4 & 426.7 & 460.6 & 479.1 & \begin{tabular}{|l|l|}
454.6941 \\
\end{tabular} & 400.6 & 446.9 & 451.1 & 454.5 & 469.9 & 417.0 \\
\hline & 1 & 752.5 & 684.9 & 796.4 & 754.3 & 776.1 & 608.4 & 901.4 & 806.1 & 741.6 & 780.3 & 800.3 & 767.251 & 684.3 & 731.0 & 738.8 & 754.3 & 776.1 & 608.4 \\
\hline & 10 & 1146.1 & 1061.5 & 1201.9 & 1130.0 & 1149.6 & 864.4 & 1300.0 & 1194.1 & 1144.2 & 1160.7 & 1180.8 & 1143.625 & 1063.4 & 1102.7 & 1108.4 & 1130.0 & 1149.6 & 864.4 \\
\hline \multirow{4}{*}{40} & 0.01 & 24.5 & 18.4 & 23.7 & & & & & & & & & & & & & & & \\
\hline & 0.1 & 50.7 & 35.1 & 46.0 & 70.4 & 82.2 & 87.2 & 85.2 & 81.2 & 63.5 & 110.6 & 119.7 & 84.02051 & 63.5 & 75.2 & 71.2 & 70.4 & 82.2 & 87.2 \\
\hline & 1 & 120.1 & 84.0 & 104.7 & 161.7 & 181.2 & 187.8 & 185.6 & 164.0 & 144.7 & 246.3 & 262.7 & \begin{tabular}{|l|}
190.725 \\
\end{tabular} & 149.1 & 165.9 & 154.6 & 161.7 & 181.2 & 187.8 \\
\hline & 10 & 269.8 & 199.3 & 234.5 & 337.2 & 366.1 & 374.2 & 376.4 & 333.7 & 310.2 & 483.8 & 505.3 & \begin{tabular}{|l|l|}
402.485 \\
\end{tabular} & 309.7 & 301.5 & 272.2 & 337.2 & 366.1 & 374.2 \\
\hline & & \multicolumn{18}{|c|}{ Phase Angle } \\
\hline \multirow{3}{*}{4} & 0.1 & 14.5 & 15.0 & 13.7 & 11.0 & 11.2 & 10.7 & 11.4 & 11.5 & 12.8 & 11.7 & 11.7 & 11.52 & 13.1 & 10.7 & 11.8 & 11.0 & 11.2 & 10.7 \\
\hline & 1 & 10.9 & 11.2 & 10.3 & 8.6 & 8.8 & 8.7 & 8.8 & 8.8 & 9.9 & 9.0 & 8.9 & 8.87 & 10.0 & 8.1 & 9.0 & 8.6 & 8.8 & 8.7 \\
\hline & 10 & 8.4 & 8.7 & 8.0 & 6.4 & 7.3 & 5.8 & 7.3 & 7.3 & 8.0 & 7.1 & 7.2 & 7.19 & 8.1 & 5.9 & 7.3 & 6.4 & 7.3 & 5.8 \\
\hline \multirow{3}{*}{20} & 0.1 & 26.5 & 27.3 & 26.0 & 24.2 & 23.9 & 29.1 & 22.9 & 23.7 & 26.3 & 24.8 & 24.7 & 24.76 & 26.8 & 24.3 & 25.2 & 24.2 & 23.9 & 29.1 \\
\hline & 1 & 20.9 & 21.6 & 20.3 & 18.9 & 18.4 & 19.8 & 17.5 & 18.5 & 20.8 & 19.2 & 18.9 & 19.09 & 21.3 & 19.8 & 20.1 & 18.9 & 18.4 & 19.8 \\
\hline & 10 & 16.0 & 16.5 & 15.4 & 14.5 & 14.4 & 19.5 & 13.3 & 14.2 & 15.7 & 14.5 & 14.3 & 14.48 & 16.2 & 13.7 & 15.6 & 14.5 & 14.4 & 19.5 \\
\hline \multirow{4}{*}{40} & 0.01 & 26.2 & 25.9 & 24.91 & & & & & & & & & & & & & & & \\
\hline & 0.1 & 30.6 & 30.7 & 30 & 31.9 & 31.3 & 33.2 & 34.0 & 31.0 & 31.3 & 30.6 & 30.6 & 31.86 & 32.5 & 32.8 & 33.3 & 31.9 & 31.3 & 33.2 \\
\hline & 1 & 33.3 & 34.7 & 33.84 & 31.7 & 30.8 & 33.2 & 33.1 & 32.2 & 32.5 & 29.5 & 28.9 & 31.86 & 32.5 & 32.9 & 33.1 & 31.7 & 30.8 & 33.2 \\
\hline & 10 & 33.1 & 35.5 & 34.28 & 28.4 & 27.6 & 25.2 & 28.6 & 29.3 & 30.2 & 25.8 & 25.3 & 29.56 & 31.5 & 33.1 & 34.8 & 28.4 & 27.6 & 25.2 \\
\hline
\end{tabular}


Table 35: Dynamic Modulus and Phase Angle for 19 mm NMAS Unique Replicate Samples

\begin{tabular}{|c|c|c|c|c|c|c|c|c|c|c|c|c|c|c|c|c|c|c|c|}
\hline \multirow{3}{*}{\multicolumn{2}{|c|}{\begin{tabular}{|c|} 
NMAS \\
Sample Type \\
Unique Sample Number \\
\end{tabular}}} & \multicolumn{18}{|c|}{19} \\
\hline & & \multicolumn{3}{|c|}{1} & \multicolumn{3}{|c|}{2} & \multicolumn{3}{|c|}{3} & \multicolumn{3}{|c|}{4} & \multicolumn{3}{|c|}{5} & \multicolumn{3}{|c|}{6} \\
\hline & & 8 & 9 & 10 & 33 а. & $33 \mathrm{~b}$. & $33 \mathrm{c}$. & 43 & 44 & 45 & 59 a. & $59 \mathrm{~b}$. & $59 \mathrm{c}$. & 73 & 74 & 75 & 91 & 92 & 93 \\
\hline 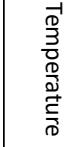 & 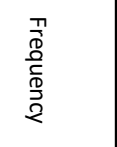 & \multicolumn{18}{|c|}{ Dynamic Modulus (ksi) } \\
\hline \multirow{3}{*}{4} & 0.1 & 989.9 & 1030.8 & 1168.6 & 1272.6 & 1264.0 & 1109.7 & 898.4 & 904.0 & 1085.3 & 1255.884 & 1184.8 & 1176.5 & 846.3 & 1129.0 & 1095.9 & 998.6 & 1054.0 & 1078.4 \\
\hline & 1 & 1432.8 & 1459.4 & 1597.3 & 1726.4 & 1729.3 & 1542.6 & 1302.2 & 1210.2 & 1522.3 & 1687.082 & 1560.9 & 1581.5 & 1215.0 & 1590.1 & 1545.1 & 1373.8 & 1420.4 & 1463.1 \\
\hline & 10 & 1891.7 & 1893.0 & 2018.9 & 2172.4 & 2211.0 & 2000.7 & 1745.8 & 1443.6 & 1980.3 & 2126.982 & 1946.8 & 1987.9 & 1637.3 & 2079.6 & 2015.4 & 1762.9 & 1779.0 & 1848.4 \\
\hline \multirow{3}{*}{20} & 0.1 & 317.5 & 357.4 & 376.5 & 340.7 & 336.2 & 313.1 & 230.3 & 208.1 & 276.7 & 370.7171 & 384.2 & 367.4 & 190.1 & 259.5 & 263.5 & 324.2 & 343.2 & 343.6 \\
\hline & 1 & 607.4 & 654.8 & 660.2 & 647.0 & 629.0 & 606.5 & 466.3 & 384.4 & 546.6 & 672.1061 & 673.8 & 650.6 & 381.6 & 504.6 & 511.7 & 595.5 & 625.8 & 635.7 \\
\hline & 10 & 1016.9 & 1041.7 & 1048.2 & 1079.2 & 1030.6 & 1011.2 & 837.4 & 613.1 & 939.8 & 1082.129 & 1043.8 & 1030.1 & 700.1 & 895.0 & 904.6 & 960.3 & 1003.4 & 1025.0 \\
\hline \multirow{4}{*}{35} & 0.01 & 18.2 & 21.4 & 30.8 & & & & & & & & & & & & & & & \\
\hline & 0.1 & 34.7 & 38.0 & 53.6 & 70.4 & 82.2 & 87.2 & 51.8 & 67.1 & 59.5 & 81.06174 & 89.8 & 82.2 & 42.6 & 58.8 & 54.4 & 76.3 & 66.1 & 73.5 \\
\hline & 1 & 82.6 & 83.8 & 110.9 & 161.7 & 181.2 & 187.8 & 126.8 & 157.7 & 146.5 & \begin{tabular}{|l|l|}
182.7479 \\
\end{tabular} & 199.3 & 178.5 & 113.5 & 137.2 & 125.9 & 170.9 & 149.1 & 166.8 \\
\hline & 10 & 195.9 & 191.5 & 237.0 & 337.2 & 366.1 & 374.2 & 292.8 & 347.7 & 331.4 & \begin{tabular}{|l|l|}
386.6713 \\
\end{tabular} & 405.8 & 367.8 & 248.5 & 257.6 & 226.0 & 357.4 & 312.3 & 350.1 \\
\hline & & \multicolumn{18}{|c|}{ Phase Angle } \\
\hline \multirow{3}{*}{4} & 0.1 & 18.3 & 17.1 & 15.1 & 14.8 & 15.0 & 16.1 & 19.1 & 22.1 & 16.9 & 14.17 & 13.6 & 14.3 & 19.0 & 16.7 & 17.3 & 15.6 & 14.8 & 15.3 \\
\hline & 1 & 13.4 & 12.4 & 10.9 & 10.8 & 11.2 & 12.1 & 14.2 & 16.6 & 12.6 & 10.45 & 10.4 & 10.8 & 14.2 & 12.5 & 12.7 & 11.5 & 10.9 & 11.3 \\
\hline & 10 & 10.1 & 9.3 & 8.5 & 8.3 & 9.0 & 8.4 & 10.6 & 13.3 & 9.4 & 8.24 & 8.2 & 8.5 & 10.7 & 9.4 & 9.6 & 8.7 & 8.4 & 8.5 \\
\hline \multirow{3}{*}{20} & 0.1 & 29.8 & 28.9 & 27.1 & 30.9 & 30.6 & 30.3 & 33.8 & 32.4 & 33.6 & 28.48 & 26.6 & 26.7 & 33.9 & 32.3 & 32.0 & 28.7 & 29.7 & 29.2 \\
\hline & 1 & 24.7 & 23.8 & 22.6 & 24.0 & 24.9 & 24.7 & 28.2 & 27.1 & 26.3 & 23.07 & 21.4 & 21.7 & 28.9 & 27.8 & 27.4 & 23.1 & 24.0 & 23.4 \\
\hline & 10 & 19.1 & 18.1 & 17.7 & 17.3 & 19.1 & 18.8 & 21.6 & 22.3 & 20.0 & 17.55 & 16.5 & 16.9 & 22.7 & 21.4 & 21.3 & 17.4 & 18.0 & 17.6 \\
\hline \multirow{4}{*}{35} & 0.01 & 23.8 & 23.0 & 24.0 & & & & & & & & & & & & & & & \\
\hline & 0.1 & 28.6 & 27.3 & 28.0 & 31.9 & 31.3 & 33.2 & 34.3 & 34.9 & 35.8 & 32.82 & 30.0 & 30.0 & 35.4 & 32.1 & 32.5 & 32.1 & 32.9 & 32.7 \\
\hline & 1 & 32.8 & 32.1 & 31.6 & 31.7 & 30.8 & 33.2 & 36.2 & 36.6 & 36.4 & 33.19 & 30.5 & 30.7 & 36.7 & 35.3 & 38.3 & 32.8 & 34.7 & 33.6 \\
\hline & 10 & 35.2 & 35.6 & 32.6 & 28.4 & 27.6 & 25.2 & 33.6 & 33.1 & 32.4 & 30.83 & 28.6 & 28.7 & 35.5 & 37.9 & 43.9 & 30.6 & 32.3 & 30.8 \\
\hline
\end{tabular}


Table 36: Dynamic Modulus and Phase Angle for 25 mm NMAS Unique Replicate Samples

\begin{tabular}{|c|c|c|c|c|c|c|c|c|c|c|c|c|c|c|c|c|c|c|c|}
\hline \multirow{3}{*}{\multicolumn{2}{|c|}{\begin{tabular}{|c|} 
NMAS \\
Sample Type \\
Unique Sample Number \\
\end{tabular}}} & \multicolumn{18}{|c|}{25} \\
\hline & & \multicolumn{3}{|c|}{1} & \multicolumn{3}{|c|}{2} & \multicolumn{3}{|c|}{3} & \multicolumn{3}{|c|}{4} & \multicolumn{3}{|c|}{5} & \multicolumn{3}{|c|}{6} \\
\hline & & 15 & 16 & 17 & 35 a. & $35 \mathrm{~b}$. & $35 \mathrm{c}$. & 49 & 50 & 51 & 63 a. & $63 \mathrm{~b}$. & $63 \mathrm{c}$. & 79 & 80 & 81 & 97 & 98 & 99 \\
\hline 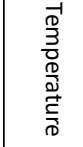 & 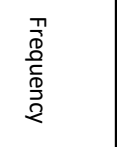 & \multicolumn{18}{|c|}{ Dynamic Modulus (ksi) } \\
\hline \multirow{3}{*}{4} & 0.1 & 1431.4 & 1430.4 & 1483.6 & 1313.5 & 1433.0 & 1454.3 & 1323.9 & 1359.3 & 1353.1 & 1412.5 & 1358.7 & \begin{tabular}{|l|l|}
1358.716 \\
\end{tabular} & 1479.7 & 1348.6 & 1558.1 & 1157.7 & 1540.7 & 1294.5 \\
\hline & 1 & 1924.2 & 1924.2 & 1975.6 & 1721.2 & 1888.0 & 1903.5 & 1788.2 & 1852.4 & 1838.9 & 1874.2 & 1815.9 & \begin{tabular}{|l|l|}
1815.876 \\
\end{tabular} & 1991.2 & 1805.4 & 2100.9 & 1544.1 & 1996.7 & 1745.1 \\
\hline & 10 & 2404.6 & 2406.0 & 2448.1 & 2152.5 & 2326.0 & 2350.5 & 2267.7 & 2336.7 & 2305.8 & 2312.8 & 2259.5 & 2259.547 & 2501.8 & 2264.0 & 2639.5 & 1927.7 & 2416.6 & 2197.0 \\
\hline \multirow{3}{*}{20} & 0.1 & 477.3 & 519.7 & 517.6 & 428.0 & 403.9 & 429.6 & 343.2 & 337.1 & 365.9 & 372.5 & 378.7 & \begin{tabular}{|l|l}
378.6942 \\
\end{tabular} & 378.3 & 408.7 & 350.6 & 363.6 & 476.7 & 456.4 \\
\hline & 1 & 876.0 & 923.3 & 900.7 & 772.9 & 738.2 & 778.4 & 660.1 & 654.0 & 703.1 & 702.4 & 707.6 & 707.6404 & 722.6 & 771.2 & 657.7 & 656.3 & 871.0 & 820.0 \\
\hline & 10 & 1374.2 & 1416.9 & 1385.4 & 1220.6 & 1180.8 & 1232.5 & 1111.1 & 1107.7 & 1163.6 & 1143.3 & 1137.8 & \begin{tabular}{|l|l|}
1137.823 \\
\end{tabular} & 1214.5 & 1289.8 & 1101.3 & 1018.5 & 1357.3 & 1253.3 \\
\hline \multirow{4}{*}{35} & 0.01 & 17.4 & 18.5 & 27.4 & & & & & & & & & & & & & & & \\
\hline & 0.1 & 33.1 & 37.4 & 56.8 & 88.9 & 66.6 & 70.9 & 66.7 & 67.9 & 68.5 & 71.3 & 72.0 & 71.95335 & 69.4 & 83.6 & 67.1 & 82.2 & 91.4 & 89.8 \\
\hline & 1 & 82.2 & 93.1 & 135.2 & 200.6 & 165.5 & 171.0 & 171.9 & 179.3 & 174.5 & 185.6 & 185.6 & \begin{tabular}{|l|l|}
185.6486 \\
\end{tabular} & 191.6 & 213.4 & 186.2 & 197.4 & 232.9 & 220.9 \\
\hline & 10 & 205.7 & 229.7 & 310.4 & 433.1 & 370.6 & 386.4 & 396.0 & 413.4 & 407.6 & 423.5 & 423.5 & \begin{tabular}{|l|}
423.511 \\
\end{tabular} & 455.1 & 481.4 & 422.1 & 414.2 & 511.4 & 468.8 \\
\hline & & \multicolumn{18}{|c|}{ Phase Angle } \\
\hline \multirow{3}{*}{4} & 0.1 & 15.0 & 14.8 & 13.7 & 14.5 & 12.8 & 11.1 & 15.1 & 14.3 & 15.1 & 14.1 & 14.4 & 14.4 & 15.2 & 14.4 & 14.7 & 14.7 & 13.5 & 15.3 \\
\hline & 1 & 10.9 & 10.8 & 9.9 & 11.3 & 9.4 & 8.8 & 11.1 & 10.4 & 10.9 & 10.2 & 10.5 & 10.5 & 11.0 & 10.7 & 10.7 & 10.4 & 9.6 & 11.1 \\
\hline & 10 & 8.3 & 8.0 & 7.2 & 7.3 & 6.6 & 6.7 & 8.6 & 7.5 & 8.2 & 7.7 & 7.9 & 7.9 & 8.3 & 8.4 & 8.2 & $\begin{array}{l}7.8 \\
\end{array}$ & 7.4 & 8.3 \\
\hline \multirow{3}{*}{20} & 0.1 & 28.4 & 27.5 & 27.3 & 28.4 & 29.2 & 29.4 & 32.9 & 32.7 & 30.9 & 31.1 & 30.1 & 30.1 & 32.0 & 30.8 & 30.7 & 29.9 & 28.9 & 28.8 \\
\hline & 1 & 22.0 & 21.4 & 21.2 & 22.4 & 23.0 & 22.1 & 25.3 & 26.0 & 24.3 & 24.7 & 23.7 & 23.7 & 25.5 & 25.1 & 24.8 & 23.7 & 22.3 & 21.8 \\
\hline & 10 & 16.4 & 15.8 & 15.7 & 16.8 & 16.9 & 15.9 & 19.1 & 19.2 & 18.0 & 18.2 & 17.7 & 17.7 & 18.9 & 18.9 & 18.7 & 17.4 & 16.2 & 15.9 \\
\hline \multirow{4}{*}{35} & 0.01 & 22.3 & 23.7 & 22.5 & & & & & & & & & & & & & & & \\
\hline & 0.1 & 28.2 & 29.3 & 28.7 & 31.2 & 32.6 & 32.3 & 34.3 & 35.6 & 38.0 & 32.8 & 31.8 & 31.8 & 35.4 & 33.8 & 36.1 & 35.6 & 33.8 & 33.7 \\
\hline & 1 & 33.8 & 34.3 & 32.9 & 33.3 & 34.7 & 34.0 & 36.8 & 37.0 & 38.7 & 34.3 & 32.6 & 32.6 & 37.2 & 35.3 & 36.1 & 35.8 & 34.0 & 33.8 \\
\hline & 10 & 37.3 & 36.7 & 34.4 & 30.7 & 32.1 & 31.5 & 32.5 & 32.7 & 32.5 & 31.6 & 30.3 & 30.3 & 34.2 & 31.9 & 32.5 & 31.9 & 30.3 & 29.6 \\
\hline
\end{tabular}



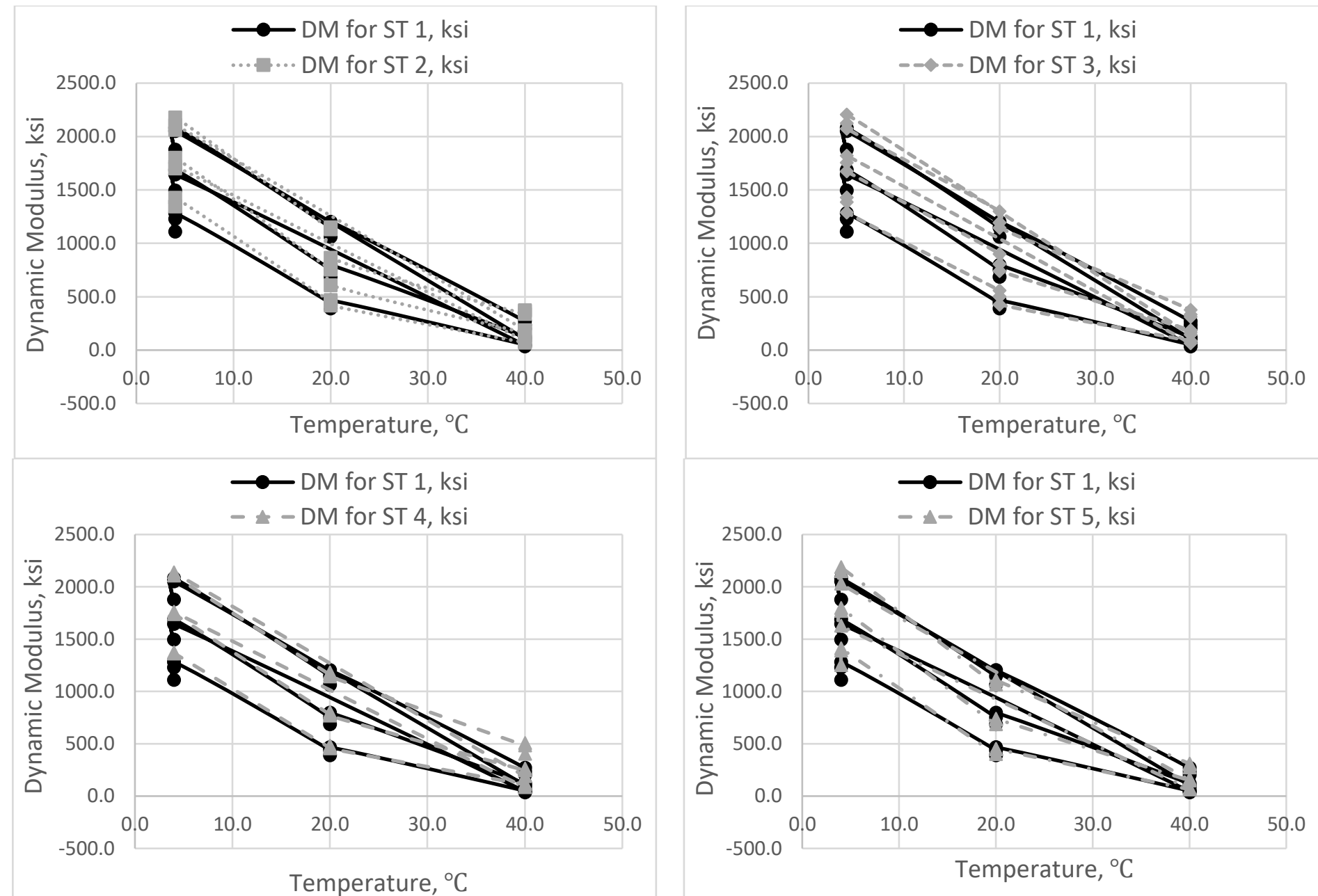

Figure 37: Comparison of Dynamic Modulus for STs fabricated with 12.5 mm NMAS 


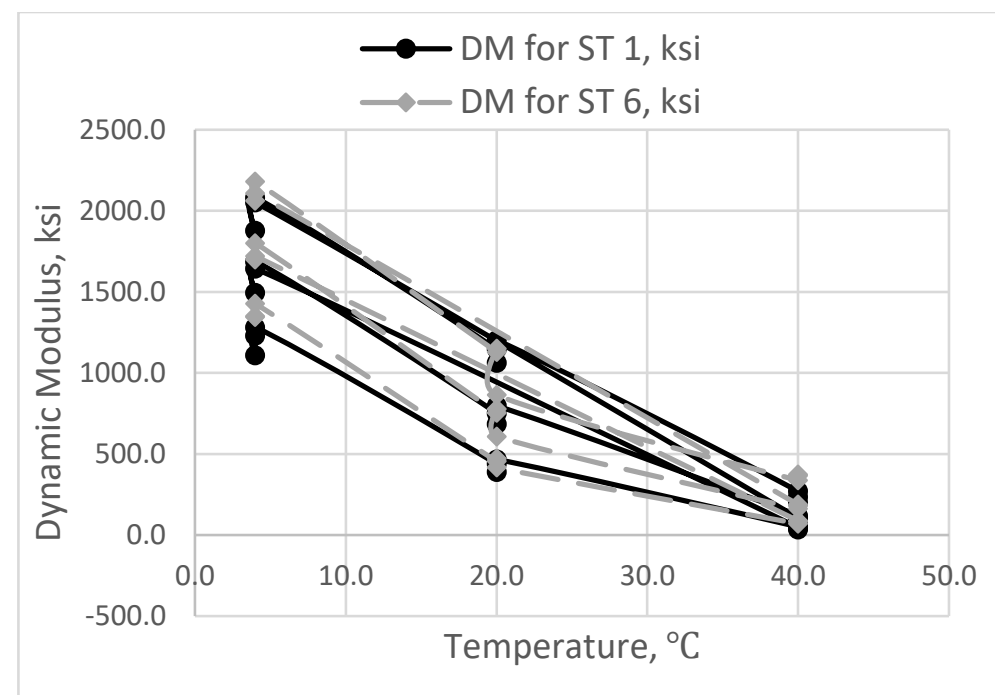

Figure 37 Cont.: Comparison of Dynamic Modulus for STs fabricated with $12.5 \mathrm{~mm}$ NMAS 

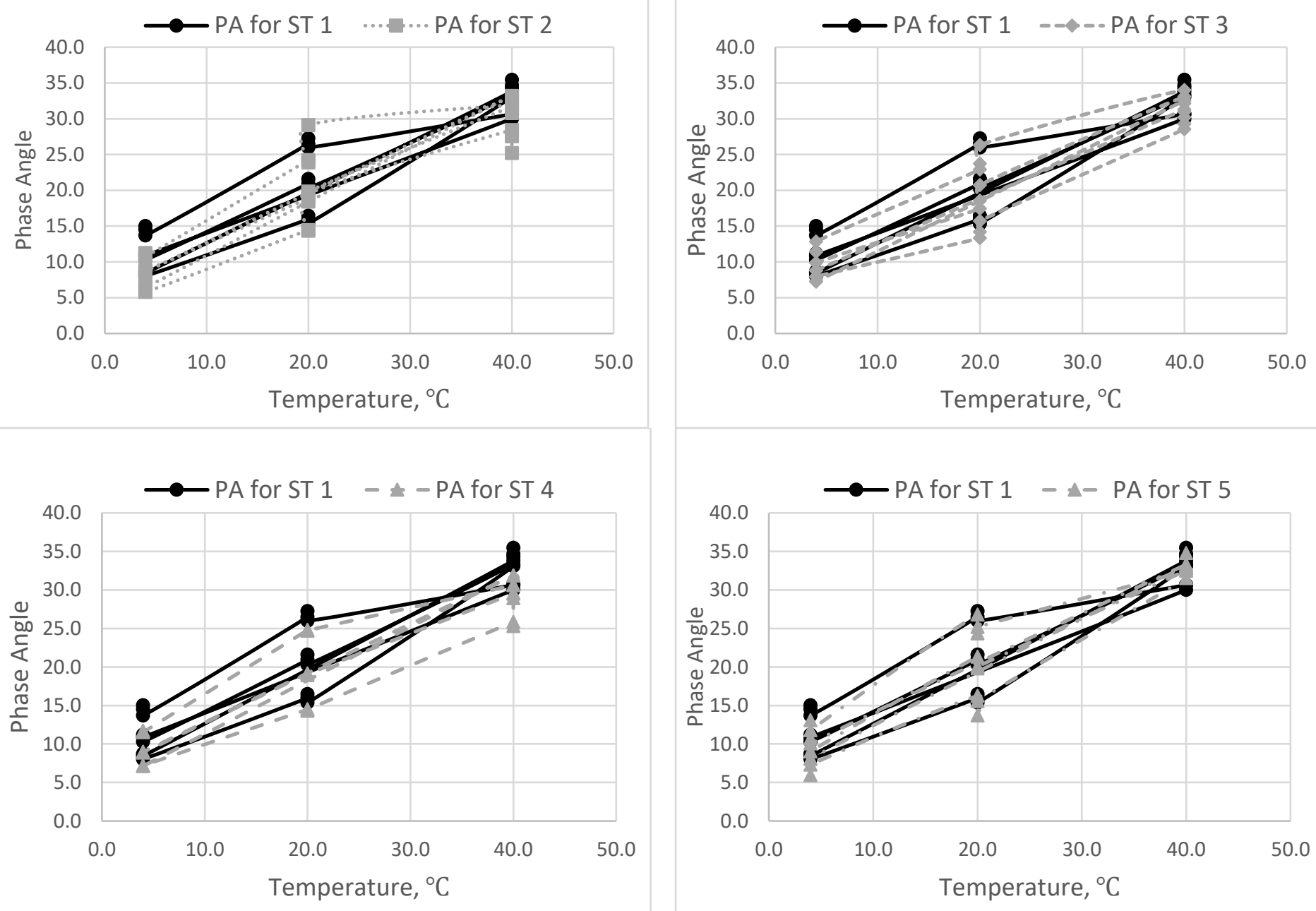

Figure 38: Comparison of Phase Angle for STs fabricated with 12.5 mm NMAS 


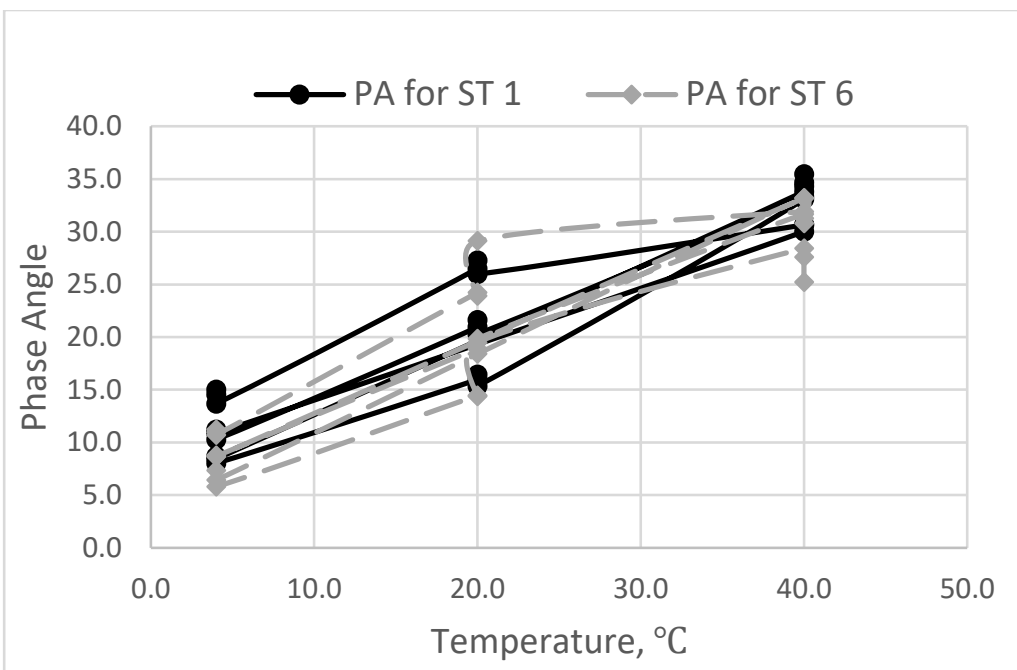

Figure 38 Cont.: Comparison of Phase Angle for STs fabricated with $12.5 \mathrm{~mm}$ NMAS 

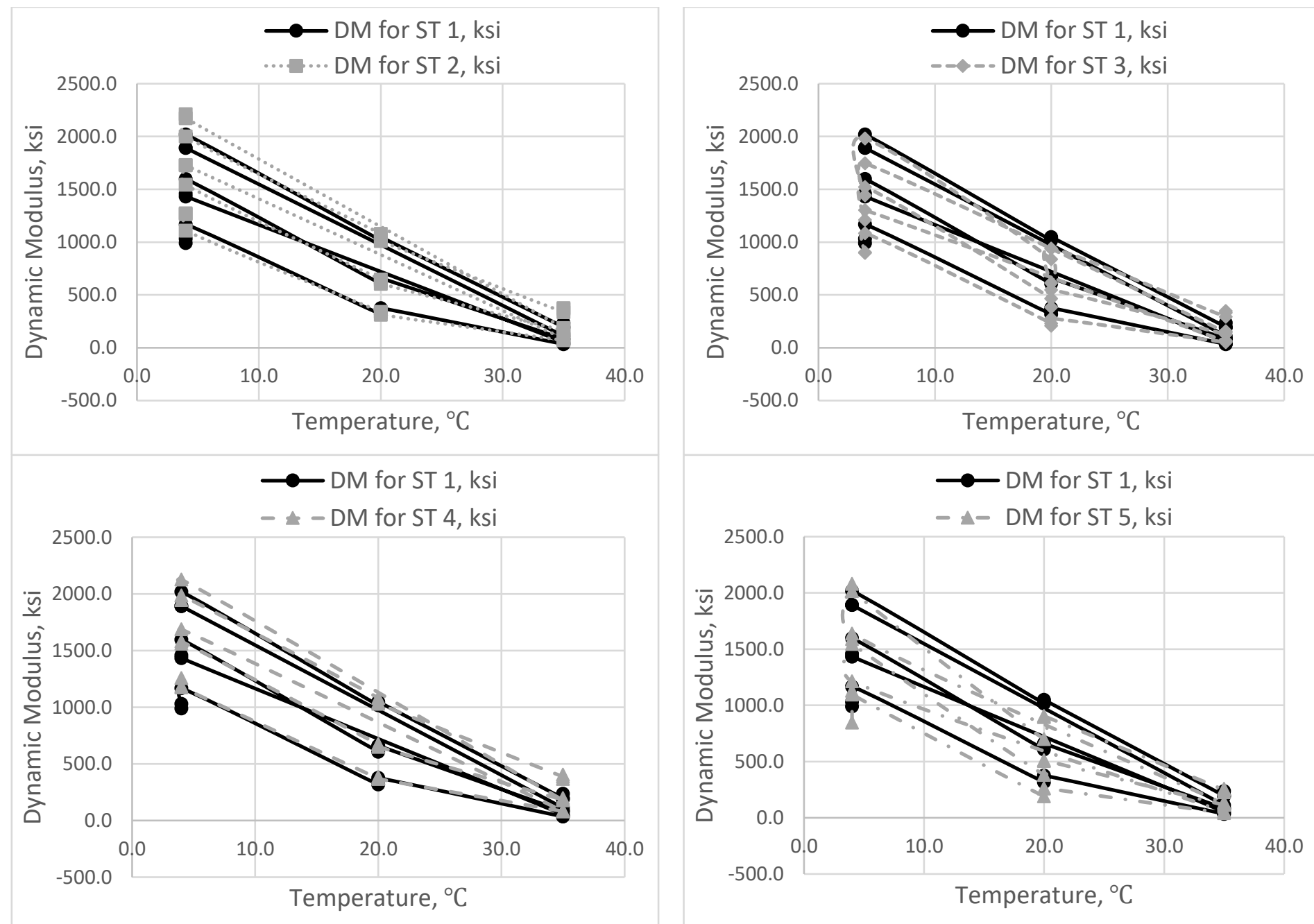

Figure 39: Comparison of Dynamic Modulus for STs fabricated with 19 mm NMAS 


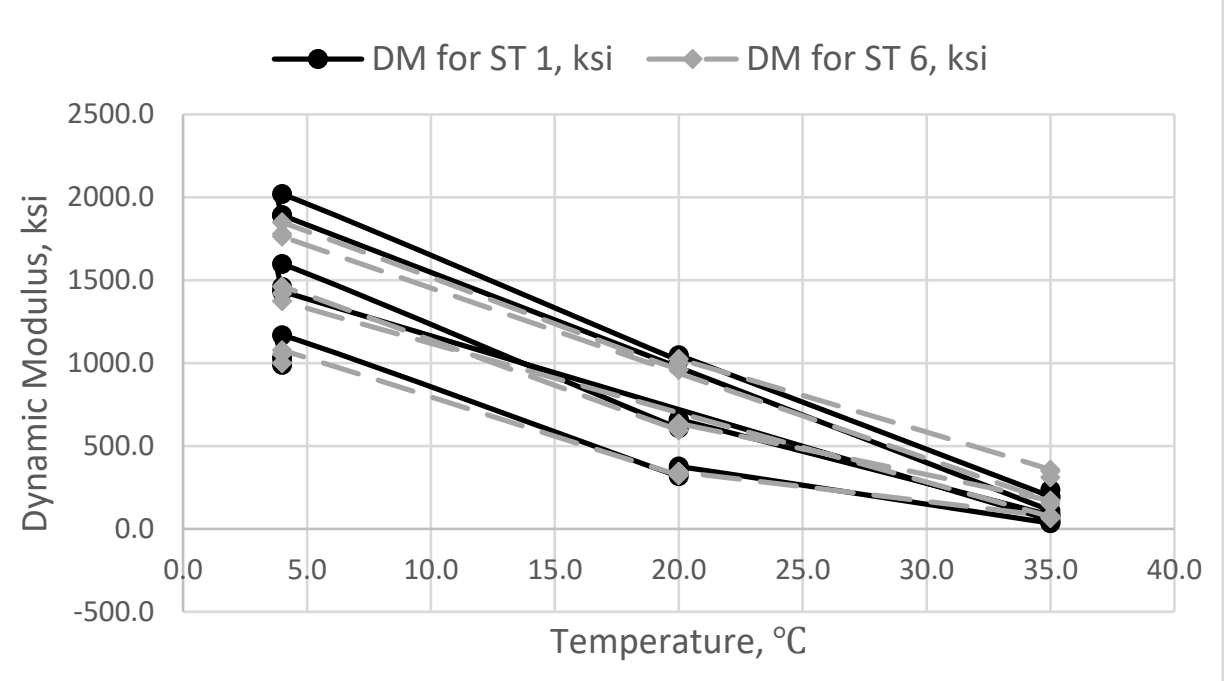

Figure 39 Cont.: Comparison of Dynamic Modulus for STs fabricated with 19 mm NMAS 

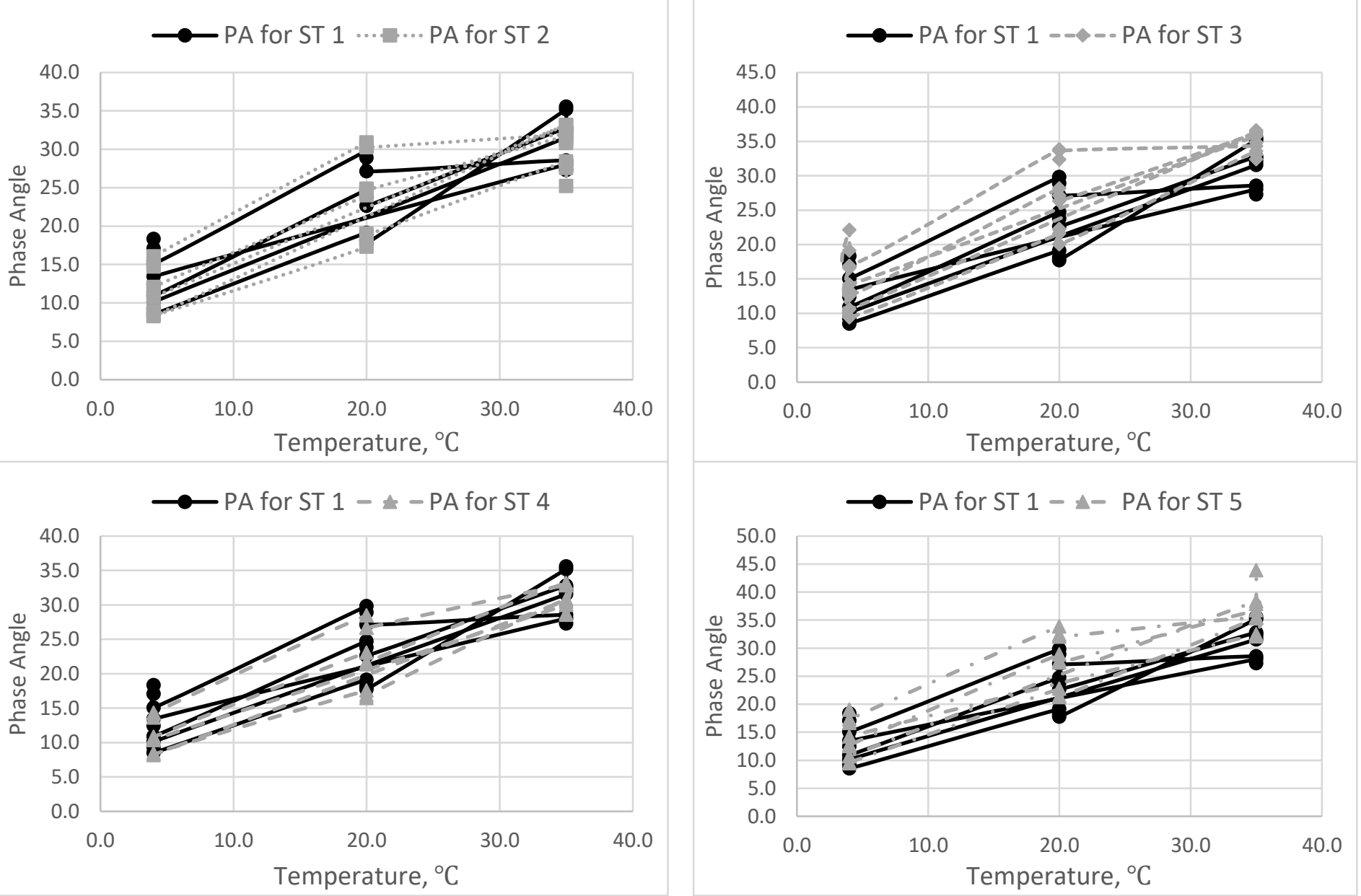

Figure 40: Comparison of Phase Angle for STs fabricated with 19 mm NMAS 


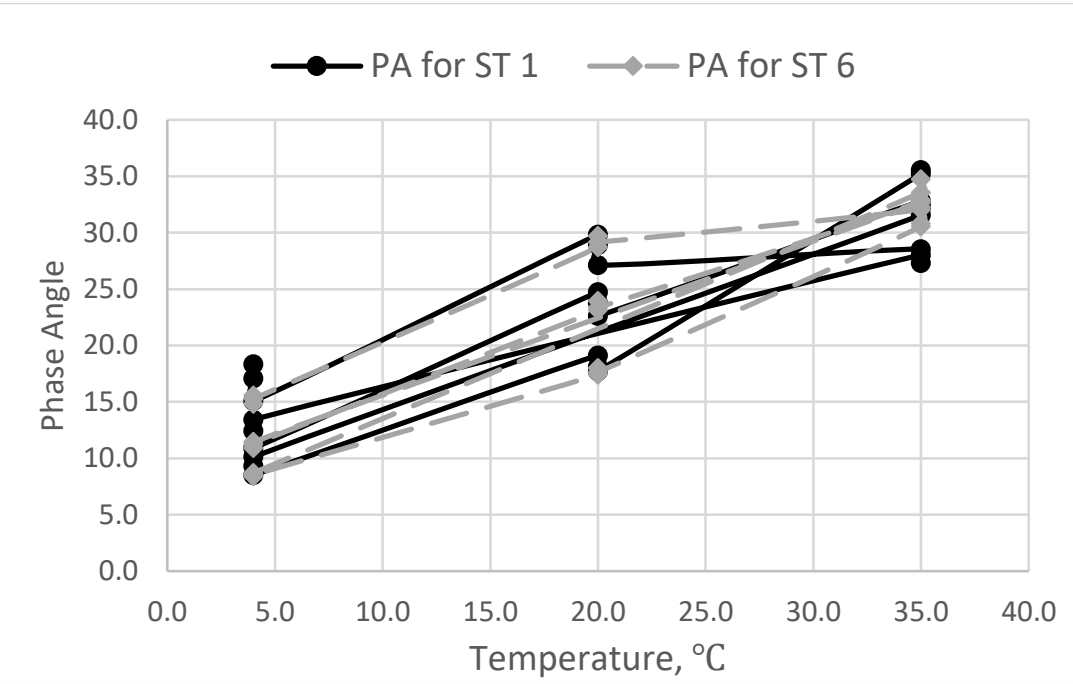

Figure 40 Cont.: Comparison of Phase Angle for STs fabricated with 19 mm NMAS 

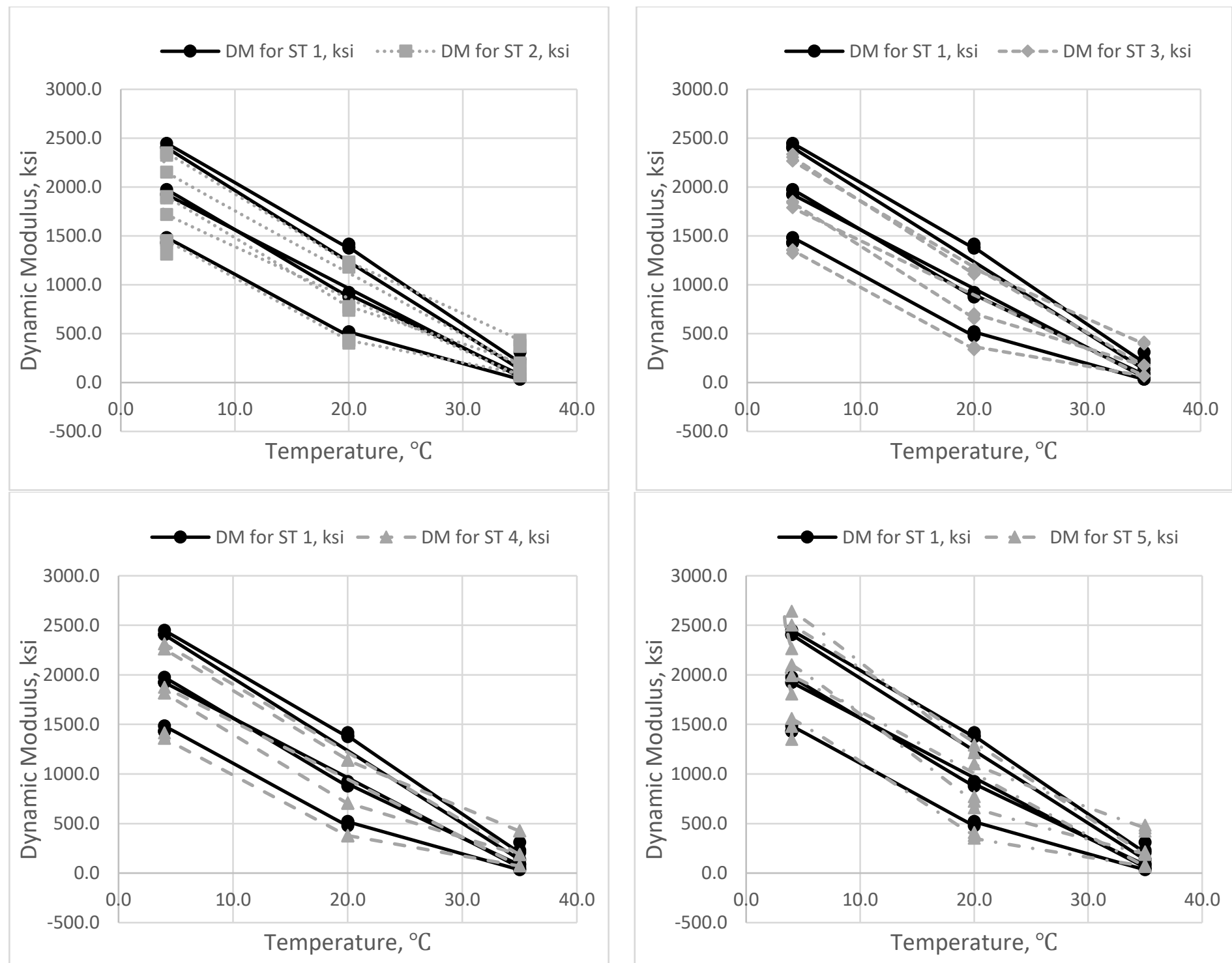

Figure 41: Comparison of Dynamic Modulus for STs fabricated with $25 \mathrm{~mm}$ NMAS 


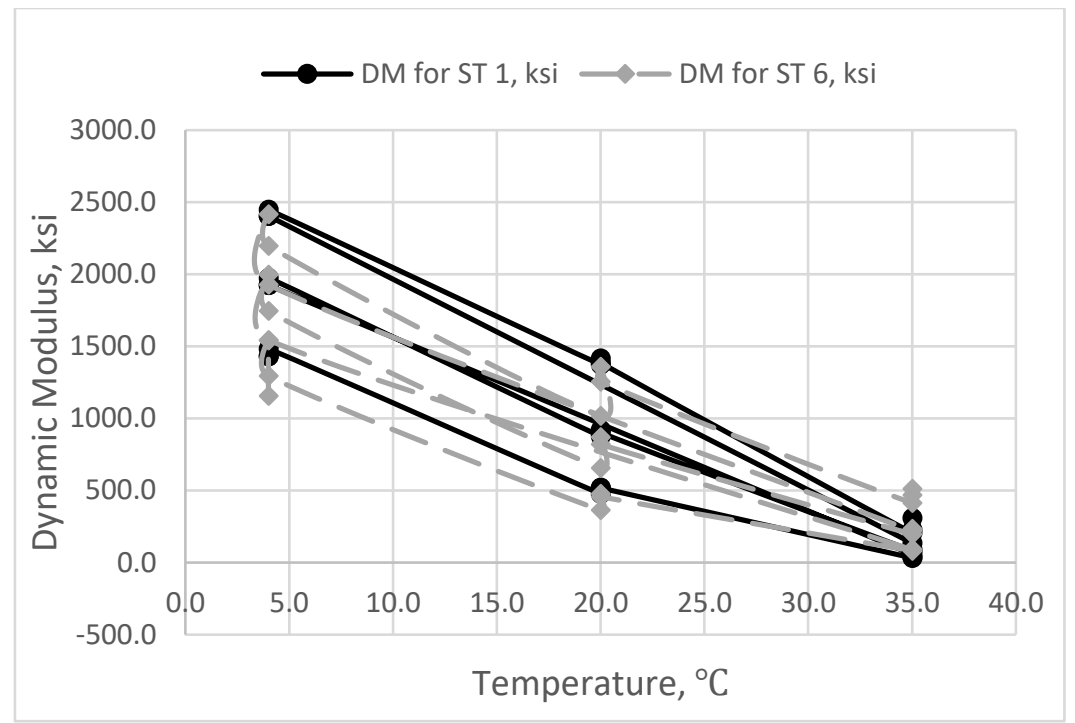

Figure 41 Cont.: Comparison of Dynamic Modulus for STs fabricated with 25 mm NMAS 

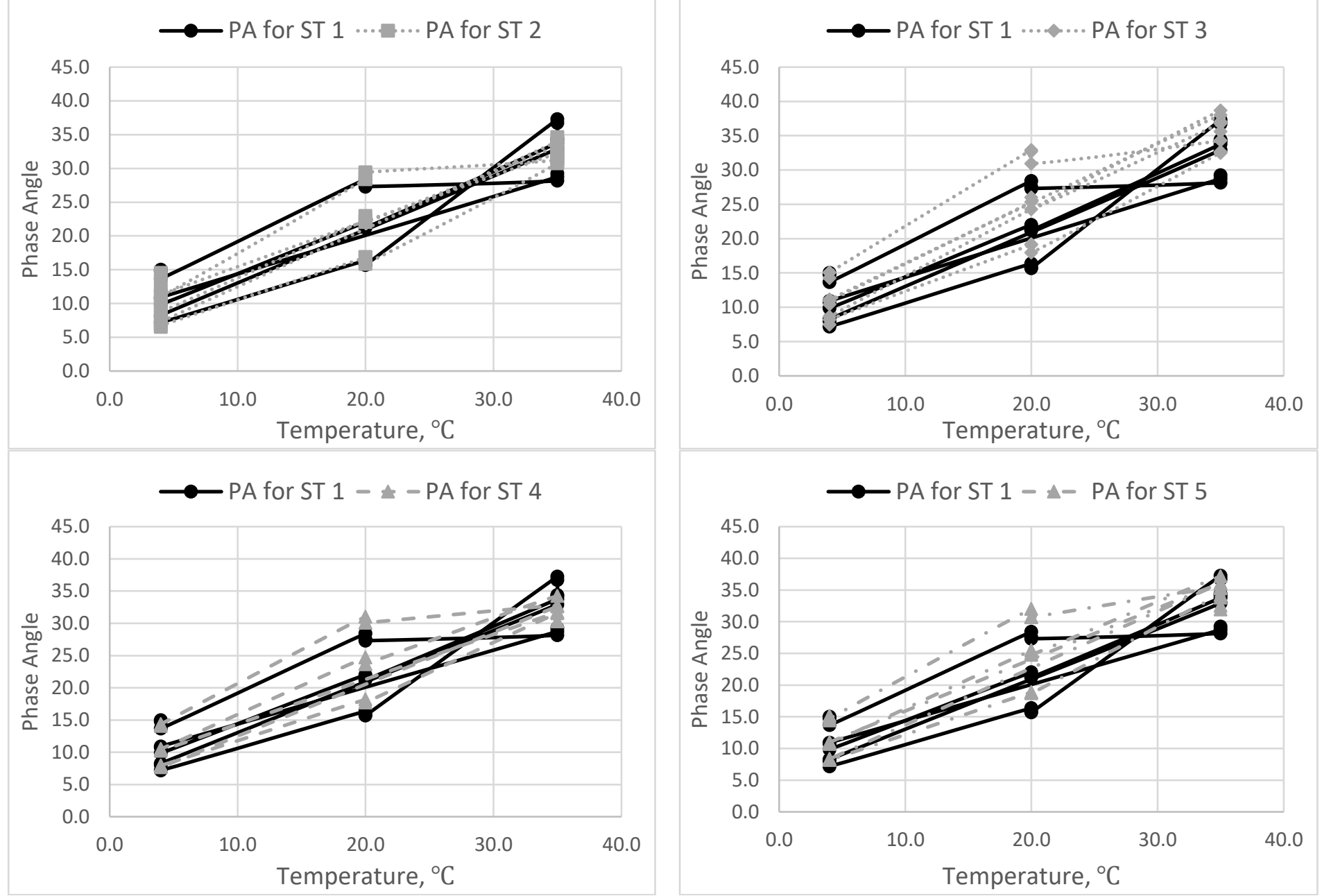

Figure 42: Comparison of Phase Angle for STs fabricated with 25 mm NMAS 


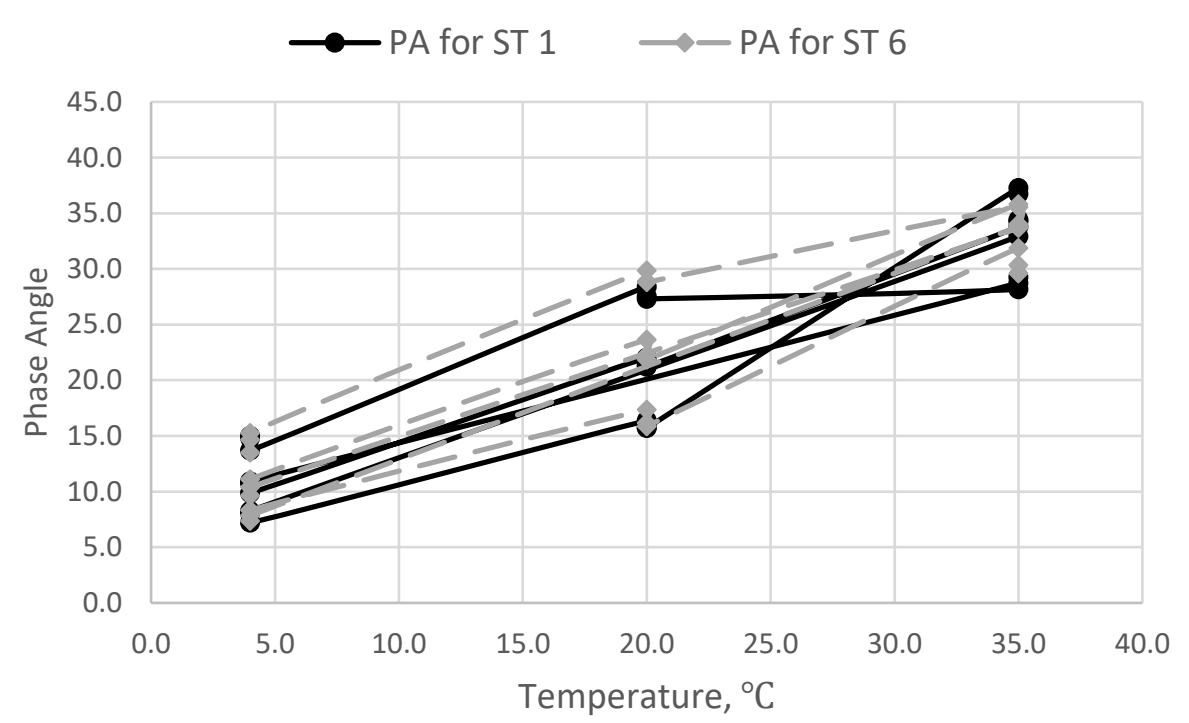

Figure 42 Cont.: Comparison of Phase Angle for STs fabricated with 25 mm NMAS 

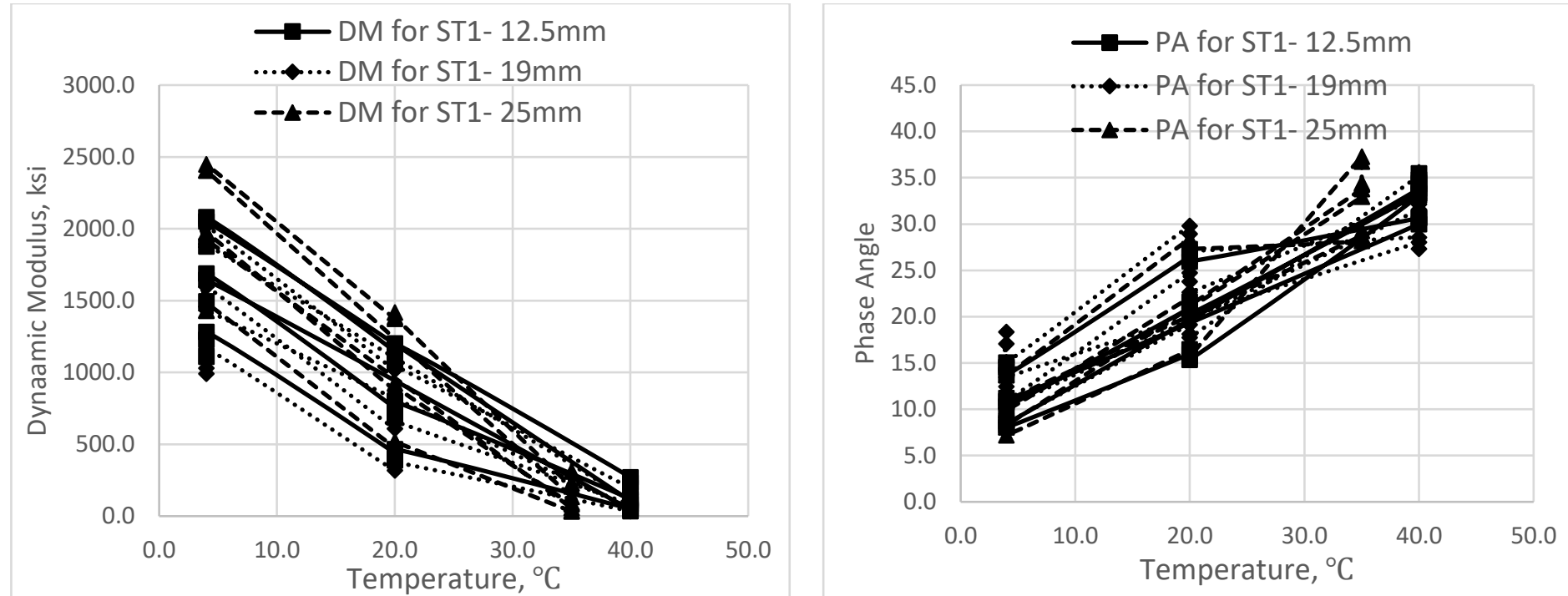

Figure 43: Comparison of Dynamic modulus and phase angle for ST 1 fabricated from different NMAS
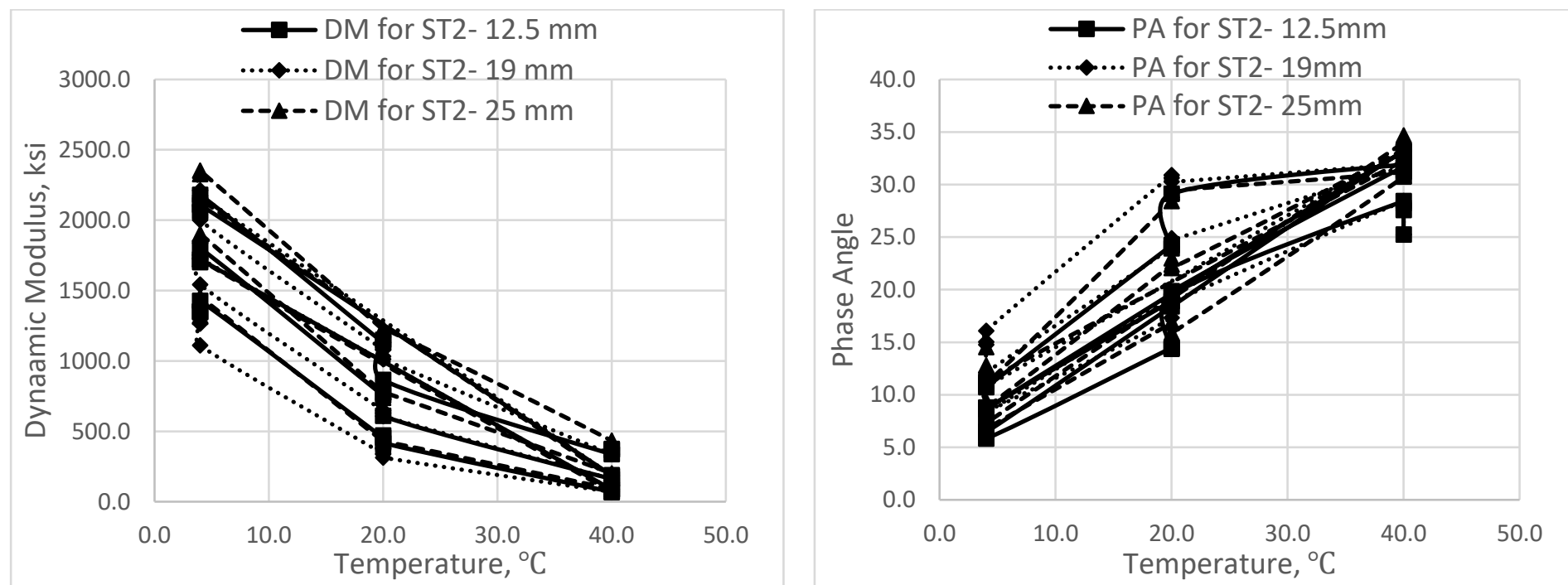

Figure 44: Comparison of Dynamic modulus and phase angle for ST 2 fabricated from different NMAS 

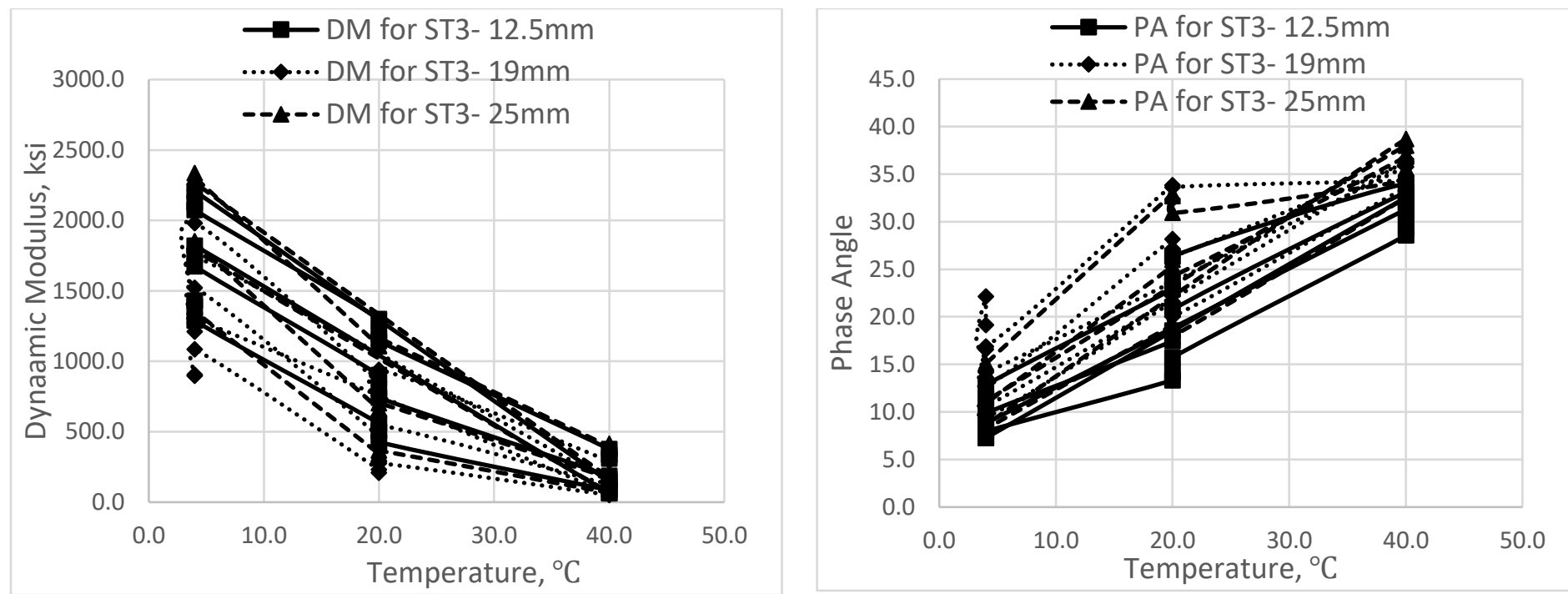

Figure 45: Comparison of Dynamic modulus and phase angle for ST 3 fabricated from different NMAS
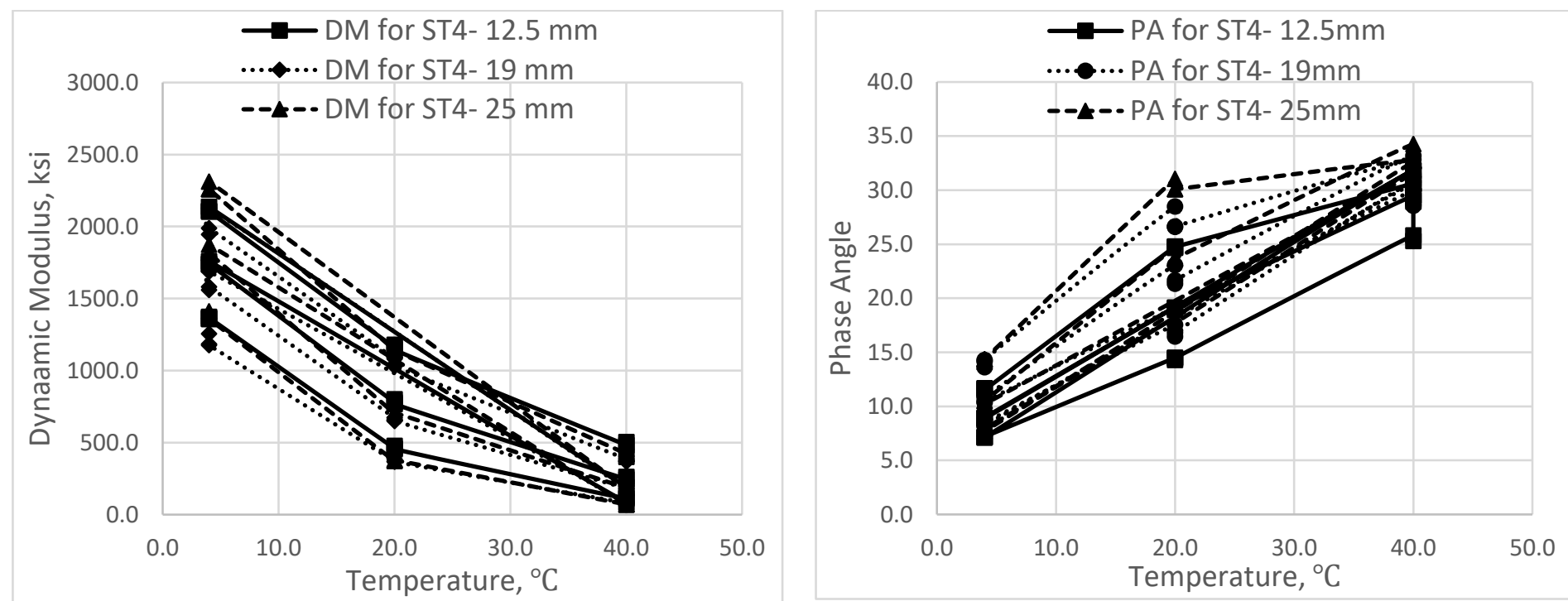

Figure 46: Comparison of Dynamic modulus and phase angle for ST 4 fabricated from different NMAS 

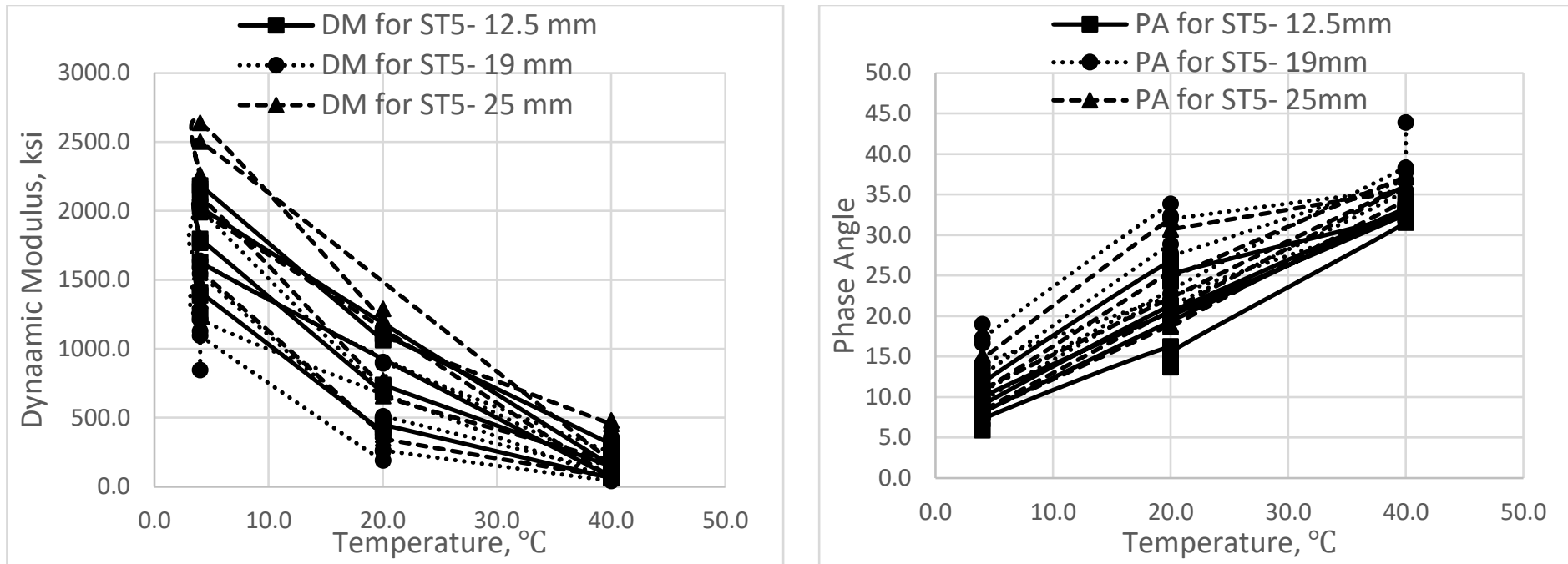

Figure 47: Comparison of Dynamic modulus and phase angle for ST 5 fabricated from different NMAS
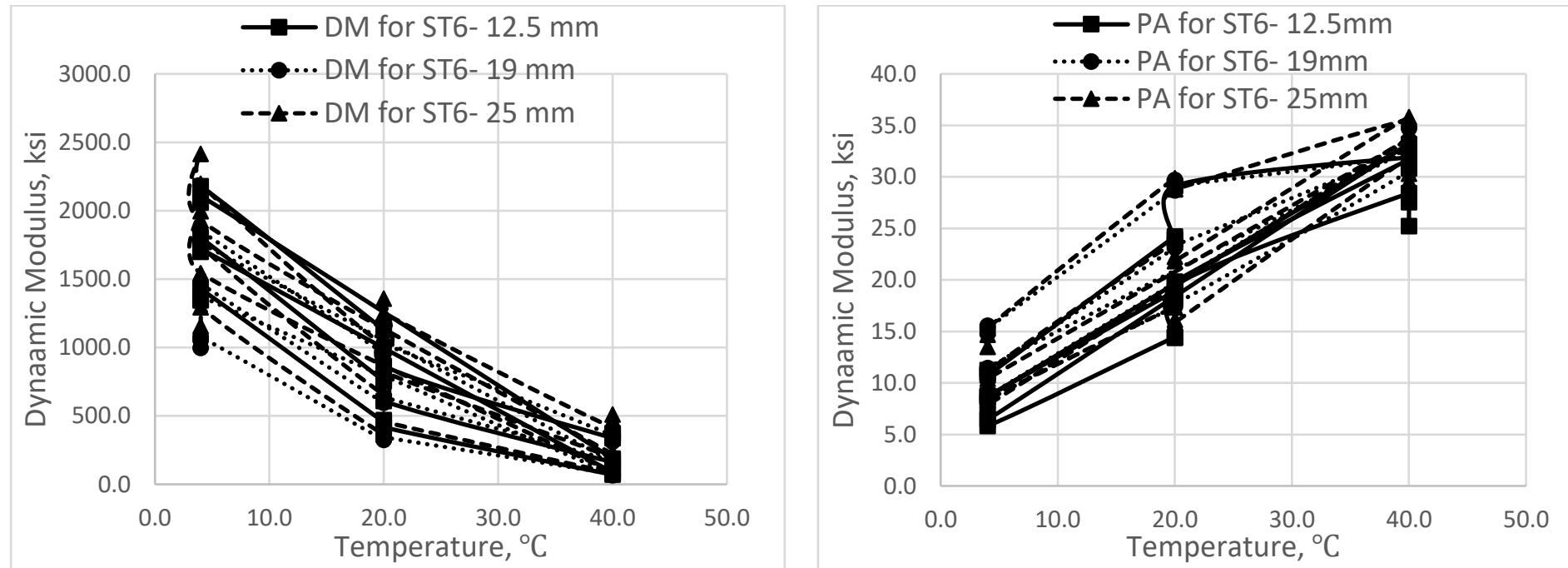

Figure 48: Comparison of Dynamic modulus and phase angle for ST 6 fabricated from different NMAS 
The comparative analysis in Figure 37 and 38 for dynamic modulus and phase angle for $12.5 \mathrm{~mm}$ NMAS mix show that ST 5 values for three different test temperatures are consistently closer to that of ST1. From Figures 39 and 40, the dynamic modulus and phase angle for $19 \mathrm{~mm}$ NMAS mix show that ST 5 values for three different test temperatures are consistently closer to that of ST1. From Figures 41 and 42, the dynamic modulus and phase angle for $25 \mathrm{~mm}$ NMAS mix show that ST 3 values for three different test temperatures are consistently closer to that of ST1. To further verify if same ST can be used for all three NMAS mixes, comparative analysis in Figure 43 to 48 is conducted for STs. Visually, ST 4 can be used for all three NMAS however, the recommendation would be to use the right sample type for a specific NMAS. This ST should have uniform air void distribution and dynamic modulus and phase angle values close to ST1. From comparative analysis ST5 performed consistently to ST1 for 12.5 and $25 \mathrm{~mm}$ NMAS and ST 4 performed consistently to ST1 for 19 mm NMAS mix.

The Student's t-test in Table 37 to 54 is used for comparison of difference between the mean of different STs and ST 1 for various combinations of temperature and frequencies in dynamic modulus test. All tests used a 95 percent confidence level, alpha of 0.05 . The null hypothesis for all tests was the means of the two data sets are equal. Failure to reject the null hypothesis indicates the means of each data set are statistically same. Rejecting the null hypothesis implies the data sets are statistically different. In the tables of t-test results, comparisons with alpha of less than 0.05 are shown with a bold font.

Table 37: t-test of Dynamic Modulus for STs fabricated from $12.5 \mathrm{~mm}$ NMAS at $4^{\circ} \mathrm{C}$

\begin{tabular}{|c|c|c|c|c|c|c|c|c|c|c|c|c|}
\hline \multirow[b]{2}{*}{ Comparison } & \multicolumn{4}{|c|}{$4^{\circ} \mathrm{C}$ and $0.1 \mathrm{~Hz}$} & \multicolumn{4}{|c|}{$4^{\circ} \mathrm{C}$ and $1 \mathrm{~Hz}$} & \multicolumn{4}{|c|}{$4^{\circ} \mathrm{C}$ and $10 \mathrm{~Hz}$} \\
\hline & 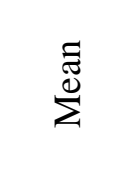 & 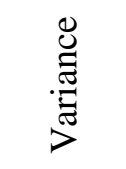 & 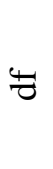 & 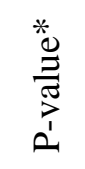 & 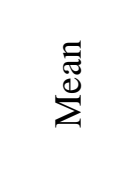 & 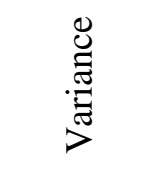 & 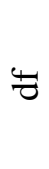 & 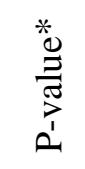 & 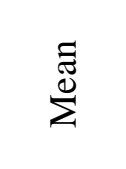 & 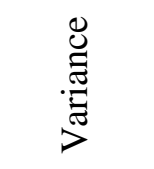 & $t$ & 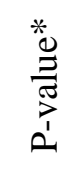 \\
\hline ST 1 & 1206.6 & 7763.2 & \multirow{2}{*}{5} & \multirow{2}{*}{0.06} & 1608.8 & 10245.9 & \multirow{2}{*}{5} & \multirow{2}{*}{0.14} & 2002.8 & 12130.9 & \multirow{2}{*}{5} & \multirow{2}{*}{0.22} \\
\hline ST 2 & 1373.8 & 2075.1 & & & 1740.6 & 2804.2 & & & 2116.3 & 3537.5 & & \\
\hline ST 1 & 1206.6 & 7763.2 & \multirow{2}{*}{5} & \multirow{2}{*}{0.07} & 1608.8 & 10245.9 & \multirow{2}{*}{5} & \multirow{2}{*}{0.12} & 2002.8 & 12130.9 & \multirow{2}{*}{5} & \multirow{2}{*}{0.17} \\
\hline ST 3 & 1368.4 & 5275.4 & & & 1750.1 & 5339.1 & & & 2135.4 & 4512.2 & & \\
\hline ST 1 & 1206.6 & 7763.2 & \multirow{2}{*}{5} & \multirow{2}{*}{0.08} & 1608.8 & 10245.9 & \multirow{2}{*}{5} & \multirow{2}{*}{0.15} & 2002.8 & 12130.9 & \multirow{2}{*}{5} & \multirow{2}{*}{0.22} \\
\hline ST 4 & 1367.2 & 30.4 & & & 1745.9 & 132.0 & & & 2116.9 & 288.7 & & \\
\hline ST 1 & 1206.6 & 7763.2 & \multirow{2}{*}{5} & \multirow{2}{*}{0.12} & 1608.8 & 10245.9 & \multirow{2}{*}{5} & \multirow{2}{*}{0.19} & 2002.8 & 12130.9 & \multirow{2}{*}{5} & \multirow{2}{*}{0.21} \\
\hline ST 5 & 1350.2 & 7578.2 & & & 1731.2 & 8221.9 & & & 2120.9 & 6836.7 & & \\
\hline ST 1 & 1206.6 & 7763.2 & \multirow[b]{2}{*}{5} & \multirow[b]{2}{*}{0.06} & 1608.8 & 10245.9 & \multirow[b]{2}{*}{5} & \multirow[b]{2}{*}{0.14} & 2002.8 & 12130.9 & \multirow[b]{2}{*}{5} & \multirow[b]{2}{*}{0.22} \\
\hline ST 6 & 1373.8 & 2075.1 & & & 1740.6 & 2804.2 & & & 2116.3 & 3537.5 & & \\
\hline
\end{tabular}

* Bold font indicates the null hypothesis of equal means is rejected. 
Table 38: t-test of Phase Angle for STs fabricated from $12.5 \mathrm{~mm}$ NMAS at $4^{\circ} \mathrm{C}$

\begin{tabular}{|c|c|c|c|c|c|c|c|c|c|c|c|c|}
\hline \multirow[b]{2}{*}{ Comparison } & \multicolumn{4}{|c|}{$4^{\circ} \mathrm{C}$ and $0.1 \mathrm{~Hz}$} & \multicolumn{4}{|c|}{$4^{\circ} \mathrm{C}$ and $1 \mathrm{~Hz}$} & \multicolumn{4}{|c|}{$4^{\circ} \mathrm{C}$ and $10 \mathrm{~Hz}$} \\
\hline & $\underset{\tilde{\Xi}}{\stackrel{\Xi}{\Sigma}}$ & $\begin{array}{l}\stackrel{\mathscr{J}}{\Xi} \\
\stackrel{\Xi}{\Xi} \\
>\end{array}$ & 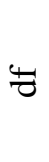 & 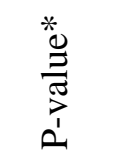 & $\stackrel{\Xi}{\stackrel{\Xi}{\Xi}}$ & 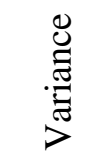 & 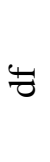 & 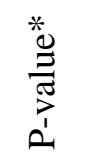 & $\sum_{\Sigma}^{\Xi \Xi}$ & $\begin{array}{l}\stackrel{\Xi}{\Xi} \\
\stackrel{\Xi}{\Xi} \\
>\end{array}$ & $t$ & 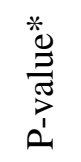 \\
\hline ST 1 & 14.4 & 0.5 & \multirow{2}{*}{5} & \multirow{2}{*}{0.004} & 10.8 & 0.2 & \multirow{2}{*}{5} & \multirow{2}{*}{0.02} & 8.4 & 0.1 & \multirow{2}{*}{5} & \multirow{2}{*}{0.03} \\
\hline ST 2 & 10.9 & 0.07 & & & 8.7 & 0.006 & & & 6.5 & 0.6 & & \\
\hline ST 1 & 14.4 & 0.5 & \multirow{2}{*}{5} & \multirow{2}{*}{0.02} & 10.8 & 0.2 & \multirow{2}{*}{5} & \multirow{2}{*}{0.02} & 8.4 & 0.1 & \multirow{2}{*}{5} & \multirow{2}{*}{0.04} \\
\hline ST 3 & 11.9 & 0.7 & & & 9.2 & 0.4 & & & 7.5 & 0.2 & & \\
\hline ST 1 & 14.4 & 0.5 & \multirow{2}{*}{5} & \multirow{2}{*}{0.02} & 10.8 & 0.2 & \multirow{2}{*}{5} & \multirow{2}{*}{0.02} & 8.4 & 0.1 & \multirow{2}{*}{5} & \multirow{2}{*}{0.02} \\
\hline ST 4 & 11.6 & 0.007 & & & 8.9 & 0.003 & & & 7.2 & 0.001 & & \\
\hline ST 1 & 14.4 & 0.5 & \multirow{2}{*}{5} & \multirow{2}{*}{0.05} & 10.8 & 0.2 & \multirow{2}{*}{5} & \multirow{2}{*}{0.07} & 8.4 & 0.1 & \multirow{2}{*}{5} & \multirow{2}{*}{0.19} \\
\hline ST 5 & 11.9 & 1.4 & & & 9.1 & 0.9 & & & 7.1 & 1.2 & & \\
\hline ST 1 & 14.4 & 0.5 & \multirow{2}{*}{5} & \multirow{2}{*}{0.004} & 10.8 & 0.2 & \multirow{2}{*}{5} & \multirow{2}{*}{0.02} & 8.4 & 0.1 & \multirow{2}{*}{5} & \multirow{2}{*}{0.22} \\
\hline ST 6 & 10.9 & 0.07 & & & 8.7 & 0.006 & & & 4.4 & 15.0 & & \\
\hline
\end{tabular}

Table 39: t-test of Dynamic Modulus for STs fabricated from $12.5 \mathrm{~mm}$ NMAS at $20^{\circ} \mathrm{C}$

\begin{tabular}{|c|c|c|c|c|c|c|c|c|c|c|c|c|}
\hline \multirow[b]{2}{*}{ Comparison } & \multicolumn{4}{|c|}{$20^{\circ} \mathrm{C}$ and $0.1 \mathrm{~Hz}$} & \multicolumn{4}{|c|}{$20^{\circ} \mathrm{C}$ and $1 \mathrm{~Hz}$} & \multicolumn{4}{|c|}{$20^{\circ} \mathrm{C}$ and $10 \mathrm{~Hz}$} \\
\hline & 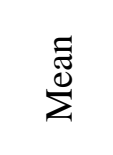 & 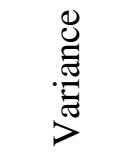 & 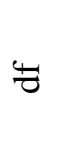 & 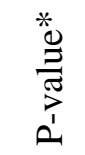 & 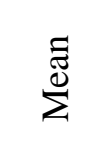 & 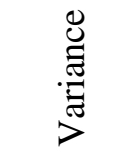 & 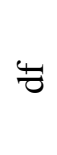 & 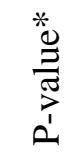 & $\underset{\Xi \Xi}{\stackrel{\Xi}{\Sigma}}$ & 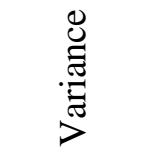 & t & 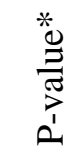 \\
\hline ST 1 & 431.8 & 1527.2 & \multirow{2}{*}{5} & \multirow{2}{*}{0.61} & 744.6 & 3156.5 & \multirow{2}{*}{5} & \multirow{2}{*}{0.64} & 1136.5 & 4996.5 & \multirow{2}{*}{5} & \multirow{2}{*}{0.44} \\
\hline ST 2 & 447.2 & 741.7 & & & 712.9 & 8312.3 & & & 1047.9 & 25369.2 & & \\
\hline ST 1 & 431.8 & 1527.2 & \multirow{2}{*}{5} & \multirow{2}{*}{0.25} & 744.6 & 3156.5 & \multirow{2}{*}{5} & \multirow{2}{*}{0.27} & 1136.5 & 4996.5 & \multirow{2}{*}{5} & \multirow{2}{*}{0.28} \\
\hline ST 3 & 495.4 & 4334.7 & & & 816.4 & 6465.0 & & & 1212.8 & 6327.3 & & \\
\hline ST 1 & 431.8 & 1527.2 & \multirow{2}{*}{5} & \multirow{2}{*}{0.29} & 744.6 & 3156.5 & \multirow{2}{*}{5} & \multirow{2}{*}{0.38} & 1136.5 & 4996.5 & \multirow{2}{*}{5} & \multirow{2}{*}{0.61} \\
\hline ST 4 & 464.8 & 161.4 & & & 782.6 & 277.4 & & & 1161.7 & 345.3 & & \\
\hline ST 1 & 431.8 & 1527.2 & \multirow{2}{*}{5} & \multirow{2}{*}{0.97} & 744.6 & 3156.5 & \multirow{2}{*}{5} & \multirow{2}{*}{0.52} & 1136.5 & 4996.5 & \multirow{2}{*}{5} & \multirow{2}{*}{0.41} \\
\hline ST 5 & 432.9 & 784.3 & & & 718.0 & 869.4 & & & 1091.5 & 599.7 & & \\
\hline ST 1 & 431.8 & 1527.2 & \multirow{2}{*}{5} & \multirow{2}{*}{0.61} & 744.6 & 3156.5 & \multirow{2}{*}{5} & \multirow{2}{*}{0.64} & 1136.5 & 4996.5 & \multirow{2}{*}{5} & \multirow{2}{*}{0.44} \\
\hline ST 6 & 447.2 & 741.7 & & & 712.9 & 8312.3 & & & 4996.5 & 25369.2 & & \\
\hline
\end{tabular}

* Bold font indicates the null hypothesis of equal means is rejected. 
Table 40: t-test of Phase Angle for STs fabricated from $12.5 \mathrm{~mm}$ NMAS at $20^{\circ} \mathrm{C}$

\begin{tabular}{|c|c|c|c|c|c|c|c|c|c|c|c|c|}
\hline \multirow[b]{2}{*}{ Comparison } & \multicolumn{4}{|c|}{$20^{\circ} \mathrm{C}$ and $0.1 \mathrm{~Hz}$} & \multicolumn{4}{|c|}{$20^{\circ} \mathrm{C}$ and $1 \mathrm{~Hz}$} & \multicolumn{4}{|c|}{$20^{\circ} \mathrm{C}$ and $10 \mathrm{~Hz}$} \\
\hline & $\stackrel{\Xi}{\Xi}$ & 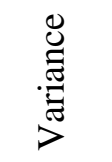 & 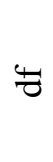 & 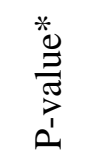 & $\sum_{\Sigma}^{\Xi}$ & $\begin{array}{l}\ddot{U} \\
\stackrel{\Xi}{\Xi} \\
\stackrel{\Xi}{\Xi} \\
>\end{array}$ & 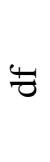 & 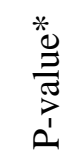 & 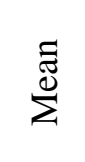 & $\begin{array}{c}\stackrel{\mathscr{E}}{\Xi} \\
\stackrel{\Xi}{\Xi} \\
>\end{array}$ & $t$ & 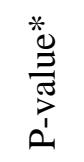 \\
\hline ST 1 & 26.6 & 0.4 & \multirow{2}{*}{5} & \multirow{2}{*}{0.68} & 20.9 & 0.4 & \multirow{2}{*}{5} & \multirow{2}{*}{0.03} & 15.9 & 0.3 & \multirow{2}{*}{5} & \multirow[t]{2}{*}{0.93} \\
\hline ST 2 & 25.8 & 8.5 & & & 19.0 & 0.5 & & & 16.1 & 8.6 & & \\
\hline ST 1 & 26.6 & 0.4 & \multirow{2}{*}{5} & \multirow{2}{*}{0.13} & 20.9 & 0.4 & \multirow{2}{*}{5} & \multirow{2}{*}{0.15} & 15.9 & 0.3 & \multirow{2}{*}{5} & \multirow[t]{2}{*}{0.14} \\
\hline ST 3 & 24.3 & 3.2 & & & 18.9 & 2.8 & & & 14.4 & 1.4 & & \\
\hline ST 1 & 26.6 & 0.4 & \multirow{2}{*}{5} & \multirow{2}{*}{0.04} & 20.9 & 0.4 & \multirow{2}{*}{5} & \multirow{2}{*}{0.04} & 15.9 & 0.3 & \multirow{2}{*}{5} & \multirow[t]{2}{*}{0.05} \\
\hline ST 4 & 24.7 & 0.004 & & & 19.1 & 0.02 & & & 14.4 & 0.01 & & \\
\hline ST 1 & 26.6 & 0.4 & \multirow{2}{*}{5} & \multirow{2}{*}{0.25} & 20.9 & 0.4 & \multirow{2}{*}{5} & \multirow{2}{*}{0.41} & 15.9 & 0.3 & \multirow{2}{*}{5} & \multirow[t]{2}{*}{$\overline{0.43}$} \\
\hline ST 5 & 25.4 & 1.6 & & & 20.4 & 0.7 & & & 15.2 & 1.8 & & \\
\hline ST 1 & 26.6 & 0.4 & \multirow{2}{*}{5} & \multirow{2}{*}{0.68} & 20.9 & 0.4 & \multirow{2}{*}{5} & \multirow{2}{*}{0.03} & 15.9 & 0.3 & \multirow{2}{*}{5} & \multirow[t]{2}{*}{0.93} \\
\hline ST 6 & 25.8 & 8.5 & & & 19.1 & 0.5 & & & 16.1 & 8.6 & & \\
\hline
\end{tabular}

* Bold font indicates the null hypothesis of equal means is rejected.

Table 41: t-test of Dynamic Modulus for STs fabricated from $12.5 \mathrm{~mm}$ NMAS at $40^{\circ} \mathrm{C}$

\begin{tabular}{|c|c|c|c|c|c|c|c|c|c|c|c|c|}
\hline \multirow[b]{2}{*}{ Comparison } & \multicolumn{4}{|c|}{$40^{\circ} \mathrm{C}$ and $0.1 \mathrm{~Hz}$} & \multicolumn{4}{|c|}{$40^{\circ} \mathrm{C}$ and $1 \mathrm{~Hz}$} & \multicolumn{4}{|c|}{$40^{\circ} \mathrm{C}$ and $10 \mathrm{~Hz}$} \\
\hline & 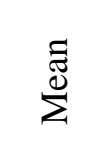 & 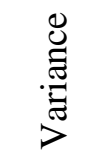 & \pm & 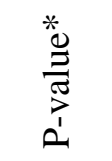 & 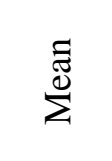 & $\begin{array}{l}\ddot{\mathscr{U}} \\
\stackrel{\Xi}{\Xi} \\
\stackrel{\nabla}{二}\end{array}$ & $t$ & 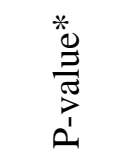 & 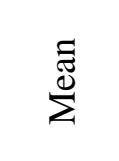 & 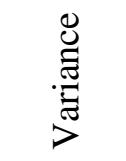 & \pm & 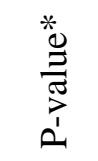 \\
\hline ST 1 & 43.9 & 79.9 & \multirow[b]{2}{*}{5} & \multirow[b]{2}{*}{0.006} & 102.9 & 328.6 & \multirow[b]{2}{*}{5} & \multirow[b]{2}{*}{0.005} & 234.5 & 1242.2 & \multirow[b]{2}{*}{5} & \multirow[b]{2}{*}{0.01} \\
\hline ST 2 & 63.6 & 74.8 & & & 176.8 & 183.9 & & & 359.2 & 377.9 & & \\
\hline ST 1 & 43.9 & 79.9 & \multirow{2}{*}{5} & \multirow{2}{*}{0.02} & 102.9 & 328.6 & \multirow{2}{*}{5} & \multirow{2}{*}{0.02} & 234.5 & 1242.2 & \multirow{2}{*}{5} & \multirow{2}{*}{0.02} \\
\hline ST 3 & 76.6 & 134.3 & & & 164.7 & 419.8 & & & 1242.2 & 1124.1 & & \\
\hline ST 1 & 43.9 & 79.9 & \multirow{2}{*}{5} & \multirow{2}{*}{0.01} & 102.9 & 328.6 & \multirow{2}{*}{5} & \multirow{2}{*}{0.01} & 234.5 & 1242.2 & \multirow{2}{*}{5} & \multirow{2}{*}{0.008} \\
\hline ST 4 & 104.8 & 343.4 & & & 233.2 & 1421.6 & & & 463.9 & 2942.6 & & \\
\hline ST 1 & 43.9 & 79.9 & \multirow{2}{*}{5} & \multirow{2}{*}{0.01} & 102.9 & 328.6 & \multirow{2}{*}{5} & \multirow{2}{*}{0.02} & 234.5 & 1242.2 & \multirow{2}{*}{5} & \multirow{2}{*}{0.08} \\
\hline ST 5 & 69.9 & 35.5 & & & 156.5 & 73.6 & & & 294.5 & 387.4 & & \\
\hline ST 1 & 43.9 & 79.9 & \multirow{2}{*}{5} & \multirow{2}{*}{0.006} & 102.9 & 328.6 & \multirow{2}{*}{5} & \multirow{2}{*}{0.0005} & 234.5 & 1242.2 & \multirow{2}{*}{5} & \multirow{2}{*}{0.01} \\
\hline ST 6 & 79.9 & 74.8 & & & 176.9 & 183.9 & & & 359.2 & 377.9 & & \\
\hline
\end{tabular}

$*$ Bold font indicates the null hypothesis of equal means is rejected. 
Table 42: t-test of Phase Angle for STs fabricated from $12.5 \mathrm{~mm}$ NMAS at $40^{\circ} \mathrm{C}$

\begin{tabular}{|c|c|c|c|c|c|c|c|c|c|c|c|c|}
\hline \multirow[b]{2}{*}{ Comparison } & \multicolumn{4}{|c|}{$40^{\circ} \mathrm{C}$ and $0.1 \mathrm{~Hz}$} & \multicolumn{4}{|c|}{$40^{\circ} \mathrm{C}$ and $1 \mathrm{~Hz}$} & \multicolumn{4}{|c|}{$40^{\circ} \mathrm{C}$ and $10 \mathrm{~Hz}$} \\
\hline & $\stackrel{\Xi \Xi}{\Sigma}$ & 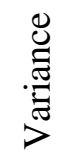 & $\frac{\pi}{7}$ & 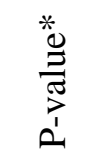 & $\stackrel{\Xi \Xi}{\Sigma}$ & $\begin{array}{l}\stackrel{\circlearrowright}{\mathscr{E}} \\
\stackrel{\Xi}{\Xi} \\
\stackrel{\Xi}{\Xi}\end{array}$ & $\tau$ & 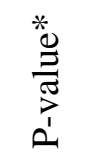 & 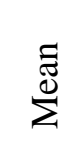 & $\begin{array}{l}\stackrel{\circlearrowright}{\Xi} \\
\stackrel{\Xi}{\Xi} \\
>\end{array}$ & $\frac{4}{7}$ & 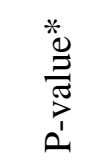 \\
\hline ST 1 & 30.4 & 0.2 & \multirow{2}{*}{5} & \multirow{2}{*}{0.07} & 33.9 & 0.5 & \multirow{2}{*}{5} & \multirow{2}{*}{0.08} & 34.2 & 1.4 & \multirow{2}{*}{5} & \multirow[t]{2}{*}{0.004} \\
\hline ST 2 & 32.1 & 0.9 & & & 31.9 & 1.5 & & & 27.1 & 2.7 & & \\
\hline ST 1 & 30.4 & 0.2 & \multirow{2}{*}{5} & \multirow{2}{*}{0.24} & 33.9 & 0.5 & \multirow{2}{*}{5} & \multirow{2}{*}{0.05} & 34.2 & 1.4 & \multirow{2}{*}{5} & \multirow[t]{2}{*}{$\overline{0.004}$} \\
\hline ST 3 & 32.1 & 2.8 & & & 32.6 & 0.2 & & & 29.3 & 0.7 & & \\
\hline ST 1 & 30.4 & 0.2 & \multirow{2}{*}{5} & \multirow{2}{*}{0.30} & 33.9 & 0.5 & \multirow{2}{*}{5} & \multirow{2}{*}{0.03} & 34.2 & 1.4 & \multirow{2}{*}{5} & \multirow[t]{2}{*}{0.02} \\
\hline ST 4 & 31.0 & 0.5 & & & 30.1 & 2.4 & & & 26.9 & 5.4 & & \\
\hline ST 1 & 30.4 & 0.2 & \multirow{2}{*}{5} & \multirow{2}{*}{0.001} & 33.9 & 0.5 & \multirow{2}{*}{5} & \multirow{2}{*}{0.08} & 34.2 & 1.4 & \multirow{2}{*}{5} & \multirow[t]{2}{*}{0.38} \\
\hline ST 5 & 32.9 & 0.2 & & & 32.9 & 0.09 & & & 33.1 & 2.6 & & \\
\hline ST 1 & 30.4 & 0.2 & \multirow{2}{*}{5} & \multirow{2}{*}{0.07} & 33.9 & 0.5 & \multirow{2}{*}{5} & \multirow{2}{*}{0.08} & 34.2 & 1.4 & \multirow{2}{*}{5} & \multirow[t]{2}{*}{0.004} \\
\hline ST 6 & 32.1 & 0.9 & & & 31.9 & 1.5 & & & 27.1 & 2.7 & & \\
\hline
\end{tabular}

* Bold font indicates the null hypothesis of equal means is rejected.

Table 43: t-test of Dynamic Modulus for STs fabricated from $19 \mathrm{~mm}$ NMAS at $4^{\circ} \mathrm{C}$

\begin{tabular}{|c|c|c|c|c|c|c|c|c|c|c|c|c|}
\hline \multirow[b]{2}{*}{ Comparison } & \multicolumn{4}{|c|}{$4^{\circ} \mathrm{C}$ and $0.1 \mathrm{~Hz}$} & \multicolumn{4}{|c|}{$4^{\circ} \mathrm{C}$ and $1 \mathrm{~Hz}$} & \multicolumn{4}{|c|}{$4^{\circ} \mathrm{C}$ and $10 \mathrm{~Hz}$} \\
\hline & $\stackrel{\Xi \Xi}{\Sigma}$ & $\begin{array}{l}\stackrel{\mathscr{J}}{\Xi} \\
\stackrel{\Xi}{\Xi} \\
\end{array}$ & 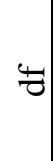 & 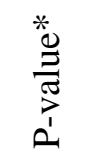 & 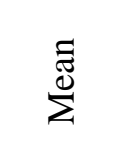 & 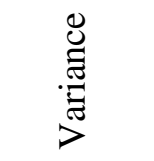 & $\frac{4}{7}$ & 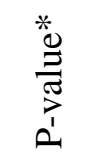 & $\sum^{\Xi}$ & 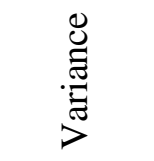 & $t$ & 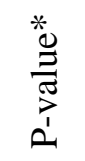 \\
\hline ST 1 & 1063.1 & 8764.5 & \multirow{2}{*}{5} & \multirow{2}{*}{0.11} & 1496.5 & 7796.8 & \multirow{2}{*}{5} & \multirow{2}{*}{0.10} & 1934.6 & 5338.4 & \multirow{2}{*}{5} & \multirow{2}{*}{0.09} \\
\hline ST 2 & 1215.4 & 8402.9 & & & 1666.1 & 11436.8 & & & 2128.0 & 12534.4 & & \\
\hline ST 1 & 1063.1 & 8764.5 & \multirow{2}{*}{5} & \multirow{2}{*}{0.29} & 1496.5 & 7796.8 & \multirow{2}{*}{5} & \multirow{2}{*}{0.25} & 1934.6 & 5338.4 & \multirow{2}{*}{5} & \multirow{2}{*}{0.32} \\
\hline ST 3 & 962.6 & 11308.7 & & & 1344.9 & 25724.9 & & & 1723.2 & 72417.0 & & \\
\hline ST 1 & 1063.1 & 8764.5 & \multirow{2}{*}{5} & \multirow{2}{*}{0.10} & 1496.5 & 7796.8 & \multirow{2}{*}{5} & \multirow{2}{*}{0.15} & 1934.6 & 5338.4 & \multirow{2}{*}{5} & \multirow{2}{*}{0.28} \\
\hline ST 4 & 1205.7 & 1902.2 & & & 1609.8 & 4582.5 & & & 2020.6 & 8913.4 & & \\
\hline ST 1 & 1063.1 & 8764.5 & \multirow{2}{*}{5} & \multirow{2}{*}{0.73} & 1496.5 & 7796.8 & \multirow{2}{*}{5} & \multirow{2}{*}{0.74} & 1934.6 & 5338.4 & \multirow{2}{*}{5} & \multirow{2}{*}{0.88} \\
\hline ST 5 & 1023.7 & 23884.8 & & & 1450.0 & 41944.7 & & & 1910.8 & 57106.5 & & \\
\hline ST 1 & 1063.1 & 8764.5 & \multirow{2}{*}{5} & \multirow{2}{*}{0.76} & 1496.5 & 7796.8 & \multirow{2}{*}{5} & \multirow{2}{*}{0.27} & 1934.6 & 5338.4 & \multirow{2}{*}{5} & \multirow{2}{*}{0.07} \\
\hline ST 6 & 1043.6 & 1671.1 & & & 1419.1 & 1996.7 & & & 1796.8 & 2060.6 & & \\
\hline
\end{tabular}

* Bold font indicates the null hypothesis of equal means is rejected. 
Table 44: t-test of Phase Angle for STs fabricated from $19 \mathrm{~mm}$ NMAS at $4^{\circ} \mathrm{C}$

\begin{tabular}{|c|c|c|c|c|c|c|c|c|c|c|c|c|}
\hline \multirow[b]{2}{*}{ Comparison } & \multicolumn{4}{|c|}{$4^{\circ} \mathrm{C}$ and $0.1 \mathrm{~Hz}$} & \multicolumn{4}{|c|}{$4^{\circ} \mathrm{C}$ and $1 \mathrm{~Hz}$} & \multicolumn{4}{|c|}{$4^{\circ} \mathrm{C}$ and $10 \mathrm{~Hz}$} \\
\hline & $\sum_{\Sigma}^{\Xi}$ & 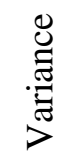 & 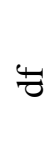 & 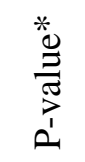 & $\stackrel{\Xi}{\Xi}$ & 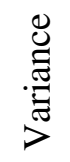 & 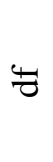 & 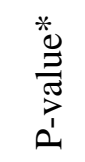 & $\stackrel{\Xi}{\Xi}$ & $\begin{array}{l}\stackrel{\Xi}{\Xi} \\
: \stackrel{\Xi}{\Xi} \\
>\end{array}$ & 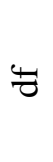 & 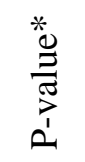 \\
\hline ST 1 & 16.8 & 2.7 & \multirow{2}{*}{5} & \multirow{2}{*}{0.23} & 12.3 & 1.6 & \multirow{2}{*}{5} & \multirow{2}{*}{0.36} & 9.3 & 0.6 & \multirow{2}{*}{5} & \multirow{2}{*}{0.21} \\
\hline ST 2 & 15.3 & 0.5 & & & 11.4 & 0.4 & & & 8.5 & 0.1 & & \\
\hline ST 1 & 16.8 & 2.7 & \multirow{2}{*}{5} & \multirow{2}{*}{0.25} & 12.3 & 1.6 & \multirow{2}{*}{5} & \multirow{2}{*}{0.21} & 9.3 & 0.6 & \multirow{2}{*}{5} & \multirow{2}{*}{0.24} \\
\hline ST 3 & 19.4 & 6.9 & & & 14.4 & 4.1 & & & 11.1 & 3.9 & & \\
\hline ST 1 & 16.8 & 2.7 & \multirow{2}{*}{5} & \multirow{2}{*}{0.10} & 12.3 & 1.6 & \multirow{2}{*}{5} & \multirow{2}{*}{0.15} & 9.3 & 0.6 & \multirow{2}{*}{5} & \multirow{2}{*}{0.16} \\
\hline ST 4 & 14.0 & 0.1 & & & 10.5 & 0.1 & & & 8.3 & 0.0 & & \\
\hline ST 1 & 16.8 & 2.7 & \multirow{2}{*}{5} & \multirow{2}{*}{0.53} & 12.3 & 1.6 & \multirow{2}{*}{5} & \multirow{2}{*}{0.30} & 9.3 & 0.6 & \multirow{2}{*}{5} & \multirow{2}{*}{0.39} \\
\hline ST 5 & 17.6 & 1.5 & & & 13.2 & 0.9 & & & 9.9 & 0.5 & & \\
\hline ST 1 & 16.8 & 2.7 & \multirow{2}{*}{5} & \multirow{2}{*}{0.24} & 12.3 & 1.6 & \multirow{2}{*}{5} & \multirow{2}{*}{0.38} & 9.3 & 0.6 & \multirow{2}{*}{5} & \multirow{2}{*}{0.24} \\
\hline ST 6 & 15.2 & 0.1 & & & 11.2 & 0.1 & & & 8.6 & 0.0 & & \\
\hline
\end{tabular}

* Bold font indicates the null hypothesis of equal means is rejected.

Table 45: t-test of Dynamic Modulus for STs fabricated from $19 \mathrm{~mm}$ NMAS at $20^{\circ} \mathrm{C}$

\begin{tabular}{|c|c|c|c|c|c|c|c|c|c|c|c|c|}
\hline \multirow[b]{2}{*}{ Comparison } & \multicolumn{4}{|c|}{$20^{\circ} \mathrm{C}$ and $0.1 \mathrm{~Hz}$} & \multicolumn{4}{|c|}{$20^{\circ} \mathrm{C}$ and $1 \mathrm{~Hz}$} & \multicolumn{4}{|c|}{$20^{\circ} \mathrm{C}$ and $10 \mathrm{~Hz}$} \\
\hline & 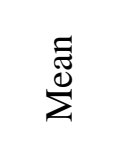 & 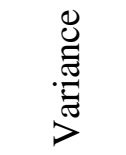 & 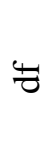 & 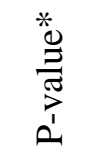 & 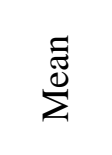 & $\begin{array}{l}\stackrel{\circlearrowright}{\Xi} \\
\stackrel{\Xi}{\Xi} \\
>\end{array}$ & 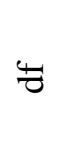 & 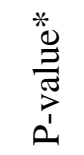 & 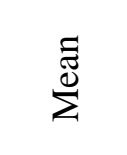 & 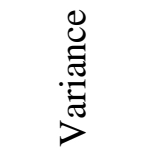 & 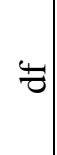 & 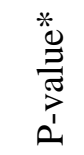 \\
\hline ST 1 & 350.5 & 907.0 & \multirow{2}{*}{5} & \multirow{2}{*}{0.37} & 640.8 & 844.2 & \multirow{2}{*}{5} & \multirow{2}{*}{0.55} & 1035.6 & 273.2 & \multirow{2}{*}{5} & \multirow{2}{*}{0.84} \\
\hline ST 2 & 330.0 & 218.6 & & & 627.5 & 411.1 & & & 1040.4 & 1227.6 & & \\
\hline ST 1 & 350.5 & 907.0 & \multirow{2}{*}{5} & \multirow{2}{*}{0.01} & 640.8 & 844.2 & \multirow{2}{*}{5} & \multirow{2}{*}{0.04} & 1035.6 & 273.2 & \multirow{2}{*}{5} & \multirow{2}{*}{0.13} \\
\hline ST 3 & 238.4 & 1225.5 & & & 465.8 & 6585.3 & & & 796.8 & 27934.6 & & \\
\hline ST 1 & 350.5 & 907.0 & \multirow{2}{*}{5} & \multirow{2}{*}{0.32} & 640.8 & 844.2 & \multirow{2}{*}{5} & \multirow{2}{*}{0.27} & 1035.6 & 273.2 & \multirow{2}{*}{5} & \multirow{2}{*}{0.43} \\
\hline ST 4 & 374.1 & 79.4 & & & 665.5 & 167.1 & & & 1052.0 & 727.9 & & \\
\hline ST 1 & 350.5 & 907.0 & \multirow{2}{*}{5} & \multirow{2}{*}{0.02} & 640.8 & 844.2 & \multirow{2}{*}{5} & \multirow{2}{*}{0.03} & 1035.6 & 273.2 & \multirow{2}{*}{5} & \multirow{2}{*}{0.10} \\
\hline ST 5 & 237.7 & 1701.5 & & & 466.0 & 5350.6 & & & 833.2 & 13318.6 & & \\
\hline ST 1 & 350.5 & 907.0 & \multirow{2}{*}{5} & \multirow{2}{*}{0.52} & 640.8 & 844.2 & \multirow{2}{*}{5} & \multirow{2}{*}{0.35} & 1035.6 & 273.2 & \multirow{2}{*}{5} & \multirow{2}{*}{0.16} \\
\hline ST 6 & 337.0 & 123.2 & & & 619.0 & 438.4 & & & 996.2 & 1084.5 & & \\
\hline
\end{tabular}

* Bold font indicates the null hypothesis of equal means is rejected. 
Table 46: t-test of Phase Angle for STs fabricated from $19 \mathrm{~mm}$ NMAS at $20^{\circ} \mathrm{C}$

\begin{tabular}{|c|c|c|c|c|c|c|c|c|c|c|c|c|}
\hline \multirow[b]{2}{*}{ Comparison } & \multicolumn{4}{|c|}{$20^{\circ} \mathrm{C}$ and $0.1 \mathrm{~Hz}$} & \multicolumn{4}{|c|}{$20^{\circ} \mathrm{C}$ and $1 \mathrm{~Hz}$} & \multicolumn{4}{|c|}{$20^{\circ} \mathrm{C}$ and $10 \mathrm{~Hz}$} \\
\hline & $\stackrel{\Xi}{\Xi}$ & 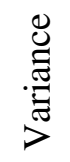 & $\Psi$ & 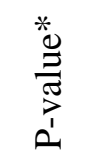 & $\sum_{\Sigma}^{\Xi}$ & 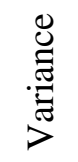 & 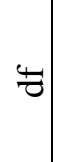 & 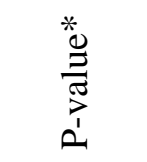 & $\sum^{\Xi}$ & 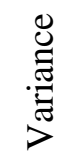 & $t$ & 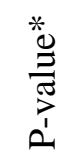 \\
\hline ST 1 & 28.6 & 1.9 & \multirow{2}{*}{5} & \multirow{2}{*}{0.13} & 18.3 & 0.5 & \multirow{2}{*}{5} & \multirow[t]{2}{*}{ 1.1E-03 } & 18.3 & 0.5 & \multirow{2}{*}{5} & \multirow[t]{2}{*}{0.92} \\
\hline ST 2 & 30.6 & 0.1 & & & 24.5 & 0.2 & & & 18.4 & 0.9 & & \\
\hline ST 1 & 28.6 & 1.9 & \multirow{2}{*}{5} & \multirow{2}{*}{0.01} & 18.3 & 0.5 & \multirow{2}{*}{5} & \multirow[t]{2}{*}{ 1.9E-04 } & 18.3 & 0.5 & \multirow{2}{*}{5} & \multirow[t]{2}{*}{0.03} \\
\hline ST 3 & 33.3 & 0.6 & & & 27.2 & 0.9 & & & 21.3 & 1.3 & & \\
\hline ST 1 & 28.6 & 1.9 & \multirow{2}{*}{5} & \multirow{2}{*}{0.24} & 18.3 & 0.5 & \multirow{2}{*}{5} & \multirow[t]{2}{*}{$5.3 \mathrm{E}-03$} & 18.3 & 0.5 & \multirow{2}{*}{5} & \multirow[t]{2}{*}{0.06} \\
\hline ST 4 & 27.3 & 1.1 & & & 22.0 & 0.8 & & & 17.0 & 0.3 & & \\
\hline ST 1 & 28.6 & 1.9 & \multirow{2}{*}{5} & \multirow{2}{*}{0.01} & 18.3 & 0.5 & \multirow{2}{*}{5} & \multirow[t]{2}{*}{ 9.3E-05 } & 18.3 & 0.5 & \multirow{2}{*}{5} & \multirow[t]{2}{*}{$\overline{0.01}$} \\
\hline ST 5 & 32.7 & 1.0 & & & 28.0 & 0.6 & & & 21.8 & 0.7 & & \\
\hline ST 1 & 28.6 & 1.9 & \multirow{2}{*}{5} & \multirow{2}{*}{0.54} & 18.3 & 0.5 & \multirow{2}{*}{5} & \multirow[t]{2}{*}{ 1.8E-03 } & 18.3 & 0.5 & \multirow{2}{*}{5} & \multirow[t]{2}{*}{0.25} \\
\hline ST 6 & 29.2 & 0.2 & & & 23.5 & 0.2 & & & 17.7 & 0.1 & & \\
\hline
\end{tabular}

* Bold font indicates the null hypothesis of equal means is rejected.

Table 47: t-test of Dynamic Modulus for STs fabricated from $19 \mathrm{~mm}$ NMAS at $35^{\circ} \mathrm{C}$

\begin{tabular}{|c|c|c|c|c|c|c|c|c|c|c|c|c|}
\hline \multirow[b]{2}{*}{ Comparison } & \multicolumn{4}{|c|}{$35^{\circ} \mathrm{C}$ and $0.1 \mathrm{~Hz}$} & \multicolumn{4}{|c|}{$35^{\circ} \mathrm{C}$ and $1 \mathrm{~Hz}$} & \multicolumn{4}{|c|}{$35^{\circ} \mathrm{C}$ and $10 \mathrm{~Hz}$} \\
\hline & 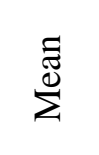 & 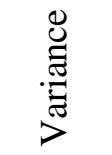 & \pm & 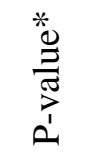 & 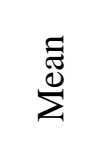 & 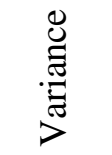 & $t$ & 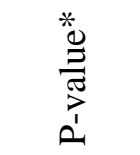 & 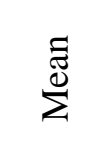 & 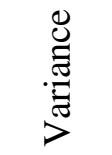 & 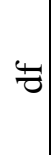 & 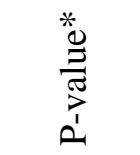 \\
\hline ST 1 & 42.1 & 101.8 & \multirow{2}{*}{5} & \multirow{2}{*}{0.01} & 92.5 & 255.6 & \multirow{2}{*}{5} & \multirow[t]{2}{*}{$2.2 \mathrm{E}-03$} & 208.1 & 629.8 & \multirow{2}{*}{5} & \multirow[t]{2}{*}{ 1.2E-03 } \\
\hline ST 2 & 79.9 & 74.8 & & & 176.9 & 184.0 & & & 359.2 & 377.8 & & \\
\hline ST 1 & 42.1 & 101.8 & \multirow{2}{*}{5} & \multirow{2}{*}{0.08} & 92.5 & 255.6 & \multirow{2}{*}{5} & \multirow[t]{2}{*}{ 1.7E-02 } & 208.1 & 629.8 & \multirow{2}{*}{5} & \multirow[t]{2}{*}{ 6.0E-03 } \\
\hline ST 3 & 59.5 & 58.0 & & & 143.7 & 244.0 & & & 324.0 & 793.0 & & \\
\hline $\begin{array}{l}\text { ST } 1 \\
\end{array}$ & 42.1 & 101.8 & \multirow{2}{*}{5} & \multirow{2}{*}{0.01} & 92.5 & 255.6 & \multirow{2}{*}{5} & \multirow[t]{2}{*}{ 1.1E-03 } & 208.1 & 629.8 & \multirow{2}{*}{5} & \multirow[t]{2}{*}{ 6.0E-04 } \\
\hline ST 4 & 84.3 & 22.5 & & & 186.9 & 120.2 & & & 386.8 & 361.0 & & \\
\hline ST 1 & 42.1 & 101.8 & \multirow{2}{*}{5} & \multirow{2}{*}{0.26} & 92.5 & 255.6 & \multirow{2}{*}{5} & \multirow{2}{*}{0.05} & 208.1 & 629.8 & \multirow{2}{*}{5} & \multirow{2}{*}{0.13} \\
\hline ST 5 & 51.9 & 70.7 & & & 125.5 & 140.7 & & & 244.0 & 264.8 & & \\
\hline ST 1 & 42.1 & 101.8 & \multirow{2}{*}{5} & \multirow{2}{*}{0.02} & 92.5 & 255.6 & \multirow{2}{*}{5} & \multirow[t]{2}{*}{ 3.6E-03 } & 208.1 & 629.8 & \multirow{2}{*}{5} & \multirow[t]{2}{*}{ 2.8E-03 } \\
\hline ST 6 & 71.9 & 27.8 & & & 162.2 & 133.8 & & & 339.9 & 586.7 & & \\
\hline
\end{tabular}

* Bold font indicates the null hypothesis of equal means is rejected. 
Table 48: t-test of Phase Angle for STs fabricated from $19 \mathrm{~mm}$ NMAS at $35^{\circ} \mathrm{C}$

\begin{tabular}{|c|c|c|c|c|c|c|c|c|c|c|c|c|}
\hline \multirow[b]{2}{*}{ Comparison } & \multicolumn{4}{|c|}{$35^{\circ} \mathrm{C}$ and $0.1 \mathrm{~Hz}$} & \multicolumn{4}{|c|}{$35^{\circ} \mathrm{C}$ and $1 \mathrm{~Hz}$} & \multicolumn{4}{|c|}{$35^{\circ} \mathrm{C}$ and $10 \mathrm{~Hz}$} \\
\hline & $\stackrel{\Xi}{\Xi}$ & $\begin{array}{l}\stackrel{\otimes}{\Xi} \\
\stackrel{\Xi}{\Xi} \\
>\end{array}$ & 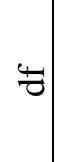 & 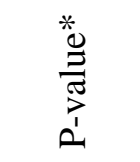 & $\underset{\Xi}{\stackrel{\Xi}{\Xi}}$ & $\begin{array}{l}\stackrel{\Xi}{\Xi} \\
\stackrel{\Xi}{\Xi} \\
>\end{array}$ & 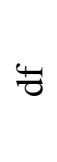 & 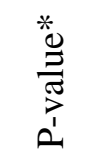 & $\sum_{\tilde{\Xi}}^{\tilde{\Xi}}$ & 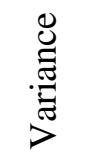 & 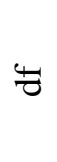 & 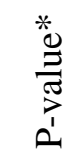 \\
\hline ST 1 & 28.0 & 0.4 & \multirow{2}{*}{5} & \multirow[t]{2}{*}{$8.2 \mathrm{E}-03$} & 32.2 & 0.4 & \multirow{2}{*}{5} & \multirow{2}{*}{0.76} & 34.4 & 2.7 & \multirow{2}{*}{5} & \multirow{2}{*}{0.01} \\
\hline ST 2 & 32.1 & 0.9 & & & 31.9 & 1.5 & & & 27.1 & 2.7 & & \\
\hline ST 1 & 28.0 & 0.4 & \multirow{2}{*}{5} & \multirow[t]{2}{*}{$2.2 \mathrm{E}-04$} & 32.2 & 0.4 & \multirow{2}{*}{5} & \multirow{2}{*}{0.01} & 34.4 & 2.7 & \multirow{2}{*}{5} & \multirow{2}{*}{0.25} \\
\hline ST 3 & 35.0 & 0.5 & & & 36.4 & 0.0 & & & 33.0 & 0.4 & & \\
\hline ST 1 & 28.0 & 0.4 & \multirow{2}{*}{5} & \multirow[t]{2}{*}{0.06} & 32.2 & 0.4 & \multirow{2}{*}{5} & \multirow{2}{*}{0.50} & 34.4 & 2.7 & \multirow{2}{*}{5} & \multirow{2}{*}{0.01} \\
\hline ST 4 & 30.9 & 2.7 & & & 31.4 & 2.3 & & & 29.4 & 1.6 & & \\
\hline ST 1 & 28.0 & 0.4 & \multirow{2}{*}{5} & \multirow{2}{*}{0.04} & 32.2 & 0.4 & \multirow{2}{*}{5} & \multirow{2}{*}{0.02} & 34.4 & 2.7 & \multirow{2}{*}{5} & \multirow{2}{*}{0.18} \\
\hline ST 5 & 33.3 & 3.3 & & & 36.8 & 2.3 & & & 39.1 & 19.0 & & \\
\hline ST 1 & 28.0 & 0.4 & \multirow{2}{*}{5} & \multirow[t]{2}{*}{$4.9 \mathrm{E}-04$} & 32.2 & 0.4 & \multirow{2}{*}{5} & \multirow{2}{*}{0.10} & 34.4 & 2.7 & \multirow{2}{*}{5} & \multirow{2}{*}{0.06} \\
\hline ST 6 & 32.5 & 0.2 & & & 33.7 & 0.9 & & & 31.2 & 0.9 & & \\
\hline
\end{tabular}

* Bold font indicates the null hypothesis of equal means is rejected.

Table 49: t-test of Dynamic Modulus for STs fabricated from $25 \mathrm{~mm}$ NMAS at $4^{\circ} \mathrm{C}$

\begin{tabular}{|c|c|c|c|c|c|c|c|c|c|c|c|c|}
\hline \multirow[b]{2}{*}{ Comparison } & \multicolumn{4}{|c|}{$4^{\circ} \mathrm{C}$ and $0.1 \mathrm{~Hz}$} & \multicolumn{4}{|c|}{$4^{\circ} \mathrm{C}$ and $1 \mathrm{~Hz}$} & \multicolumn{4}{|c|}{$4^{\circ} \mathrm{C}$ and $10 \mathrm{~Hz}$} \\
\hline & 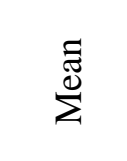 & 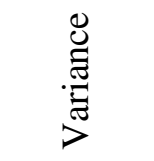 & 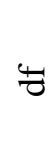 & 葛 & 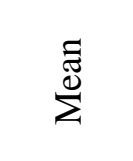 & 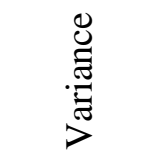 & 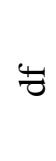 & 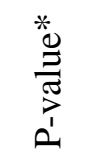 & $\frac{\Xi}{\Xi^{\oplus}}$ & 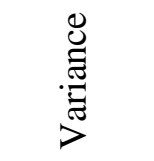 & $\frac{4}{4}$ & 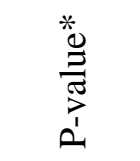 \\
\hline ST 1 & 1448.4 & 926.8 & \multirow{2}{*}{5} & \multirow{2}{*}{0.38} & 1941.3 & 878.7 & \multirow{2}{*}{5} & \multirow{2}{*}{0.23} & 2419.6 & 610.7 & \multirow{2}{*}{5} & \multirow{2}{*}{0.15} \\
\hline ST 2 & 1400.2 & 5761.9 & & & 1837.5 & 10216.5 & & & 2276.3 & 11647.7 & & \\
\hline ST 1 & 1448.4 & 926.8 & \multirow{2}{*}{5} & \multirow{2}{*}{0.02} & 1941.3 & 878.7 & \multirow{2}{*}{5} & \multirow{2}{*}{0.01} & 2419.6 & 610.7 & \multirow{2}{*}{5} & \multirow[t]{2}{*}{ 9.1E-03 } \\
\hline ST 3 & 1345.4 & 356.9 & & & 1826.5 & 1147.9 & & & 2303.4 & 1195.9 & & \\
\hline ST 1 & 1448.4 & 926.8 & \multirow{2}{*}{5} & \multirow{2}{*}{0.05} & 1941.3 & 878.7 & \multirow{2}{*}{5} & \multirow{2}{*}{0.01} & 2419.6 & 610.7 & \multirow{2}{*}{5} & \multirow[t]{2}{*}{$3.3 \mathrm{E}-03$} \\
\hline ST 4 & 1376.7 & 965.1 & & & 1835.3 & 1133.2 & & & 2277.3 & 944.4 & & \\
\hline ST 1 & 1448.4 & 926.8 & \multirow{2}{*}{5} & \multirow{2}{*}{0.85} & 1941.3 & 878.7 & \multirow{2}{*}{5} & \multirow{2}{*}{0.81} & 2419.6 & 610.7 & \multirow{2}{*}{5} & \multirow{2}{*}{0.70} \\
\hline ST 5 & 1462.1 & 11211.9 & & & 1965.8 & 22304.7 & & & 2468.5 & 36082.9 & & \\
\hline ST 1 & 1448.4 & 926.8 & \multirow{2}{*}{5} & \multirow{2}{*}{0.41} & 1941.3 & 878.7 & \multirow{2}{*}{5} & \multirow{2}{*}{0.31} & 2419.6 & 610.7 & \multirow{2}{*}{5} & \multirow{2}{*}{0.23} \\
\hline ST 6 & 1331.0 & 37680.2 & & & 1762.0 & 51439.6 & & & 2180.5 & 59967.7 & & \\
\hline
\end{tabular}

* Bold font indicates the null hypothesis of equal means is rejected. 
Table 50: t-test of Phase Angle for STs fabricated from $25 \mathrm{~mm}$ NMAS at $4^{\circ} \mathrm{C}$

\begin{tabular}{|c|c|c|c|c|c|c|c|c|c|c|c|c|}
\hline \multirow[b]{2}{*}{ Comparison } & \multicolumn{4}{|c|}{$4^{\circ} \mathrm{C}$ and $0.1 \mathrm{~Hz}$} & \multicolumn{4}{|c|}{$4^{\circ} \mathrm{C}$ and $1 \mathrm{~Hz}$} & \multicolumn{4}{|c|}{$4^{\circ} \mathrm{C}$ and $10 \mathrm{~Hz}$} \\
\hline & $\sum_{\Sigma}^{\Xi}$ & $\begin{array}{c}\mathscr{U} \\
\stackrel{\Xi}{\Xi} \\
\stackrel{\Xi}{\Xi} \\
>\end{array}$ & 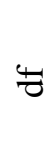 & 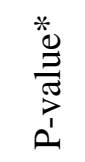 & 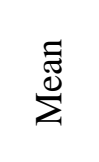 & 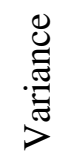 & $t$ & 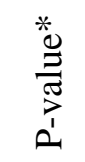 & $\sum_{\Sigma}^{\Xi \Xi}$ & 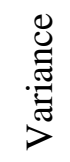 & 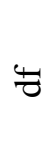 & 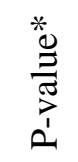 \\
\hline ST 1 & 14.5 & 12.8 & \multirow{2}{*}{5} & \multirow{2}{*}{.28} & 10.5 & 9.8 & \multirow{2}{*}{5} & \multirow{2}{*}{0.44} & 7.8 & 0.3 & \multirow{2}{*}{5} & \multirow{2}{*}{0.09} \\
\hline ST 2 & 0.5 & 3.0 & & & 0.3 & 1.7 & & & 6.9 & 0.1 & & \\
\hline ST 1 & 14.5 & 12.8 & \multirow{2}{*}{5} & \multirow{2}{*}{0.58} & 10.5 & 9.8 & \multirow{2}{*}{5} & \multirow{2}{*}{0.50} & 7.8 & 0.3 & \multirow{2}{*}{5} & \multirow{2}{*}{0.60} \\
\hline ST 3 & 14.8 & 0.2 & & & 10.8 & 0.1 & & & 8.1 & 0.3 & & \\
\hline ST 1 & 14.5 & 12.8 & \multirow{2}{*}{5} & \multirow{2}{*}{0.65} & 10.5 & 9.8 & \multirow{2}{*}{5} & \multirow{2}{*}{0.75} & 7.8 & 0.3 & \multirow{2}{*}{5} & \multirow{2}{*}{0.98} \\
\hline ST 4 & 14.3 & 0.0 & & & 10.4 & 0.0 & & & 7.9 & 0.0 & & \\
\hline ST 1 & 14.5 & 12.8 & \multirow{2}{*}{5} & \multirow{2}{*}{0.59} & 10.5 & 9.8 & \multirow{2}{*}{5} & \multirow{2}{*}{0.46} & 7.8 & 0.3 & \multirow{2}{*}{5} & \multirow{2}{*}{0.30} \\
\hline ST 5 & 14.8 & 0.1 & & & 10.8 & 0.0 & & & 8.3 & 0.0 & & \\
\hline ST 1 & 14.5 & 12.8 & \multirow{2}{*}{5} & \multirow{2}{*}{0.98} & 10.5 & 9.8 & \multirow{2}{*}{5} & \multirow{2}{*}{0.81} & 7.8 & 0.3 & \multirow{2}{*}{5} & \multirow{2}{*}{1.00} \\
\hline ST 6 & 14.5 & 0.8 & & & 10.4 & 0.5 & & & 7.8 & 0.2 & & \\
\hline
\end{tabular}

Table 51: t-test of Dynamic Modulus for STs fabricated from $25 \mathrm{~mm}$ NMAS at $20^{\circ} \mathrm{C}$

\begin{tabular}{|c|c|c|c|c|c|c|c|c|c|c|c|c|}
\hline \multirow[b]{2}{*}{ Comparison } & \multicolumn{4}{|c|}{$20^{\circ} \mathrm{C}$ and $0.1 \mathrm{~Hz}$} & \multicolumn{4}{|c|}{$20^{\circ} \mathrm{C}$ and $1 \mathrm{~Hz}$} & \multicolumn{4}{|c|}{$20^{\circ} \mathrm{C}$ and $10 \mathrm{~Hz}$} \\
\hline & 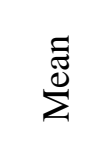 & 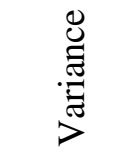 & 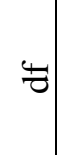 & 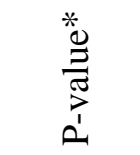 & $\underset{\Xi}{\stackrel{\Xi}{\Sigma}}$ & 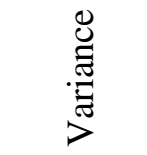 & 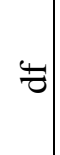 & 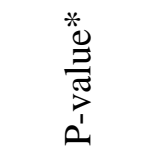 & $\sum_{\Sigma}^{\Xi}$ & $\begin{array}{l}\ddot{U} \\
\stackrel{\Xi}{\Xi} \\
\stackrel{\Xi}{\Xi}\end{array}$ & 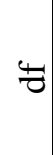 & 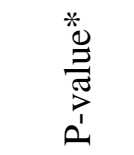 \\
\hline ST 1 & 504.9 & 570.6 & \multirow{2}{*}{5} & \multirow{2}{*}{$1.4 \mathrm{E}-02$} & 900.0 & 559.2 & \multirow{2}{*}{5} & \multirow[t]{2}{*}{$1.8 \mathrm{E}-03$} & 1392.2 & 488.9 & \multirow{2}{*}{5} & \multirow[t]{2}{*}{$8.6 \mathrm{E}-04$} \\
\hline ST 2 & 420.5 & 206.9 & & & 763.2 & 474.3 & & & 1211.3 & 735.6 & & \\
\hline ST 1 & 504.9 & 570.6 & \multirow{2}{*}{5} & \multirow[t]{2}{*}{$2.4 \mathrm{E}-03$} & 900.0 & 559.2 & \multirow{2}{*}{5} & \multirow[t]{2}{*}{$3.8 \mathrm{E}-04$} & 1392.2 & 488.9 & \multirow{2}{*}{5} & \multirow[t]{2}{*}{$2.8 \mathrm{E}-04$} \\
\hline ST 3 & 348.7 & 231.4 & & & 672.4 & 718.4 & & & 1127.5 & 983.8 & & \\
\hline ST 1 & 504.9 & 570.6 & \multirow{2}{*}{5} & \multirow[t]{2}{*}{$1.2 \mathrm{E}-02$} & 900.0 & 559.2 & \multirow{2}{*}{5} & \multirow[t]{2}{*}{$5.0 \mathrm{E}-03$} & 1392.2 & 488.9 & \multirow{2}{*}{5} & \multirow[t]{2}{*}{$2.6 \mathrm{E}-03$} \\
\hline ST 4 & 376.6 & 13.0 & & & 705.9 & 9.1 & & & 1139.7 & 10.1 & & \\
\hline ST 1 & 504.9 & 570.6 & \multirow{2}{*}{5} & \multirow[t]{2}{*}{ 4.4E-03 } & 900.0 & 559.2 & \multirow{2}{*}{5} & \multirow[t]{2}{*}{ 1.4E-02 } & 1392.2 & 488.9 & \multirow{2}{*}{5} & \multirow{2}{*}{0.08} \\
\hline ST 5 & 379.2 & 846.3 & & & 717.2 & 3238.0 & & & 1201.9 & 9008.1 & & \\
\hline ST 1 & 504.9 & 570.6 & \multirow{2}{*}{5} & \multirow{2}{*}{0.15} & 900.0 & 559.2 & \multirow{2}{*}{5} & \multirow{2}{*}{0.22} & 1392.2 & 488.9 & \multirow{2}{*}{5} & \multirow{2}{*}{0.21} \\
\hline ST 6 & 432.3 & 3637.8 & & & 782.4 & 12580.4 & & & 1209.7 & 30124.1 & & \\
\hline
\end{tabular}

* Bold font indicates the null hypothesis of equal means is rejected. 
Table 52: t-test of Phase Angle for STs fabricated from $25 \mathrm{~mm}$ NMAS at $20^{\circ} \mathrm{C}$

\begin{tabular}{|c|c|c|c|c|c|c|c|c|c|c|c|c|}
\hline \multirow[b]{2}{*}{ Comparison } & \multicolumn{4}{|c|}{$20^{\circ} \mathrm{C}$ and $0.1 \mathrm{~Hz}$} & \multicolumn{4}{|c|}{$20^{\circ} \mathrm{C}$ and $1 \mathrm{~Hz}$} & \multicolumn{4}{|c|}{$20^{\circ} \mathrm{C}$ and $10 \mathrm{~Hz}$} \\
\hline & 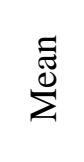 & 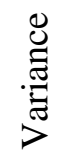 & 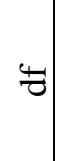 & 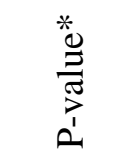 & $\stackrel{\Xi}{\Xi}$ & 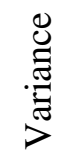 & $\tau$ & 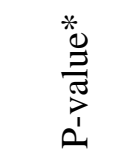 & 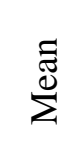 & $\begin{array}{c}\stackrel{\mathscr{\Xi}}{\Xi} \\
\stackrel{\Xi}{\Xi} \\
\end{array}$ & 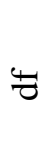 & 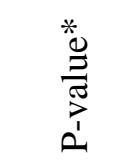 \\
\hline ST 1 & 27.8 & 0.3 & \multirow{2}{*}{5} & \multirow{2}{*}{0.04} & 21.5 & 0.2 & \multirow{2}{*}{5} & \multirow{2}{*}{0.06} & 16.0 & 16.5 & \multirow{2}{*}{5} & \multirow[t]{2}{*}{0.24} \\
\hline ST 2 & 29.0 & 0.3 & & & 22.5 & 0.2 & & & 0.1 & 0.3 & & \\
\hline ST 1 & 27.8 & 0.3 & \multirow{2}{*}{5} & \multirow[t]{2}{*}{ 8.5E-03 } & 21.5 & 0.2 & \multirow{2}{*}{5} & \multirow[t]{2}{*}{ 7.4E-03 } & 16.0 & 16.5 & \multirow{2}{*}{5} & \multirow[t]{2}{*}{ 8.4E-03 } \\
\hline ST 3 & 32.2 & 1.2 & & & 25.2 & 0.8 & & & 18.8 & 0.5 & & \\
\hline ST 1 & 27.8 & 0.3 & \multirow{2}{*}{5} & \multirow[t]{2}{*}{ 4.7E-03 } & 21.5 & 0.2 & \multirow{2}{*}{5} & \multirow[t]{2}{*}{$4.1 E-03$} & 16.0 & 16.5 & \multirow{2}{*}{5} & \multirow[t]{2}{*}{ 1.9E-03 } \\
\hline ST 4 & 30.4 & 0.3 & & & 24.0 & 0.3 & & & 17.9 & 0.1 & & \\
\hline ST 1 & 27.8 & 0.3 & \multirow{2}{*}{5} & \multirow[t]{2}{*}{$3.4 \mathrm{E}-03$} & 21.5 & 0.2 & \multirow{2}{*}{5} & \multirow[t]{2}{*}{ 4.1E-04 } & 16.0 & 16.5 & \multirow{2}{*}{5} & \multirow[t]{2}{*}{ 5.8E-03 } \\
\hline ST 5 & 31.2 & 0.6 & & & 25.1 & 0.1 & & & 18.8 & 0.0 & & \\
\hline ST 1 & 27.8 & 0.3 & \multirow{2}{*}{5} & \multirow{2}{*}{0.05} & 21.5 & 0.2 & \multirow{2}{*}{5} & \multirow{2}{*}{0.18} & 16.0 & 16.5 & \multirow{2}{*}{5} & \multirow[t]{2}{*}{0.39} \\
\hline ST 6 & 29.2 & 0.4 & & & 22.6 & 0.9 & & & 16.5 & 0.6 & & \\
\hline
\end{tabular}

* Bold font indicates the null hypothesis of equal means is rejected.

Table 53: t-test of Dynamic Modulus for STs fabricated from $25 \mathrm{~mm}$ NMAS at $35^{\circ} \mathrm{C}$

\begin{tabular}{|c|c|c|c|c|c|c|c|c|c|c|c|c|}
\hline \multirow[b]{2}{*}{ Comparison } & \multicolumn{4}{|c|}{$35^{\circ} \mathrm{C}$ and $0.1 \mathrm{~Hz}$} & \multicolumn{4}{|c|}{$35^{\circ} \mathrm{C}$ and $1 \mathrm{~Hz}$} & \multicolumn{4}{|c|}{$35^{\circ} \mathrm{C}$ and $10 \mathrm{~Hz}$} \\
\hline & $\sum_{\Sigma}^{\Xi}$ & 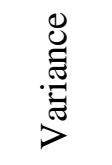 & $\Psi$ & 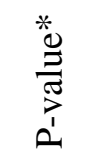 & 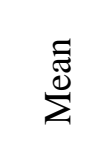 & 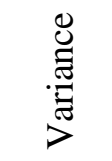 & 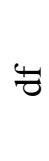 & 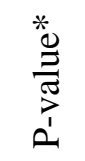 & $\underset{\Xi}{\stackrel{\Xi}{\Xi}}$ & 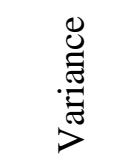 & 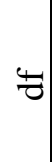 & 葛 \\
\hline ST 1 & 42.4 & 159.7 & \multirow{2}{*}{5} & \multirow{2}{*}{0.03} & 103.5 & 785.3 & \multirow{2}{*}{5} & \multirow{2}{*}{0.02} & 248.6 & 3008.1 & \multirow{2}{*}{5} & \multirow{2}{*}{0.03} \\
\hline ST 2 & 75.5 & 140.2 & & & 179.0 & 356.3 & & & 396.7 & 1056.5 & & \\
\hline ST 1 & 42.4 & 159.7 & \multirow{2}{*}{5} & \multirow{2}{*}{0.07} & 103.5 & 785.3 & \multirow{2}{*}{5} & \multirow{2}{*}{0.05} & 248.6 & 3008.1 & \multirow{2}{*}{5} & \multirow{2}{*}{0.04} \\
\hline ST 3 & 67.7 & 0.8 & & & 175.2 & 14.1 & & & 405.6 & 78.5 & & \\
\hline ST 1 & 42.4 & 159.7 & \multirow{2}{*}{5} & \multirow{2}{*}{0.06} & 103.5 & 785.3 & \multirow{2}{*}{5} & \multirow{2}{*}{0.04} & 248.6 & 3008.1 & \multirow{2}{*}{5} & \multirow{2}{*}{0.03} \\
\hline ST 4 & 71.7 & 0.1 & & & 185.6 & 0.0 & & & 423.5 & 0.0 & & \\
\hline ST 1 & 42.4 & 159.7 & \multirow{2}{*}{5} & \multirow{2}{*}{0.03} & 103.5 & 785.3 & \multirow{2}{*}{5} & \multirow{2}{*}{0.01} & 248.6 & 3008.1 & \multirow{2}{*}{5} & \multirow{2}{*}{0.01} \\
\hline ST 5 & 73.4 & 80.4 & & & 197.1 & 206.3 & & & 452.9 & 883.6 & & \\
\hline ST 1 & 42.4 & 159.7 & \multirow{2}{*}{5} & \multirow{2}{*}{0.01} & 103.5 & 785.3 & \multirow{2}{*}{5} & \multirow{2}{*}{0.01} & 248.6 & 3008.1 & \multirow{2}{*}{5} & \multirow{2}{*}{0.01} \\
\hline ST 6 & 87.8 & 24.0 & & & 217.1 & 326.6 & & & 464.8 & 2372.6 & & \\
\hline
\end{tabular}

* Bold font indicates the null hypothesis of equal means is rejected. 
Table 54: t-test of Phase Angle for STs fabricated from $25 \mathrm{~mm}$ NMAS at $35^{\circ} \mathrm{C}$

\begin{tabular}{|c|c|c|c|c|c|c|c|c|c|c|c|c|}
\hline \multirow[b]{2}{*}{ Comparison } & \multicolumn{4}{|c|}{$35^{\circ} \mathrm{C}$ and $0.1 \mathrm{~Hz}$} & \multicolumn{4}{|c|}{$35^{\circ} \mathrm{C}$ and $1 \mathrm{~Hz}$} & \multicolumn{4}{|c|}{$35^{\circ} \mathrm{C}$ and $10 \mathrm{~Hz}$} \\
\hline & $\stackrel{\Xi}{\Xi}$ & $\begin{array}{l}\stackrel{\mathscr{J}}{\Xi} \\
\stackrel{\Xi}{\Xi} \\
>\end{array}$ & 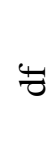 & 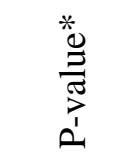 & $\stackrel{\Xi}{\Xi}$ & 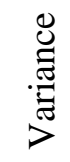 & $\frac{5}{7}$ & 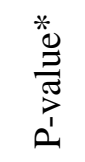 & $\sum_{\Sigma}^{\Xi}$ & 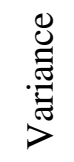 & $\Psi$ & 葛 \\
\hline ST 1 & 28.7 & 0.3 & \multirow{2}{*}{5} & \multirow[t]{2}{*}{ 3.0E-03 } & 33.7 & 0.5 & \multirow{2}{*}{5} & \multirow{2}{*}{0.58} & 36.1 & 2.3 & \multirow{2}{*}{5} & \multirow{2}{*}{0.02} \\
\hline ST 2 & 32.0 & 0.5 & & & 34.0 & 0.4 & & & 31.5 & 0.5 & & \\
\hline ST 1 & 28.7 & 0.3 & \multirow{2}{*}{5} & \multirow[t]{2}{*}{ 2.3E-02 } & 33.7 & 0.5 & \multirow{2}{*}{5} & \multirow{2}{*}{0.01} & 36.1 & 2.3 & \multirow{2}{*}{5} & \multirow{2}{*}{0.06} \\
\hline ST 3 & 36.0 & 3.5 & & & 37.5 & 1.1 & & & 32.6 & 0.0 & & \\
\hline ST 1 & 28.7 & 0.3 & \multirow{2}{*}{5} & \multirow[t]{2}{*}{$1.8 \mathrm{E}-03$} & 33.7 & 0.5 & \multirow{2}{*}{5} & \multirow{2}{*}{0.51} & 36.1 & 2.3 & \multirow{2}{*}{5} & \multirow{2}{*}{0.01} \\
\hline ST 4 & 32.1 & 0.3 & & & 33.2 & 0.9 & & & 30.7 & 0.5 & & \\
\hline ST 1 & 28.7 & 0.3 & \multirow{2}{*}{5} & \multirow{2}{*}{ 3.3E-03 } & 33.7 & 0.5 & \multirow{2}{*}{5} & \multirow{2}{*}{0.02} & 36.1 & 2.3 & \multirow{2}{*}{5} & \multirow{2}{*}{0.04} \\
\hline ST 5 & 35.1 & 1.4 & & & 36.2 & 0.9 & & & 32.9 & 1.4 & & \\
\hline ST 1 & 28.7 & 0.3 & \multirow{2}{*}{5} & \multirow[t]{2}{*}{ 3.9E-03 } & 33.7 & 0.5 & \multirow{2}{*}{5} & \multirow{2}{*}{0.33} & 36.1 & 2.3 & \multirow{2}{*}{5} & \multirow{2}{*}{0.01} \\
\hline ST 6 & 34.3 & 1.1 & & & 34.5 & 1.2 & & & 30.6 & 1.4 & & \\
\hline
\end{tabular}

* Bold font indicates the null hypothesis of equal means is rejected.

The t-test results of dynamic modulus and phase values for same combinations of temperature and frequency does not follow the same pattern. Meaning, if dynamic modulus is statistically significant, phase angle may/may not be statistically significant. The results from t- test analysis are:

- For 12.5 mm NMAS mix at $4^{\circ} \mathrm{C}$ - different frequencies, combination of ST1 with all STs are statistically same for dynamic modulus. For phase angle, ST1-ST5 are statistically same at two of three frequencies. At $20^{\circ} \mathrm{C}$ - different frequencies, ST1-ST3 and ST1-ST5 are statistically same for both dynamic modulus and phase angle. At $40^{\circ} \mathrm{C}$ - different frequencies, none was statistically same to ST1 for dynamic modulus values however two of three frequencies for ST1-ST 5 and ST1-ST 6 were statistically same for phase angle.

- For $19 \mathrm{~mm}$ NMAS mix at $4^{\circ} \mathrm{C}$ - different frequencies, combination of ST1 with all STs are statistically same for dynamic modulus and phase angle. At $20^{\circ} \mathrm{C}$ and different frequencies, ST1-ST2, ST1-ST4 and ST1-ST6 are statistically same for dynamic modulus. For phase angle, ST1-ST2, ST1-ST4, and ST1-ST6 are statistically same for two of three frequencies. At $35^{\circ} \mathrm{C}$ - different frequencies, ST1-ST5 for dynamic modulus values is statistically same. For phase angle, ST1-ST2 and ST1-ST6 are statistically same at different frequencies. 
- For $25 \mathrm{~mm}$ NMAS mix at $4^{\circ} \mathrm{C}$ - different frequencies, ST1-all STs are statistically same for dynamic modulus and phase angle. At $20^{\circ} \mathrm{C}$ and different frequencies, ST1- ST6 are statistically same for dynamic modulus and phase angle. At $35^{\circ} \mathrm{C}$ for dynamic modulus, ST1-ST3 is statistically same for two of three frequencies. For phase angle, ST1-ST3 is statistically same for one of three frequencies.

ANOVA in Table 55 to 57 is used for comparing different STs for various combinations of temperature and frequencies in dynamic modulus test. All tests used a 95 percent confidence level, alpha of 0.05. The null hypothesis states no difference in means of ST1, ST2, ST3, ST4, ST5, and ST6. Failure to reject the null hypothesis indicates the means of ST1, ST2, ST3, ST4, ST5, and ST6 are statistically same. Rejecting the null hypothesis implies the data sets are statistically different. In the tables of ANOVA results, comparisons with alpha of less than 0.05 are shown with a bold font.

Table 55: ANOVA of STs for Dynamic Modulus and Phase Angle from 12.5 mm NMAS

\begin{tabular}{|c|c|c|c|c|c|c|}
\hline \multirow{3}{*}{$\begin{array}{c}\text { ANOVA } \\
\text { Comparison of ST1, } \\
\text { ST2, ST3, ST4, ST5, } \\
\text { and ST6 }\end{array}$} & \multicolumn{6}{|c|}{$12.5 \mathrm{~mm}$} \\
\hline & \multicolumn{3}{|c|}{ Dynamic Modulus } & \multicolumn{3}{|c|}{ Phase Angle } \\
\hline & $\tilde{\hbar}$ & 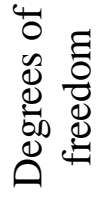 & 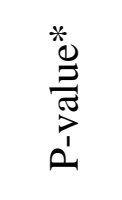 & $\tilde{\varkappa}$ & 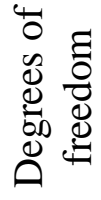 & 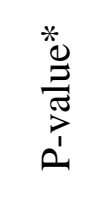 \\
\hline $4^{\circ} \mathrm{C}$ and $0.1 \mathrm{~Hz}$ & 84487.0 & \multirow{9}{*}{17} & 0.13 & 19.3 & \multirow{9}{*}{17} & 0.02 \\
\hline $4^{\circ} \mathrm{C}$ and $1 \mathrm{~Hz}$ & 77811.0 & & 0.27 & 8.1 & & 0.03 \\
\hline $4^{\circ} \mathrm{C}$ and $10 \mathrm{~Hz}$ & 77011.5 & & 0.37 & 8.9 & & 0.26 \\
\hline $20^{\circ} \mathrm{C}$ and $0.1 \mathrm{~Hz}$ & 10966.1 & & 0.91 & 40.7 & & 0.87 \\
\hline $20^{\circ} \mathrm{C}$ and $1 \mathrm{~Hz}$ & 52118.5 & & 0.77 & 10.9 & & 0.40 \\
\hline $20^{\circ} \mathrm{C}$ and $10 \mathrm{~Hz}$ & $\begin{array}{c}146983 . \\
6\end{array}$ & & 0.65 & 42.9 & & 0.75 \\
\hline $40^{\circ} \mathrm{C}$ and $0.1 \mathrm{~Hz}$ & 5082.3 & & 0.03 & 14.2 & & 0.15 \\
\hline $40^{\circ} \mathrm{C}$ and $1 \mathrm{~Hz}$ & 22411.3 & & 0.02 & 26.6 & & 0.23 \\
\hline $40^{\circ} \mathrm{C}$ and $10 \mathrm{~Hz}$ & 72719.7 & & 0.005 & 160.1 & & 0.007 \\
\hline
\end{tabular}


Table 56: ANOVA of STs for Dynamic Modulus and Phase Angle from 19 mm NMAS

\begin{tabular}{|c|c|c|c|c|c|c|}
\hline \multirow{3}{*}{$\begin{array}{c}\text { ANOVA } \\
\text { Comparison of } \\
\text { ST1, ST2, } \\
\text { ST3, ST4, } \\
\text { ST5, and ST6 }\end{array}$} & \multicolumn{6}{|c|}{$19 \mathrm{~mm}$} \\
\hline & \multicolumn{3}{|c|}{ Dynamic Modulus } & \multicolumn{3}{|c|}{ Phase Angle } \\
\hline & $\tilde{N}$ & 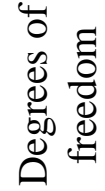 & 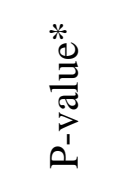 & $\tilde{n}$ & 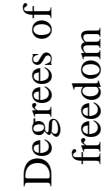 & 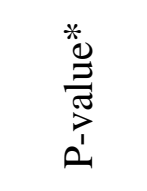 \\
\hline $4^{\circ} \mathrm{C}$ and $0.1 \mathrm{~Hz}$ & 91055.8 & \multirow{9}{*}{17} & 0.29 & 53.3 & \multirow{9}{*}{17} & 0.14 \\
\hline $4^{\circ} \mathrm{C}$ and $1 \mathrm{~Hz}$ & $\begin{array}{c}174073 . \\
9\end{array}$ & & 0.32 & 29.8 & & 0.15 \\
\hline $4^{\circ} \mathrm{C}$ and $10 \mathrm{~Hz}$ & $\begin{array}{c}394952 . \\
2\end{array}$ & & 0.33 & 20.7 & & 0.22 \\
\hline $\begin{array}{c}20^{\circ} \mathrm{C} \text { and } 0.1 \\
\mathrm{~Hz}\end{array}$ & 33590.8 & & 0.004 & 61.5 & & $5.1 \mathrm{E}-04$ \\
\hline $20^{\circ} \mathrm{C}$ and $1 \mathrm{~Hz}$ & 82609.6 & & 0.03 & 52.9 & & $9.8 \mathrm{E}-05$ \\
\hline $\begin{array}{c}20^{\circ} \mathrm{C} \text { and } 10 \\
\mathrm{~Hz}\end{array}$ & $\begin{array}{c}167783 . \\
8\end{array}$ & & 0.15 & 38.7 & & 0.002 \\
\hline $\begin{array}{c}35^{\circ} \mathrm{C} \text { and } 0.1 \\
\mathrm{~Hz}\end{array}$ & 2729.9 & & 0.004 & 70.5 & & 2.9E-04 \\
\hline $35^{\circ} \mathrm{C}$ and $1 \mathrm{~Hz}$ & 12595.2 & & 0.002 & 76.3 & & 0.007 \\
\hline $\begin{array}{c}35^{\circ} \mathrm{C} \text { and } 10 \\
\mathrm{~Hz}\end{array}$ & 52286.3 & & 0.001 & 277.8 & & 0.005 \\
\hline & & old & ct & thes & & \\
\hline
\end{tabular}


Table 57: ANOVA of STs for Dynamic Modulus and Phase Angle from 25 mm NMAS

\begin{tabular}{|c|c|c|c|c|c|c|}
\hline \multirow{3}{*}{$\begin{array}{c}\text { ANOVA } \\
\text { Comparison of } \\
\text { ST1, ST2, } \\
\text { ST3, ST4, } \\
\text { ST5, and ST6 }\end{array}$} & \multicolumn{6}{|c|}{$25 \mathrm{~mm}$} \\
\hline & \multicolumn{3}{|c|}{ Dynamic Modulus } & \multicolumn{3}{|c|}{ Phase Angle } \\
\hline & $\tilde{\varkappa}$ & 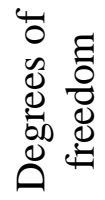 & 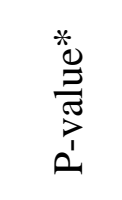 & $\tilde{n}$ & 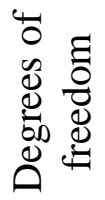 & 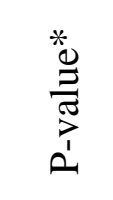 \\
\hline $4^{\circ} \mathrm{C}$ and $0.1 \mathrm{~Hz}$ & 75322.6 & \multirow{9}{*}{17} & 0.78 & 15.0 & \multirow{9}{*}{17} & 0.09 \\
\hline $4^{\circ} \mathrm{C}$ and $1 \mathrm{~Hz}$ & $\begin{array}{c}107670 . \\
1 \\
\end{array}$ & & 0.78 & 5.5 & & 0.14 \\
\hline $4^{\circ} \mathrm{C}$ and $10 \mathrm{~Hz}$ & $\begin{array}{c}150888 . \\
0\end{array}$ & & 0.66 & 4.2 & & 0.09 \\
\hline $\begin{array}{c}20^{\circ} \mathrm{C} \text { and } 0.1 \\
\mathrm{~Hz}\end{array}$ & 42506.3 & & 0.002 & 25.1 & & 0.002 \\
\hline $20^{\circ} \mathrm{C}$ and $1 \mathrm{~Hz}$ & 92665.3 & & 0.007 & 26.9 & & 0.003 \\
\hline $\begin{array}{c}20^{\circ} \mathrm{C} \text { and } 10 \\
\mathrm{~Hz}\end{array}$ & $\begin{array}{c}134755 . \\
5\end{array}$ & & 0.04 & 19.0 & & 0.002 \\
\hline $\begin{array}{c}35^{\circ} \mathrm{C} \text { and } 0.1 \\
\mathrm{~Hz}\end{array}$ & 2302.4 & & 0.02 & 78.5 & & 0.002 \\
\hline $35^{\circ} \mathrm{C}$ and $1 \mathrm{~Hz}$ & 15542.5 & & 0.004 & 37.2 & & 0.002 \\
\hline $\begin{array}{c}35^{\circ} \mathrm{C} \text { and } 10 \\
\mathrm{~Hz}\end{array}$ & 63464.2 & & 0.005 & 43.6 & & 0.003 \\
\hline
\end{tabular}

The results from t-test analysis are:

- All the STs are statistically same in DM and PA for $12.5 \mathrm{~mm}$ NMAS mix at $20^{\circ} \mathrm{C}$ at 0.1 , 1 and $10 \mathrm{HZ}$

- All the STs are statistically same in DM and PA for 19 mm NMAS mix at $4^{\circ} \mathrm{C}$ at $0.1,1$ and $10 \mathrm{HZ}$

- All the STs are statistically same in DM and PA for $25 \mathrm{~mm}$ NMAS mix at $4^{\circ} \mathrm{C}$ at $0.1,1$, and 10HZ.

MANOVA in Table 58 to 66 is used for comparing ST1 and other STs for all three different NMAS for various combinations of temperature and frequencies in dynamic modulus test. This is performed because for all NMAS mixes up to $37.5 \mathrm{~mm}$, ST1 is used to perform dynamic modulus test per current 
AASHTO standard. Therefore, MANOVA is performed to verify if jointly ST1 and other STs are statistically same for all NMAS mixes.

All tests used a 95 percent confidence level, alpha of 0.05 . The null hypothesis states no difference in means of ST1 and other ST for all three different NMAS mixes. Failure to reject the null hypothesis indicates no significant difference between $12.5 \mathrm{~mm}, 19 \mathrm{~mm}$, and $25 \mathrm{~mm}$ when considered jointly on ST1 and other ST. Rejecting the null hypothesis implies the data sets are statistically different for all three different NMAS mixes. In the tables of MANOVA results, comparisons with alpha of less than 0.05 are shown with a bold font.

The MANOVA results show that a significant difference between $12.5 \mathrm{~mm}, 19 \mathrm{~mm}$, and $25 \mathrm{~mm}$ when considered jointly on ST1 and other STs for various combinations of temperature and frequencies in dynamic modulus test. This confirms that specific ST must be used to test dynamic modulus test of a given NMAS mix. 
Table 58: MANOVA of NMAS and STs for Dynamic Modulus and Phase Angle at $4^{\circ} \mathrm{C}$ and $0.1 \mathrm{~Hz}$

\begin{tabular}{|c|c|c|c|c|c|c|c|c|c|}
\hline \multirow[b]{3}{*}{$\sum_{z}^{\infty}$} & \multicolumn{9}{|c|}{$4^{\circ} \mathrm{C}$ and $0.1 \mathrm{~Hz}$} \\
\hline & \multicolumn{5}{|c|}{ Dynamic Modulus } & \multicolumn{4}{|c|}{ Phase Angle } \\
\hline & 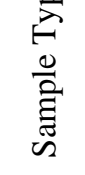 & $\stackrel{\Xi}{\stackrel{\Xi}{\Xi}}$ & के & t & 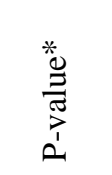 & $\stackrel{\varpi}{\Sigma}^{\stackrel{\Xi}{\Sigma}}$ & 合 & 岁 & 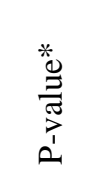 \\
\hline 12.5 & \multirow{3}{*}{ ST1 } & 1206.6 & 88.0 & \multirow{6}{*}{17} & \multirow{6}{*}{0.006} & 14.4 & .6 & \multirow{6}{*}{17} & \multirow{6}{*}{0.027} \\
\hline 19 & & 1063.1 & 93.6 & & & 16.8 & 1.6 & & \\
\hline 25 & & 1448.4 & 30.4 & & & 14.5 & .7 & & \\
\hline 12.5 & \multirow{3}{*}{$\mathrm{ST} 2$} & 1373.7 & 45.5 & & & 10.9 & .2 & & \\
\hline 19 & & 1215.4 & 91.6 & & & 15.3 & .7 & & \\
\hline 25 & & 1400.2 & 75.8 & & & 12.8 & 1.7 & & \\
\hline 12.5 & \multirow{3}{*}{ ST1 } & 1206.6 & 88.0 & \multirow{6}{*}{17} & \multirow{6}{*}{0.000} & 14.4 & .6 & \multirow{6}{*}{17} & \multirow{6}{*}{0.022} \\
\hline 19 & & 1063.1 & 93.6 & & & 16.8 & 1.6 & & \\
\hline 25 & & 1448.4 & 30.4 & & & 14.5 & .7 & & \\
\hline 12.5 & \multirow{3}{*}{ ST3 } & 1368.4 & 72.6 & & & 11.9 & .7 & & \\
\hline 19 & & 962.6 & 106.3 & & & 19.3 & 2.6 & & \\
\hline 25 & & 1345.4 & 18.9 & & & 14.8 & .4 & & \\
\hline 12.5 & \multirow{3}{*}{ ST1 } & 1206.6 & 88.0 & \multirow{6}{*}{17} & \multirow{6}{*}{0.000} & 14.4 & .6 & \multirow{6}{*}{17} & \multirow{6}{*}{0.022} \\
\hline 19 & & 1063.1 & 93.6 & & & 16.8 & 1.6 & & \\
\hline 25 & & 1448.4 & 30.4 & & & 14.5 & .7 & & \\
\hline 12.5 & \multirow{3}{*}{ ST4 } & 1368.4 & 72.6 & & & 11.900 & .7 & & \\
\hline 19 & & 962.6 & 106.3 & & & 19.367 & 2.6 & & \\
\hline 25 & & 1345.4 & 18.9 & & & 14.833 & .4 & & \\
\hline 12.5 & \multirow{3}{*}{ ST1 } & 1206.6 & 88.0 & \multirow{6}{*}{17} & \multirow{6}{*}{0.007} & 14.4 & .6 & \multirow{6}{*}{17} & \multirow{6}{*}{0.008} \\
\hline 19 & & 1063.1 & 93.6 & & & 16.8 & 1.6 & & \\
\hline 25 & & 1448.4 & 30.4 & & & 14.5 & .7 & & \\
\hline 12.5 & & 1350.2 & 87.1 & & & 11.867 & 1.2 & & \\
\hline 19 & ST5 & 1023.7 & 154.6 & & & 17.667 & 1.1 & & \\
\hline 25 & & 1462.1 & 105.8 & & & 14.767 & .4 & & \\
\hline 12.5 & & 1206.6 & 88.0 & & & 14.4 & .6 & & \\
\hline 19 & ST1 & 1063.1 & 93.6 & & & 16.8 & 1.6 & & \\
\hline 25 & & 1448.4 & 30.4 & & & 14.5 & .7 & & \\
\hline 12.5 & & 1373.7 & 45.6 & 17 & 0.004 & 10.967 & .2 & 17 & 0.001 \\
\hline 19 & ST6 & 1043.7 & 40.9 & & & 15.233 & .4 & & \\
\hline 25 & & 1330.9 & 194.1 & & & 14.500 & .9 & & \\
\hline & & & & & & othesis & & & \\
\hline
\end{tabular}


Table 59: MANOVA of NMAS and STs for Dynamic Modulus and Phase Angle at $4^{\circ} \mathrm{C}$ and $1 \mathrm{~Hz}$

\begin{tabular}{|c|c|c|c|c|c|c|c|c|c|}
\hline & \multicolumn{9}{|c|}{$4^{\circ} \mathrm{C}$ and $1 \mathrm{~Hz}$} \\
\hline & \multicolumn{5}{|c|}{ Dynamic Modulus } & \multicolumn{4}{|c|}{ Phase Angle } \\
\hline$\sum_{Z}^{\infty}$ & 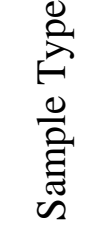 & $\sum_{\Sigma}^{\Xi}$ & $\hat{\Omega}$ & $t$ & $\frac{\stackrel{*}{O}}{\stackrel{*}{J}}$ & $\sum_{\Sigma}^{\Xi}$ & $\hat{n}$ & $t$ & 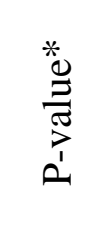 \\
\hline 12.5 & & 1608.7 & 101.1 & \multirow{6}{*}{17} & \multirow{6}{*}{0.010} & 10.8 & .4 & \multirow{6}{*}{17} & \multirow{6}{*}{0.044} \\
\hline 19 & ST1 & 1496.5 & 88.3 & & & 12.2 & 1.2 & & \\
\hline 25 & & 1941.3 & 29.6 & & & 10.5 & .5 & & \\
\hline 12.5 & \multirow{3}{*}{ ST2 } & 1740.6 & 52.9 & & & 8.7 & .1 & & \\
\hline 19 & & 1666.1 & 106.9 & & & 11.3 & .6 & & \\
\hline 25 & & 1837.5 & 101.0 & & & 9.8 & 1.3 & & \\
\hline 12.5 & \multirow{3}{*}{ ST1 } & 1608.7 & 101.1 & \multirow{6}{*}{17} & \multirow{6}{*}{0.001} & 10.8 & .4 & \multirow{6}{*}{17} & \multirow{6}{*}{0.028} \\
\hline 19 & & 1496.5 & 88.3 & & & 12.2 & 1.2 & & \\
\hline 25 & & 1941.3 & 29.6 & & & 10.5 & .5 & & \\
\hline 12.5 & \multirow{3}{*}{ ST3 } & 1750.1 & 73.0 & & & 9.1 & .6 & & \\
\hline 19 & & 1344.9 & 160.3 & & & 14.4 & 2.0 & & \\
\hline 25 & & 1826.5 & 33.8 & & & 10.8 & .3 & & \\
\hline 12.5 & \multirow{3}{*}{ ST1 } & 1608.7 & 101.1 & \multirow{6}{*}{17} & \multirow{6}{*}{0.001} & 10.8 & .4 & \multirow{6}{*}{17} & \multirow{6}{*}{0.028} \\
\hline 19 & & 1496.5 & 88.3 & & & 12.2 & 1.2 & & \\
\hline 25 & & 1941.3 & 29.6 & & & 10.5 & .5 & & \\
\hline 12.5 & \multirow{3}{*}{ ST4 } & 1750.1 & 73.0 & & & 9.1 & .6 & & \\
\hline 19 & & 1344.9 & 160.3 & & & 14.4 & 2.0 & & \\
\hline 25 & & 1826.5 & 33.8 & & & 10.8 & .3 & & \\
\hline 12.5 & \multirow{3}{*}{ ST1 } & 1608.7 & 101.1 & \multirow{6}{*}{17} & \multirow{6}{*}{0.008} & 10.8 & .4 & \multirow{6}{*}{17} & \multirow{6}{*}{0.010} \\
\hline 19 & & 1496.5 & 88.3 & & & 12.2 & 1.2 & & \\
\hline 25 & & 1941.3 & 29.6 & & & 10.5 & .5 & & \\
\hline 12.5 & \multirow{3}{*}{ ST5 } & 1731.2 & 90.6 & & & 9.0 & .9 & & \\
\hline 19 & & 1450.0 & 204.8 & & & 13.1 & .9 & & \\
\hline 25 & & 1965.8 & 149.3 & & & 10.8 & .2 & & \\
\hline 12.5 & \multirow{3}{*}{ ST1 } & 1608.7 & 101.1 & \multirow{6}{*}{17} & & 10.8 & .4 & & \\
\hline 19 & & 1496.5 & 88.3 & & & 12.2 & 1.2 & & \\
\hline 25 & & 1941.3 & 29.6 & & 0003 & 10.5 & .5 & 17 & 0005 \\
\hline 12.5 & & 1740.6 & 52.9 & & 0.003 & 8.7 & .1 & 17 & 0.005 \\
\hline 19 & ST6 & 1419.1 & 44.6 & & & 11.2 & .3 & & \\
\hline 25 & & 1761.9 & 226.7 & & & 10.3 & .7 & & \\
\hline
\end{tabular}


Table 60: MANOVA of NMAS and STs for Dynamic Modulus and Phase Angle at $4^{\circ} \mathrm{C}$ and $10 \mathrm{~Hz}$

\begin{tabular}{|c|c|c|c|c|c|c|c|c|c|}
\hline \multirow{3}{*}{$\sum_{\sum^{2}}^{\infty}$} & \multicolumn{9}{|c|}{$4^{\circ} \mathrm{C}$ and $10 \mathrm{~Hz}$} \\
\hline & \multicolumn{5}{|c|}{ Dynamic Modulus } & \multicolumn{4}{|c|}{ Phase Angle } \\
\hline & 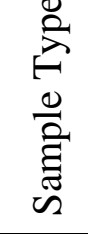 & $\sum^{\varpi ే}$ & के & 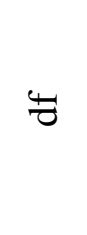 & 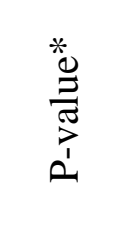 & $\stackrel{\varpi}{\varpi^{\varpi}}$ & $\theta$ & 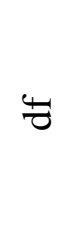 & 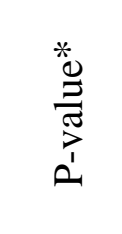 \\
\hline 12.5 & \multirow{3}{*}{ ST1 } & 2002.8 & 110.1 & \multirow{6}{*}{17} & \multirow{6}{*}{0.007} & 8.3 & .35 & \multirow{6}{*}{17} & \multirow{6}{*}{0.025} \\
\hline 19 & & 1934.5 & 73.1 & & & 9.3 & .80 & & \\
\hline 25 & & 2419.5 & 24.7 & & & 7.8 & .56 & & \\
\hline 12.5 & \multirow{3}{*}{ ST2 } & 2116.2 & $\begin{array}{c}59.47 \\
1\end{array}$ & & & 6.5 & .75 & & \\
\hline 19 & & 2128.0 & 111.9 & & & 8.5 & .37 & & \\
\hline 25 & & 2276.3 & 107.9 & & & 6.8 & .37 & & \\
\hline 12.5 & \multirow{3}{*}{ ST1 } & 2002.8 & 110.1 & \multirow{6}{*}{17} & \multirow{6}{*}{0.007} & 8.3 & .35 & \multirow{6}{*}{17} & \multirow{6}{*}{0.056} \\
\hline 19 & & 1934.5 & 73.1 & & & 9.3 & .80 & & \\
\hline 25 & & 2419.5 & 24.7 & & & 7.8 & .56 & & \\
\hline 12.5 & \multirow{3}{*}{ ST3 } & 2116.2 & 59.4 & & & 7.5 & .40 & & \\
\hline 19 & & 2128.0 & 111.9 & & & 11.1 & 1.9 & & \\
\hline 25 & & 2276.3 & 107.9 & & & 8.1 & .5 & & \\
\hline 12.5 & \multirow{3}{*}{ ST1 } & 2002.8 & 110.1 & \multirow{6}{*}{17} & \multirow{6}{*}{0.001} & 8.3 & .35 & \multirow{6}{*}{17} & \multirow{6}{*}{0.056} \\
\hline 19 & & 1934.5 & 73.1 & & & 9.3 & .80 & & \\
\hline 25 & & 2419.5 & 24.7 & & & 7.8 & .56 & & \\
\hline 12.5 & \multirow{3}{*}{ ST4 } & 2135.4 & 67.1 & & & 7.5 & .40 & & \\
\hline 19 & & 1723.2 & 269.1 & & & 11.1 & 1.9 & & \\
\hline 25 & & 2303.4 & 34.5 & & & 8.1 & .56 & & \\
\hline 12.5 & \multirow{3}{*}{ ST1 } & 2002.8 & 110.1 & \multirow{6}{*}{17} & \multirow{6}{*}{0.005} & 8.3 & .35 & \multirow{6}{*}{17} & \multirow{6}{*}{0.021} \\
\hline 19 & & 1934.5 & 73.1 & & & 9.3 & .80 & & \\
\hline 25 & & 2419.5 & 24.7 & & & 7.8 & .56 & & \\
\hline 12.5 & & 2120.9 & 82.6 & & & 7.1 & 1.1 & & \\
\hline 19 & ST5 & 1910.7 & 238.9 & & & 9.9 & .70 & & \\
\hline 25 & & 2468.4 & 189.9 & & & 8.3 & .10 & & \\
\hline 12.5 & & 2002.8 & 110.1 & & & 8.3 & .35 & & \\
\hline 19 & ST1 & 1934.5 & 73.1 & & & 9.3 & .80 & & \\
\hline 25 & & 2419.5 & 24.7 & & & 7.8 & .56 & & \\
\hline 12.5 & & 2116.2 & 59.4 & 17 & 0.002 & 6.500 & .75 & 17 & 0.011 \\
\hline 19 & ST6 & 1796.7 & 45.4 & & & 8.533 & .15 & & \\
\hline 25 & & 2180.4 & 244.8 & & & 7.8 & .45 & & \\
\hline & & & * Bol & $=1$ & 10 & pothes & & & \\
\hline
\end{tabular}


Table 61: MANOVA of NMAS and STs for Dynamic Modulus and Phase Angle at $20^{\circ} \mathrm{C}$ and $0.1 \mathrm{~Hz}$

\begin{tabular}{|c|c|c|c|c|c|c|c|c|c|}
\hline & \multicolumn{9}{|c|}{$20^{\circ} \mathrm{C}$ and $0.1 \mathrm{~Hz}$} \\
\hline & \multicolumn{5}{|c|}{ Dynamic Modulus } & \multicolumn{4}{|c|}{ Phase Angle } \\
\hline$\sum_{z}^{\infty}$ & 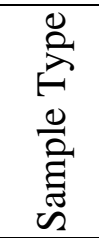 & 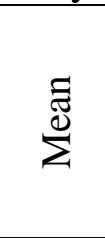 & $\hat{\Omega}$ & $\frac{4}{7}$ & 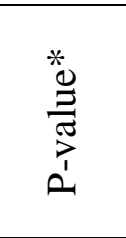 & $\sum^{\tilde{\Xi}}$ & $\hat{s}$ & $t$ & 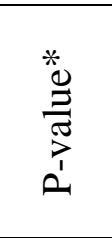 \\
\hline 12.5 & & 431.7 & 39.1 & \multirow{6}{*}{17} & \multirow{6}{*}{0.000} & 26.6 & .65 & \multirow{6}{*}{17} & \multirow{6}{*}{0.053} \\
\hline 19 & ST1 & 350.4 & 30.1 & & & 28.6 & 1.37 & & \\
\hline 25 & & 504.9 & 23.9 & & & 27.7 & .58 & & \\
\hline 12.5 & \multirow{3}{*}{ ST2 } & 447.1 & 27.2 & & & 25.7 & 2.91 & & \\
\hline 19 & & 330.0 & 14.8 & & & 30.6 & .30 & & \\
\hline 25 & & 420.5 & 14.3 & & & 29.0 & .52 & & \\
\hline 12.5 & \multirow{3}{*}{ ST1 } & 431.7 & 39.1 & \multirow{6}{*}{17} & \multirow{6}{*}{0.000} & 26.6 & .65 & \multirow{6}{*}{17} & \multirow{6}{*}{0.003} \\
\hline 19 & & 350.4 & 30.1 & & & 28.6 & 1.37 & & \\
\hline 25 & & 504.9 & 23.9 & & & 27.7 & .58 & & \\
\hline 12.5 & \multirow{3}{*}{ ST3 } & 495.3 & 65.8 & & & 24.3 & 1.7 & & \\
\hline 19 & & 238.3 & 35.0 & & & 33.2 & .75 & & \\
\hline 25 & & 348.7 & 15.1 & & & 32.1 & 1.1 & & \\
\hline 12.5 & \multirow{3}{*}{ ST1 } & 431.7 & 39.1 & \multirow{6}{*}{17} & \multirow{6}{*}{0.000} & 26.6 & .65 & \multirow{6}{*}{17} & \multirow{6}{*}{0.001} \\
\hline 19 & & 350.4 & 30.1 & & & 28.6 & 1.37 & & \\
\hline 25 & & 504.9 & 23.9 & & & 27.7 & .58 & & \\
\hline 12.5 & \multirow{3}{*}{ ST4 } & 495.3 & 65.8 & & & 24.3 & 1.7 & & \\
\hline 19 & & 238.3 & 35.0 & & & 33.26 & .75 & & \\
\hline 25 & & 348.7 & 15.1 & & & 32.16 & 1.10 & & \\
\hline 12.5 & \multirow{3}{*}{ ST1 } & 431.7 & 39.1 & \multirow{6}{*}{17} & \multirow{6}{*}{0.000} & 26.6 & .65 & \multirow{6}{*}{17} & \multirow{6}{*}{0.004} \\
\hline 19 & & 350.4 & 30.1 & & & 28.6 & 1.37 & & \\
\hline 25 & & 504.9 & 23.9 & & & 27.7 & .58 & & \\
\hline 12.5 & \multirow{3}{*}{ ST5 } & 432.8 & 28.0 & & & 25.4 & 1.26 & & \\
\hline 19 & & 237.7 & 41.2 & & & 32.73 & 1.02 & & \\
\hline 25 & & 379.2 & 29.1 & & & 31.16 & .72 & & \\
\hline 12.5 & \multirow{3}{*}{ ST1 } & 431.7 & 39.1 & \multirow{6}{*}{17} & & 26.6 & .65 & & \\
\hline 19 & & 350.4 & 30.1 & & & 28.6 & 1.37 & & \\
\hline 25 & & 504.9 & 23.9 & & & 27.7 & .58 & & \\
\hline 12.5 & & 447.1 & 27.2 & & 0.007 & 25.73 & 2.91 & 17 & 0.078 \\
\hline 19 & ST6 & 337.0 & 11.1 & & & 29.20 & .50 & & \\
\hline 25 & & 432.2 & 60.2 & & & 29.20 & .60 & & \\
\hline
\end{tabular}


Table 62: MANOVA of NMAS and STs for Dynamic Modulus and Phase Angle at $20^{\circ} \mathrm{C}$ and $1 \mathrm{~Hz}$

\begin{tabular}{|c|c|c|c|c|c|c|c|c|c|}
\hline \multirow{3}{*}{$\sum_{Z}^{\infty}$} & \multicolumn{9}{|c|}{$20^{\circ} \mathrm{C}$ and $1 \mathrm{~Hz}$} \\
\hline & \multicolumn{5}{|c|}{ Dynamic Modulus } & \multicolumn{4}{|c|}{ Phase Angle } \\
\hline & 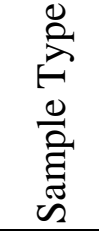 & 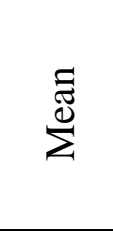 & $\hat{\Omega}$ & $t$ & 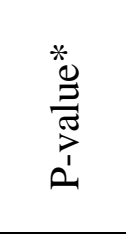 & 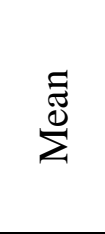 & $\hat{\sim}$ & 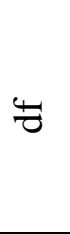 & 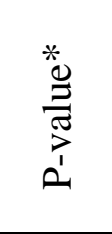 \\
\hline 12.5 & \multirow{3}{*}{ ST1 } & 744.6 & 56.1 & \multirow{6}{*}{17} & \multirow{6}{*}{0.003} & 20.9 & .65 & \multirow{6}{*}{17} & \multirow{6}{*}{0.000} \\
\hline 19 & & 640.8 & 29.1 & & & 23.7 & 1.05 & & \\
\hline 25 & & 640.8 & 29.1 & & & 21.5 & .41 & & \\
\hline 12.5 & \multirow{3}{*}{ ST2 } & 712.9 & 91.1 & & & 19.03 & .71 & & \\
\hline 19 & & 627.5 & 20.3 & & & 24.5 & .47 & & \\
\hline 25 & & 763.2 & 21.8 & & & 22.50 & .45 & & \\
\hline 12.5 & \multirow{3}{*}{ ST1 } & 744.6 & 56.1 & \multirow{6}{*}{17} & \multirow{6}{*}{0.005} & 20.9 & .65 & \multirow{6}{*}{17} & \multirow{6}{*}{0.001} \\
\hline 19 & & 640.8 & 29.1 & & & 23.7 & 1.05 & & \\
\hline 25 & & 744.6 & 56.1 & & & 21.5 & .41 & & \\
\hline 12.5 & \multirow{3}{*}{ ST3 } & 816.3 & 80.3 & & & 18.9 & 1.6 & & \\
\hline 19 & & 465.7 & 81.1 & & & 27.2 & .95 & & \\
\hline 25 & & 672.4 & 26.7 & & & 25.2 & .85 & & \\
\hline 12.5 & \multirow{3}{*}{ ST1 } & 744.6 & 56.1 & \multirow{6}{*}{17} & \multirow{6}{*}{0.005} & 20.9 & .65 & \multirow{6}{*}{17} & \multirow{6}{*}{0.001} \\
\hline 19 & & 640.8 & 29.1 & & & 23.7 & 1.05 & & \\
\hline 25 & & 744.6 & 56.1 & & & 21.5 & .41 & & \\
\hline 12.5 & \multirow{3}{*}{ ST4 } & 816.3 & 80.3 & & & 18.9 & 1.69 & & \\
\hline 19 & & 465.7 & 81.1 & & & 27.2 & .95 & & \\
\hline 25 & & 672.4 & 26.7 & & & 25.2 & .85 & & \\
\hline 12.5 & \multirow{3}{*}{ ST1 } & 744.6 & 56.1 & \multirow{6}{*}{17} & \multirow{6}{*}{0.002} & 20.9 & .65 & \multirow{6}{*}{17} & \multirow{6}{*}{0.000} \\
\hline 19 & & 640.8 & 29.1 & & & 23.7 & 1.05 & & \\
\hline 25 & & 744.6 & 56.1 & & & 21.5 & .41 & & \\
\hline 12.5 & & 718.03 & 29.4 & & & 20.40 & .79 & & \\
\hline 19 & ST5 & 465.96 & 73.1 & & & 28.03 & .77 & & \\
\hline 25 & & 717.16 & 56.9 & & & 25.13 & .35 & & \\
\hline 12.5 & & 744.6 & 56.1 & & & 20.9 & .65 & & \\
\hline 19 & ST1 & 640.8 & 29.1 & & & 23.7 & 1.05 & & \\
\hline 25 & & 744.6 & 56.1 & & & 21.5 & .41 & 17 & \\
\hline 12.5 & & 712.9 & 91.18 & 17 & 0.040 & 19.03 & .70 & $1 /$ & 0.001 \\
\hline 19 & ST6 & 619.0 & 20.94 & & & 23.50 & .45 & & \\
\hline 25 & & 782.4 & 112.17 & & & 22.60 & .98 & & \\
\hline & & & $* \mathbf{I}$ & & & thes & & & \\
\hline
\end{tabular}


Table 63: MANOVA of NMAS and STs for Dynamic Modulus and Phase Angle at $20^{\circ} \mathrm{C}$ and $10 \mathrm{~Hz}$

\begin{tabular}{|c|c|c|c|c|c|c|c|c|c|}
\hline \multirow{3}{*}{$\sum_{Z}^{\infty}$} & \multicolumn{9}{|c|}{$20^{\circ} \mathrm{C}$ and $10 \mathrm{~Hz}$} \\
\hline & \multicolumn{5}{|c|}{ Dynamic Modulus } & \multicolumn{4}{|c|}{ Phase Angle } \\
\hline & 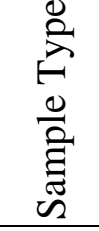 & 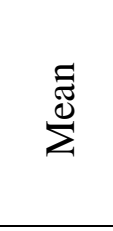 & $\hat{\Omega}$ & 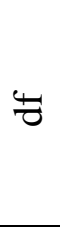 & 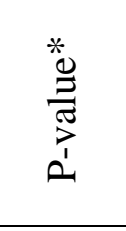 & $\stackrel{\Xi}{\Xi}_{\Sigma}^{\Xi}$ & $\hat{\sim}$ & 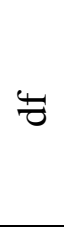 & 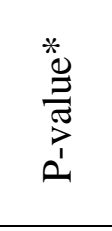 \\
\hline 12.5 & \multirow{3}{*}{ ST1 } & 1136.5 & 70.6 & \multirow{6}{*}{17} & \multirow{6}{*}{0.000} & 15.9 & .55 & \multirow{6}{*}{17} & \multirow{6}{*}{0.006} \\
\hline 19 & & 1035.6 & 16.5 & & & 18.3 & .72 & & \\
\hline 25 & & 1392.2 & 22.1 & & & 15.9 & .37 & & \\
\hline 12.5 & \multirow{3}{*}{ ST2 } & 1048.0 & 159.3 & & & 16.1 & 2.9 & & \\
\hline 19 & & 1040.3 & 35.0 & & & 18.4 & .96 & & \\
\hline 25 & & 1211.3 & 27.1 & & & 16.5 & .55 & & \\
\hline 12.5 & \multirow{3}{*}{ ST1 } & 1136.5 & 70.6 & \multirow{6}{*}{17} & \multirow{6}{*}{0.000} & 15.9 & .55 & \multirow{6}{*}{17} & \multirow{6}{*}{0.000} \\
\hline 19 & & 1035.6 & 16.5 & & & 18.3 & .72 & & \\
\hline 25 & & 1392.2 & 22.1 & & & 15.9 & .37 & & \\
\hline 12.5 & \multirow{3}{*}{ ST3 } & 1212.7 & 79.6 & & & 14.4 & 1.2 & & \\
\hline 19 & & 796.8 & 167.1 & & & 21.3 & 1.1 & & \\
\hline 25 & & 1127.5 & 31.3 & & & 18.7 & .665 & & \\
\hline 12.5 & \multirow{3}{*}{ ST1 } & 1136.5 & 70.6 & \multirow{6}{*}{17} & \multirow{6}{*}{0.000} & 15.9 & .55 & \multirow{6}{*}{17} & \multirow{6}{*}{0.000} \\
\hline 19 & & 1035.6 & 16.5 & & & 18.3 & .72 & & \\
\hline 25 & & 1392.2 & 22.1 & & & 15.9 & .37 & & \\
\hline 12.5 & \multirow{3}{*}{ ST4 } & 1212.7 & 79.6 & & & 14.4 & 1.2 & & \\
\hline 19 & & 796.7 & 167.1 & & & 21.3 & 1.1 & & \\
\hline 25 & & 1127.5 & 31.3 & & & 18.7 & .66 & & \\
\hline 12.5 & \multirow{3}{*}{ ST1 } & 1136.5 & 70.6 & \multirow{6}{*}{17} & \multirow{6}{*}{0.001} & 15.9 & .55 & \multirow{6}{*}{17} & \multirow{6}{*}{0.000} \\
\hline 19 & & 1035.6 & 16.5 & & & 18.3 & .72 & & \\
\hline 25 & & 1392.2 & 22.1 & & & 15.9 & .37 & & \\
\hline 12.5 & & 1091.5 & 24.5 & & & 15.1 & 1.3 & & \\
\hline 19 & ST5 & 833.2 & 115.4 & & & 21.8 & .78 & & \\
\hline 25 & & 1201.9 & 94.9 & & & 18.8 & .11 & & \\
\hline 12.5 & & 1136.5 & 70.6 & & & 15.9 & .55 & & \\
\hline 19 & ST1 & 1035.6 & 16.5 & & & 18.3 & .72 & & \\
\hline 25 & & 1392.2 & 22.1 & 17 & 00 & 15.9 & .37 & 17 & 0017 \\
\hline 12.5 & & 1048.0 & 159.3 & 17 & 0.002 & 16.1 & 2.9 & 11 & 0.017 \\
\hline 19 & ST6 & 996.2 & 32.9 & & & 17.6 & .30 & & \\
\hline 25 & & 1209.7 & 173.6 & & & 16.5 & .79 & & \\
\hline & & & $*$ & & & othe & & & \\
\hline
\end{tabular}


Table 64: MANOVA of NMAS and STs for Dynamic Modulus and Phase Angle at $35^{\circ} \mathrm{C}$ and $0.1 \mathrm{~Hz}$

\begin{tabular}{|c|c|c|c|c|c|c|c|c|c|}
\hline \multirow[b]{3}{*}{$\sum_{Z}^{\infty}$} & \multicolumn{9}{|c|}{$35^{\circ} \mathrm{C}$ and $0.1 \mathrm{~Hz}$} \\
\hline & \multicolumn{5}{|c|}{ Dynamic Modulus } & \multicolumn{4}{|c|}{ Phase Angle } \\
\hline & 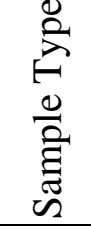 & $\sum_{\Sigma}^{\Xi}$ & $\theta$ & 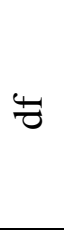 & 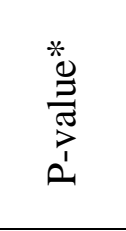 & $\stackrel{\Xi}{\Xi}_{\Sigma}^{\Xi}$ & $\hat{\varepsilon}$ & 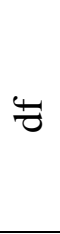 & 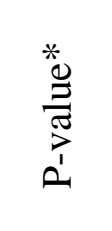 \\
\hline 19 & \multirow{2}{*}{ ST1 } & 42.1 & 10.1 & \multirow{4}{*}{11} & \multirow{4}{*}{0.903} & 27.9 & .65 & \multirow{4}{*}{11} & \multirow{4}{*}{0.350} \\
\hline 25 & & 42.4 & 12.6 & & & 28.7 & .55 & & \\
\hline 19 & \multirow{2}{*}{ ST2 } & 79.9 & 8.62 & & & 32.1 & .97 & & \\
\hline 25 & & 75.4 & 11.8 & & & 32.0 & .73 & & \\
\hline 19 & \multirow{2}{*}{ ST1 } & 42.1 & 10.1 & \multirow{4}{*}{11} & \multirow{4}{*}{0.388} & 27.9 & .65 & \multirow{4}{*}{11} & \multirow{4}{*}{0.438} \\
\hline 25 & & 42.4 & 12.6 & & & 28.7 & .55 & & \\
\hline 19 & \multirow{2}{*}{ ST3 } & 59.4 & 7.6 & & & 35.0 & .75 & & \\
\hline 25 & & 67.7 & .91 & & & 35.9 & 1.8 & & \\
\hline 19 & \multirow{2}{*}{ ST1 } & 42.1 & 10.1 & \multirow{4}{*}{11} & \multirow{4}{*}{0.388} & 27.9 & .65 & \multirow{4}{*}{11} & \multirow{4}{*}{0.438} \\
\hline 25 & & 42.4 & 12.6 & & & 28.7 & .55 & & \\
\hline 19 & \multirow{2}{*}{ ST4 } & 59.4 & 7.6 & & & 35.0 & .75 & & \\
\hline 25 & & 67.7 & .91 & & & 35.9 & 1.8 & & \\
\hline 19 & & 42.1 & 10.1 & \multirow{4}{*}{11} & \multirow{4}{*}{0.165} & 27.9 & .65 & \multirow{4}{*}{11} & \multirow{4}{*}{0.399} \\
\hline 25 & S11 & 42.4 & 12.6 & & & 28.7 & .55 & & \\
\hline 19 & \multirow{2}{*}{ ST5 } & 51.9 & 8.3 & & & 33.3 & 1.8 & & \\
\hline 25 & & 73.3 & 8.9 & & & 35.1 & 1.1 & & \\
\hline 19 & \multirow{2}{*}{ ST1 } & 42.1 & 10.1 & \multirow{4}{*}{11} & \multirow{4}{*}{0.090} & 27.9 & .65 & \multirow{4}{*}{11} & \multirow{4}{*}{0.030} \\
\hline 25 & & 42.4 & 12.6 & & & 28.7 & .55 & & \\
\hline 19 & ST6 & 71.9 & 5.2 & & & 32.5 & .41 & & \\
\hline 25 & 516 & 87.8 & 4.9 & & & 34.3 & 1.0 & & \\
\hline
\end{tabular}


Table 65: MANOVA of NMAS and STs for Dynamic Modulus and Phase Angle at $35^{\circ} \mathrm{C}$ and $1 \mathrm{~Hz}$

\begin{tabular}{|c|c|c|c|c|c|c|c|c|c|}
\hline \multirow[b]{3}{*}{$\sum_{Z}^{\infty}$} & \multicolumn{9}{|c|}{$35^{\circ} \mathrm{C}$ and $1 \mathrm{~Hz}$} \\
\hline & \multicolumn{5}{|c|}{ Dynamic Modulus } & \multicolumn{4}{|c|}{ Phase Angle } \\
\hline & 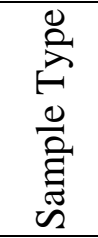 & $\sum_{\bar{\Xi}}^{\stackrel{\Xi}{\Sigma}}$ & $\theta$ & $\frac{4}{\sigma}$ & 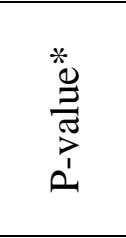 & $\sum_{\Sigma}^{\tilde{E}}$ & $\theta$ & 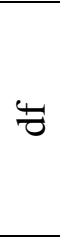 & 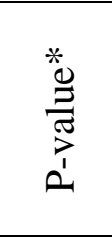 \\
\hline 19 & \multirow{2}{*}{ ST1 } & 92.4 & 16.0 & \multirow{4}{*}{11} & \multirow{4}{*}{0.860} & 32.1 & .60 & \multirow{4}{*}{11} & \multirow{4}{*}{0.080} \\
\hline 25 & & 103.5 & 27.9 & & & 33.6 & .70 & & \\
\hline 19 & \multirow{2}{*}{ ST2 } & 176.9 & 13.5 & & & 31.9 & 1.2 & & \\
\hline 25 & & 179.0 & 18.8 & & & 34.0 & .70 & & \\
\hline 19 & \multirow{2}{*}{ ST1 } & 92.4 & 16.0 & \multirow{4}{*}{11} & \multirow{4}{*}{0.130} & 32.1 & .60 & \multirow{4}{*}{11} & \multirow{4}{*}{0.026} \\
\hline 25 & & 103.5 & 27.9 & & & 33.6 & .70 & & \\
\hline 19 & \multirow{2}{*}{ ST3 } & 143.6 & 15.6 & & & 36.4 & .20 & & \\
\hline 25 & & 175.2 & 3.7 & & & 37.5 & 1.0 & & \\
\hline 19 & \multirow{2}{*}{ ST1 } & 92.4 & 16.0 & \multirow{4}{*}{11} & \multirow{4}{*}{0.130} & 32.1 & .60 & \multirow{4}{*}{11} & \multirow{4}{*}{0.026} \\
\hline 25 & & 103.5 & 27.9 & & & 33.6 & .70 & & \\
\hline 19 & \multirow{2}{*}{ ST4 } & 143.6 & 15.6 & & & 36.4 & .20 & & \\
\hline 25 & & 175.2 & 3.7 & & & 37.5 & 1.0 & & \\
\hline 19 & \multirow{2}{*}{ ST1 } & 92.4 & 16.0 & \multirow{4}{*}{11} & \multirow{4}{*}{0.019} & 32.1 & .60 & \multirow{4}{*}{11} & \multirow{4}{*}{0.192} \\
\hline 25 & & 103.5 & 27.9 & & & 33.6 & .70 & & \\
\hline 19 & \multirow{2}{*}{ ST5 } & 125.5 & 11.8 & & & 36.7 & 1.5 & & \\
\hline 25 & & 197.0 & 14.4 & & & 36.2 & .95 & & \\
\hline 19 & \multirow{2}{*}{ ST1 } & 92.4 & 16.0 & \multirow{4}{*}{11} & \multirow{4}{*}{0.065} & 32.1 & .60 & \multirow{4}{*}{11} & \multirow{4}{*}{0.167} \\
\hline 25 & & 103.5 & 27.9 & & & 33.6 & .70 & & \\
\hline 19 & ST6 & 162.2 & 11.5 & & & 33.7 & .95 & & \\
\hline 25 & S16 & 217.0 & 18.0 & & & 34.5 & 1.1 & & \\
\hline
\end{tabular}


Table 66: MANOVA of NMAS and STs for Dynamic Modulus and Phase Angle at $35^{\circ} \mathrm{C}$ and $10 \mathrm{~Hz}$

\begin{tabular}{|c|c|c|c|c|c|c|c|c|c|}
\hline \multirow[b]{3}{*}{$\sum_{Z}^{\infty}$} & \multicolumn{9}{|c|}{$35^{\circ} \mathrm{C}$ and $10 \mathrm{~Hz}$} \\
\hline & \multicolumn{5}{|c|}{ Dynamic Modulus } & \multicolumn{4}{|c|}{ Phase Angle } \\
\hline & 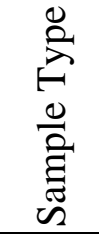 & $\sum_{\bar{\Xi}}^{\Xi}$ & $\hat{n}$ & 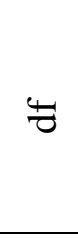 & 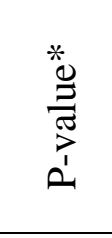 & $\sum_{\Sigma}^{\Xi}$ & $\hat{s}$ & 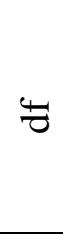 & 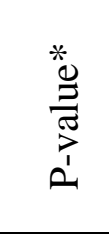 \\
\hline 19 & \multirow{2}{*}{ ST1 } & 208.1 & 25.0 & \multirow{4}{*}{11} & \multirow{4}{*}{0.269} & 34.4 & 34.4 & \multirow{4}{*}{11} & \multirow{4}{*}{0.072} \\
\hline 25 & & 248.6 & 54.8 & & & 1.6 & 1.6 & & \\
\hline 19 & \multirow{2}{*}{ ST2 } & 359.1 & 19.4 & & & 36.1 & 36.1 & & \\
\hline 25 & & 396.7 & 32.4 & & & 1.5 & 1.5 & & \\
\hline 19 & \multirow{2}{*}{ ST1 } & 208.1 & 25.0 & \multirow{4}{*}{11} & \multirow{4}{*}{0.057} & 34.4 & 34.4 & \multirow{4}{*}{11} & \multirow{4}{*}{0.016} \\
\hline 25 & & 248.6 & 54.8 & & & 1.6 & 1.6 & & \\
\hline 19 & \multirow{2}{*}{ ST3 } & 323.9 & 28.1 & & & 36.1 & 36.1 & & \\
\hline 25 & & 405.6 & 8.8 & & & 1.5 & 1.5 & & \\
\hline 19 & ST1 & 208.1 & 25.0 & \multirow{4}{*}{11} & \multirow{4}{*}{0.057} & 34.4 & 34.4 & \multirow{4}{*}{11} & \multirow{4}{*}{0.15} \\
\hline 25 & 311 & 248.6 & 54.8 & & & 1.6 & 1.6 & & \\
\hline 19 & \multirow{2}{*}{ ST4 } & 323.9 & 28.1 & & & 36.1 & 36.1 & & \\
\hline 25 & & 405.6 & 8.8 & & & 1.5 & 1.5 & & \\
\hline 19 & CT1 & 208.1 & 25.0 & \multirow{4}{*}{11} & \multirow{4}{*}{0.001} & 33.0 & 33.0 & \multirow{4}{*}{11} & \multirow{4}{*}{0.261} \\
\hline 25 & 311 & 248.6 & 54.8 & & & .60 & .60 & & \\
\hline 19 & \multirow{2}{*}{ ST5 } & 244.0 & 16.2 & & & 32.5 & 32.5 & & \\
\hline 25 & & 452.8 & 29.71 & & & .11 & .11 & & \\
\hline 19 & \multirow{2}{*}{ ST1 } & 208.1 & 25.0 & \multirow{4}{*}{11} & \multirow{4}{*}{0.091} & 34.4 & 34.4 & \multirow{4}{*}{11} & \multirow{4}{*}{0.229} \\
\hline 25 & & 248.6 & 54.8 & & & 1.6 & 1.6 & & \\
\hline 19 & \multirow{2}{*}{ ST6 } & 339.9 & 24.2 & & & 36.1 & 36.1 & & \\
\hline 25 & & 464.8 & 48.7 & & & 1.5 & 1.5 & & \\
\hline
\end{tabular}

\subsection{WITHIN SAMPLE AIR VOID VARIABILITY ANALYSIS}

The Analysis 4 evaluates air void uniformity using samples tested for dynamic modulus test in AMPT. The air void uniformity is conducted using AASHTO R 83-17 with the exception that all six STs are tested. The AASHTO standard specifies only ST1, which is 100X150 mm samples cored from the center of SGC pill to be tested for air void uniformity. It is beneficial to investigate uniformity at $7.0 \pm 0.5$ percent of all replicates for six different STs. Each ST was cut into equal thirds: 


\begin{tabular}{|c|c|}
\hline Sample Types & Approximate Height of Thirds, $\mathrm{mm}$ \\
\hline ST 1 & $50 \mathrm{~mm}$ \\
\hline ST 2 & \\
\cline { 1 - 1 } ST 3 & \\
ST 4 & 36.7 \\
\hline ST 5 & \\
\cline { 1 - 1 } ST 6 & \\
\hline
\end{tabular}

The VTM of thirds and intact samples were measured using SSD method and the Gmb was computed. The air void data for comparison of sample third to intact sample for each NMAS is shown in Table 67, 68, and 69.

Table 67: Comparison of Sample Third Air Void to Entire Sample Air Void for $12.5 \mathrm{~mm}$ NMAS mix

\begin{tabular}{|c|c|c|c|c|c|c|c|c|c|}
\cline { 2 - 9 } \multicolumn{1}{c|}{} & \multicolumn{3}{c|}{ VTM \% } & \multicolumn{3}{c|}{ Ranking } & \multicolumn{2}{c|}{} \\
\hline & Sample & T & B & T & M & B & $\begin{array}{c}\text { Thirds } \\
\text { Average, } \\
\%\end{array}$ & $\begin{array}{c}\text { Intact } \\
\text { Sample, } \\
\%\end{array}$ & $\begin{array}{c}\text { Difference, } \\
\%\end{array}$ \\
\hline 1 & 7.5 & 6.9 & 8.0 & 0 & -1 & 1 & 7.4 & 7.4 & 0.0 \\
\hline 2 & 8.2 & 7.0 & 7.4 & 1 & -1 & 0 & 7.5 & 7.4 & 0.1 \\
\hline 3 & 8.1 & 6.8 & 6.5 & 1 & 0 & -1 & 7.1 & 7.1 & 0.0 \\
\hline 31 a. & 8.0 & 7.0 & 6.3 & 1 & 0 & -1 & 7.1 & 6.9 & 0.2 \\
\hline 31 b. & 7.5 & 6.3 & 7.2 & 1 & -1 & 0 & 7.0 & 6.9 & 0.1 \\
\hline 31 c. & 7.8 & 6.5 & 6.3 & 1 & 0 & -1 & 6.9 & 6.6 & 0.3 \\
\hline 37 & 7.1 & 6.8 & 6.3 & 1 & 0 & -1 & 6.7 & 6.7 & 0.0 \\
\hline 38 & 7.8 & 7.4 & 7.0 & 1 & 0 & -1 & 7.4 & 7.4 & 0.0 \\
\hline 39 & 7.2 & 6.8 & 7.1 & 1 & -1 & 0 & 7.0 & 7.0 & 0.0 \\
\hline 55 a. & 7.6 & 8.0 & 7.2 & 0 & 1 & -1 & 7.6 & 7.5 & 0.1 \\
\hline 55 b. & 8.0 & 7.7 & 7.1 & 1 & 0 & -1 & 7.6 & 7.5 & 0.1 \\
\hline 55 c. & 7.2 & 8.0 & 7.2 & 0 & 1 & 0 & 7.5 & 7.5 & 0.0 \\
\hline 67 & 7.6 & 7.5 & 7.3 & 1 & 0 & -1 & 7.5 & 7.5 & 0.0 \\
\hline 68 & 7.5 & 7.6 & 7.3 & 0 & 1 & -1 & 7.5 & 7.5 & 0.0 \\
\hline 69 & 7.6 & 8.0 & 7.6 & 0 & 1 & 0 & 7.7 & 7.5 & 0.2 \\
\hline 85 & 7.3 & 7.1 & 7.0 & 1 & 0 & -1 & 7.2 & 7.2 & 0.0 \\
\hline 86 & 7.7 & 7.6 & 7.5 & 1 & 0 & -1 & 7.6 & 7.4 & 0.2 \\
\hline 87 & 7.5 & 7.4 & 7.5 & 1 & 0 & 1 & 7.5 & 7.5 & 0.0 \\
\hline
\end{tabular}


Table 68: Comparison of Sample Third Air Void to Entire Sample Air Void for 19 mm NMAS mix

\begin{tabular}{|c|c|c|c|c|c|c|c|c|c|}
\hline & \multicolumn{3}{|c|}{ VTM \% } & \multicolumn{3}{|c|}{ Ranking } & \multirow[b]{2}{*}{$\begin{array}{c}\text { Thirds } \\
\text { Average, } \\
\%\end{array}$} & \multirow[b]{2}{*}{$\begin{array}{c}\text { Intact } \\
\text { Sample, \% }\end{array}$} & \multirow[b]{2}{*}{$\begin{array}{c}\text { Difference, } \\
\%\end{array}$} \\
\hline Sample & $\mathrm{T}$ & M & B & $\mathrm{T}$ & M & B & & & \\
\hline 8 & 6.1 & 6.4 & 7.6 & -1 & 0 & 1 & 6.7 & 6.7 & 0.0 \\
\hline 9 & 6.6 & 5.8 & 7.2 & 0 & -1 & 1 & 6.5 & 6.5 & 0.0 \\
\hline 10 & 6.6 & 7.3 & 5.8 & 0 & 1 & -1 & 6.6 & 6.6 & 0.0 \\
\hline 33 a. & 7.9 & 6.0 & 6.3 & 1 & -1 & 0 & 6.7 & 6.5 & 0.2 \\
\hline $33 \mathrm{~b}$. & 8.1 & 6.3 & 6.1 & 1 & 0 & -1 & 6.8 & 6.6 & 0.2 \\
\hline $33 \mathrm{c}$. & 7.0 & 6.6 & 6.5 & 1 & 0 & -1 & 6.7 & 6.5 & 0.2 \\
\hline 43 & 8.2 & 7.3 & 7.2 & 1 & 0 & -1 & 7.5 & 7.4 & 0.1 \\
\hline 44 & 7.8 & 6.5 & 7.4 & 1 & -1 & 0 & 7.2 & 7.0 & 0.2 \\
\hline 45 & 8.1 & 7.2 & 7.6 & 1 & -1 & 0 & 7.6 & 7.4 & 0.2 \\
\hline 59 a. & 6.4 & 7.2 & 6.9 & -1 & 1 & 0 & 6.9 & 6.6 & 0.3 \\
\hline $59 \mathrm{~b}$. & 7.1 & 8.1 & 7.3 & -1 & 1 & 0 & 7.5 & 7.5 & 0.0 \\
\hline $59 \mathrm{c}$. & 7.8 & 8.3 & 7.3 & 0 & 1 & -1 & 7.8 & 7.5 & 0.3 \\
\hline 73 & 7.3 & 7.9 & 7.4 & -1 & 1 & 0 & 7.5 & 7.5 & 0.0 \\
\hline 74 & 6.7 & 7.1 & 6.6 & 0 & 1 & -1 & 6.8 & 6.5 & 0.3 \\
\hline 75 & 7.9 & 7.5 & 7.1 & 1 & 0 & -1 & 7.5 & 7.2 & 0.3 \\
\hline 91 & 7.1 & 6.5 & 7.4 & 0 & -1 & 1 & 7.0 & 7.0 & 0.0 \\
\hline 92 & 8.0 & 7.2 & 7.4 & 1 & -1 & 0 & 7.5 & 7.5 & 0.0 \\
\hline 93 & 7.2 & 6.8 & 6.9 & 1 & -1 & 0 & 6.9 & 6.9 & 0.0 \\
\hline
\end{tabular}


Table 69: Comparison of Sample Third Air Void to Entire Sample Air Void for 25 mm NMAS mix

\begin{tabular}{|c|c|c|c|c|c|c|c|c|c|}
\cline { 2 - 9 } \multicolumn{1}{c|}{} & \multicolumn{3}{c|}{ VTM \% } & \multicolumn{3}{c|}{ Ranking } & \multicolumn{2}{c|}{} \\
\hline Sample & $\mathrm{T}$ & $\mathrm{M}$ & $\mathrm{B}$ & $\mathrm{T}$ & $\mathrm{M}$ & $\mathrm{B}$ & $\begin{array}{c}\text { Thirds } \\
\text { Average, } \%\end{array}$ & $\begin{array}{c}\text { Intact } \\
\text { Sample, \% }\end{array}$ & $\begin{array}{c}\text { Difference, } \\
\%\end{array}$ \\
\hline 15 & $5.8 \%$ & $6.1 \%$ & $8.4 \%$ & -1 & 0 & 1 & 6.8 & 6.8 & 0.0 \\
\hline 16 & $6.8 \%$ & $5.3 \%$ & $8.0 \%$ & -1 & 0 & 1 & 6.7 & 6.7 & 0.0 \\
\hline 17 & $5.5 \%$ & $6.6 \%$ & $8.2 \%$ & -1 & 0 & 1 & 6.8 & 6.5 & 0.3 \\
\hline 35 a. & $7.4 \%$ & $6.5 \%$ & $8.5 \%$ & 0 & -1 & 1 & 7.5 & 7.5 & 0.0 \\
\hline 35 b. & $7.7 \%$ & $8.3 \%$ & $6.2 \%$ & 0 & 1 & -1 & 7.4 & 7.2 & 0.2 \\
\hline 35 c. & $8.5 \%$ & $7.0 \%$ & $6.3 \%$ & 1 & 0 & -1 & 7.3 & 7.2 & 0.1 \\
\hline 49 & $6.8 \%$ & $7.1 \%$ & $6.2 \%$ & 0 & 1 & -1 & 6.7 & 6.5 & 0.2 \\
\hline 50 & $7.4 \%$ & $7.0 \%$ & $6.4 \%$ & 1 & 0 & -1 & 6.9 & 6.5 & 0.4 \\
\hline 51 & $7.0 \%$ & $7.1 \%$ & $6.2 \%$ & 0 & 1 & -1 & 6.8 & 6.5 & 0.3 \\
\hline 63 a. & $7.0 \%$ & $6.7 \%$ & $6.1 \%$ & 1 & 0 & -1 & 6.6 & 6.5 & 0.1 \\
\hline 63 b. & $6.7 \%$ & $7.2 \%$ & $6.4 \%$ & 0 & 1 & -1 & 6.7 & 6.5 & 0.2 \\
\hline 63 c. & $6.9 \%$ & $7.4 \%$ & $6.8 \%$ & 0 & 1 & -1 & 7.0 & 7.0 & 0.0 \\
\hline 79 & $7.3 \%$ & $8.6 \%$ & $7.2 \%$ & 0 & 1 & -1 & 7.7 & 7.5 & 0.2 \\
\hline 80 & $6.0 \%$ & $7.2 \%$ & $7.8 \%$ & -1 & 0 & 1 & 7.0 & 6.8 & 0.2 \\
\hline 81 & $6.9 \%$ & $8.1 \%$ & $7.3 \%$ & -1 & 1 & 0 & 7.4 & 7.1 & 0.3 \\
\hline 97 & $7.3 \%$ & $6.5 \%$ & $6.7 \%$ & 1 & -1 & 0 & 6.8 & 6.8 & 0.0 \\
\hline 98 & $7.0 \%$ & $6.7 \%$ & $6.5 \%$ & 1 & 0 & -1 & 6.7 & 6.7 & 0.0 \\
\hline 99 & $7.8 \%$ & $7.1 \%$ & $7.3 \%$ & 1 & -1 & 0 & 7.4 & 7.2 & 0.2 \\
\hline
\end{tabular}

The analysis of thirds and intact samples show that in some cases the air void of sections were higher than the air void of intact samples. The possible reasoning is when the thirds were sawed, an aggregate might have chipped towards the end from the edge. This gap from the chipped aggregate might have caused an increase in air void.

With most compactive efforts in the middle section, the section should have lowest air void content. Although few samples demonstrated this behavior, it was not the same for all the samples. The segments were assigned 1,0, or -1 to highest, second highest, and lowest air void measurement for each sample third. In $12.5 \mathrm{~mm}$ NMAS mix, ST 6 showed consistent results with lowest air voids in the lower section and highest in the top section. Additionally, ST 6 had the lowest variation in air void between thirds. In 19 mm NMAS mix, ST 2, ST 3, and ST 4 showed consistent results. However, ST 4 had the lowest variation in air void between the thirds. ST 4 demonstrated lowest air voids in the top section and highest in the middle section. In 25 mm NMAS mix, ST 1 was most consistent with the ranking. The 
lowest air voids were observed in the top section and the highest air voids in the top section. ST 6 showed lowest variation in the air voids between the thirds.

The results for air void uniformity are shown in Table 70 to 72 . All tests used a 95 percent confidence level, alpha of 0.05 . The null hypothesis for all tests was the mean $\mathrm{G}_{\mathrm{mb}}$ of the two data sets are equal. Failure to reject the null hypothesis indicates the mean $\mathrm{G}_{\mathrm{mb}}$ of each data set are statistically same. The precision is calculated per AASHTO T 312-15. The precision values show that the comparison of thirds that does not fall within the precision range should only be considered for statistical analysis. And therefore, if the comparison of third is within the limits of precision, they are statistically same. Per AASHTO T 312-15, the precision of percent relative density for $12.5 \mathrm{~mm}$ NMAS should be within the range of 0.3 and 0.9 . The precision of percent relative density for $19 \mathrm{~mm}$ and $25 \mathrm{~mm}$ NMAS should be within the range of 0.5 and 1.4

Table 70: Statistical Analysis of Air Void Variability for Different STs in 12.5 mm NMAS Mix

\begin{tabular}{|c|c|c|c|c|c|c|c|c|}
\hline & \multicolumn{8}{|c|}{$12.5 \mathrm{~mm}$ NMAS } \\
\hline Comparison & $\begin{array}{c}\text { Unique Sample } \\
\text { Number }\end{array}$ & Gmb & $\begin{array}{l}\text { Mean } \\
\text { f } \\
\text { arison }\end{array}$ & Precision & $\begin{array}{l}\text { Varia } \\
\text { comb }\end{array}$ & $\begin{array}{l}\text { nce of } \\
\text { arison }\end{array}$ & & \\
\hline T-M & 1 & 2.26 & 2.28 & $1.1 \%$ & $9.2 \mathrm{E}-05$ & 6.1E-06 & & 0.02 \\
\hline M-B & 2 & 2.28 & 2.27 & $0.5 \%$ & $6.1 \mathrm{E}-06$ & 3.4E-04 & & 0.21 \\
\hline B-T & 3 & 2.27 & 2.26 & $0.6 \%$ & $3.4 \mathrm{E}-04$ & $9.2 \mathrm{E}-05$ & & 0.16 \\
\hline T-M & 31 a. & 2.26 & 2.29 & $1.2 \%$ & $4.0 \mathrm{E}-05$ & $7.2 \mathrm{E}-05$ & & 0.01 \\
\hline M-B & $31 \mathrm{~b}$. & 2.29 & 2.29 & $0.0 \%$ & $7.2 \mathrm{E}-05$ & $1.5 \mathrm{E}-04$ & & 0.47 \\
\hline B-T & $31 \mathrm{c}$. & 2.29 & 2.26 & $1.2 \%$ & $1.5 \mathrm{E}-04$ & 4.0E-05 & & 0.02 \\
\hline T-M & 37 & 2.27 & 2.28 & $0.4 \%$ & $7.5 \mathrm{E}-05$ & $7.2 \mathrm{E}-05$ & & 0.13 \\
\hline M-B & 38 & 2.28 & 2.28 & $0.2 \%$ & $7.2 \mathrm{E}-05$ & $1.2 \mathrm{E}-04$ & & 0.31 \\
\hline B-T & 39 & 2.28 & 2.27 & $0.6 \%$ & $1.2 \mathrm{E}-04$ & 7.5E-05 & 5 & 0.09 \\
\hline T-M & 55 a. & 2.26 & 2.26 & $0.2 \%$ & $1.8 \mathrm{E}-04$ & 1.7E-05 & & 0.35 \\
\hline M-B & $55 \mathrm{~b}$. & 2.26 & 2.28 & $0.8 \%$ & $1.7 \mathrm{E}-05$ & $2.4 \mathrm{E}-06$ & & 0.06 \\
\hline B-T & $55 \mathrm{c}$. & 2.28 & 2.26 & $0.5 \%$ & $2.4 \mathrm{E}-06$ & $1.8 \mathrm{E}-04$ & & 0.21 \\
\hline T-M & 67 & 2.26 & 2.26 & $0.2 \%$ & $1.1 \mathrm{E}-06$ & $5.0 \mathrm{E}-05$ & & 0.23 \\
\hline M-B & 68 & 2.26 & 2.27 & $0.3 \%$ & $5.0 \mathrm{E}-05$ & $1.5 \mathrm{E}-05$ & & 0.10 \\
\hline B-T & 69 & 2.27 & 2.26 & $0.2 \%$ & $1.5 \mathrm{E}-05$ & $1.1 \mathrm{E}-06$ & & 0.12 \\
\hline T-M & 85 & 2.27 & 2.27 & $0.1 \%$ & $1.9 \mathrm{E}-05$ & $3.8 \mathrm{E}-05$ & & 0.27 \\
\hline M-B & 86 & 2.27 & 2.27 & $0.1 \%$ & $3.8 \mathrm{E}-05$ & 4.4E-05 & & 0.37 \\
\hline B-T & 87 & 2.27 & 2.27 & $0.2 \%$ & 4.4E-05 & $1.9 \mathrm{E}-05$ & & 0.19 \\
\hline
\end{tabular}

* Bold font indicates the null hypothesis of equal means is rejected. 
Table 71: Statistical Analysis of Air Void Variability for Different STs in 19 mm NMAS Mix

\begin{tabular}{|c|c|c|c|c|c|c|c|c|}
\hline & \multicolumn{8}{|c|}{$19 \mathrm{~mm}$ NMAS } \\
\hline Comparison & $\begin{array}{l}\text { Unique Sample } \\
\text { Number }\end{array}$ & \multicolumn{2}{|c|}{$\begin{array}{c}\text { Gmb Mean } \\
\text { of } \\
\text { comparison }\end{array} \mid$} & Precision & \multicolumn{2}{|c|}{$\begin{array}{l}\text { Variance of } \\
\text { comparison }\end{array}$} & & \\
\hline T-M & 8 & 2.34 & 2.35 & $0.5 \%$ & $5.6 \mathrm{E}-05$ & $9.1 \mathrm{E}-05$ & \multirow{18}{*}{5} & 0.10 \\
\hline M-B & 9 & 2.35 & 2.32 & $1.5 \%$ & $9.1 \mathrm{E}-05$ & 2.7E-05 & & 5.4E-03 \\
\hline B-T & 10 & 2.32 & 2.34 & $1.1 \%$ & 2.7E-05 & $5.6 \mathrm{E}-05$ & & 4.6E-03 \\
\hline $\mathrm{T}-\mathrm{M}$ & $33 \mathrm{a}$. & 2.31 & 2.34 & $1.5 \%$ & 1.9E-04 & $5.0 \mathrm{E}-05$ & & 0.02 \\
\hline M-B & $33 \mathrm{~b}$. & 2.34 & 2.34 & $0.0 \%$ & 5.0E-05 & $2.1 \mathrm{E}-05$ & & 0.50 \\
\hline B-T & $33 \mathrm{c}$. & 2.34 & 2.31 & $1.5 \%$ & 2.1E-05 & $1.9 \mathrm{E}-04$ & & 0.03 \\
\hline T-M & 43 & 2.30 & 2.33 & $1.3 \%$ & 2.6E-05 & $1.5 \mathrm{E}-04$ & & 0.10 \\
\hline M-B & 44 & 2.33 & 2.31 & $0.7 \%$ & $1.5 \mathrm{E}-04$ & 1.4E-05 & & 0.16 \\
\hline B-T & 45 & 2.31 & 2.30 & $0.5 \%$ & $1.4 \mathrm{E}-05$ & 2.6E-05 & & 0.06 \\
\hline T-M & 59 a. & 2.32 & 2.30 & $0.8 \%$ & $1.4 \mathrm{E}-04$ & $2.0 \mathrm{E}-05$ & & 0.14 \\
\hline M-B & $59 \mathrm{~b}$. & 2.30 & 2.32 & $1.0 \%$ & 2.0E-05 & $3.1 \mathrm{E}-07$ & & 0.05 \\
\hline B-T & $59 \mathrm{c}$. & 2.32 & 2.32 & $0.1 \%$ & $3.1 \mathrm{E}-07$ & 1.4E-04 & & 0.40 \\
\hline $\mathrm{T}-\mathrm{M}$ & 73 & 2.32 & 2.32 & $0.0 \%$ & 4.1E-04 & $5.4 \mathrm{E}-05$ & & 0.49 \\
\hline M-B & 74 & 2.32 & 2.33 & $0.5 \%$ & $5.4 \mathrm{E}-05$ & $5.7 \mathrm{E}-05$ & & 0.13 \\
\hline B-T & 75 & 2.33 & 2.32 & $0.5 \%$ & 5.7E-05 & $4.1 \mathrm{E}-04$ & & 0.29 \\
\hline T-M & 91 & 2.31 & 2.33 & $0.6 \%$ & 2.1E-04 & $4.0 \mathrm{E}-05$ & & 0.21 \\
\hline M-B & 92 & 2.33 & 2.32 & $0.2 \%$ & 4.0E-05 & $9.1 \mathrm{E}-05$ & & 0.32 \\
\hline B-T & 93 & 2.32 & 2.31 & $0.4 \%$ & 9.1E-05 & $2.1 \mathrm{E}-04$ & & 0.25 \\
\hline
\end{tabular}

* Bold font indicates the null hypothesis of equal means is rejected. 
Table 72: Statistical Analysis of Air Void Variability for Different STs in 25 mm NMAS Mix

\begin{tabular}{|c|c|c|c|c|c|c|c|c|}
\hline \multirow{3}{*}{\begin{tabular}{|c|} 
Comparison \\
$\mathrm{T}-\mathrm{M}$ \\
\end{tabular}} & \multicolumn{8}{|c|}{$25 \mathrm{~mm}$ NMAS } \\
\hline & \multirow{2}{*}{$\begin{array}{c}\begin{array}{c}\text { Unique Sample } \\
\text { Number }\end{array} \\
15\end{array}$} & \multicolumn{2}{|c|}{$\begin{array}{c}\text { Gmb Mean } \\
\text { of } \\
\text { comparison }\end{array} \mid$} & \multirow{2}{*}{$\begin{array}{c}\text { Precision } \\
0.0 \%\end{array}$} & \multicolumn{2}{|c|}{$\begin{array}{l}\text { Variance of } \\
\text { comparison }\end{array}$} & $\mathrm{df}$ & \multirow{2}{*}{$\frac{\text { P-Value* }}{0.49}$} \\
\hline & & 2.39 & 2.39 & & $3.1 \mathrm{E}-04$ & 2.8E-04 & \multirow{18}{*}{5} & \\
\hline M-B & 16 & 2.39 & 2.34 & $2.4 \%$ & $2.8 \mathrm{E}-04$ & 3.0E-05 & & 0.02 \\
\hline B-T & 17 & 2.34 & 2.39 & $2.4 \%$ & $3.0 \mathrm{E}-05$ & $3.1 \mathrm{E}-04$ & & 0.02 \\
\hline T-M & $35 \mathrm{a}$. & 2.37 & 2.36 & $0.3 \%$ & $2.0 \mathrm{E}-05$ & 8.9E-05 & & 0.19 \\
\hline M-B & $35 \mathrm{~b}$. & 2.36 & 2.38 & $0.7 \%$ & $8.9 \mathrm{E}-05$ & 6.2E-05 & & 0.04 \\
\hline $\mathrm{B}-\mathrm{T}$ & $35 \mathrm{c}$. & 2.38 & 2.37 & $0.5 \%$ & $6.2 \mathrm{E}-05$ & 2.0E-05 & & 0.06 \\
\hline T-M & 49 & 2.37 & 2.36 & $0.1 \%$ & $5.2 \mathrm{E}-05$ & $1.6 \mathrm{E}-06$ & & 0.39 \\
\hline M-B & 50 & 2.36 & 2.39 & $0.9 \%$ & $1.6 \mathrm{E}-06$ & 1.2E-05 & & 1.1E-03 \\
\hline B-T & 51 & 2.39 & 2.37 & $0.8 \%$ & $1.2 \mathrm{E}-05$ & 5.2E-05 & & 0.01 \\
\hline T-M & 63 a. & 2.35 & 2.36 & $0.6 \%$ & $2.0 \mathrm{E}-04$ & 5.7E-04 & & 0.22 \\
\hline M-B & $63 \mathrm{~b}$. & 2.36 & 2.37 & $0.3 \%$ & 5.7E-04 & 1.1E-03 & & 0.40 \\
\hline B-T & $63 \mathrm{c}$. & 2.37 & 2.35 & $0.9 \%$ & $1.1 \mathrm{E}-03$ & 2.0E-04 & & 0.20 \\
\hline $\mathrm{T}-\mathrm{M}$ & 79 & 2.37 & 2.34 & $1.3 \%$ & $2.8 \mathrm{E}-04$ & 3.3E-04 & & 0.05 \\
\hline M-B & 80 & 2.34 & 2.36 & $0.6 \%$ & $3.3 \mathrm{E}-04$ & 5.5E-05 & & 0.16 \\
\hline $\mathrm{B}-\mathrm{T}$ & 81 & 2.36 & 2.37 & $0.8 \%$ & $5.5 \mathrm{E}-05$ & 2.8E-04 & & 0.10 \\
\hline T-M & 97 & 2.36 & 2.37 & $0.6 \%$ & 1.0E-04 & 4.6E-05 & & 0.06 \\
\hline M-B & 98 & 2.37 & 2.37 & $0.1 \%$ & $4.6 \mathrm{E}-05$ & 1.2E-04 & & 0.43 \\
\hline B-T & 99 & 2.37 & 2.36 & $0.6 \%$ & $1.2 \mathrm{E}-04$ & 1.0E-04 & & 0.09 \\
\hline
\end{tabular}

$*$ Bold font indicates the null hypothesis of equal means is rejected.

The issues with lack of uniformity is evident in ST 1 and ST 2 in 12.5 and 19 mm NMAS mixes. In 25 mm NMAS mix, the lack of uniformity was observed in ST 1, ST 2, and ST 3. The issue with ST 1 was probably due to bigger dimension compared to other dimensions. Although samples were cored and trimmed from SGC pill, bigger dimension of ST would still show non uniformity of air voids. The issue with ST 2 and ST 3 is the smallest dimension compared to other dimensions. The smallest dimensions probably did not account the maximum aggregate size within the mix. Since ST 3 was seen to have uniform air void distribution in 12.5 and 19 mm NMAS mixes. This confirms that the correct sample dimension not only depends on maximum aggregate size of asphalt mix but also depends on the inscribed circle the sample was fabricated within. 


\subsection{WITHIN INSCRIBED CIRCLES AIR VOID VARIABILITY ANALYSIS}

The Analysis 5 statistically compares STs for six different inscribed circles each for three different NMAS mixes. This analysis is performed to analyze if staying within $100 \mathrm{~mm}$ inscribed circle is statistically same to other inscribed circles. The comparisons of data set using t-test are shown in Table 73.

The results for air void comparisons from 6 different inscribed circles for each of the 3 NMAS mix are shown in Table 74 to 76 . All tests used a 95 percent confidence level, alpha of 0.05 . The null hypothesis for all tests was the mean air void of the two data sets are equal. Failure to reject the null hypothesis indicates the mean air void of each data set are statistically same. Rejecting the null hypothesis implies the data sets are statistically different. In the tables of t-test results, comparisons with alpha of less than 0.05 are shown with a bold font.

Table 73: Data Set Comparisons for Different Inscribed Circles used in Sample Types

\begin{tabular}{|c|c|c|c|c|c|c|}
\hline $\mathrm{ST}$ & 1 & 2 & 3 & 4 & 5 & 6 \\
\hline 1 & $\mathrm{X}$ & & 0 & & 0 & 0 \\
\hline 2 & & $\mathrm{X}$ & 0 & & & \\
\hline 3 & & & $\mathrm{X}$ & & 0 & 0 \\
\hline 4 & & & & $\mathrm{X}$ & 0 & \\
\hline 5 & & & & & $\mathrm{X}$ & 0 \\
\hline 6 & & & & & & $\mathrm{X}$ \\
\hline
\end{tabular}


Table 74: Statistical Analysis of Air Void Variability within different inscribed Circles of Sample Types in 12.5 mm NMAS Mix

\begin{tabular}{|c|c|c|c|c|c|c|c|}
\hline \multirow{3}{*}{$\frac{\text { Comparison }}{\mathrm{T}-\mathrm{T}}$} & \multicolumn{7}{|c|}{$12.5 \mathrm{~mm}$ NMAS } \\
\hline & t- Test & \multicolumn{2}{|c|}{$\begin{array}{l}\text { VTM Mean of } \\
\text { comparison, \% }\end{array}$} & \multicolumn{2}{|c|}{ Variance of comparison } & $d f$ & \multirow{2}{*}{$\begin{array}{c}\begin{array}{c}\mathrm{P}- \\
\text { Value* }\end{array} \\
0.13\end{array}$} \\
\hline & \multirow{3}{*}{ ST1-ST3 } & 8.1 & 7.5 & 7.5E-07 & $1.8 \mathrm{E}-05$ & \multirow{24}{*}{5} & \\
\hline M-M & & 6.9 & 7.1 & $2.0 \mathrm{E}-06$ & 1.7E-05 & & 0.30 \\
\hline B-B & & 7.0 & 7.1 & 4.1E-05 & 3.7E-07 & & 0.44 \\
\hline $\mathrm{T}-\mathrm{T}$ & \multirow{3}{*}{ ST1-ST5 } & 8.1 & 7.5 & 7.5E-07 & $3.1 \mathrm{E}-07$ & & 7.6E-03 \\
\hline M-M & & 6.9 & 7.8 & 2.0E-06 & 7.9E-06 & & 0.07 \\
\hline B-B & & 7.0 & 7.4 & 4.1E-05 & $3.8 \mathrm{E}-06$ & & 0.25 \\
\hline $\mathrm{T}-\mathrm{T}$ & \multirow{3}{*}{ ST1-ST6 } & 8.1 & 7.6 & 7.5E-07 & $1.2 \mathrm{E}-06$ & & 0.02 \\
\hline M-M & & 6.9 & 7.5 & $2.0 \mathrm{E}-06$ & $2.0 \mathrm{E}-06$ & & 0.02 \\
\hline B-B & & 7.0 & 7.5 & $4.1 \mathrm{E}-05$ & $5.1 \mathrm{E}-08$ & & 0.23 \\
\hline $\mathrm{T}-\mathrm{T}$ & \multirow{3}{*}{ ST2-ST3 } & 7.7 & 7.5 & $6.8 \mathrm{E}-06$ & $1.8 \mathrm{E}-05$ & & 0.32 \\
\hline M-M & & 6.4 & 7.1 & $2.1 \mathrm{E}-06$ & $1.7 \mathrm{E}-05$ & & 0.14 \\
\hline B-B & & 6.7 & 7.1 & 4.0E-05 & 3.7E-07 & & 0.31 \\
\hline $\mathrm{T}-\mathrm{T}$ & \multirow{3}{*}{ ST3-ST5 } & 7.5 & 7.5 & $1.8 \mathrm{E}-05$ & $3.1 \mathrm{E}-07$ & & 0.41 \\
\hline M-M & & 7.1 & 7.8 & 1.7E-05 & 7.9E-06 & & 0.08 \\
\hline B-B & & 7.1 & 7.4 & 3.7E-07 & $3.8 \mathrm{E}-06$ & & 0.11 \\
\hline $\mathrm{T}-\mathrm{T}$ & \multirow{3}{*}{ ST3-ST6 } & 7.5 & 7.6 & $1.8 \mathrm{E}-05$ & $1.2 \mathrm{E}-06$ & & 0.35 \\
\hline M-M & & 7.1 & 7.5 & 1.7E-05 & $2.0 \mathrm{E}-06$ & & 0.19 \\
\hline B-B & & 7.1 & 7.5 & 3.7E-07 & 5.1E-08 & & 0.03 \\
\hline $\mathrm{T}-\mathrm{T}$ & \multirow{3}{*}{ ST4-ST5 } & 7.6 & 7.5 & $3.1 \mathrm{E}-05$ & $3.1 \mathrm{E}-07$ & & 0.43 \\
\hline M-M & & 7.9 & 7.8 & $2.9 \mathrm{E}-06$ & 7.9E-06 & & 0.47 \\
\hline B-B & & 7.1 & 7.4 & 4.0E-07 & $3.8 \mathrm{E}-06$ & & 0.14 \\
\hline $\mathrm{T}-\mathrm{T}$ & \multirow{3}{*}{ ST5-ST6 } & 7.5 & 7.6 & $3.1 \mathrm{E}-07$ & $1.2 \mathrm{E}-06$ & & 0.29 \\
\hline M-M & & 7.8 & 7.5 & 7.9E-06 & 2.0E-06 & & 0.21 \\
\hline B-B & & 7.4 & 7.5 & $3.8 \mathrm{E}-06$ & $5.1 \mathrm{E}-08$ & & 0.41 \\
\hline
\end{tabular}

$*$ Bold font indicates the null hypothesis of equal means is rejected. 
Table 75: Statistical Analysis of Air Void Variability within different inscribed Circles of Sample Types in 19 mm NMAS Mix

\begin{tabular}{|c|c|c|c|c|c|c|c|}
\hline \multirow{3}{*}{$\begin{array}{c}\begin{array}{c}\text { Compariso } \\
\mathrm{n}\end{array} \\
\mathrm{T}-\mathrm{T}\end{array}$} & \multicolumn{7}{|c|}{19 mm NMAS } \\
\hline & t- Test & \multicolumn{2}{|c|}{$\begin{array}{l}\text { VTM Mean of } \\
\text { comparison, \% }\end{array}$} & \multicolumn{2}{|c|}{ Variance of comparison } & $d f$ & \multirow{2}{*}{$\begin{array}{c}\begin{array}{c}\mathrm{P}- \\
\text { Value* }\end{array} \\
\mathbf{0 . 0 3}\end{array}$} \\
\hline & \multirow{3}{*}{ ST1-ST3 } & 6.6 & 8.0 & 1.7E-07 & $4.1 \mathrm{E}-06$ & \multirow{24}{*}{5} & \\
\hline M-M & & 6.5 & 6.8 & $1.2 \mathrm{E}-04$ & $2.5 \mathrm{E}-05$ & & 0.40 \\
\hline B-B & & 6.5 & 7.5 & 1.1E-04 & $2.3 \mathrm{E}-06$ & & 0.21 \\
\hline $\mathrm{T}-\mathrm{T}$ & \multirow{3}{*}{ ST1-ST5 } & 6.6 & 7.3 & $1.7 \mathrm{E}-07$ & $6.5 \mathrm{E}-05$ & & 0.21 \\
\hline M-M & & 6.5 & 7.3 & $1.2 \mathrm{E}-04$ & 8.6E-06 & & 0.26 \\
\hline B-B & & 6.5 & 6.8 & $1.1 \mathrm{E}-04$ & $9.1 E-06$ & & 0.37 \\
\hline $\mathrm{T}-\mathrm{T}$ & \multirow{3}{*}{ ST1-ST6 } & 6.6 & 7.6 & 1.7E-07 & $3.3 E-05$ & & 0.13 \\
\hline M-M & & 6.5 & 7.0 & $1.2 \mathrm{E}-04$ & $6.4 \mathrm{E}-06$ & & 0.34 \\
\hline B-B & & 6.5 & 7.2 & $1.1 \mathrm{E}-04$ & $1.5 \mathrm{E}-05$ & & 0.28 \\
\hline $\mathrm{T}-\mathrm{T}$ & \multirow{3}{*}{ ST2-ST3 } & 7.6 & 8.0 & $5.5 \mathrm{E}-05$ & 4.1E-06 & & 0.30 \\
\hline M-M & & 6.4 & 6.8 & $3.4 \mathrm{E}-06$ & $2.5 \mathrm{E}-05$ & & 0.24 \\
\hline B-B & & 6.3 & 7.5 & 6.6E-06 & $2.3 \mathrm{E}-06$ & & 0.01 \\
\hline $\mathrm{T}-\mathrm{T}$ & \multirow{3}{*}{ ST3-ST5 } & 8.0 & 7.3 & 4.1E-06 & $6.5 \mathrm{E}-05$ & & 0.23 \\
\hline M-M & & 6.8 & 7.3 & 2.5E-05 & 8.6E-06 & & 0.18 \\
\hline B-B & & 7.5 & 6.8 & 2.3E-06 & $9.1 \mathrm{E}-06$ & & 0.11 \\
\hline $\mathrm{T}-\mathrm{T}$ & \multirow{3}{*}{ ST3-ST6 } & 8.0 & 7.6 & 4.1E-06 & $3.3 \mathrm{E}-05$ & & 0.26 \\
\hline M-M & & 6.8 & 7.0 & $2.5 \mathrm{E}-05$ & $6.4 \mathrm{E}-06$ & & 0.37 \\
\hline B-B & & 7.5 & 7.2 & 2.3E-06 & $1.5 \mathrm{E}-05$ & & 0.23 \\
\hline $\mathrm{T}-\mathrm{T}$ & \multirow{3}{*}{ ST4-ST5 } & 7.4 & 7.3 & 2.3E-05 & $6.5 \mathrm{E}-05$ & & 0.44 \\
\hline M-M & & 8.2 & 7.3 & $3.2 \mathrm{E}-06$ & 8.6E-06 & & 0.03 \\
\hline B-B & & 7.3 & 6.8 & 5.0E-08 & $9.1 \mathrm{E}-06$ & & 0.13 \\
\hline $\mathrm{T}-\mathrm{T}$ & \multirow{3}{*}{ ST5-ST6 } & 7.3 & 7.6 & $6.5 \mathrm{E}-05$ & $3.3 \mathrm{E}-05$ & & 0.38 \\
\hline M-M & & 7.3 & 7.0 & 8.6E-06 & $6.4 \mathrm{E}-06$ & & 0.19 \\
\hline B-B & & 6.8 & 7.2 & $9.1 \mathrm{E}-06$ & $1.5 \mathrm{E}-05$ & & 0.23 \\
\hline
\end{tabular}

* Bold font indicates the null hypothesis of equal means is rejected. 
Table 76: Statistical Analysis of Air Void Variability within different inscribed Circles of Sample Types in 25 mm NMAS Mix

\begin{tabular}{|c|c|c|c|c|c|c|c|}
\hline \multirow{3}{*}{$\begin{array}{c}\text { Comparison } \\
\mathrm{T}-\mathrm{T}\end{array}$} & \multicolumn{7}{|c|}{$25 \mathrm{~mm}$ NMAS } \\
\hline & t- Test & \multicolumn{2}{|c|}{$\begin{array}{l}\text { VTM Mean of } \\
\text { comparison, \% }\end{array}$} & \multicolumn{2}{|c|}{ Variance of comparison } & $\mathrm{df}$ & $\begin{array}{c}\text { P- } \\
\text { Value* }\end{array}$ \\
\hline & \multirow{3}{*}{$\begin{array}{l}\text { ST1- } \\
\text { ST3 }\end{array}$} & 6.1 & 7.2 & $8.5 \mathrm{E}-05$ & $7.9 \mathrm{E}-06$ & \multirow{24}{*}{5} & 0.19 \\
\hline M-M & & 6.0 & 7.1 & 8.3E-05 & $3.4 \mathrm{E}-07$ & & 0.17 \\
\hline B-B & & 8.1 & 6.3 & 2.0E-06 & $2.5 \mathrm{E}-06$ & & 3.5E-03 \\
\hline $\mathrm{T}-\mathrm{T}$ & \multirow{3}{*}{$\begin{array}{l}\text { ST1- } \\
\text { ST5 }\end{array}$} & 6.1 & 6.4 & 8.5E-05 & $3.5 \mathrm{E}-05$ & & 0.37 \\
\hline $\mathrm{M}-\mathrm{M}$ & & 6.0 & 7.6 & 8.3E-05 & $3.6 \mathrm{E}-05$ & & 0.08 \\
\hline B-B & & 8.1 & 7.5 & 2.0E-06 & $9.5 \mathrm{E}-06$ & & 0.13 \\
\hline $\mathrm{T}-\mathrm{T}$ & \multirow{3}{*}{$\begin{array}{l}\text { ST1- } \\
\text { ST6 }\end{array}$} & 6.1 & 7.4 & $8.5 \mathrm{E}-05$ & $3.2 \mathrm{E}-05$ & & 0.12 \\
\hline M-M & & 6.0 & 6.9 & 8.3E-05 & $6.2 \mathrm{E}-06$ & & 0.20 \\
\hline B-B & & 8.1 & 6.9 & $2.0 \mathrm{E}-06$ & 3.3E-05 & & 0.11 \\
\hline $\mathrm{T}-\mathrm{T}$ & \multirow{3}{*}{$\begin{array}{l}\text { ST2- } \\
\text { ST3 }\end{array}$} & 6.8 & 7.2 & $3.5 \mathrm{E}-06$ & 7.9E-06 & & 0.14 \\
\hline M-M & & 7.3 & 7.1 & $2.3 \mathrm{E}-06$ & $3.4 \mathrm{E}-07$ & & 0.15 \\
\hline B-B & & 6.6 & 6.3 & 7.1E-06 & $2.5 \mathrm{E}-06$ & & 0.17 \\
\hline $\mathrm{T}-\mathrm{T}$ & \multirow{3}{*}{$\begin{array}{l}\text { ST3- } \\
\text { ST5 }\end{array}$} & 7.2 & 6.4 & 7.9E-06 & $3.5 \mathrm{E}-05$ & & 0.18 \\
\hline M-M & & 7.1 & 7.6 & $3.4 \mathrm{E}-07$ & $3.6 \mathrm{E}-05$ & & 0.20 \\
\hline B-B & & 6.3 & 7.5 & $2.5 \mathrm{E}-06$ & 9.5E-06 & & 0.06 \\
\hline $\mathrm{T}-\mathrm{T}$ & \multirow{3}{*}{$\begin{array}{l}\text { ST3- } \\
\text { ST6 }\end{array}$} & 7.2 & 7.4 & 7.9E-06 & $3.2 \mathrm{E}-05$ & & 0.34 \\
\hline M-M & & 7.1 & 6.9 & $3.4 \mathrm{E}-07$ & $6.2 \mathrm{E}-06$ & & 0.25 \\
\hline B-B & & 6.3 & 6.9 & $2.5 \mathrm{E}-06$ & $3.3 \mathrm{E}-05$ & & 0.19 \\
\hline $\mathrm{T}-\mathrm{T}$ & \multirow{3}{*}{$\begin{array}{l}\text { ST4- } \\
\text { ST5 }\end{array}$} & 8.1 & 6.4 & $2.9 \mathrm{E}-05$ & $3.5 \mathrm{E}-05$ & & 0.05 \\
\hline M-M & & 7.7 & 7.6 & $8.2 \mathrm{E}-05$ & $3.6 \mathrm{E}-05$ & & 0.48 \\
\hline B-B & & 6.3 & 7.5 & $9.5 \mathrm{E}-07$ & $9.5 \mathrm{E}-06$ & & 0.06 \\
\hline $\mathrm{T}-\mathrm{T}$ & \multirow{3}{*}{$\begin{array}{l}\text { ST5- } \\
\text { ST6 }\end{array}$} & 6.4 & 7.4 & $3.5 \mathrm{E}-05$ & $3.2 \mathrm{E}-05$ & & 0.12 \\
\hline M-M & & 7.6 & 6.9 & $3.6 \mathrm{E}-05$ & $6.2 \mathrm{E}-06$ & & 0.18 \\
\hline B-B & & 7.5 & 6.9 & $9.5 \mathrm{E}-06$ & $3.3 E-05$ & & 0.15 \\
\hline
\end{tabular}

$*$ Bold font indicates the null hypothesis of equal means is rejected.

The results show that air voids obtained from data set comparisons: ST3-ST5 and ST5-ST6 are statistically same from all the three NMAS mixes. This concludes that air voids of sampled fabricated from within $38 \mathrm{~mm}$ inscribed circle are statistically same to $50 \mathrm{~mm}$ inscribed circle. Also, means of air voids fabricated from within $50 \mathrm{~mm}$ inscribed circle are statistically same to $75 \mathrm{~mm}$ inscribed circle. Although in $12.5 \mathrm{~mm}$ and $25 \mathrm{~mm}$, ST2-ST3 and ST4-ST5 comparisons were statistically same. This tells us that the air voids obtained from $104 \mathrm{~mm}$ inscribed circle is same as $38 \mathrm{~mm}$ inscribed circle. And air 
voids obtained from $120 \mathrm{~mm}$ inscribed circle is same as $50 \mathrm{~mm}$ inscribed circle. Therefore, more variability in air void distribution is caused axially than radially. 


\section{CHAPTER 5. SUMMARY AND RECOMMENDATIONS}

This research used the Asphalt Mixture Performance Tester to evaluate six sample types, including six different inscribed circles and four different sample dimensions using three NMAS mix types, $12.5 \mathrm{~mm}, 19 \mathrm{~mm}$, and $25 \mathrm{~mm}$. All samples are fabricated at air voids of $7 \% \pm 0.5 \%$.

Dynamic modulus, rutting, and fatigue tests were performed in AMPT and the data were analyzed using FlexPAVE. In addition to FlexPAVE, statistical analysis such as ANOVA, MANOVA and t-tests were performed. In addition, sample air void variability was investigated by cutting the sample into three equal thirds and fabricating different STs from within different inscribed circles.

Five different analysis were performed in this research and the first analysis evaluated rankings of different STs by comparing them to ST1. For data analysis all STs are compared to ST1 because it is full size AASHTO standard dimension used for up to $37.5 \mathrm{~mm}$ NMAS mixes for dynamic modulus and rutting test and for up to $25 \mathrm{~mm}$ NMAS mixes for fatigue test. This analysis used results from master curve to capture time and frequency relationship to dynamic modulus for different STs. The master curves were also used to examine rutting and fatigue potential of different STs for different mixes.

The comparison of fitting parameters and dynamic modulus values for STs using Master Solver shows ST6 performed very closely to ST1 for all the three different NMAS mixes. This is attributed to the same aspect ratio of ST5 and ST6. The rutting potential from dynamic modulus master curve shows ST3 performed closely to ST1 for all the three different NMAS mixes. The evaluation of fatigue potential from dynamic modulus master curve shows ST6 to be consistently close to that of ST 1 for all three NMAS mixes.

The results of $S_{\text {app }}$ to evaluate fatigue from FlexMAT Cracking shows ST 4 to be consistently close to ST 1 for all three NMAS mixes. The damage characterization curves show ST 5 in $12.5 \mathrm{~mm}$ NMAS mix, ST 4 in 19 mm NMAS mix, and ST 4 in 25 mm NMAS mix to be close to ST1.

The RSI results to evaluate rutting from FlexMAT Rutting shows wide different between ST 4 and ST1 in $12.5 \mathrm{~mm}$ and 19 mm NMAS mixes. However, closest RSI comparison between ST 1 and ST 4 was observed in 25 mm NMAS mix.

One of the other outputs for ranking analysis obtained from FlexPAVE is percentage damage and total rut depth at the end of 20 years. The percentage damage at the end of the 20 years value for model 5 
values were consistently close to model 1 for all three structures. The total rut depth at the end of 20 years for model 2 and 10 values were same as that of model 1 for all three structures.

The Analysis 2 analyzed twelve models for each of the three pavement structures in the FlexPAVE software. Upon analyzing percentage damage at the end of the 20 years values, model 5 values were consistently close to model 1 for all the three structures. Also, the total rut depth at the end of 20 years for model 2 and 10 values were same as that of model 1 for all three structures. This concludes that for both percentage damage and total rut depth at the end of 20 years, sample dimension 50X110 mm provided results closest to $100 \mathrm{X} 150 \mathrm{~mm}$ sample dimension.

The statistical analysis in Analysis 3 evaluates dynamic modulus and phase angle values for six different STs fabricated form three different NMAS. The dynamic modulus and phase angle for $12.5 \mathrm{~mm}$ NMAS mix show that ST 5 values for all three different test temperatures to be consistently closer to that of ST1. The dynamic modulus and phase angle for 19 mm NMAS mix show that ST 4 values for all three different test temperatures to be consistently closer to that of ST1. The dynamic modulus and phase angle for $25 \mathrm{~mm}$ NMAS mix show that ST 4 values for all three different test temperatures to be consistently closer to that of ST1.

The t-test results of dynamic modulus and phase values for same combinations of temperature and frequency does not follow the same pattern. Meaning, if dynamic modulus is statistically significant, phase angle may/may not be statistically significant. For $12.5 \mathrm{~mm}$ NMAS mix, over ST1-ST5 were statistically same. For 19 mm NMAS ST1-ST6 were statistically same. ST1- ST6 and ST1-ST3 were statistically same for $25 \mathrm{~mm}$. The MANOVA results showed that when considered ST1 and other STs jointly for three different NMAS mixes, there is a statistical difference for different temperatures and frequencies in dynamic modulus test. This confirms that specific ST must be used to test a give NMAS mix in dynamic modulus test.

The air void variability analysis within samples of each ST in Analysis 4 show ST6 to have consistent results between replicates with most uniform air voids for $12.5 \mathrm{~mm}$ NMAS mix. In $19 \mathrm{~mm}$ NMAS mix, ST 2, ST 3, and ST 4 showed consistent results between replicates. Out of different STs, ST 4 showed the most uniform air void distribution for 19 mm NMAS mix. In 25 mm NMAS mix, ST 1 showed consistent results between replicates. Additionally, ST 6 showed the most uniform air void distribution for $25 \mathrm{~mm}$ NMAS mix. 
The air void variability for STs fabricated from within different inscribed circle in Analysis 5 show ST3-ST5 and ST5-ST6 are statistically same for all the three different NMAS mixes. This concludes that air voids of sampled fabricated from within $38 \mathrm{~mm}$ inscribed circle are statistically same to $50 \mathrm{~mm}$ inscribed circle. Also, means of air voids fabricated from within $50 \mathrm{~mm}$ inscribed circle are statistically same to $75 \mathrm{~mm}$ inscribed circle. Although in $12.5 \mathrm{~mm}$ and $25 \mathrm{~mm}$, ST2-ST3 and ST4-ST5 comparisons were statistically same. This tells us that the air voids obtained from $104 \mathrm{~mm}$ inscribed circle is same as $38 \mathrm{~mm}$ inscribed circle. And air voids obtained from $120 \mathrm{~mm}$ inscribed circle is same as 50 $\mathrm{mm}$ inscribed circle. Therefore, more variability in air void distribution is caused axially than radially. For the ease of fabrication, ST4 is recommended in Analysis 5.

In summary, recommended sample size for different analysis are:

Table 77: Recommendations of this Research for AASHTO Tests performed in AMPT

\begin{tabular}{|c|c|c|c|}
\hline Test & $12.5 \mathrm{~mm}$ & $19 \mathrm{~mm}$ & $25 \mathrm{~mm}$ \\
\hline Dynamic Modulus & $\begin{array}{c}\text { ST5- 50X110 mm } \\
\text { from 50 mm } \\
\text { inscribed circle }\end{array}$ & \multicolumn{2}{|c|}{$\begin{array}{c}\text { ST6- 75X110 mm from 75 mm inscribed } \\
\text { circle }\end{array}$} \\
\hline DTC Fatigue & \multicolumn{2}{|c|}{ ST4- 50X110 mm inscribed from 120 mm inscribed circle } \\
\hline SSR & $\begin{array}{c}\text { ST1- 100X150 mm } \\
\text { from 100 mm } \\
\text { inscribed circle }\end{array}$ & $\begin{array}{c}\text { ST4- 50X110 mm inscribed from 120 mm } \\
\text { inscribed circle }\end{array}$ \\
\hline Air Void Uniformity & $\begin{array}{c}\text { ST6- 75X110 mm } \\
\text { from 75 mm } \\
\text { inscribed circle }\end{array}$ & $\begin{array}{c}\text { ST4- 50X110 mm } \\
\text { inscribed from 120 } \\
\text { mm inscribed circle }\end{array}$ & $\begin{array}{c}\text { ST6- 75X110 mm } \\
\text { from 75 mm } \\
\text { inscribed circle }\end{array}$ \\
\hline
\end{tabular}

This research presents state of art contribution by evaluating RVE of four sample dimensions fabricated from six different inscribed circles and three different NMAS mixes. This research has distinctively evaluated different inscribed circles and in parallel analyzed sample uniformity within different STs. The results are conclusive that more air void variability occurs axially than radially. Additionally, in this research, the unique in-depth laboratory analysis of RVE has concluded variability within different STs in three different AMPT tests and NMAS mixes. The variability is determined by comparing and ranking all STs to ST1 as it is a full size AASHTO standard dimension. This research concludes that the STs that have more uniform air void distribution performs comparable to ST1 and have low variability. ST5 for $12.5 \mathrm{~mm}$ and ST4 or ST6 for $19 \mathrm{~mm}$ and $25 \mathrm{~mm}$ NMAS mixes have uniform air void distribution from statistical analysis and performed comparable to ST1 from ranking analysis. Thus, from the evaluation of lab experiments and statistically analysis, STs should be unique to each NMAS mix. 


\section{References}

Aguilar, A. M. 2015. "Analysis of the Air Voids within Superpave Gyratory Compacted Hot Mix Asphalt Specimens." Graduate Theses, Dissertations, and Problem Reports. 5034.

Blankenship, P. and Anderson, R. M. 2010. "Laboratory Investigation of HMA Modulus, Flow Number and Flexural Fatigue on Samples of Varying Density." Journal of the Association of Asphalt Paving Technologists. Volume 79. pp 497-518.

Bennert, T. 2009. "Dynamic Modulus of Hot Mix Asphalt." New Jersey Department of Transportation Bureau of Research and Technology and U.S. Department of Transportation Federal Highway Administration. FHWA-NJ-2009-011.

Bonaquist, R., Christensen, D. W., and Stump, W. 2003. "NCHRP Report 513 Simple Performance Tester for Superpave Mix Design.” Washington, D.C.: Transportation Research Board.

Bonaquist, R. 2011. "MasterSolver: Version 2.2 [Software]." Available from http://apps.trb.org/cmsfeed/TRBNetProjectDisplay.asp?ProjectID=963.

Bowers, B. F., Diefenderfer, B. K., and Diefenderfer, S. D. 2015. "Evaluation of Dynamic Modulus in Asphalt Paving Mixtures Utilizing Small-Scale Specimen Geometries.” Asphalt Paving Technology: Association of Asphalt Paving Technologists-Proceedings of the Technical Sessions. Volume 84. pp 497-526.

Castorena, C., Kim, Y. R., Pape, S., and Lee, K. 2017. "Development of Small Specimen Geometry for Asphalt Mixture Performance Testing.” NCHRP Innovative Deserving Exploratory Analysis (IDEA) Programs N181 Final Report. Washington, D.C.: Transportation Research Board.

Chehab, G. R., O’Quinn, E., and Kim, R. Y. 2000. “Speciment Geometry for Direct Tension Test Based on Mechanical Tests and Air void Variation in Asphalt Cencerete Specimens Compacted by Superpave Gyratory Compactor.” Transportation Research Record. Volume 1723(1). pp 125-132.

Chilukwa, N. and Lungu, R. 2019. "Determination of Layers Responsible for Rutting Failure in a Pavement Structure. Infrastructures."

Christensen, D. W., and Bonaquist, R. 2009. "Analysis of HMA Fatigue Data Using the Concepts of Reduced Loading Cycles and Endurance Limit." Journal of the Association of Asphalt Paving Technologists. Volume 78.

Dalton, L. 2016. "Evaluating the protocol of the spectrum of hot mix asphalt mixes produced in West Virginia." Graduate Theses, Dissertations, and Problem Reports. 5431.

Faridmehr, I., Osman, M., Adnan, A., Nejad, A., Hodjati, R., and Amin, M. 2014. "Correlation between Engineering Stress-Strain and True Stress-Strain Curve.” American Journal of Civil Engineering and Architecture. DOI: 10.12691/ajcea-2-1-6.

Federal Highway Administration. 2013. "Asphalt Mixture Performance Tester. Office of Pavement Technology.” FHWA-HIF-13-005.

Federal Highway Administration. 2019." Highway Finance Data Collection- Our Nation's Highways: 2011." U.S. Department of Transportation. Retrieved from: https://www.fhwa.dot.gov/policyinformation/pubs/hf/pl11028/chapter1.cfm. Date Access: 04/12/2020 
FlexPAVE 1.1 Alpha. 2018. "LAYERED VISCOELASTIC PAVEMENT ANALYSIS FOR CRITICAL DISTRESSES (User Guide).” Department of Civil, Construction, and Environmental Engineering. North Carolina State University.

Ghanbari, A., Underwood, B. S., and Kim, Y. R. 2020. "Development of a Rutting Index Parameter Based on the Stress Sweep Rutting Test and Permanent Deformation Shift Model.” International Journal of Pavement Engineering.

Hou, T. 2009. "Fatigue Performance Prediction of North Carolina Mixtures Using Simplified Viscoelastic Continuum Damage Model. MS thesis.” North Carolina State University, Raleigh.

Hou, T., Underwood, B.S., and Kim, R. 2010. "Fatigue Performance Prediction of North Carolina Mixtures Using the Simplified Viscoelastic Continuum Damage Model." Journal of the Association of Asphalt Paving Technologists. Volume 79.

Karr, G. J. 2018. "Developnment of a New Test Specimen with Tapered Cross-Section for Use in Uniaxial Fatigue Testing of Hot Mix." UNIVERSITY OF HAWAI'I AT MĀNOA.

Kim, D. and Kim, Y. R. 2017. "Developnment of Stress Sweep Rutting (SSR) test for permanent deformation characterization of asphalt mixture." Construction and Building Materials. Volume 154. pp 373-383.

Kim, Y. K. 2018. "FlexMAT Cracking v1.1.2” [Software].

Kim, Y. K. 2018. "FlexMAT Rutting v1.1.2” [Software].

Kutay, M. E., Gibson, N., Youtcheff, J., \& Dongré, R. 2009. “Use of Small Samples to Predict Fatigue Lives of Field Cores: Newly Developed Formulation Based on Viscoelastic Continuum Damage Theory." Transportation Research Record. National Research Council. Volume 2127. pp 90-97.

Lee, Hyung. 2013. "Developnment of a New Solution for Viscoelastic Wave Propogation of Pavement Structures and its Use in Dynamic Backcalculation."

Lee, J. S., Norouzi, A., and Kim, Y. R. 2017a. "Determining Specimen Geometry of Cylindrical Specimens for Direct Tension Fatigue Testing of Asphalt Concrete." Journal of Testing and Evaluation. pp 613-623.

Lee, K., Pape, S., Castorena, C., and Kim, Y. R. 2017b. "Evaluation of Small Specimen Geometries for Asphalt Mixture Performance Testing and Pavement Performance Prediction.” Transportation Research Record. pp 74-82.

Li, X. and Gibson, N. H. 2012. "Using small scale specimens for AMPT dynamic modulus and fatigue tests." Asphalt Paving Technology: Association of Asphalt Paving Technologists-Proceedings of the Technical Sessions. Volume 82. pp 579-615.

Mitchell, M. R., Link, R. E., Kim, Y. R., Lee, J. and Teixeira, J. 2010. "Geometrical Evaluation and Experimental Verification to Determine Representative Volume Elements of Heterogeneous Asphalt Mixtures." Civil Engineering Faculty Publications. Volume 81.

Park H. J. and Kim, Y. R. 2013. "Investigation of Primary Causes of Fatigue Cracking in Asphalt Pavement in North Carolina." North Carolina Department of Transportation Office of ResearchFHWA/NC/2010-01.

Pellinen, T., and M.Witczak. (2002) "Use of Stiffness of Hot-Mix Asphalt as a Simple Performance Test." Transportation Research Record 1789.1: 80-90. 
Romero, P., and Masad, E. 2001. "Relationship between the Representative Volume Element and Mechanical Properties of Asphalt Concrete.” Journal of Materials in Civil Engineering. Volume 13.

Sabouri, M. 2014. "Development of a Unified Fatigue Failure Criterion for Asphalt Mixtures and Its Applications to Reclaimed Asphalt Pavement (RAP) Mixtures.” Ph. D. dissertation. North Carolina State University, Raleigh.

Sabouri, M. and Kim, Y. R. 2014. "Development of a failure criterion for asphalt mixtures under different modes of fatigue loading." Washington, D.C.: Transportation Research Board. Volume 2447. pp $117-125$.

Sun, Y., Gu, B., Gao, L., Li, L., Guo, R., Yue, Q., \& Wang, J. 2018. "Viscoelastic Mechanical Responses of HMAP under Moving Load.” Materials (Basel, Switzerland) Volume 11(12). DOI: $10.3390 / \mathrm{ma} 11122490$

Thyagarajan, S., Tashman, L., and Masad, E. 2010. "The heterogeneity and mechanical response of hot mix asphalt laboratory specimens." International Journal of Pavement Engineering.

Underwood, B. S., Kim, Y. R., and Guddati, M. N. 2009. "Improved Calculation Method of Damage Parameter in Viscoelastic Continuum Damage Model." International Journal of Pavement Engineering. Volume 11(6). pp 495-476.

Underwood, B. S., Baek, C., and Kim, Y. R. 2012. "Simplified Viscoelastic Continuum Damage Model as Platform for Asphalt Concrete Fatigue Analysis." Washington, D.C.: Transportation Research Board. Volume 2296. pp 36-45.

Wang, Y. D., Keshavarzi, B., and Kim, Y. R. 2018. "Fatigue Performance Prediction of Asphalt Pavements with FlexPAVE ${ }^{\mathrm{TM}}$, the S-VECD Model, and $\mathrm{D}^{\mathrm{R}}$ Failure Criterion Washington, D.C.: Transportation Research Board. Volume 2672(40). pp 217-227.

Wang, Y. 2019. "Development of the Framework of Performance-Engineered Mixture Design for Asphalt Concrete." Transportation Research Record: Journal of the Transportation Research Board.

Wang, Y., and Kim, Y. R. 2019. "Development of a pseudo strain energy-based fatigue failure criterion for asphalt mixtures." International Journal of Pavement Engineering. Volume 20 (10). pp 1182 1192.

Weissman, S. L. 1999. "Selection of laboratory test specimen dimension for permanent deformation of asphalt concrete pavements." Washington, D.C.: Transportation Research Board. Volume 1681. pp 113-120.

West Virginia Division of Highways Performance Audit. 2016." Prepared for the Joint Committee on Government and Highways of the West Virginia Legislature." Retrieved from: http://www.wvlegislature.gov/legisdocs/reports/agency/PA/PA_2016_572.pdf. Date Access: $04 / 12 / 2020$

West Virginia Department of Transportation Division of Highways, Material Control, Soils and Testing Division Material Procedure. MP 401.02.22. 2011. "Guide to Designing Hot Mix Asphalt Using the Marshall Design Method." Retrieved from: https://ransportation.wv.gov/highways/mcst/Material\%20Procedures/MP\%20401\%2002\%2022.p df. Date Access: 04/12/2019 
Witczak, M. W., Bonaquist, R., Von Quintus, H., and Kaloush, K. 2000. "Specimen Geometry and Aggregate Size Effects in Uniaxial Compression and Constant Height Shear Tests." Journal of the Association of Asphalt Paving Technologists. Volume 69.

Witczak, M., Mamlouk, M., Souliman, M., and Zeiada, W. 2013. NCHRP Report 9-44A, "Validating an Endurance Limit for HMA Pavements: Laboratory Experiment and Algorithm Development." Washington, D.C.: Transportation Research Board.

Zapata, C. E. and Houston, W. N. 2008. "NCHRP Report 602. Calibration and Validation of the Enhanced Integrated Climatic Model for Pavement Design.” Washington, D.C.: Transportation Research Board.

Zhang, J. 2012. "Development of Failure Criteria for Asphalt Concrete Mixtures under Fatigue Loading." MS thesis. North Carolina State University, Raleigh.

Zhang, W., Cui, B., Gu, X., and Dong, Q. 2018. “Comparison of Relaxation Modulus Converted from Frequency- and Time-Dependent Viscoelastic Functions through Numerical Methods.” Applied Sciences. Volume 8(12). DOI: 10.3390/app8122447. 


\section{Test Methods Used}

American Association of State Highway and Transportation Officials. Superpave Volumetric Design for Asphalt Mixtures. AASHTO R 35-17. Washington, D.C.: AASHTO, 2001.

American Association of State Highway and Transportation Officials. Standard Practice for Preparation of Cylindrical Performance Test Specimens Using the Superpave Gyratory Compactor (SGC). AASHTO PP 60. Washington, D.C.: AASHTO, 2014.

American Association of State Highway and Transportation Officials. Standard Method of Test for Determining the Dynamic Modulus and Flow Number for Asphalt Mixtures Using the Asphalt Mixture Performance Tester (AMPT). AASHTO TP 79. Washington, D.C.: AASHTO, 2015.

American Association of State Highway and Transportation Officials. Standard Practice for Preparation of Cylindrical Performance Test Specimens Using the Superpave Gyratory Compactor (SGC). AASHTO R 83-17. Washington, D.C.: AASHTO, 2017.

American Association of State Highway and Transportation Officials. Developing Dynamic Modulus Master Curves for Asphalt Mixtures Using the Asphalt Mixture Performance Tester (AMPT). AASHTO R 84. Washington, D.C.: AASHTO, 2017.

American Association of State Highway and Transportation Officials. Standard Method of Test for Specific Gravity and Absorption of Fine Aggregate. AASHTO T 84-13. Washington, D.C.: AASHTO, 2017.

American Association of State Highway and Transportation Officials. Provisional Standard Practice for Preparation of Small Cylindrical Performance Test Specimens Using the Superpave Gyratory Compactor (SGC) or Field Cores. AASHTO PP 99-19. Washington, D.C.: AASHTO, 2019.

American Association of State Highway and Transportation Officials. Standard Method of Test for Determining the Damage Characteristic Curve of Asphalt Mixtures from Direct Tension Cyclic Fatigue Tests. AASHTO TP 107-18. Washington, D.C.: AASHTO, 2018.

American Association of State Highway and Transportation Officials. Provisional Standard Method of Test for Determining the Dynamic Modulus for Asphalt Mixtures Using Small Specimens in the Asphalt Mixture Performance Tester (AMPT). AASHTO TP 132-19. Washington, D.C.: AASHTO, 2019.

American Association of State Highway and Transportation Officials. Provisional Standard Method of Test for Determining the Damage Characteristic Curve and Failure Criterion Using Small Specimens in the Asphalt Mixture Performance Tester (AMPT) Cyclic Fatigue Test. AASHTO TP 133-19. Washington, D.C.: AASHTO, 2019.

American Association of State Highway and Transportation Officials. Provisional Standard Method of Test for Stress Sweep Rutting (SSR) Test Using Asphalt Mixture Performance Tester (AMPT). AASHTO TP 134-19. Washington, D.C.: AASHTO, 2019.

American Association of State Highway and Transportation Officials. Standard Method of Test for Bulk Specific Gravity $\left(G_{m b}\right)$ of Compacted Hot Mix Asphalt $(H M A)$ Using Saturated Surface-Dry Specimens. AASHTO T 166. Washington, D.C.: AASHTO, 2018.

American Association of State Highway and Transportation Officials. Standard Method of Test for Theoretical Maximum Specific Gravity $\left(G_{m m}\right)$ and Density of Hot-Mix Asphalt (HMA). AASHTO T 209. Washington, D.C.: AASHTO, 2019. 
American Association of State Highway and Transportation Officials. Standard Method of Test for Percent Air Void in Compacted Dense and Open Asphalt Mixtures. AASHTO T 269-14. Washington, D.C.: AASHTO, 2018.

American Association of State Highway and Transportation Officials. Determining the Fatigue Life of Compacted Hot Mix Asphalt (HMA) Subjected to Repeated Flexural Bending. AASHTO T 32107. Washington, D.C.: AASHTO, 2015.

American Association of State Highway and Transportation Officials. Standard Method of Test for Determining the Dynamic Modulus and Flow Number for Asphalt Mixtures Using the Asphalt Mixture Performance Tester (AMPT). AASHTO T 378. Washington, D.C.: AASHTO, 2017.

ASTM International. Standard Test Method for Dynamic Modulus of Asphalt Mixtures. ASTM International. ASTM D-3497-79. West Conshohocken, PA: ASTM International, 2003. 


\section{Appendix A - General AMPT Test Setup}

The general description of AMPT test setup is shown in Figure 49.

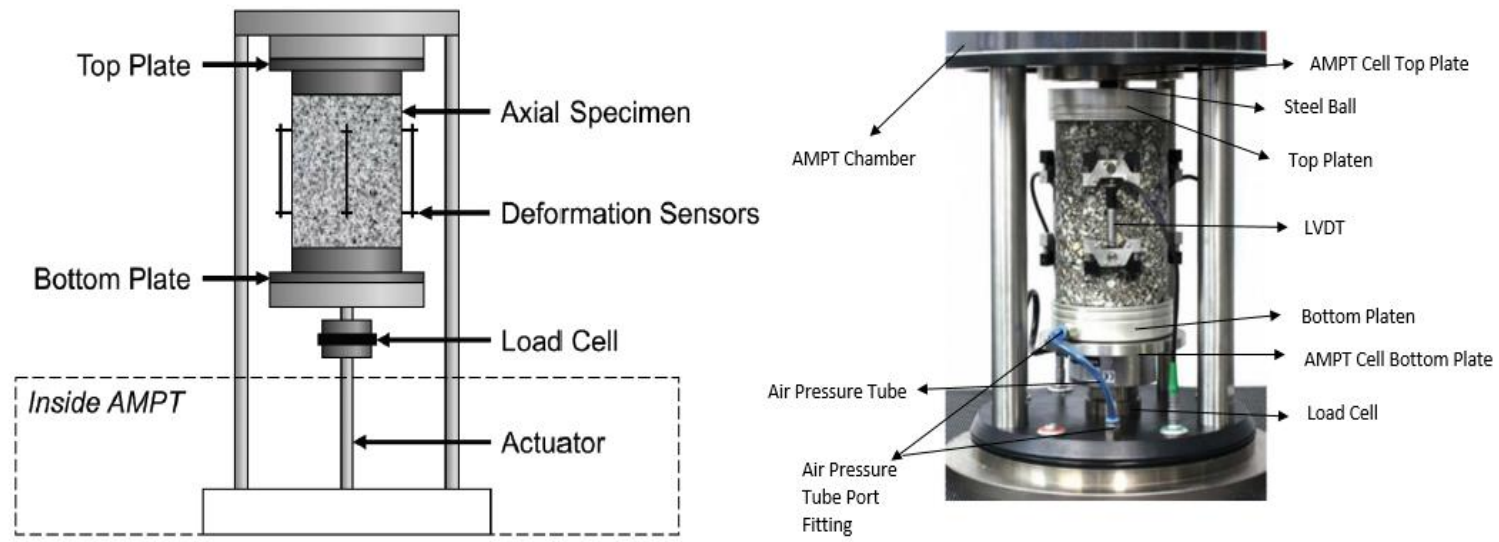

Figure 49: General Schematic of AMPT Test Setup

The AMPT testing takes place inside AMPT chamber. In this research, an external conditioning chamber (such as fridge, freezer, and oven) is used for achieving specimen test temperature. AMPT chamber is also temperature conditioned at the target test temperature. However, in getting the specimen ready for testing, the specimen temperature is altered as user removes test specimen from external conditioning chamber to setup inside AMPT chamber. Once specimen is setup for testing, AMPT chamber is closed to bring back the specimen to the test temperature. Table 77 provides vertical space description available in AMPT testing chamber for testing.

Table 78: Description of Vertical Space in AMPT Testing Chamber for Dynamic Modulus, DTC Fatigue, and SSR Test

\begin{tabular}{|c|c|}
\hline Test Description & Original size $(\mathrm{mm})$ \\
\hline Actuator Movement & 30 \\
\hline Maximum Distance between AMPT Top and Bottom Load Plate & 215 \\
\hline Minimum Distance between AMPT Top and Bottom Load Platen & 185 \\
\hline
\end{tabular}

The actuator controls the movement of load cell from $-15 \mathrm{~mm}$ to $+15 \mathrm{~mm}$, providing $30 \mathrm{~mm}$ of

extra space after specimen and top and bottom platen are placed inside the chamber. The purpose of placing ball bearing between top platen and top load platform is an attempt to account for loading eccentricity. The steel ball is used only in dynamic modulus and SSR test and not used in DTC test as it could have a negative effect if screws are overtightened leading to debonding the platen from specimen. LVDT measures specimen deformation in AMPT testing. One end of drainage tube is attached to port fitting inside AMPT chamber and another end is connected to platen for only SSR test. Drainage tube helps specimen to be vented to atmospheric pressure. 


\section{Appendix B -Design Mix Sheet}

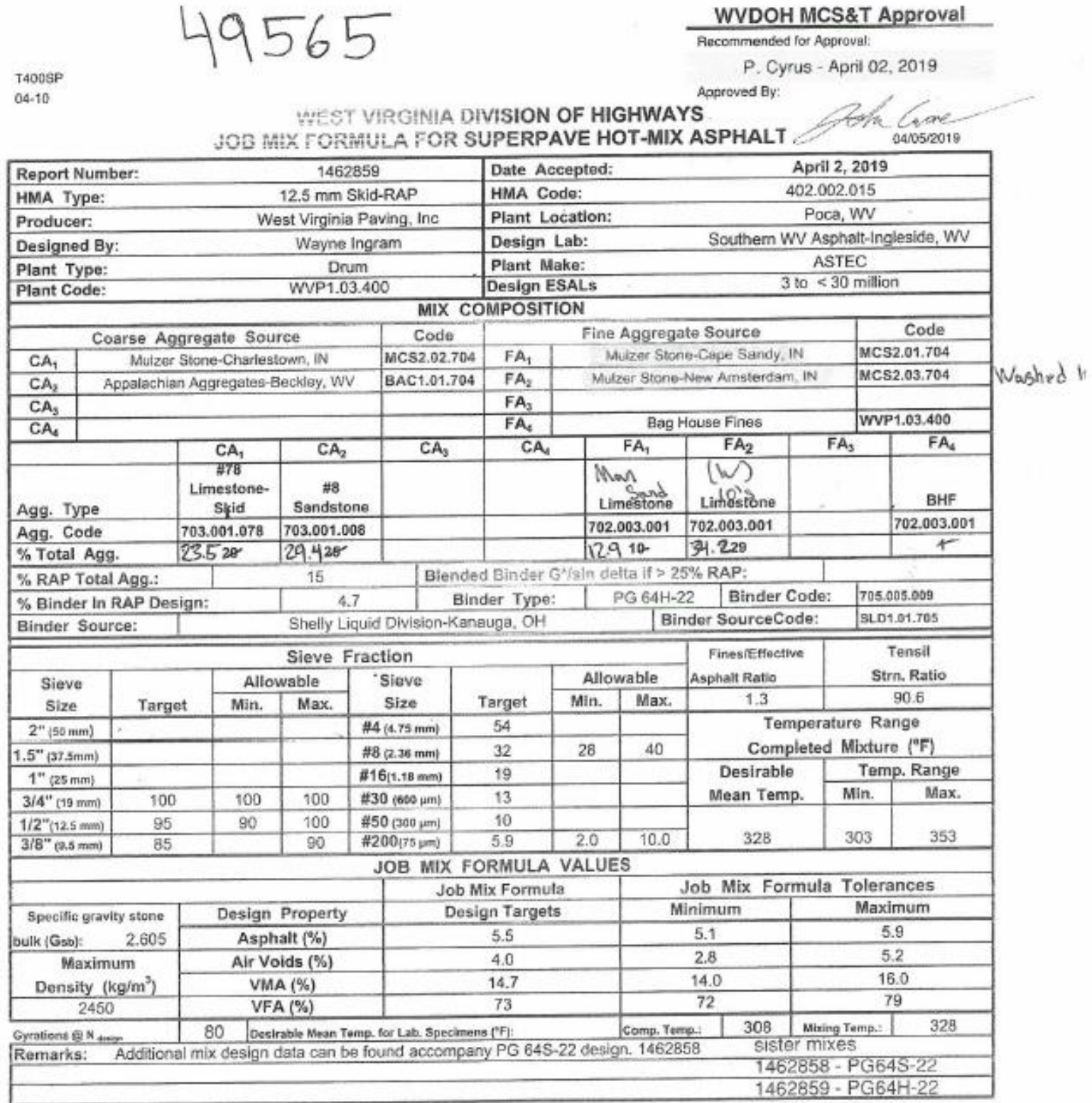




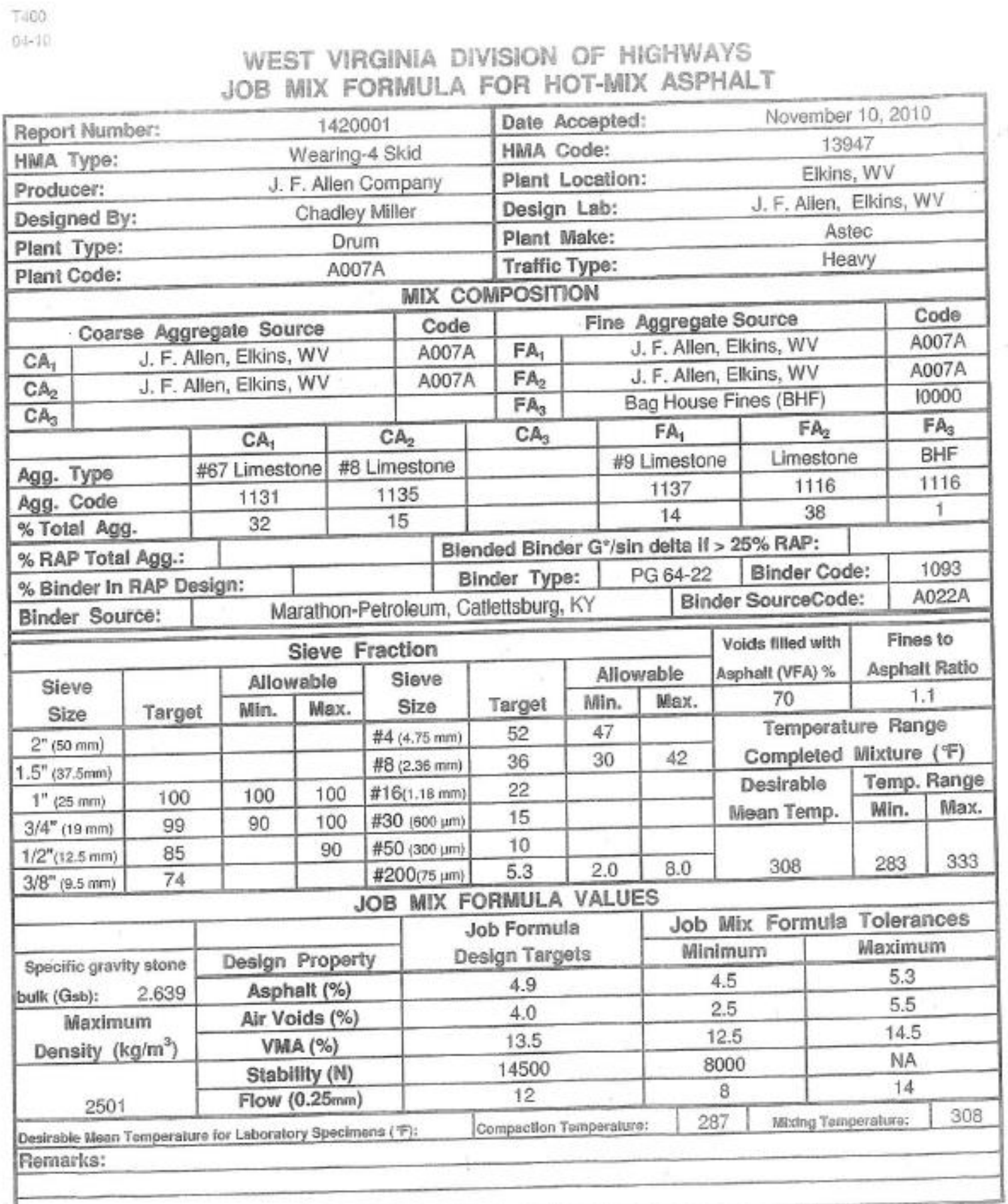




\section{WEST VIRGINIA DIVISION OF HIGHWAYS JOB MIX FORMULA FOR SUPERPAVE HOT-MIX ASPHALT}

\begin{tabular}{|lc|lc|}
\hline Report Number: & Date Accepted: & \\
\hline HMA Type: & 25 mm - RAP & HMA Code: & 401.002 .007 \\
\hline Producer: & J. F. Allen Company & Plant Location: & Lorentz, WV \\
\hline Designed By: & Chadley Miller & Design Lab: & J. F. Allen (Elkins, WV) \\
\hline Plant Type: & Batch & Plant Make: & McCarter \\
\hline Plant Code: & JFA1.02.400 & Design ESALs: & 3 to $<30$ Million (3) \\
\hline
\end{tabular}

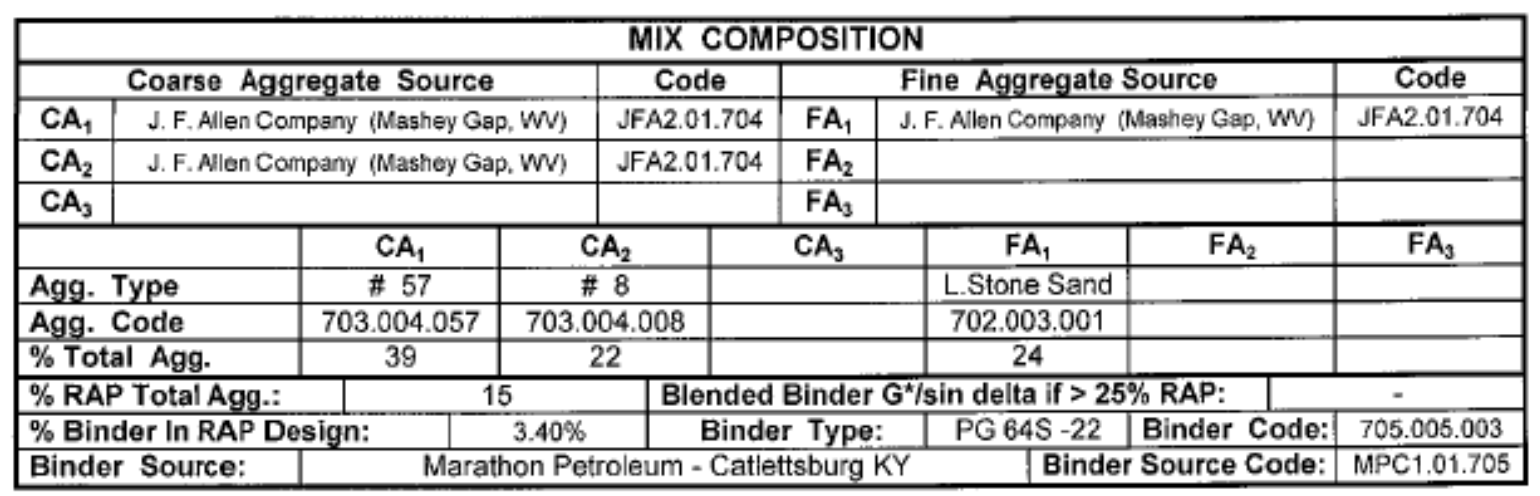

\begin{tabular}{|c|c|c|c|c|c|c|c|c|c|c|c|}
\hline \multicolumn{8}{|c|}{ Sieve Fraction } & \multirow{3}{*}{\multicolumn{2}{|c|}{$\begin{array}{c}\begin{array}{c}\text { Fines / Effective } \\
\text { Asphalt Ratio }\end{array} \\
1.0 \\
\end{array}$}} & \multirow{3}{*}{\multicolumn{2}{|c|}{\begin{tabular}{|c|}
$\begin{array}{c}\text { Tensile } \\
\text { Strn. Ratio }\end{array}$ \\
84.2 \\
\end{tabular}}} \\
\hline \multirow{2}{*}{$\begin{array}{l}\text { Sieve } \\
\text { Size }\end{array}$} & \multirow[b]{2}{*}{ Target } & \multicolumn{2}{|c|}{ Allowable } & \multirow{2}{*}{$\begin{array}{l}\text { Sieve } \\
\text { Size }\end{array}$} & \multirow[b]{2}{*}{ Target } & \multicolumn{2}{|c|}{ Allowable } & & & & \\
\hline & & Min. & Max. & & & Min. & Max. & & & & \\
\hline $2^{\prime \prime}(50 \mathrm{~mm})$ & & & & $\# 4(4.75 \mathrm{~mm})$ & 45 & & & \multirow{2}{*}{\multicolumn{4}{|c|}{$\begin{array}{c}\text { Temperature Range } \\
\text { Completed Mixture }\left({ }^{\circ} \mathrm{F}\right)\end{array}$}} \\
\hline $1.5^{\prime \prime}(37.5 \mathrm{~mm})$ & 100 & 100 & 100 & $\# 8(2.36 \mathrm{~mm})$ & 31 & 25 & 37 & & & & \\
\hline $1^{1 "}(25 \mathrm{~mm})$ & 98 & 90 & 100 & $\# 16(1.18 \mathrm{~mm})$ & 20 & & & \multirow{2}{*}{$\begin{array}{l}\text { Desirable } \\
\text { Mean Temp. }\end{array}$} & \multicolumn{3}{|c|}{ Temp. Range } \\
\hline $3 / 4^{\prime \prime}(19 \mathrm{~mm})$ & 89 & & 90 & $\# 30$ (600 prm) & 13 & & & & Min & & Max. \\
\hline $1 / 2^{\prime \prime}(12.5 \mathrm{~mm})$ & 74 & & & $\# 50$ (300 um) & 8 & & & & & & \\
\hline $3 / 8^{\prime \prime}(9.5 \mathrm{~mm})$ & 66 & & & $\# 200(75 \mu \mathrm{m})$ & 3.9 & 2.0 & 7.0 & 309 & 284 & & 334 \\
\hline
\end{tabular}

\begin{tabular}{|c|c|c|c|c|c|}
\hline \multicolumn{6}{|c|}{ JOB MIX FORMULA VALUES } \\
\hline & & Job Mix Formula & \multicolumn{3}{|c|}{ Job Mix Formula Tolerances } \\
\hline Specific Gravity Stone & Design Property & Design Targets & Minimum & \multicolumn{2}{|c|}{ Maximum } \\
\hline Bulk (Gsb): 2.686 & Asphalt (\%) & 4.4 & 4.0 & \multicolumn{2}{|c|}{4.8} \\
\hline \multirow{2}{*}{$\begin{array}{c}\text { Maximum } \\
\text { Density }\left(\mathrm{kg} / \mathrm{m}^{3}\right)\end{array}$} & Air Voids (\%) & 4.0 & 2.8 & \multicolumn{2}{|c|}{5.2} \\
\hline & VMA (\%) & 13.1 & 12.1 & \multicolumn{2}{|c|}{14.1} \\
\hline 2545 & VFA $(\%)$ & 70 & 68 & \multicolumn{2}{|c|}{76} \\
\hline Gyrations esente & \multicolumn{2}{|c|}{ Desirable Mean Temp. for Lab. Specimens ('F): } & \begin{tabular}{|l|l|} 
Comp. Temp. : & 288 \\
\end{tabular} & Mixing Temp. : & 309 \\
\hline
\end{tabular}

Remarks: 


\section{Appendix C-Mix Verification}

\section{MARSHALL VERIFICATION}

Steps for verification of Marshall mix (per AASHTO R 68) using Marshall compaction hammer are:

- Three replicate specimens are prepared by weighing $1200 \mathrm{~g}$ for each replicate into three separate pans.

- The asphalt mix in the pan is heated to $5^{\circ} \mathrm{C}$ above compaction temperature of $153^{\circ} \mathrm{C}\left(308^{\circ} \mathrm{F}\right)$ such that when the batch is taken out to be poured in the mold, the temperature drop brings the mixture close to the compaction temperature. This can be done with infrared no touch thermometer.

- The Marshall mold and funnel are placed in a separate oven and heated to compaction temperature of $153^{\circ} \mathrm{C}$. Additionally, spatula and Marshall hammer are heated on a hot plate.

- Insert thermometer in the mix to monitor temperature.

- When mix is reached $5^{\circ} \mathrm{C}$ above compaction temperature of $153^{\circ} \mathrm{C}$, pan with heated mix is removed from the oven. Place a filter paper at the bottom of mold and carefully pour the mix into the mold using heated funnel. Use spatula to scrape fines from the pans and empty it into the mold.

- Use heated spatula to spade the mix 15 times around the perimeter and 10 times in the interior region.

- Place the filter paper on the top of the heated asphalt mix.

- Apply 75 blows with the heated Marshall hammer on top side. Stop and rotate the mold to apply 75 blows on the bottom side.

Marshall traffic detail is obtained from T-400 and number of blows on each side of sample is selected (per MP 401.02.22) as:

\begin{tabular}{|c|c|c|}
\hline Marshall traffic & Number of blows & ESAL \\
\hline Medium & 50 & $10^{4}$ to $10^{6}$ \\
\hline Heavy & 75 & $10^{6}<$ \\
\hline
\end{tabular}

- After compaction, carefully extract the specimen and place on a flat surface to be cooled overnight.

- VTM is calculated after pills are cooled. VTM must be within the job mix tolerance found in T400 sheet.

\section{SUPERPAVE VERIFICATION}

Steps for verification of Superpave mix (per AASHTO T 312) using PINE compactor are: 
- Three replicate specimens are prepared by weighing $4500 \mathrm{~g}$ to $4700 \mathrm{~g}$ for each replicate into separate pans.

- Superpave pill is compacted to $150 \mathrm{X} 115 \mathrm{~mm}$ at number of design gyrations stated in T 400 .

- The asphalt mix in the pan is heated to $5^{\circ} \mathrm{C}$ above compaction temperature for each mix such that when the batch is taken out to be poured in the mold, the temperature drop brings the mixture close to the compaction temperature. The compaction temperature number of design gyrations for each mix is also obtained from T-400 as:

\begin{tabular}{|c|c|c|}
\hline NMAS, $\mathrm{mm}$ & Compaction Temperature, ${ }^{\circ} \mathrm{C}$ & Number of Gyrations \\
\hline 12.5 & 164 & \multirow{2}{*}{80} \\
\hline 25 & 153 & \\
\hline
\end{tabular}

- The Superpave mold and funnel is placed in a separate oven and heated to compaction temperature. Additionally, spatula, is heated on a hot plate.

- Insert thermometer in the mix to monitor temperature.

- When mix is reached $5^{\circ} \mathrm{C}$ above compaction temperature, pan with heated mix is removed from the oven. Place a filter paper at the bottom of mold and carefully pour the mix into the mold using heated funnel. Use spatula to scrape fines from the pans and empty it into the mold.

- Place a filter paper on top of the heated asphalt mix and gently place the top plate of mold.

- Both mixes are gyrated to 80 gyrations per T-400. The Pine compactor applies a pressure of 600 $\pm 18 \mathrm{kPa}$ on the specimen during compaction.

- After compaction, carefully extract the specimen and place on a flat surface to be cooled overnight.

- VTM is calculated after pills are cooled. VTM must be within the job mix tolerance found in T400 sheet. 


\section{Appendix D Custom Parts Fabricated for Research}

Table 78 provides summary and description of fabricated custom parts for 50X110 mm and 75X110 mm dynamic modulus and SSR test specimens.

Table 79: Summary of Custom Parts for Dynamic Modulus and SSR Test for This Research

\begin{tabular}{|c|c|c|c|}
\hline Test & $\begin{array}{c}\text { Dimension, } \\
\mathrm{mm}\end{array}$ & Custom Part Description & $\begin{array}{c}\text { Custom Part } \\
\text { Drawing }\end{array}$ \\
\hline \multirow[t]{5}{*}{$\begin{array}{l}\text { Dynamic } \\
\text { Modulus } \\
\text { and SSR }\end{array}$} & \multirow[t]{5}{*}{$50 \times 110$} & $\begin{array}{l}\text { Bottom Platen cross section. The bottom } \\
\text { platen is in direct contact with AMPT cell } \\
\text { bottom plate. }\end{array}$ & Figure 50 \\
\hline & & Bottom Platen top view. & Figure 51 \\
\hline & & $\begin{array}{l}\text { Stem cross section. The stem is a new term } \\
\text { used throughout this research for } 50 \mathrm{X} 110 \mathrm{~mm} \\
\text { specimen in both dynamic modulus and SSR } \\
\text { test. Stem has port fitting for drainage tube in } \\
\text { SSR test that helps specimen to be vented to } \\
\text { atmospheric pressure. The drainage tube is } \\
\text { not used for dynamic modulus test however } \\
\text { can be left in place. Stem has dowel pin that } \\
\text { slides into the bottom platen to provide extra } \\
\text { height of } 40 \mathrm{~mm} \text { (1.575 inch) to specimen and } \\
\text { platen. Stem is detachable from bottom } \\
\text { platen. The specimen sits on the stem inside } \\
\text { AMPT chamber. }\end{array}$ & Figure 52 \\
\hline & & $\begin{array}{l}\text { Top platen with ball cross section with steel } \\
\text { ball. }\end{array}$ & Figure 53 \\
\hline & & Top platen top view. & Figure 54 \\
\hline \multirow[t]{5}{*}{$\begin{array}{l}\text { Dynamic } \\
\text { Modulus }\end{array}$} & \multirow[t]{5}{*}{$75 X 110$} & $\begin{array}{l}\text { Bottom Platen cross section. The bottom } \\
\text { platen is in direct contact with AMPT cell } \\
\text { bottom plate. }\end{array}$ & Figure 56 \\
\hline & & Bottom Platen top view. & Figure 57 \\
\hline & & $\begin{array}{l}\text { Stem cross section. The stem is a new term } \\
\text { used throughout this research for } 75 \mathrm{X} 110 \mathrm{~mm} \\
\text { specimen in only dynamic modulus test. Stem } \\
\text { has dowel pin that slides into the bottom } \\
\text { platen to provide extra height of } 40 \mathrm{~mm} \\
\text { (1.575 inch) to specimen and platen. Stem is } \\
\text { detachable from bottom platen. The specimen } \\
\text { sits on the stem inside AMPT chamber. }\end{array}$ & Figure 58 \\
\hline & & $\begin{array}{l}\text { Top platen with ball cross section with steel } \\
\text { ball. }\end{array}$ & Figure 59 \\
\hline & & Top platen top view. & Figure 60 \\
\hline
\end{tabular}


For different specimens tested in this research, only 50X110 mm (dynamic modulus and SSR test) is designed to have a stem.

Table 80: Summary of New Custom Test Parts for DTC Fatigue Test for This Research

\begin{tabular}{|c|c|c|c|}
\hline Test & $\begin{array}{l}\text { Dimension, } \\
\mathrm{mm}\end{array}$ & Custom Part Description & $\begin{array}{l}\text { Custom Part } \\
\text { Drawing }\end{array}$ \\
\hline \multirow[t]{12}{*}{$\begin{array}{c}\text { DTC } \\
\text { Fatigue }\end{array}$} & $50 \times 110$ & $\begin{array}{l}\text { Gauge point gluing Jig spacer cross section. } \\
\text { The spacer has dowel pin that slides into the } \\
\text { center hole of jig. }\end{array}$ & Figure 64 \\
\hline & \multirow[t]{5}{*}{$75 X 110$} & $\begin{array}{l}\text { Gauge point gluing Jig spacer cross section. } \\
\text { The spacer has dowel pin that slides into the } \\
\text { center hole of jig. }\end{array}$ & Figure 65 \\
\hline & & $\begin{array}{l}\text { Top fatigue end platen gluing adapter cross } \\
\text { section }\end{array}$ & Figure 66 \\
\hline & & $\begin{array}{c}\text { Top fatigue end platen gluing adapter top } \\
\text { view }\end{array}$ & Figure 68 \\
\hline & & $\begin{array}{c}\text { Bottom fatigue end platen gluing adapter } \\
\text { cross section }\end{array}$ & Figure 69 \\
\hline & & $\begin{array}{l}\text { Bottom fatigue end platen gluing adapter } \\
\text { top view }\end{array}$ & Figure 70 \\
\hline & \multirow[t]{2}{*}{$50 X 110$} & $\begin{array}{l}\text { Top and bottom platens cross-section. The } \\
\text { platen shown is same for both top and } \\
\text { bottom. }\end{array}$ & Figure 71 \\
\hline & & Top and bottom platens top view. & Figure 72 \\
\hline & \multirow[t]{2}{*}{$75 X 110$} & $\begin{array}{l}\text { Top and bottom platens cross-section. The } \\
\text { platen shown is same for both top and } \\
\text { bottom. }\end{array}$ & Figure 73 \\
\hline & & Top and bottom platens top view. & Figure 74 \\
\hline & \multirow[t]{2}{*}{$\begin{array}{l}50 \times 110 \text { and } \\
75 \times 110\end{array}$} & $\begin{array}{l}\text { Top and bottom spacer cross-section. The } \\
\text { spacer provides additional height to } 110 \\
\text { mm height specimens and provides same } \\
\text { bolt pattern as the load platform and platen. } \\
\text { Spacer is bolted to top and bottom AMPT } \\
\text { cell plate. The specimen glued to platen is } \\
\text { then bolted to top and bottom spacer. }\end{array}$ & Figure 75 \\
\hline & & Top and bottom spacer top view & Figure 76 \\
\hline
\end{tabular}

In DTC fatigue, top and bottom platen are glued to 100X130 mm specimen, which is then attached to the top and bottom of AMPT cell plate. The 38X110 mm, 50X110 mm, and 75X110 mm specimens are glued to platen, which is then attached to spacers with bolts. The spacers serve two purpose in the fabrication: 
1. It provides additional height for small specimens so that the actuator does not have to go complete $30 \mathrm{~mm}$ upwards.

2. It provides same bolt pattern as the load platform and platen. Load platform and platen have two different bolt patterns. By placing spacer between them, the AMPT cell plate can be attached to the spacer which can be attached to the platen.

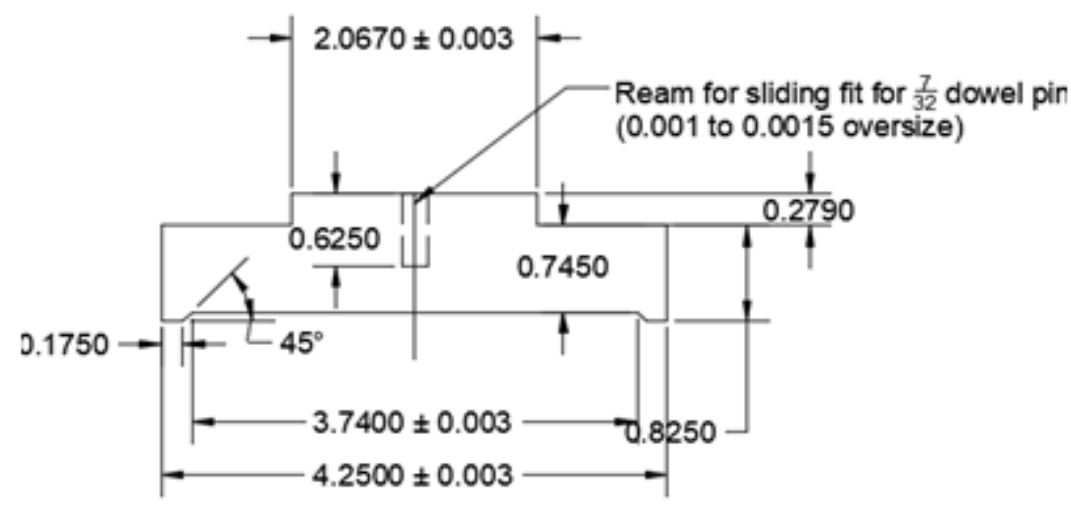

Figure 50: Dynamic Modulus and SSR Test Bottom Platen Cross Section for 50X110 mm Specimen

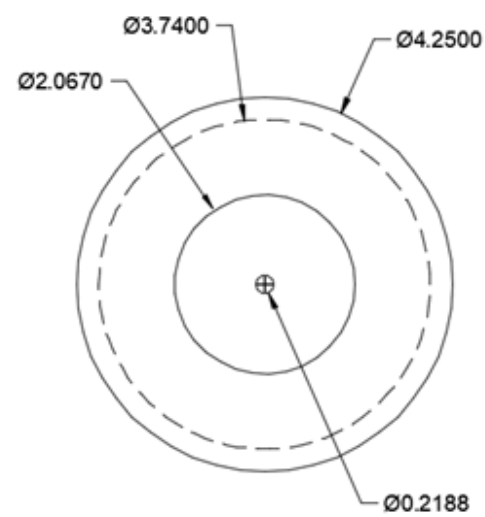

Figure 51: Dynamic Modulus and SSR Test Bottom Platen Top View for 50X110 mm Specimen
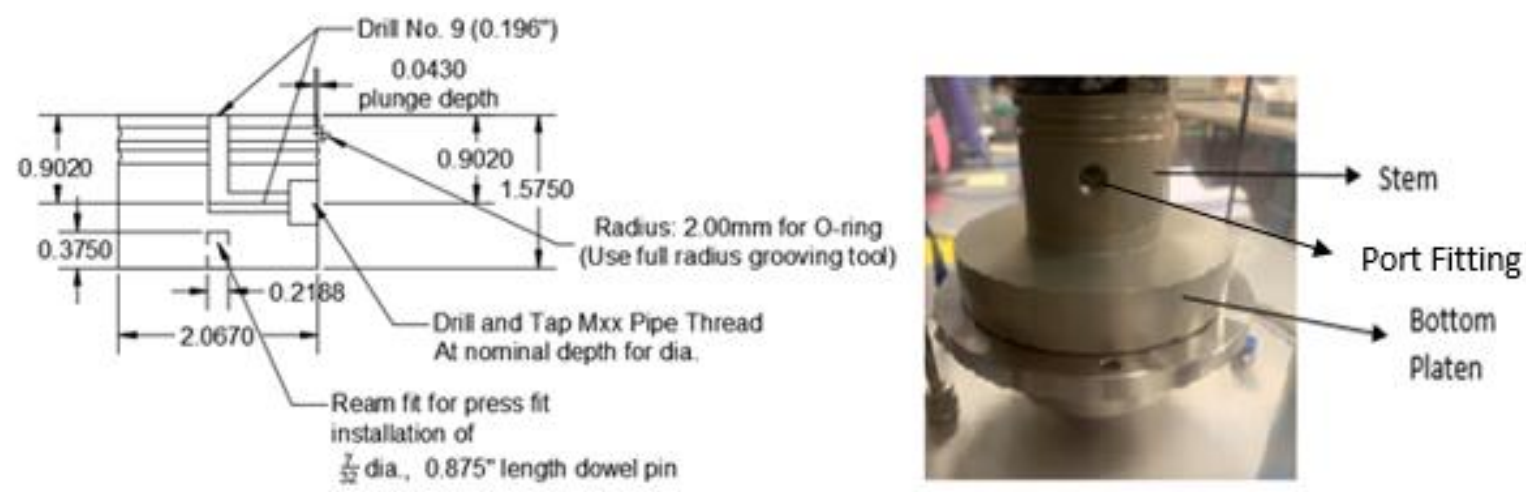

Figure 52: Dynamic Modulus and SSR Test Stem Cross Section for 50X110 mm Specimen 


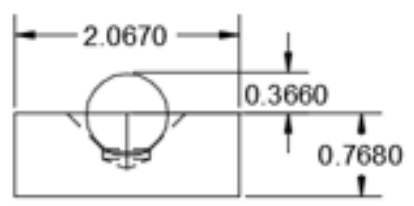

Figure 53: Dynamic Modulus and SSR Test Top Platen Cross Section for 50X110 mm Specimen

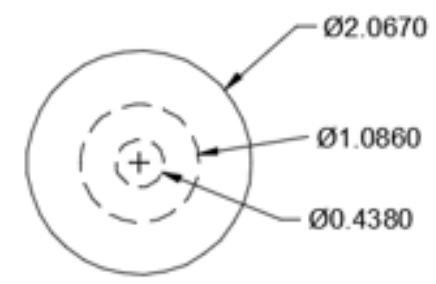

Figure 54: Dynamic Modulus and SSR Test Top Platen Top View for 50X110 mm Specimen
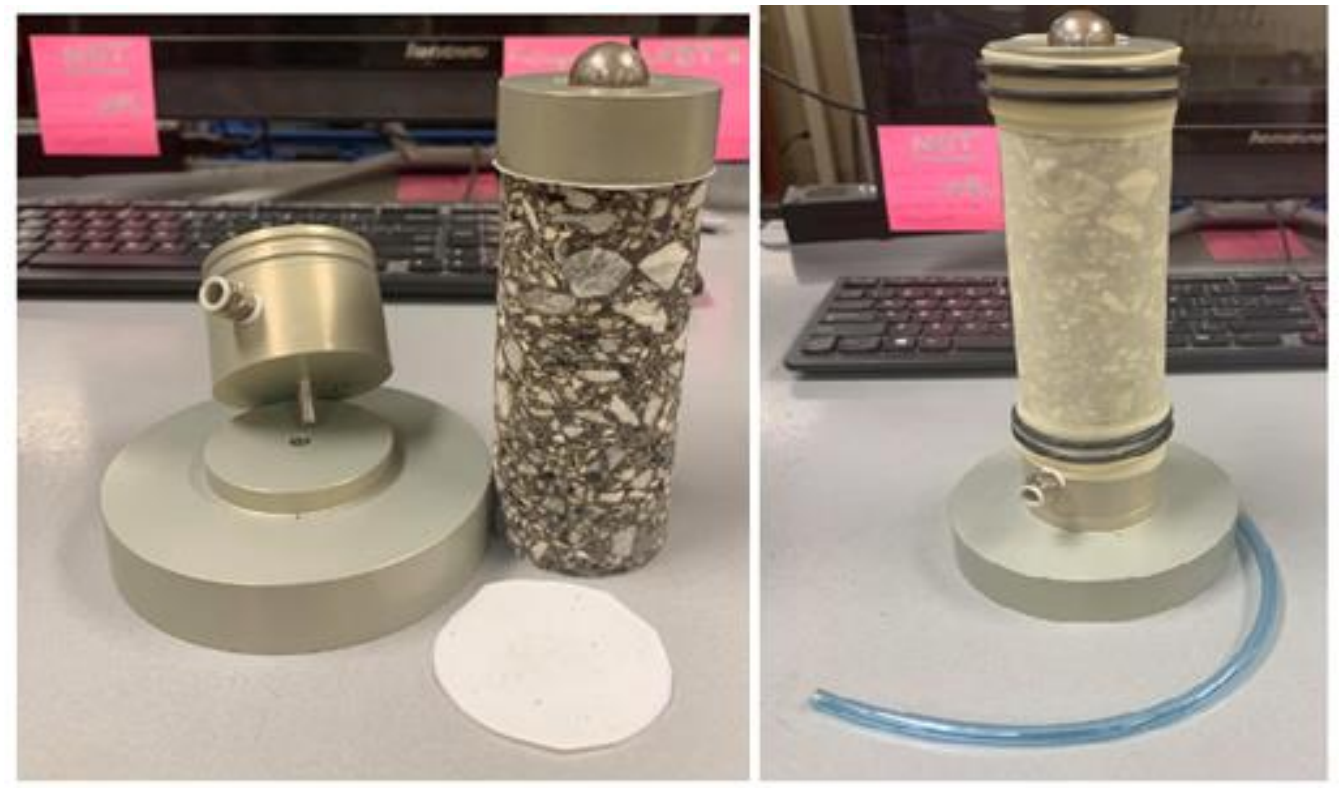

Figure 55: Dynamic Modulus and SSR Test Setup for 50X110 mm Specimen

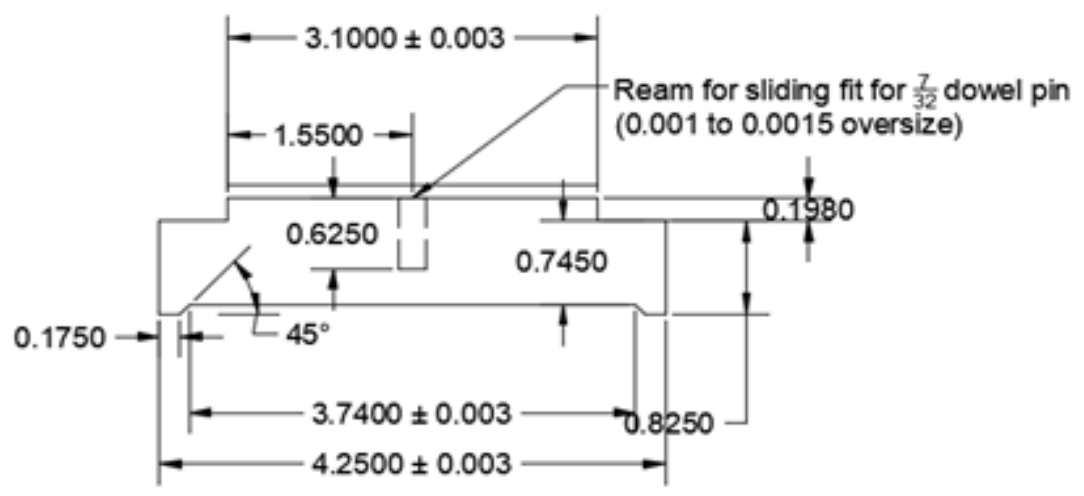

Figure 56: Dynamic Modulus Test Bottom Platen Cross Section for $75 X 110 \mathrm{~mm}$ Specimen 


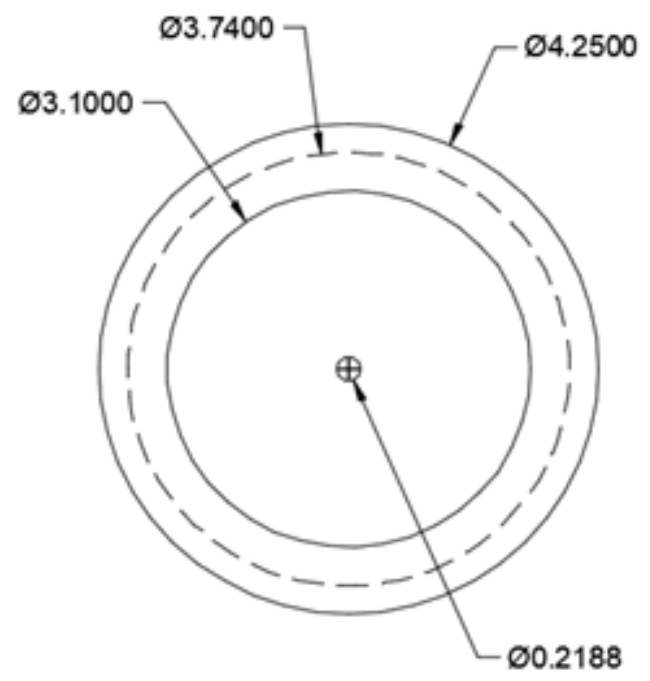

Figure 57: Dynamic Modulus Test Bottom Platen Top View for $75 X 110 \mathrm{~mm}$ Specimen

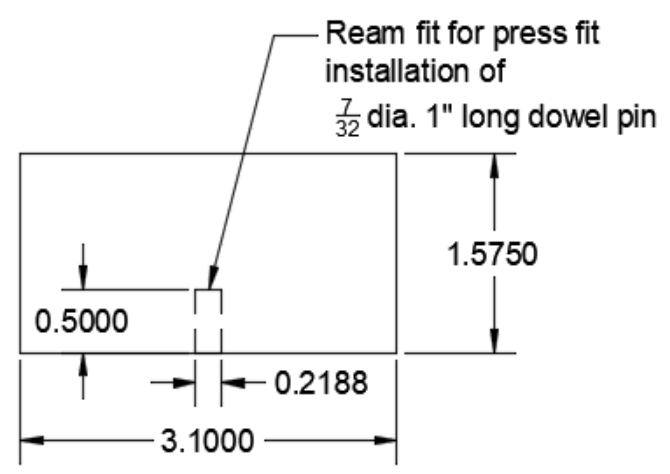

Figure 58: Dynamic Modulus Test Stem Cross Section for 75X110 mm Specimen

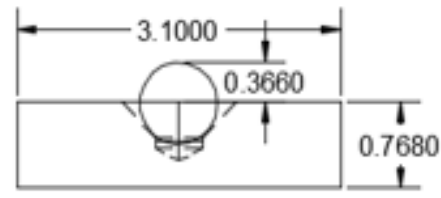

Figure 59: Dynamic Modulus Test Top Platen Cross Section for 75X110 mm Specimen 


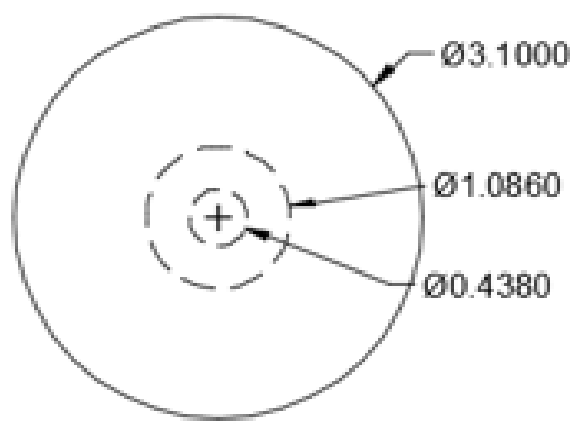

Figure 60: Dynamic Modulus Test Top Platen Top View for 75X110 mm Specimen

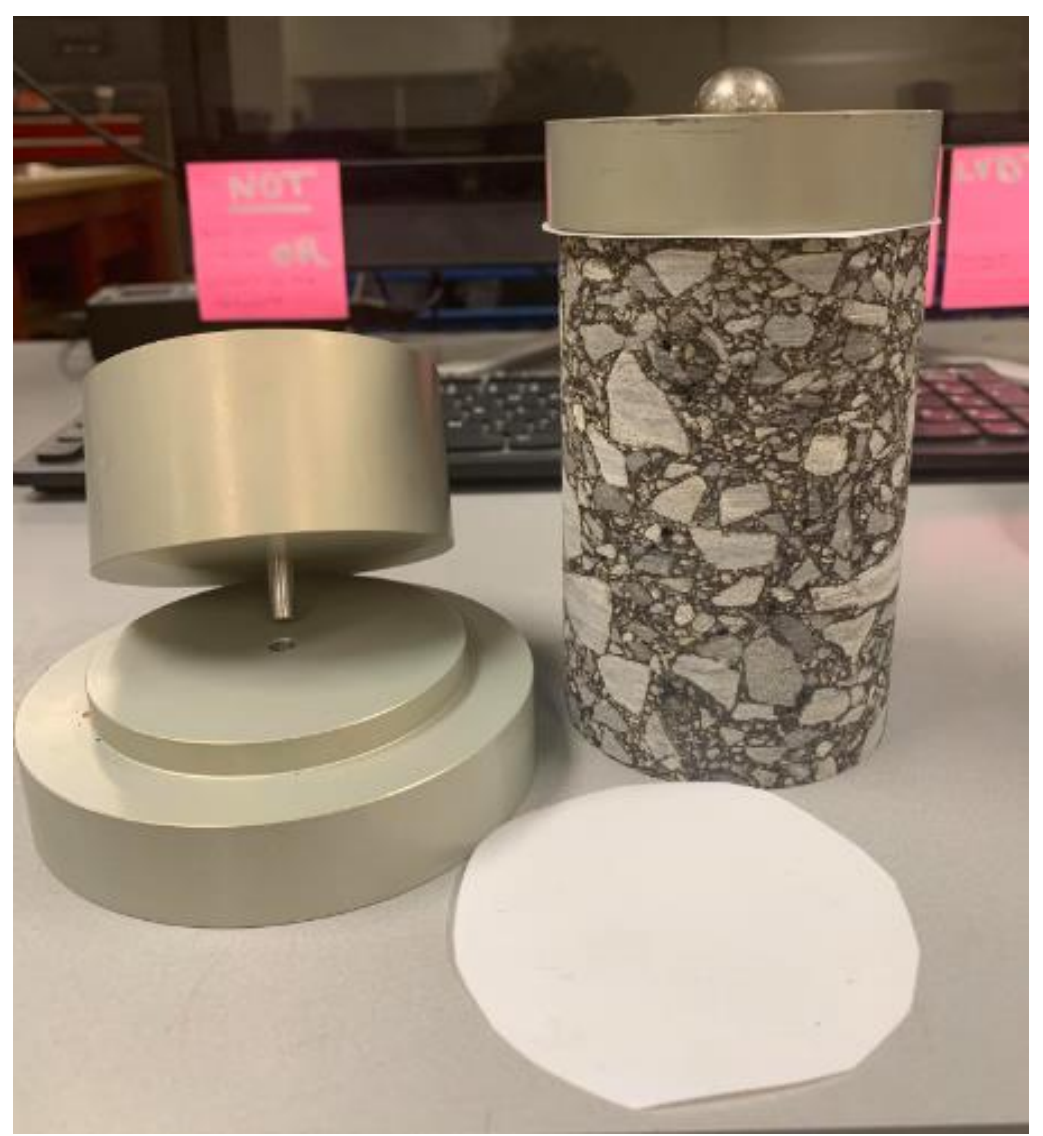

Figure 61: Dynamic Modulus Test Setup for 75X110 mm Specimen 


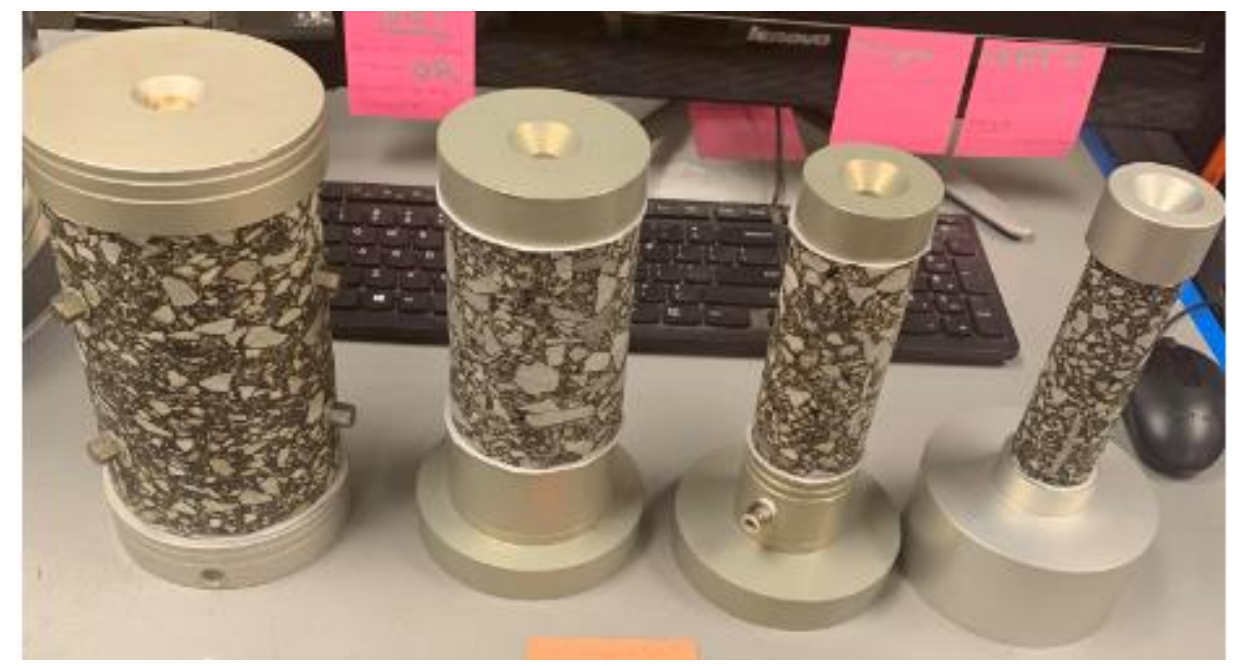

Figure 62: Dynamic Modulus Test Setup Comparison of Four Different Specimen Geometries

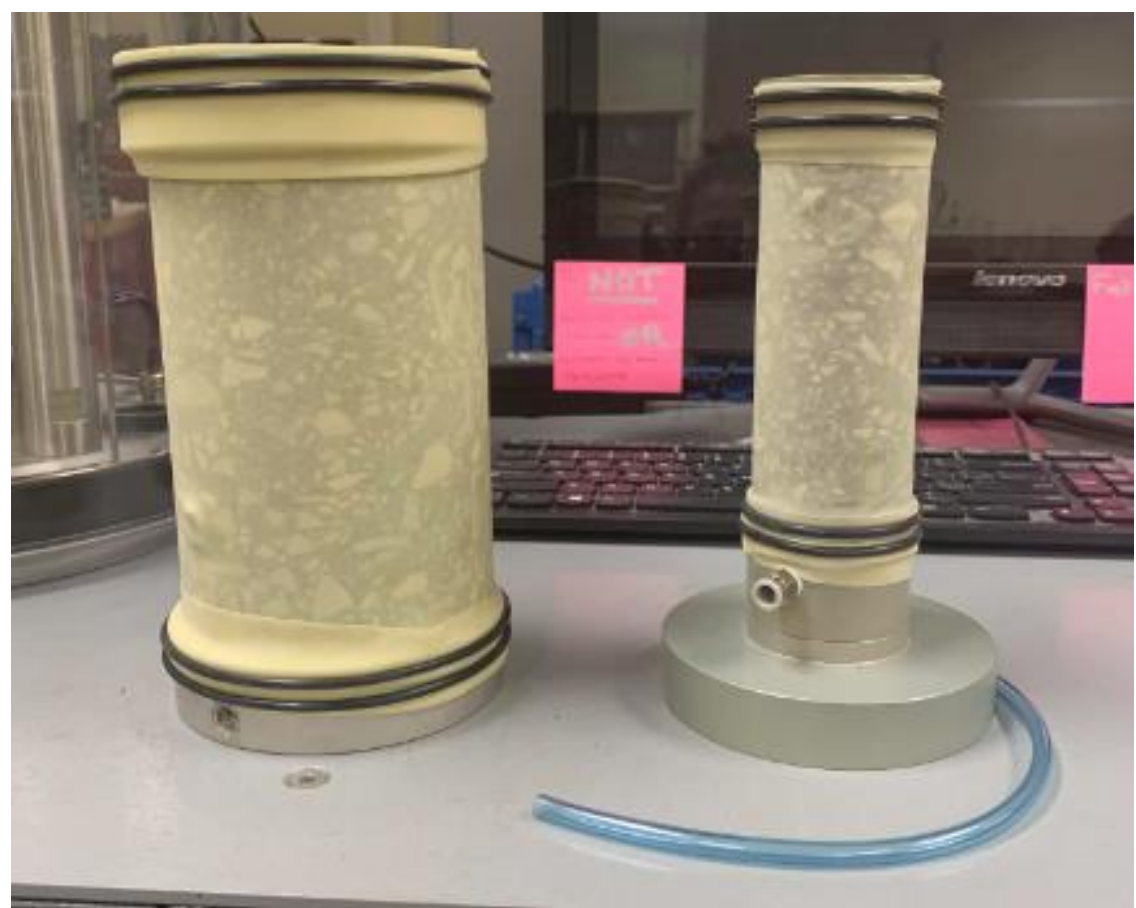

Figure 63: SSR Test Setup Comparison for of Different Specimen Geometries 


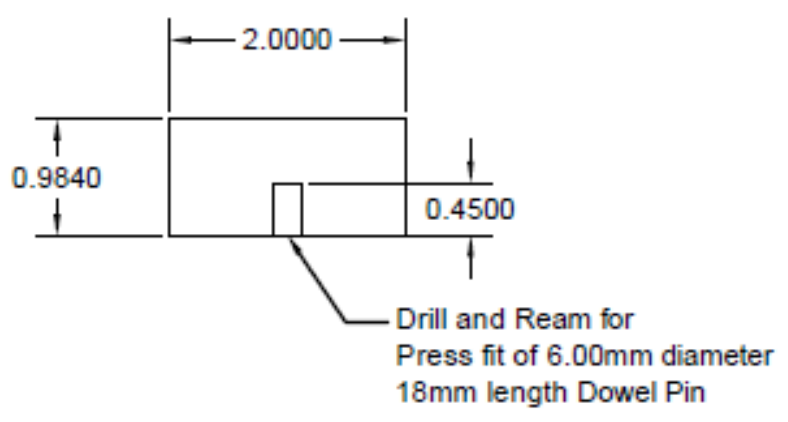

Figure 64: Gauge point gluing Jig spacer cross section for $50 X 110 \mathrm{~mm}$ Specimen

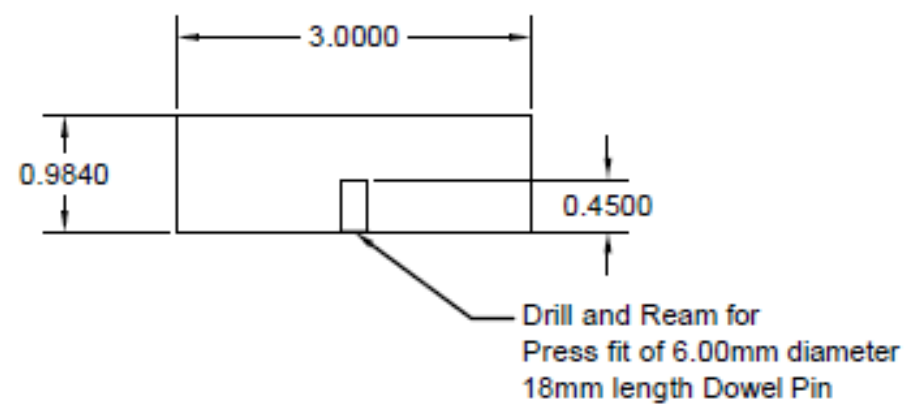

Figure 65: Gauge point gluing Jig spacer cross section for $75 X 110 \mathrm{~mm}$ Specimen

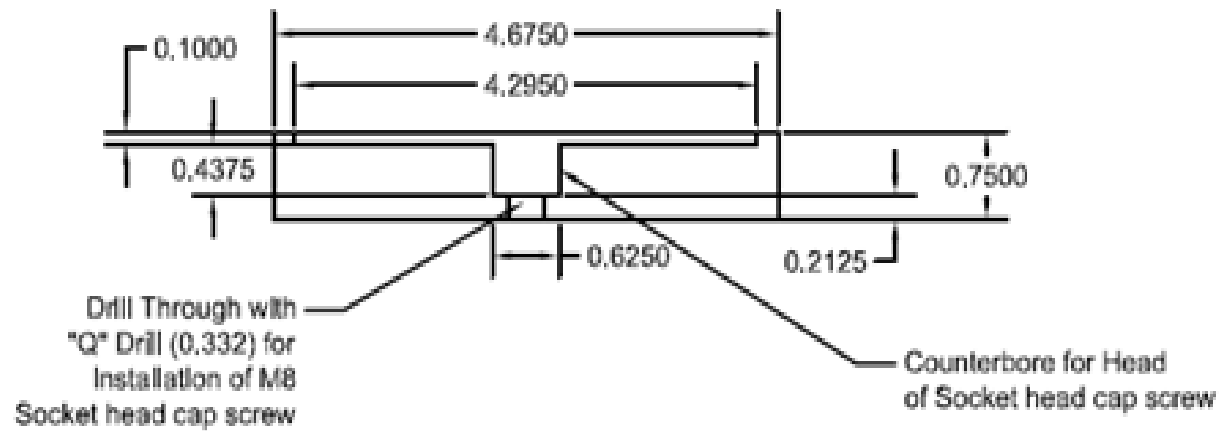

Figure 66: Top Fatigue End Platen Gluing Adapter Cross Section for $75 X 110$ mm Specimen 


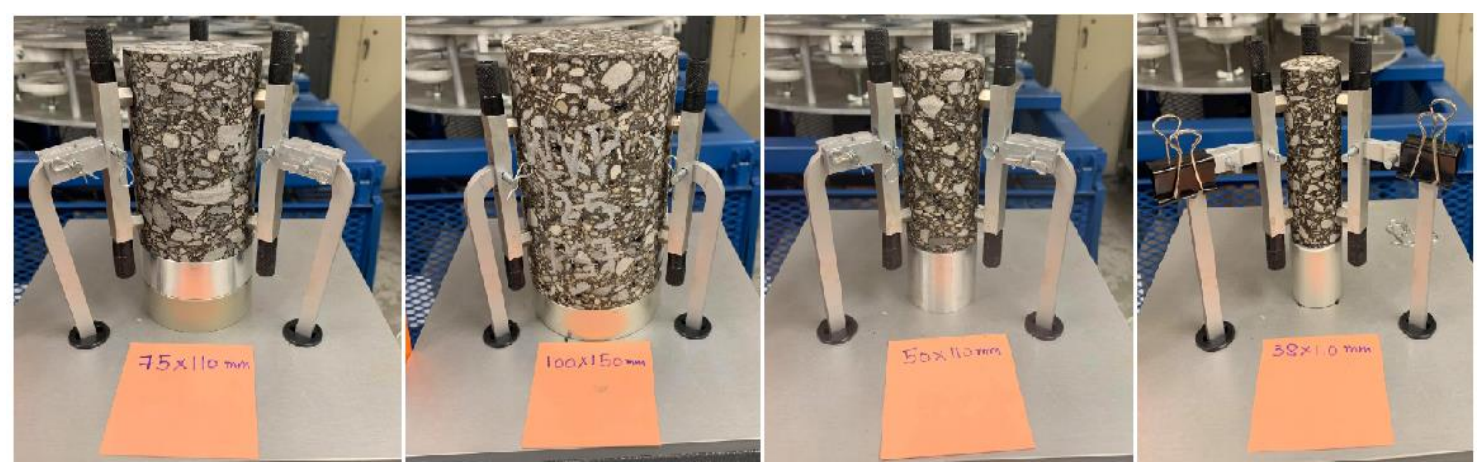

Figure 67: Gauge point gluing Jig Comparison of Four Different Specimen Geometries

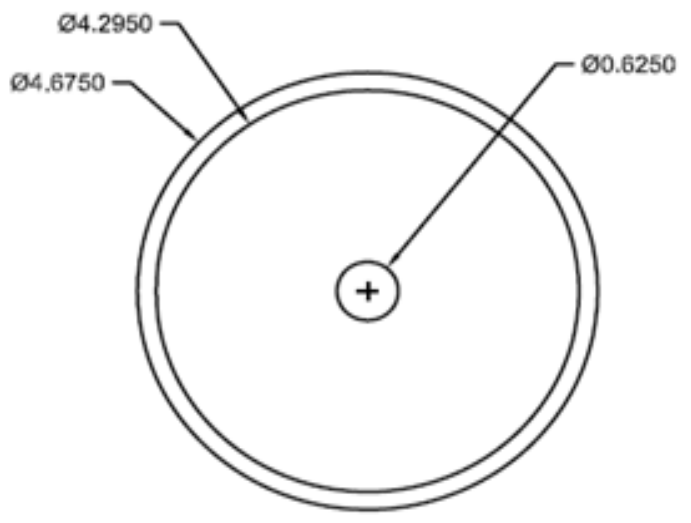

Figure 68: Top Fatigue End Platen Gluing Adapter Top View for 75X110 mm Specimen

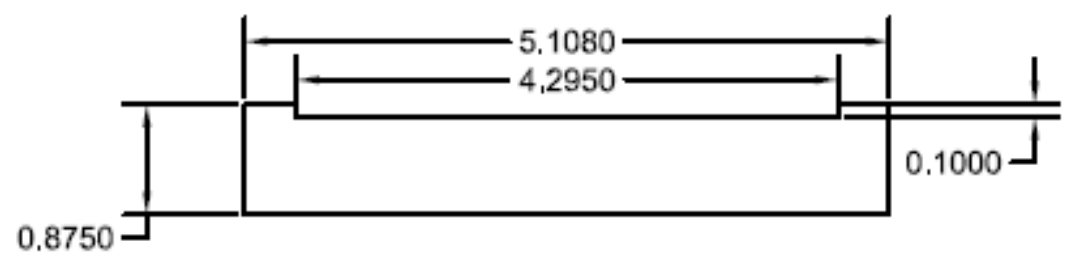

Figure 69: Bottom Fatigue End Platen Gluing Adapter Cross Section for 75X110 mm Specimen 


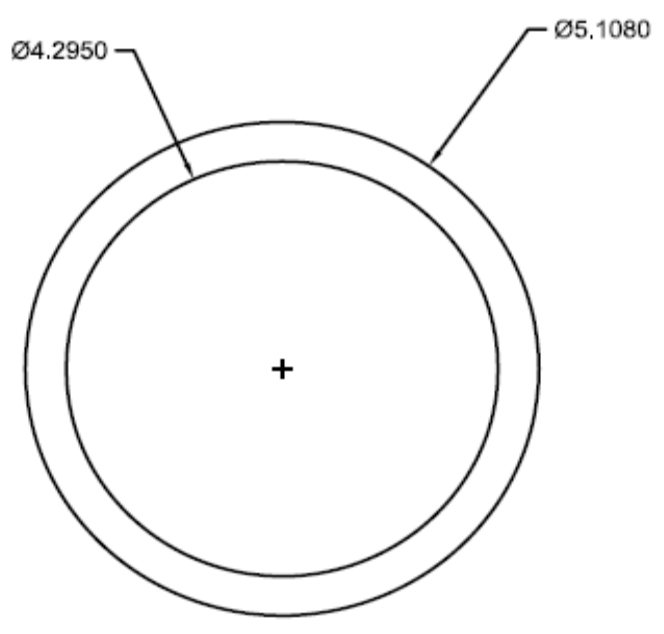

Figure 70: Bottom Fatigue End Platen Gluing Adapter Top View for $75 X 110 \mathrm{~mm}$ Specimen

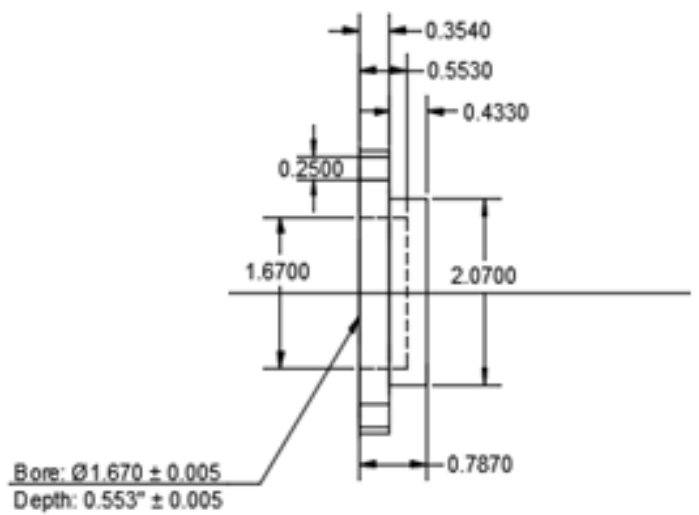

Figure 71: DTC Fatigue Top and Bottom Platens Cross-Section for $50 X 110 \mathrm{~mm}$ Specimen
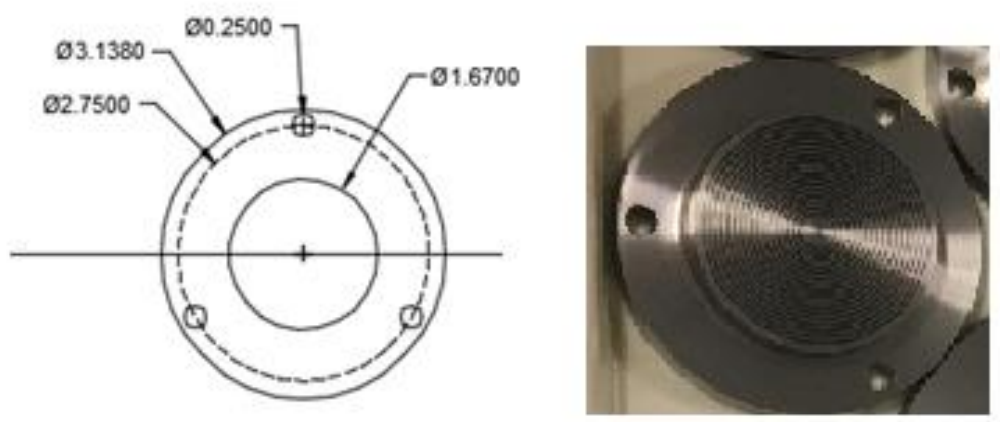

Figure 72: DTC Fatigue Top and Bottom Platens Top View for 50X110 mm Specimen 


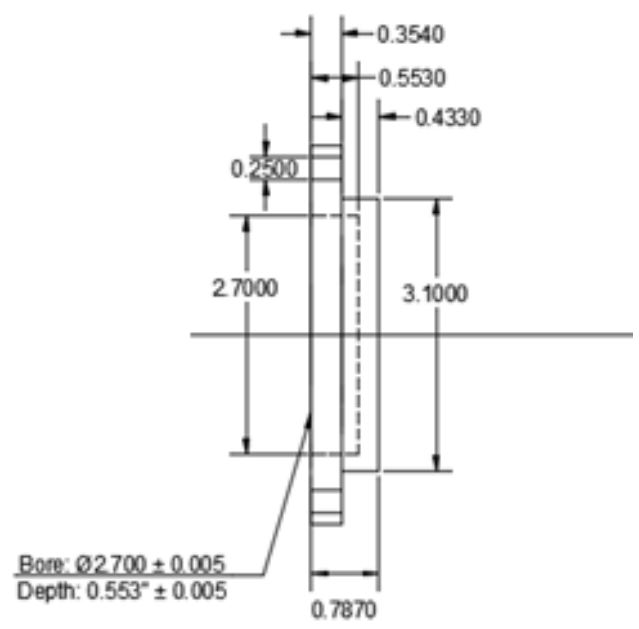

Figure 73: DTC Fatigue Top and Bottom Platens Cross-Section for $75 X 110 \mathrm{~mm}$ Specimen
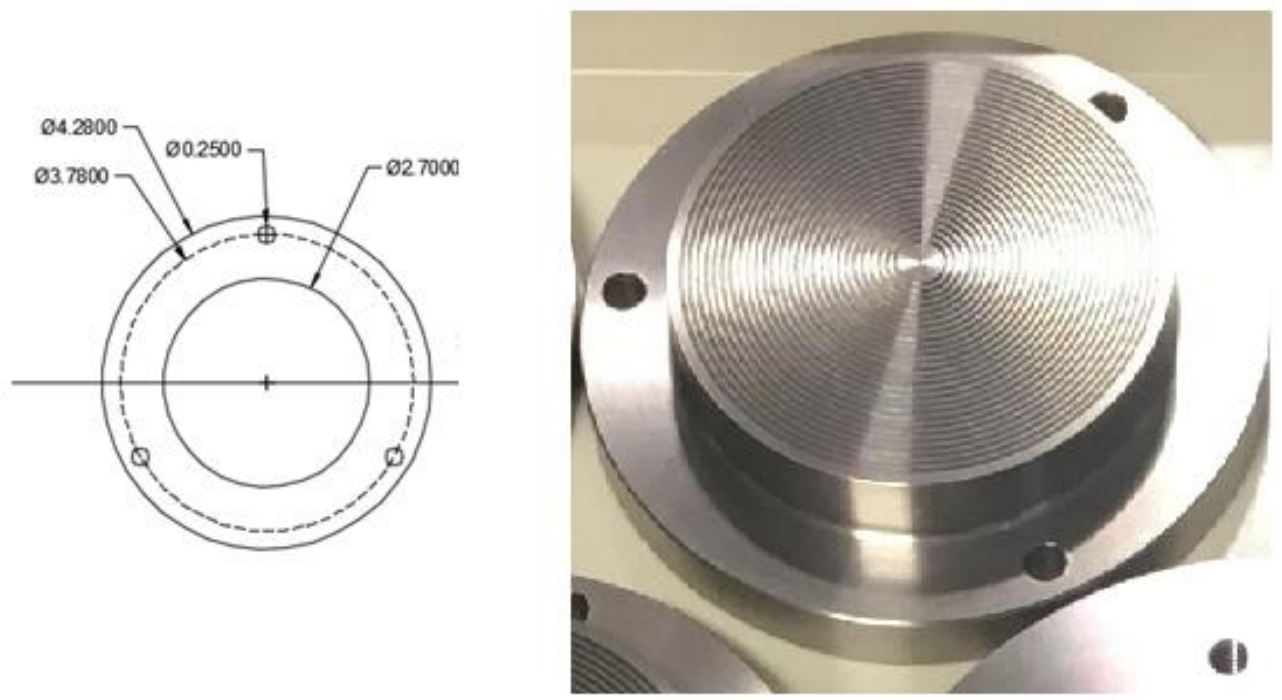

Figure 74: DTC Fatigue Top and Bottom Platens Top View for $75 X 110 \mathrm{~mm}$ Specimen 


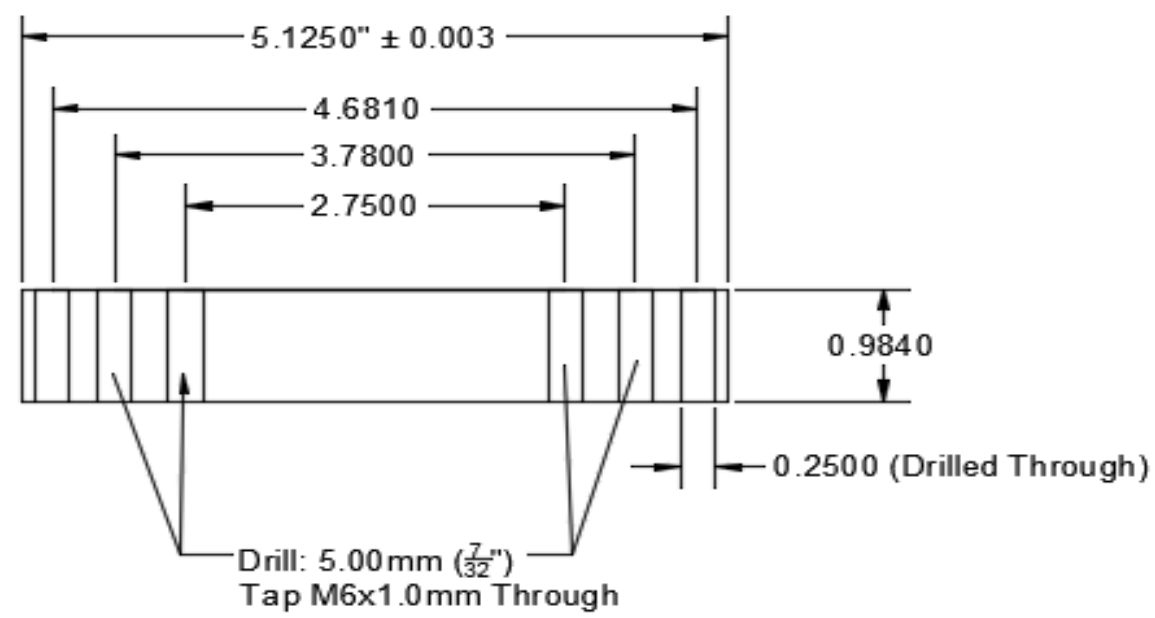

Figure 75: DTC Fatigue Top and Bottom Spacer Cross Section for 50X110 mm and 75X110 mm Specimen
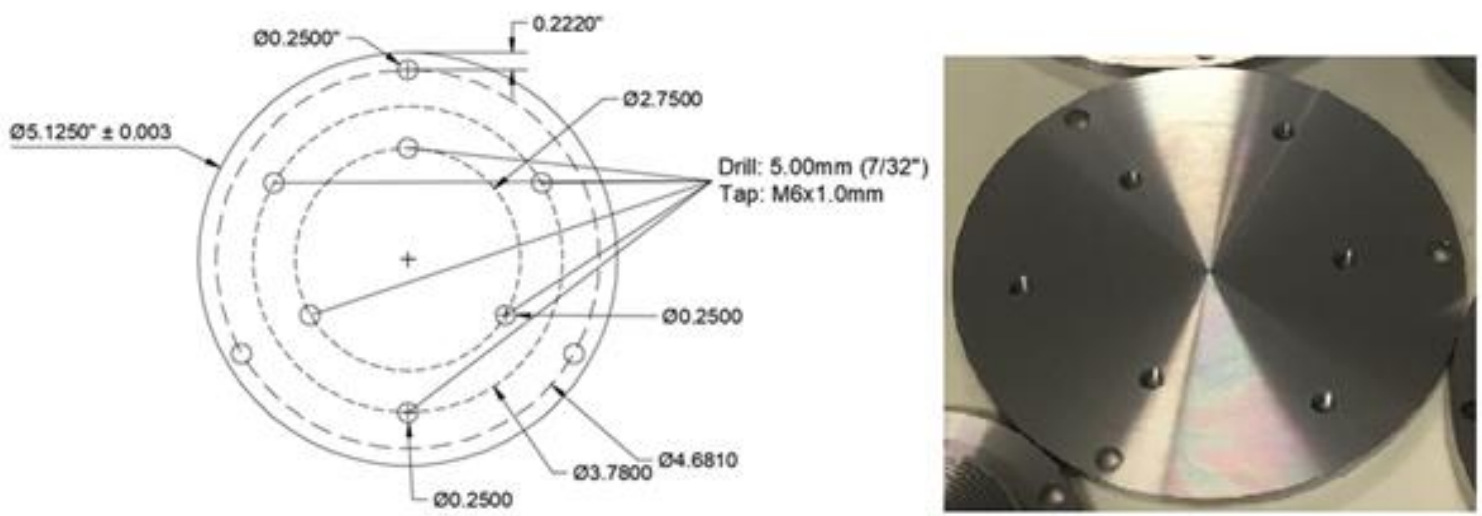

Figure 76: DTC Fatigue Top and Bottom Spacer Top View for 50X110 mm and 75X110 mm Specimen 

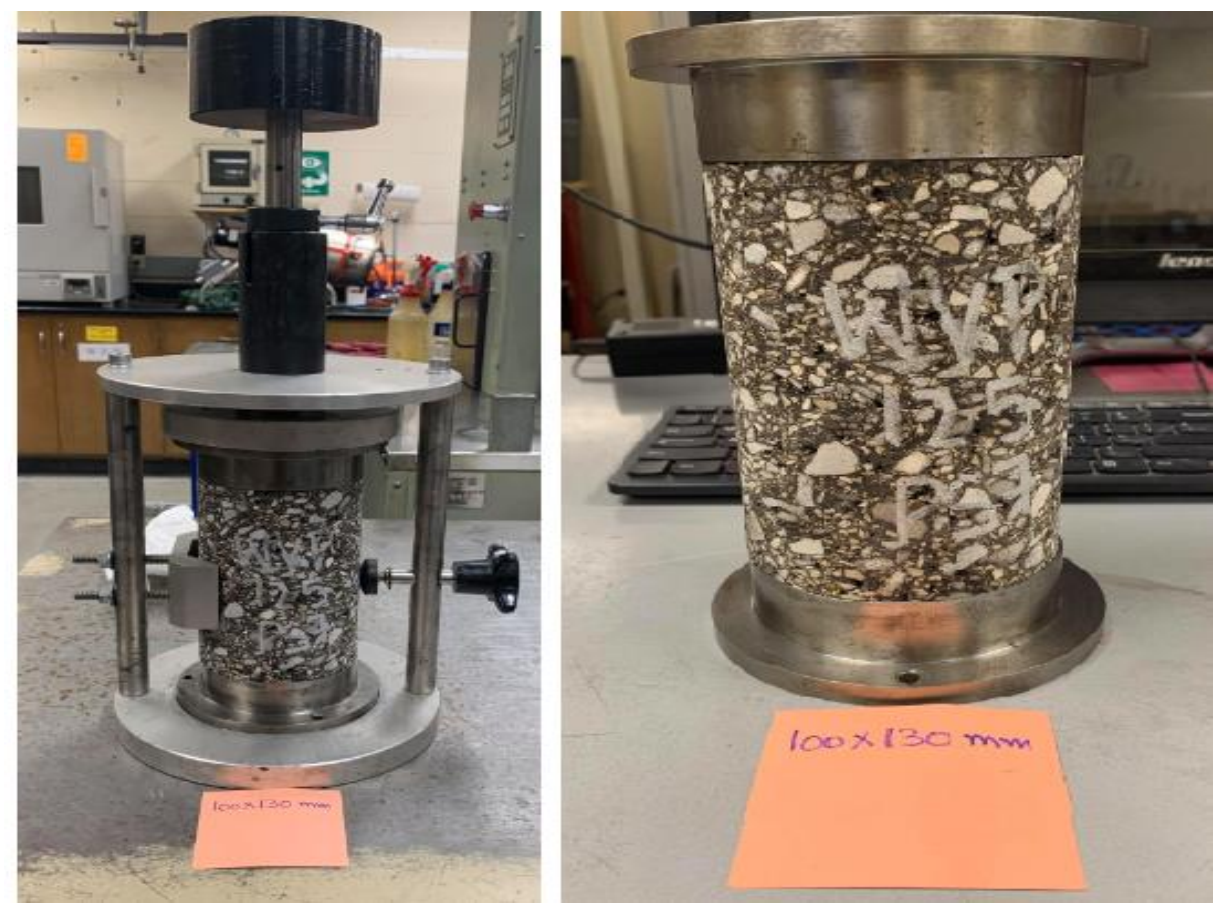

Figure 77: DTC Fatigue End Platens Gluing Jig and Test Set Up for 100X130 mm Specimen8

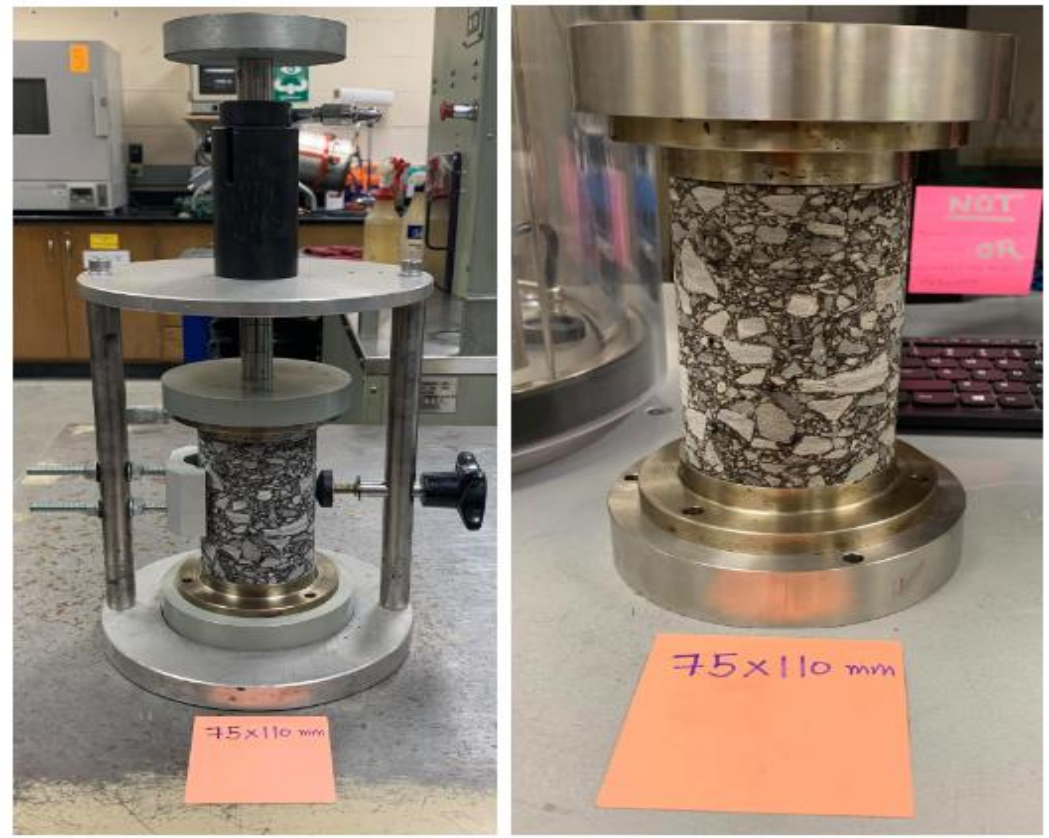

Figure 78: DTC Fatigue End Platens Gluing Jig and Test Set Up for $75 X 110$ mm Specimen ${ }^{7}$

${ }^{8}$ The figures are for depiction purposes only and epoxy is not used to glue the end platens and specimen. For the real test, all specimens were tested per AASHTO TP 107-18 and AASHTO TP 133-19 

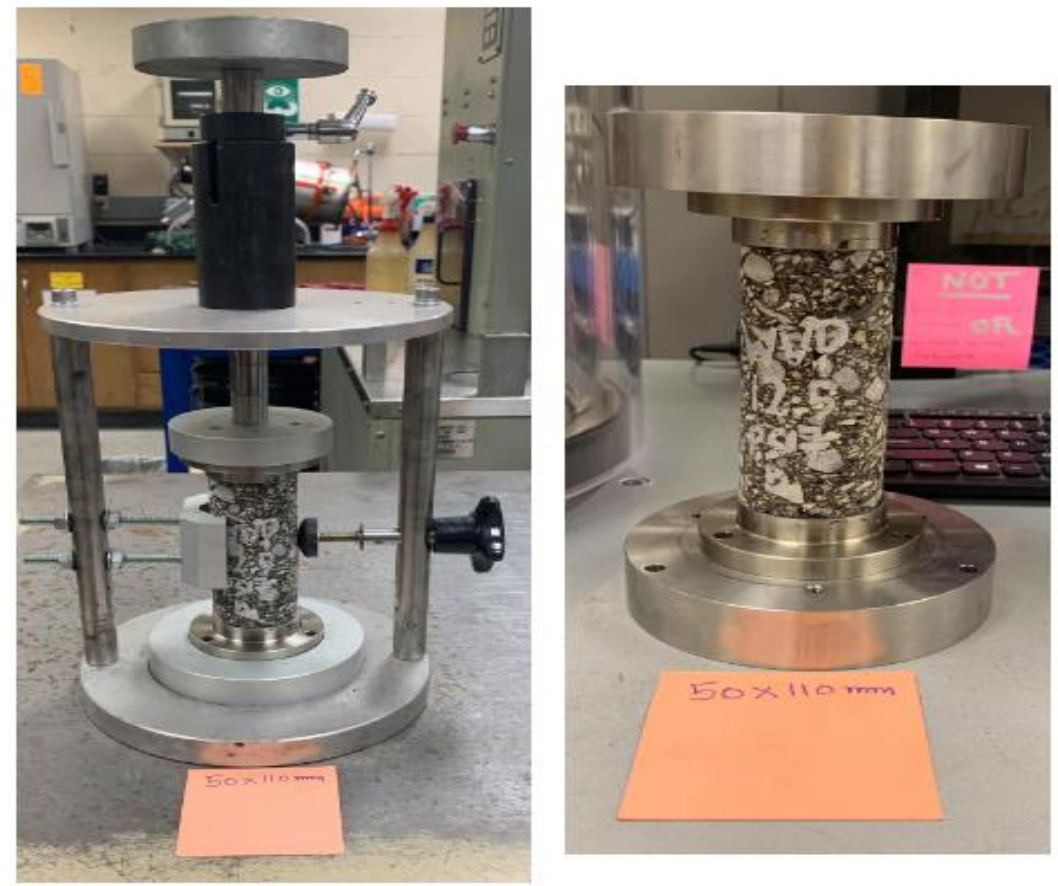

Figure 79: DTC Fatigue End Platens Gluing Jig and Test Set Up for 50X110 mm Specimen ${ }^{7}$
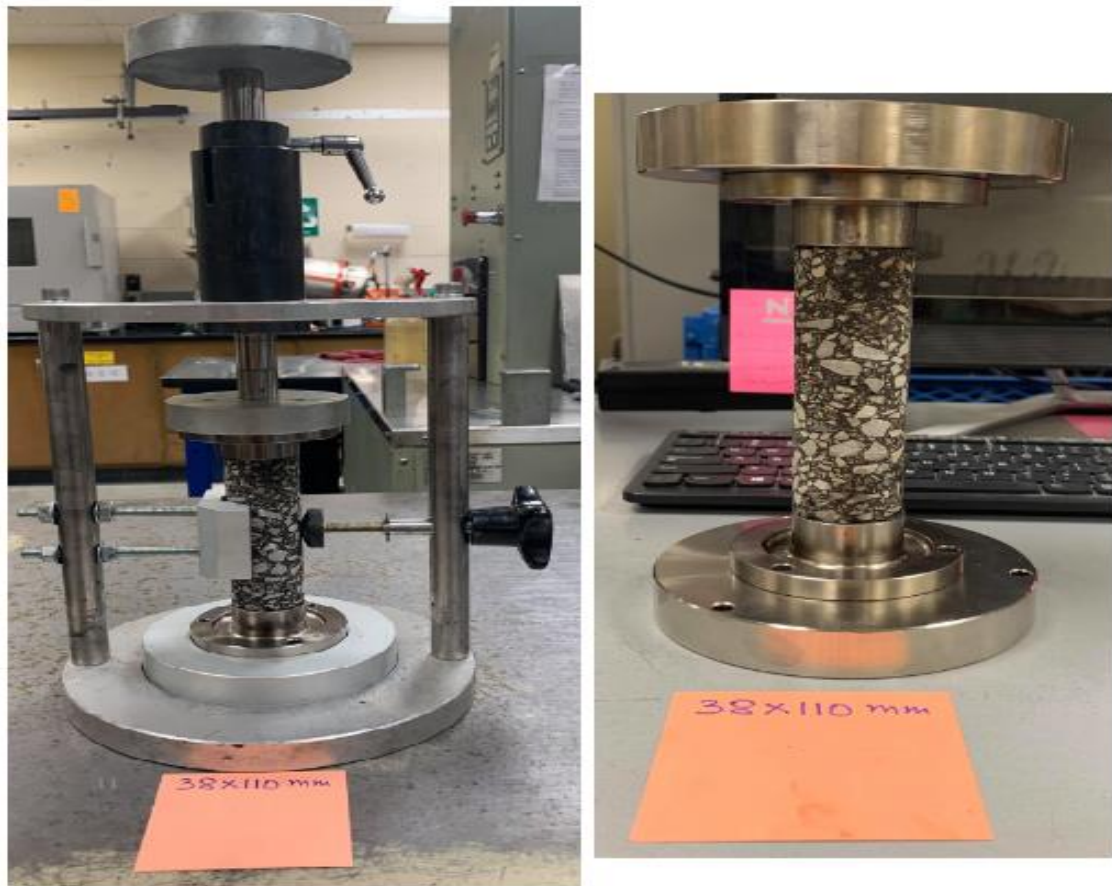

Figure 80: DTC Fatigue End Platens Gluing Jig and Test Set Up for $38 X 110$ mm Specimen ${ }^{7}$ 


\section{Appendix E Test Procedure Steps}

\section{Steps for dynamic modulus testing}

- Place the test specimens in environment chamber for temperature conditioning. The temperature conditioning for all specimen geometries is done using 100X150 mm dummy specimen with the assumption that if the core of $100 \mathrm{X} 150 \mathrm{~mm}$ specimen has reached the test temperature, other specimen geometries is also at the same temperature.

- The AMPT chamber is also temperature conditioned at the target test temperature with top and bottom platens, top and bottom Teflon end-friction reducers cut slightly larger than the loading platen, steel ball, and stem (required for 50X110 mm and 75X110 mm specimens).

- When specimens reach target test temperature, remove them from the external temperature and quickly place them inside the AMPT chamber.

- Assemble specimen bottom to top: bottom platen, stem (if applicable), specimen, top platen, and steel ball.

- Install the LVDTs on the gauge points.

- Close the AMPT chamber and allow the temperature to return to target test temperature.

- This procedure above shall be completed within 5 minutes. After AMPT chamber returns to target test temperature, typically specimen sits in the chamber for 20 minutes before dynamic modulus test begins.

- Enter specimen identification number and input software prompts to begin the test. The AMPT will automatically unload when test is completed.

- Save the AMPT test files and repeat same steps for all replicates.

\section{Steps for air void uniformity (per AASHTO R 83-17)}

- Label top, middle, and bottom of specimen and saw into three equal sections.

- Determine $G_{m b}$ of each slide per AASHTO T 166

- Assemble a summary table to calculate mean and variance of $G_{m b}$

- Perform statistical analysis of means.

\section{Steps for DTC Fatigue testing using AMPT}

- Place the test specimens in environment chamber for temperature conditioning. The temperature conditioning for all specimen geometries is done using 100X150 mm dummy specimen with the 
assumption that if the core of $100 \mathrm{X} 150 \mathrm{~mm}$ specimen has reached the test temperature, other specimen geometries is also at the same temperature.

- The AMPT chamber is also temperature conditioned at the target test temperature with spacers (required for 38X110 mm, 50X110 mm and 75X110 mm specimens).

- When specimens reach target test temperature, remove them from the external temperature and quickly place them inside the AMPT chamber.

- Assemble specimen bottom to top: bottom platen, stem (if applicable), specimen, and top platen. This is bolted to top and bottom AMPT cell plate.

- Caution needs to be taken when attaching glued small specimen geometry of 38X110 mm, $50 \times 110 \mathrm{~mm}$, and 75X110 mm and platens to AMPT cell plate. As very little shear can cause debonding of specimen from the platens. The steps followed for attaching glued specimen and platen to the AMPT cell plate are:

1. The actuator that controls the movement of load cell should be at $-15 \mathrm{~mm}$, providing $30 \mathrm{~mm}$ space inside the chamber.

2. Bolt the spacers on top and bottom of the AMPT cell plate for $38 X 110 \mathrm{~mm}, 50 \mathrm{X} 110 \mathrm{~mm}$, and 75X110 mm samples.

3. Support the glued sample and platen from the bottom and avoid lifting the specimens from the ends as that would cause tension stress on the glue, causing debonding.

4. Place the glued sample and platen on the spacer and bold the lower platen to the lower spacer.

5. Move the actuator up so that the top platen bolts are aligned with top spacer bolts.

6. Stop and start the bolts such that when actuator is moved up the spacer holes and top platen holes are still aligned. Do not tighten the bolts as that would cause tension and shear, ultimately leading to debonding.

7. Bring the actuator up and stop when the seating load reaches $0.09 \mathrm{kN}(20 \mathrm{lb}$.) for $100 \mathrm{X} 130$ $\mathrm{mm}$ specimen and $0.01 \mathrm{kN}$ (2.25 lb.) for $38 \mathrm{X} 110 \mathrm{~mm}, 50 \mathrm{X} 110 \mathrm{~mm}$, and $75 \mathrm{X} 110 \mathrm{~mm}$ specimens.

8. Start tightening the bolts of top spacer and top platen such that the seating load is maintained. If seating load goes in negative, glued specimen and platen are in tension which will lead to debonding.

9. If specimens are debonded from the platen, specimens are discarded, and new test specimen is fabricated.

- Install the LVDTs on the gauge points.

- Close the AMPT chamber and allow the temperature to return to target test temperature. 
- This procedure above shall be completed within 5 minutes. After AMPT chamber returns to target test temperature, typically specimen sits in the chamber for 20 minutes before dynamic modulus test begins.

- Enter specimen identification number and input software prompts to begin the test. The AMPT will automatically unload when test is completed.

- $\quad$ Save the AMPT test files and repeat same steps for all replicates.

\section{Steps for SSR testing using AMPT}

- Place the test specimens in environment chamber for temperature conditioning. The temperature conditioning for all specimen geometries is done using 100X150 mm dummy specimen with the assumption that if the core of $100 \mathrm{X} 150 \mathrm{~mm}$ specimen has reached the test temperature, other specimen geometries is also at the same temperature.

- The AMPT chamber is also temperature conditioned at the target test temperature with top and bottom platens, top and bottom two greased double latex end-friction reducers cut slightly larger than the loading platen, membrane, O-ring seals, steel ball, and stem (required for 50X110 mm specimens).

- When specimens reach target test temperature, remove them from the external temperature and quickly place them inside the AMPT chamber.

- Assemble specimen bottom to top: bottom platen, stem (if applicable), bottom greased double latex end-friction reducers, specimen, top greased double latex end-friction reducers, top platen, membrane, O-ring seal, and steel ball

- Close the AMPT chamber and allow the temperature to return to target test temperature.

- This procedure above shall be completed within 5 minutes. After AMPT chamber returns to target test temperature, typically specimen sits in the chamber for 1 hour at $69 \mathrm{kPa}$ (10 psi) confining pressure before SSR test begins.

- Enter specimen identification number and input software prompts to begin the test. The AMPT will automatically unload when test is completed.

- $\quad$ Save the AMPT test files and repeat same steps for all replicates. 


\section{Appendix F FlexPAVE Models Inputs}

Three pavement structures with different STs for data analysis in FlexPAVE, are shown as:

\begin{tabular}{|c|c|c|c|}
\hline Model & $\begin{array}{c}\text { Dynamic } \\
\text { Modulus } \\
\text { Specimen, mm }\end{array}$ & $\begin{array}{c}\text { DTC Fatigue } \\
\text { Specimen, } \\
\text { mm }\end{array}$ & $\begin{array}{c}\text { SSR } \\
\text { Specimen, } \\
\text { mm }\end{array}$ \\
\hline 1 & ST1 ST1 & ST1 \\
\hline 2 & ST2 & ST1 \\
\hline 3 & ST3 ST1 \\
\hline 4 & ST5 & ST4 \\
\hline 5 & ST6 & ST1 \\
\hline 6 & ST1 & ST4 \\
\hline 7 & ST2 & ST4 \\
\hline 8 & ST3 & ST1 \\
\hline 9 & ST4 & ST1 \\
\hline 10 & ST5 & ST4 \\
\hline 11 & ST6 & ST \\
\hline 12 & ST & ST \\
\hline
\end{tabular}

The terminology of Run X.Y is used in this analysis where, X stands for one of the three different structures and $\mathrm{Y}$ stands for one of the twelve different models.

\section{Run 1.1}

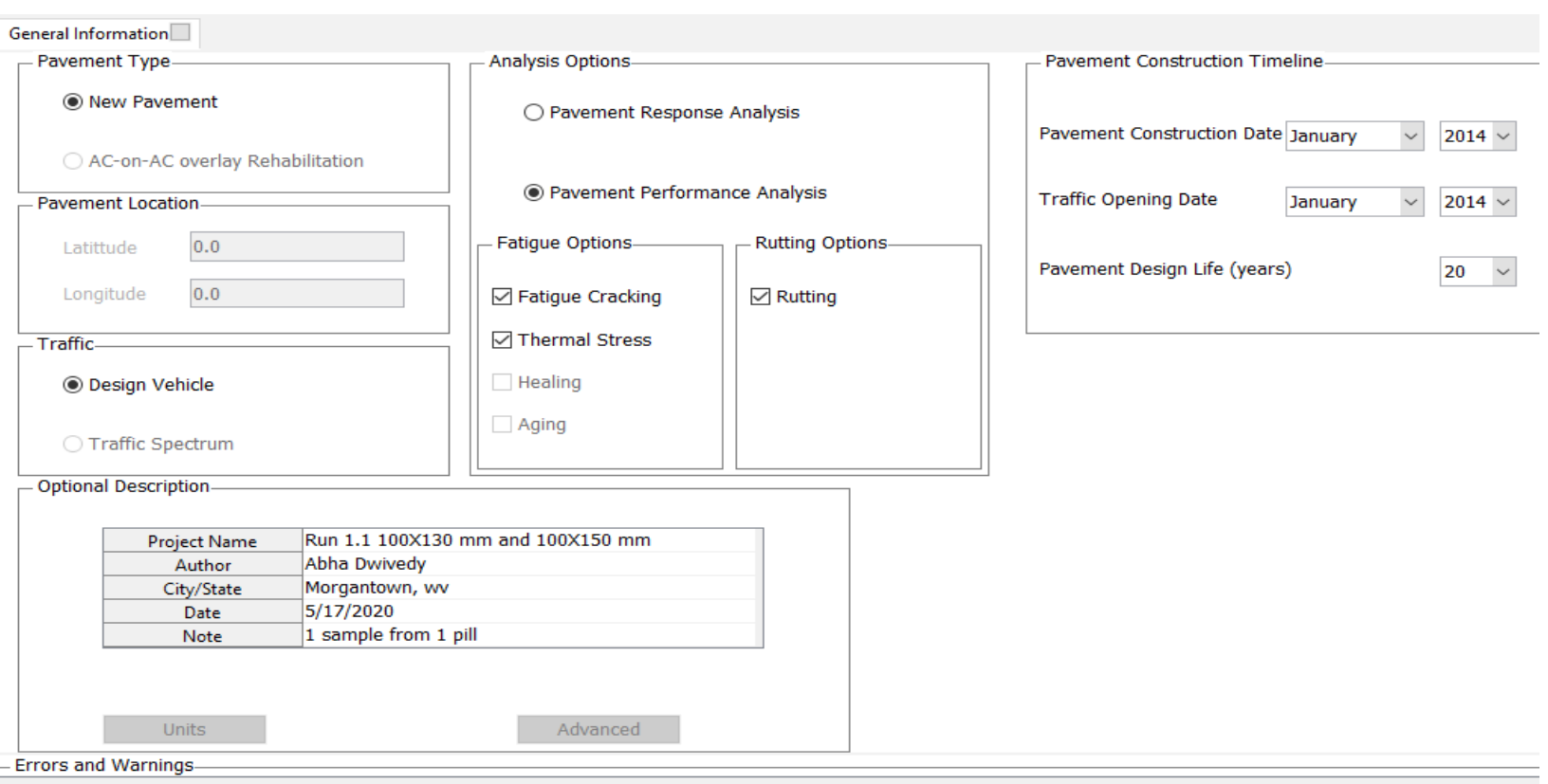

Figure 81: General Information for Run 1.1 

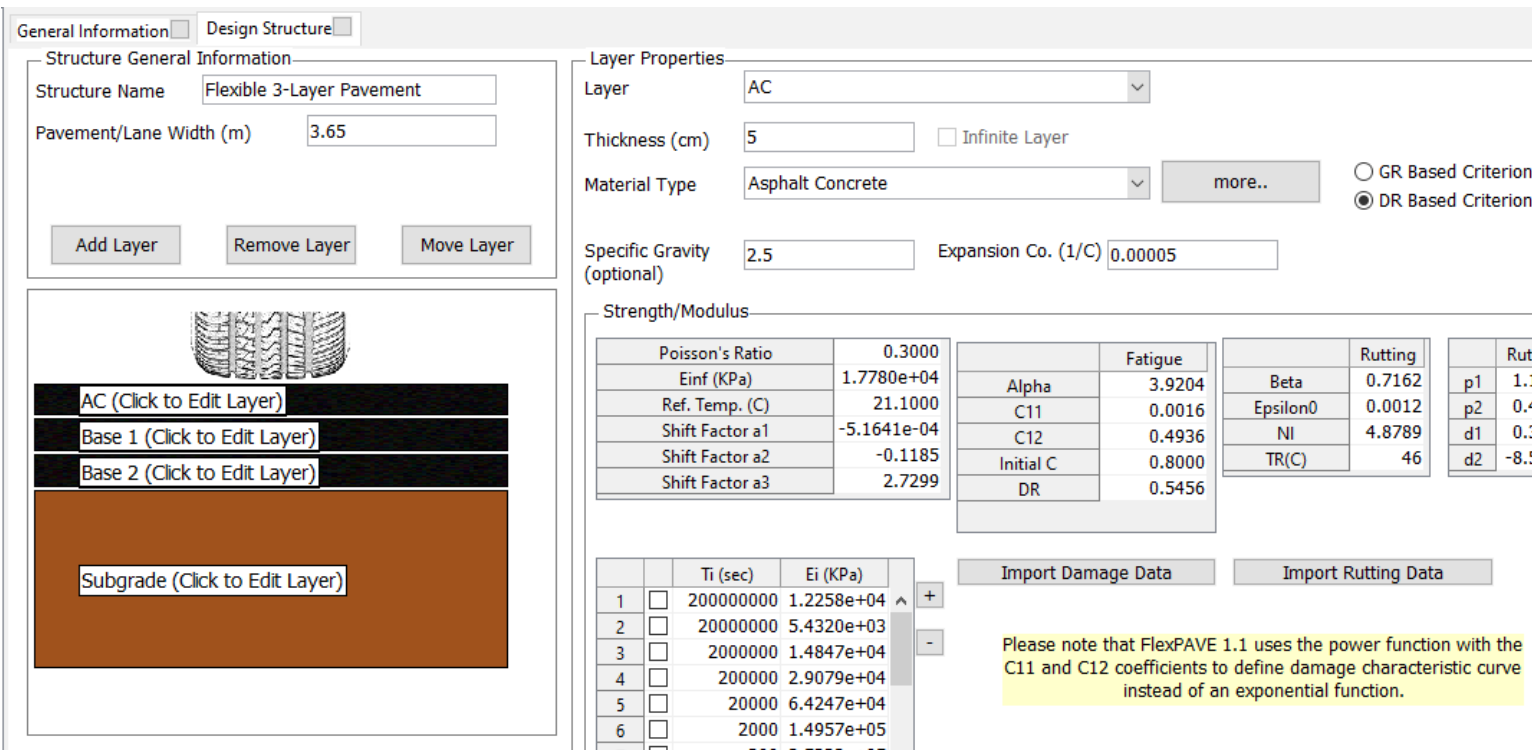

(optional)

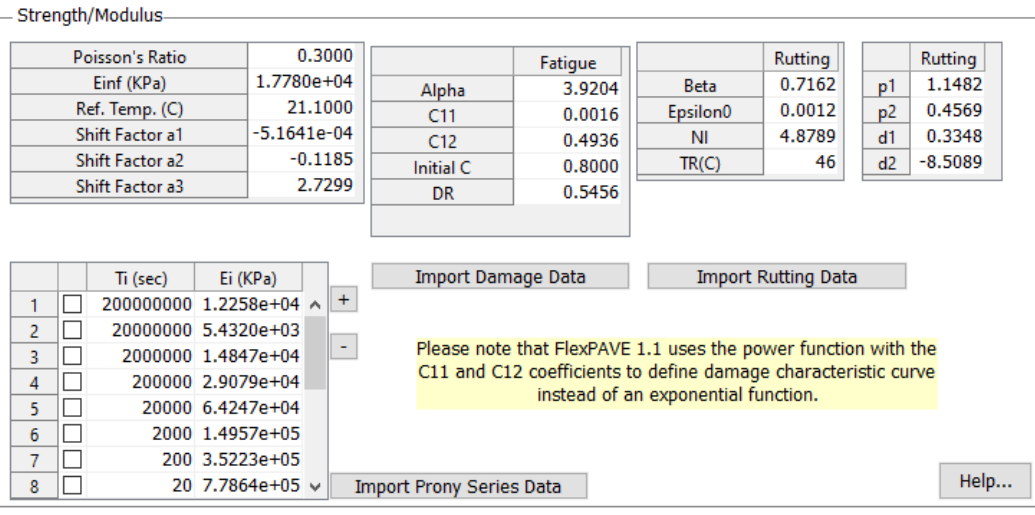

\section{Figure 82: Design Structure of AC Layer for Run 1.1}
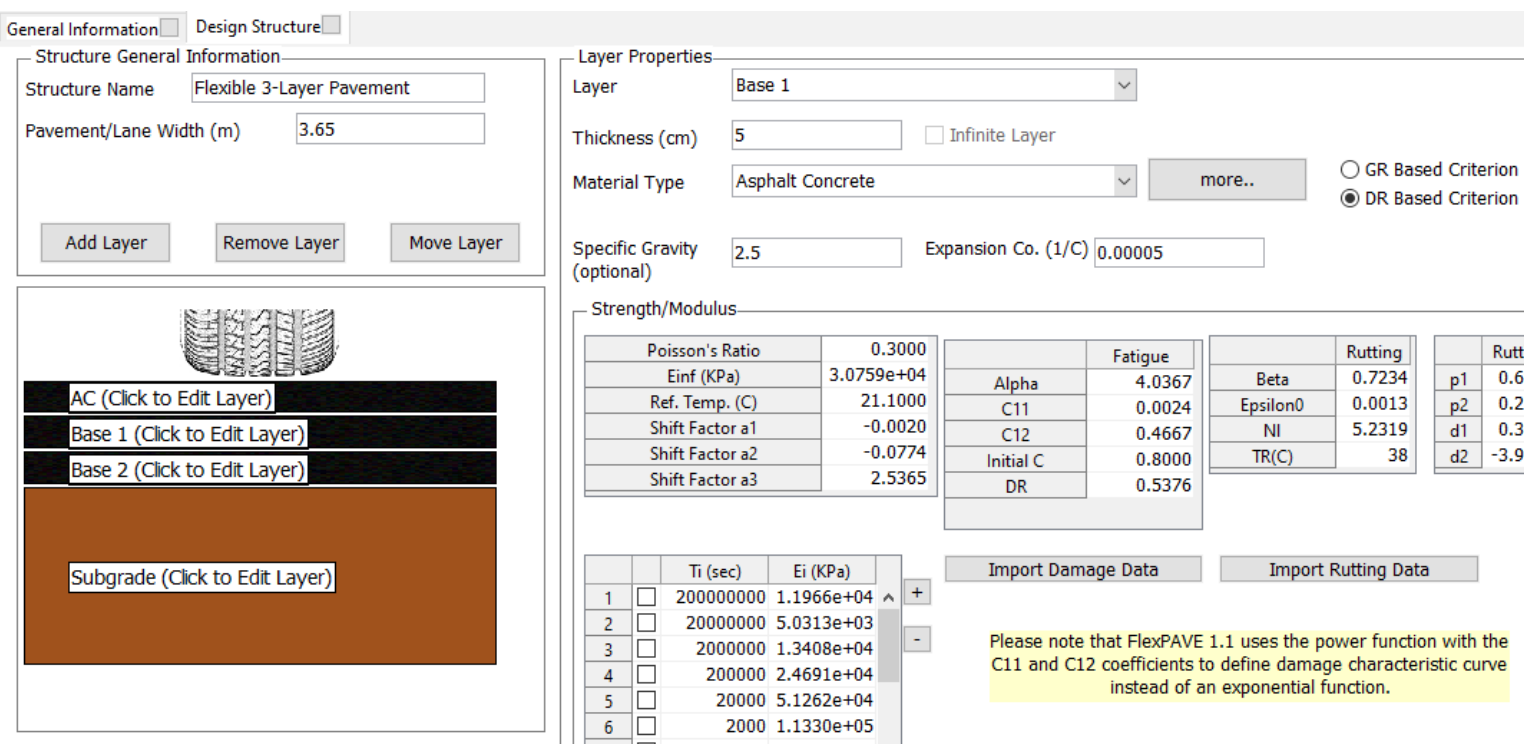

Figure 83: Design Structure of Base 1 Layer for Run 1.1 

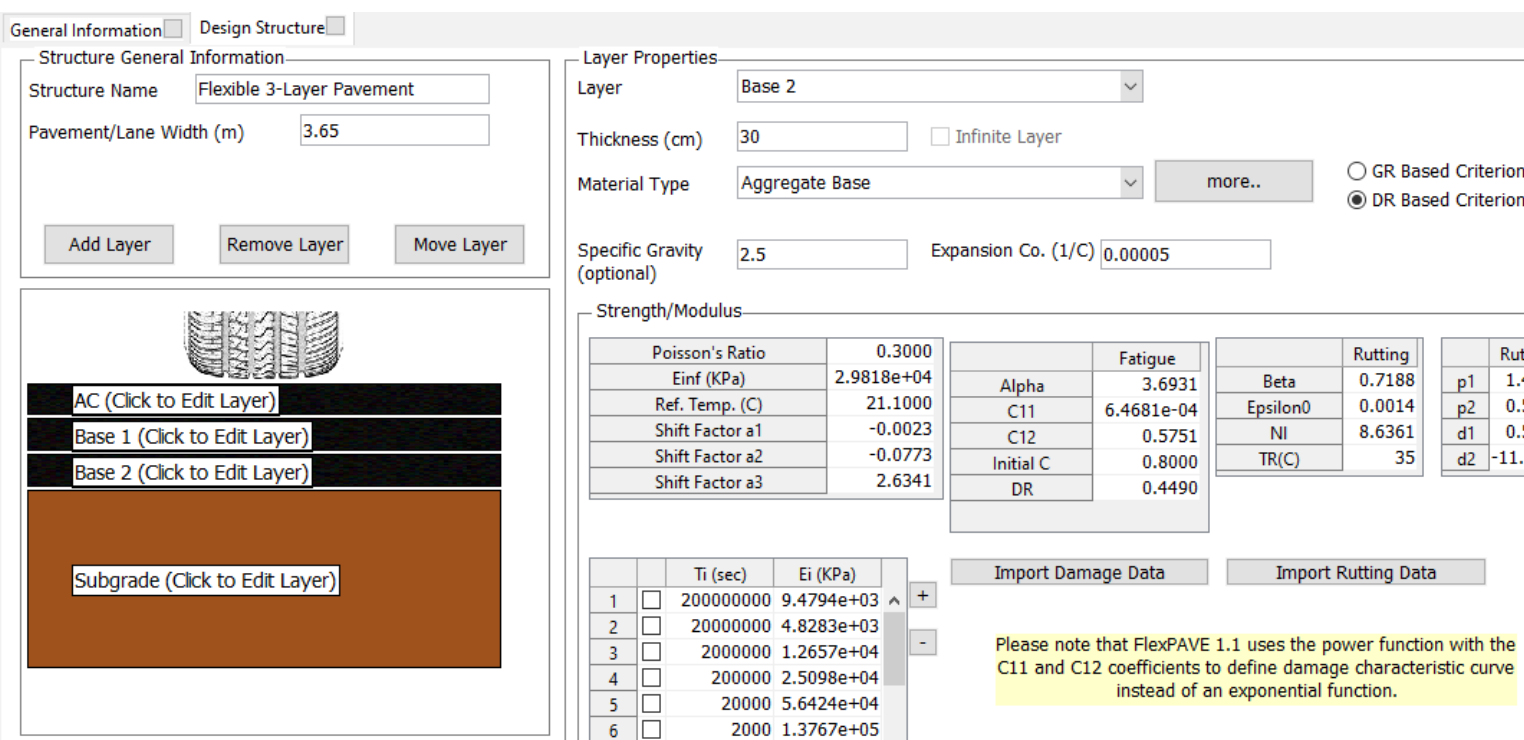

(optional)

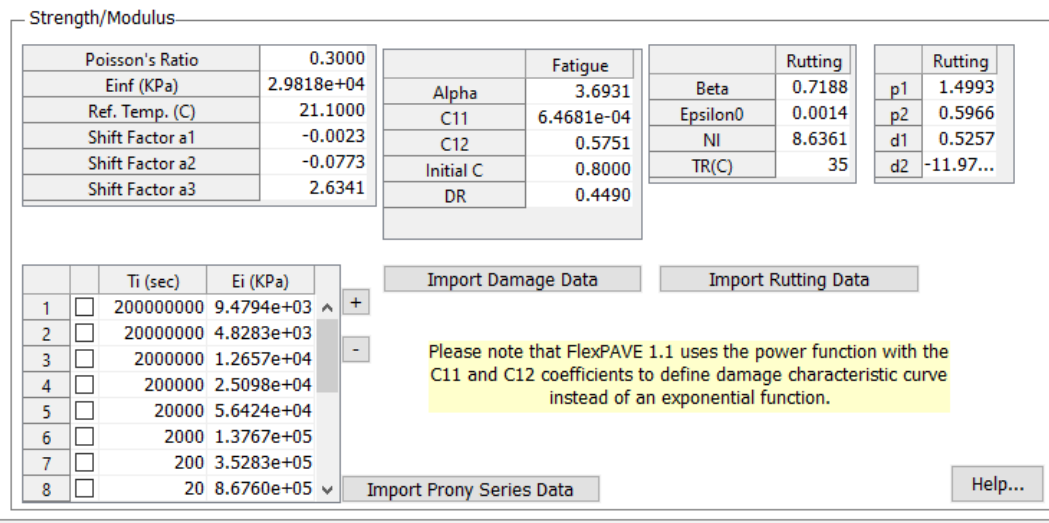

Errors and Warnings

\section{Figure 84: Design Structure of Base 2 Layer for Run 1.1}

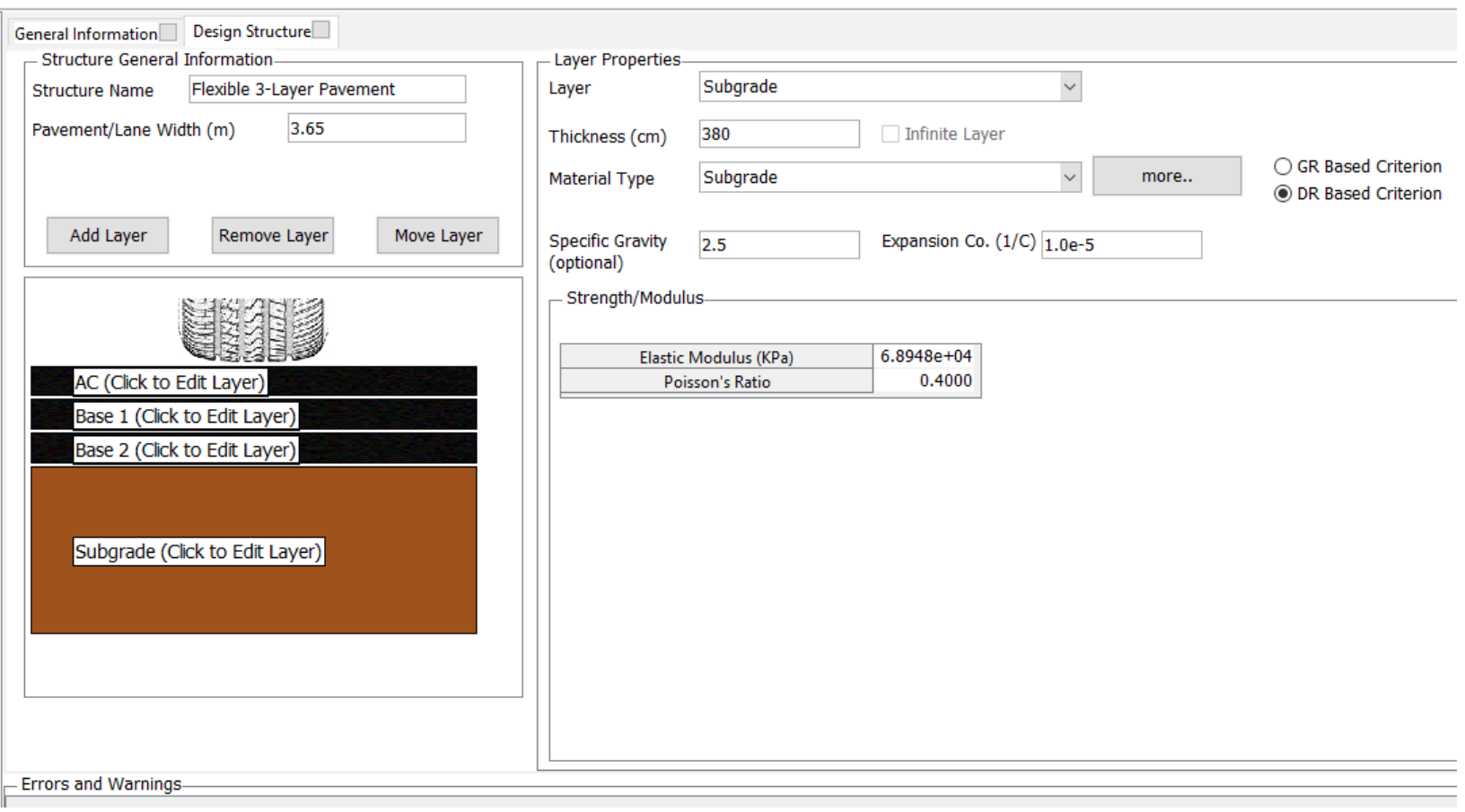

Figure 85: Design Structure of Subgrade Layer for Run 1.1 


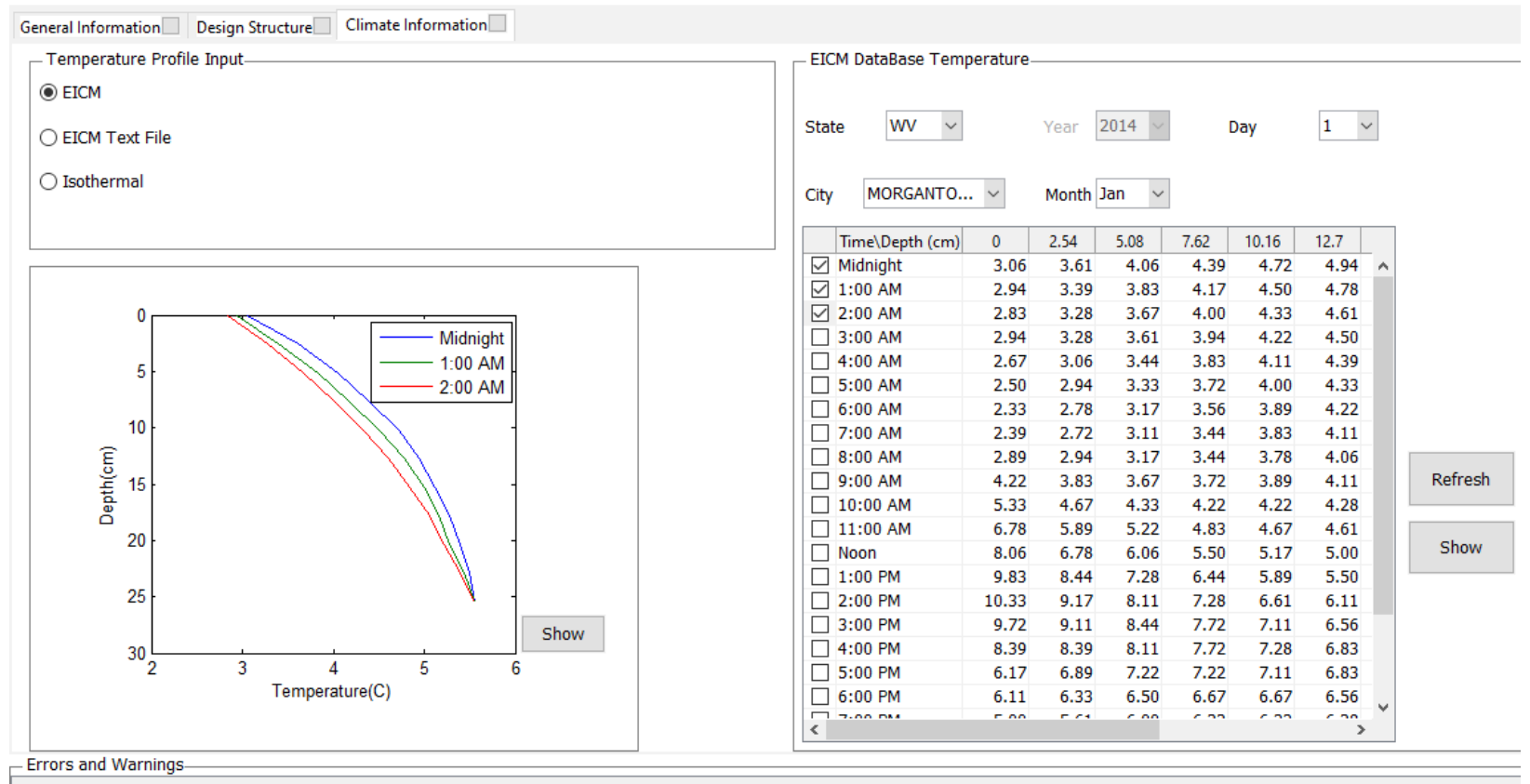

\section{Figure 86: Climate Data for Run 1.1}

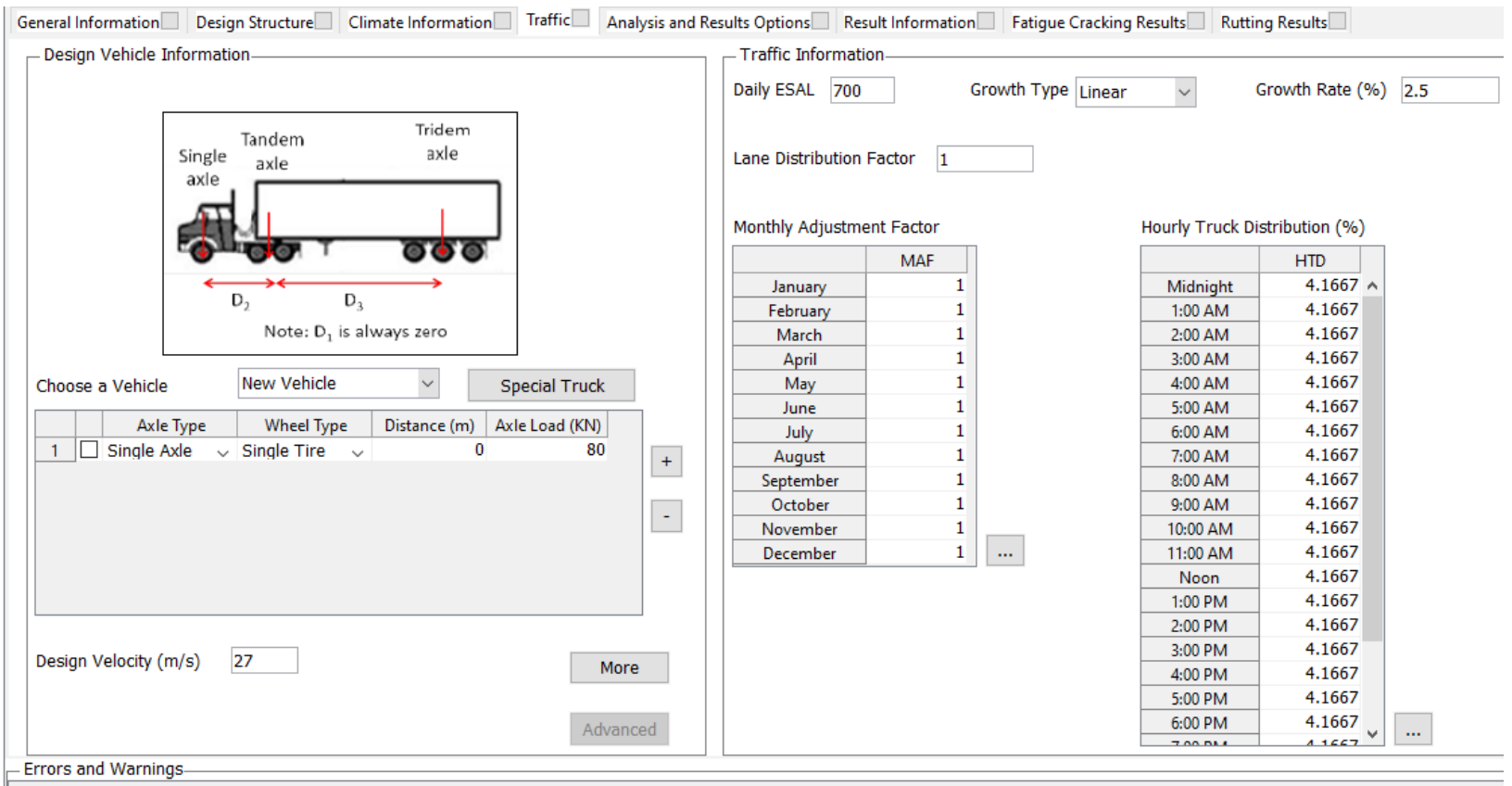




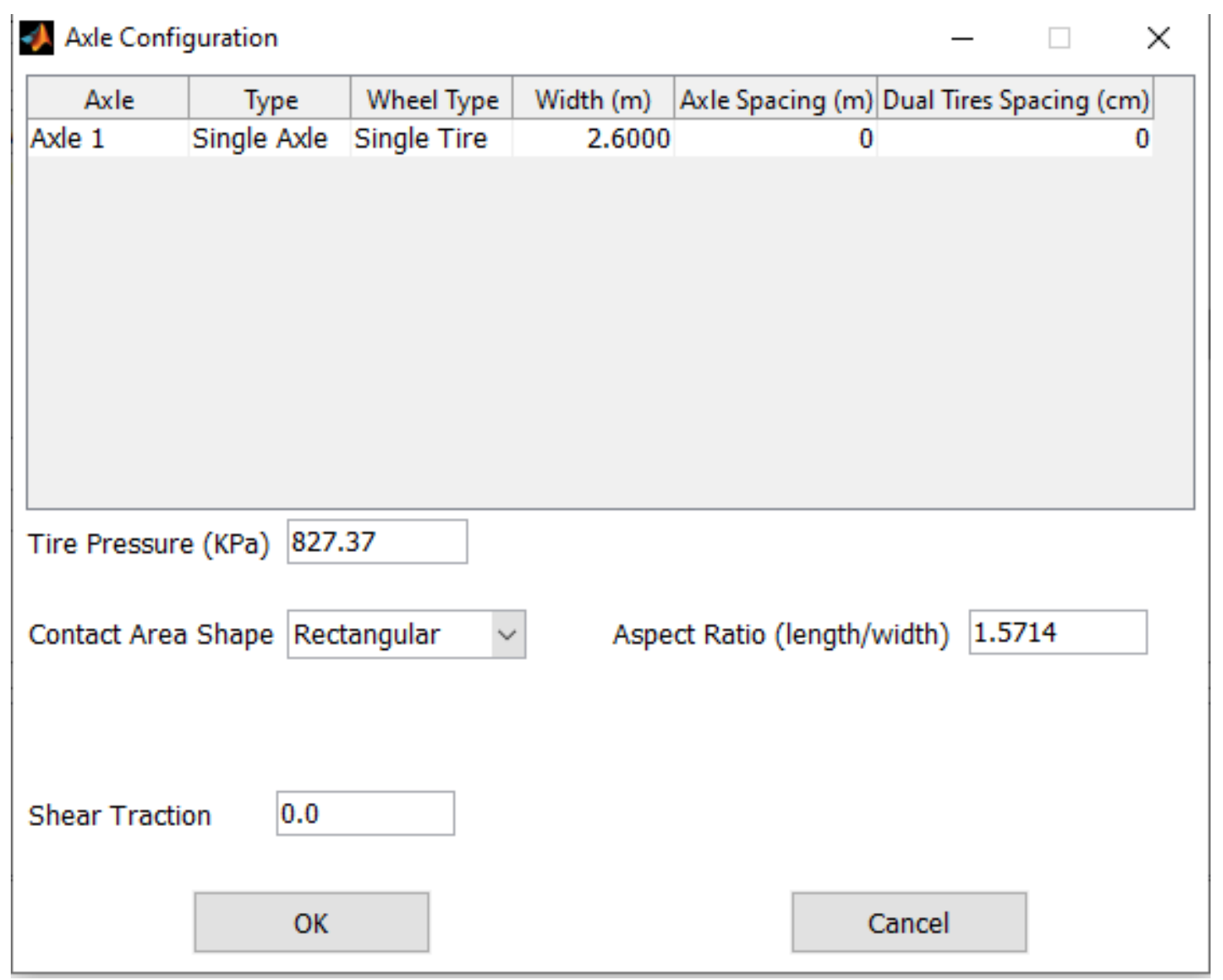

Figure 87: Traffic Data for Run 1.1

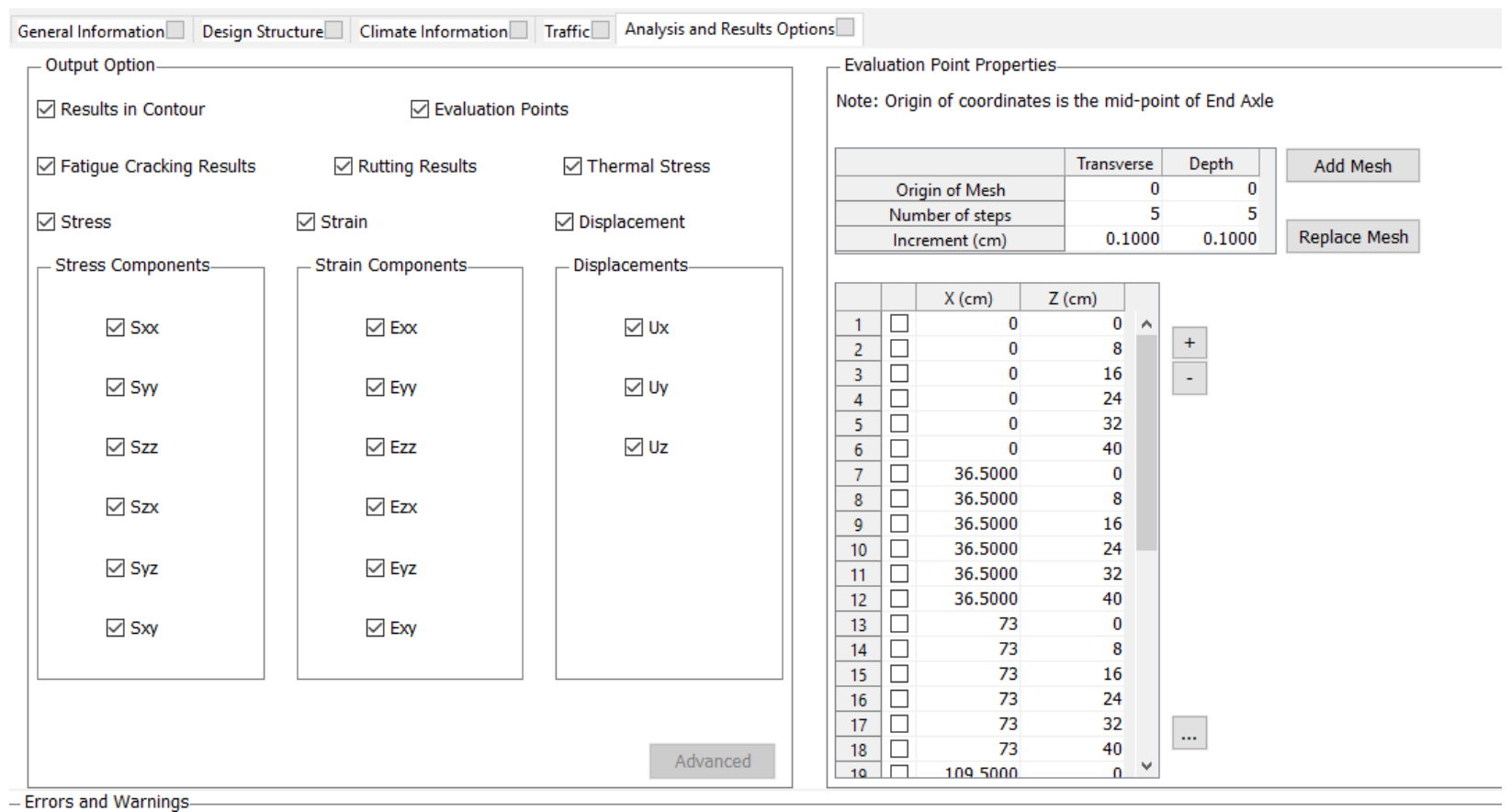

Figure 88: Output and Analysis Options for Run 1.1 


\section{Run 1.2}

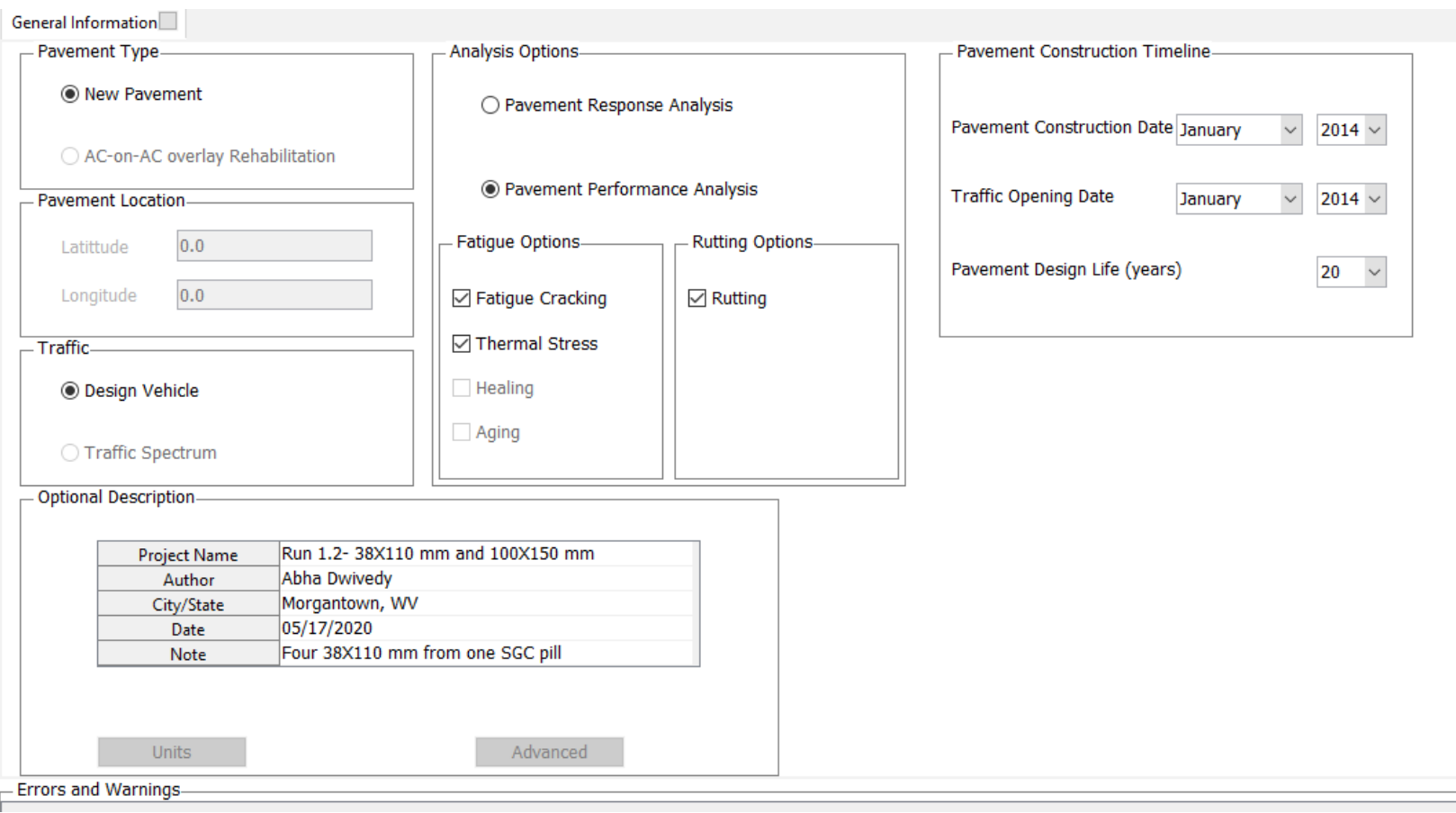

\section{Figure 89: General Information for Run 1.2}

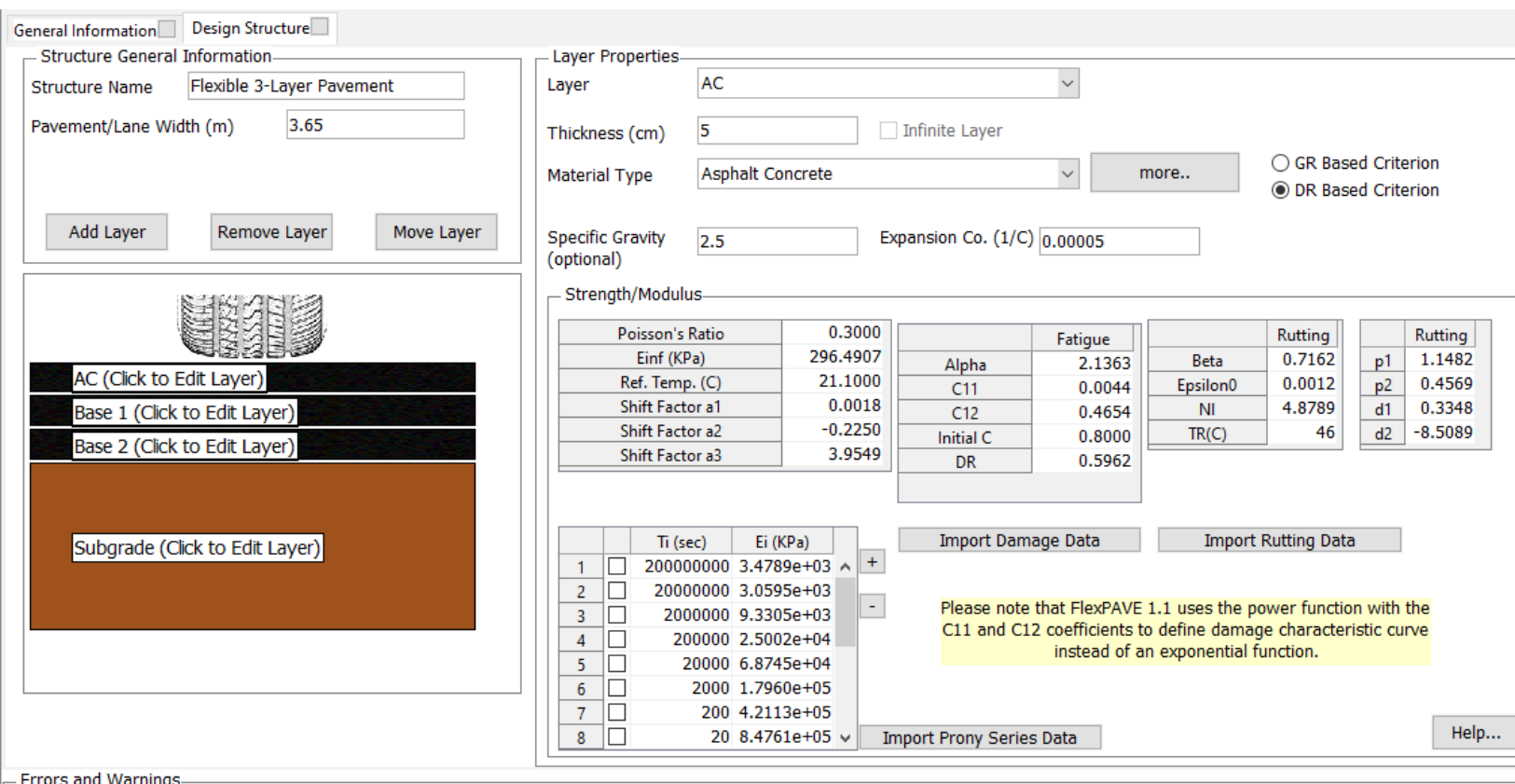

Figure 90: Design Structure of AC Layer for Run 1.2 


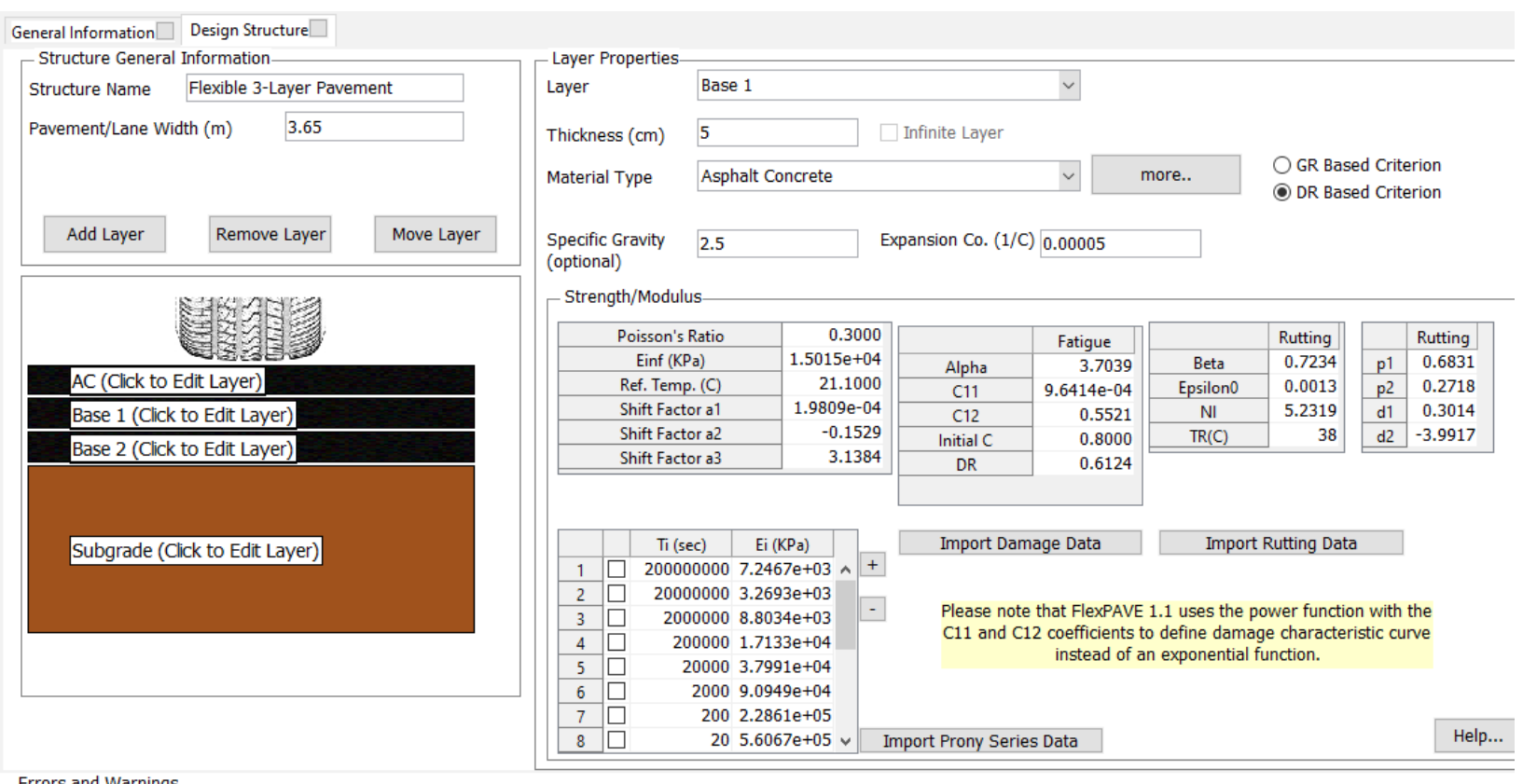

\section{Figure 91: Design Structure of Base 1 Layer for Run 1.2}

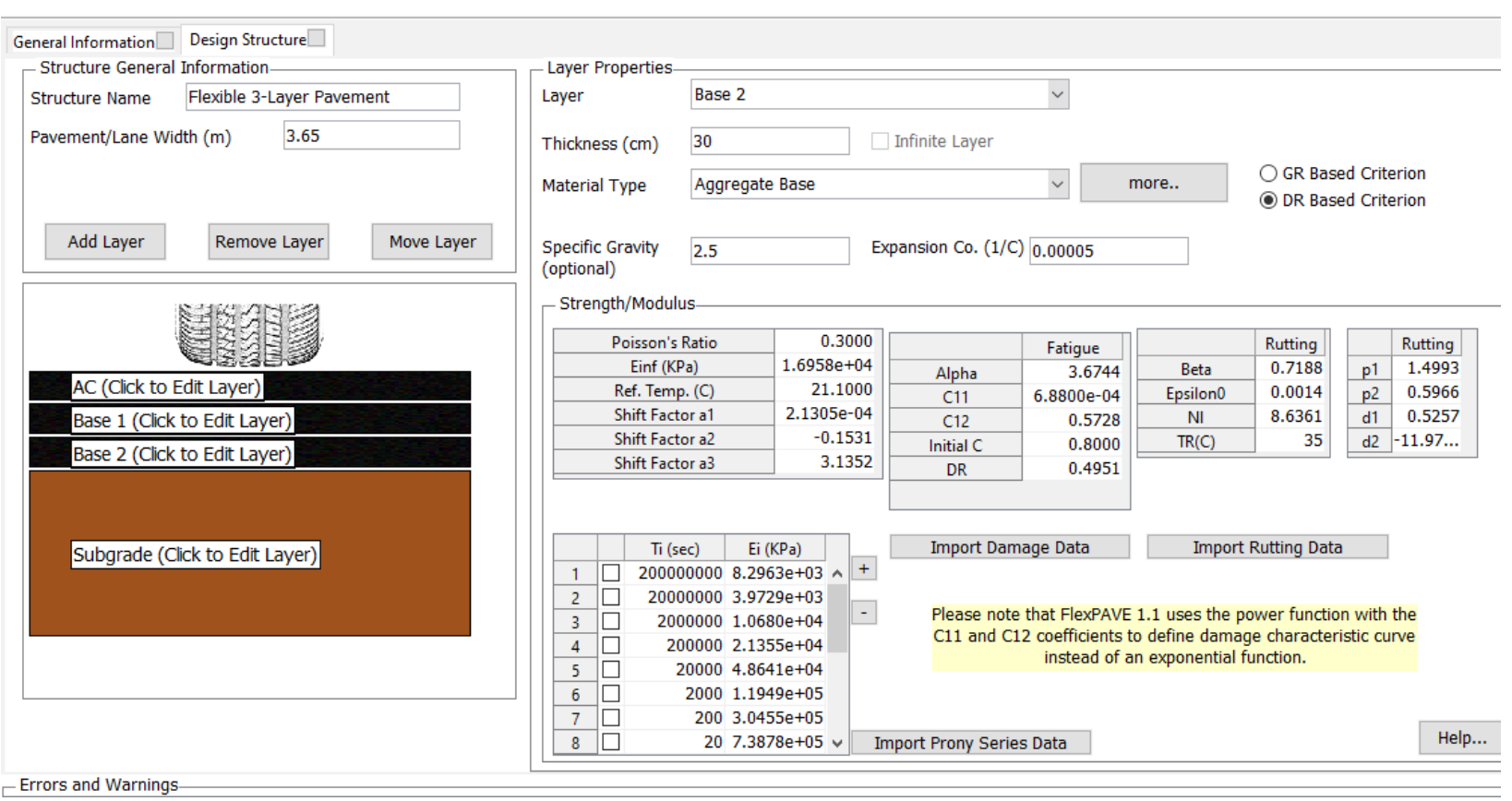

Figure 92: Design Structure of Base 2 Layer for Run 1.2 


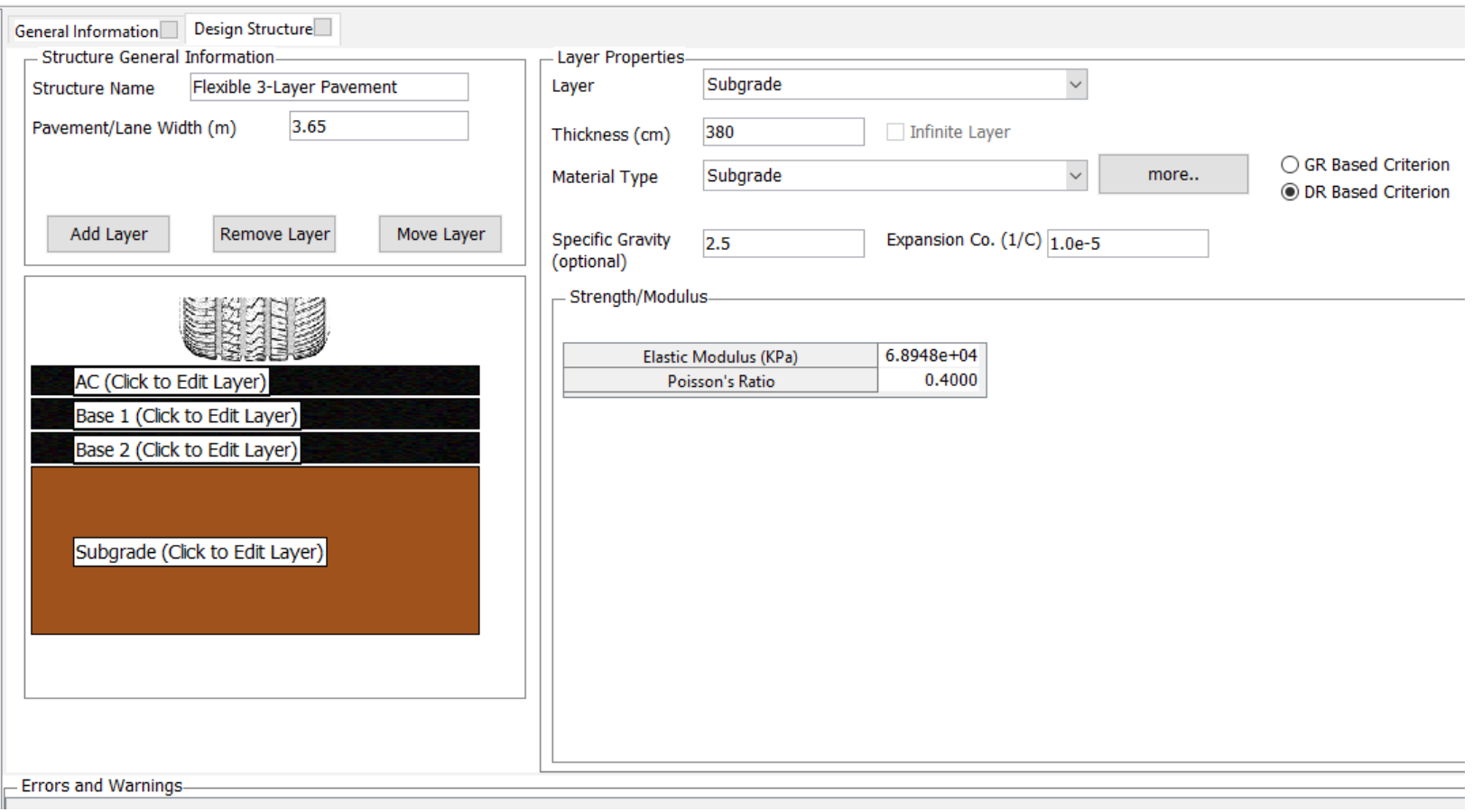

Figure 93: Design Structure of Subgrade Layer for Run 1.2

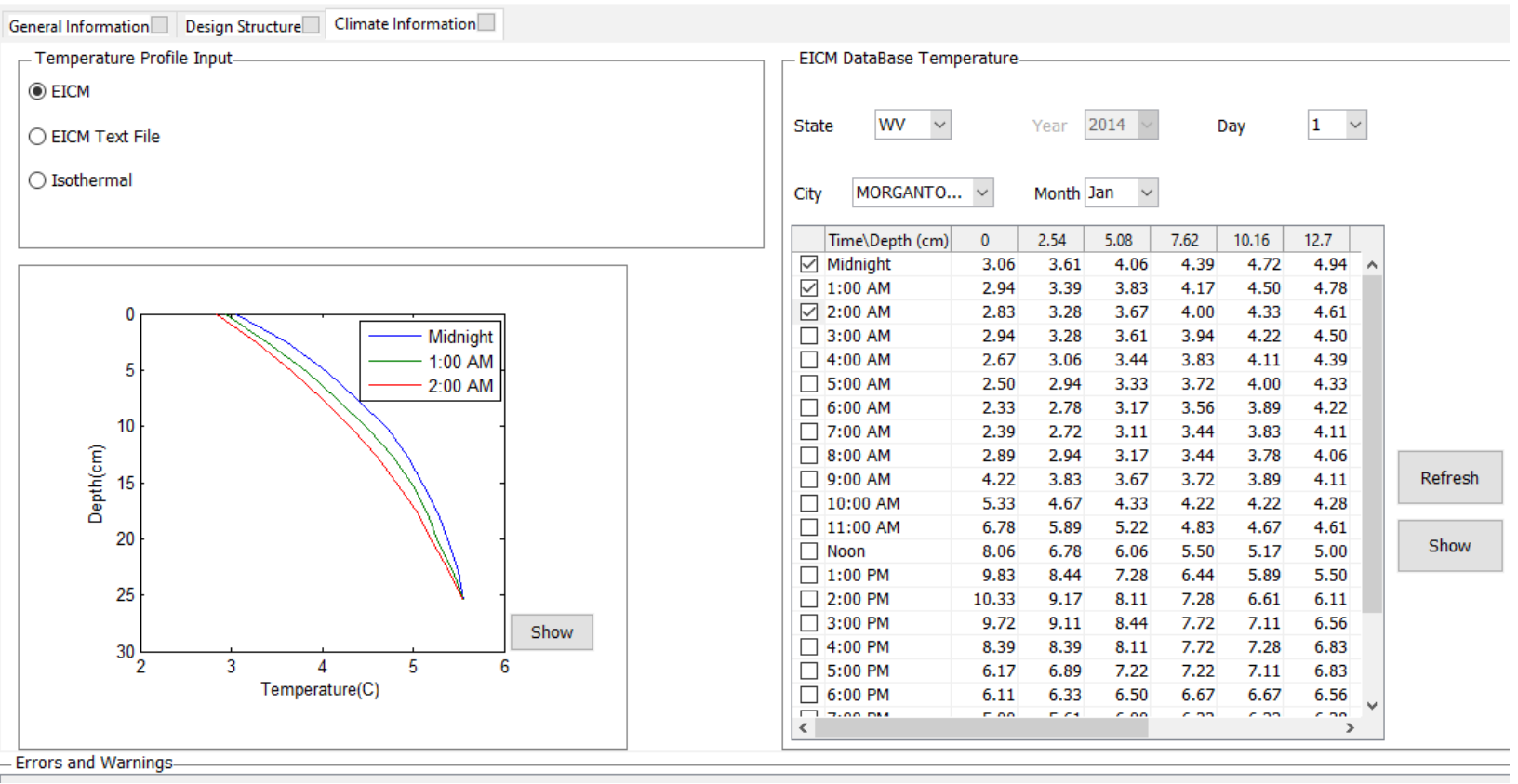

Figure 94: Climate Data for Run 1.2 


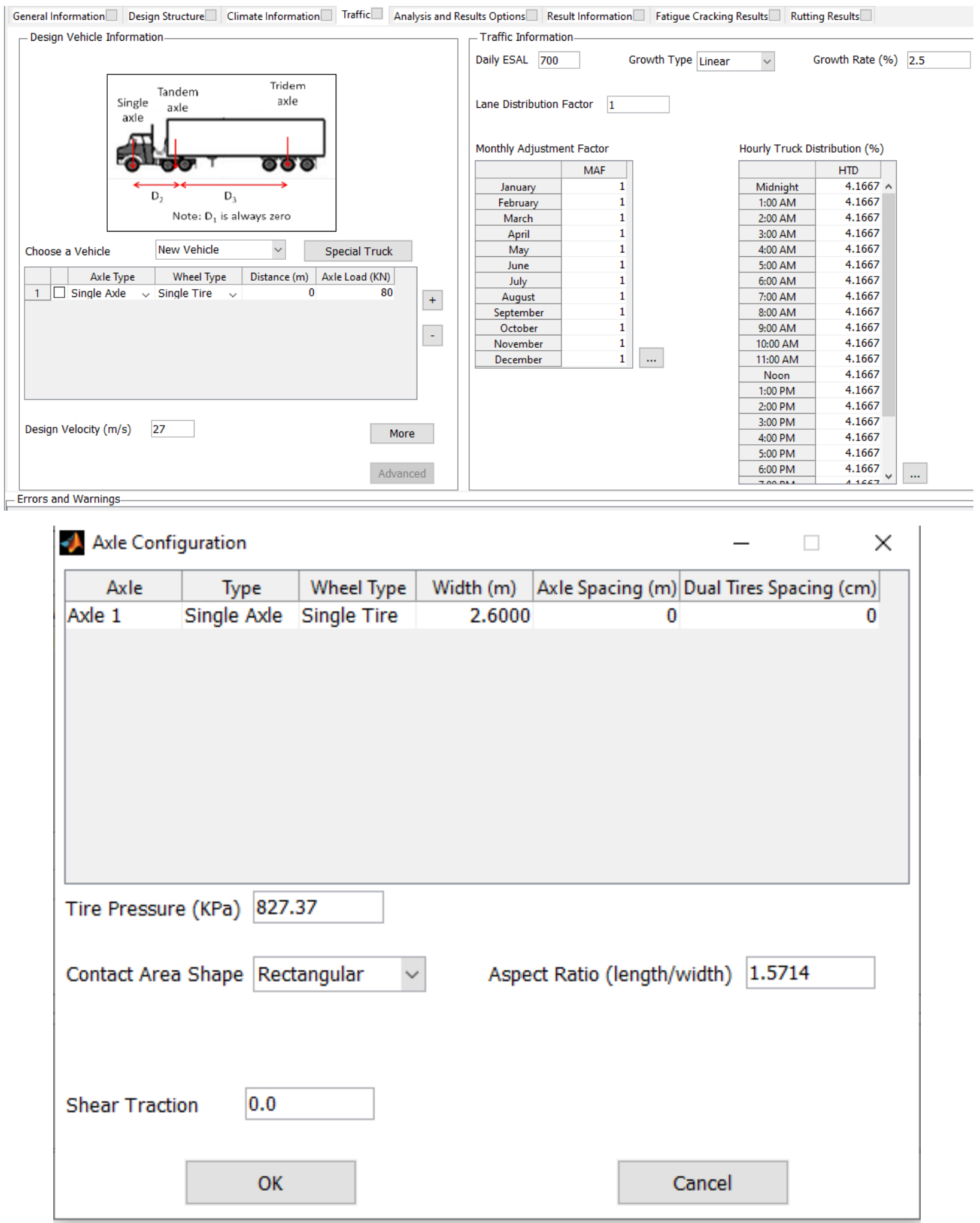

Figure 95: Traffic Data for Run 1.2 


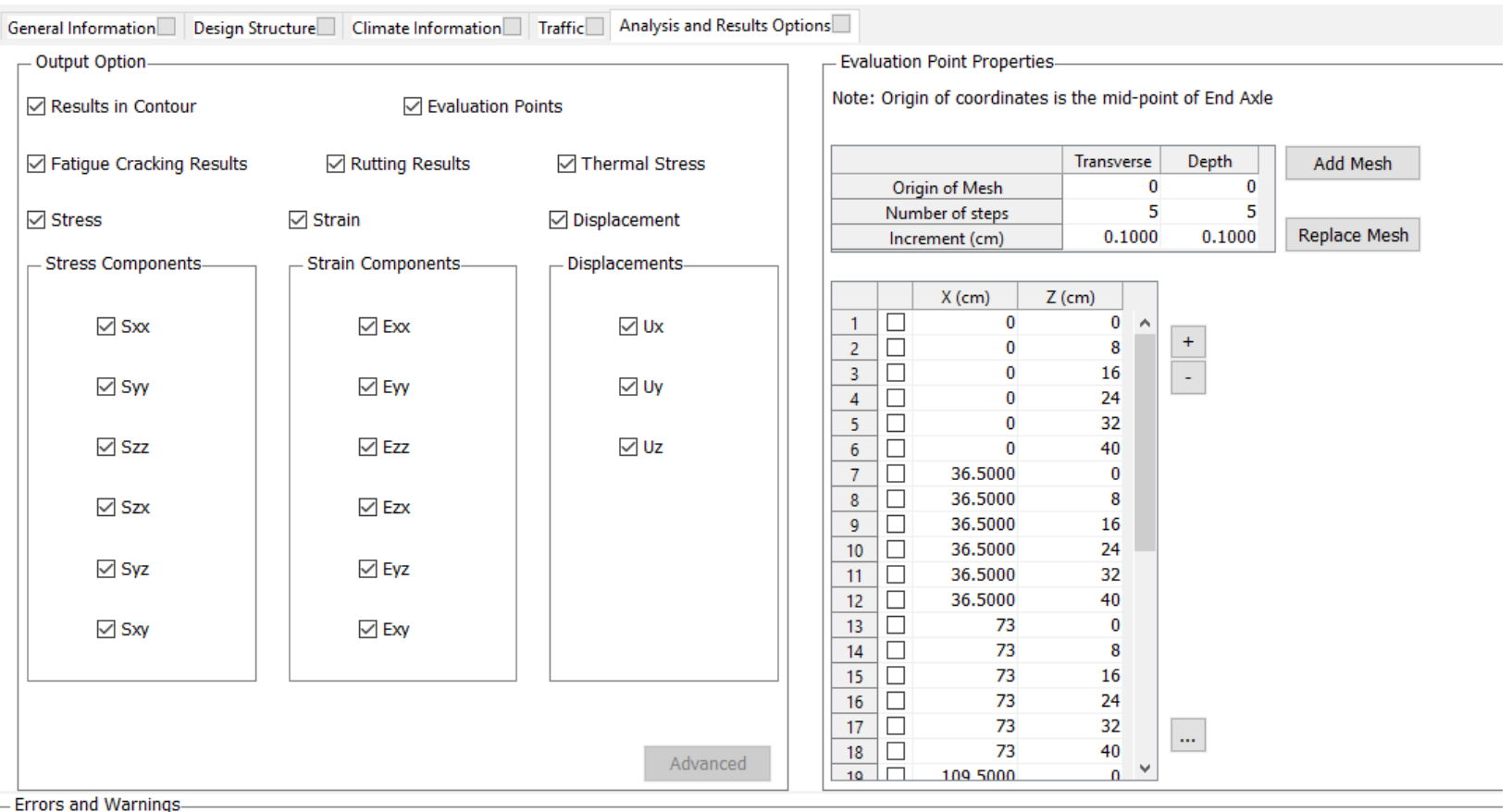

Figure 96: Output and Analysis Options for Run 1.2

\section{Run 1.3}

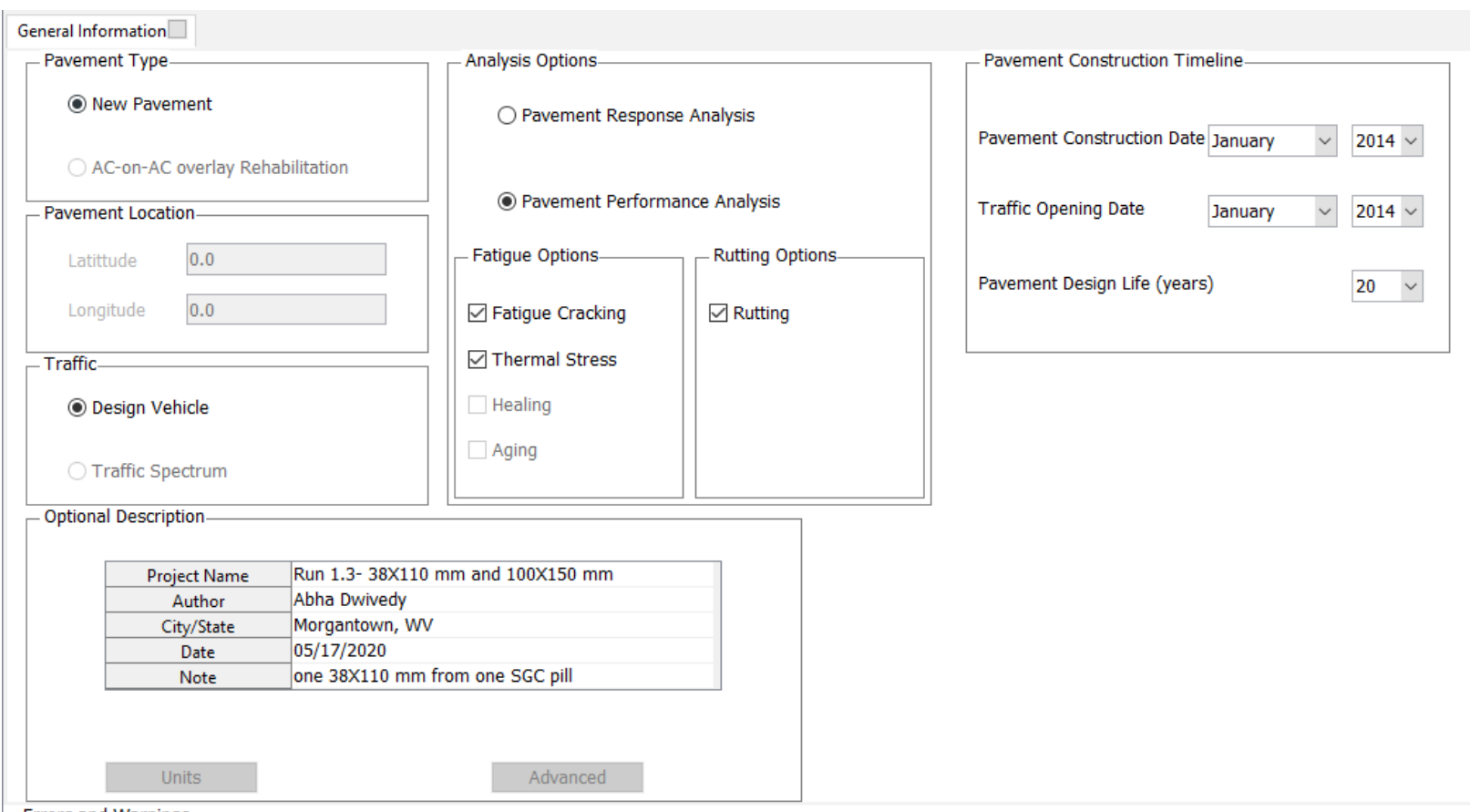

Figure 97: General Information for Run 1.3 

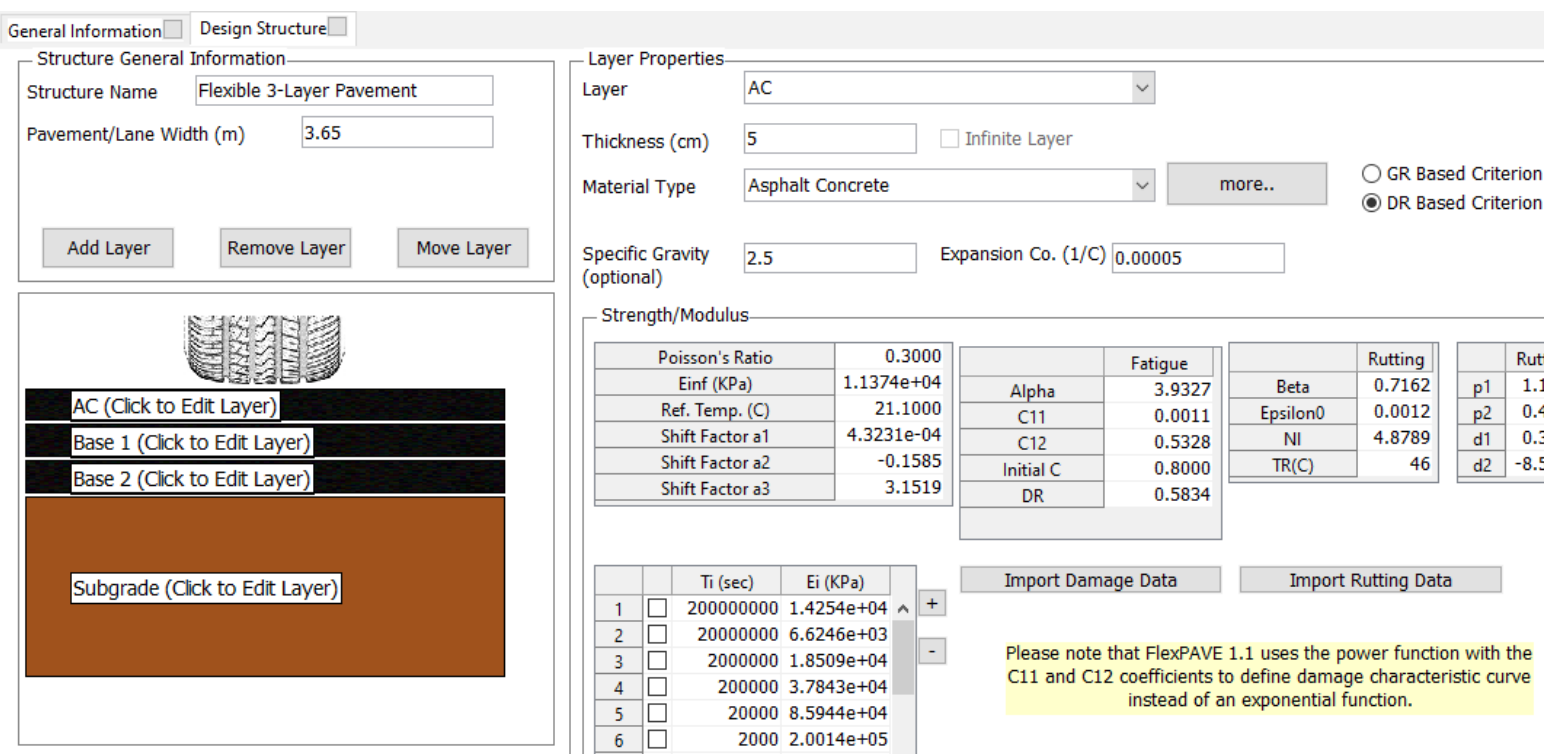

Figure 98: Design Structure of AC Layer for Run 1.3

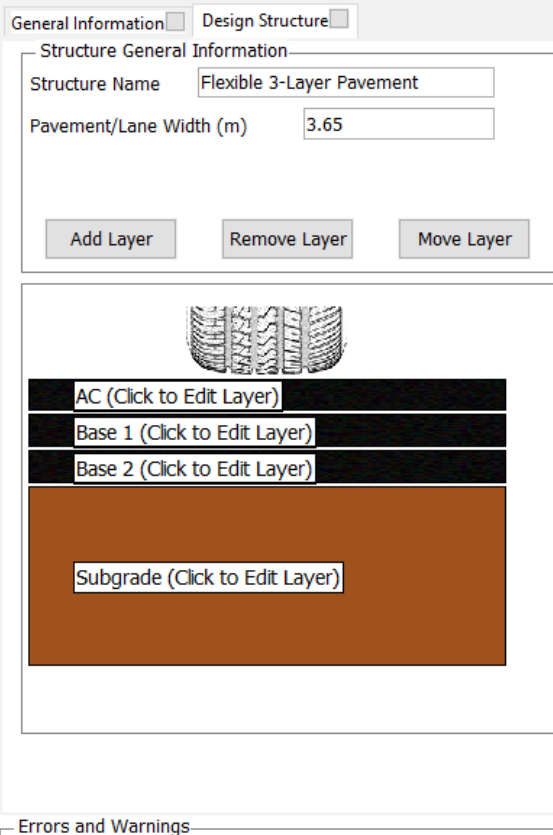

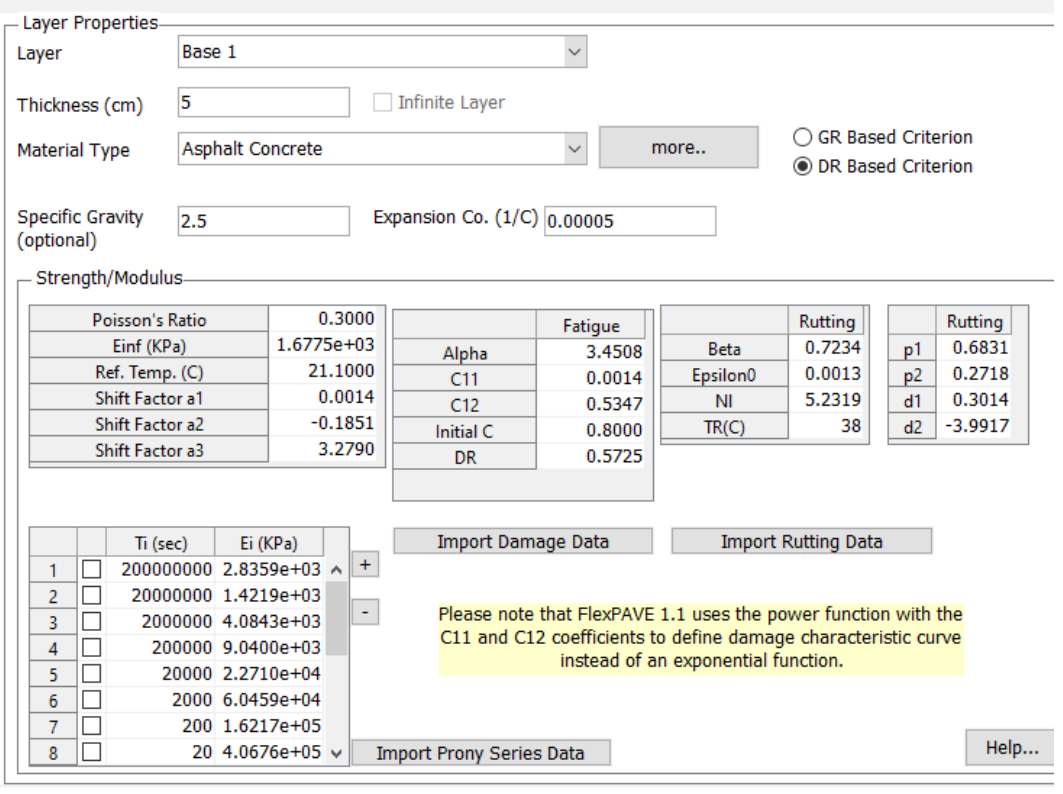

Figure 99: Design Structure of Base 1 Layer for Run 1.3 


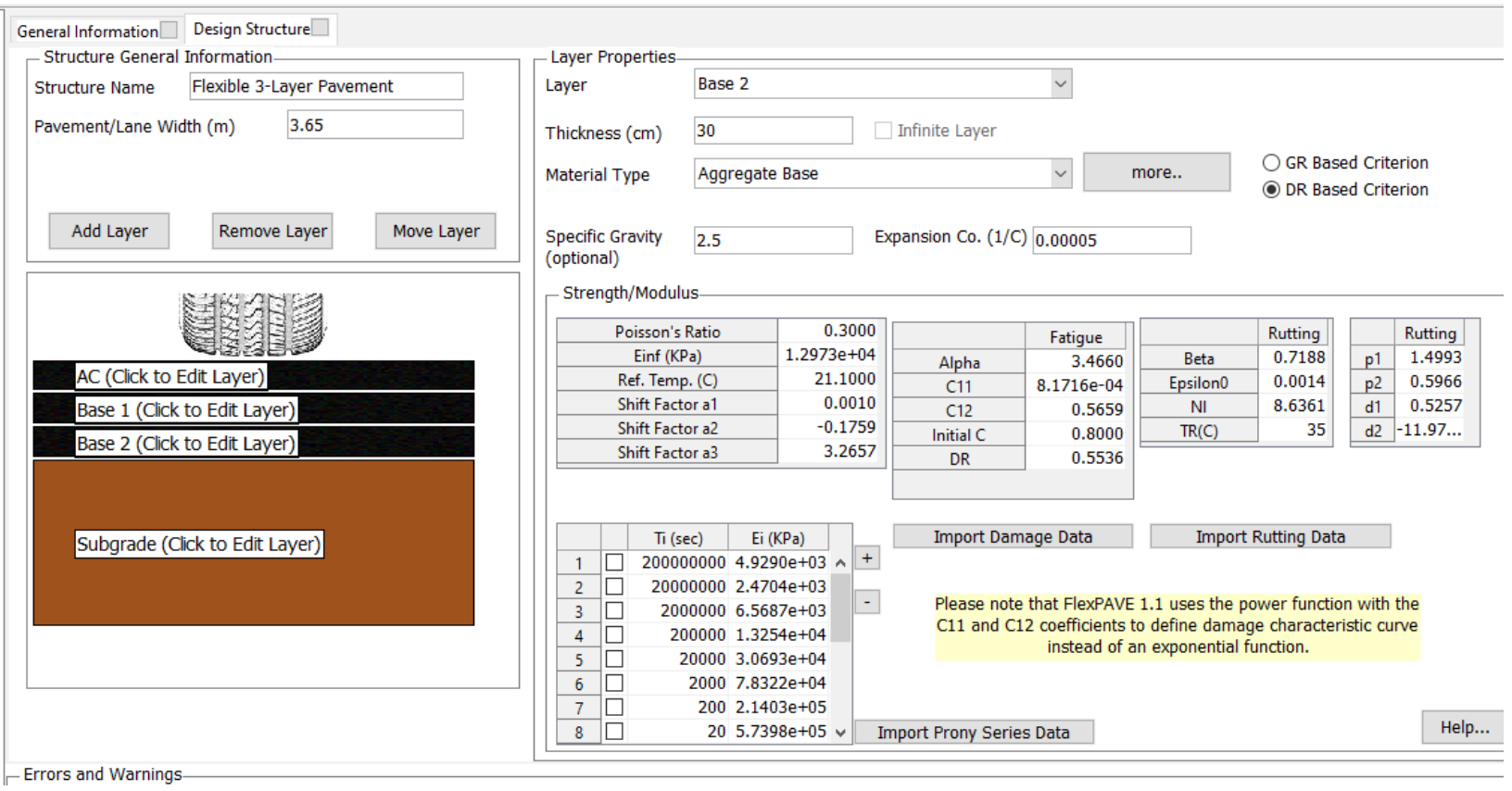

Figure 100: Design Structure of Base 2 Layer for Run 1.3

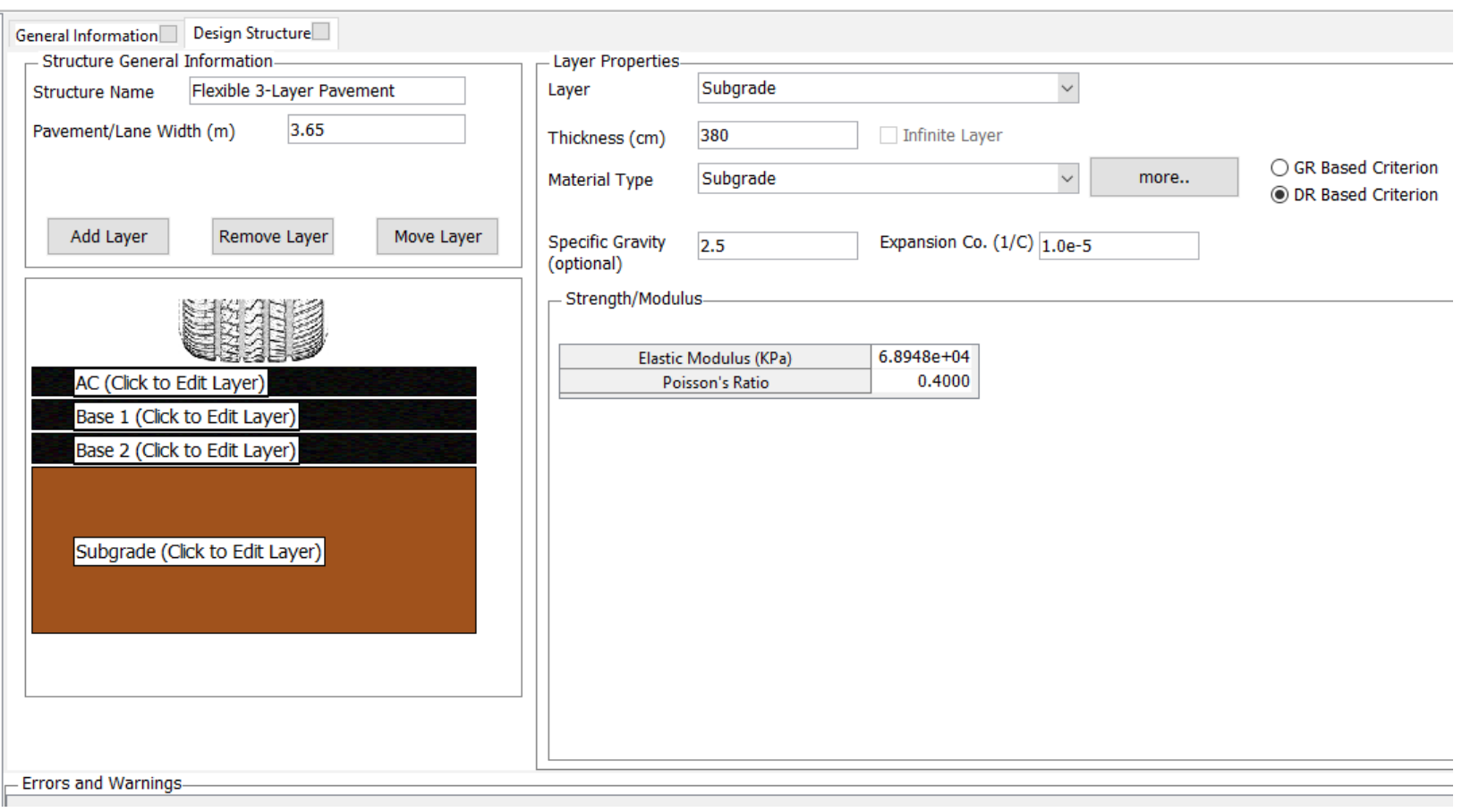

Figure 101: Design Structure of Subgrade Layer for Run 1.3 


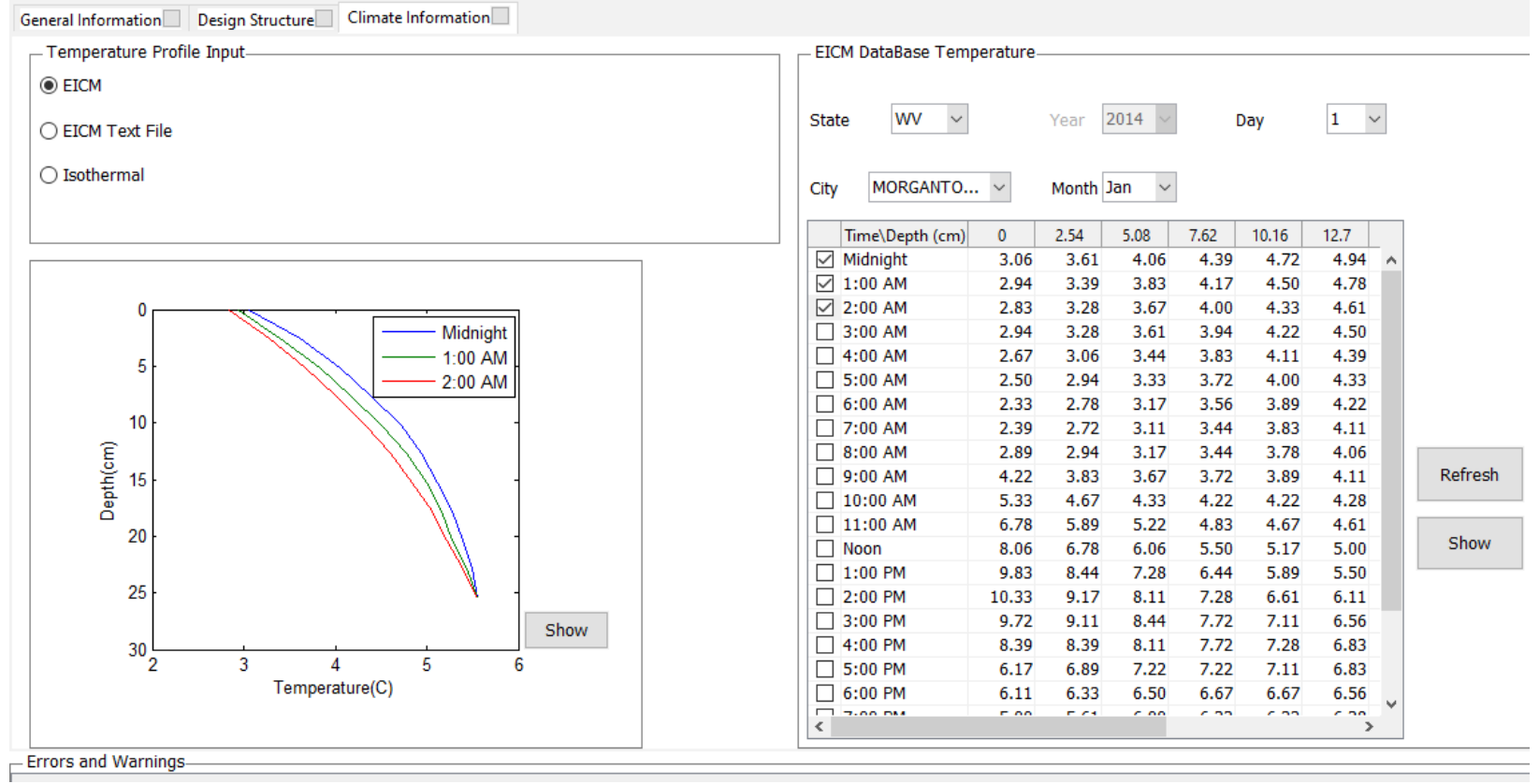

Figure 102: Climate Data for Run 1.3 


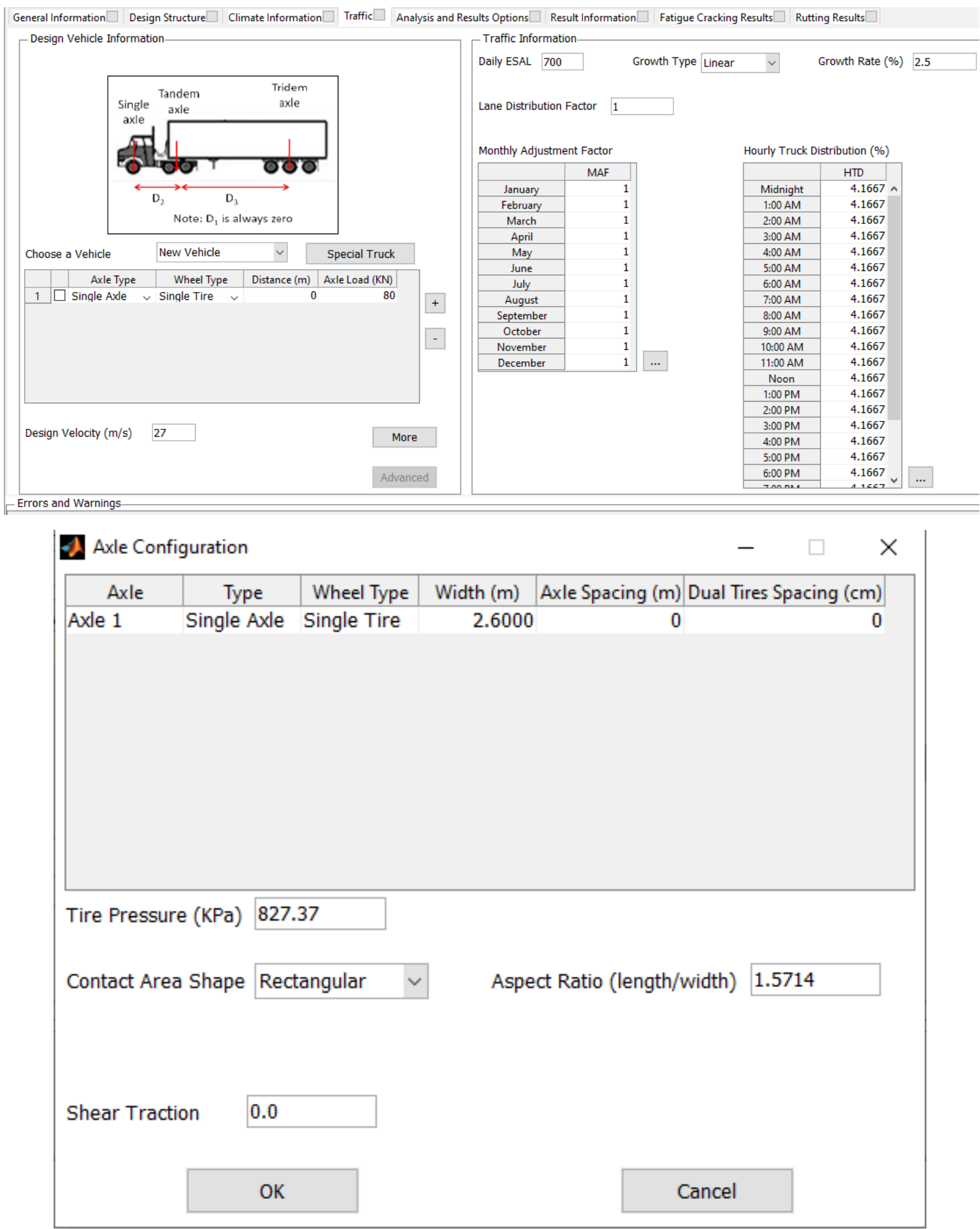

Figure 103: Traffic Data for Run 1.3 


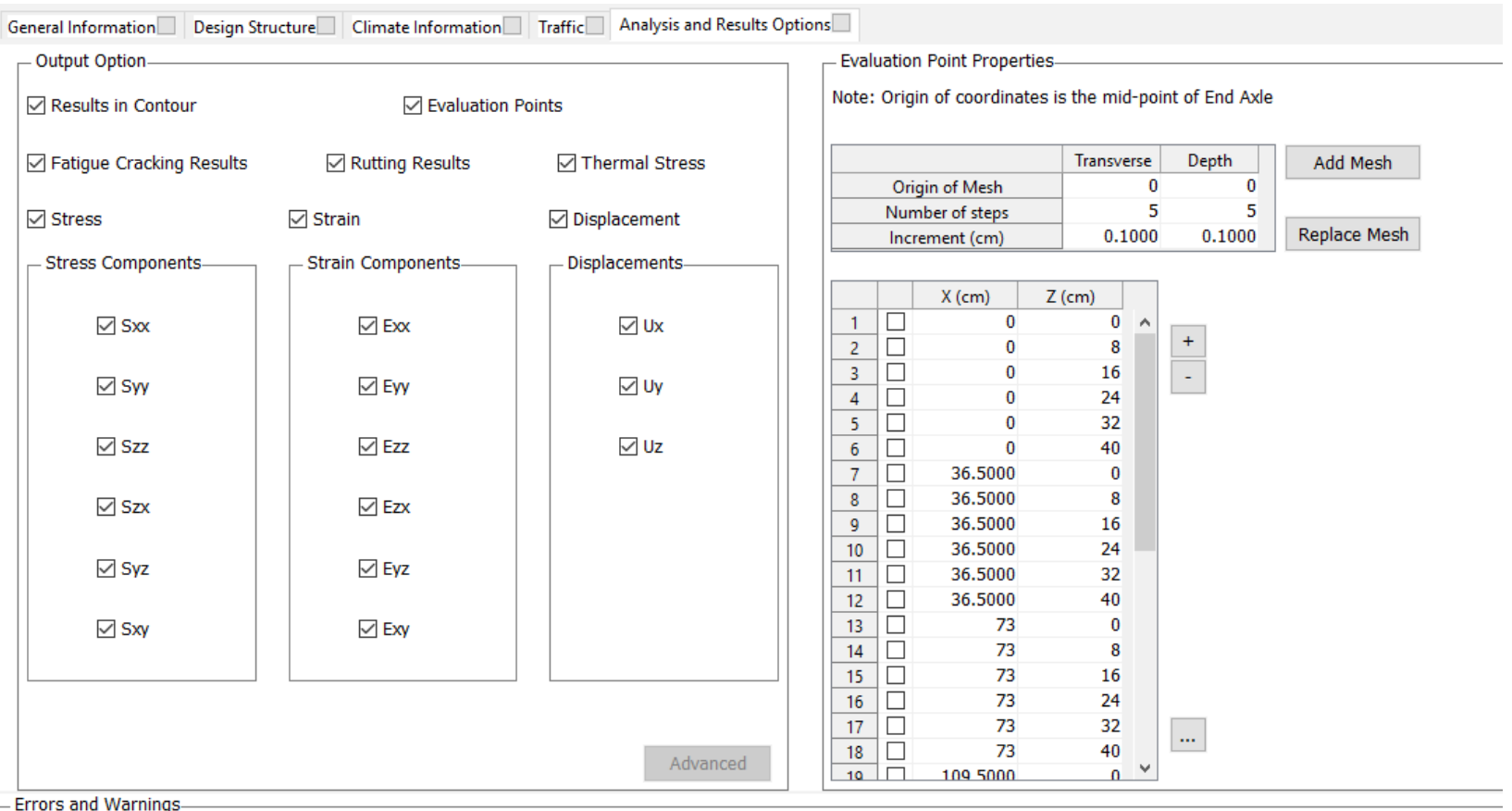

Figure 104: Output and Analysis Options for Run 1.3

\section{Run 1.4}

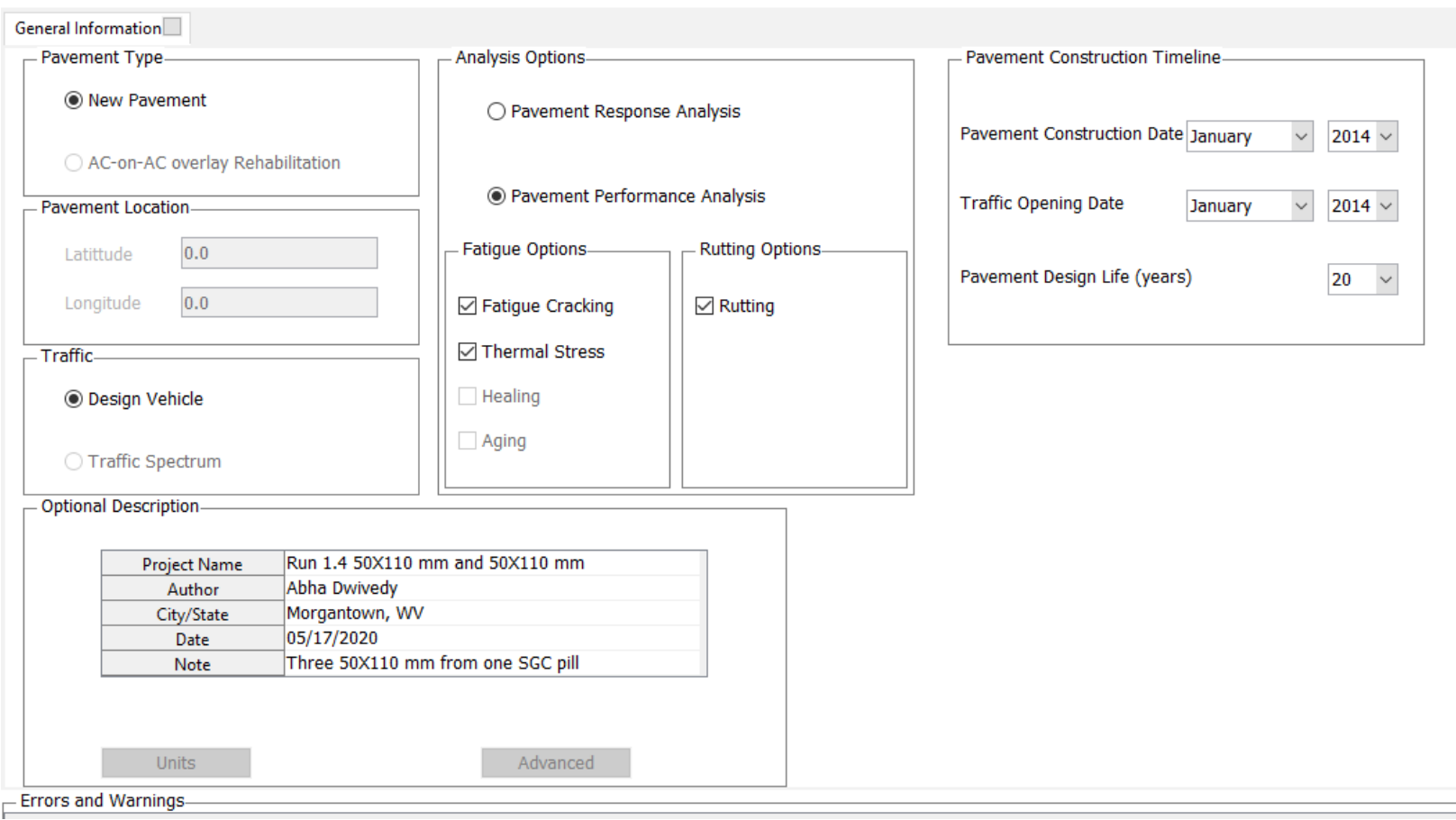

Figure 105: General Information for Run 1.4 


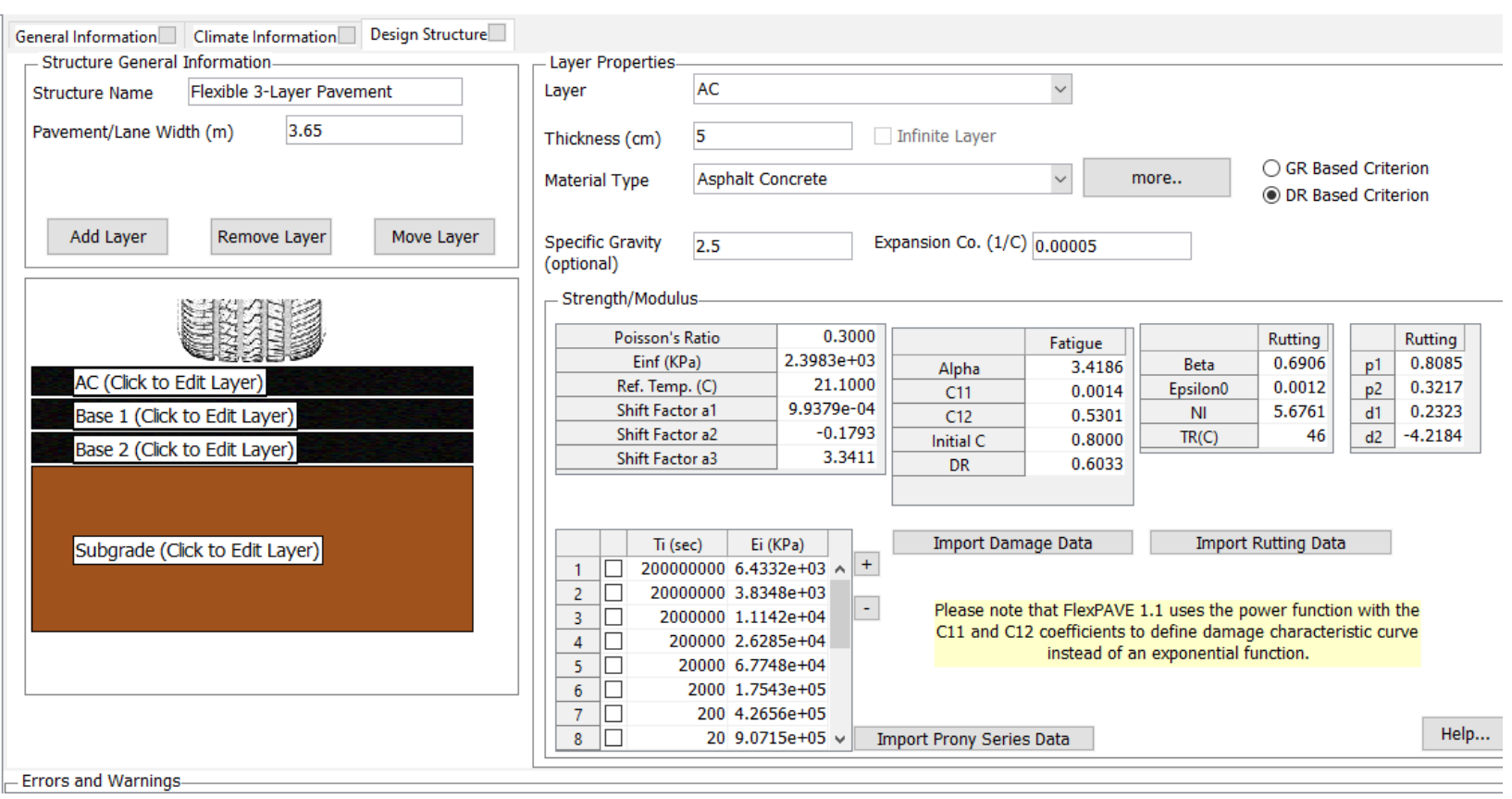

Figure 106: Design Structure of AC Layer for Run 1.4

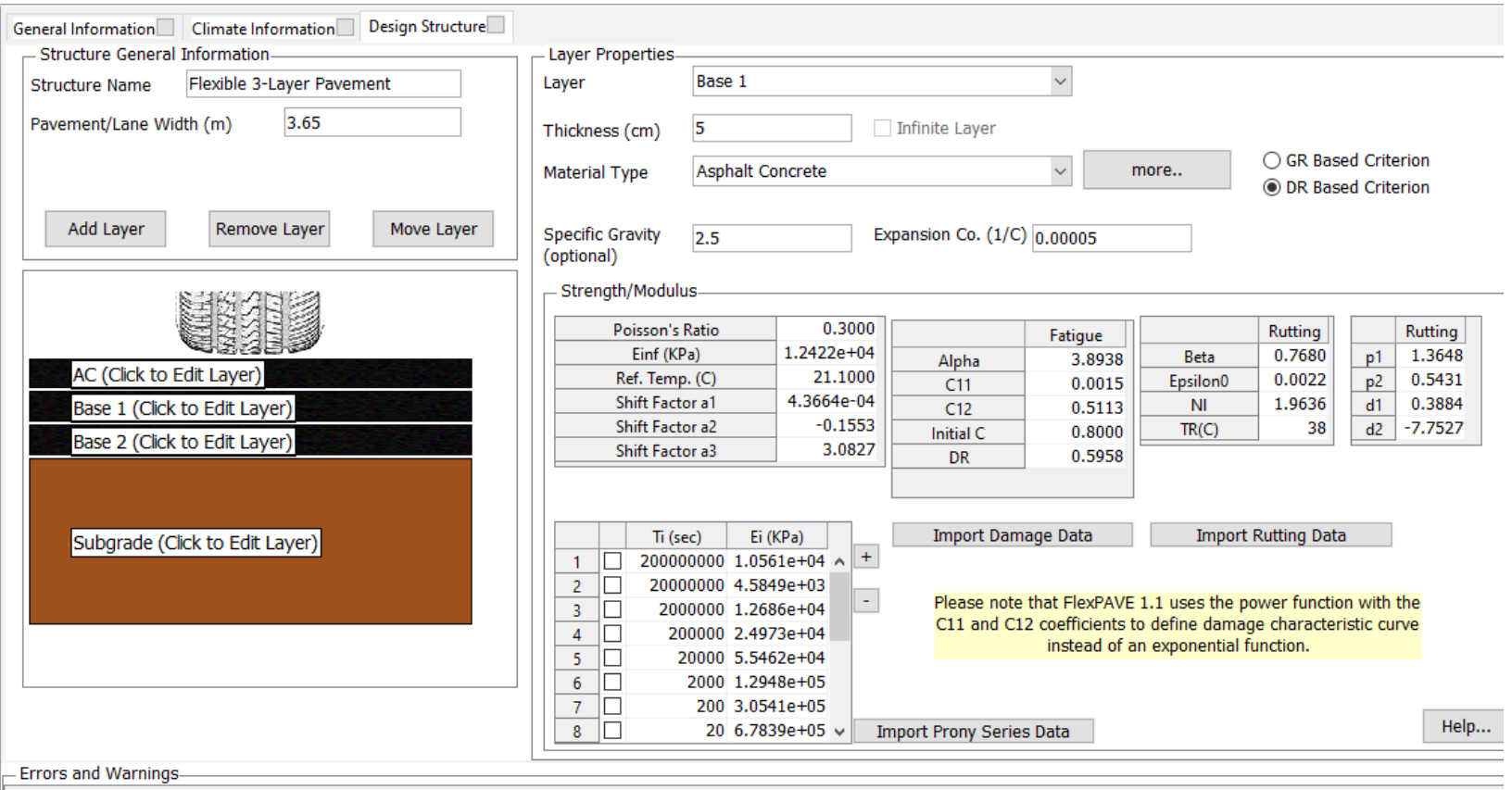

Figure 107: Design Structure of Base 1 Layer for Run 1.4 


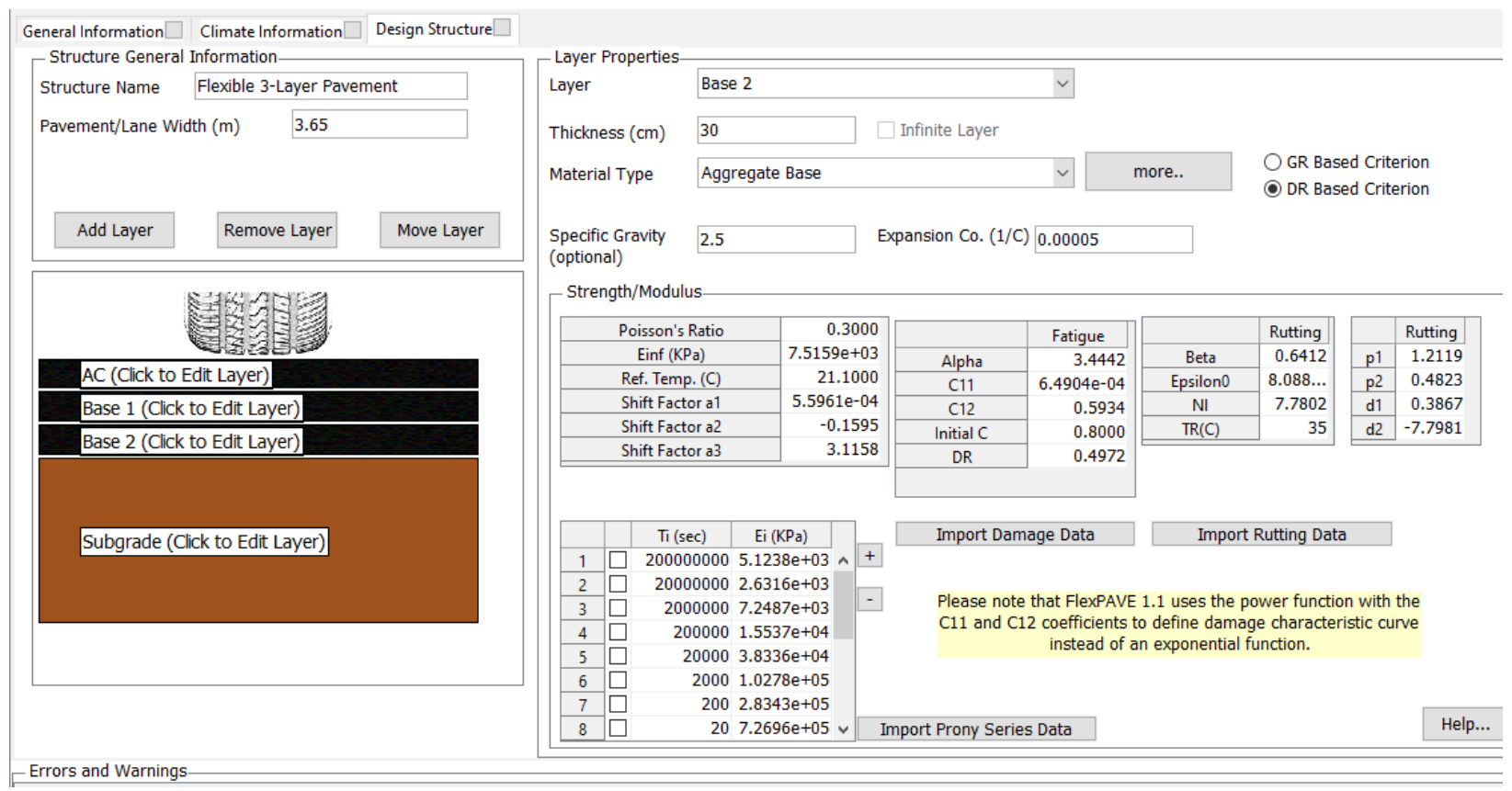

Figure 108: Design Structure of Base 2 Layer for Run 1.4

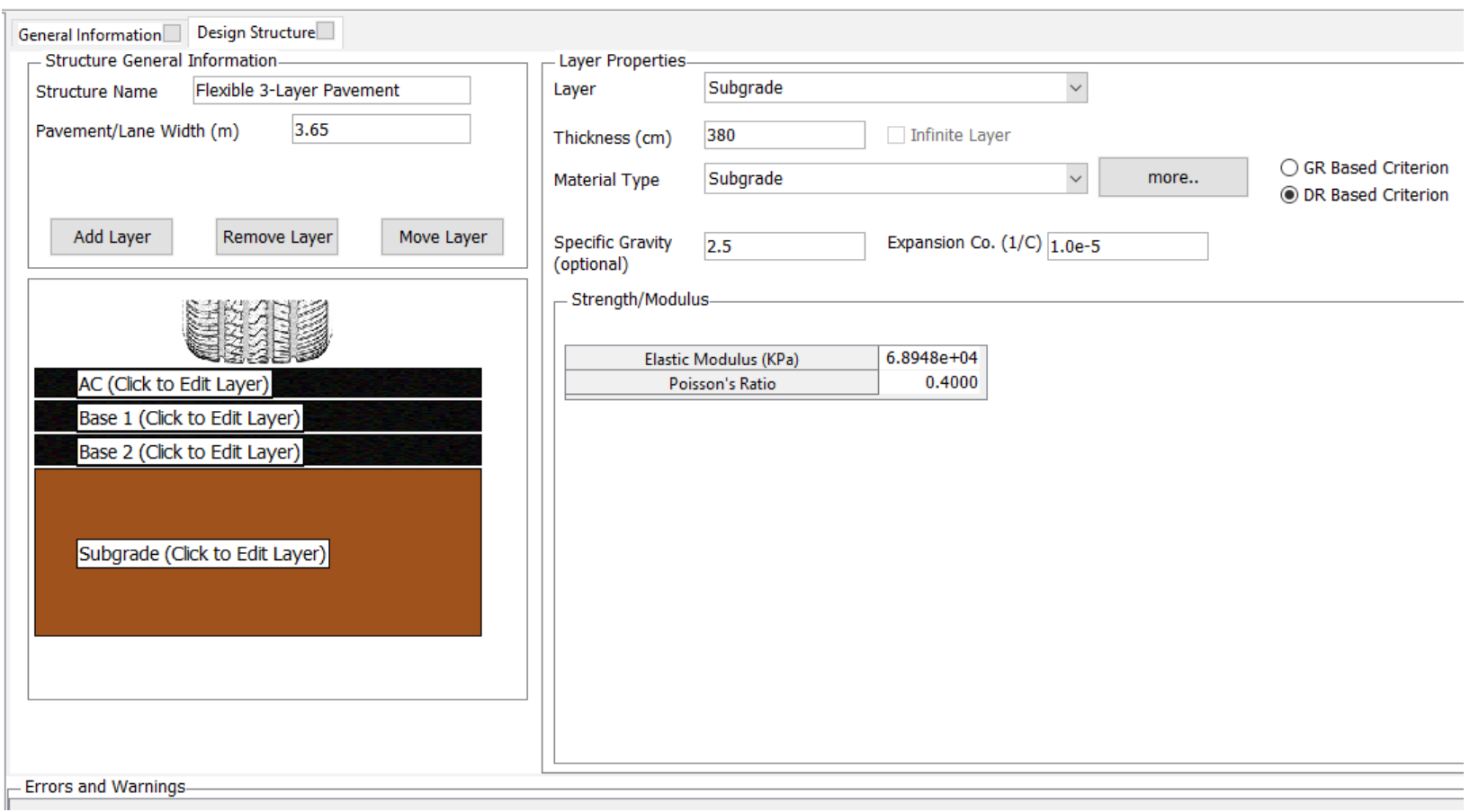

Figure 109: Design Structure of Subgrade Layer for Run 1.4 


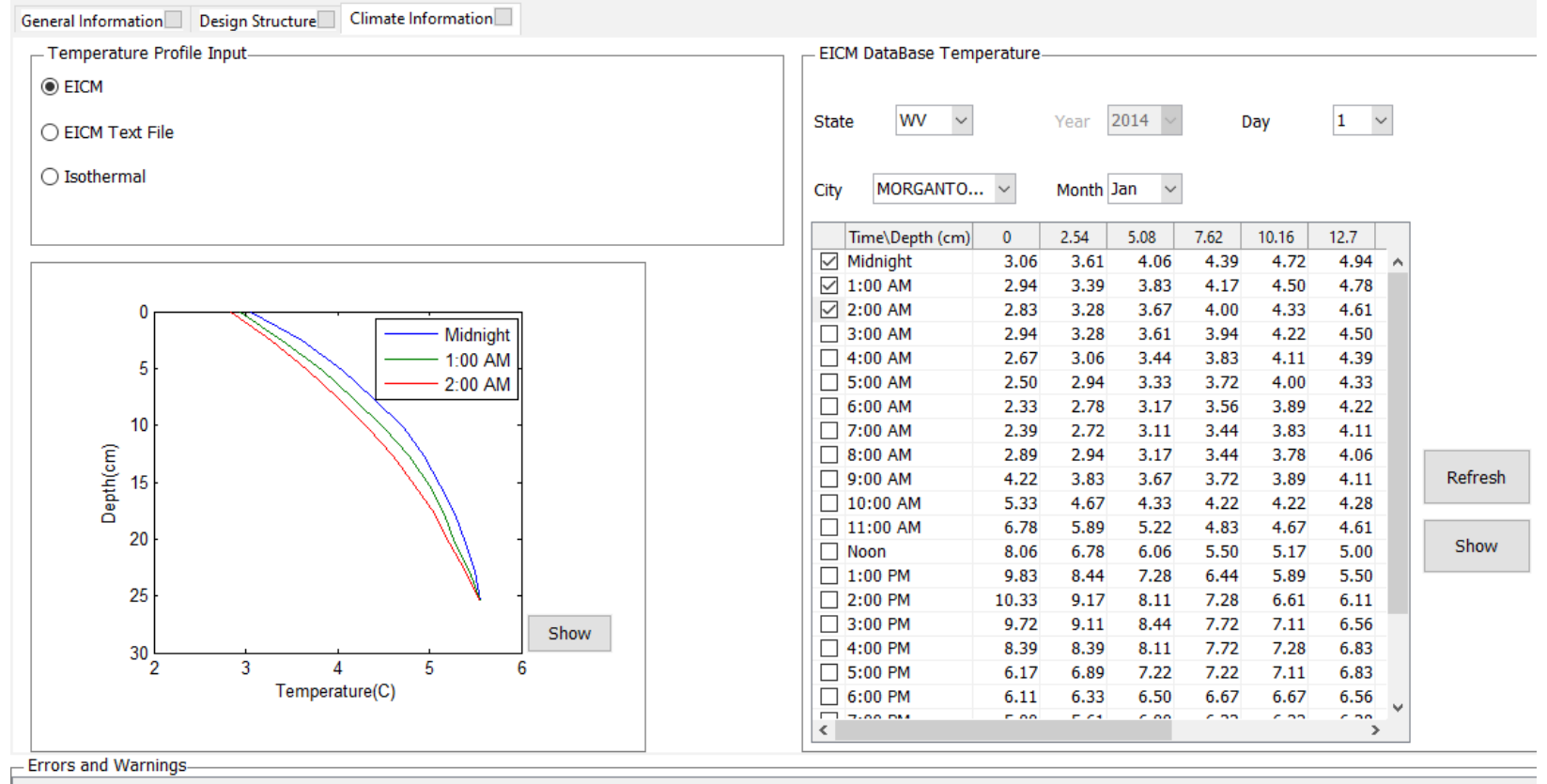

Figure 110: Climate Data for Run 1.4 

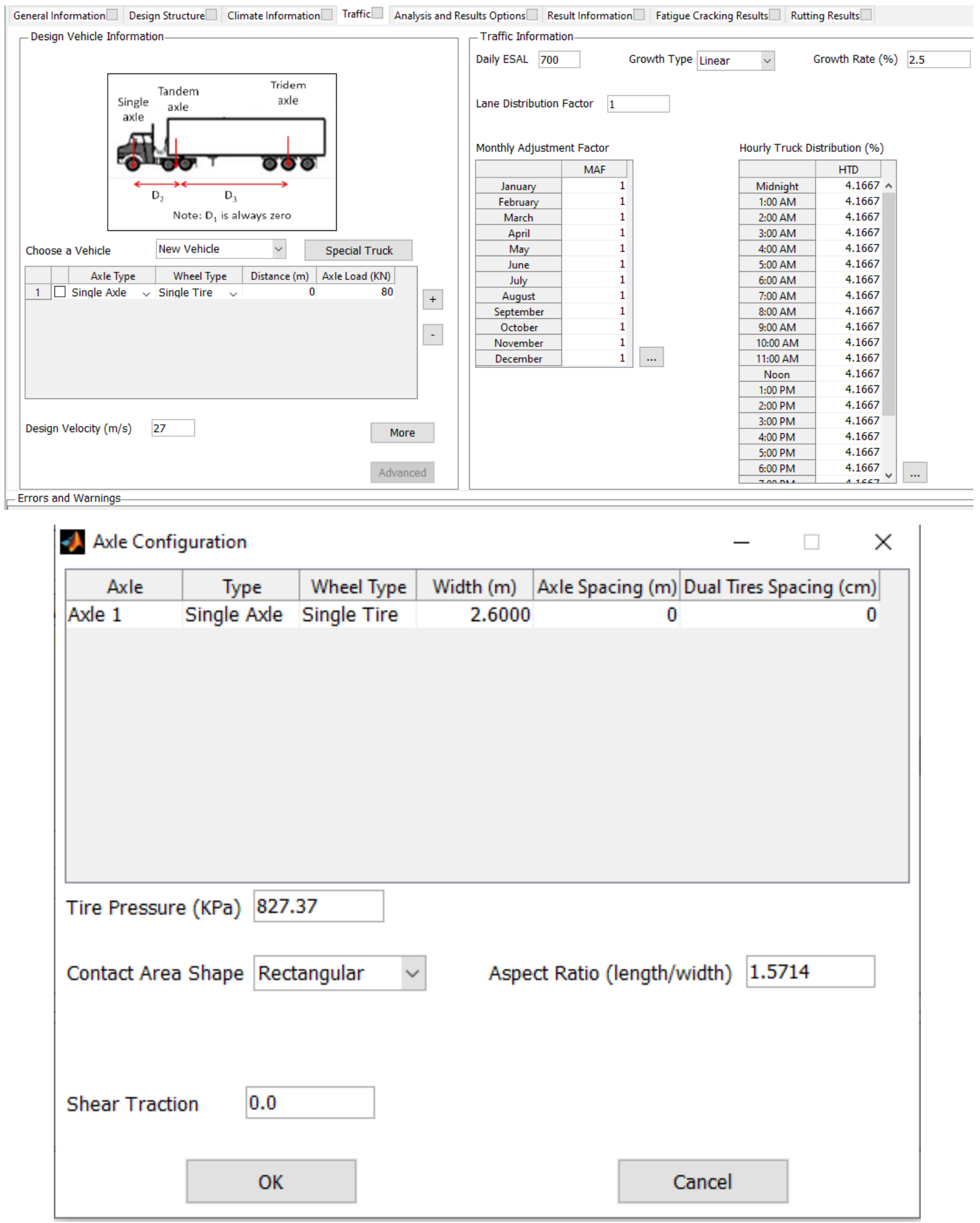

Figure 111: Traffic Data for Run 1.4 


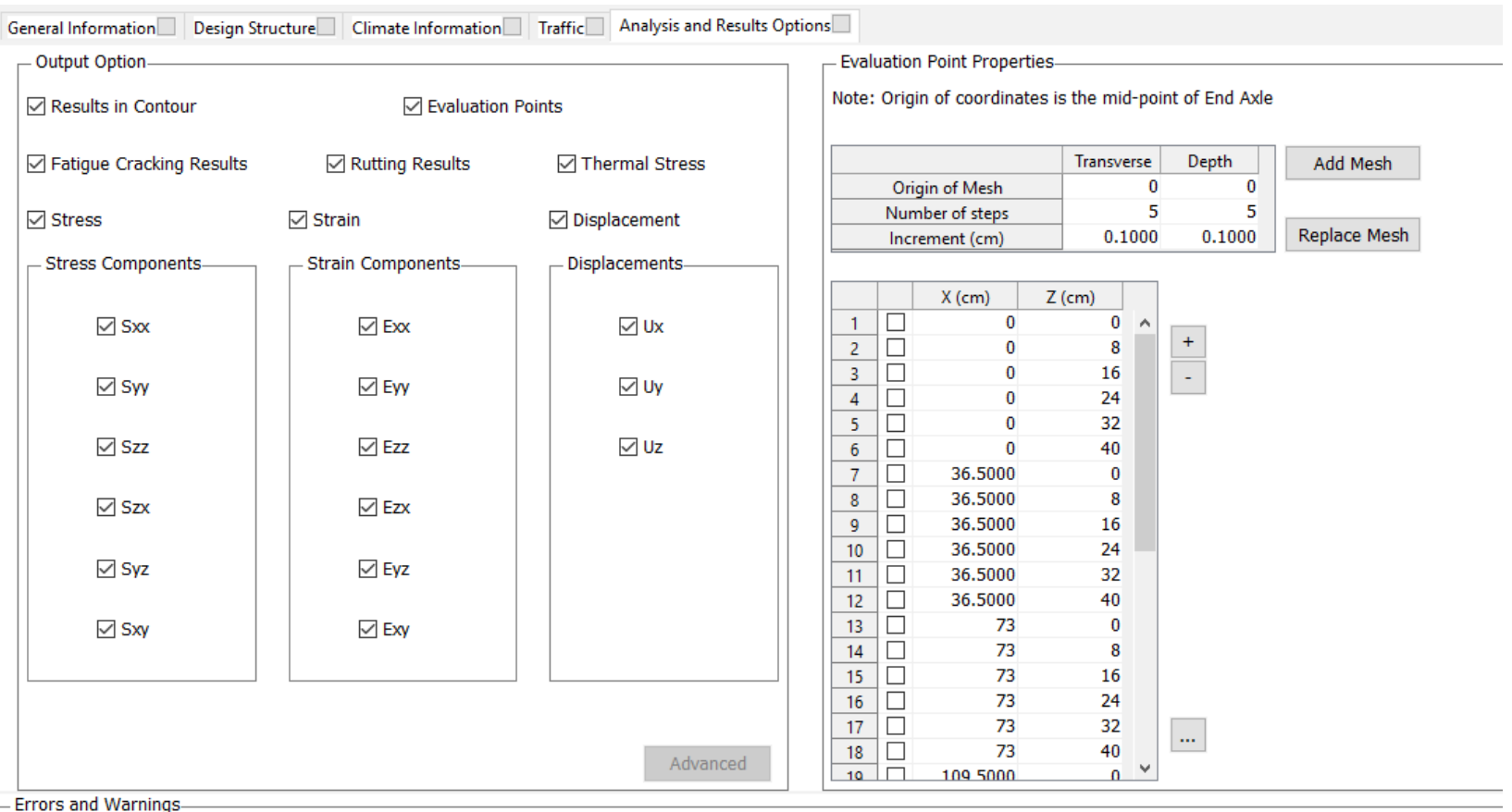

Figure 112: Output and Analysis Options for Run 1.4

\section{Run 1.5}

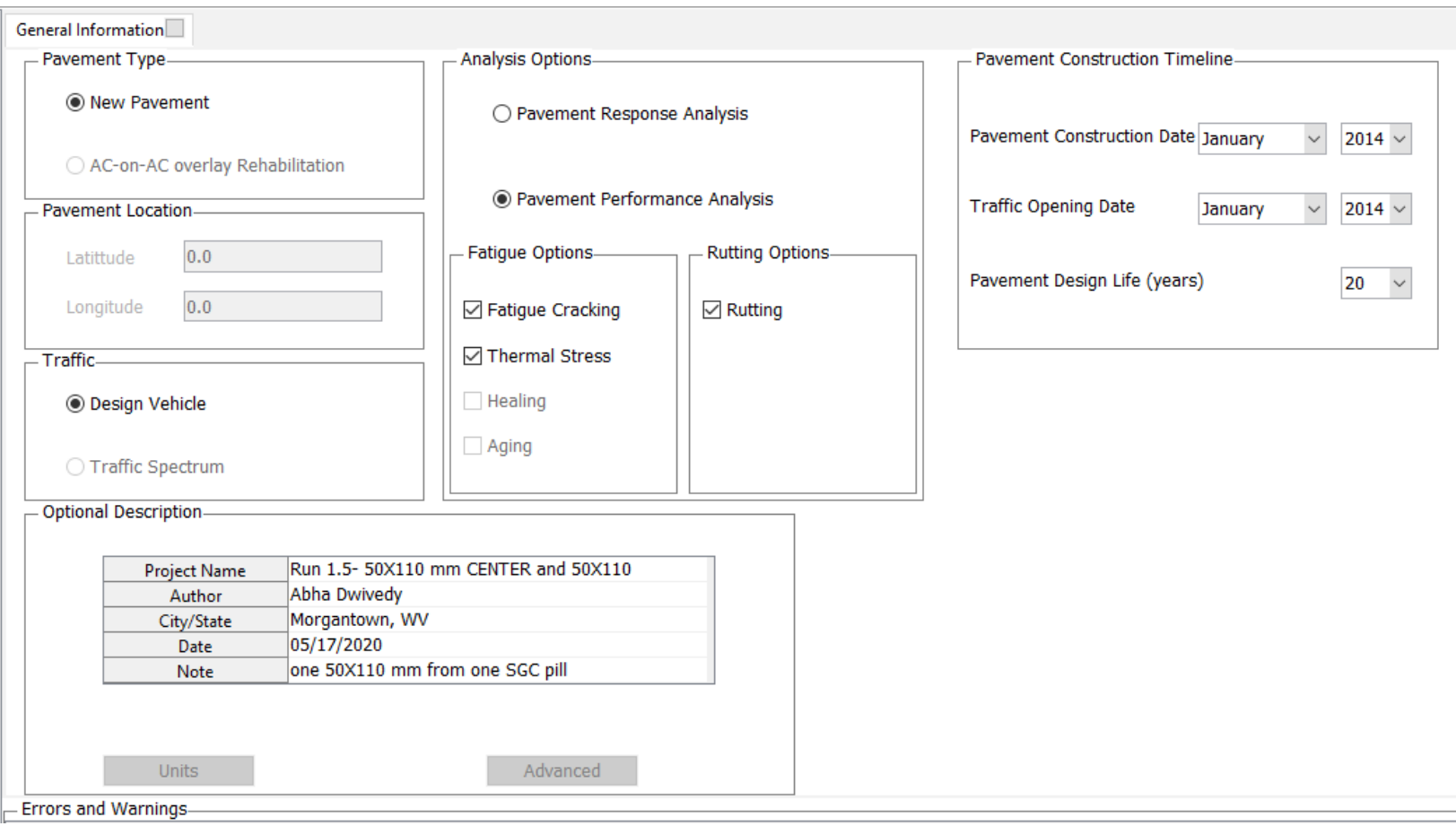

Figure 113: General Information for Run 1.5 


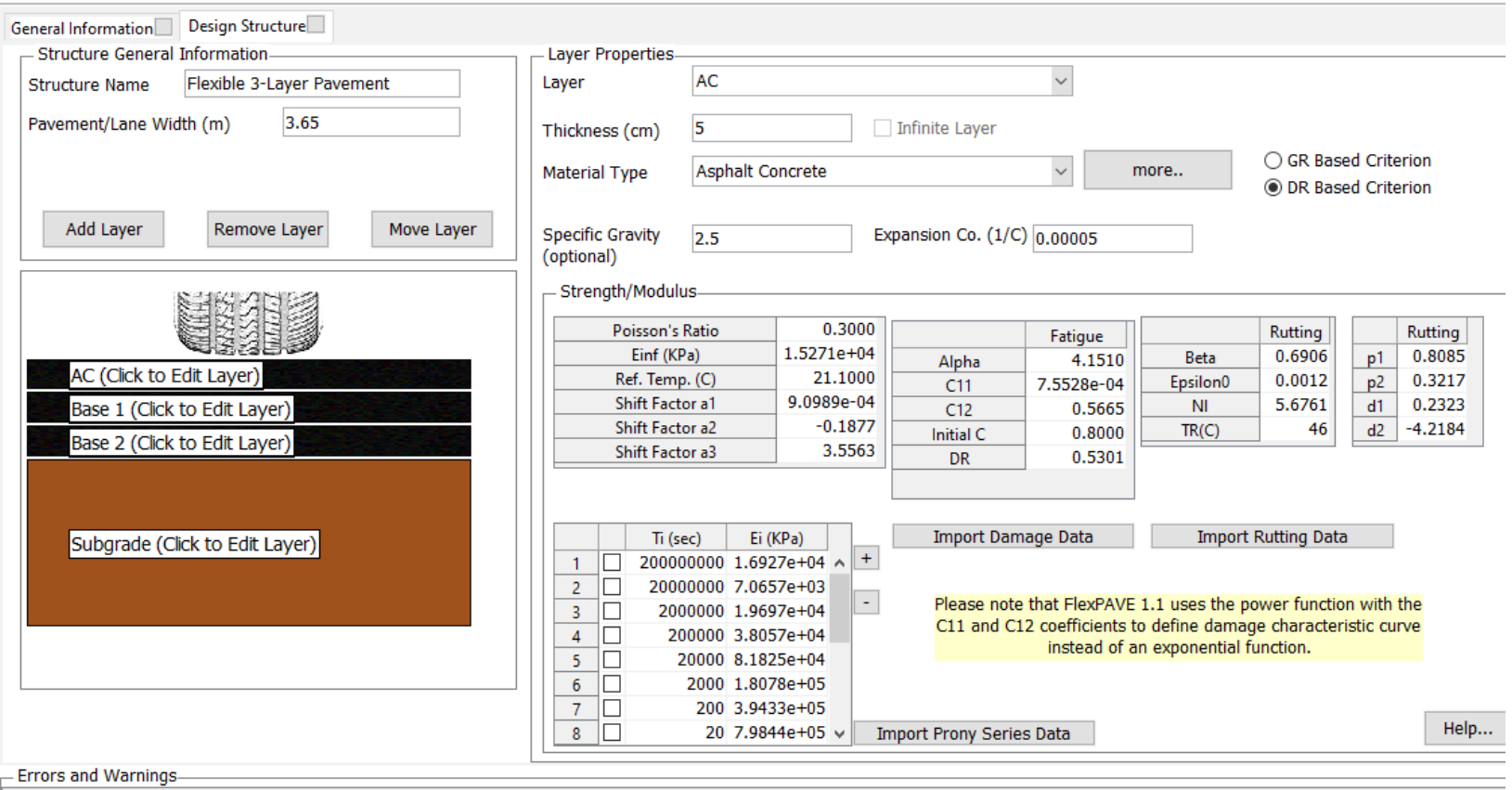

\section{Figure 114: Design Structure of AC Layer for Run 1.5}

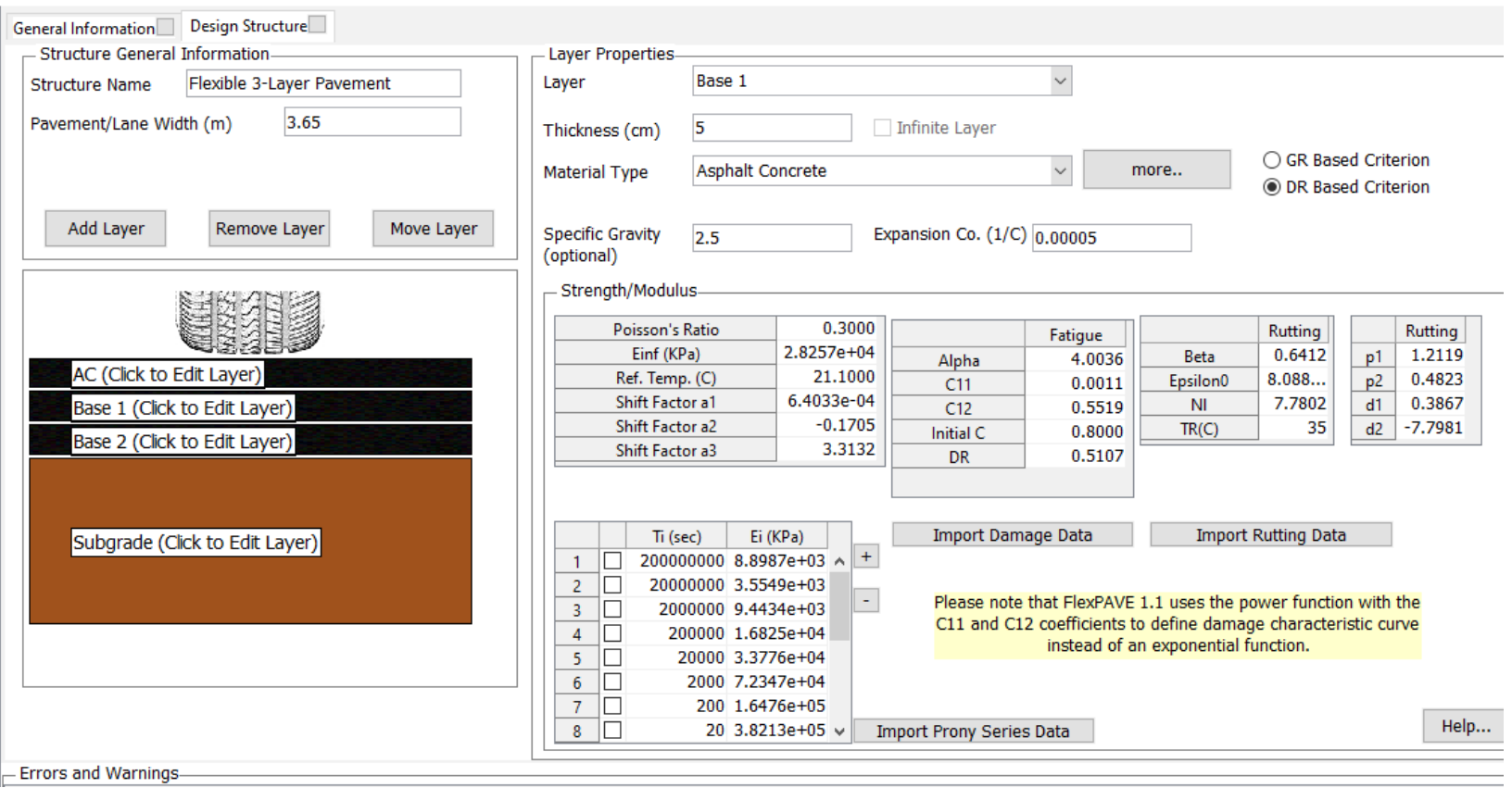

Figure 115: Design Structure of Base 1 Layer for Run 1.5 


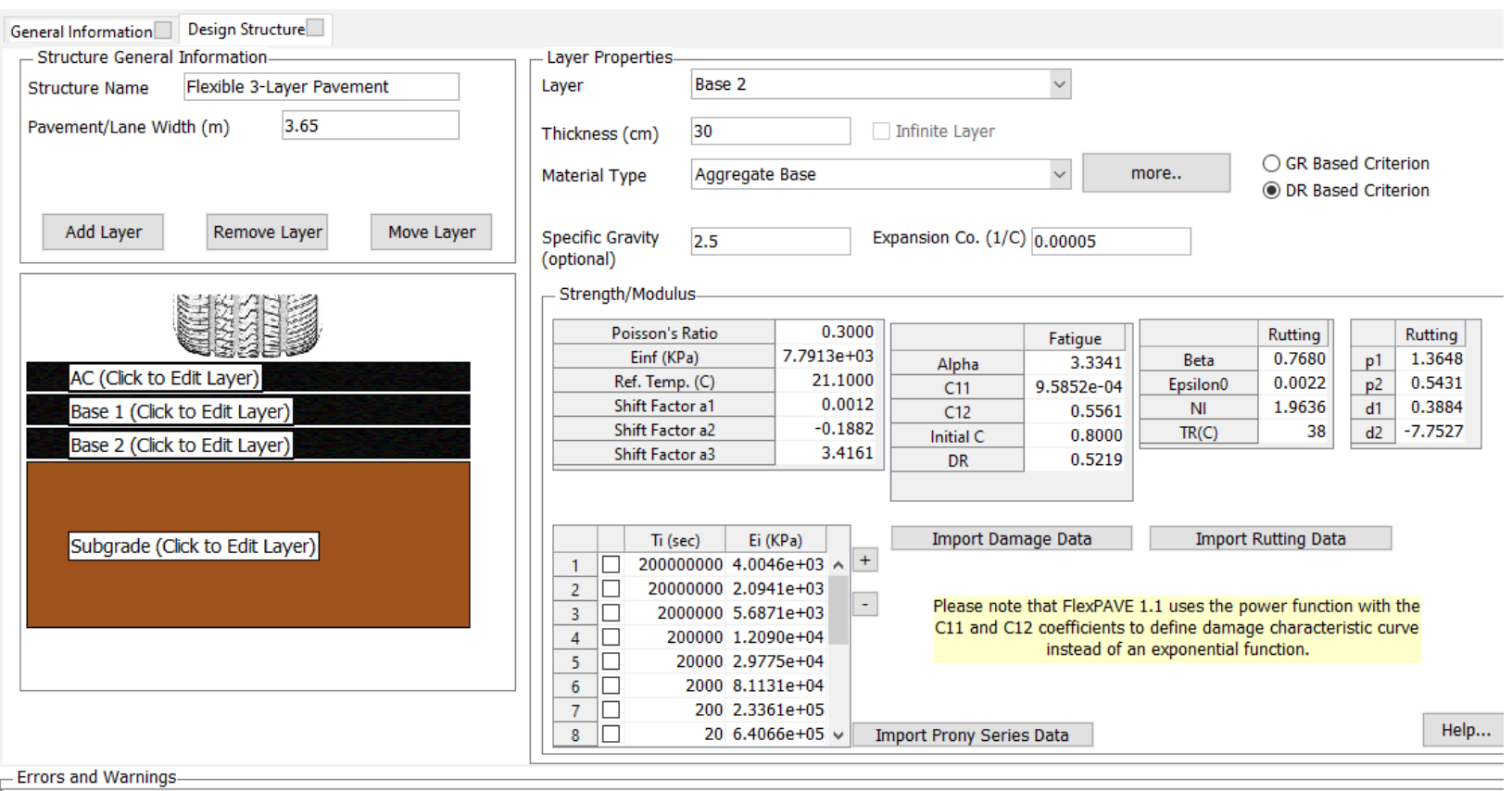

Figure 116: Design Structure of Base 2 Layer for Run 1.5

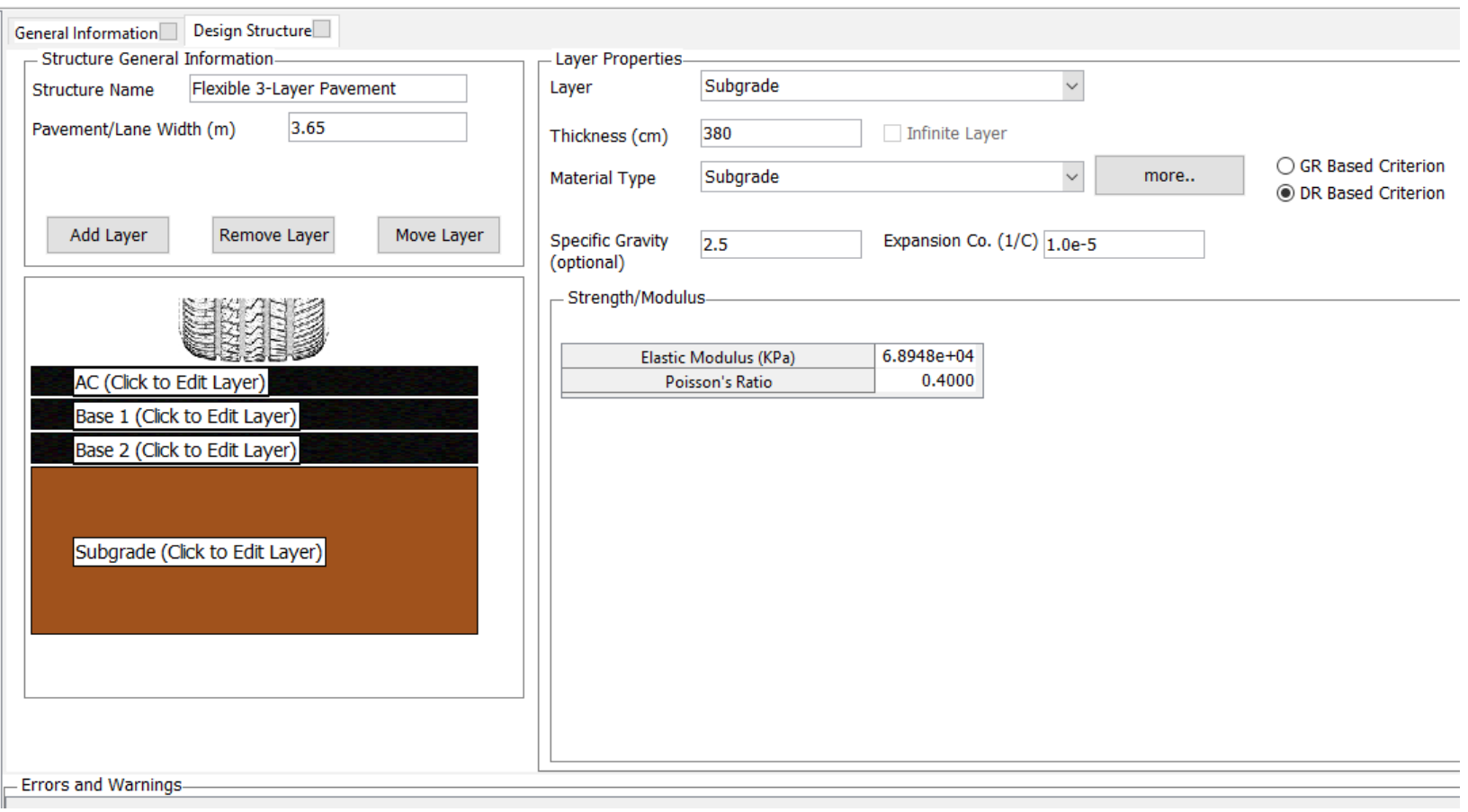

Figure 117: Design Structure of Subgrade Layer for Run 1.5 


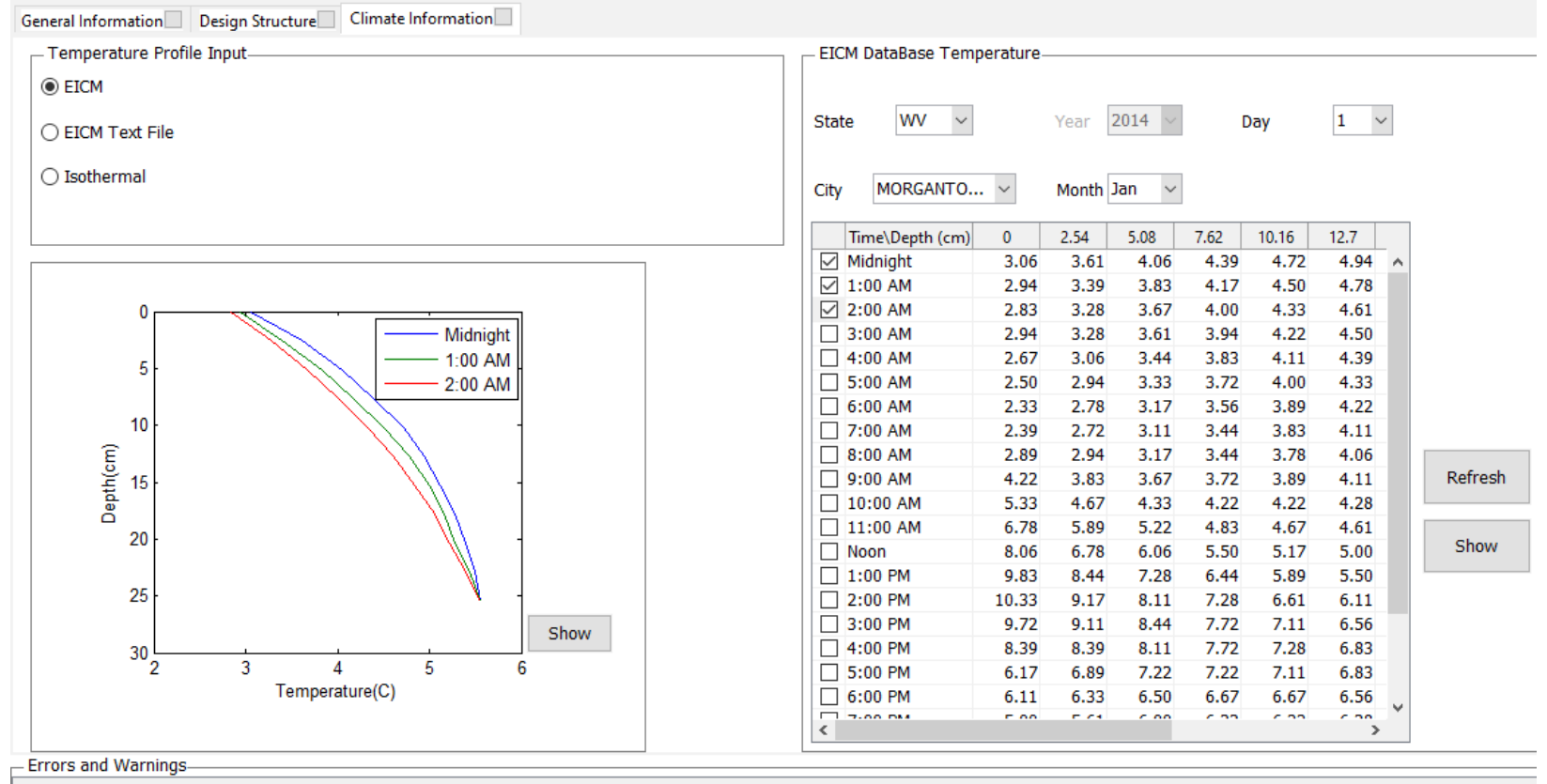

Figure 118: Climate Data for Run 1.5 

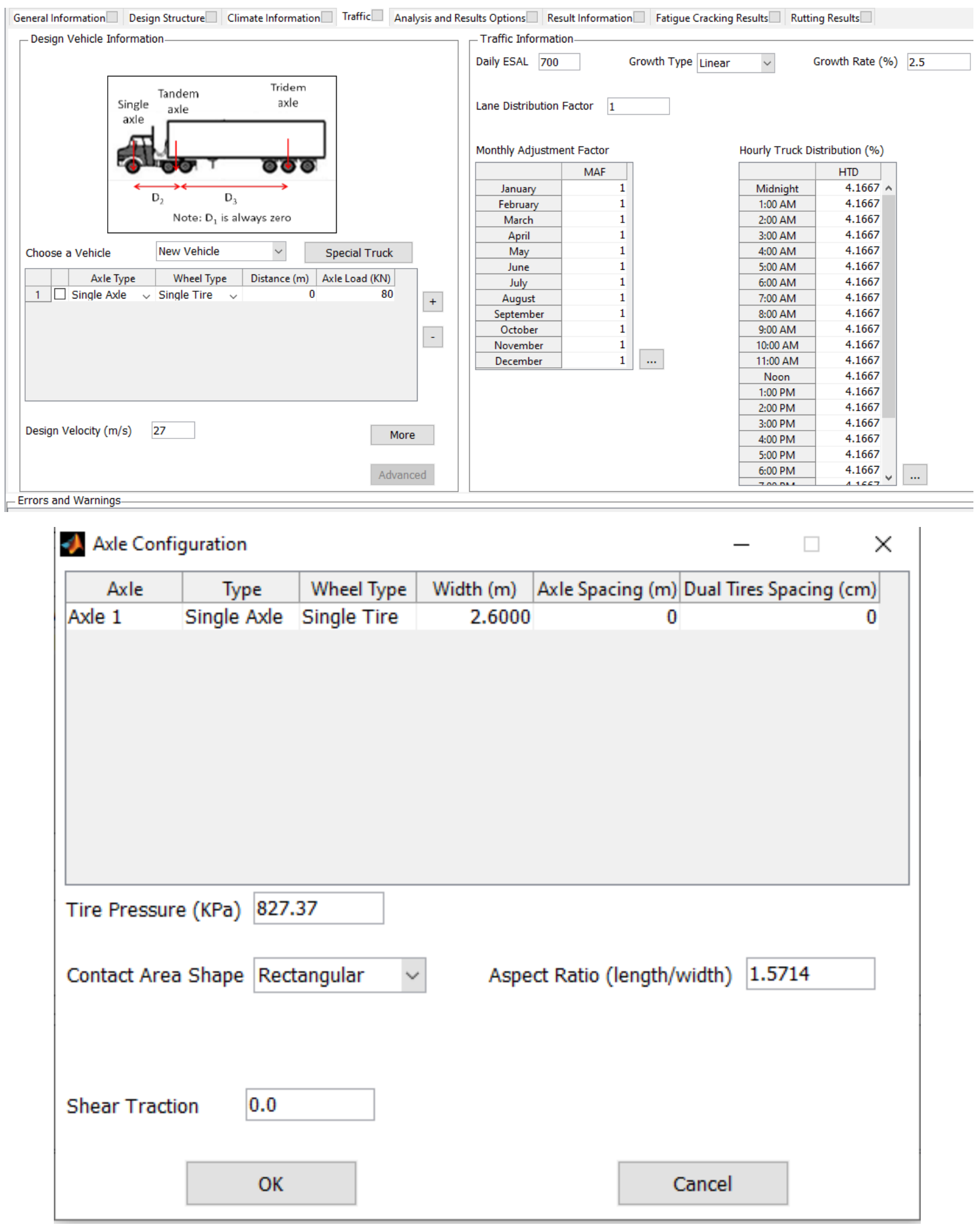

Figure 119: Traffic Data for Run 1.5 


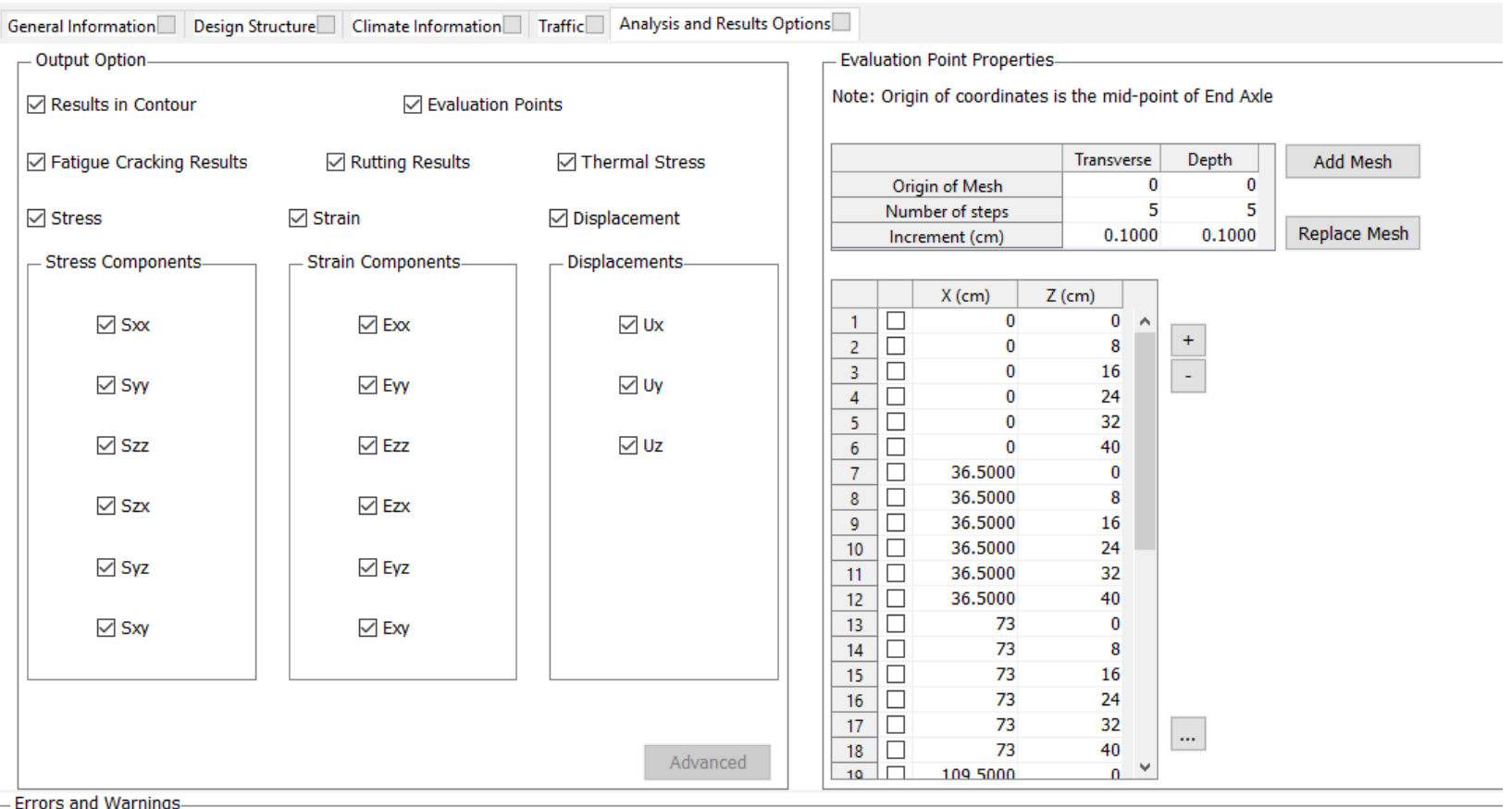

Figure 120: Output and Analysis Options for Run 1.5

\section{Run 1.6}

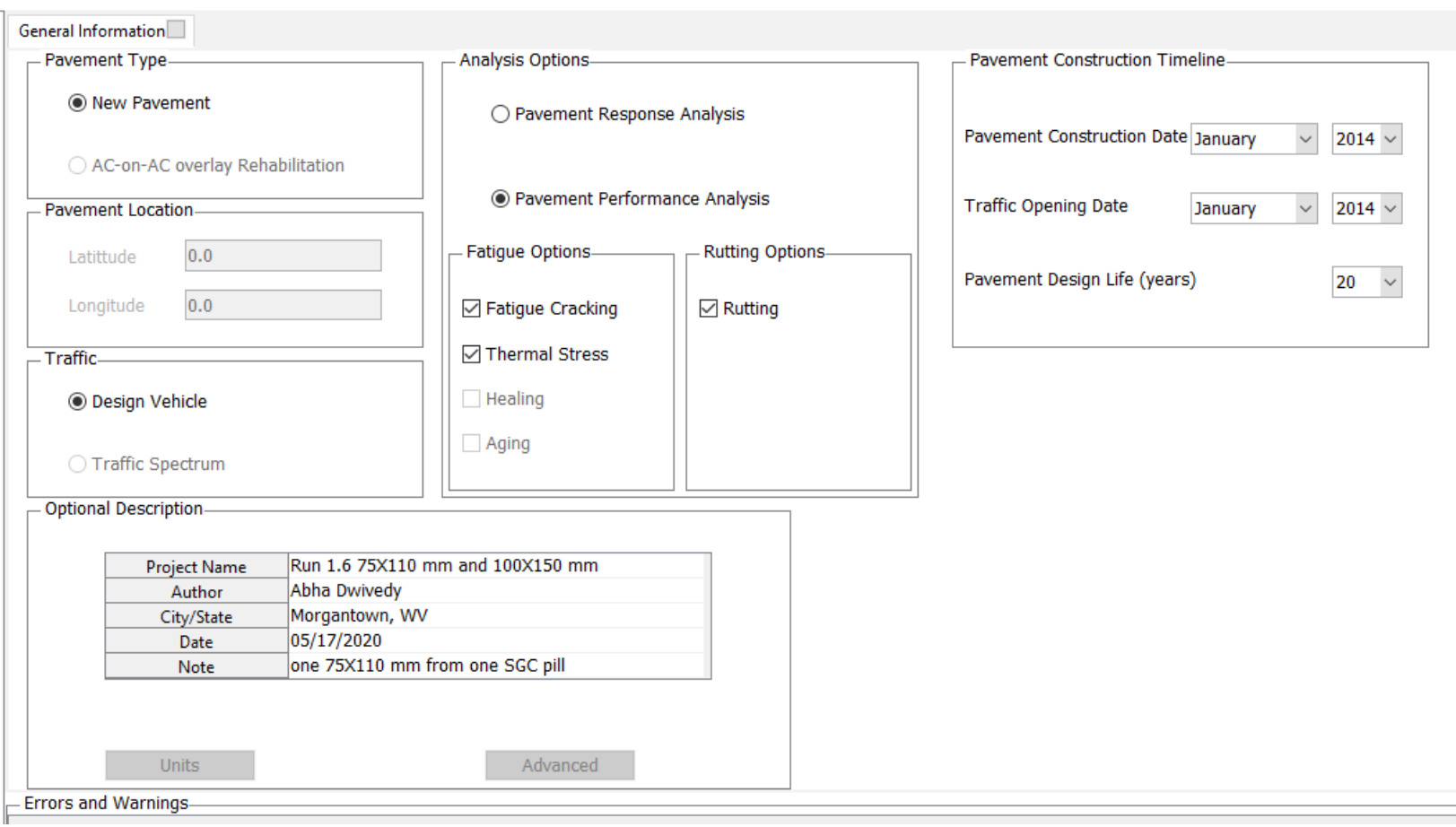

Figure 121: General Information for Run 1.6 


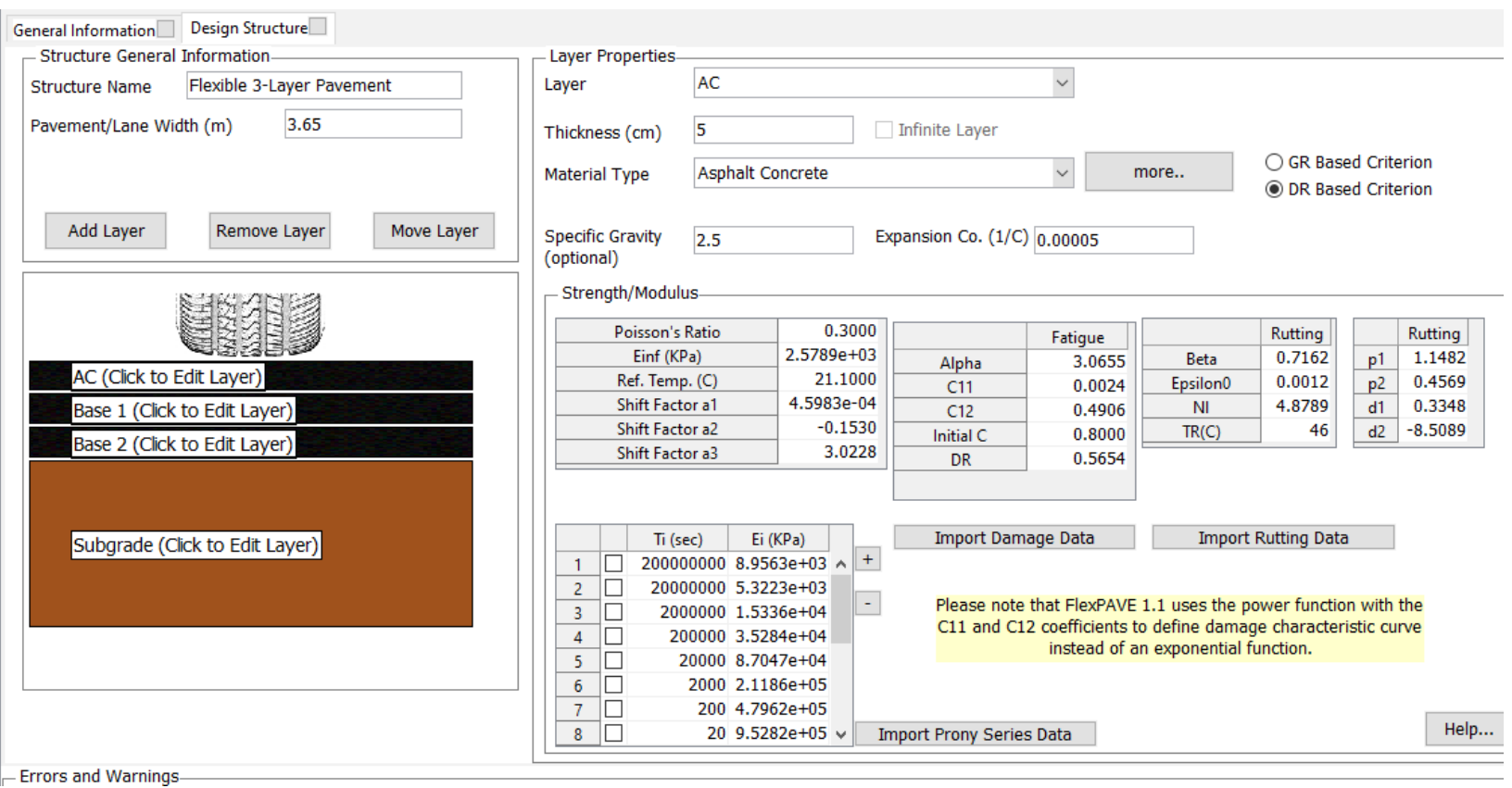

Figure 122: Design Structure of AC Layer for Run 1.6

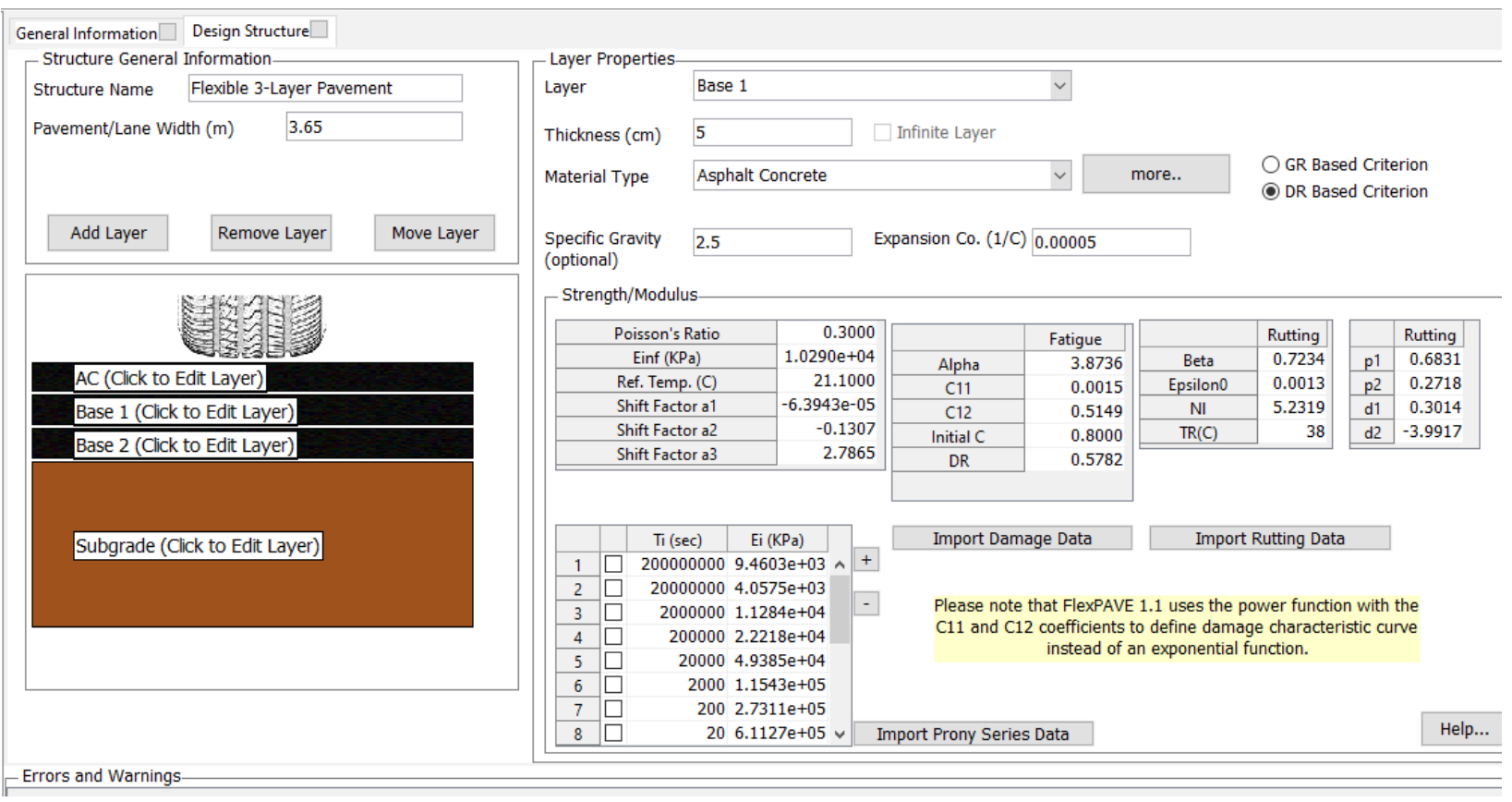

Figure 123: Design Structure of Base 1 Layer for Run 1.6 


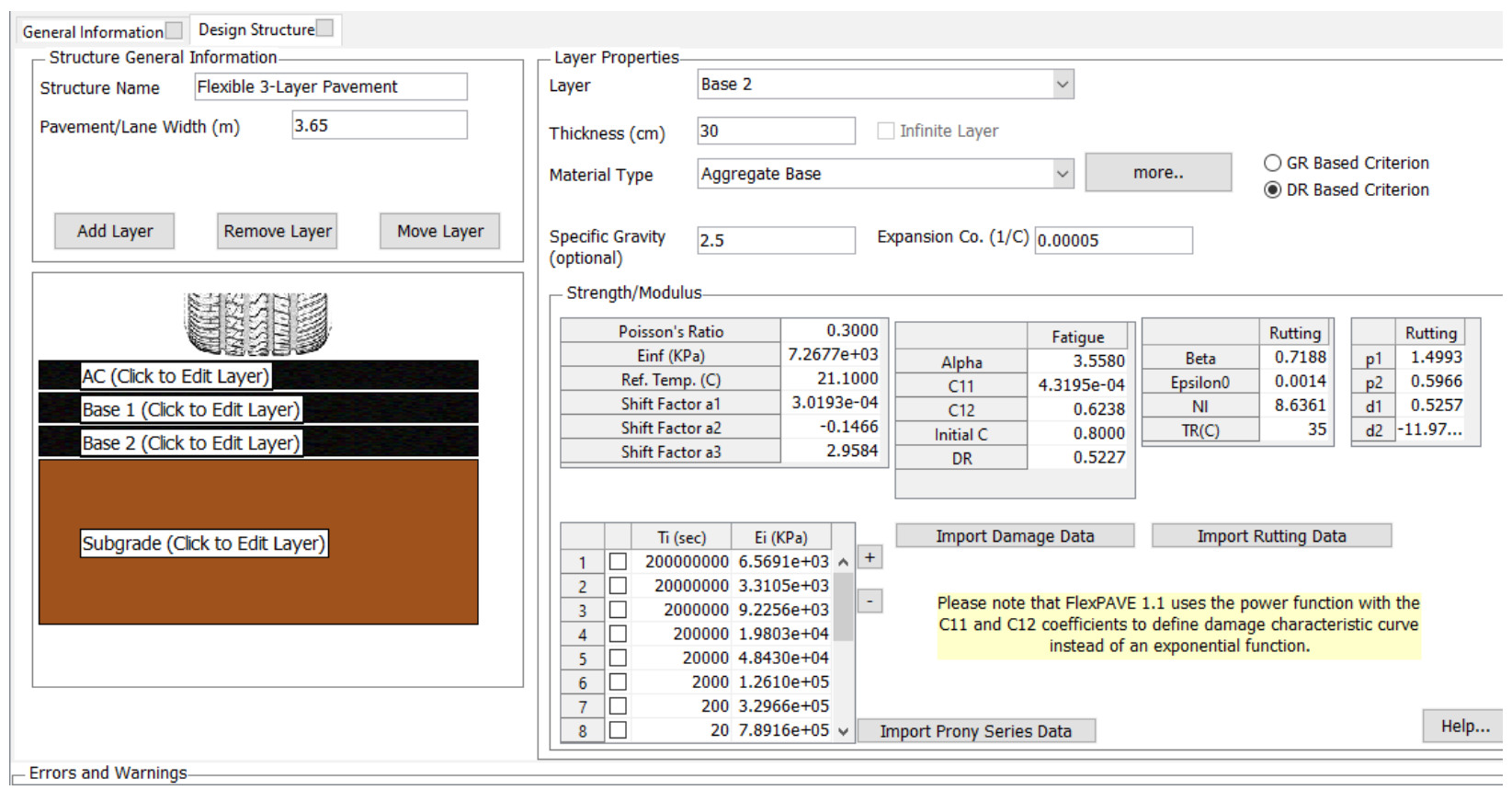

Figure 124: Design Structure of Base 2 Layer for Run 1.6

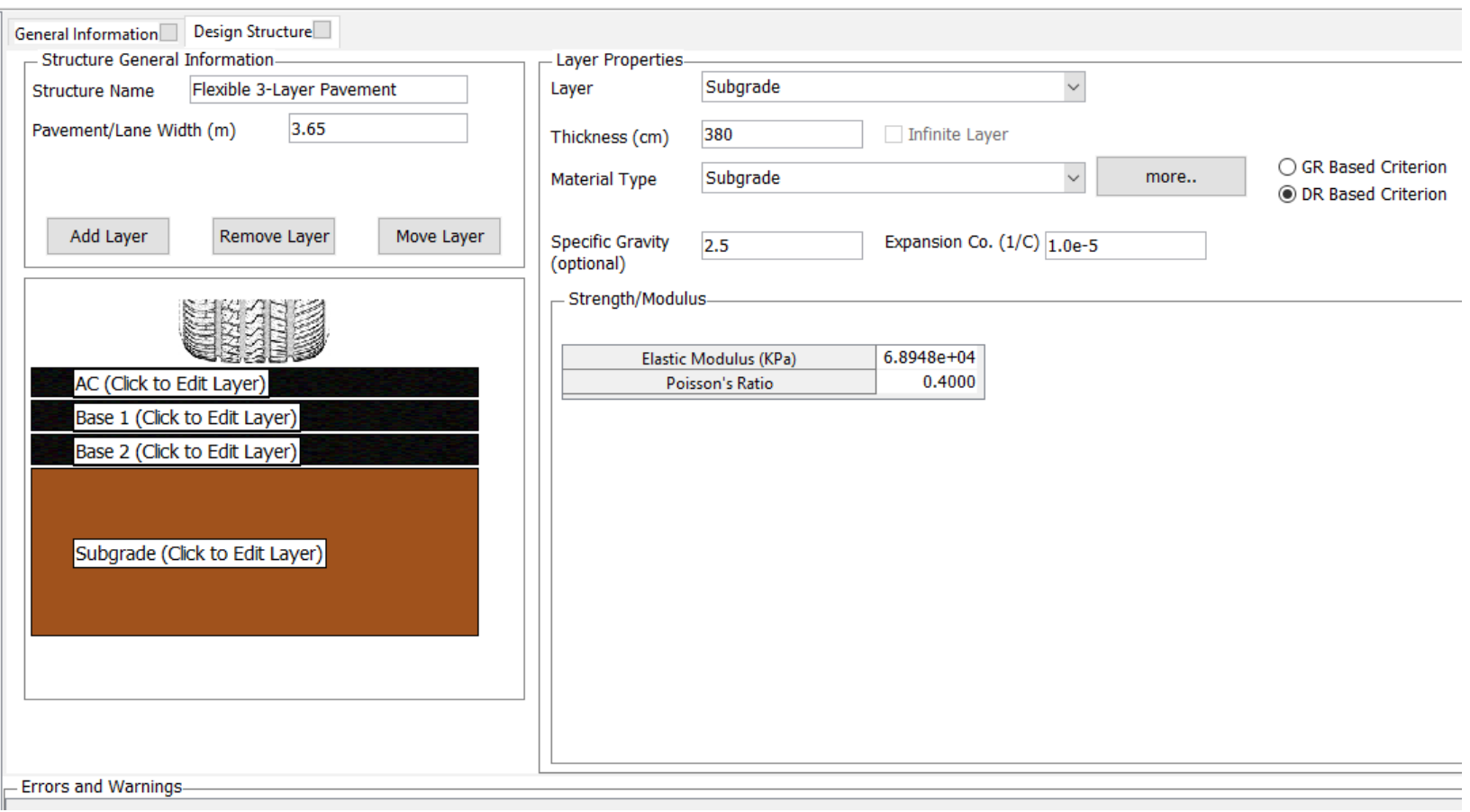

Figure 125: Design Structure of Subgrade Layer for Run 1.6 


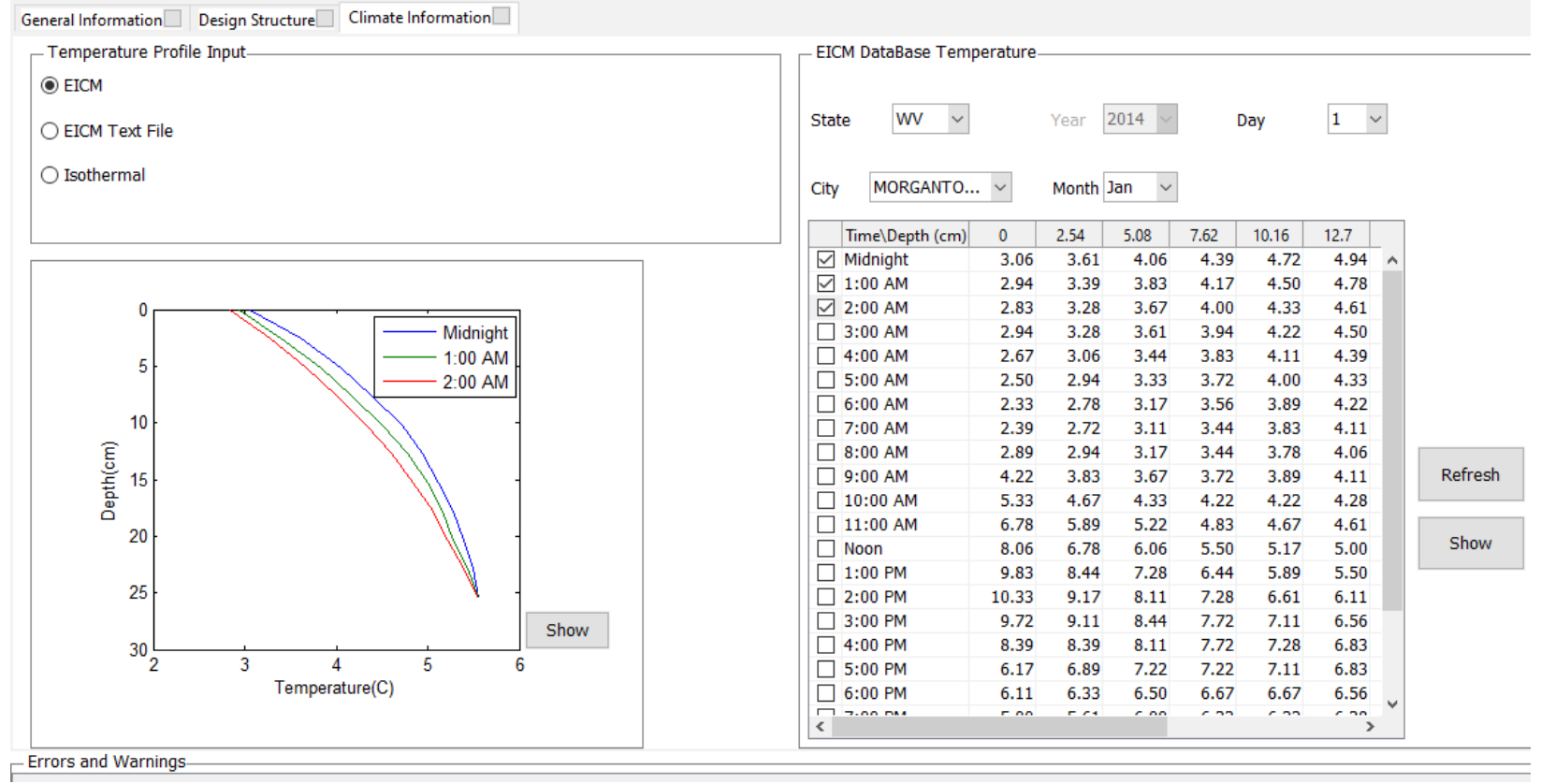

Figure 126: Climate Data for Run 1.6 


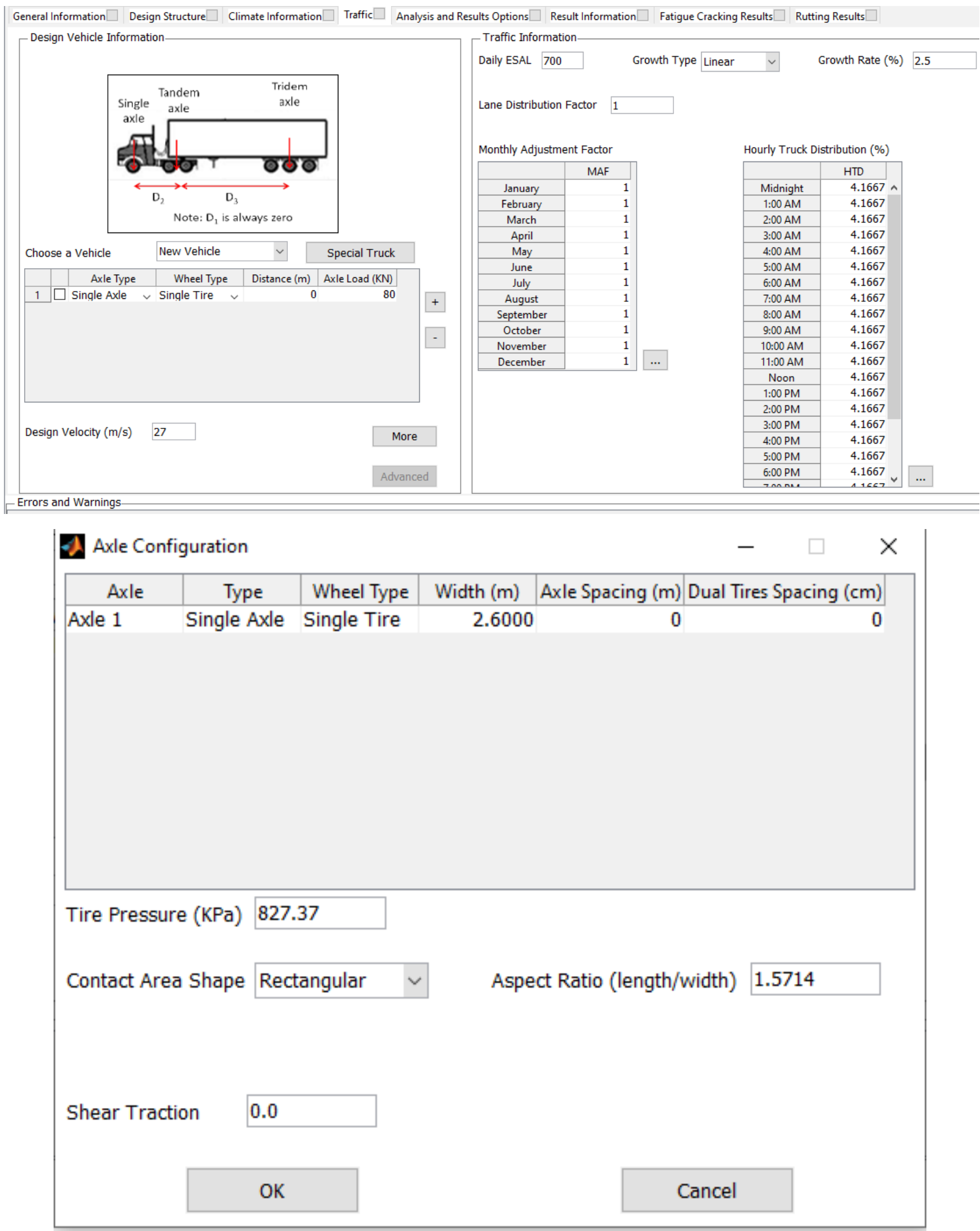

Figure 127: Traffic Data for Run 1.6 


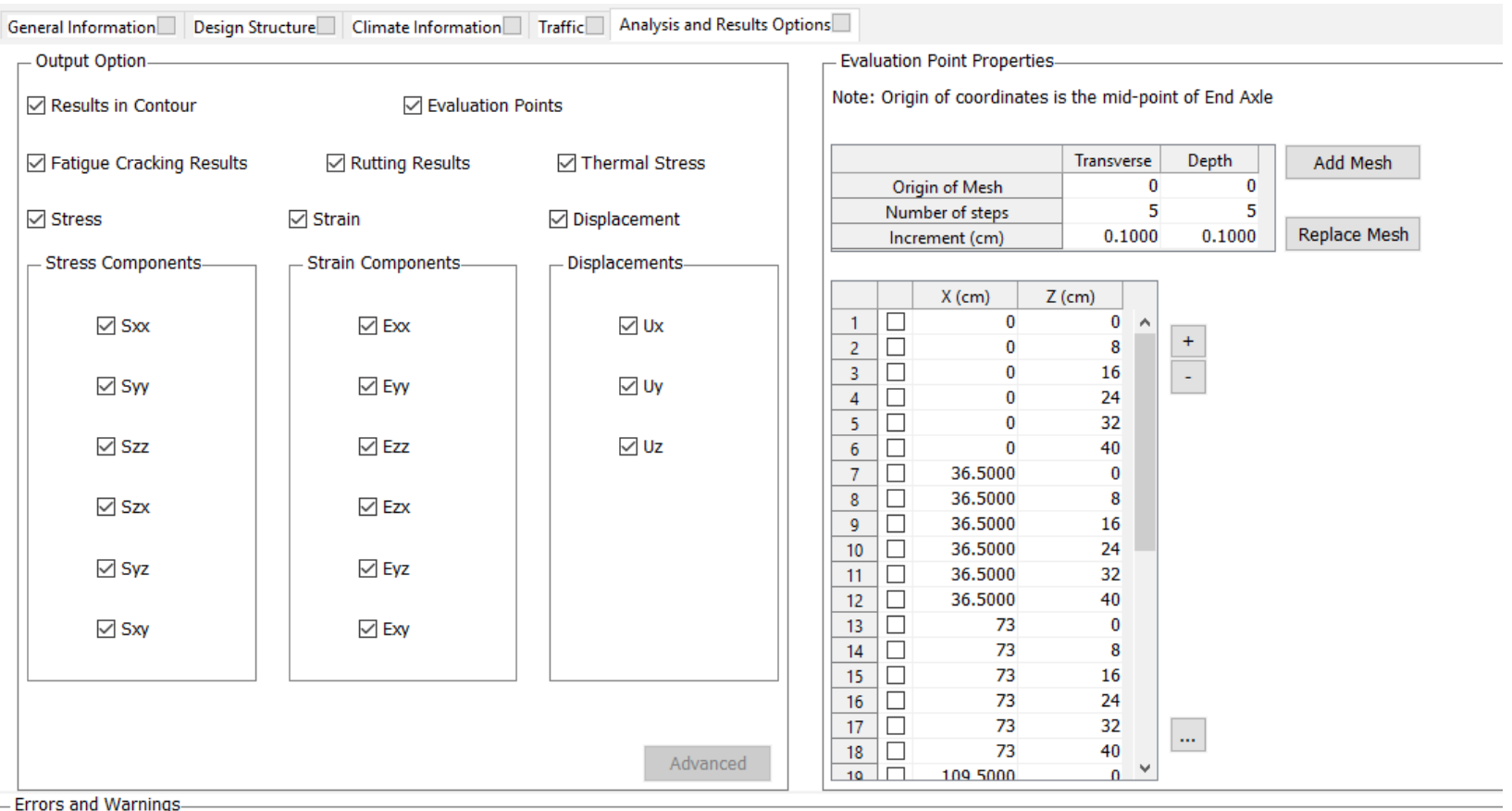

Figure 128: Output and Analysis Options for Run 1.6

\section{Run 1.7}

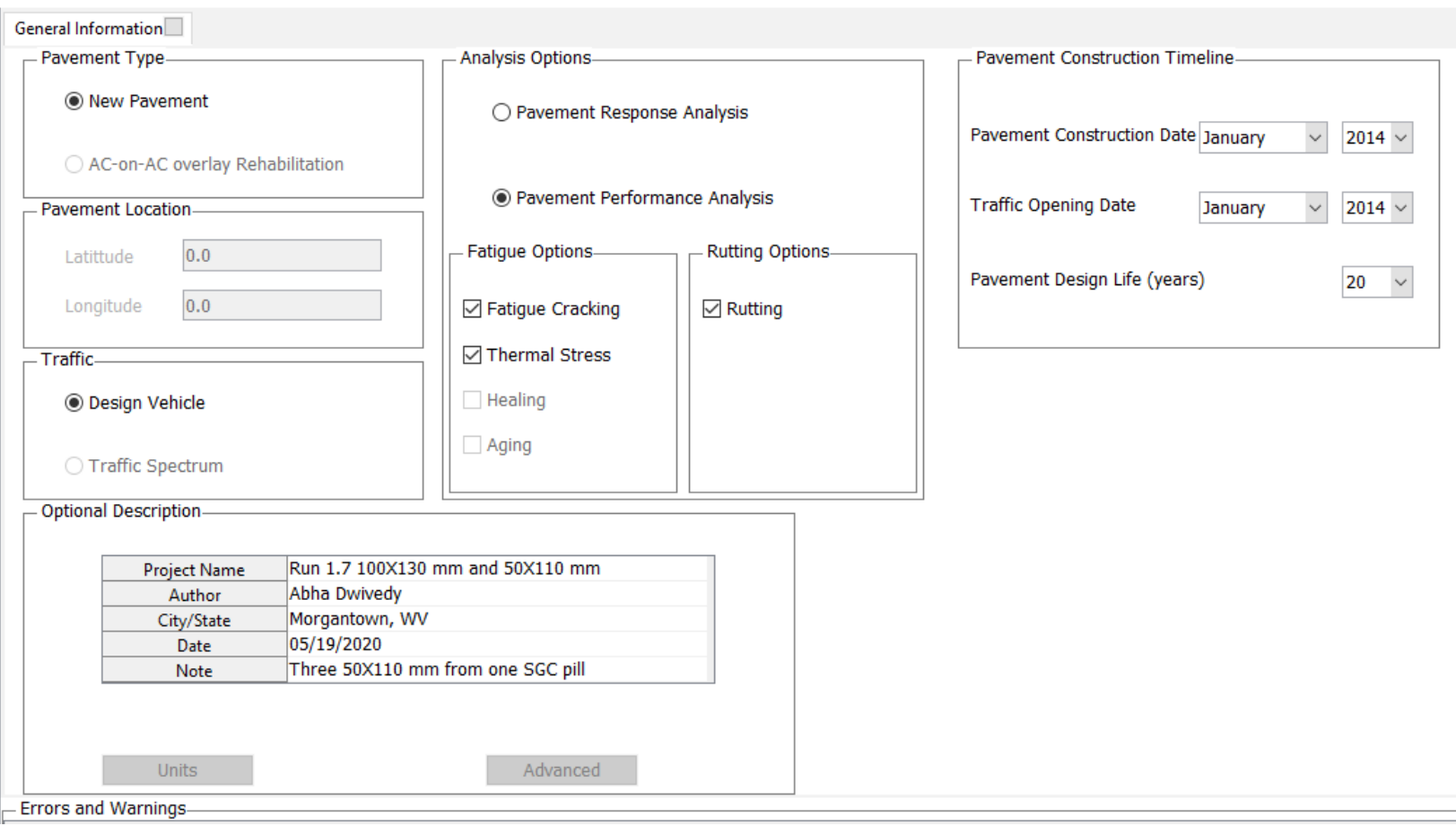

Figure 129: General Information for Run 1.7 


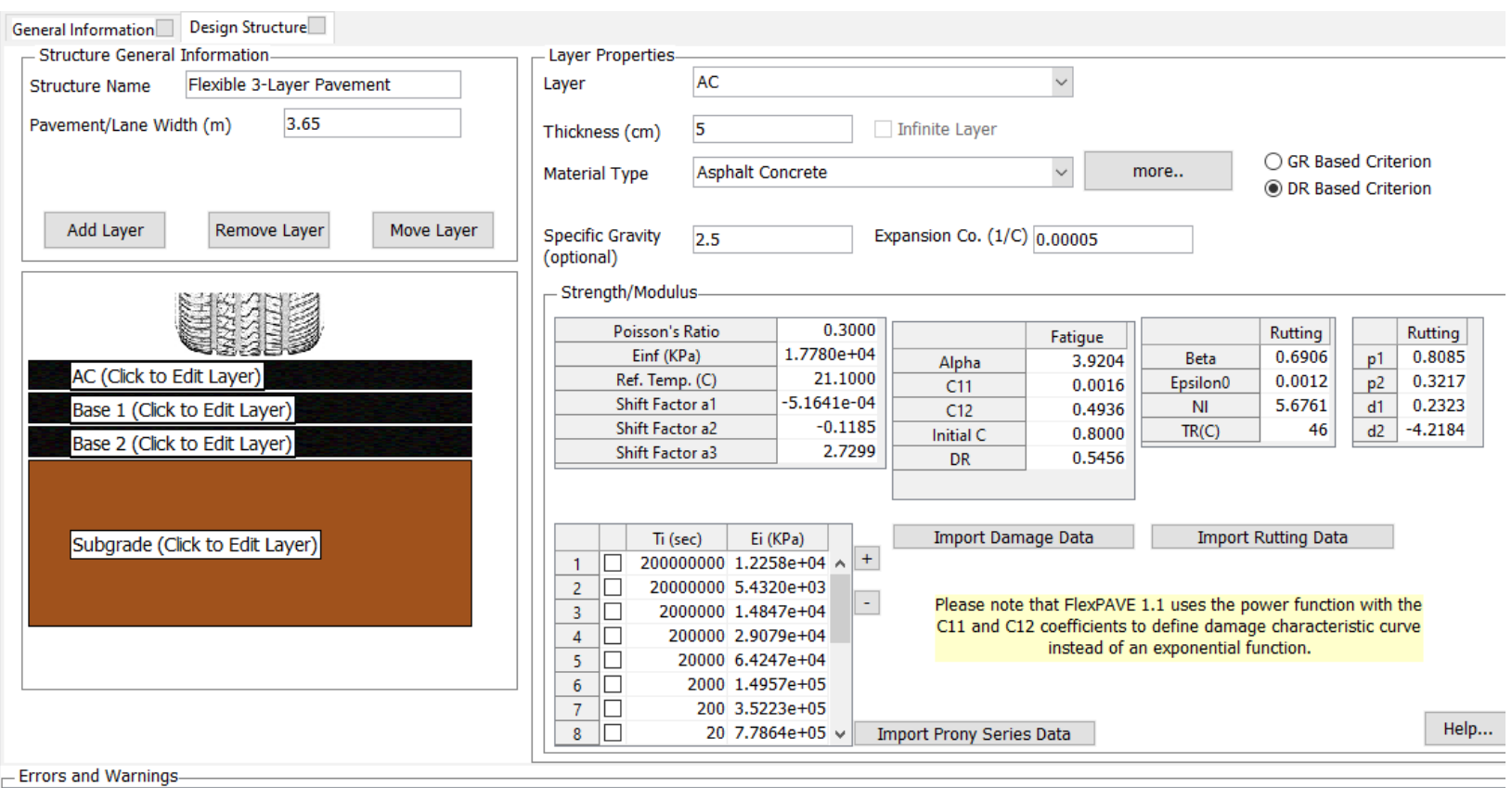

Figure 130: Design Structure of AC Layer for Run 1.7

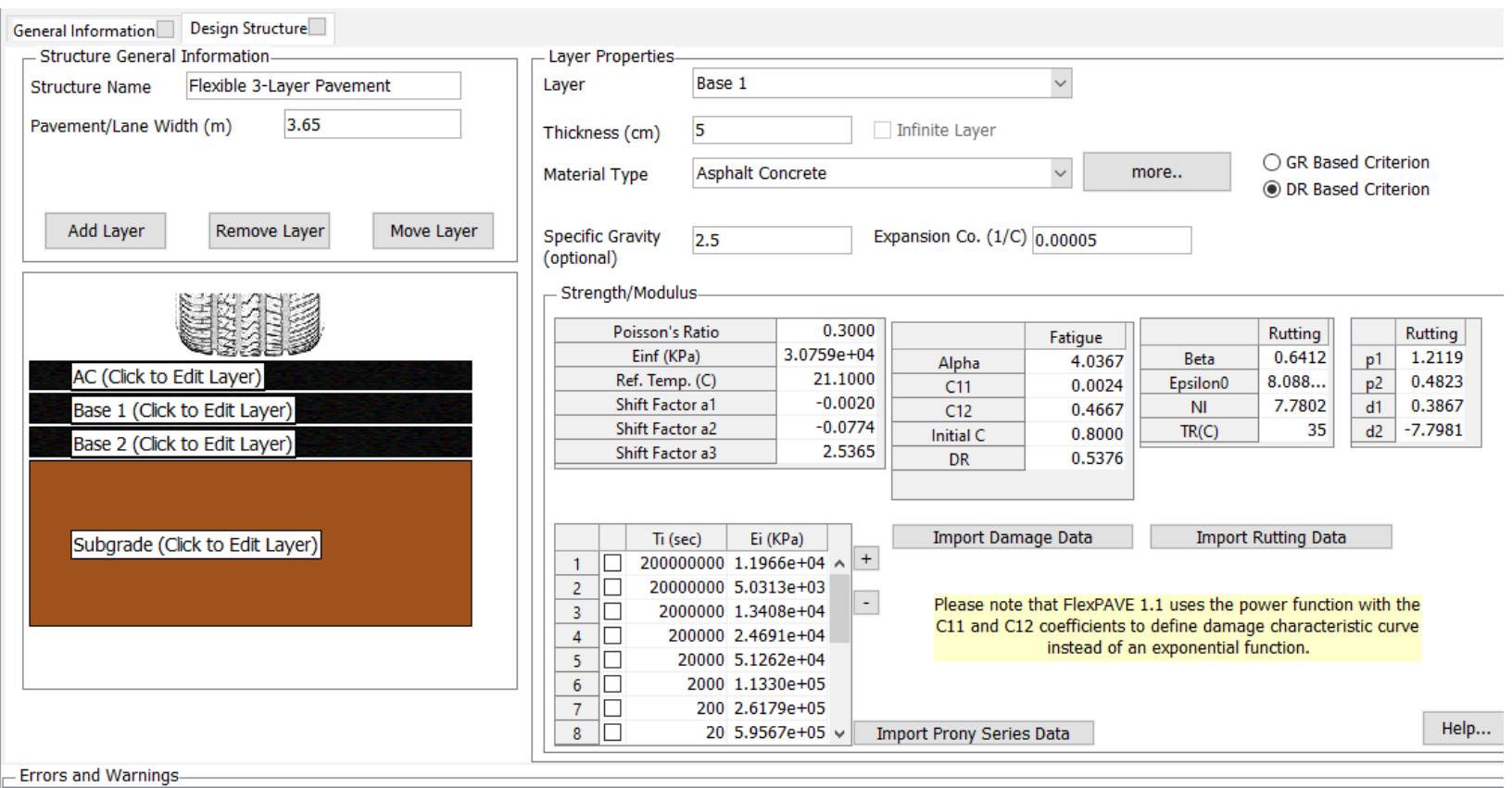

Figure 131: Design Structure of Base 1 Layer for Run 1.7 


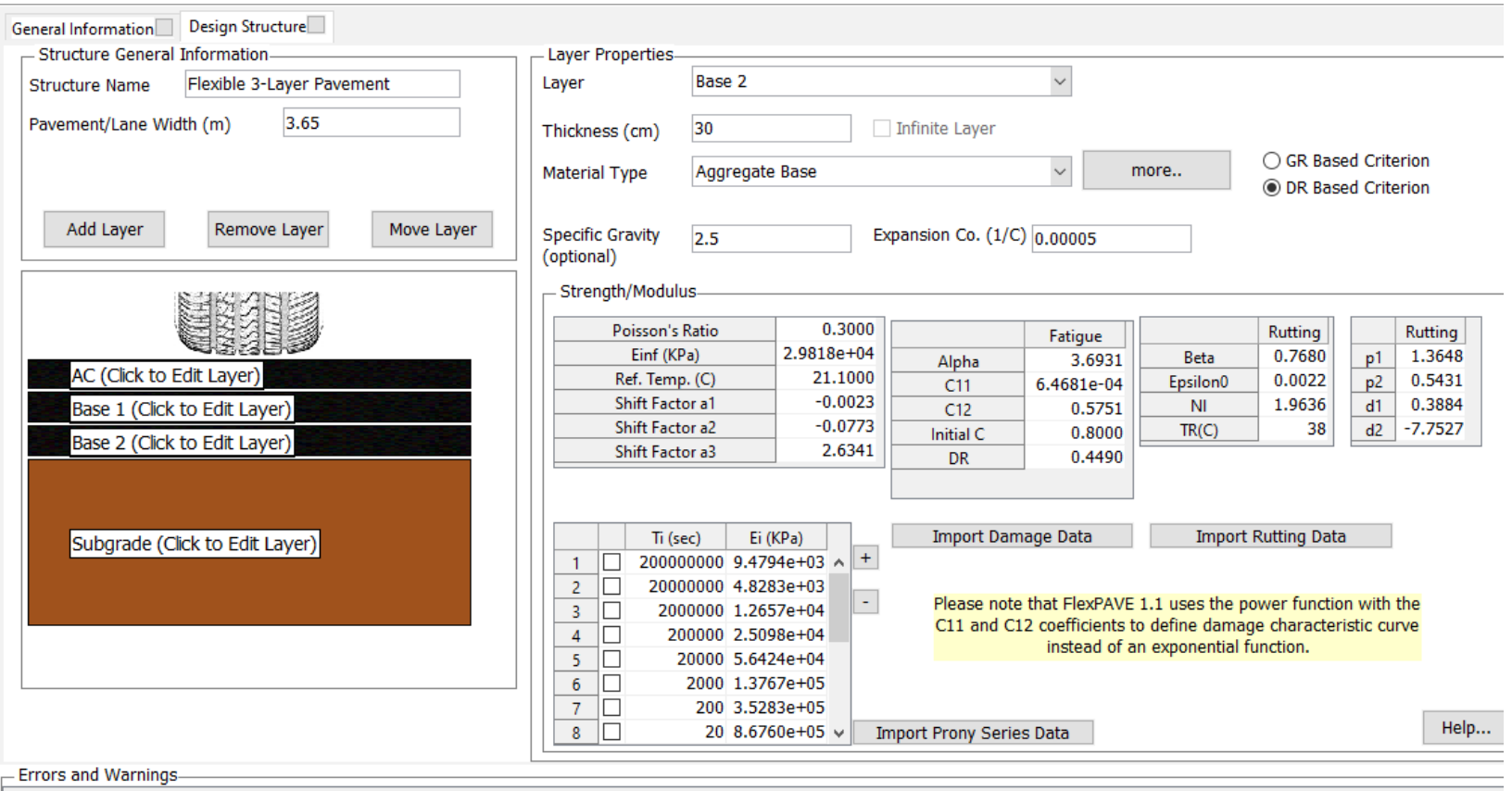

Figure 132: Design Structure of Base 2 Layer for Run 1.7

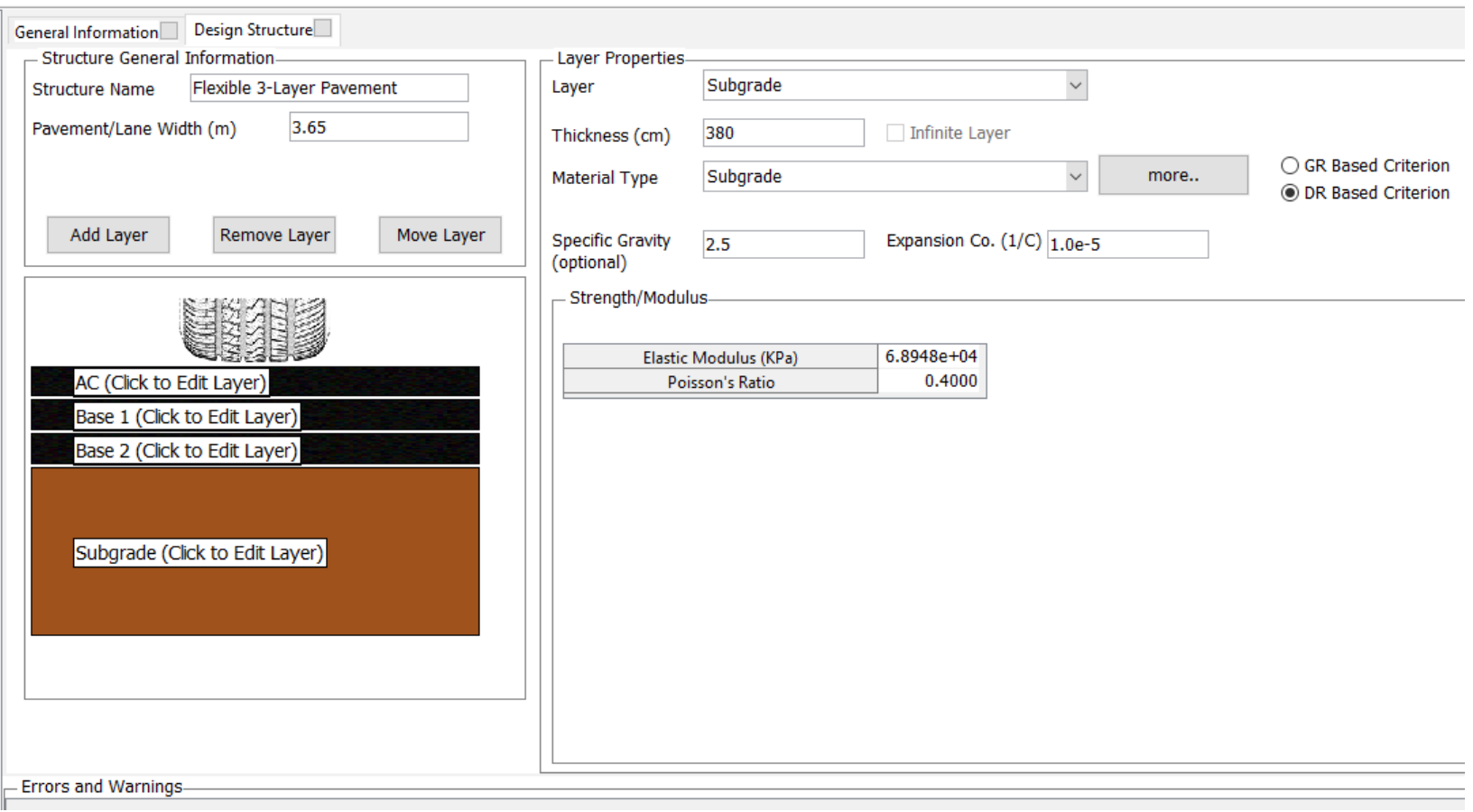

Figure 133: Design Structure of Subgrade Layer for Run 1.7 


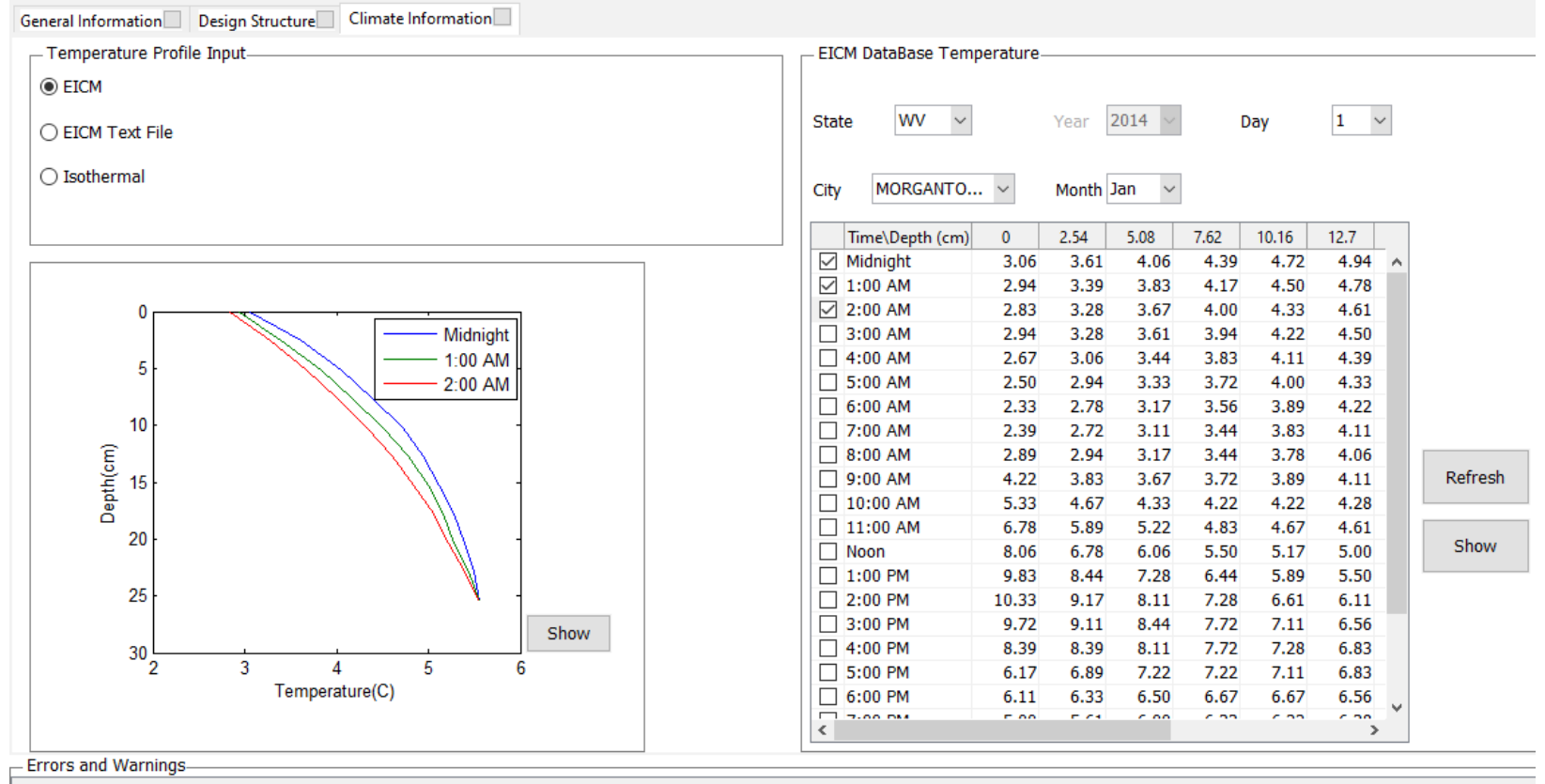

Figure 134: Climate Data for Run 1.7 


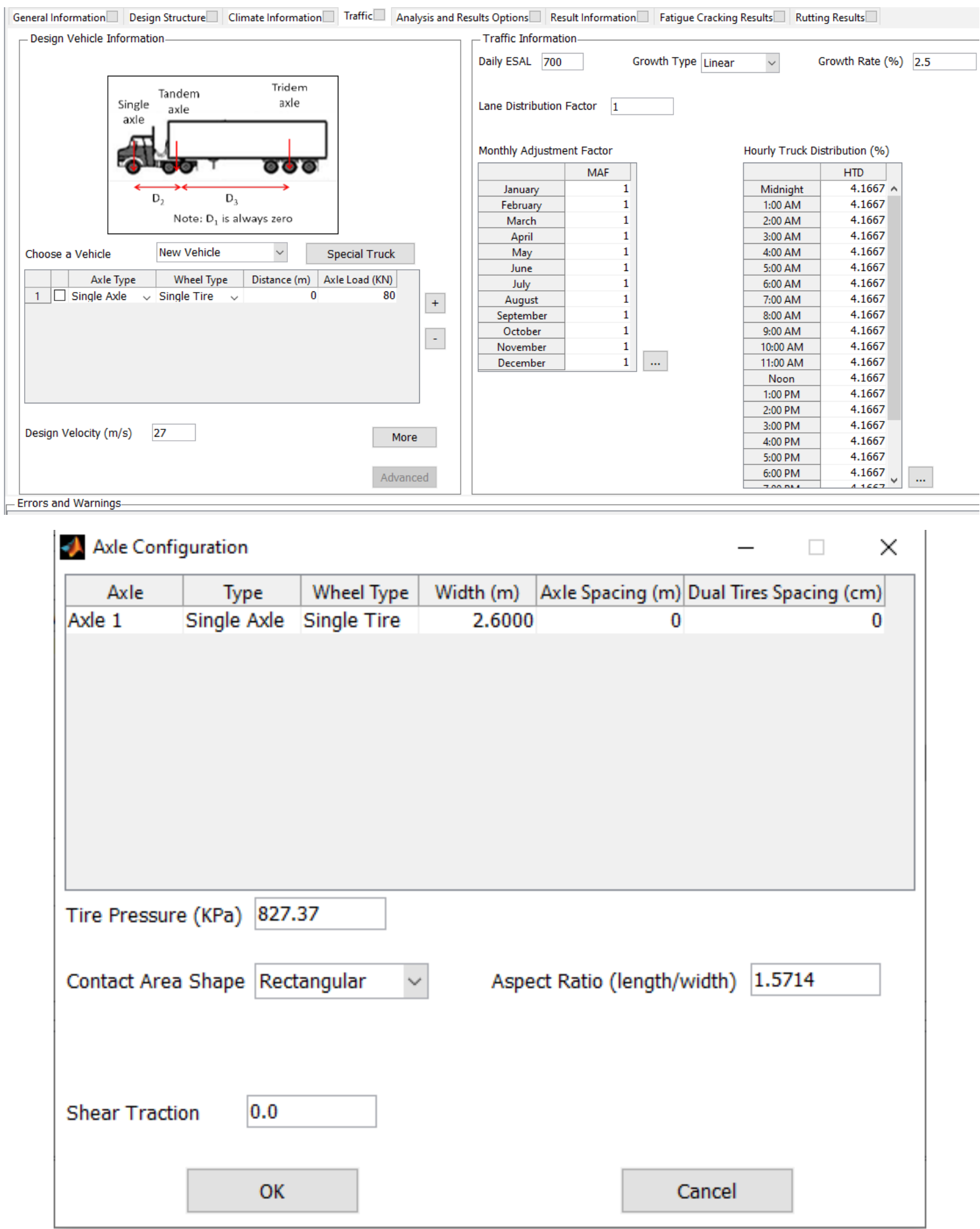

Figure 135: Traffic Data for Run 1.7 


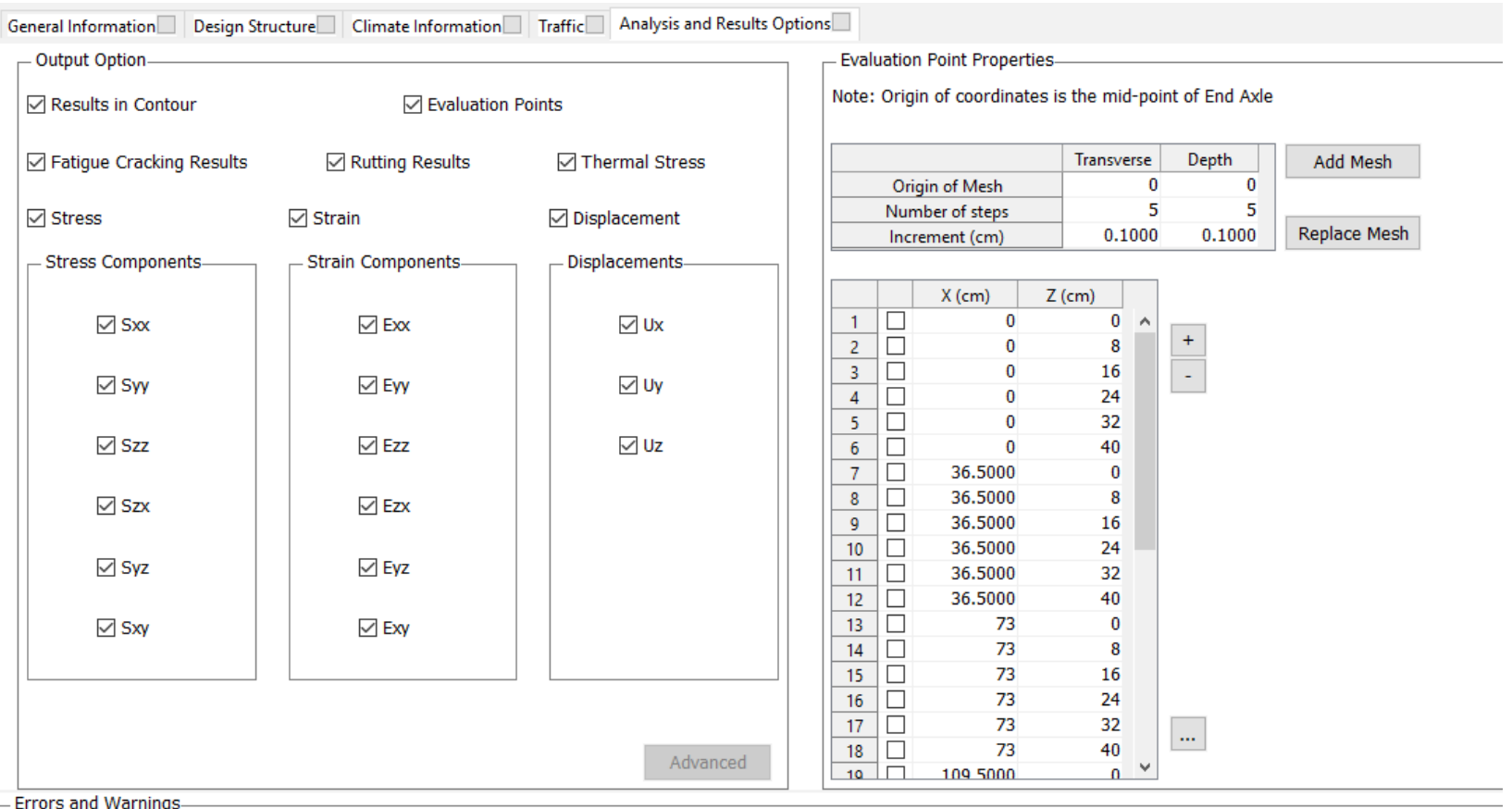

Figure 136: Output and Analysis Options for Run 1.7

\section{Run 1.8}

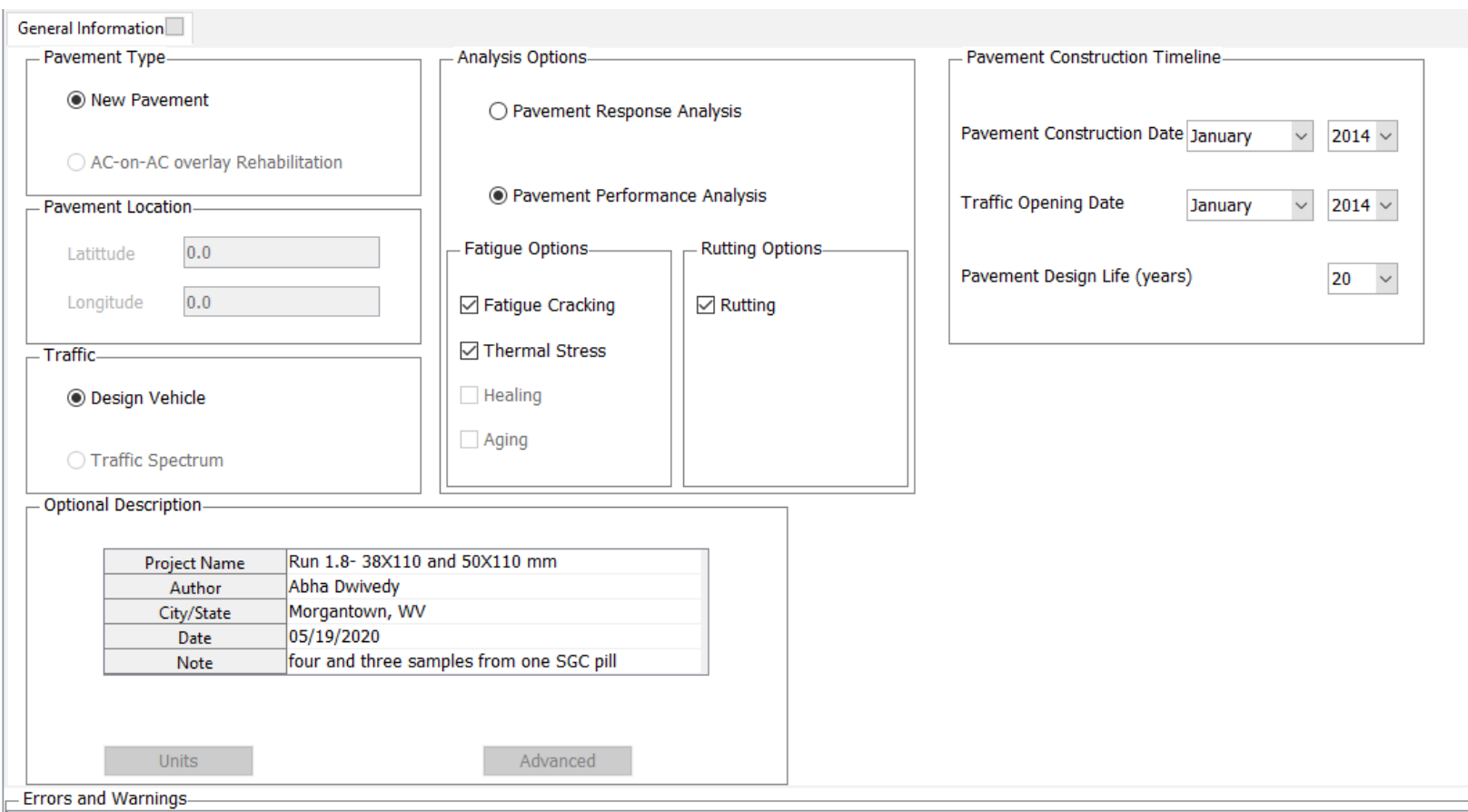

Figure 137: General Information for Run 1.8 

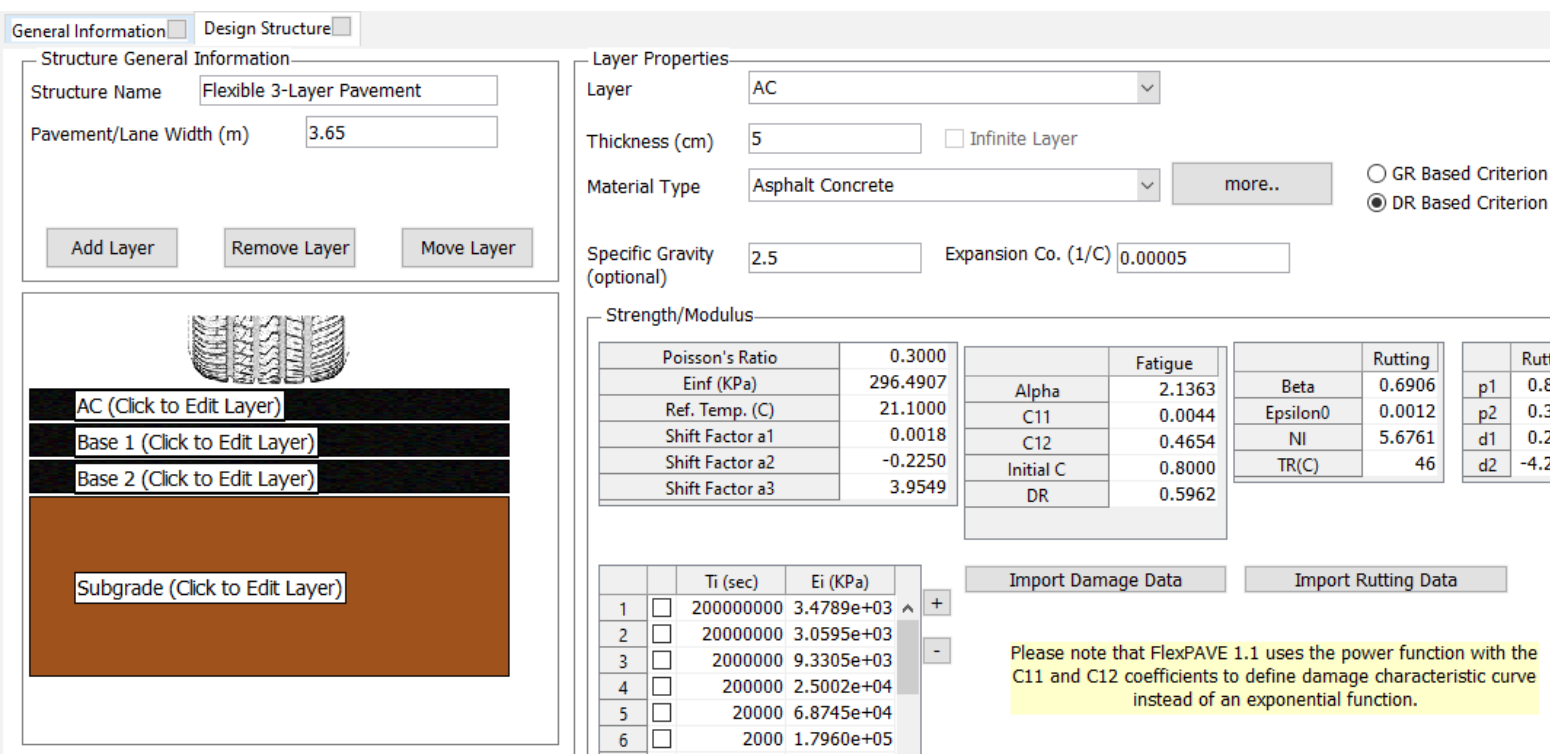

\begin{tabular}{|c|c|c|c|c|c|c|c|}
\hline Poisson's Ratio & 0.3000 & & Fatigue & & Rutting & & Rutting \\
\hline Einf $(\mathrm{KPa})$ & 296.4907 & Alpha & 2.1363 & Beta & 0.6906 & $p 1$ & 0.8085 \\
\hline Ref. Temp. (C) & 21.1000 & $\mathrm{C} 11$ & 0.0044 & Epsilon 0 & 0.0012 & $p^{2}$ & 0.3217 \\
\hline Shift Factor a1 & 0.0018 & $\mathrm{C} 12$ & 0.4654 & $\mathrm{NI}$ & 5.6761 & d1 & 0.2323 \\
\hline Shift Factor a2 & -0.2250 & Initial C & 0.8000 & $\operatorname{TR}(\mathrm{C})$ & 46 & d2 & -4.2184 \\
\hline Shift Factor a3 & 3.9549 & DR & 0.5962 & & & & \\
\hline
\end{tabular}

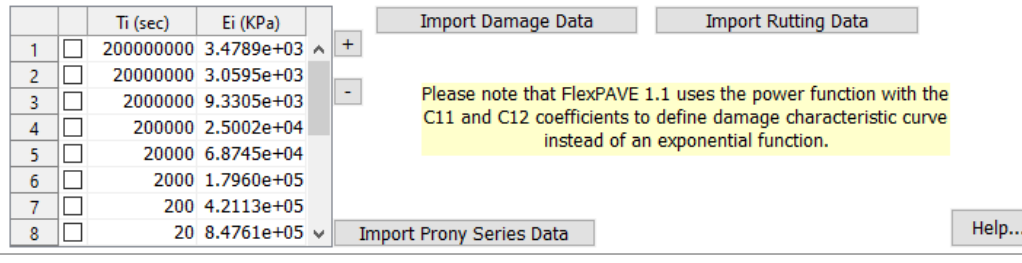

- Errors and Warnings

Figure 138: Design Structure of AC Layer for Run 1.8

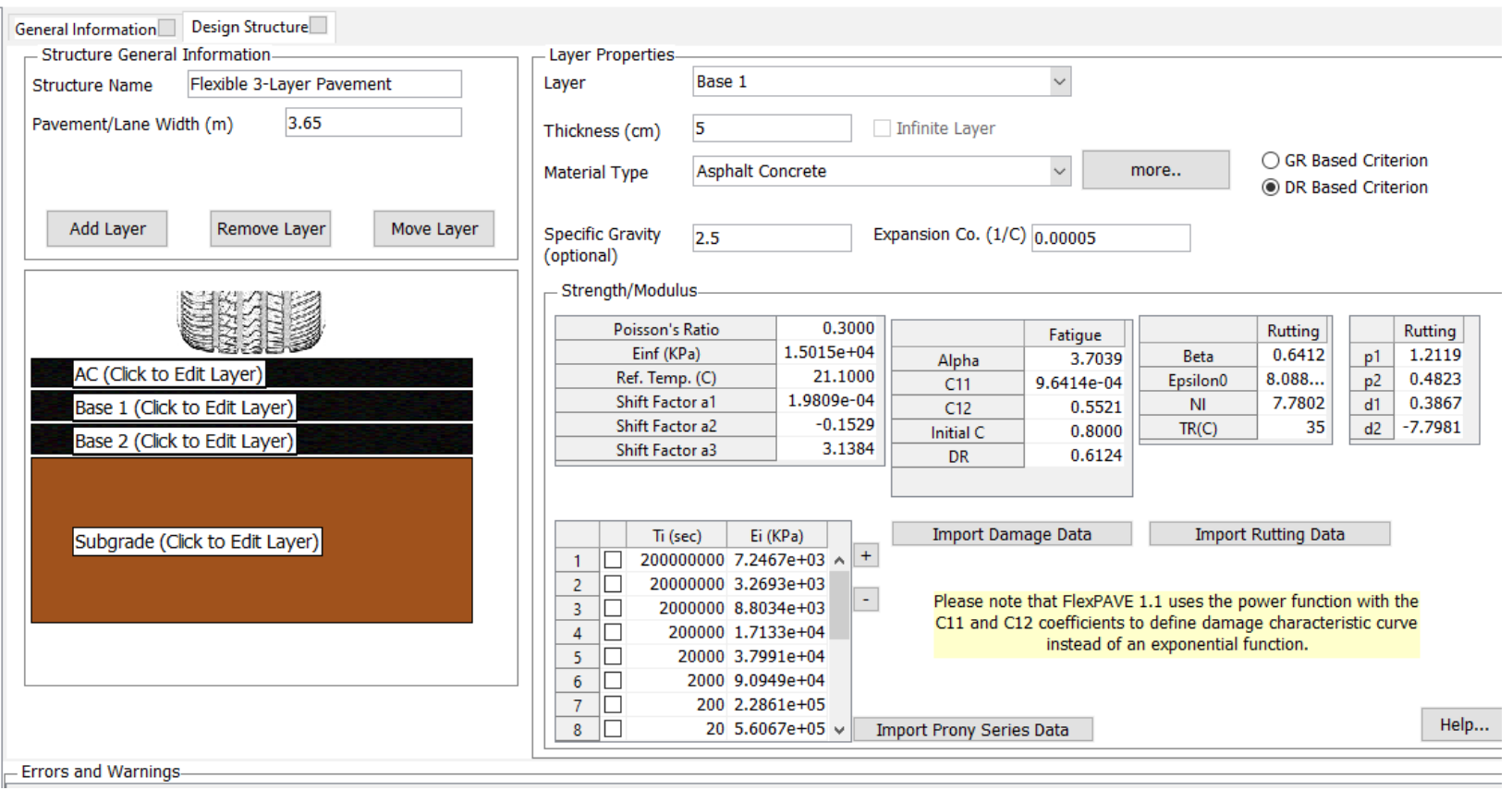

Figure 139: Design Structure of Base 1 Layer for Run 1.8 


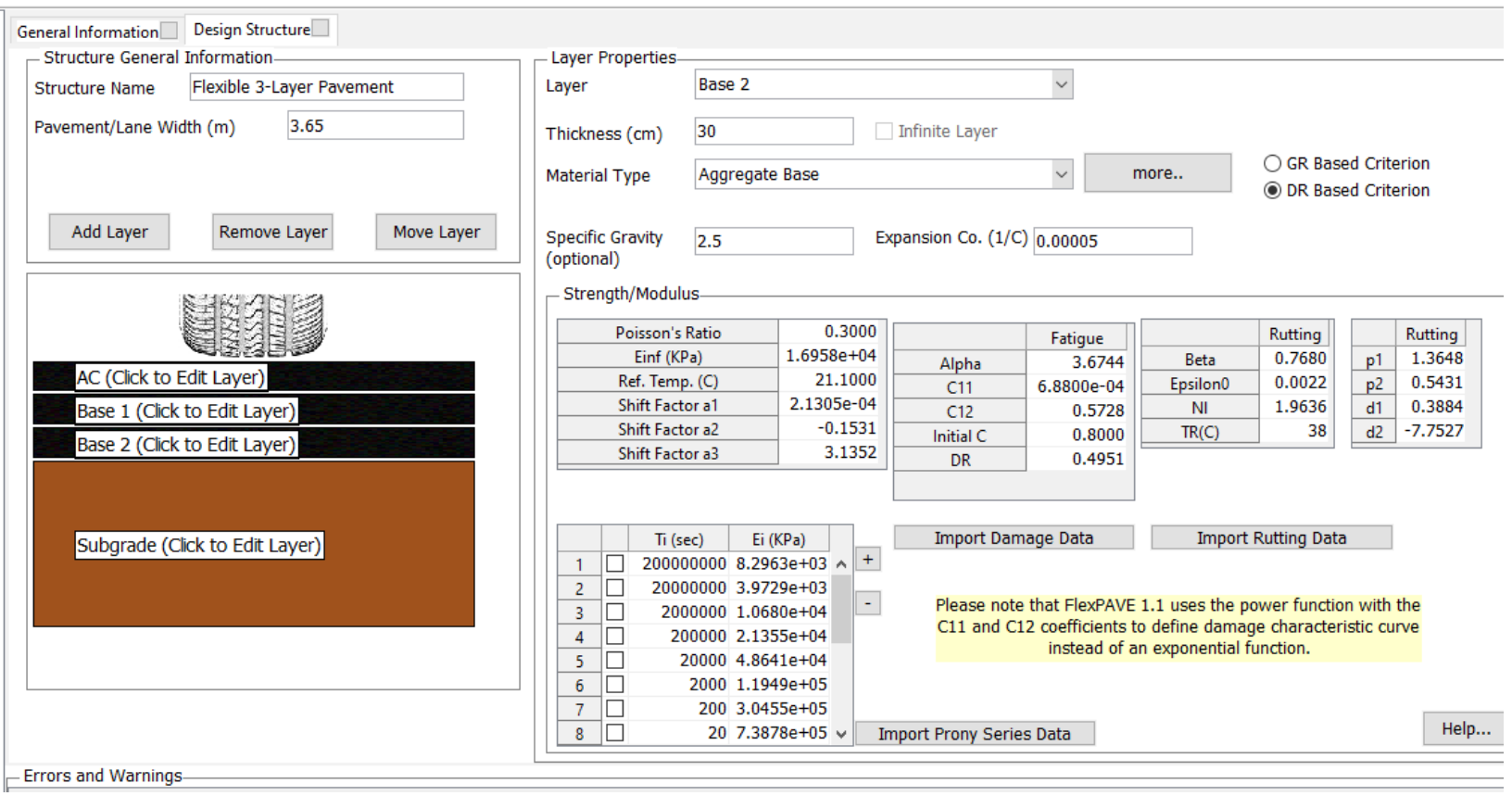

Figure 140: Design Structure of Base 2 Layer for Run 1.8

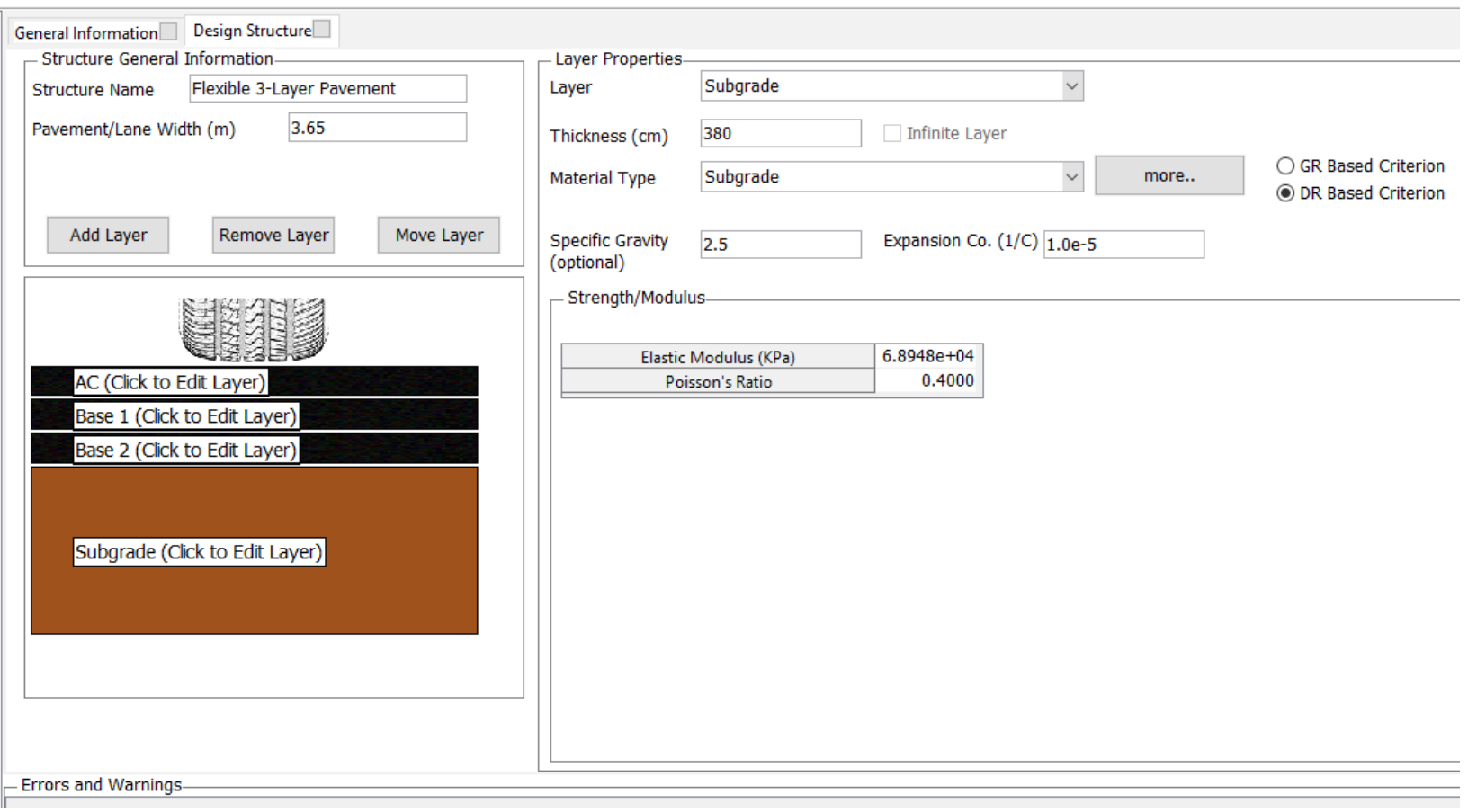

Figure 141: Design Structure of Subgrade Layer for Run 1.8 


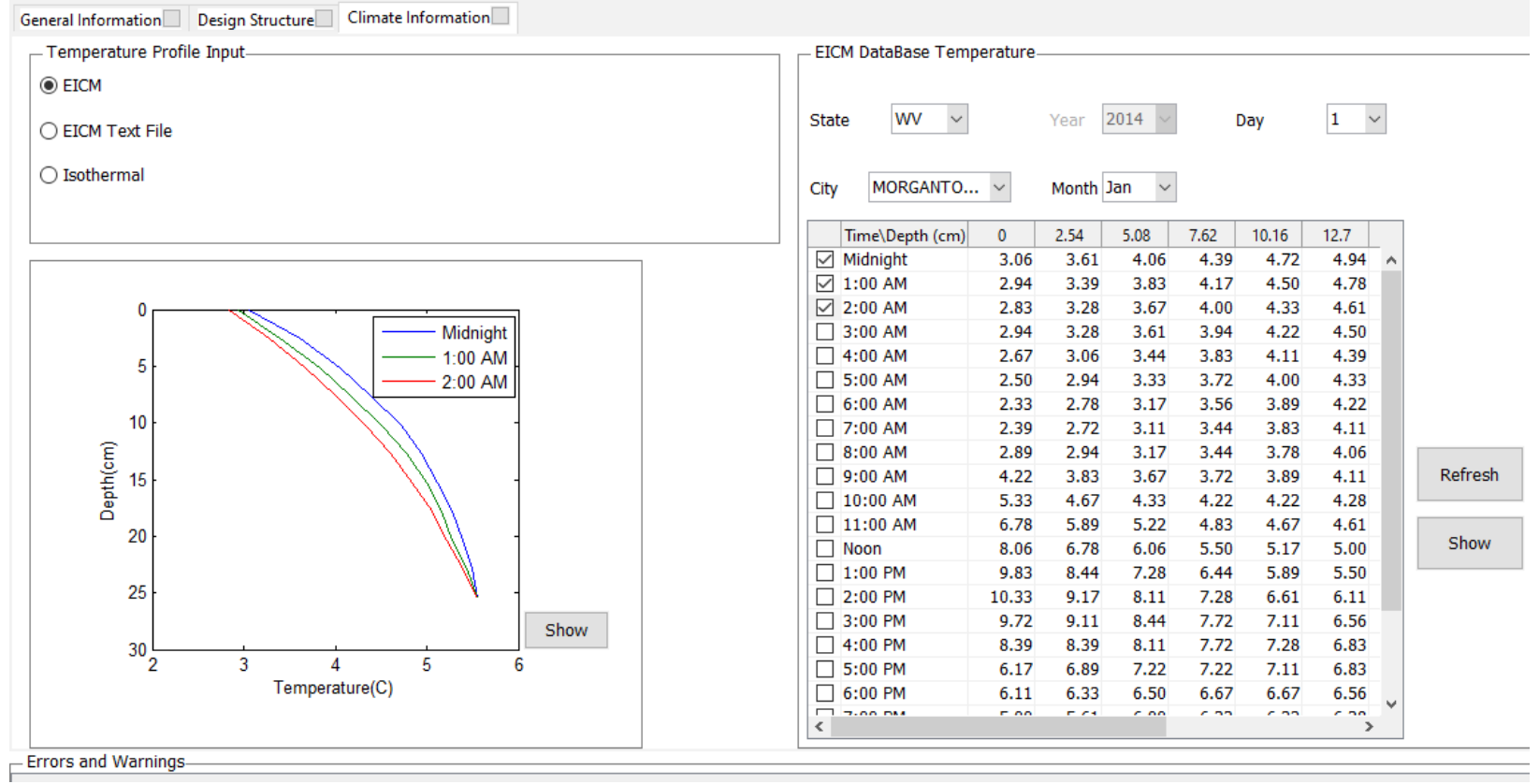

Figure 142: Climate Data for Run 1.8 


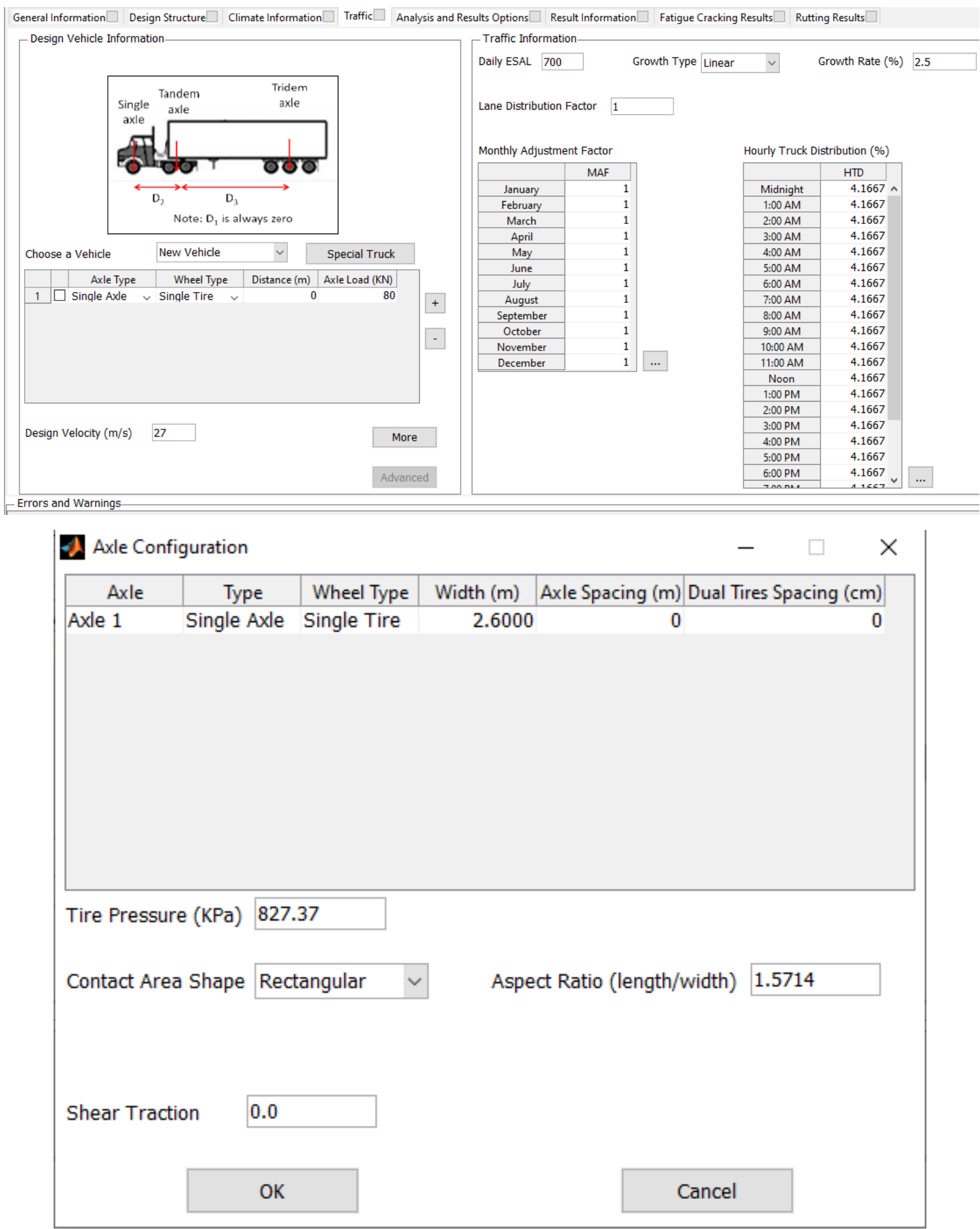

Figure 143: Traffic Data for Run 1.8 


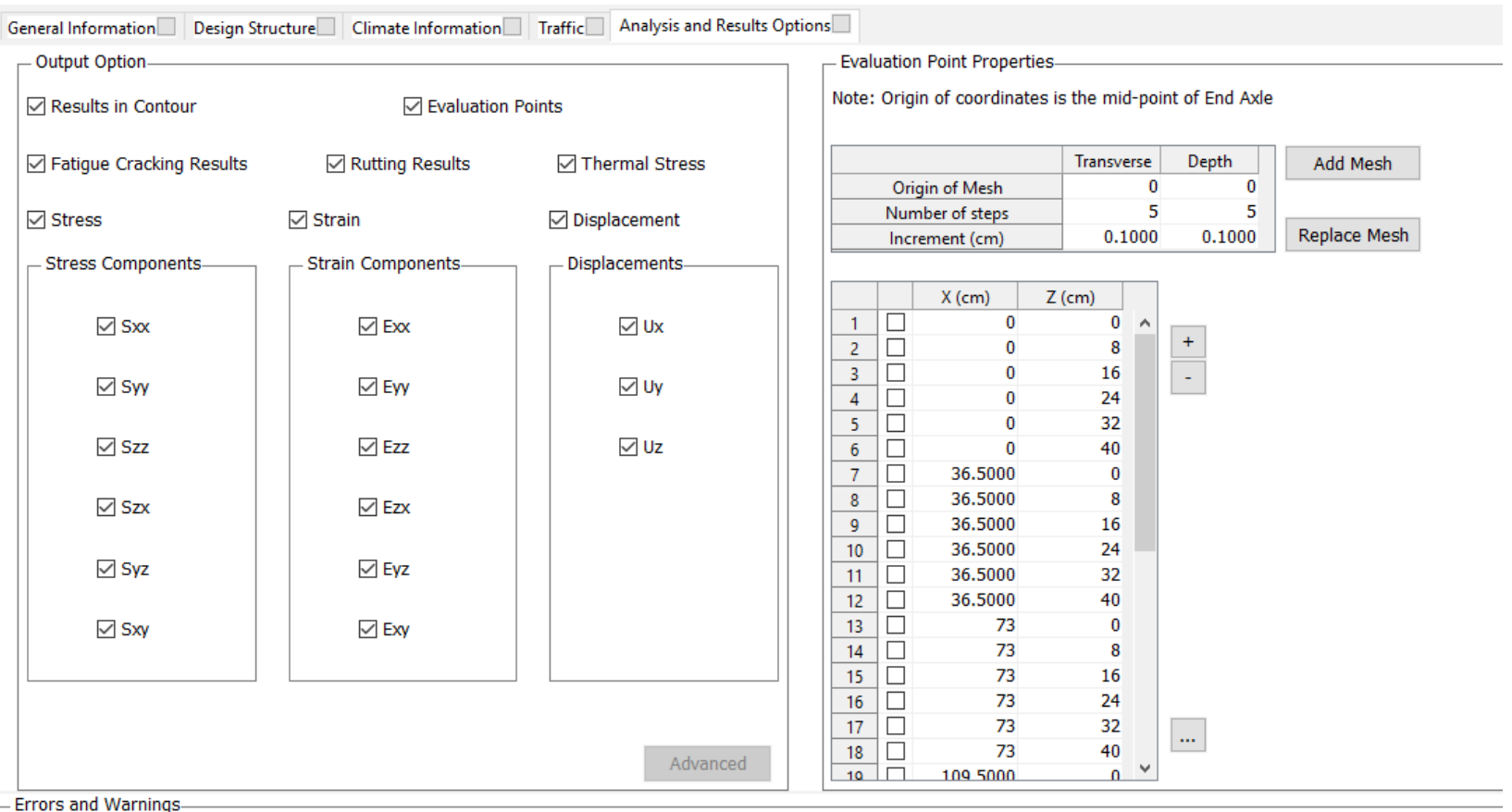

Figure 144: Output and Analysis Options for Run 1.8

\section{Run 1.9}

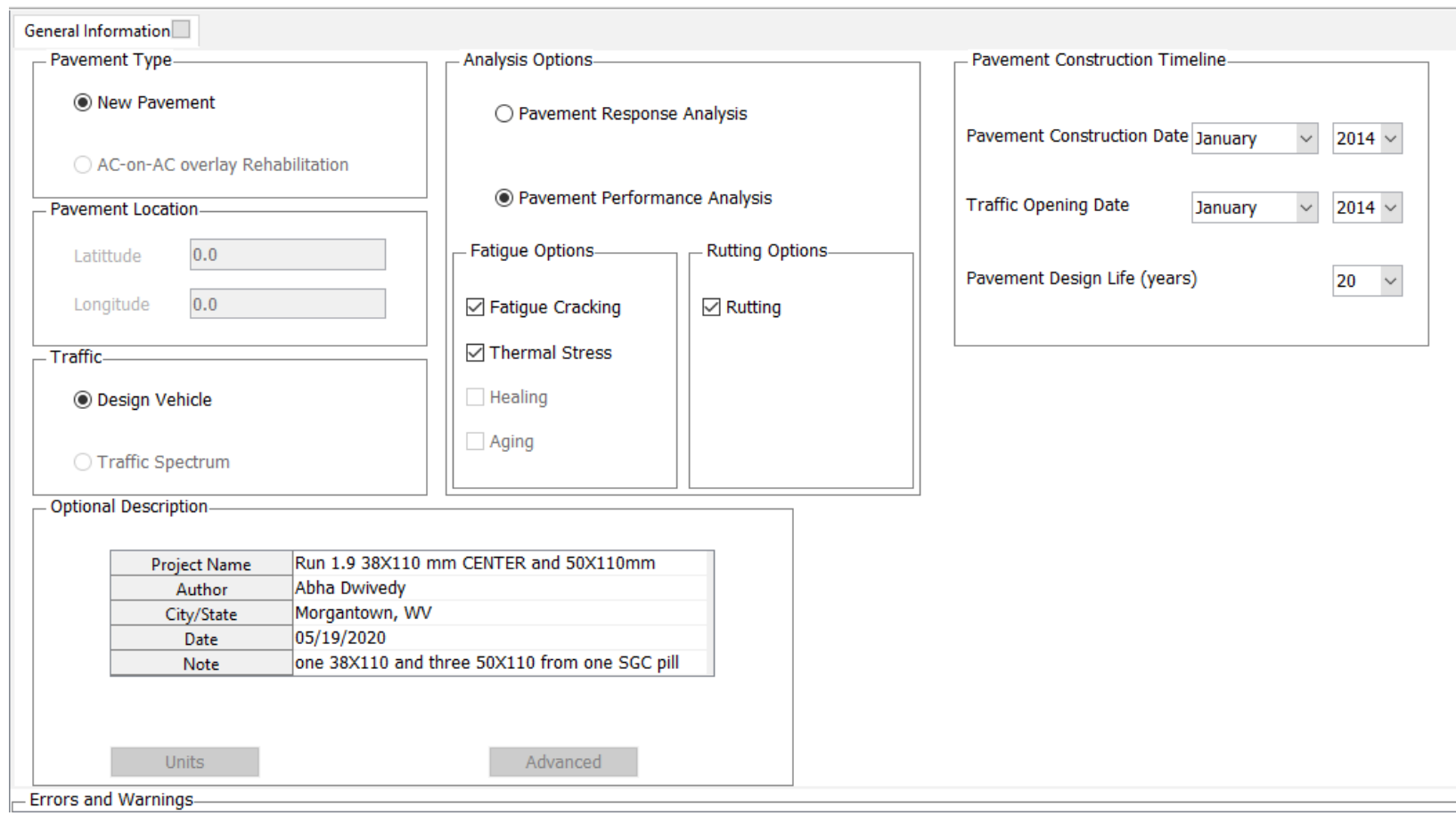

Figure 145: General Information for Run 1.9 


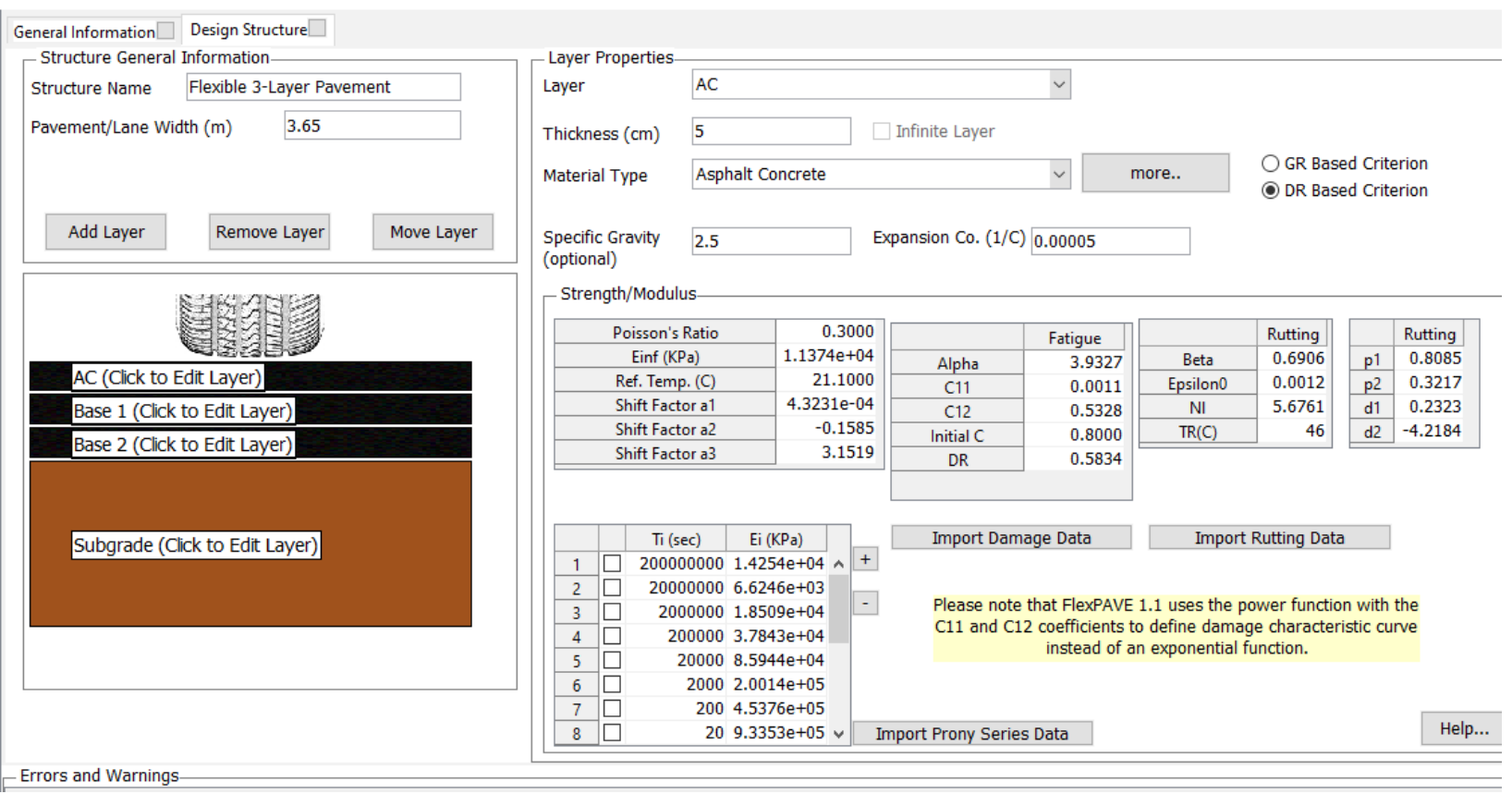

Figure 146: Design Structure of AC Layer for Run 1.9

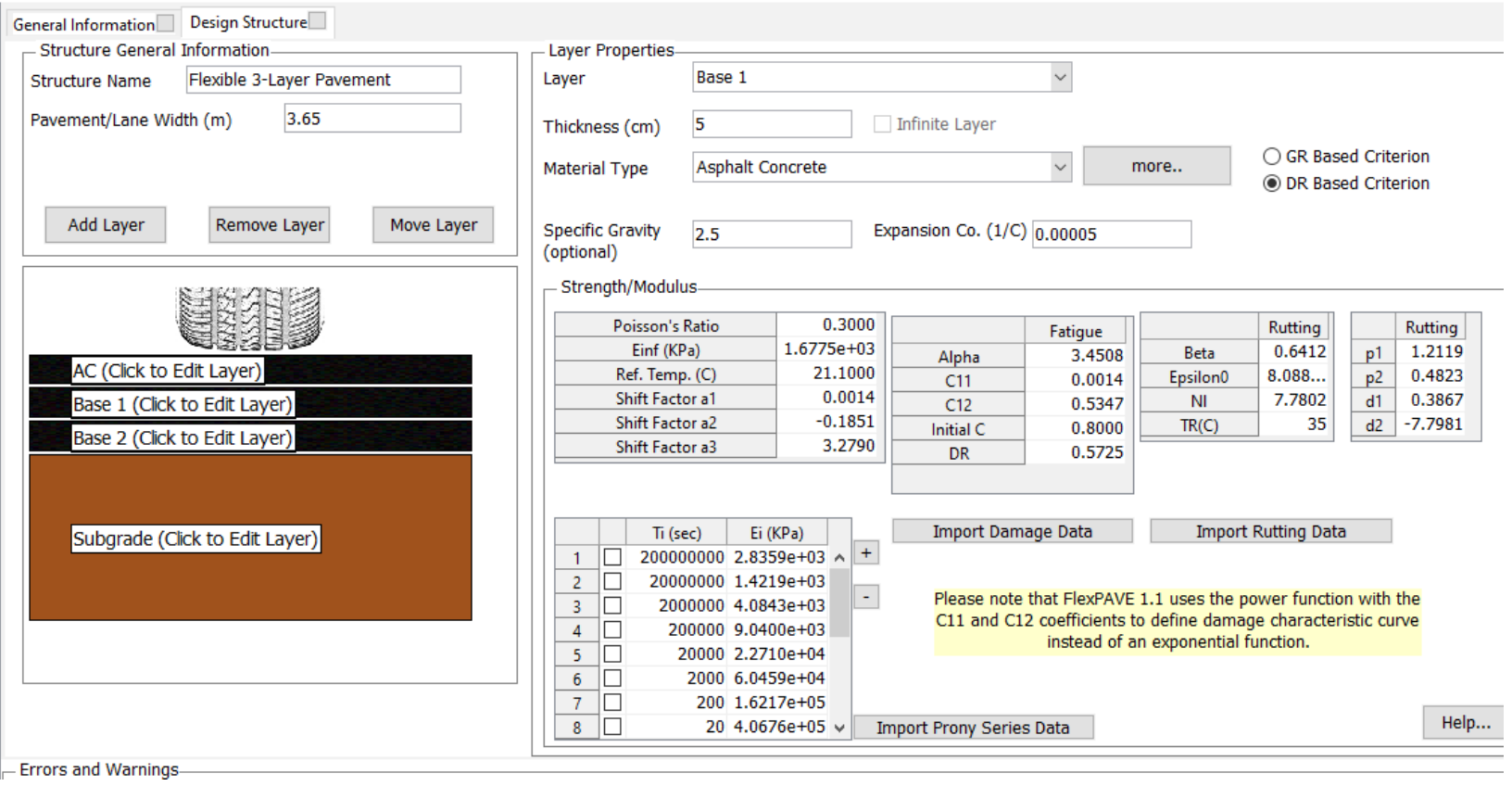

Figure 147: Design Structure of Base 1 Layer for Run 1.9 


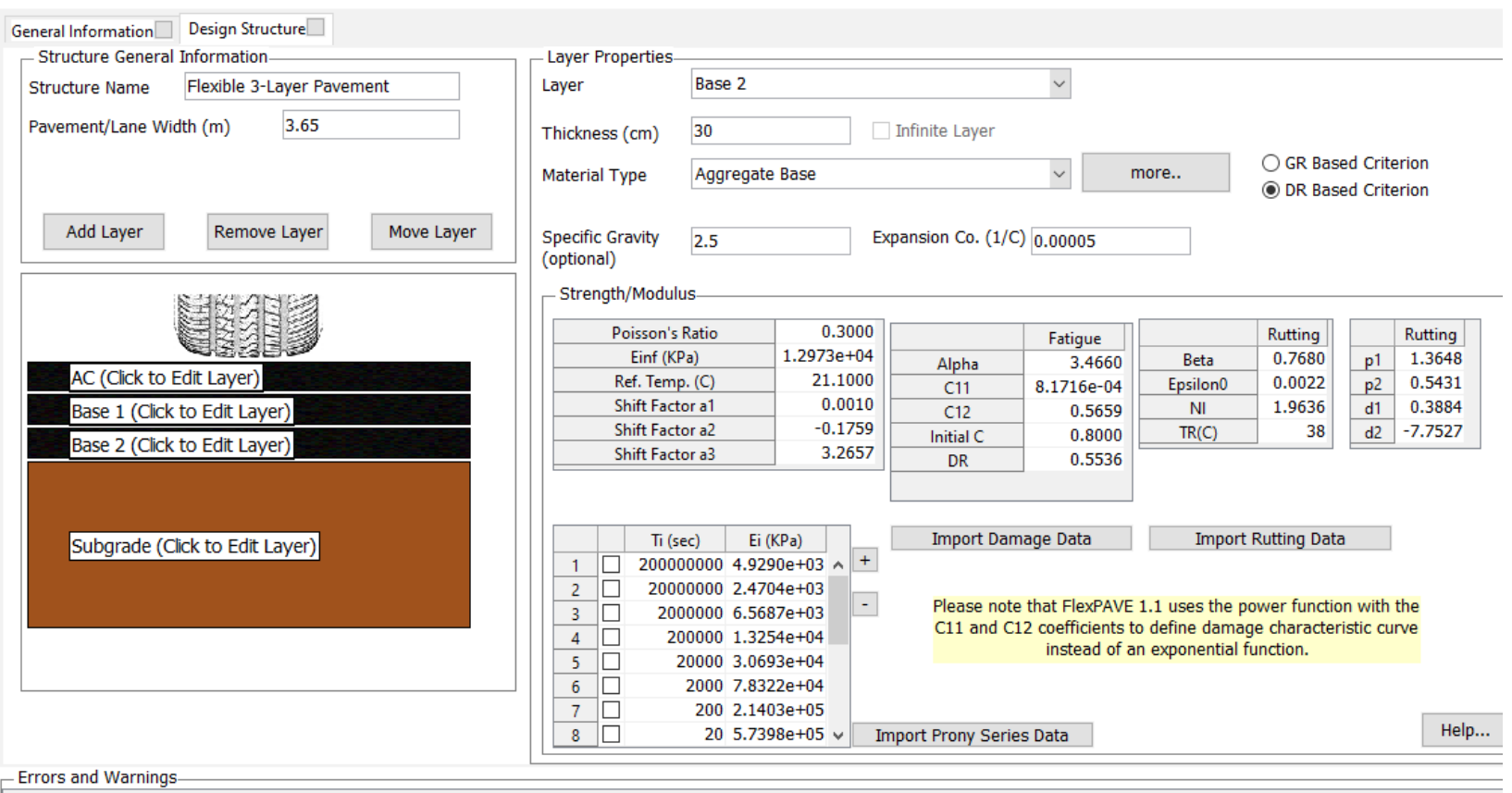

Figure 148: Design Structure of Base 2 Layer for Run 1.9

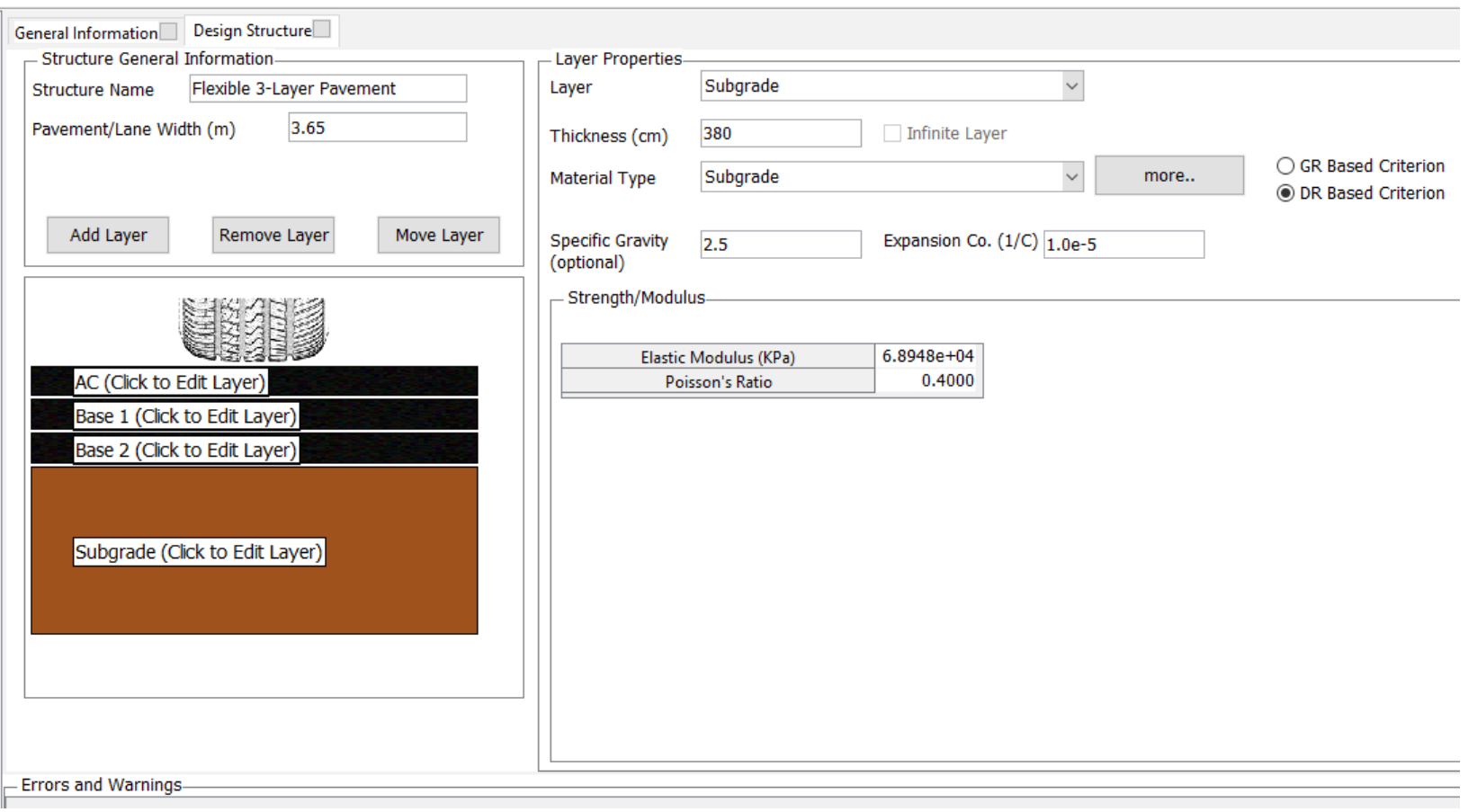

Figure 149: Design Structure of Subgrade Layer for Run 1.9 


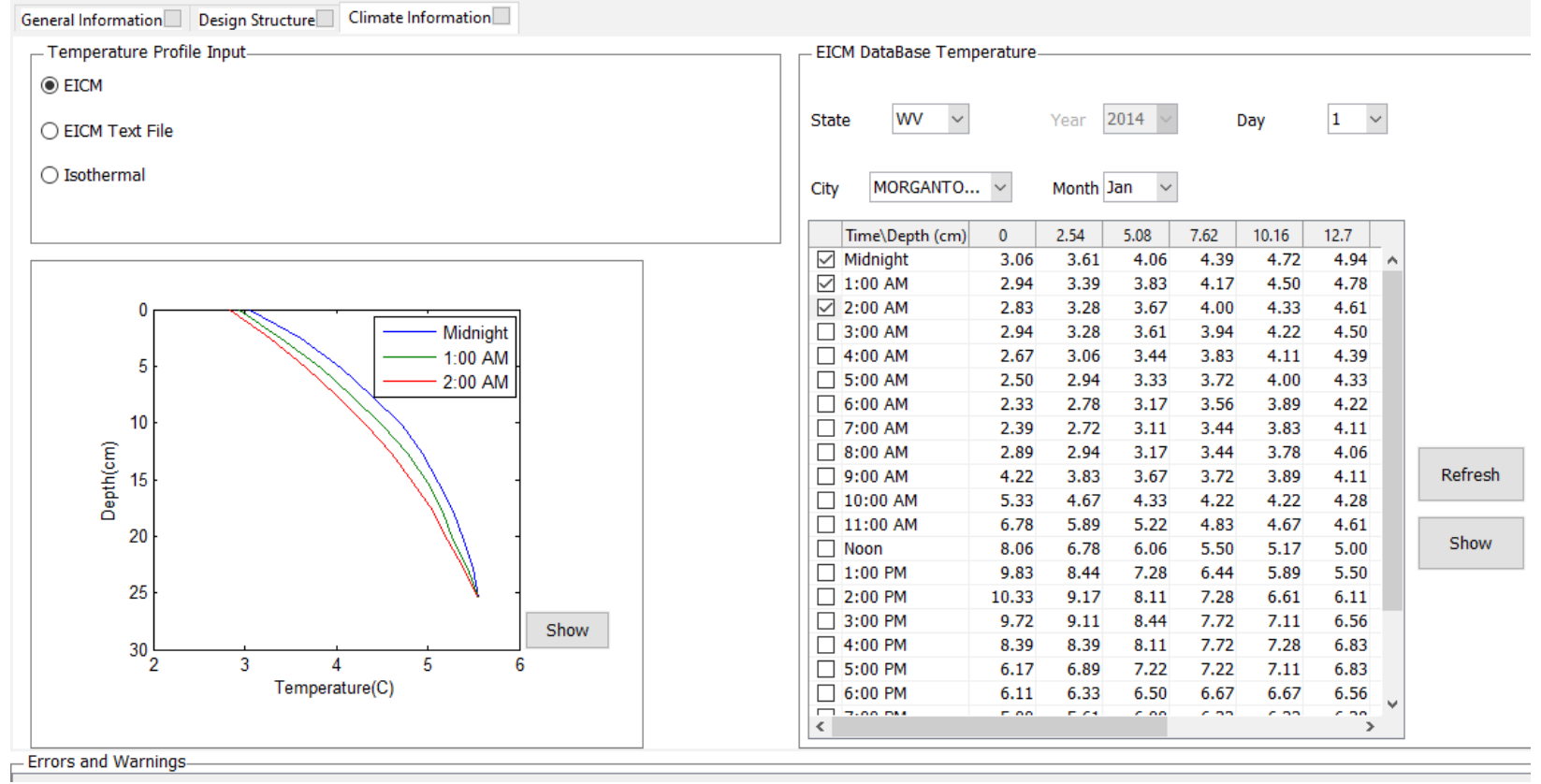

Figure 150: Climate Data for Run 1.9 

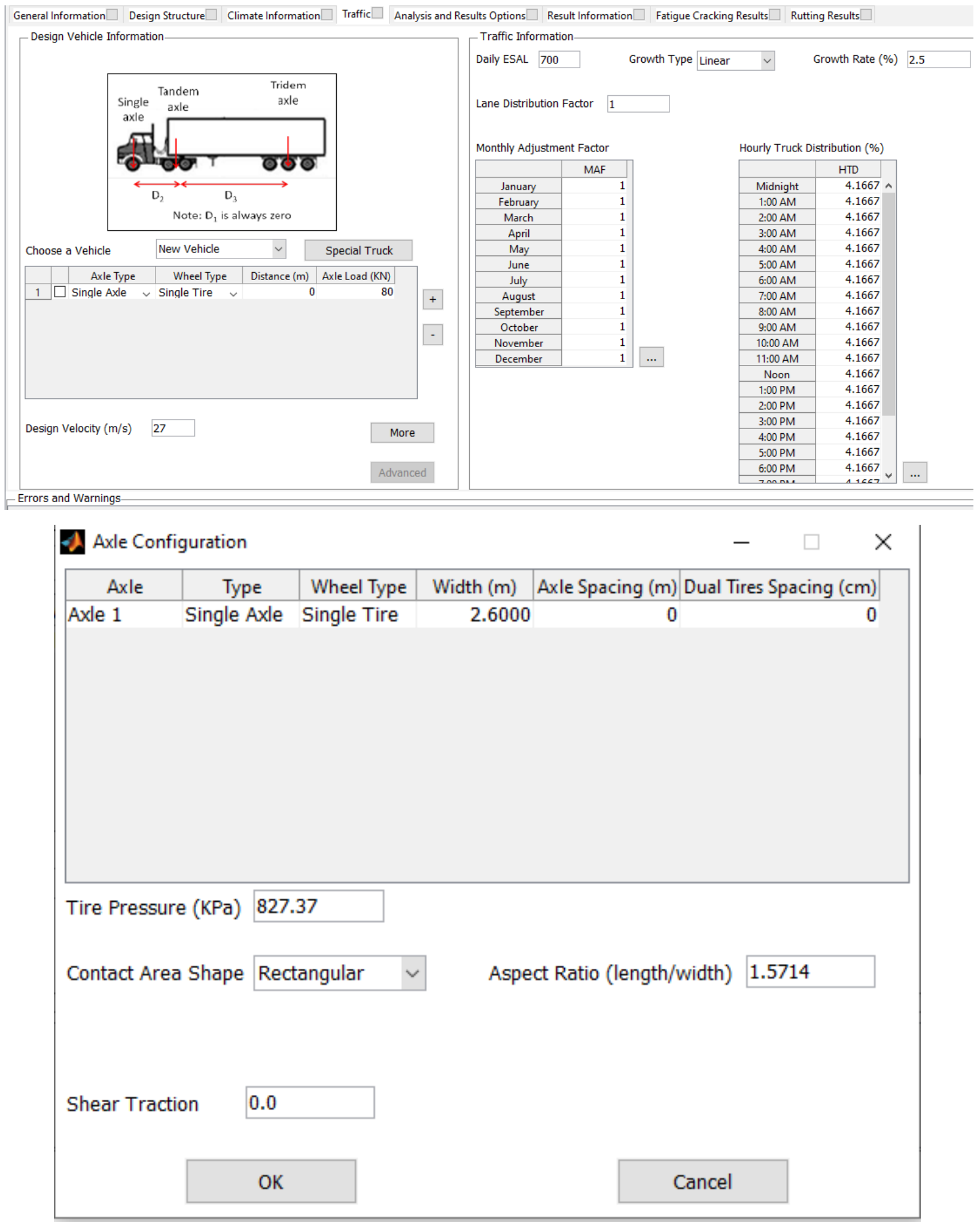

Figure 151: Traffic Data for Run 1.9 


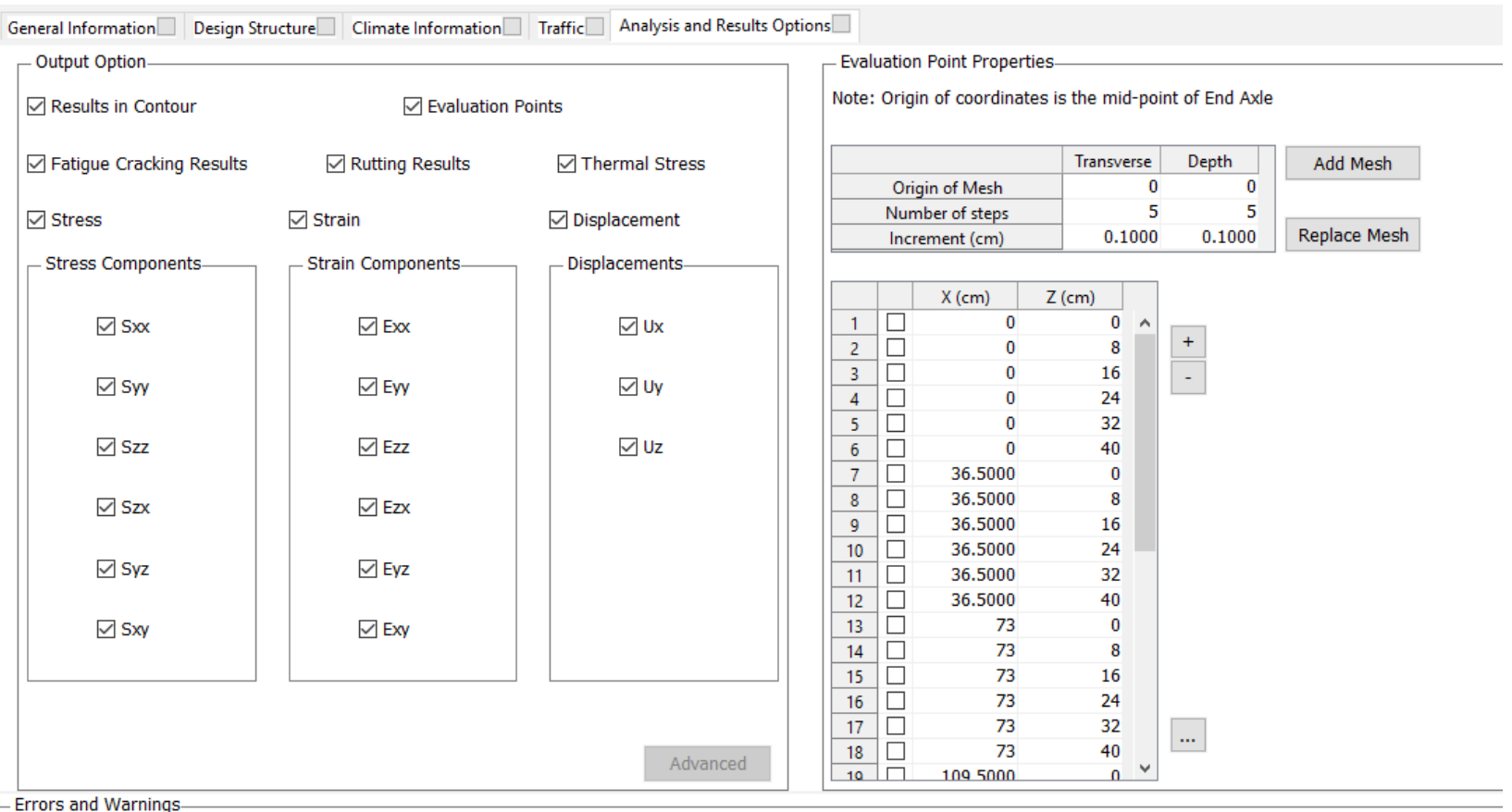

Figure 152: Output and Analysis Options for Run 1.9

\section{Run 1.10}

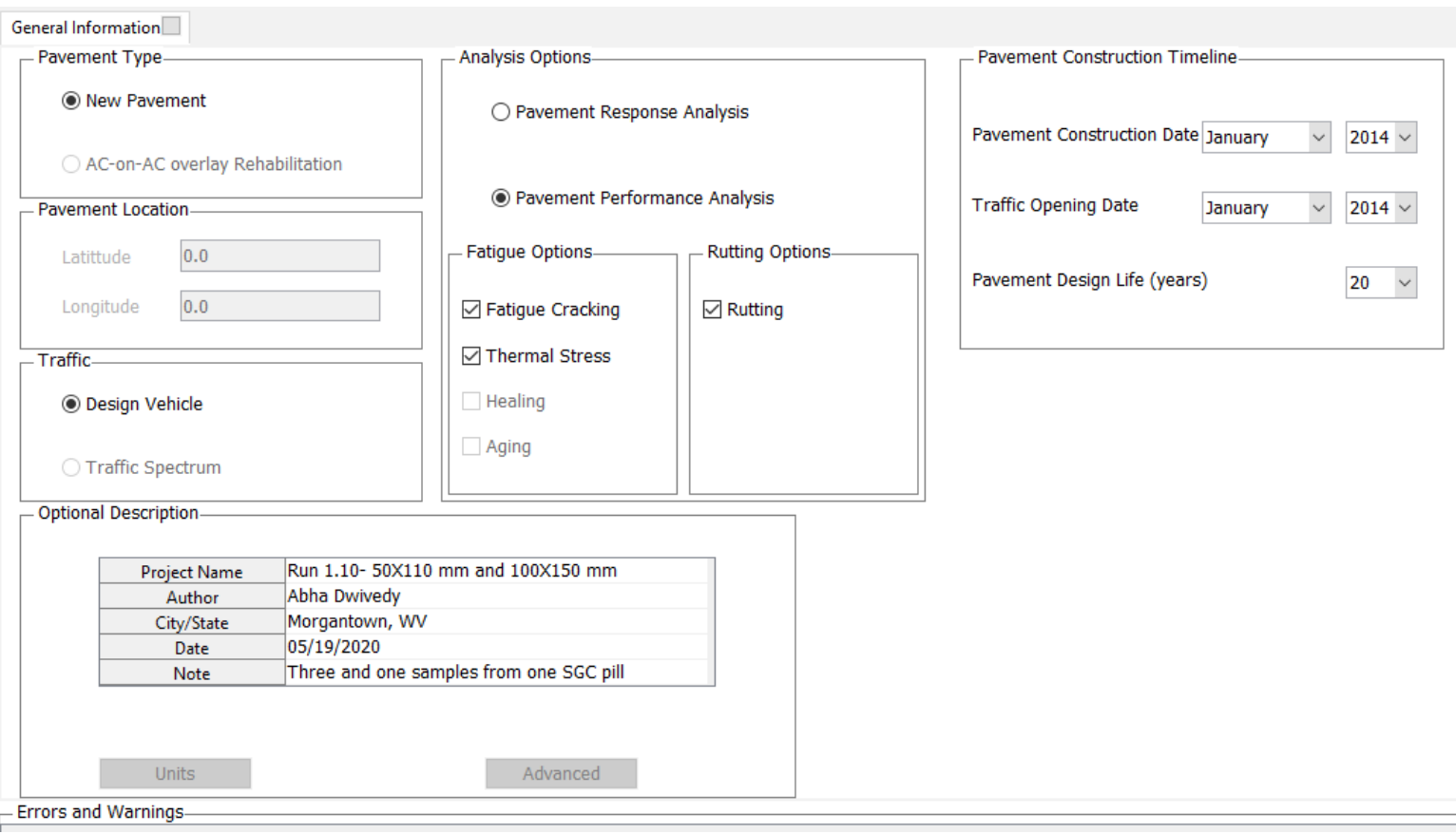

Figure 153: General Information for Run 1.10 


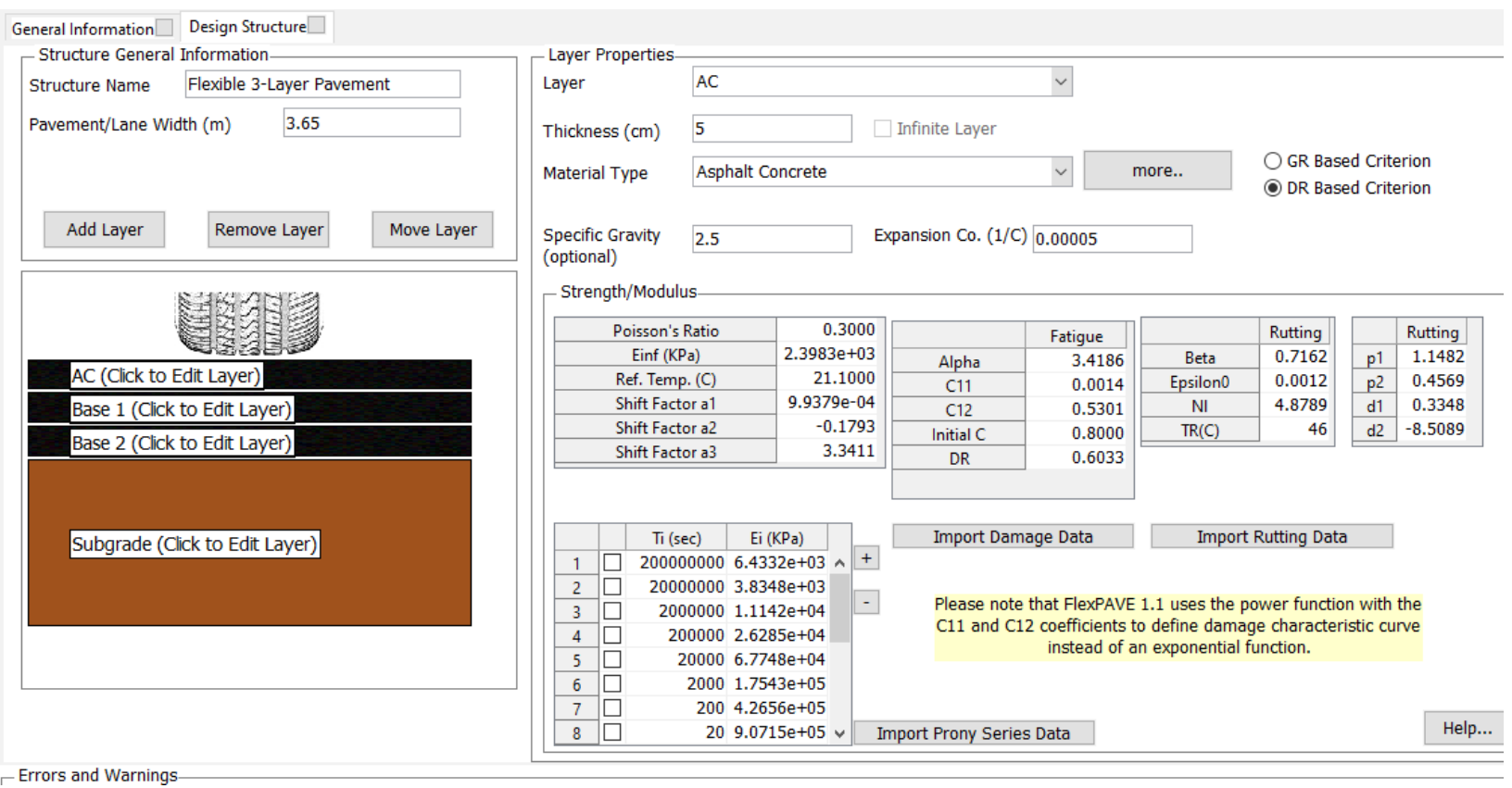

Figure 154: Design Structure of AC Layer for Run 1.10

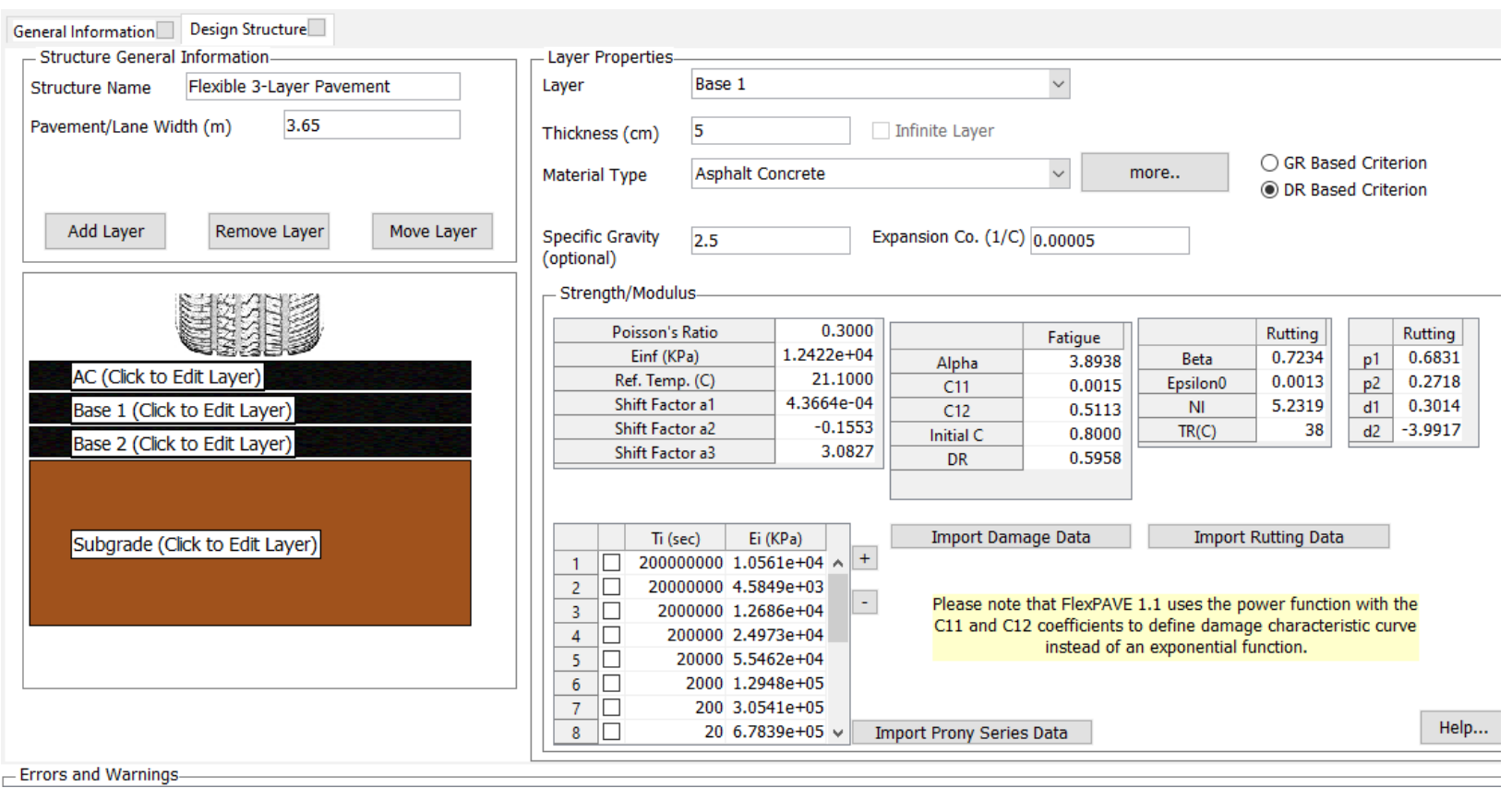

Figure 155: Design Structure of Base 1 Layer for Run 1.10 


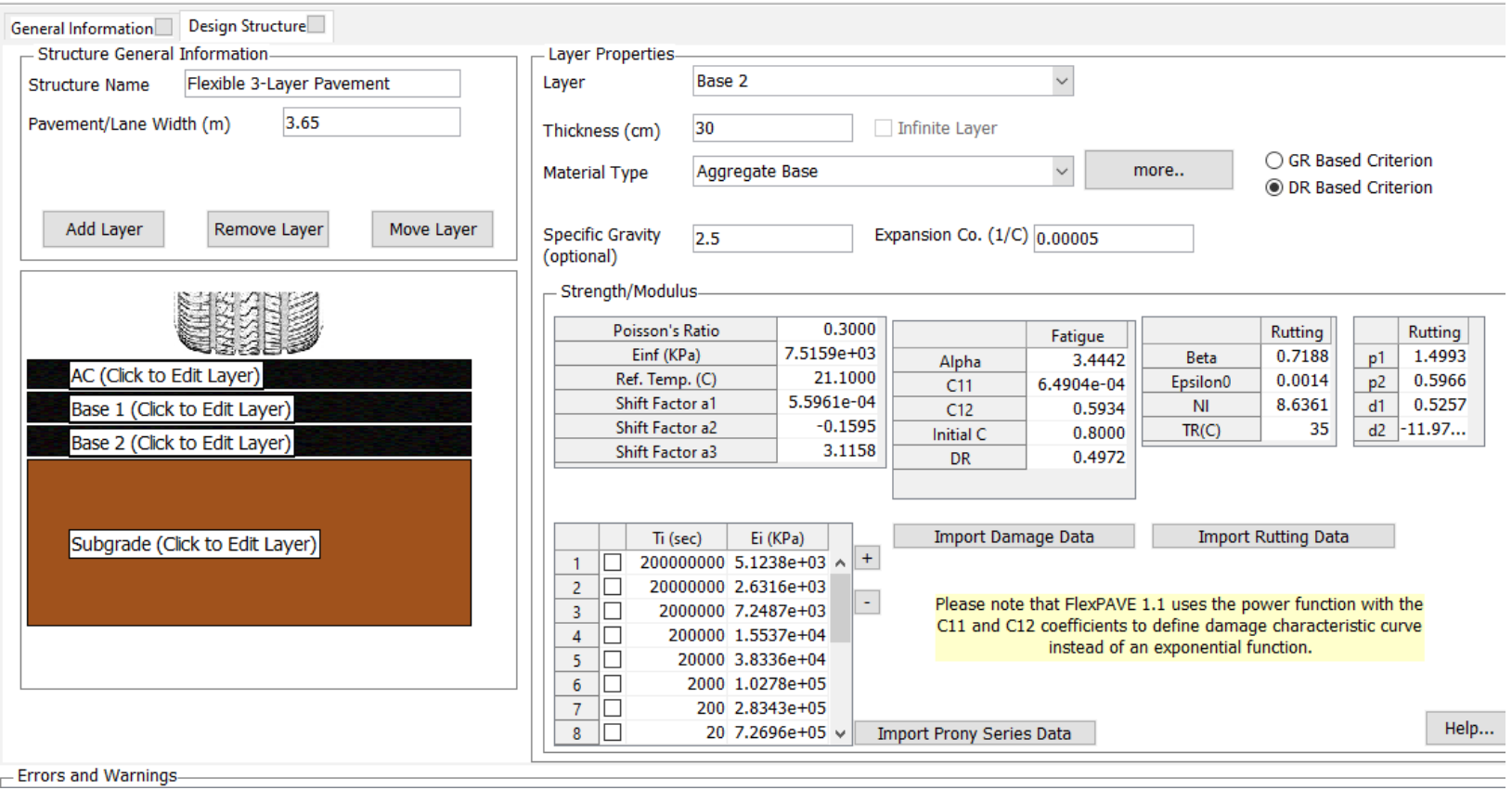

Figure 156: Design Structure of Base 2 Layer for Run 1.10

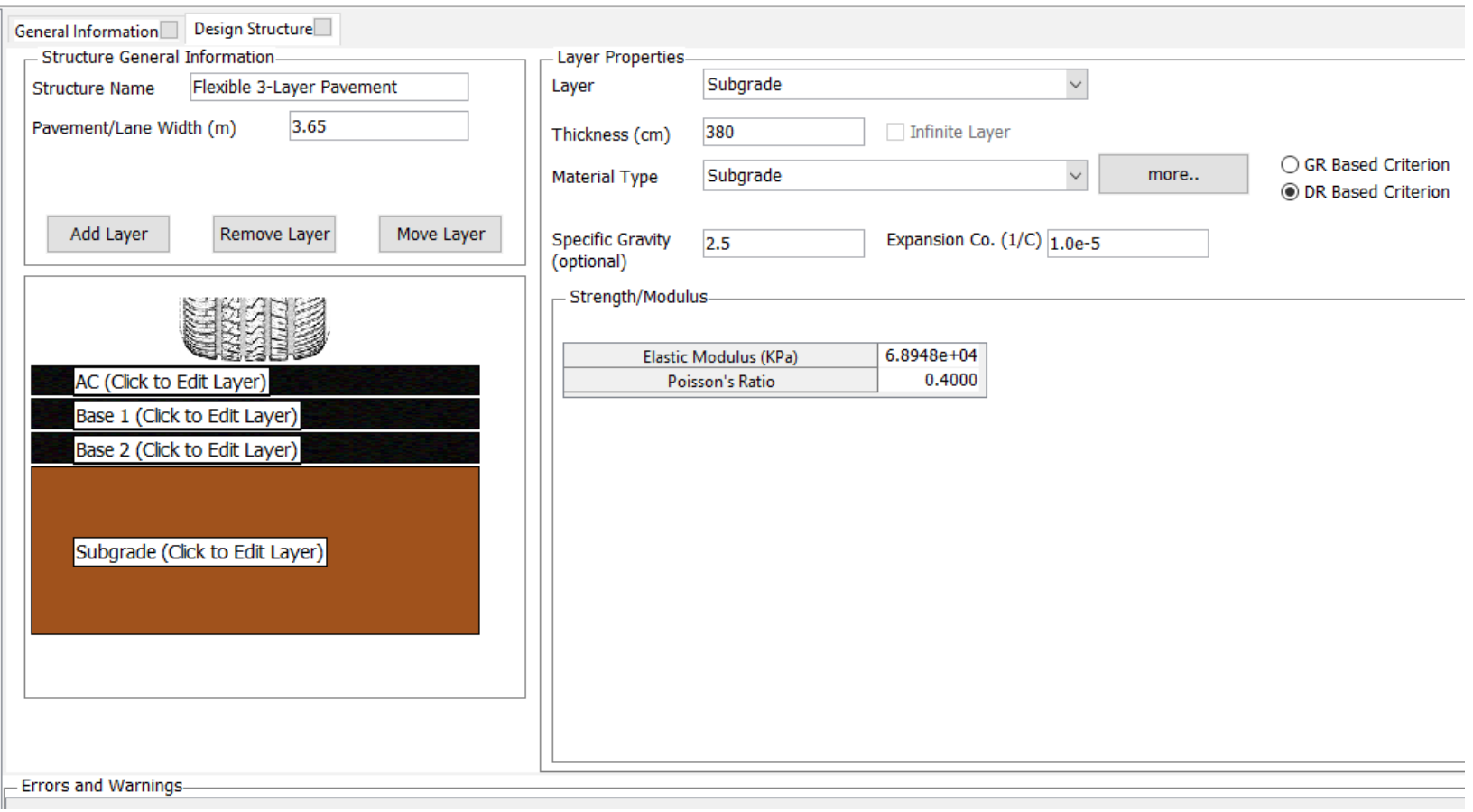

Figure 157: Design Structure of Subgrade Layer for Run 1.10 


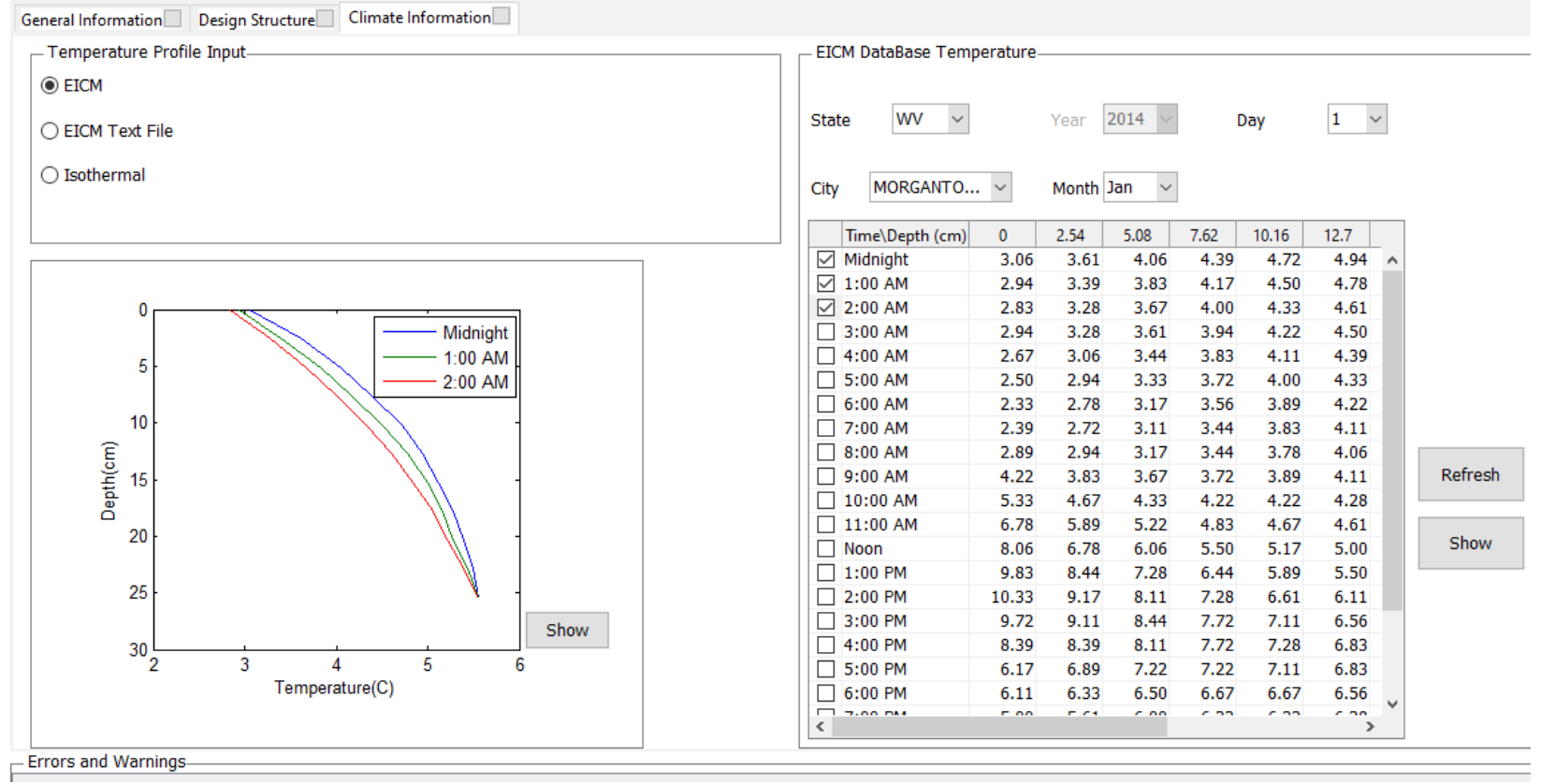

Figure 158: Climate Data for Run 1.10 

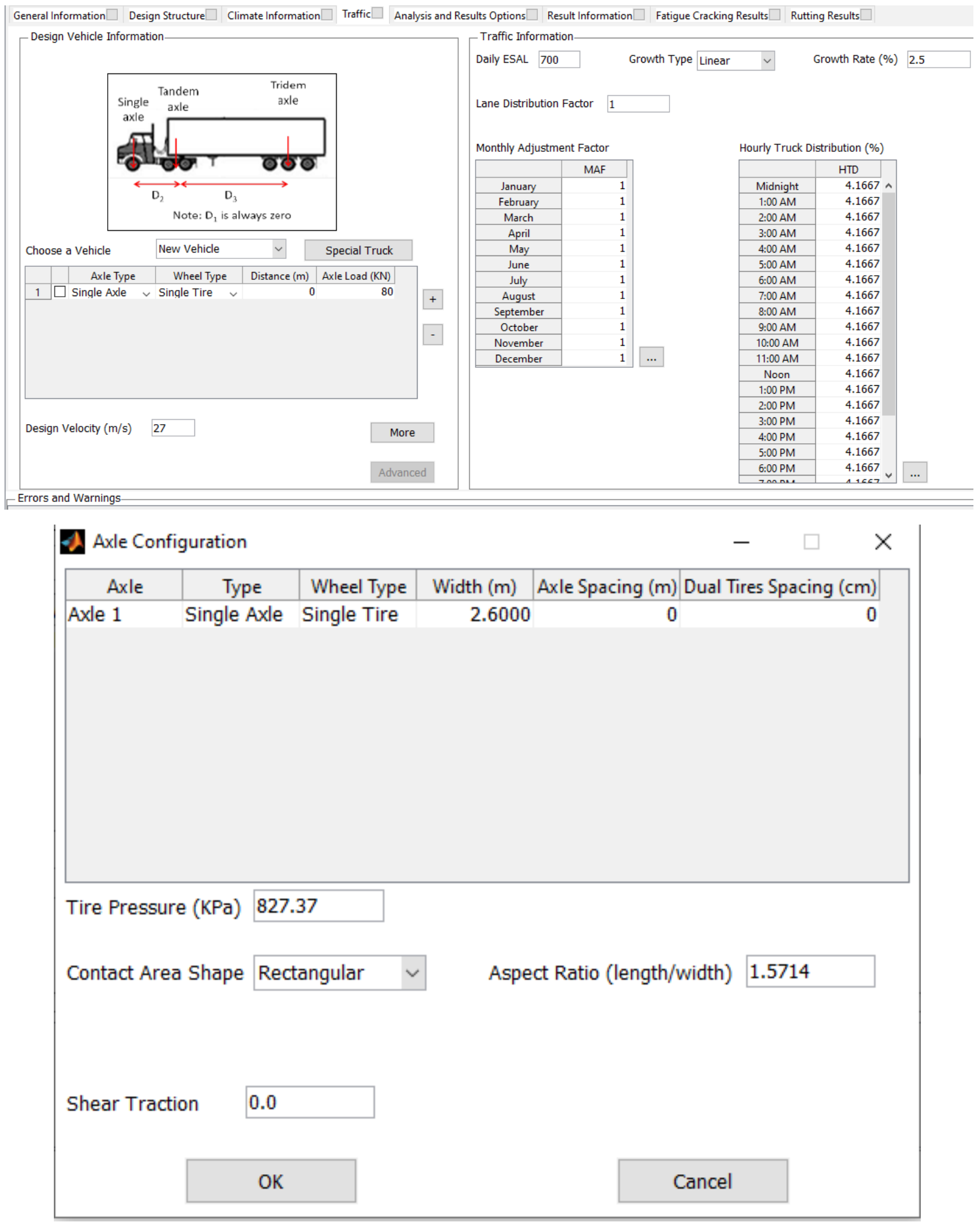

Figure 159: Traffic Data for Run 1.10 


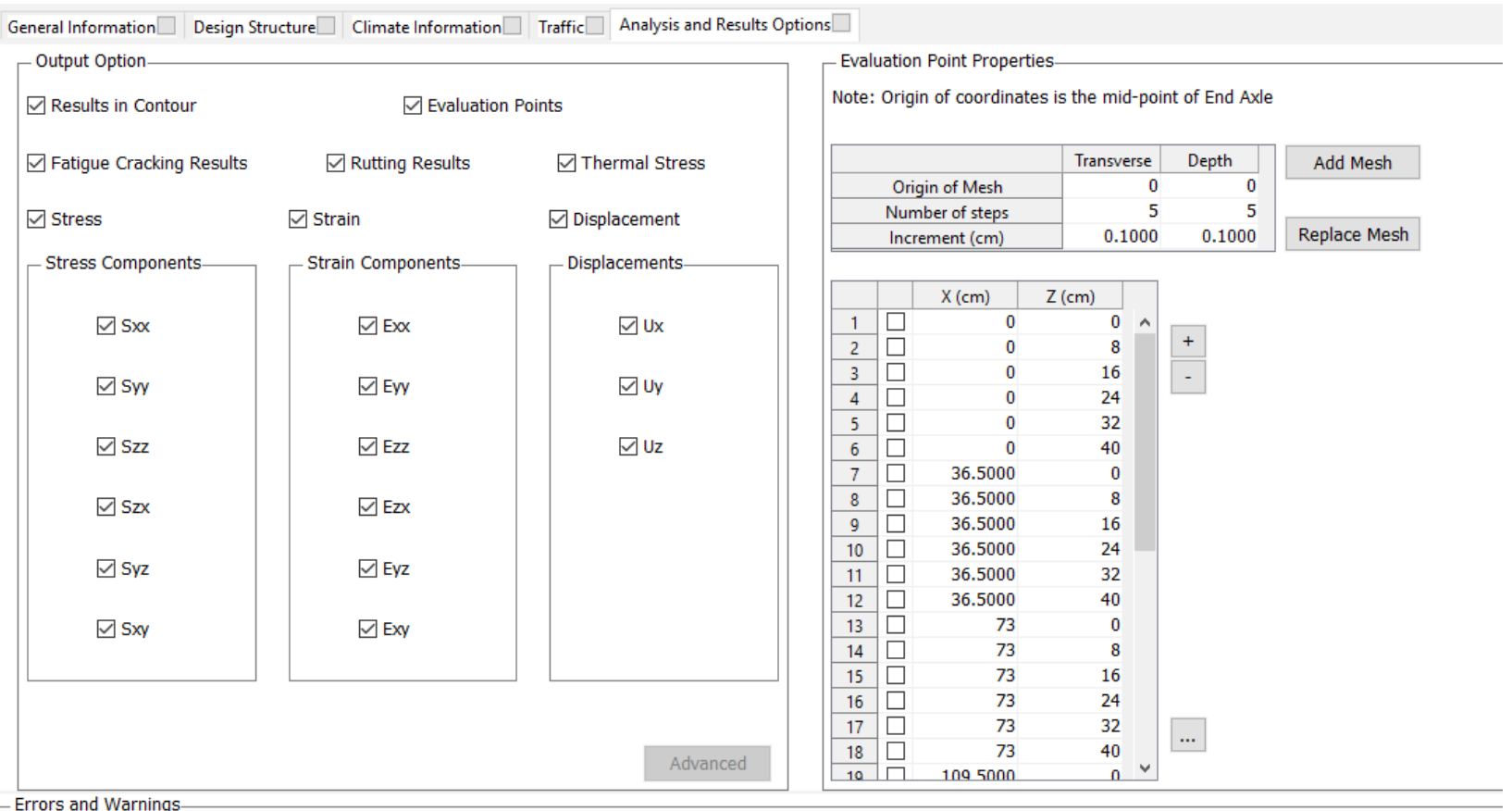

Figure 160: Output and Analysis Options for Run 1.10

\section{Run 1.11}

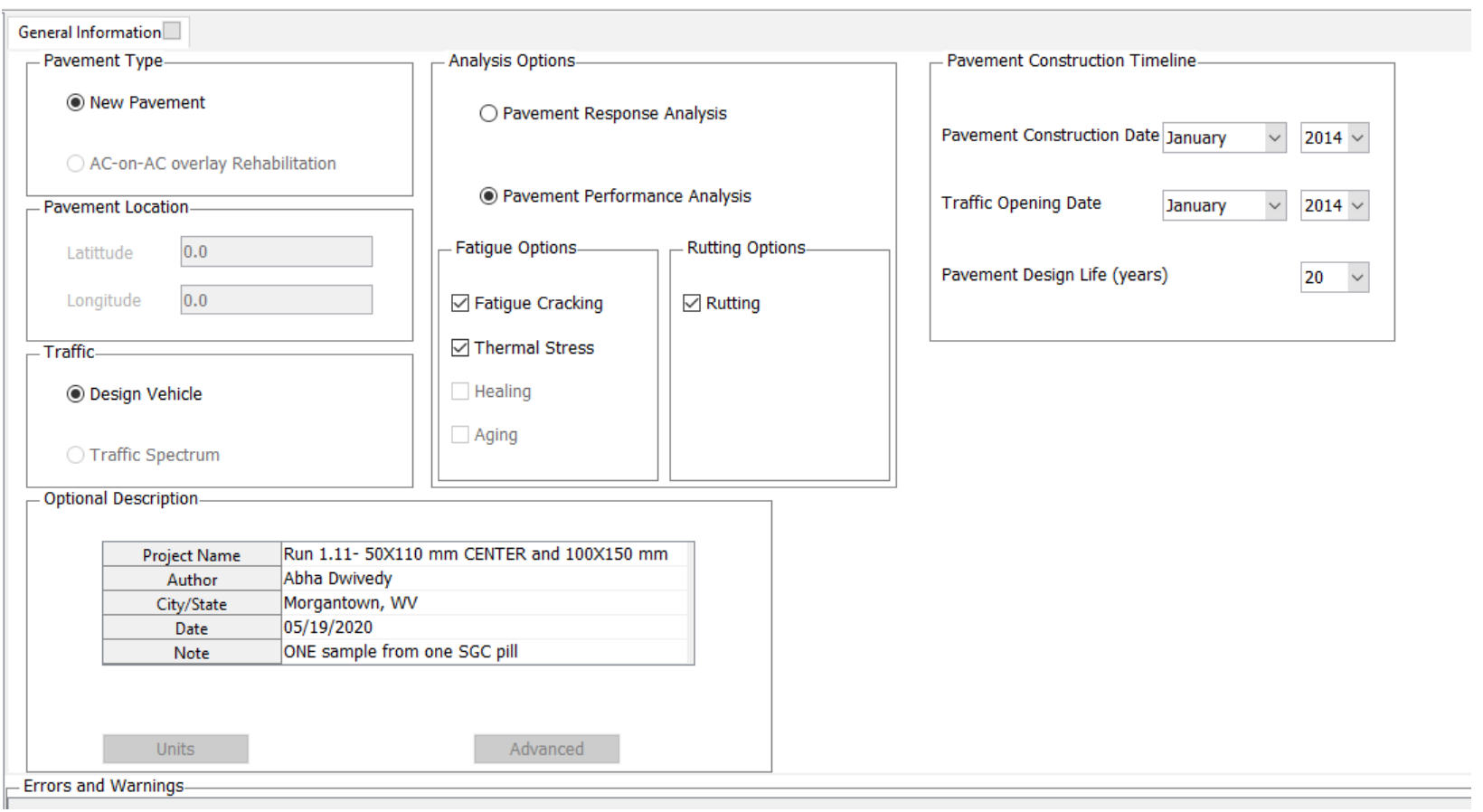

Figure 161: General Information for Run 1.11 


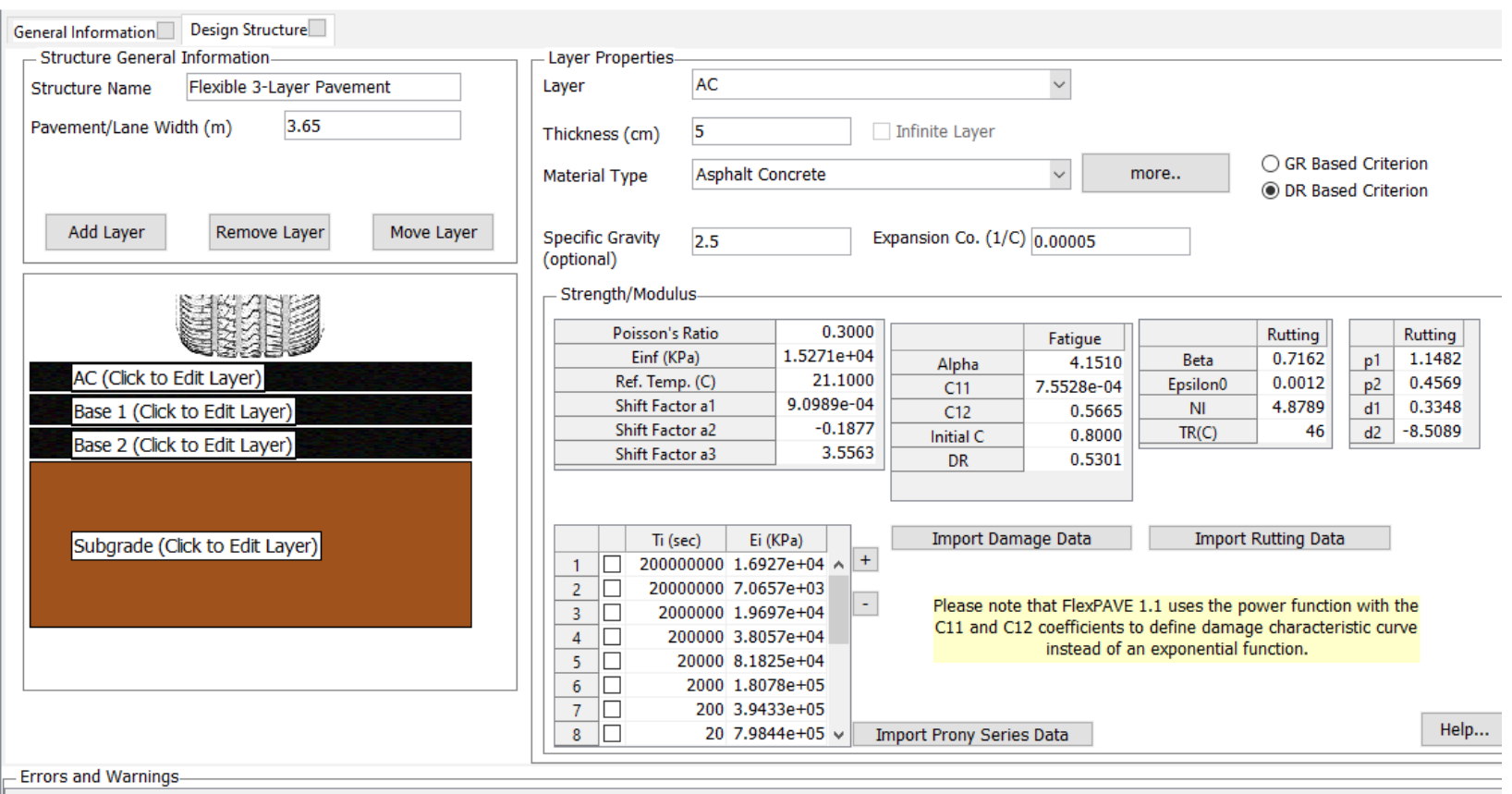

Figure 162: Design Structure of AC Layer for Run 1.11

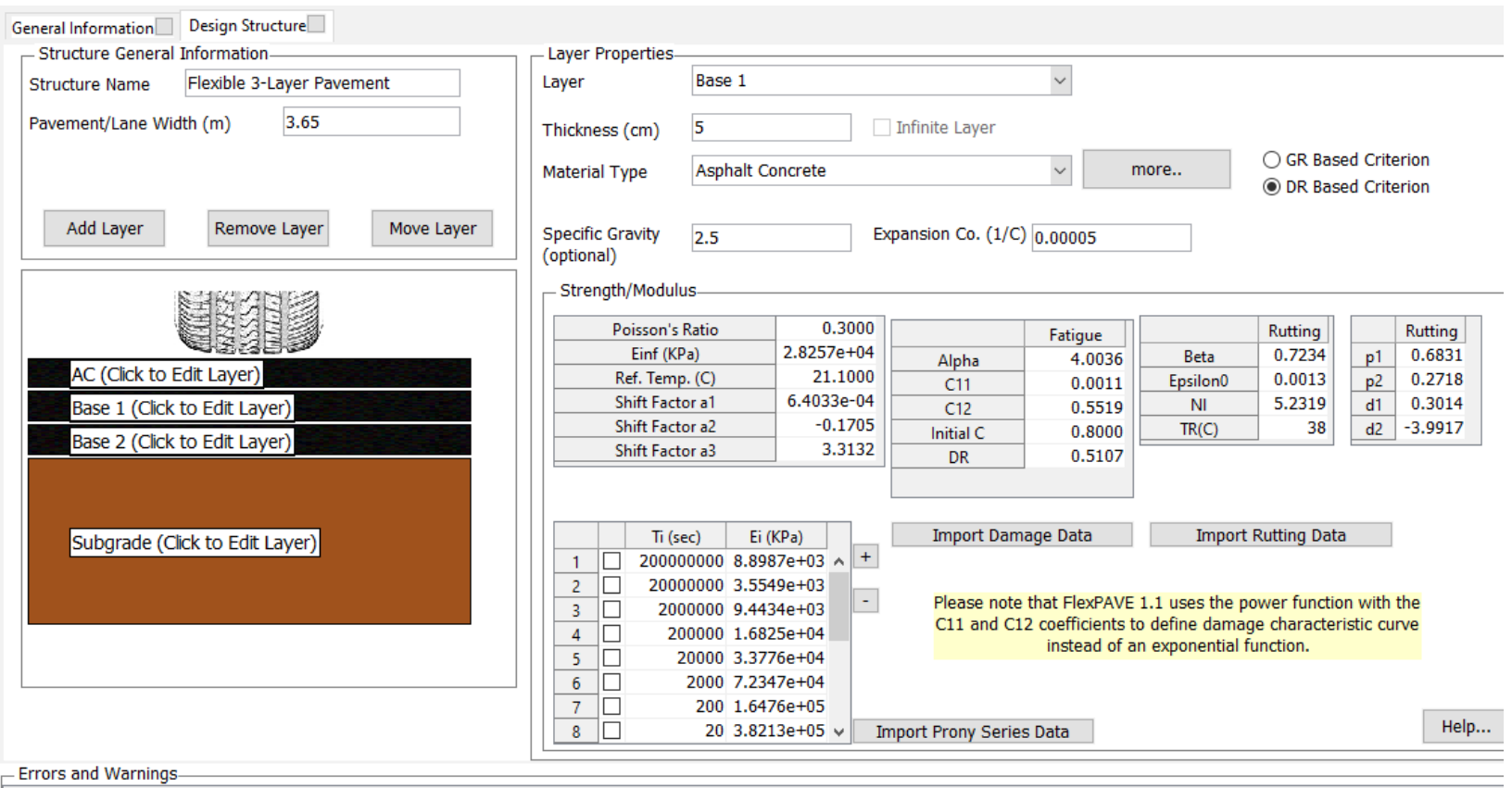

Figure 163: Design Structure of Base 1 Layer for Run 1.11 


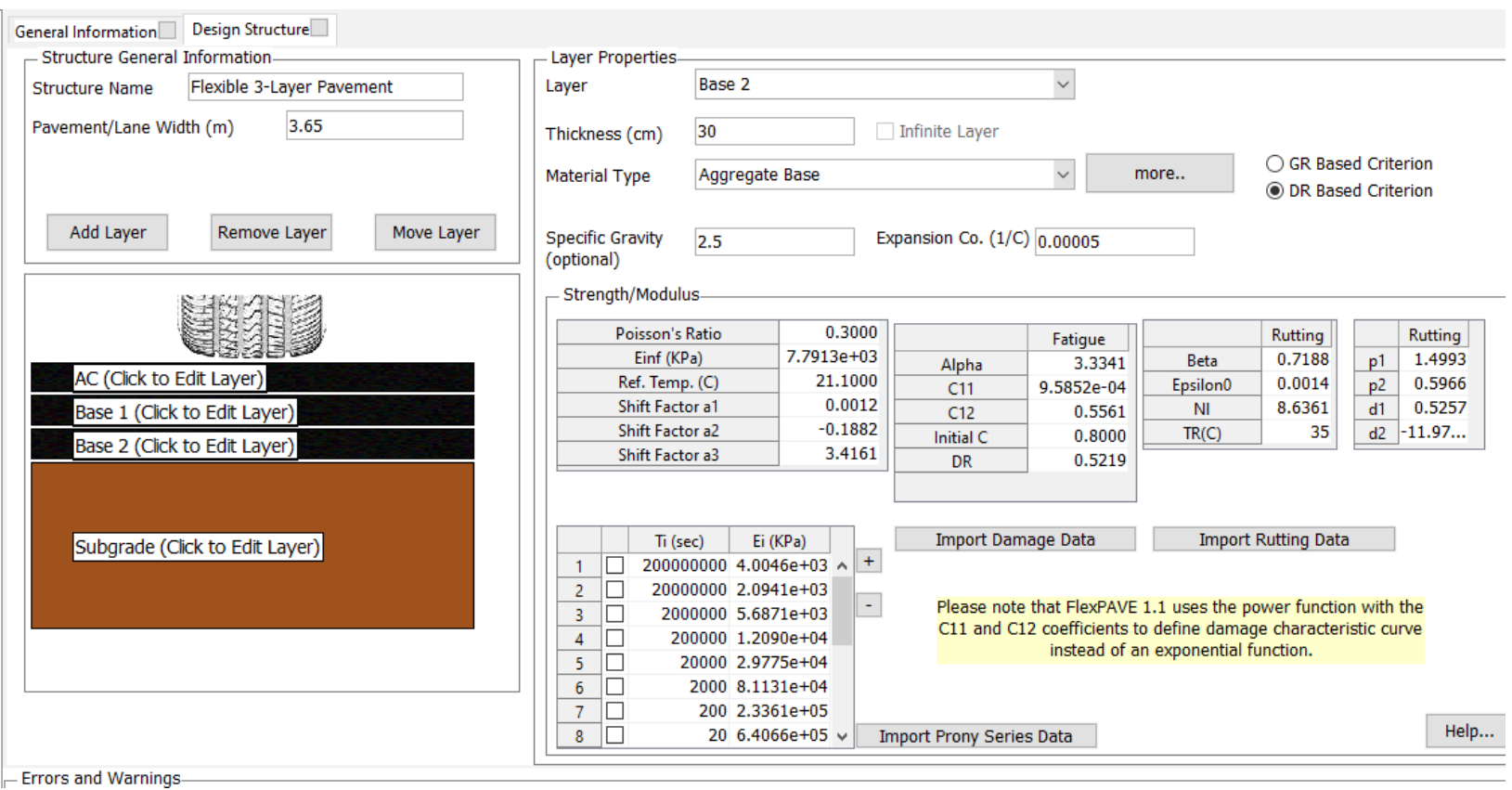

Figure 164: Design Structure of Base 2 Layer for Run 1.11

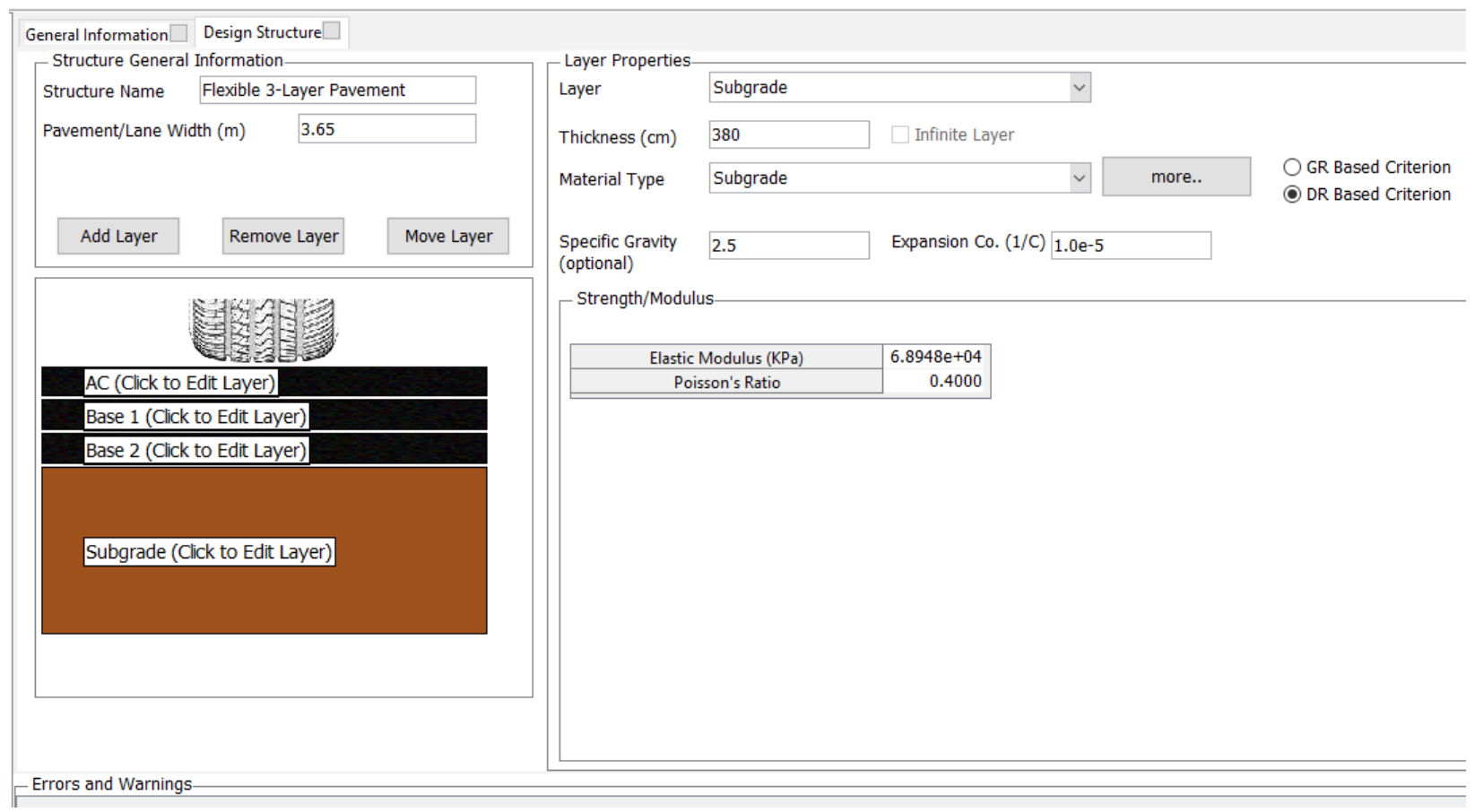

Figure 165: Design Structure of Subgrade Layer for Run 1.11 


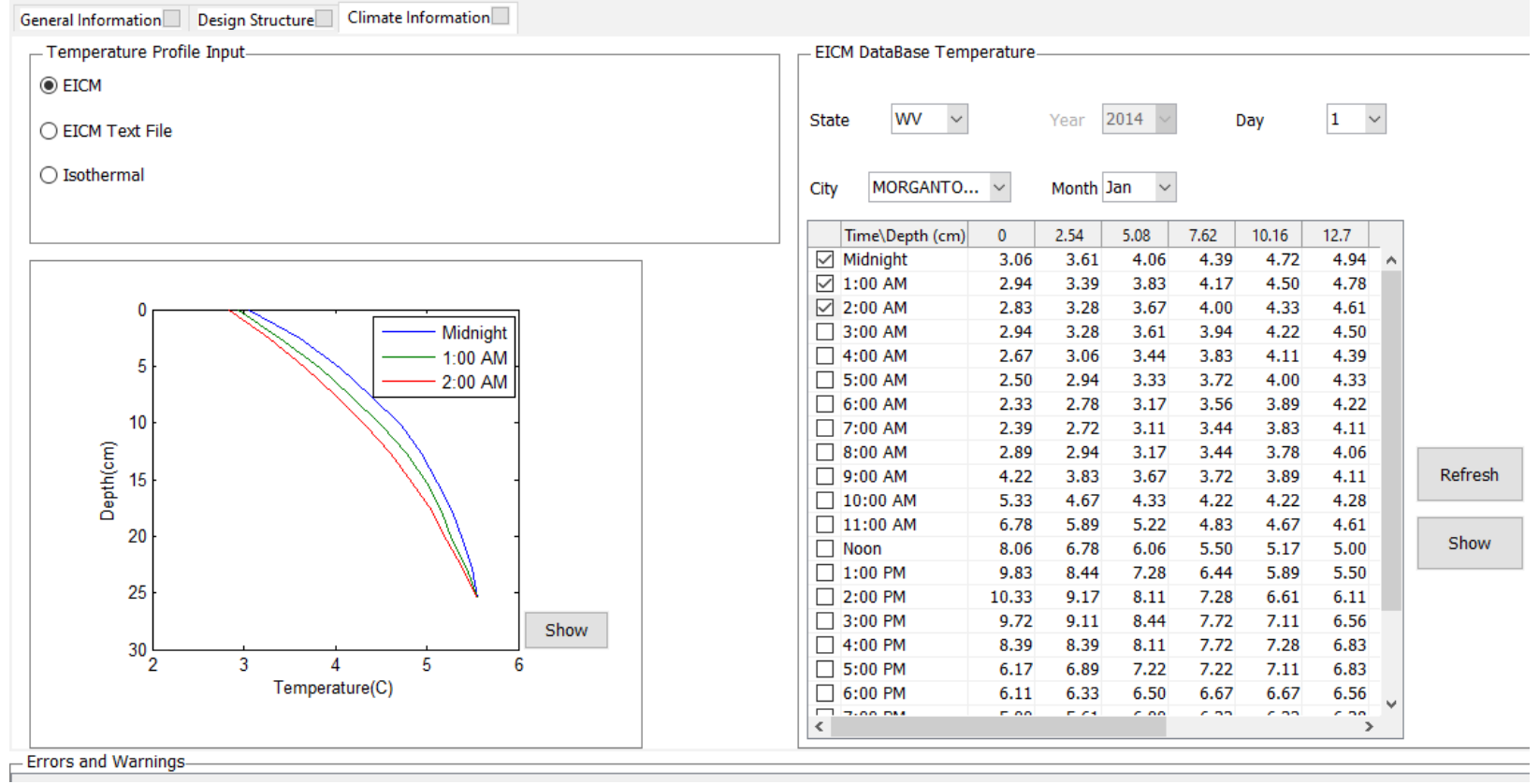

Figure 166: Climate Data for Run 1.11 

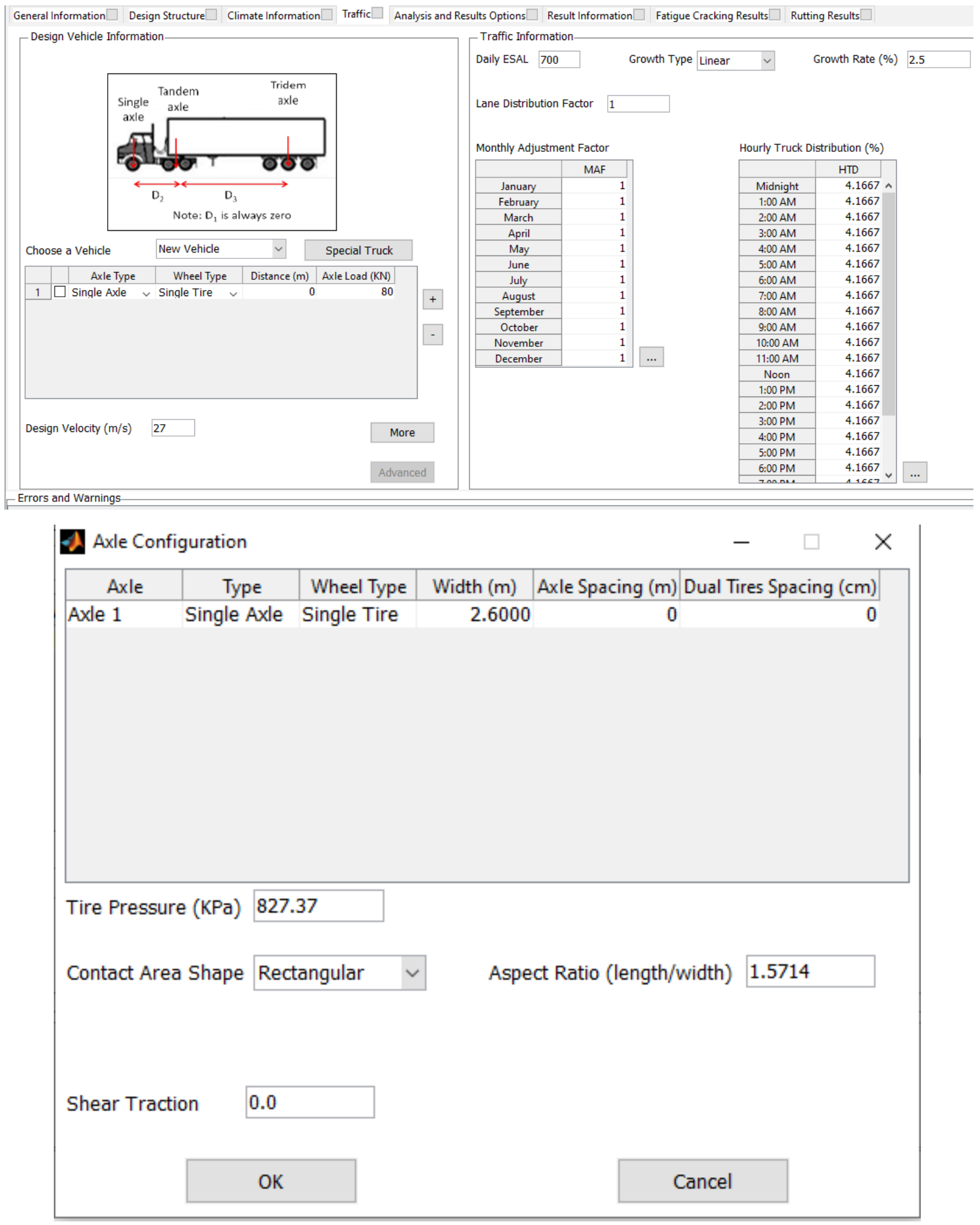

Figure 167: Traffic Data for Run 1.11 


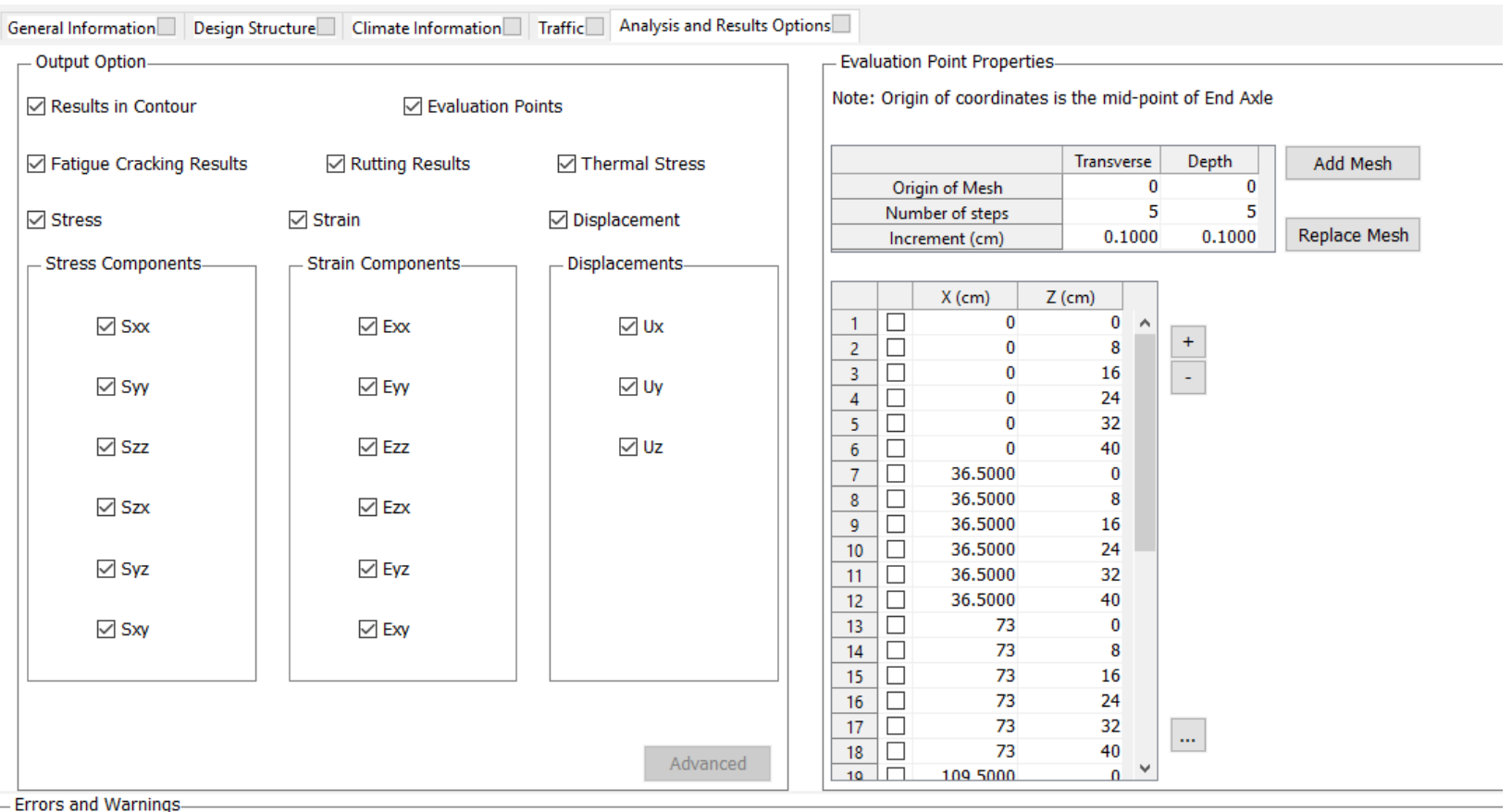

Figure 168: Output and Analysis Options for Run 1.11

\section{Run 1.12}

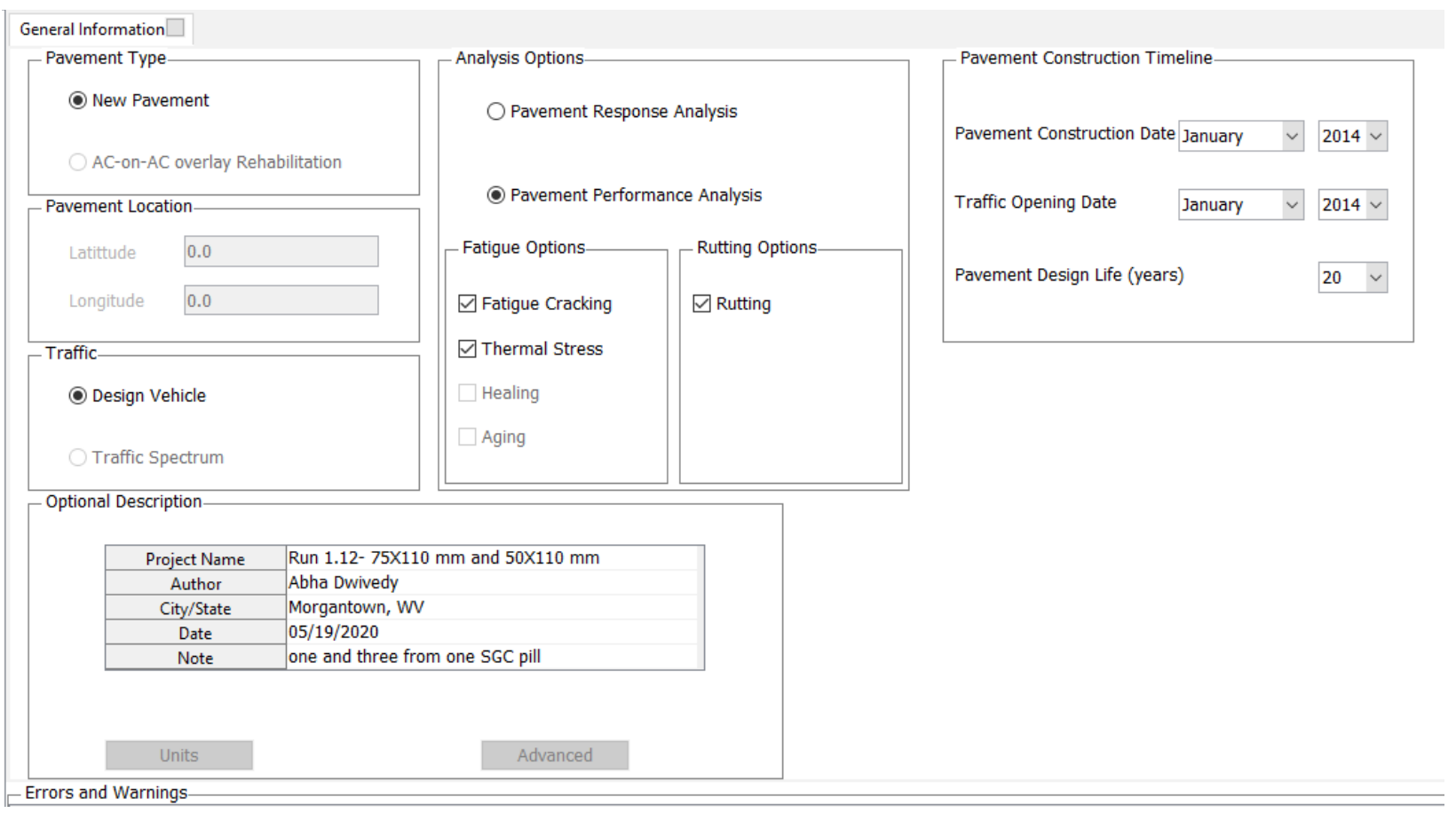

Figure 169: General Information for Run 1.12 


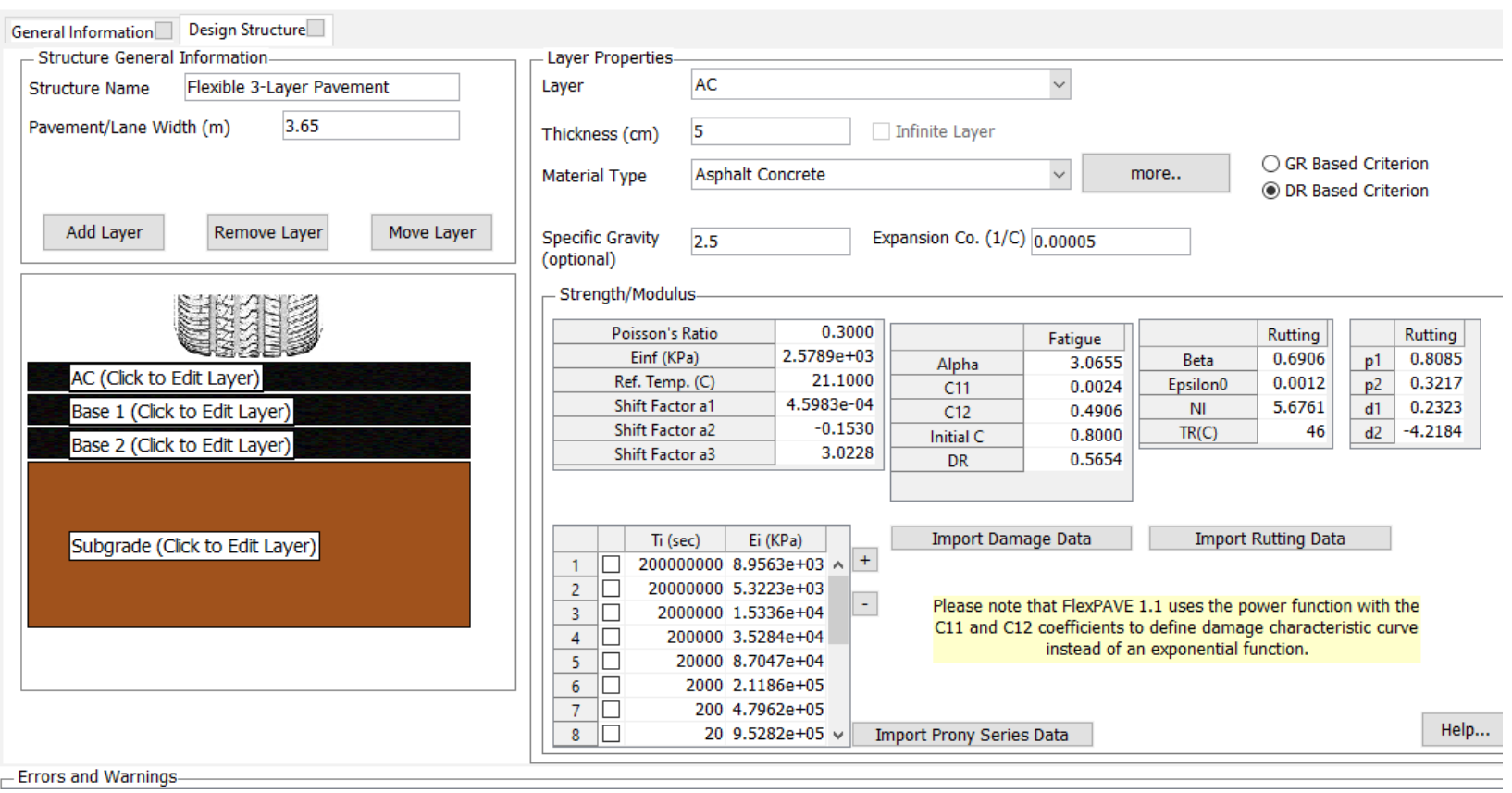

Figure 170: Design Structure of AC Layer for Run 1.12

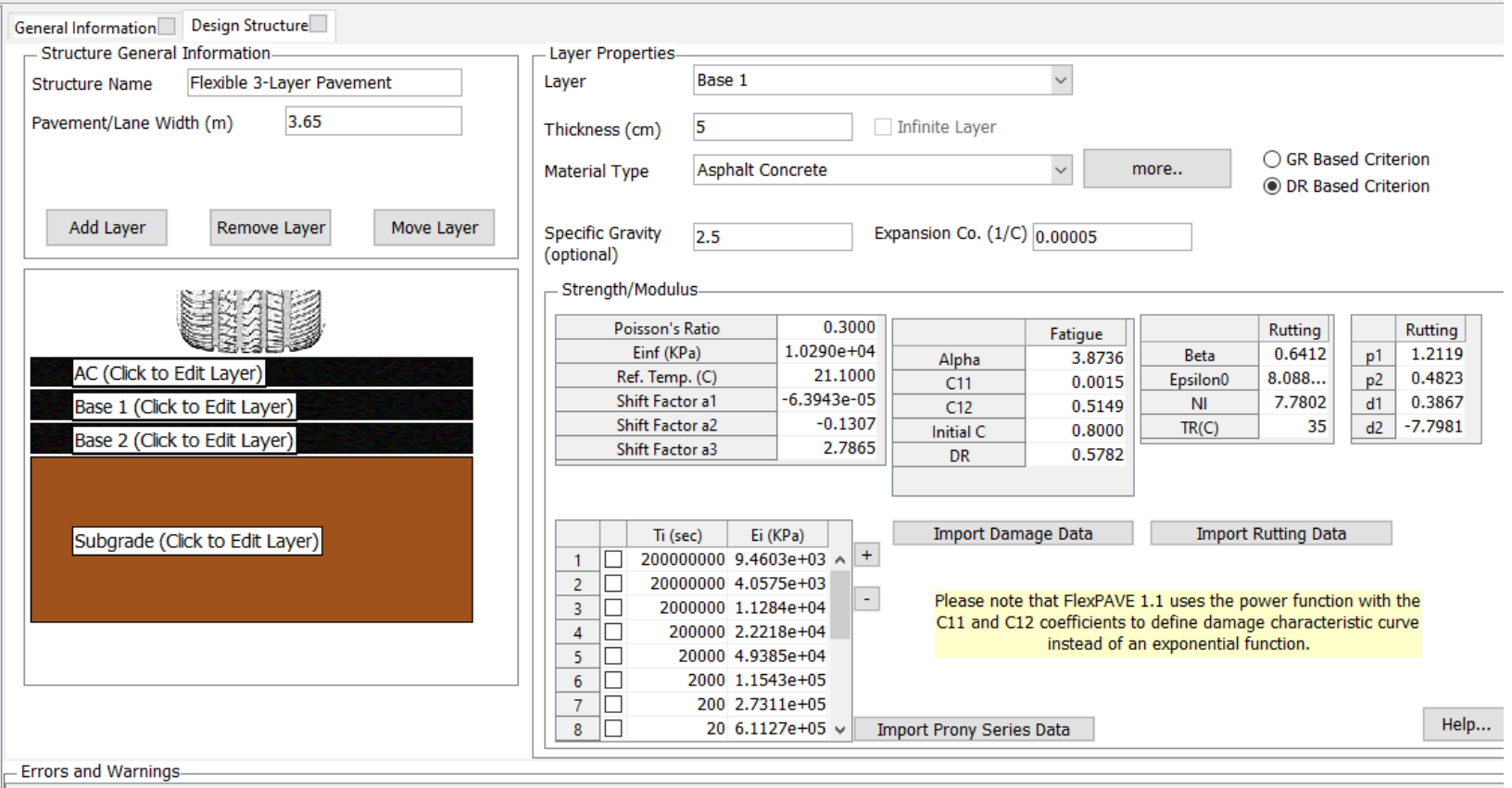

Figure 171: Design Structure of Base 1 Layer for Run 1.12 


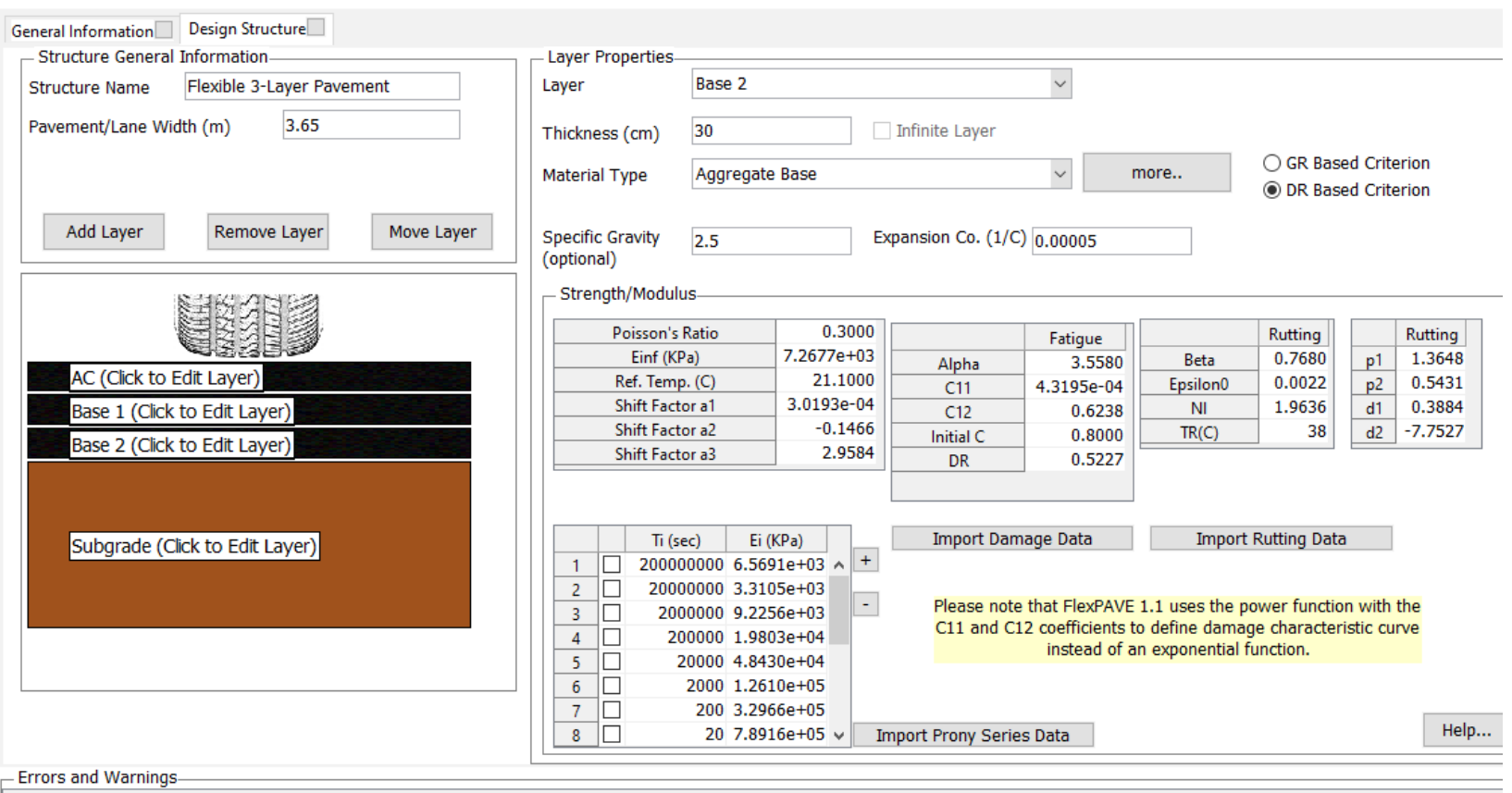

Figure 172: Design Structure of Base 2 Layer for Run 1.12

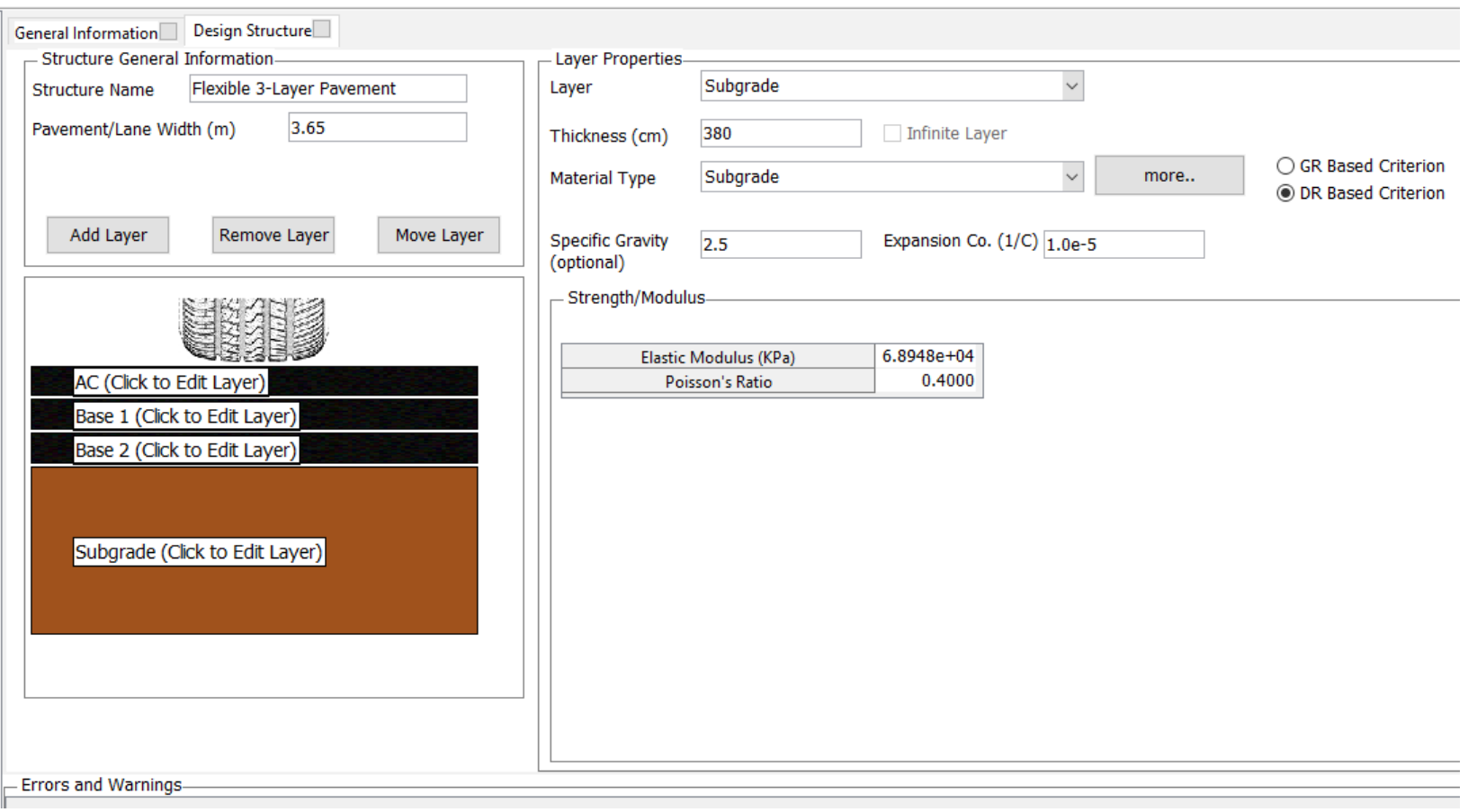

Figure 173: Design Structure of Subgrade Layer for Run 1.12 


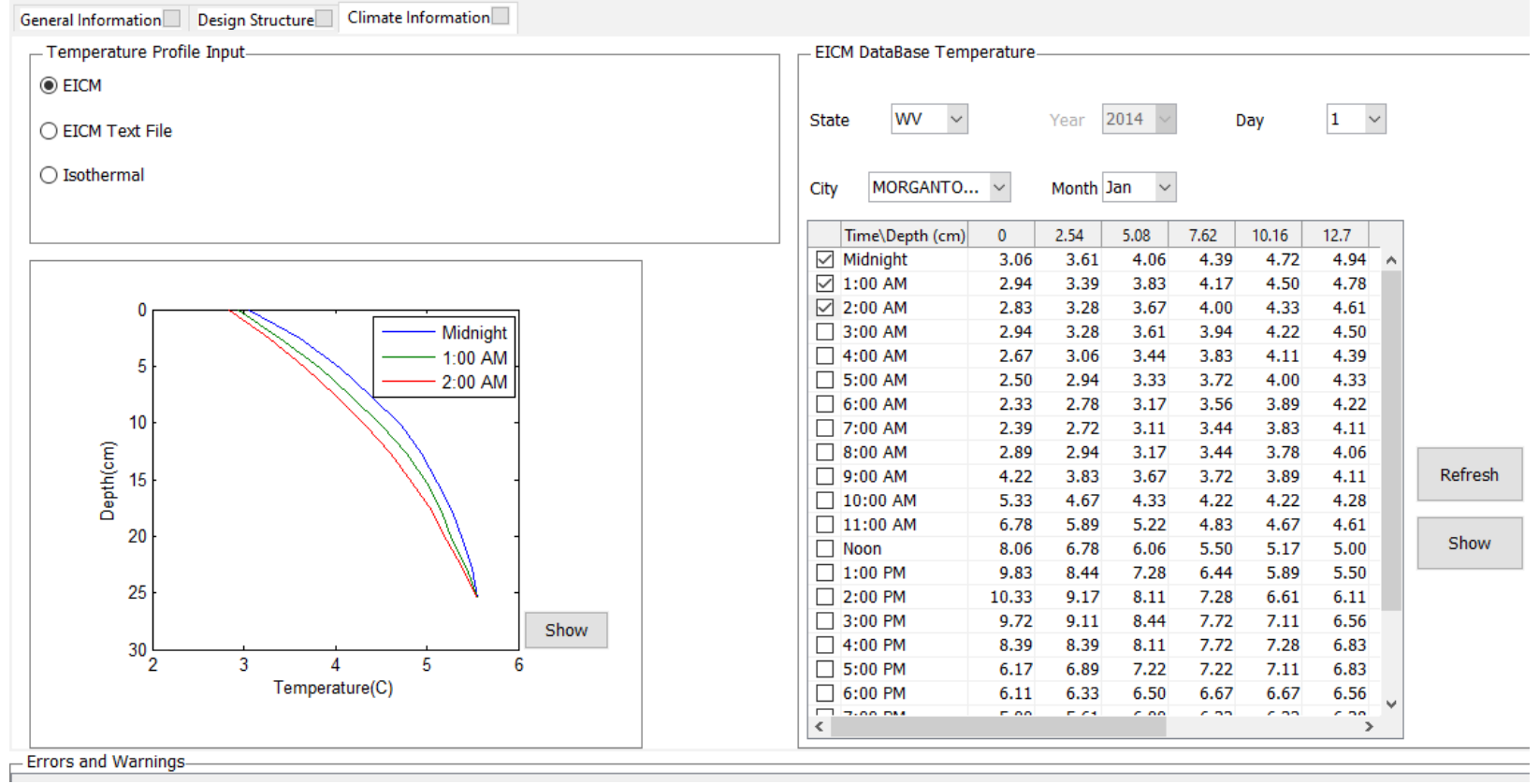

Figure 174: Climate Data for Run 1.12 


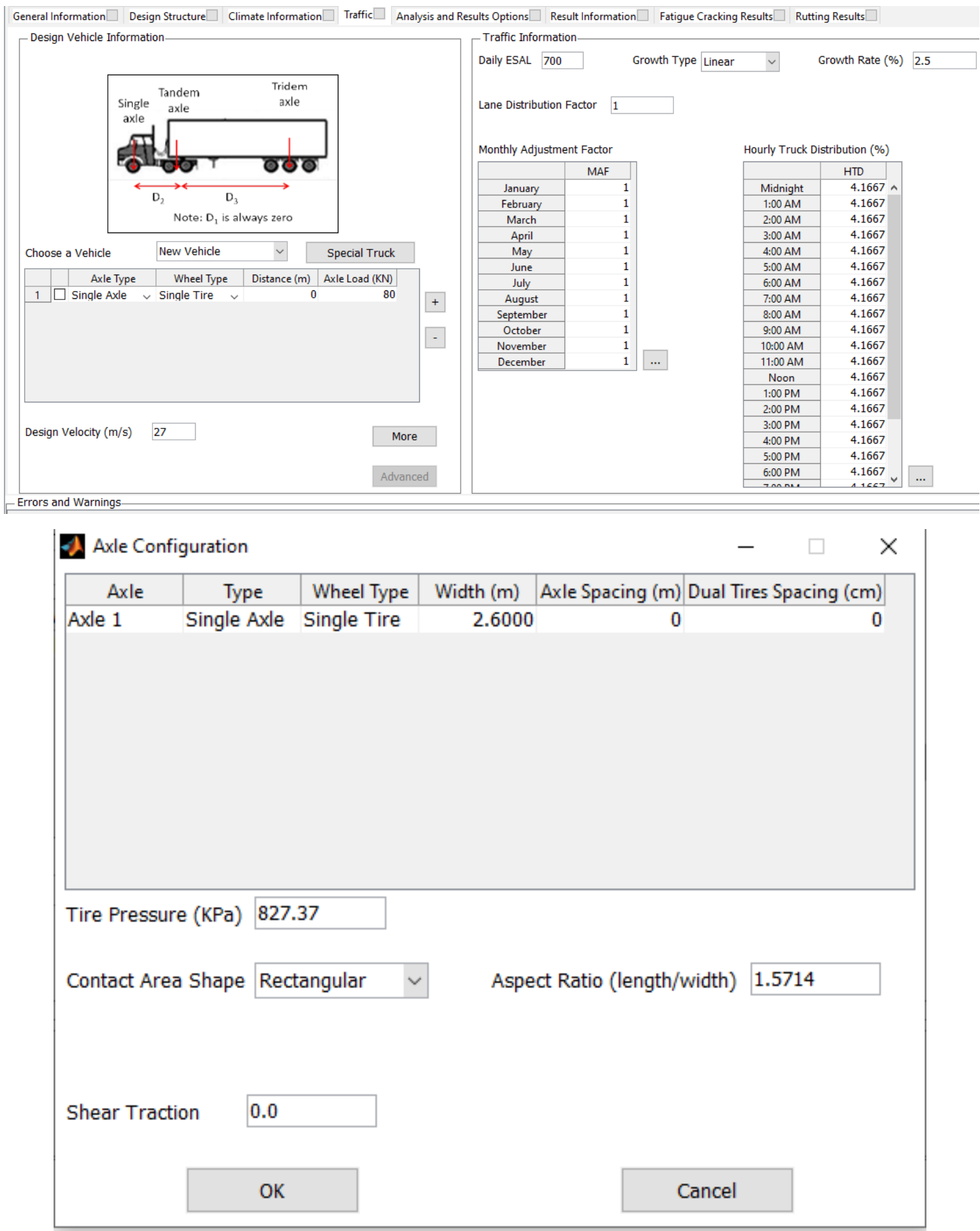

Figure 175: Traffic Data for Run 1.12 


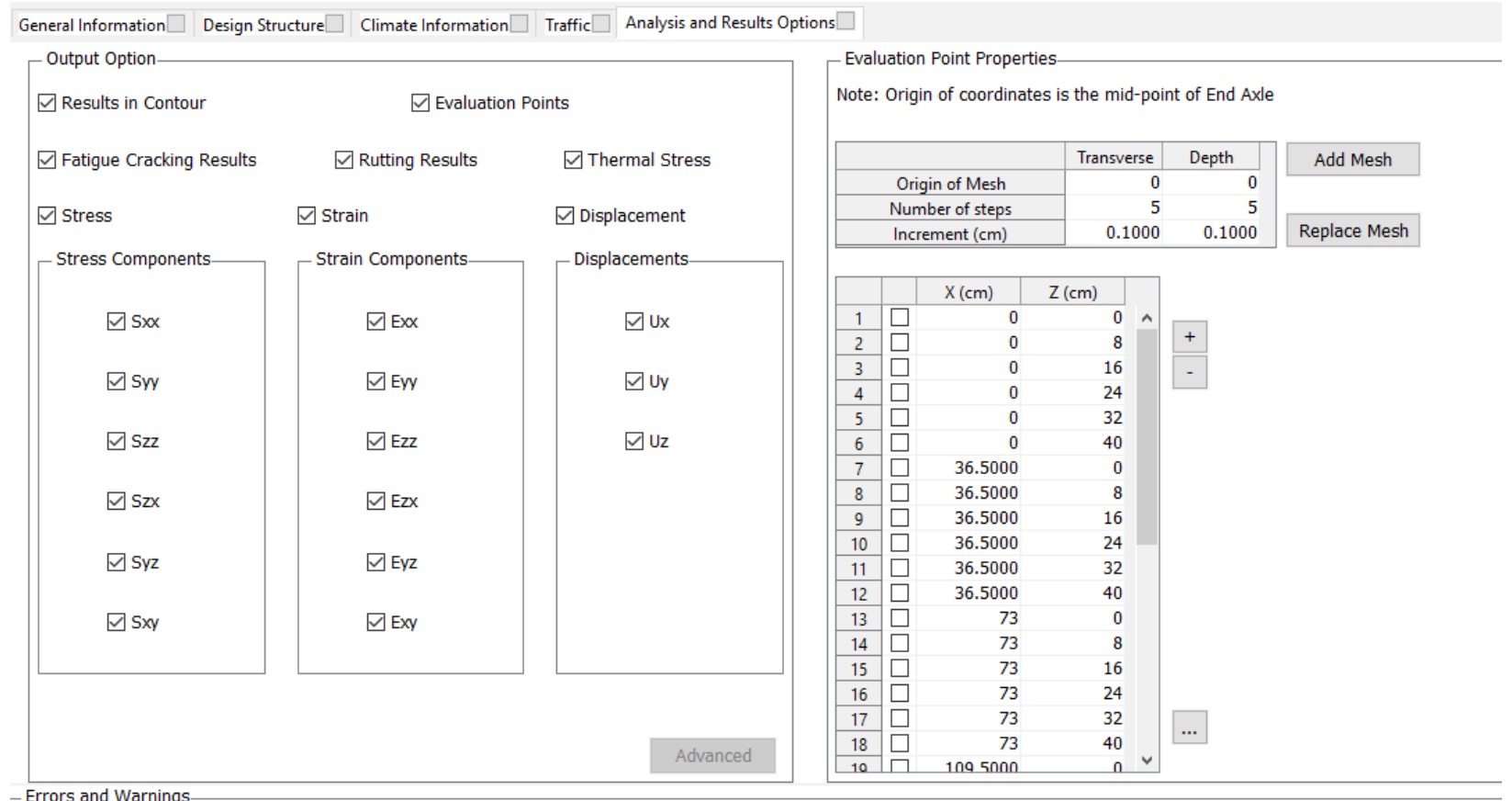

Figure 176: Output and Analysis Options for Run 1.12 


\section{Run 2.1}

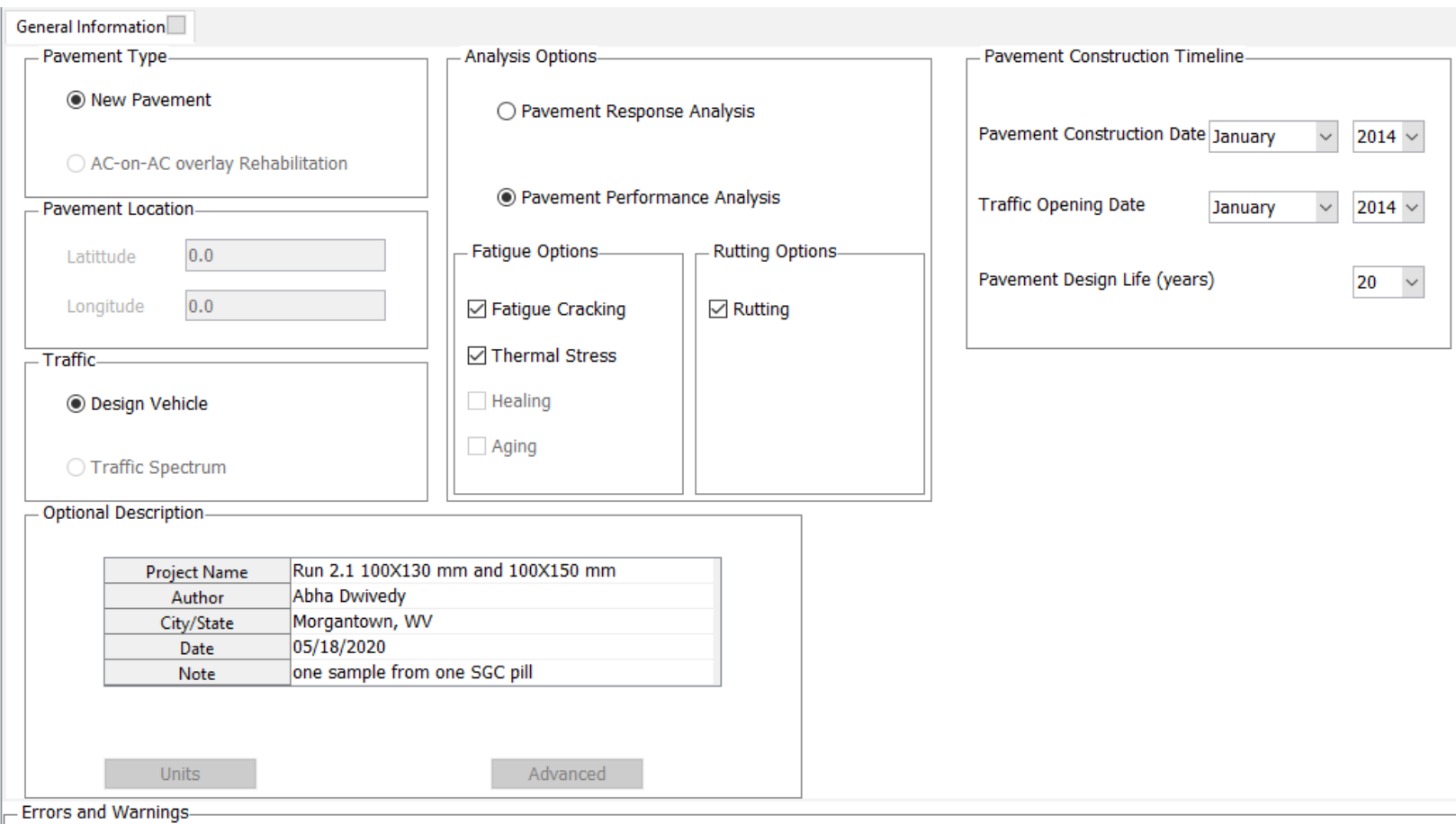

\section{Figure 177: General Information for Run 2.1}

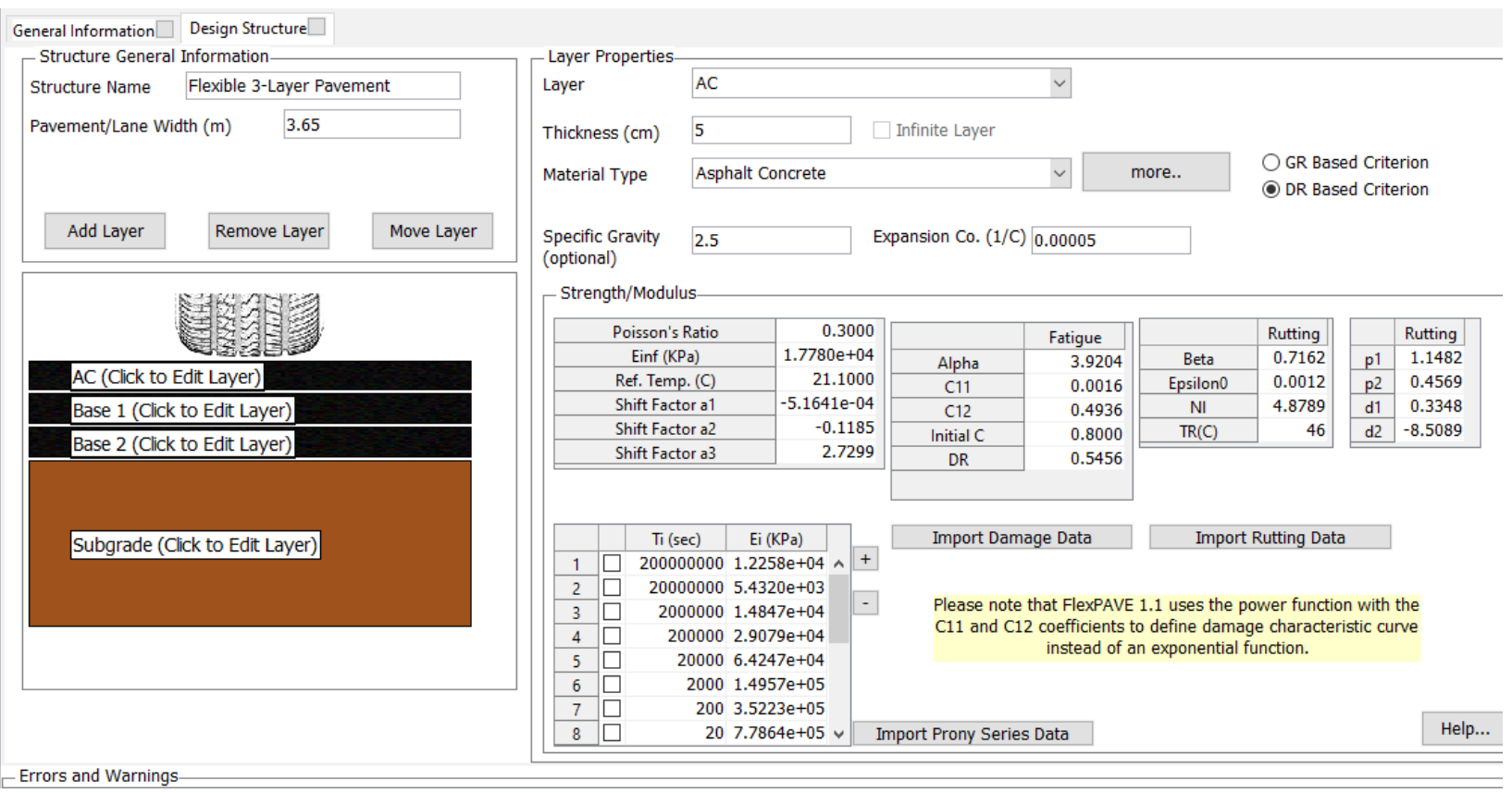

Figure 178: Design Structure of AC Layer for Run 2.1 


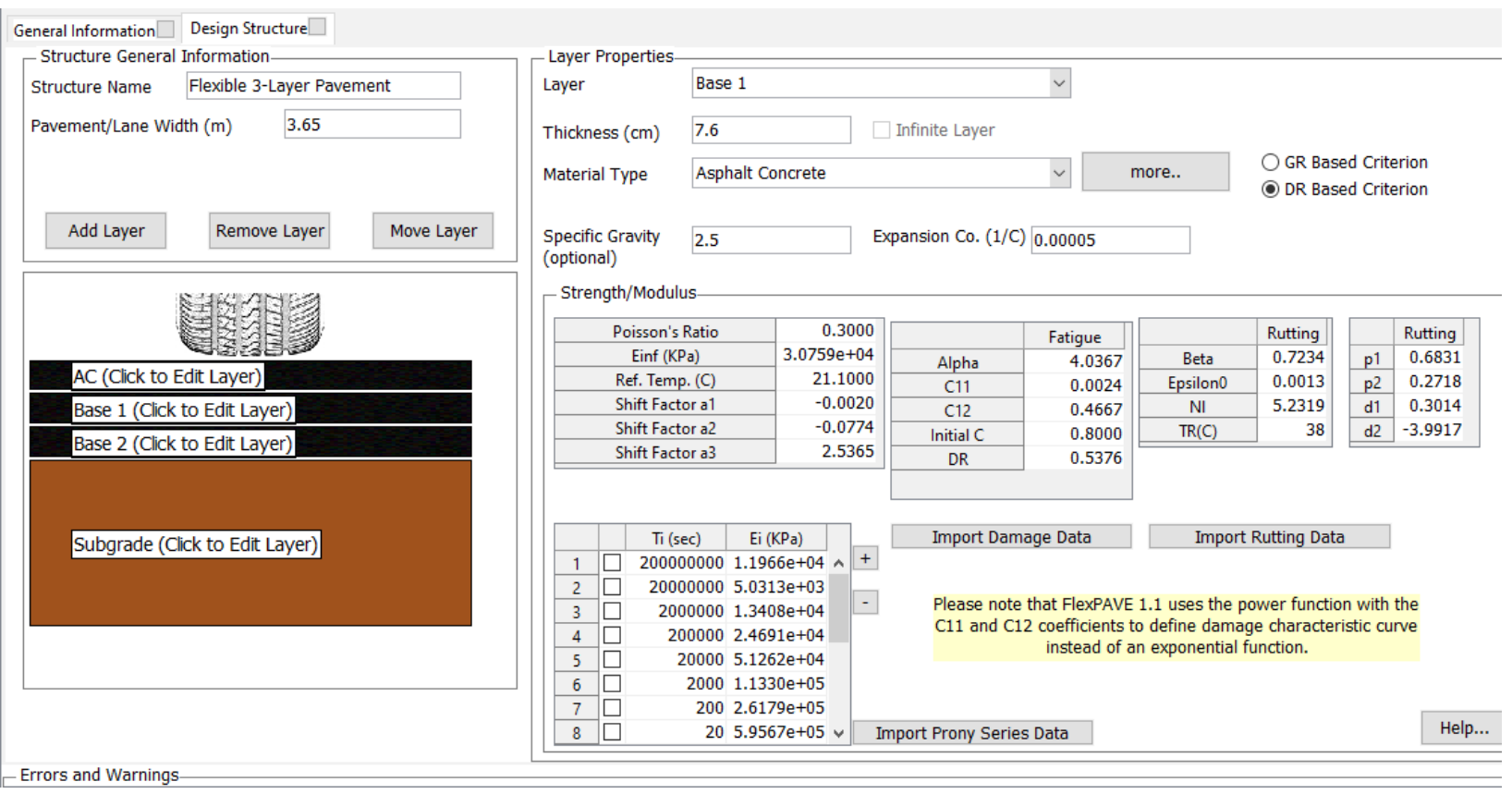

Figure 179: Design Structure of Base 1 Layer for Run 2.1

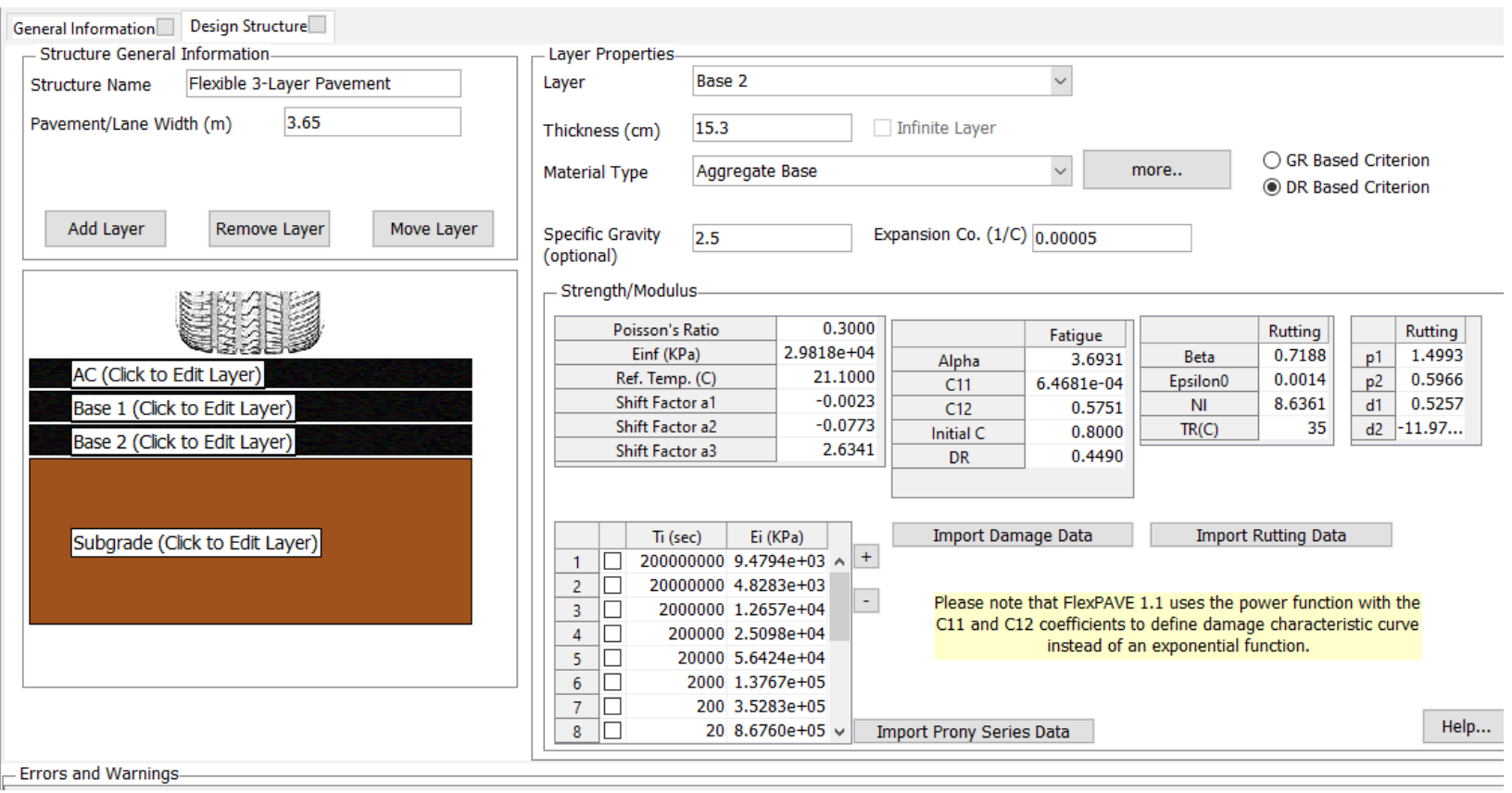

Figure 180: Design Structure of Base 2 Layer for Run 2.1 


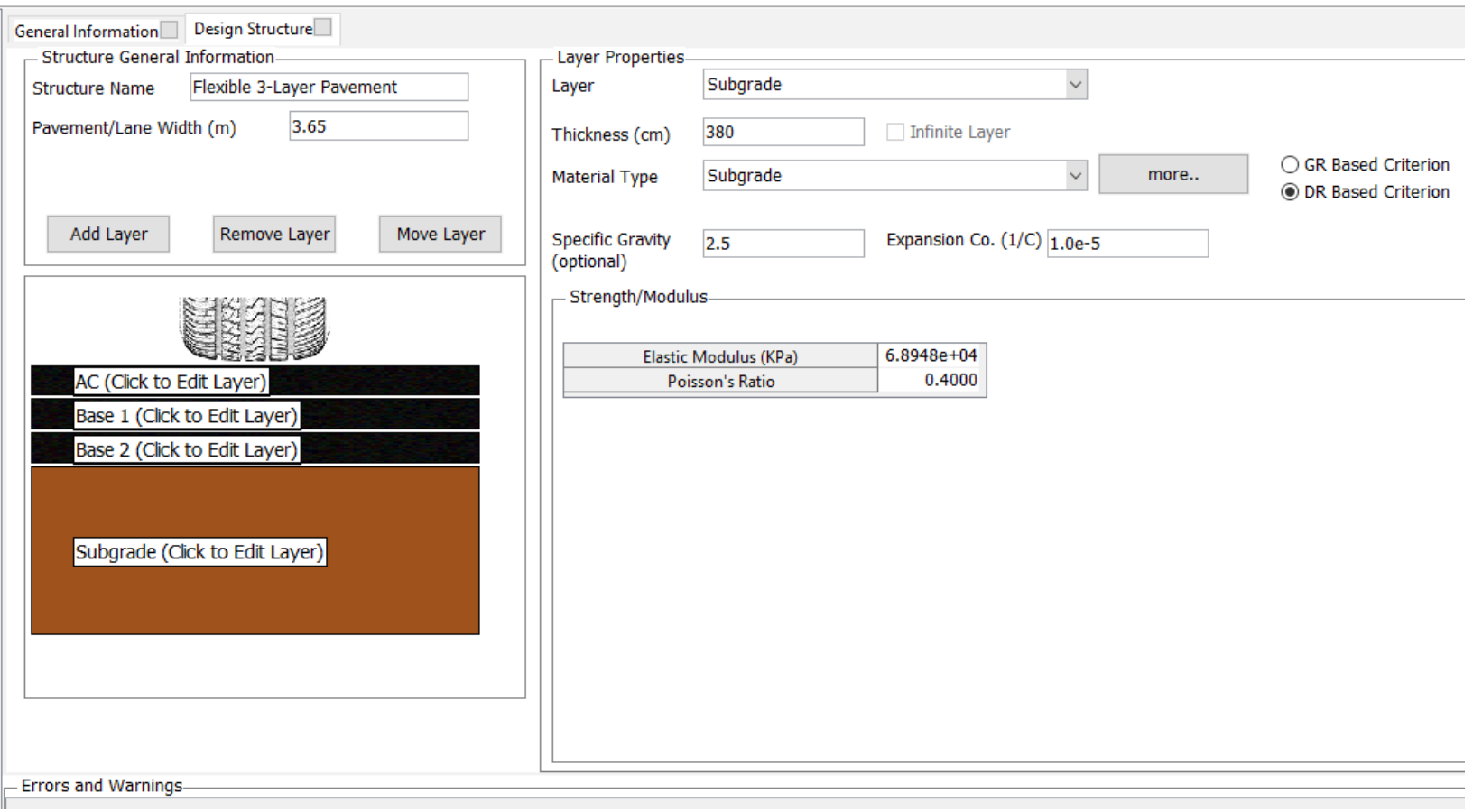

Figure 181: Design Structure of Subgrade Layer for Run 2.1

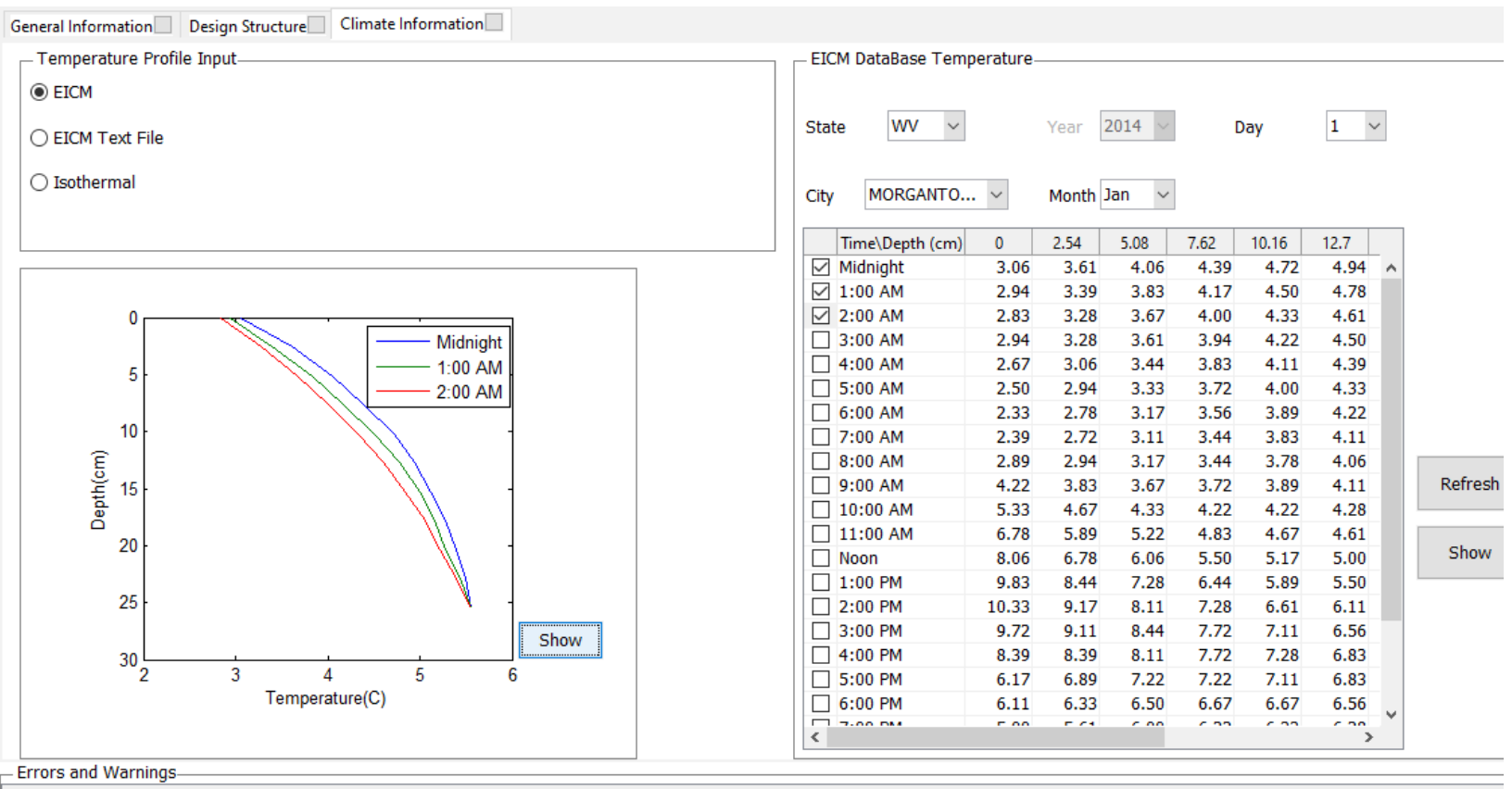

Figure 182: Climate Data for Run 2.1 


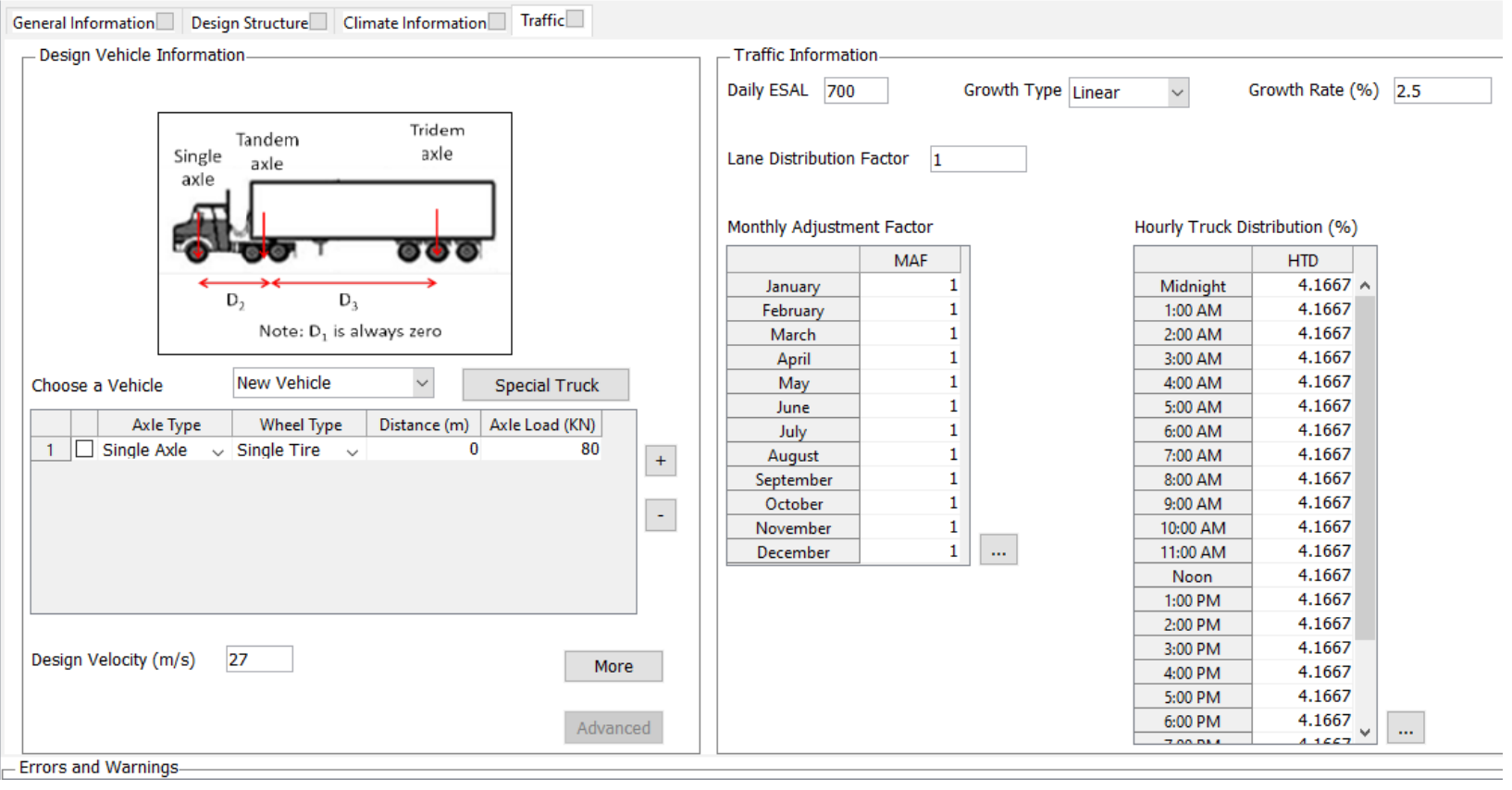

Axle Configuration

$-\quad \square \quad \times$

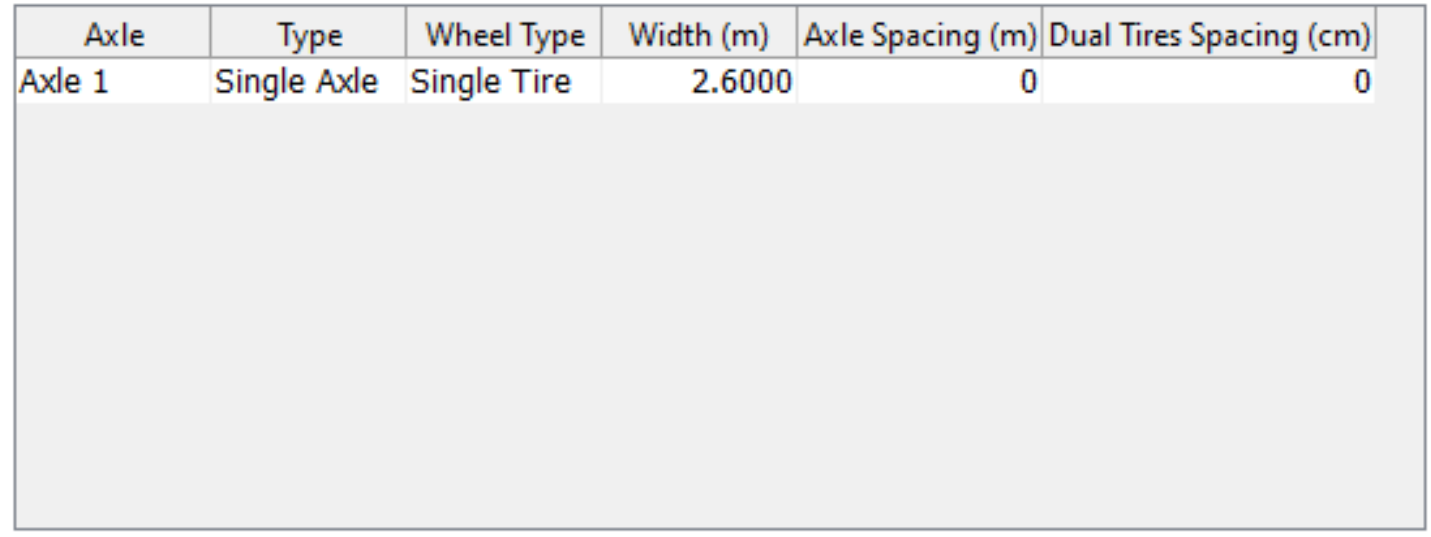

Tire Pressure (KPa) 827.37

Contact Area Shape Rectangular

Aspect Ratio (length/width) 1.5714

Shear Traction $\quad 0.0$

OK

Cancel

Figure 183: Traffic Data for Run 2.1 


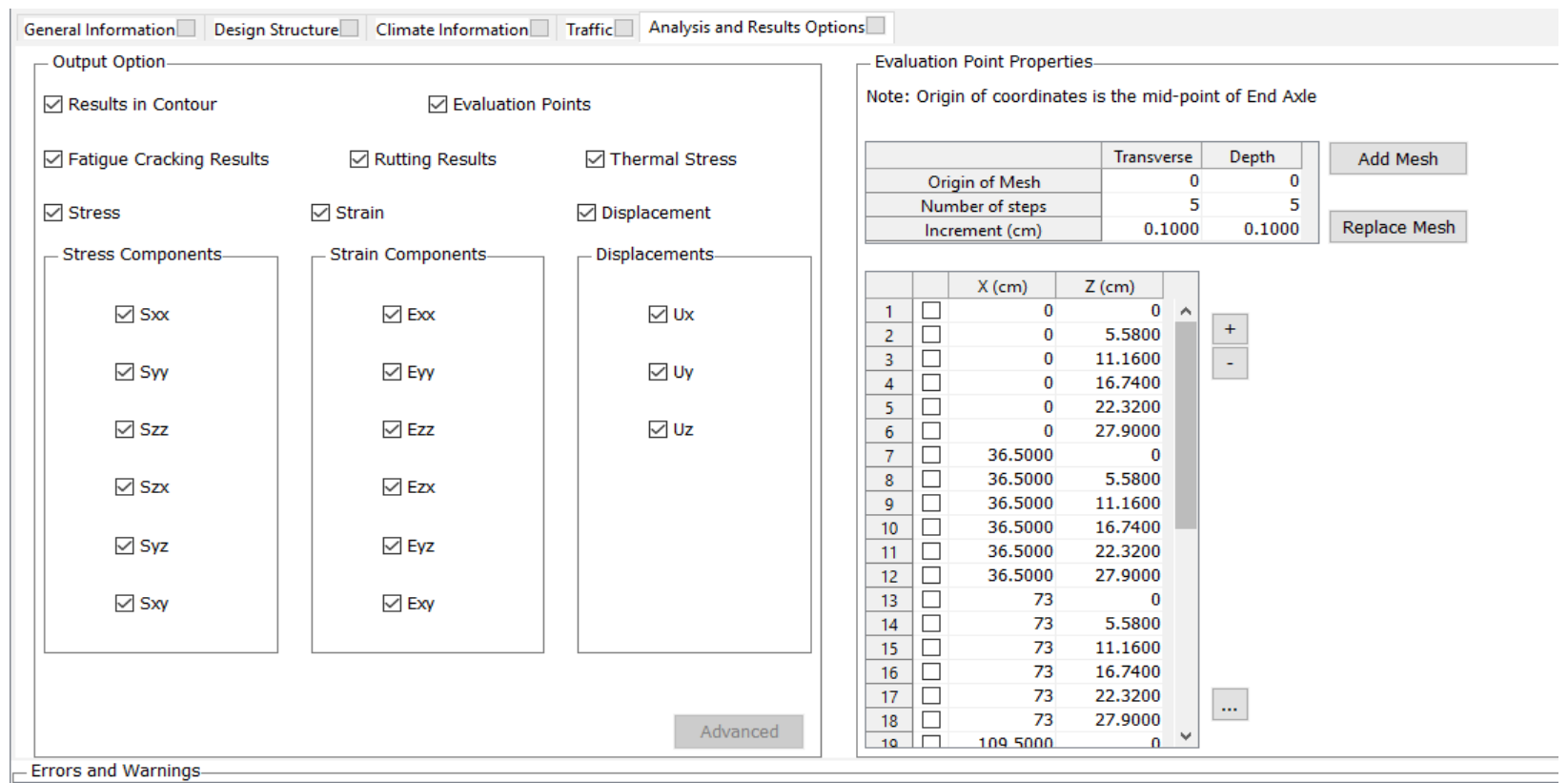

Figure 184: Output and Analysis Options for Run 2.1

\section{Run 2.2}

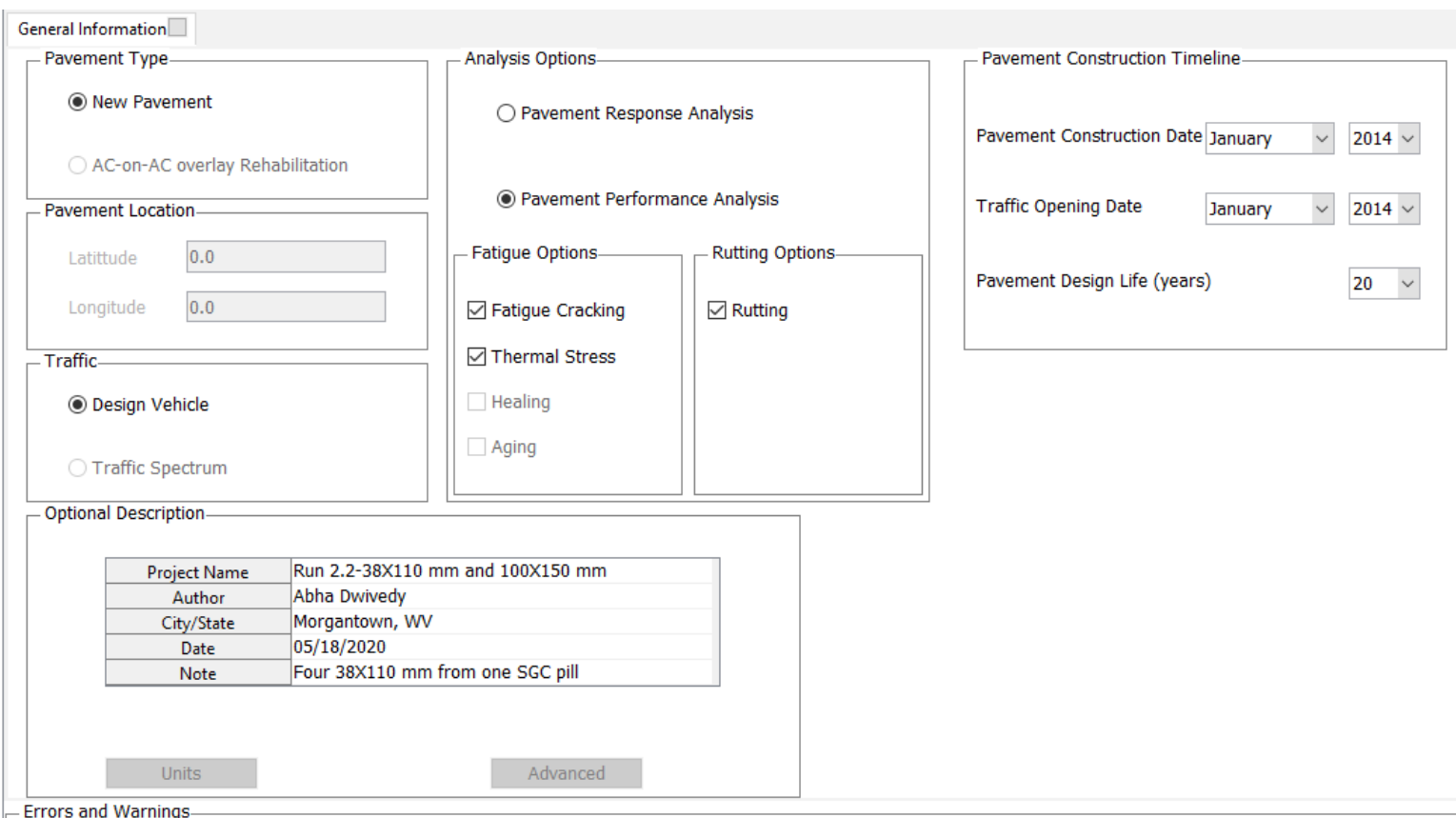

Figure 185: General Information for Run 2.2 


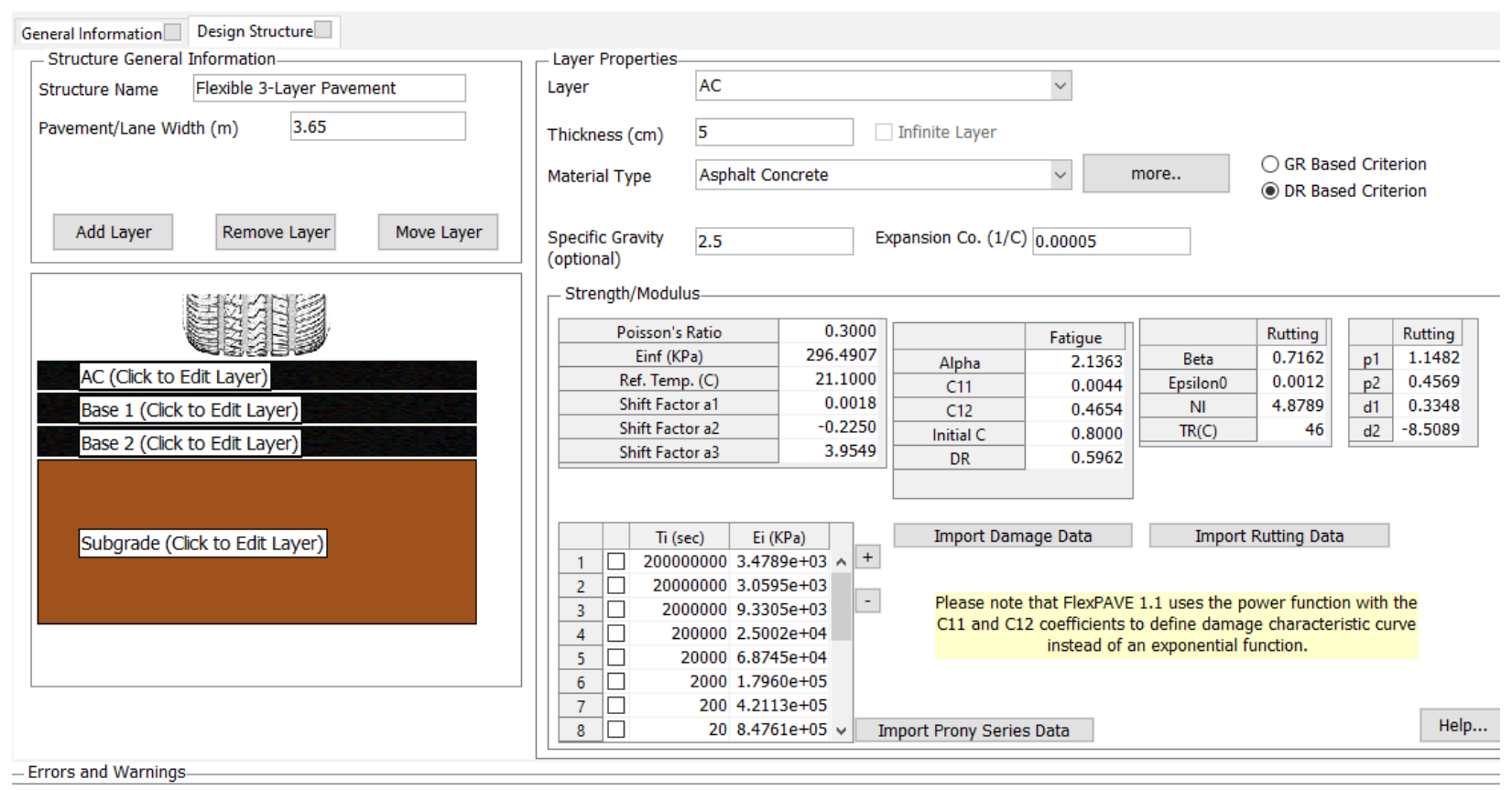

Figure 186: Design Structure of AC Layer for Run 2.2

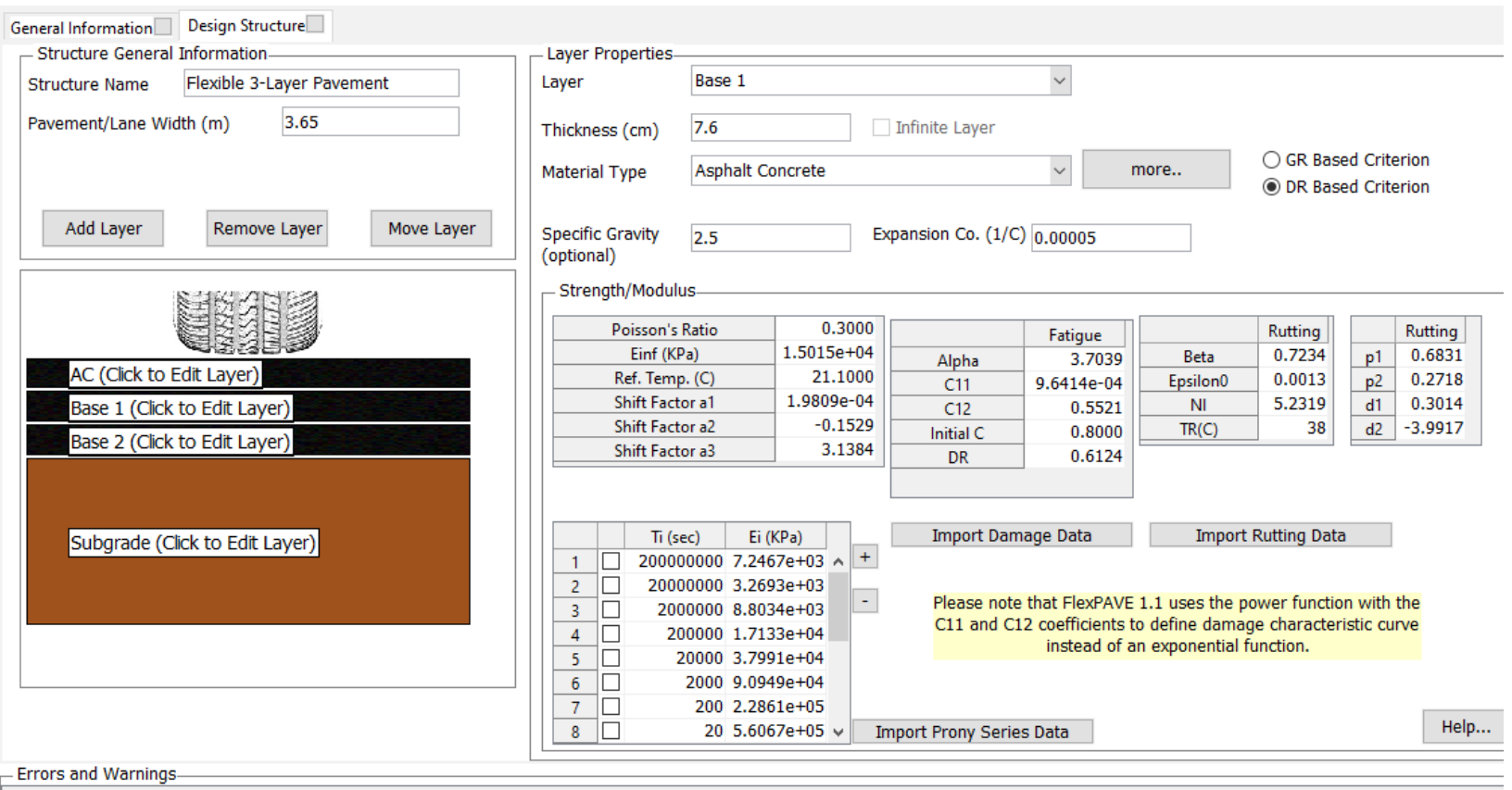

Figure 187: Design Structure of Base 1 Layer for Run 2.2 

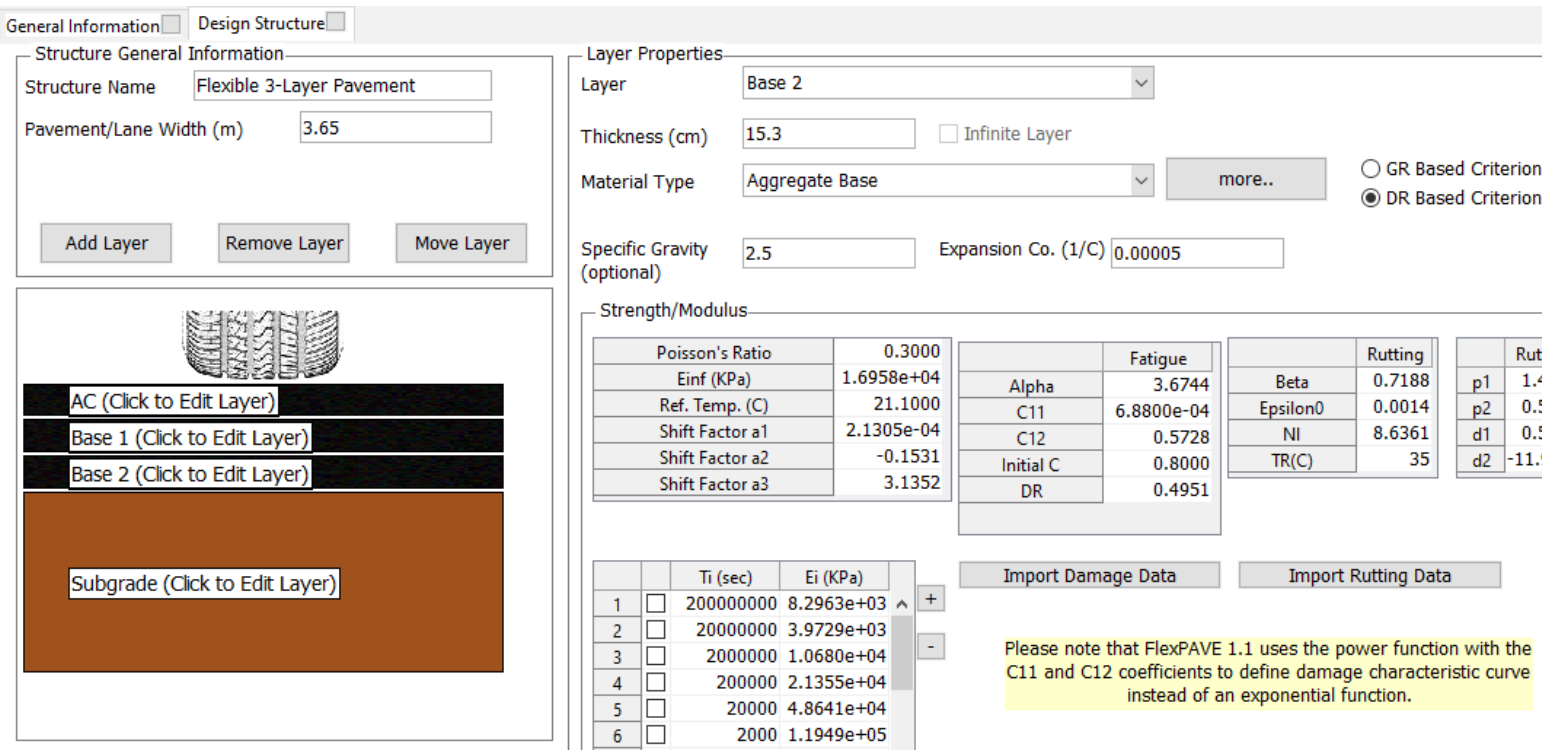

\begin{tabular}{|c|c|c|c|c|c|c|c|}
\hline Poisson's Ratio & 0.3000 & & Fatigue & & Rutting & & Rutting \\
\hline Einf ( $\mathrm{KPa})$ & $1.6958 \mathrm{e}+04$ & Alpha & 3.6744 & Beta & 0.7188 & $p 1$ & 1.4993 \\
\hline Ref. Temp. (C) & 21.1000 & $\mathrm{C} 11$ & $6.8800 \mathrm{e}-04$ & Epsilono & 0.0014 & $p^{2}$ & 0.5966 \\
\hline Shift Factor a1 & $2.1305 e-04$ & $\mathrm{C} 12$ & 0.5728 & $\mathrm{NI}$ & 8.6361 & d1 & 0.5257 \\
\hline Shift Factor a2 & -0.1531 & Initial C & 0.8000 & $\operatorname{TR}(\mathrm{C})$ & 35 & d2 & $-11.97 \ldots$ \\
\hline Shift Factor a3 & 3.1352 & $\mathrm{DR}$ & 0.4951 & & & & \\
\hline
\end{tabular}

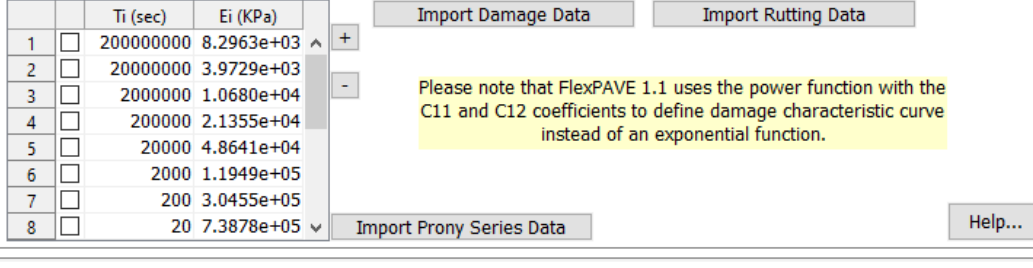

-Errors and Warnings

Figure 188: Design Structure of Base 2 Layer for Run 2.2

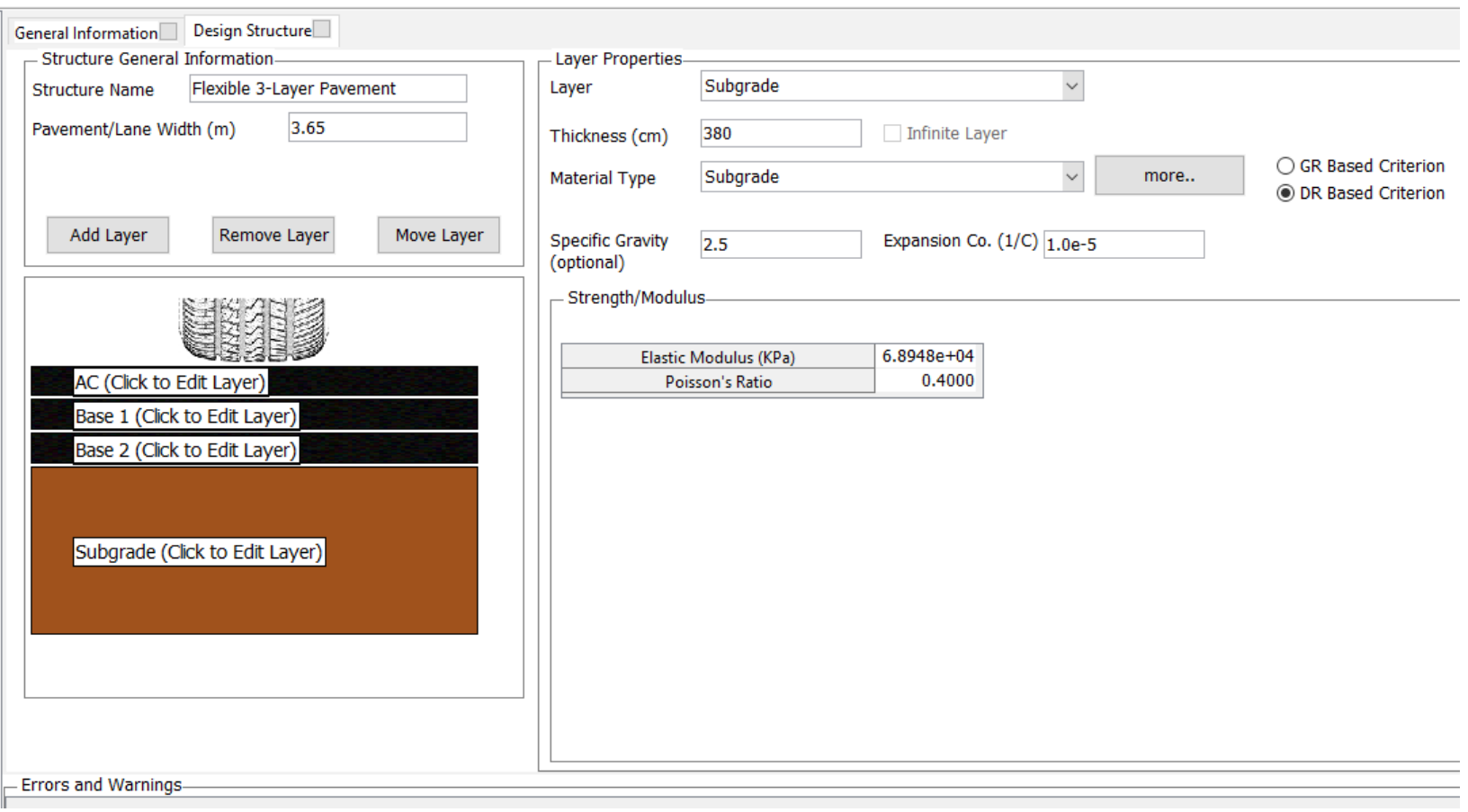

Figure 189: Design Structure of Subgrade Layer for Run 2.2 


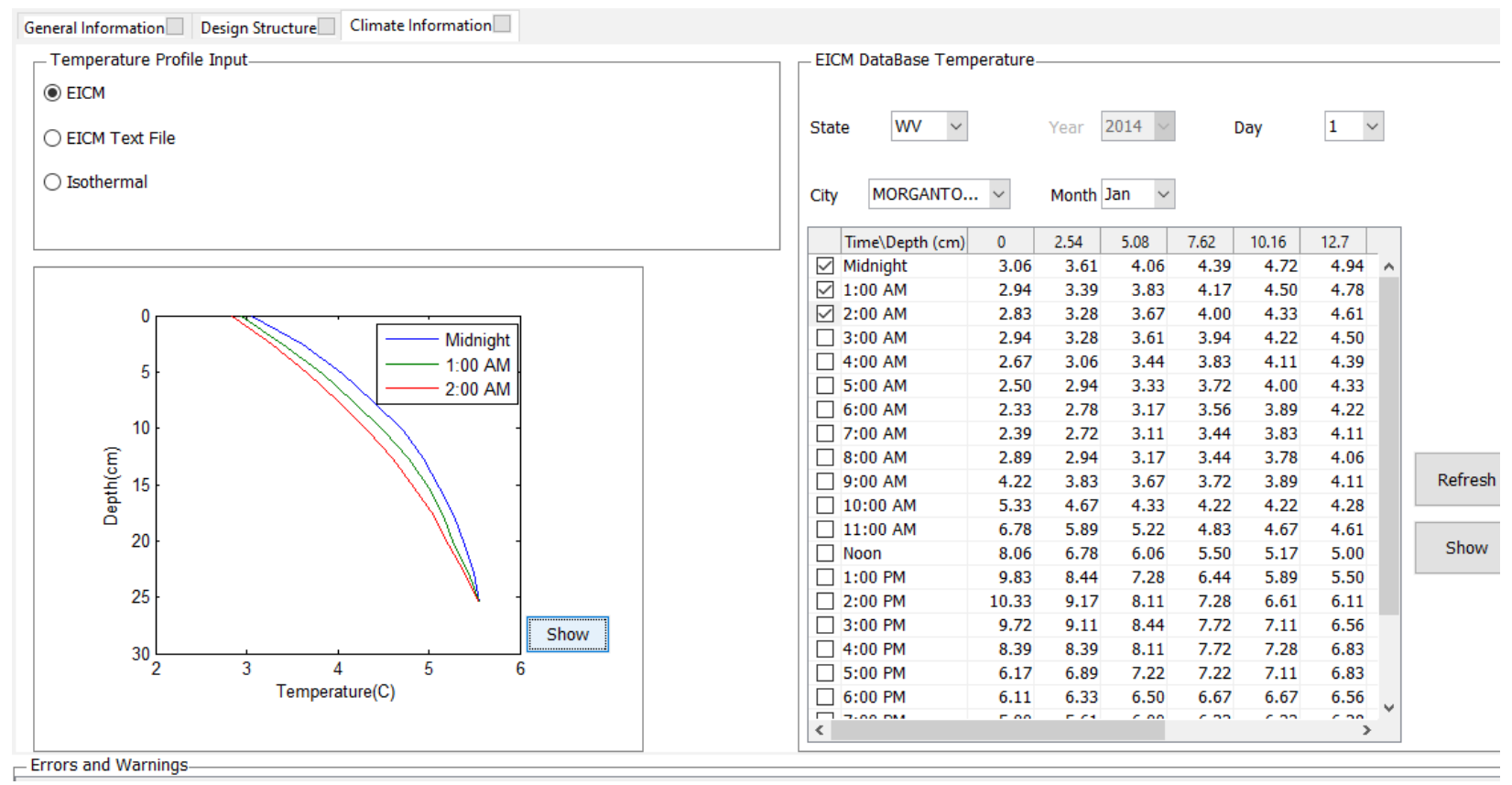

Figure 190: Climate Data for Run 2.2 


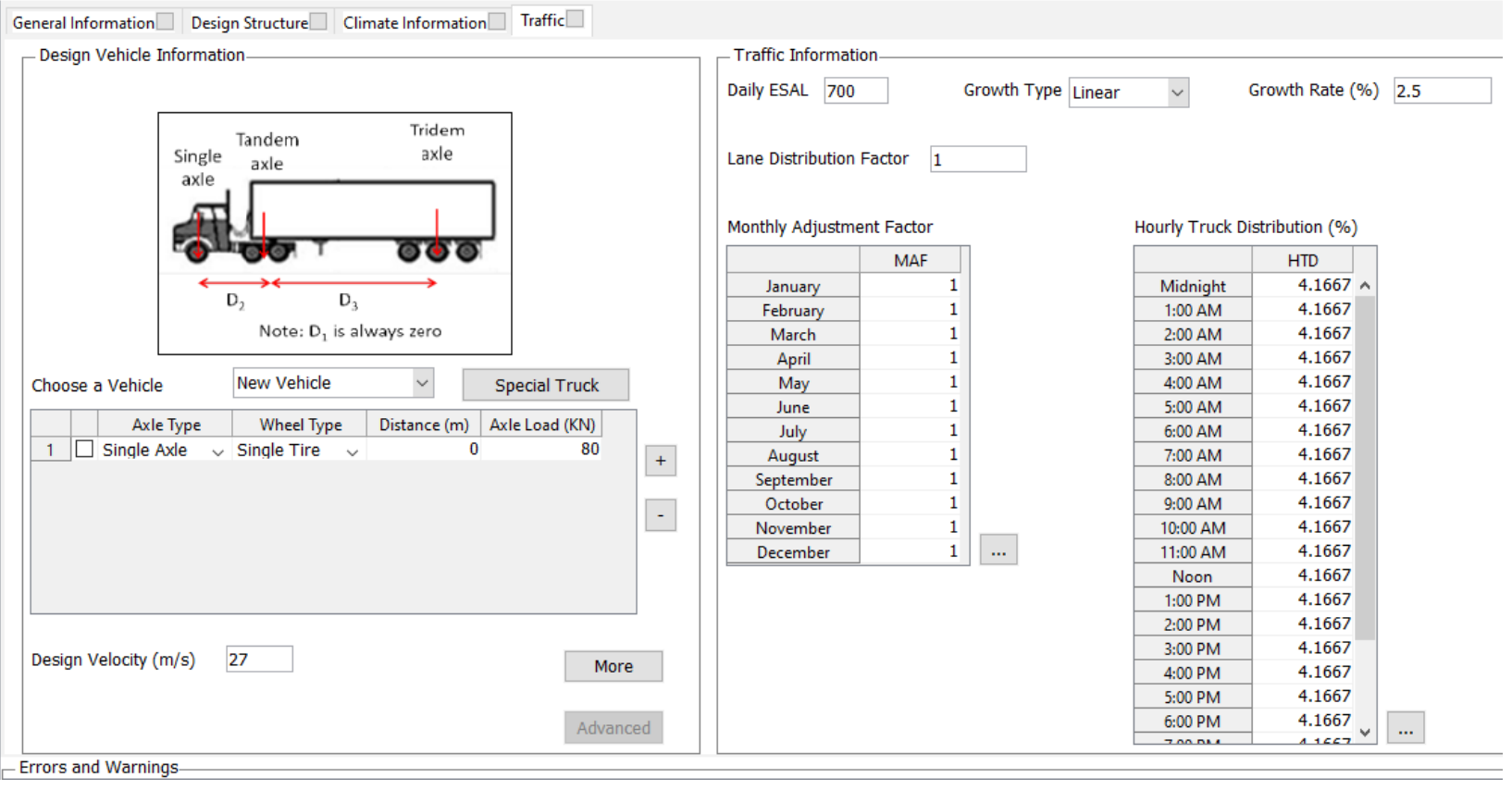

Axle Configuration

$-\quad \square \quad \times$

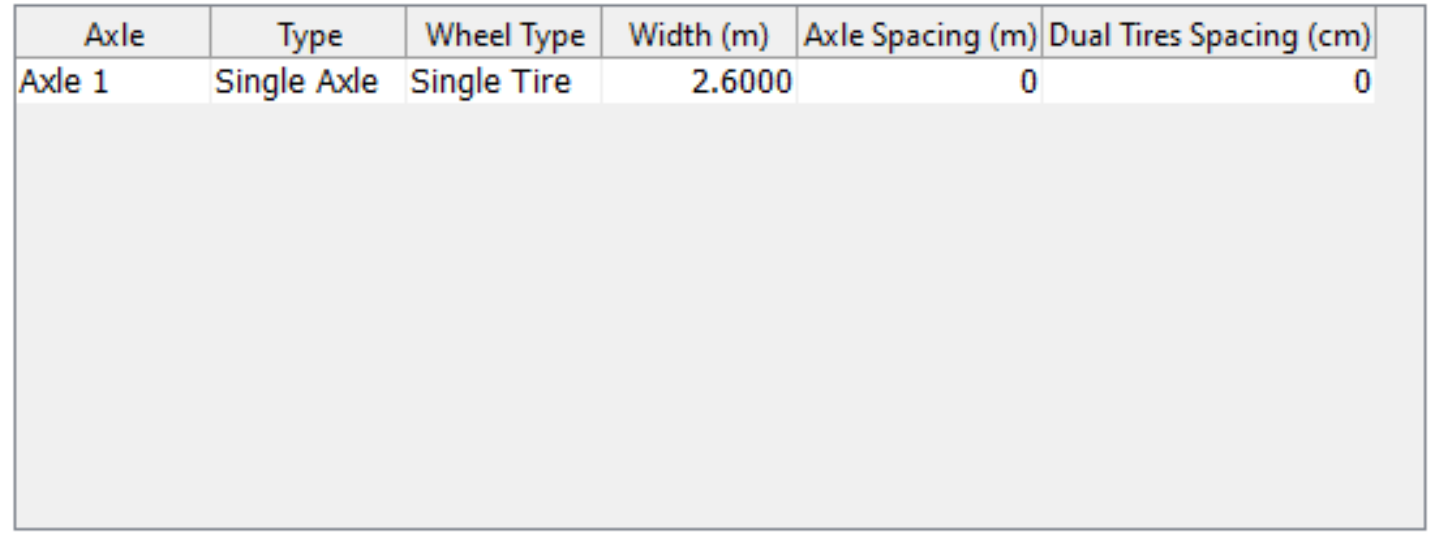

Tire Pressure (KPa) 827.37

Contact Area Shape Rectangular

Aspect Ratio (length/width) 1.5714

Shear Traction $\quad 0.0$

OK

Cancel

Figure 191: Traffic Data for Run 2.2 


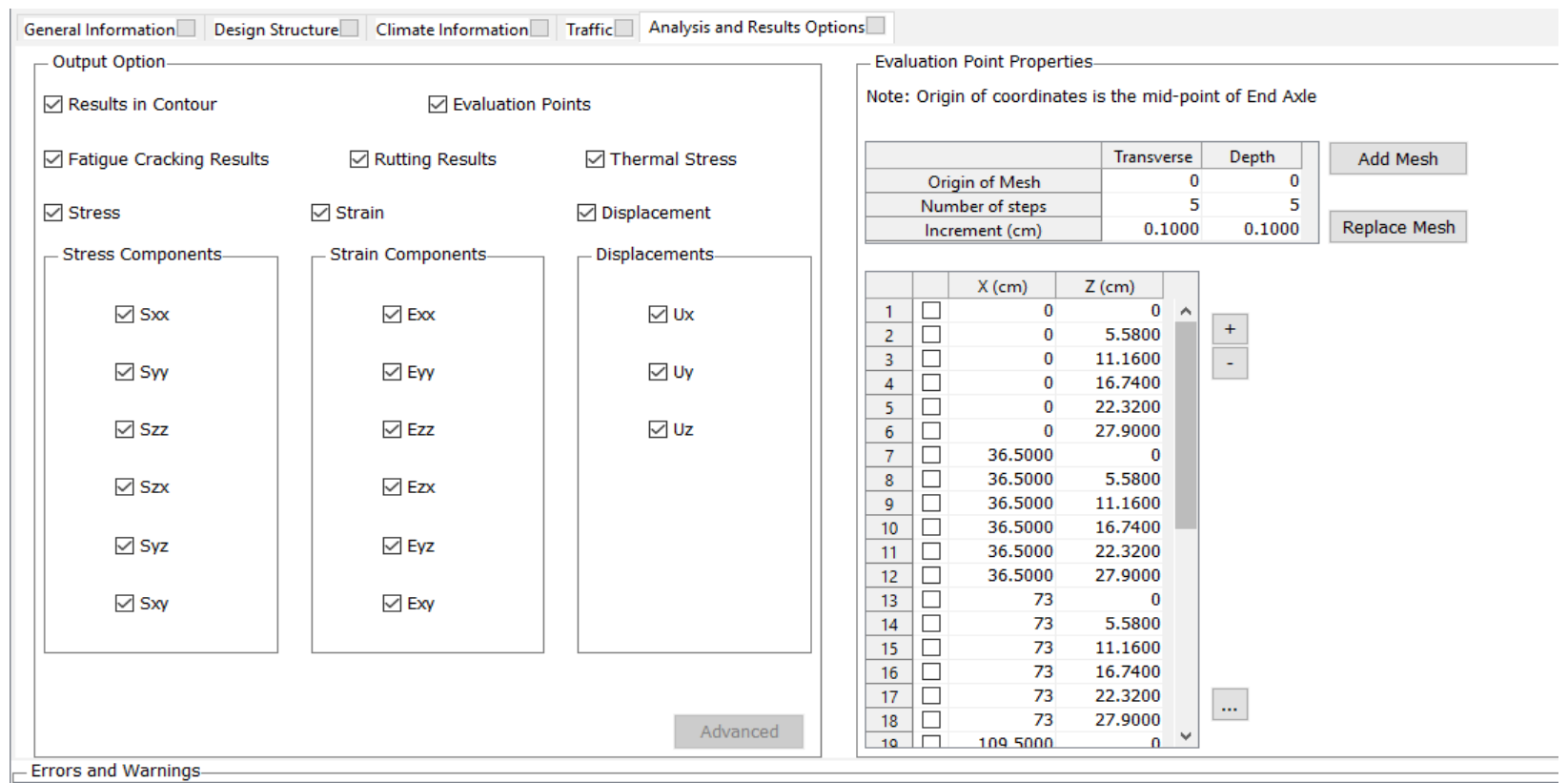

Figure 192: Output and Analysis Options for Run 2.2

\section{Run 2.3}

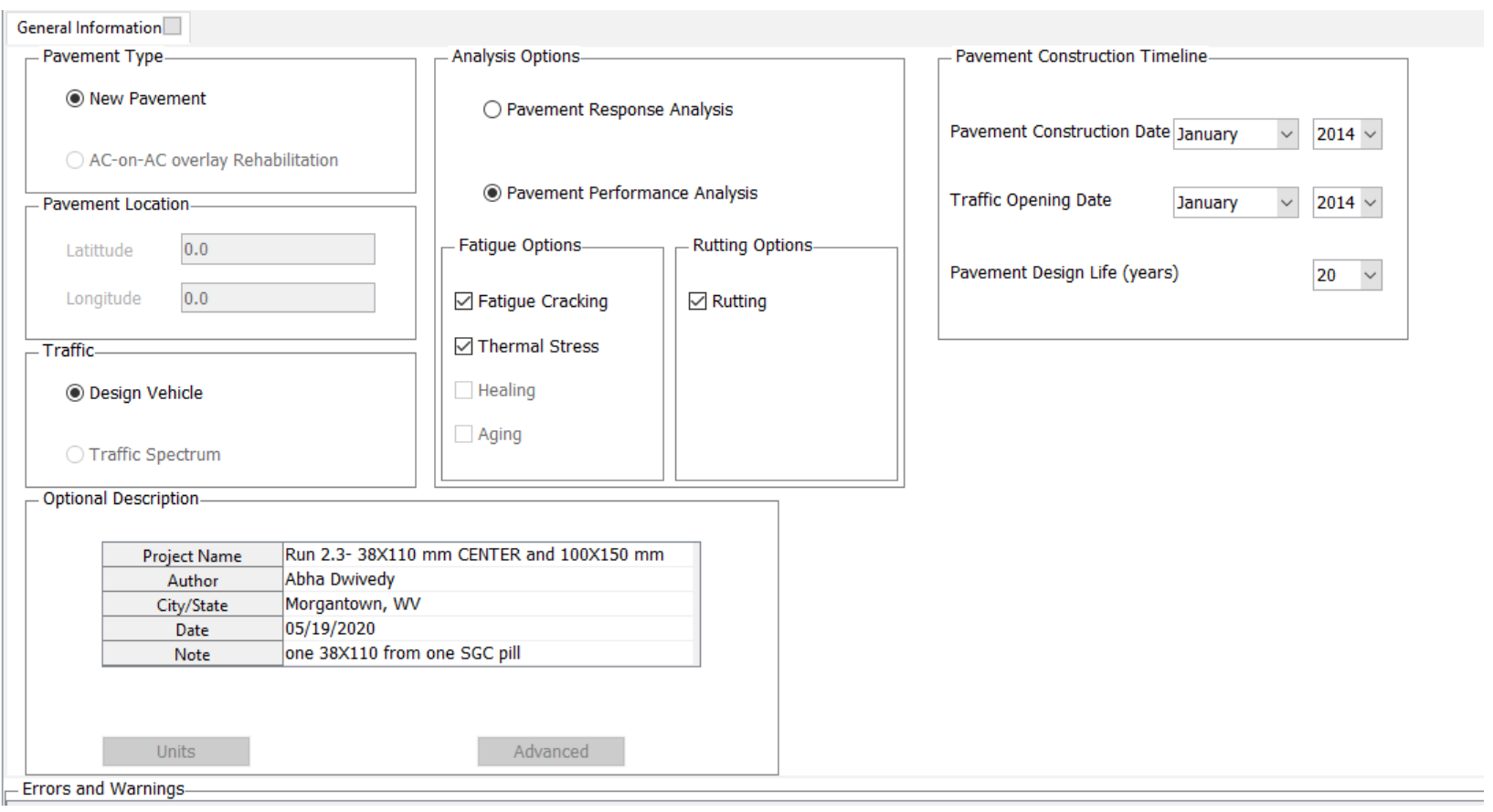

Figure 193: General Information for Run 2.3 


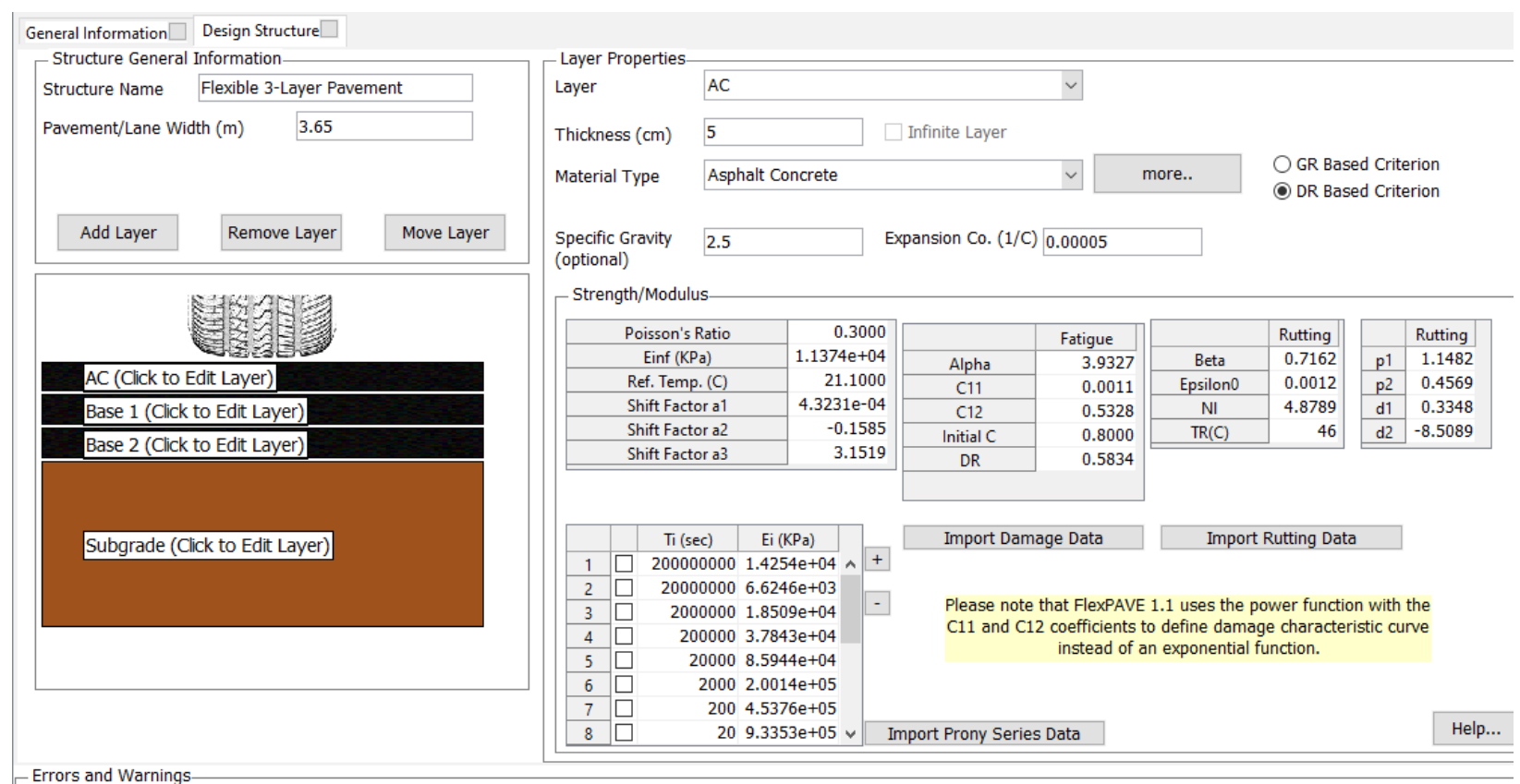

Figure 194: Design Structure of AC Layer for Run 2.3

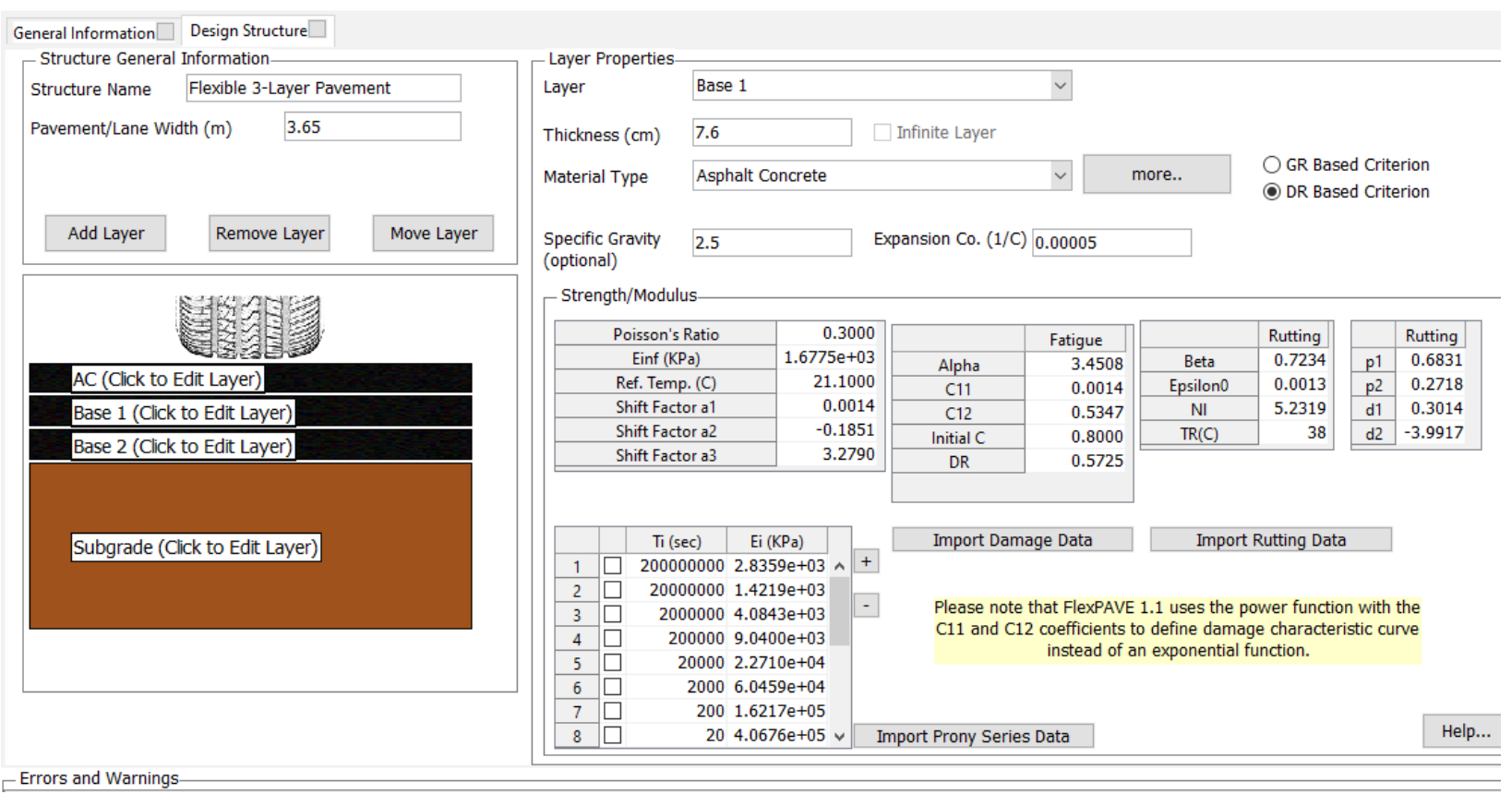

Figure 195: Design Structure of Base 1 Layer for Run 2.3 


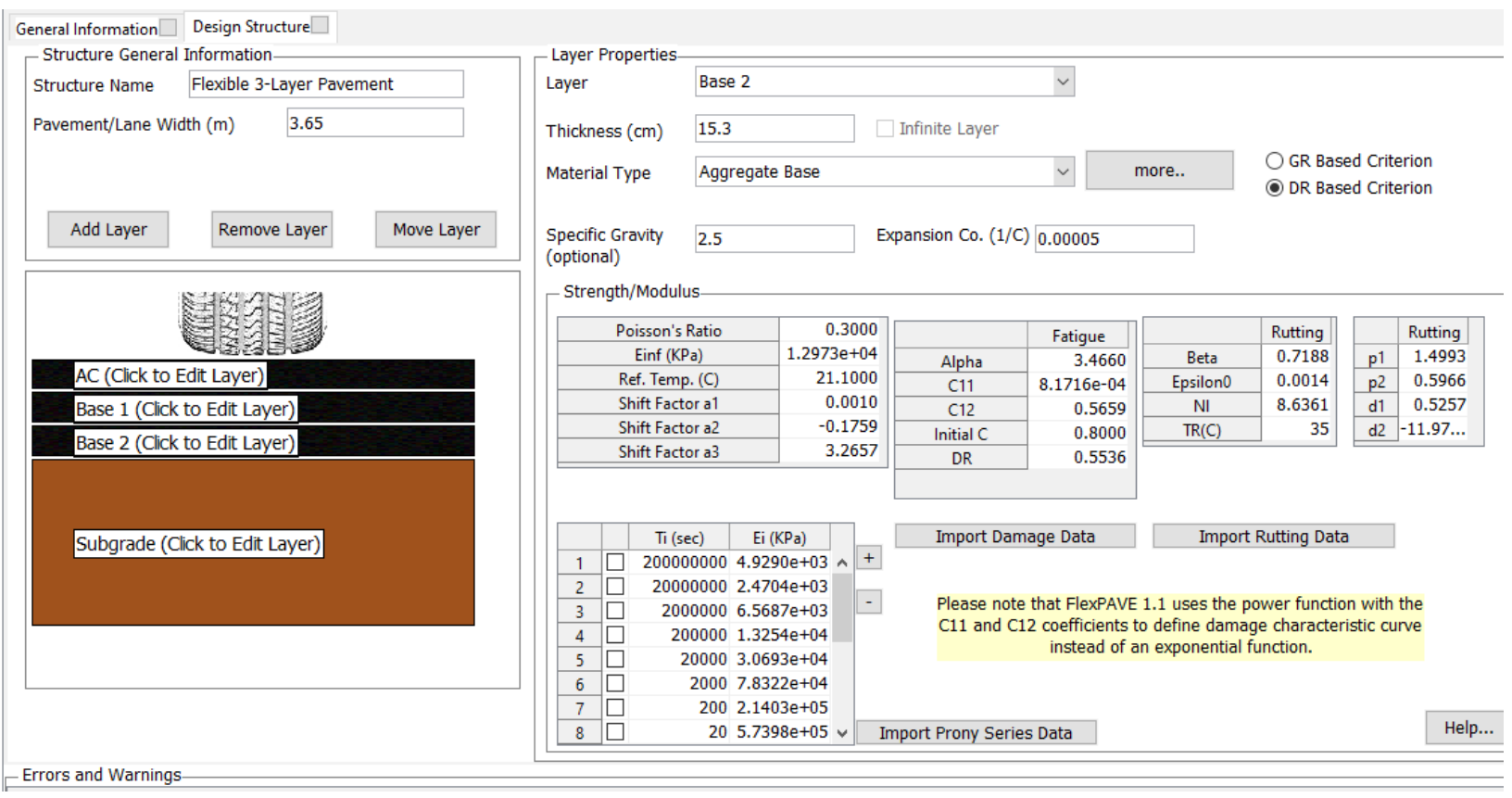

Figure 196: Design Structure of Base 2 Layer for Run 2.3

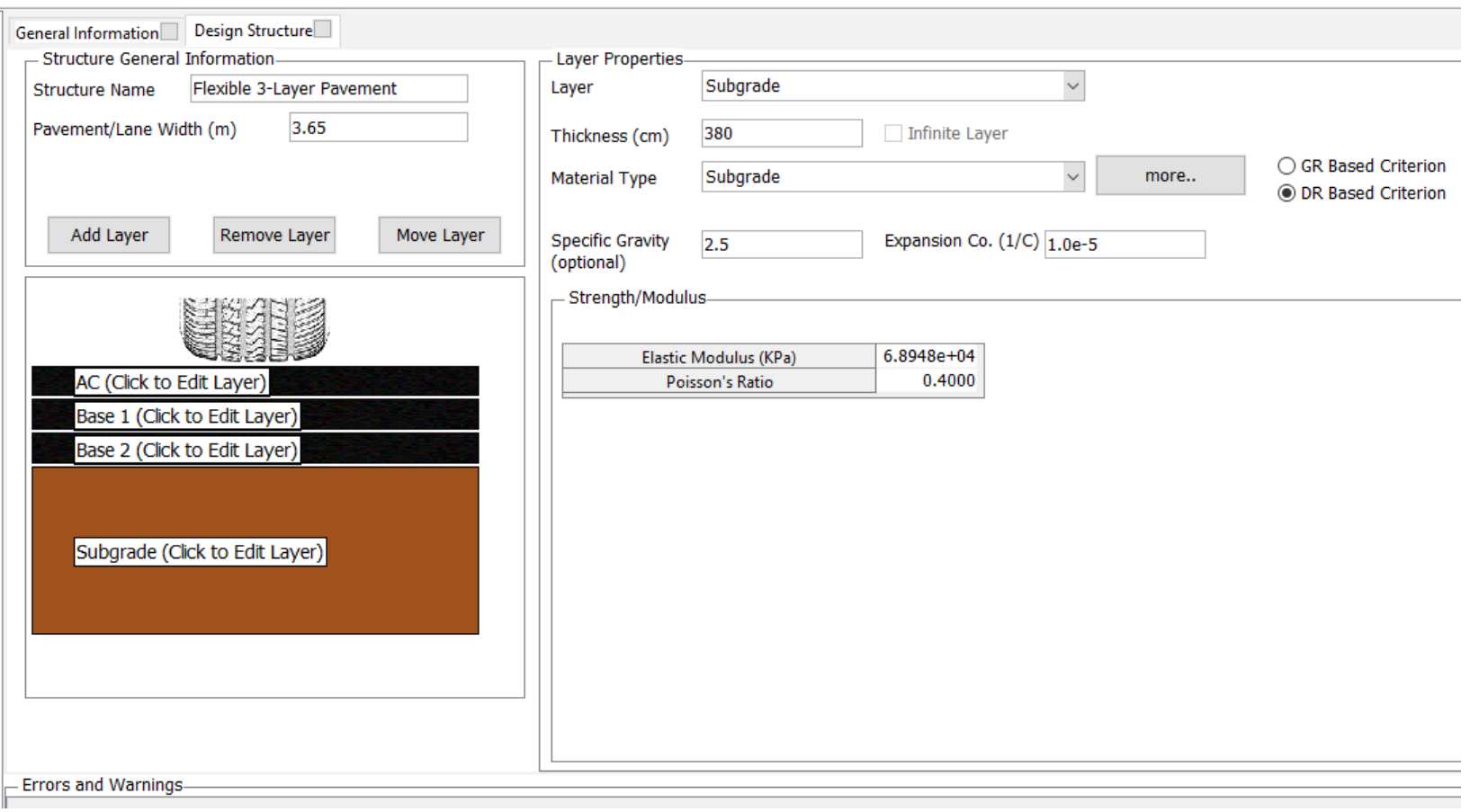

Figure 197: Design Structure of Subgrade Layer for Run 2.3 


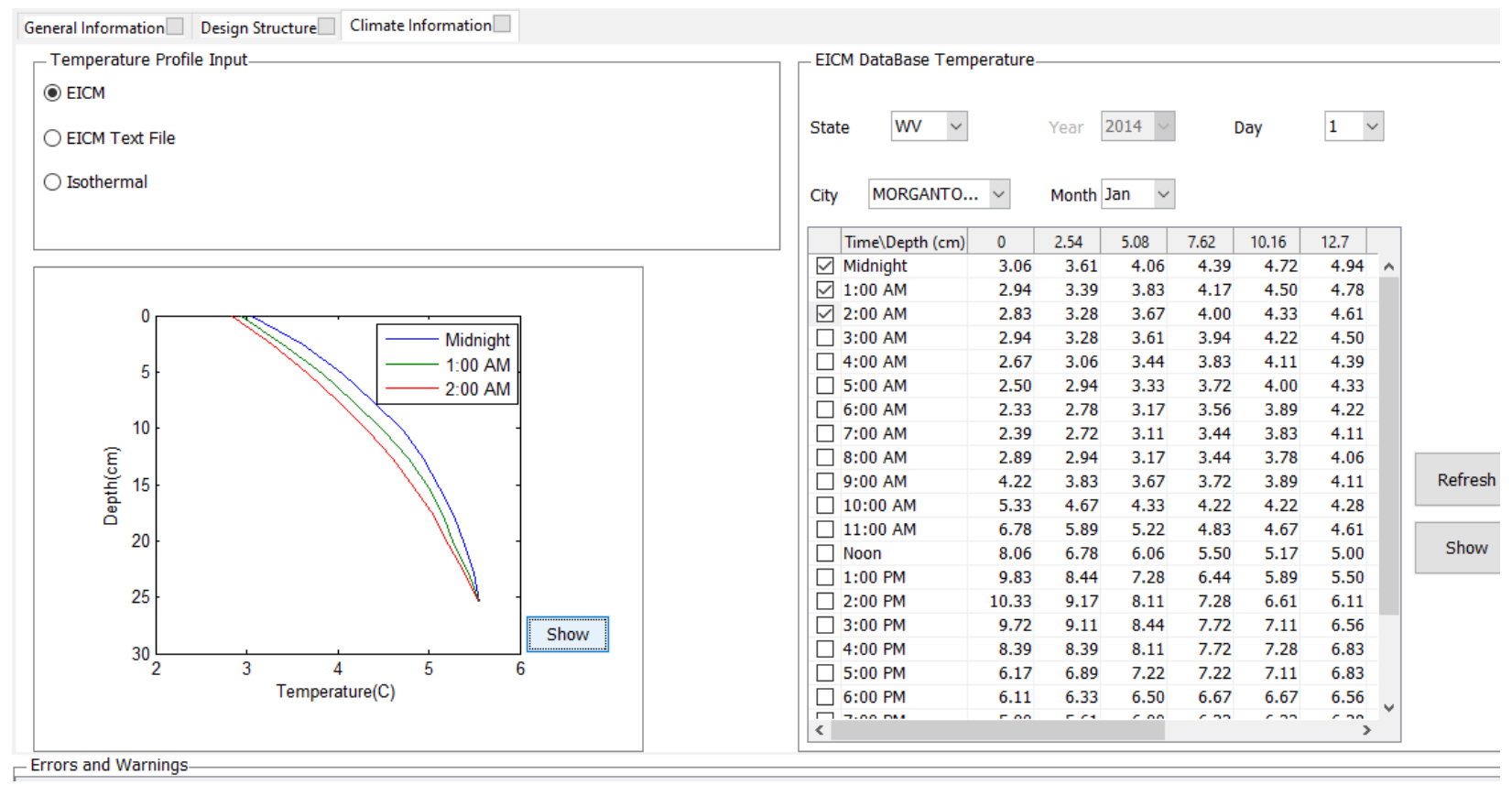

Figure 198: Climate Data for Run 2.3 


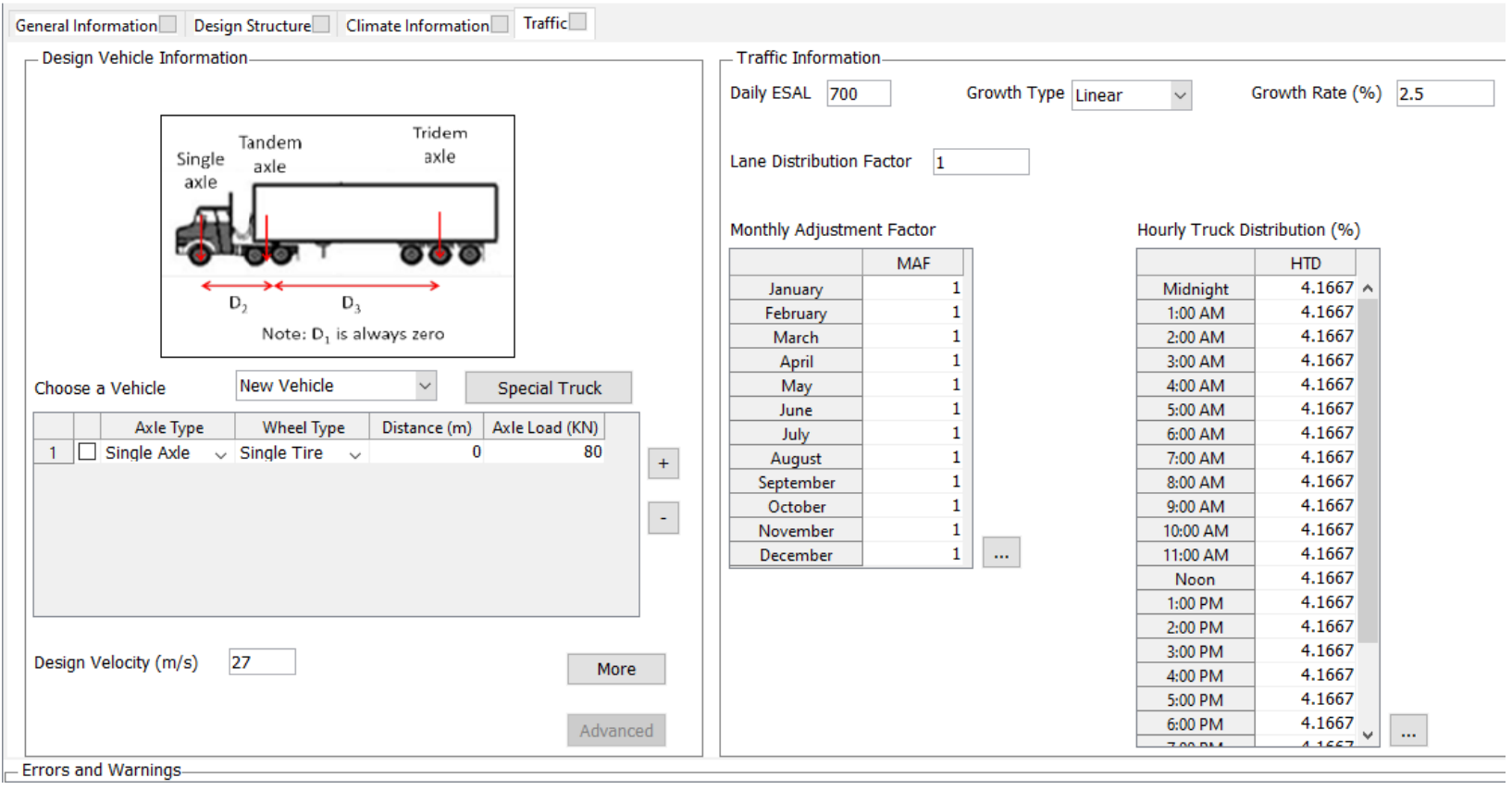

Axle Configuration

$-\quad \square \quad \times$

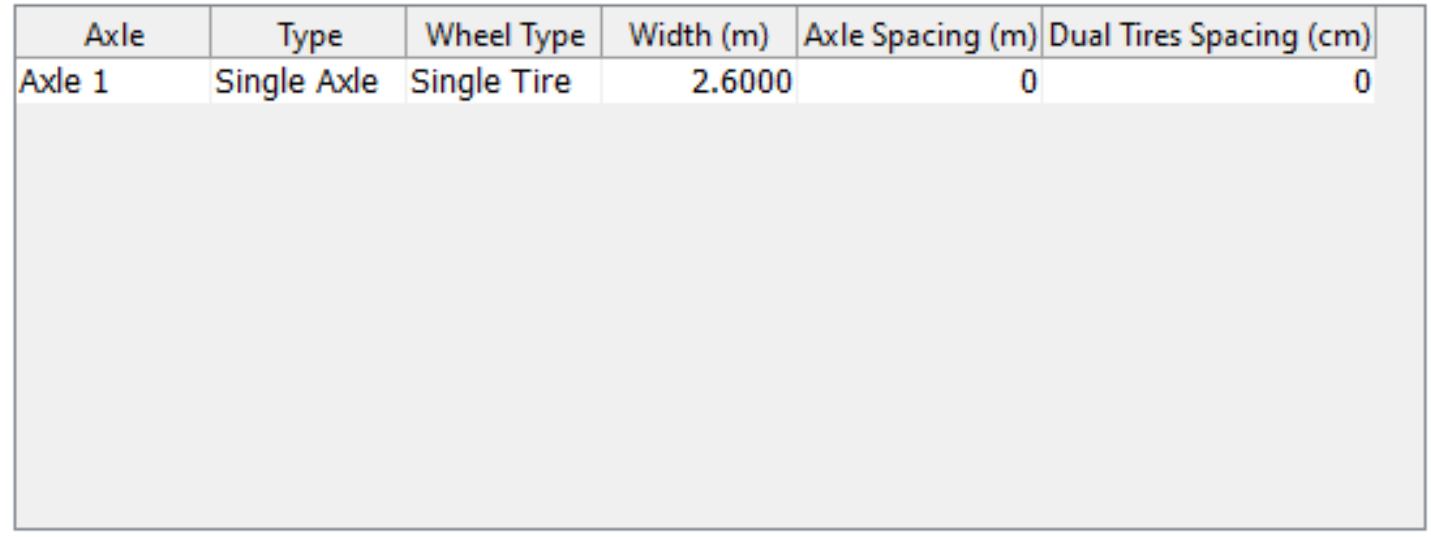

Tire Pressure (KPa) 827.37

Contact Area Shape Rectangular

Aspect Ratio (length/width) 1.5714

Shear Traction $\quad 0.0$

OK

Cancel

Figure 199: Traffic Data for Run 2.3 


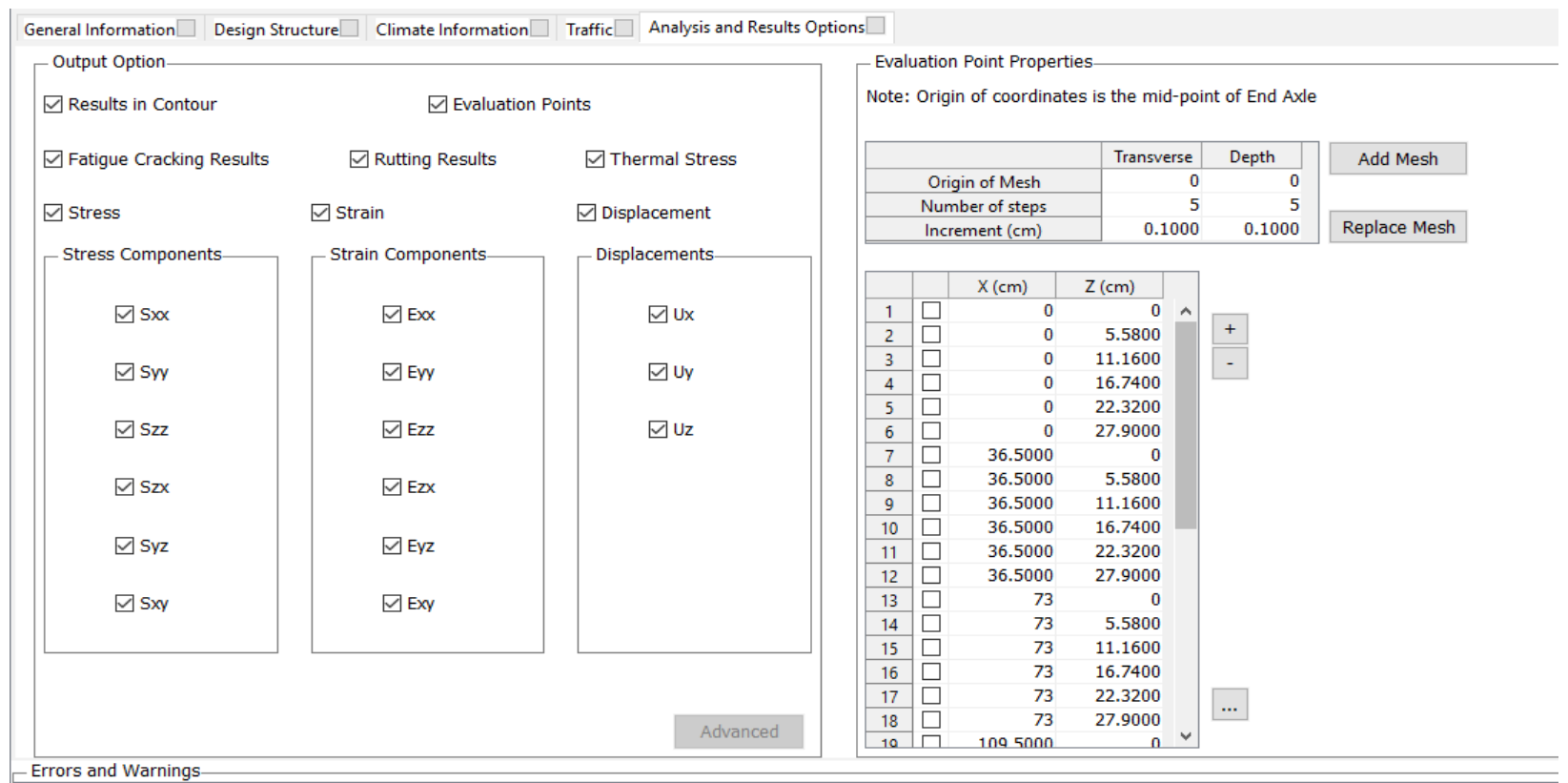

Figure 200: Output and Analysis Options for Run 2.3

\section{Run 2.4}

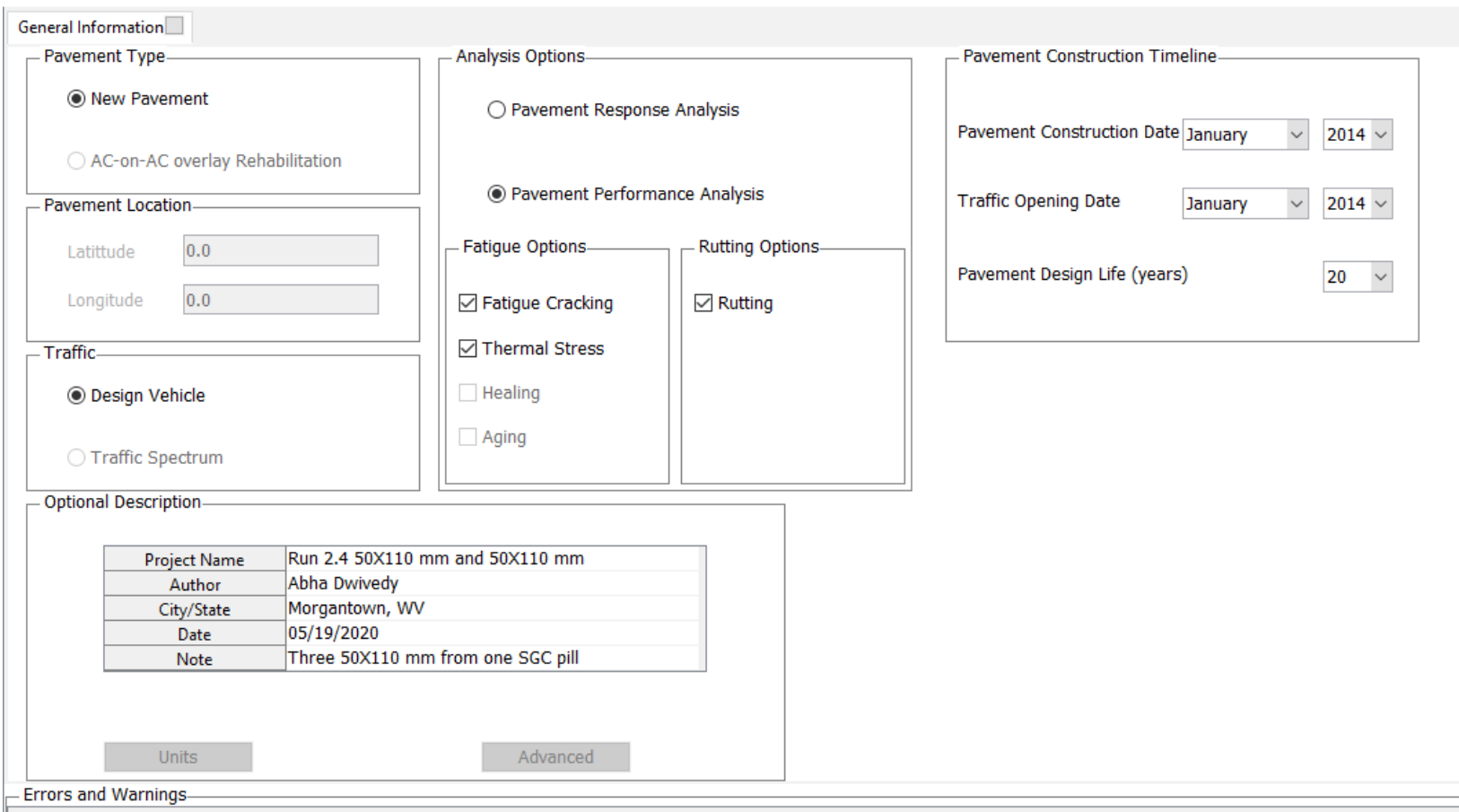

Figure 201: General Information for Run 2.4 


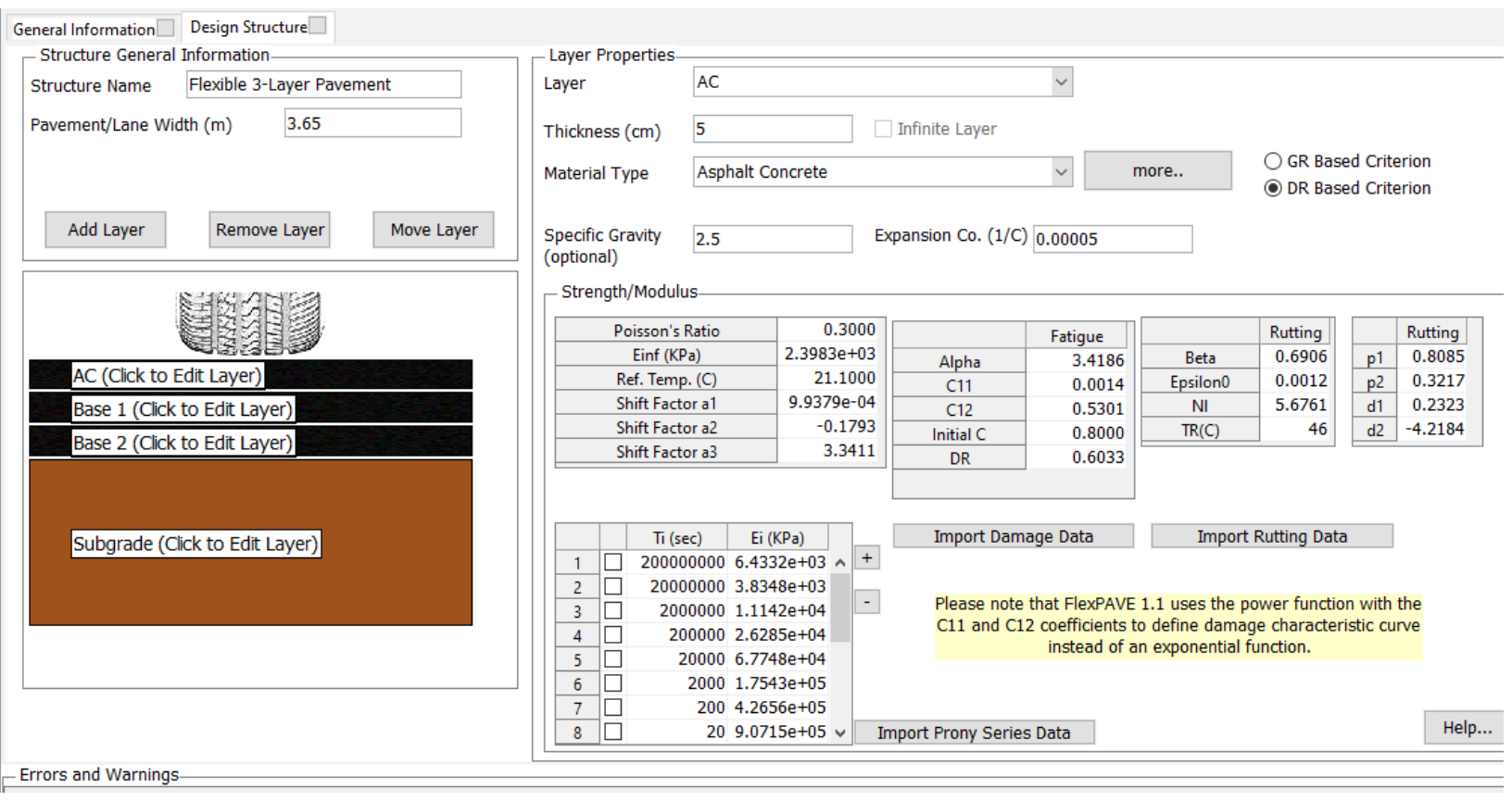

Figure 202: Design Structure of AC Layer for Run 2.4

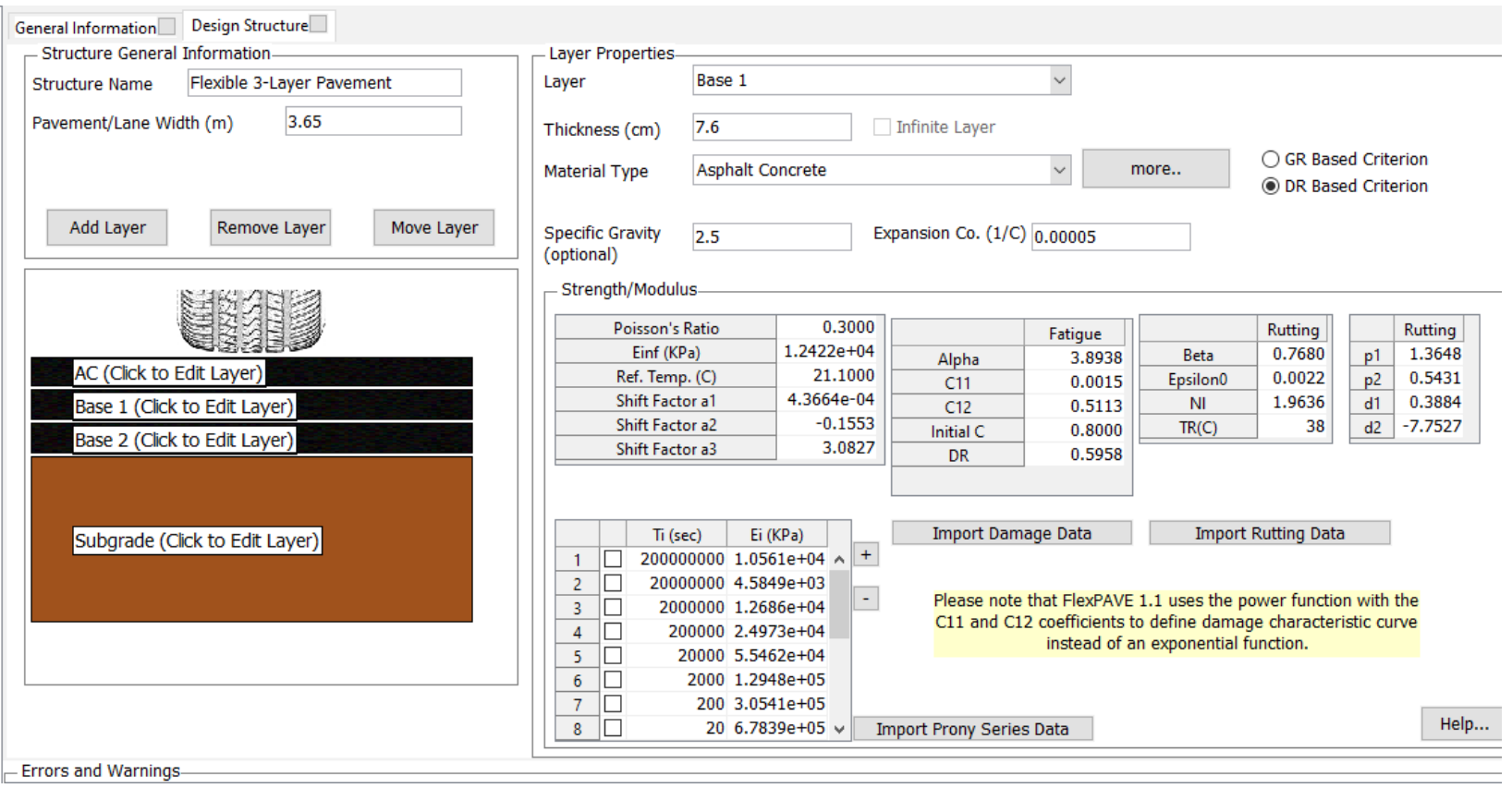

Figure 203: Design Structure of Base 1 Layer for Run 2.4 


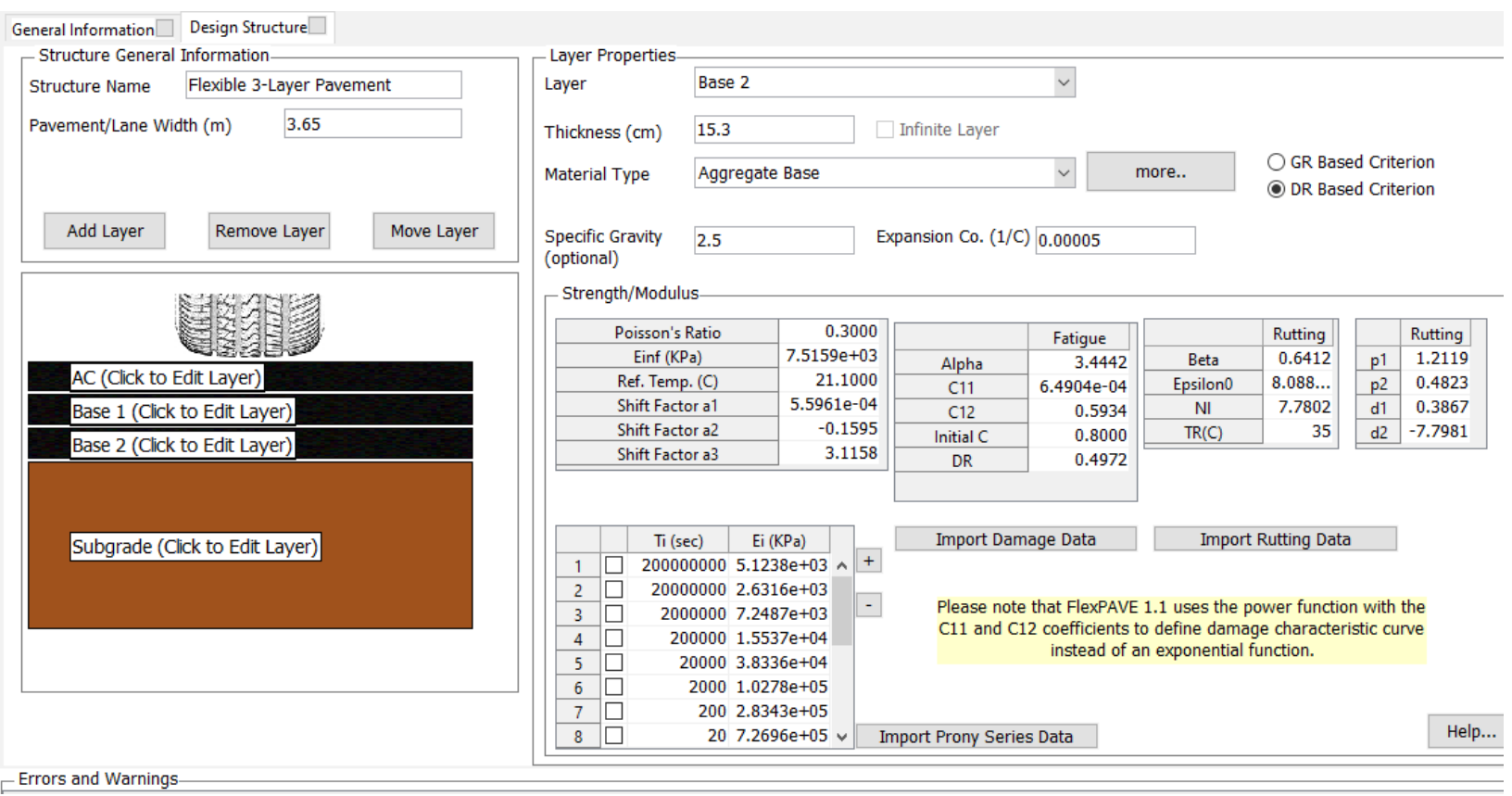

Figure 204: Design Structure of Base 2 Layer for Run 2.4

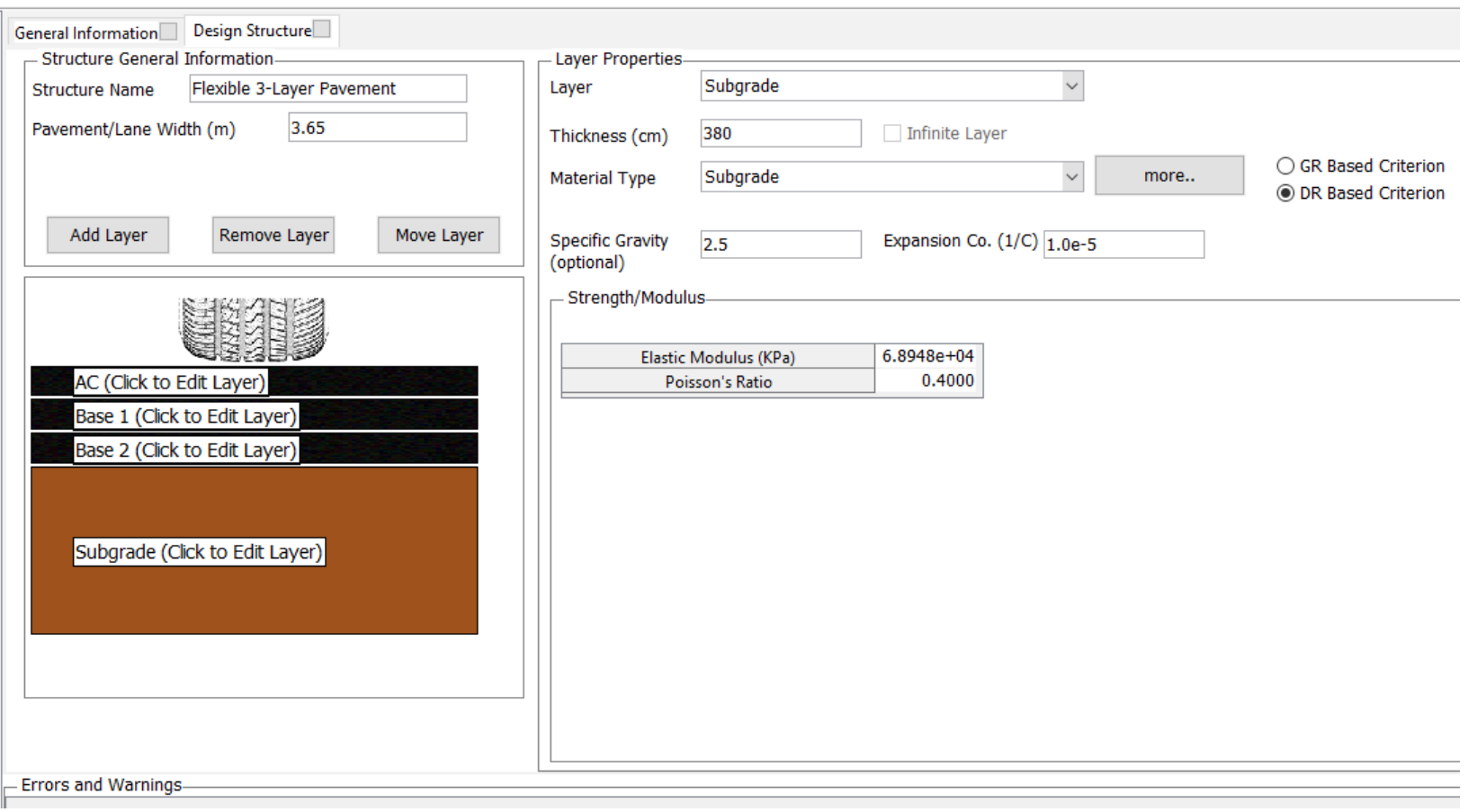

Figure 205: Design Structure of Subgrade Layer for Run 2.4 


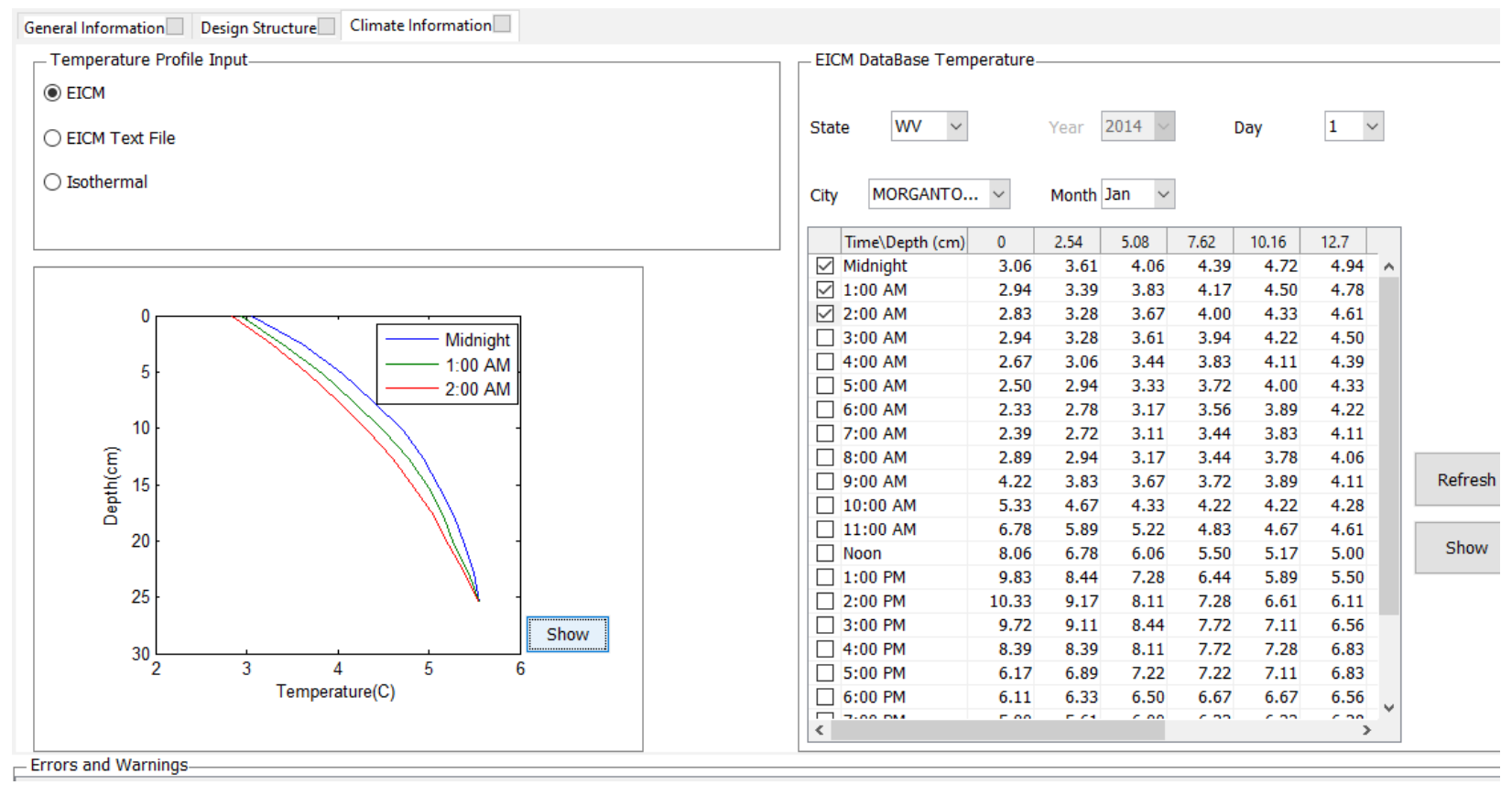

Figure 206: Climate Data for Run 2.4 


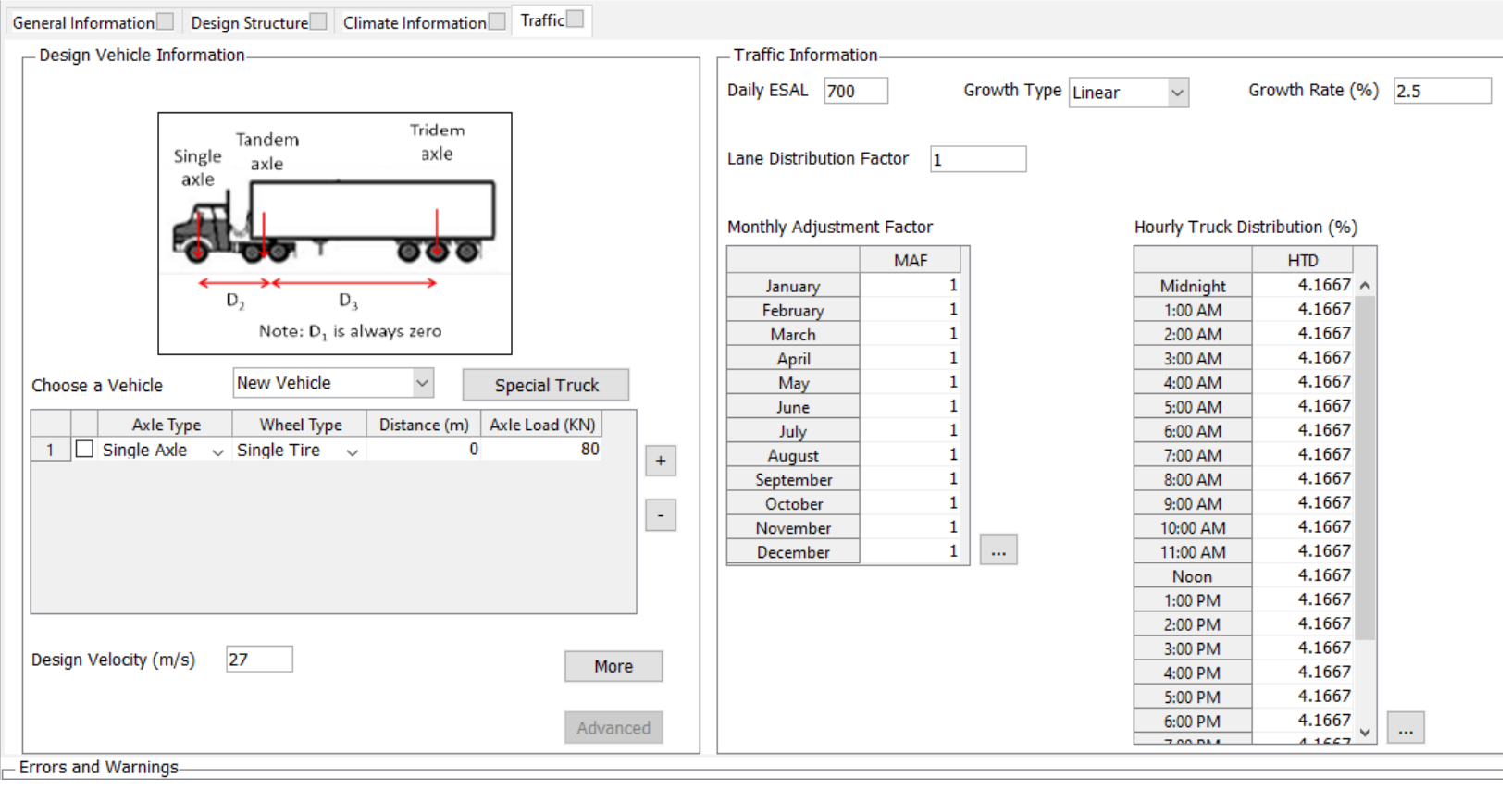

Axle Configuration

$-\quad \square \quad \times$

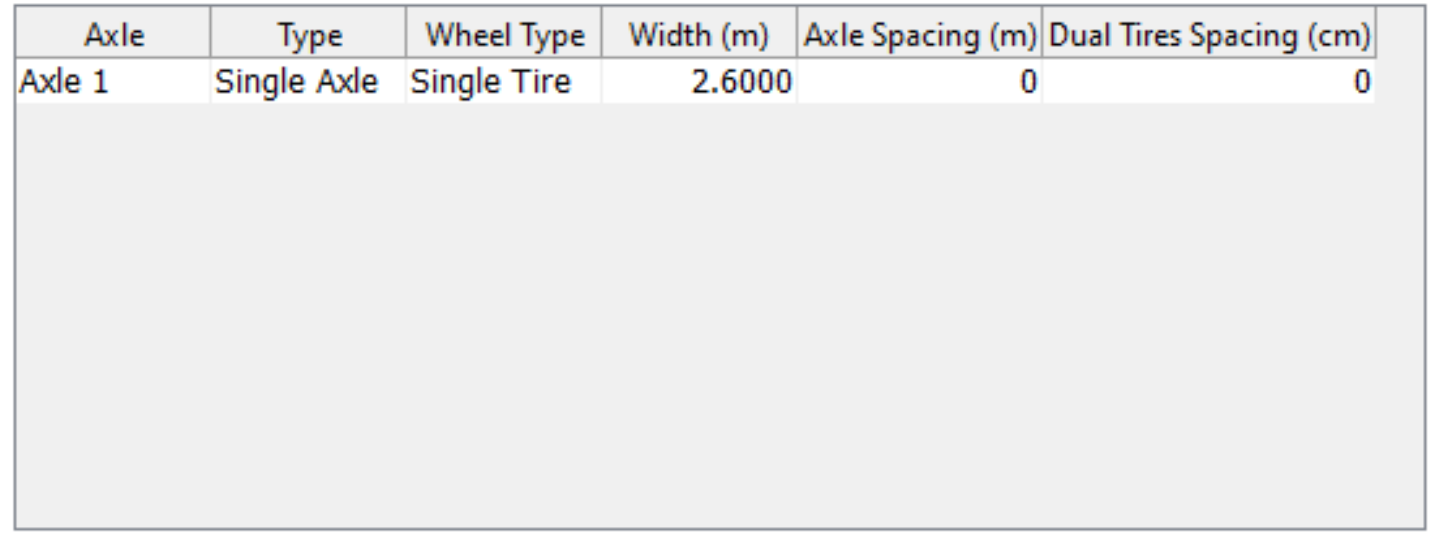

Tire Pressure (KPa) 827.37

Contact Area Shape Rectangular

Aspect Ratio (length/width) 1.5714

Shear Traction $\quad 0.0$

OK

Cancel

Figure 207: Traffic Data for Run 2.4 


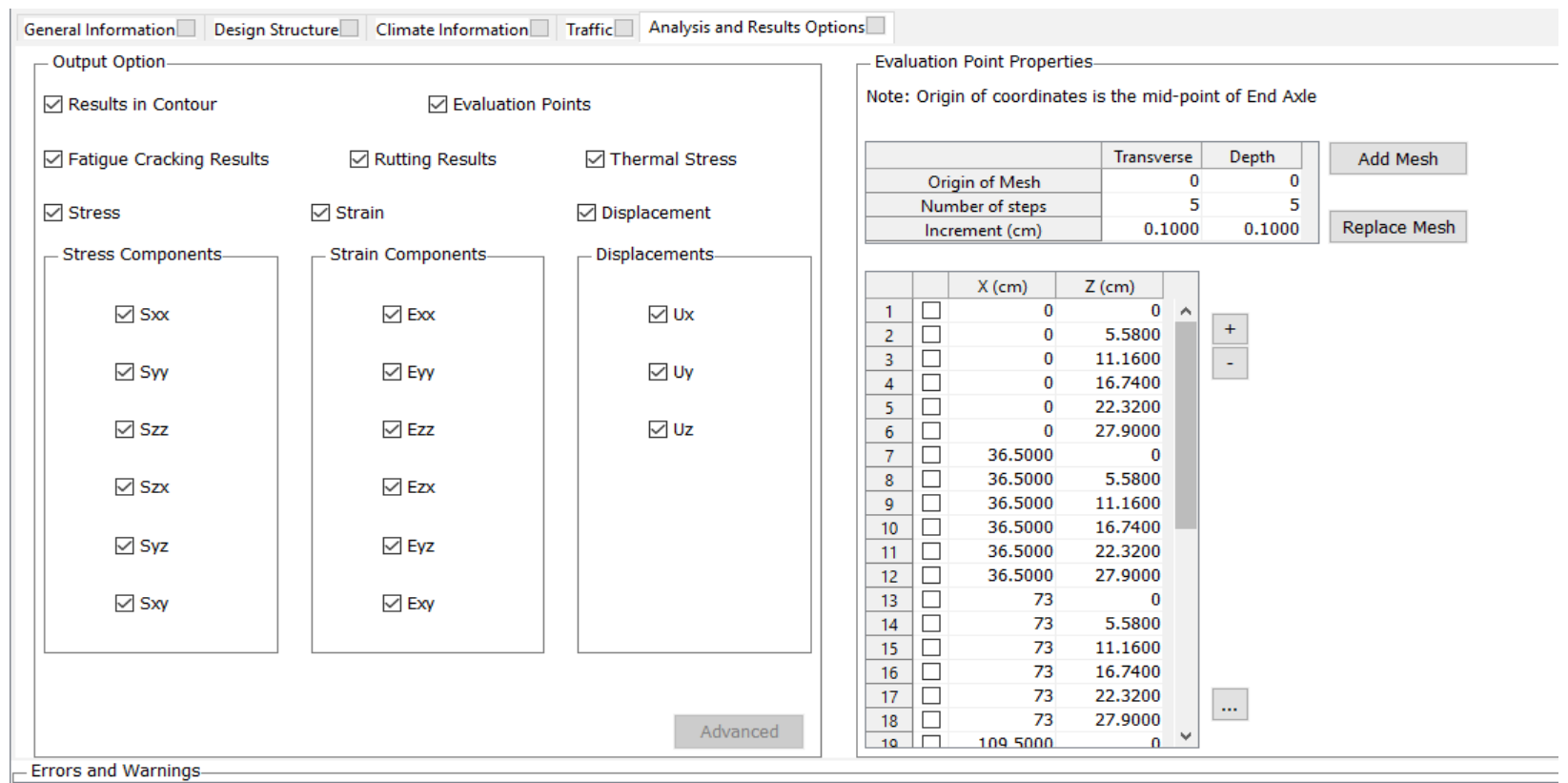

Figure 208: Output and Analysis Options for Run 2.4

\section{Run 2.5}

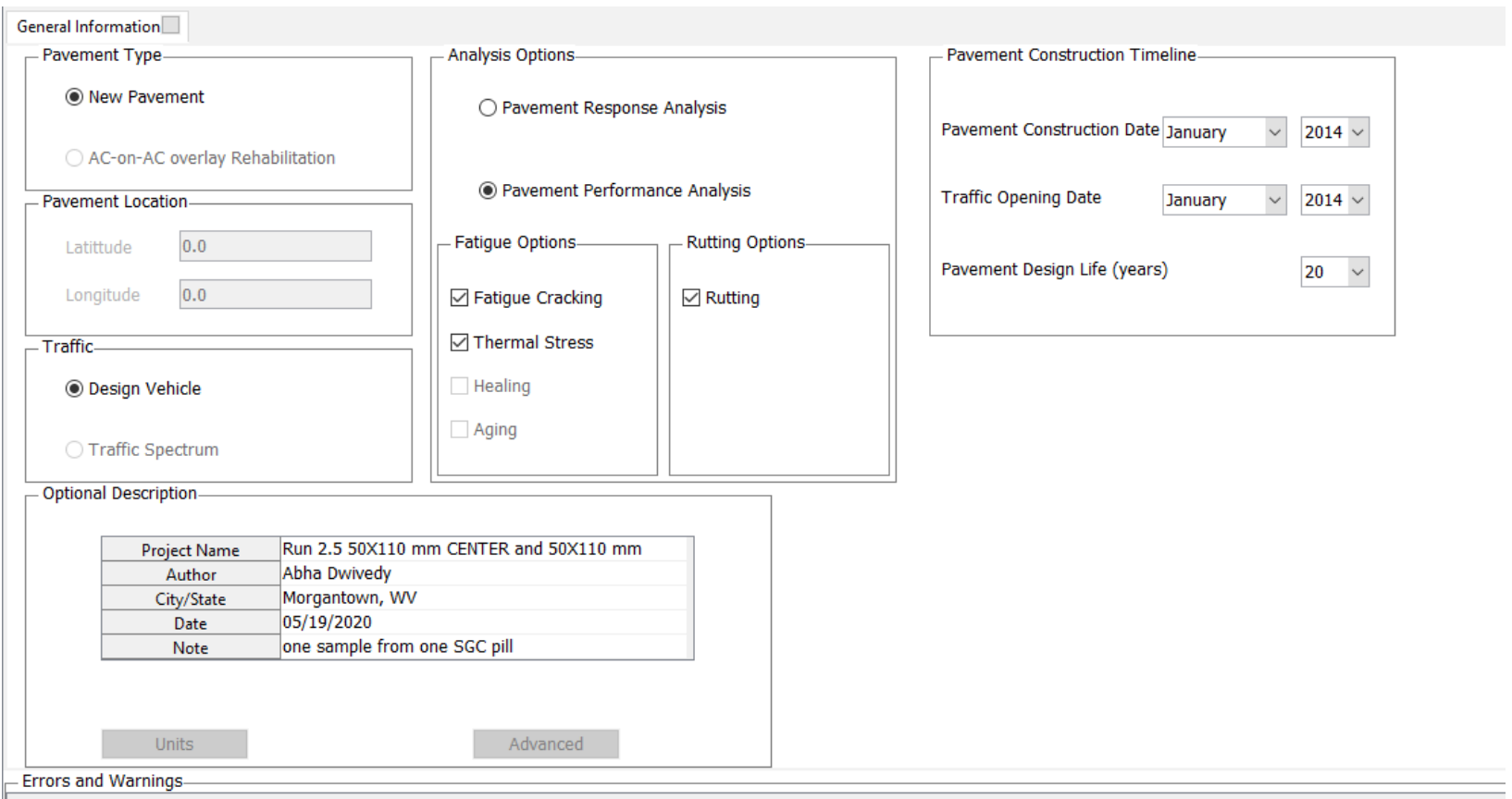

Figure 209: General Information for Run 2.5 


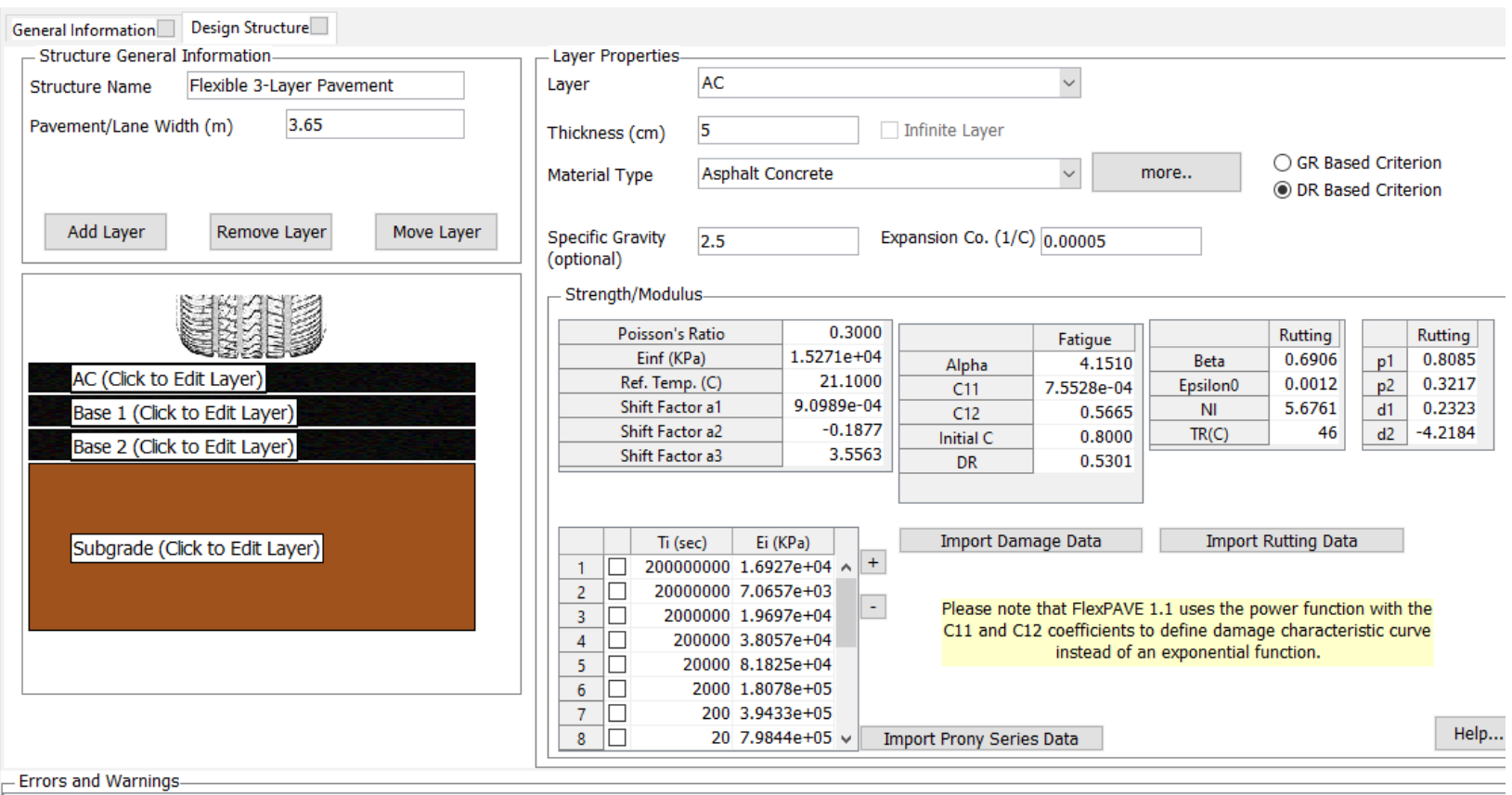

Figure 210: Design Structure of AC Layer for Run 2.5

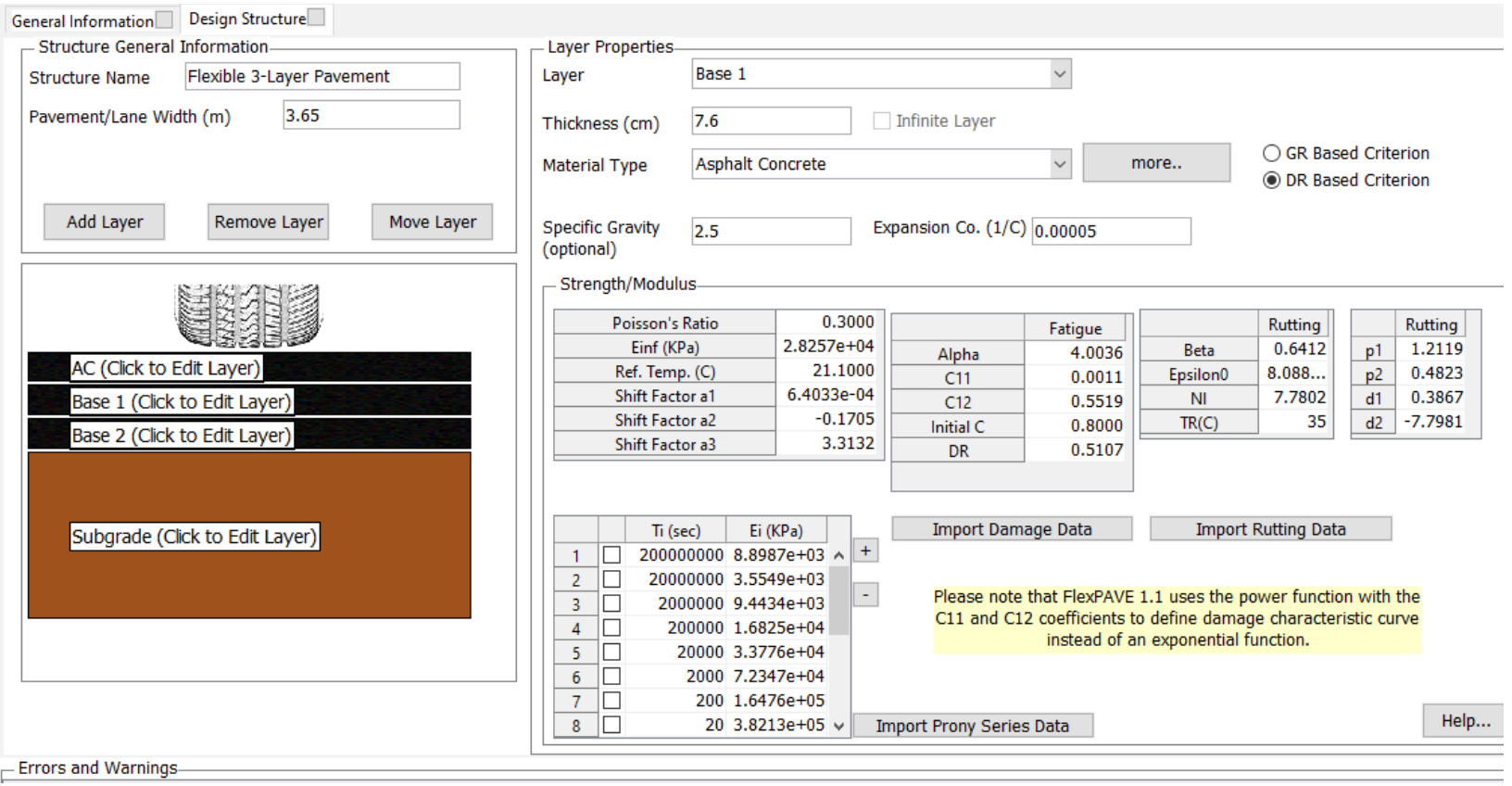

Figure 211: Design Structure of Base 1 Layer for Run 2.5 


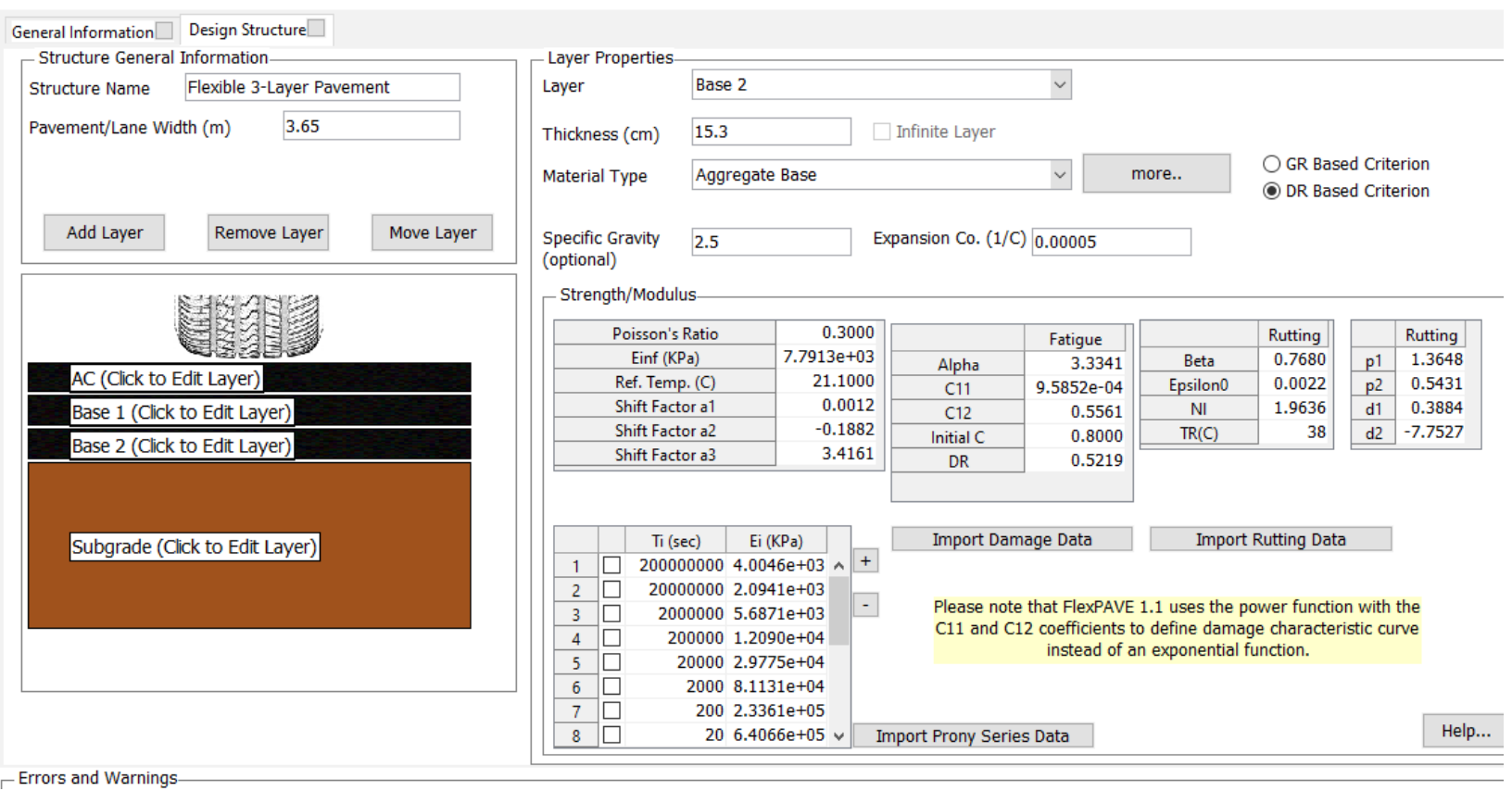

Figure 212: Design Structure of Base 2 Layer for Run 2.5

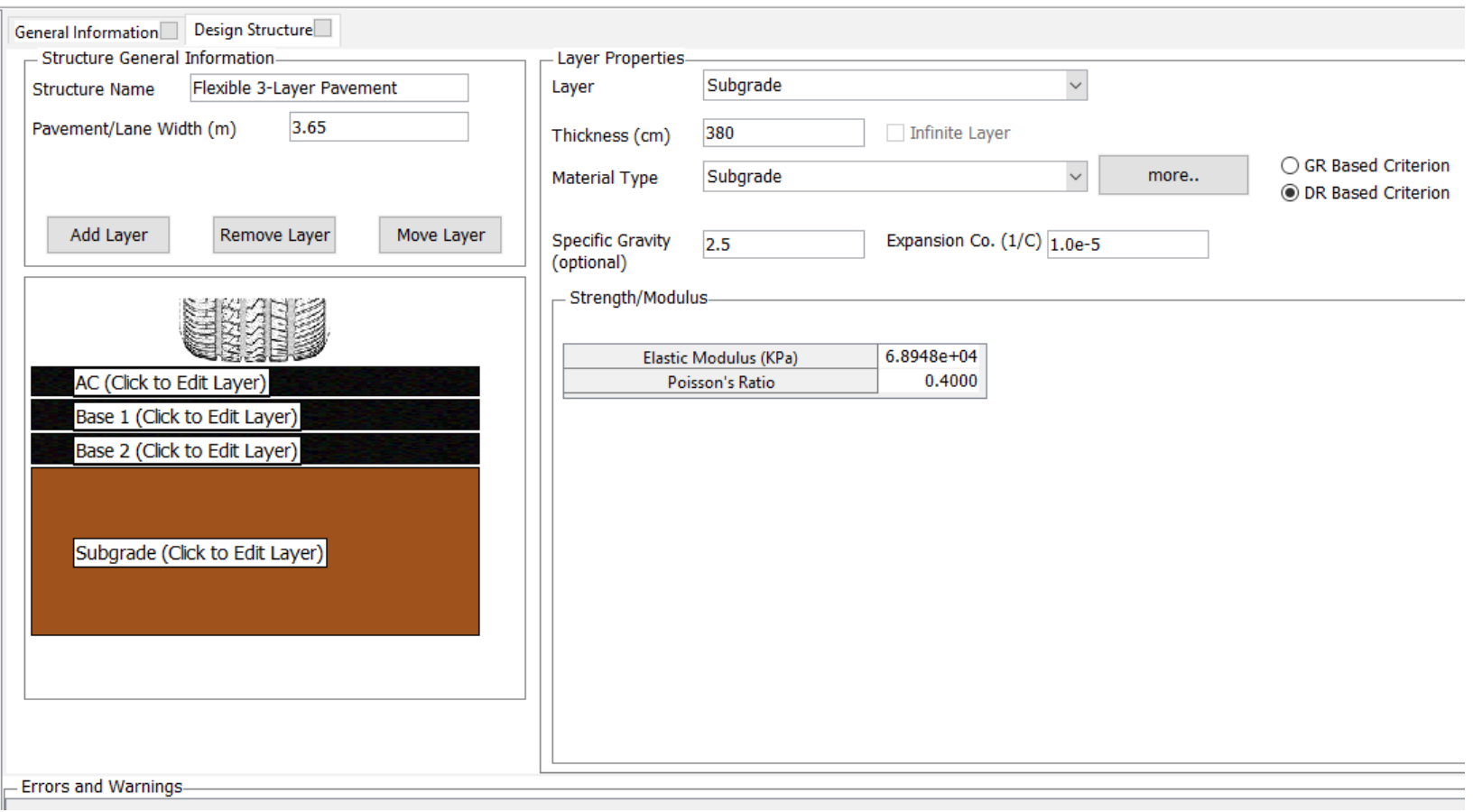

Figure 213: Design Structure of Subgrade Layer for Run 2.5 


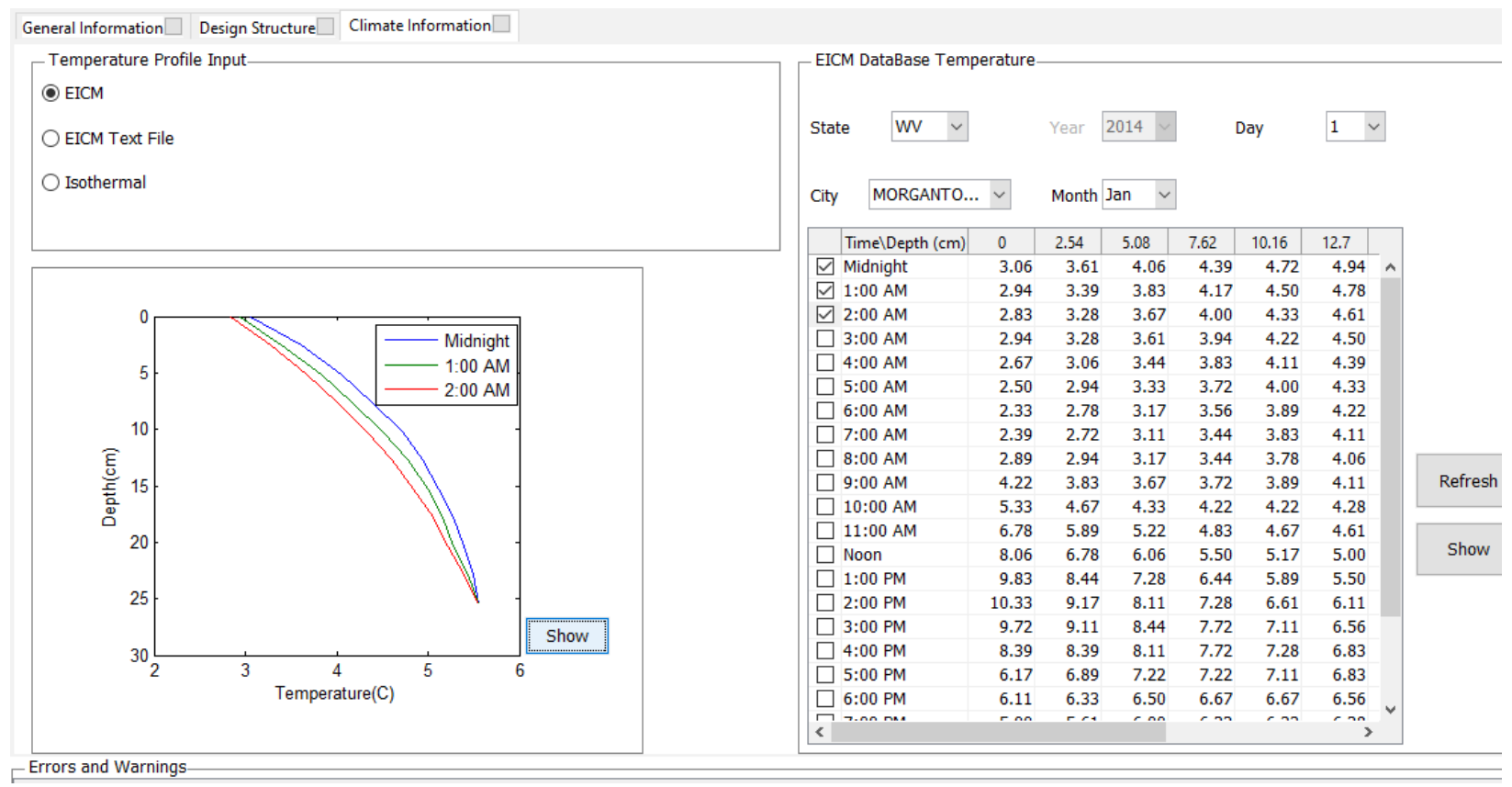

Figure 214: Climate Data for Run 2.5 

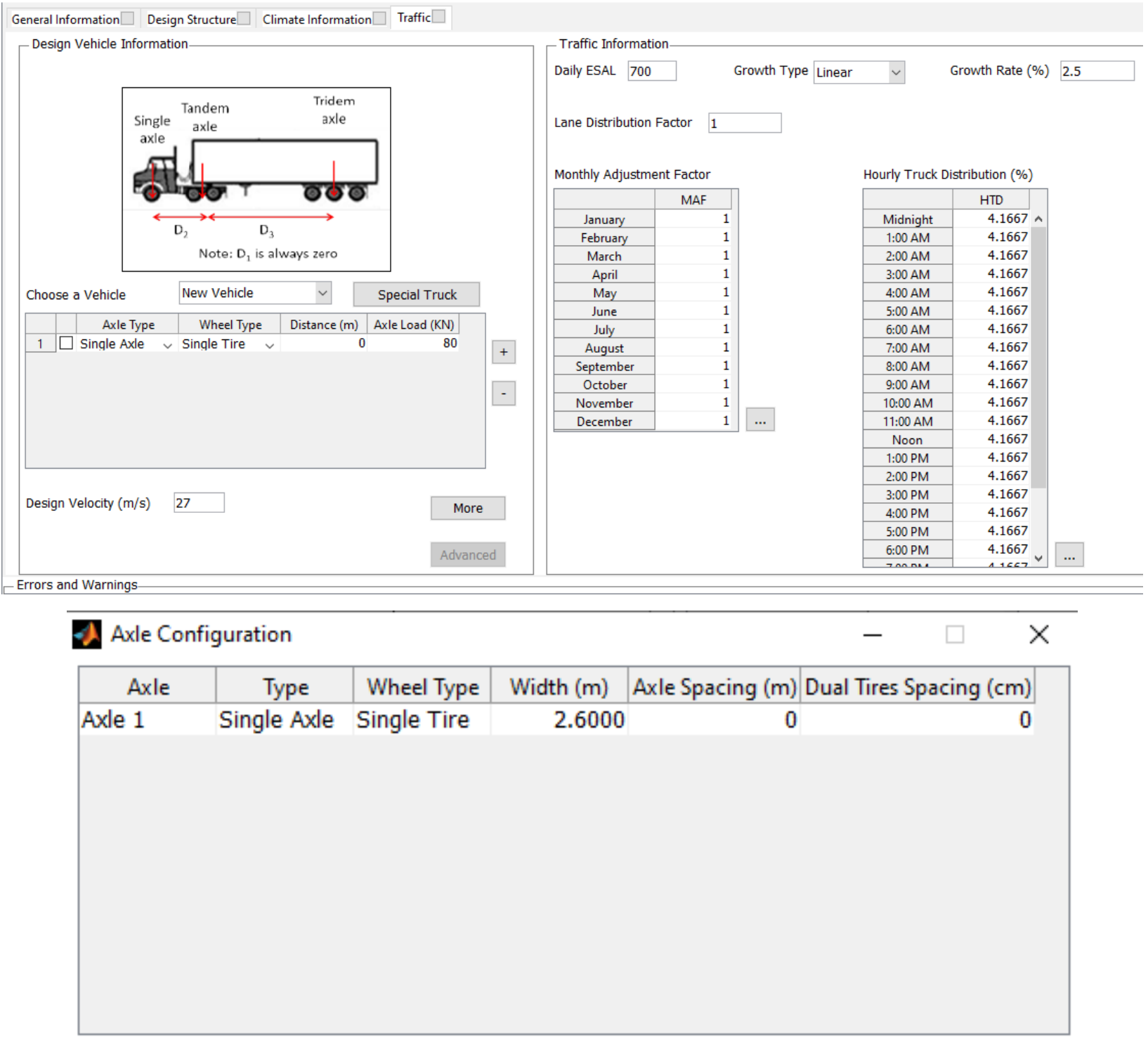

Tire Pressure (KPa) 827.37

\begin{tabular}{|c|c|c|c|c|}
\hline Contact Area Shape & Rectangular & $\checkmark$ & Aspect Ratio (length/widt) & 1.5714 \\
\hline
\end{tabular}

Shear Traction 0.0

OK

Cancel

Figure 215: Traffic Data for Run 2.5 


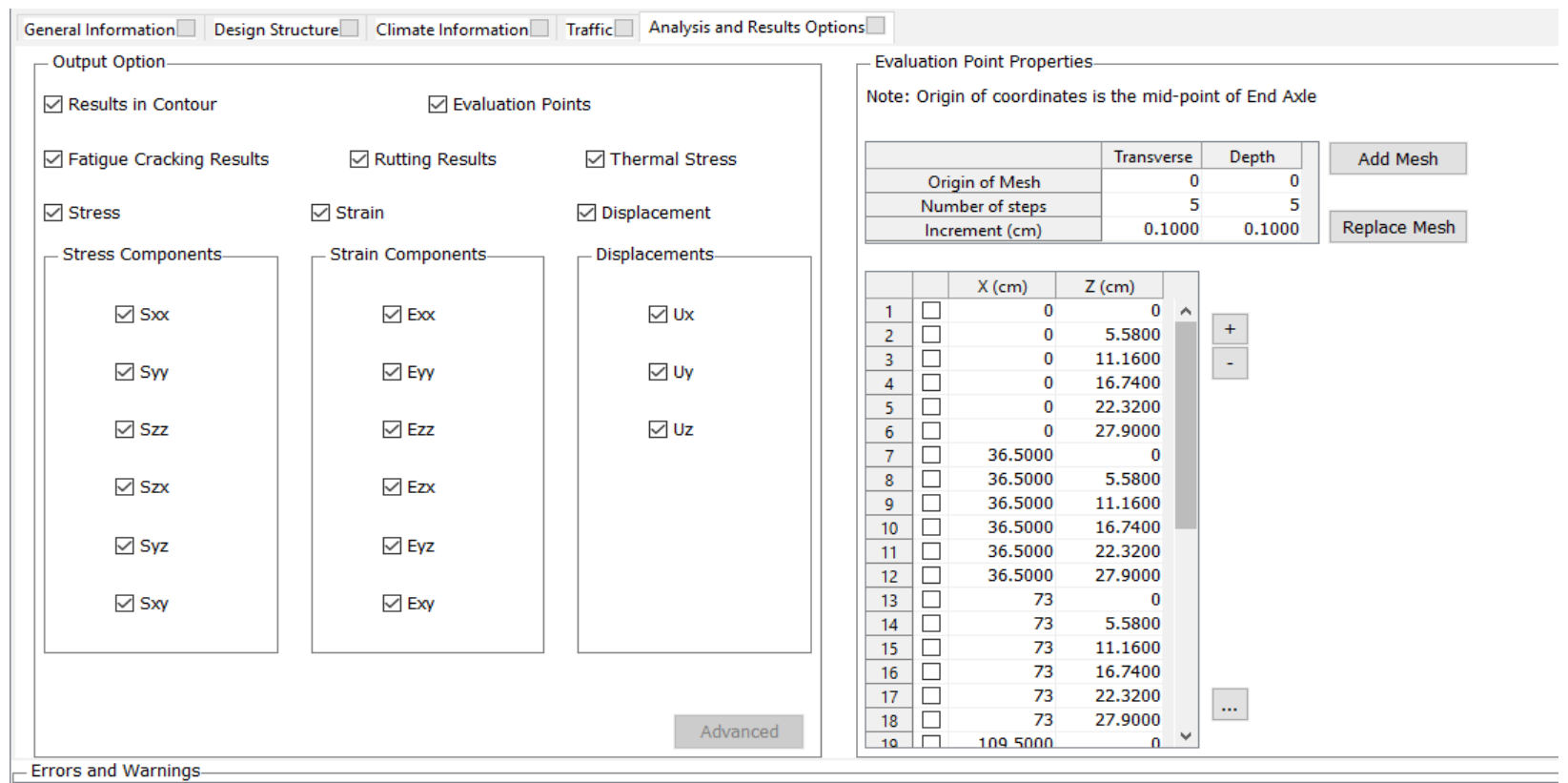

Figure 216: Output and Analysis Options for Run 2.5

\section{Run 2.6}

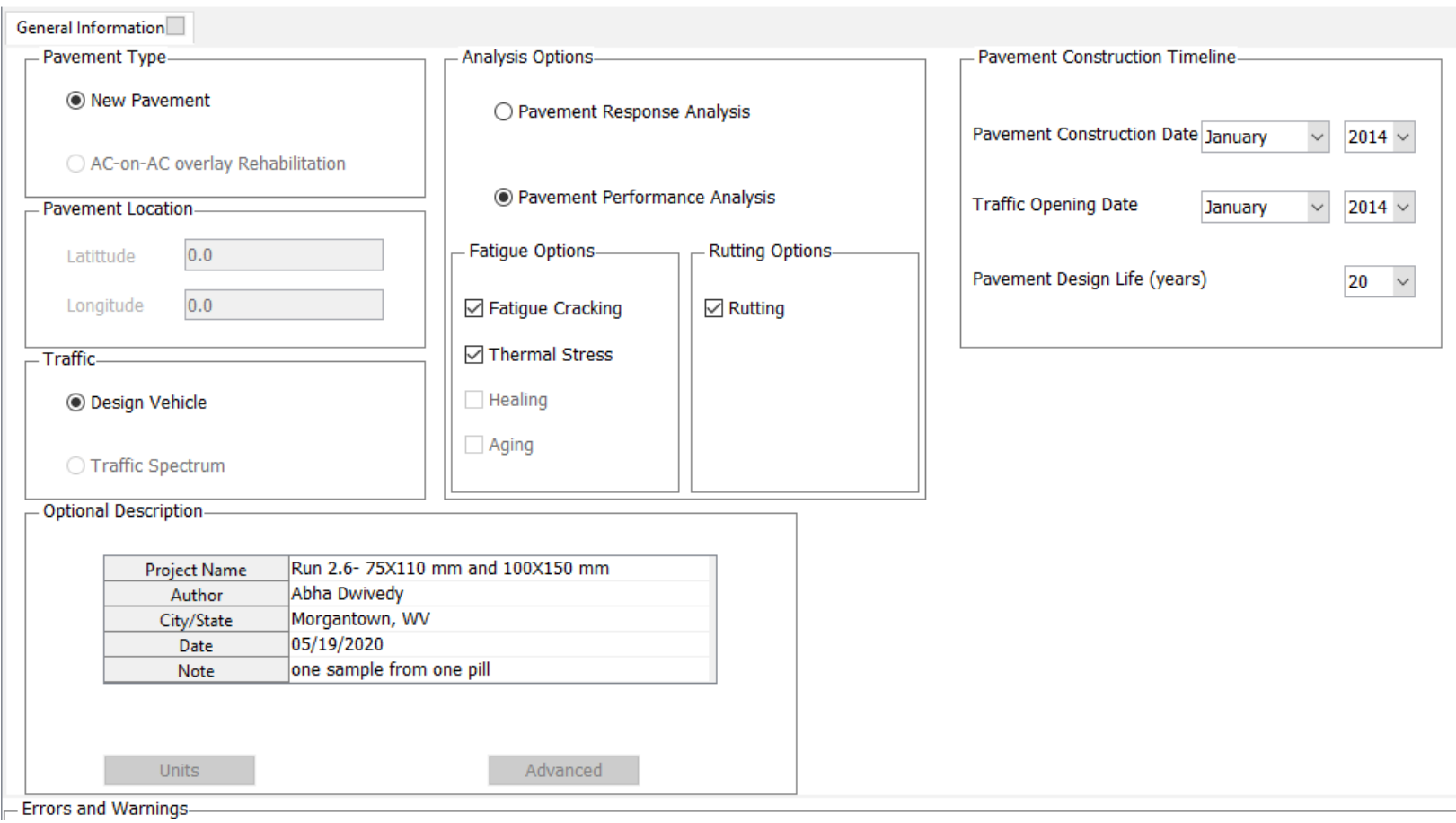

Figure 217: General Information for Run 2.6 


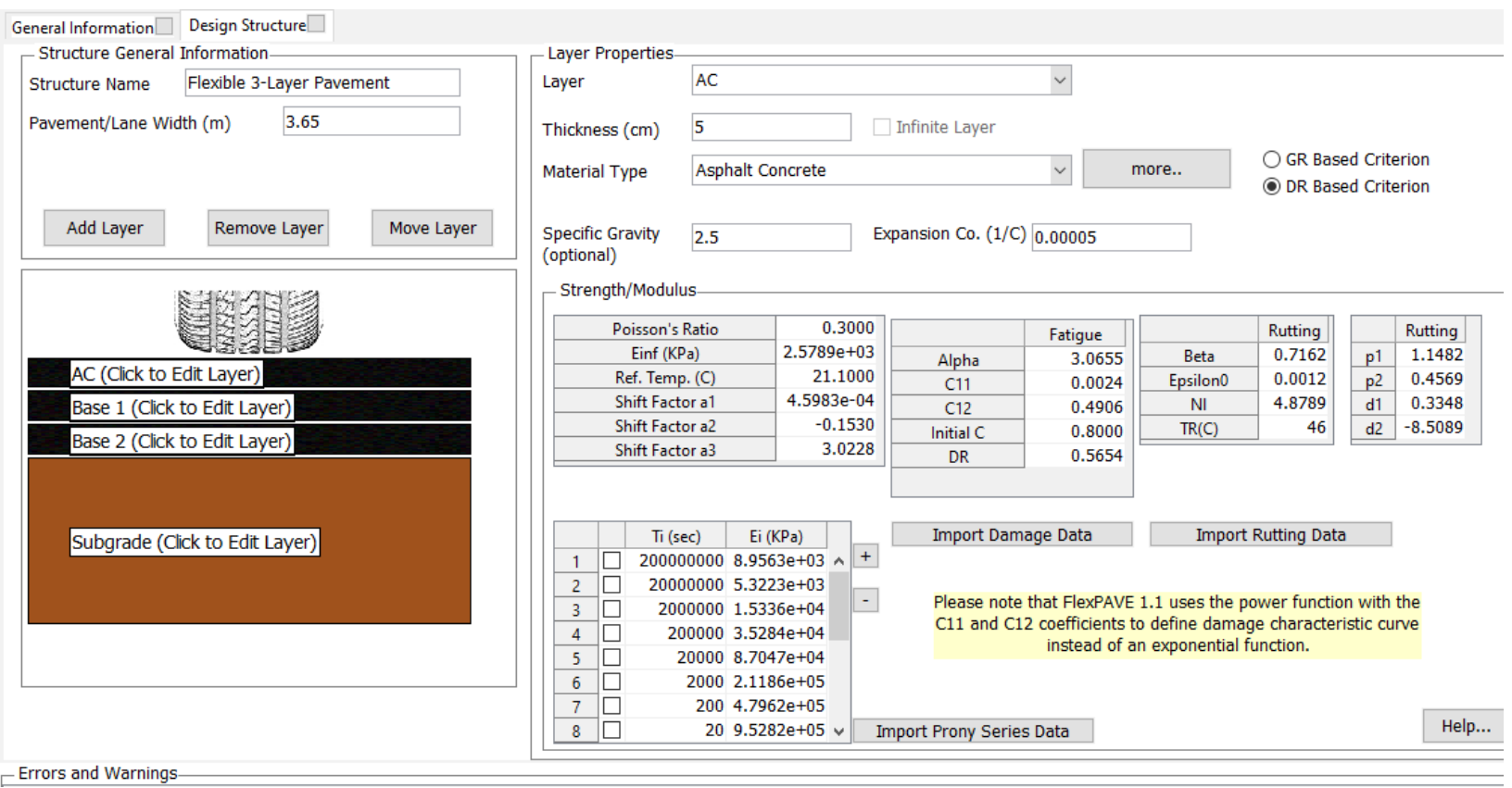

Figure 218: Design Structure of AC Layer for Run 2.6

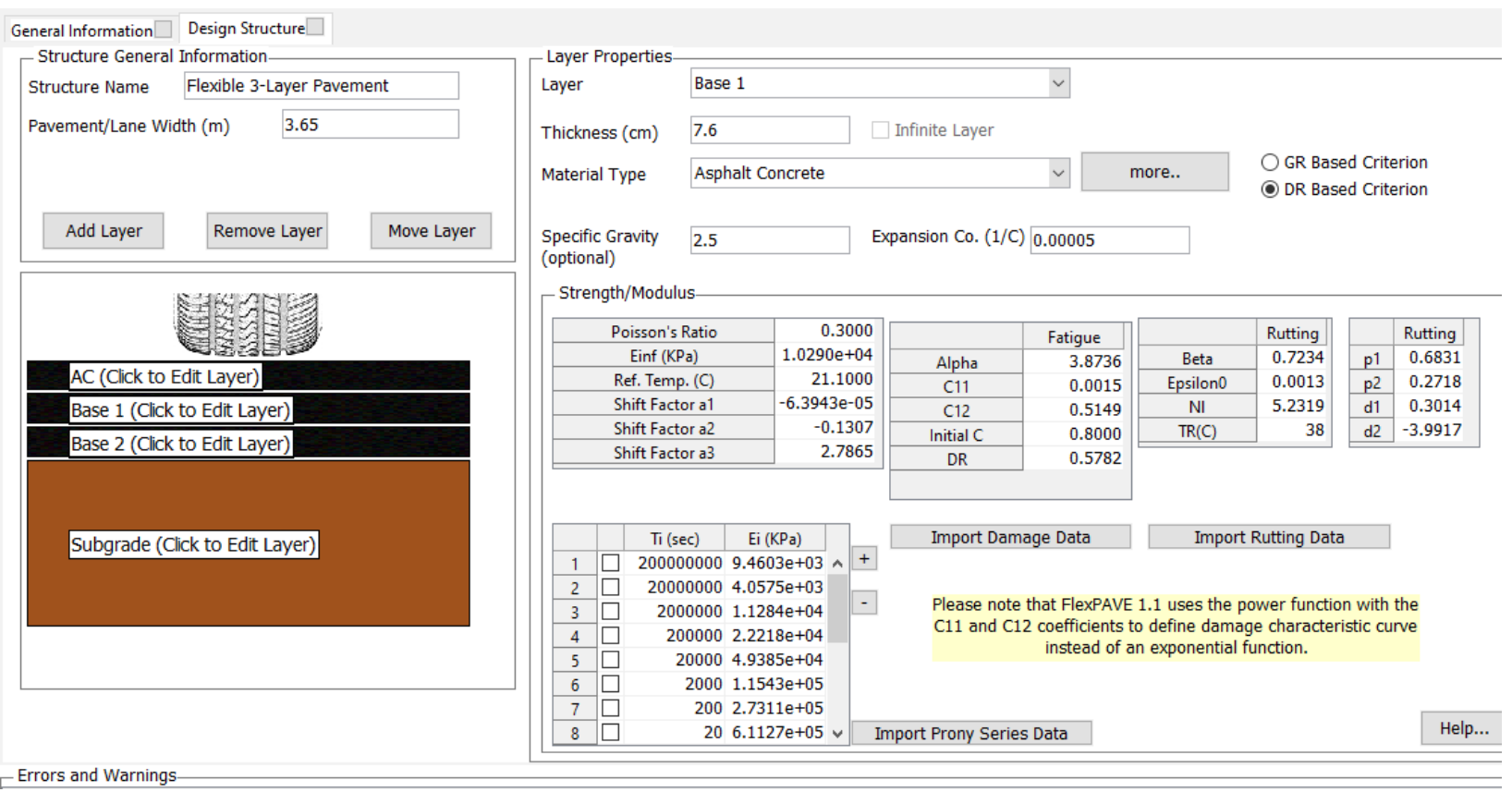

Figure 219: Design Structure of Base 1 Layer for Run 2.6 


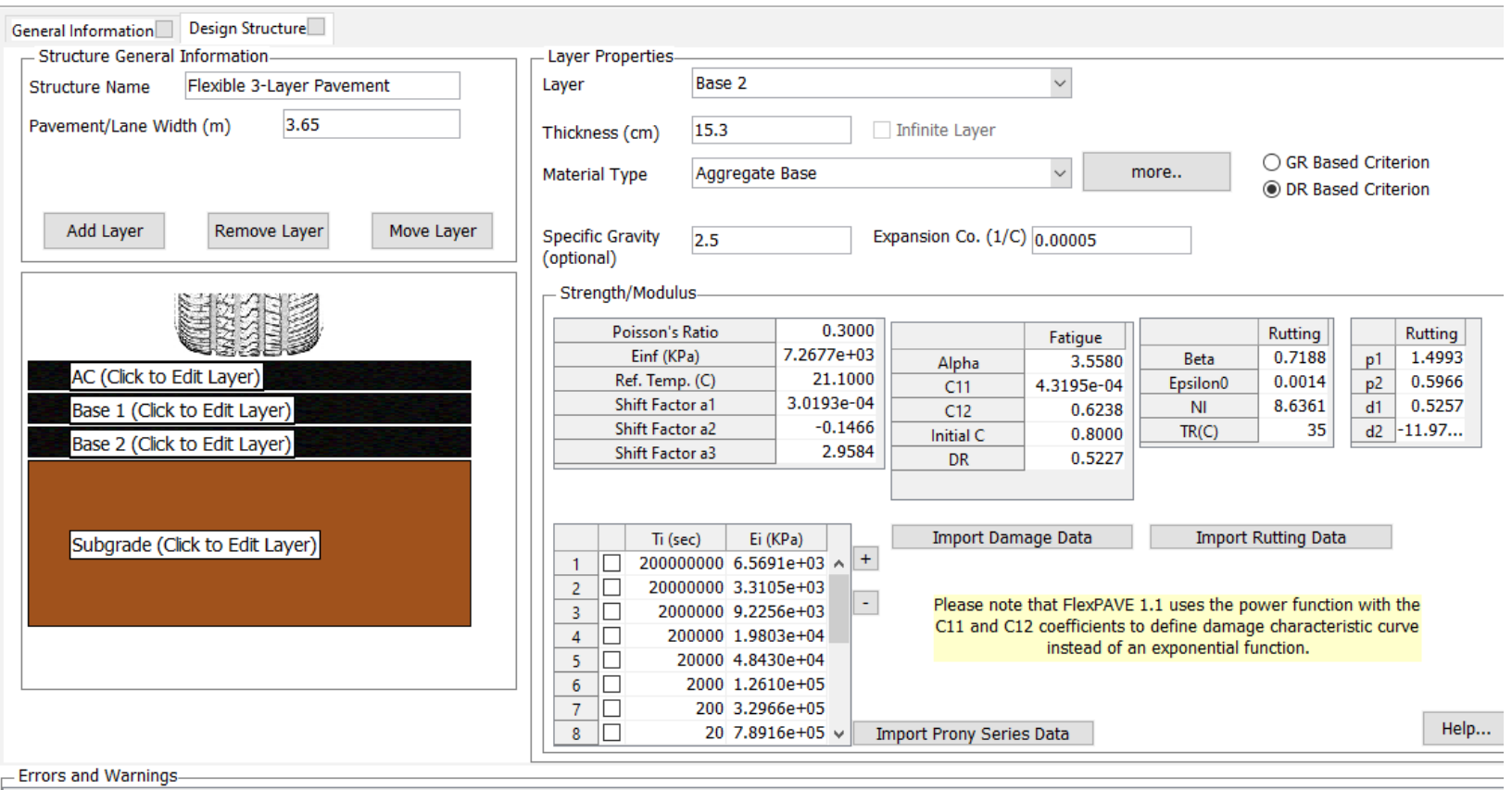

Figure 220: Design Structure of Base 2 Layer for Run 2.6

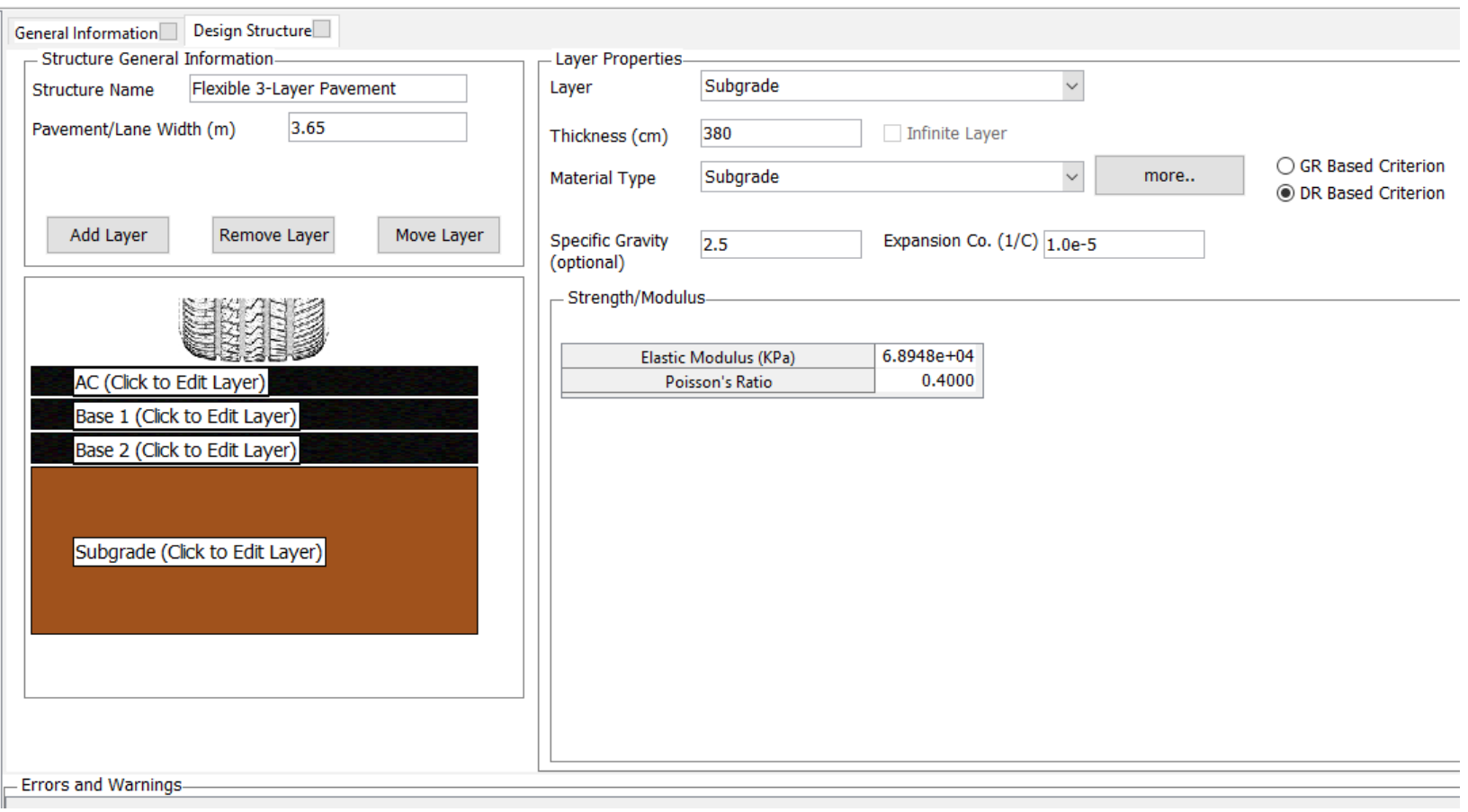

Figure 221: Design Structure of Subgrade Layer for Run 2.6 


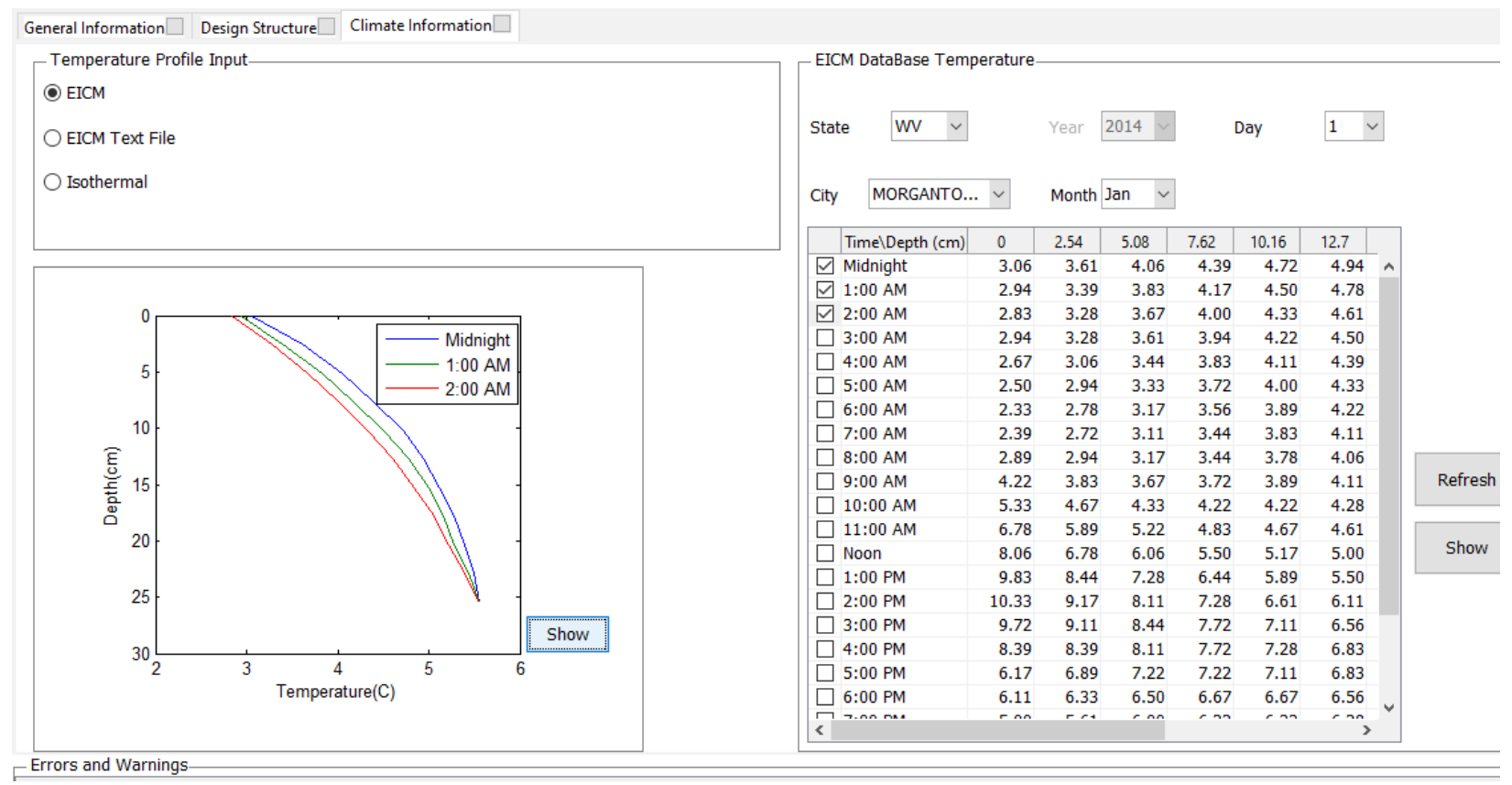

Figure 222: Climate Data for Run 2.6 


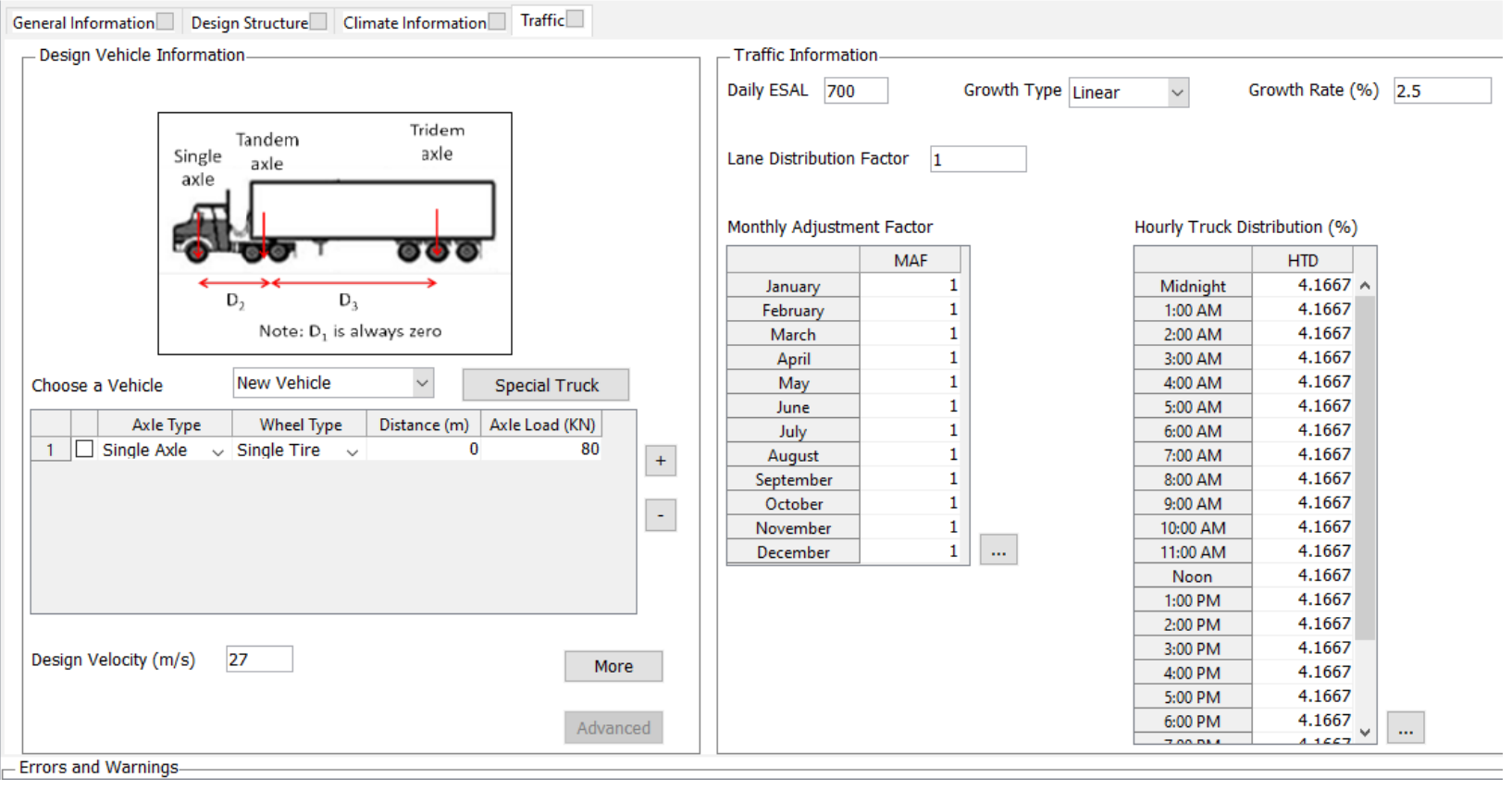

Axle Configuration

$-\quad \square \quad \times$

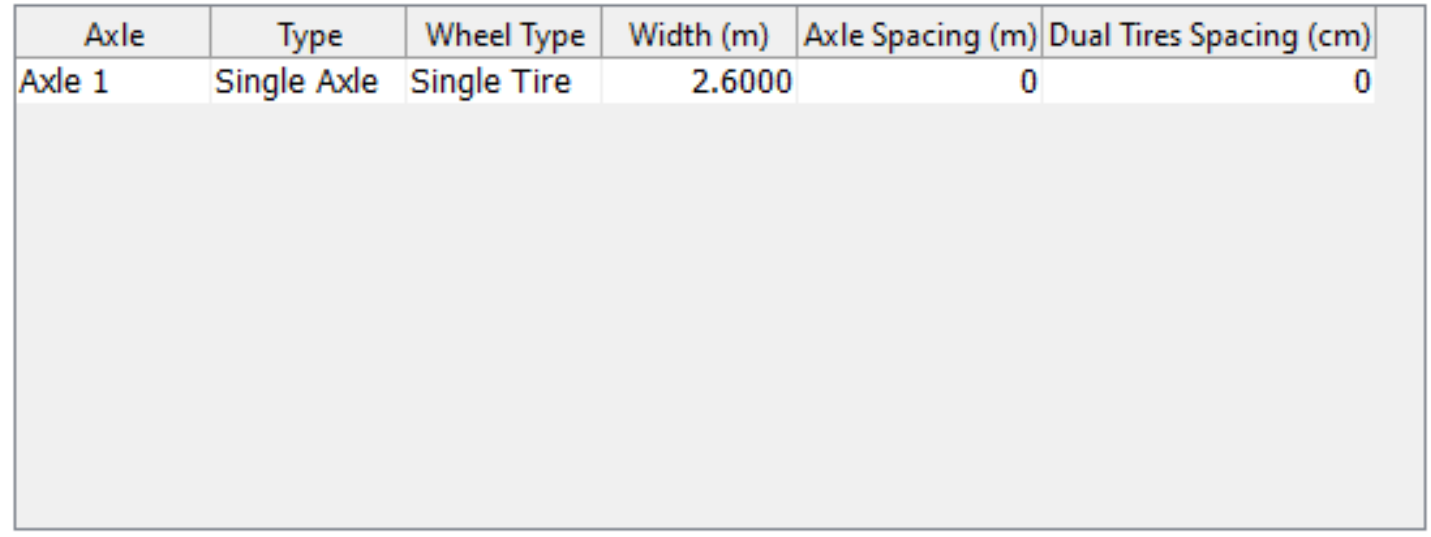

Tire Pressure (KPa) 827.37

Contact Area Shape Rectangular

Aspect Ratio (length/width) 1.5714

Shear Traction $\quad 0.0$

OK

Cancel

Figure 223: Traffic Data for Run 2.6 


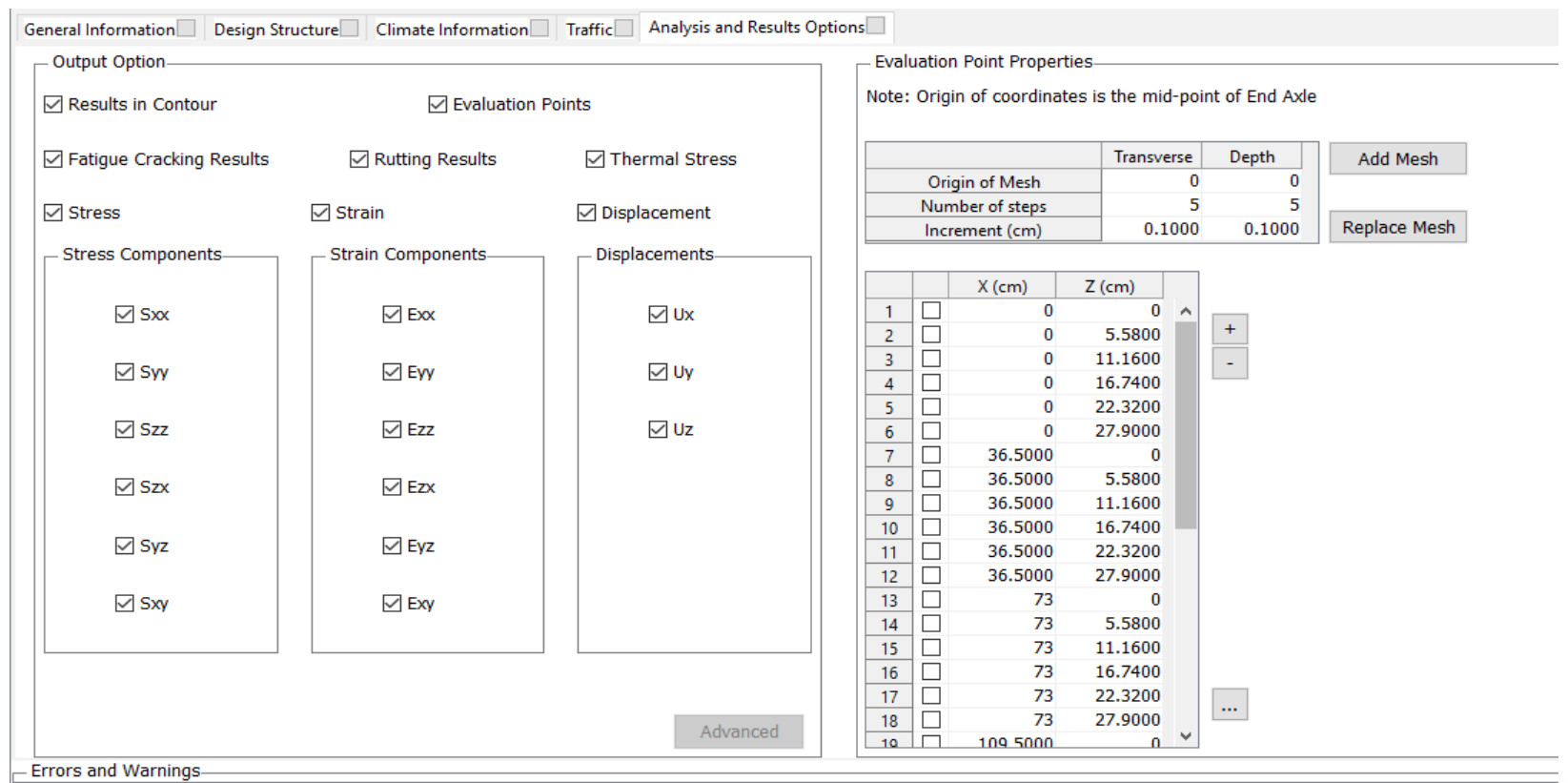

Figure 224: Output and Analysis Options for Run 2.6

\section{Run 2.7}

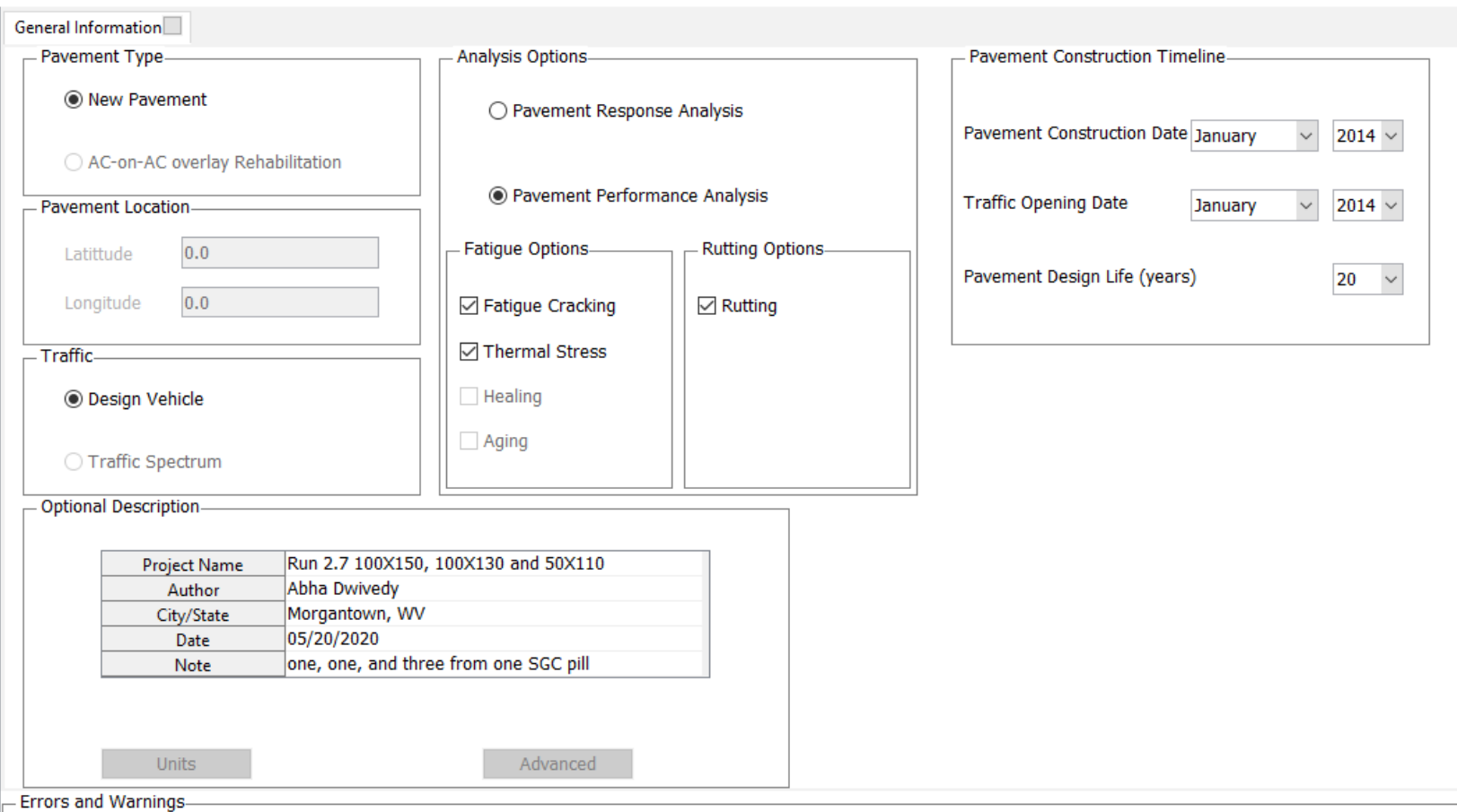

Figure 225: General Information for Run 2.7 

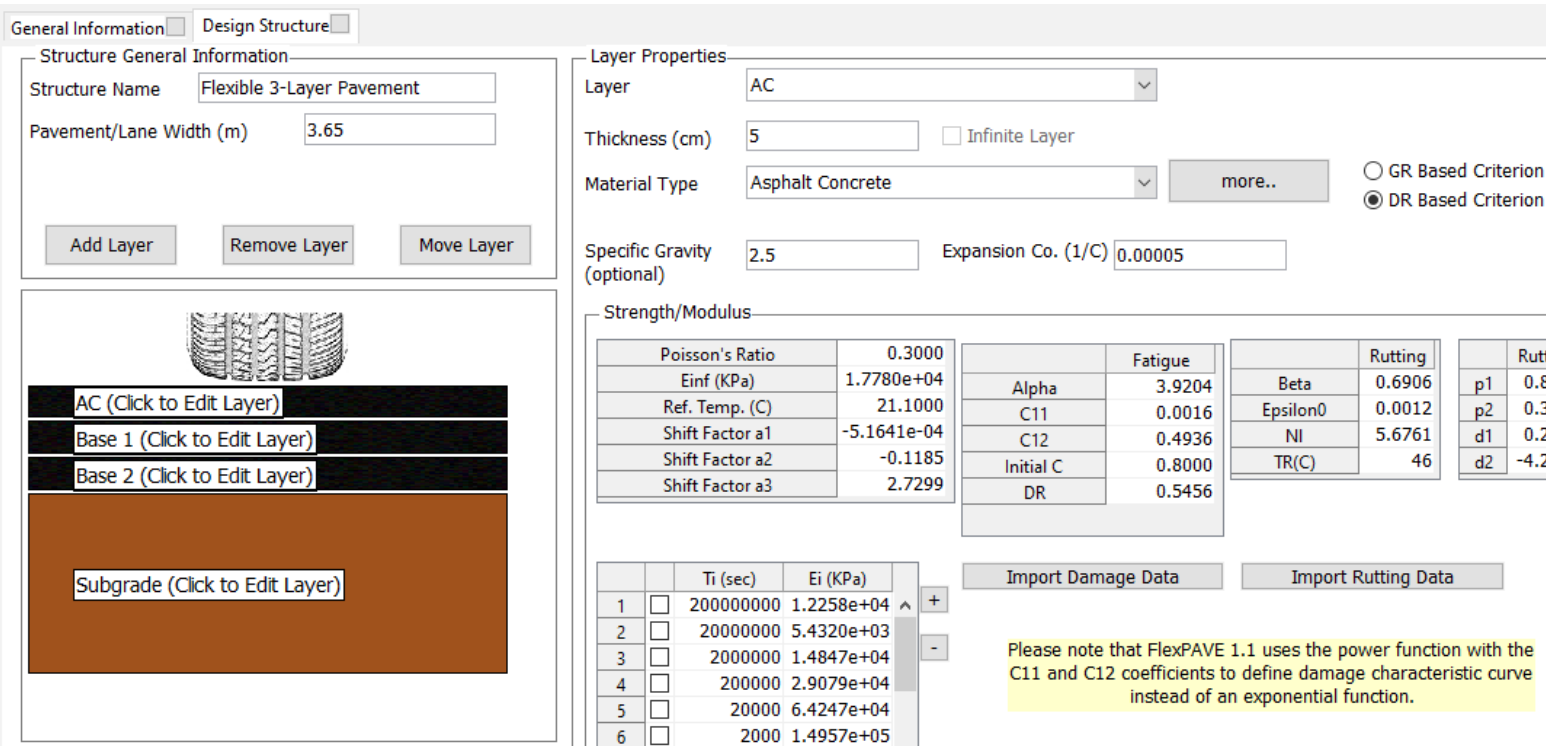

\begin{tabular}{|r|}
0.3000 \\
$1.7780 \mathrm{e}+04$ \\
21.1000 \\
$-5.1641 \mathrm{e}-04$ \\
-0.1185 \\
2.7299 \\
\hline
\end{tabular}

\section{\begin{tabular}{|r|r|}
\hline & \multicolumn{1}{|c|}{ Fatigue } \\
\hline Alpha & 3.9204 \\
\cline { 1 - 1 } & 0.0011 \\
\hline
\end{tabular}} 0.0016

0.8000 Initial DR

\section{Import Damage Data}

Import Rutting Data

$2000000001.2258 \mathrm{e}+04 \wedge+$

$200000005.4320 \mathrm{e}+03$

$20000001.4847 \mathrm{e}+04$

$2000002.9079 \mathrm{e}+04$

$200006.4247 \mathrm{e}+04$

$20001.4957 \mathrm{e}+05$

$2003.5223 \mathrm{e}+05$

\begin{tabular}{l|l|l}
\hline $207.7864 e+05 ~ \checkmark$ & Import Prony Series Data \\
\hline
\end{tabular}

Errors and Warning

Figure 226: Design Structure of AC Layer for Run 2.7

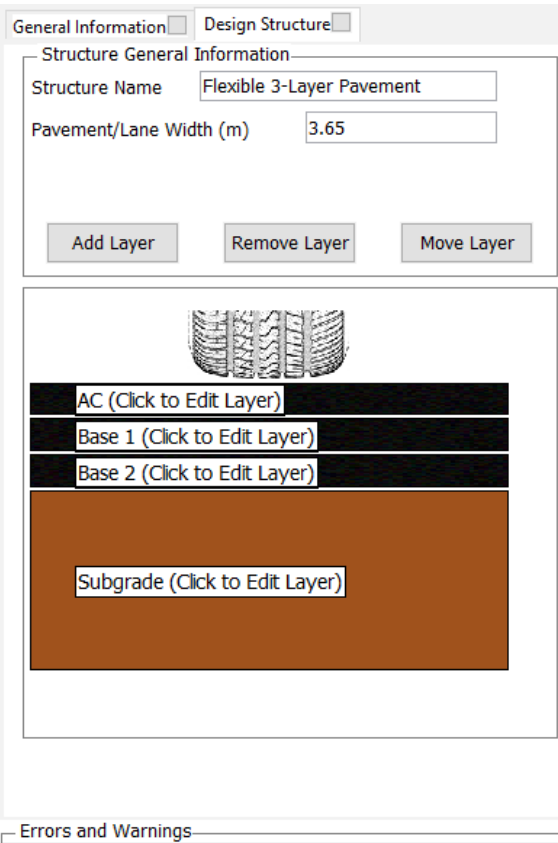

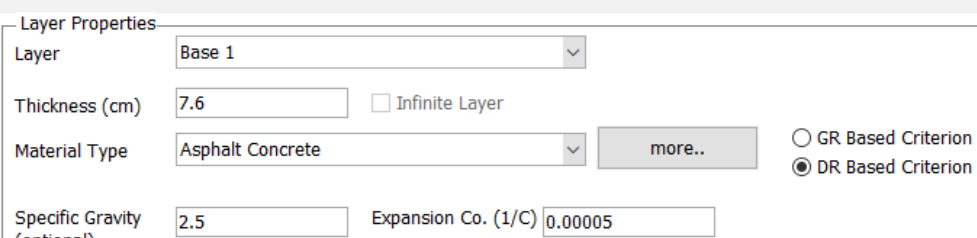

(optional)

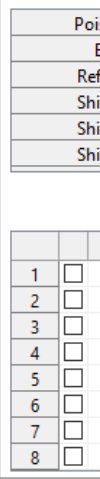

Poisson's Ratio Einf $(\mathrm{KPa})$

Ref. Temp. (C)

Shift Factor a

Shift Factor a2

Shift Factor a3

$\begin{array}{r}0.3000 \\ 3.0759 \mathrm{e}+04 \\ 21.1000 \\ -0.0020 \\ -0.0774 \\ 2.5365\end{array} \mid$

\begin{tabular}{|c|r|}
\hline & Fatigue \\
\hline Alpha & 4.0367 \\
\cline { 1 - 1 } C11 & 0.0024 \\
\cline { 1 - 1 } C12 & 0.4667 \\
\cline { 1 - 1 } Initial C & 0.8000 \\
\cline { 1 - 1 } DR & 0.5376 \\
\cline { 1 - 1 } & \\
\hline
\end{tabular}

Import Damage Data

$966 \mathrm{e}+04 \wedge+$

$200000005.0313 e+03$

$20000001.3408 \mathrm{e}+04$

$2000002.4691 \mathrm{e}+04$

$200005.1262 \mathrm{e}+04$

$20001.1330 \mathrm{e}+05$

$2002.6179 \mathrm{e}+05$

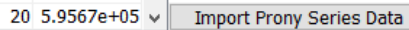

Please note that FlexPAVE 1.1 uses the power function with the

$\mathrm{C} 11$ and $\mathrm{C} 12$ coefficients to define damage characteristic curve
$\mathrm{Ti}(\mathrm{sec}) \quad \mathrm{Ei}(\mathrm{KPa})$

Import Rutting Data instead of an exponential function.

\section{Figure 227: Design Structure of Base 1 Layer for Run 2.7}




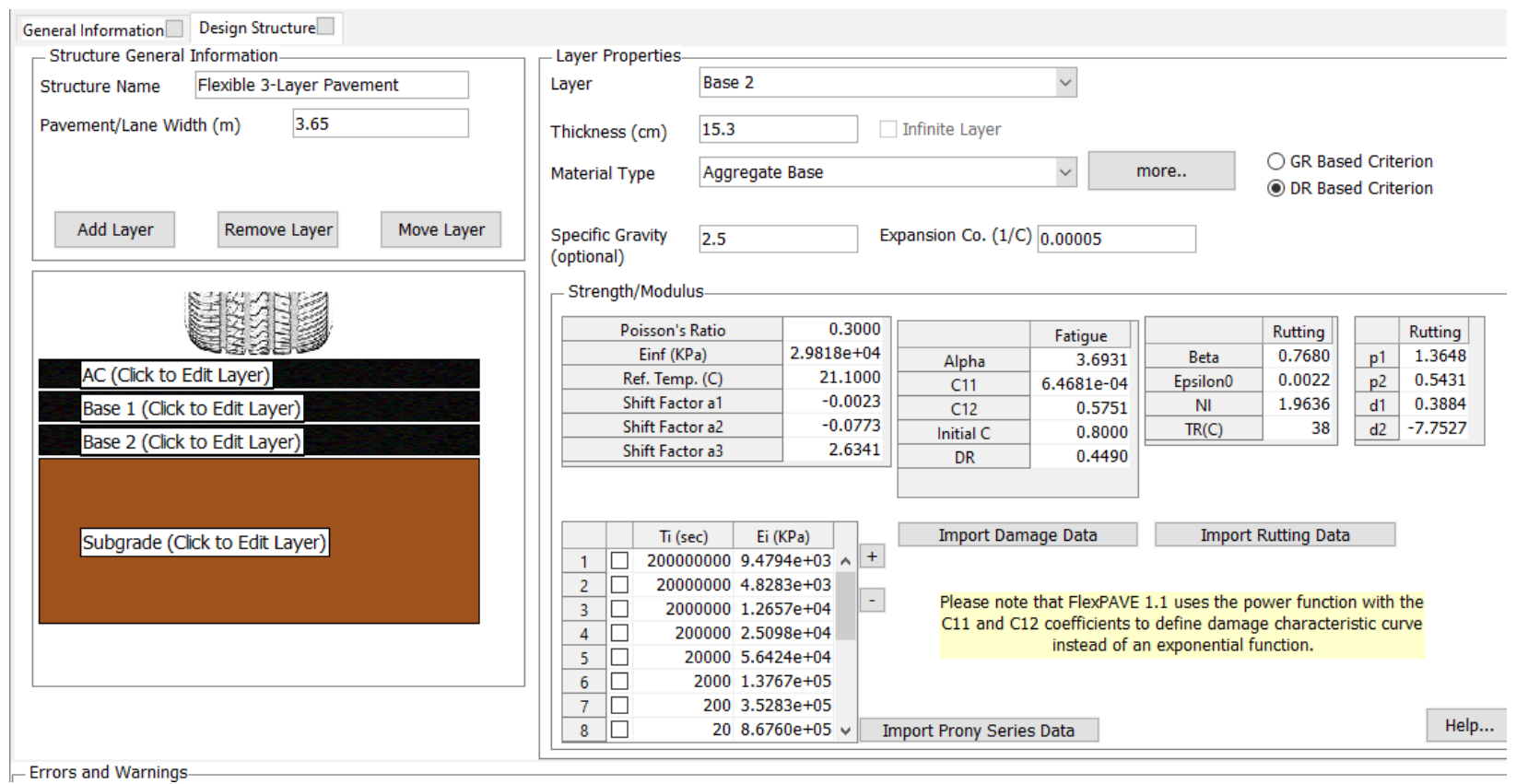

Figure 228: Design Structure of Base 2 Layer for Run 2.7

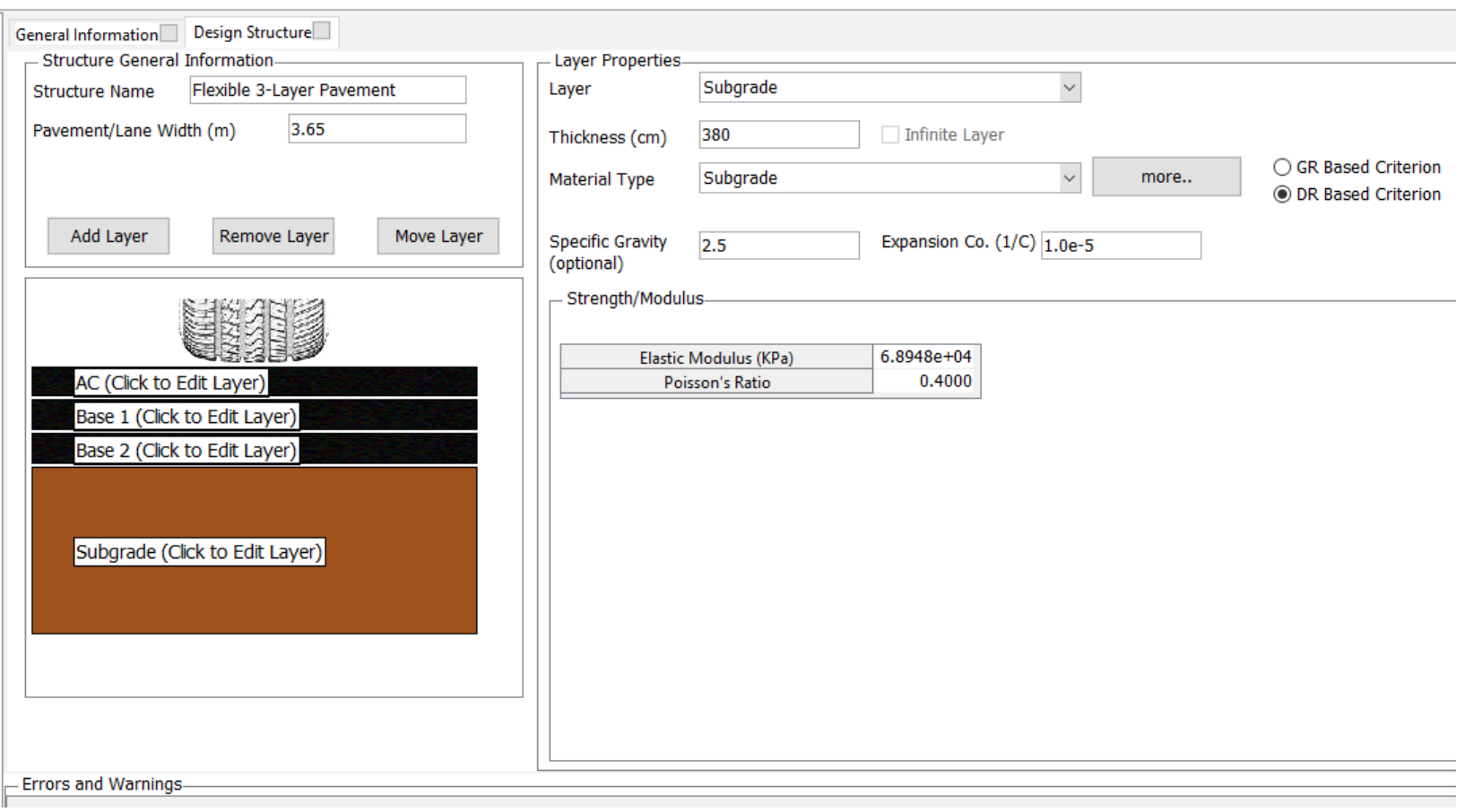

Figure 229: Design Structure of Subgrade Layer for Run 2.7 


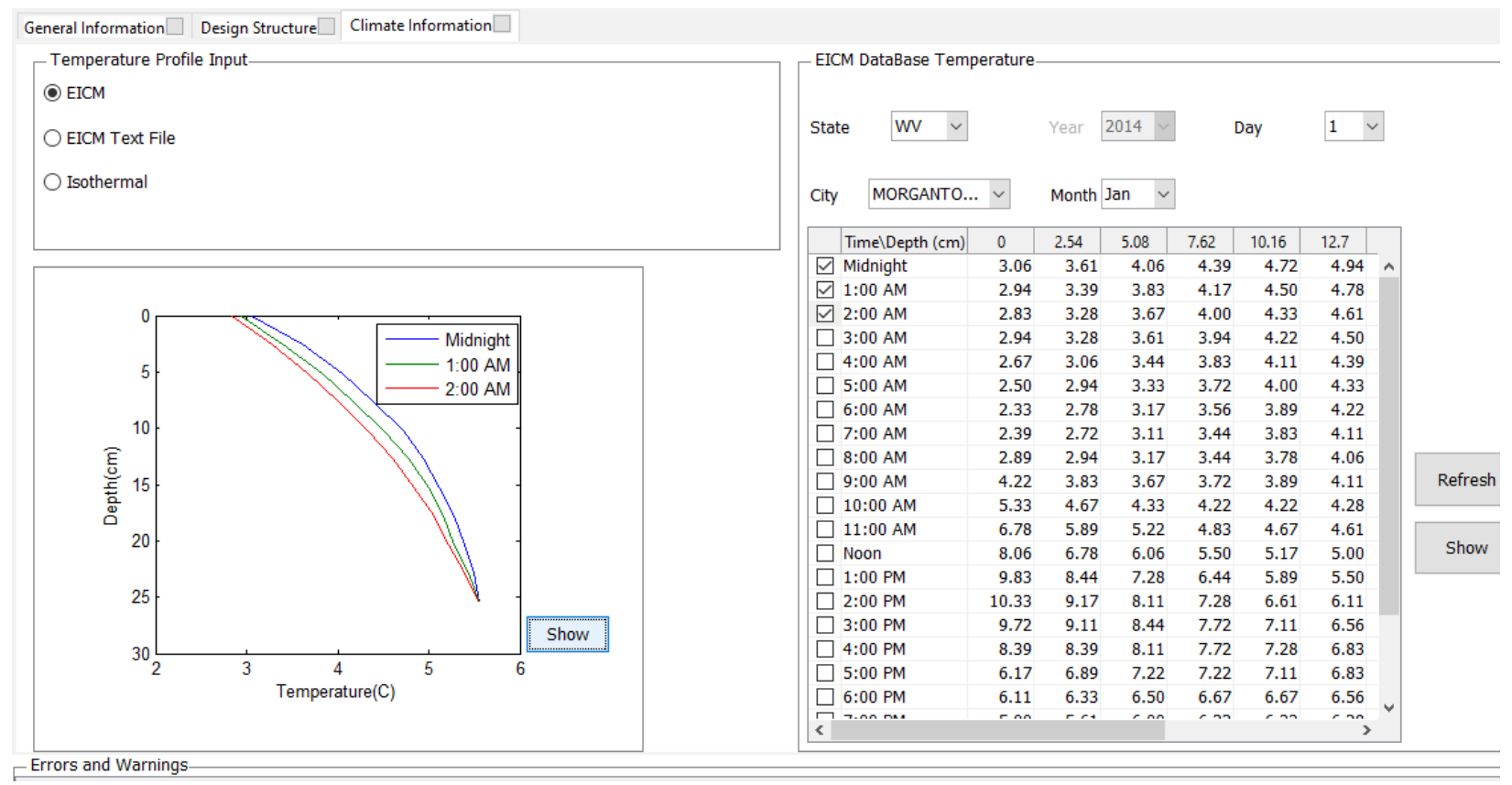

Figure 230: Climate Data for Run 2.7 

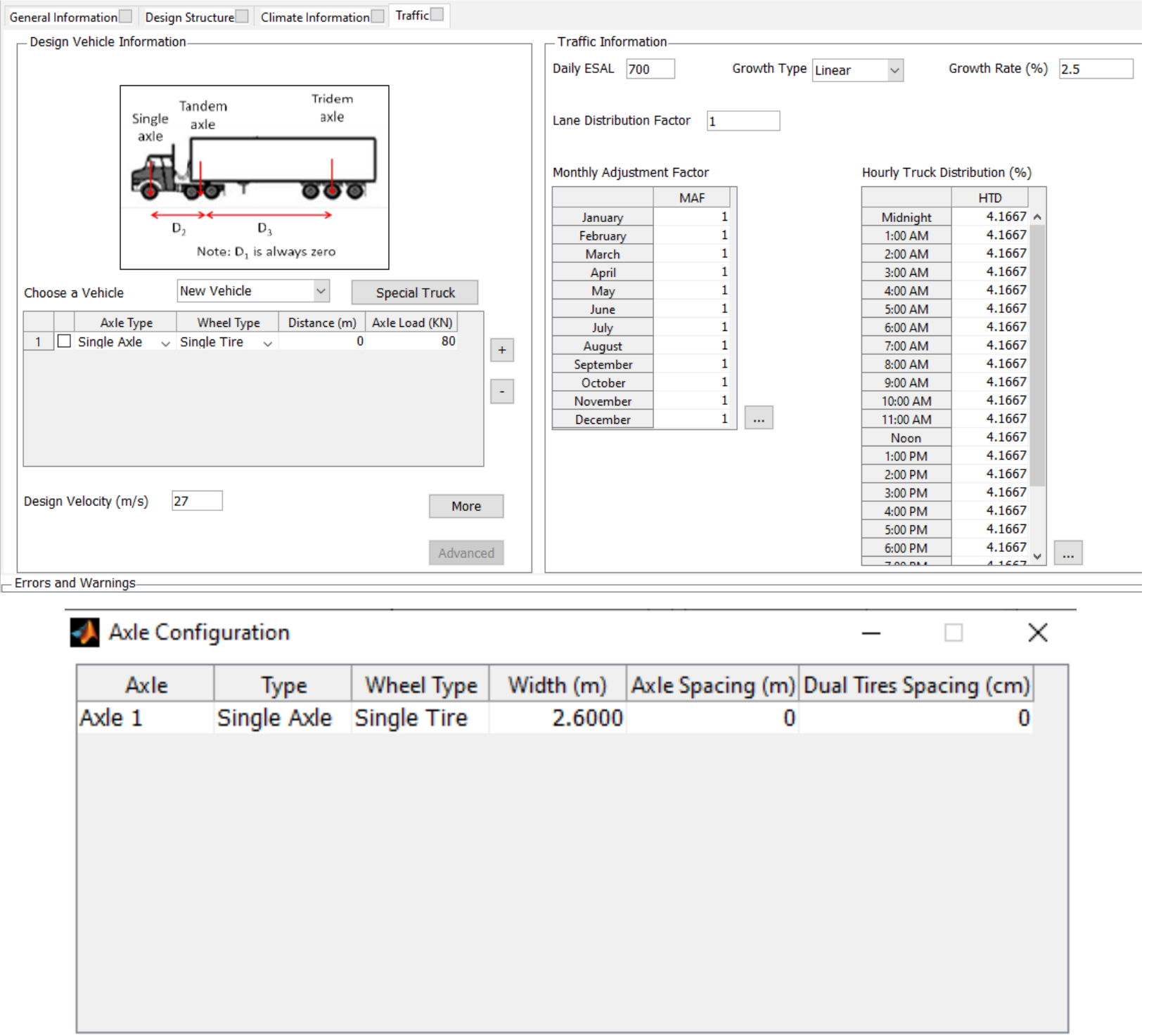

Tire Pressure (KPa) 827.37

\begin{tabular}{|c|c|c|c|c|}
\hline Contact Area Shape & Rectangular & $\checkmark$ & Aspect Ratio (length/widt) & 1.5714 \\
\hline
\end{tabular}

Shear Traction 0.0

OK

Cancel

Figure 231: Traffic Data for Run 2.7 


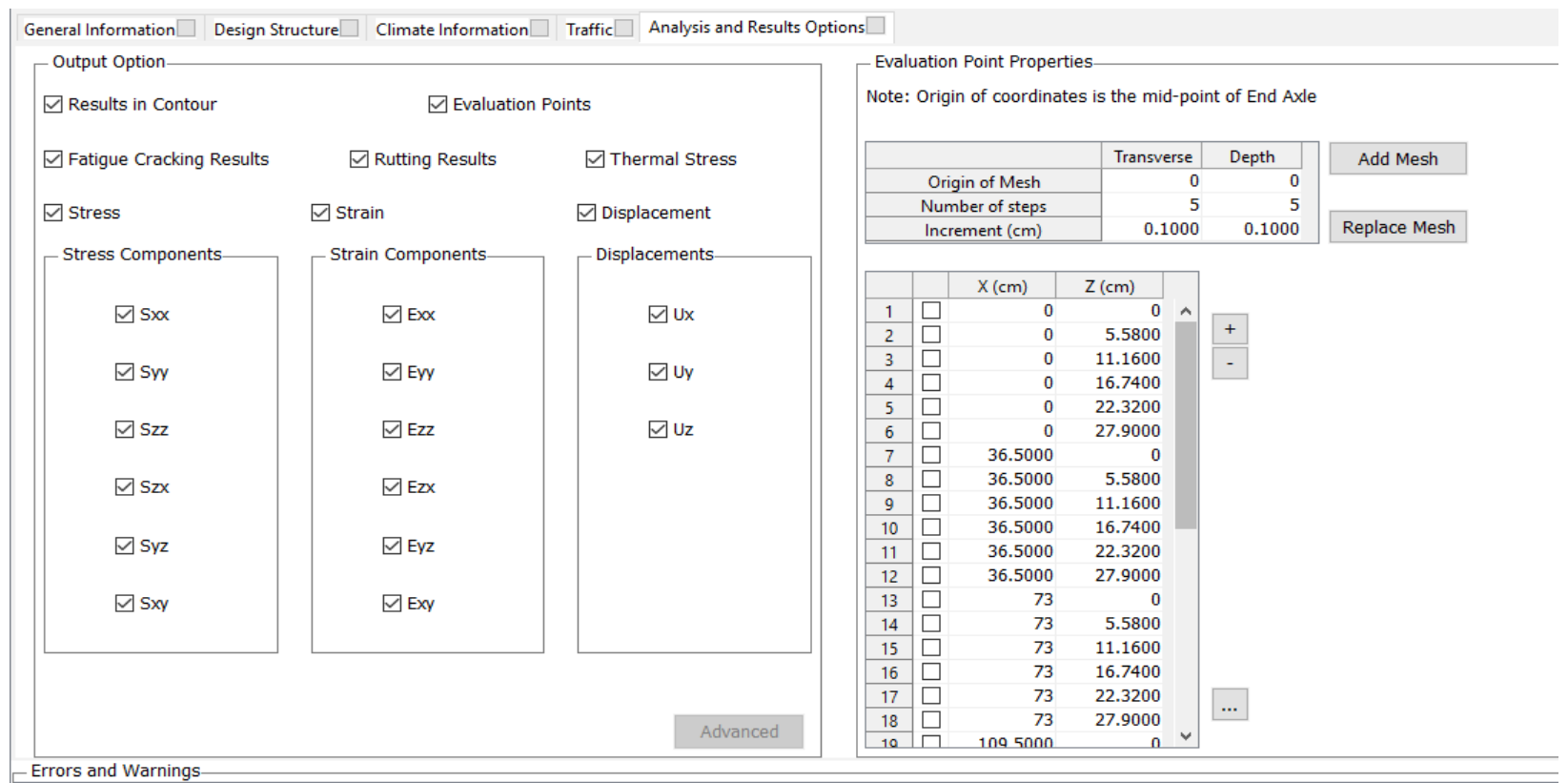

Figure 232: Output and Analysis Options for Run 2.7

\section{Run 2.8}

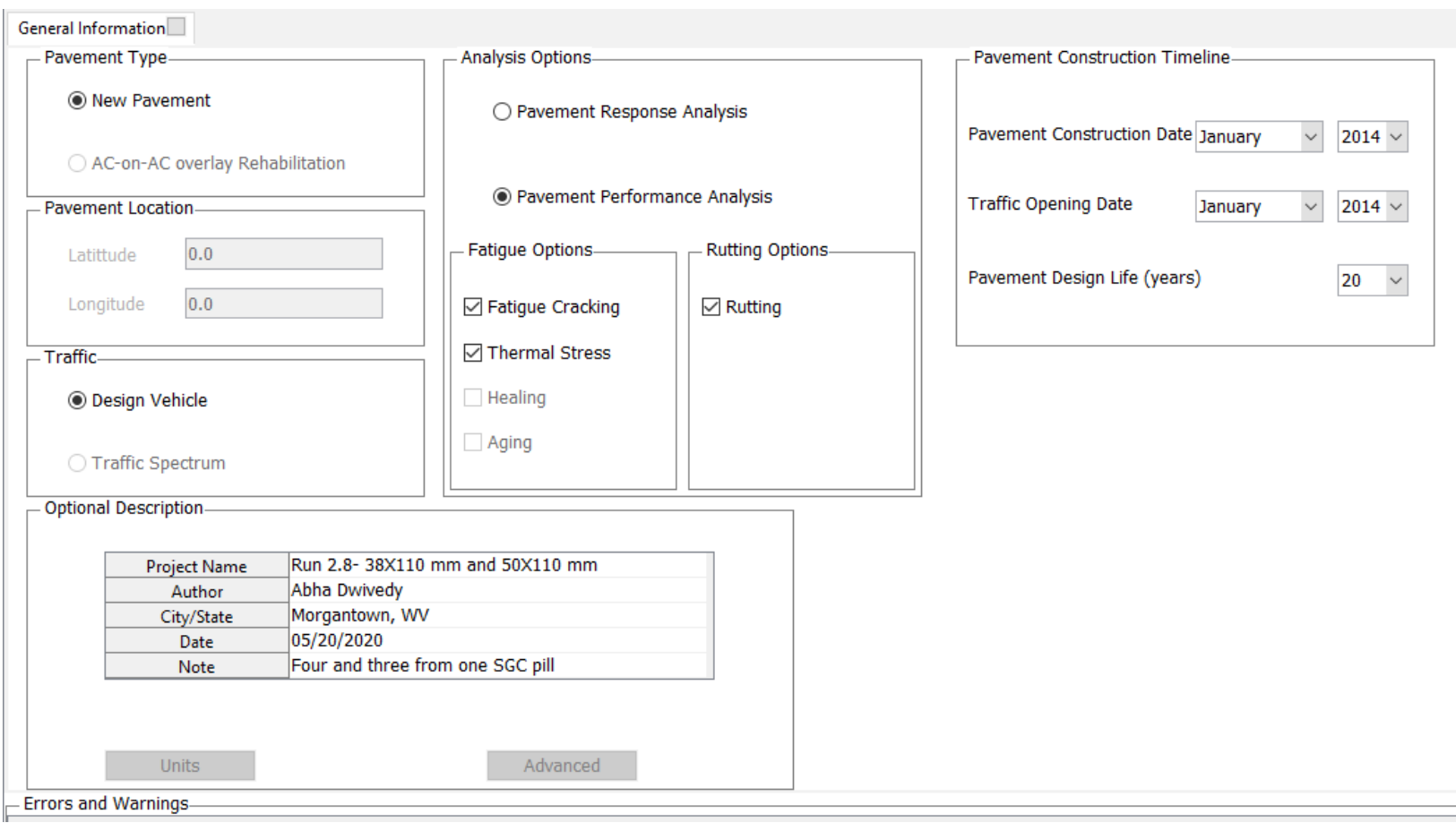

Figure 233: General Information for Run 2.8 


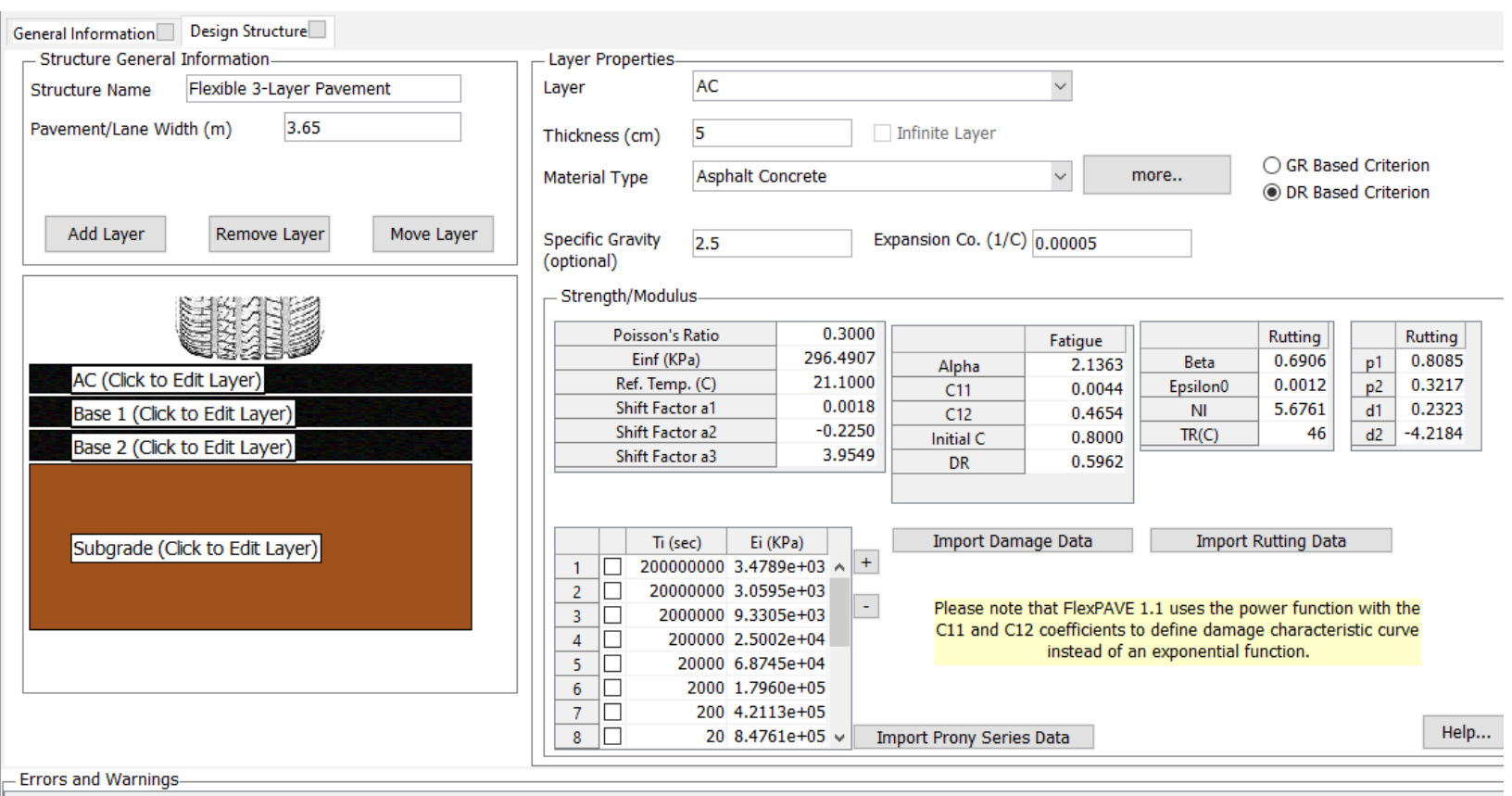

Figure 234: Design Structure of AC Layer for Run 2.8

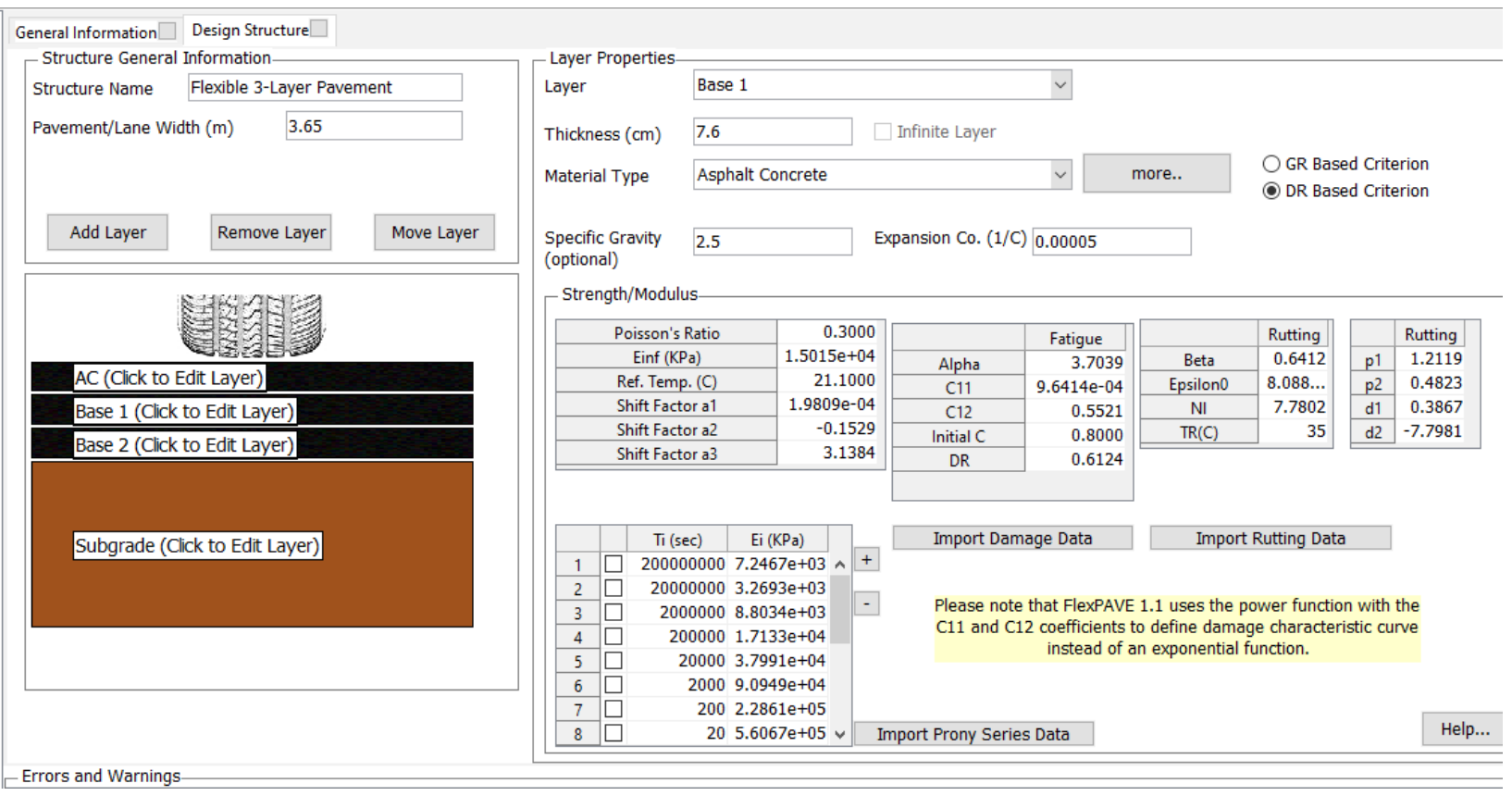

Figure 235: Design Structure of Base 1 Layer for Run 2.8 


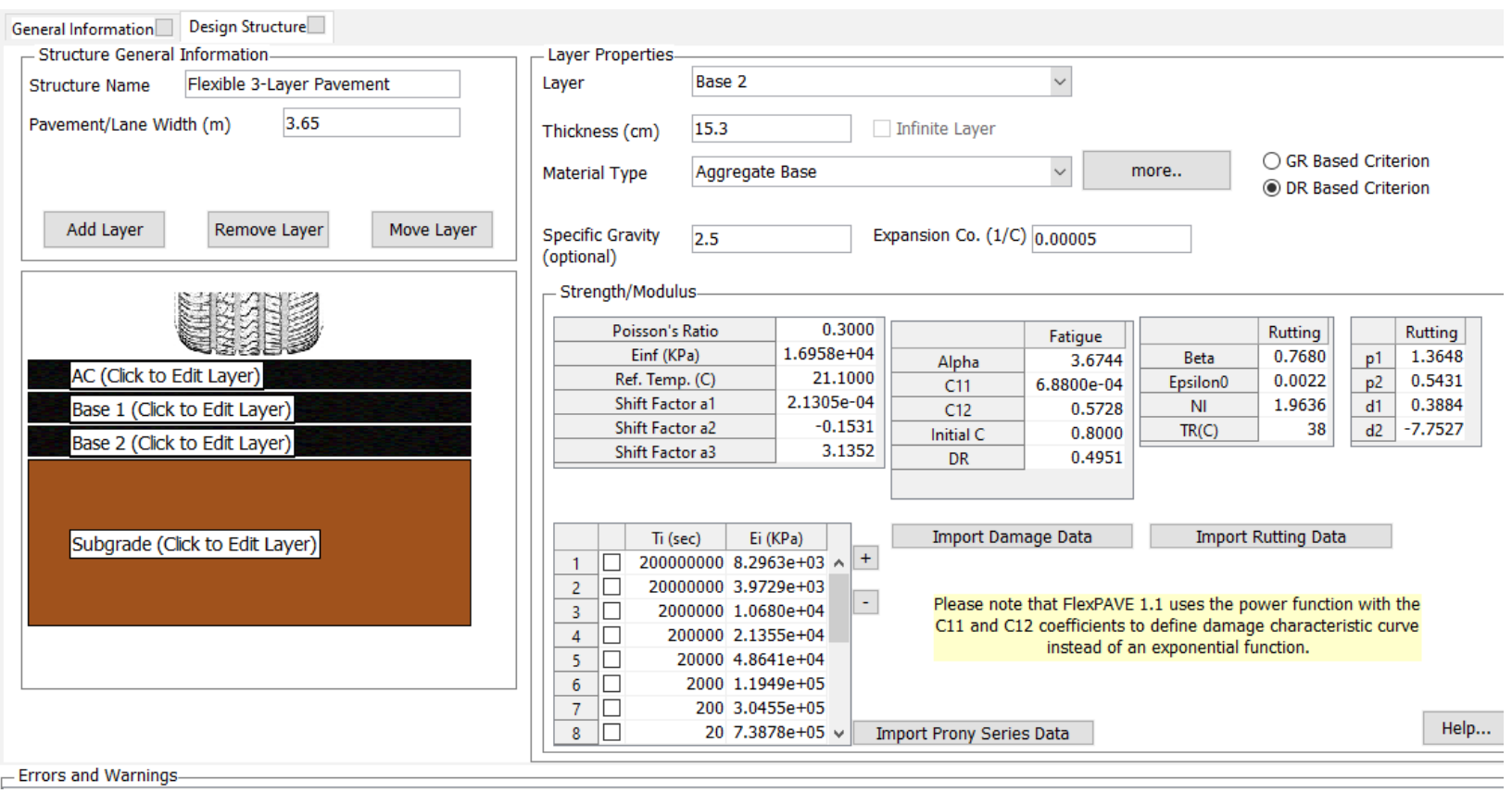

Figure 236: Design Structure of Base 2 Layer for Run 2.8

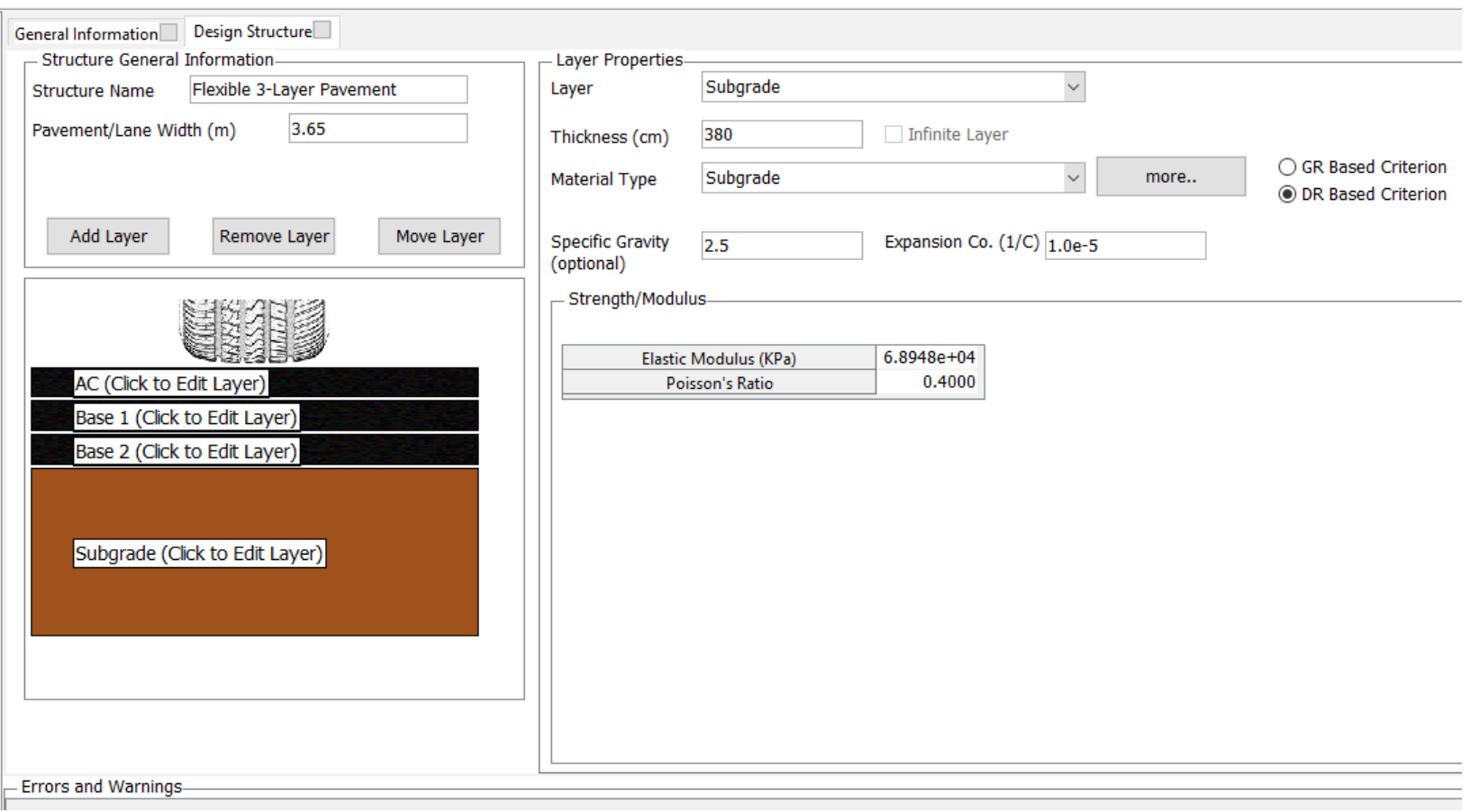

Figure 237: Design Structure of Subgrade Layer for Run 2.8 


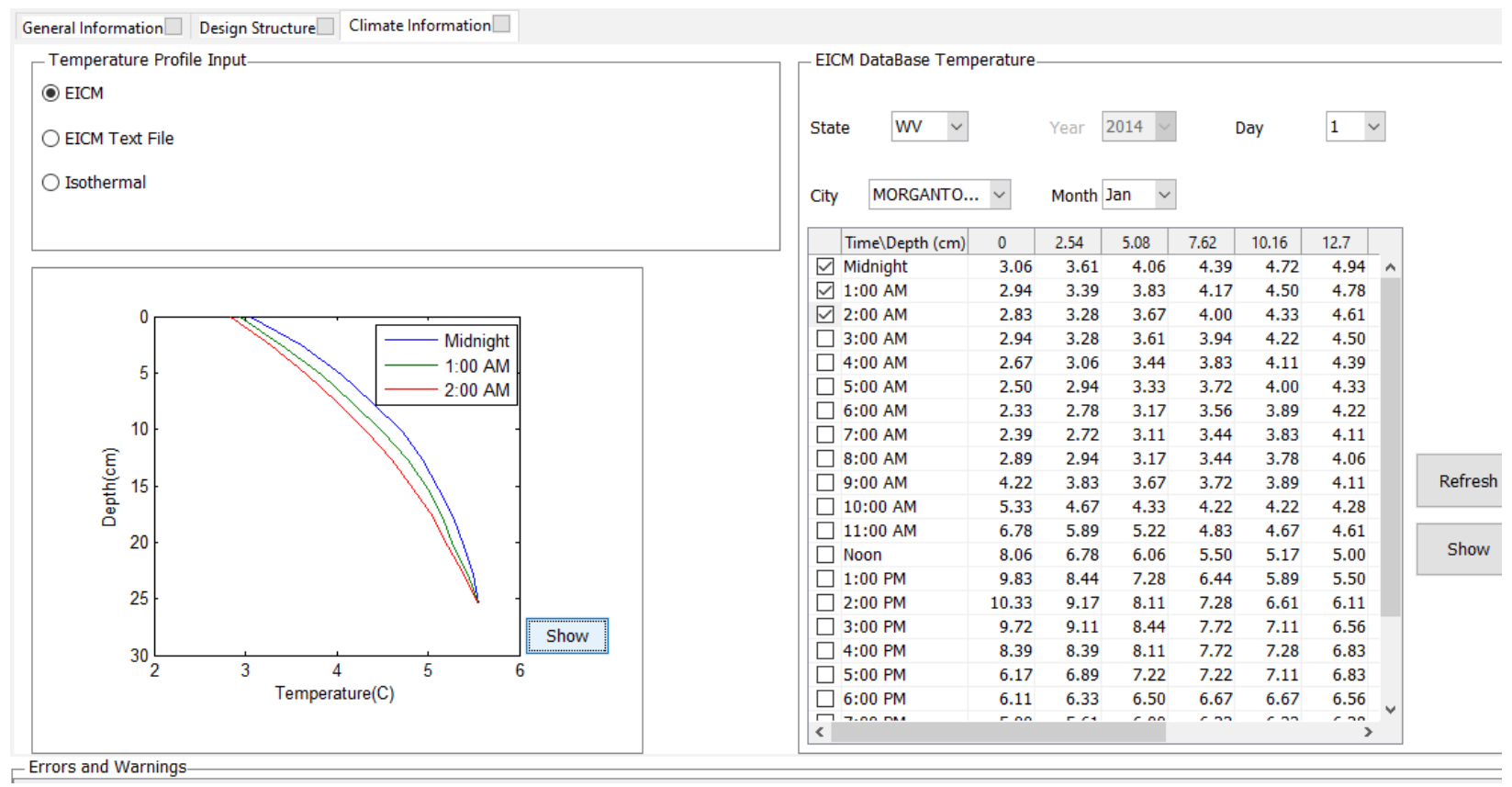

Figure 238: Climate Data for Run 2.8 


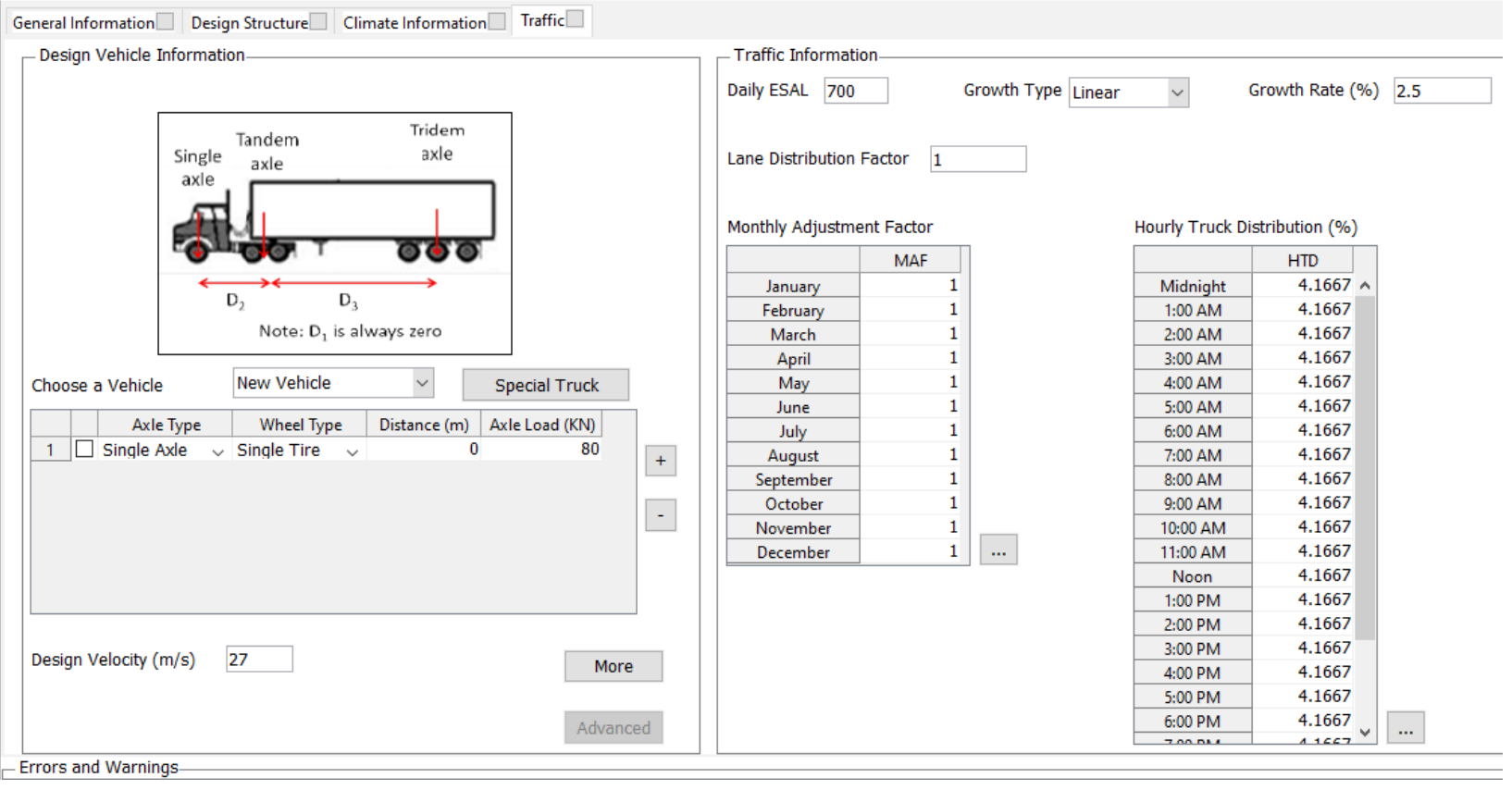

Axle Configuration

$-\quad \square \quad \times$

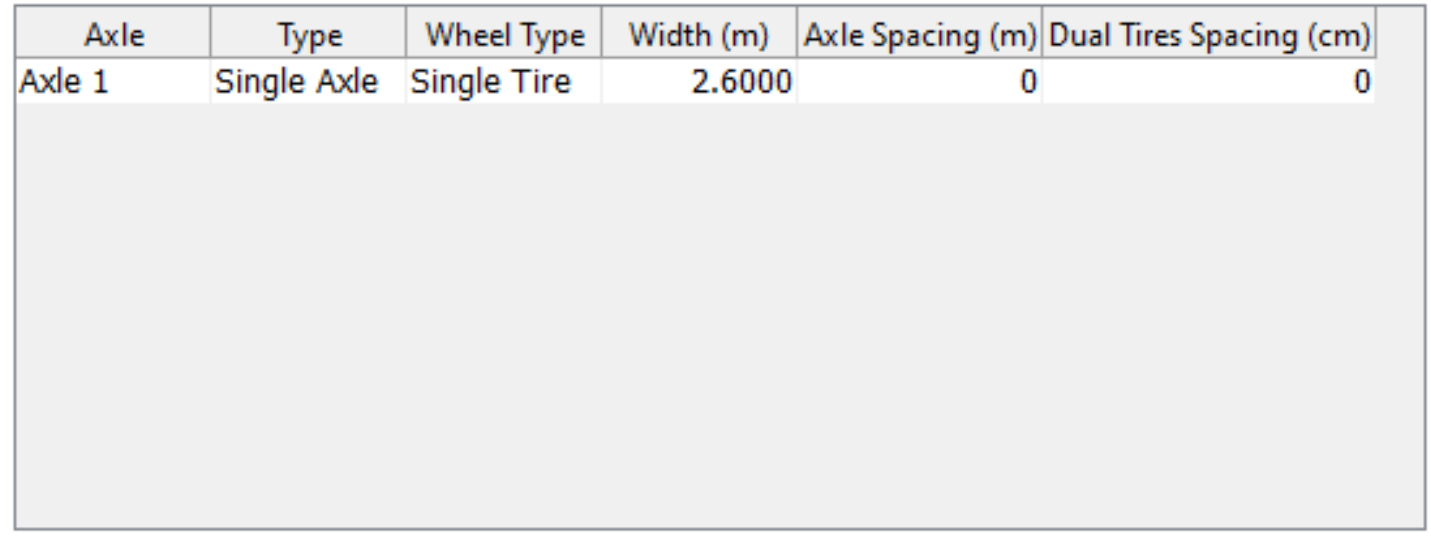

Tire Pressure (KPa) 827.37

Contact Area Shape Rectangular

Aspect Ratio (length/width) 1.5714

Shear Traction $\quad 0.0$

OK

Cancel

Figure 239: Traffic Data for Run 2.8 


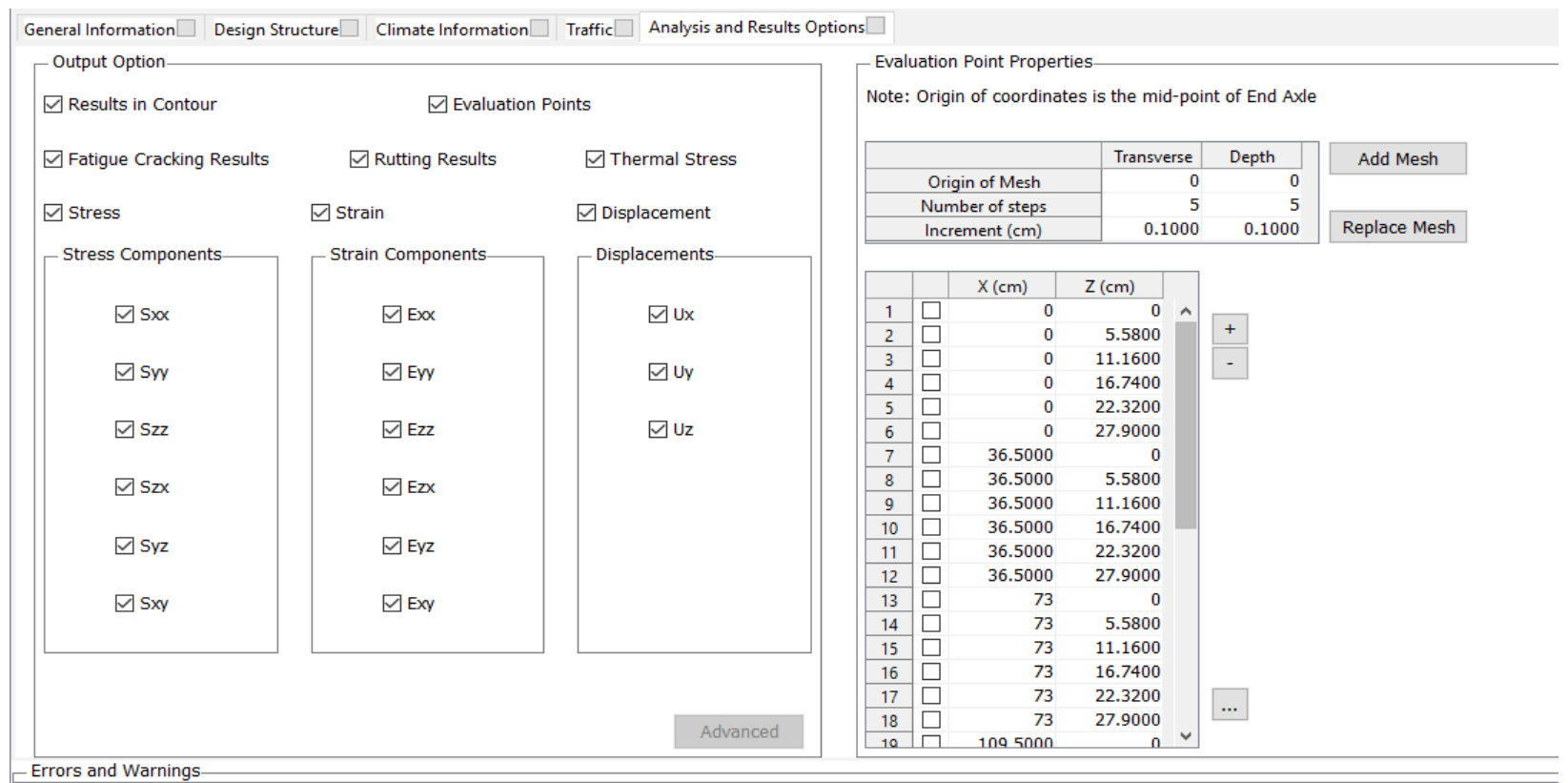

Figure 240: Output and Analysis Options for Run 2.8

\section{Run 2.9}

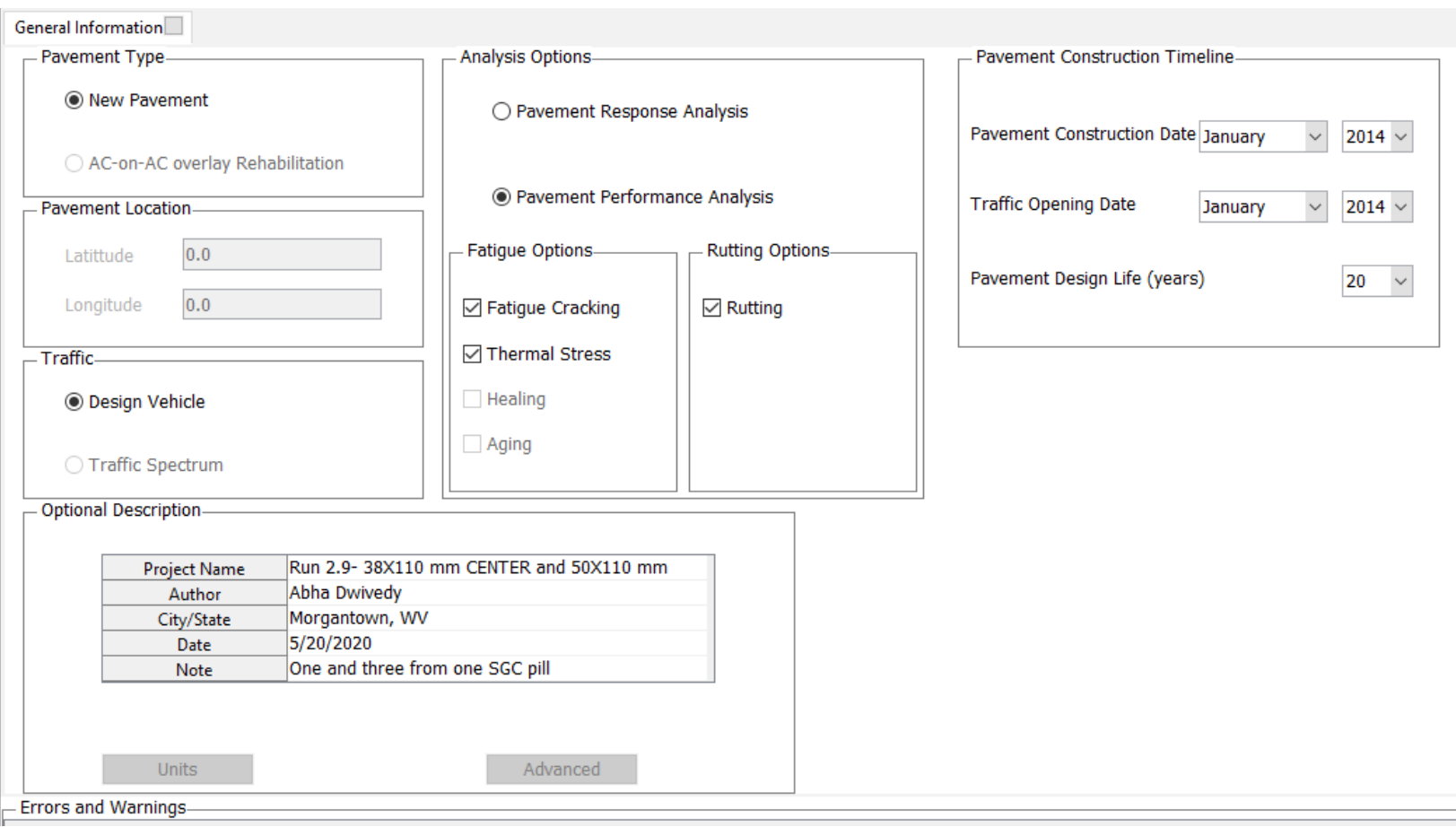

Figure 241: General Information for Run 2.9 

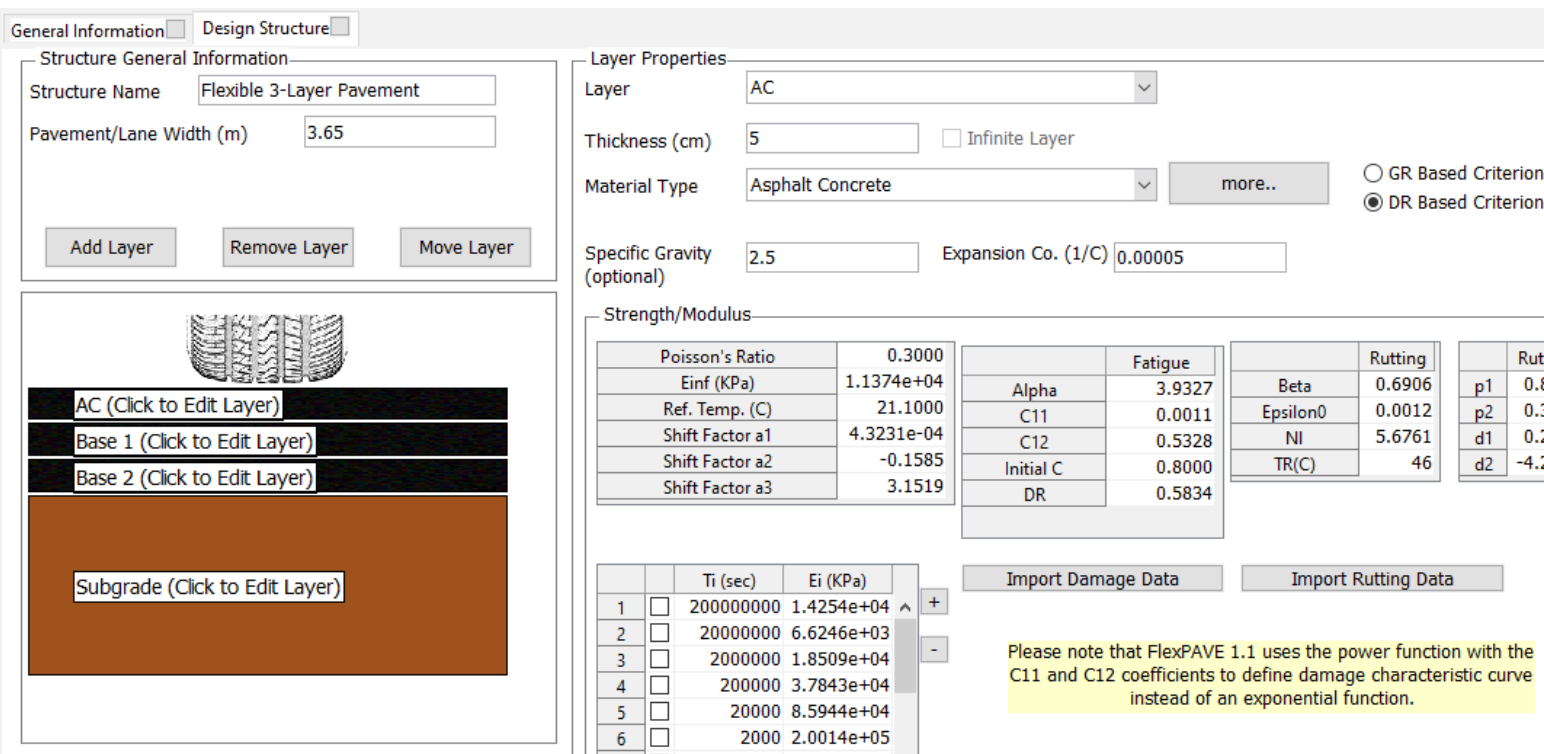
Shift Factor a3

\begin{tabular}{|r|}
0.3000 \\
$1.1374 \mathrm{e}+04$ \\
21.1000 \\
$4.3231 \mathrm{e}-04$ \\
-0.1585 \\
3.1519 \\
\hline
\end{tabular}

\begin{tabular}{|c|c|c|}
\hline & Fatigue & \\
\hline Alpha & 3.9327 & Beta \\
\hline C11 & 0.0011 & Epsilon0 \\
\hline
\end{tabular}
\begin{tabular}{l|l|l|} 
Rutting & Rutting \\
\hline
\end{tabular} \begin{tabular}{|l|r|r|}
\hline 0.6906 & $p 1$ & 0.8085 \\
\hline
\end{tabular} 3.1519 nitial

$\operatorname{TR}(\mathrm{C})$ \begin{tabular}{|l|l|l|}
\hline 0.0012 & $\mathrm{p} 2$ & 0.3217 \\
\cline { 2 - 3 } & $\mathrm{d}$ & 0.2323 \\
\hline
\end{tabular} \begin{tabular}{l|l|l|l|}
\hline .0012 & $\mathrm{p} 2$ & 0.3217 \\
\cline { 2 - 3 } & $\mathrm{d} 1$ & 0.2323 \\
\hline & & 0.2161
\end{tabular}

Figure 242: Design Structure of AC Layer for Run 2.9

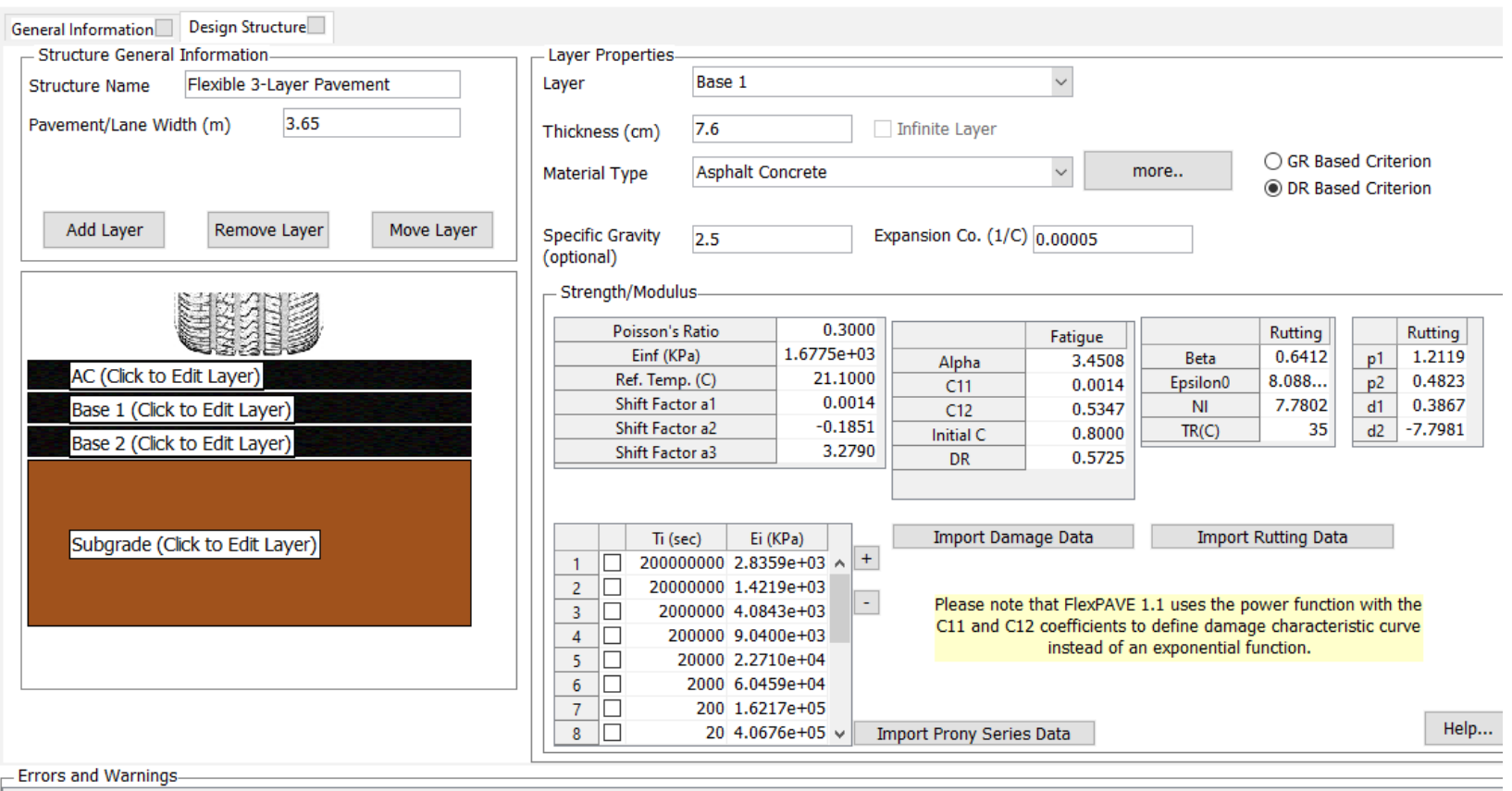

Figure 243: Design Structure of Base 1 Layer for Run 2.9 


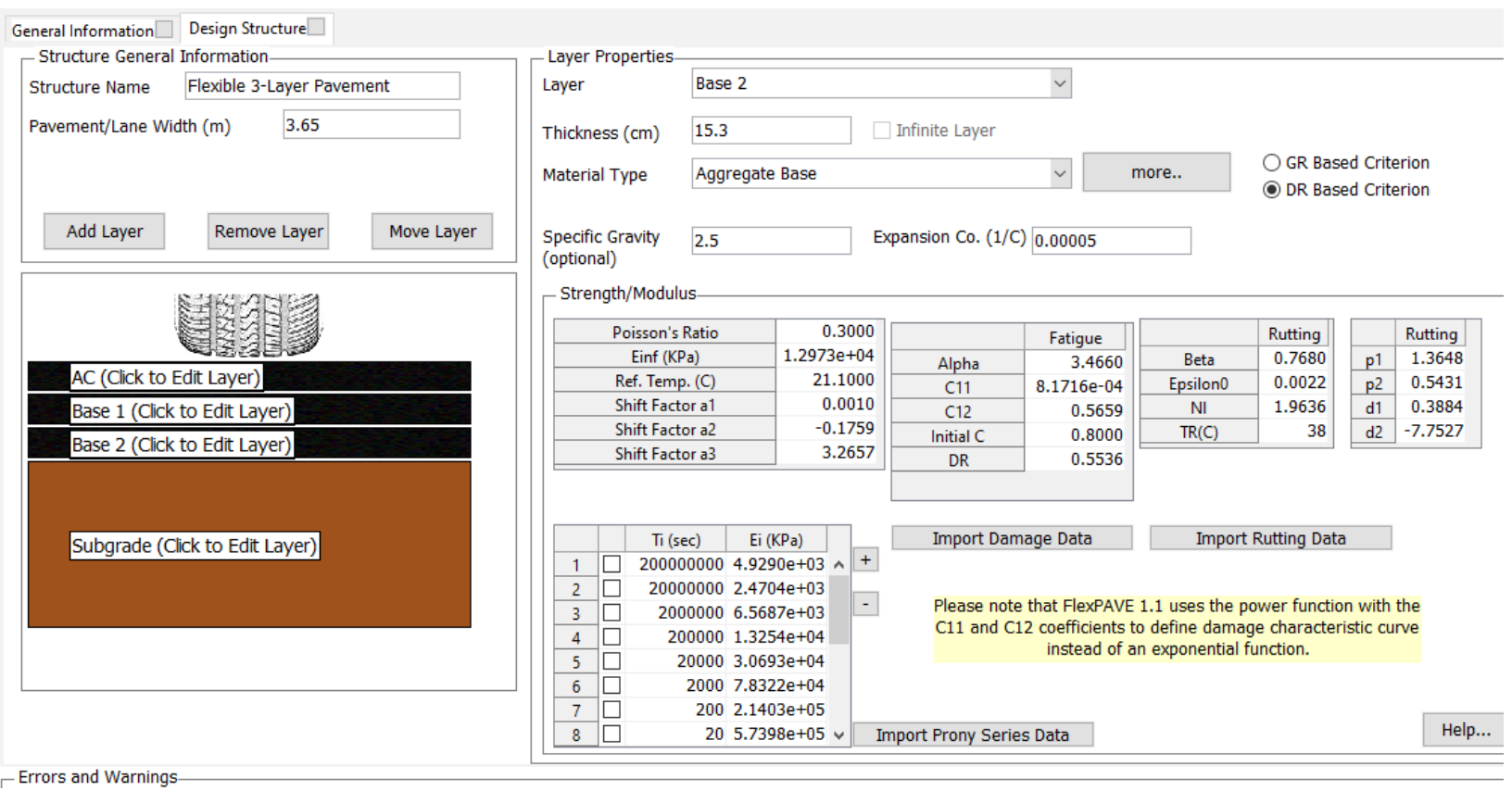

Figure 244: Design Structure of Base 2 Layer for Run 2.9

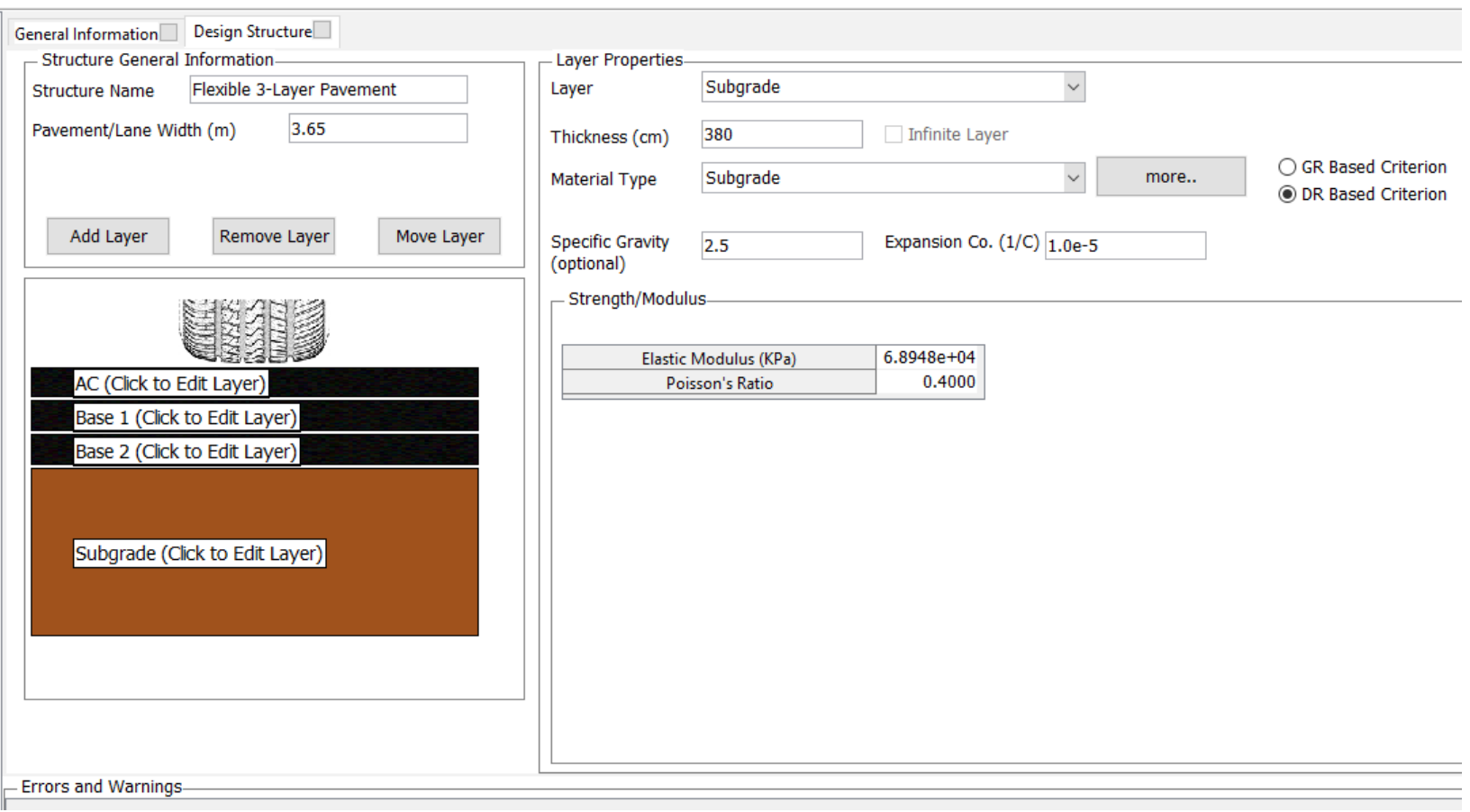

Figure 245: Design Structure of Subgrade Layer for Run 2.9 


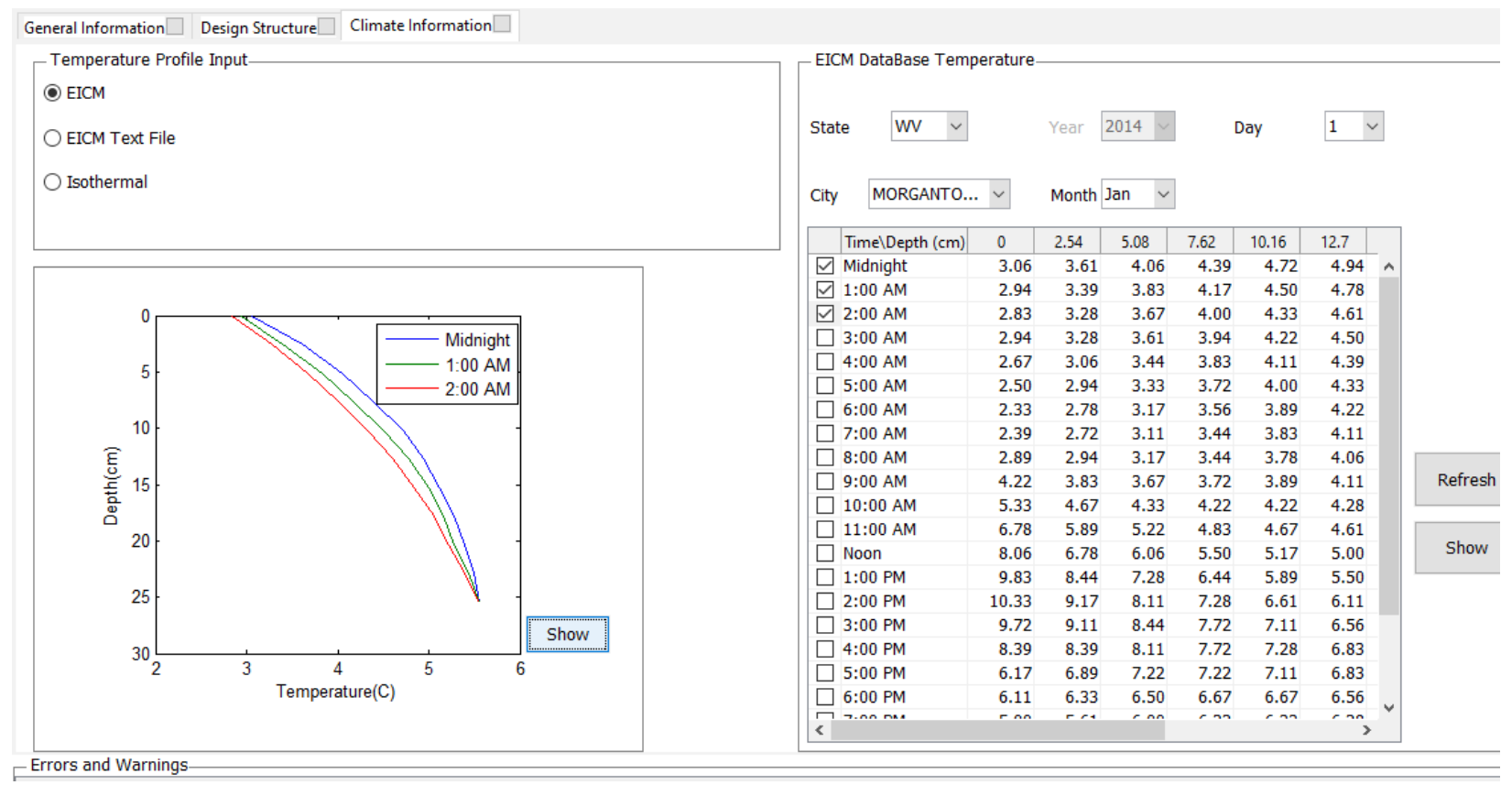

Figure 246: Climate Data for Run 2.9 


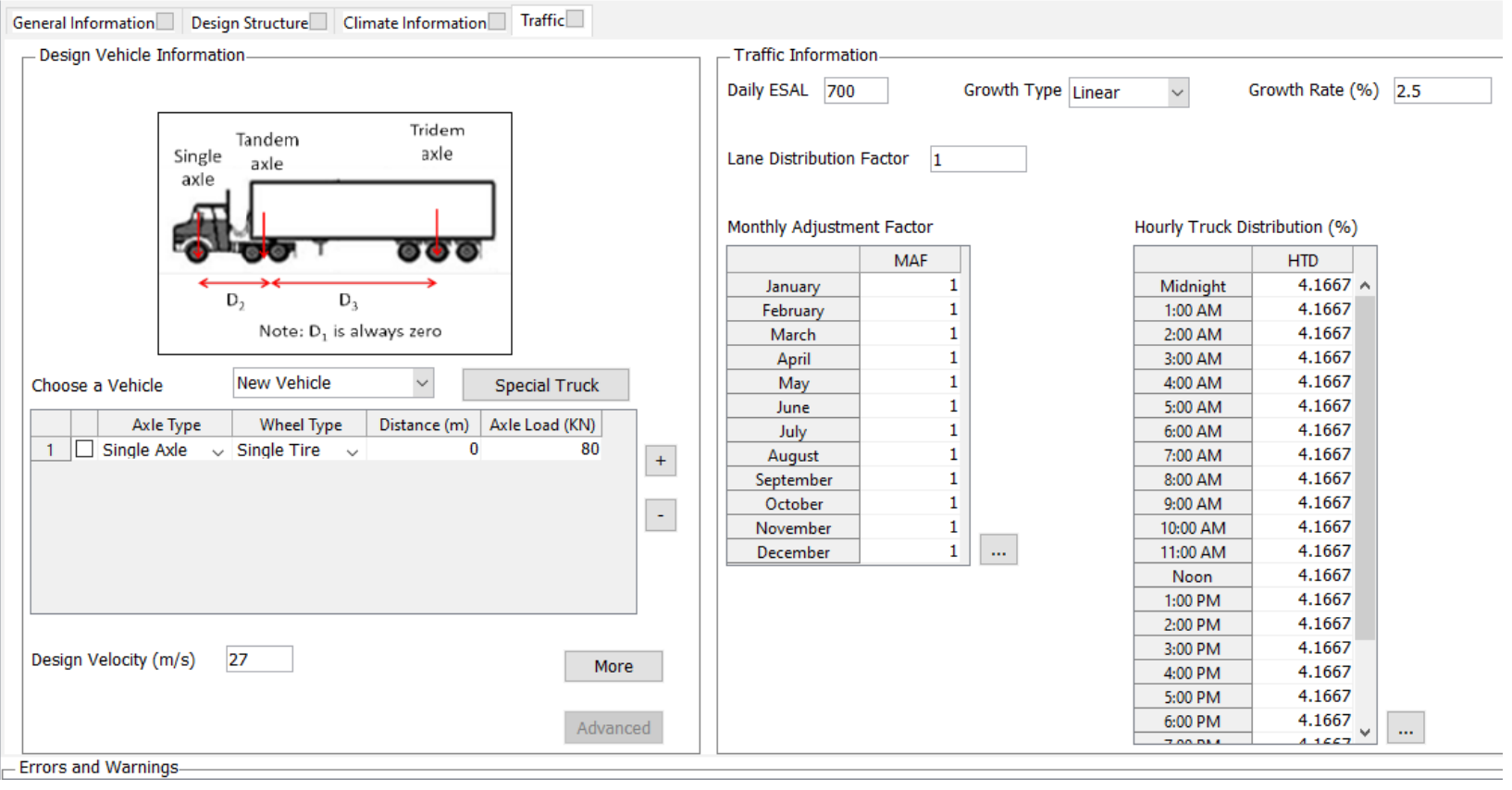

Axle Configuration

$-\quad \square \quad \times$

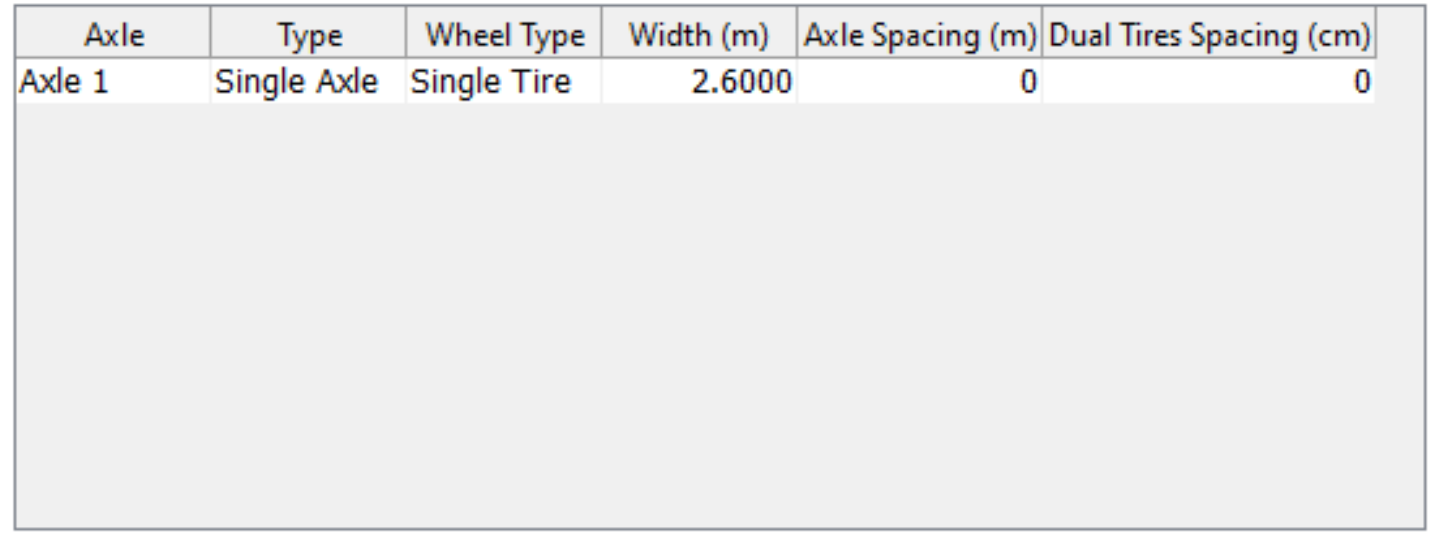

Tire Pressure (KPa) 827.37

Contact Area Shape Rectangular

Aspect Ratio (length/width) 1.5714

Shear Traction $\quad 0.0$

OK

Cancel

Figure 247: Traffic Data for Run 2.9 


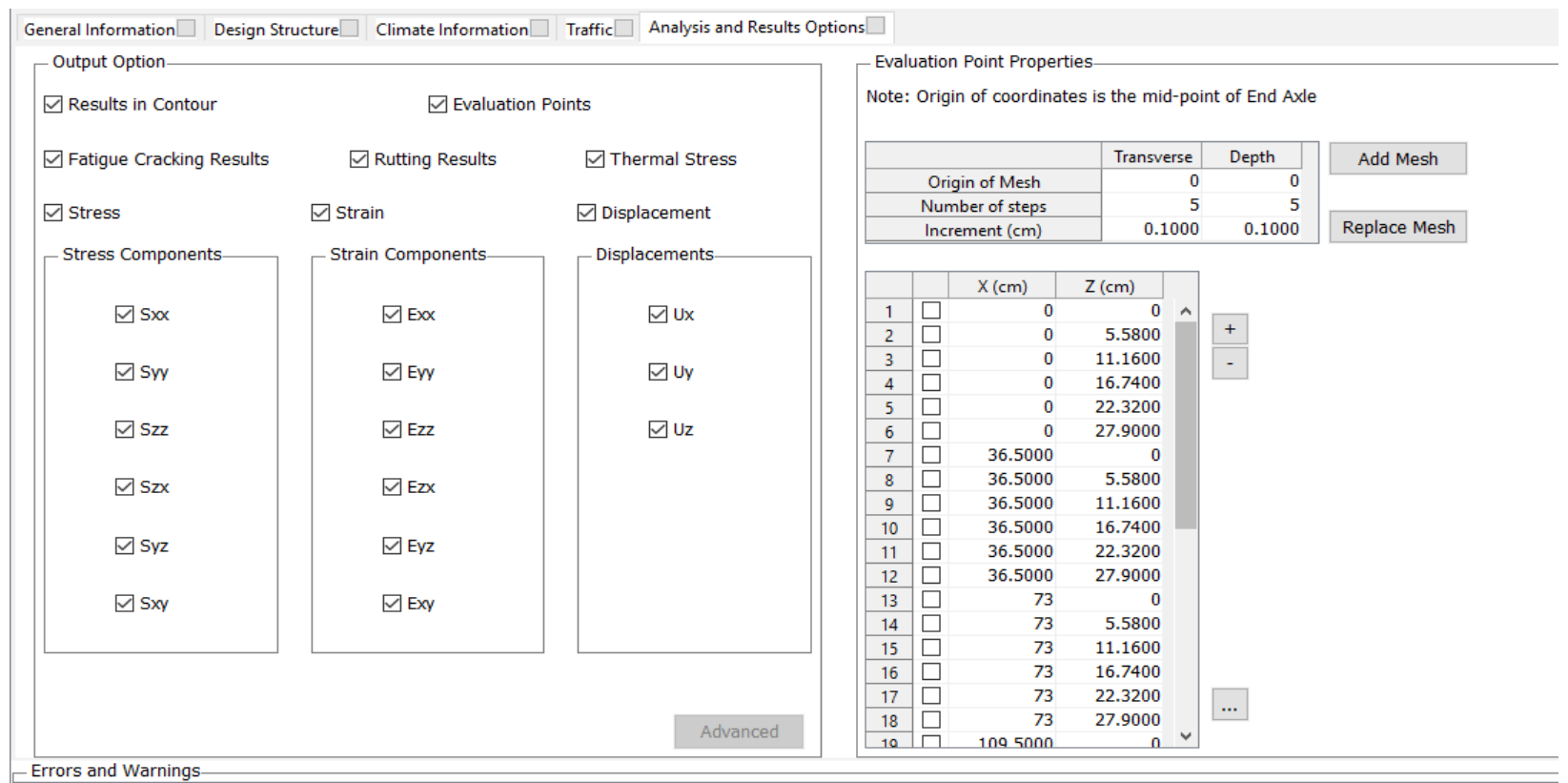

Figure 248: Output and Analysis Options for Run 2.9

Run 2.10

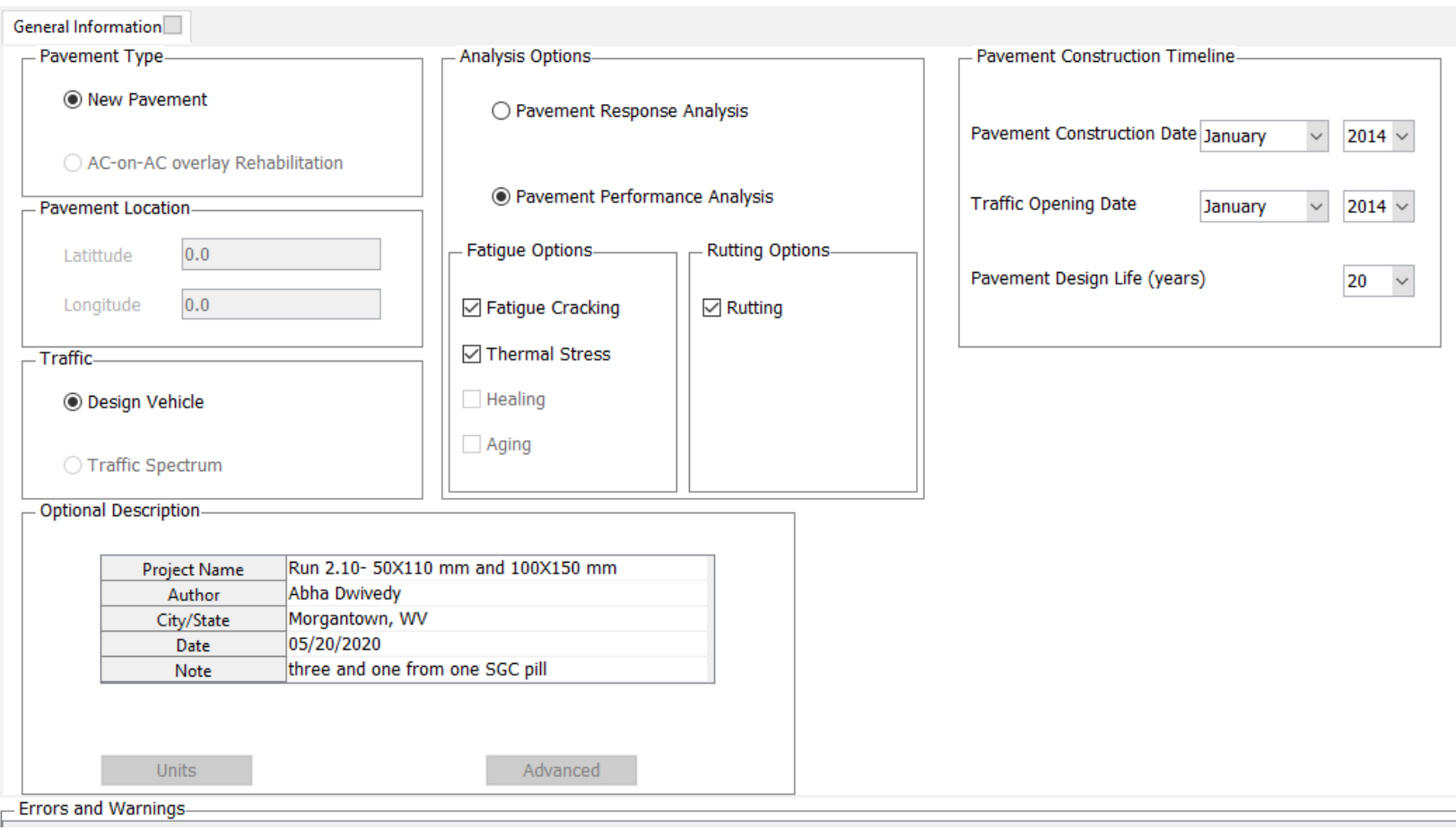

Figure 249: General Information for Run 2.10 


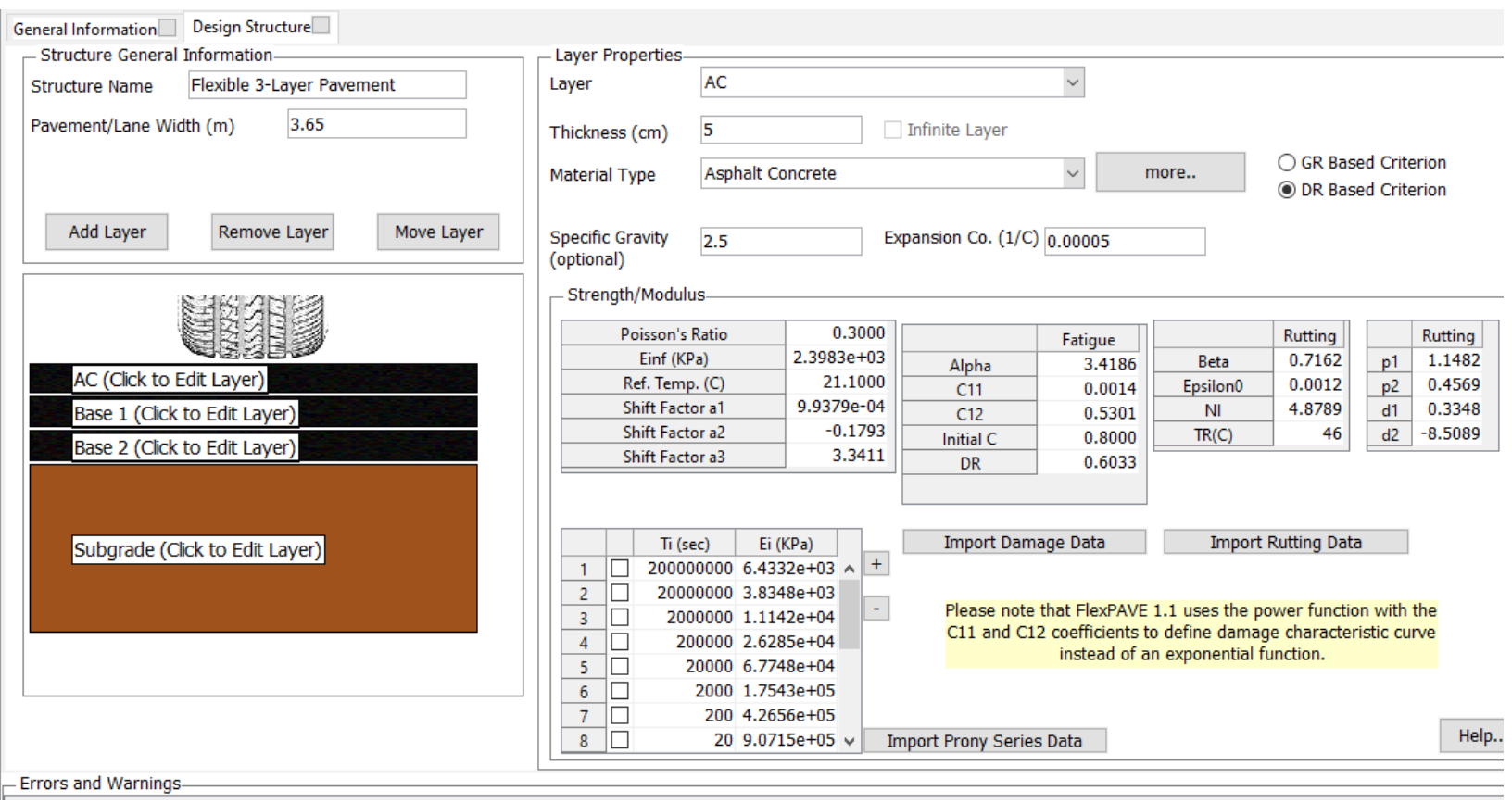

Figure 250: Design Structure of AC Layer for Run 2.10

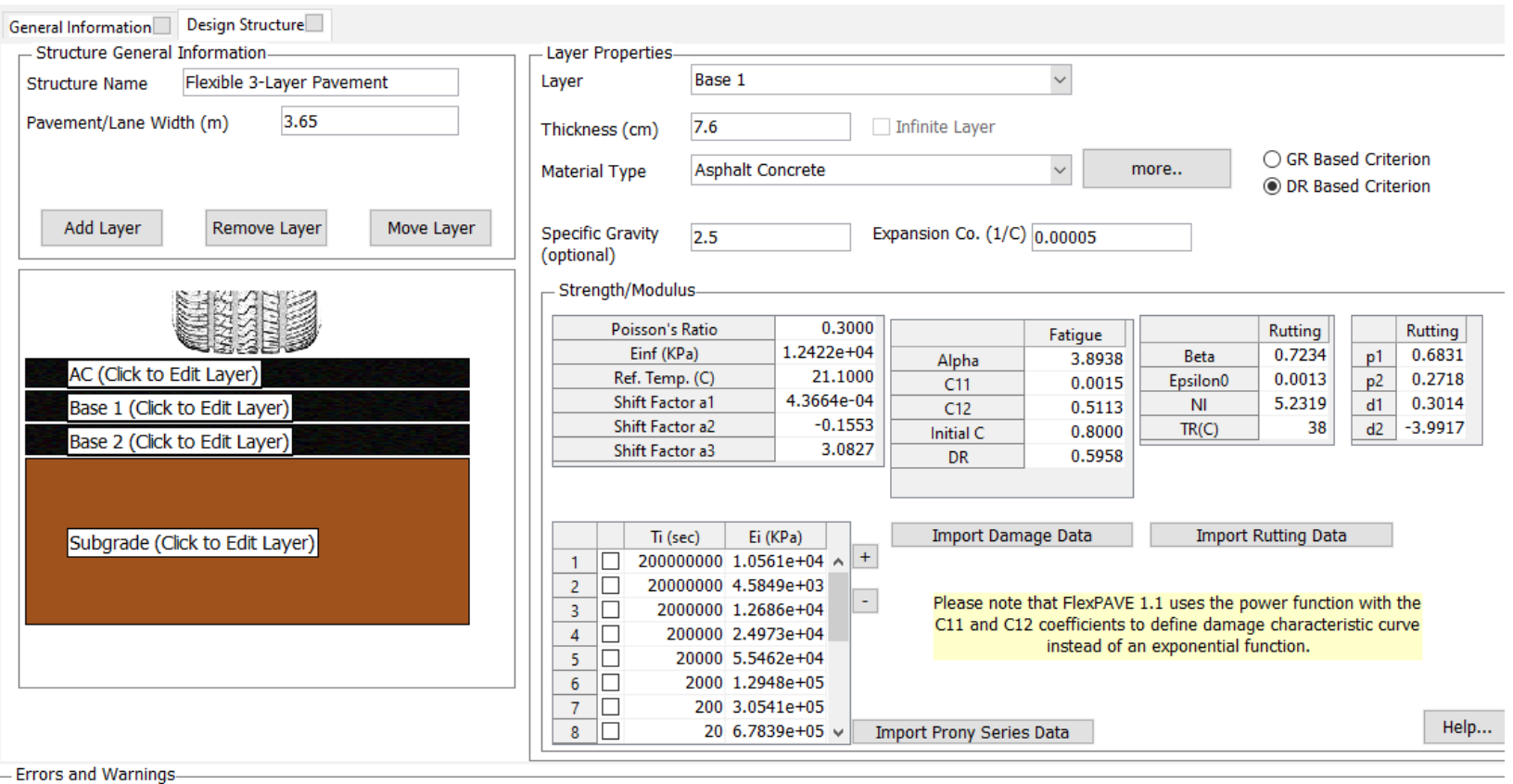

- Errors and Warning

Figure 251: Design Structure of Base 1 Layer for Run 2.10 


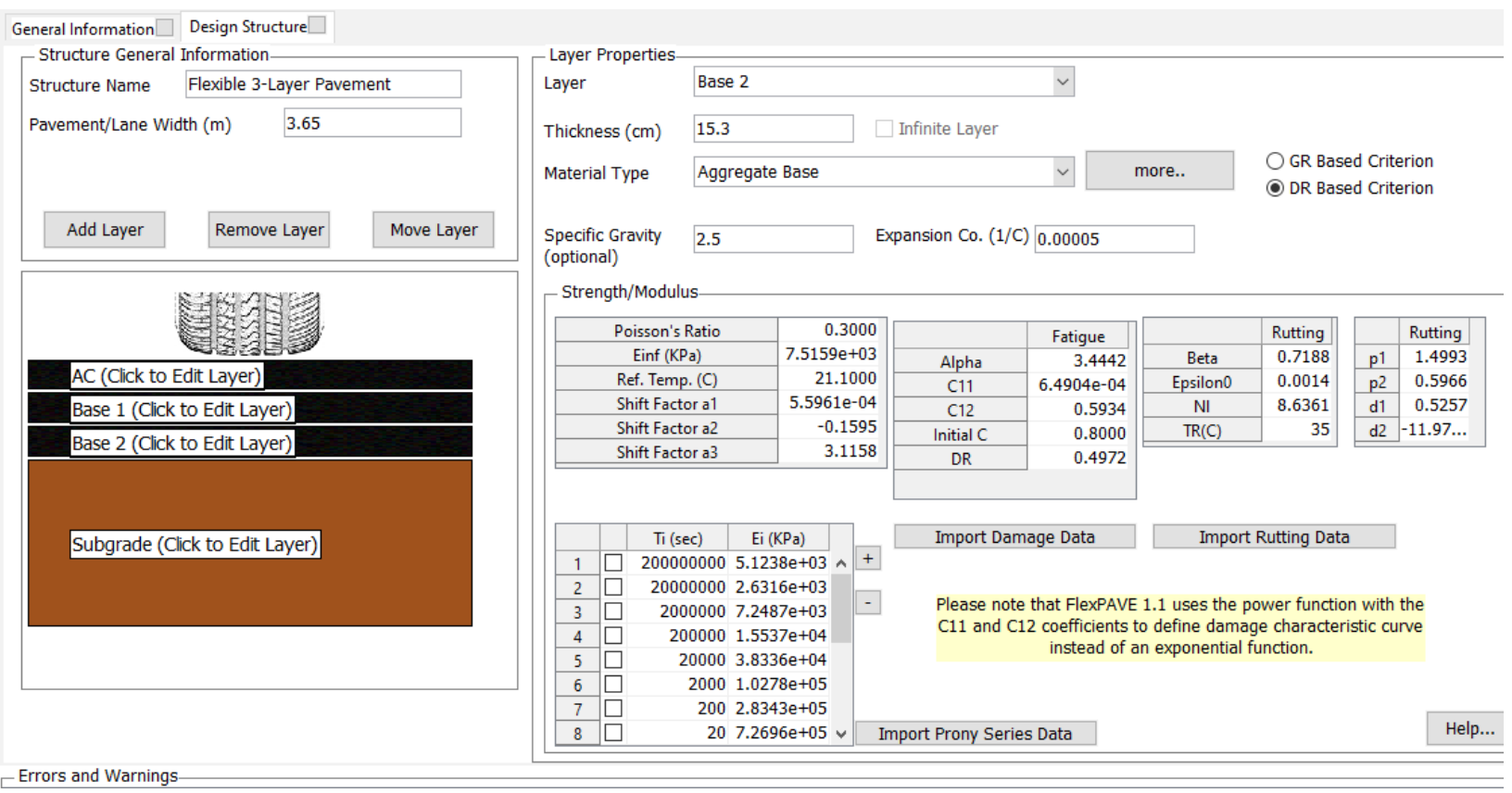

Figure 252: Design Structure of Base 2 Layer for Run 2.10

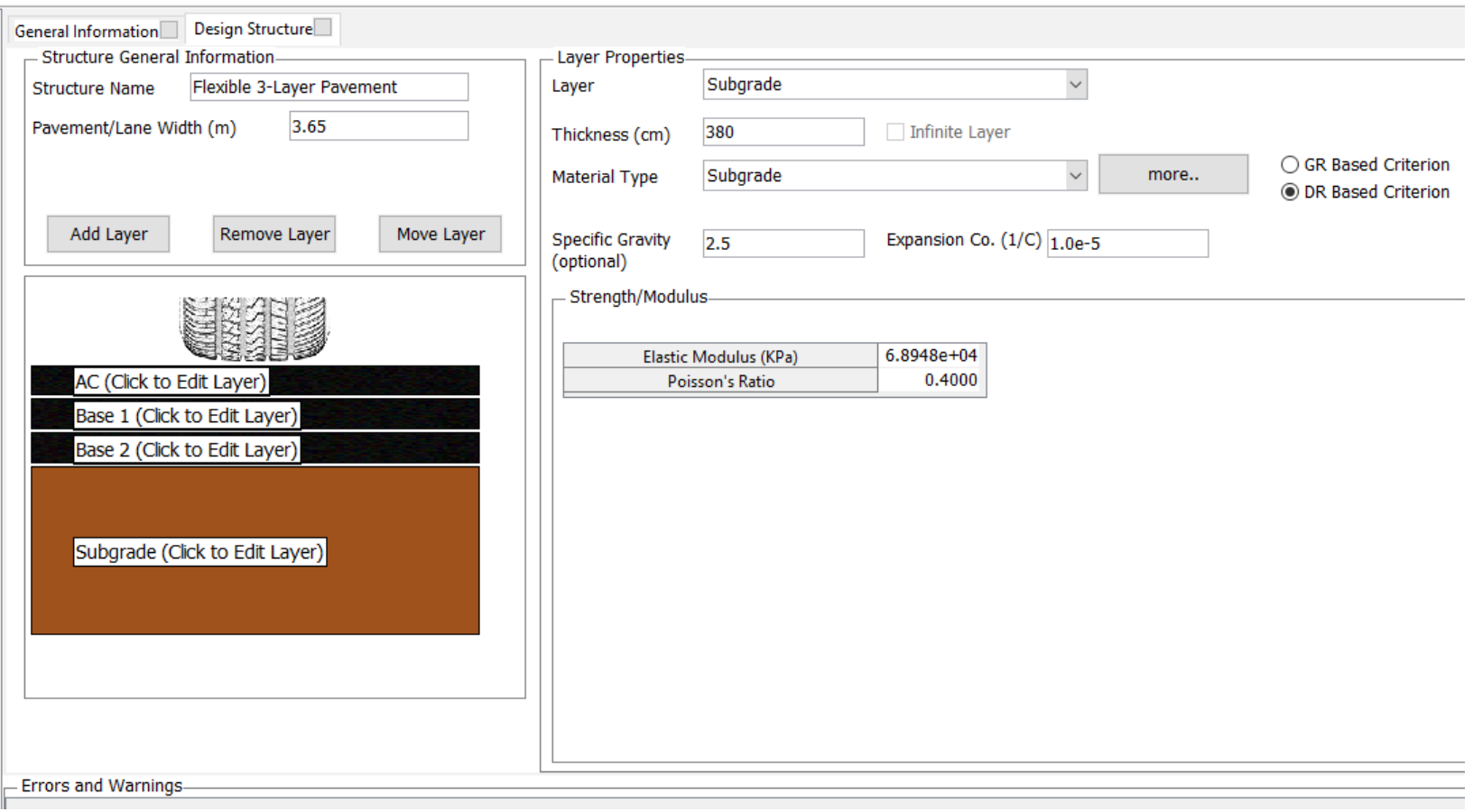

Figure 253: Design Structure of Subgrade Layer for Run 2.10 


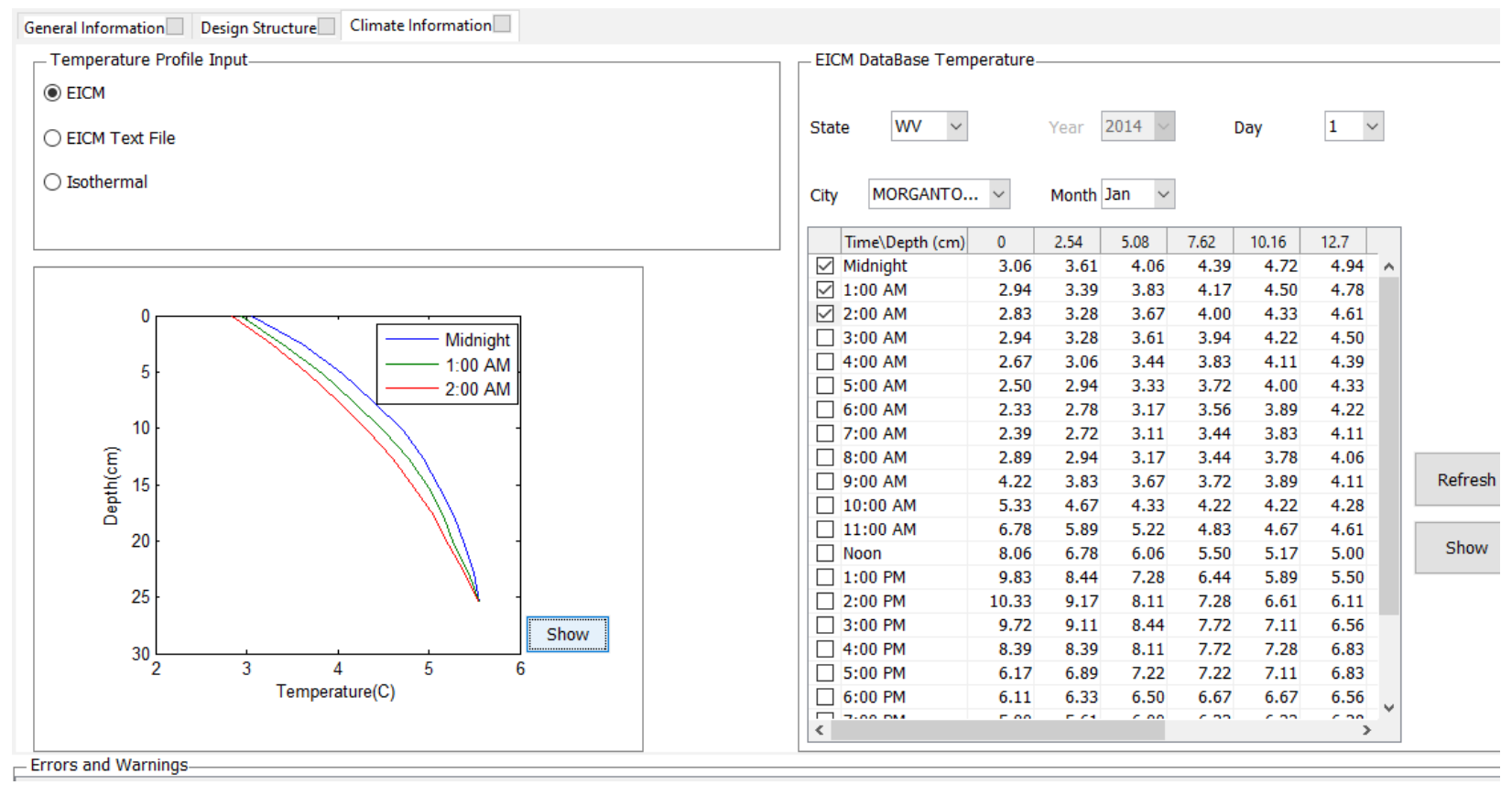

Figure 254: Climate Data for Run 2.10 


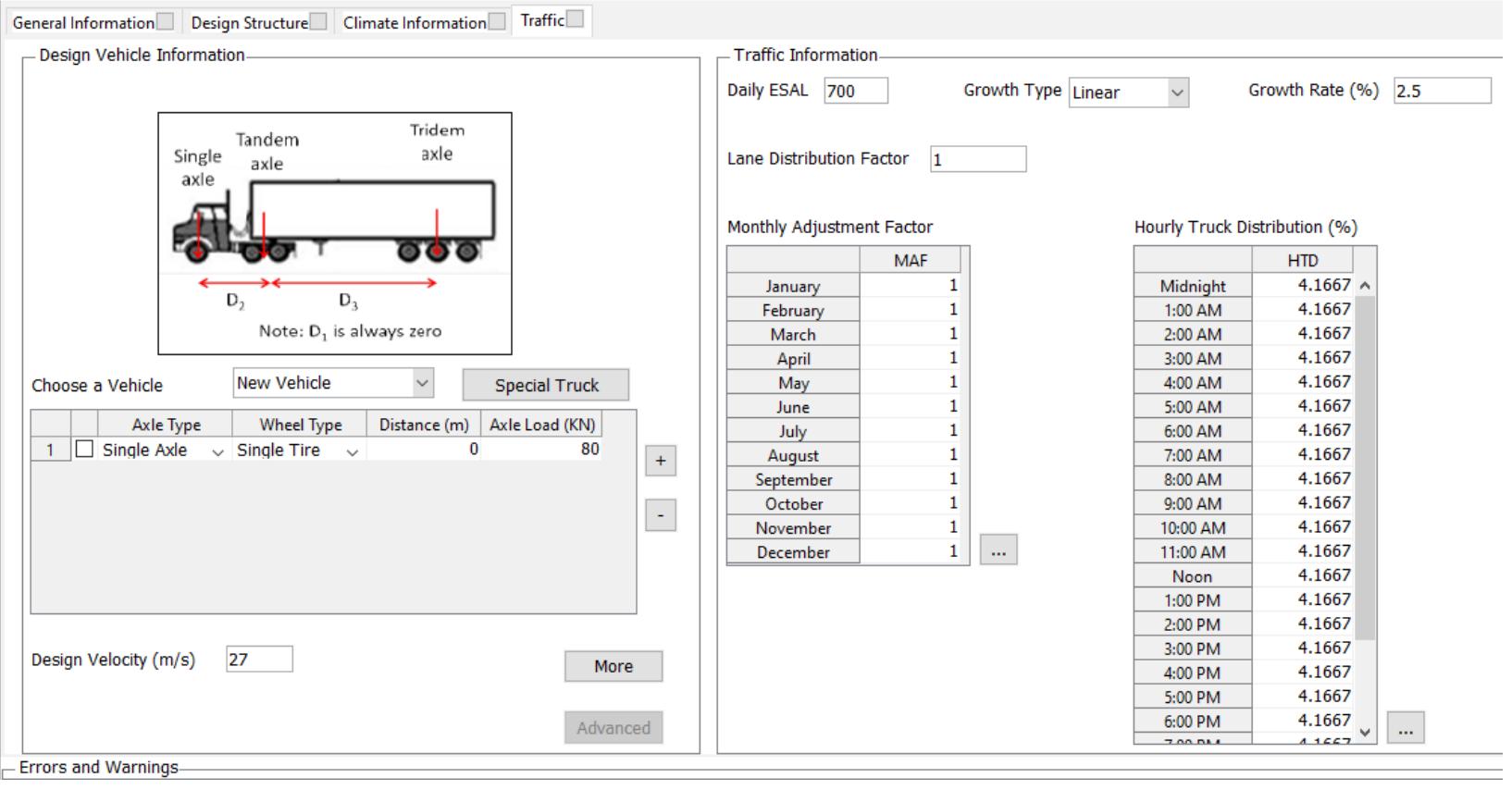

Axle Configuration

$-\quad \square \quad \times$

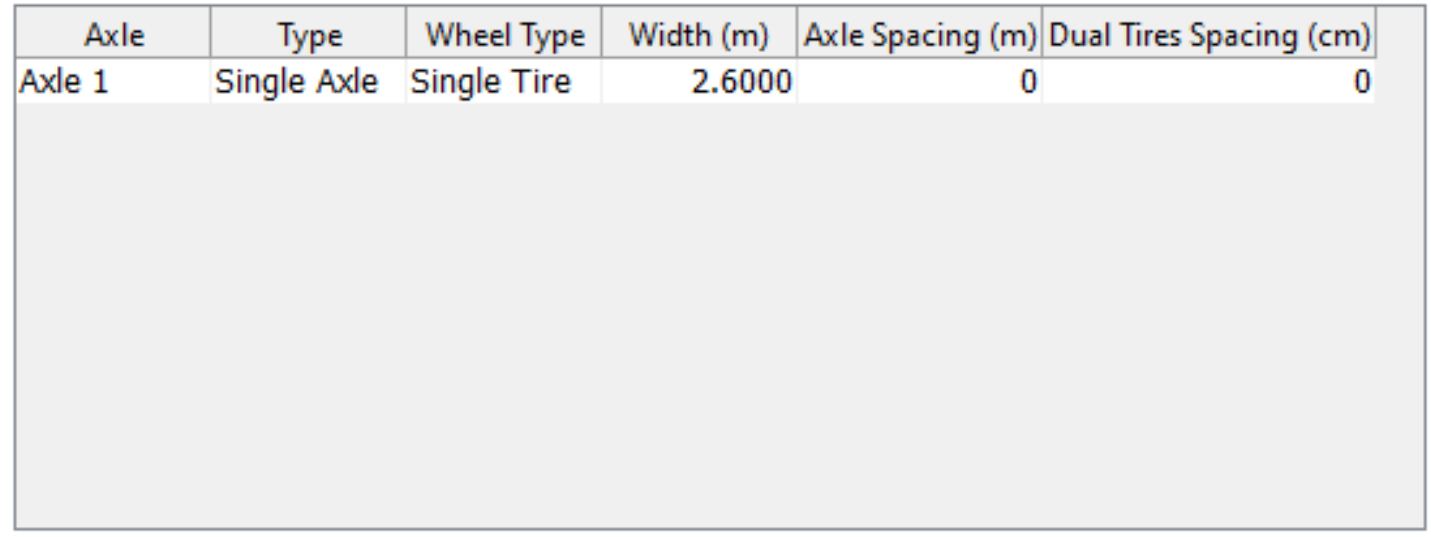

Tire Pressure (KPa) 827.37

Contact Area Shape Rectangular

Aspect Ratio (length/width) 1.5714

Shear Traction $\quad 0.0$

OK

Cancel

Figure 255: Traffic Data for Run 2.10 


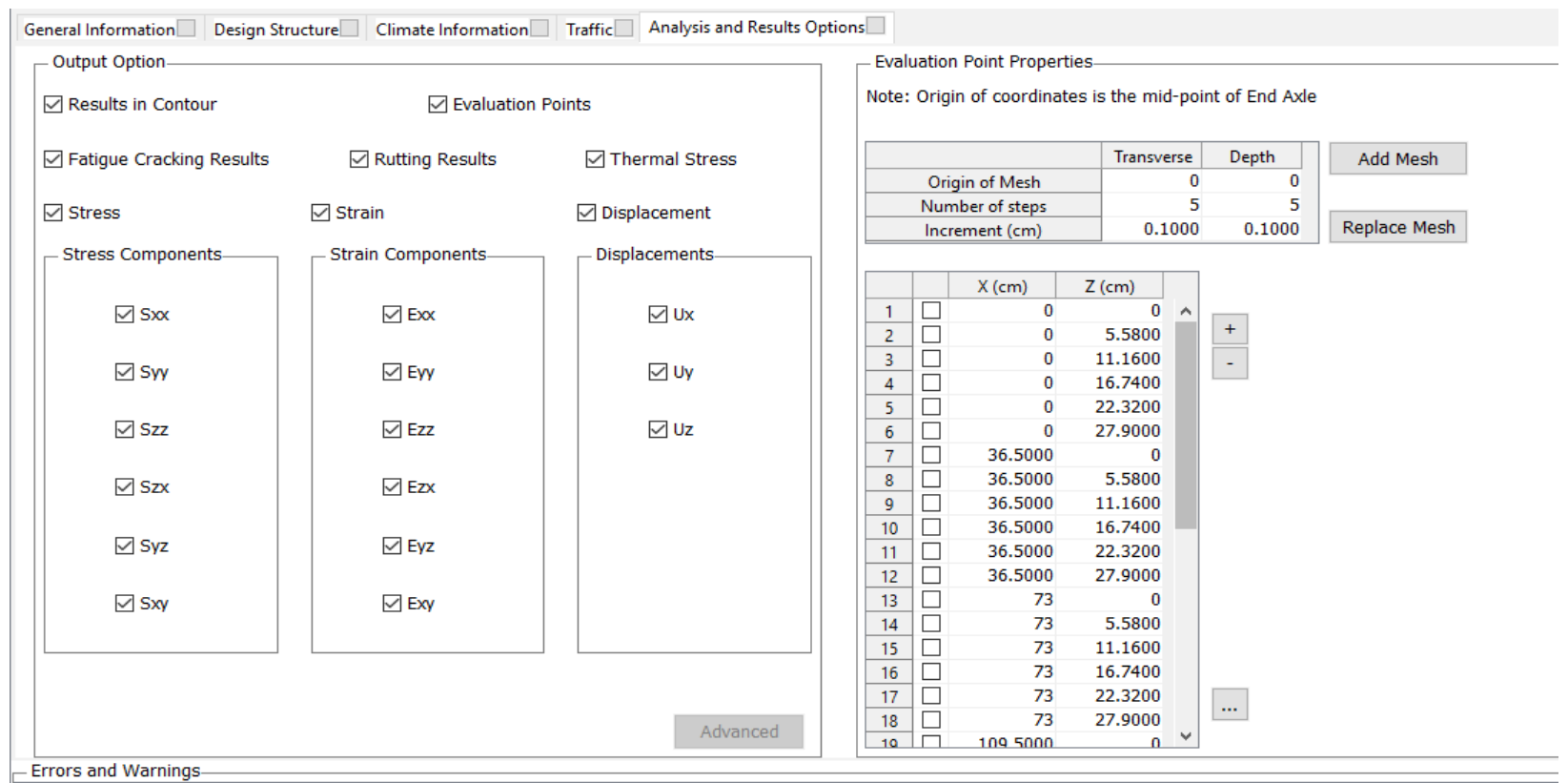

Figure 256: Output and Analysis Options for Run 2.10

\section{Run 2.11}

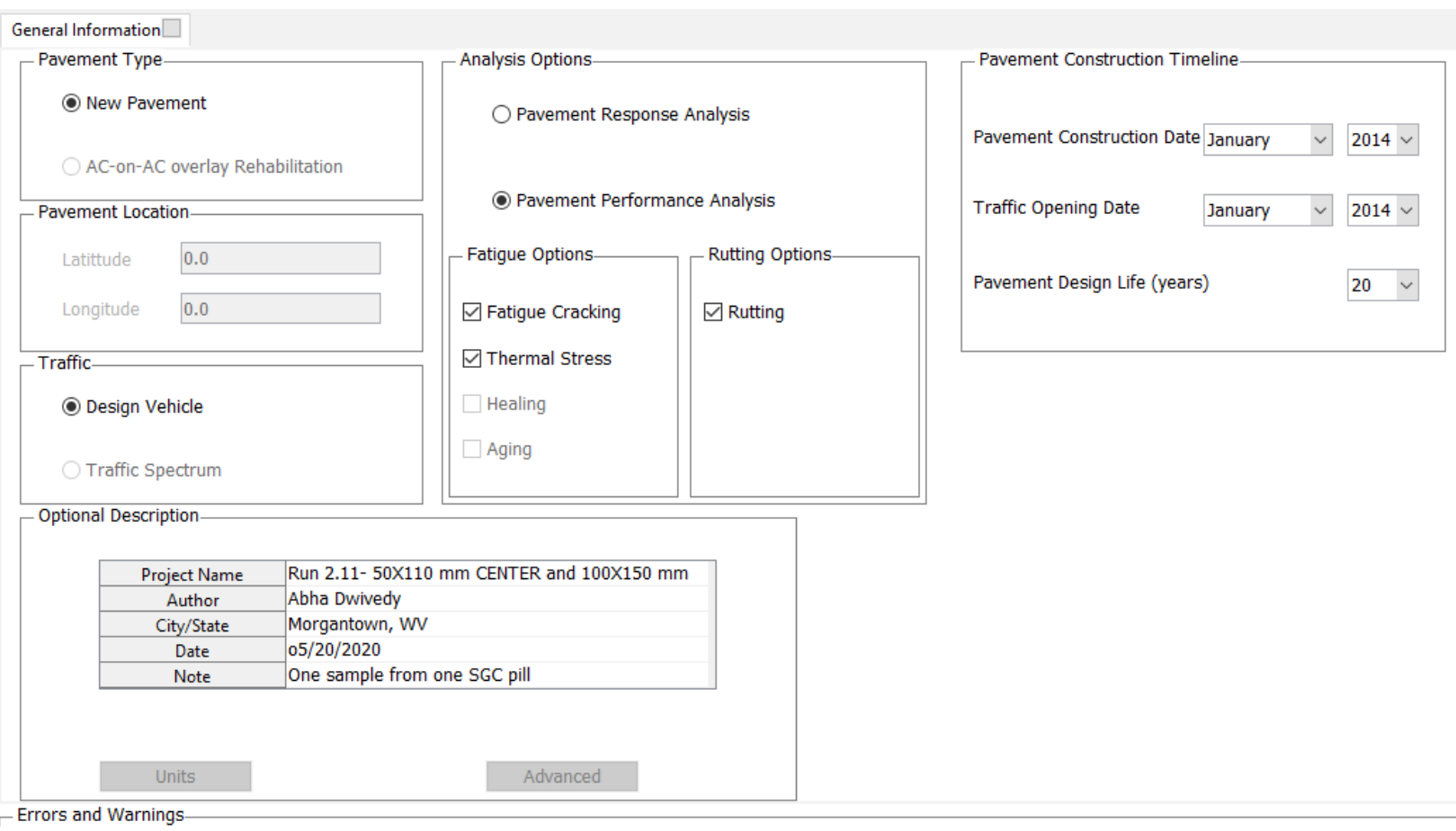

Figure 257: General Information for Run 2.11 

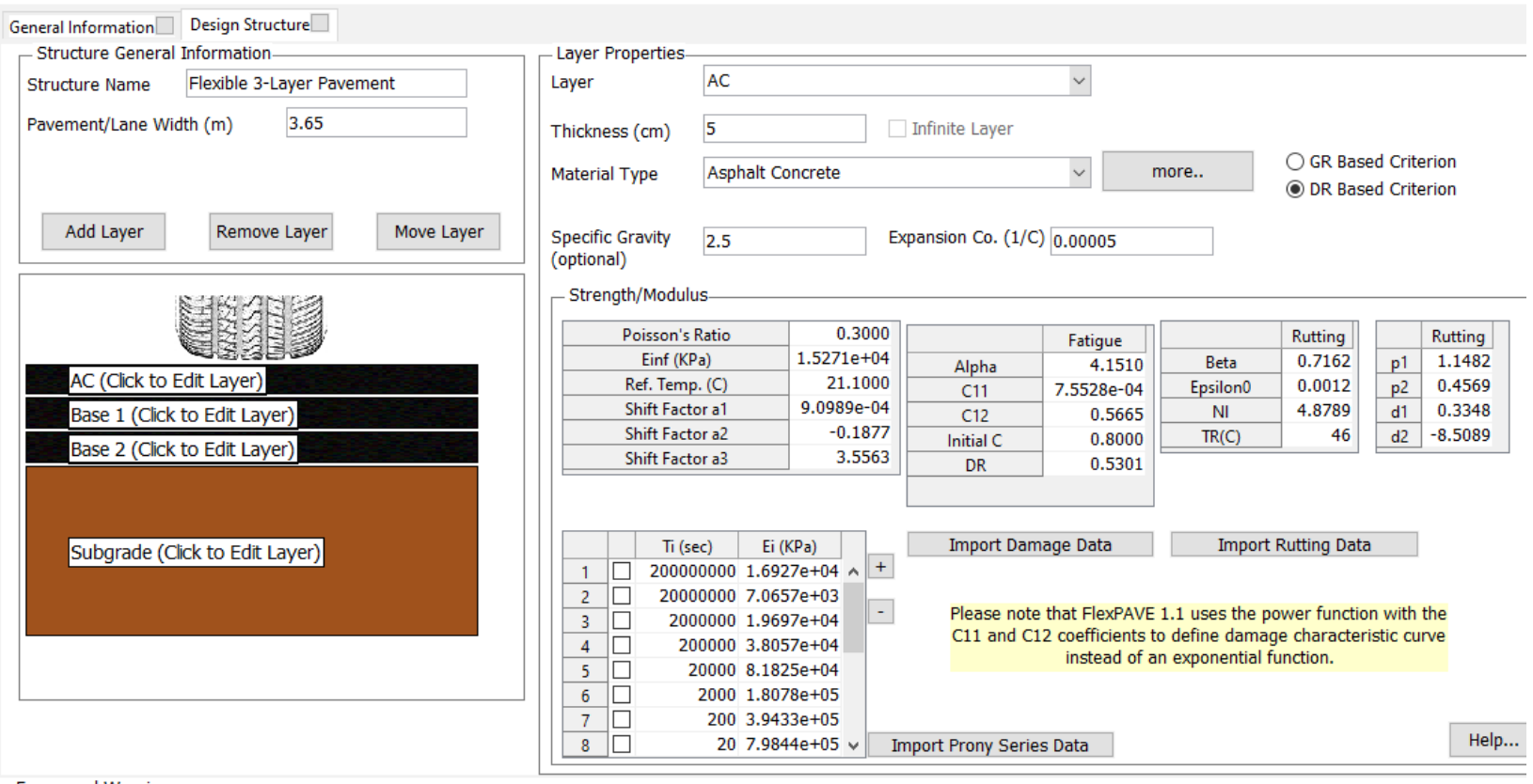

Errors and Warnings

\section{Figure 258: Design Structure of AC Layer for Run 2.11}

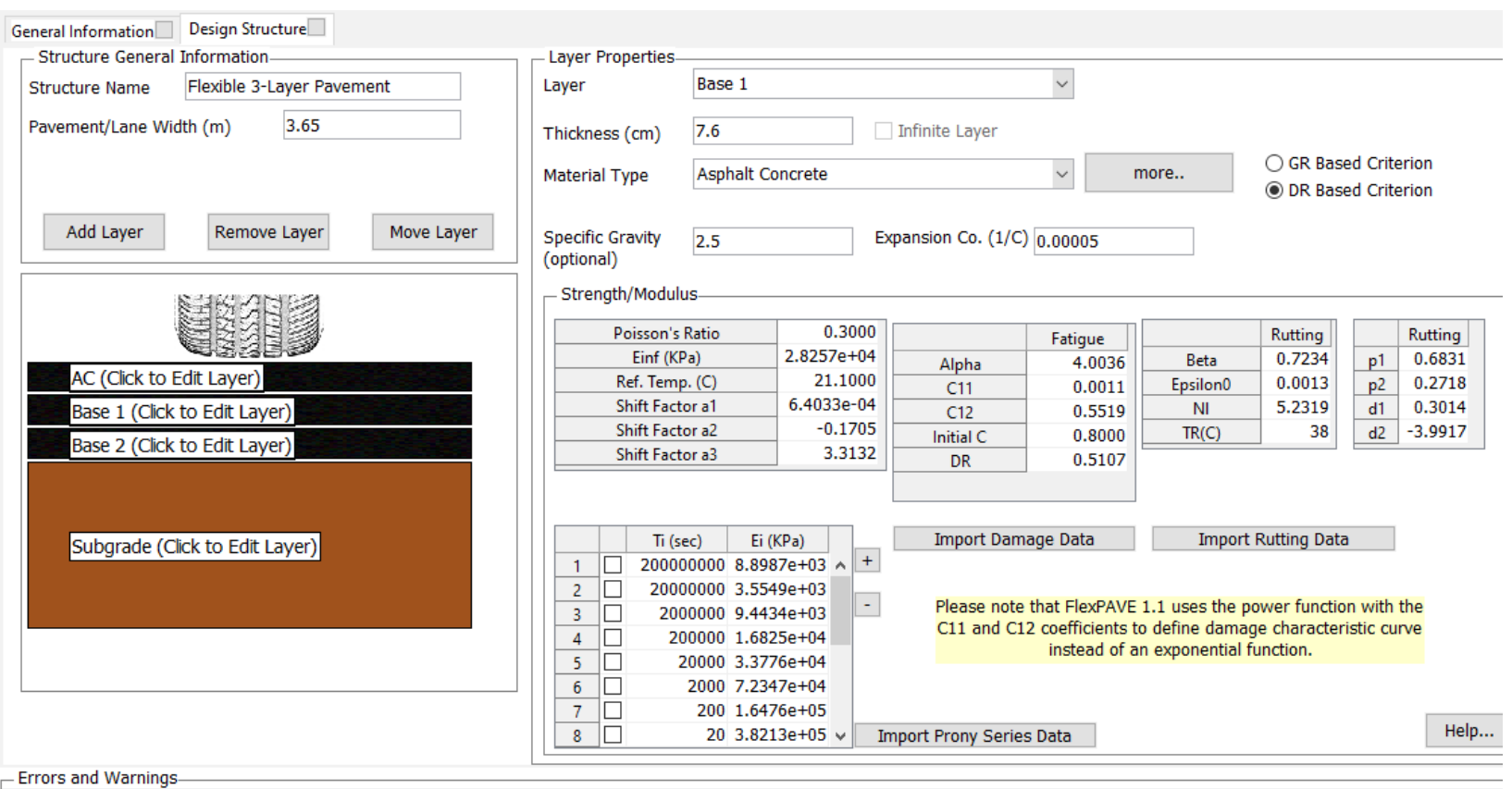

Figure 259: Design Structure of Base 1 Layer for Run 2.11 


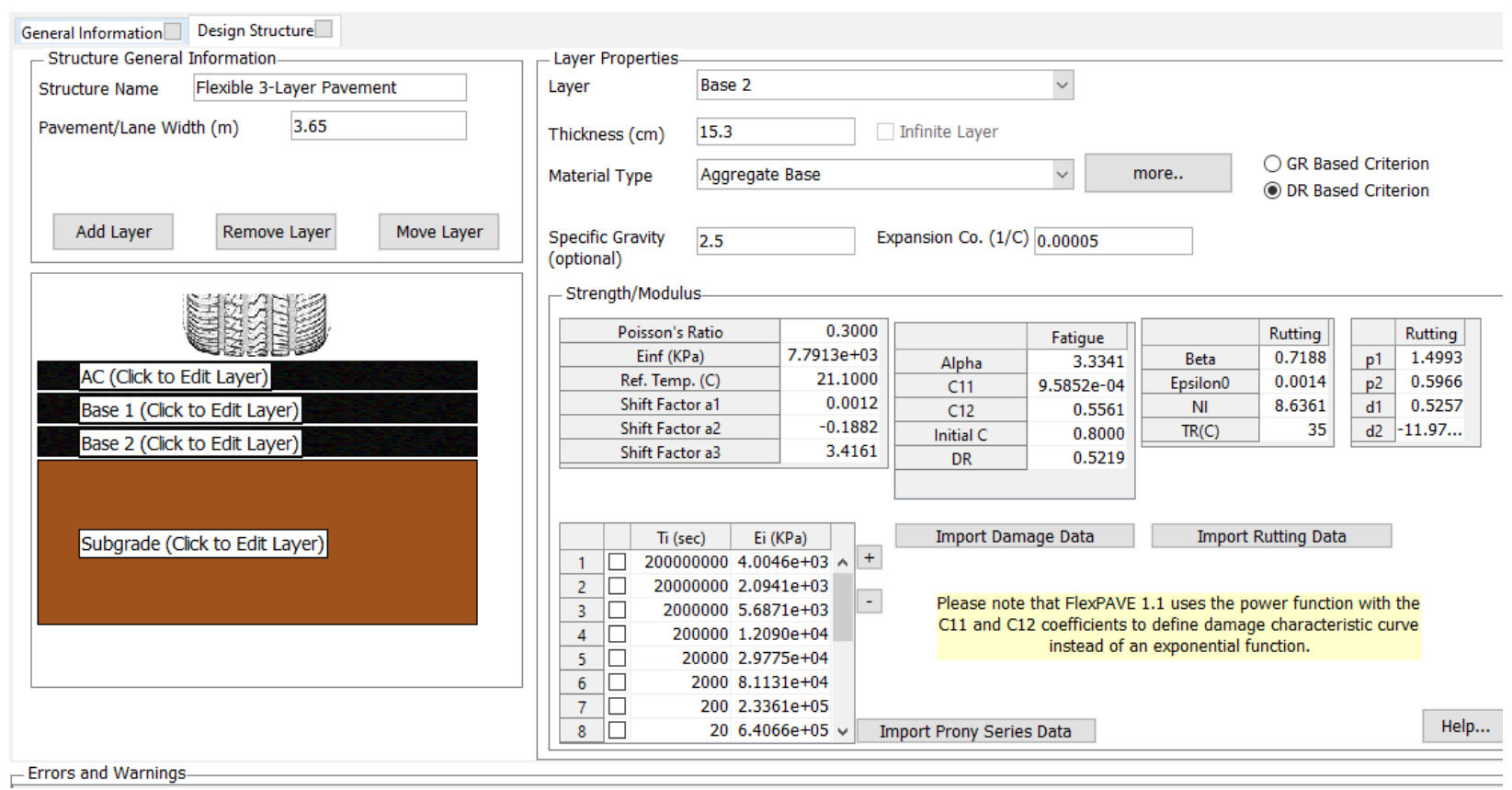

Figure 260: Design Structure of Base 2 Layer for Run 2.11

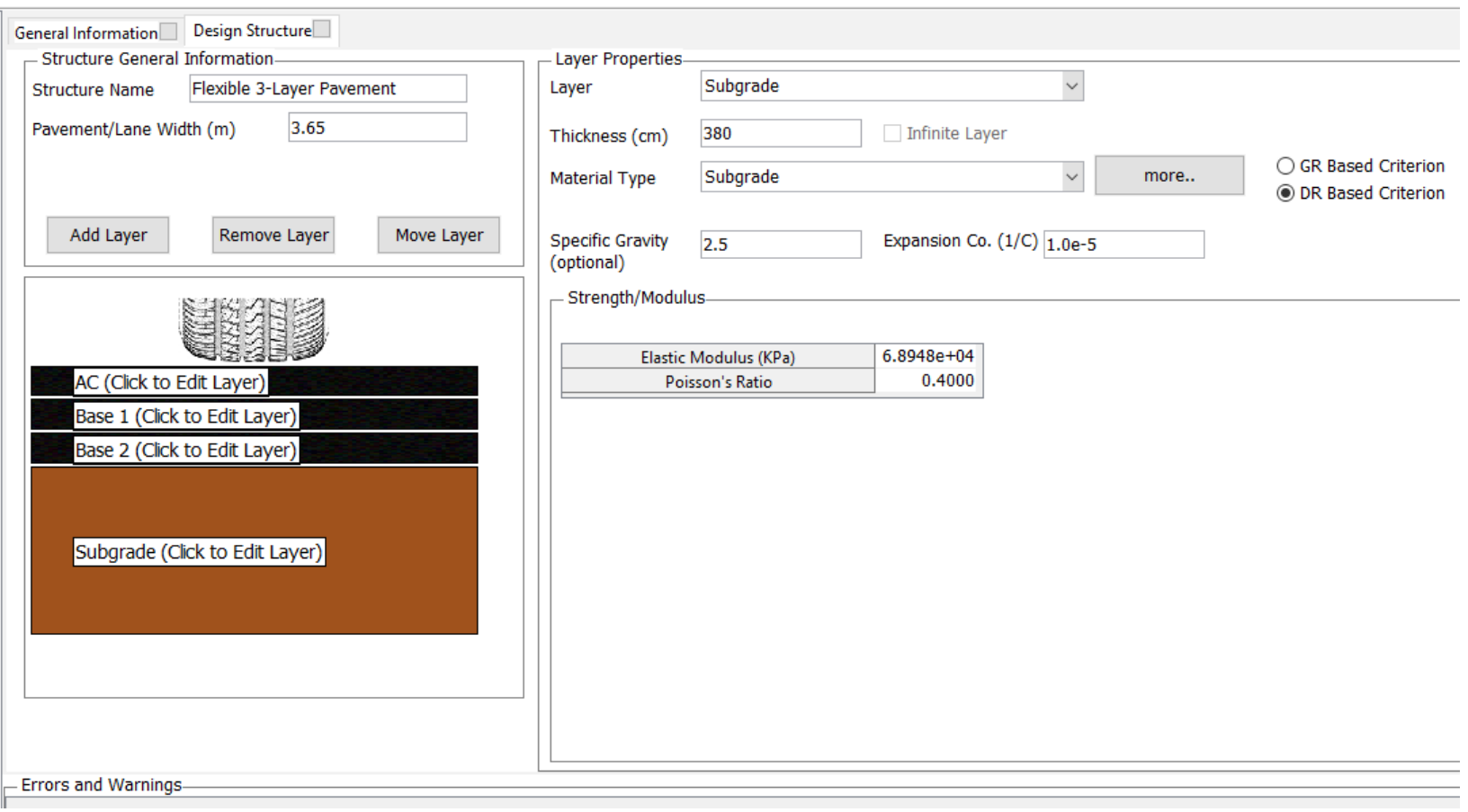

Figure 261: Design Structure of Subgrade Layer for Run 2.11 


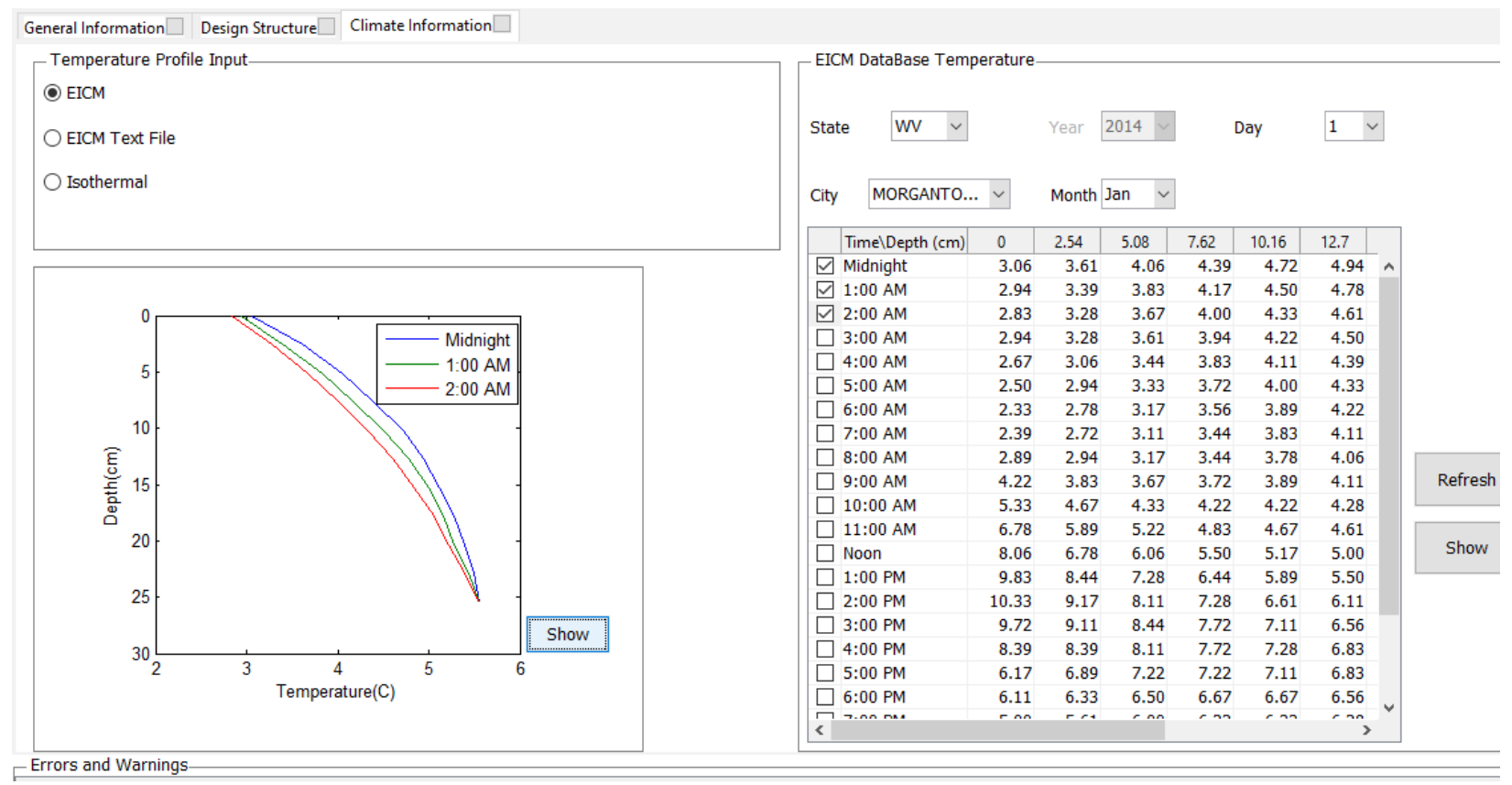

Figure 262: Climate Data for Run 2.11 


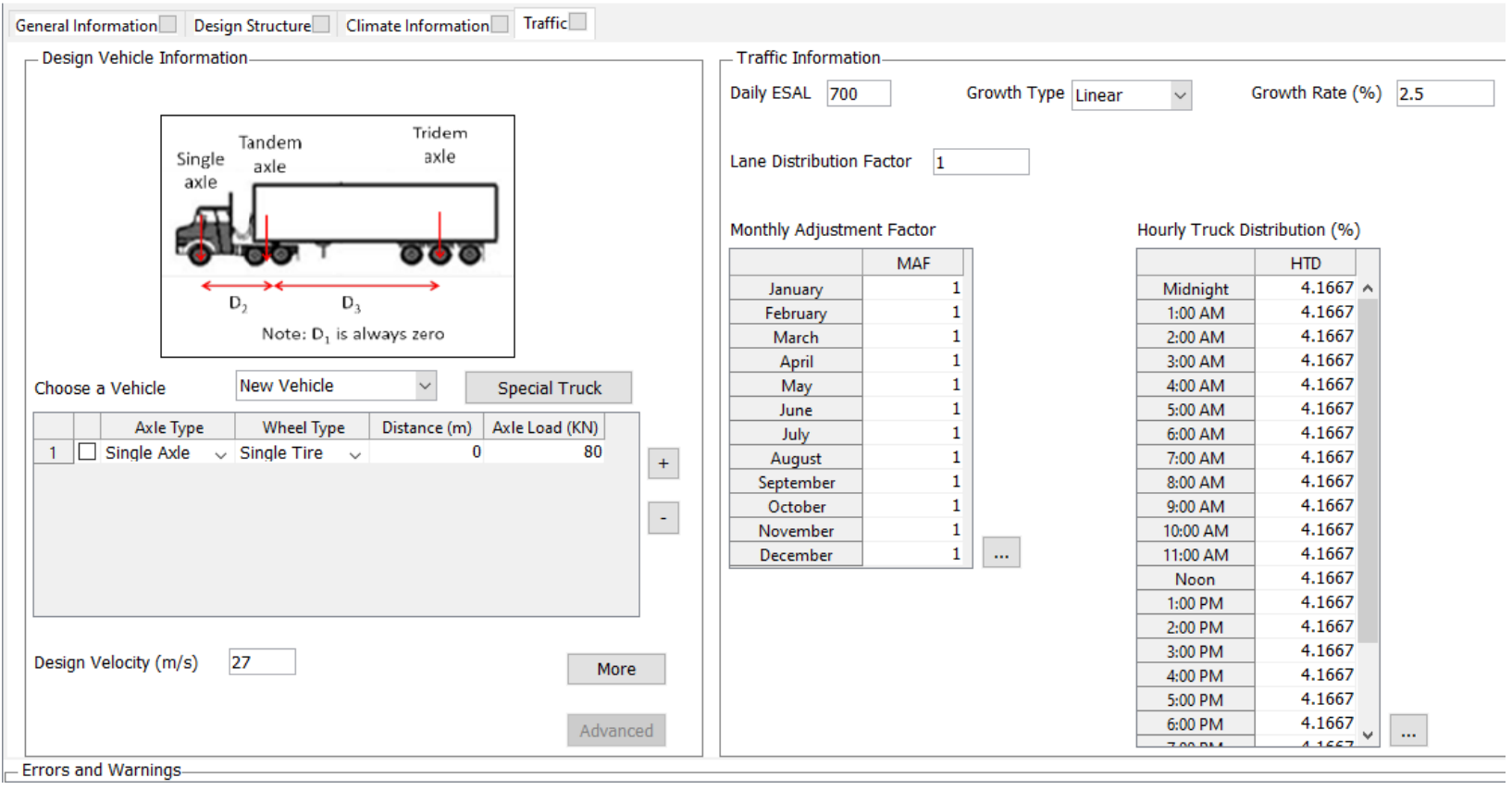

Axle Configuration

$-\quad \square \quad \times$

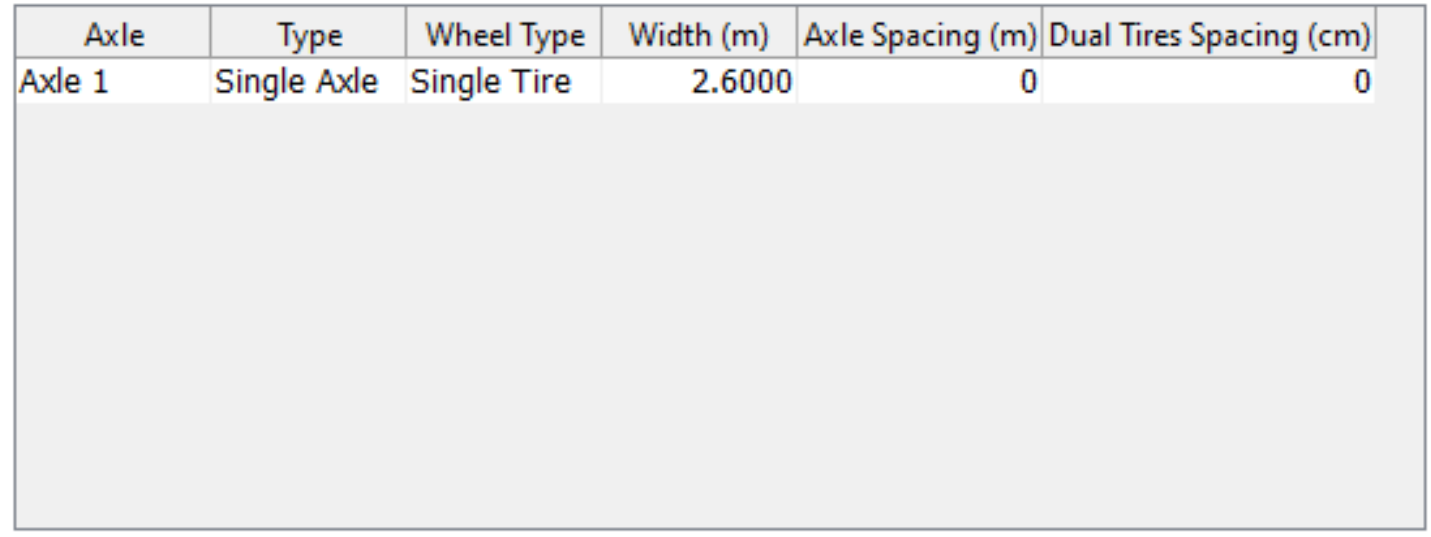

Tire Pressure (KPa) 827.37

Contact Area Shape Rectangular

Aspect Ratio (length/width) 1.5714

Shear Traction $\quad 0.0$

OK

Cancel

Figure 263: Traffic Data for Run 2.11 


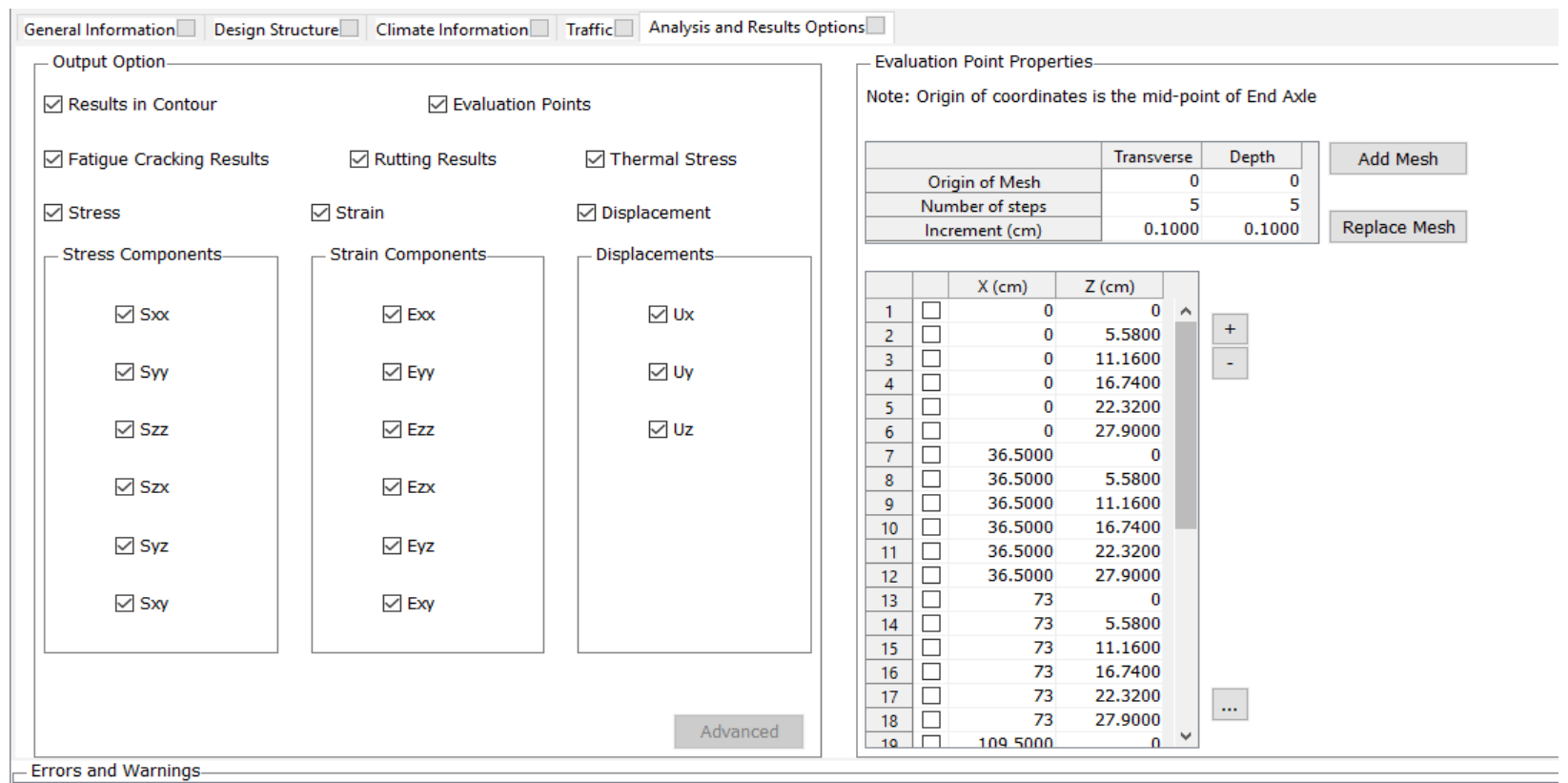

Figure 264: Output and Analysis Options for Run 2.11

Run 2.12

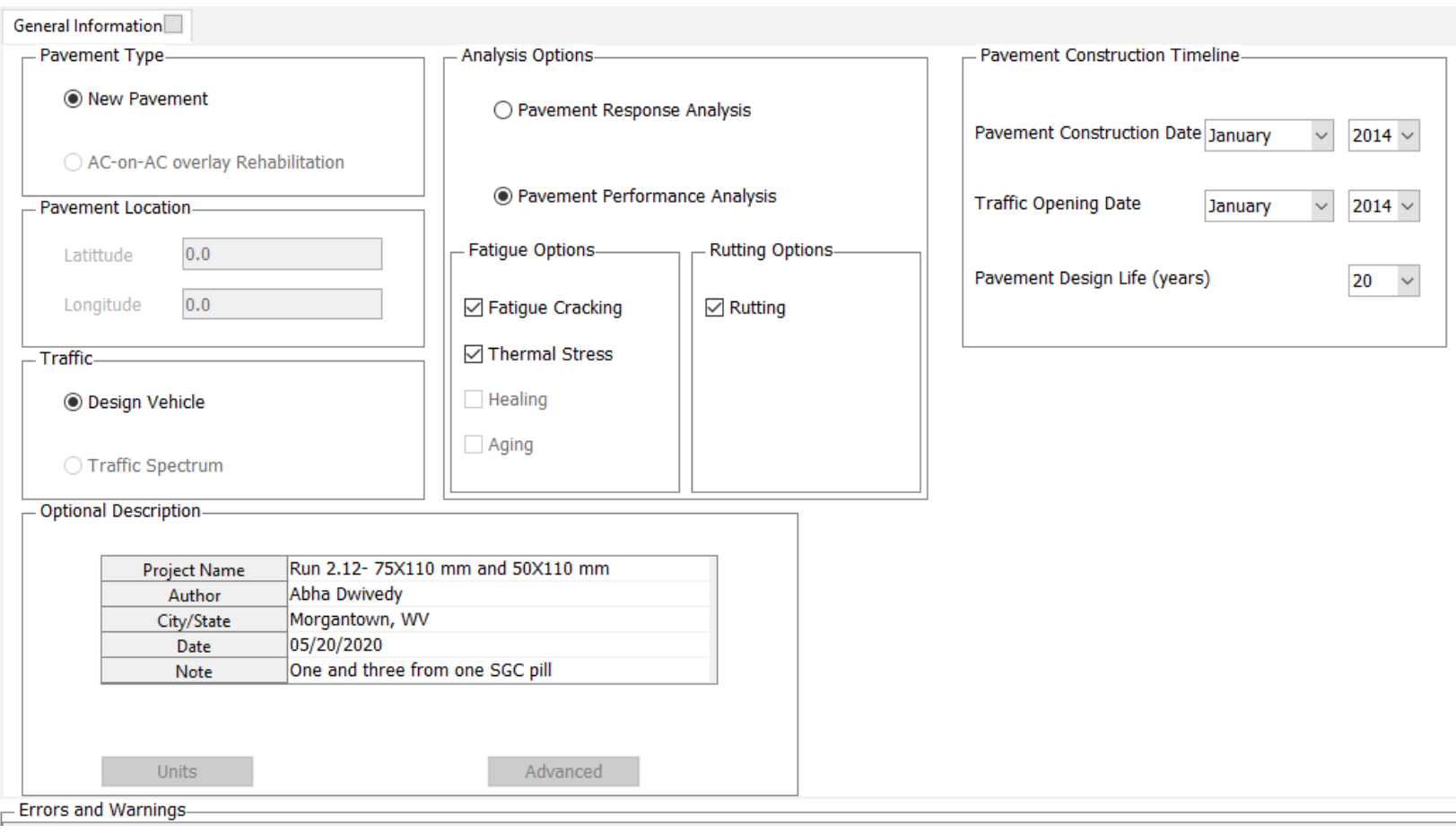

Figure 265: General Information for Run 2.12 


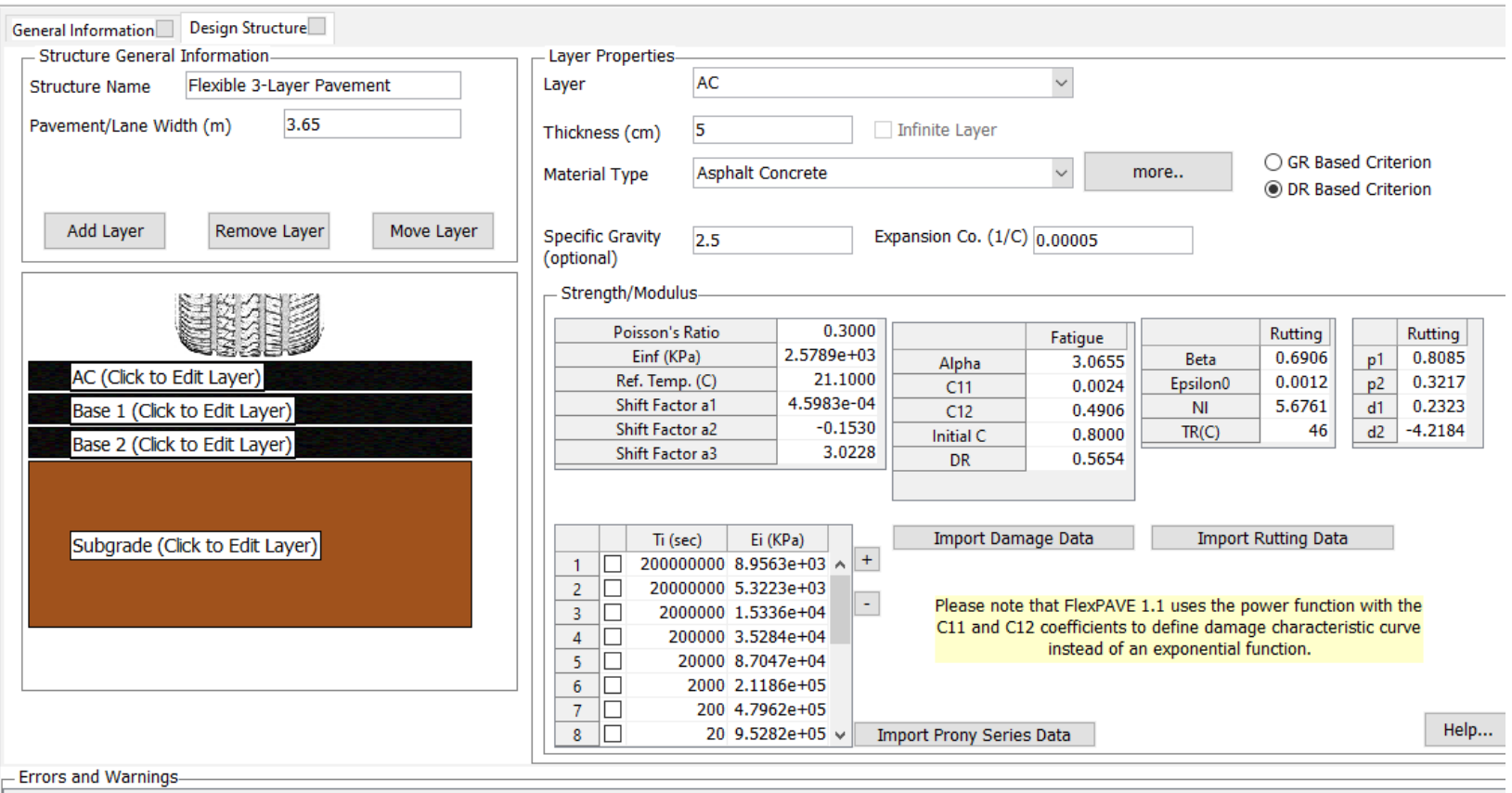

Figure 266: Design Structure of AC Layer for Run 2.12
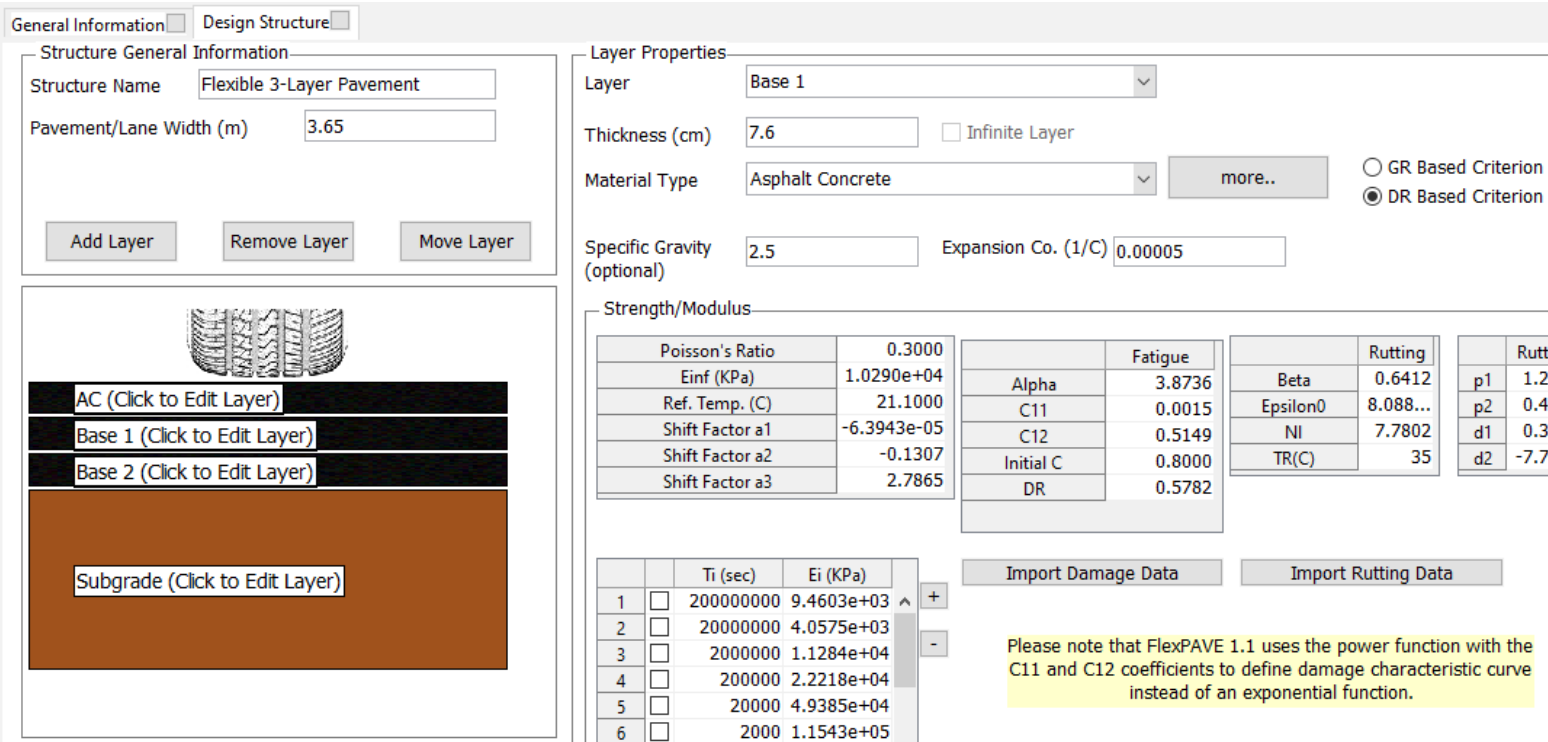

\section{(C) DR Based Criterion}

\begin{tabular}{|c|c|c|c|c|c|c|c|}
\hline Poisson's Ratio & 0.3000 & & Fatigue & & Rutting & & Rutting \\
\hline Einf (KPa) & $1.0290 \mathrm{e}+04$ & Alpha & 3.8736 & Beta & 0.6412 & p1 & 1.2119 \\
\hline Ref. Temp. (C) & 21.1000 & $\mathrm{C} 11$ & 0.0015 & Epsilon0 & $8.088 \ldots$ & $p^{2}$ & 0.4823 \\
\hline Shift Factor a1 & $-6.3943 e-05$ & $\mathrm{C} 12$ & 0.5149 & $\mathrm{NI}$ & 7.7802 & d1 & 0.3867 \\
\hline Shift Factor a2 & -0.1307 & Initial C & 0.8000 & $\operatorname{TR}(\mathrm{C})$ & 35 & d2 & -7.7981 \\
\hline Shift Factor a3 & 2.7865 & DR & 0.5782 & & & & \\
\hline
\end{tabular}

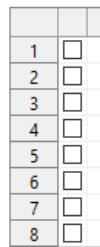

Ti $(\mathrm{sec}) \quad \mathrm{Ei}(\mathrm{KPa})$ $2000000009.4603 \mathrm{e}+03 \wedge+$ 0000000.4603 $20000001.1284 \mathrm{e}+04$ $2000002.2218 \mathrm{e}+04$ $200004.9385 e+04$ $20001.1543 e+05$ $2002.7311 \mathrm{e}+05$ \begin{tabular}{l|l}
20 & $6.1127 e+05 \checkmark$ \\
& Import Prony Series Data
\end{tabular}

Please note that FlexPAVE 1.1 uses the power function with the $\mathrm{C} 11$ and $\mathrm{C} 12$ coefficients to define damage characteristic curve instead of an exponential function.

Errors and Warnings

Figure 267: Design Structure of Base 1 Layer for Run 2.12 

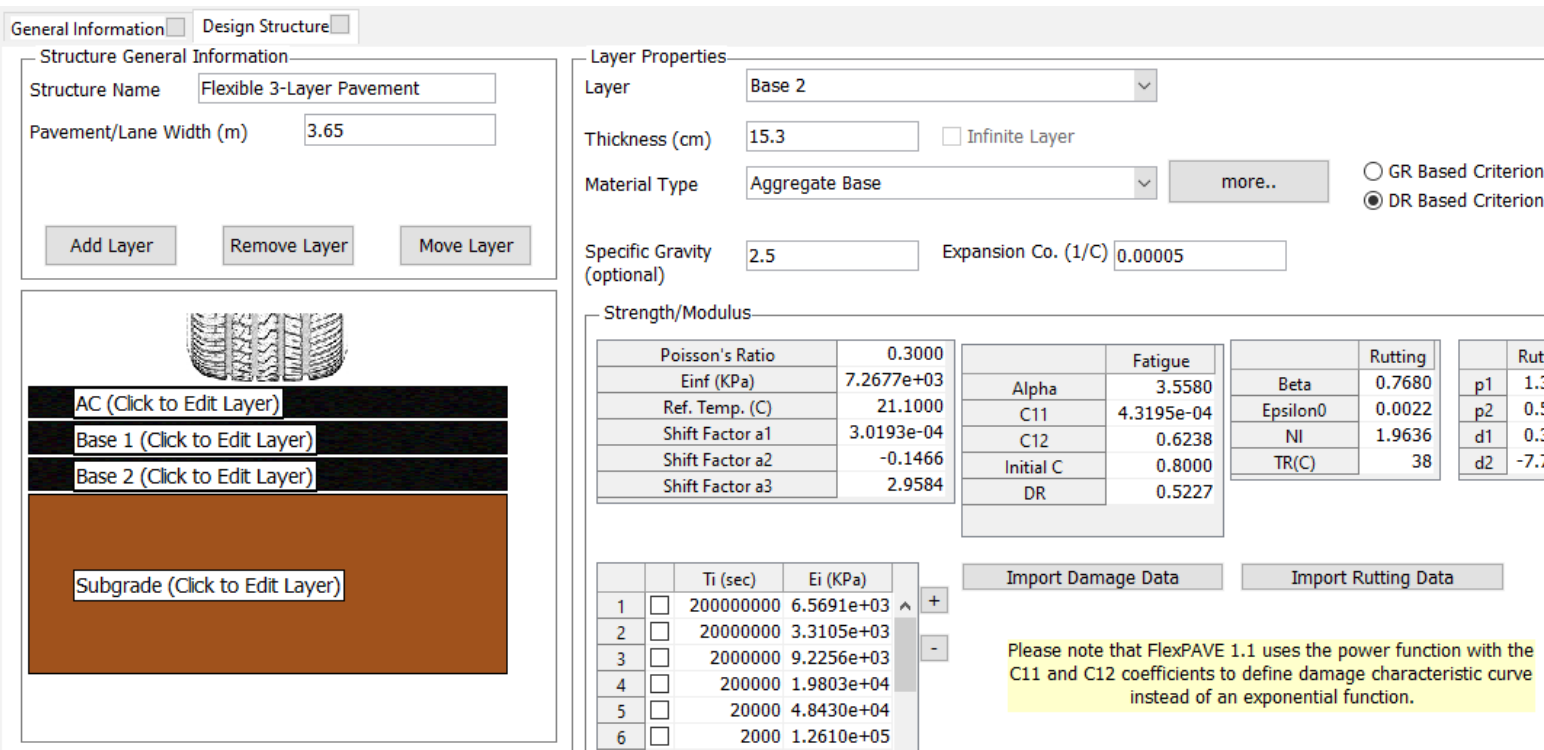

\begin{tabular}{|r|}
0.3000 \\
$7.2677 \mathrm{e}+03$ \\
21.1000 \\
$3.0193 \mathrm{e}-04$ \\
-0.1466 \\
2.9584 \\
\hline
\end{tabular}

\begin{tabular}{|c|c|}
\hline Alpha & \\
C11 & 4. \\
\hline C12 & \\
\hline Initial C & \\
\cline { 1 - 1 } DR & \\
\hline
\end{tabular}

\begin{tabular}{|r|}
\hline Fatigue \\
\hline 3.5580 \\
$4.3195 \mathrm{e}-04$ \\
0.6238 \\
0.8000 \\
0.5227 \\
\hline
\end{tabular}

\begin{tabular}{|c|c|c|c|}
\hline & Rutting & & Rutting \\
\hline Beta & 0.7680 & $\mathrm{p} 1$ & 1.3648 \\
\hline Epsilon0 & 0.0022 & $p^{2}$ & 0.5431 \\
\hline $\mathrm{NI}$ & 1.9636 & d1 & 0.3884 \\
\hline $\operatorname{TR}(\mathrm{C})$ & 38 & $\mathrm{~d} 2$ & -7.7527 \\
\hline
\end{tabular}

Errors and Warning

Figure 268: Design Structure of Base 2 Layer for Run 2.12

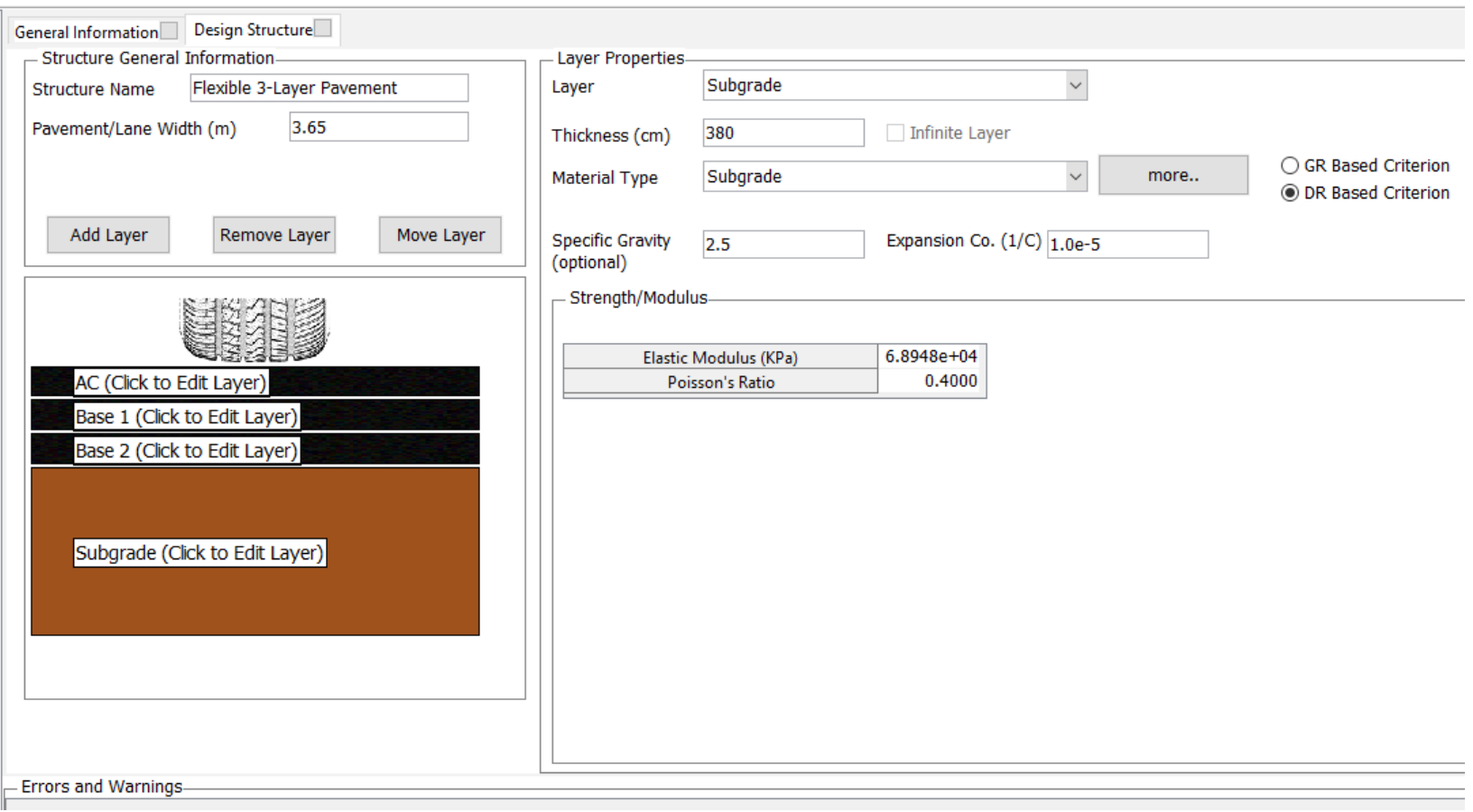

Figure 269: Design Structure of Subgrade Layer for Run 2.12 


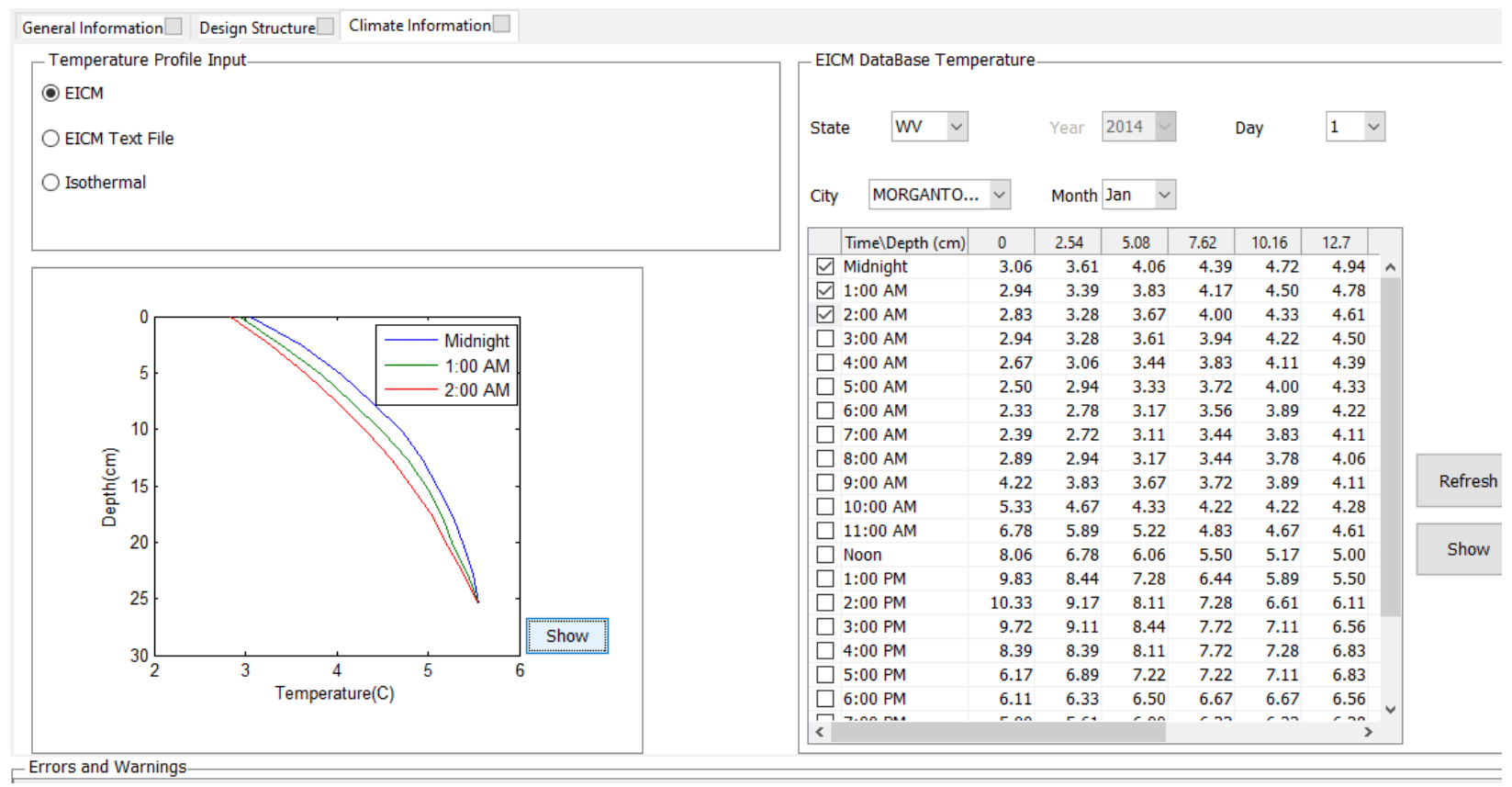

Figure 270: Climate Data for Run 2.12 

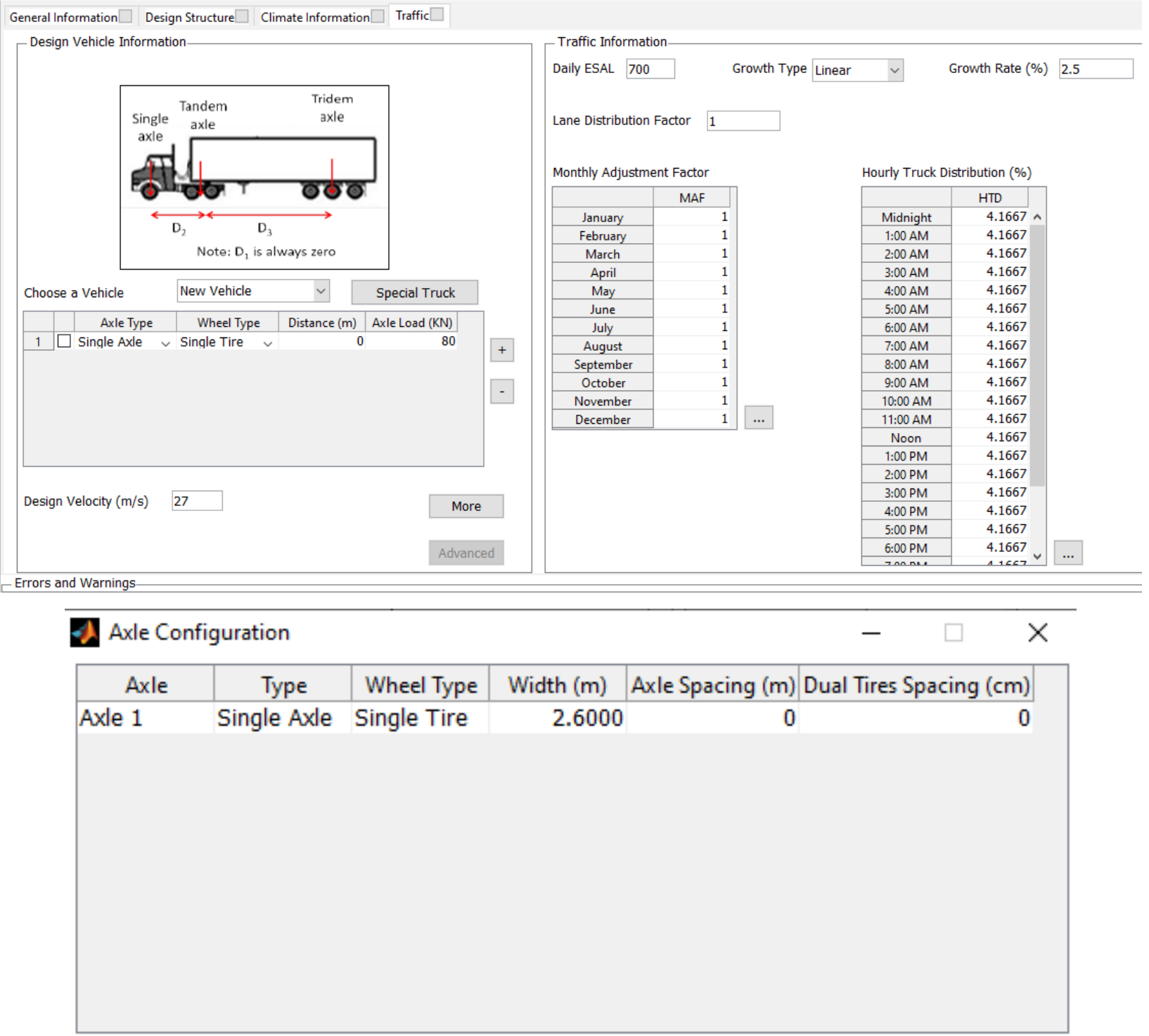

Tire Pressure (KPa) 827.37

\begin{tabular}{|c|c|c|c|c|}
\hline Contact Area Shape & Rectangular & $\checkmark$ & Aspect Ratio (length/widt) & 1.5714 \\
\hline
\end{tabular}

Shear Traction 0.0

OK

Cancel

Figure 271: Traffic Data for Run 2.12 


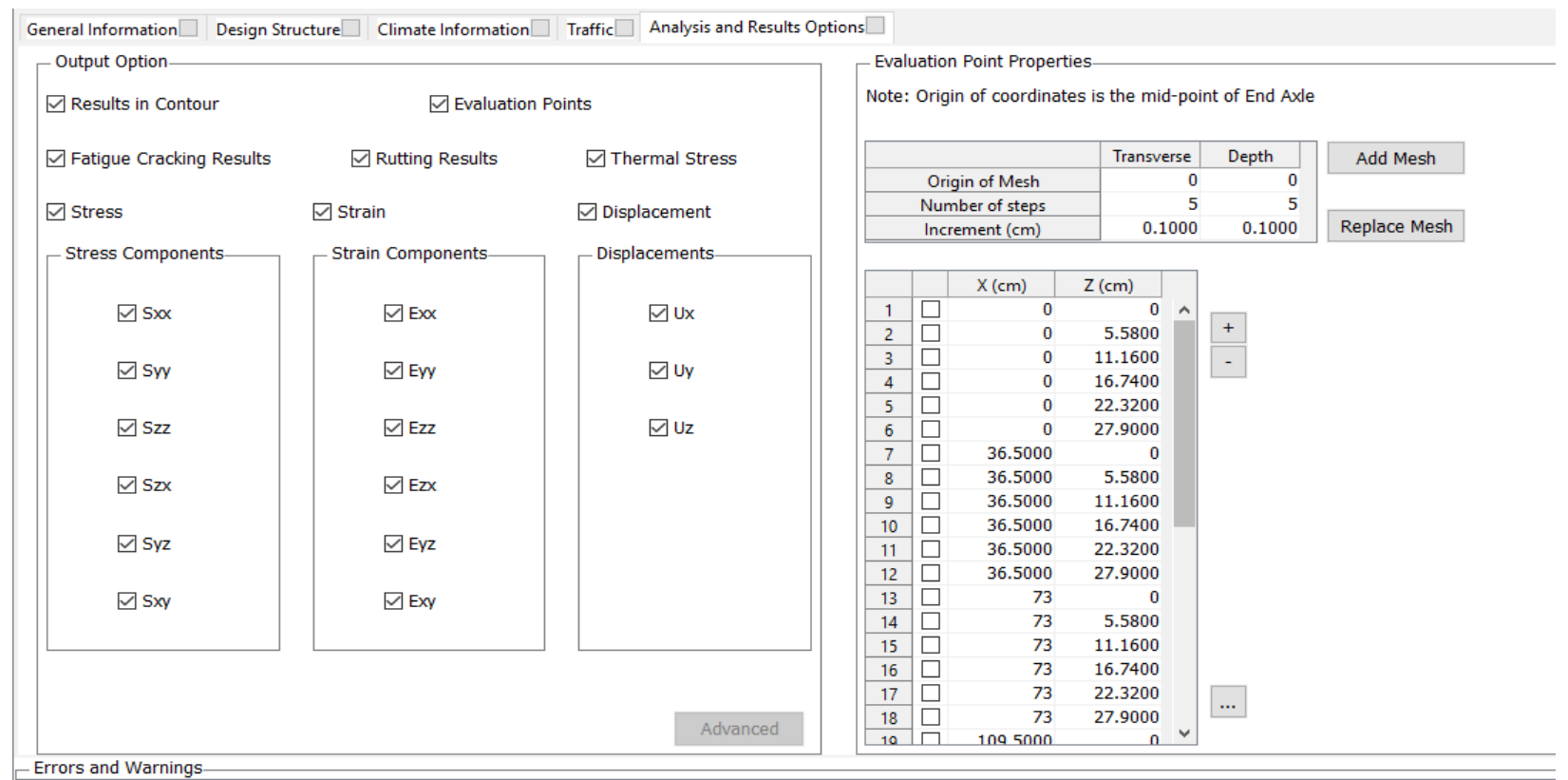

Figure 272: Output and Analysis Options for Run 2.12 


\section{Run 3.1}

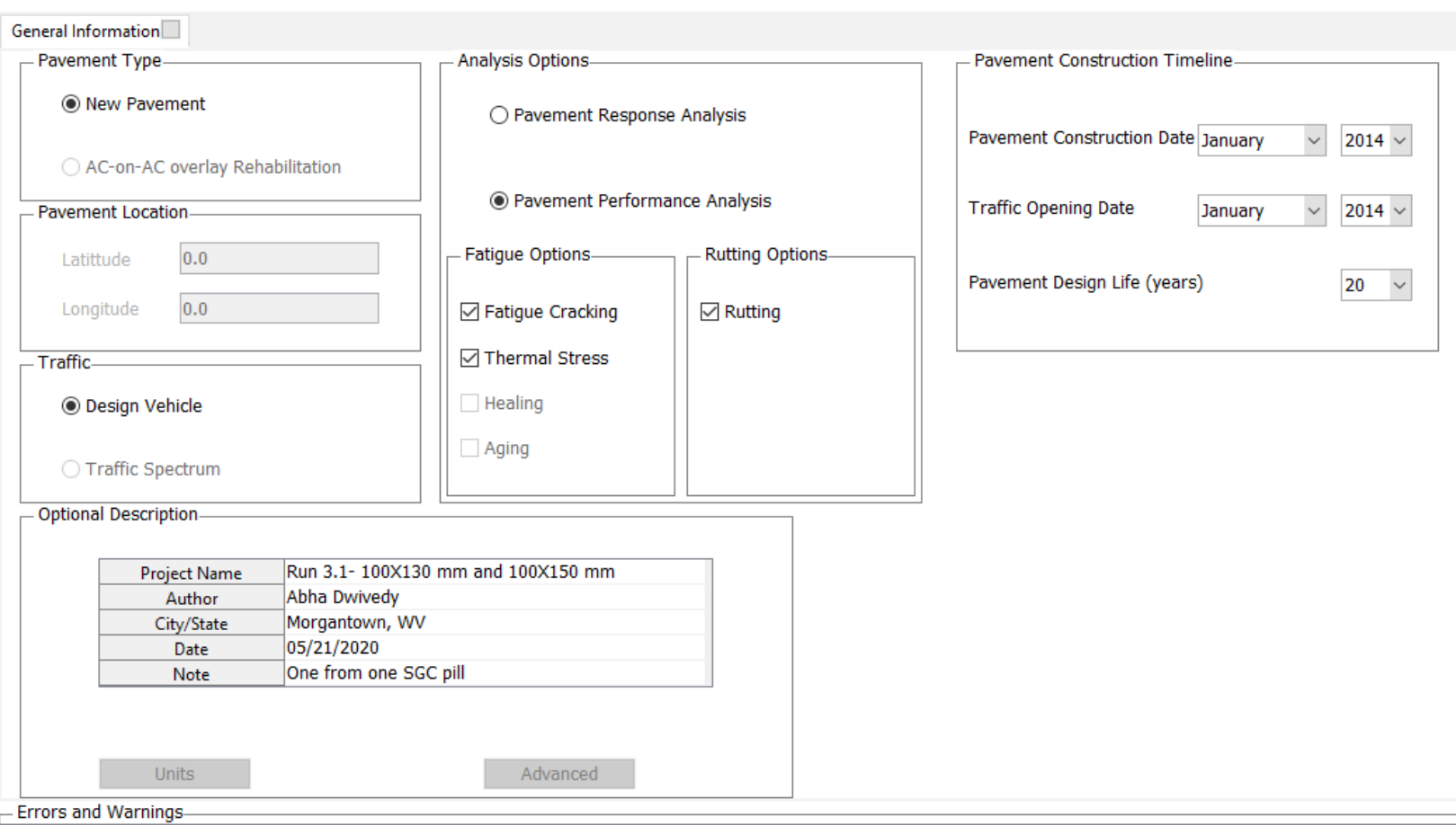

\section{Figure 273: General Information for Run 3.1}

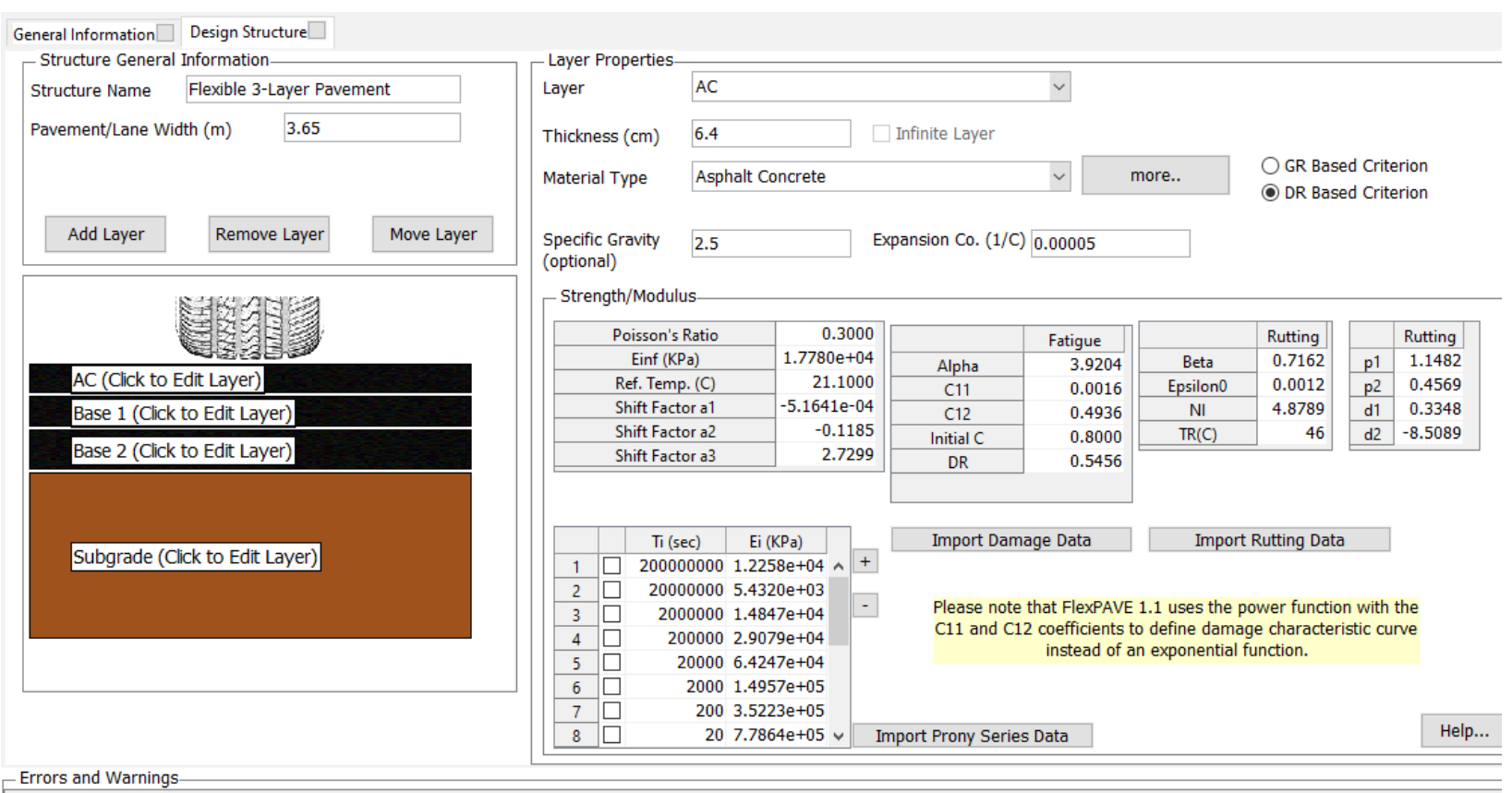

Figure 274: Design Structure of AC Layer for Run 3.1 


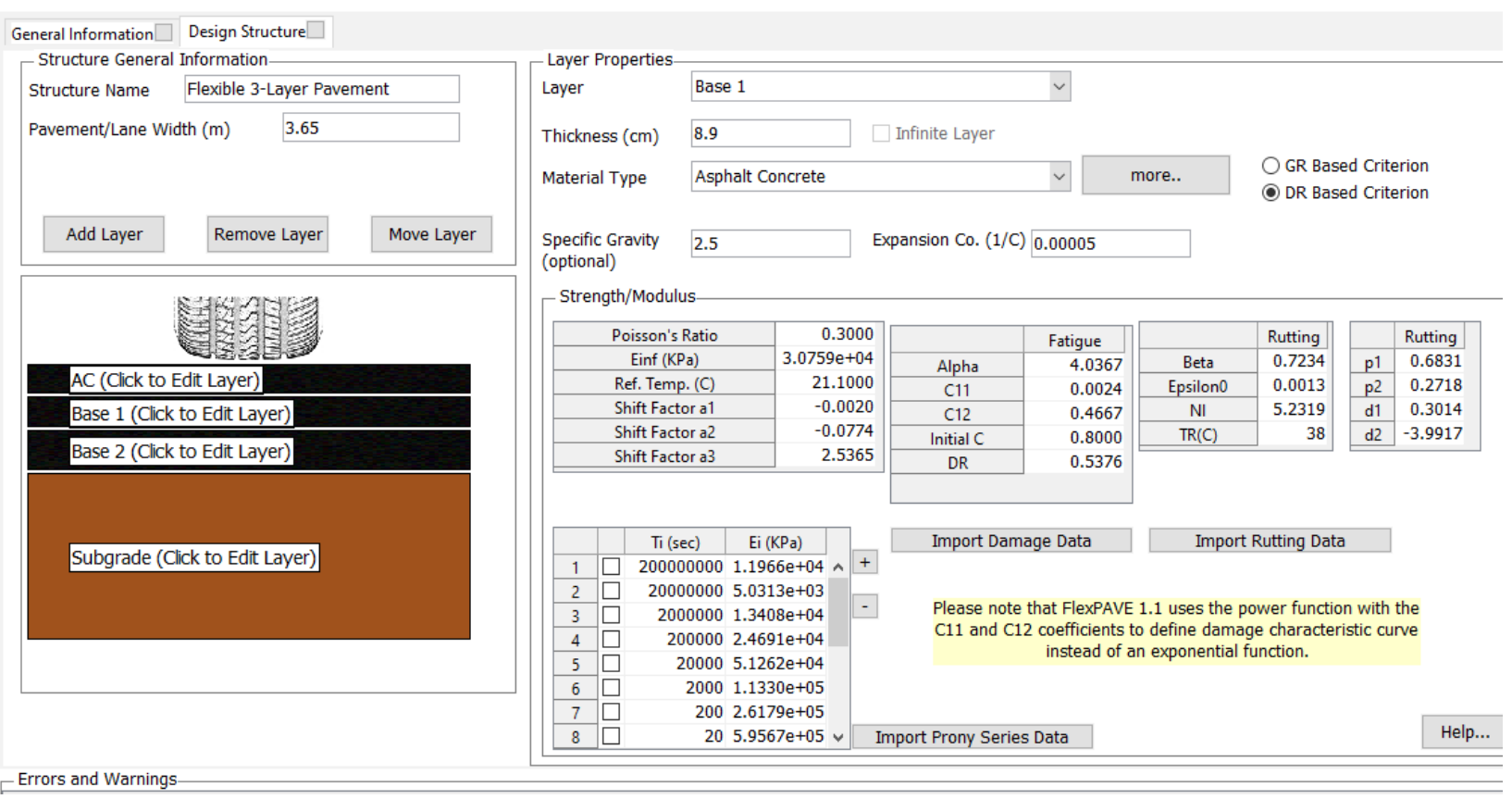

Figure 275: Design Structure of Base 1 Layer for Run 3.1

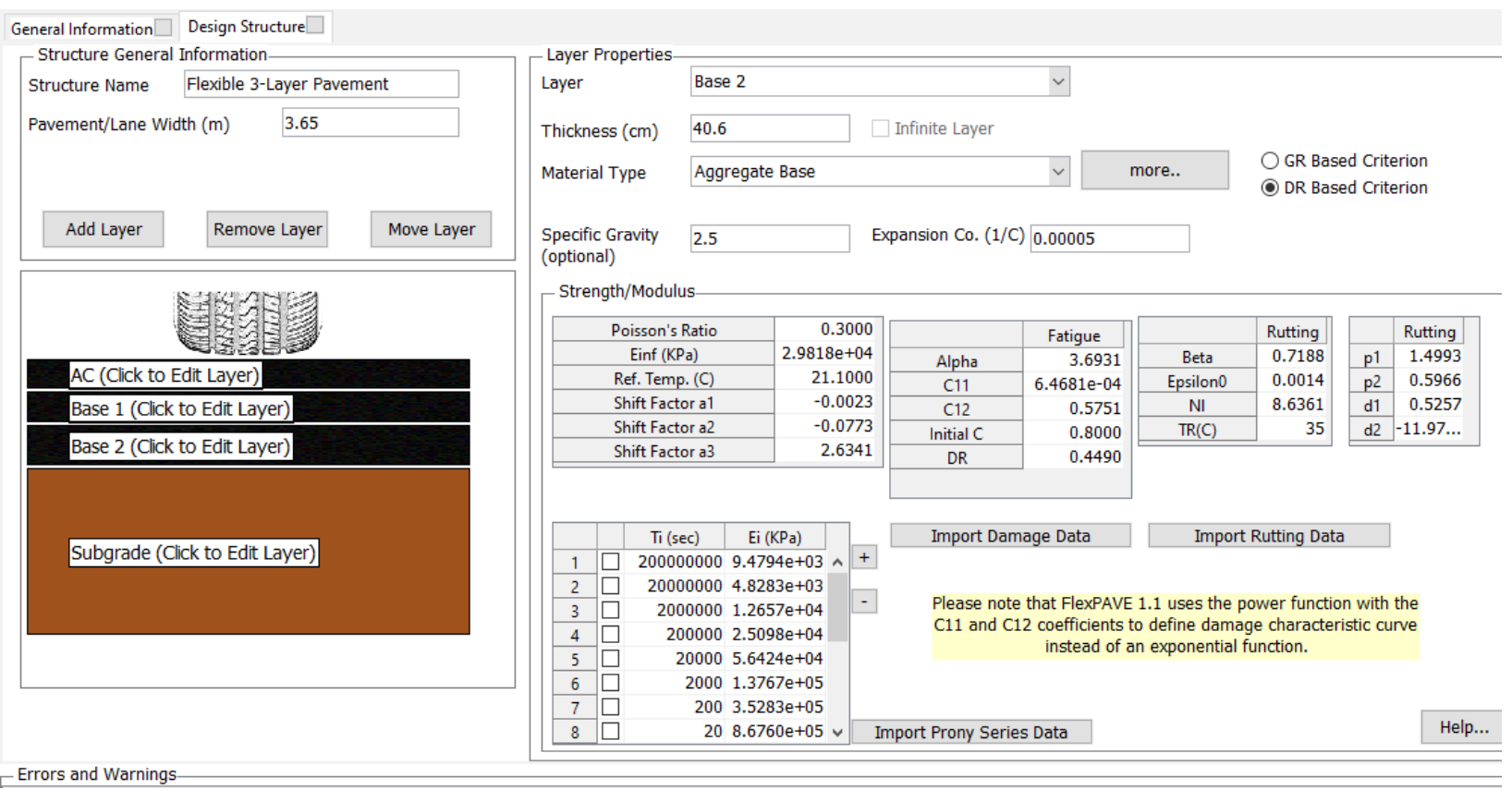

Figure 276: Design Structure of Base 2 Layer for Run 3.1 


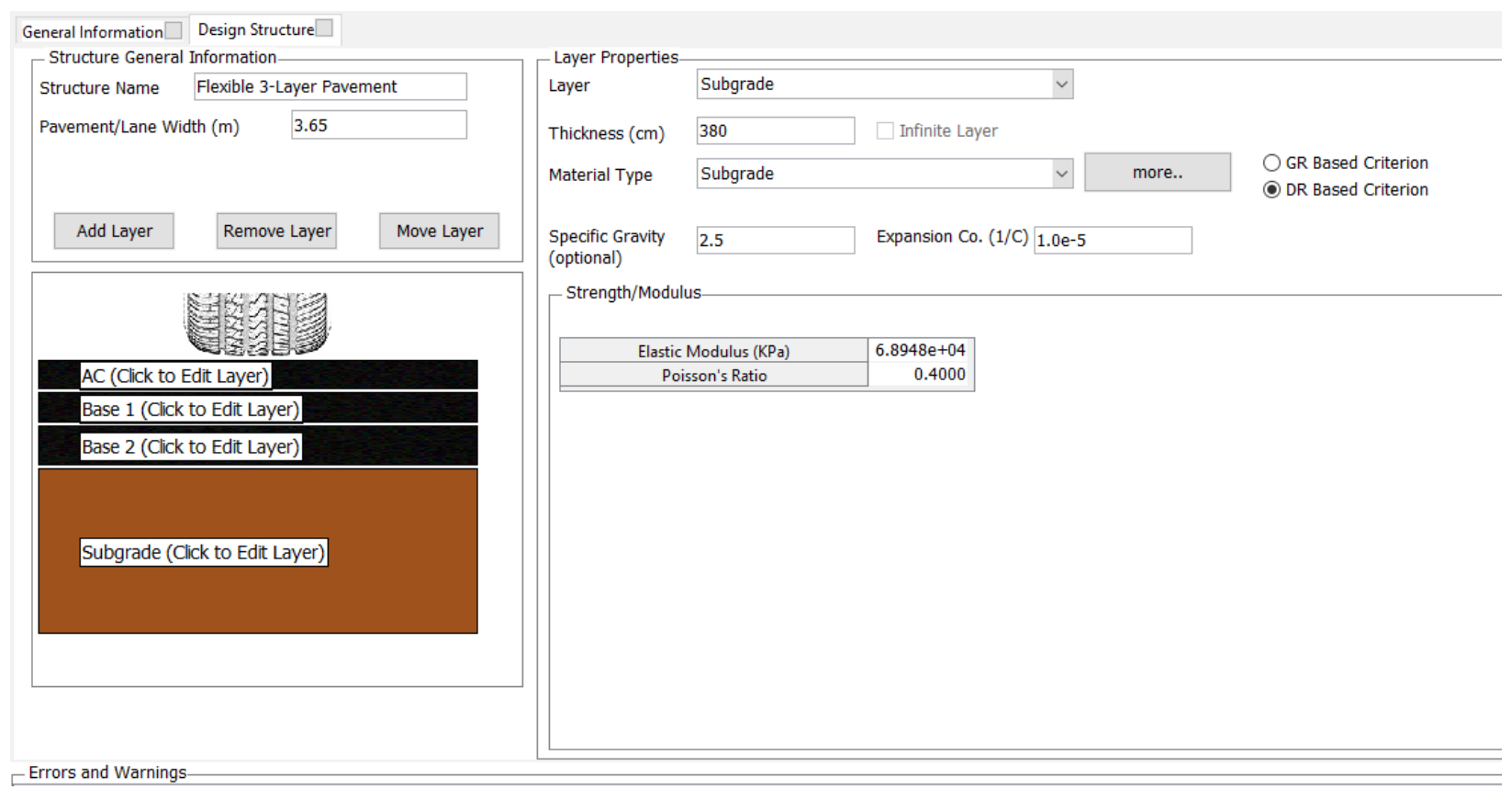

Figure 277: Design Structure of Subgrade Layer for Run 3.1

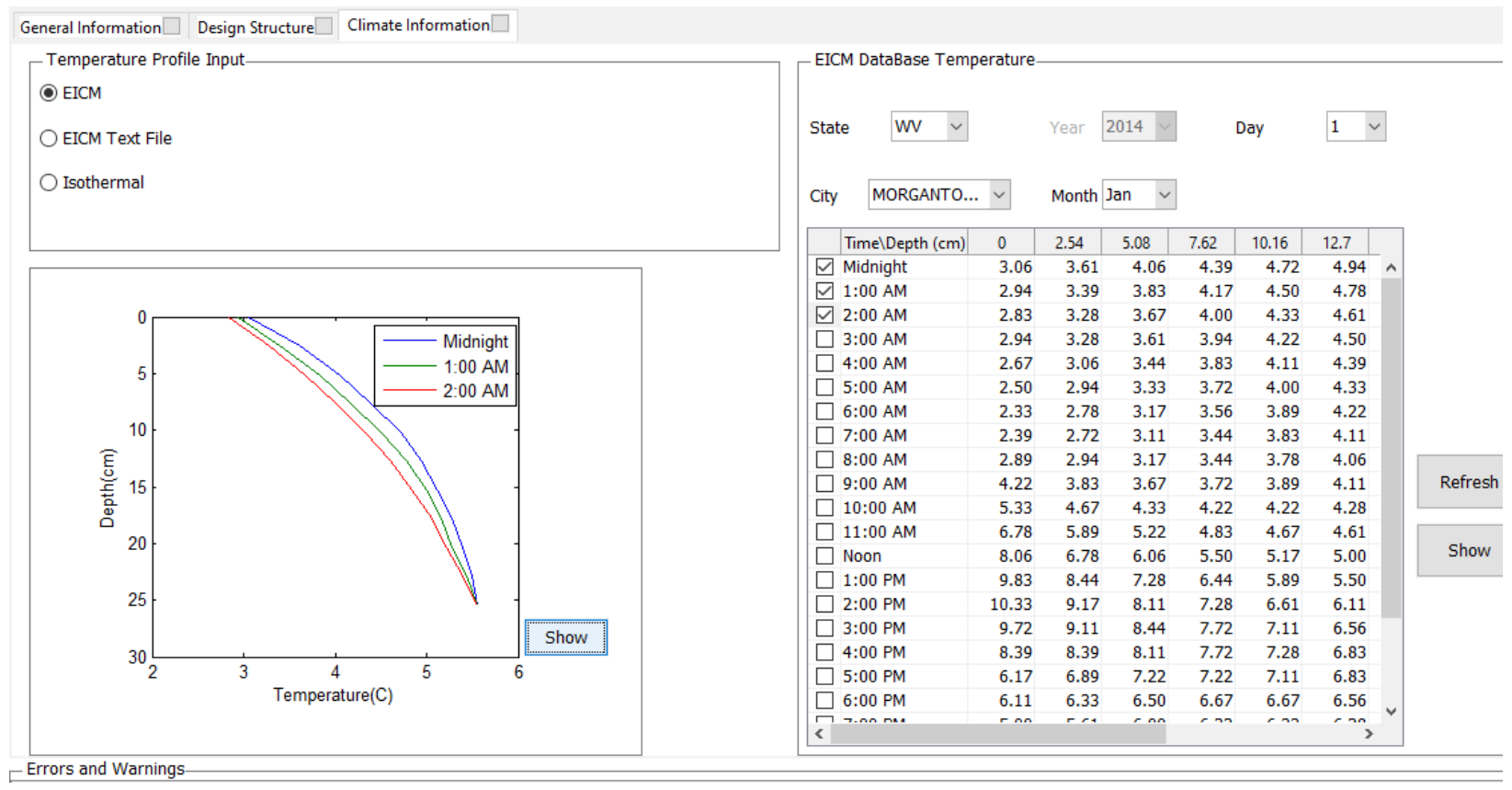

Figure 278: Climate Data for Run 3.1 


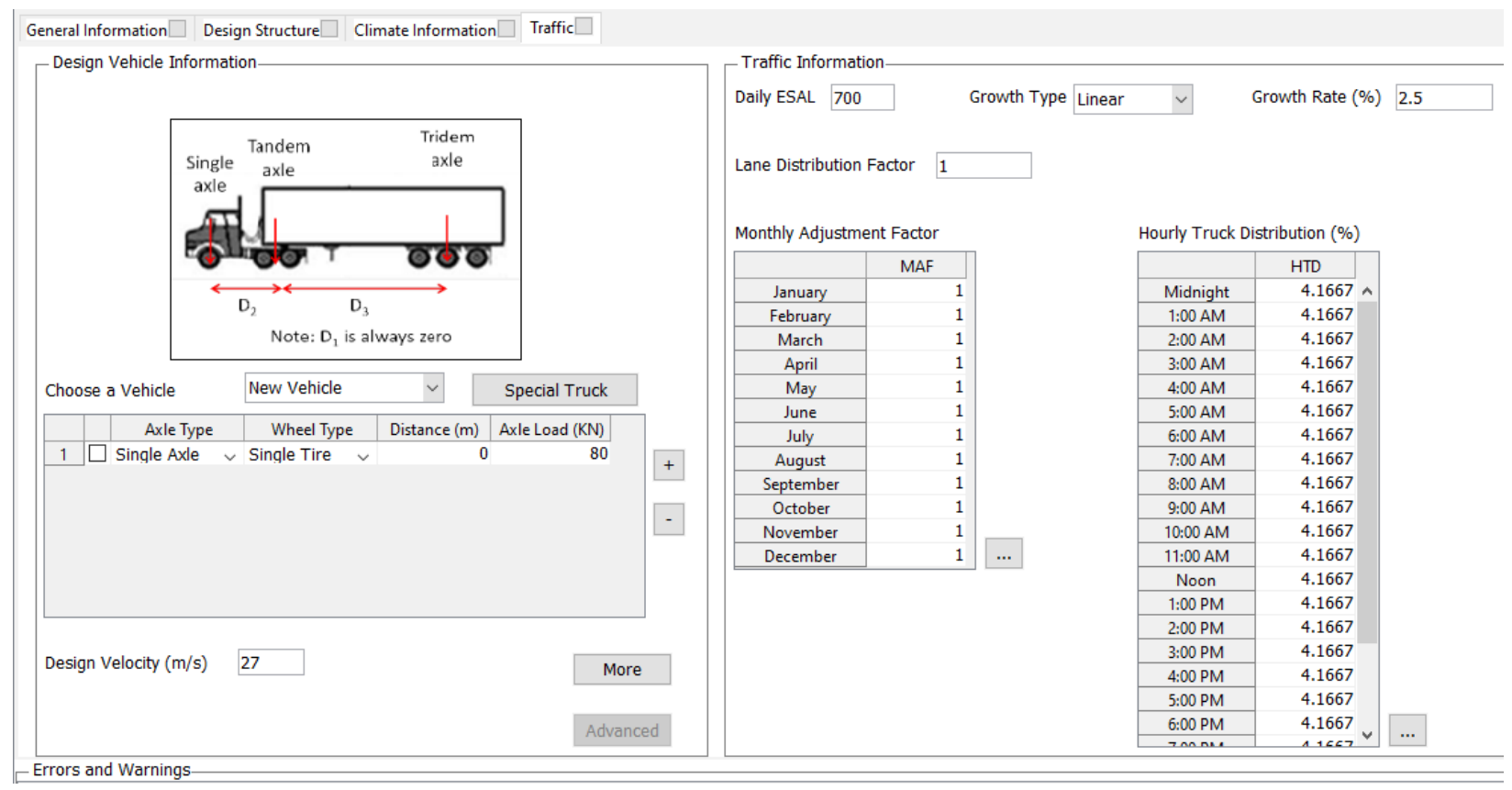

Axle Configuration

$-\square \times$

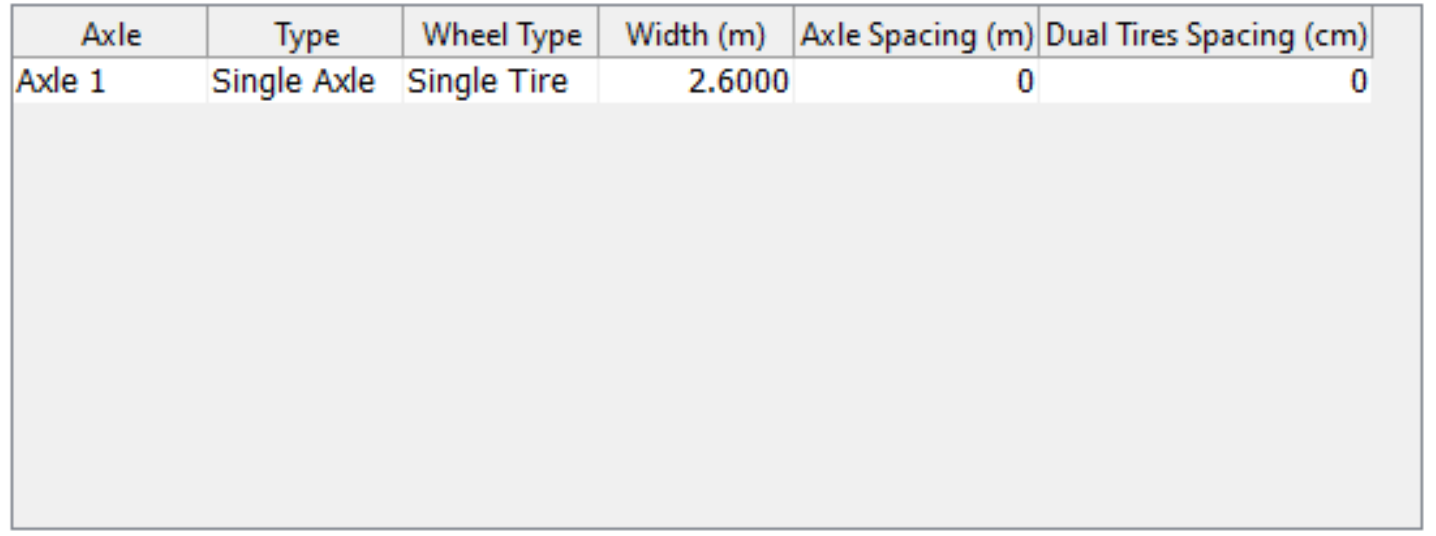

Tire Pressure (KPa) 827.37

Contact Area Shape Rectangular $\vee \quad$ Aspect Ratio (length/width) 1.5714

Shear Traction $\quad 0.0$

OK

Cancel

Figure 279: Traffic Data for Run 3.1 


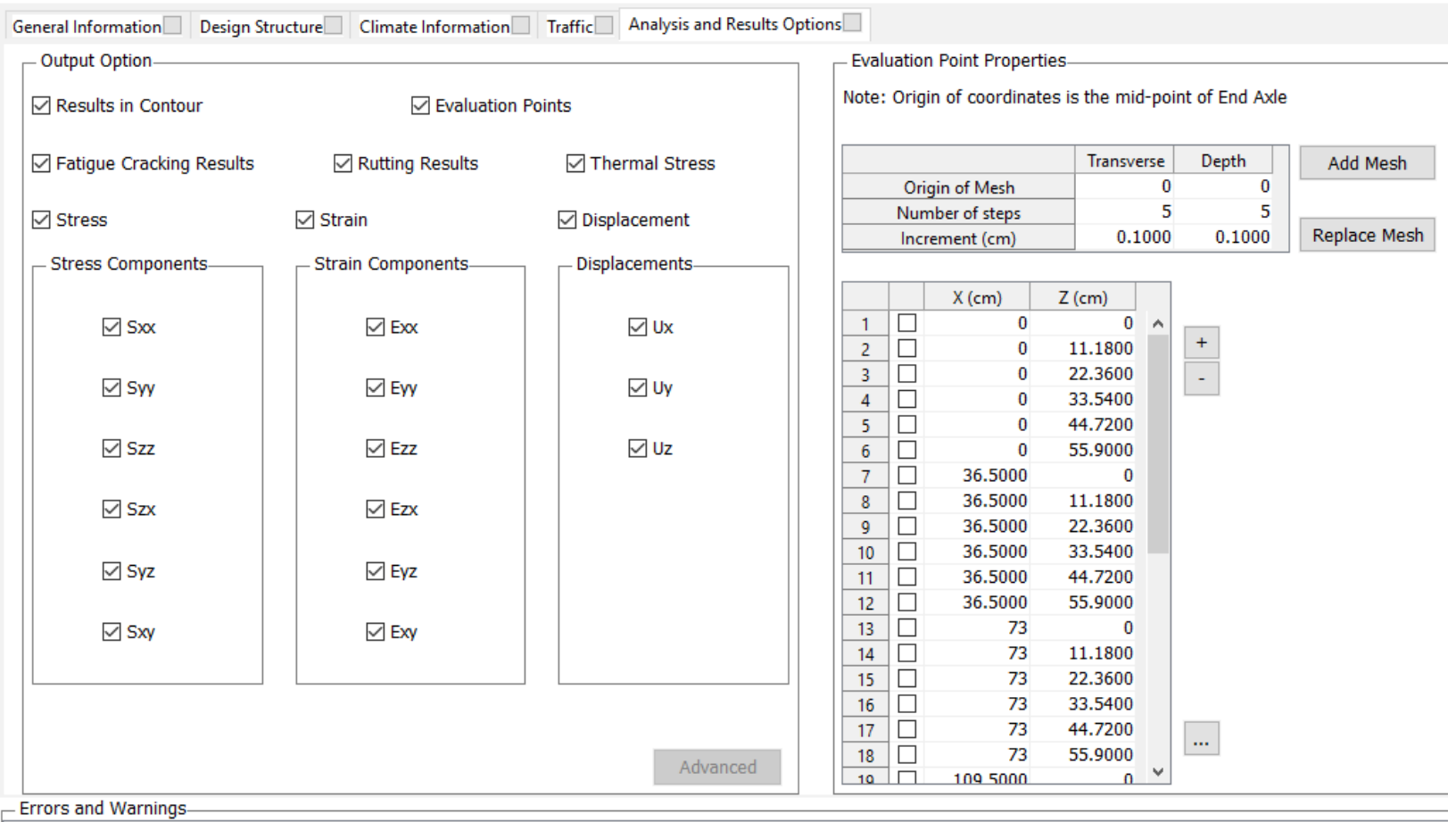

Figure 280: Output and Analysis Options for Run 3.1

\section{Run 3.2}

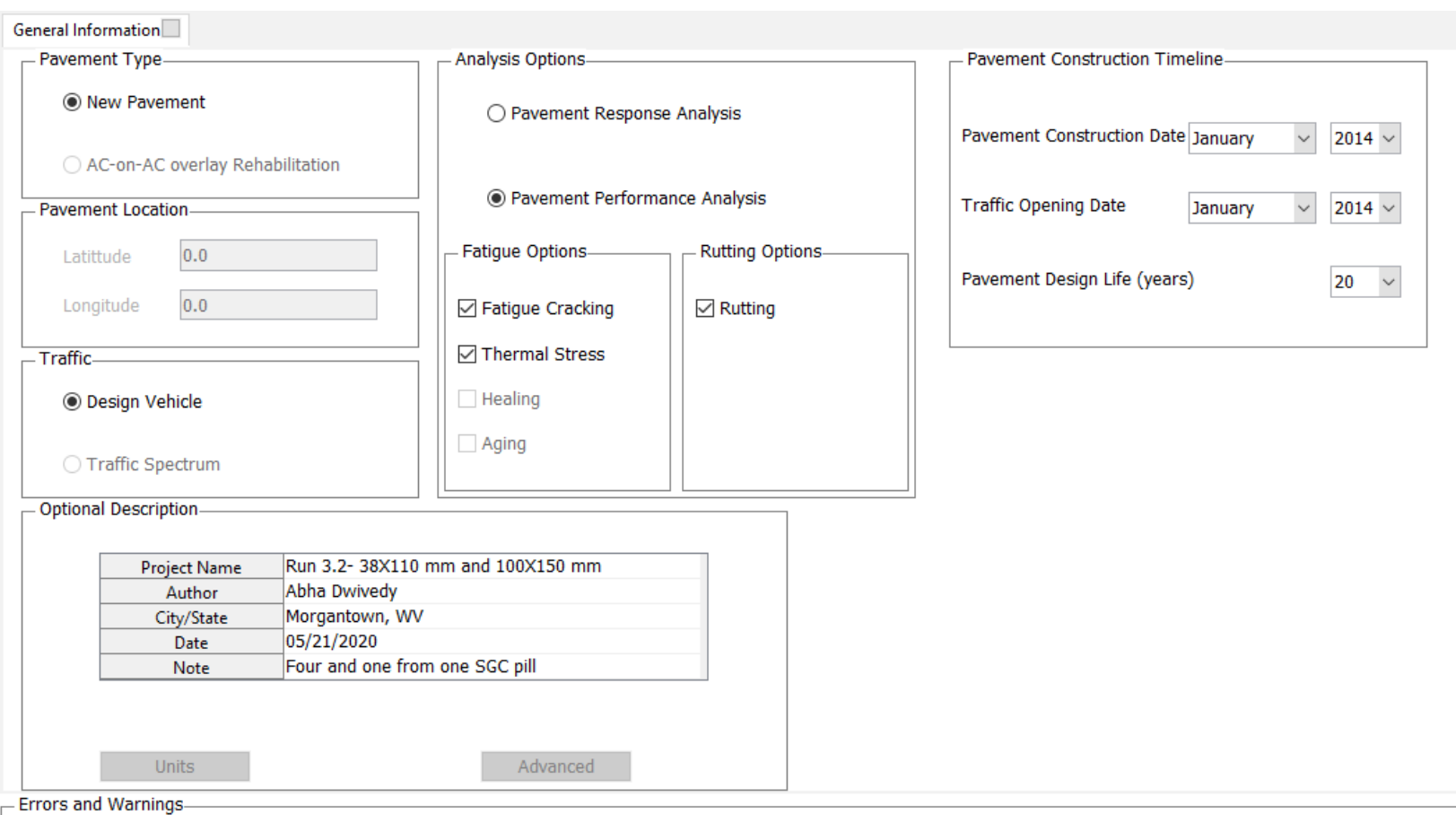

Figure 281: General Information for Run 3.2 


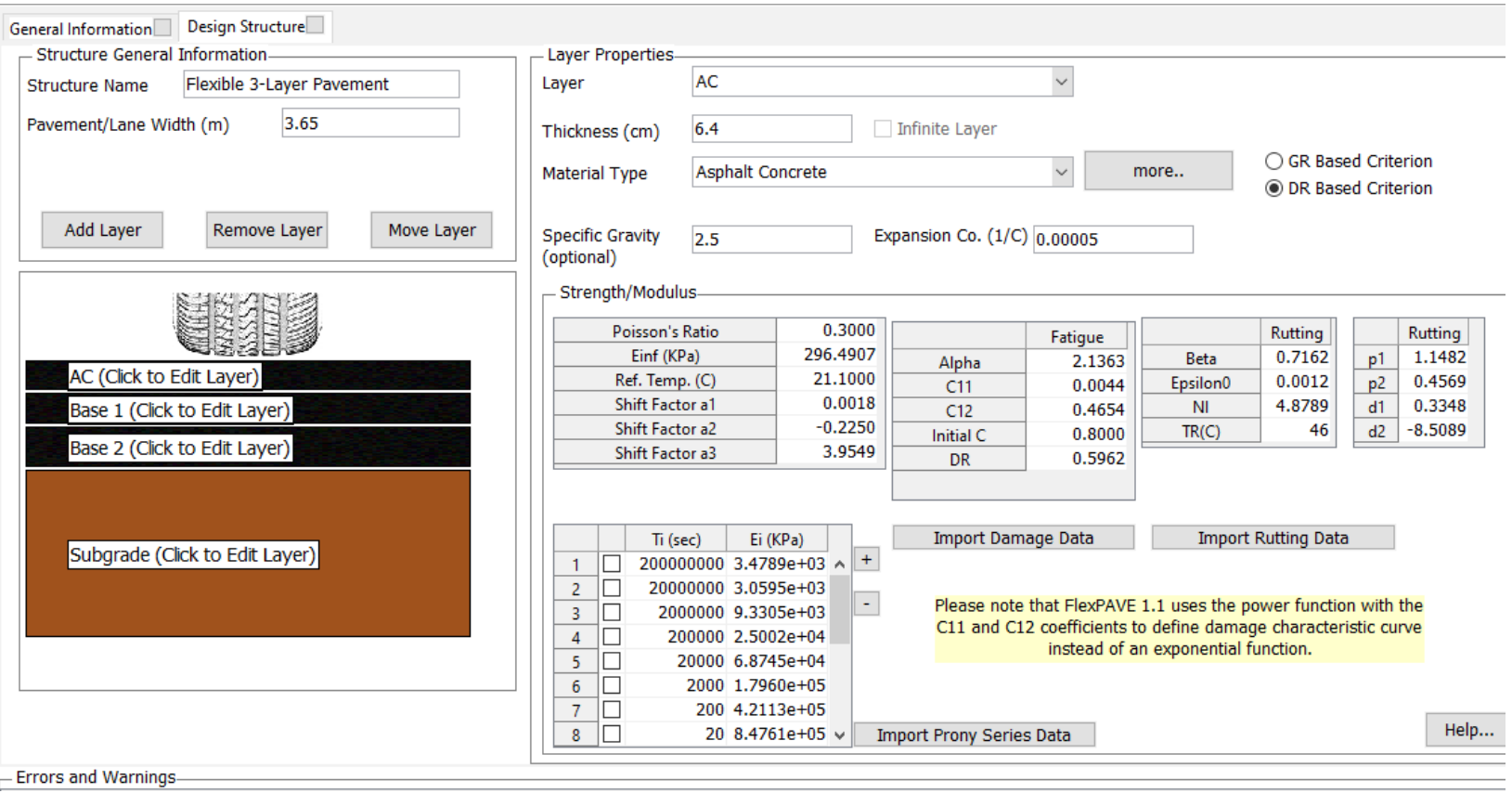

Figure 282: Design Structure of AC Layer for Run 3.2

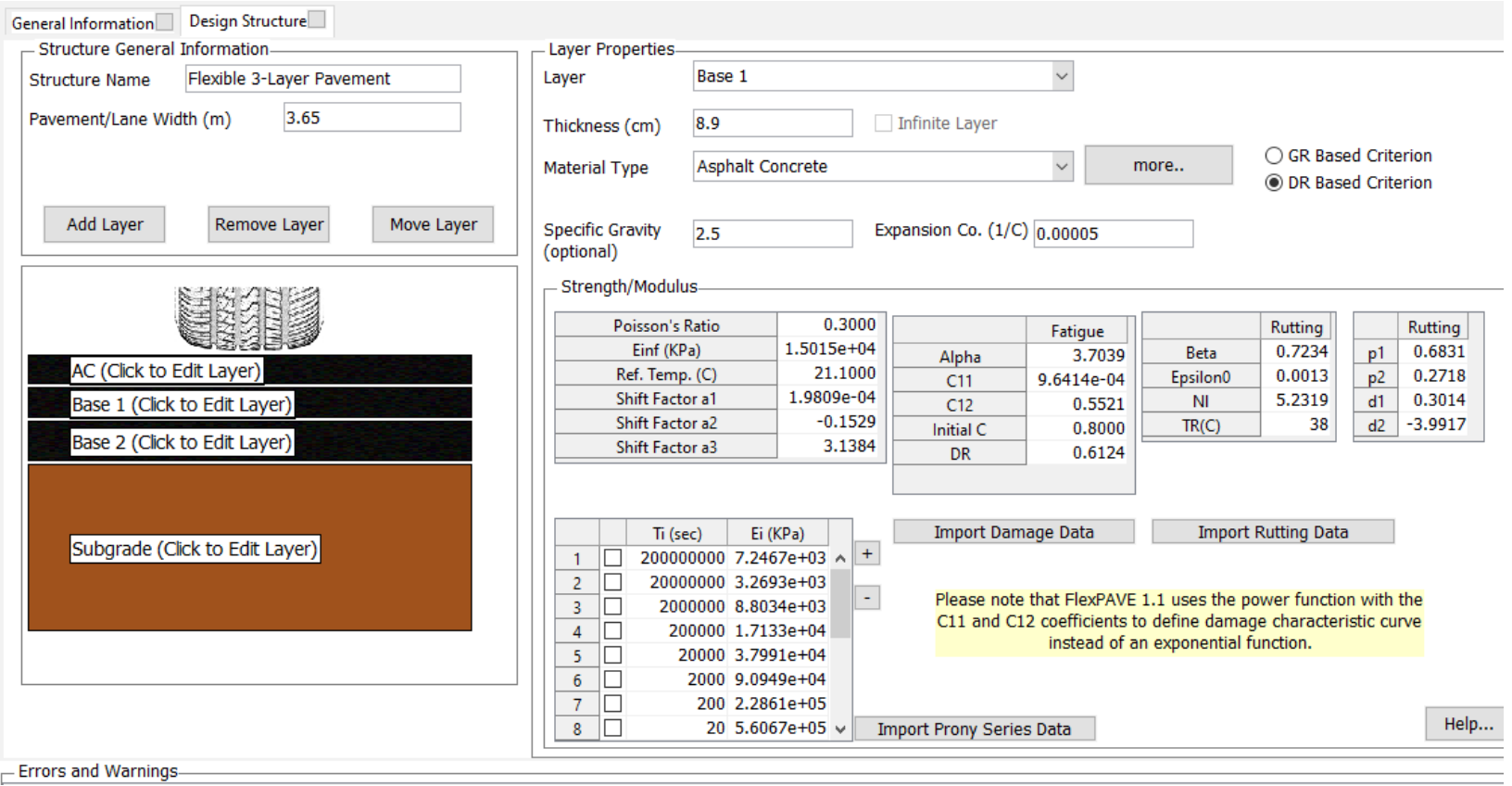

Figure 283: Design Structure of Base 1 Layer for Run 3.2 


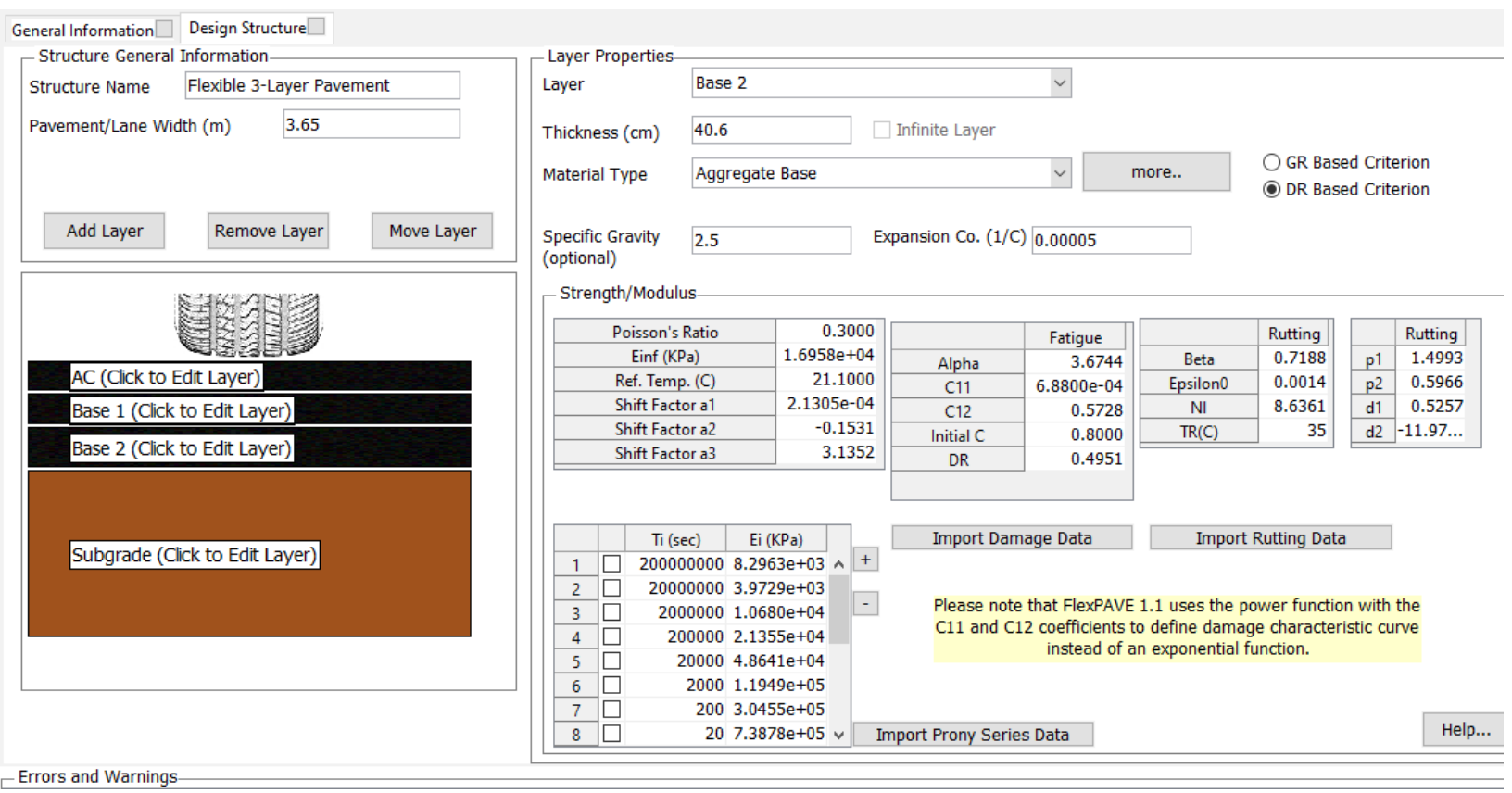

Figure 284: Design Structure of Base 2 Layer for Run 3.2

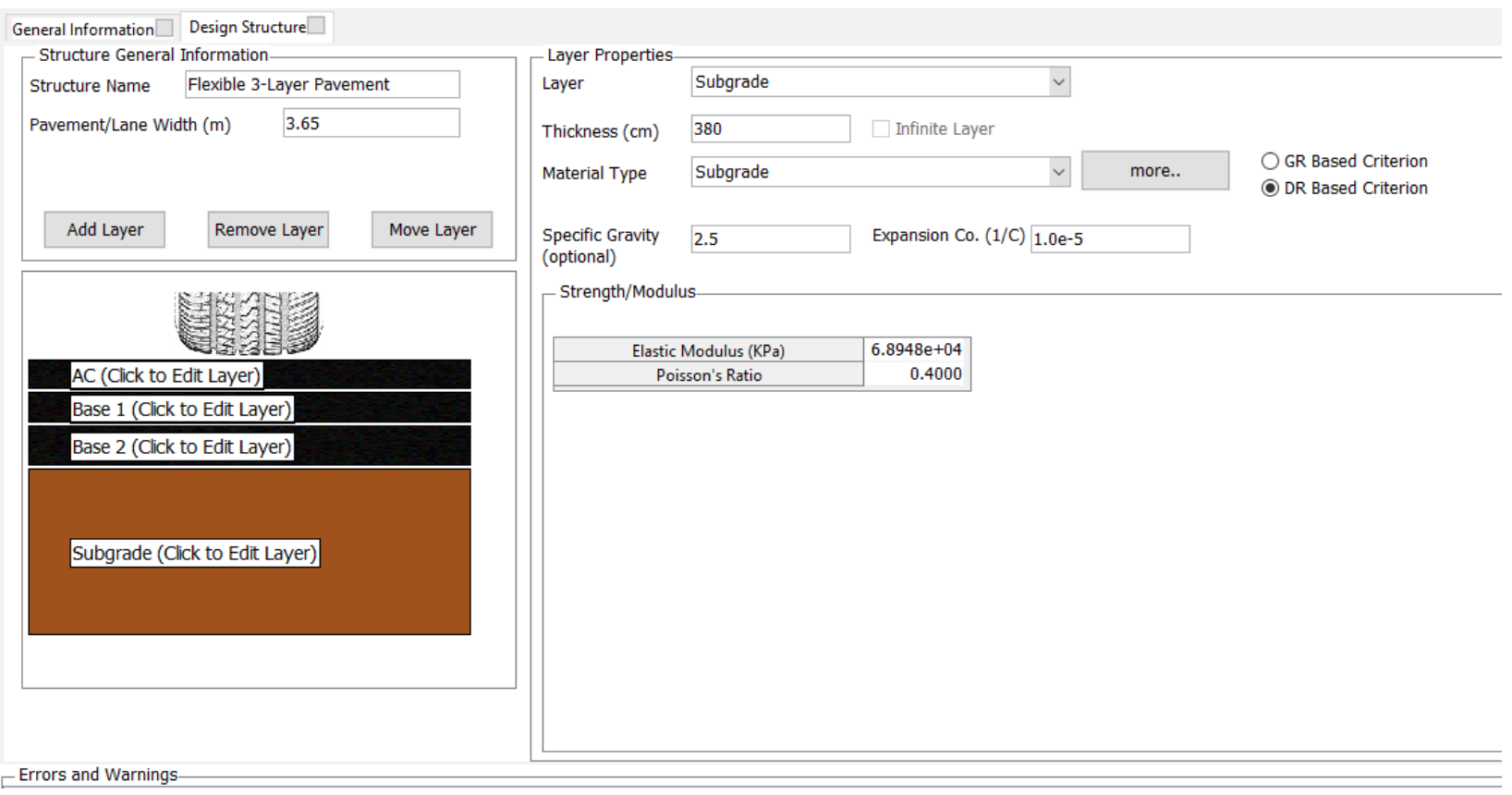

Figure 285: Design Structure of Subgrade Layer for Run 3.2 


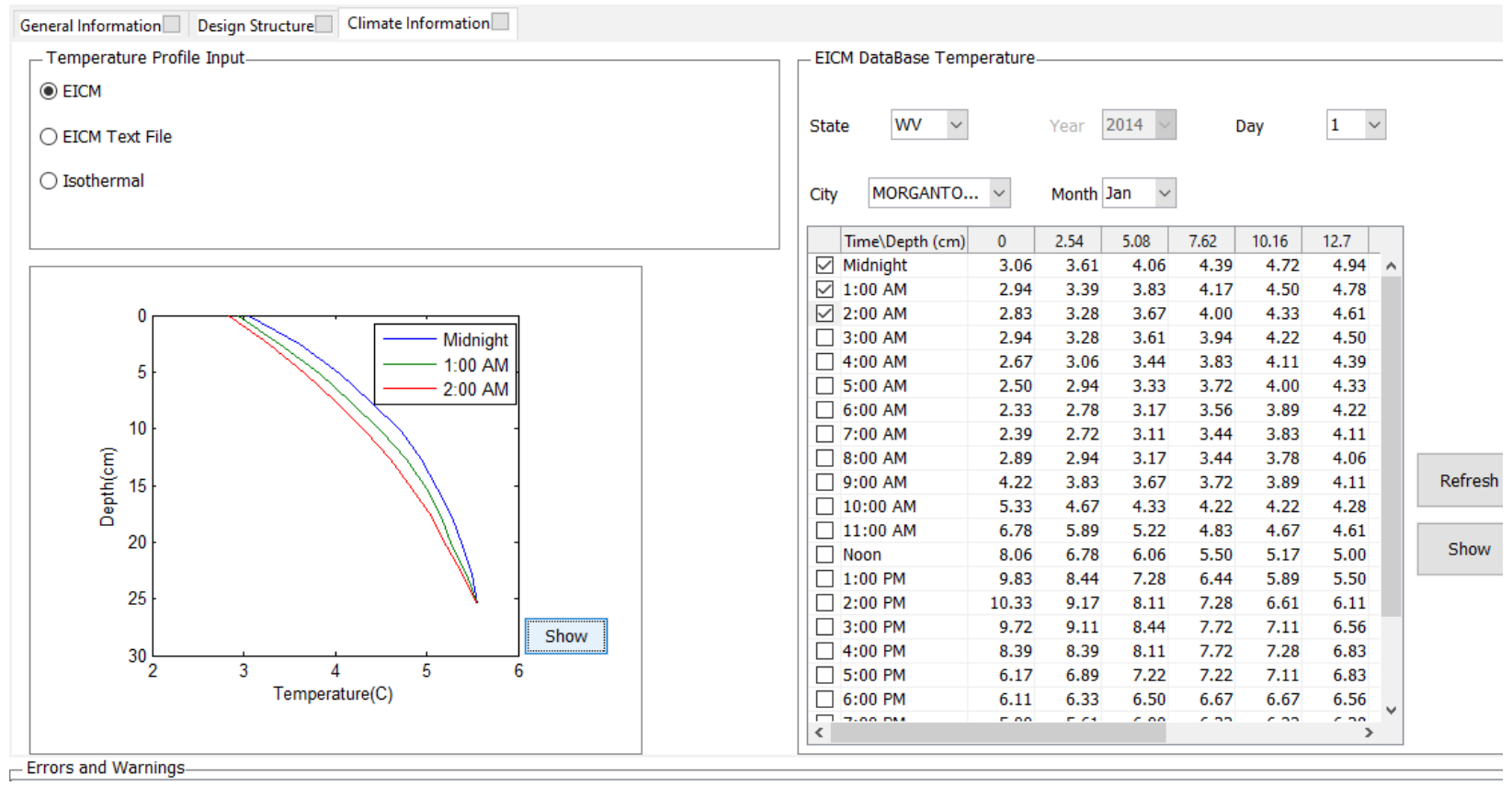

Figure 286: Climate Data for Run 3.2 


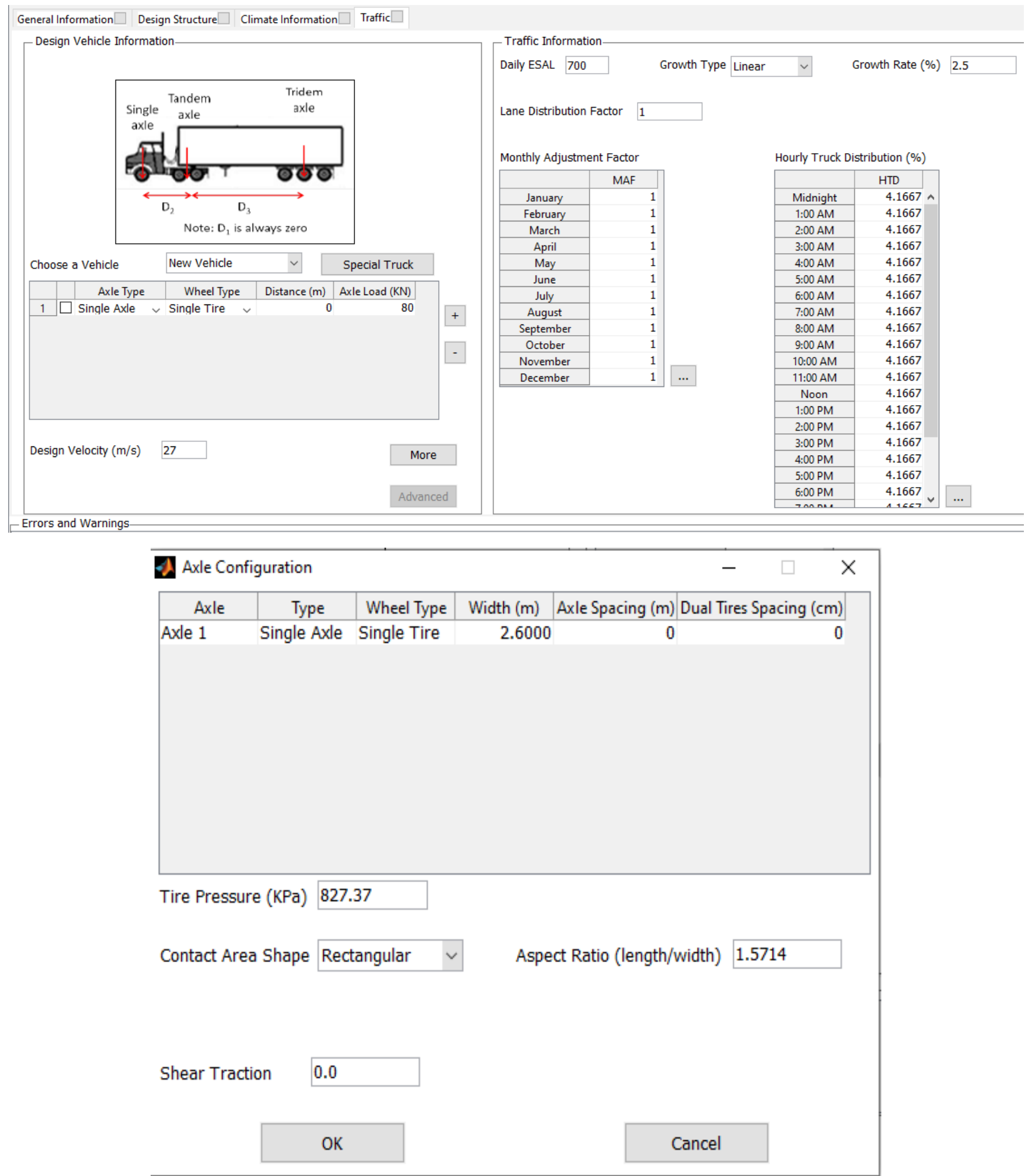

Figure 287: Traffic Data for Run 3.2 


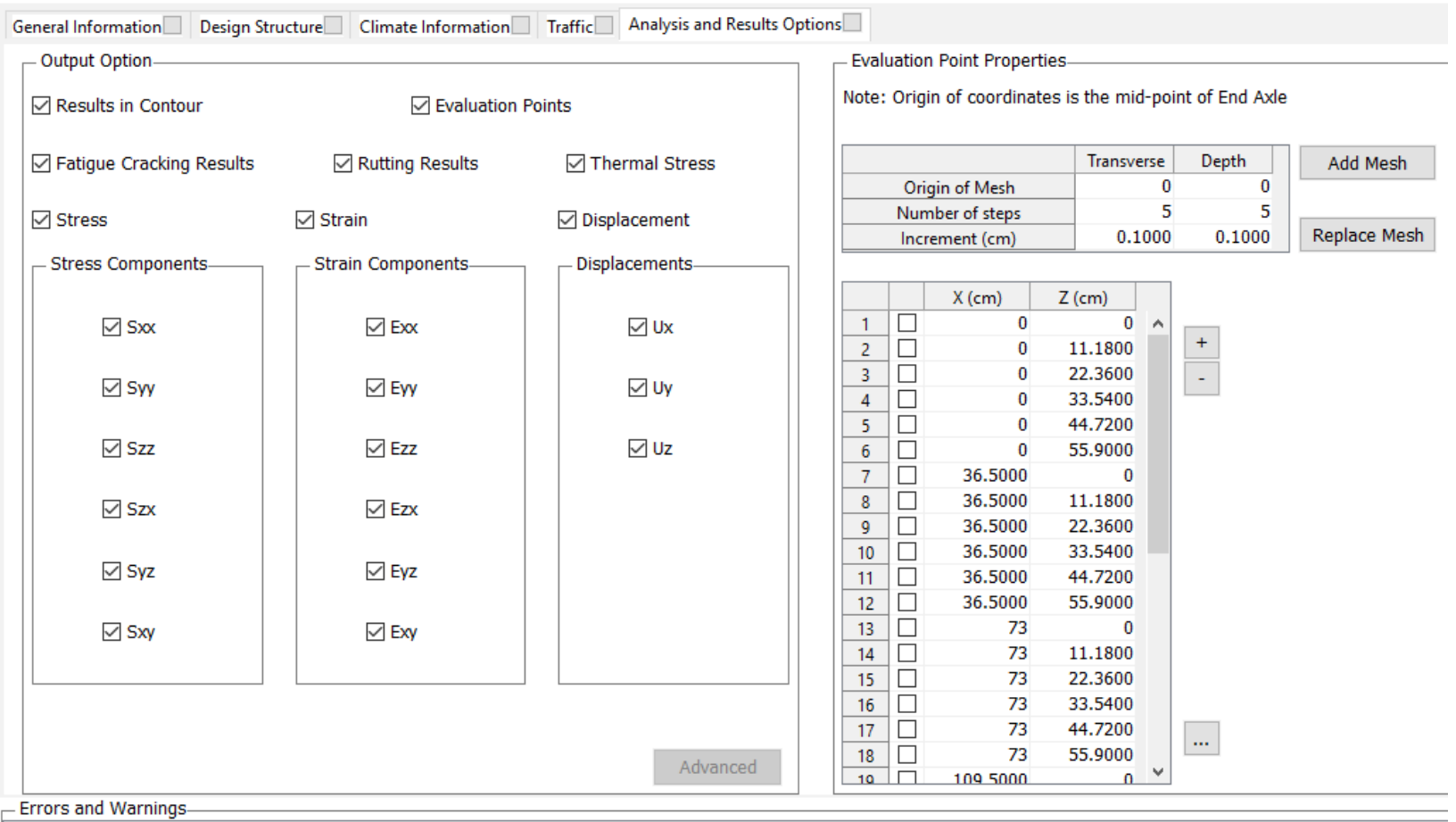

Figure 288: Output and Analysis Options for Run 3.2

\section{Run 3.3}

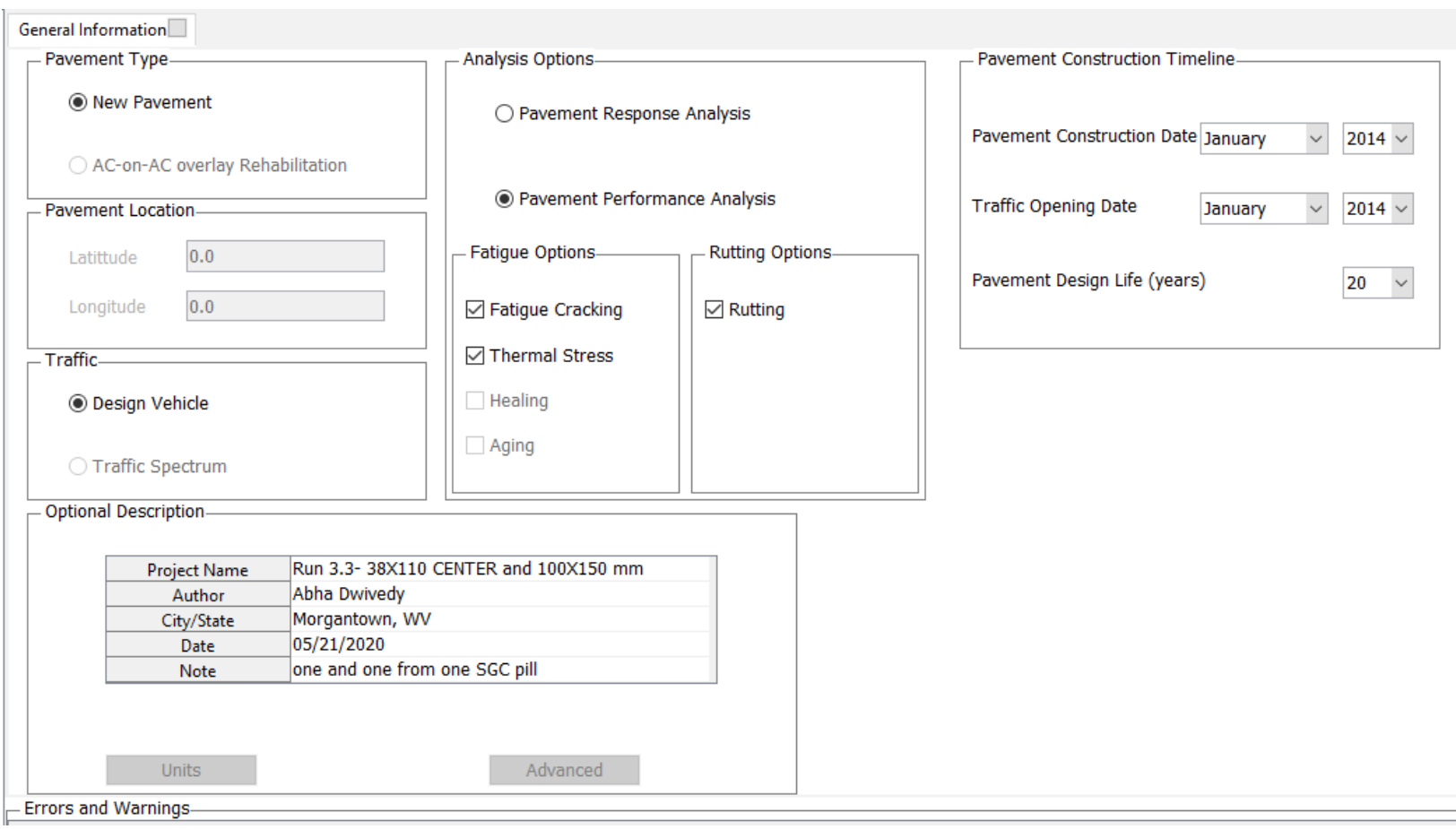

Figure 289: General Information for Run 3.3 


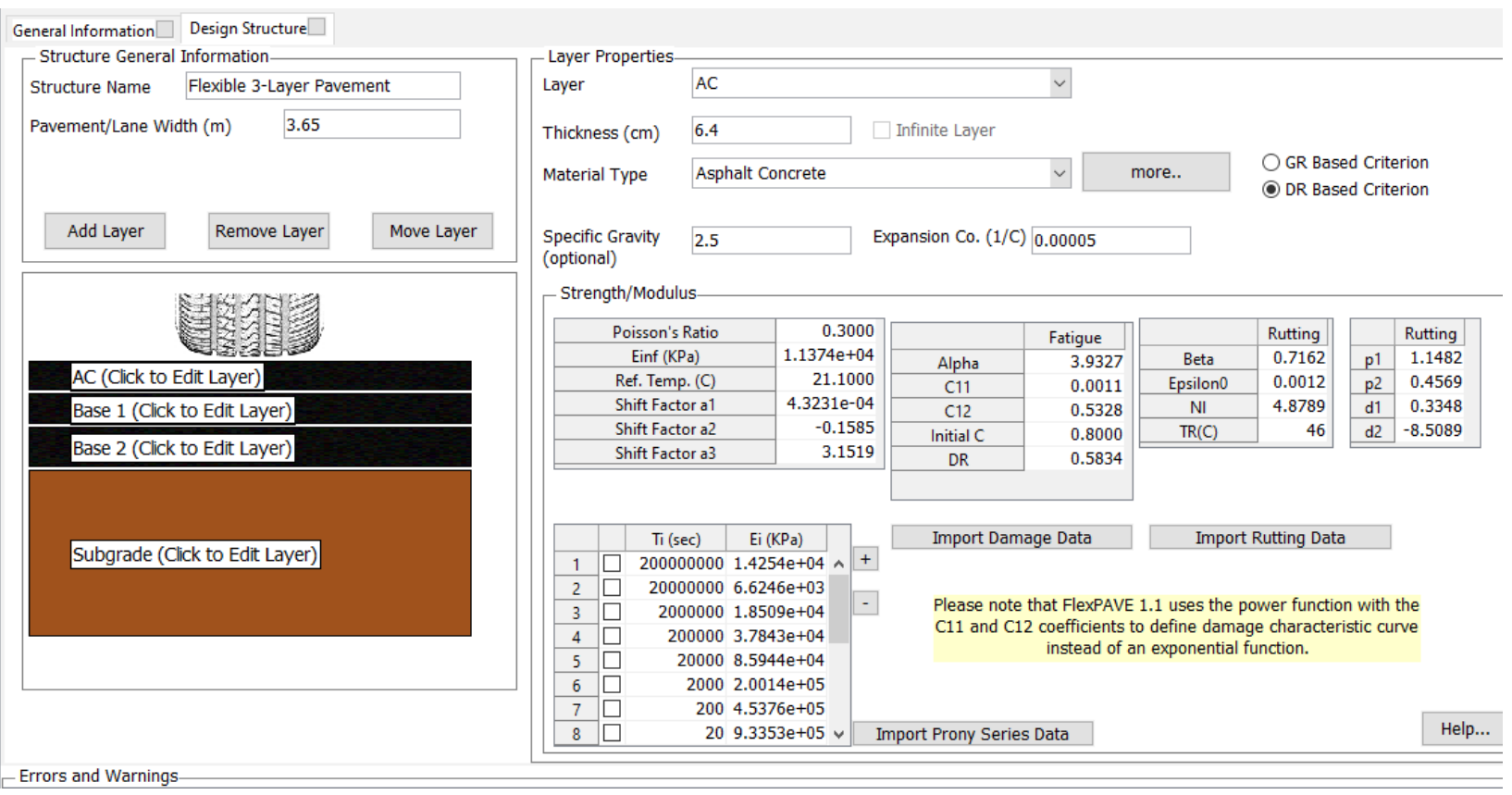

Figure 290: Design Structure of AC Layer for Run 3.3

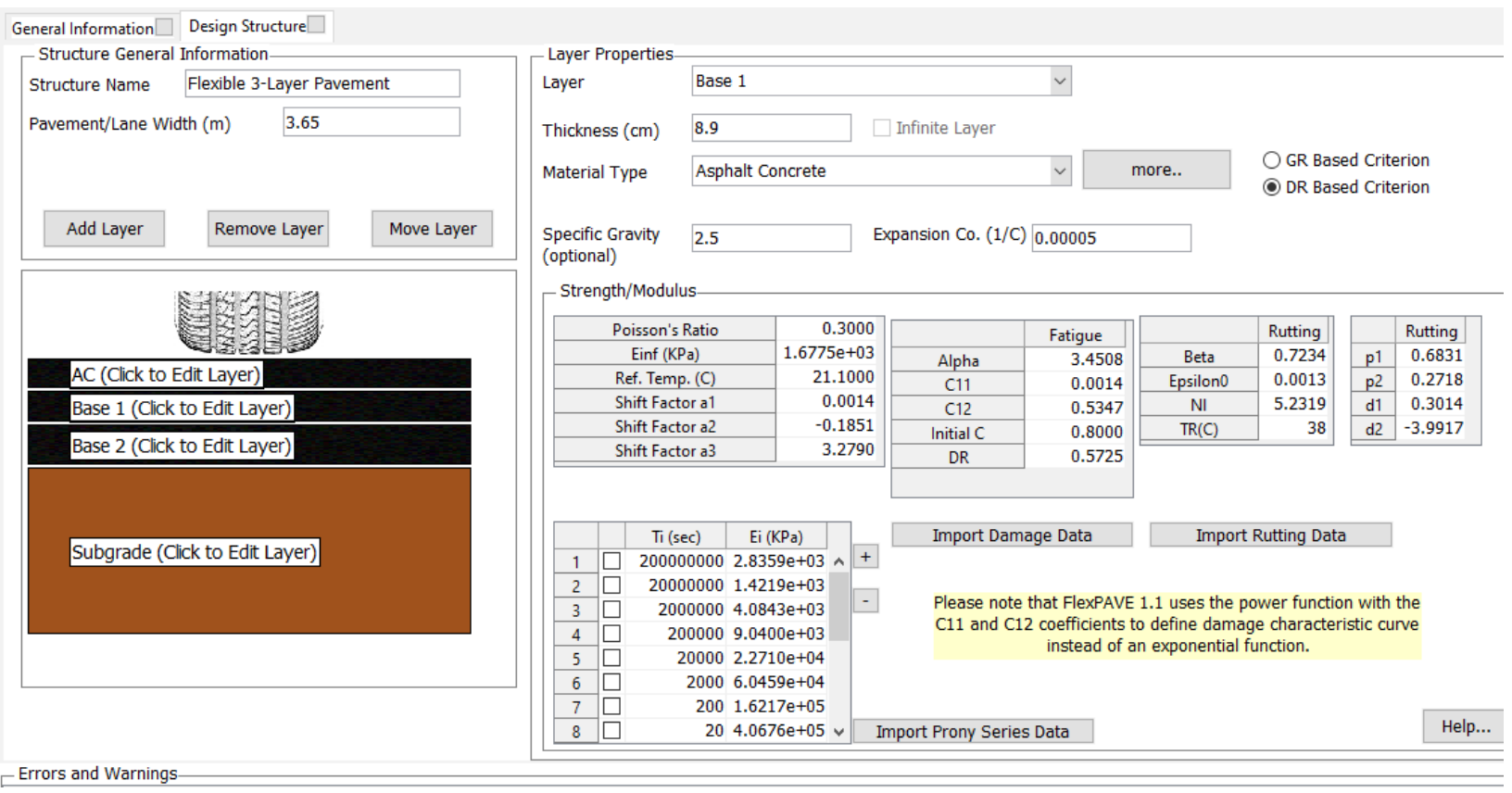

Figure 291: Design Structure of Base 1 Layer for Run 3.3 


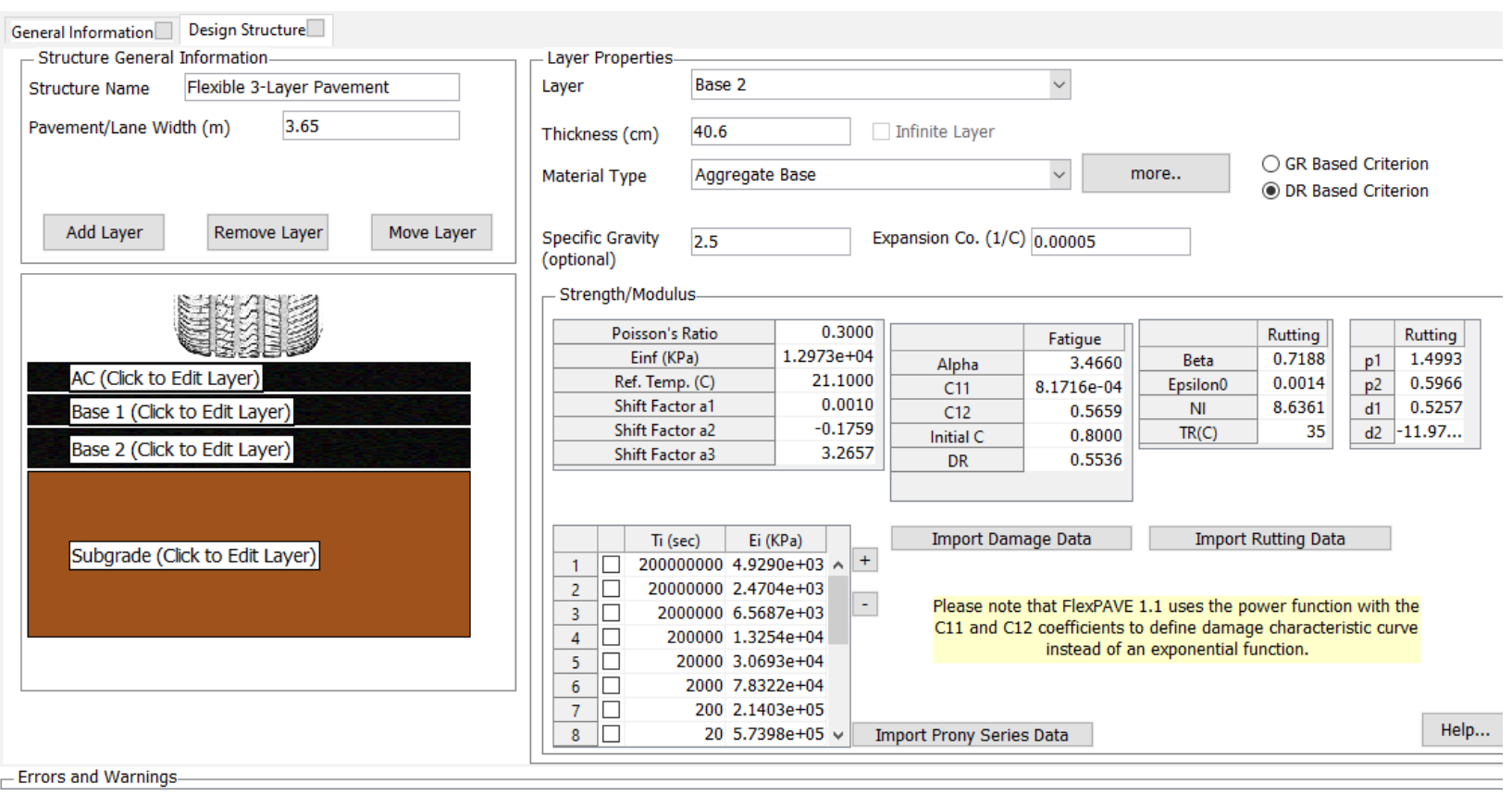

Figure 292: Design Structure of Base 2 Layer for Run 3.3

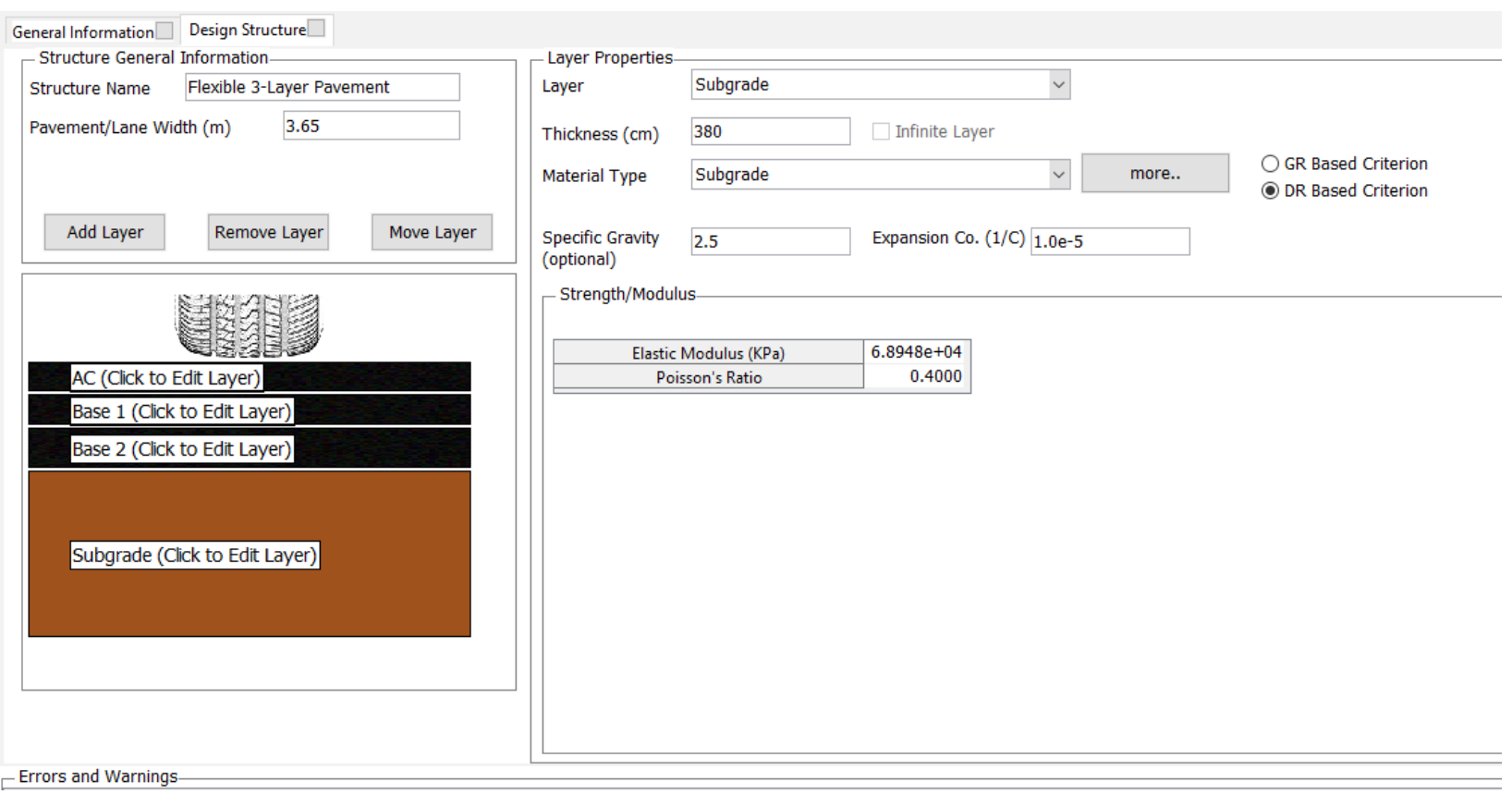

Figure 293: Design Structure of Subgrade Layer for Run 3.3 


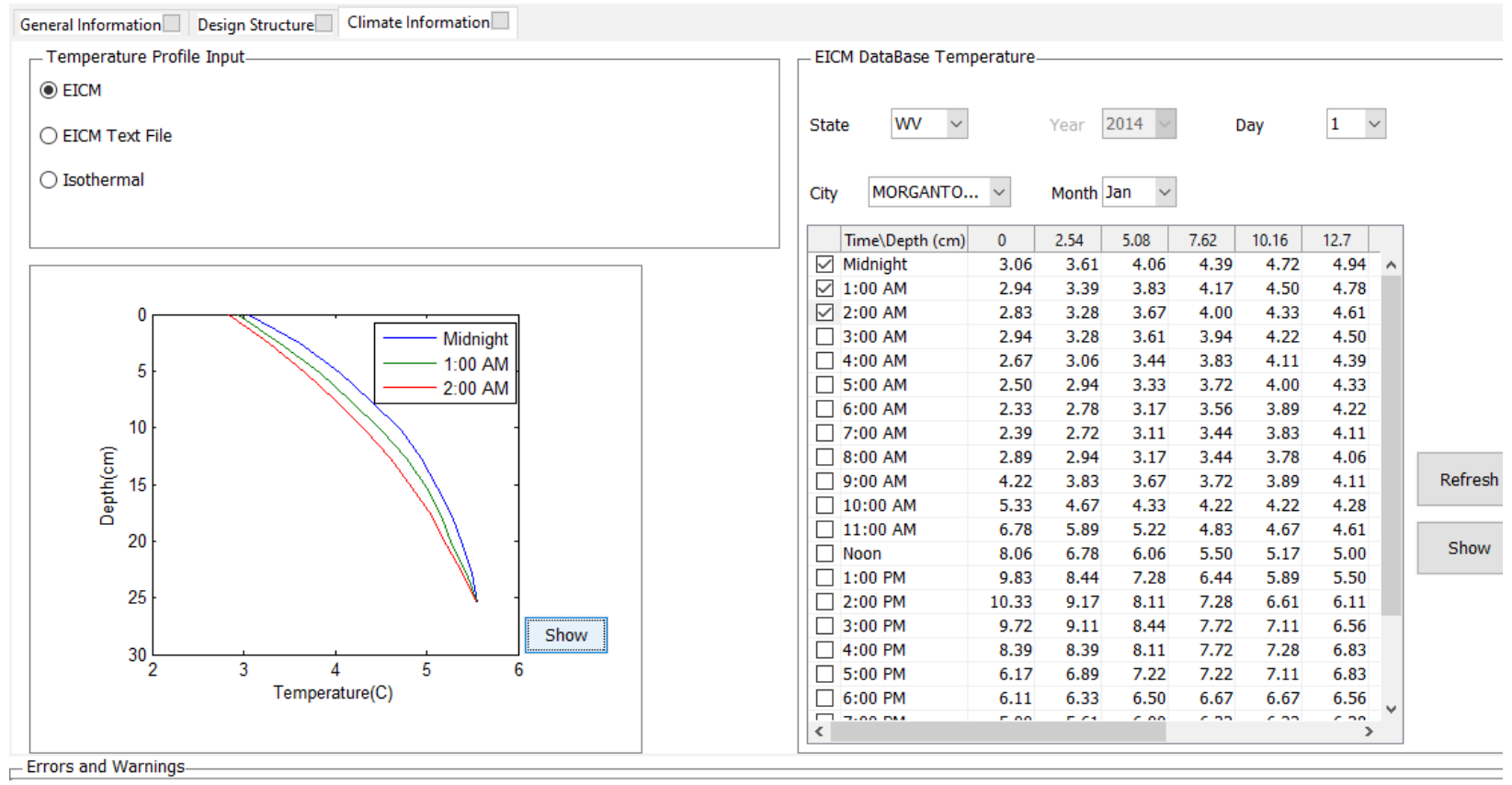

Figure 294: Climate Data for Run 3.3 


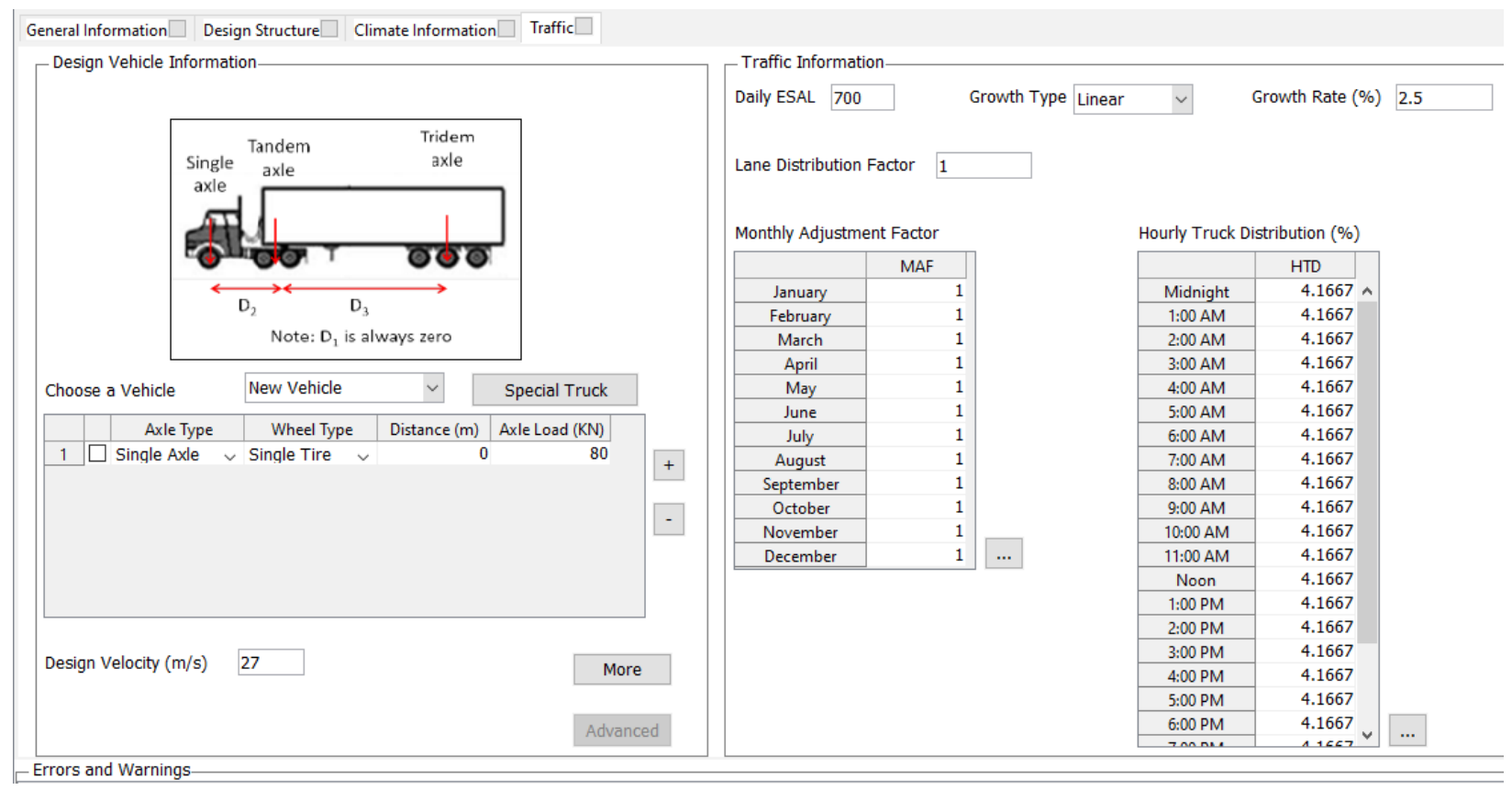

Axle Configuration

$-\square \times$

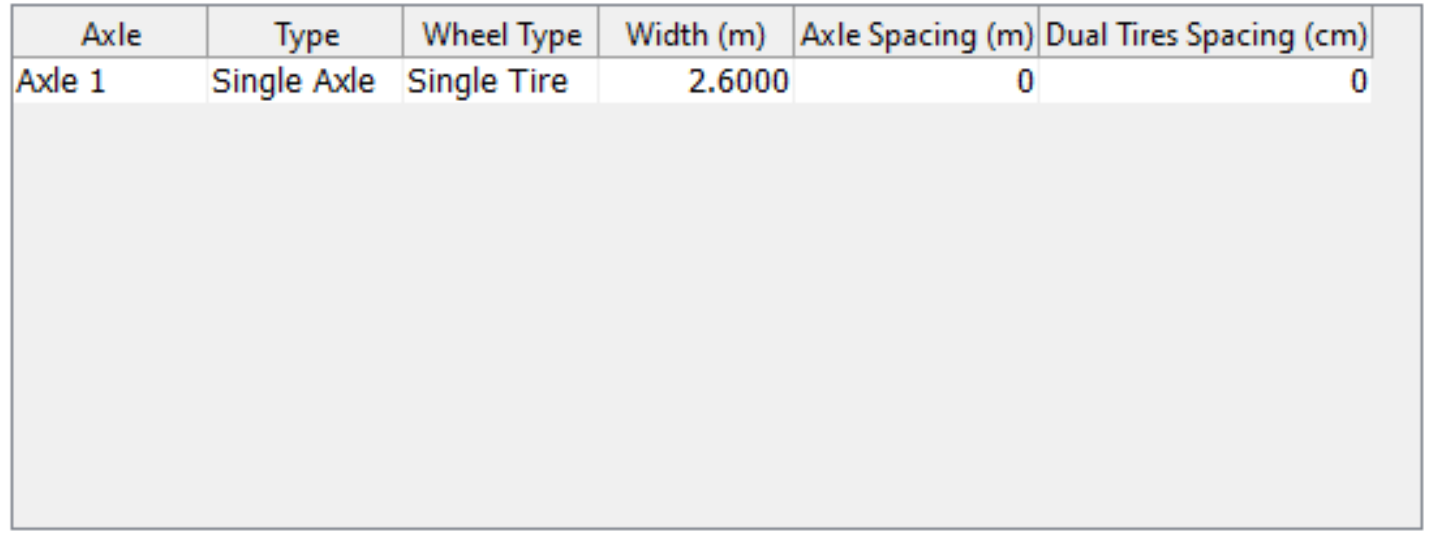

Tire Pressure (KPa) 827.37

Contact Area Shape Rectangular $\vee \quad$ Aspect Ratio (length/width) 1.5714

Shear Traction $\quad 0.0$

OK

Cancel

Figure 295: Traffic Data for Run 3.3 


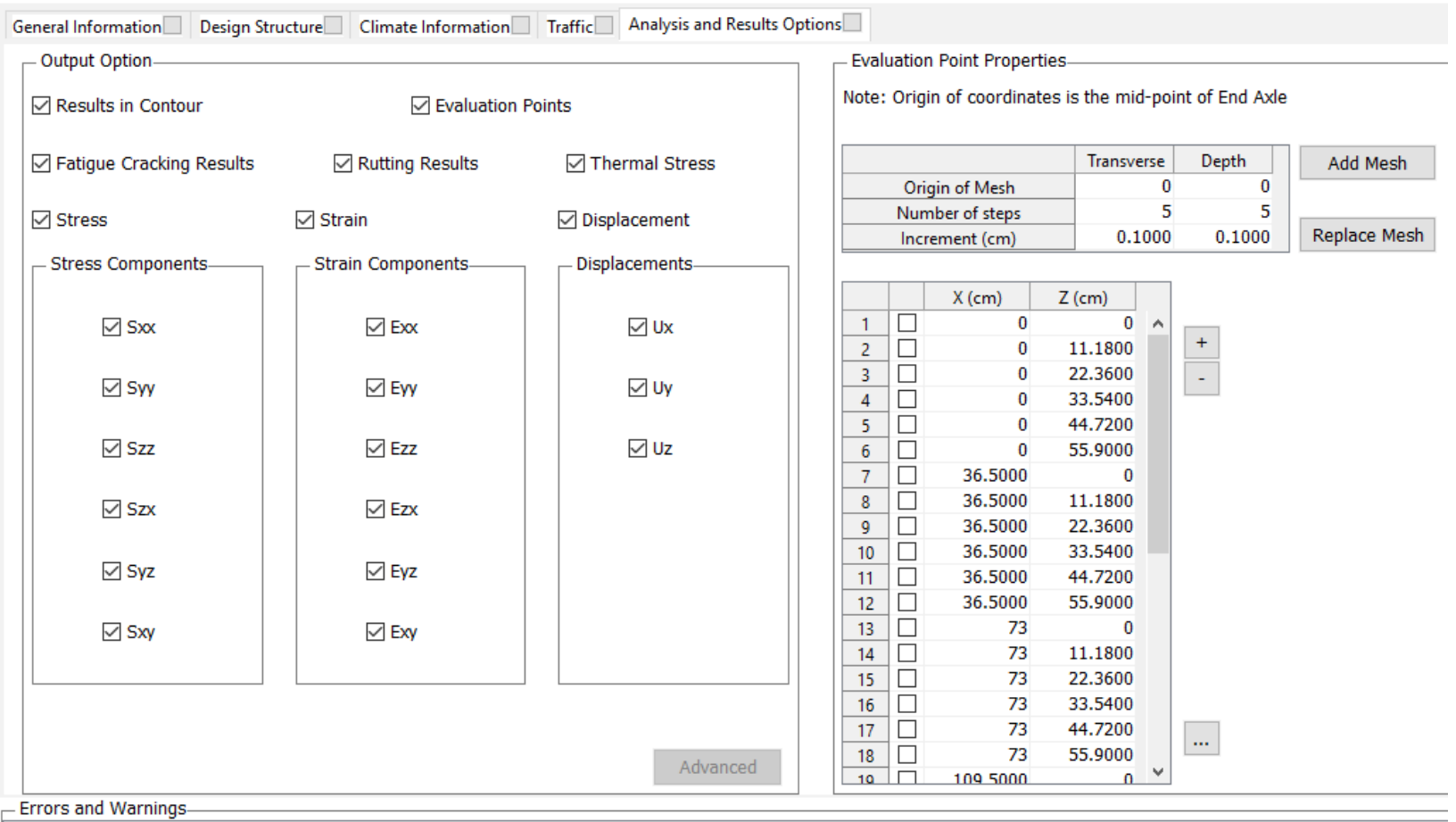

Figure 296: Output and Analysis Options for Run 3.3

\section{Run 3.4}

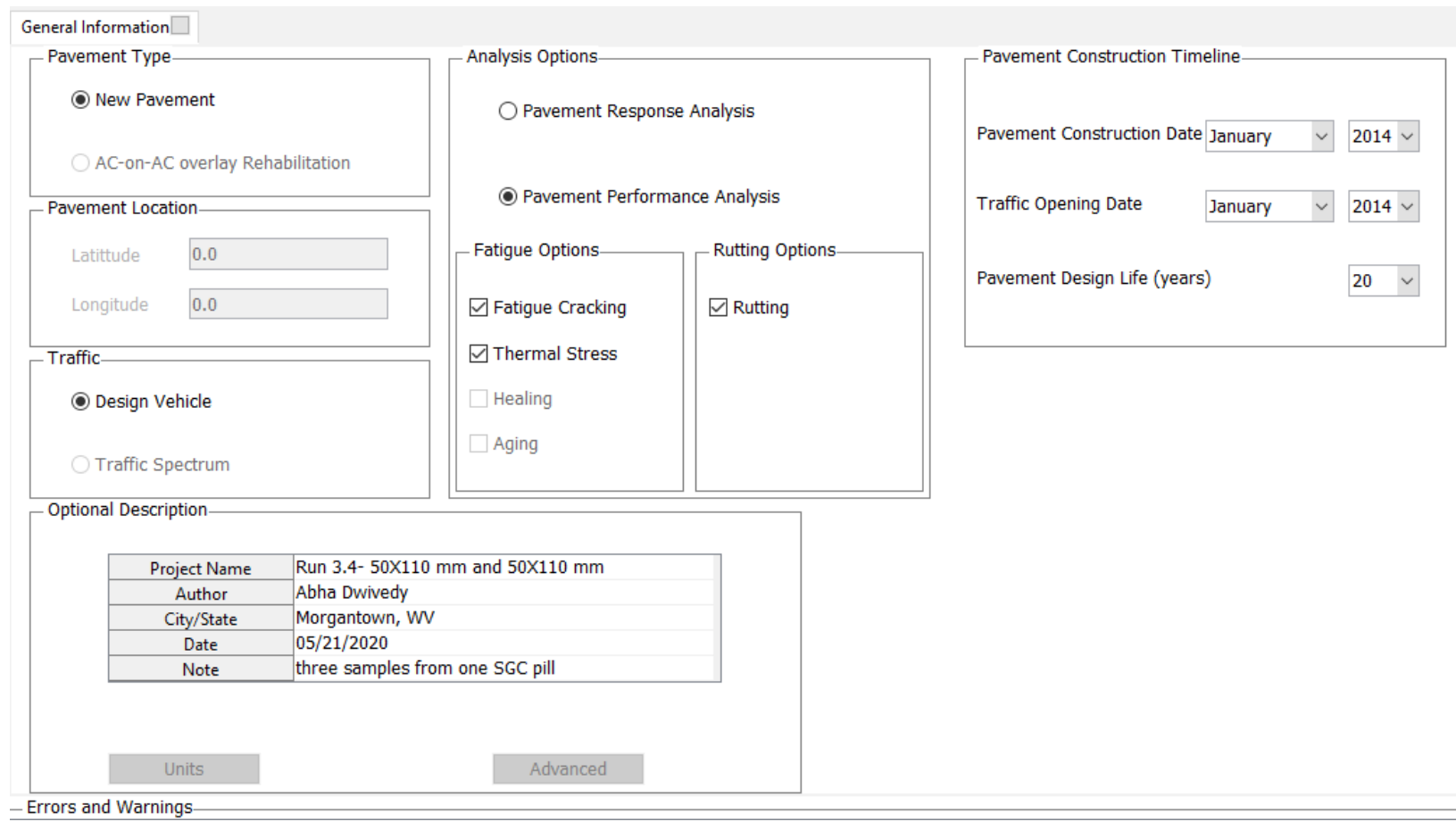

Figure 297: General Information for Run 3.4 


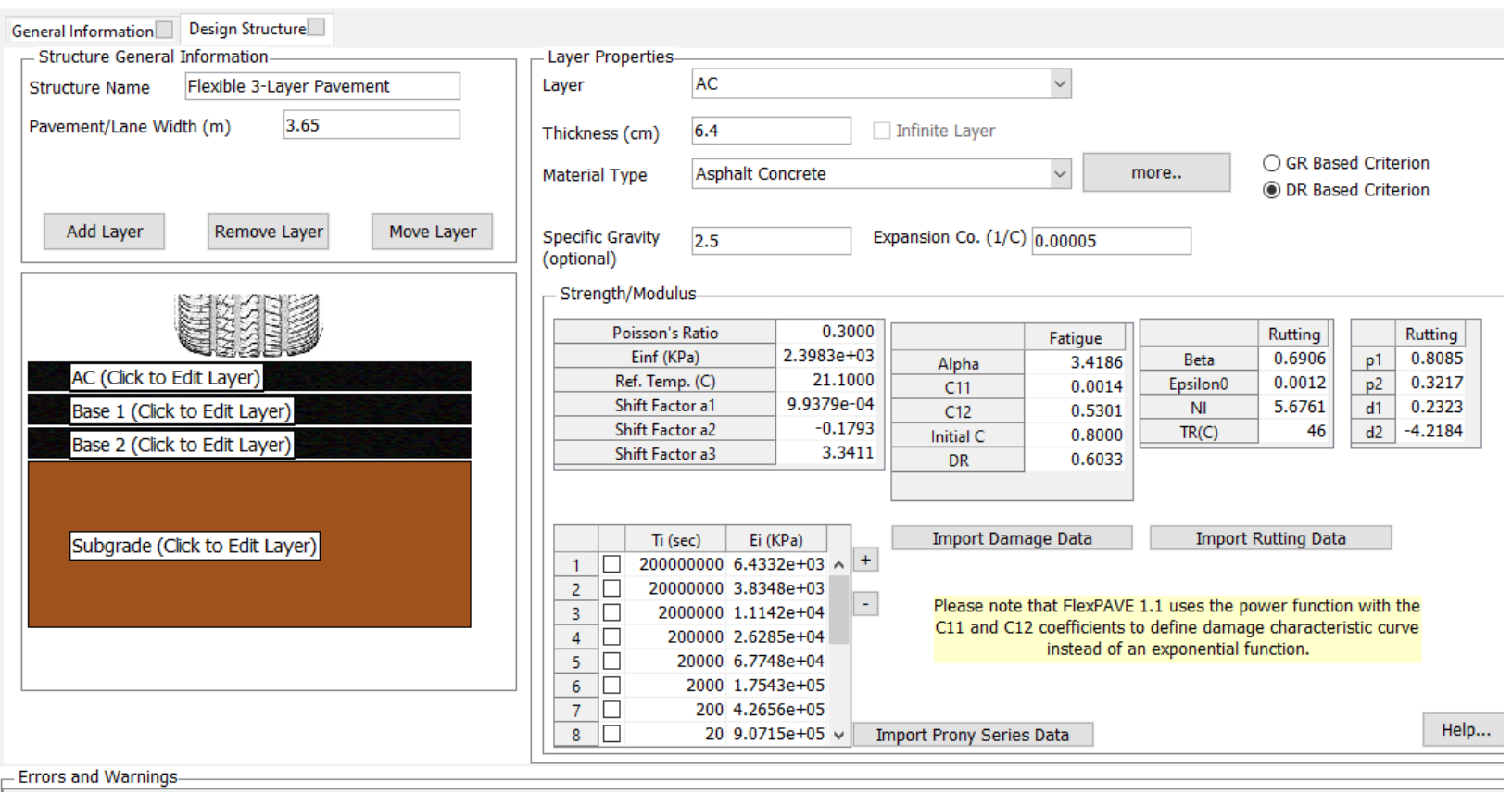

Figure 298: Design Structure of AC Layer for Run 3.4

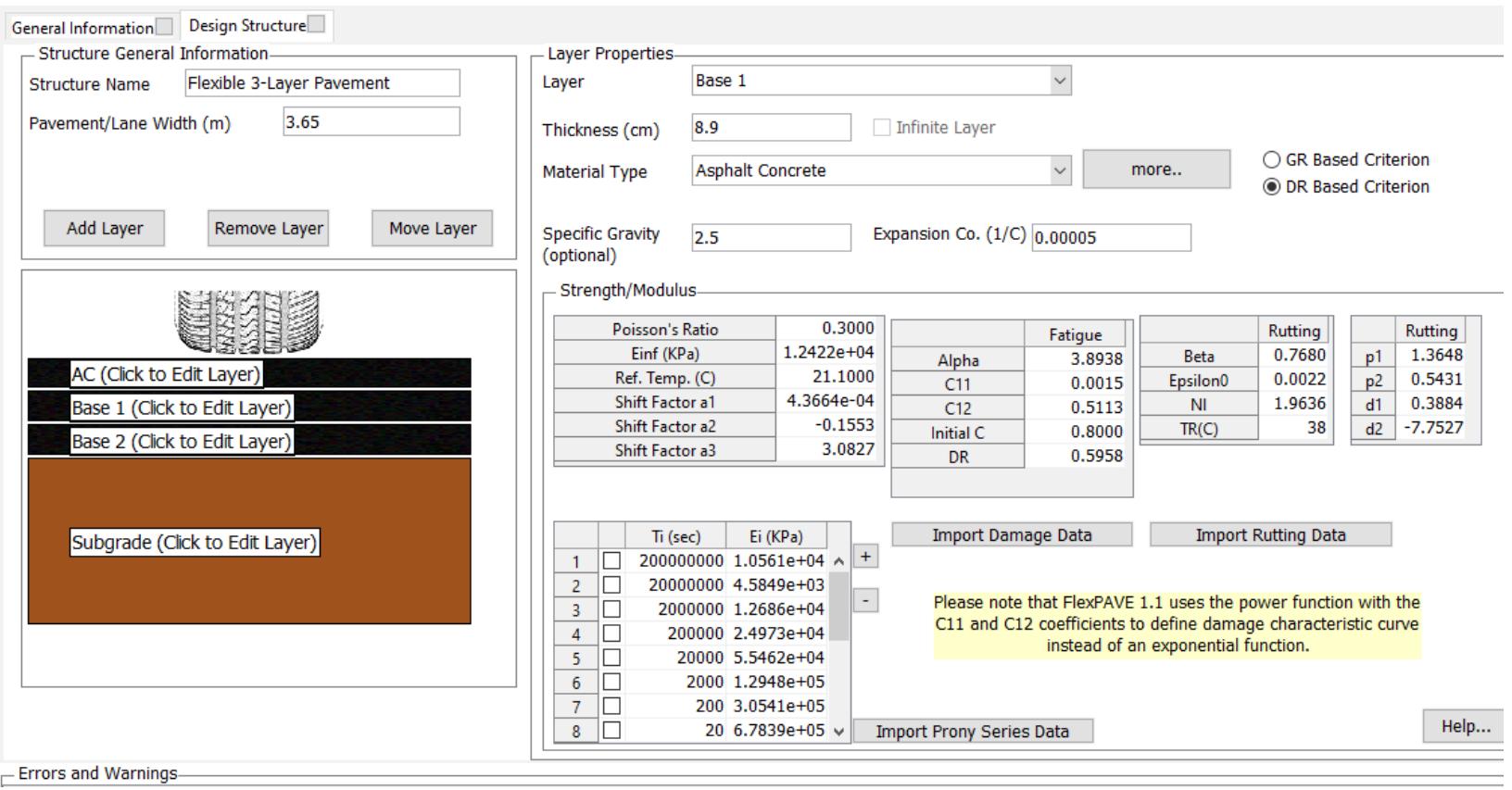

Figure 299: Design Structure of Base 1 Layer for Run 3.4 


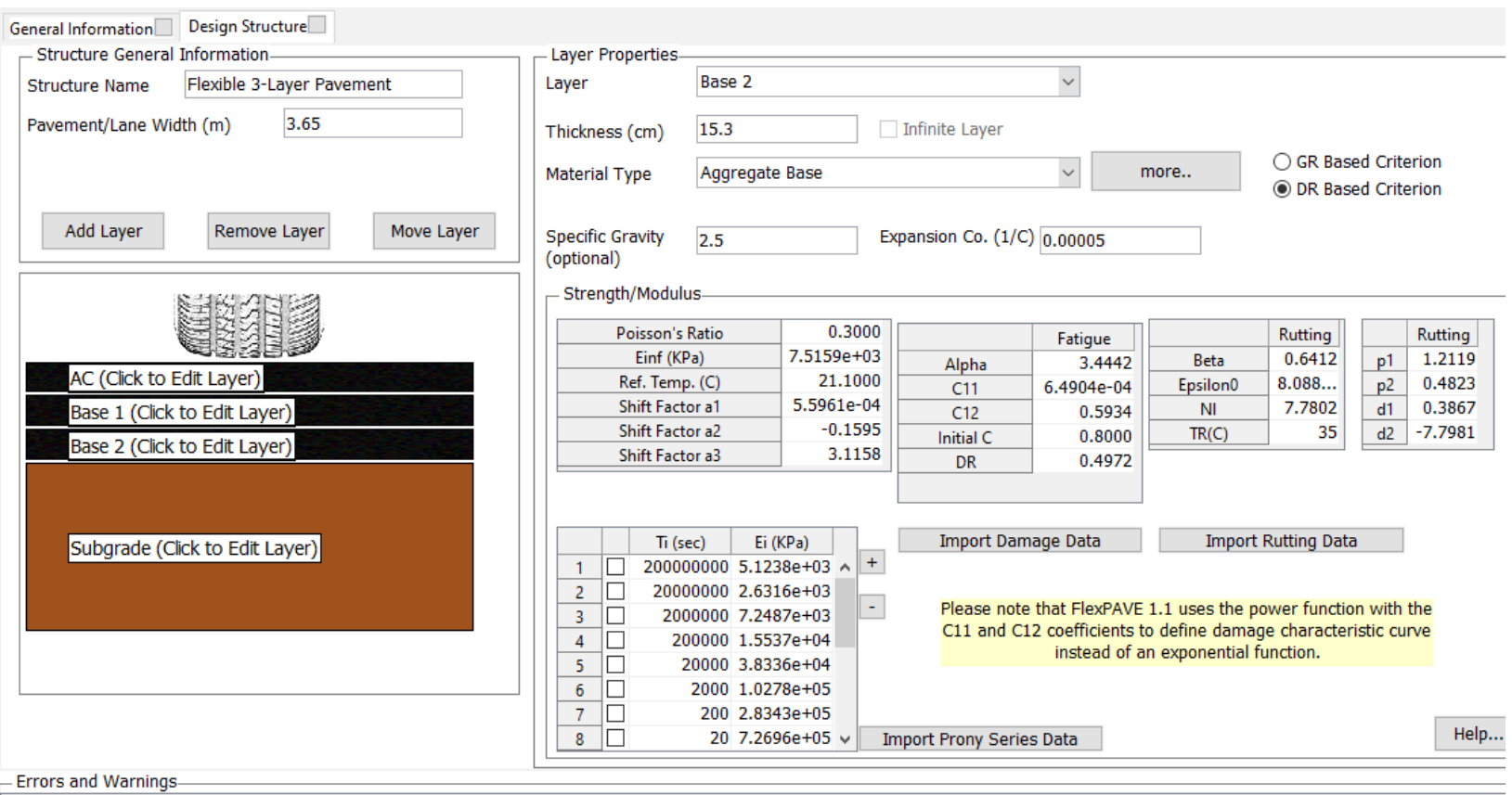

Figure 300: Design Structure of Base 2 Layer for Run 3.4

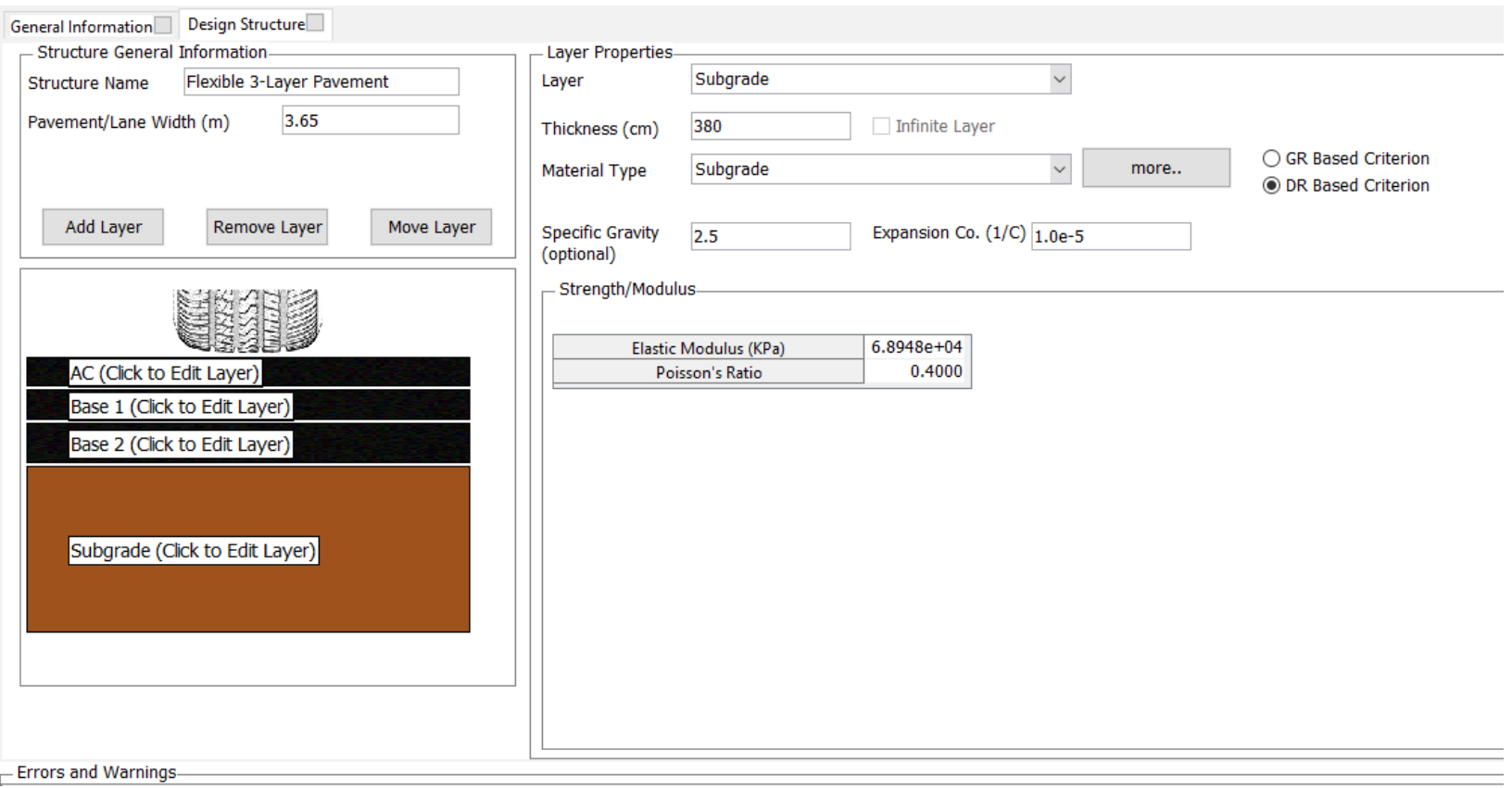

Figure 301: Design Structure of Subgrade Layer for Run 3.4 


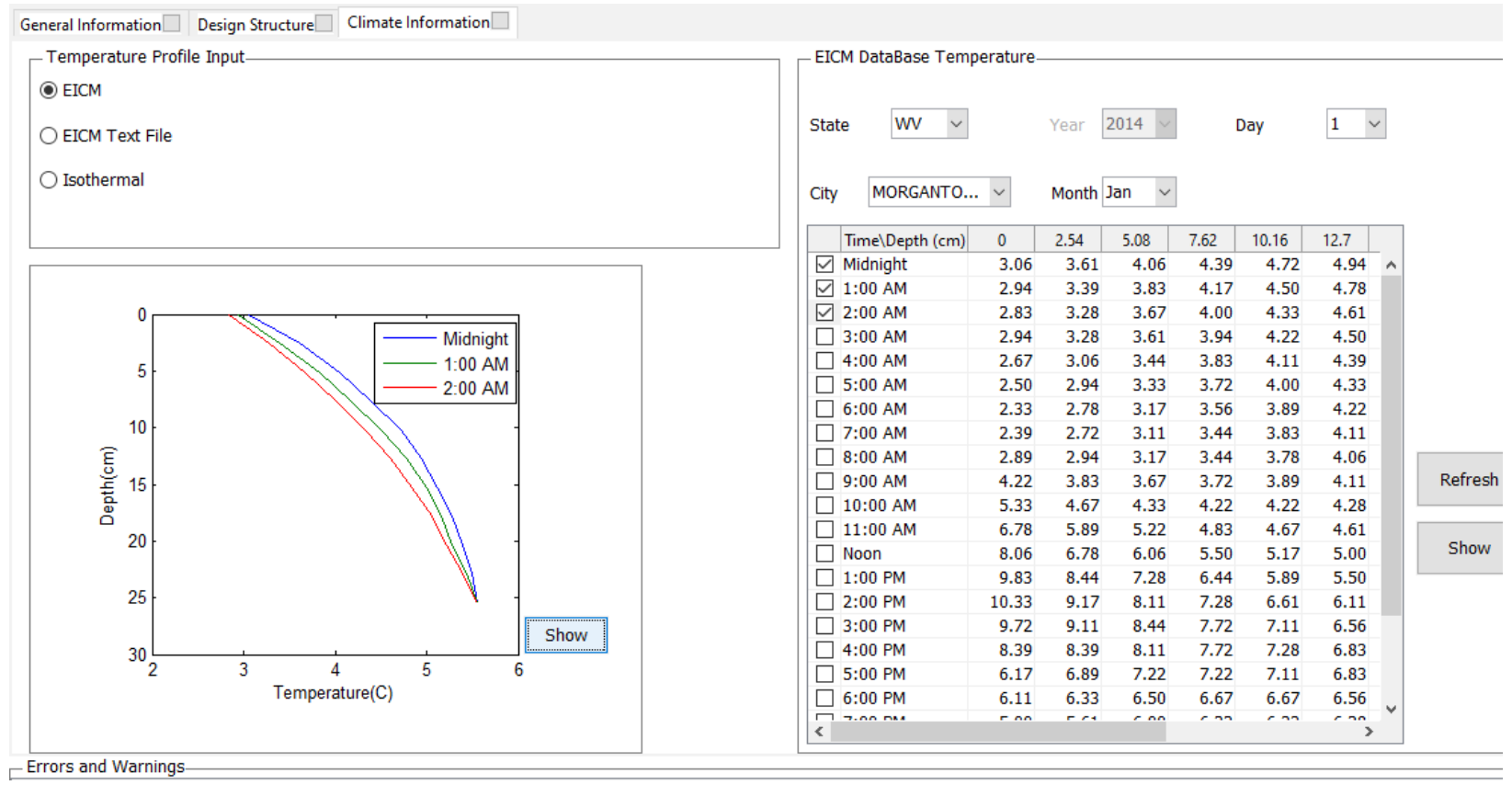

Figure 302: Climate Data for Run 3.4 


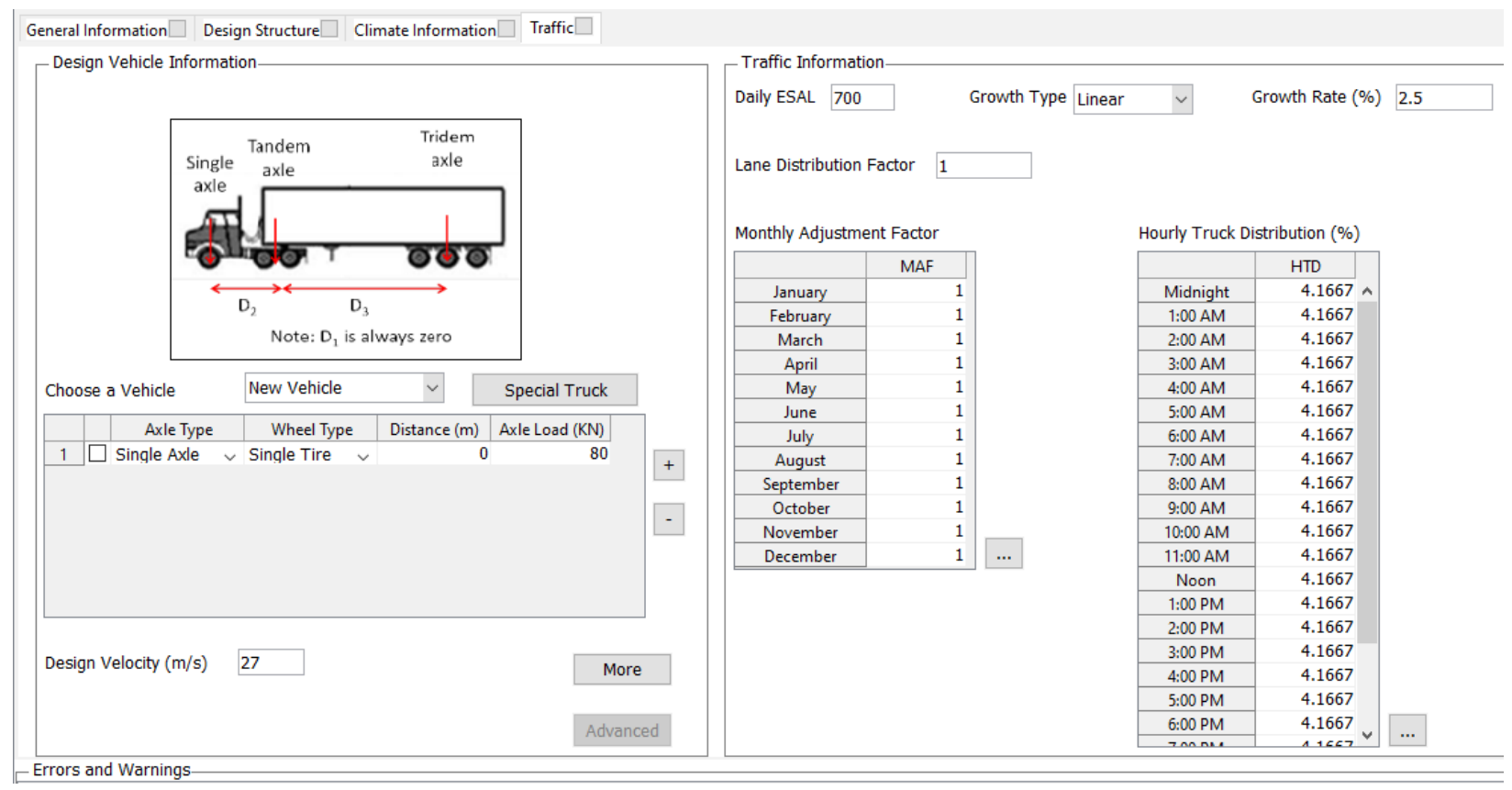

Axle Configuration

$-\square \times$

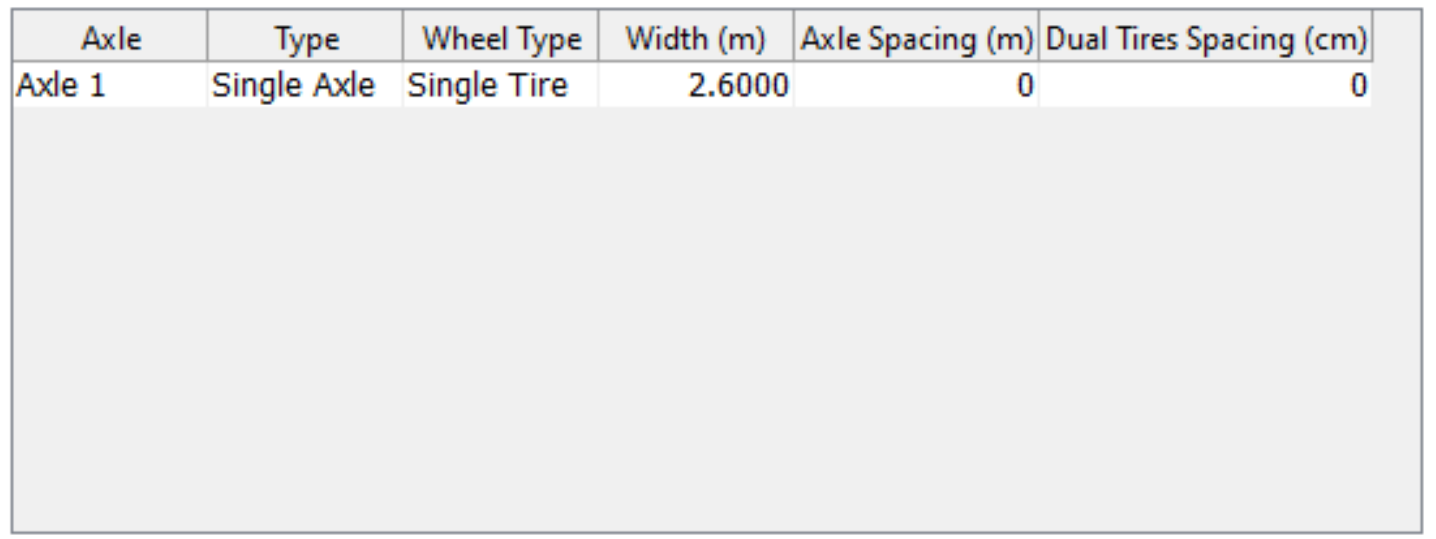

Tire Pressure (KPa) 827.37

Contact Area Shape Rectangular $\vee \quad$ Aspect Ratio (length/width) 1.5714

Shear Traction $\quad 0.0$

OK

Cancel

Figure 303: Traffic Data for Run 3.4 


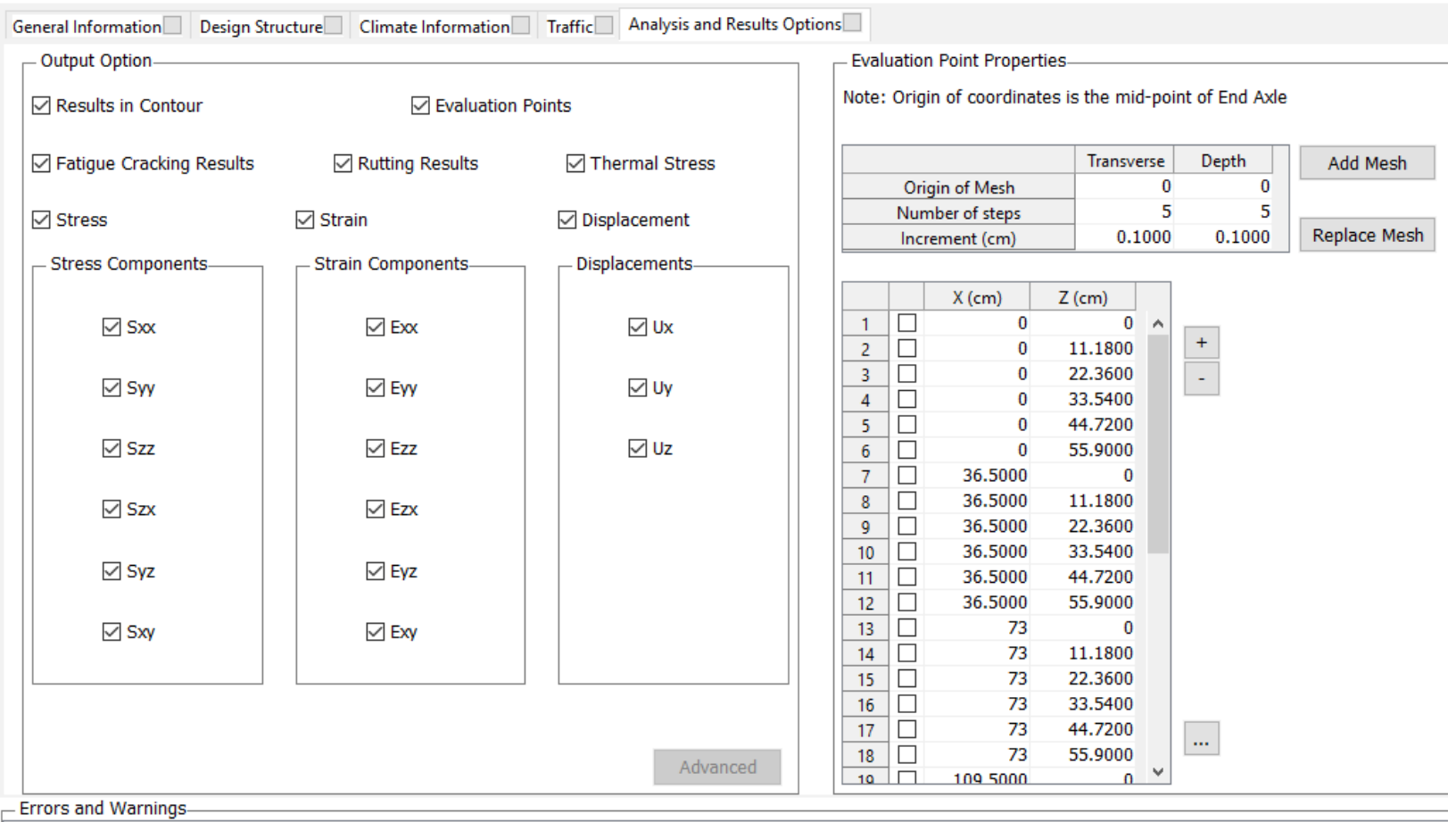

Figure 304: Output and Analysis Options for Run 3.4

\section{Run 3.5}

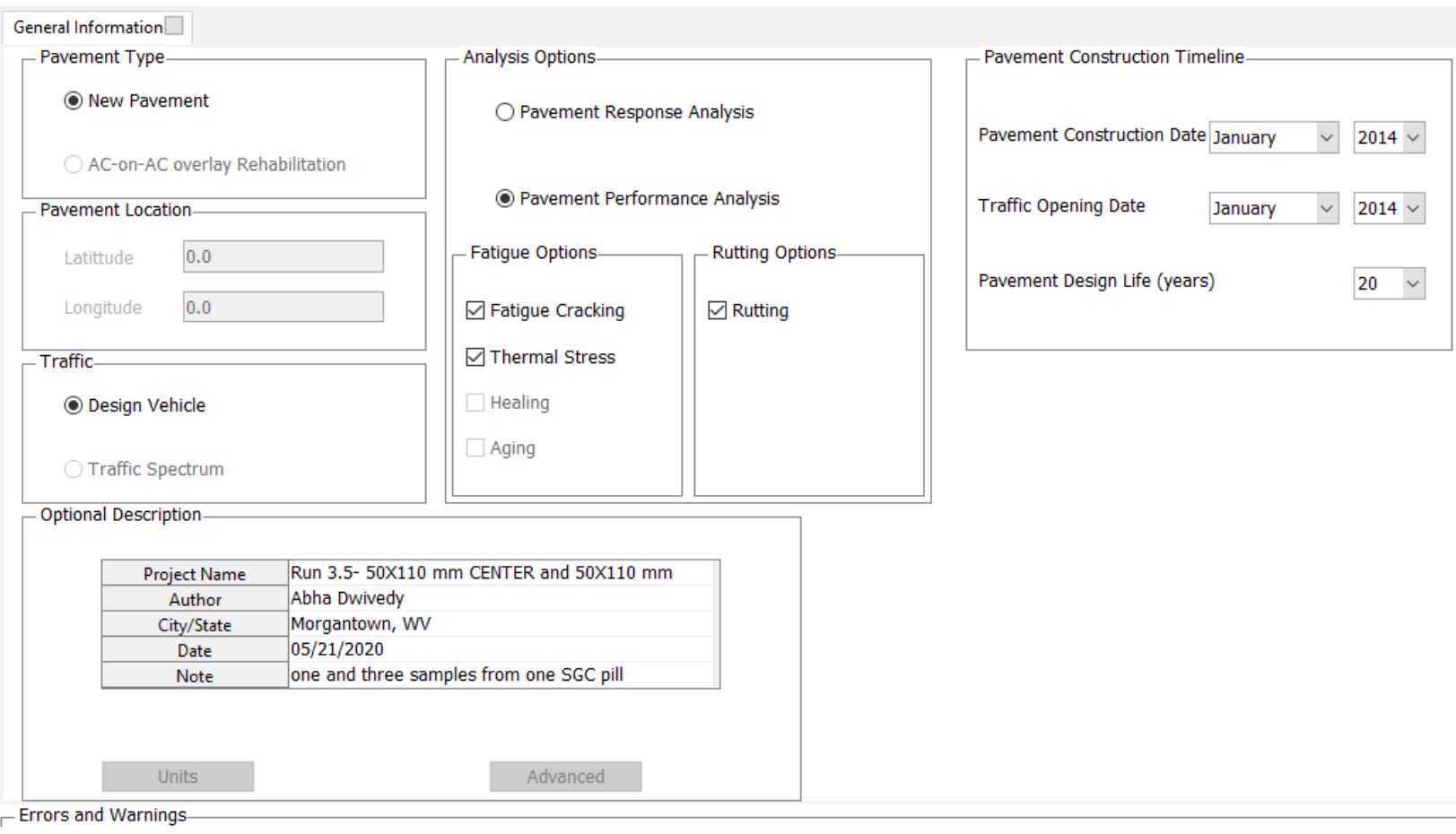

Figure 305: General Information for Run 3.5 


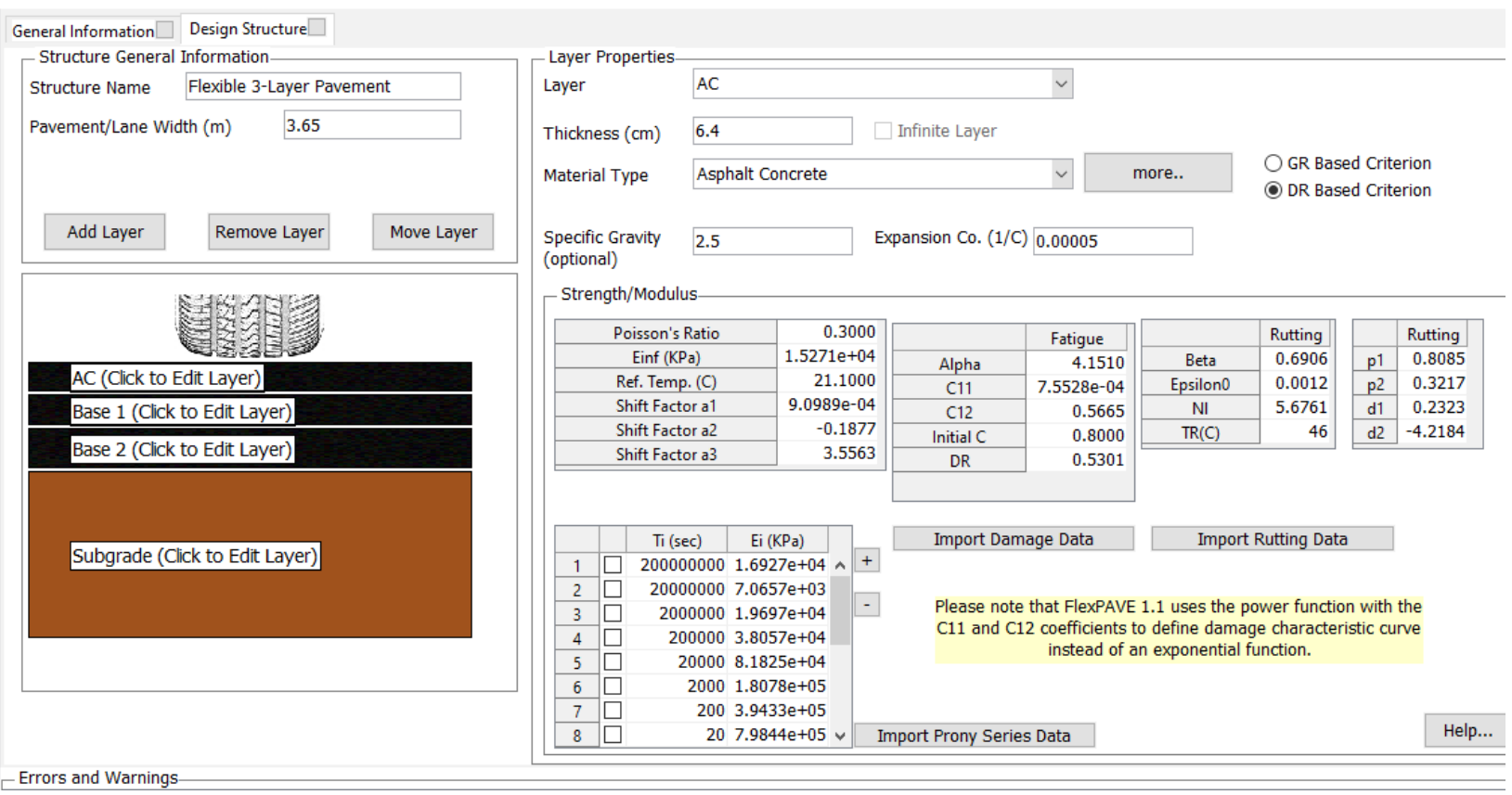

Figure 306: Design Structure of AC Layer for Run 3.5

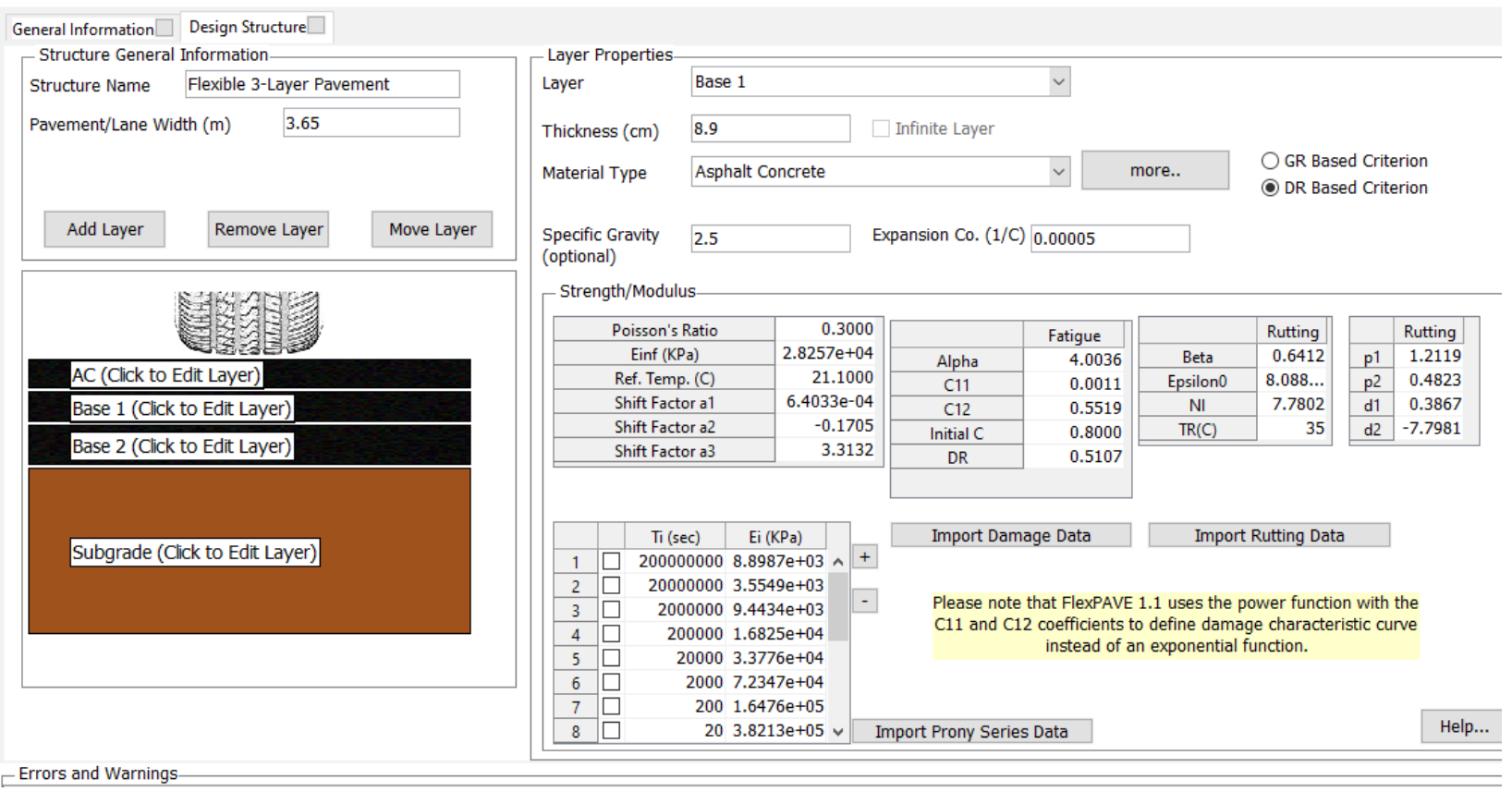

Figure 307: Design Structure of Base 1 Layer for Run 3.5 


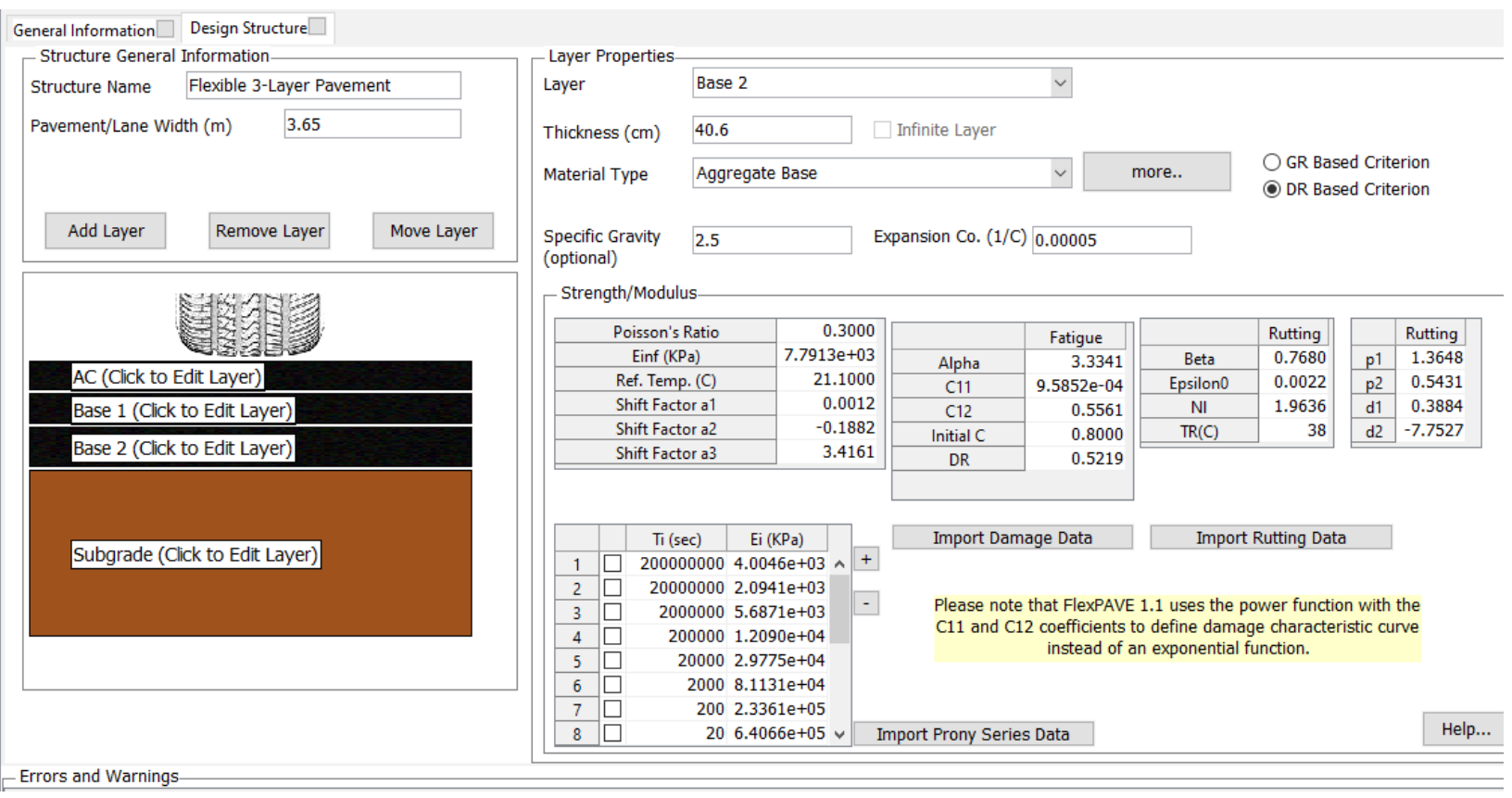

Figure 308: Design Structure of Base 2 Layer for Run 3.5

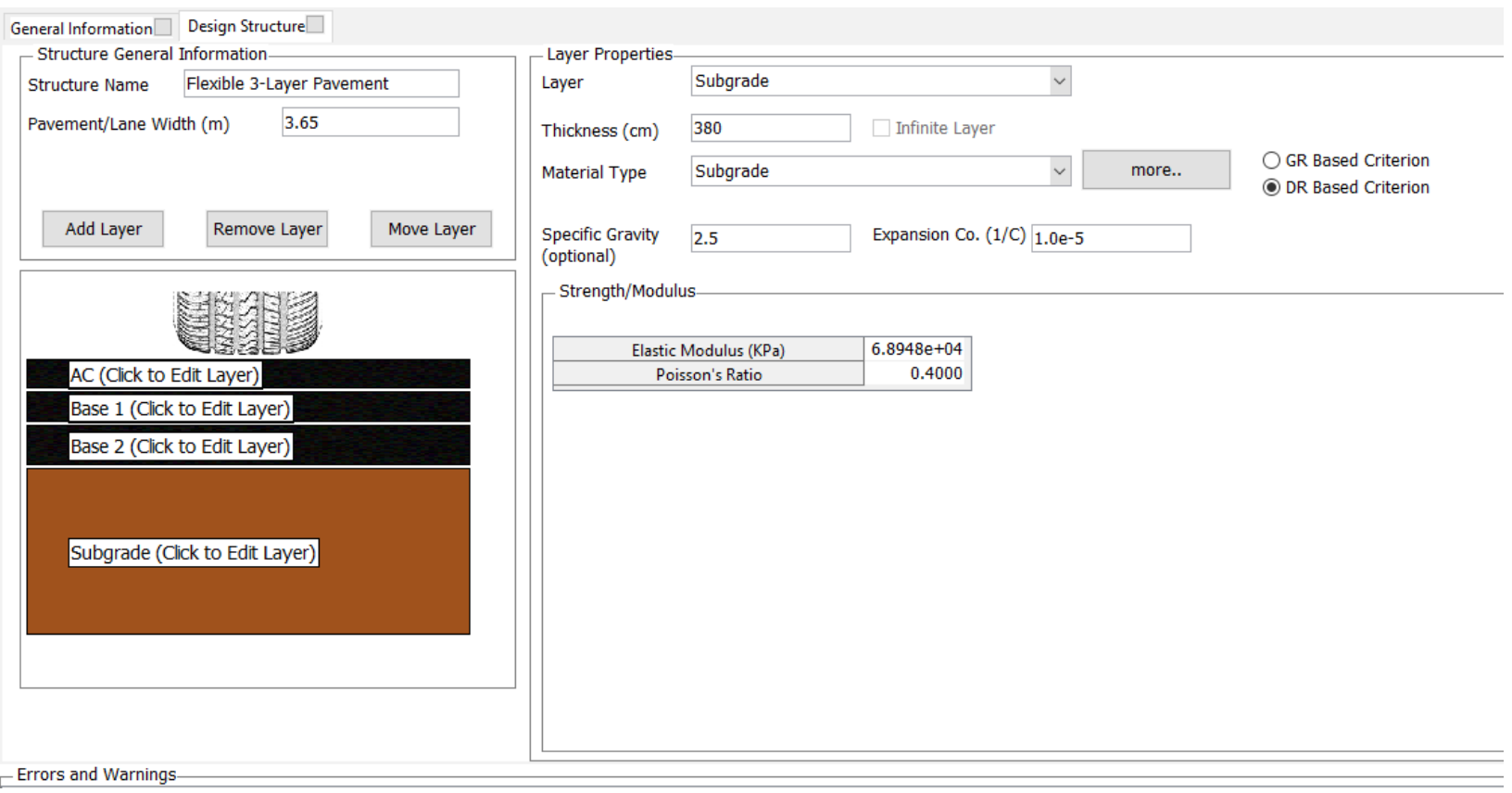

Figure 309: Design Structure of Subgrade Layer for Run 3.5 


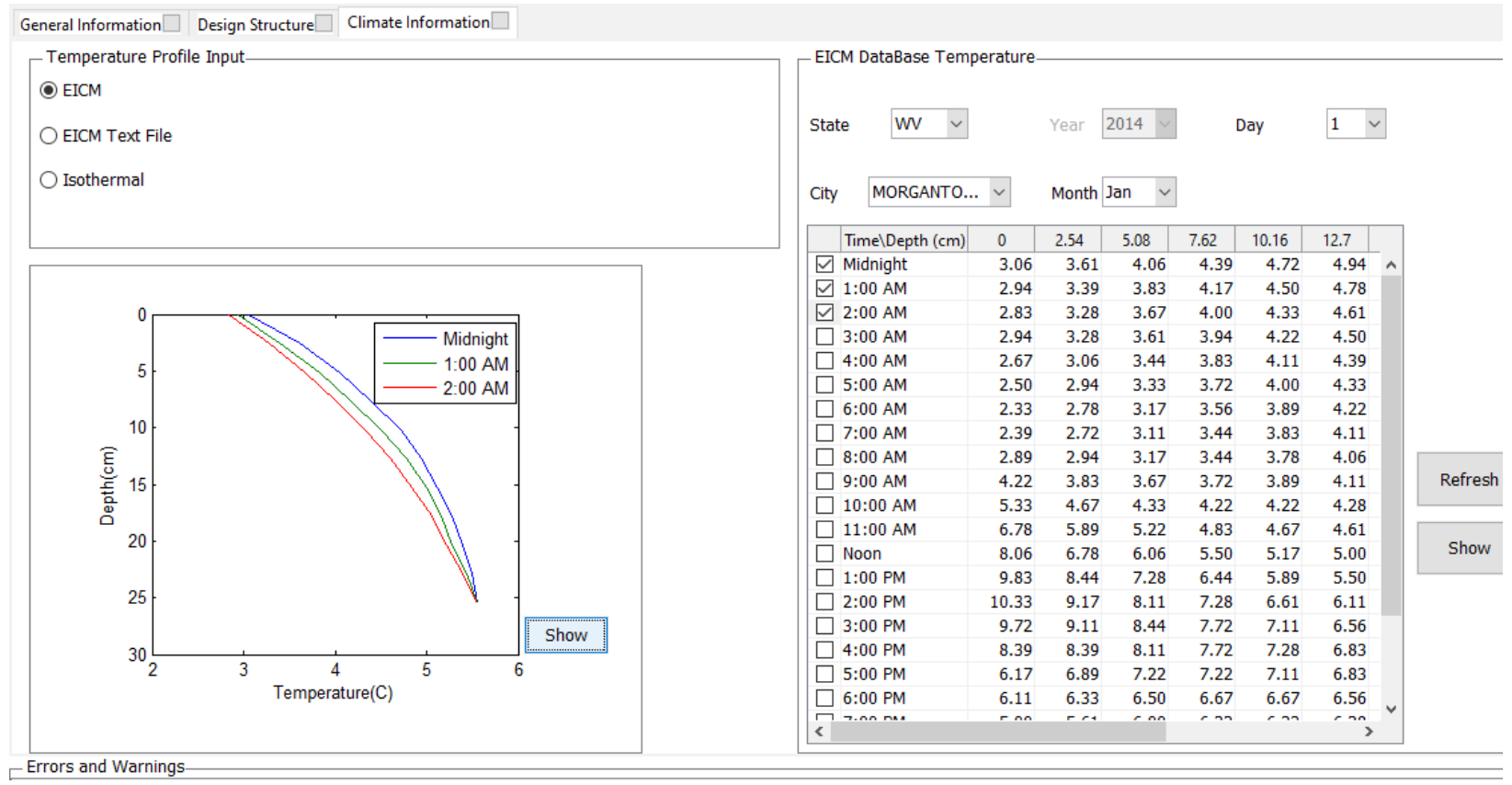

Figure 310: Climate Data for Run 3.5 


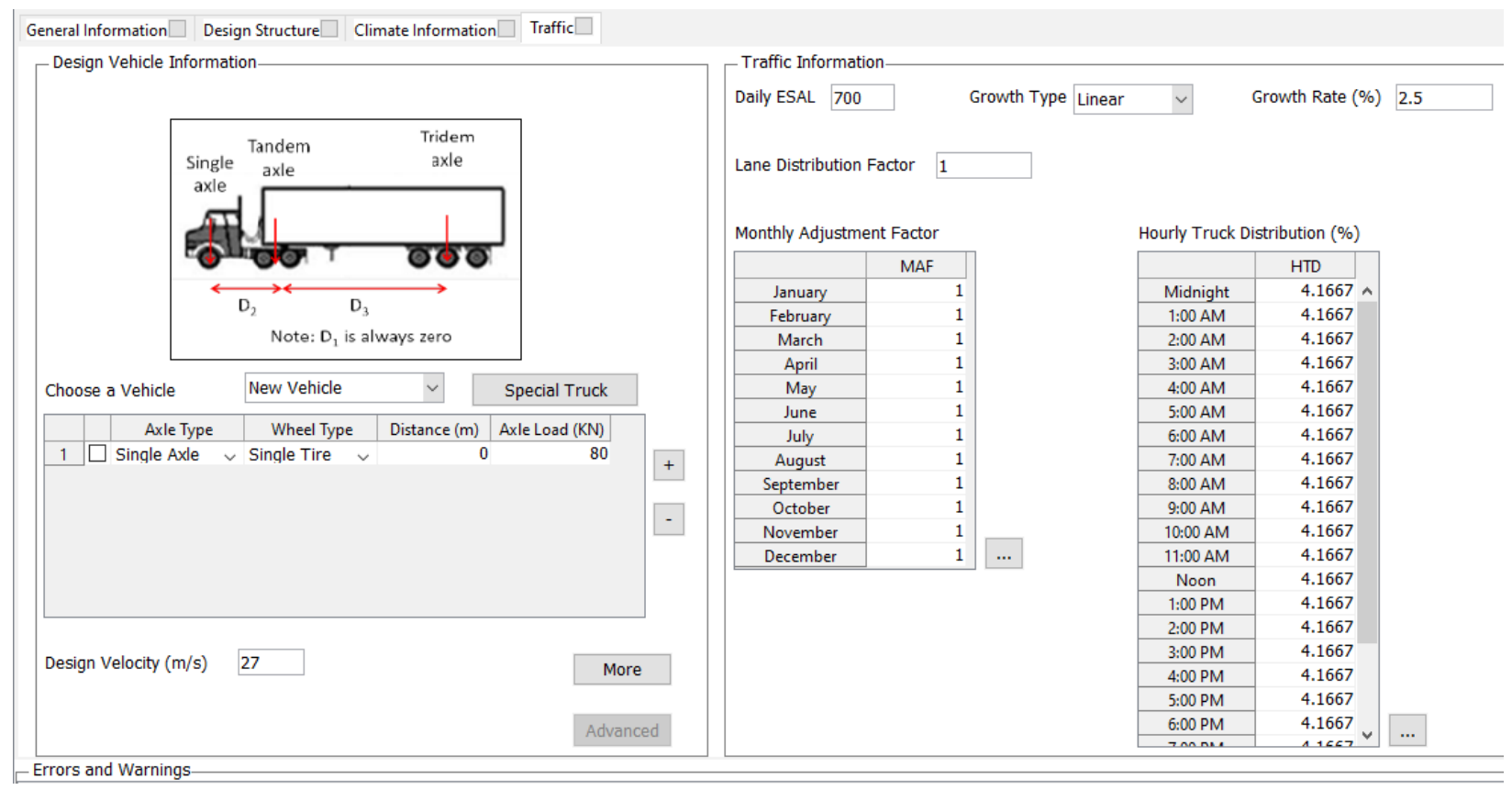

Axle Configuration

$-\square \times$

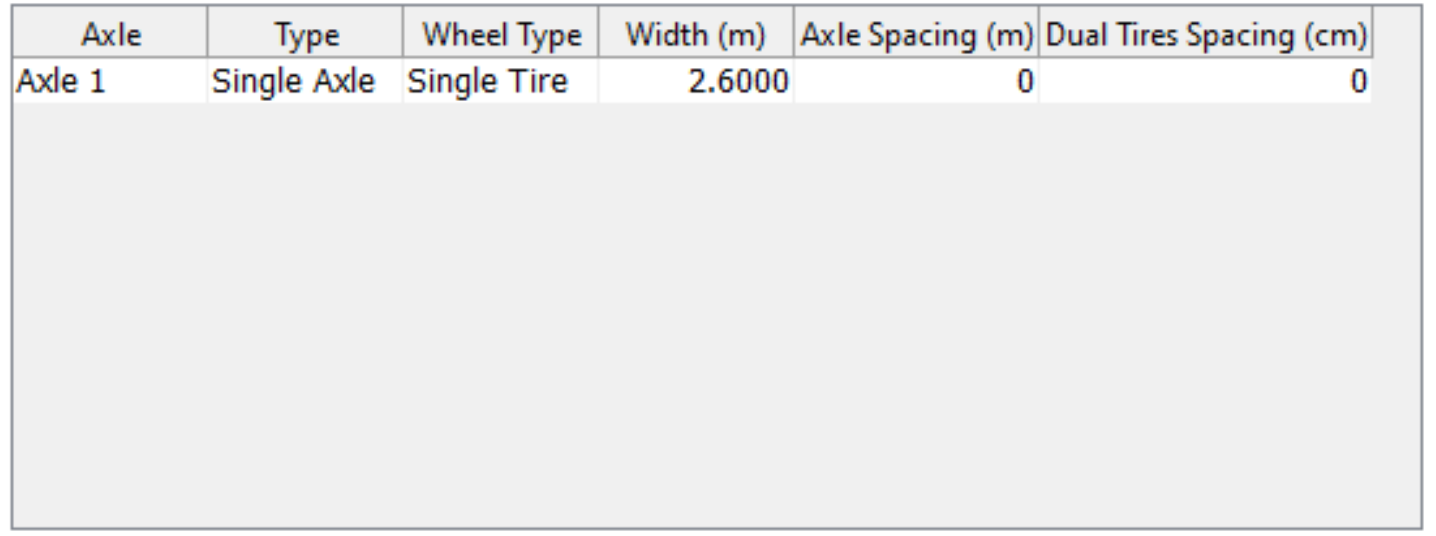

Tire Pressure (KPa) 827.37

Contact Area Shape Rectangular $\vee \quad$ Aspect Ratio (length/width) 1.5714

Shear Traction $\quad 0.0$

OK

Cancel

Figure 311: Traffic Data for Run 3.5 


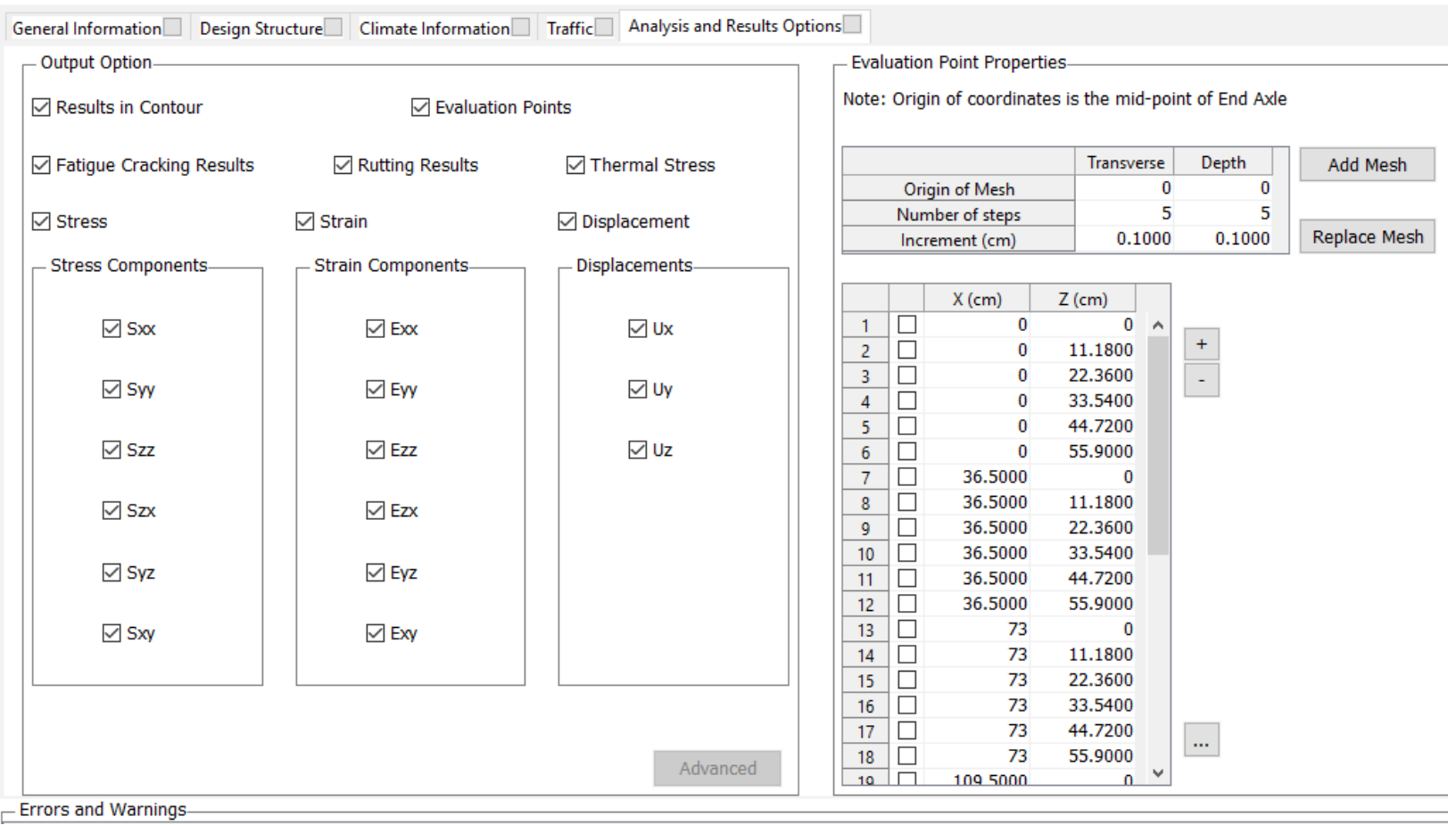

Figure 312: Output and Analysis Options for Run 3.5

\section{Run 3.6}

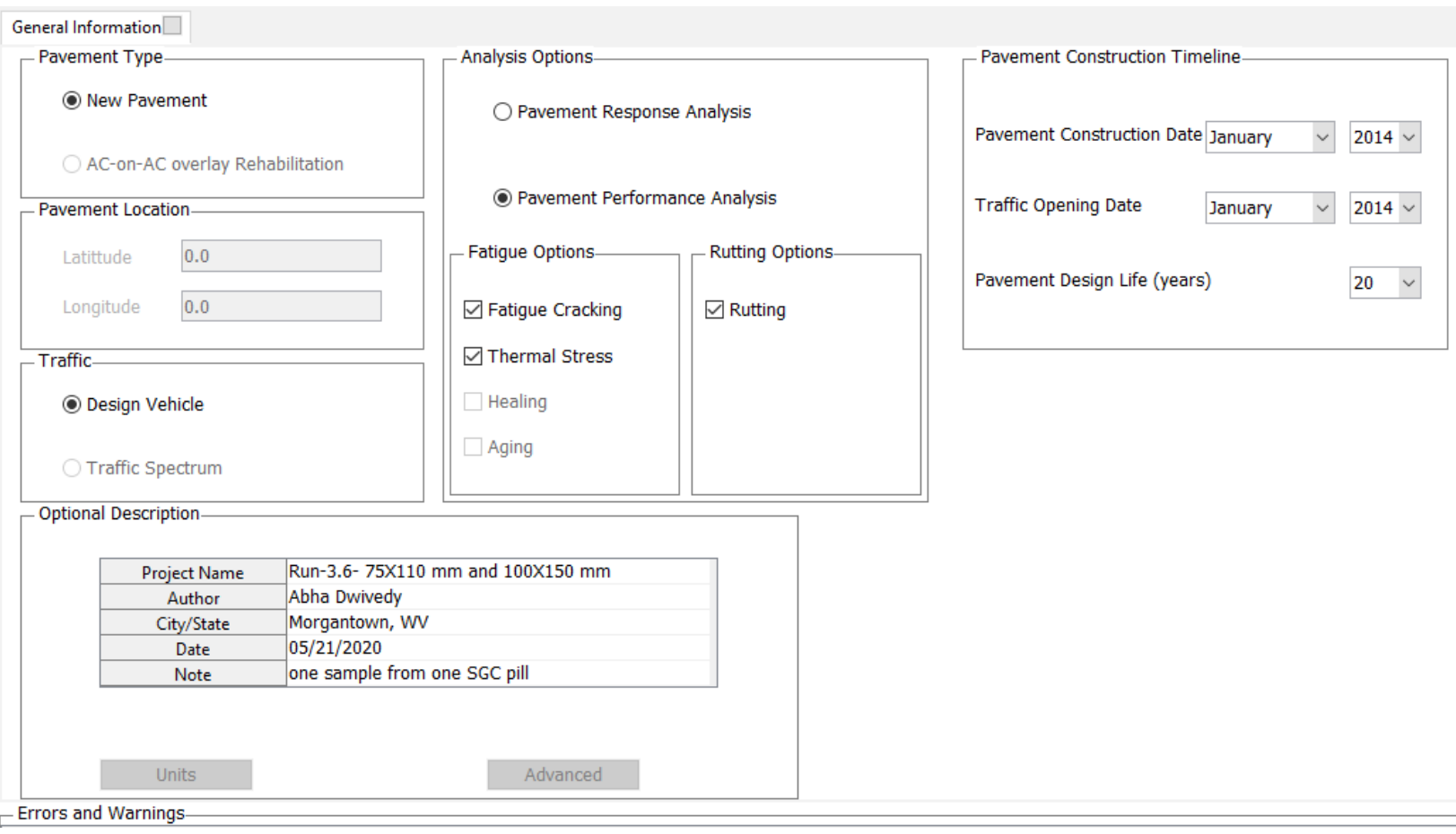

Figure 313: General Information for Run 3.6 


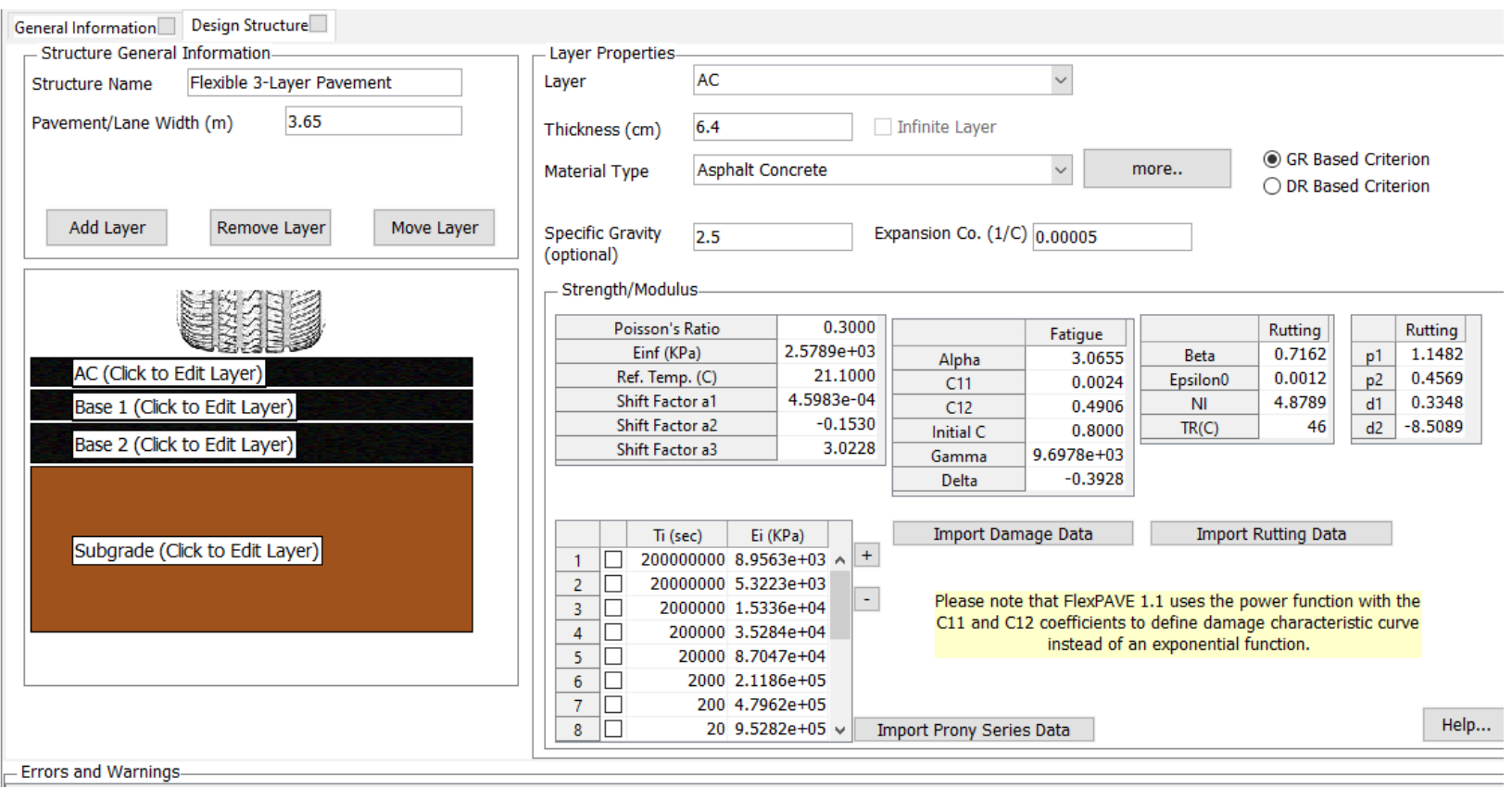

Figure 314: Design Structure of AC Layer for Run 3.6

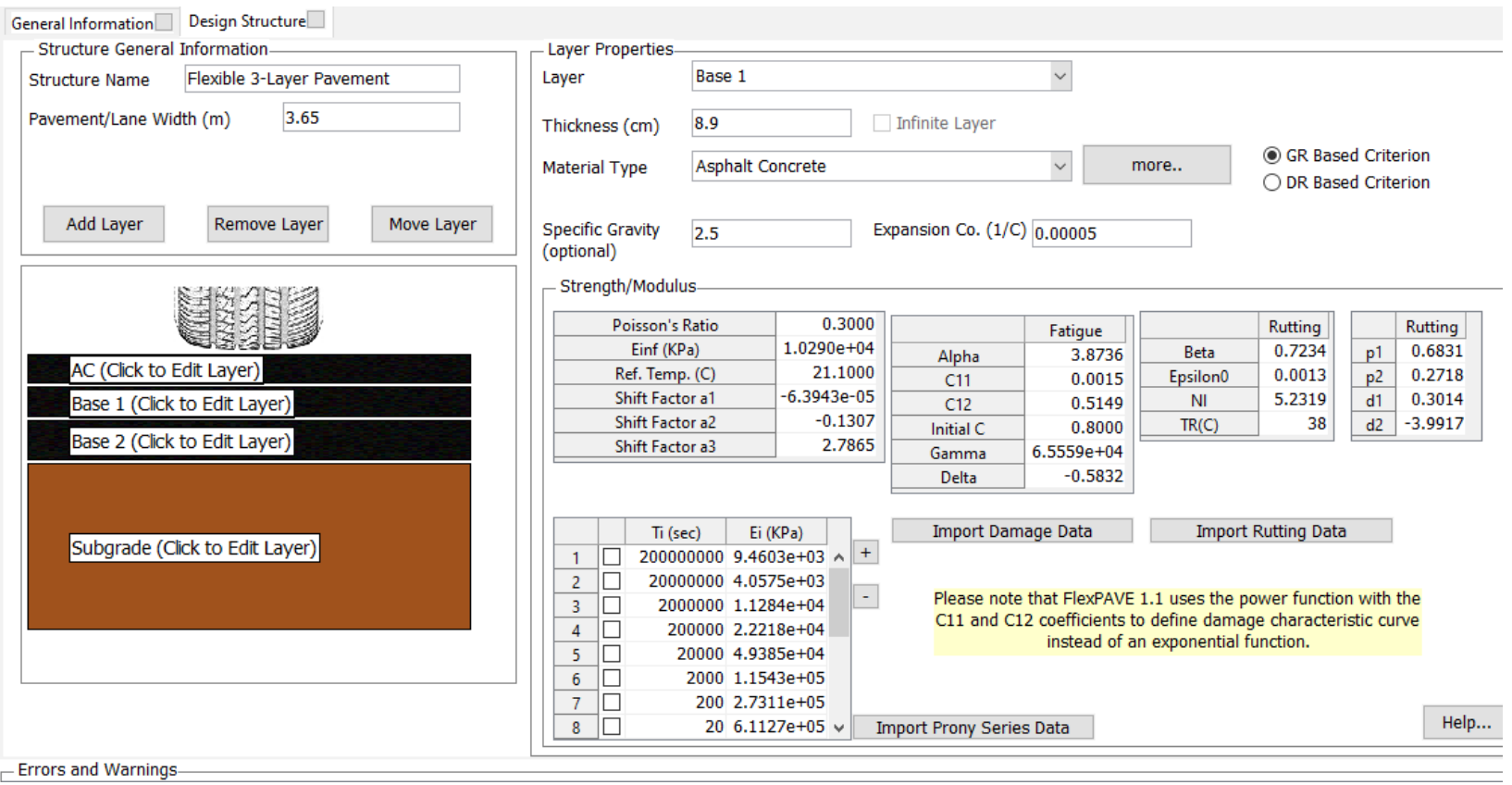

Figure 315: Design Structure of Base 1 Layer for Run 3.6 


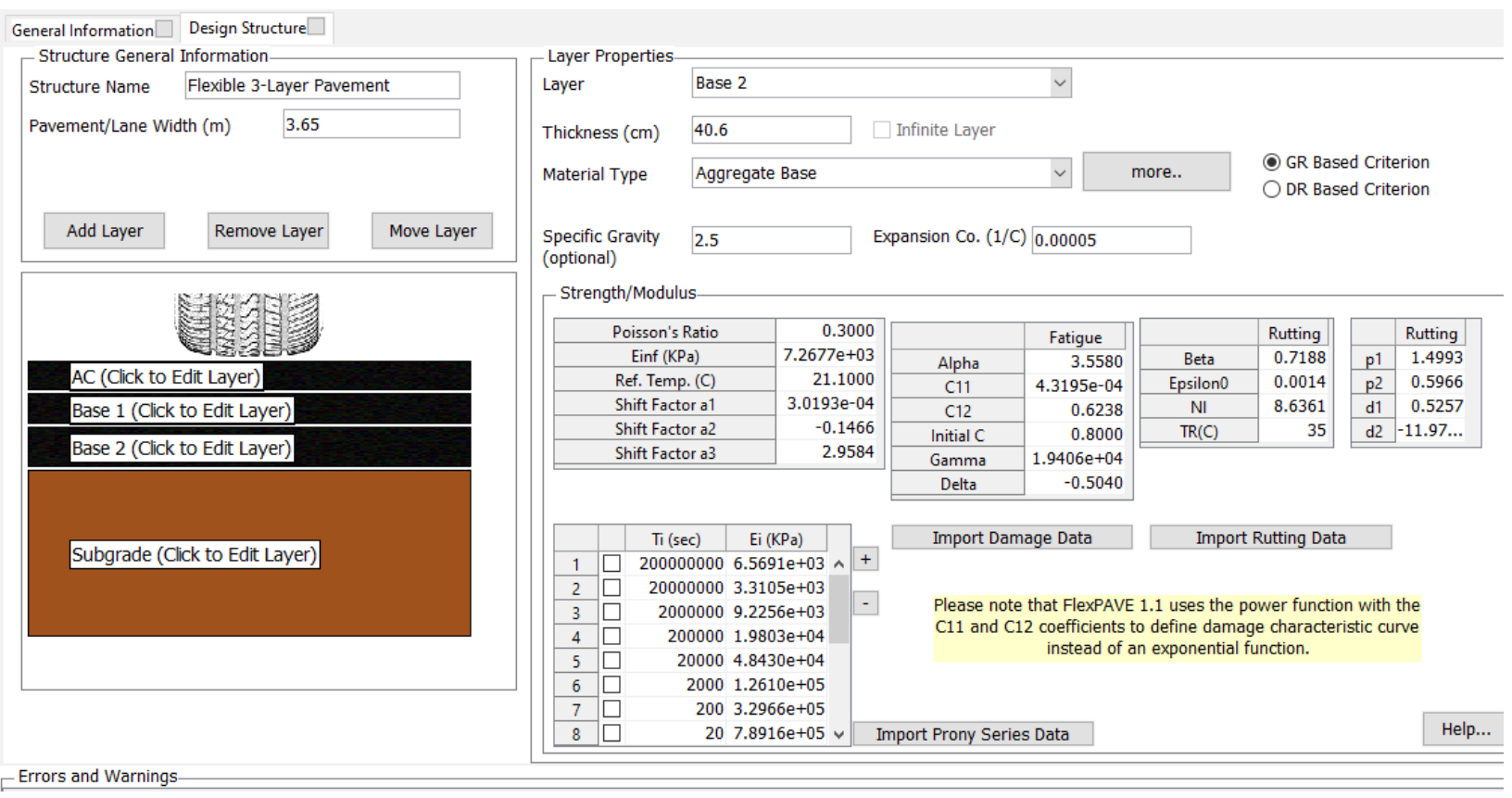

Figure 316: Design Structure of Base 2 Layer for Run 3.6

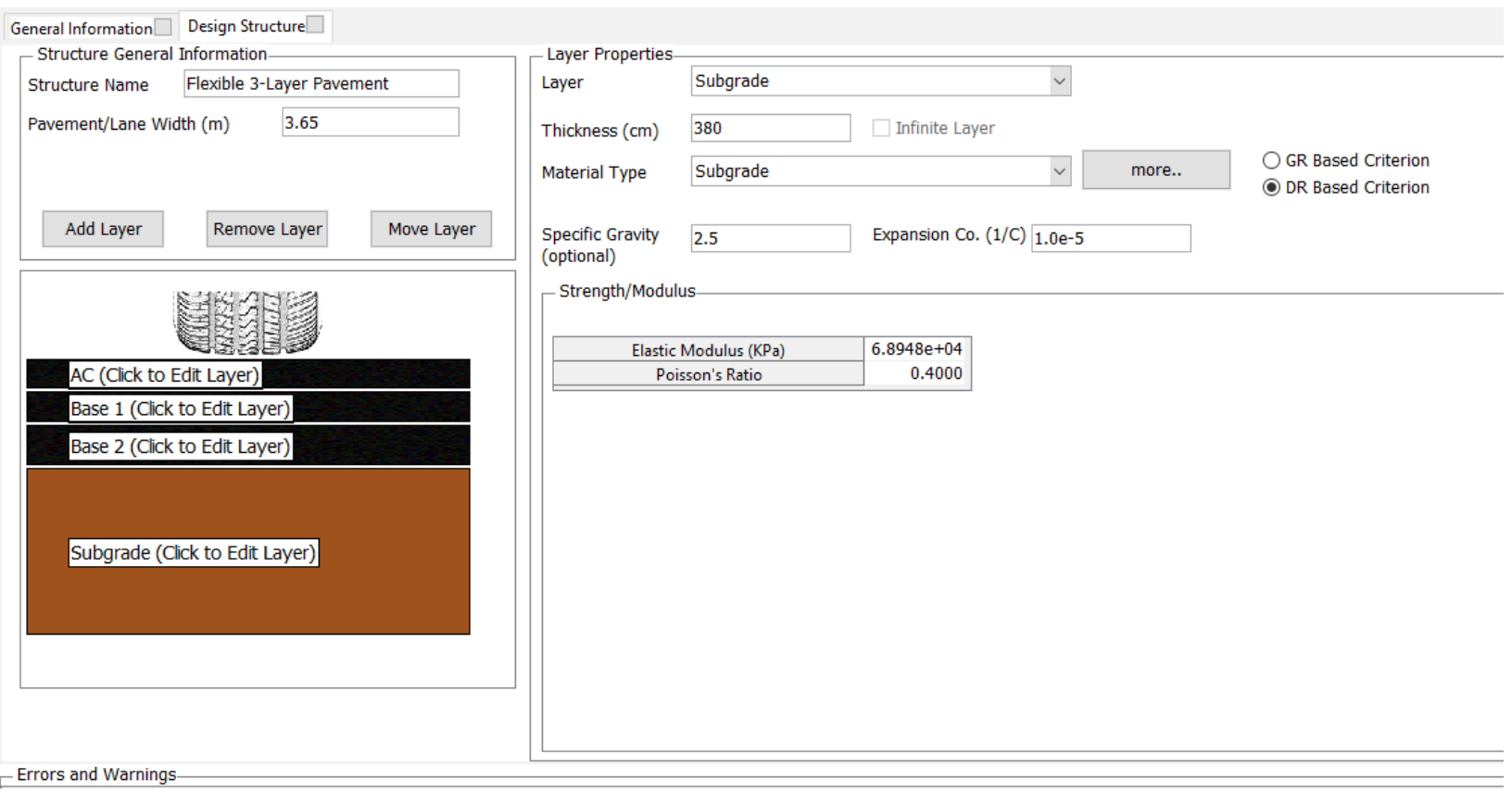

Figure 317: Design Structure of Subgrade Layer for Run 3.6 


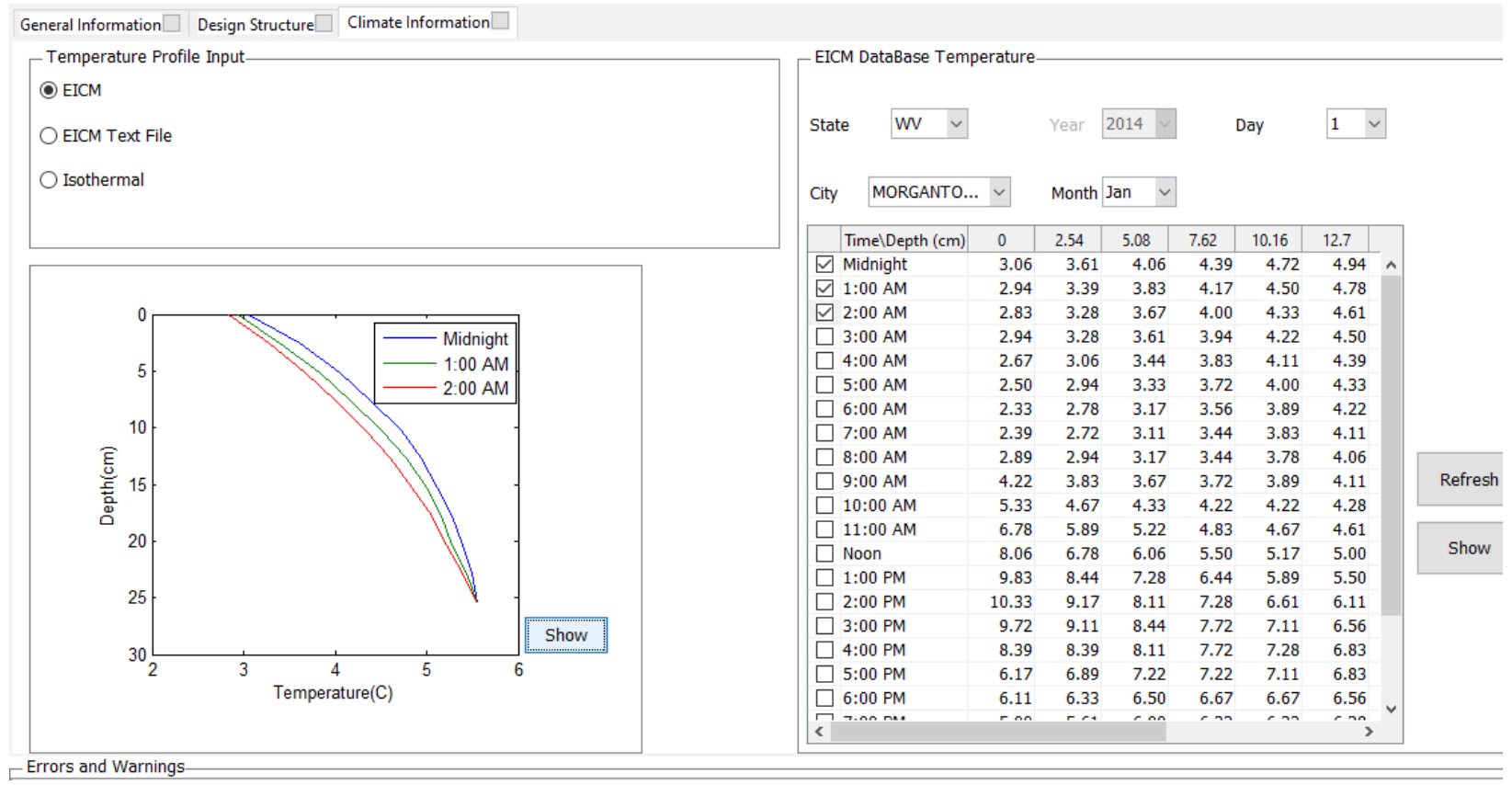

Figure 318: Climate Data for Run 3.6 


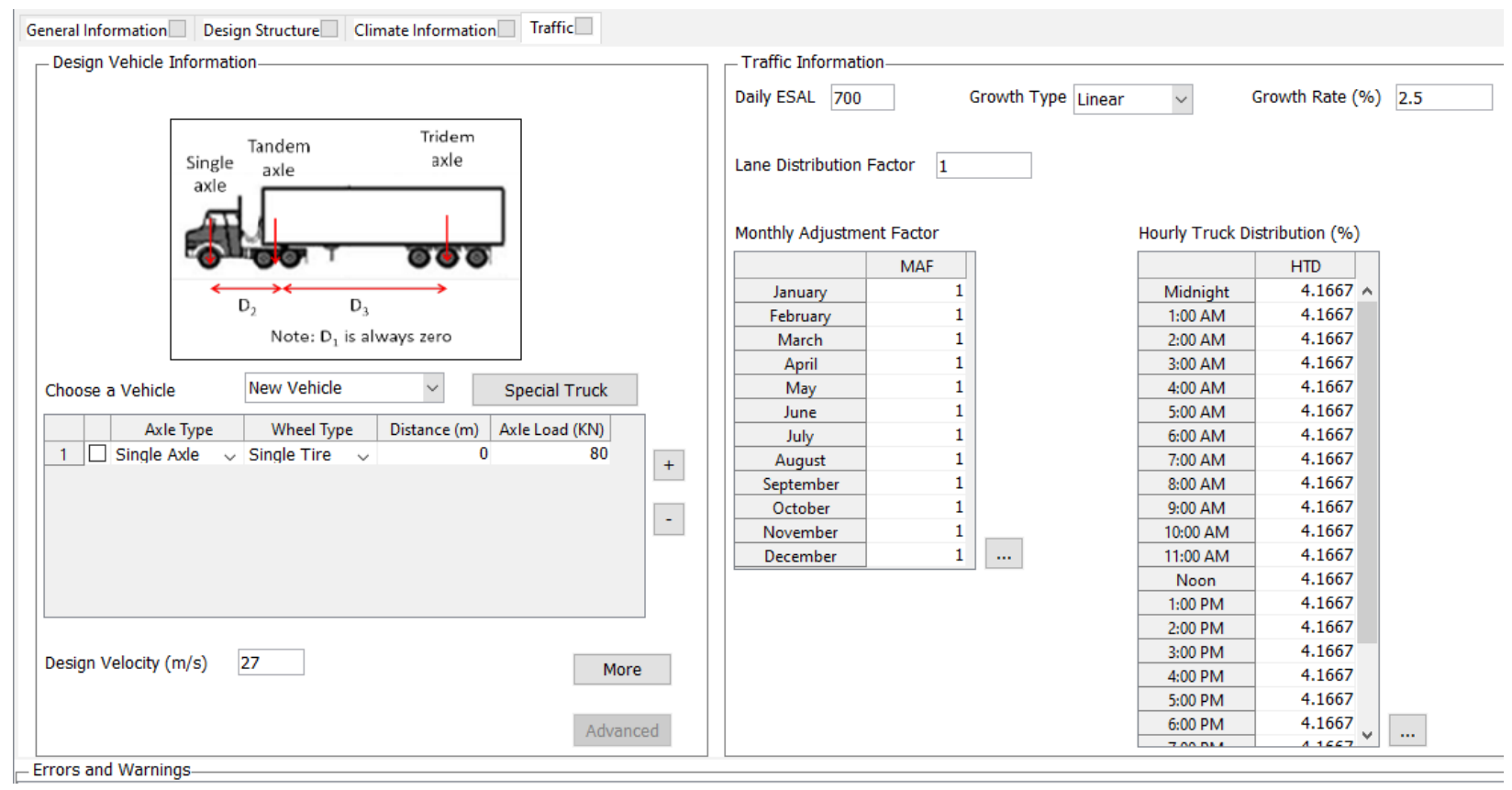

Axle Configuration

$-\square \times$

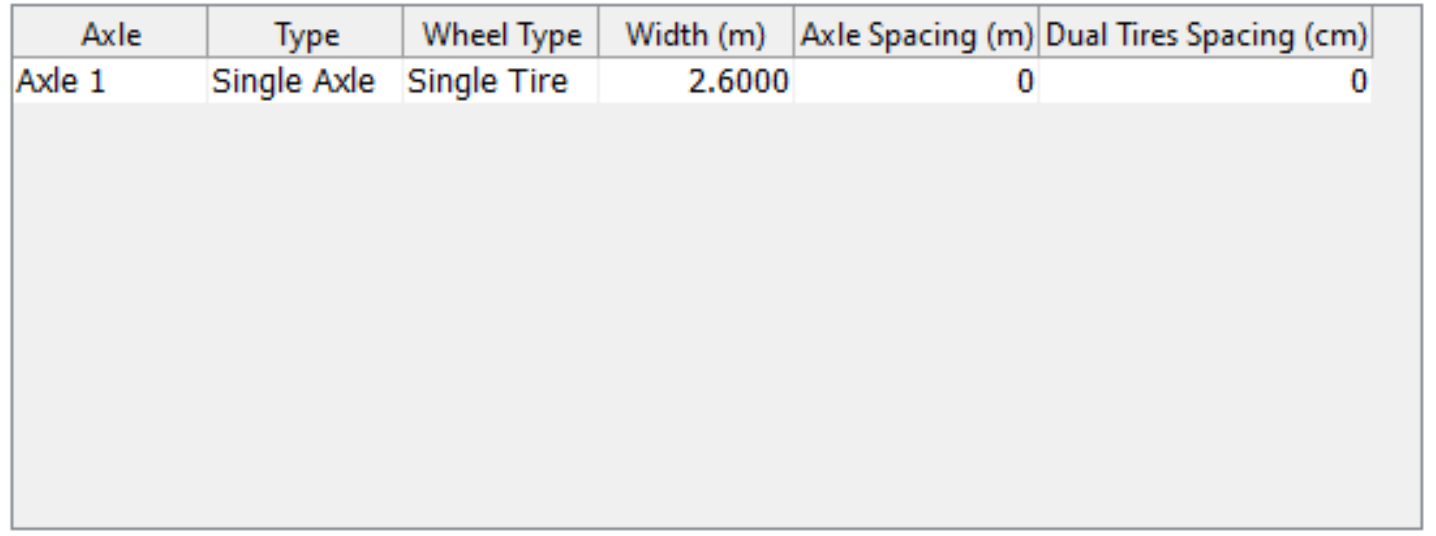

Tire Pressure (KPa) 827.37

Contact Area Shape Rectangular $\vee \quad$ Aspect Ratio (length/width) 1.5714

Shear Traction $\quad 0.0$

OK

Cancel

Figure 319: Traffic Data for Run 3.6 


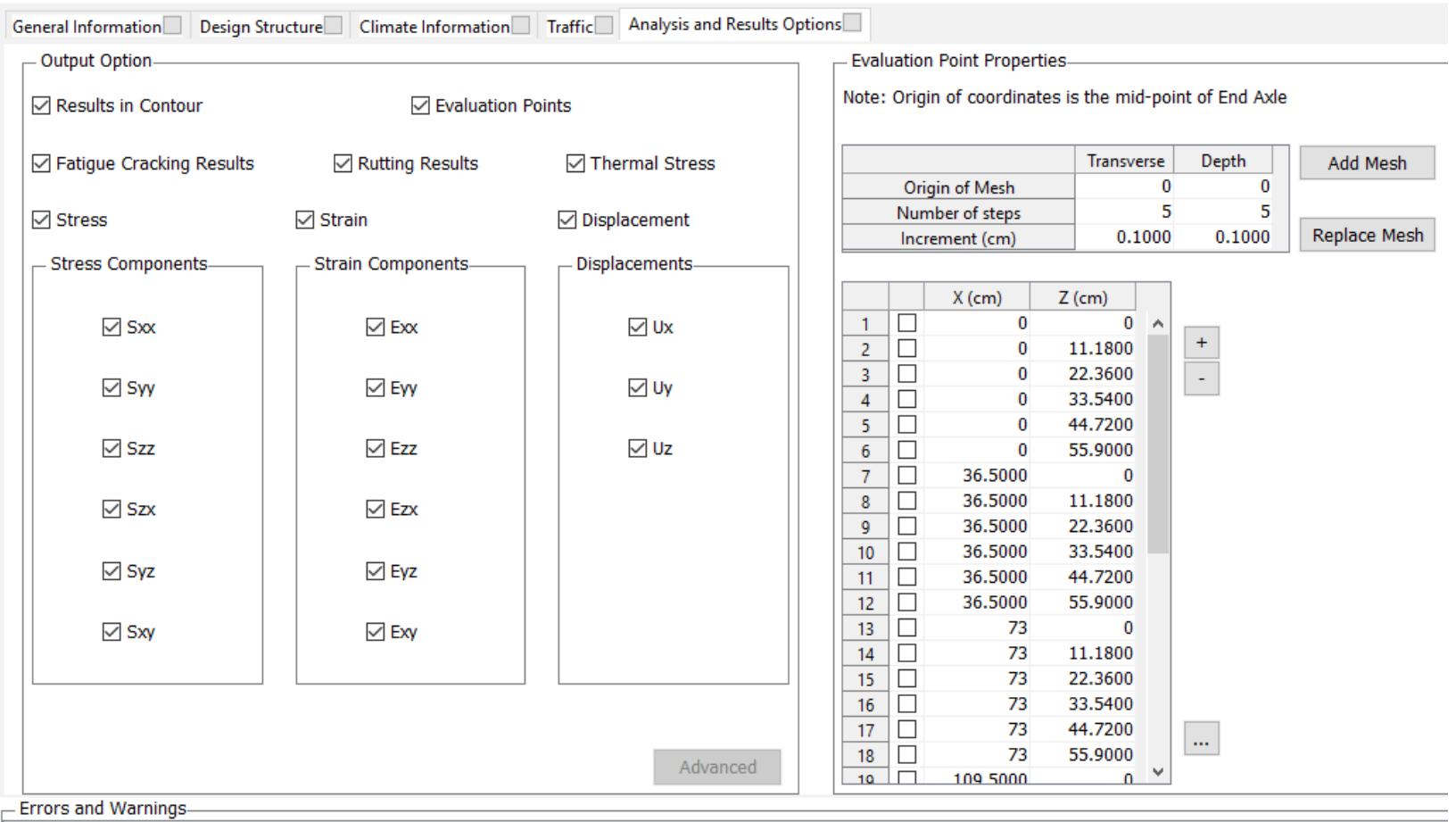

Figure 320: Output and Analysis Options for Run 3.6

\section{Run 3.7}

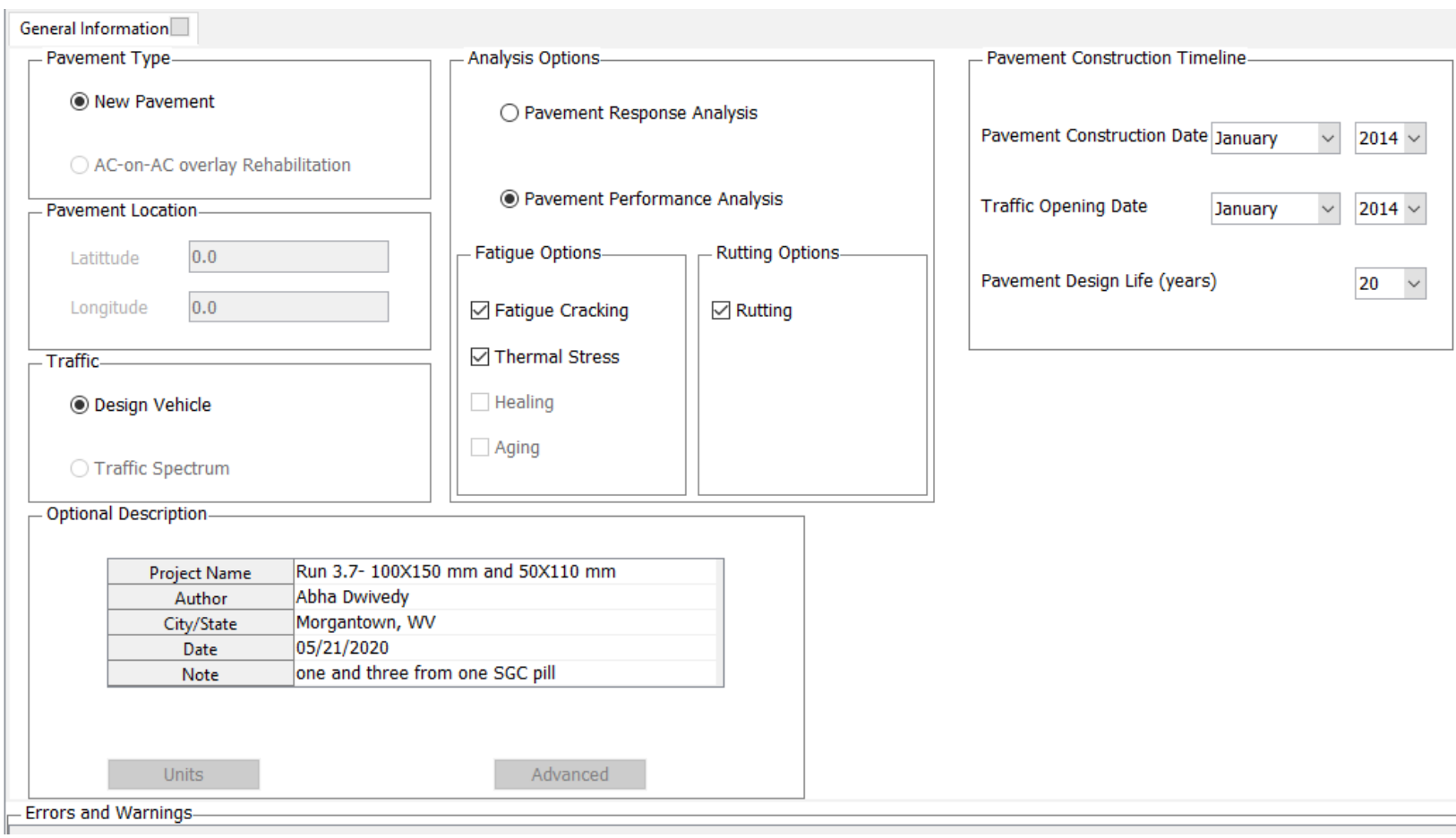

Figure 321: General Information for Run 3.7 


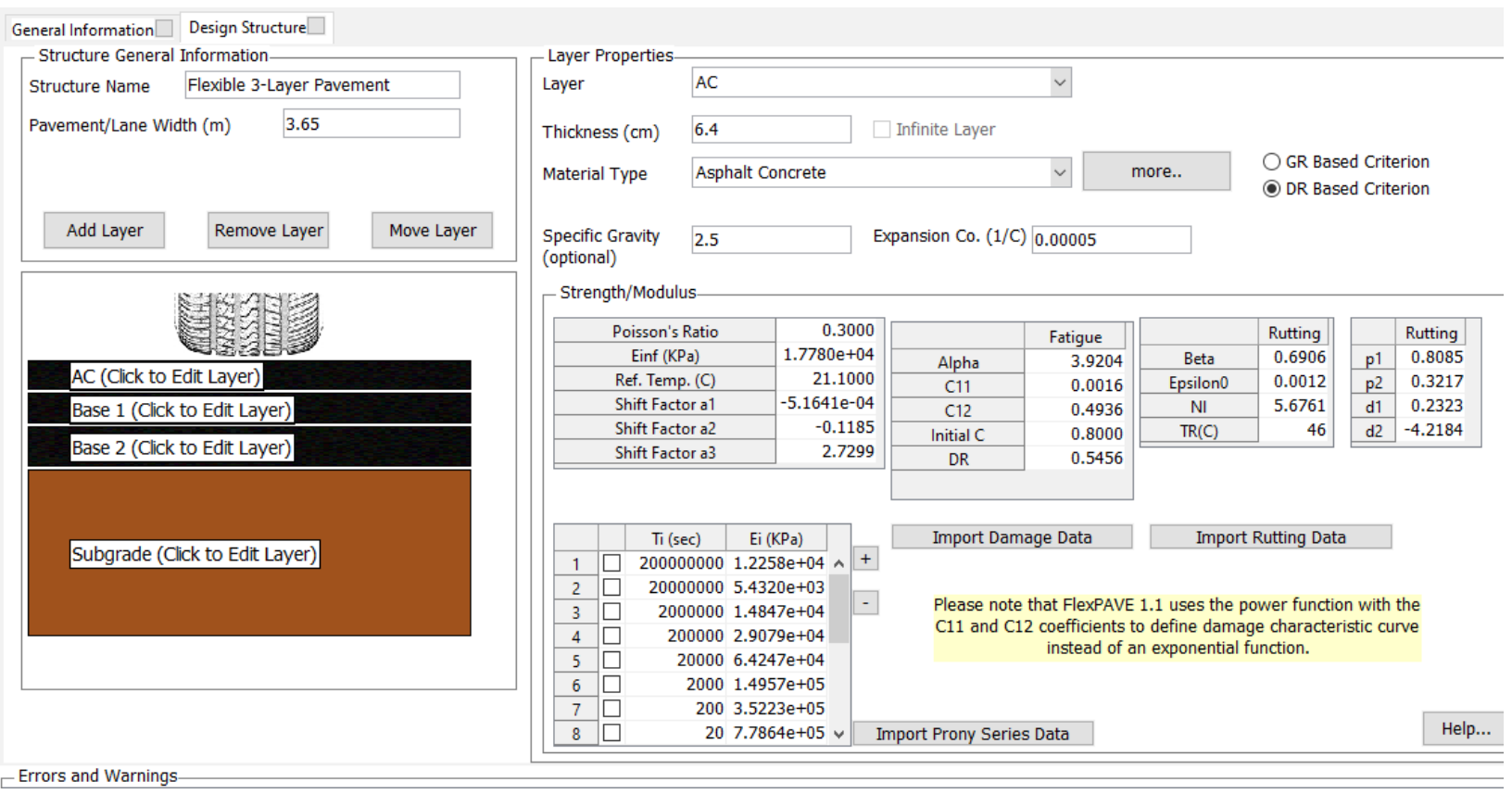

Figure 322: Design Structure of AC Layer for Run 3.7

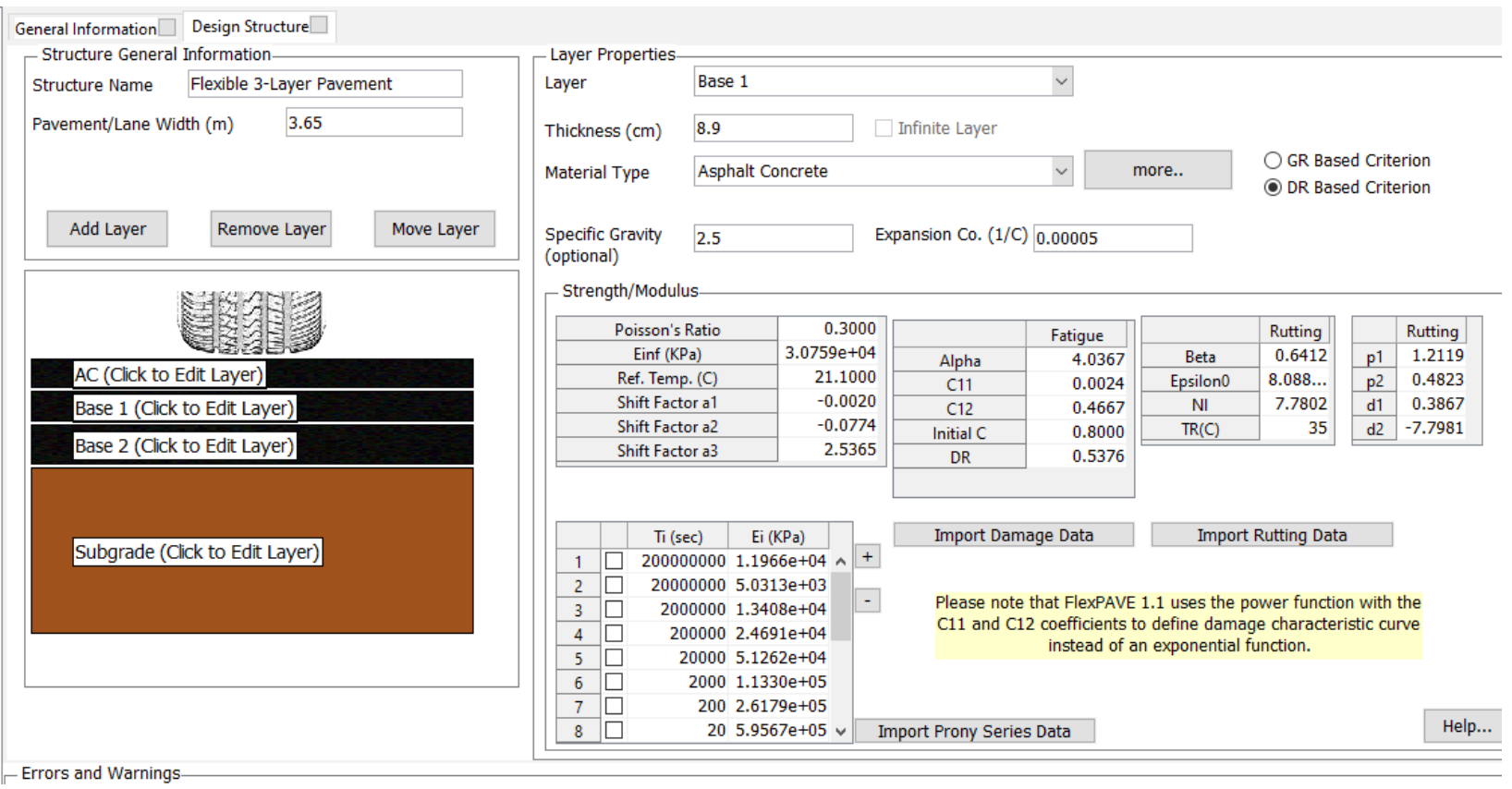

Figure 323: Design Structure of Base 1 Layer for Run 3.7 

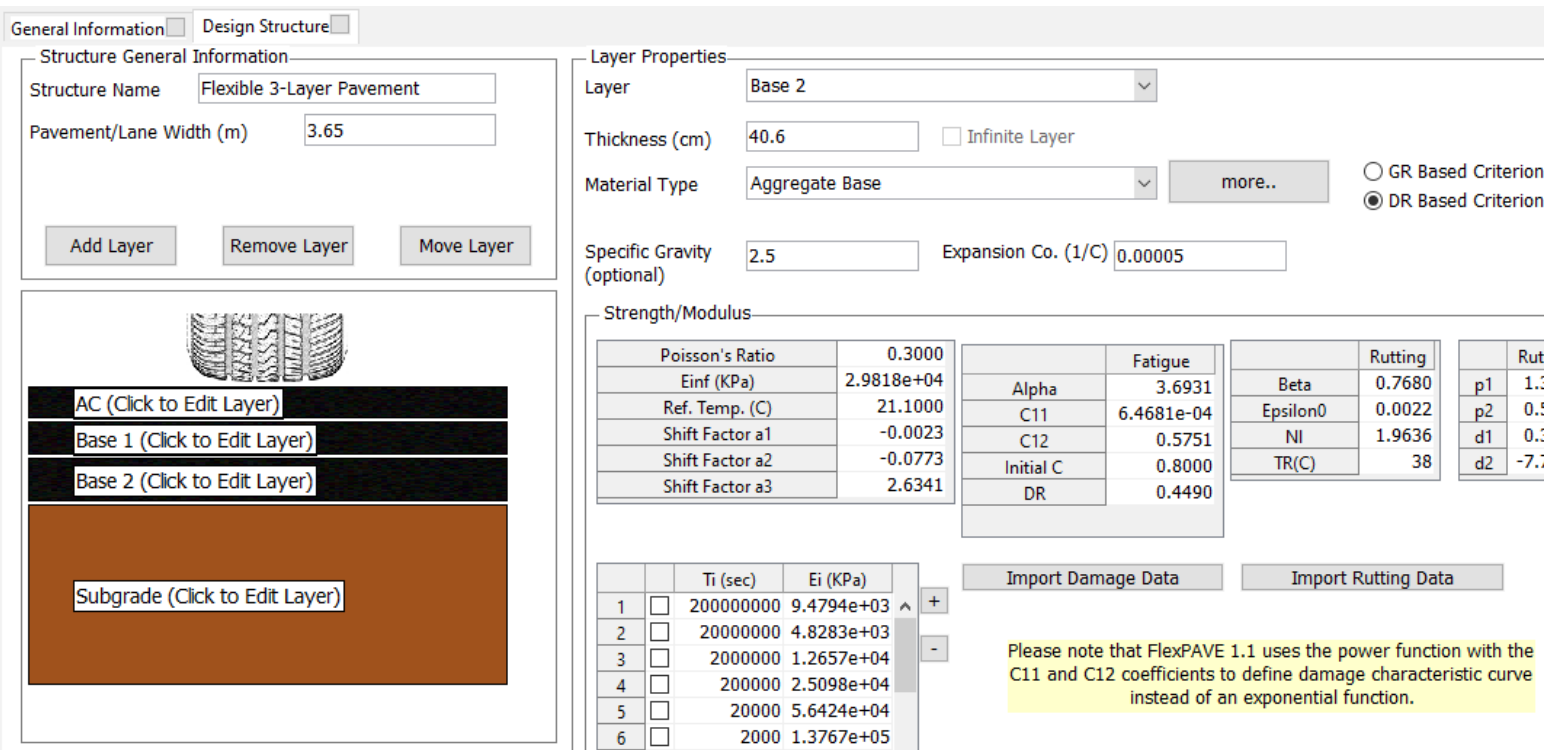

\begin{tabular}{r|r|}
0.3000 \\
$2.9818 \mathrm{e}+04$ \\
21.1000 \\
-0.0023 \\
-0.0773 \\
2.6341 \\
\hline
\end{tabular}

\begin{tabular}{|c|}
\hline Alpha \\
\hline $\mathrm{C} 11$ \\
\hline $\mathrm{C} 12$ \\
\hline Initial C \\
\hline DR \\
\hline
\end{tabular}

\begin{tabular}{|r|}
\hline \multicolumn{1}{|c|}{ Fatigue } \\
\hline 3.6931 \\
$6.4681 \mathrm{e}-04$ \\
0.5751 \\
0.8000 \\
0.4490 \\
\hline
\end{tabular}

\begin{tabular}{|c|c|c|c|}
\hline & Rutting & & Rutting \\
\hline Beta & 0.7680 & p1 & 1.3648 \\
\hline Epsilon0 & 0.0022 & $p^{2}$ & 0.5431 \\
\hline $\mathrm{NI}$ & 1.9636 & d1 & 0.3884 \\
\hline $\operatorname{TR}(C)$ & 38 & $\mathrm{~d} 2$ & -7.7527 \\
\hline
\end{tabular}

$20000001.2657 \mathrm{e}+04$

$2000002.5098 \mathrm{e}+04$

$200005.6424 \mathrm{e}+04$

$20001.3767 \mathrm{e}+05$

$2003.5283 e+05$

-Errors and Warning

\section{Figure 324: Design Structure of Base 2 Layer for Run 3.7}

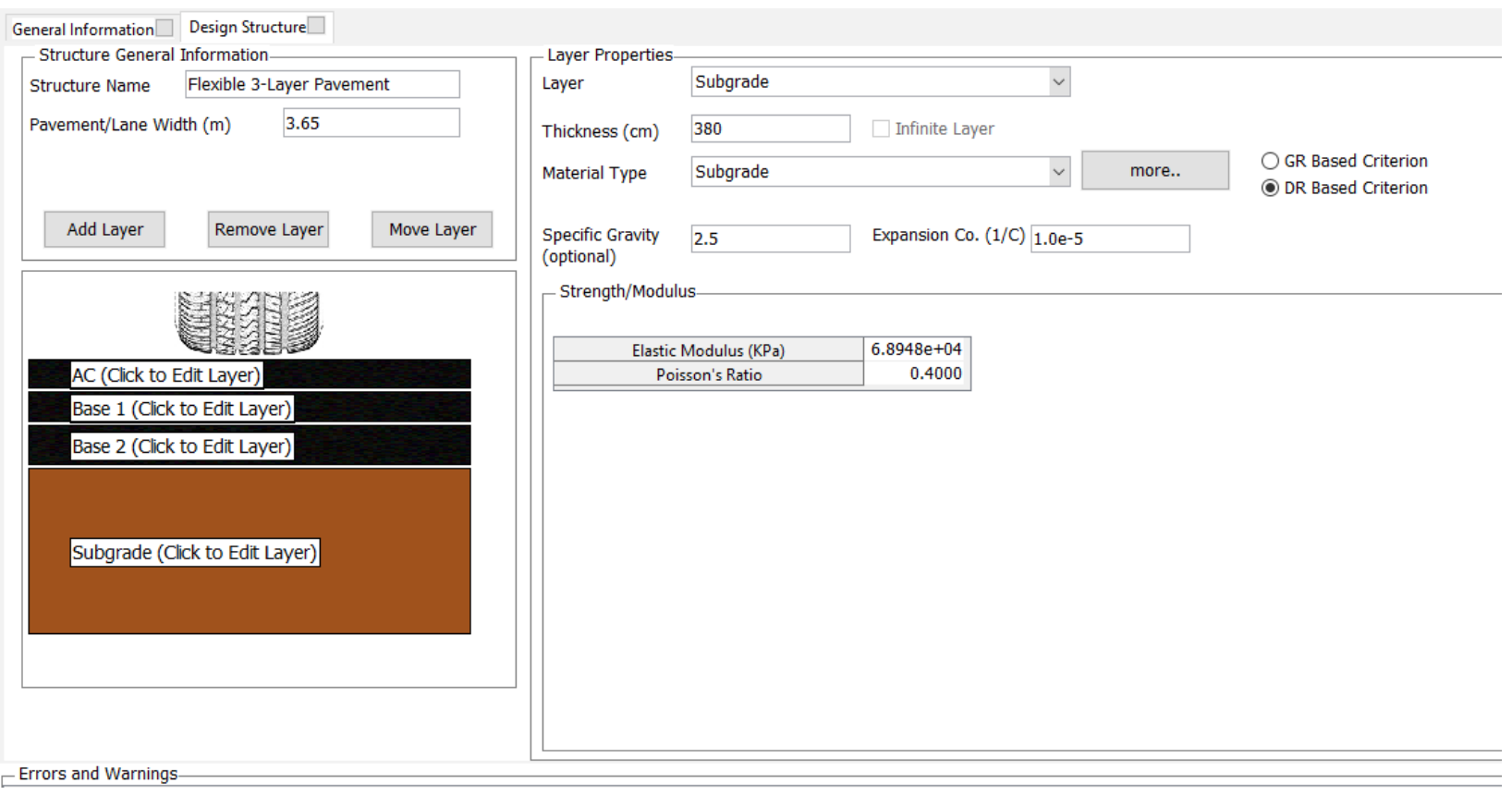

Figure 325: Design Structure of Subgrade Layer for Run 3.7 


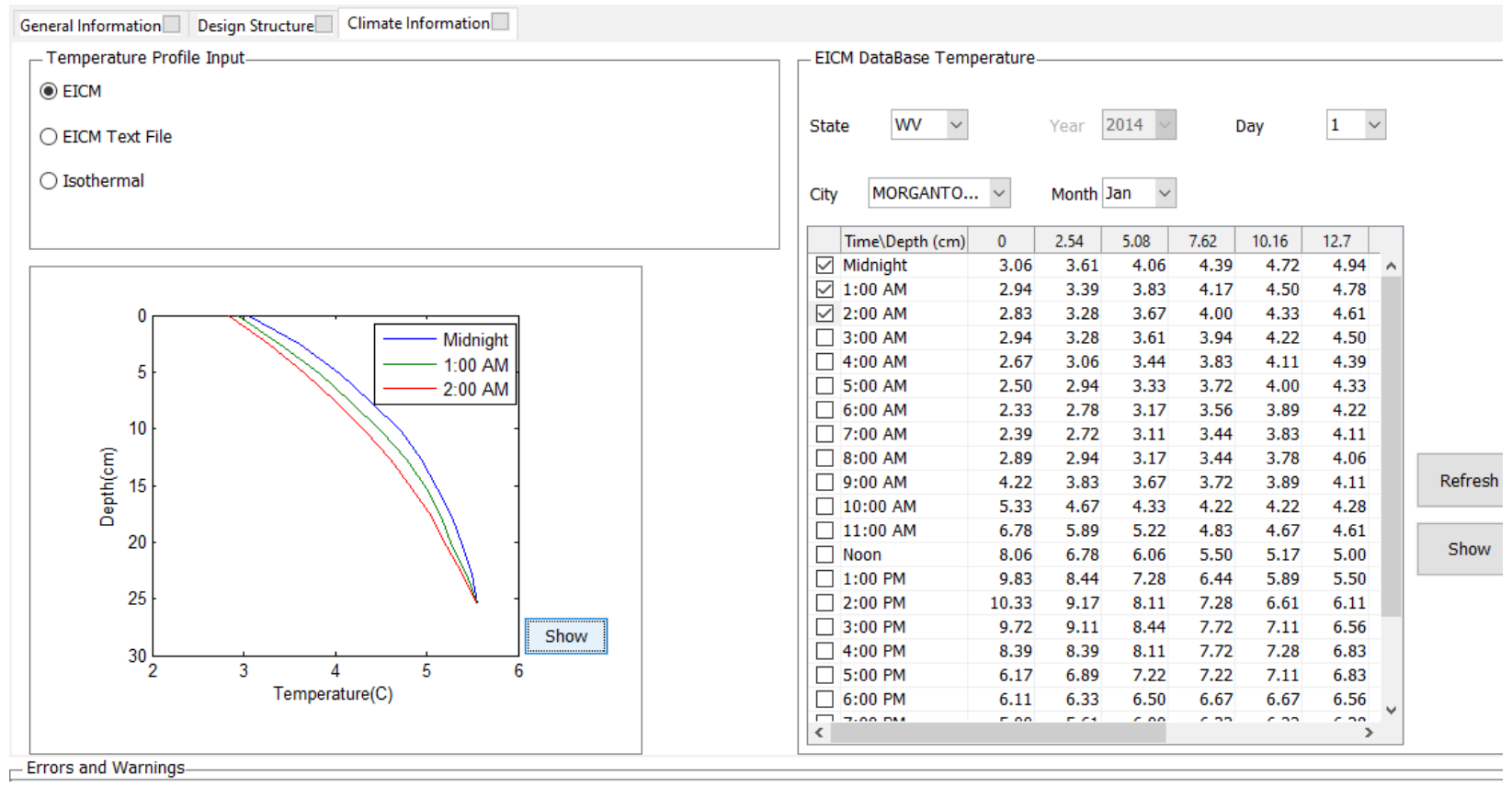

Figure 326: Climate Data for Run 3.7 


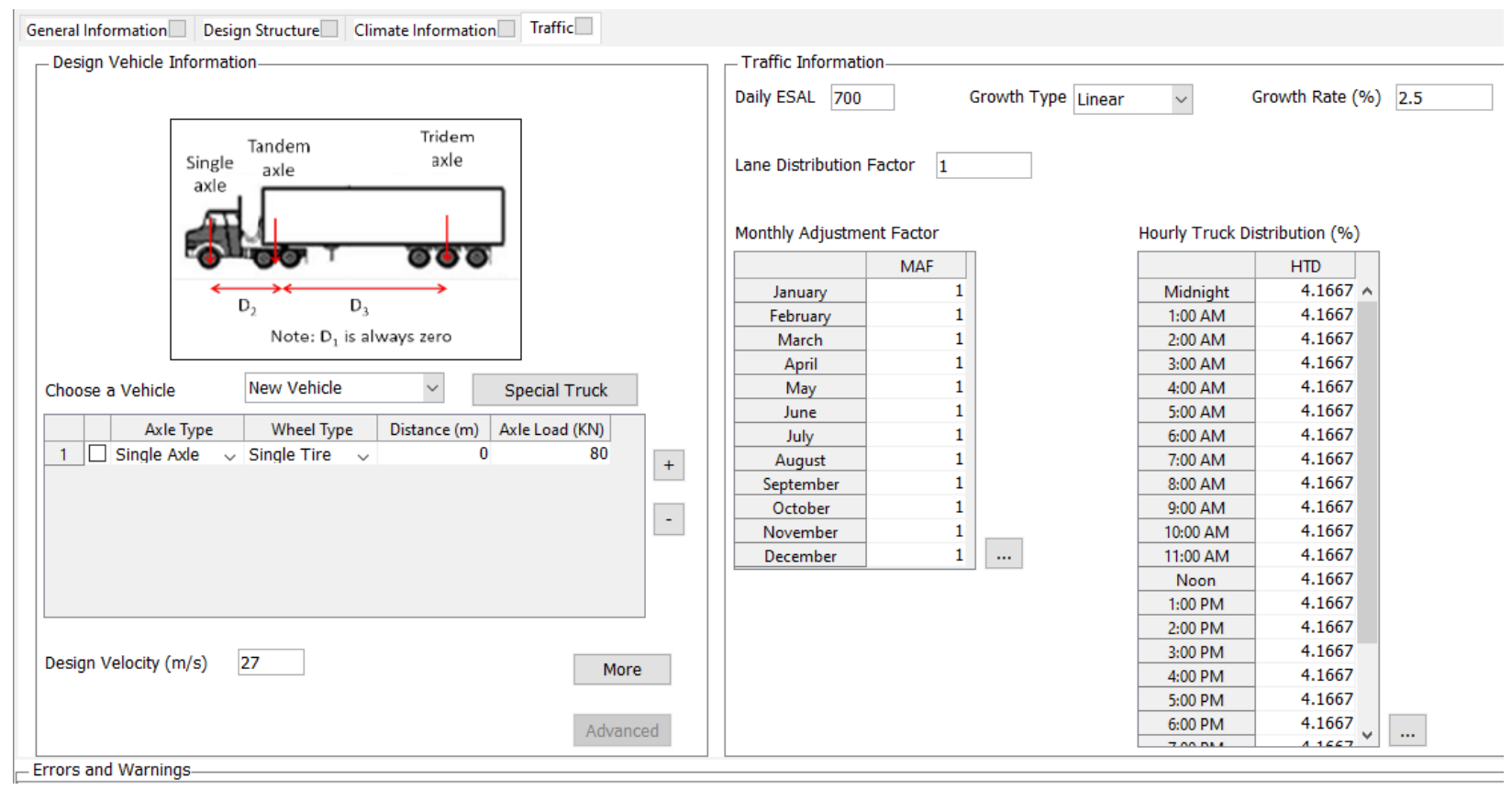

Axle Configuration

$-\square \times$

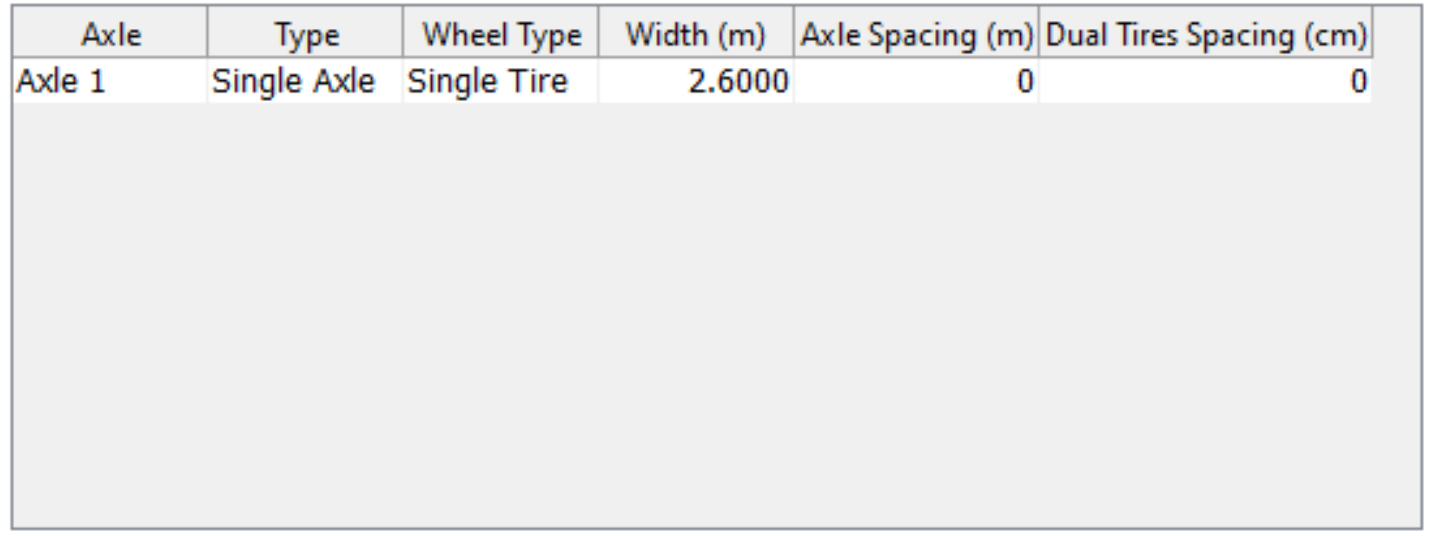

Tire Pressure (KPa) 827.37

Contact Area Shape Rectangular $\vee \quad$ Aspect Ratio (length/width) 1.5714

Shear Traction $\quad 0.0$

OK

Cancel

Figure 327: Traffic Data for Run 3.7 


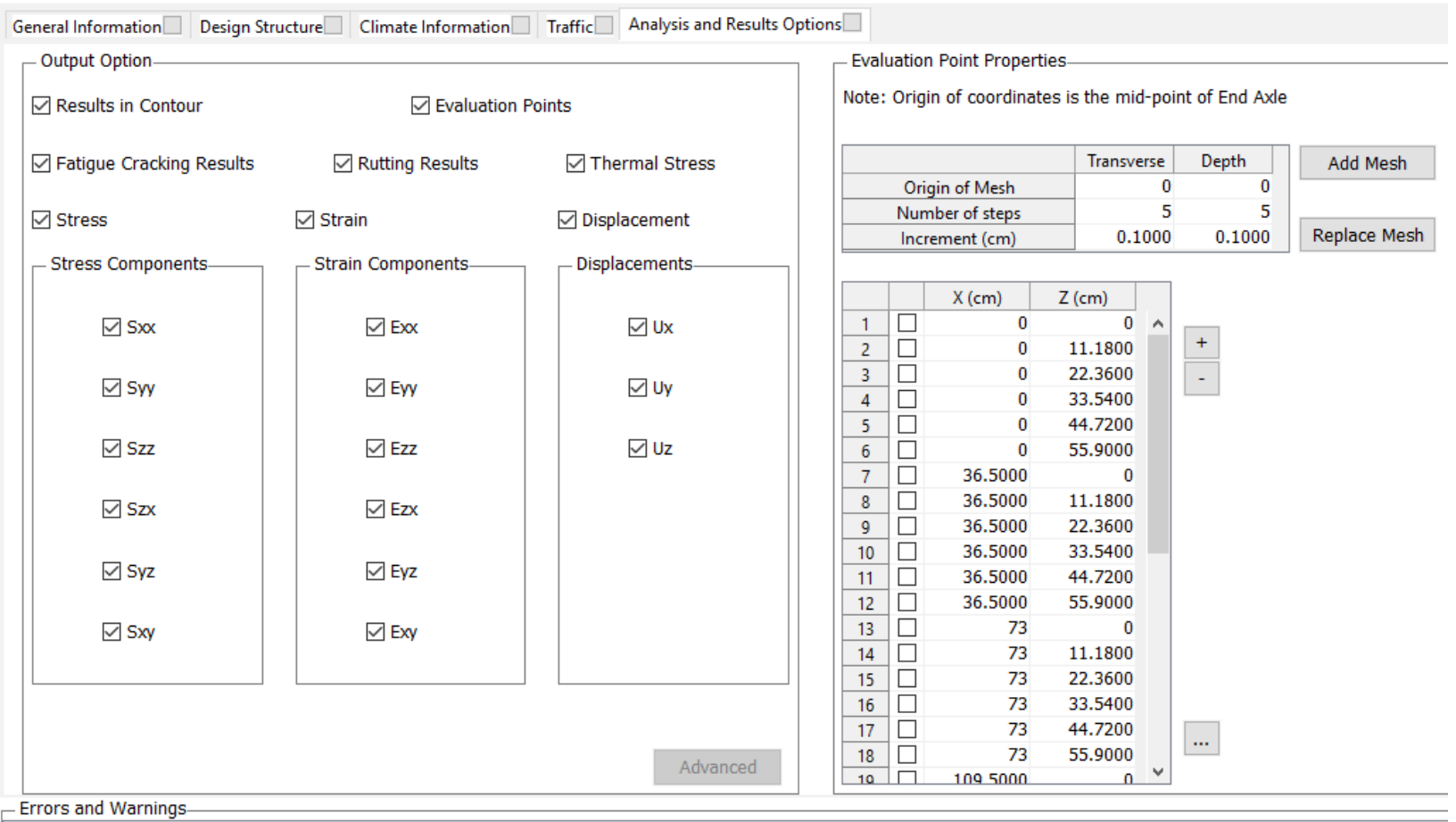

Figure 328: Output and Analysis Options for Run 3.7

\section{Run 3.8}

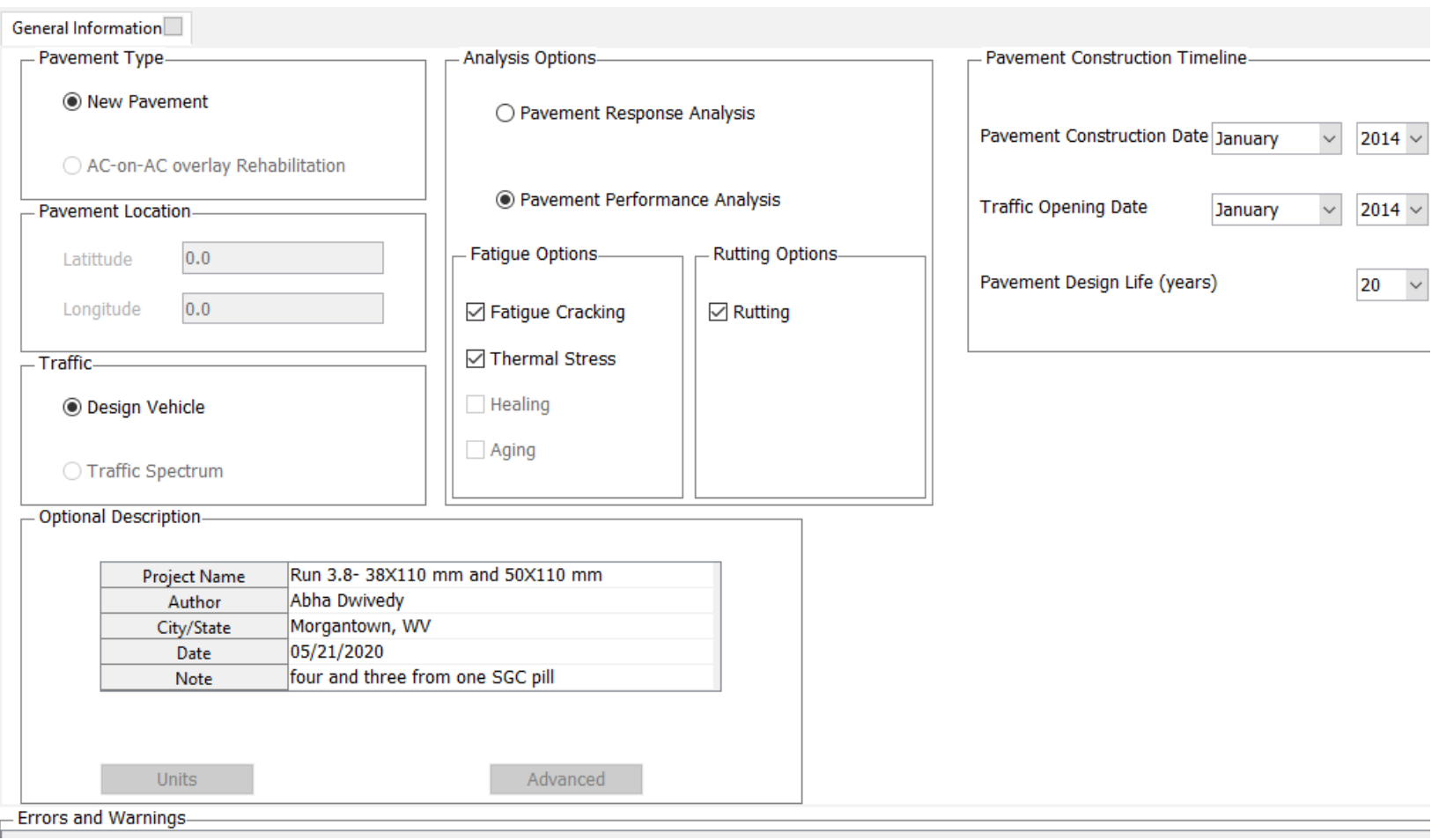

Figure 329: General Information for Run 3.8 

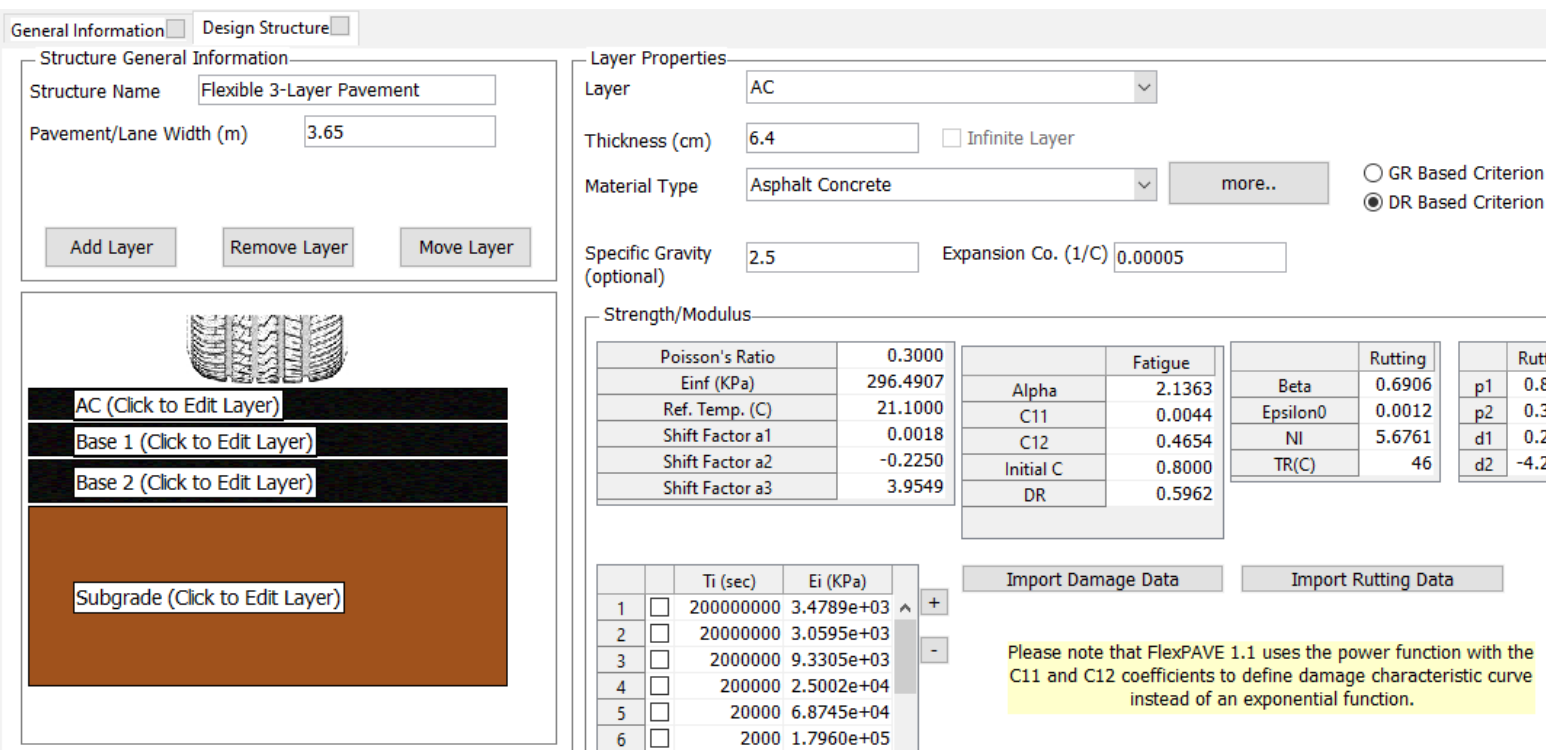

\begin{tabular}{|c|c|c|}
\hline \multirow{2}{*}{$\begin{array}{r}0.3000 \\
296.4907\end{array}$} & & Fatigue \\
\hline & Alpha & 2.1363 \\
\hline \multirow{2}{*}{$\begin{array}{r}21.1000 \\
0.0018\end{array}$} & $\mathrm{C} 11$ & 0.0044 \\
\hline & $\mathrm{C} 12$ & 0.4654 \\
\hline \multirow{2}{*}{$\begin{array}{r}-0.2250 \\
3.9549\end{array}$} & Initial C & 0.8000 \\
\hline & DR & 0.5962 \\
\hline
\end{tabular}

Ti $(\mathrm{sec}) \quad \mathrm{Ei}(\mathrm{KPa})$ $2000000003.4789 \mathrm{e}+03$ ^ + $200000003.0595 \mathrm{e}+03$ $20000009.3305 \mathrm{e}+03$ $2000002.5002 \mathrm{e}+04$ $200006.8745 \mathrm{e}+04$ $2000 \quad 1.7960 \mathrm{e}+05$ $2004.2113 e+05$

Errors and Warning

\section{Figure 330: Design Structure of AC Layer for Run 3.8}

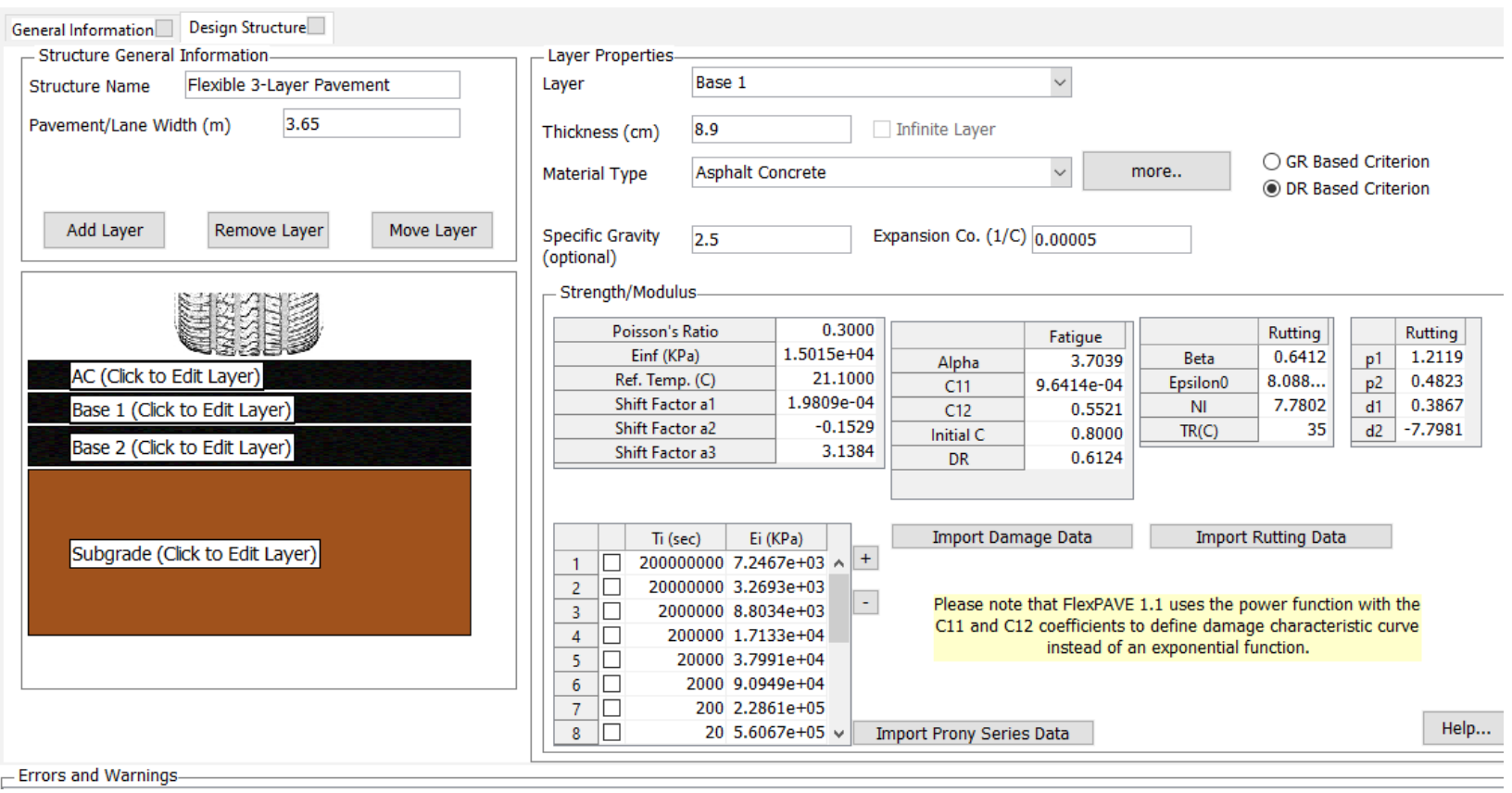

Figure 331: Design Structure of Base 1 Layer for Run 3.8 


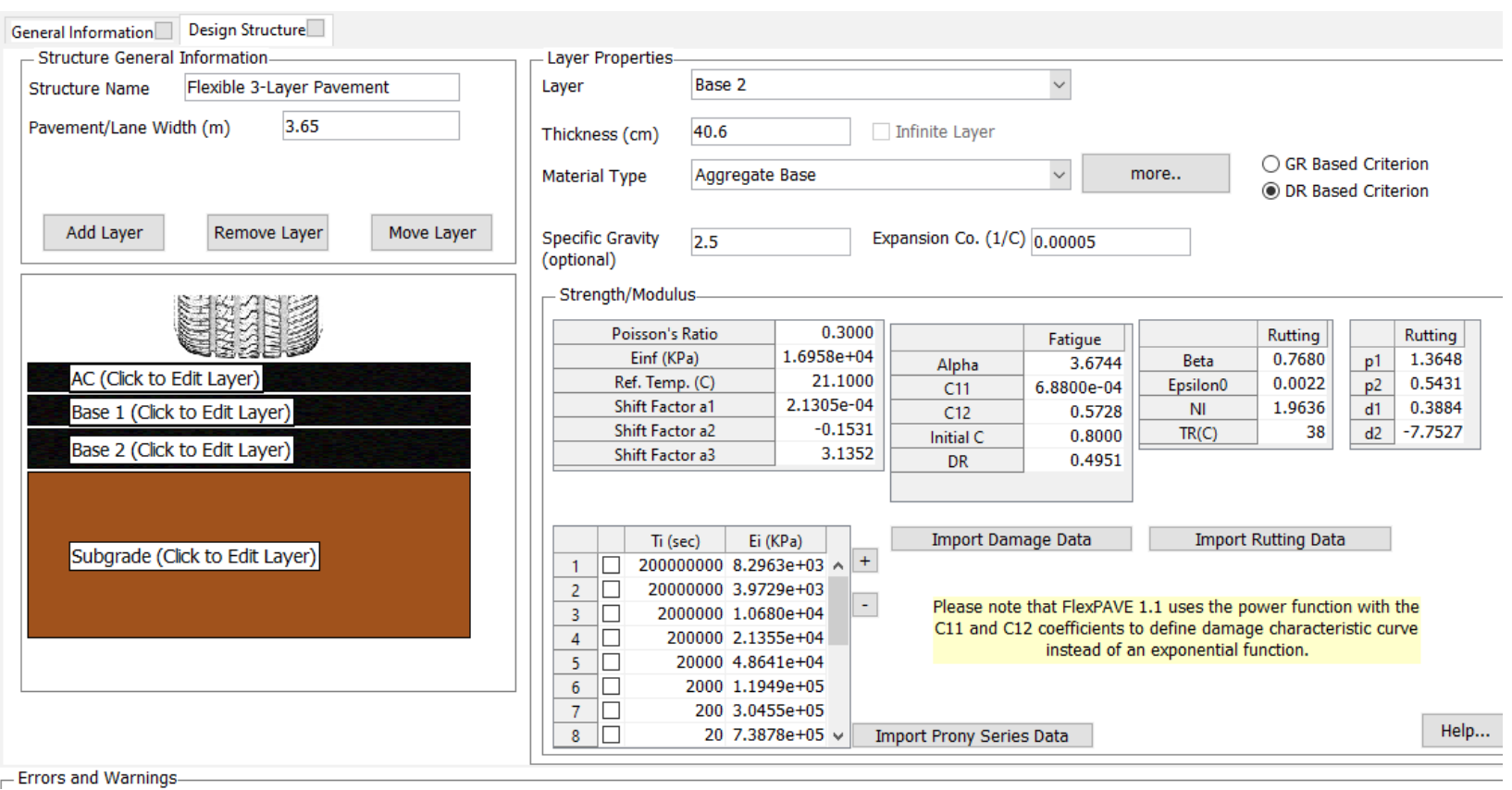

Figure 332: Design Structure of Base 2 Layer for Run 3.8

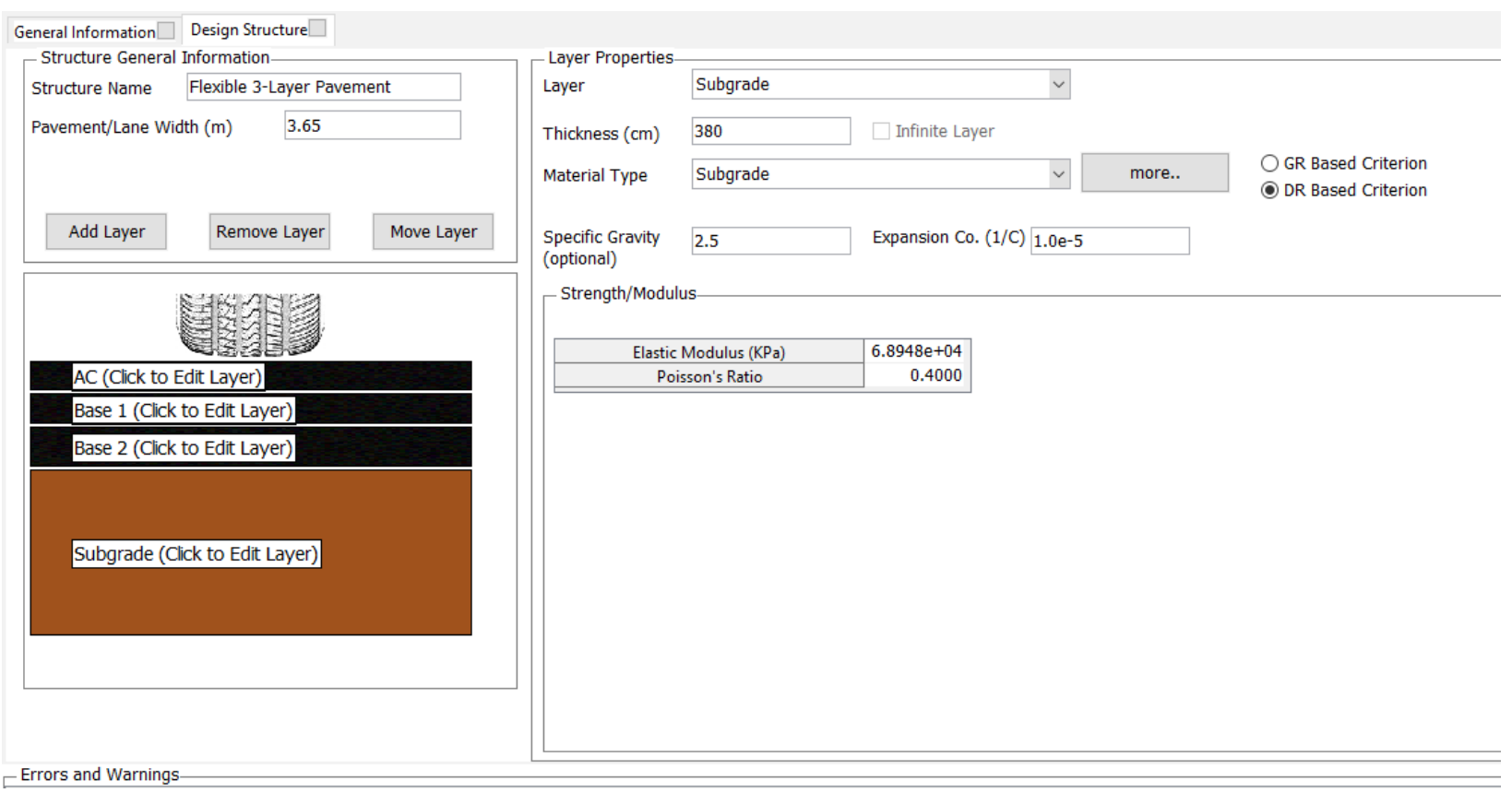

Figure 333: Design Structure of Subgrade Layer for Run 3.8 


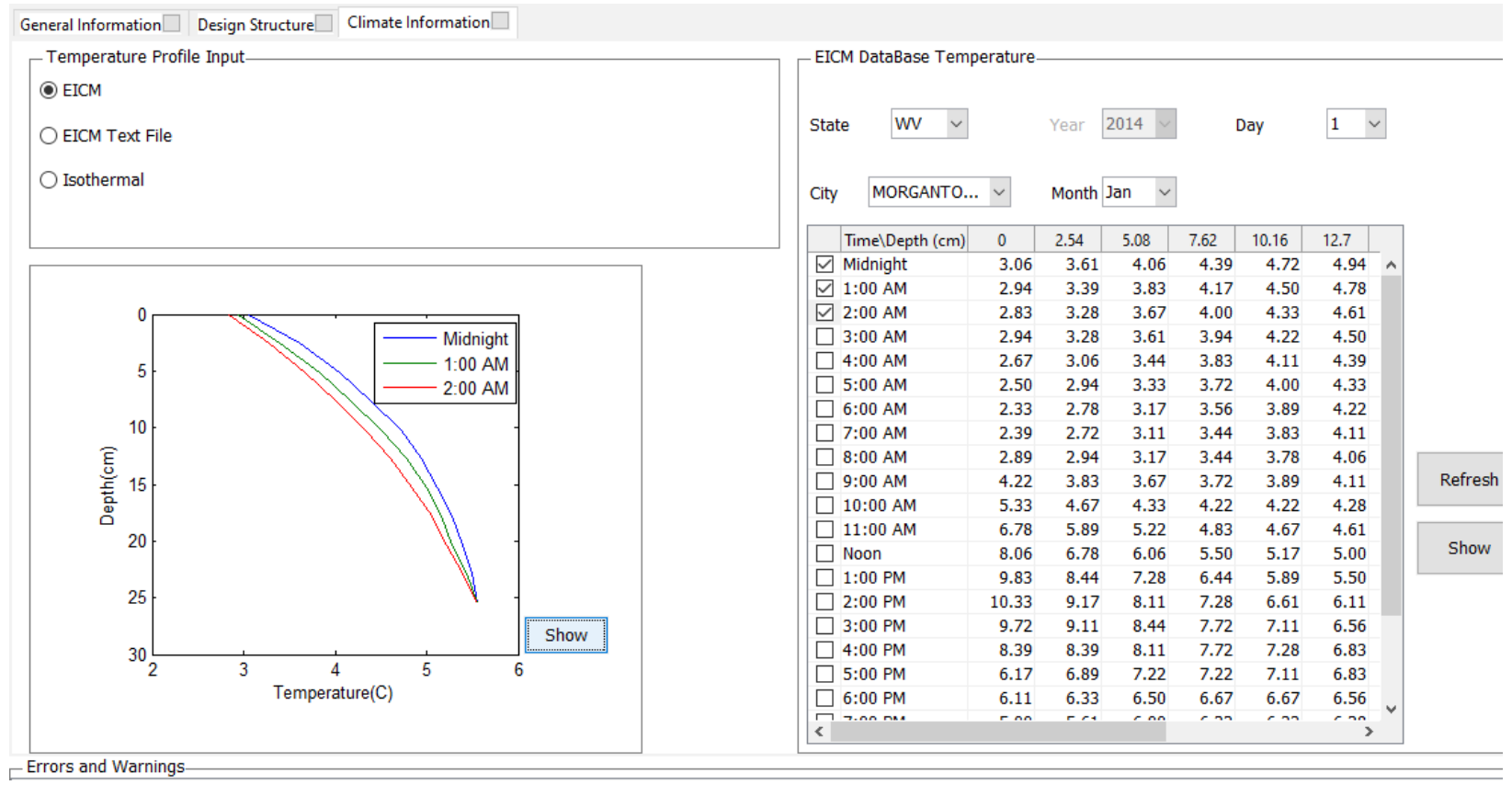

Figure 334: Climate Data for Run 3.8 


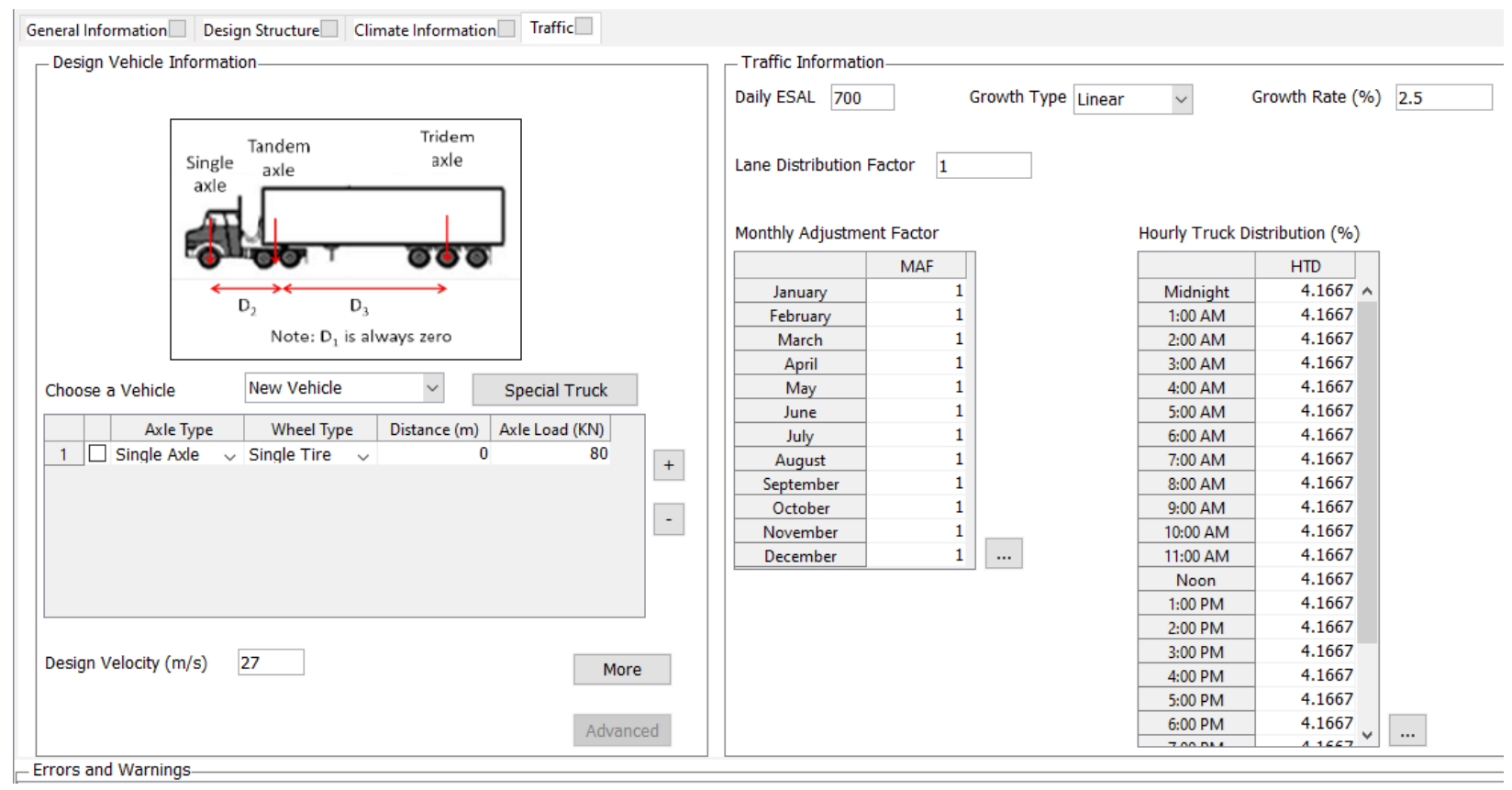

Axle Configuration

$-\square \times$

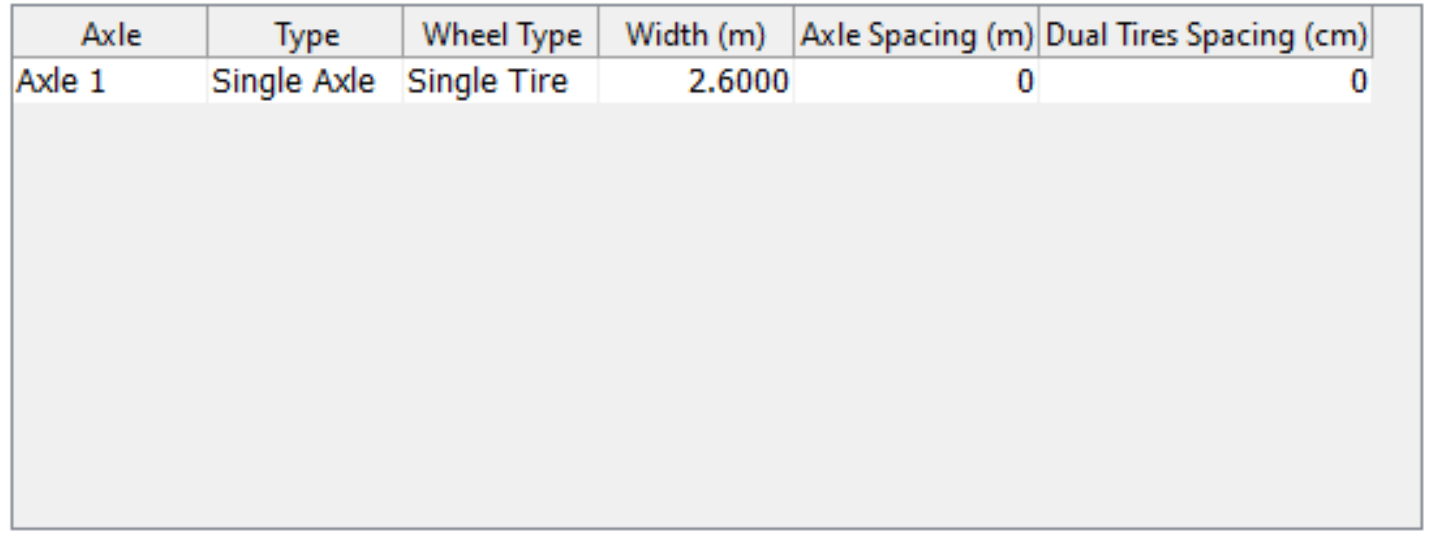

Tire Pressure (KPa) 827.37

Contact Area Shape Rectangular $\vee \quad$ Aspect Ratio (length/width) 1.5714

Shear Traction $\quad 0.0$

OK

Cancel

Figure 335: Traffic Data for Run 3.8 


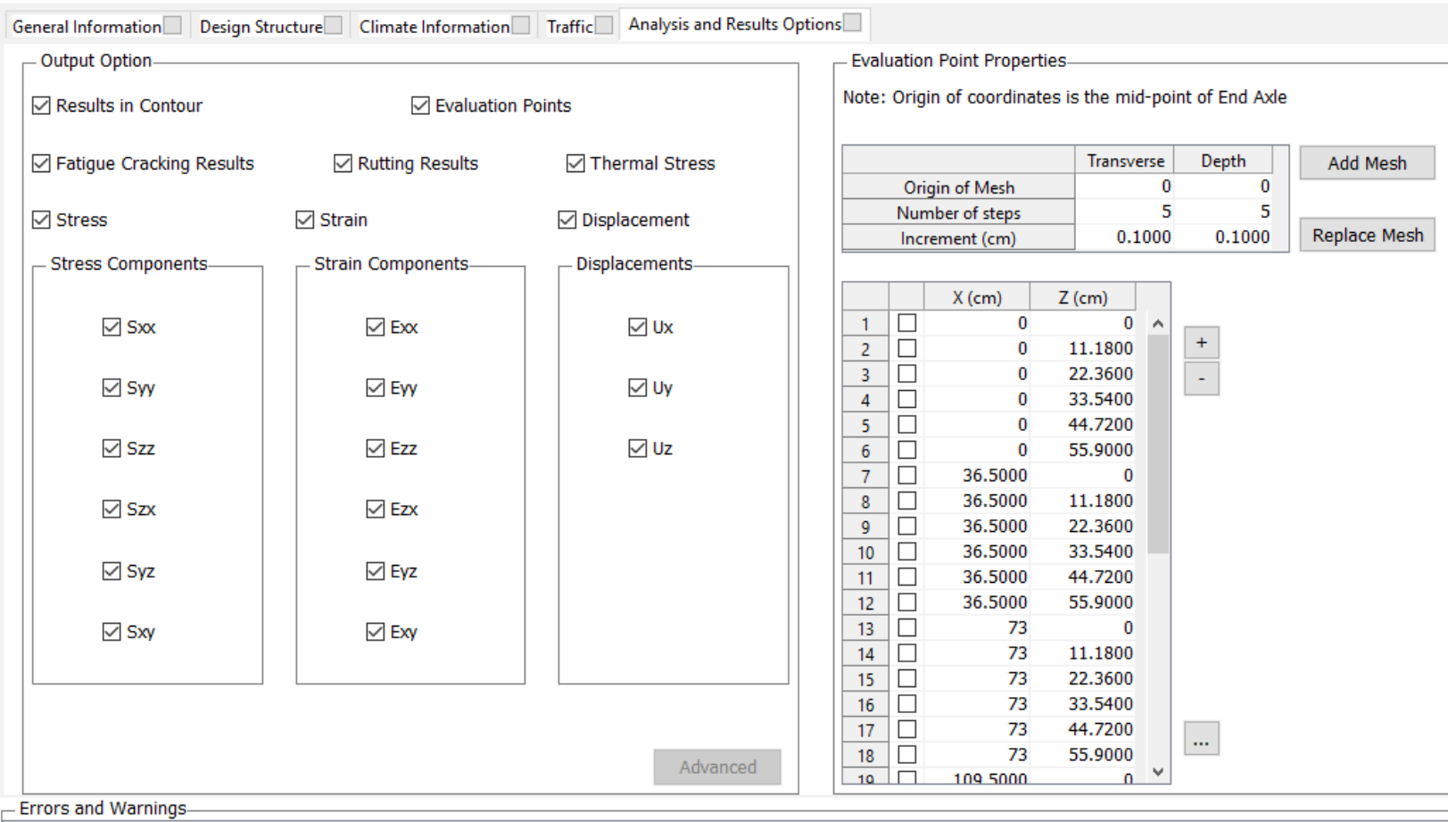

Figure 336: Output and Analysis Options for Run 3.8

\section{Run 3.9}

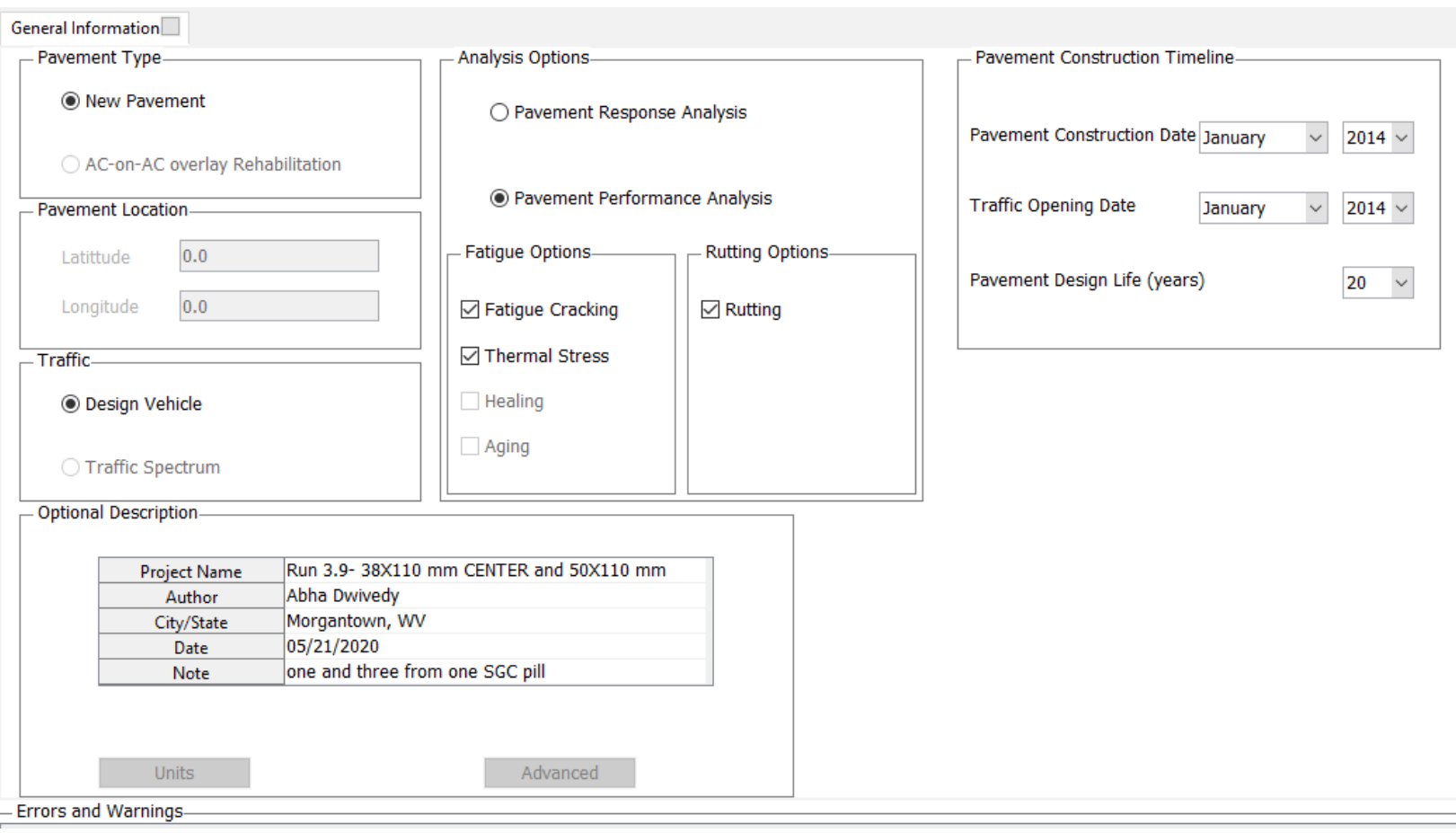

Figure 337: General Information for Run 3.9 


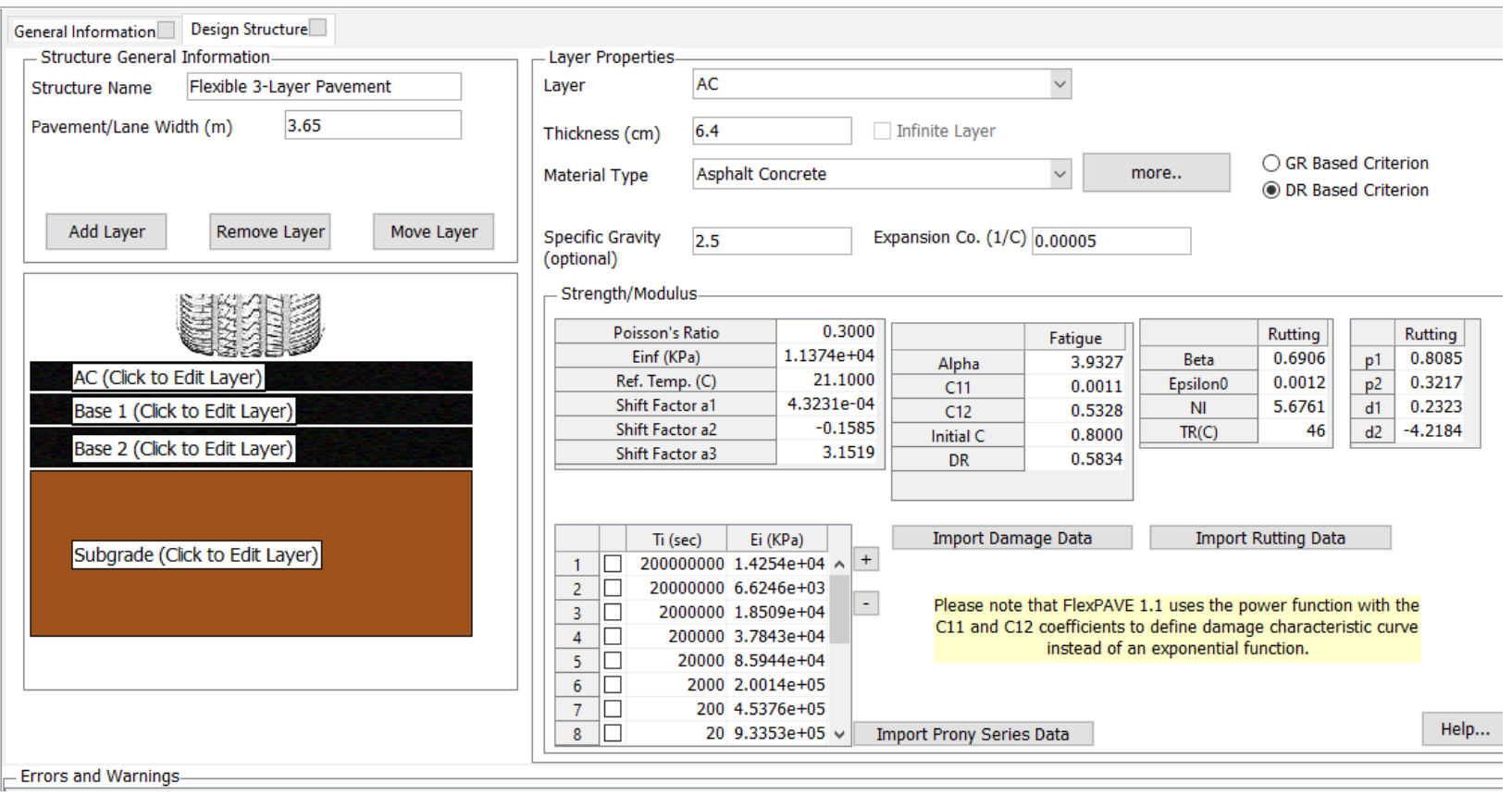

Figure 338: Design Structure of AC Layer for Run 3.9

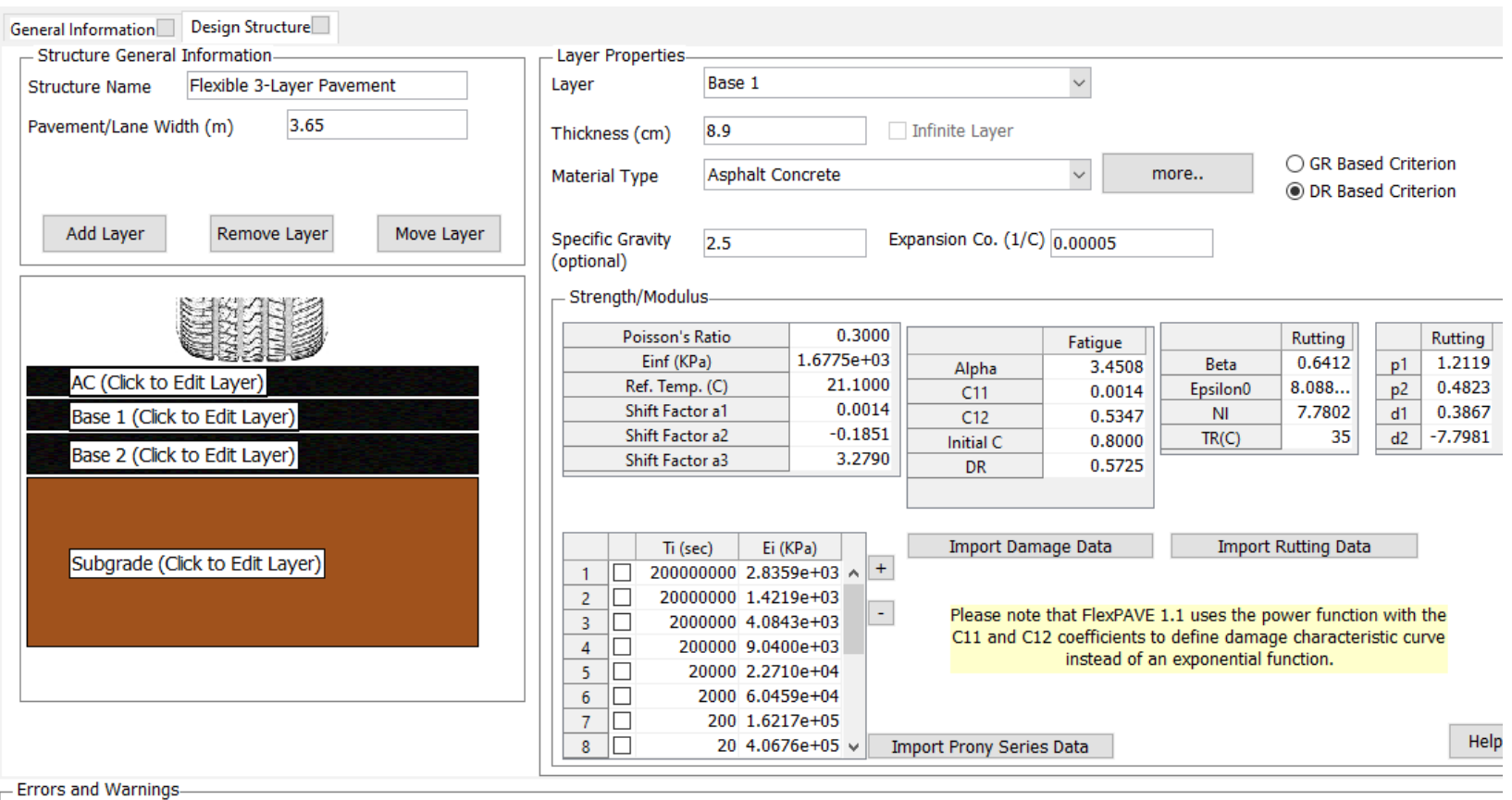

Figure 339: Design Structure of Base 1 Layer for Run 3.9 


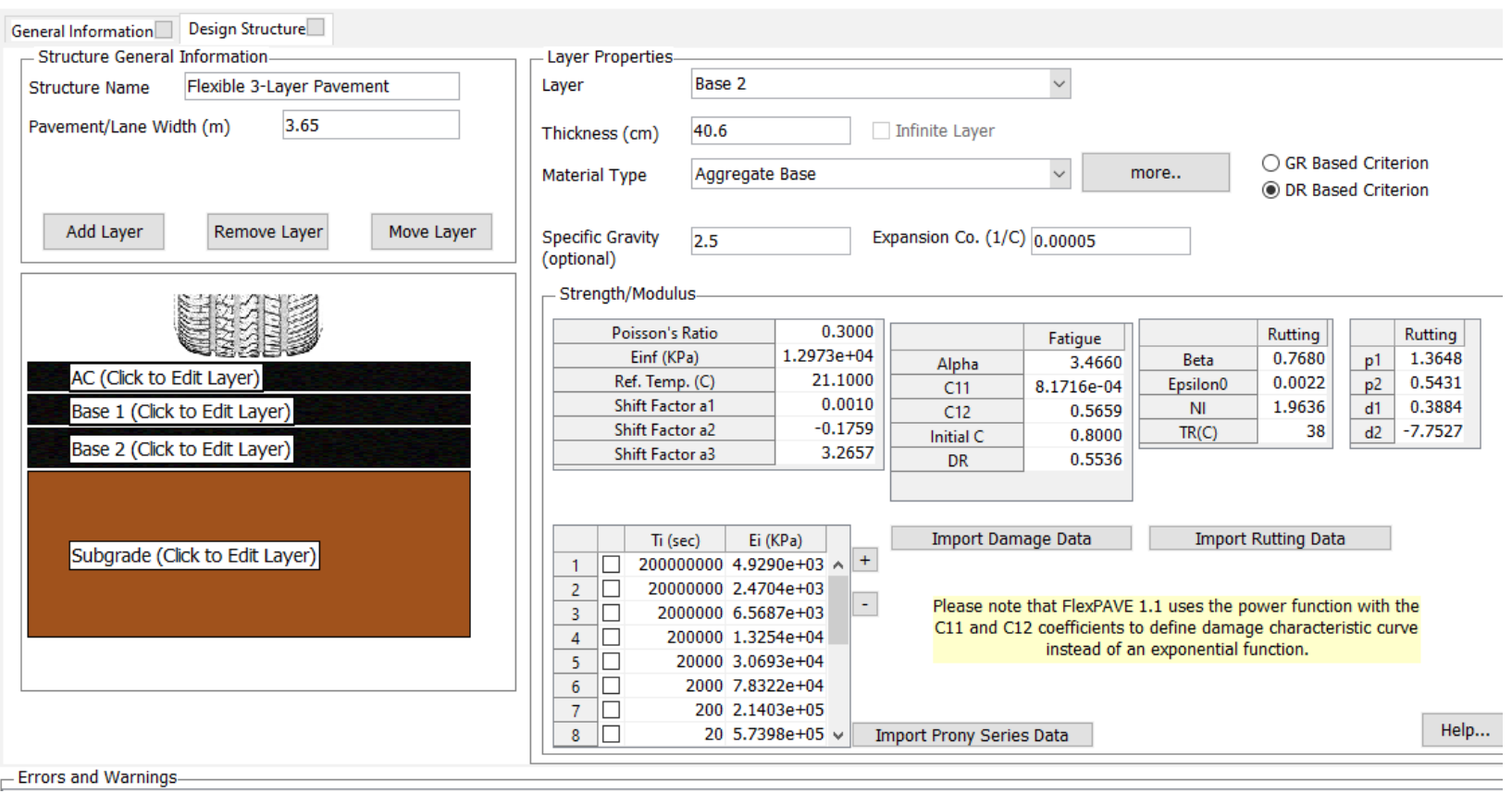

Figure 340: Design Structure of Base 2 Layer for Run 3.9

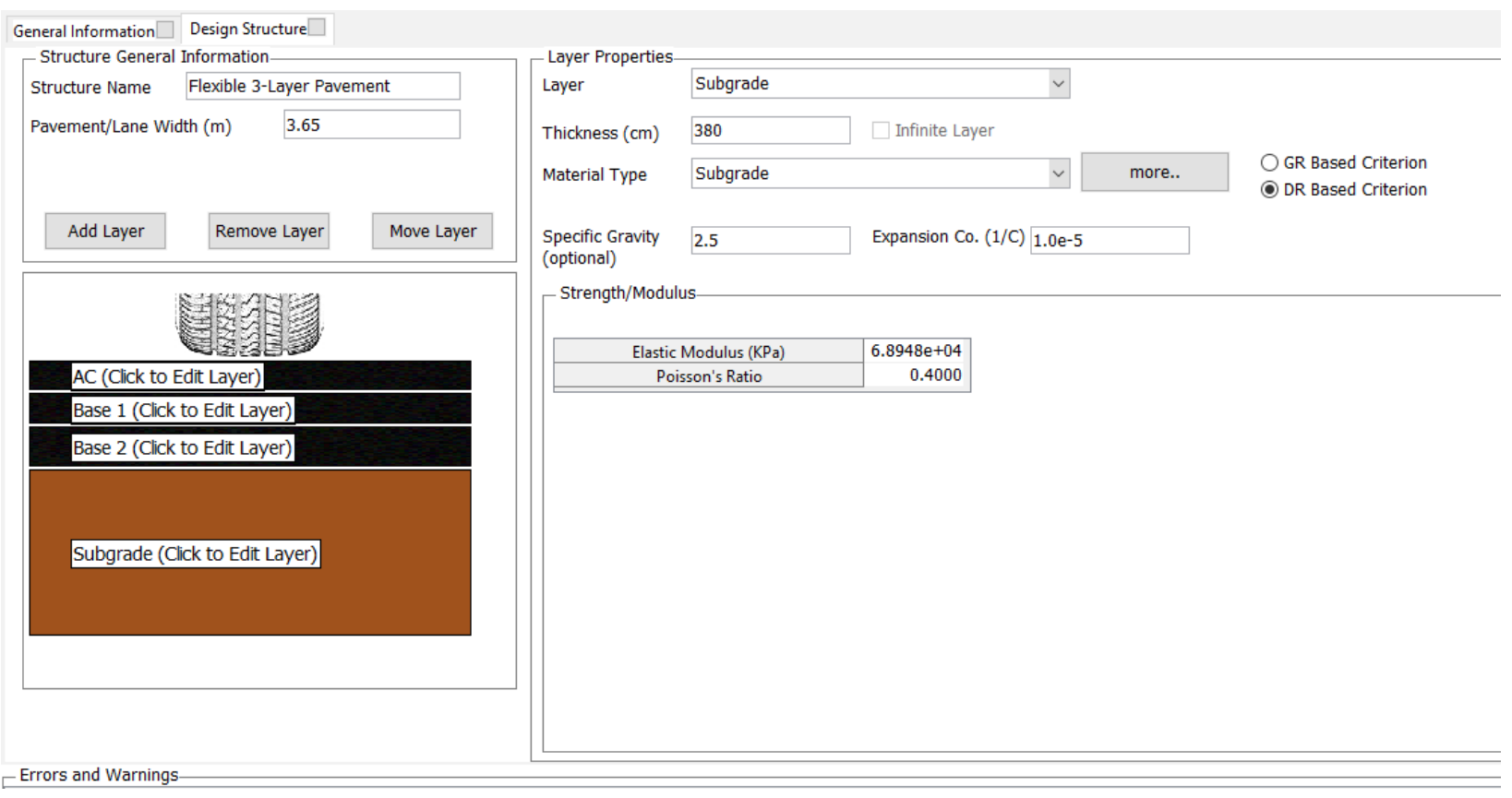

Figure 341: Design Structure of Subgrade Layer for Run 3.9 


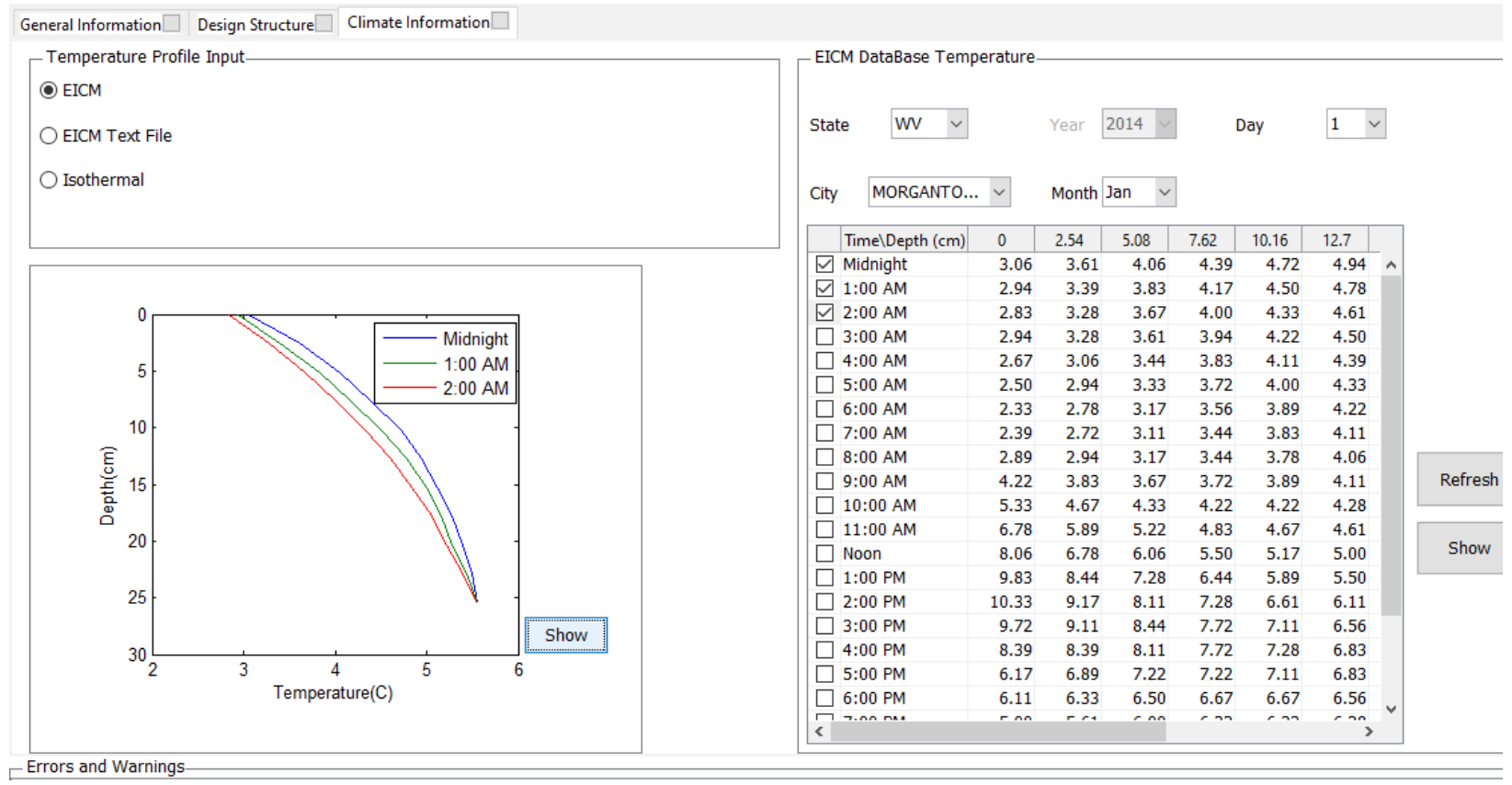

Figure 342: Climate Data for Run 3.9 


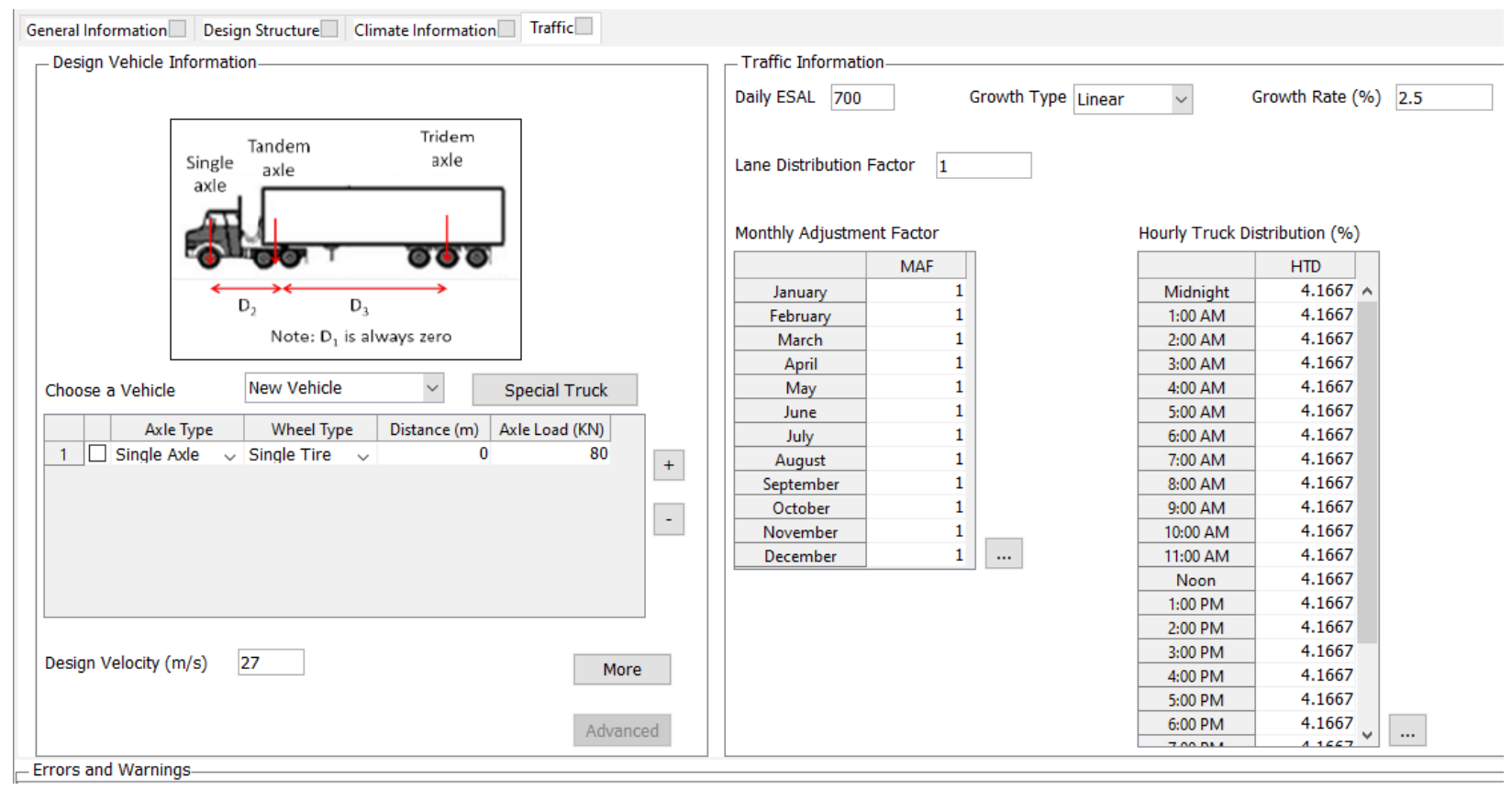

Axle Configuration

$-\square \times$

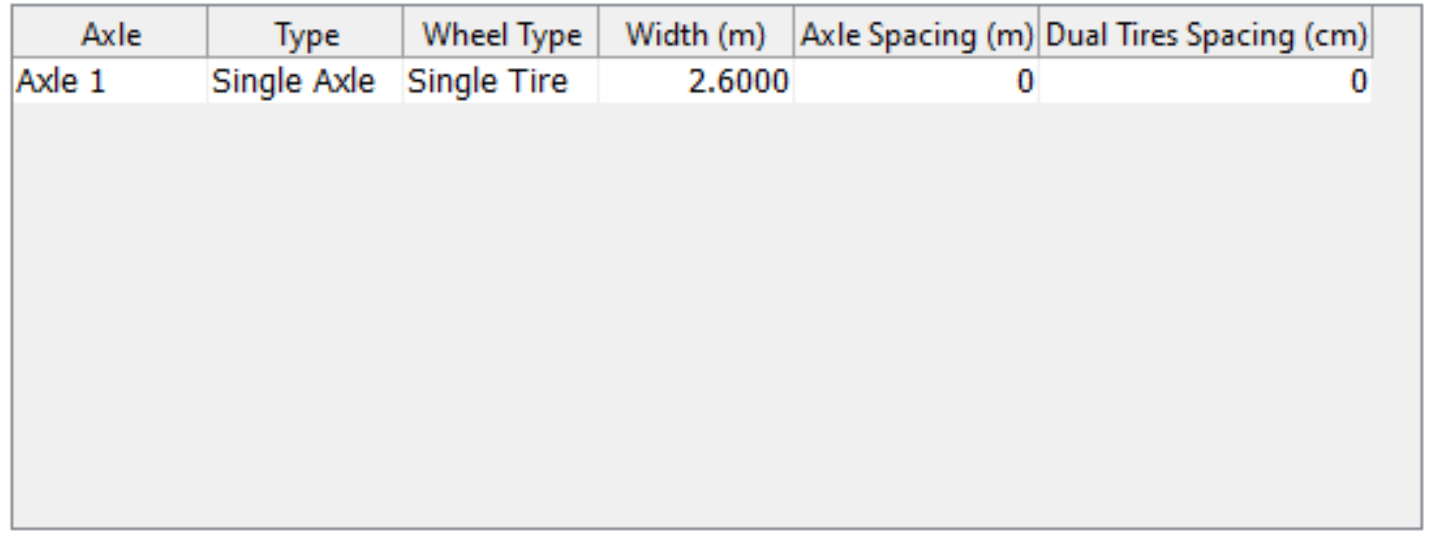

Tire Pressure (KPa) 827.37

Contact Area Shape Rectangular $\vee \quad$ Aspect Ratio (length/width) 1.5714

Shear Traction $\quad 0.0$

OK

Cancel

Figure 343: Traffic Data for Run 3.9 


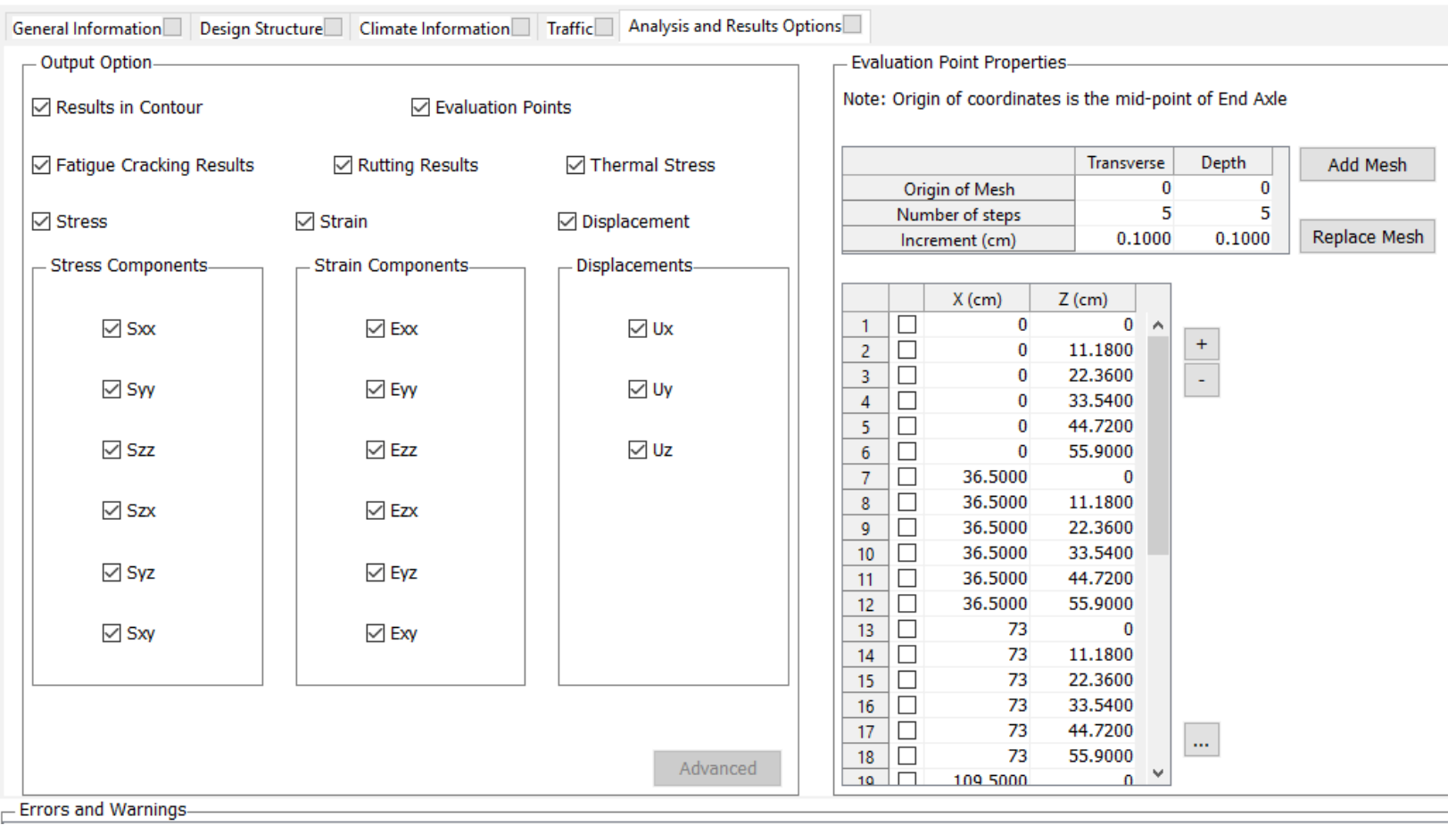

Figure 344: Output and Analysis Options for Run 3.9

\section{Run 3.10}

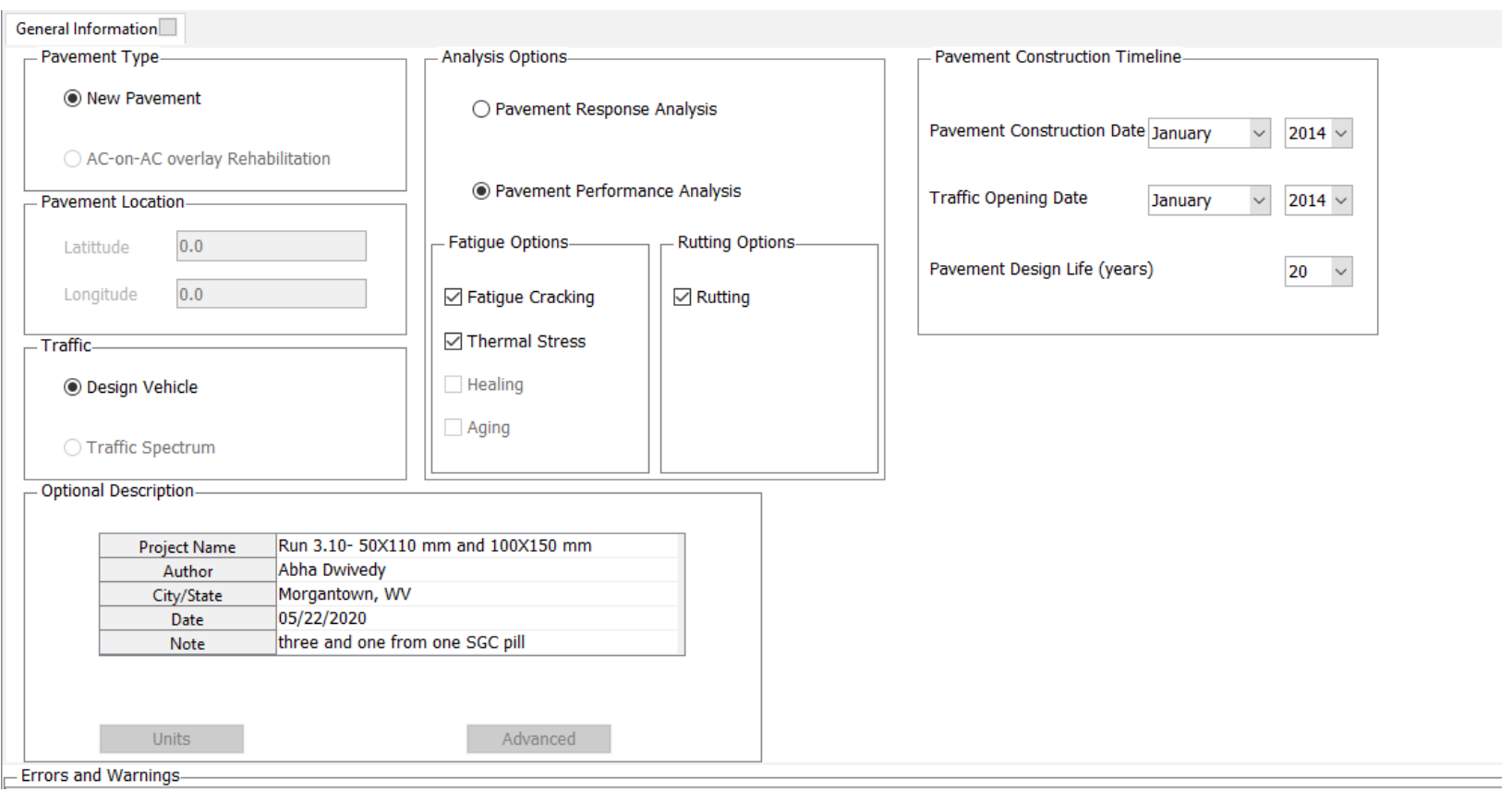

Figure 345: General Information for Run 3.10 

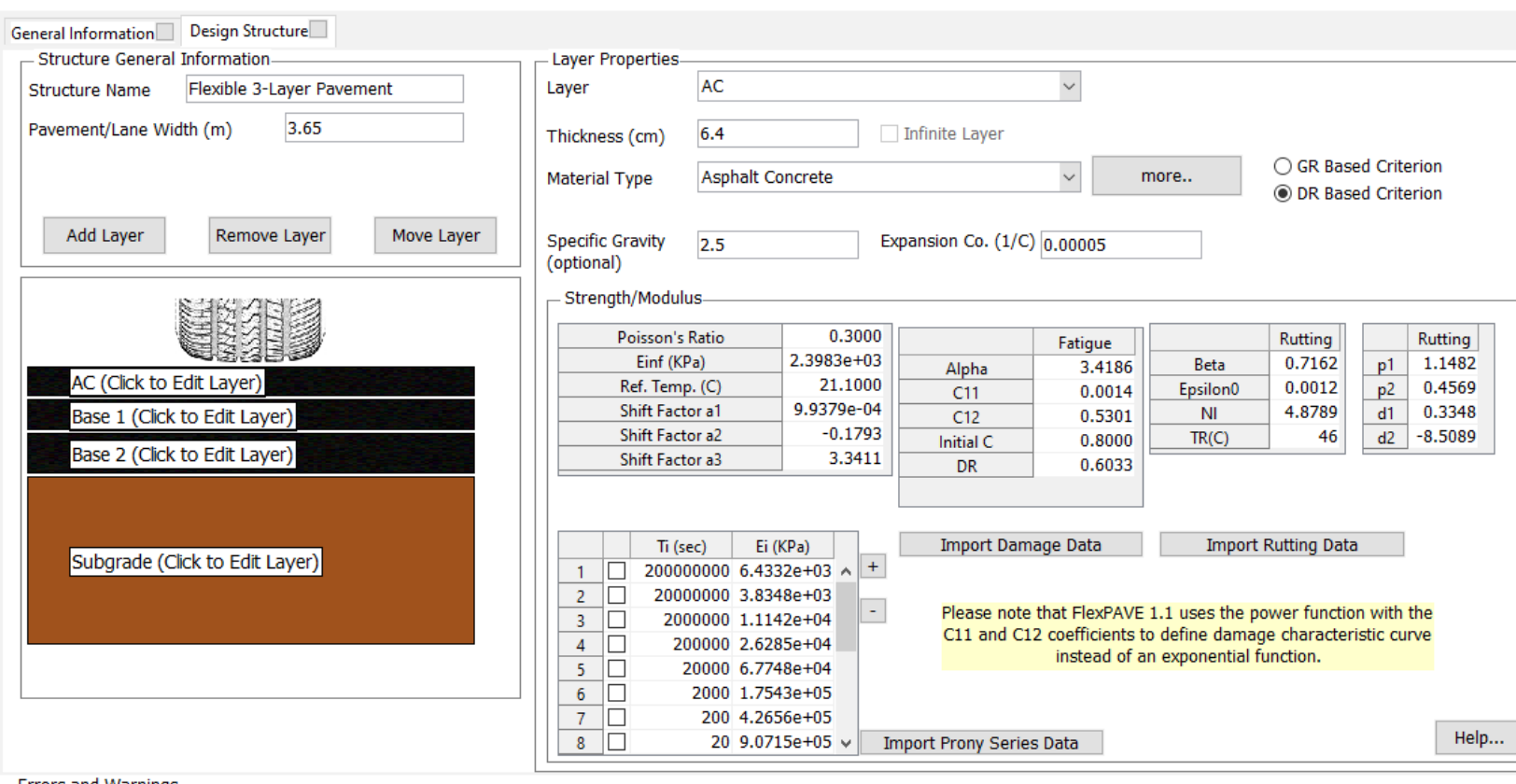

Errors and Warnings-

Figure 346: Design Structure of AC Layer for Run 3.10

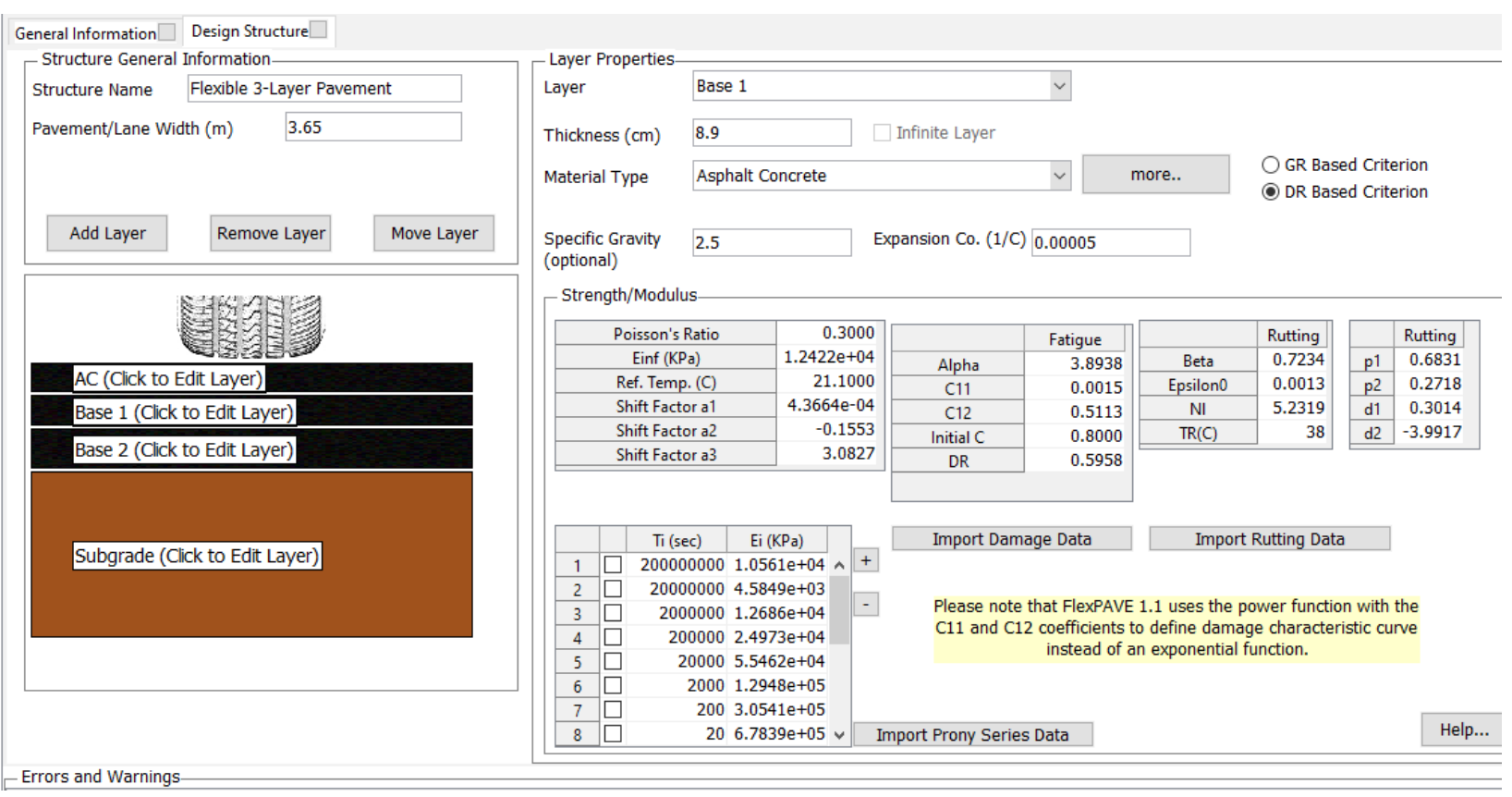

Figure 347: Design Structure of Base 1 Layer for Run 3.10 


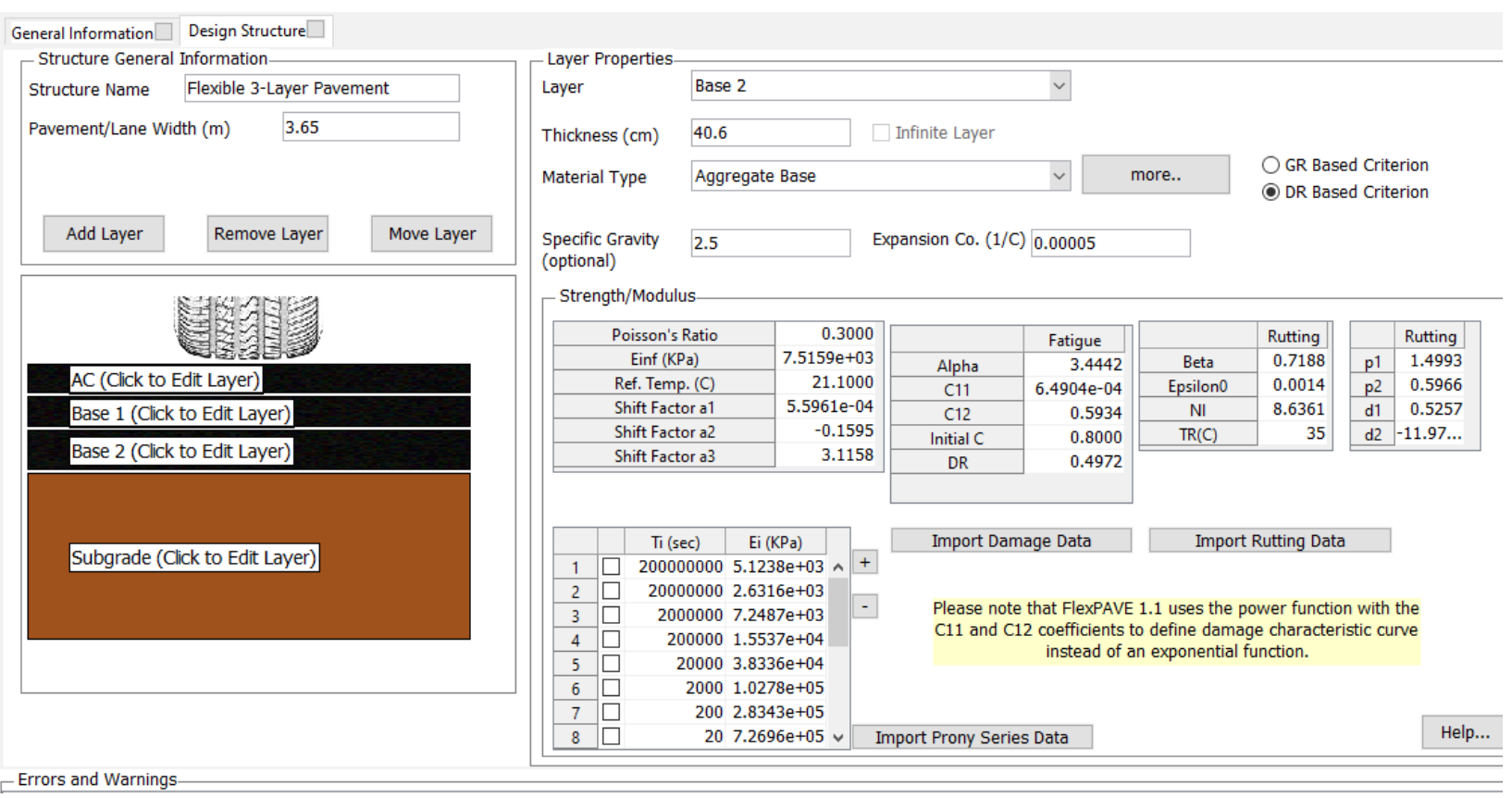

Figure 348: Design Structure of Base 2 Layer for Run 3.10

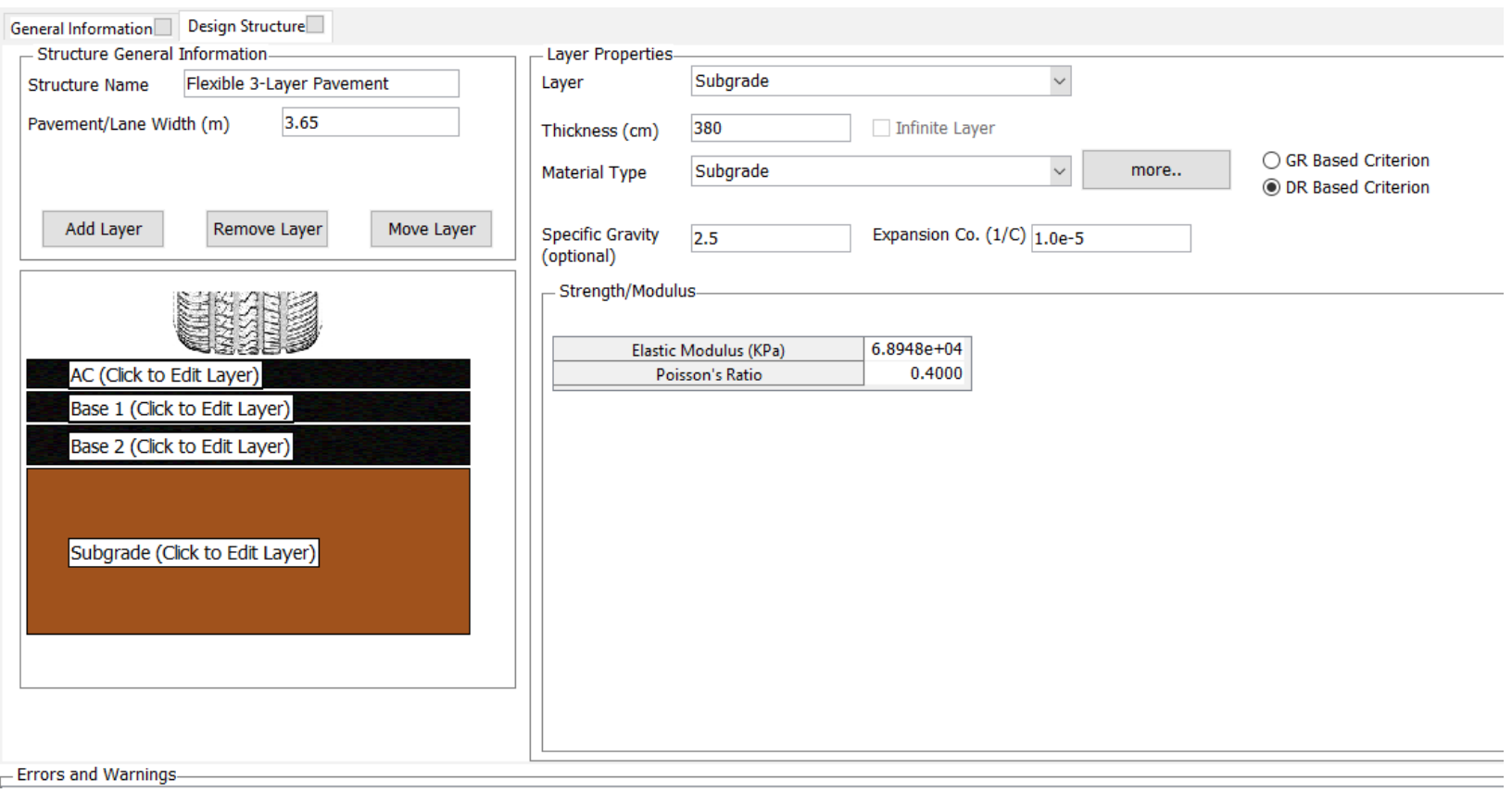

Figure 349: Design Structure of Subgrade Layer for Run 3.10 


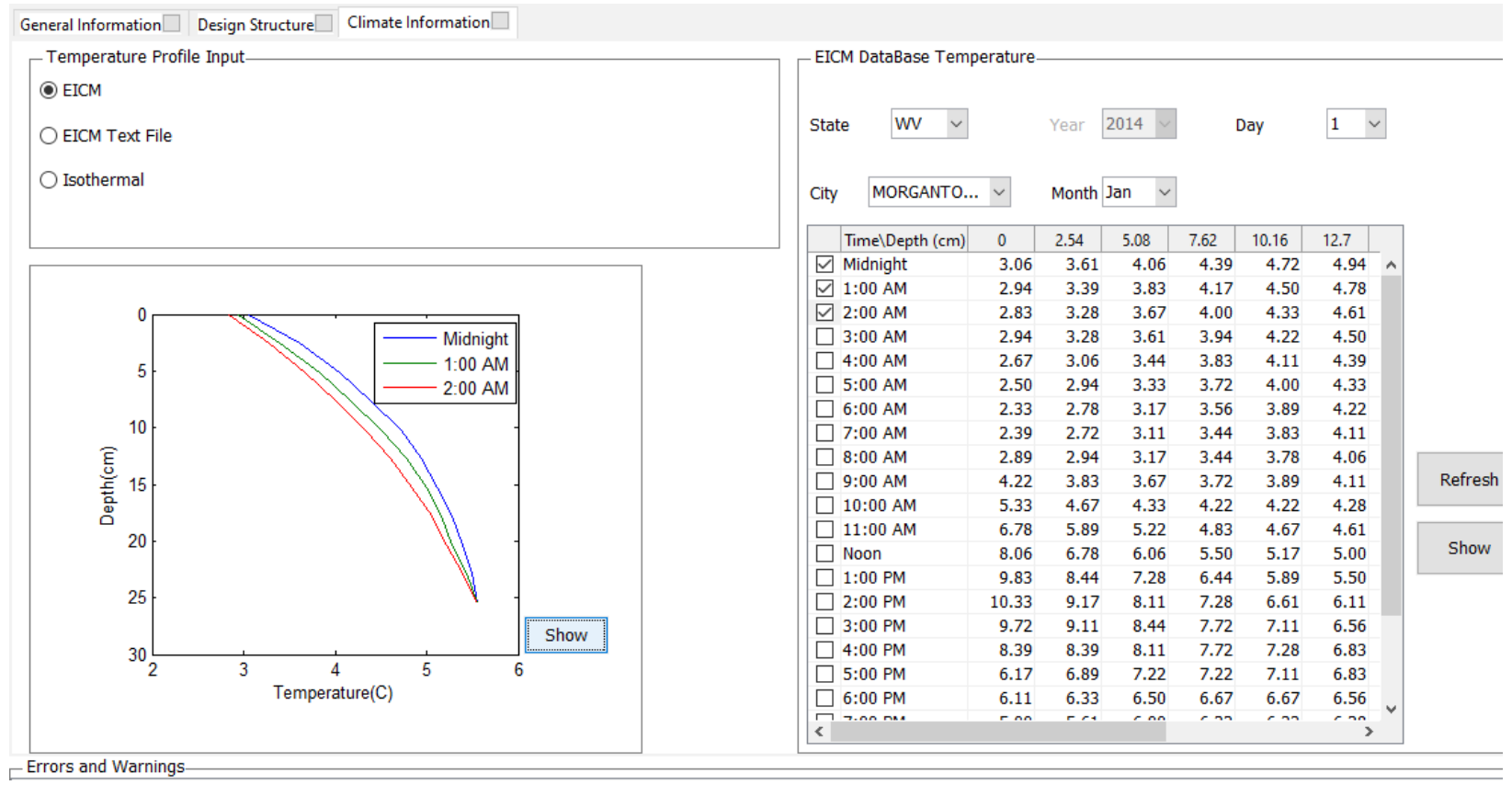

Figure 350: Climate Data for Run 3.10 


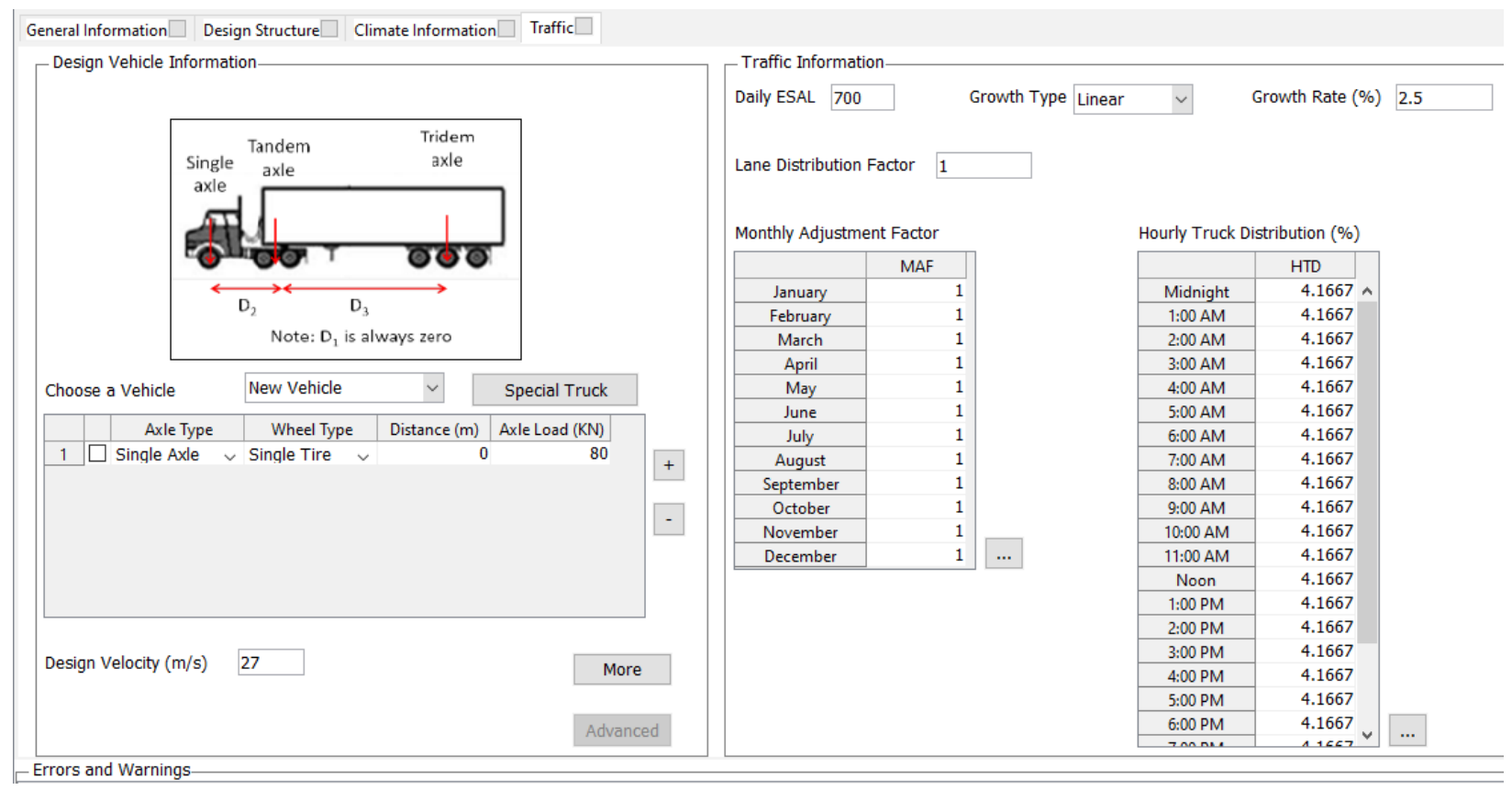

Axle Configuration

$-\square \times$

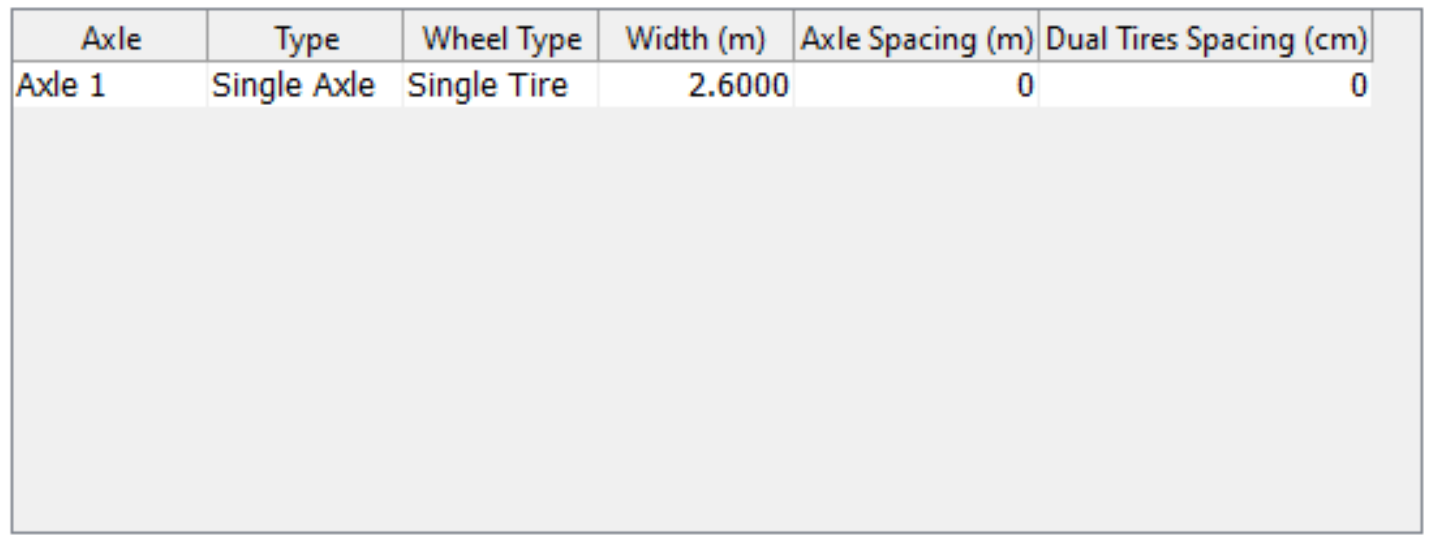

Tire Pressure (KPa) 827.37

Contact Area Shape Rectangular $\vee \quad$ Aspect Ratio (length/width) 1.5714

Shear Traction $\quad 0.0$

OK

Cancel

Figure 351: Traffic Data for Run 3.10 


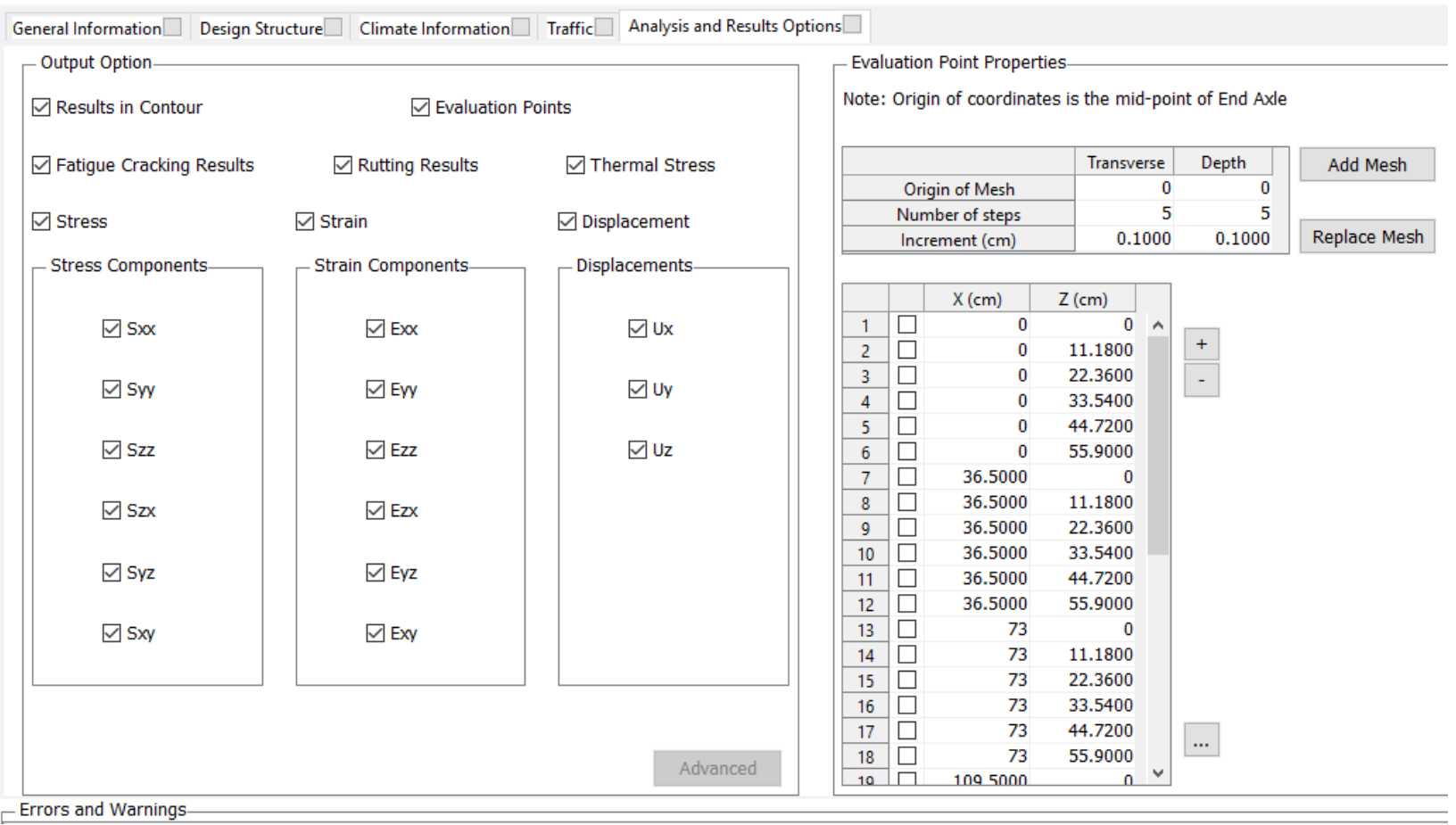

Figure 352: Output and Analysis Options for Run 3.10

\section{Run 3.11}

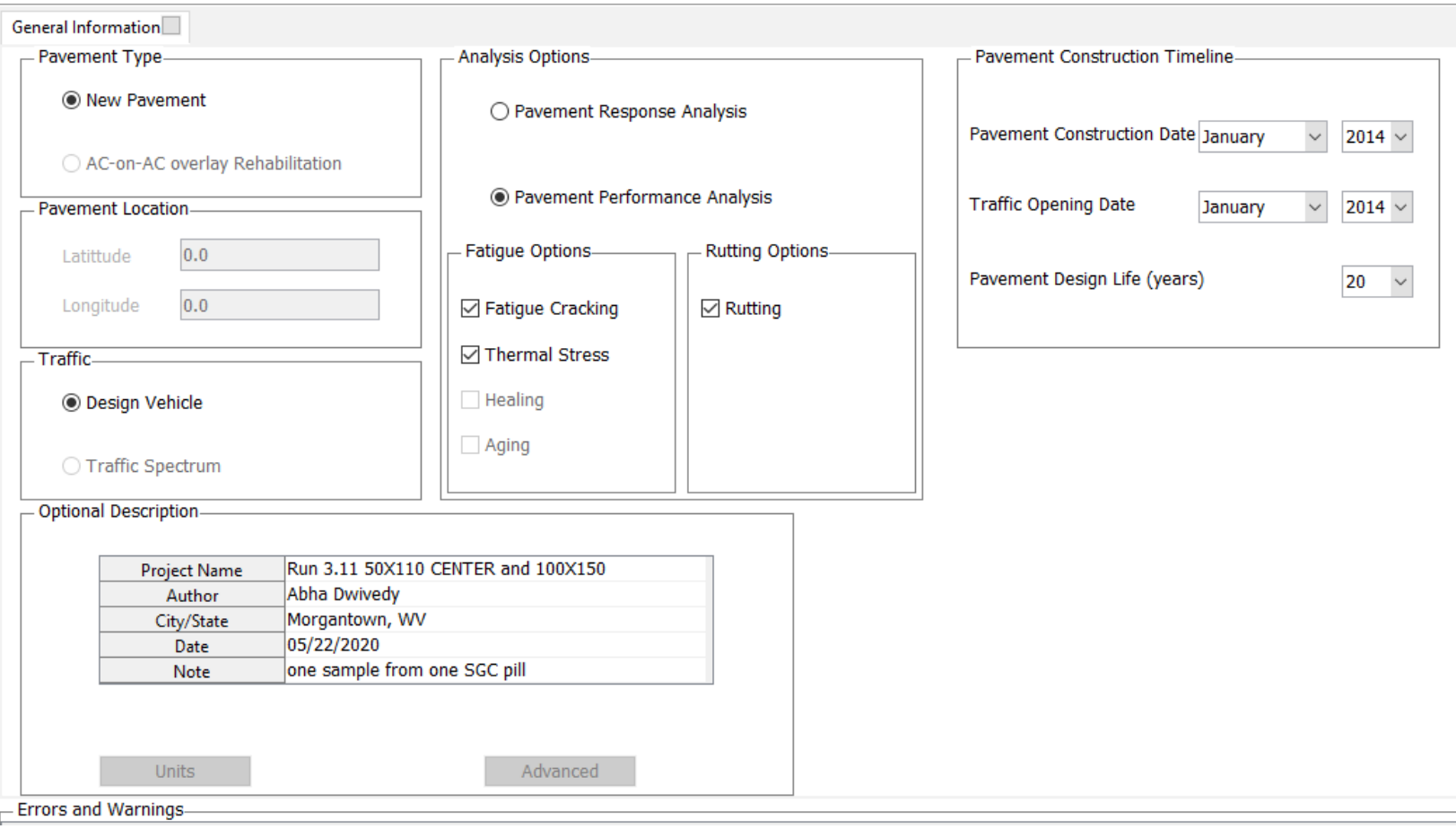

Figure 353: General Information for Run 3.11 


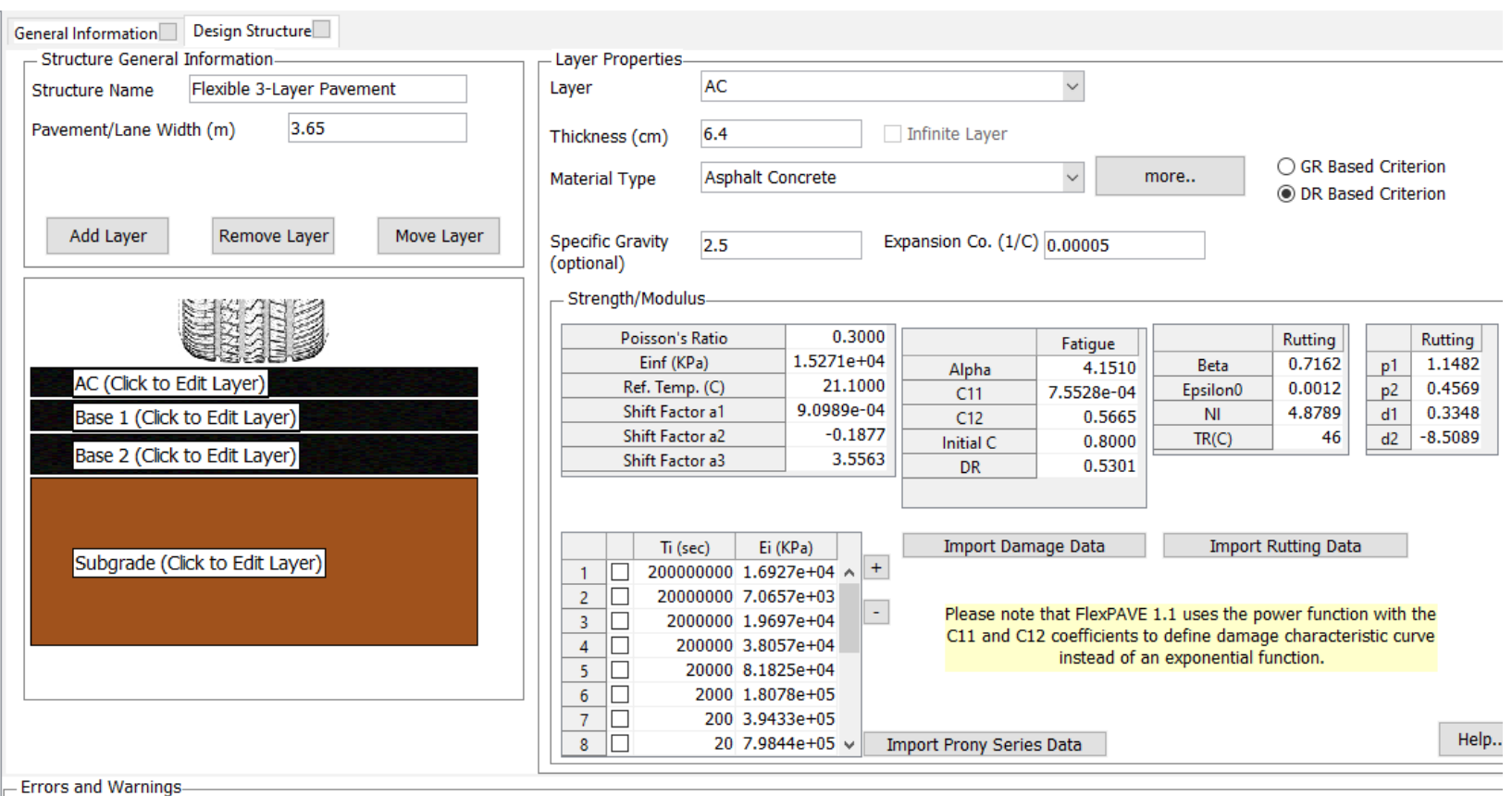

\section{Figure 354: Design Structure of AC Layer for Run 3.11}

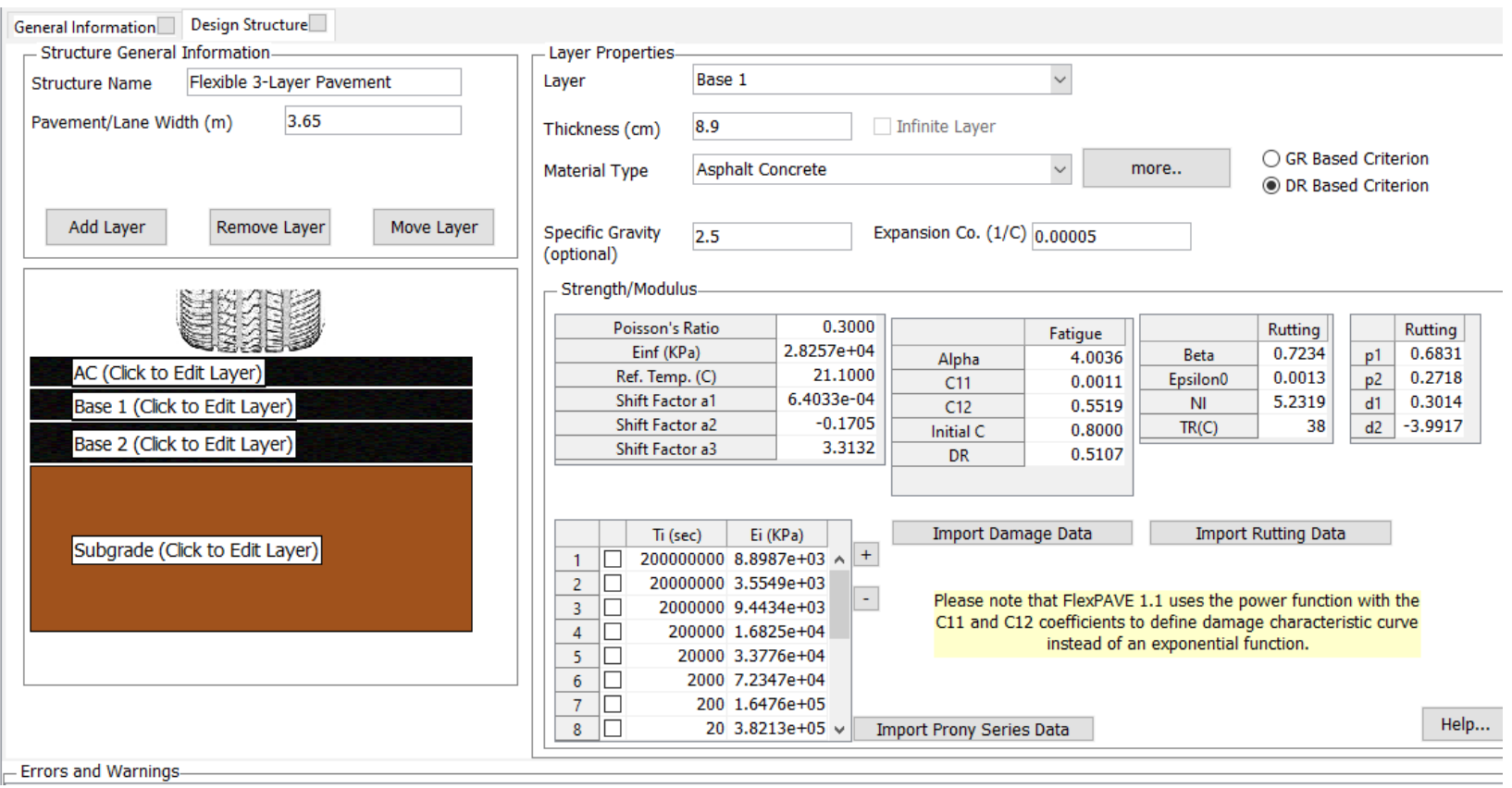

Figure 355: Design Structure of Base 1 Layer for Run 3.11 


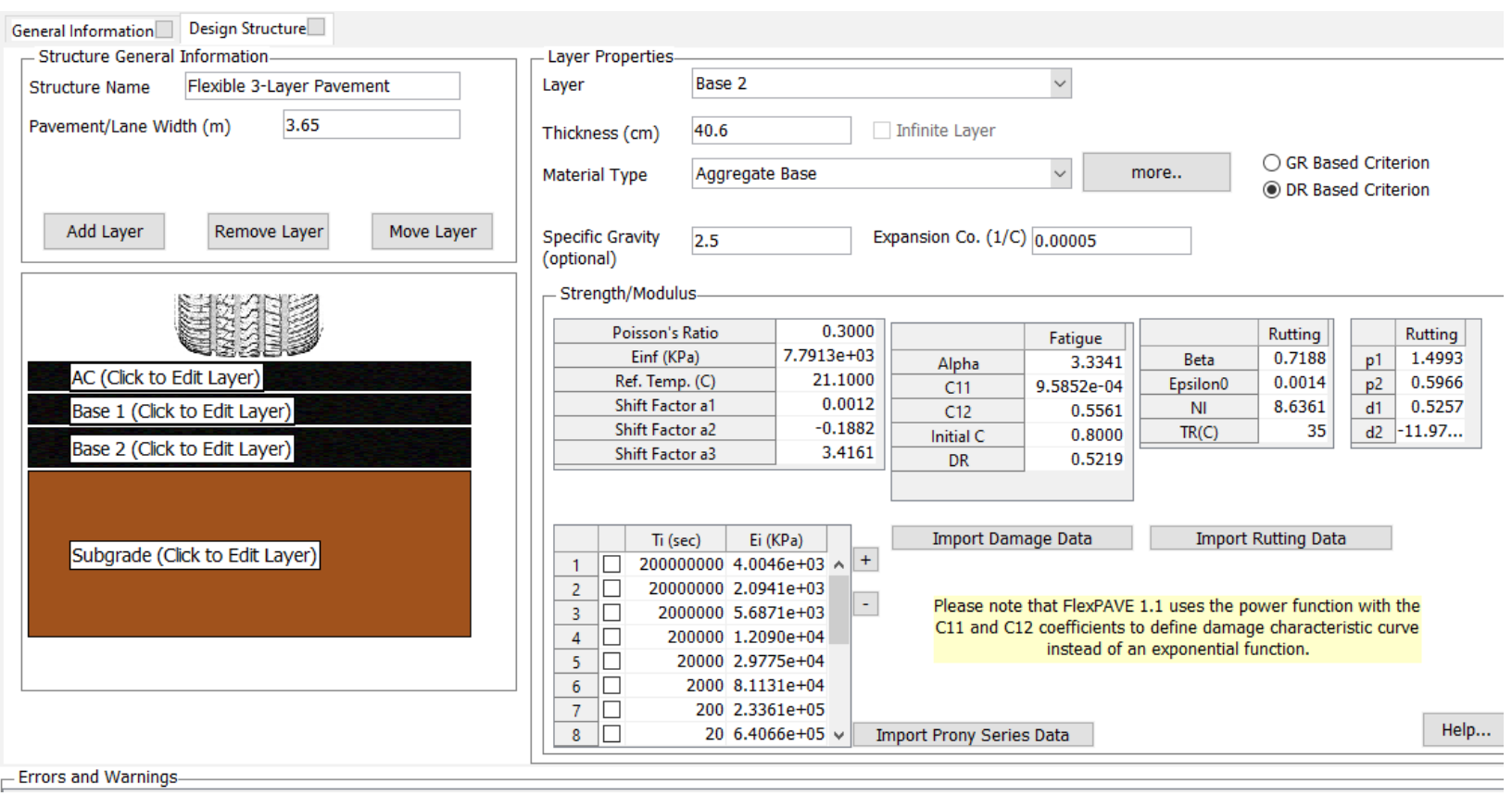

Figure 356: Design Structure of Base 2 Layer for Run 3.11

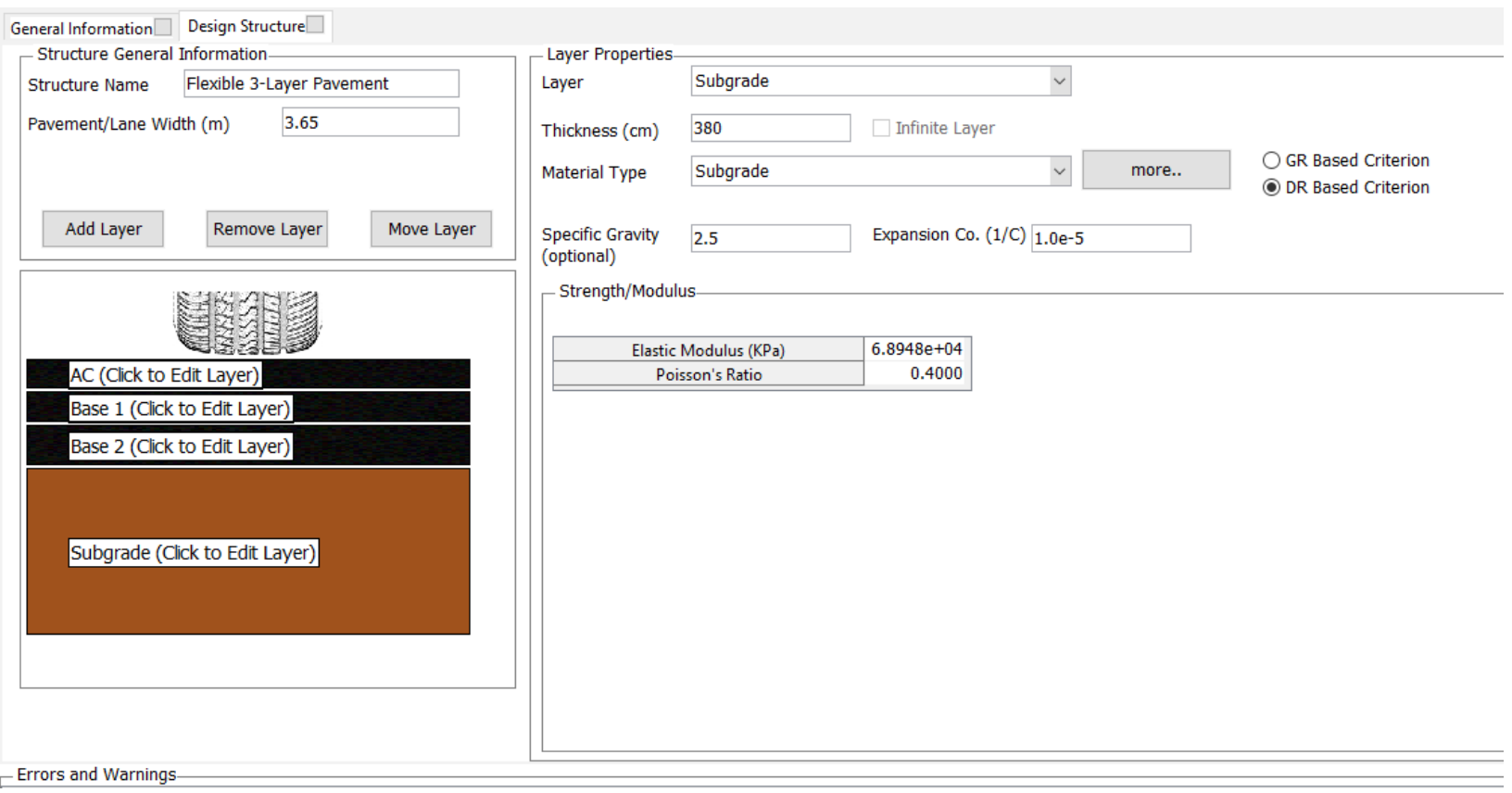

Figure 357: Design Structure of Subgrade Layer for Run 3.11 


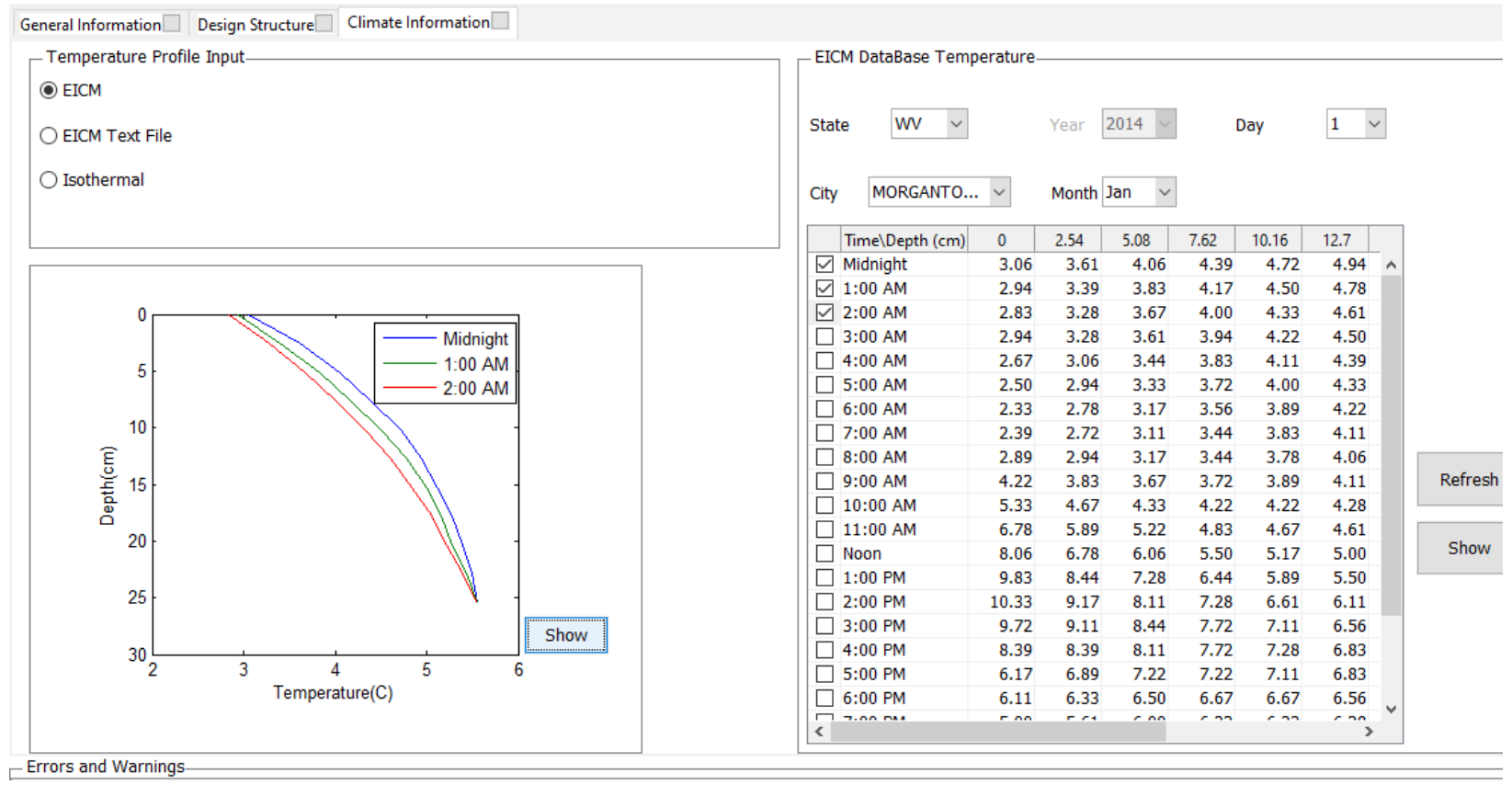

Figure 358: Climate Data for Run 3.11 


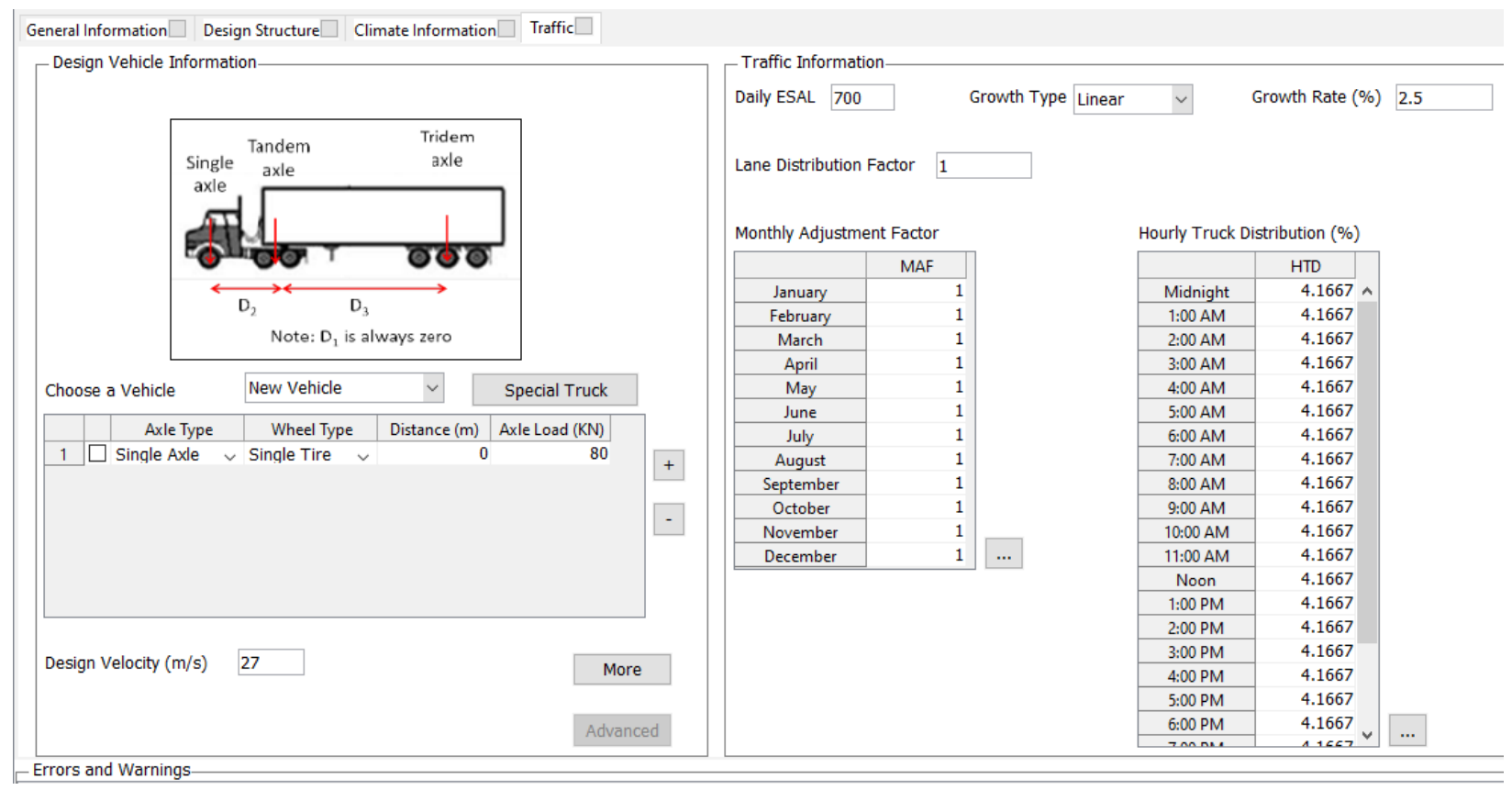

Axle Configuration

$-\square \times$

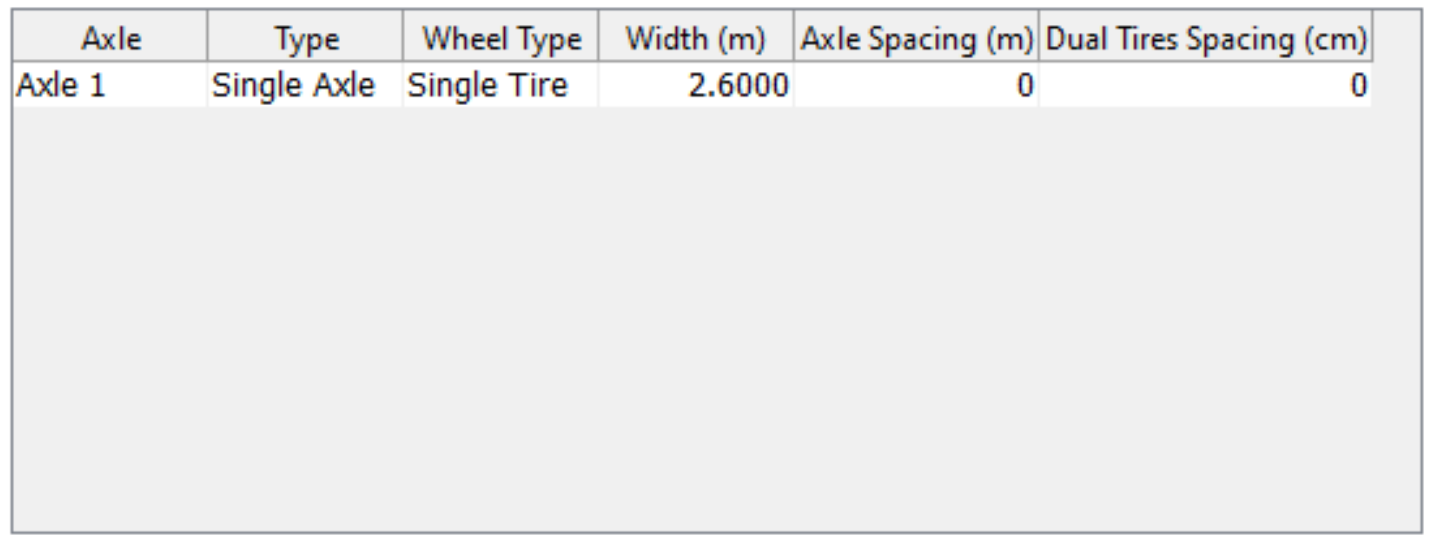

Tire Pressure (KPa) 827.37

Contact Area Shape Rectangular $\vee \quad$ Aspect Ratio (length/width) 1.5714

Shear Traction $\quad 0.0$

OK

Cancel

Figure 359: Traffic Data for Run 3.11 


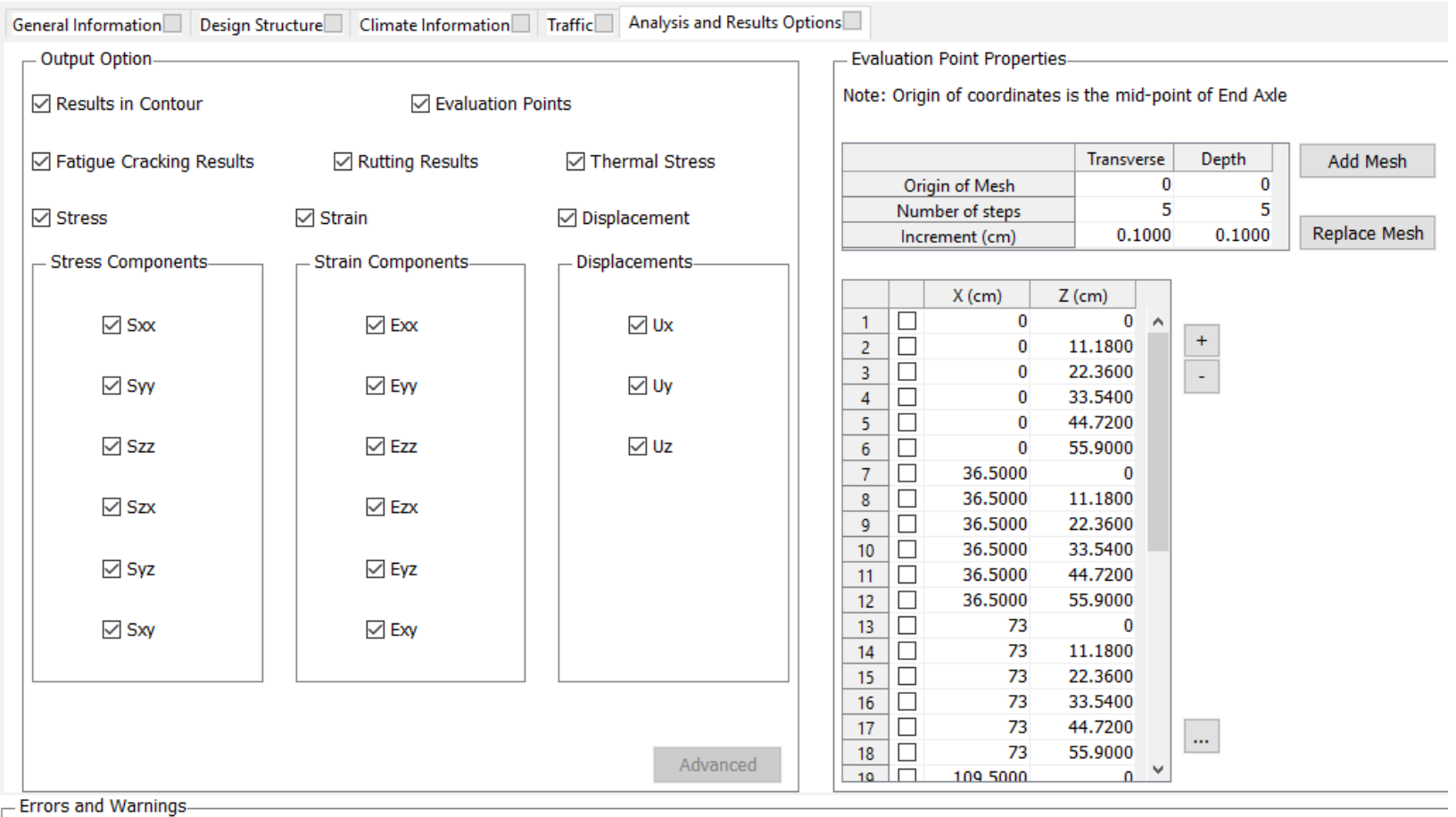

Figure 360: Output and Analysis Options for Run 3.11

\section{Run 3.12}

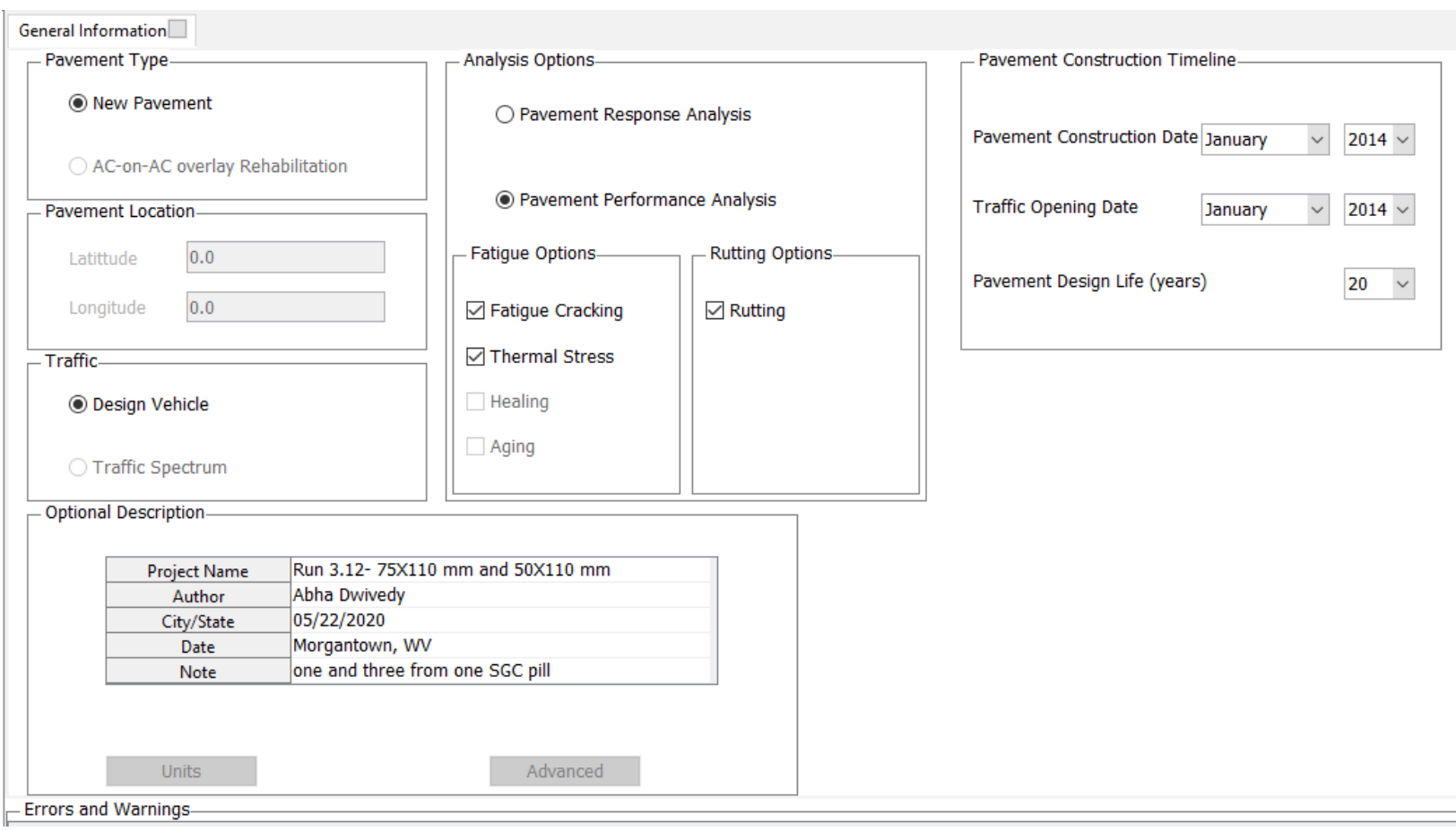

Figure 361: General Information for Run 3.12 


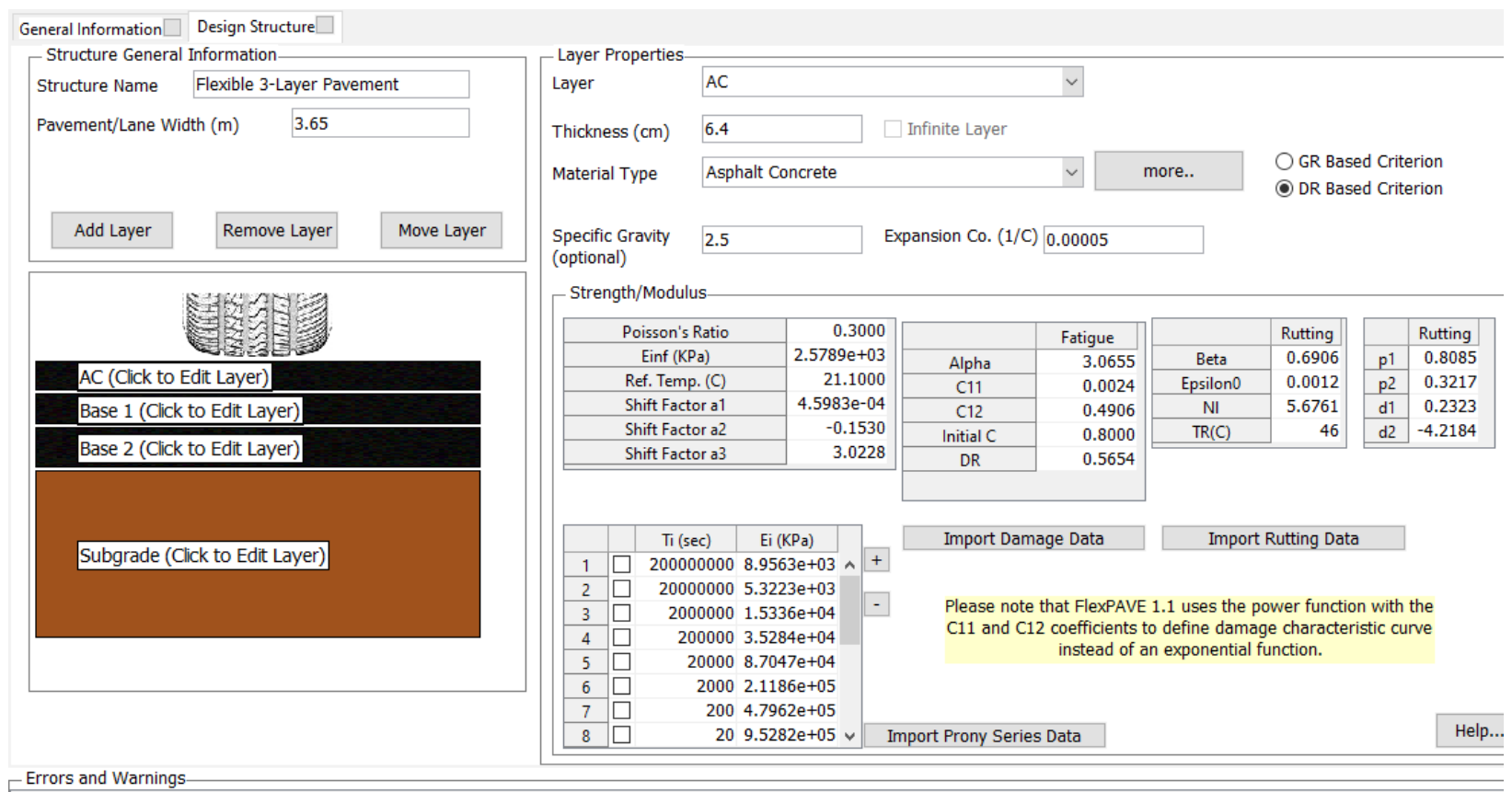

Figure 362: Design Structure of AC Layer for Run 3.12

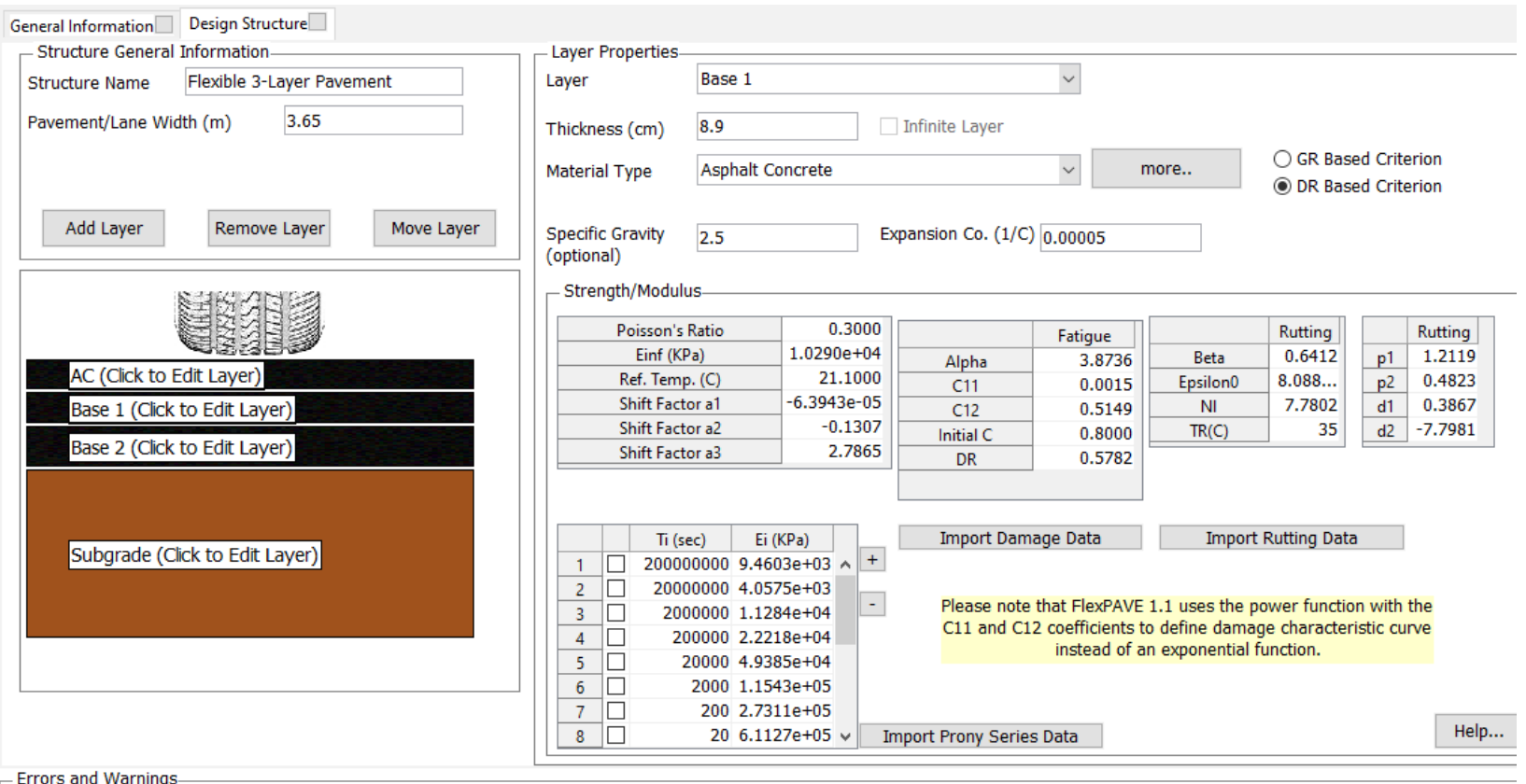

Figure 363: Design Structure of Base 1 Layer for Run 3.12 

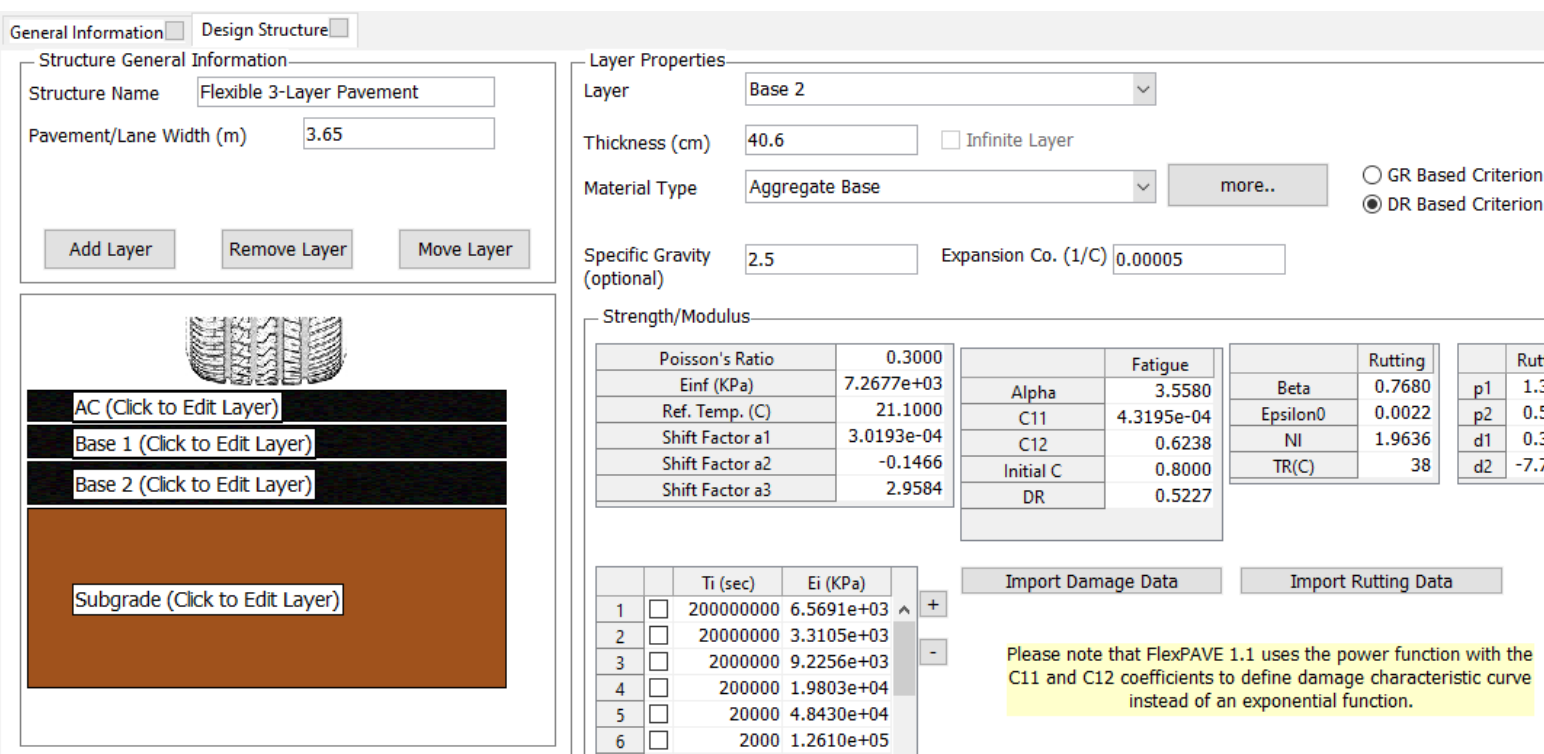

Alpha
C11
C12
Initial C
DR

\begin{tabular}{r|}
\multicolumn{1}{|c|}{ Fatigue } \\
3.5580 \\
$4.3195 \mathrm{e}-04$ \\
0.6238 \\
0.8000 \\
0.5227
\end{tabular}

\section{Ti $(\mathrm{sec}) \quad \mathrm{Ei}(\mathrm{KPa})$}

$2000000006.5691 \mathrm{e}+03 \wedge+$

$200000003.3105 \mathrm{e}+03$

$20000009.2256 \mathrm{e}+03$

$2000001.9803 \mathrm{e}+04$

$200004.8430 \mathrm{e}+04$

$2000 \quad 1.2610 \mathrm{e}+05$

$2003.2966 \mathrm{e}+05$

\begin{tabular}{l|l}
$207.8916 \mathrm{e}+05 \checkmark$ Import Prony Series Data \\
\hline
\end{tabular}

Errors and Warning

\section{Figure 364: Design Structure of Base 2 Layer for Run 3.12}

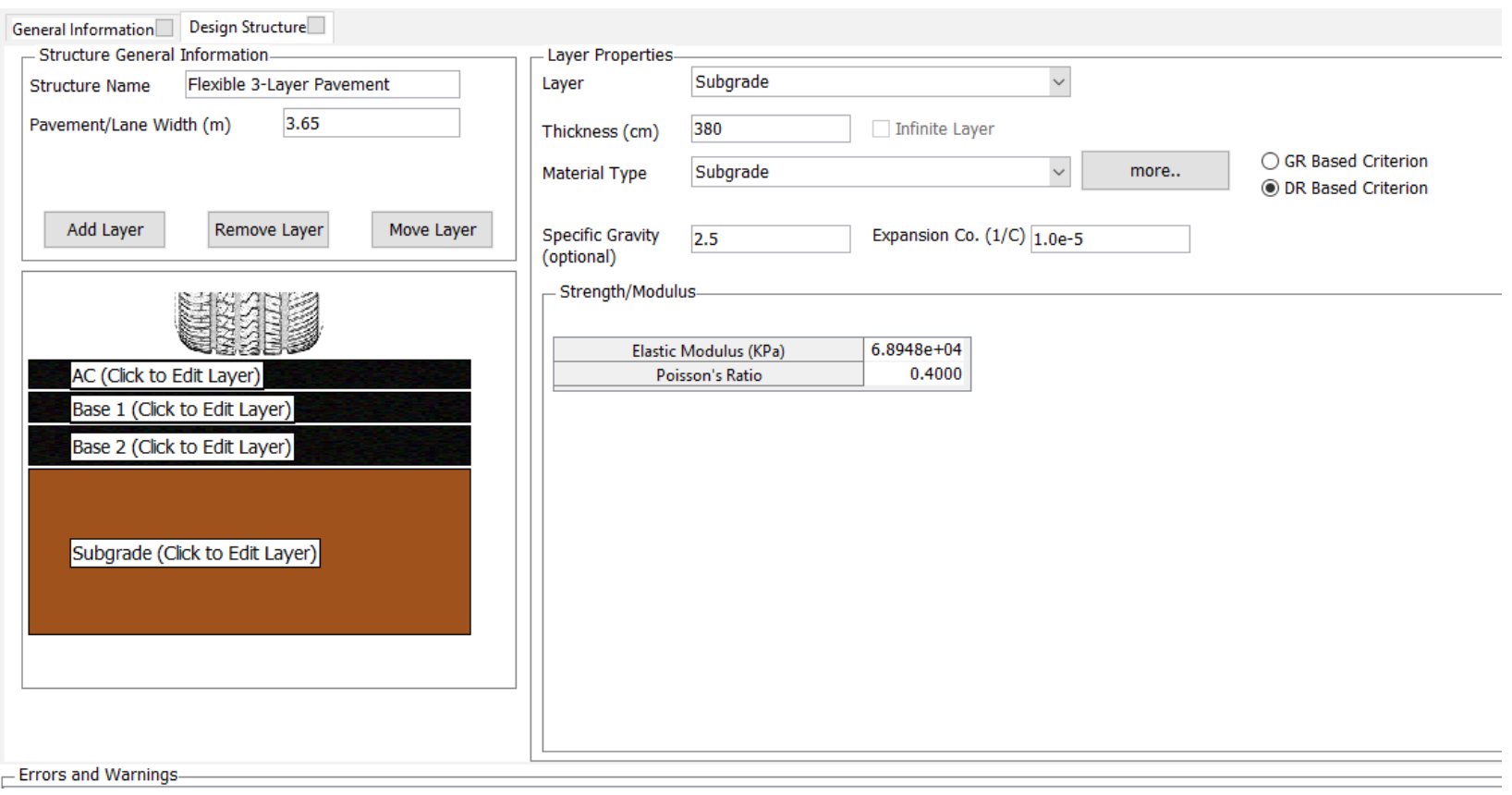

Figure 365: Design Structure of Subgrade Layer for Run 3.12 


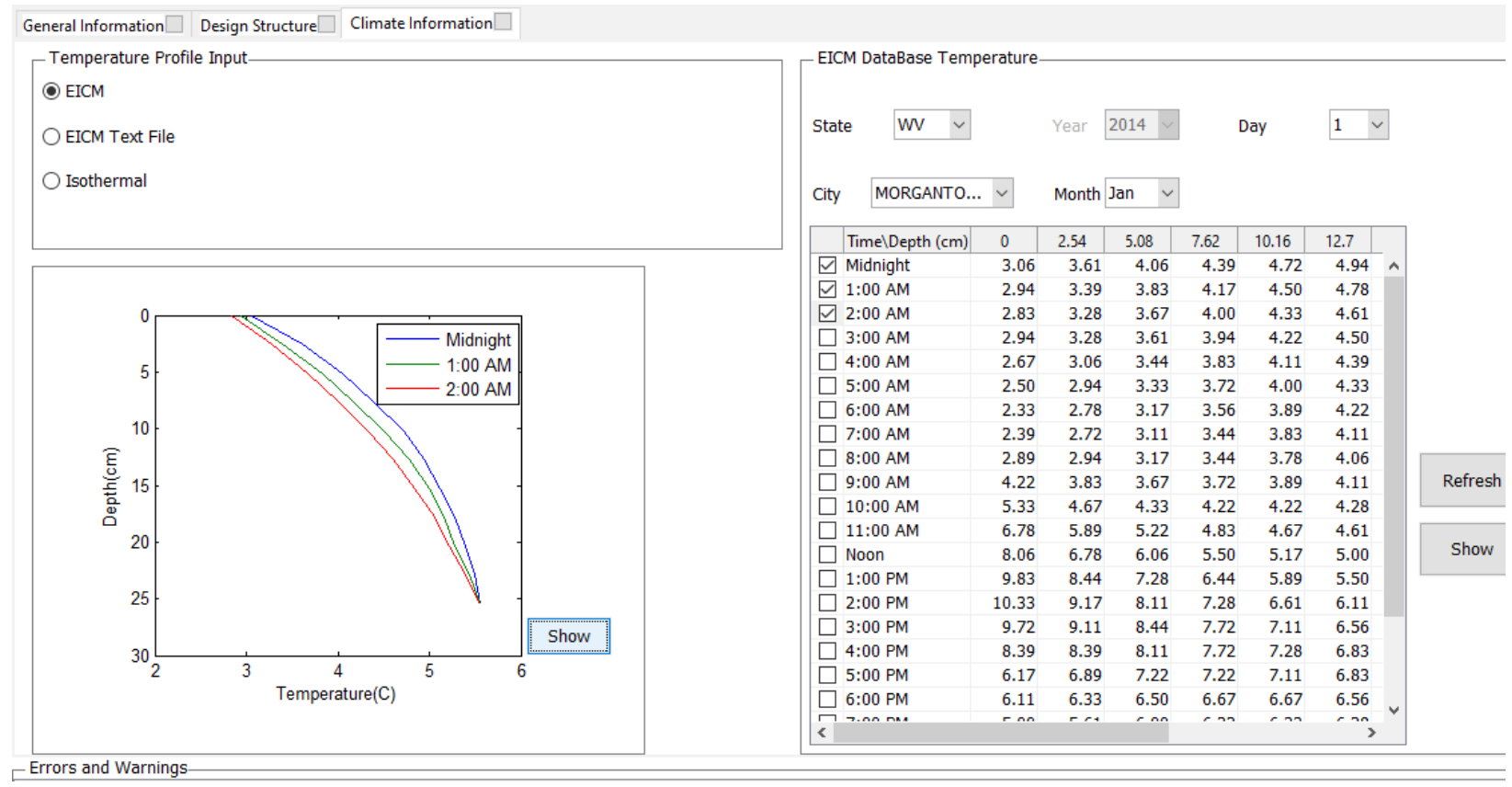

Figure 366: Climate Data for Run 3.12 


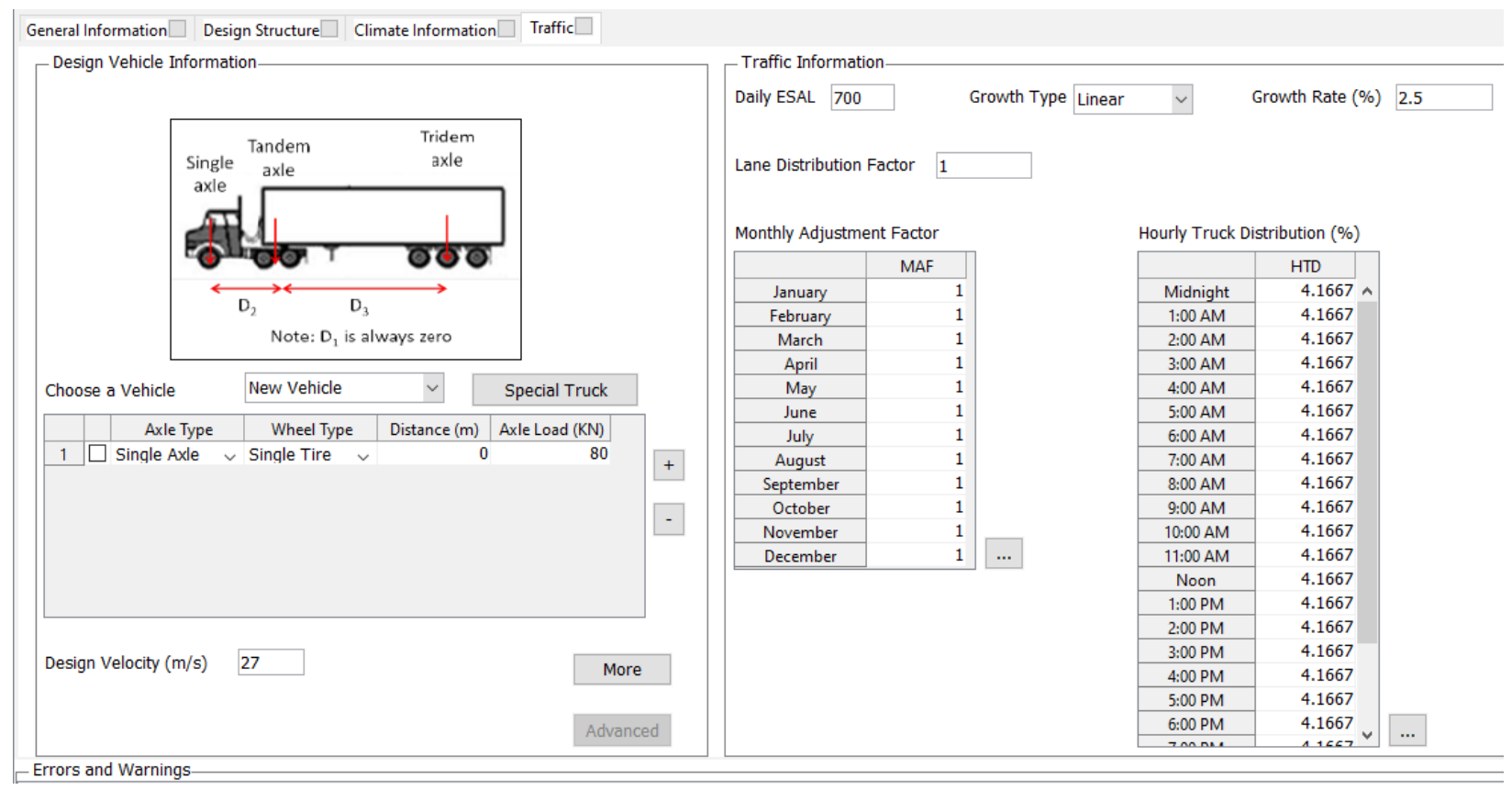

Axle Configuration

$-\square \times$

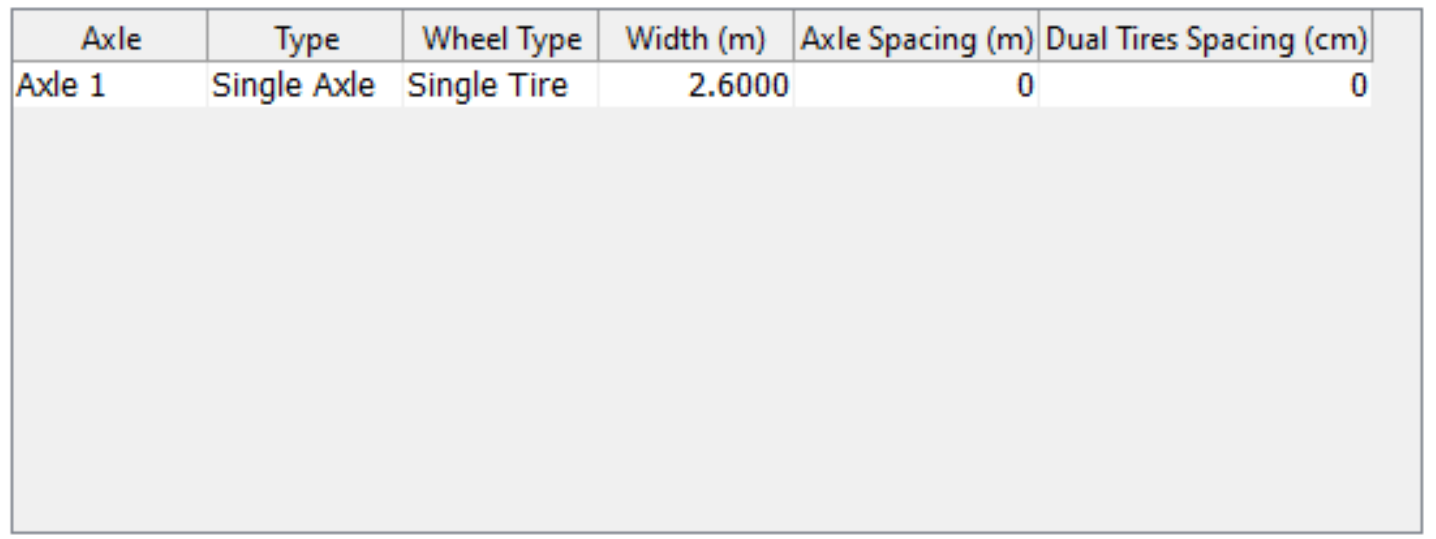

Tire Pressure (KPa) 827.37

Contact Area Shape Rectangular $\vee \quad$ Aspect Ratio (length/width) 1.5714

Shear Traction $\quad 0.0$

OK

Cancel

Figure 367: Traffic Data for Run 3.12 


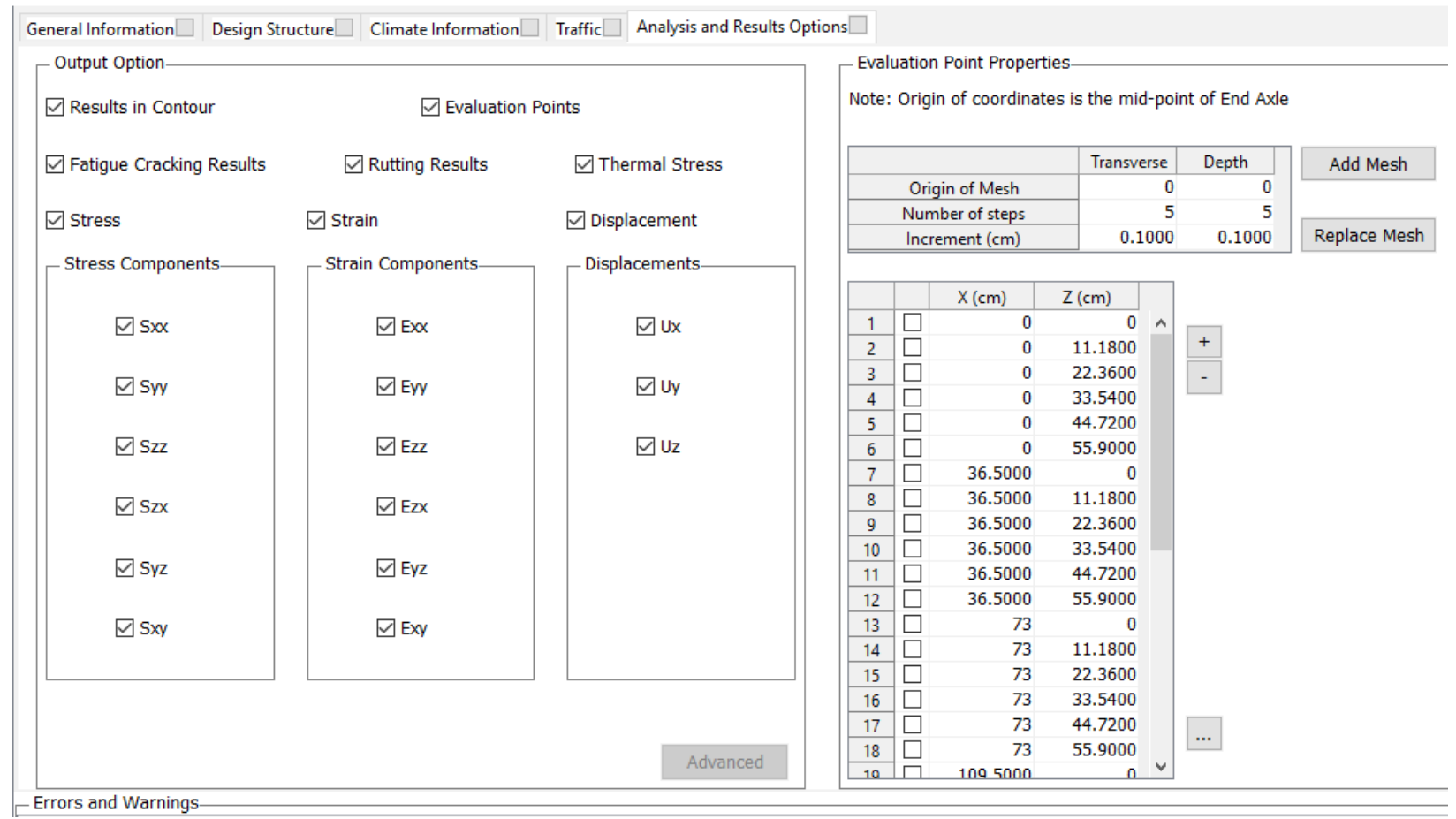

Figure 368: Output and Analysis Options for Run 3.12 